\title{
Entwurf robuster modellbasierter Fehlerisolationsfilter
}

Vom Fachbereich

Elektrotechnik und Informationstechnik der Technischen Universität Darmstadt zur Erlangung des akademischen Grades eines Doktor-Ingenieurs (Dr.-Ing.) genehmigte Dissertation

VOn

\section{Dipl.-Ing. Arne Wahrburg}

geboren am 02. März 1985 in Lüdenscheid

Referent: Prof. Dr.-Ing. J. Adamy

Korreferent: Prof. Dr.-Ing. habil. B. Lohmann Tag der Einreichung: 30. August 2013

Tag der mündlichen Prüfung: 12. Dezember 2013 



\section{Vorwort}

Die vorliegende Dissertation entstand in meiner Zeit als wissenschaftlicher Mitarbeiter am Fachgebiet Regelungstheorie und Robotik an der TU Darmstadt von Mai 2010 bis September 2013.

Bei meinem Doktorvater, Herrn Prof. Adamy, bedanke ich mich herzlich für die Betreuung der Arbeit und seine Unterstützung. Er hat mir in meiner Zeit als Doktorand die Möglichkeit gegeben, meine wissenschaftlichen Ideen in einer hervorragenden Arbeitsatmosphäre mit großem Freiraum zu verfolgen und zu entwickeln.

Herrn Prof. Lohmann danke ich für die freundliche Übernahme des Korreferats sowie seine Anmerkungen und Anregungen zur Arbeit.

Den überwiegenden Teil meiner Promotionszeit wurde ich durch ein Stipendium der Deutsche Telekom Stiftung gefördert. Dies umfasste nicht nur finanzielle Unterstützung, sondern insbesondere auch vielfältige Seminare und den Austausch mit anderen Stipendiaten. Für diese wertvolle Förderung möchte ich mich herzlich bedanken.

Ebenfalls sehr zum Gelingen der Arbeit beigetragen hat die einmalige Atmosphäre in der Kaffeeküche. In ihr wurde nicht nur die vielmehr freundschaftliche als kollegiale Stimmung gepflegt, sondern auch so manches fachliche Problem diskutiert, gelöst oder überhaupt erst angeregt. Allen Kolleginnen und Kollegen hierfür ein herzliches Dankeschön.

Ganz besonders gilt dies für Stefan Gering, Jochen Grieser, Dominik Haumann, Klaus Kefferpütz und Dieter Lens, die viel Zeit und Engagement für die kritische Durchsicht der Arbeit und zahlreiche inhaltliche Diskussionen und wertvolle Anregungen aufbrachten.

Nicht zuletzt gebührt mein ganz besonderer Dank meiner Familie. Meine Eltern haben mir nicht nur das Studium und damit den Weg zur Promotion ermöglicht, sondern standen und stehen mir stets als Ratgeber zur Seite. Ebenso bedanke ich mich ganz herzlich bei Tina, die mir ein großer Rückhalt ist und mir immer wieder Motivation und Zuversicht gibt. 
Für meine Eltern

und in liebevollem Gedenken an meine Schwester. 


\section{Inhaltsverzeichnis}

Abkürzungen und Symbole $\quad$ X

$\begin{array}{ll}\text { Verzeichnis der Beispielsysteme } & \text { XVII }\end{array}$

$\begin{array}{ll}\text { Kurzfassung } & \text { XIX }\end{array}$

1 Einleitung 1

1.1 Ziele und Beiträge der Arbeit . . . . . . . . . . . . 3

1.2 Aufbau der Arbeit . . . . . . . . . . . . . 3

2 Modellbasierte Fehlerdiagnose: ein Überblick $\quad 7$

2.1 Grundbegriffe . . . . . . . . . . . . . . 8

2.1.1 Typen von Fehlern . . . . . . . . . . . . . 99

2.1.2 Teilaufgaben der Fehlerdiagnose . . . . . . . . 15

2.2 Grundkonzepte der Fehlerdiagnose . . . . . . . . . . 17

2.2.1 Residuenerzeugung . . . . . . . . . . . . . 18

2.2 .2 Residuenauswertung . . . . . . . . . . . . 22

2.3 Ziele der modellbasierten Fehlerisolation . . . . . . . . . . 22

2.4 Ansätze zur modellbasierten Fehlerdiagnose . . . . . . . . . 31

2.5 Existierende Ansätze für modellbasierte Fehlerisolationsfilter 35

2.5.1 Fehlerisolation durch Rückführung auf Detektionsprobleme .................. 35

2.5.2 Fehlerisolation durch Lösung von Identifikationsproblemen . . . . . . . . . . . . . . 4 41

2.5.3 Direkte Ansätze zur Fehlerisolation . . . . . . . . . 43

3 Mathematische und regelungstechnische Grundlagen $\quad 45$

3.1 Signal- und Systemnormen _. . . . . . . . . . . 45

3.2 Lineare Matrixungleichungen . . . . . . . . . . . . . . . . 49

3.2 .1 Rechenregeln für LMIs . . . . . . . . . . . . . . 52

3.2 .2 Spezielle LMIs . . . . . . . . . . . . . . . . . . 54

3.3 Nullstellen linearer Mehrgrößensysteme . . . . . . . . . . 60

3.4 Lineare Entkopplungsregler . . . . . . . . . . . . . 66 


\section{Entwurf von Fehlerisolationsbeobachtern für nominale} Systeme

4.1 Problemstellung. . . . . . . . . . . . . . . . . . . . . . . . 69

4.2 Stand der Technik . . . . . . . . . . . . . 72

4.3 Beiträge dieses Kapitels . . . . . . . . . . . . . . . . . 74

4.4 Dualität zu Entkopplungsregelungen . . . . . . . . . 75

4.5 Vollständige Fehlerisolation in quadratischen Systemen . . . 78

4.5.1 Entwurf im Zeitbereich . . . . . . . . . . . . 80

4.5.2 Entwurf mittels Vollständiger Modaler Synthese . . 85

4.5.3 Beispiel CE 150 Modellhubschrauber . . . . . . . . 95

4.5.4 Fazit . . . . . . . . . . . . . . . 97

4.6 Erweiterte Dynamik der Diagonalelemente . . . . . . . . . 98

4.6.1 Idee des Verfahrens . . . . . . . . . . . . . . . . 98

4.6.2 Dualität zwischen dynamischen Entkopplungsrege-

lungen und dynamischen Fehlerisolationsbeobachtern 100

4.6.3 Entwurf im Laplace-Bereich . . . . . . . . . . . . . . 104

4.6.4 Entwurf im Zeitbereich . . . . . . . . . . . . . . 105

4.6.5 Beispiel CE 150 Modellhubschrauber . . . . . . . . 108

4.6 .6 Fazit . . . . . . . . . . . . . . . . . 109

4.7 Vollständige Fehlerisolation für statisch nicht isolierbare

Systeme . . . . . . . . . . . . . . . . 109

4.7.1 Entwurf mittels virtueller Systemerweiterungen . . . 110

4.7.2 Realisierung mithilfe dynamisch erweiterter Beobachter . . . . . . . . . . . . . 119

4.7.3 Zusammenfassung des Entwurfsverfahrens . . . . . . 121

4.7.4 Alternatives Entwurfsverfahren . . . . . . . . . . . 124

4.7.5 Beispiel DHC-2 Beaver . . . . . . . . . . . . . . . 124

4.7 .6 Fazit . . . . . . . . . . . . . . . . 127

4.8 Vollständige Fehlerisolation für nicht minimalphasige Systeme 127

4.8.1 Entwurf mittels virtueller Systemerweiterungen . . . 128

4.8.2 Realisierung mithilfe dynamisch erweiterter Beobachter . . . . . . . . . . . . 135

4.8.3 Zusammenfassung des Entwurfsverfahrens . . . . . . 139

4.8.4 Beispiel Verladebrücke . . . . . . . . . . . . . . . . 142

4.8.5 Beispiel DHC-2 Beaver . . . . . . . . . . . . . . 146

4.8 .6 Fazit . . . . . . . . . . . . . . . . . . 148

4.9 Vollständige Fehlerisolation in nichtquadratischen Systemen 149

4.9.1 Entwurf im Zeitbereich . . . . . . . . . . . . 150

4.9.2 Entwurf mittels Vollständiger Modaler Synthese . . 154

4.9.3 Beispiel Hydrauliksystem . . . . . . . . . . . . 161 
4.9.4 Dualität zur Control Allocation . . . . . . . . . . 162

4.9.5 Entwurf für erweiterte Diagonalelemente mittels Vollständiger Modaler Synthese . . . . . . . . . . 165

4.9 .6 Fazit . . . . . . . . . . . . . . . 170

4.10 Partielle Fehlerisolation . . . . . . . . . . . 170

4.10.1 Eine alternative Formulierung des Entwurfsverfahrens für FFIOs . . . . . . . . . . . . . . 172

4.10.2 Entwurf vollständiger Beobachter zur teilweisen Fehlerisolation . . . . . . . . . . . . 174

4.10 .3 Beispiel Verladebrücke . . . . . . . . . . . . . . . 181

4.10 .4 Fazit . . . . . . . . . . . . . . . . 183

4.11 Zusammenfassung des Kapitels . . . . . . . . . . . . 183

5 Optimierung der Robustheit von Fehlerisolationsbeobachtern

5.1 Stand der Technik . . . . . . . . . . . . . 186

5.2 Beiträge dieses Kapitels . . . . . . . . . . . . . . . . 188

5.3 Steigerung der Robustheit in quadratischen Systemen . . . 190

5.3.1 Optimierung durch Anpassung der vorgegebenen Residuendynamik . . . . . . . . . . . . 190

5.3.2 Optimierung durch Relaxierung der strukturellen Anforderungen . . . . . . . . . . . 200

5.3.3 Unterdrückung hochfrequenter Störungen . . . . . . 208

5.3 .4 Fazit . . . . . . . . . . . . . . . . 213

5.4 Steigerung der Robustheit in nichtquadratischen Systemen . 213

5.4.1 Robustheit bezüglich beliebiger exogener Störungen 214

5.4.2 Erweiterung durch Nutzung aller Freiheitsgrade . . . 219

5.4.3 Robustheit bezüglich exogener Störungen in beschränkten Frequenzbereichen . . . . . . . . . . . . 224

5.4.4 Robustheit bezüglich unsicherer Systemparameter . 242

5.4 .5 Fazit . . . . . . . . . . . . . . 260

5.5 Steigerung der Robustheit bei partieller Fehlerisolation . . . 262

5.5.1 Abhängigkeit der FIO-Parametrierung von den Kopplungszahlen . . . . . . . . . . . . . . 263

5.5.2 Entwurfsverfahren bei affiner Abhängigkeit der Beobachtermatrix von den dynamischen Kopplungszahlen . . . . . . . . . . . . . . 271

5.5.3 Entwurfsverfahren auf Basis der Vollständigen Modalen Synthese . . . . . . . . . . . . . 275

5.5.4 Akademisches Beispiel . . . . . . . . . . . . . 277 
5.5.5 Beispiel Verladebrücke . . . . . . . . . . . . 280

5.5 .6 Fazit . . . . . . . . . . . . . . . . 283

5.6 Zusammenfassung des Kapitels . . . . . . . . . . . 283

6 Allgemeine Fehlerisolationsfilter $\mathbf{2 8 5}$

6.1 Stand der Technik und Beiträge dieses Kapitels . . . . . . . 286

6.2 Grundidee des Entwurfs . . . . . . . . . . . . . . . 288

6.3 Wahl eines zulässigen Referenzmodells . . . . . . . . . . 290

6.4 Lösung des Entwurfsproblems bei nominaler Systemdynamik 296

6.4.1 Linearisierende Variablentransformation . . . . . . . 299

6.4.2 Fehlerisolationsfilter reduzierter Ordnung . . . . . . 313

6.4 .3 Beispiele . . . . . . . . . . . . . . 317

6.4.4 Fazit . . . . . . . . . . . . . . 320

6.5 Robustheitsaspekte beim Entwurf von Fehlerisolationsfiltern 320

6.5.1 Robuste Fehlerisolationsfilter bei nominaler Systemdynamik . . . . . . . . . . . . . . 320

6.5.2 Robuste Fehlerisolationsfilter für unsichere Systeme 329

6.5.3 Fazit . . . . . . . . . . . . . . . . 337

6.6 Zusammenfassung des Kapitels . . . . . . . . . . . . . 338

$\begin{array}{lll}7 & \text { Zusammenfassung und Ausblick } & 340\end{array}$

A Mathematische Sätze und Lemmata 344

B Dynamische Entkopplungsregler für lineare Systeme $\quad 356$

B.1 Entwurf im Laplace-Bereich . . . . . . . . . . . . . . . 358

B.2 Entwurf im Zeitbereich . . . . . . . . . . . . . . . 361

C Parameter der Beispielsysteme $\quad \mathbf{3 7 0}$

C.1 Beispiele zum Entwurf von FIOs für nominale Systeme . . . 370

C.1.1 Quadratisches System, CE 150 Modellhubschrauber 370

C.1.2 Erweiterte Diagonalelemente, CE 150 Modellhubschrauber . . . . . . . . . . . . 370

C.1.3 Statisch nicht isolierbares System, DHC-2 Beaver . . 371

C.1.4 Nicht minimalphasiges System, Verladebrücke . . . . 371

C.1.5 Doppelt erweitertes System, DHC-2 Beaver . . . . . 371

C.1.6 Nichtquadratisches System, Hydraulik . . . . . . . . 371

C.1.7 Teilweise Fehlerisolation, Verladebrücke . . . . . . 372

C.2 Beispiele zum Entwurf robuster FIOs . . . . . . . . . . . 373

C.2.1 Quadratisches System, CE 150 Modellhubschrauber 373 
C.2.2 Quadratisches System, Verladebrücke . . . . . . . 373

C.2.3 Nichtquadratisches System, Hydraulik . . . . . . . . 374

C.2.4 Nichtquadratisches System, Hydraulik (beschränkter Frequenzbereich) . . . . . . . . . . . 374

C.2.5 Nichtquadratisches System, akademisches Beispiel (unsichere Parameter) . . . . . . . . . . . 375

C.2.6 Partielle Fehlerisolation . . . . . . . . . . . . . . 375

C.3 Beispiele zum Entwurf von FIFs . . . . . . . . . 376

C.3.1 FIFs für nominale Systeme . . . . . . . . . . 376

C.3.2 Robustheit von FIFs bezüglich exogener Störungen . 379

C.3.3 Robustheit von FIFs bezüglich unsicherer Parameter . 380

D Veröffentlichungen und studentische Arbeiten 381

$\begin{array}{ll}\text { Literaturverzeichnis } & 382\end{array}$ 


\section{Abkürzungen und Symbole}

\section{Abkürzungen}

BMI

Bilinear Matrix Inequality, bilineare Matrixungleichung

BRL Bounded-Real Lemma

DFIO

Dynamically extended Fault Isolation Observer, dynamisch erweiterter Fehlerisolationsbeobachter

FFI

FFIO

Full Fault Isolation, vollständige Fehlerisolation Full Fault Isolation Observer, Beobachter zur vollständigen Fehlerisolation

FIF

Fault Isolation Filter, Fehlerisolationsfilter

FIO

Fault Isolation Observer, Fehlerisolationsbeobachter

GKYP

Generalized Kalman-Yakubovich-Popov Lemma, verallgemeinertes Kalman-Yakubovich-PopovLemma

LMI

MIMO

Linear Matrix Inequality, lineare Matrixungleichung

Multiple Input, Multiple Output, Mehrgrößensystem

$\mathrm{NMPH}$ nicht minimalphasig

PFI

PFIO

Partial Fault Isolation, teilweise Fehlerisolation Partial Fault Isolation Observer, Beobachter zur teilweisen Fehlerisolation

SISO

SNI

Single Input, Single Output, Eingrößensystem

SVO

UIO statisch nicht (fehler-) isolierbar

Set-Valued Observer, mengenbasierter Beobachter

Unknown Input Observer, Beobachter für Systeme mit unbekannten Eingängen 


\section{Allgemeine Notation}

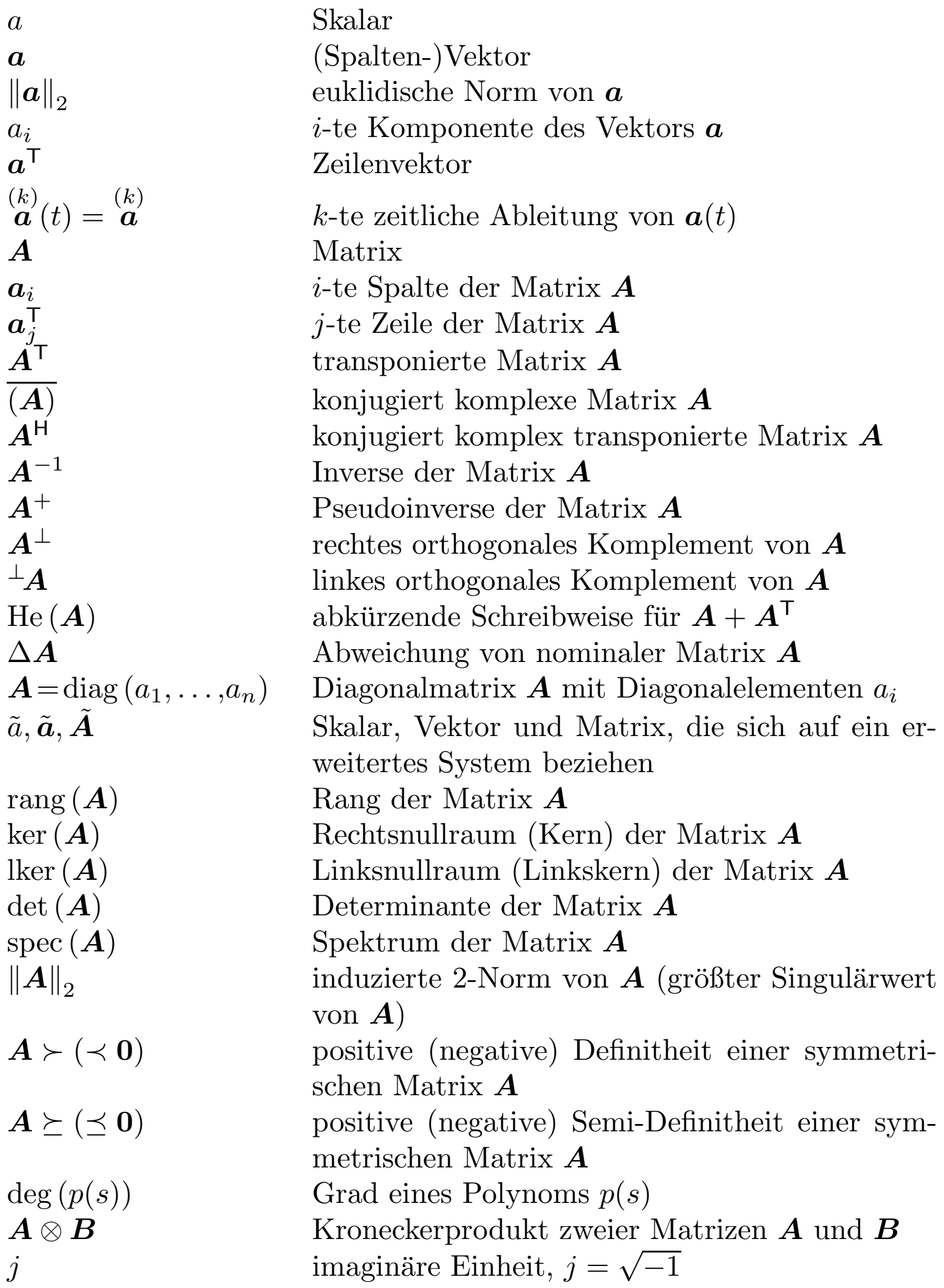




\begin{tabular}{|c|c|}
\hline 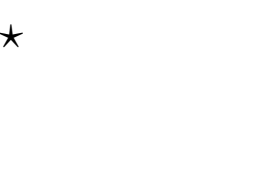 & $\begin{array}{l}\text { Platzhalter für Elemente beziehungsweise } \\
\text { Blöcke, die sich aus der Symmetrie von Matri- } \\
\text { zen ergeben }\end{array}$ \\
\hline $\boldsymbol{I}_{n}$ & Einheitsmatrix des Dimension $n$ \\
\hline $\mathbf{0}_{n \times m}$ & $n \times m$-dimensionale Nullmatrix \\
\hline$\phi_{i}$ & Einheitsvektor mit $i$-tem Element gleich 1 \\
\hline $\boldsymbol{G}_{\boldsymbol{u y}}(s)$ & $\begin{array}{l}\text { Übertragungsmatrix zwischen Eingängen } \boldsymbol{u} \text { und } \\
\text { Ausgängen } \boldsymbol{u}\end{array}$ \\
\hline$g_{i, j}(s)$ & $\begin{array}{l}\text { Element in der } i \text {-ten Zeile und } j \text {-ten Spalte einer } \\
\text { Übertragungsmatrix } \boldsymbol{G}(s)\end{array}$ \\
\hline$\left\|\boldsymbol{G}_{\boldsymbol{u y} \boldsymbol{y}}(s)\right\|_{\infty}$ & $\mathcal{H}_{\infty}$-Norm der Übertragungsmatrix $\boldsymbol{G}_{\boldsymbol{u} \boldsymbol{y}}(s)$ \\
\hline$\left\|\boldsymbol{G}_{\boldsymbol{u y}}(s)\right\|_{\infty}^{\Omega}$ & $\begin{array}{l}\mathcal{H}_{\infty} \text {-Norm der Übertragungsmatrix } \boldsymbol{G}_{\boldsymbol{u y}}(s) \text { im } \\
\text { Frequenzbereich } \Omega\end{array}$ \\
\hline$\left\|\boldsymbol{G}_{\boldsymbol{u} \boldsymbol{y}}(s)\right\|_{1}$ & $\mathcal{H}_{-}$-Index der Übertragungsmatrix $\boldsymbol{G}_{u y}(s)$ \\
\hline$\left\|\boldsymbol{G}_{\boldsymbol{u} \boldsymbol{y}}(s)\right\|_{-}^{\Omega}$ & $\begin{array}{l}\mathcal{H}_{-}-\text {Index der Übertragungsmatrix } \boldsymbol{G}_{u \boldsymbol{u}}(s) \text { im } \\
\text { Frequenzbereich } \Omega\end{array}$ \\
\hline$\lambda$ & Eigenwert einer Matrix \\
\hline$\sigma$ & Singulärwert einer Matrix \\
\hline $\bar{\sigma}$ & größter Singulärwert einer Matrix \\
\hline$\underline{\sigma}$ & kleinster Singulärwert einer Matrix \\
\hline & (invariante) Nullstelle eines Systems \\
\hline$\|\boldsymbol{u}(t)\|_{L}$ & $\mathcal{L}_{2}$-Norm eines Signals $\boldsymbol{u}(t)$ \\
\hline$(\boldsymbol{A}, \boldsymbol{B}, \boldsymbol{C}, \boldsymbol{D})$ & Abkürzung für $\boldsymbol{G}(s)=\boldsymbol{C}(s \boldsymbol{I}-\boldsymbol{A})^{-1} \boldsymbol{B}+\boldsymbol{D}$ \\
\hline$(A, B, C)$ & Abkürzung für $\boldsymbol{G}(s)=\boldsymbol{C}(s \boldsymbol{I}-\boldsymbol{A})^{-1} \boldsymbol{B}$ \\
\hline C & Menge der komplexen Zahlen \\
\hline $\mathbb{N}$ & Menge der natürlichen Zahlen \\
\hline $\mathbb{R}$ & Menge der reellen Zahlen \\
\hline
\end{tabular}

\section{Symbole}

Es werden lediglich diejenigen Symbole aufgeführt, die übergreifend in der gesamten Arbeit Anwendung finden. Abweichende Bedeutungen werden in den jeweiligen Abschnitten erläutert.
$k_{d, i j l}$
dynamische Kopplungszahl (partielle Fehleriso- lation, $j$-ter Pol der $i$-ten Spalte wird in $l$-te Spalte gekoppelt)
$k_{s, i j}$
statische Kopplungszahl (partielle Fehlerisola- tion, Verkopplung in $i$-ter Zeile und $j$-ter Spalte) 


$\begin{array}{ll}n & \text { Dimension des Zustandsvektors } \\ n_{d} & \text { Dimension des Störvektors } \\ n_{f} & \text { Dimension des Fehlervektors } \\ n_{k_{d}} & \text { Anzahl der dynamischen Kopplungszahlen (par- } \\ & \text { tielle Fehlerisolation) } \\ n_{k_{s}} & \text { Anzahl der statischen Kopplungszahlen (partiel- } \\ & \text { le Fehlerisolation) } \\ n_{u} & \text { Dimension des Eingangsvektors } \\ n_{y} & \text { Dimension des Ausgangsvektors } \\ n_{\zeta} & \text { Dimension der internen Beobachterdynamik } \\ n_{\text {filt }} & \text { Dimension der Filterdynamik } \\ n_{\text {ref }} & \text { Dimension des Referenzmodells } \\ q_{i, j} & \text { Nennerkoeffizient einer Übertragungsfunktion } \\ z_{i, j} & \text { Zählerkoeffizient einer Übertragungsfunktion } \\ N & \text { Anzahl der Eckpunkte eines Polytops } \\ \boldsymbol{d}_{\boldsymbol{f}} & \text { Störvektor } \\ \boldsymbol{k}_{d} & \text { Fehlervektor } \\ & \text { Vektor der dynamischen Kopplungszahlen (par- } \\ \boldsymbol{k}_{s} & \text { tielle Fehlerisolation) } \\ & \text { Vektor der statischen Kopplungszahlen (partiel- } \\ \boldsymbol{p}_{i}^{\top} & \text { le Fehlerisolation) } \\ \boldsymbol{r} & \text { Parametervektor zum Linkseigenvektor } \boldsymbol{w}_{B_{i}}^{\top} \\ \boldsymbol{u} & \text { Residuenvektor } \\ \boldsymbol{v}_{B_{i j}} & \text { Eingangsvektor } \\ \boldsymbol{w}_{B_{i j}}^{\top} & \text { Rechtseigenvektor des Beobachters zum Eigen- } \\ \tilde{\boldsymbol{w}}_{B_{i j}, l}^{\top} & \text { wert } \lambda_{B_{i j}} \\ & \text { Linkseigenvektor des Beobachters zum Eigen- } \\ \boldsymbol{w}_{B_{i j}, l, b}^{\top} & \text { wert } \lambda_{B_{i j}} \\ & \text { Linkseigenvektor des Gesamtsystems aus Stre- } \\ & \text { cke und Beobachter zum Eigenwert } \lambda_{B_{i j}} \text { im Eck- } \\ \boldsymbol{w}_{B_{i j}, l, s}^{\top} & \text { punkt } l \\ & \text { mit den Beobachterzuständen korrespondieren- } \\ & \text { der Teil des Linkseigenvektors des Gesamtsys- } \\ & \text { tems aus Strecke und Beobachter zum Eigenwert } \\ & \lambda_{B_{i j}} \text { im Eckpunkt } l \\ & \text { } \text { it den Streckenzuständen korrespondierender } \\ & \text { aus Strecke und Beobachter zum Eigenwert } \lambda_{B_{i j}} \\ & \\ & \text { im Eckpunt } l \\ & \\ & \end{array}$




$$
\begin{aligned}
& \boldsymbol{x} \\
& \hat{\boldsymbol{x}} \\
& \boldsymbol{x}_{f} \\
& \boldsymbol{x}_{\mathrm{ref}} \\
& \boldsymbol{y} \\
& \boldsymbol{z}_{i j}^{\top}
\end{aligned}
$$

\section{A}

$\boldsymbol{A}_{f}$

$\boldsymbol{A}_{\mathrm{ref}}$

$B$

$\boldsymbol{B}_{d}$

$\boldsymbol{B}_{f, u}$

$\boldsymbol{B}_{f, y}$

$\boldsymbol{B}_{\mathrm{ref}, d}$

$\boldsymbol{B}_{\mathrm{ref}, f}$

$\boldsymbol{B}_{\mathrm{ref}, u}$

$C$

$C_{f}$

$C_{\text {ref }}$

$D$

$\boldsymbol{D}_{d}$

$\boldsymbol{D}_{f, u}$

$\boldsymbol{D}_{f, y}$

$D_{\text {ref, } d}$

$D_{\text {ref, } f}$

$\boldsymbol{D}_{\text {ref }, u}$

$D^{*}$

$\boldsymbol{E}_{a}$

$\boldsymbol{E}_{s}$

F
Zustandsvektor

Schätzvektor (Zustandsvektor des Beobachters)

interner Filterzustand

Zustandsvektor des Referenzmodells

Ausgangsvektor

zusätzliche Freiheitsgrade bezüglich des Linkseigenvektors $\boldsymbol{w}_{B_{i j}}^{\top}$ in nichtquadratischen Systemen Systemmatrix

Dynamikmatrix eines allgemeinen Filters

Dynamikmatrix des Referenzmodells

Eingangsmatrix

Störeingangsmatrix (Systemdynamik)

Wirkung der Stellgrößen auf die Filterdynamik

Wirkung der Ausgangsgrößen auf die Filterdynamik

Wirkung der Störgrößen auf die Dynamik des Referenzmodells

Wirkung der Fehler auf die Dynamik des Referenzmodells

Wirkung der Stellgrößen auf die Dynamik des Referenzmodells

Ausgangsmatrix

Ausgangsmatrix eines allgemeinen Filters

Ausgangsmatrix des Referenzmodells

Durchgriffsmatrix

Störeingangsmatrix (Messgleichung)

Wirkung der Stellgrößen auf den Filterausgang

Wirkung der Ausgangsgrößen auf den Filterausgang

Wirkung der Störgrößen auf den Ausgang des Referenzmodells

Wirkung der Fehler auf den Ausgang des Referenzmodells

Wirkung der Stellgrößen auf den Ausgang des Referenzmodells

Fehlerdetektionsmatrix

Aktorfehlermatrix

Sensorfehlermatrix

Vorfiltermatrix, Permutationsmatrix 


\begin{tabular}{|c|c|}
\hline $\boldsymbol{H}$ & $\begin{array}{l}\text { Matrix zu Berechnung von Linearkombinationen } \\
\text { des Ausgangsvektors }\end{array}$ \\
\hline $\boldsymbol{K}$ & Reglermatrix \\
\hline $\boldsymbol{L}$ & Beobachtermatrix \\
\hline $\boldsymbol{L}_{p}$ & $\begin{array}{l}\text { Matrix der proportionalen Rückführung der } \\
\text { Ausgangsdifferenz eines dynamisch erweiterten } \\
\text { Beobachters }\end{array}$ \\
\hline $\boldsymbol{L}_{i}$ & $\begin{array}{l}\text { Matrix zur Rückführung der internen Zustände } \\
\text { eines dynamisch erweiterten Beobachters }\end{array}$ \\
\hline $\boldsymbol{P}$ & Parametervektormatrix \\
\hline$Q_{L}$ & $\begin{array}{l}\text { Matrix der Nennerkoeffizienten bei vollständiger } \\
\text { Fehlerisolation }\end{array}$ \\
\hline$Q_{V}$ & $\begin{array}{l}\text { Matrix der Zählerkoeffizienten bei vollständiger } \\
\text { Fehlerisolation }\end{array}$ \\
\hline $\boldsymbol{R}_{L}$ & $\begin{array}{l}\text { zusätzliche Freiheitsgrade bezüglich der Beob- } \\
\text { achtermatrix }\end{array}$ \\
\hline $\boldsymbol{R}_{V}$ & $\begin{array}{l}\text { zusätzliche Freiheitsgrade bezüglich der Nachfil- } \\
\text { termatrix }\end{array}$ \\
\hline$S$ & Diagonalmatrix \\
\hline $\boldsymbol{V}$ & Nachfiltermatrix \\
\hline$V_{y}$ & $\begin{array}{l}\text { Nachfiltermatrix für die Ausgangsdifferenz bei } \\
\text { dynamisch erweitertem Fehlerisolationsbeobach- } \\
\text { ter }\end{array}$ \\
\hline$V_{\zeta}$ & $\begin{array}{l}\text { Nachfiltermatrix für den internen Beobachter- } \\
\text { zustand bei dynamisch erweitertem Fehlerisola- } \\
\text { tionsbeobachter }\end{array}$ \\
\hline $\boldsymbol{V}_{B}$ & Rechtseigenvektormatrix des Beobachters \\
\hline $\boldsymbol{W}_{B}$ & Linkseigenvektormatrix des Beobachters \\
\hline$Z_{V}$ & $\begin{array}{l}\text { zusätzliche Freiheitsgrade bezüglich der Nachfil- } \\
\text { termatrix (Vollständige Modale Synthese) }\end{array}$ \\
\hline$\gamma$ & Schranke zur Abschätzung einer Norm \\
\hline$\alpha$ & $\begin{array}{l}\text { Dimension der dynamischen Erweiterung (sta- } \\
\text { tisch nicht isolierbares System), obere Schranke } \\
\text { für den Realteil von Eigenwerten }\end{array}$ \\
\hline$\alpha_{d}$ & Skalar zur Gewichtung der Störgrößen \\
\hline$\alpha_{f}$ & Skalar zur Gewichtung der Fehler \\
\hline$\alpha_{u}$ & Skalar zur Gewichtung der Stellgrößen \\
\hline$\delta$ & Gesamtfehlerindex \\
\hline$\delta_{i}$ & Fehlerdetektionsindex \\
\hline
\end{tabular}


$\zeta$

$\kappa$

$\lambda_{B_{i}}$

$\mu$

$\varrho$

$\phi$

$\zeta$

$\xi$

$\chi$

$\Gamma$

$\Theta$

$\boldsymbol{\Pi}(\eta)=\boldsymbol{\Pi}_{\eta}$

$\Phi$

K

$\mathbf{P}$

$\mathcal{L}$

$\mathcal{V}$
Schrittweitenparameter beim Path-FollowingVerfahren

Rangdefizit der Fehlerdetektionsmatrix

Beobachtereigenwert

Anzahl der (invarianten) Nullstellen eines Systems

obere Schranke für den Betrag von Eigenwerten

obere Schranke für den Winkel von Eigenwerten zur negativen reellen Achse

interner Zustandsvektor eines dynamisch erweiterten Beobachters

Beobachterfehler

Vektor zur Beschreibung der konvexen Kombination von Eckpunkten eines Polytops

Eingangsmatrix der internen Dynamik eines dynamisch erweiterten Beobachters

Abkürzung für $\boldsymbol{C}(\boldsymbol{A}-\boldsymbol{L} \boldsymbol{C})^{-1}\left(\boldsymbol{E}_{a}-\boldsymbol{L} \boldsymbol{E}_{s}\right)-\boldsymbol{E}_{s}$ Rosenbrock'sche Systemmatrix an der Stelle $\eta$

Matrix der internen Dynamik eines dynamisch erweiterten Beobachters

verallgemeinerter Regler (Standard-Regelungsproblem)

verallgemeinerte Strecke (Standard-Regelungsproblem)

Menge von Beobachtermatrizen

Menge von Nachfiltermatrizen 


\section{Verzeichnis der Beispielsysteme}

\section{Akademische Beispielsysteme}

- Entwurf von FIOs in quadratischen Systemen, Abschnitte 4.5.1 und 4.5.2

- Entwurf von FIOs in nichtquadratischen Systemen, Abschnitte 4.9.1 und 4.9.2

- Entwurf von Beobachtern zur teilweisen Fehlerisolation, Abschnitt 4.10 .2

- Optimierung der Robustheit von FIOs bezüglich exogener Störungen bei teilweiser Fehlerisolation, Abschnitt 5.5.4

- Optimierung der Robustheit von FIOs bezüglich unsicherer Systemparameter, Abschnitt 5.4.4

- Optimierung der Robustheit von FIFs bezüglich unsicherer Systemparameter, Abschnitt 6.5.2

\section{Modellhubschrauber CE 150}

- Beobachterbasierte Fehlerisolation in quadratischen Systemen, Abschnitt 4.5.3

- Beobachterbasierte Fehlerisolation in quadratischen Systemen mit erweiterten Diagonalelementen, Abschnitte 4.6.5 und 5.3.3

- Optimierung der Robustheit von FIOs in quadratischen Systemen durch Veränderung der Residuendynamik, Abschnitt 5.3.1 


\section{Verladebrücke}

- Beobachterbasierte Fehlerisolation in nicht minimalphasigen Systemen, Abschnitt 4.8.4

- Entwurf von Beobachtern zur teilweisen Fehlerisolation, Abschnitt 4.10 .3

- Optimierung der Robustheit von FIOs bezüglich exogener Störungen durch Relaxierung der strukturellen Anforderungen, Abschnitt 5.3.2

- Optimierung der Robustheit von FIOs bezüglich exogener Störungen bei teilweiser Fehlerisolation, Abschnitt 5.5.5

\section{Hydraulisch-mechanisches System}

- Beobachterbasierte Fehlerisolation in nichtquadratischen Systemen, Abschnitt 4.9.3

- Optimierung der Robustheit von FIOs bezüglich exogener Störungen bei vollständiger Fehlerisolation in nichtquadratischen Systemen, Abschnitte 5.4.1 und 5.4.2

- Optimierung der Robustheit von FIOs bezüglich exogener Störungen in begrenzten Frequenzbereichen bei vollständiger Fehlerisolation in nichtquadratischen Systemen, Abschnitt 5.4.3

- Entwurf von Fehlerisolationsfiltern für nominale Systeme, Abschnitt 6.4 .3

- Optimierung der Robustheit von FIFs bezüglich exogener Störungen, Abschnitt 6.5.1

\section{DHC-2 Beaver}

- Beobachterbasierte Fehlerisolation in statisch nicht isolierbaren Systemen, Abschnitt 4.7.5

- Nichtkompensation invarianter Nullstellen mithilfe von dynamisch erweiterten FIOs, Abschnitt 4.8.5

- Entwurf allgemeiner FIFs für nominale Systeme, Abschnitt 6.4.3 


\section{Kurzfassung}

Stetig steigende Anforderungen an technische Systeme hinsichtlich ihrer Geschwindigkeit, ihrer Genauigkeit sowie ihrer Energie- und Kosteneffizienz bedingen eine immer größere Systemkomplexität. Mit dieser geht eine steigende Anzahl möglicher Fehlerquellen im System einher, was die Notwendigkeit fortgeschrittener Diagnoseverfahren begründet. Vor diesem Hintergrund hat diese Arbeit den Entwurf robuster modellbasierter Fehlerisolationsfilter für lineare zeitinvariante Systeme zum Ziel. Diese erlauben eine zuverlässige Lokalisierung von Fehlern auf Basis eines quantitativen Systemmodells.

Zunächst wird der Entwurf beobachterbasierter Fehlerisolationsfilter betrachtet. Dazu wird die Dualität zum Entkopplungsreglerentwurf herausgearbeitet und systematisch genutzt, was es erlaubt, bekannte Verfahren wie den Entkopplungsreglerentwurf nach Falb und Wolovich und die Vollständige Modale Synthese auf das Problem der Fehlerisolation zu übertragen. Darüber hinaus wird die Klasse der mittels Fehlerisolationsbeobachtern handhabbaren linearen Systeme erweitert und es erfolgt eine Verallgemeinerung für nichtquadratische Systeme mit zusätzlichen Messgrößen. Dabei ergeben sich zusätzliche Entwurfsfreiheitsgrade.

Für die praktische Anwendung von Diagnoseverfahren ist ihre Robustheit sowohl gegenüber exogenen Störungen als auch unsicheren Parametern von essentieller Bedeutung. Beide Aspekte werden im Rahmen der Arbeit betrachtet und es erfolgt eine auf linearen Matrixungleichungen basierende Optimierung der Robustheit der Fehlerisolationsbeobachter, welche die herausgearbeiteten Freiheitsgrade nutzt.

Schließlich werden Fehlerisolationsfilter allgemeinerer Struktur betrachtet, die gegenüber den beobachterbasierten Methoden einen vereinheitlichenden Entwurf liefern. Dieser erfolgt optimierungsbasiert und erlaubt eine einfache Auslegung, bei der lediglich intuitive Parameter einzustellen sind. 


\section{Abstract}

Increasing demands regarding speed, accuracy, energy-efficiency, and costs drive the complexity of technical system. This comes along with a rising number of potential faults in a system creating a need for advanced fault diagnosis methods. To this end, this thesis is focused on the design of robust model-based fault isolation filters for linear time-invariant plants. These allow to reliably localize faults based on quantitative system models.

First, observer-based fault isolation filters are considered. Duality to non-interacting controller design is established and systematically exploited by transferring well-known methods (Falb and Wolovich's noninteracting controller design as well as the parametric eigenstructure assignment approach) to the problem of fault isolation. Furthermore, the class of systems, which are tractable using fault isolation observers, is extended and the methods are generalized to non-square systems with additional measurements. This gives rise to additional degrees of freedom in the design.

Since robustness with respect to both exogenous disturbances and uncertain parameters is of utmost importance for practical applications, both aspects are taken into account in this thesis. Optimization-based methods are deduced with the aid of linear matrix inequalities to optimize robustness of the fault isolation observers employing the free design parameters.

Lastly, fault isolation filters with a generalized structure are discussed. Compared to the observer-based approaches they enable a unifying design framework. The method is optimization-based and allows to design the filters by means of tuning only few intuitive parameters. 


\section{Einleitung}

Unser Alltag ist geprägt von einem ständig steigenden Automatisierungsgrad. Dies betrifft nicht nur die industrielle Produktion, in der Roboter in immer mehr Anwendungsbereichen Einzug halten, sondern auch das alltägliche Leben. Hier werden zunehmend Aufgaben von Computern übernommen. Als Beispiel sind Assistenzsysteme zu nennen, die in der Luftfahrttechnik in Form von Autopiloten seit geraumer Zeit etabliert sind und derzeit unter anderem als Spurhaltesysteme und automatische Notbremssysteme Schritt für Schritt in der Automobilindustrie zum Einsatz kommen. Auch in anderen Bereichen wie zum Beispiel der mobilen Telekommunikation, der Unterhaltungselektronik oder der Haushaltsgeräte werden zunehmend Aufgaben automatisiert.

Doch nicht nur die Anzahl automatisierter Systeme nimmt stetig zu, auch die Anforderungen, die derartige Systeme erfüllen müssen, steigen. Getrieben von ökonomischen und ökologischen Randbedingungen sind eine möglichst hohe Zuverlässigkeit und Produktqualität bei gleichzeitiger Senkung der Kosten und Emissionen zu erreichen. Oftmals haben diese konkurrierenden Anforderungen eine gesteigerte Komplexität des Gesamtsystems zur Folge, da sich nur damit die entsprechenden Ziele erreichen lassen. Ein Beispiel aus dem Bereich der Verbrennungsmotoren ist die selektive katalytische Reduktion (SCR), bei der Stickoxidemissionen in Abgasen von Dieselmotoren gesenkt werden. Der zusätzliche Katalysator mit der Einspritzung von Harnstofflösung wird benötigt, um strenge Abgasnormen zu erfüllen, er erhöht jedoch die Komplexität des Gesamtsystems. Darüber hinaus existieren zahlreiche Systeme, die in ihrer Natur äußerst komplex sind, wie zum Beispiel Energieverteilungssysteme oder Verkehrsmanagementsysteme.

Mit steigender Komplexität ergeben sich gleichermaßen neue Fehlerquellen. Dabei können Fehlfunktionen sowohl innerhalb zusätzlicher Komponenten als auch im Zusammenspiel verschiedener Teilsysteme auftreten, sie können die Aktorik, den Prozess selbst oder die Sensorik betreffen. Aufgrund der komplexen und vielfältigen Abhängigkeiten innerhalb eines Systems können bereits vermeintlich unbedeutende Fehler drastische Konsequenzen für das Gesamtsystem haben. Dies kann von einer niedrigeren 
Performance über ein undefiniertes und unvorhersehbares Verhalten bis hin zur Instabilität beziehungsweise dem Totalausfall des Systems führen.

Das Gebiet der Fehlerdiagnose befasst sich daher mit der rechtzeitigen und zuverlässigen Erkennung, Lokalisierung und Quantifizierung von Fehlern innerhalb eines Systems. Dies erfüllt unterschiedliche Zwecke: Zunächst liefert es Informationen über aufgetretene Fehler und erleichtert somit die Wartung. Darüber hinaus stellt die Fehlerdiagnose die Grundlage dafür dar, die Steuerung beziehungsweise Regelung eines Systems zu rekonfigurieren und beispielsweise in einen sicheren Betriebsmodus zu wechseln. Derartige Maßnahmen werden unter dem Begriff der fehlertoleranten Regelung zusammengefasst.

Der klassische Ansatz zur Fehlerdiagnose basiert auf der redundanten Realisierung von Hardwarekomponenten. Als Beispiel hierfür sind mehrfach verbaute Sensoren zu nennen. Diese kommen unter anderem in der Geschwindigkeitsmessung von Flugzeugen zum Einsatz, für die mehrere Staudrucksonden herangezogen werden. Allerdings bringt ein solcher Ansatz zusätzliche Kosten mit sich und erhöht den benötigten Bauraum, weswegen er nicht immer möglich beziehungsweise wirtschaftlich sinnvoll ist. Seit den 1970er Jahren hat sich daher, angetrieben zunächst vor allem durch Entwicklungen im Luft- und Raumfahrtbereich [257], das Feld der modellbasierten Fehlerdiagnose etabliert. Dabei wird auf ein dynamisches Modell des betrachteten Systems und der möglichen Fehler zurückgegriffen, um die Fehler zu erkennen und zu lokalisieren. Grundsätzlich erfolgt somit eine Verlagerung des zusätzlichen Aufwandes von der Hardware- hin zur kostengünstigeren Softwareseite. Auf Grundlage eines Prozessmodells werden messbare Größen gefiltert, um auf aufgetretene Fehler zurückschließen zu können. Einen ausführlichen Überblick über die Entwicklungen der vergangenen Jahrzehnte bieten die Bücher [32, 58, 111]. Ziel ist es entweder, das gleiche Sicherheitsniveau bei verringertem Hardwareaufwand zu erreichen, oder aber mit gleichem Hardwareaufwand das Sicherheitsniveau durch die Einbeziehung eines Prozessmodells weiter zu steigern. Neben der Frage, wie sich Filter zu diesem Zweck entwerfen lassen, spielt die Frage der Robustheit für die praktische Anwendung solcher Filter eine große Rolle. Die beim Entwurf verwendeten Modelle stellen zwangsläufig eine Abstraktion beziehungsweise Vereinfachung der realen Zusammenhänge dar, sie unterliegen damit gewissen Unsicherheiten. Für den praktischen Einsatz ist es jedoch von größter Wichtigkeit, dass die Filter trotz derartiger Unsicherheiten und Störungen zuverlässig funktionieren und einen Rückschluss auf die Fehler zulassen. 


\subsection{Ziele und Beiträge der Arbeit}

Ziel der Arbeit ist die Entwicklung von Verfahren zum Entwurf modellbasierter Fehlerisolationsfilter, die eine Lokalisierung von Fehlern auf Grundlage gemessener Ausgangsgrößen sowie eines quantitativen Prozessmodells erlauben. Wesentliches Augenmerk liegt dabei

1. auf der Vergrößerung der handhabbaren Systemklasse gegenüber existierenden Resultaten,

2. auf dem Entwurf von Fehlerisolationsfiltern niedriger Ordnung, die mit einem geringen Implementierungsaufwand einhergehen, sowie

3. auf der Steigerung der Robustheit von Fehlerisolationsfiltern gegenüber Störungen und Parameterunsicherheiten.

Neben einem umfassenden Überblick über die modellbasierte Fehlerdiagnose und einer Einordnung von Fehlerisolationsfiltern in diese Thematik liefert die vorliegende Arbeit in allen drei Punkten neue Verfahren und Ergebnisse, die in den entsprechenden Kapiteln ausführlich diskutiert werden.

Grundphilosophie bei der Bewältigung der genannten Teilziele ist es, die Problemstellung soweit wie möglich auf bekannte Probleme der Regelungstechnik zurückzuführen, um auf Resultate aus anderen Bereichen zurückgreifen zu können. Dies betrifft insbesondere die systematische Ausnutzung der engen Verwandtschaft zum Problem des linearen Entkopplungsreglerentwurfes.

Ein weiterer Schwerpunkt liegt auf dem einfachen Entwurf der Fehlerisolationsfilter. Dazu wird angestrebt, die Filter weitgehend automatisiert zu entwerfen und die Steigerung der Robustheit durch das automatisierte Lösen von Optimierungsproblemen in einfacher Art und Weise zu ermöglichen. Die Anzahl der beim Entwurf einzustellenden Parameter sollte dabei möglichst gering sein und sich insbesondere durch eine intuitive Wahl der Entwurfsparameter auszeichnen.

\subsection{Aufbau der Arbeit}

Die Arbeit gliedert sich in sieben Kapitel, der Aufbau ist in Abbildung 1.1 skizziert. Nach den einführenden Betrachtungen wird in Kapitel 2 ein Überblick über den Stand der Technik im Bereich der modellbasierten 


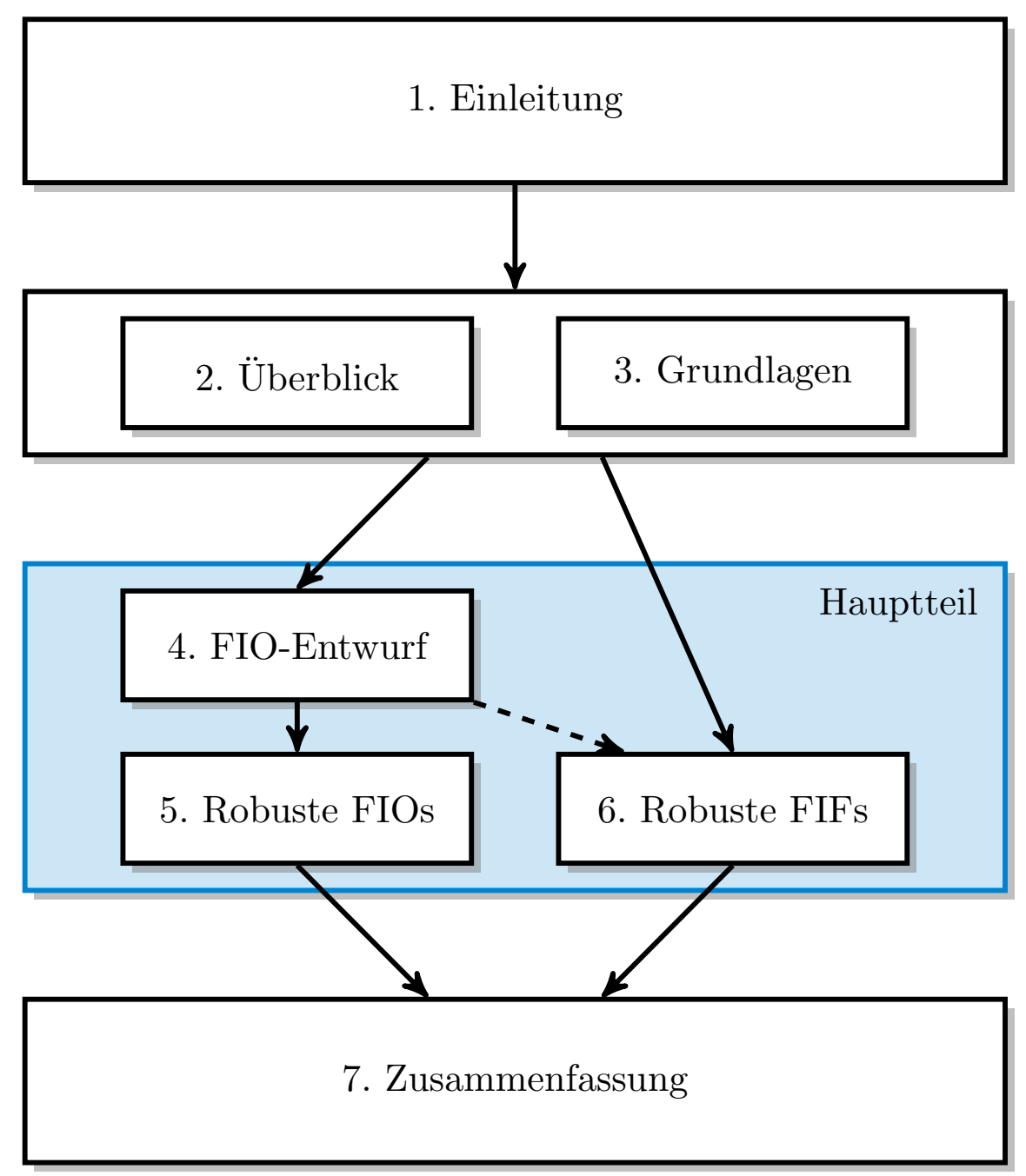

Abbildung 1.1: Aufbau der Arbeit

Fehlerisolation gegeben. Dabei erfolgt auch die Einordnung in den Gesamtkontext der Fehlerdiagnose und die Klärung der Grundbegriffe.

Kapitel 3 rekapituliert anschließend mathematische und regelungstechnische Grundlagen, die zum Verständnis der Arbeit nötig sind. Es bildet gemeinsam mit Kapitel 2 das Fundament für die erarbeiteten neuen Ergebnisse.

Diese finden sich im Hauptteil der Arbeit in den Kapiteln 4 bis 6 . Kapitel 4 befasst sich mit dem Entwurf von Fehlerisolationsbeobachtern $\left(\right.$ FIOs $^{1)}$ ) für Systeme, deren Parameter exakt bekannt sind und die keinerlei Störungen unterliegen. Grundlage dabei ist die Erkenntnis, dass

1) In der weitgehend englischsprachigen Literatur werden Fehlerisolationsbeobachter als fault isolation observers bezeichnet. Aus Gründen der Konsistenz wird daher die Abkürzung FIO (beziehungsweise FIOs im Plural) verwendet. 
es sich bei dem Entwurfsproblem für derartige Systeme um das duale Problem zum bekannten linearen Entkopplungsreglerentwurf handelt (Abschnitt 4.4). In der systematischen Ausnutzung dieser Tatsache ist der wesentliche Beitrag des Kapitels zu sehen. Es ergeben sich daraus zwei unterschiedliche Entwurfsverfahren, die in Abschnitt 4.5 vorgestellt werden. Das erste basiert auf dem klassischen Entkopplungsreglerentwurf nach Falb und Wolovich [67] und das zweite auf der Vollständigen Modalen Synthese [185]. Durch dynamische Erweiterungen des Beobachters lässt sich zum einen die Dynamik der erzeugten Residuen beeinflussen (Abschnitt 4.6), zum anderen lassen sich die Existenzbedingungen für den Entwurf von FIOs abschwächen. Damit gelingt der Entwurf für statisch nicht isolierbare Systeme (Abschnitt 4.7) und für nicht minimalphasige Systeme (Abschnitt 4.8), wodurch die Klasse der handhabbaren linearen Systeme wesentlich erweitert wird. Abschnitt 4.9 behandelt Systeme, die über zusätzliche Messgrößen verfügen. In solchen so genannten nichtquadratischen Systemen ergeben sich zusätzliche Freiheitsgrade, die explizit charakterisiert und diskutiert werden. Zum Abschluss des Kapitels finden sich neue Resultate zur beobachterbasierten Fehlerisolation unter abgeschwächten strukturellen Anforderungen. Dies wird in Abschnitt 4.10 unter dem Stichwort der partiellen Fehlerisolation behandelt.

Durch die ausführliche Behandlung der Entwurfsverfahren für störungsfreie, nominale Systeme wird weiterhin die Grundlage für Kapitel 5 gelegt. Darin werden verschiedene Robustheitsaspekte betrachtet und Entwürfe erarbeitet, welche die robuste Generierung von Residuen unter dem Einfluss exogener Störungen sowie unsicherer Parameter ermöglichen. In Abschnitt 5.3 werden Systeme mit ebenso vielen Messgrößen wie potentiellen Fehlern behandelt (quadratische Systeme). Abschnitt 5.4 erweitert die Betrachtungen auf nichtquadratische Systeme. Bei derartigen Systemen ergeben sich wie bereits angedeutet zusätzliche Freiheitsgrade, die systematisch zur Optimierung der Robustheit ausgenutzt werden. Die Freiheitsgrade, die sich bei partieller Fehlerisolation ergeben, werden in Abschnitt 5.5 zur Robustheitssteigerung ausgenutzt.

Während in den Kapiteln 4 und 5 die Struktur eines Beobachters für die Fehlerisolation herangezogen wird, wird in Kapitel 6 ein allgemeinerer Ansatz gewählt. Es kommen allgemeine dynamische Filter $\left(\mathrm{FIFs}^{2)}\right)$ zum Einsatz, für die kein analytischer, sondern ein optimierungsbasierter Entwurf vorgeschlagen wird (Abschnitt 6.4). In Abschnitt 6.5 werden Robustheits-

${ }^{2)}$ Die Abkürzung erklärt sich aus dem in der englischsprachigen Literatur gängigen Begriff der fault isolation filters. 
aspekte für allgemeine Fehlerisolationsfilter behandelt. Dabei zeigt sich, dass die Optimierung der Robustheit und die Ausnutzung zusätzlicher Freiheitsgrade in sehr geradliniger Art und Weise möglich ist und dass somit nicht nur ein allgemeinerer, sondern auch ein vereinheitlichender Entwurf gegeben ist.

Die Ergebnisse werden in Kapitel 7 zusammengefasst, ebenso wird dort ein Ausblick auf sich anschließende Problemstellungen gegeben. 


\section{Modellbasierte Fehlerdiagnose: ein Überblick}

Die Fehlerdiagnose ist wie bereits erwähnt seit den 1970er Jahren ein aktives Forschungsgebiet. Dementsprechend haben sich zahlreiche Forschungsrichtungen und Ansätze herauskristallisiert und es existiert eine Fülle von Literatur zu diesem Thema. Die umfassenden Bücher $[16,32,58,111]$ sowie die Übersichtsartikel [78, 84, 109, 110, 219-221] bieten in dieser Hinsicht eine detaillierte Zusammenfassung der Entwicklung und des Standes der Technik.

In diesem Kapitel werden zunächst gemäß Abbildung 2.1 die Grundbegriffe und Teilprobleme der Fehlerdiagnose eingeführt und erörtert (Abschnitt 2.1). Auf dieser Grundlage werden in Abschnitt 2.2 unterschied-

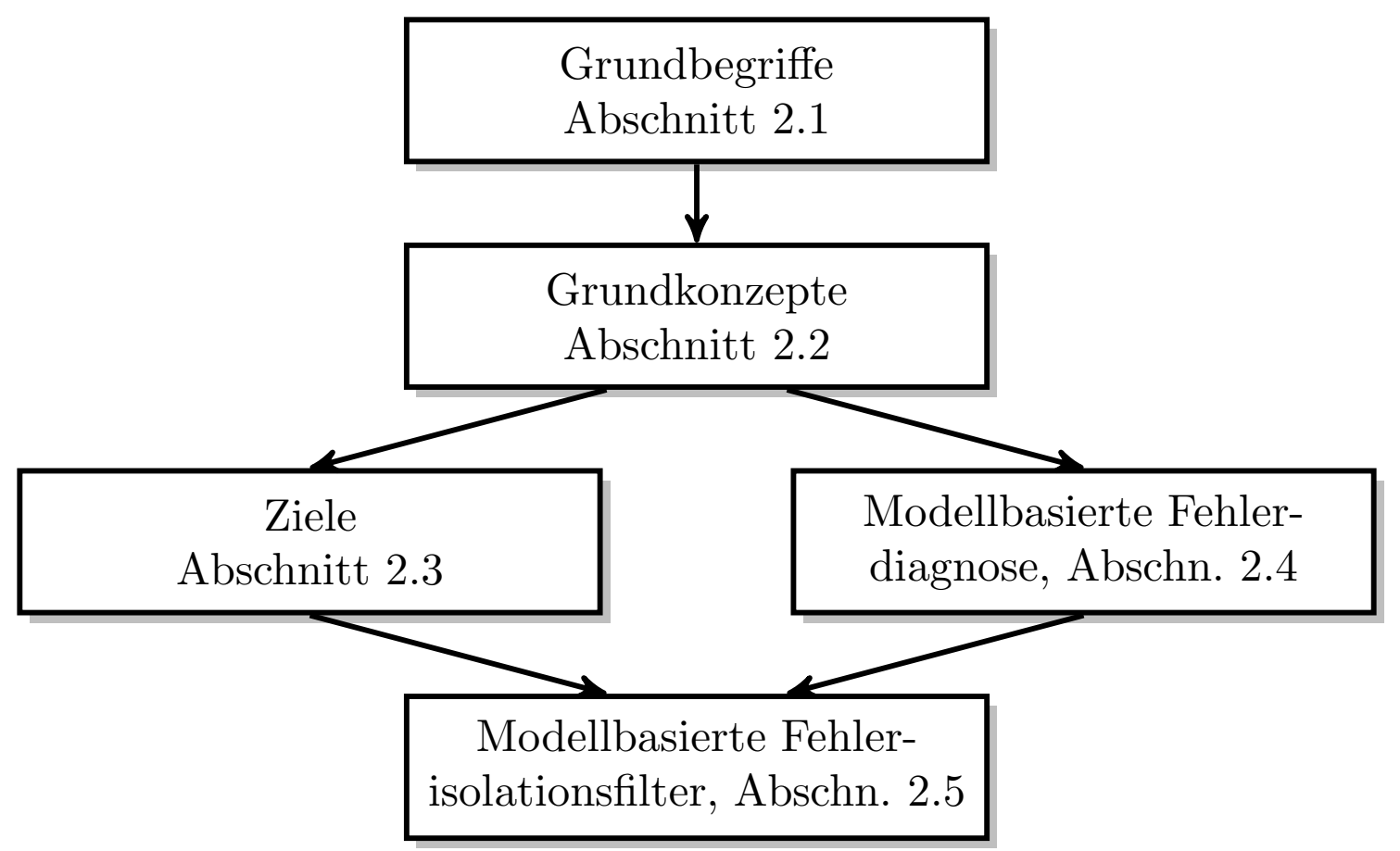

Abbildung 2.1: Aufbau des 2. Kapitels 
liche Konzepte der Fehlerdiagnose zusammengefasst. Dies erlaubt es zum einen, die wesentlichen Entwurfsziele für die im Rahmen dieser Arbeit entwickelten Verfahren zu präzisieren (Abschnitt 2.3). Zum anderen lassen sich die Ergebnisse dadurch wie in Abschnitt 2.4 beschrieben in den Gesamtkontext der modellbasierten Fehlerdiagnose einordnen. Abschnitt 2.5 bietet schließlich einen detaillierten Überblick zu existierenden Verfahren der beobachter- beziehungsweise filterbasierten Fehlerisolation.

\subsection{Grundbegriffe}

Als einheitlicher Standard für die Definition wesentlicher Grundbegriffe hat sich im Bereich der Fehlerdiagnose der Kanon des SAfEProcessKomitees der IFAC ${ }^{1)}$ etabliert. Auch die Begriffsdefinitionen in der vorliegenden Arbeit folgen im Wesentlichen diesen in [109] veröffentlichten Vorschlägen. Zentral ist dabei der Begriff des Fehlers.

Definition 2.1 (Fehler). Ein Fehler ist eine unerlaubte Abweichung wenigstens einer charakteristischen Eigenschaft oder eines Parameters von seiner Standardausprägung.

Diese sehr allgemeine Definition umfasst eine Vielzahl unterschiedlicher Fehlerklassen, sowohl hinsichtlich der Fehlerursache (prozessinterne Fehler und Fehler durch externe Einflüsse), des Fehlerortes (Fehler in Aktorik, Prozess oder Sensorik) und der Dynamik des Fehlers (zum Beispiel sprungförmig, kriechend oder periodisch). Die Fehler gilt es mittels der Fehlerdiagnose zu erkennen, zu lokalisieren, in ihrer Amplitude abzuschätzen und in ihrer Ursache zu bestimmen. Dies grenzt sie entscheidend ab von Störungen.

Definition 2.2 (Störung). Eine Störung ist eine unbekannte Eingangsgröße, die auf das System einwirkt.

Es sei angemerkt, dass diese Definition den einfacheren Fall messbarer Störgrößen ausschließt, deren Einfluss sich durch eine Störgrößenaufschaltung abschwächen beziehungsweise sogar kompensieren ließe. Störungen sind also ebenso wie Fehler prinzipiell nicht bekannt. Sie sollen jedoch nicht erkannt werden, vielmehr gilt es, ihren Einfluss bei der Fehlerdiagnose zu unterdrücken. Grundsätzlich wird also angestrebt, Fehler trotz des Einflusses von Störungen zu diagnostizieren. Ein wesentliches Hilfsmittel dafür sind die sogenannten Residuen.

\footnotetext{
1) International Federation of Automatic Control
} 
Definition 2.3 (Residuen). Residuen sind Fehlerindikatoren.

Diese Fehlerindikatoren ergeben sich häufig aus der Diskrepanz zwischen zwei Signalen, zum Beispiel der Differenz von gemessenen und geschätzten Ausgangssignalen. Dementsprechend sind Residuen in [109] definiert als Fehlerindikatoren, die sich aus einer Differenz zwischen gemessenen Signalen und modellbasierten Berechnungen ergeben. Definition 2.3 weicht bewusst davon ab und fasst den Begriff des Residuums allgemeiner auf. Näher begründet wird dies in Kapitel 6 beim Entwurf allgemeiner Fehlerisolationsfilter.

Im Folgenden wird näher auf verschiedene Typen von Fehlern sowie ihre Modellierung eingegangen.

\subsubsection{Typen von Fehlern}

Die in einem System auftretenden Fehler lassen sich hinsichtlich verschiedener Merkmale charakterisieren. Zunächst können sie wie in Abbildung 2.2 gezeigt bezüglich des Streckenteils unterschieden werden, in dem sie auftreten. Daraus ergibt sich die Unterscheidung in Aktorfehler, Prozessfehler und Sensorfehler.

Aktorfehler entstehen daraus, dass ein Stellglied nicht die vom Regler oder der Steuerung vorgegebene Stellgröße produziert. Für eine lineare Strecke der Darstellung $\dot{\boldsymbol{x}}=\boldsymbol{A} \boldsymbol{x}+\boldsymbol{B} \boldsymbol{u}$ führt dies zu

$$
\dot{\boldsymbol{x}}=\boldsymbol{A} \boldsymbol{x}+\boldsymbol{B}\left(\boldsymbol{u}_{\mathrm{reg}}+\Delta \boldsymbol{u}\right)=\boldsymbol{A} \boldsymbol{x}+\boldsymbol{B} \boldsymbol{u}_{\mathrm{reg}}+\underbrace{\boldsymbol{B}}_{\boldsymbol{E}_{\text {aktor }}} \underbrace{\Delta \boldsymbol{u}}_{\text {aktor }} .
$$

Offensichtlich besteht der Fehler (beziehungsweise die Fehler) in $\Delta \boldsymbol{u}$, al-

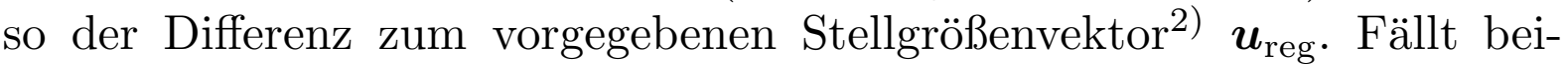
spielsweise ein Aktor komplett aus und gibt die Stellgröße 0 aus, so ist $\Delta u_{i}=-u_{\mathrm{reg}, i}$. Die Aktorfehler wirken über die gleiche Eingangsmatrix auf die Streckendynamik wie die Stellgrößen $\left(\boldsymbol{E}_{\text {aktor }}=\boldsymbol{B}\right)$.

Treten im Prozess selbst Fehler auf, so spricht man von Prozessfehlern. Beispiele dafür sind ein Leck in einem Tank oder eine zu detektierende einwirkende Kraft in einem mechanischen System. Prozessfehler wirken auf die Systemdynamik wie zusätzliche, unbekannte Eingangsgrößen, es ist also

$$
\dot{\boldsymbol{x}}=\boldsymbol{A} \boldsymbol{x}+\boldsymbol{B} \boldsymbol{u}+\boldsymbol{E}_{\text {prozess }} \boldsymbol{f}_{\text {prozess }},
$$

${ }^{2)}$ Gemeint ist hier eine Differenz aufgrund eines Defektes. Differenzen zwischen vorgegebener und tatsächlicher Stellgröße aufgrund von Stellgrößenbeschränkungen werden nicht betrachtet. 


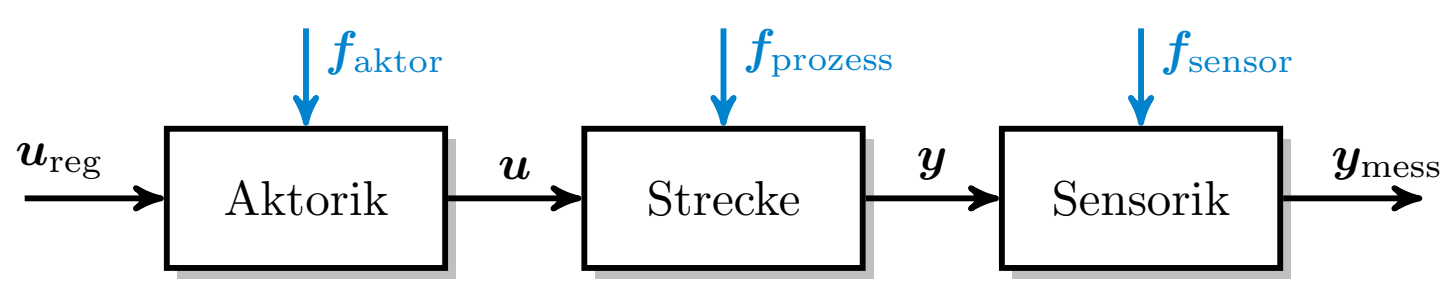

Abbildung 2.2: Aktor-, Prozess- und Sensorfehler

wobei sich die Matrix $\boldsymbol{E}_{\text {prozess }}$ aus der Modellbildung ergibt. Prozessfehler wirken in der gleichen Art und Weise auf die Strecke, wie es additive Störungen der Streckendynamik tun. Es ist daher ein entscheidender Schritt in der Modellbildung beziehungsweise in der Konzeption eines Fehlerdiagnosesystems, festzulegen, ob eine Einflussgröße als Störung oder als Prozessfehler aufgefasst werden soll. Daraus ergibt sich, ob die Größe zu detektieren und isolieren ist (falls sie als Fehler aufgefasst wird), oder ob ihr Einfluss durch das Diagnosesystem unterdrückt werden soll (falls sie als Störung aufgefasst wird). Aus dem Vergleich von (2.1) und (2.2) wird weiterhin deutlich, dass Prozessfehler strukturell genauso auf die Streckendynamik wirken wie Aktorfehler. Sie unterscheiden sich lediglich durch die Fehlereingangsmatrix und man kann zusammenfassend schreiben

$$
\dot{\boldsymbol{x}}=\boldsymbol{A} \boldsymbol{x}+\boldsymbol{B} \boldsymbol{u}_{\mathrm{reg}}+\left[\begin{array}{ll}
\boldsymbol{B} & \boldsymbol{E}_{\text {prozess }}
\end{array}\right]\left[\begin{array}{c}
\boldsymbol{f}_{\text {aktor }} \\
\boldsymbol{f}_{\text {prozess }}
\end{array}\right] .
$$

Vereinfachend wird daher im Rahmen dieser Arbeit der Begriff Aktorfehler übergreifend für alle auf die Streckendynamik einwirkenden Fehler verwendet, er schließt also Prozessfehler ausdrücklich mit ein.

Neben der Aktorik und dem Prozess selbst stellt die Sensorik den dritten wesentlichen Teil des Gesamtsystems dar. Geht man weiterhin von einer linearen Strecke aus, lässt sich die Ausgangsgleichung durch $\boldsymbol{y}=\boldsymbol{C} \boldsymbol{x}+\boldsymbol{D u}$ beschreiben. Treten nun in den Sensoren Fehler auf, so weichen die Messgrößen ab und es ist

$$
\boldsymbol{y}_{\text {mess }}=\boldsymbol{C x}+\boldsymbol{D} \boldsymbol{u}+\boldsymbol{E}_{\text {sensor }} \boldsymbol{f}_{\text {sensor }} .
$$

In der Literatur werden oftmals Systeme betrachtet, die lediglich Fehlern unterliegen, die auf die Streckendynamik einwirken (also Aktor- und Prozessfehlern). Dies ist dadurch begründet, dass sich nach [169] Sensorfehler als sogenannte Pseudoaktorfehler modellieren lassen. Dazu nimmt man an, dass die Dynamik der Fehler mit einem fiktiven $\tilde{\boldsymbol{f}}$ beschrieben werden kann 
durch

$$
\begin{aligned}
\dot{\zeta} & =\Phi \zeta+\Lambda \tilde{f}, \\
f_{\text {sensor }} & =\Gamma \zeta .
\end{aligned}
$$

Setzt man dies in das mit Sensorfehlern behaftete System ein, so ergibt sich das erweiterte System ${ }^{3)}$

$$
\begin{aligned}
{\left[\begin{array}{c}
\dot{\boldsymbol{x}} \\
\dot{\boldsymbol{\zeta}}
\end{array}\right] } & =\left[\begin{array}{cc}
\boldsymbol{A} & \mathbf{0} \\
\mathbf{0} & \boldsymbol{\Phi}
\end{array}\right]\left[\begin{array}{l}
\boldsymbol{x} \\
\boldsymbol{\zeta}
\end{array}\right]+\left[\begin{array}{c}
\boldsymbol{B} \\
\mathbf{0}
\end{array}\right] \boldsymbol{u}+\left[\begin{array}{l}
\mathbf{0} \\
\boldsymbol{\Lambda}
\end{array}\right] \tilde{\boldsymbol{f}}, \\
\boldsymbol{y}_{\text {mess }} & =\left[\begin{array}{ll}
\boldsymbol{C} & \boldsymbol{E}_{\text {sensor }} \boldsymbol{\Gamma}
\end{array}\right]\left[\begin{array}{l}
\boldsymbol{x} \\
\boldsymbol{\zeta}
\end{array}\right]+\boldsymbol{D} \boldsymbol{u} .
\end{aligned}
$$

Offensichtlich treten in diesem System keine Sensorfehler auf, sondern lediglich Aktorfehler. Dadurch lassen sich Systeme mit Sensorfehlern in erweiterte Systeme umwandeln, die ausschließlich Aktorfehlern unterliegen. Dadurch müssen Sensorfehler prinzipiell bei der Entwicklung von Entwurfsverfahren nicht berücksichtigt werden, was die Betrachtungen zum Teil vereinfacht. Das Vorgehen weist allerdings auch zwei Nachteile auf. Zum einen ergibt sich ein erweitertes Gesamtsystem höherer Ordnung, so dass im Allgemeinen auch der Implementierungsaufwand für das zu entwerfende Diagnosesystem steigt. Zum anderen setzt (2.5) voraus, dass sich die Dynamik der Sensorfehler modellieren lässt. Weicht die tatsächliche Fehlerdynamik davon ab, wird die Qualität des Diagnosesystems beeinträchtigt. Außerdem stellen $\boldsymbol{\Phi}, \boldsymbol{\Lambda}$ und $\boldsymbol{\Gamma}$ zusätzliche Entwurfsparameter dar, die auszulegen sind. Daher werden Sensorfehler im Rahmen der vorliegenden Arbeit nicht in Pseudoaktorfehler umgewandelt. Aufgrund dieser direkten Behandlung ist keine Annahme über die Dynamik der Sensorfehler nötig.

Fasst man (2.3) und (2.4) zusammen, so erhält man als kompakte Darstellung

$$
\begin{gathered}
\dot{\boldsymbol{x}}=\boldsymbol{A} \boldsymbol{x}+\boldsymbol{B} \boldsymbol{u}_{\mathrm{reg}}+\underbrace{\left[\begin{array}{lll}
\boldsymbol{B} & \boldsymbol{E}_{\text {prozess }} & \mathbf{0}
\end{array}\right]}_{\boldsymbol{E}_{a}} \boldsymbol{f}, \\
\boldsymbol{y}_{\mathrm{mess}}=\boldsymbol{C} \boldsymbol{x}+\boldsymbol{D} \boldsymbol{u}_{\mathrm{reg}}+\underbrace{\left[\begin{array}{lll}
\boldsymbol{D} & \boldsymbol{0} & \boldsymbol{E}_{\text {sensor }}
\end{array}\right]}_{\boldsymbol{E}_{s}} \boldsymbol{f} .
\end{gathered}
$$

3) Mit $\mathbf{0}$ wird in dieser Arbeit eine Nullmatrix passender Dimension abgekürzt. 
Dabei werden alle auftretenden Fehler in dem Fehlervektor

$$
\boldsymbol{f}=\left[\begin{array}{c}
\boldsymbol{f}_{\text {aktor }} \\
\boldsymbol{f}_{\text {prozess }} \\
\boldsymbol{f}_{\text {sensor }}
\end{array}\right]
$$

zusammengefasst. Von dieser Systemdarstellung wird bei den Entwurfsverfahren im Rahmen dieser Arbeit stets ausgegangen.

Bei den bisher diskutierten Arten von Fehlern handelt es sich jeweils um additive Fehler. Eine weitere Art von Fehlern, die auftreten können, sind multiplikative Fehler. Diese werden im Wesentlichen dadurch hervorgerufen, dass sich Parameter der Systemmatrizen ändern, was sich durch

$$
\begin{aligned}
& \dot{\boldsymbol{x}}=\left(\boldsymbol{A}+\sum_{i=1}^{n_{f, A}} f_{A, i} \cdot \Delta \boldsymbol{A}_{i}\right) \boldsymbol{x}+\left(\boldsymbol{B}+\sum_{i=1}^{n_{f, B}} f_{B, i} \cdot \Delta \boldsymbol{B}_{i}\right) \boldsymbol{u}, \\
& \boldsymbol{y}=\left(\boldsymbol{C}+\sum_{i=1}^{n_{f, C}} f_{C, i} \cdot \Delta \boldsymbol{C}_{i}\right) \boldsymbol{x}+\left(\boldsymbol{D}+\sum_{i=1}^{n_{f, D}} f_{D, i} \cdot \Delta \boldsymbol{D}_{i}\right) \boldsymbol{u}
\end{aligned}
$$

beschreiben lässt. Dabei gibt $n_{f, A}$ die Anzahl der möglichen Fehler in der Systemmatrix $\boldsymbol{A}$ an, $n_{f, B}$ die Anzahl der Fehler in der Eingangsmatrix $\boldsymbol{B}, n_{f, C}$ die Anzahl der Fehler in der Ausgangsmatrix $\boldsymbol{C}$ und $n_{f, D}$ die Anzahl der Fehler in der Durchgangsmatrix $\boldsymbol{D}$. Fasst man die Einzelfehler zu Vektoren wie $\boldsymbol{f}_{A}=\left[\begin{array}{lll}f_{A, 1} & \ldots & f_{A, n_{f, A}}\end{array}\right]^{\top}$ zusammen, so lässt sich der verallgemeinerte Fehlervektor

$$
\boldsymbol{f}=\left[\begin{array}{l}
\boldsymbol{f}_{A} \otimes \boldsymbol{x} \\
\boldsymbol{f}_{B} \otimes \boldsymbol{u} \\
\boldsymbol{f}_{C} \otimes \boldsymbol{x} \\
\boldsymbol{f}_{D} \otimes \boldsymbol{u}
\end{array}\right] \in \mathbb{R}^{n\left(n_{f, A}+n_{f, C}\right)+n_{u}\left(n_{f, B}+n_{f, D}\right)}
$$

aufstellen, wobei $n$ die Dimension des Zustandsvektors $\boldsymbol{x}$ und $n_{u}$ die Dimension des Eingangsgrößenvektors $\boldsymbol{u}$ ist. ${ }^{4)}$ Es wird also zum Beispiel jedes Element des Vektors $\boldsymbol{f}_{A}$ mit dem gesamten Zustandsvektor $\boldsymbol{x}$ multipliziert,

4) Dabei stellt $\otimes$ das Kroneckerprodukt dar, welches für zwei Matrizen $\boldsymbol{A} \in \mathbb{R}^{n \times m}$ und $\boldsymbol{B}$ definiert ist als

$$
\boldsymbol{A} \otimes \boldsymbol{B}=\left[\begin{array}{ccc}
a_{11} & \cdots & a_{1 m} \\
\vdots & & \vdots \\
a_{n 1} & \cdots & a_{n m}
\end{array}\right] \otimes \boldsymbol{B}=\left[\begin{array}{ccc}
a_{11} \boldsymbol{B} & \cdots & a_{1 m} \boldsymbol{B} \\
\vdots & & \vdots \\
a_{n 1} \boldsymbol{B} & \cdots & a_{n m} \boldsymbol{B}
\end{array}\right]
$$


woraus sich die Dimension von $\boldsymbol{f}$ erklärt. Damit lässt sich (2.6) schreiben als

$$
\begin{aligned}
& \dot{\boldsymbol{x}}=\boldsymbol{A} \boldsymbol{x}+\boldsymbol{B} \boldsymbol{u}+\underbrace{\left[\begin{array}{lllllll}
\Delta \boldsymbol{A}_{1} & \cdots & \Delta \boldsymbol{A}_{n_{f, A}} & \Delta \boldsymbol{B}_{1} & \cdots & \Delta \boldsymbol{B}_{n_{f, B}} & \mathbf{0}
\end{array}\right]}_{\boldsymbol{E}_{a}} \boldsymbol{f}, \\
& \boldsymbol{y}=\boldsymbol{C} \boldsymbol{x}+\boldsymbol{D} \boldsymbol{u}+\underbrace{\left[\begin{array}{lllllll}
\mathbf{0} & \Delta \boldsymbol{C}_{1} & \cdots & \Delta \boldsymbol{C}_{n_{f, C}} & \Delta \boldsymbol{D}_{1} & \cdots & \Delta \boldsymbol{D}_{n_{f, B}}
\end{array}\right]}_{\boldsymbol{E}_{s}} \boldsymbol{f} .
\end{aligned}
$$

Somit lassen sich multiplikative Fehler als additive Fehler darstellen. Dabei ist jedoch zu beachten, dass multiplikative Fehler im Gegensatz zu additiven Fehlern die Stabilität des Systems beeinflussen können. Bei der Umformulierung als additive Fehler ist dementsprechend zu überprüfen, ob $\boldsymbol{A}+\sum_{i=1}^{n_{f, A}} f_{A, i} \cdot \Delta \boldsymbol{A}_{i}$ für alle möglichen Fehler $\boldsymbol{f}_{A}$ eine Hurwitz-Matrix ${ }^{5)}$ ist. Wie in [58] angemerkt können auch multiplikative Fehler in $\boldsymbol{B}, \boldsymbol{C}$ und $\boldsymbol{D}$ die Stabilität beeinträchtigen, wenn anstatt einer offenen Strecke der geschlossene Regelkreis betrachtet wird.

Ebenso wie bei der Modellbildung entschieden werden muss, ob bestimmte Einflussgrößen auf das System als Prozessfehler oder als zu unterdrückende Störungen aufgefasst werden sollen, ist eine Entscheidung bezüglich der in (2.6) auftretenden Größen $\sum_{i=1}^{n_{f, A}} f_{A, i} \cdot \Delta \boldsymbol{A}_{i}$, $\sum_{i=1}^{n_{f, B}} f_{B, i} \cdot \Delta \boldsymbol{B}_{i}, \sum_{i=1}^{n_{f, C}} f_{C, i} \cdot \Delta \boldsymbol{C}_{i}$ und $\sum_{i=1}^{n_{f, D}} f_{D, i} \cdot \Delta \boldsymbol{D}_{i}$ zu treffen. Diese können einerseits wie oben beschrieben als zu erkennende multiplikative Fehler verstanden werden. Andererseits ist es aber auch möglich, sie als Unsicherheiten in der Modellbildung aufzufassen, deren Einfluss es zu unterdrücken gilt. Diese grundlegende Entscheidung ist zu Beginn des Entwurfes eines Diagnosesystems zu treffen.

Eine weiteres Merkmal, anhand dessen sich Fehler klassifizieren lassen, ist die Fehlerdynamik. Typische Fälle sind sprungförmige Fehler, wie sie beispielsweise durch den Bruch einer Feder oder einen vergleichbaren Ausfall gegeben sind. Eine weitere Klasse stellen rampenförmige Fehler dar, wie sie bei einem temperaturbedingten, langsamen Ansteigen eines Sensoroffsets auftreten. Da für die im Rahmen dieser Arbeit verwendeten Verfahren überwiegend keine Annahmen über die Fehlerdynamik nötig sind, werden die Fehler in dieser Hinsicht nicht differenziert. Mit den entwickelten Methoden ist eine Detektion und Isolation von Fehlern beliebiger Dynamik möglich.

Anhand des in Abbildung 2.3 gezeigten schematischen Dreitanksystems sollen die unterschiedlichen Fehlertypen beispielhaft verdeutlicht werden.

${ }^{5)}$ Eine Matrix heißt Hurwitz-Matrix, wenn alle ihre Eigenwerte in der offenen linken s-Halbebene liegen. 


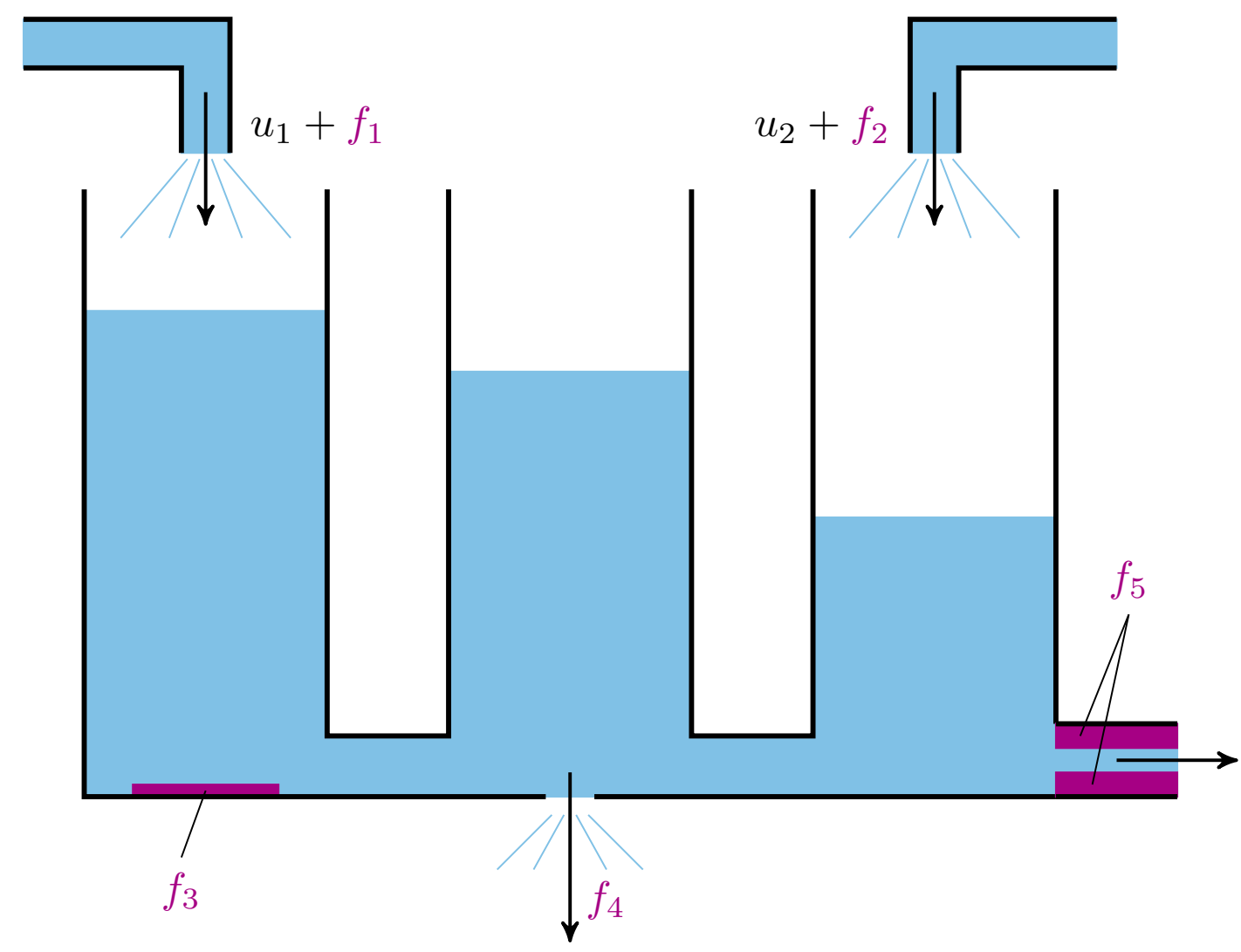

Abbildung 2.3: Beispiele für Fehlertypen in einem Dreitanksystem

Das System besteht aus drei miteinander gekoppelten Tanks, die über Rohre verbunden sind. Mittels zweier Pumpen können Zuflüsse in den ersten und dritten Tank eingestellt werden. Im dritten Tank befindet sich ein Abfluss und die Füllhöhe der drei Tanks wird gemessen.

Bei dem im Bild mit $f_{1}=\Delta u_{1}$ und $f_{2}=\Delta u_{2}$ gekennzeichneten Fehlern handelt es sich um additive Aktorfehler. Sie resultieren daraus, dass die jeweilige Pumpe nicht den angeforderten Volumenstrom produziert. An dieser Stelle wird deutlich, dass beim Entwurf eines Fehlerdiagnosesystems die festgelegten Systemgrenzen und das Abstraktionsniveau eine wesentliche Rolle spielen. Im betrachteten Beispiel werden die Pumpen als Aktoren des Dreitanksystems aufgefasst. Bei einer detaillierteren Betrachtung könnte man entweder ein separates Diagnosesystem für jede Pumpe entwerfen oder aber das Modell der Pumpendynamik in das Gesamtsystem miteinbeziehen.

Der Fehler $f_{3}$ stellt einen additiven Sensorfehler dar, der aus einem Offset der gemessenen Füllhöhe resultiert. Weiterhin ist im zweiten Tank ein mögliches Leck modelliert, was sich durch den additiven Prozessfehler $f_{4}$ äußert. Zuletzt ist mit $f_{5}$ ein multiplikativer Prozessfehler skizziert. Dieser 


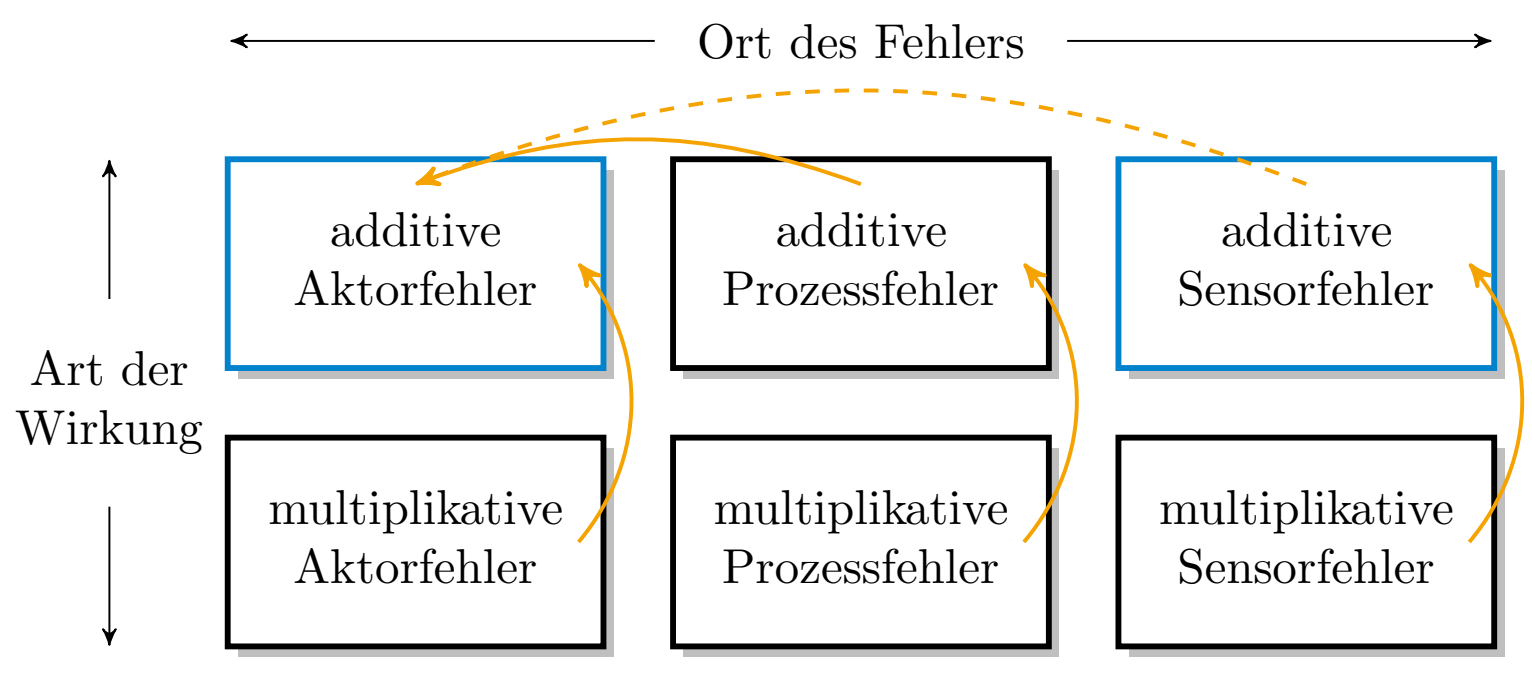

Abbildung 2.4: Klassifikation verschiedener Fehlertypen

kommt durch Ablagerungen im Abflussrohr und einem damit einhergehenden verringerten Querschnitt zustande. Selbstverständlich ist eine Vielzahl weiterer Fehler denkbar, die vorgestellten Beispiele erheben keinen Anspruch auf Vollständigkeit. Sie dienen lediglich dazu, verschiedene Typen von Fehlern zu demonstrieren.

Abbildung 2.4 fasst obige Ausführungen allgemein zusammen und verdeutlicht, dass sich die diskutierten Fehlerarten letztendlich auf additive Aktor- und Sensorfehler zurückführen lassen. Diese blau gekennzeichneten Typen sind die im Rahmen dieser Arbeit betrachteten Fehler. Bei den weiteren Betrachtungen wird stets angenommen, dass alle anderen Fehlertypen in diese beiden Arten von Fehlern überführt wurden. Unter Rückgriff auf Pseudoaktorfehler ist wie erörtert auch die ausschließliche Betrachtung von additiven Aktorfehlern möglich, was durch den strichlierten gelben Pfeil angedeutet wird.

\subsubsection{Teilaufgaben der Fehlerdiagnose}

Das Gebiet der Fehlerdiagnose umfasst im Wesentlichen drei Teilaufgaben, die gemäß Abbildung 2.5 hierarchisch gegliedert sind. Die Definition der Teilaufgaben ist erneut an [109] angelehnt. Die hierarchisch erste Teilaufgabe stellt die Fehlerdetektion dar. 


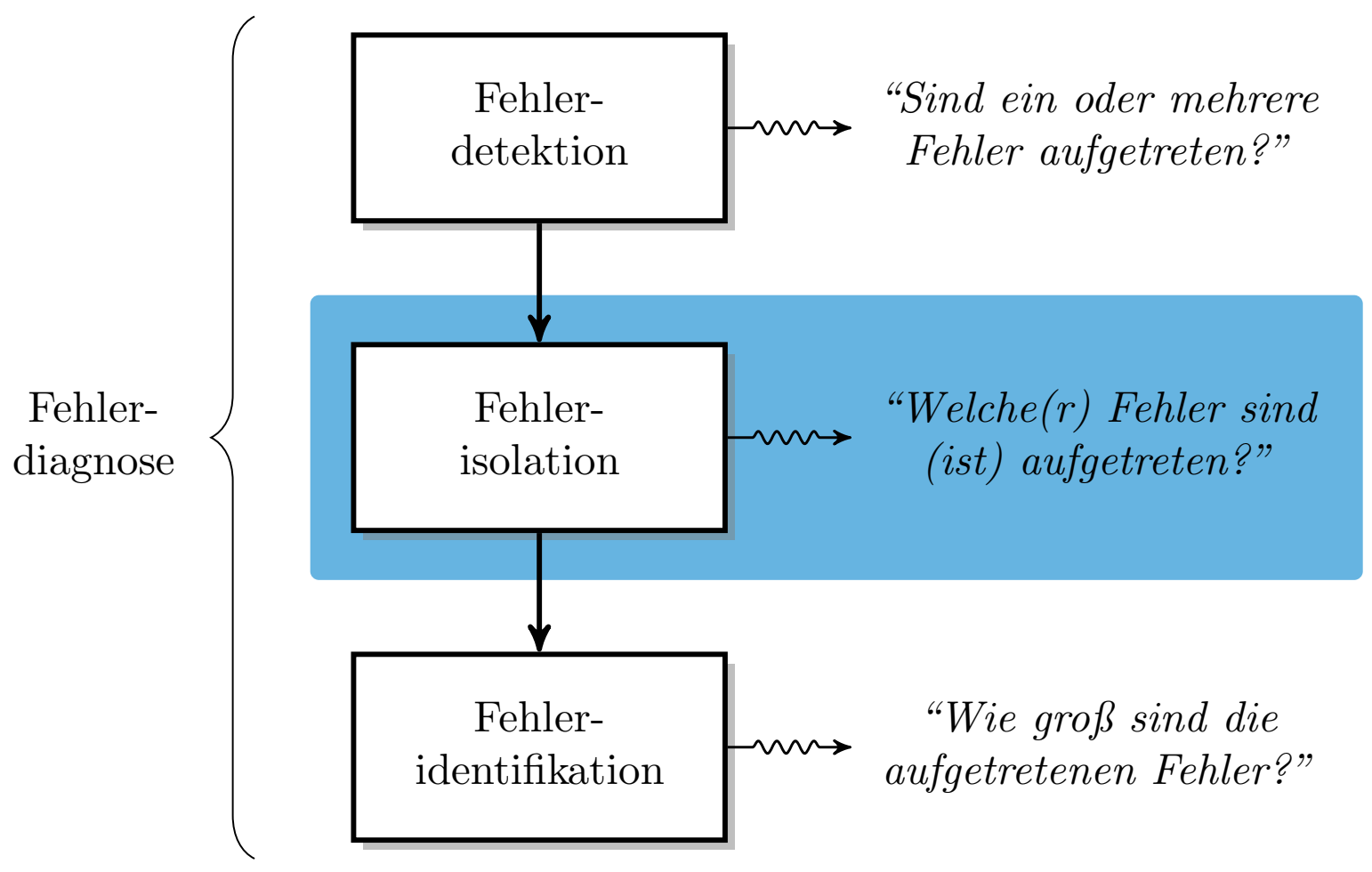

Abbildung 2.5: Aufgaben der Fehlerdiagnose

Definition 2.4 (Fehlerdetektion). Gegenstand der Fehlerdetektion ist die Erkennung von Fehlern in einem System und das Feststellen des Zeitpunktes, zu dem der oder die Fehler aufgetreten sind.

Es wird also die Frage geklärt, ob ein oder mehrere Fehler im System aufgetreten sind. Somit wird ermittelt, ob das System fehlerfrei arbeitet. Die Fehlerisolation geht demgegenüber einen Schritt weiter.

Definition 2.5 (Fehlerisolation). Gegenstand der Fehlerisolation ist die Ermittlung der Art, des Ortes und des Zeitpunktes des Auftretens eines bestimmten Fehlers oder mehrerer Fehler.

Die Fehlerisolation beschäftigt sich damit, welcher Fehler beziehungsweise welche Fehler im System aktiv sind. Sie kann somit als eine Lokalisierung der Fehler verstanden werden, die offensichtlich den Schritt der Fehlererkennung beinhaltet beziehungsweise hierarchisch auf diesen folgt. Als nachfolgende Ebene kann die Fehleridentifikation verstanden werden.

Definition 2.6 (Fehleridentifikation). Gegenstand der Fehleridentifikation ist die Ermittlung der Fehleramplitude und des Zeitverhaltens eines Fehlers oder mehrerer Fehler. 
Hier wird ermittelt, wie groß der aufgetretene Fehler beziehungsweise die aufgetretenen Fehler sind. Darüber hinaus wird häufig auch die Ermittlung der Fehlerursache der Fehleridentifikation zugeschlagen [58].

Die verschiedenen Teilaufgaben lassen sich am Beispiel der LeckageErkennung in einem Rohrleitungssystem verdeutlichen. Während in der Fehlererkennung ermittelt wird, ob ein oder mehrere Lecks vorhanden sind, werden das beziehungsweise die Lecks in der Fehlerisolation lokalisiert. In der Fehleridentifikation wird schließlich die Größe des beziehungsweise der Lecks ermittelt.

Die vorliegende Arbeit befasst sich wie in Abbildung 2.5 gekennzeichnet vorrangig mit der Fehlerisolation, was wie erwähnt eine Fehlerdetektion impliziert. Unter gewissen Voraussetzungen ist mit den entwickelten Verfahren auch eine Fehleridentifikation möglich. Auf diese Tatsache wird in Kapitel 4 eingegangen.

Neben den Kernaufgaben der Fehlerdiagnose (Fehlerdetektion, -isolation und -identifikation) existieren weitere Problemfelder, die hier nur kurz angerissen werden. Ein wesentlicher Schritt, der vor den drei genannten Teilaufgaben geleistet werden muss, ist die Ermittlung möglicher Fehler, die im System auftreten können. Dies geht einher mit einer Modellbildung des Systems und der Analyse der Auswirkungen möglicher Fehler [209]. Auf Basis einer erfolgreichen Fehleridentifikation ist schließlich eine Rekonfiguration der Regelung möglich, um so eine fehlertolerante Regelung zu realisieren $[4,16,160]$.

\subsection{Grundkonzepte der Fehlerdiagnose}

Grundidee der Fehlerdiagnose ist es, aus verfügbaren Daten Indikatoren zu erzeugen, die einen Rückschluss auf die einwirkenden Fehler erlauben. Diese Fehlerindikatoren werden gemäß Definition 2.3 Residuen genannt und üblicherweise mit $\boldsymbol{r}$ bezeichnet. Für eine erfolgreiche Fehlerdiagnose sind die Residuen nicht nur zu erzeugen, sondern auch entsprechend auszuwerten (s. Abbildung 2.6) um letztendlich Alarme auszulösen. Auf beide Aspekte wird in den folgenden Abschnitten eingegangen.

Dabei wird grundsätzlich zwischen passiver und aktiver Diagnose unterschieden. Bei der passiven Residuenerzeugung werden die Stellgrößen $\boldsymbol{u}$ lediglich vom Regler oder der Steuerung beeinflusst und nicht vom Diagnosesystem. Im Gegensatz dazu werden bei der aktiven Fehlerdiagnose gezielt bestimmte Eingangssignale $\boldsymbol{u}$ vorgegeben, die eine Fehlererkennung, -isolation und -identifikation erleichtern. Aktuelle Ergebnisse zur aktiven 


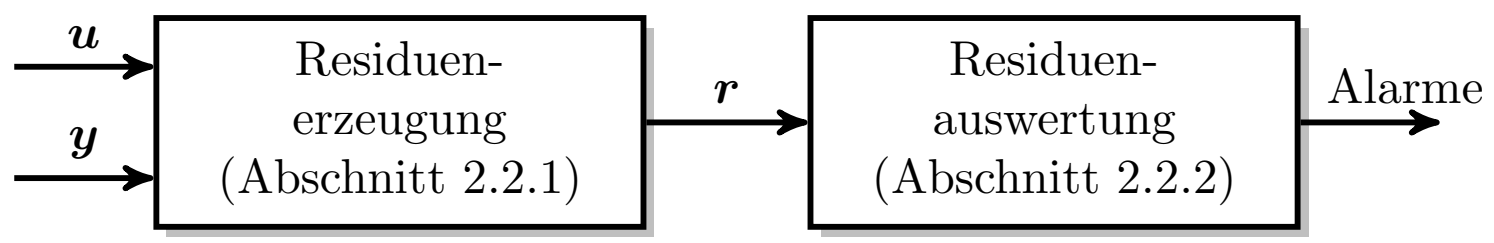

Abbildung 2.6: Auswertung generierter Residuen

Fehlerdiagnose finden sich in $[159,177]$. Gegenstand der vorliegenden Arbeit ist jedoch die passive Fehlerisolation.

\subsubsection{Residuenerzeugung}

Um die grundlegenden Aufgaben der Fehlerdiagnose, das heißt Fehlerdetektion, Fehlerisolation und Fehleridentifikation, zu bewerkstelligen, gibt es verschiedene Grundkonzepte $[32,58,78,87,111]$. Diese lassen sich gliedern in

- Verfahren basierend auf Hardwareredundanz,

- signalbasierte Methoden und

- modellbasierte Ansätze.

Zur Einordnung der im Rahmen dieser Arbeit entwickelten Verfahren werden diese grundlegenden Ansätze im Folgenden kurz erörtert, ausführlichere Betrachtungen finden sich zum Beispiel in [118].

\section{Fehlerdiagnose durch Hardwareredundanz}

Zunächst ist es möglich, kritische Systeme beziehungsweise Teilsysteme mehrfach zu verbauen. Wirkt wie in Abbildung 2.7 dargestellt ein Fehler auf das System, nicht jedoch auf das zusätzlich realisierte redundante System, so unterscheiden sich die Ausgangssignale der beiden Teilsysteme. Der Softwareteil ist vergleichsweise klein, es findet lediglich ein Abgleich der generierten Ausgangsgrößen statt. Die Differenz dieser Signale der beiden Systeme stellt die generierten Residuen dar. Ein klassisches Beispiel für eine solche Hardwareredundanz sind redundante Sensoren. Wird eine Größe von drei Sensoren gemessen, lässt sich ein fehlerhafter Sensor mit hoher Sicherheit durch eine Mehrheitsentscheidung erkennen und isolieren. 


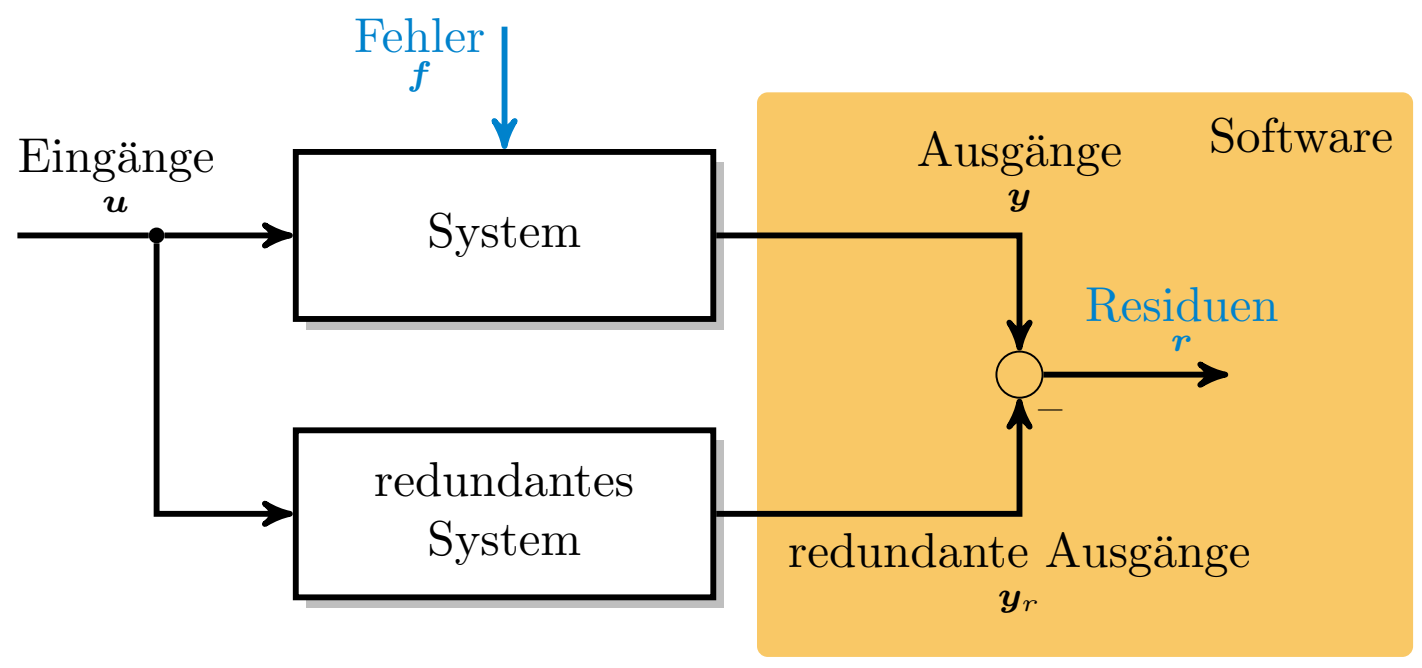

Abbildung 2.7: Fehlerdiagnose durch Hardwareredundanz

Vorteil der hardwareredundanten Fehlerdiagnose ist ihre hohe $\mathrm{Zu}$ verlässigkeit, wodurch sich ihr Einsatz bei hoch sicherheitskritischen Anwendungen erklärt. Eine absolute Sicherheit können jedoch auch redundante Sensoren nicht bieten, wie das Beispiel des Absturzes des Air-FranceFluges 447 zeigt, bei dem mehrere Geschwindigkeitssensoren gleichzeitig ausfielen [22].

Außerdem steht den Vorteilen ein hoher Aufwand gegenüber, da Investitionen in zusätzliche Hardware nötig sind. Darüber hinaus benötigen hardwareredundante Lösungen zusätzlichen Bauraum, der je nach Anwendung einen kritischen Faktor darstellen kann [204, Abschnitt 1.2].

\section{Signalbasierte Fehlerdiagnose}

Der Nachteil zusätzlicher Hardware wird bei der signalbasierten Fehlerdiagnose, die in Abbildung 2.8 skizziert ist, umgangen. Hierbei werden lediglich durch eine Filterung der Systemausgänge (und teilweise auch der Eingänge) Merkmale generiert, die einen Rückschluss auf die einwirkenden Fehler erlauben [11]. Typische Beispiele zur Erzeugung der Merkmale sind Bandpassfilter oder Spektralanalysen [204, Abschnitt 1.3].

Bei der signalbasierten Fehlerdiagnose kommt der Auswertung der generierten Merkmale eine große Bedeutung zu. Neben Mittelwert- und Varianzschätzungen und weiteren stochastischen Methoden kommen dabei häufig Verfahren aus dem Bereich der Mustererkennung zum Einsatz, insbesondere für die Isolation von Fehlern. Neben der sogenannten Principal Component Analysis [61] werden dabei Support Vector Ma- 


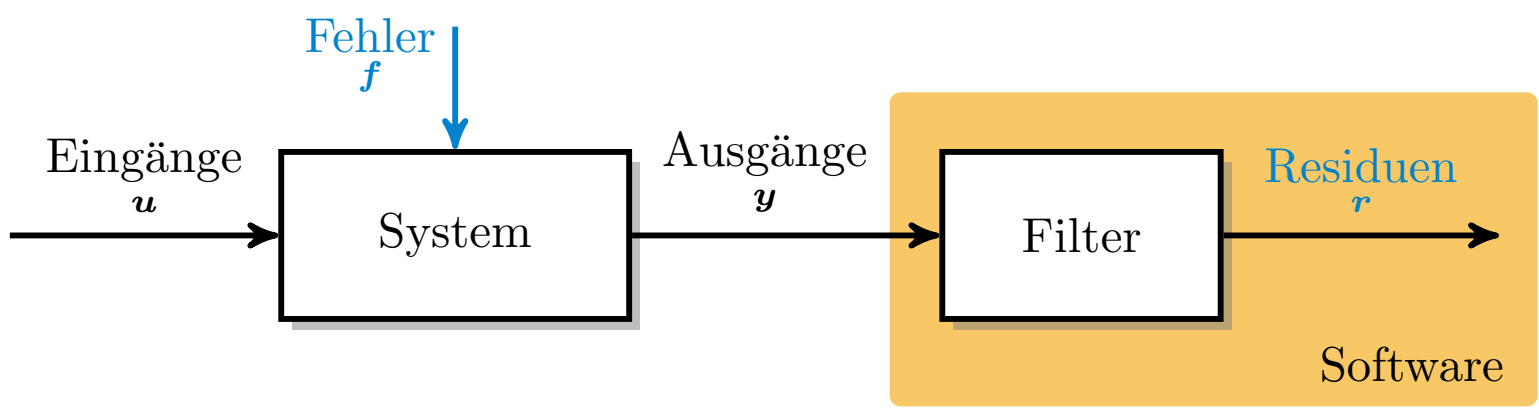

Abbildung 2.8: Signalbasierte Fehlerdiagnose

chines [256], statische Neuro-Fuzzy-Systeme [166] und fortgeschrittene Clustering-Algorithmen [10] angewendet. Der zusätzliche Aufwand wird also in den Softwareteil verlagert, was gegenüber redundanter Hardware wesentlich kostengünstiger ist. Allerdings sind signalbasierte Methoden häufig beschränkt auf stationäre Zusammenhänge oder auf höhere Abstraktionsebenen von Prozessen [55].

\section{Modellbasierte Fehlerdiagnose}

Als eine Verbindung der Vorteile beider Ansätze kann die modellbasierte Fehlerdiagnose aufgefasst werden. Sie beruht ebenfalls auf der Generierung redundanter Größen. Diese werden jedoch nicht durch redundante Hardware erzeugt, sondern durch ein Prozessmodell (s. Abbildung 2.9), welches beschreibt, wie sich das fehlerfreie System verhält. Es wird daher auch häufig von analytischer Redundanz gesprochen.

Grundsätzlich ist dabei zu unterscheiden, ob quantitatives Prozesswissen in Form expliziter Systemmodellgleichungen vorliegt oder lediglich qualitatives Wissen. Liegt qualitatives Wissen vor, so lässt sich die Dynamik mithilfe rekurrenter Fuzzy-Systeme beschreiben. Diese erlauben es, die Dynamik von Systemen wissensbasiert zu modellieren [2, 3, 71, 120, 198], was es ermöglicht, rekurrente Fuzzy-Systeme auch zur Fehlererkennung und -isolation einzusetzen [198, 199]. Dies ist insbesondere dann sinnvoll, wenn eine analytische Beschreibung des Modellverhaltens nicht oder nur mit sehr großem Aufwand möglich ist, Expertenwissen über das qualitative Verhalten des Systems jedoch vorhanden ist. Dies impliziert, dass keine Einschränkung auf Systeme mit linearer Dynamik besteht. Im Rahmen dieser Arbeit wird jedoch stets davon ausgegangen, dass explizites, quantitatives Wissen über den Prozess zur Verfügung steht, sich die Dynamik also durch ein System von Differentialgleichungen beschreiben lässt. Der 


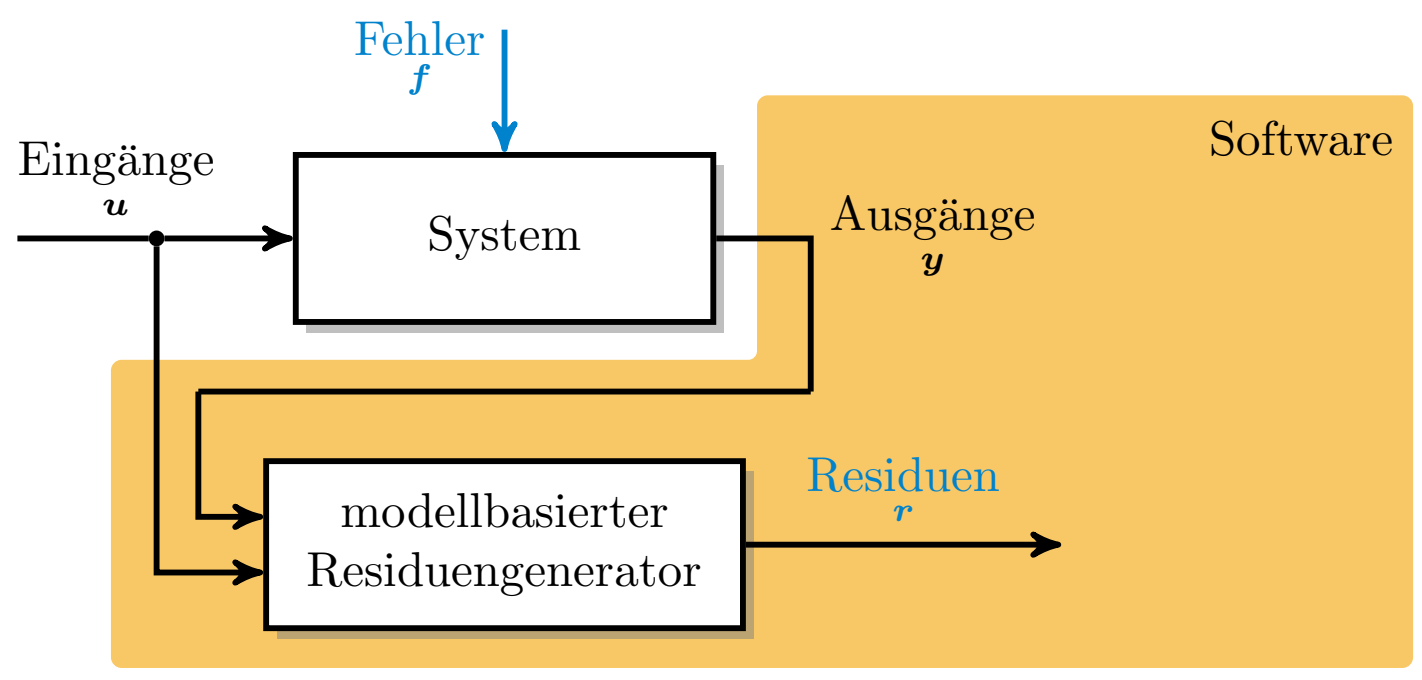

Abbildung 2.9: Modellbasierte Fehlerdiagnose

Begriff der modellbasierten Fehlerdiagnose zielt demnach im Weiteren stets auf die Fehlerdiagnose mittels explizitem, quantitativem Modellwissen ab.

Steht das gemessene Verhalten des Systems nicht im Einklang mit dem Verhalten des Prozessmodells, so kann auf den beziehungsweise die Fehler zurückgeschlossen werden. Modellbasierte Ansätze sind ebenso wie signalbasierte Methoden kostengünstig zu realisieren, da sich der Aufwand prinzipiell auf die Softwareseite beschränkt. Durch die analytische Redundanz ist dabei gleichzeitig eine robuste Fehlerdiagnose und eine Erfüllung aller wesentlichen Aufgaben (Detektion, Isolation, Identifikation) möglich. Eine vergleichende Betrachtung signal- und modellbasierter Verfahren findet sich in [94] sowie in [219-221]. Darüber hinaus zeigen jüngste Entwicklungen, wie sich Verfahren, die ursprünglich für signalbasierte Methoden entwickelt wurden, für den automatisierten Entwurf modellbasierter Residuengeneratoren heranziehen lassen [55, 248].

Es ist allerdings zu betonen, dass eine zuverlässige Fehlerdiagnose ein geeignetes und möglichst genau bekanntes Prozessmodell erfordert. Die Robustheit der Fehlerdiagnose diesbezüglich macht einen wesentlichen Teil der Problemstellung der vorliegenden Arbeit aus. Ein Überblick über relevante Literatur aus dem Bereich der modellbasierten Fehlerdiagnose wird in Abschnitt 2.4 gegeben, da zuvor die Ziele der modellbasierten Fehlerisolation präzisiert werden müssen. 


\subsubsection{Residuenauswertung}

Den Abbildungen 2.7 bis 2.9 ist gemeinsam, dass sie lediglich die Generierung von Residuen zeigen. Für den praktischen Einsatz von Fehlerdiagnoseverfahren ist darüber hinaus die Residuenauswertung von großer Bedeutung. Dieser Schritt schließt sich der Residuenerzeugung an. Es wird dort zum Beispiel wie in Abbildung 2.6 dargestellt die Entscheidung darüber getroffen, ob ein bestimmter Alarm ausgelöst wird. Diese Arbeit beschränkt sich weitgehend auf die Residuengenerierung, weshalb die Residuenauswertung im Folgenden lediglich kurz beschrieben wird.

Eine einfache und häufig verwendete Technik für die Residuenauswertung sind statische Schwellenwerte. Überschreitet ein Residuum einen solchen Schwellenwert, wird ein Alarm ausgelöst. Problematisch ist dabei die Wahl der Schwellenwerte - ein zu niedriger Wert führt zu häufigen Fehlalarmen, ein zu hoher Wert führt dazu, dass Alarme fälschlicherweise nicht ausgelöst werden. Weiterhin kann es zu einem An- und Abschalten des Alarms kommen, wenn das Residuum um den Grenzwert schwingt. Dieses letztere unerwünschte Verhalten lässt sich durch eine Hysterese beheben.

Die Idee der systematischen Auslegung von Grenzwerten geht auf [66] zurück. Erweiterungen hat das Konzept in [40, 41, 57, 75, 103, 116, 153, 178] erfahren, wobei vielfach dynamische Grenzwerte zum Einsatz kommen, die einen besseren Kompromiss zwischen Fehlalarmen und nicht gemeldeten kritischen Situationen ermöglichen. Eine ausführliche Übersicht über normbasierte und statistische Verfahren zur Residuenauswertung findet sich in [58, Kapitel 9 und 10].

\subsection{Ziele der modellbasierten Fehlerisolation}

In diesem Abschnitt werden die Ziele der modellbasierten Fehlerisolation aufgeführt und miteinander in Bezug gesetzt. Sie ergeben sich aus den grundsätzlichen Anforderungen an das Diagnosesystem: Es muss in der Lage sein, Fehler zeitnah und mit hoher Zuverlässigkeit zu erkennen und zu isolieren. Dies bedeutet, dass Fehler (im Verhältnis zur Systemdynamik) schnell erkannt werden müssen. Die Zuverlässigkeit drückt sich zum einen in einer niedrigen Fehlalarmrate aus, zum anderen in einer niedrigen Rate nicht registrierter kritischer Situationen.

Aus diesen übergeordneten Überlegungen ergeben sich die in Abbildung 2.10 zusammengefassten Ziele. Diese werden im Folgenden präzisiert, wobei Abbildung 2.10 näher erläutert wird. 


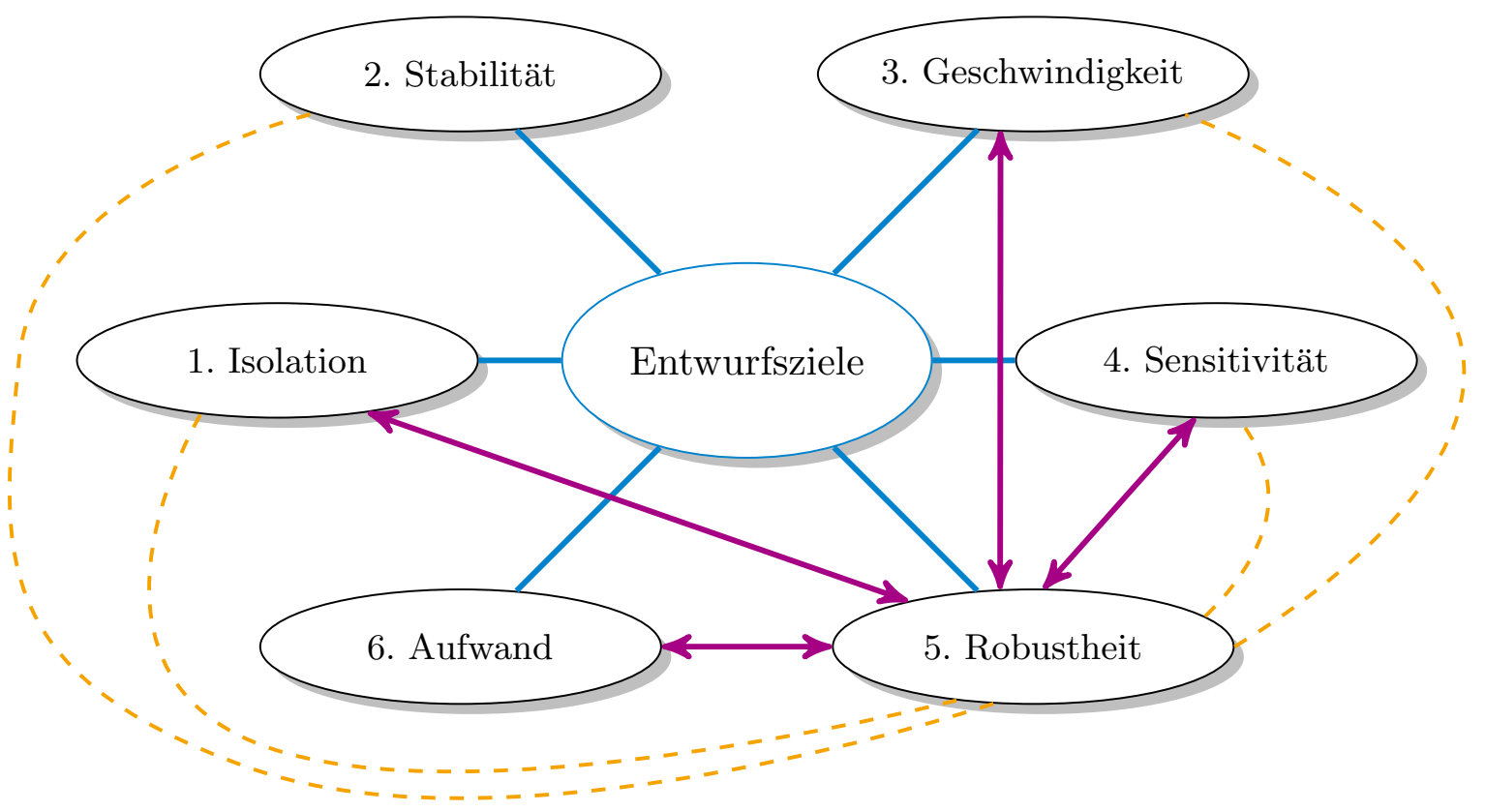

Abbildung 2.10: Entwurfsziele der modellbasierten Fehlerisolation

\section{Strukturierung der Residuen}

Grundidee der Fehlerisolation ist es, die Residuen derart zu generieren, dass anhand dieser Fehlerindikatoren nicht nur eine Fehlererkennung, sondern eine Lokalisierung der Fehler möglich wird. Systematisch verfolgt wurde dieser Ansatz erstmals in [13, 117], wobei dem erzeugten Residuenvektor für jede mögliche Fehlerkonfiguration eine feste Richtung aufgeprägt wird. Somit ist durch Auswertung der Richtung des Residuenvektors ein Rückschluss auf den beziehungsweise die einwirkenden Fehler möglich. Beispielhaft sind in Abbildung 2.11 zwei Residuenvektoren $\boldsymbol{r}_{f_{1}}^{*}$ und $\boldsymbol{r}_{f_{2}}^{*}$ eingezeichnet, die sich jeweils bei der Einwirkung des Fehlers $f_{1}$ beziehungsweise $f_{2}$ auf das System ergeben. Da die Residuenvektoren linear unabhängig sind, kann im stationären Fall gezielt auf einen der beiden Fehler zurückgeschlossen werden.

Ein Spezialfall obigen Sachverhaltes ist offensichtlich dadurch gegeben, dass die erzeugten Residuenvektoren für unterschiedliche Fehler orthogonal zueinander sind, wie dies ebenfalls in Abbildung 2.11 durch die Vektoren $\boldsymbol{r}_{f_{1}}$ und $\boldsymbol{r}_{f_{2}}$ dargestellt ist. Sind in linearen Systemen die Residuenvektoren nicht nur im stationären Fall, sondern auch bei transienten Vorgängen orthogonal zueinander, so weist die Übertragungsmatrix, welche die Fehler mit den Residuen verknüpft, Diagonalgestalt auf. Diese Matrix wird mit 


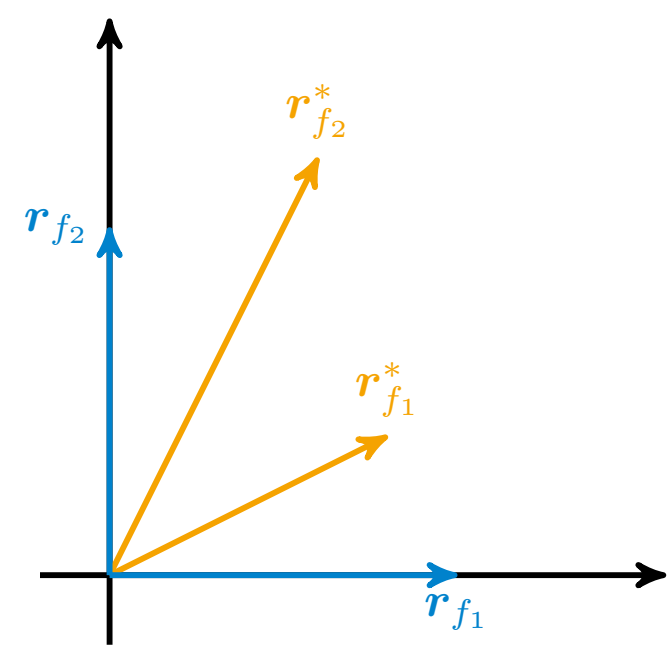

Abbildung 2.11: Richtung der Residuenvektoren bei unterschiedlichen Fehlerkonfigurationen

$\boldsymbol{G}_{\boldsymbol{r} \boldsymbol{f}}(s)$ bezeichnet. Gilt

$$
\boldsymbol{G}_{\boldsymbol{r} \boldsymbol{f}}(s)=\left[\begin{array}{cccc}
g_{1,1}(s) & 0 & \cdots & 0 \\
0 & \ddots & \ddots & \vdots \\
\vdots & \ddots & \ddots & 0 \\
0 & \cdots & 0 & g_{n_{f}, n_{f}}(s)
\end{array}\right]=\operatorname{diag}\left(g_{1,1}(s), \ldots, g_{n_{f}, n_{f}}(s)\right)
$$

so wird von vollständiger Fehlerisolation (engl. full fault isolation, FFI) gesprochen, da dann der $i$-te Fehler ausschließlich das ihm zugeordnete $i$-te Residuum beeinflusst. Somit kann von dem entsprechenden Residuum $r_{i}$ eindeutig auf den zugehörigen Fehler $f_{i}$ zurückgeschlossen werden. Dieser kann selbst dann von allen anderen Fehlern isoliert werden, wenn diese gleichzeitig auftreten beziehungsweise zur gleichen Zeit aktiv sind, was sich aus der Orthogonalität der Residuenvektoren bei unterschiedlichen einwirkenden Fehlern ergibt. Wird lediglich der eingeschwungene $\mathrm{Zu}-$ stand betrachtet, so ist eine Diagonalstruktur von $\boldsymbol{G}_{\boldsymbol{r} \boldsymbol{f}}(s=0)$ hinreichend für eine Fehlerisolation. Unter der vollständigen Fehlerisolation wird im Rahmen dieser Arbeit jedoch stets die vollständige Diagonalisierung von $\boldsymbol{G}_{\boldsymbol{r} \boldsymbol{f}}(s)$ für alle $s \in \mathbb{C}$ verstanden.

Ein einfaches Schema zur Auswertung von Residuen, die (2.7) genügen, ist in Abbildung 2.12 dargestellt. Dabei wird zur Residuenauswertung lediglich ein einfacher, statischer Schwellenwert verwendet. Wie in Abschnitt 2.2.2 diskutiert, existieren in dieser Hinsicht fortgeschrittenere Verfahren. Überschreitet ein Residuum $r_{i}$ betragsmäßig einen festgelegten 

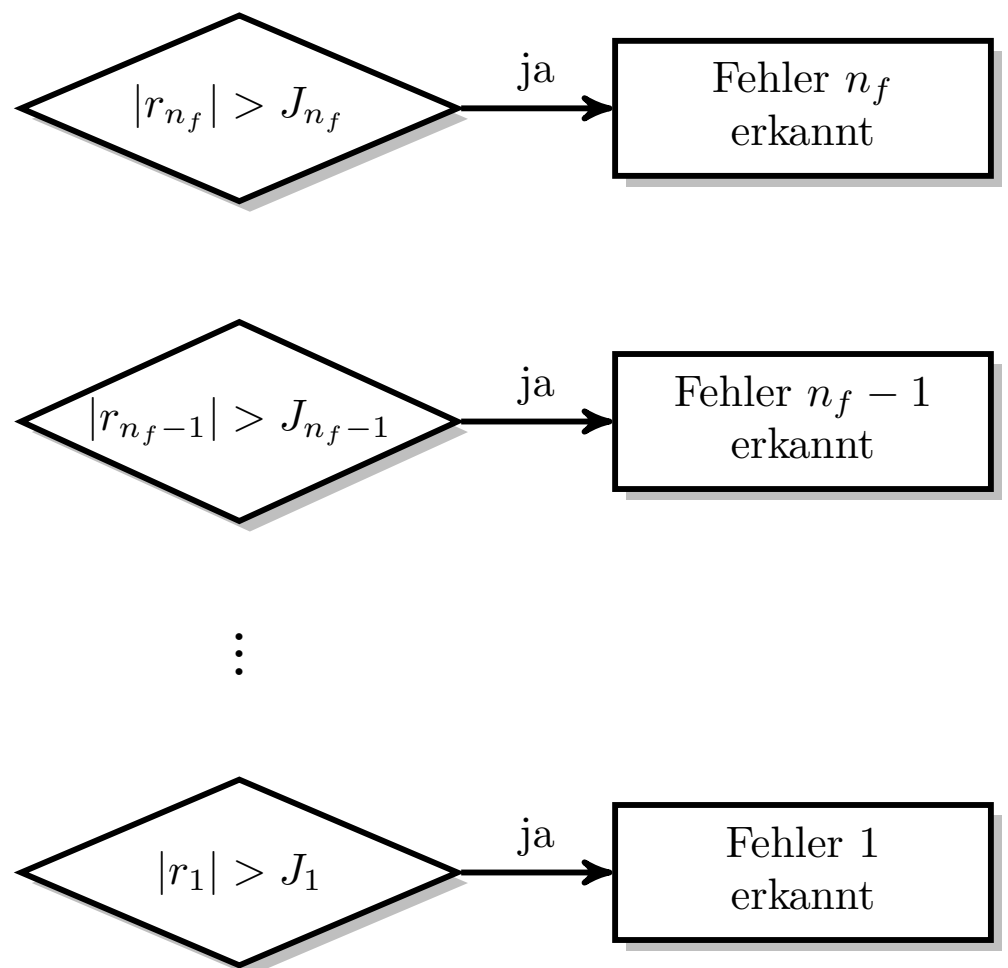

Abbildung 2.12: Ablaufplan der Auswertelogik bei vollständiger Fehlerisolation

Wert $J_{i}$, so wird ein entsprechender Alarm ausgelöst. Die Auswertung der einzelnen Residuen kann dabei aufgrund der Diagonalstruktur von $\boldsymbol{G}_{\boldsymbol{r} \boldsymbol{f}}(s)$ in (2.7) parallel erfolgen. Liegen alle Residuen unterhalb des jeweiligen Schwellenwertes, so ist das System fehlerfrei.

Offensichtlich bedingt die Forderung nach (2.7) starke strukturelle Einschränkungen der Matrix $\boldsymbol{G}_{\boldsymbol{r} \boldsymbol{f}}(s)$. Wie im Verlauf der Arbeit gezeigt wird, führt dies zum einen zu relativ restriktiven Bedingungen für die Existenz von Fehlerisolationsbeobachtern beziehungsweise -filtern. Zum anderen wird ein Großteil der zur Verfügung stehenden Entwurfsfreiheitsgrade zur Einhaltung der strukturellen Beschränkungen benötigt. In der praktischen Anwendung sind jedoch oftmals weniger restriktive Strukturen von $\boldsymbol{G}_{\boldsymbol{r} \boldsymbol{f}}(s)$ ausreichend.

Dies ist beispielsweise dann der Fall, wenn vorausgesetzt werden kann, dass Fehler in einem System nicht gleichzeitig auftreten können. Bedingt sein kann dies einerseits durch kausale Abhängigkeiten zwischen verschiedenen Fehlern. Andererseits ist es bei einer entsprechend schnell ausgelegten Fehlerisolation plausibel, dass ein aufgetretener Fehler behoben wird, bevor ein weiterer Fehler auftritt. Wird somit vorausgesetzt, dass lediglich ein einziger Fehler in einem System zu einem Zeitpunkt aktiv sein kann, so 
ist eine eindeutige Fehlerisolation auch dann möglich, wenn $\boldsymbol{G}_{\boldsymbol{r} \boldsymbol{f}}(s)$ eine obere Dreiecksstruktur aufweist, das heißt wenn

$$
\boldsymbol{G}_{\boldsymbol{r} \boldsymbol{f}}(s)=\left[\begin{array}{cccc}
g_{1,1}(s) & g_{1,2}(s) & \cdots & g_{1, n_{f}}(s) \\
0 & g_{2,2}(s) & \ddots & \vdots \\
\vdots & \ddots & \ddots & g_{n_{f}-1, n_{f}}(s) \\
0 & \cdots & 0 & g_{n_{f}, n_{f}}(s)
\end{array}\right]
$$

gilt.

Wird erkannt, dass das Residuum $r_{n_{f}}$ aktiv ist, das heißt $\left|r_{n_{f}}\right|>J_{n_{f}}$, so wird der Fehler $f_{n_{f}}$ isoliert und die Auswertung ist abgeschlossen. Ist $\left|r_{n_{f}}\right| \leq J_{n_{f}}$, so wird $r_{n_{f}-1}$ betrachtet. Wird lediglich der stationäre Fall betrachtet, so ist eine eindeutige Isolation gleichzeitig aktiver Fehler auch mittels (2.8) möglich. Entsprechend der Dreiecksstruktur von $\boldsymbol{G}_{\boldsymbol{r} \boldsymbol{f}}(s)$ ist

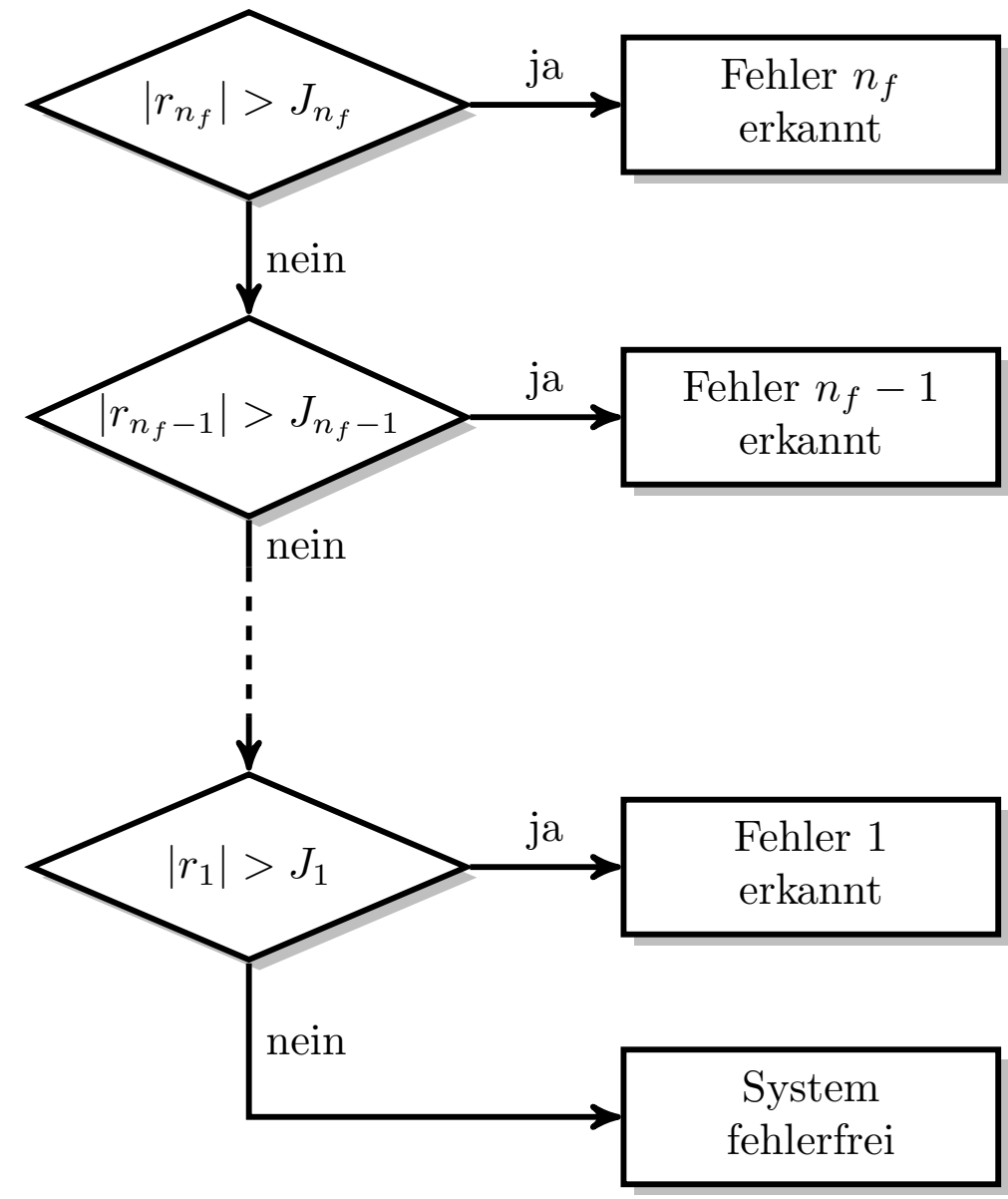

Abbildung 2.13: Ablaufplan der Auswertelogik bei teilweiser Fehlerisolation 


$$
\begin{aligned}
r_{n_{f}-1} & =g_{n_{f}-1, n_{f}-1}(s) \cdot f_{n_{f}-1}+g_{n_{f}-1, n_{f}}(s) \cdot f_{n_{f}}, \\
r_{n_{f}} & =g_{n_{f}, n_{f}}(s) \cdot f_{n_{f}} .
\end{aligned}
$$

Durch Umformen dieser Gleichungen nach $f_{n_{f}-1}$ und Auswertung für $s=0$ ergibt sich, dass bei der Auswertung von $r_{n_{f}-1}$ die Bedingung

$$
\left|\frac{r_{n_{f}-1}}{g_{n_{f}-1, n_{f}-1}(s=0)}-\frac{g_{n_{f}-1, n_{f}}(s=0) \cdot r_{n_{f}}}{g_{n_{f}-1, n_{f}-1}(s=0) \cdot g_{n_{f}, n_{f}}(s=0)}\right| \leq J_{n_{f}-1}
$$

zu überprüfen ist. Es wird also der stationäre Einfluss des Fehlers $f_{n_{f}}$ auf das Residuum $r_{n_{f}-1}$ heraus gerechnet, bevor der Vergleich mit dem Schwellenwert stattfindet. Dies wird jedoch nicht weiter verfolgt, da wie bereits erwähnt im Rahmen dieser Arbeit stets eine Fehlerisolation für alle $s \in \mathbb{C}$ angestrebt wird.

Abbildung 2.13 veranschaulicht, dass die Auswertung der Residuen im Gegensatz zur vollständigen Fehlerisolation (vgl. Abbildung 2.12) sequentiell erfolgt. Nach dem beschriebenen Schema werden gegebenenfalls alle Residuen ausgewertet und der aktive Fehler wird nicht nur erkannt, sondern auch eindeutig isoliert. Eine vergleichbare Auswertelogik ist natürlich auch für den Fall einer unteren Dreieckstruktur von $\boldsymbol{G}_{\boldsymbol{r} \boldsymbol{f}}(s)$ realisierbar. Da $\boldsymbol{G}_{\boldsymbol{r} \boldsymbol{f}}(s)$ in (2.8) nicht vollständig diagonalisiert ist, wird in Anlehnung an die Ergebnisse zur teilweisen Entkopplung [136] in dieser Arbeit von teilweiser oder partieller Fehlerisolation (engl. partial fault isolation, PFI) gesprochen. Eine geometrische Interpretation des Sachverhaltes ist in Abbildung 2.14 für ein System mit drei Fehlern dargestellt. Das Auftreten des Fehlers $f_{3}$ ruft einen Residuenvektor $\boldsymbol{r}_{f_{3}}$ hervor, der ausschließlich eine

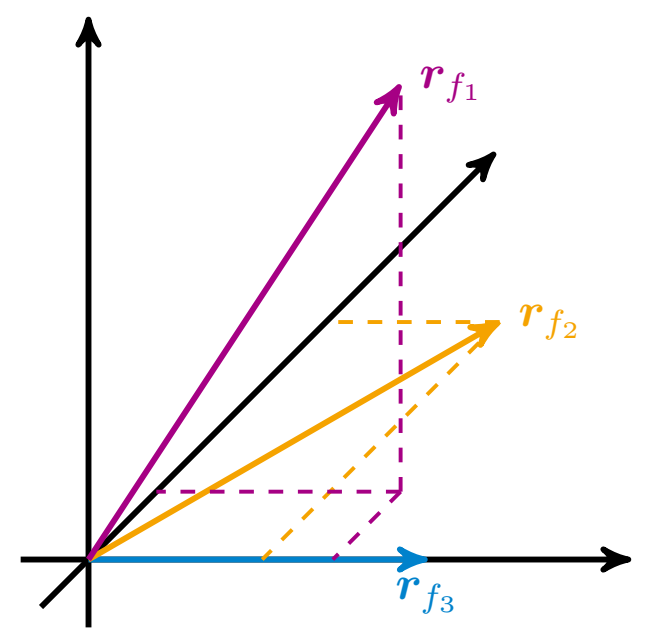

Abbildung 2.14: Richtung der Residuenvektoren bei teilweiser Fehlerisolation 
Komponente in einer Koordinatenrichtung besitzt. Der Fehler $f_{2}$ bedingt einen Residuenvektor $\boldsymbol{r}_{f_{2}}$, der Komponenten in zwei Koordinatenrichtungen enthält. Der Fehler $f_{1}$ erzeugt schließlich einen Residuenvektor $\boldsymbol{r}_{f_{1}}$, der darüber hinaus auch über eine Komponente in der dritten Koordinatenrichtung verfügt.

Die vollständige Diagonalisierung von $\boldsymbol{G}_{\boldsymbol{r} \boldsymbol{f}}(s)$ wie in (2.7) und die Dreiecksstruktur aus (2.8) stellen dabei zwei Extreme dar. Während (2.7) zur Isolation notwendig ist, falls alle Fehler im System gleichzeitig auftreten können, ist (2.8) hinreichend, falls jeweils nur ein Fehler zu einem Zeitpunkt aktiv sein kann. Selbstverständlich sind Zwischenstufen möglich, falls einige Fehler zeitgleich auftreten können, dies jedoch für bestimmte Fehler ausgeschlossen ist. Auf diese Situation wird im Abschnitt zur teilweisen Fehlerisolation (Abschnitt 4.10) näher eingegangen.

\section{Stabilität}

Für die Realisierung des entworfenen Filters beziehungsweise Beobachters ist es unerlässlich, dass die Dynamikmatrix des Filters eine Hurwitz-Matrix ist. Im weiteren Verlauf der Arbeit wird gezeigt, dass dies insbesondere bei einer entsprechenden Strukturierung von $\boldsymbol{G}_{\boldsymbol{r} \boldsymbol{f}}(s)$ nicht immer garantiert ist. Dies rührt daher, dass das Filter eine interne Dynamik aufweisen kann, die anhand von $\boldsymbol{G}_{\boldsymbol{r} \boldsymbol{f}}(s)$ nicht ersichtlich ist.

\section{Geschwindigkeit}

Ein weiterer wesentlicher Punkt ist eine ausreichende Geschwindigkeit bei der Erkennung beziehungsweise Isolation der Fehler. Nur wenn diese zeitnah isoliert werden, können rechtzeitig entsprechende Gegenmaßnahmen eingeleitet werden, die eventuellen Folgefehlern oder Schäden vorbeugen. Dies kann das Umschalten auf einen sicheren Betriebszustand bedeuten oder auch eine Rekonfiguration der Regelung. Die Geschwindigkeit der Fehlerdetektion ist dabei stets im Verhältnis zur Dynamik des betrachteten Systems zu setzen. Üblicherweise wird man die Dynamik des Fehlerdiagnosesystems daher schneller auslegen als die der betrachteten Strecke.

\section{Sensitivität}

Der Begriff der Sensitivität beschreibt, wie stark die generierten Residuen auf einwirkende Fehler reagieren, sie bezieht sich also auf die Übertragungsmatrix $\boldsymbol{G}_{\boldsymbol{r} \boldsymbol{f}}(s)$. Eine hohe Sensitivität bewirkt, dass selbst 
kleine Fehleramplituden zu großen und damit leicht detektierbaren Amplituden der Residuen führen. Im Bereich des Reglerentwurfs wird meist eine stationäre Verstärkung von 1 zwischen Führungs- und geregelten Ausgangsgrößen angestrebt, um gewünschte Sollgrößen einzuregeln. Bei der Fehlerisolation ist dies nicht immer erstrebenswert. Im Sinne einer hohen Sensitivität ist es einerseits oftmals wünschenswert, eine stationäre Verstärkung deutlich größer als $1 \mathrm{zu}$ realisieren. Andererseits konvergiert bei einer stationären Verstärkung von 1 und sprungförmigen Fehlern das jeweilige Residuum asymptotisch gegen den Wert des Fehlers. Somit ist über die Isolation des Fehlers hinaus auch eine Identifikation möglich, da die Größe des Fehlers mitbestimmt wird.

\section{Robustheit}

Sind alle bisher formulierten Entwurfsziele erfüllt, lässt sich für exakt bekannte und störungsfreie Systeme bereits eine ideale Fehlerisolation erreichen. Es ist jedoch zu beachten, dass das beim Entwurf verwendete Modell des Systems inhärent sowohl eine Abstraktion als auch eine Vereinfachung des tatsächlichen Verhaltens darstellt. Darüber hinaus können Parameter des Systems Alterungseffekten unterliegen oder aus anderen Gründen unsicherheitsbehaftet sein. Für den praktischen Einsatz modellbasierter Fehlerisolationssysteme ist daher die Anforderung nach einer möglichst hohen Robustheit gegenüber derartigen Modellunsicherheiten von größter Bedeutung. Sie betrifft zum einen die bisher aufgeführten Ziele der Strukturierung der Residuen, der Stabilität, der Erkennungsgeschwindigkeit und der Sensitivität, was durch die strichlierten gelben Verbindungen in Abbildung 2.10 angedeutet wird. Alle diese Entwurfsziele beziehen sich auf das Ein-/Ausgangsverhalten zwischen den auftretenden Fehlern und den generierten Residuen, welches durch $\boldsymbol{G}_{\boldsymbol{r} \boldsymbol{f}}(s)$ beschrieben wird. Zum anderen stellt sich die Robustheitsanforderung aber auch bezüglich des Einflusses der Stellgrößen des Systems auf die Residuen. Im Idealfall sollten diese keinerlei Wirkung auf die generierten Fehlerindikatoren haben, das heißt es wird $\boldsymbol{G}_{\boldsymbol{r u}}(s)=\mathbf{0}$ angestrebt.

Neben den angesprochenen Modellunsicherheiten unterliegen reale Systeme immer auch exogenen Störeinflüssen, die mit $\boldsymbol{d}$ bezeichnet werden und deren Einfluss auf die Residuen ebenfalls zu unterdrücken ist. Dies beinhaltet zum Beispiel Sensorrauschen, es können aber auch Störungen auftreten, die auf die Systemdynamik wirken. Ein Beispiel hierfür sind vernachlässigte Nichtlinearitäten, die als exogene Störungen modelliert werden. Auch hinsichtlich der Störungen wird $\boldsymbol{G}_{\boldsymbol{r} \boldsymbol{d}}(s)=\mathbf{0}$ angestrebt. 
Zusammenfassend sind der Robustheitsanforderung die folgenden Punkte zuzuordnen:

1. Einhaltung der Stabilität des Filters sowie der Vorgaben hinsichtlich Strukturierung der Residuen, Erkennungsgeschwindigkeit und Sensitivität trotz vorhandener Modellunsicherheiten $\left(\boldsymbol{G}_{\boldsymbol{r} \boldsymbol{f}}(s)\right)$,

2. Unterdrückung des Einflusses der Stellgrößen auf die generierten Residuen trotz vorhandener Modellunsicherheiten $\left(\boldsymbol{G}_{\boldsymbol{r} \boldsymbol{u}}(s)\right)$,

3. Unterdrückung des Einflusses exogener Störgrößen auf die generierten Residuen $\left(\boldsymbol{G}_{\boldsymbol{r} \boldsymbol{d}}(s)\right)$.

Dabei wird stets angenommen, dass die exogenen Störungen $\boldsymbol{d}$ nicht messbar sind und dass sie auch nicht durch ein Störmodell modelliert werden können. In einem solchen Fall wäre es stets möglich, mittels einer geeigneten Störgrößenaufschaltung den Einfluss der Störungen auf die erzeugten Residuen zu unterdrücken beziehungsweise abzuschwächen.

\section{Entwurfs- und Implementierungsaufwand}

Zusätzlich zu den genannten Zielen wird ein möglichst geringer Entwurfsund insbesondere Implementierungsaufwand angestrebt. Ein niedriger Entwurfsaufwand schließt dabei sowohl die Transparenz der Verfahren als auch den rechnerischen Aufwand ein, was die Anwendbarkeit der entwickelten Methoden erhöht. Ein geringer Implementierungsaufwand ist trotz immer schnellerer Computer und steigender verfügbarer Rechenkapazitäten wünschenswert, da gerade in eingebetteten Systemen Speicherkapazität und Rechenleistung für Online-Diagnosesysteme häufig eingeschränkt sind.

\section{Zielkonflikte}

Häufig steht die Robustheitsforderung im Zielkonflikt mit anderen Entwurfszielen, was in Abbildung 2.10 durch die magentafarbenen Pfeile gekennzeichnet ist. Typischerweise konkurriert die Robustheitsforderung insbesondere mit der Forderung nach einer hohen Fehlersensitivität [32], sodass eine Abwägung beziehungsweise Optimierung hinsichtlich zu definierender Gütemaße erfolgen muss. Ebenso wird im weiteren Verlauf der Arbeit deutlich, dass zur Sicherstellung einer Struktur gemäß (2.7) beziehungsweise (2.8) ein Teil der zur Verfügung stehenden Entwurfsfreiheitsgrade herangezogen werden muss. Dadurch reduziert sich die Anzahl der Freiheitsgrade, die zur Optimierung der Robustheit zur Verfügung stehen. 
Insbesondere bei hochfrequenten exogenen Störsignalen kann sich eine zu hohe Erkennungsgeschwindigkeit negativ auf die Robustheit auswirken. Nicht zuletzt sind die beobachterbasierten Verfahren, welche die Robustheit berücksichtigen, mit zusätzlichem Entwurfsaufwand gegenüber dem nominalen Fall verbunden.

Im Rahmen dieser Arbeit wird der Ansatz verfolgt, bei gegebenen Anforderungen hinsichtlich der Strukturierung der Residuen, der Erkennungsgeschwindigkeit und der Fehlersensitivität die Robustheit zu optimieren. Auf die Quantifizierung der bisher lediglich qualitativ beschriebenen Ziele wird im weiteren Verlauf der Arbeit näher eingegangen.

\subsection{Ansätze zur modellbasierten Fehlerdiagnose}

Wie in Abschnitt 2.2 erörtert existieren verschiedene Grundkonzepte für die modellbasierte Fehlerdiagnose. Die modellbasierten Ansätze lassen sich

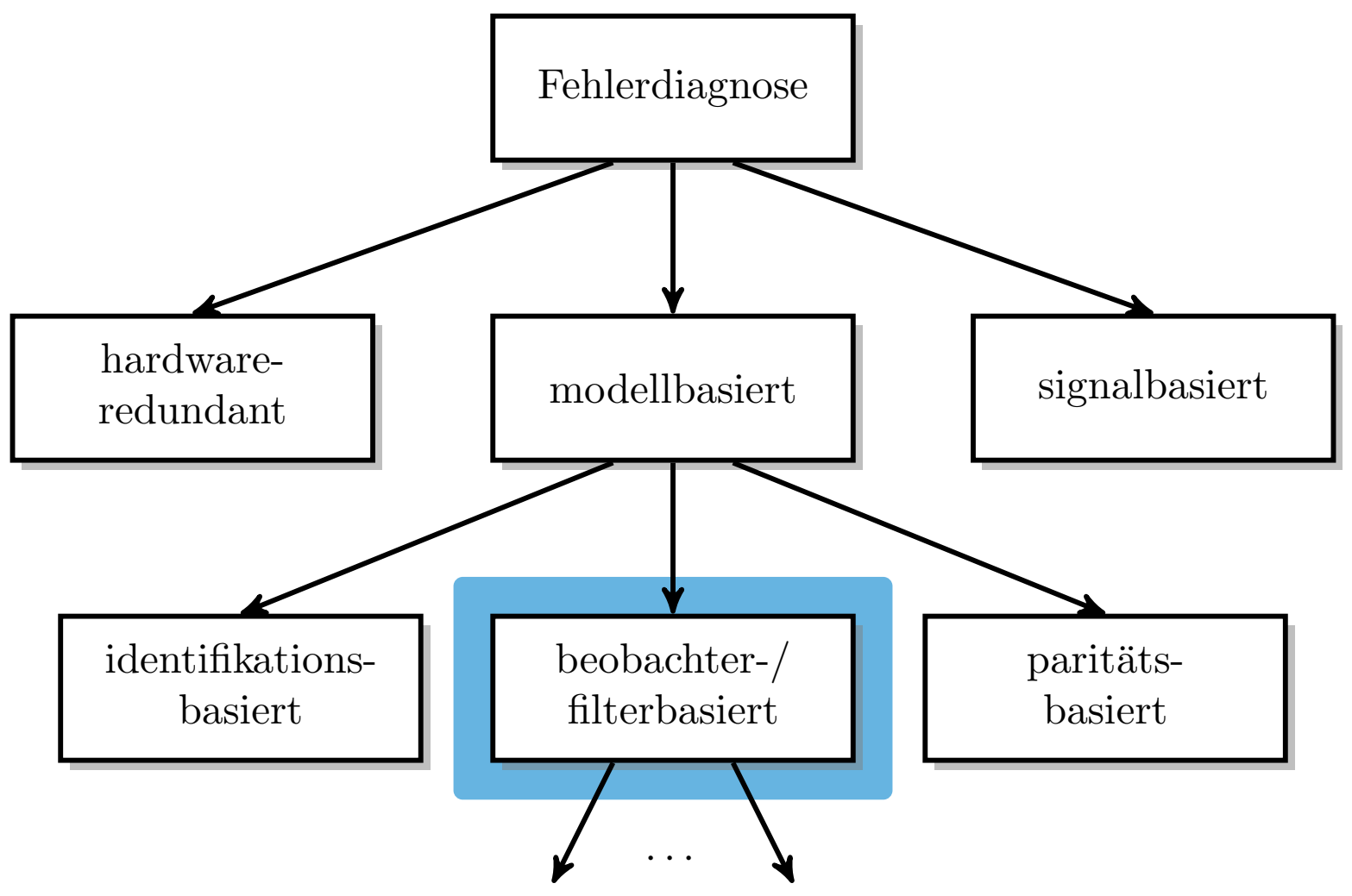

siehe Abschnitt 2.5

Abbildung 2.15: Zur Einordnung beobachterbasierter Fehlerdiagnosesysteme 
wie in Abbildung 2.15 gezeigt weiter unterteilen in

- identifikationsbasierte Ansätze,

- Ansätze basierend auf Paritätsgleichungen und

- beobachter- beziehungsweise filterbasierte Ansätze.

Eine ausführliche, vergleichende Betrachtung der Ansätze findet sich in [32, Abschnitt 2.13], an dieser Stelle werden lediglich die jeweils grundlegenden Konzepte von identifikations- und paritätsbasierten Verfahren erörtert.

Die in dieser Arbeit entwickelten Verfahren sind dem in Abbildung 2.15 hervorgehobenen Bereich der beobachter- beziehungsweise filterbasierten Fehlerdiagnose zuzuordnen. Daher werden bestehende Konzepte und Ansätze dazu in Abschnitt 2.5 in ausführlicher Form gesondert dargestellt.

\section{Identifikationsbasierte Ansätze zur Fehlerdiagnose}

Eine ausführliche Übersicht zu identifikationsbasierten Methoden findet sich unter anderem in [110, 111, 204]. Grundgedanke ist, dass sich auftretende Fehler im Ein-/Ausgangsverhalten eines Systems niederschlagen. Für ein lineares zeitdiskretes SISO-System lässt sich dieses Verhalten durch

$$
\begin{array}{r}
a_{n} y_{k-n}+a_{n-1} y_{k-n+1}+\ldots+a_{1} y_{k-1}+y_{k} \\
=b_{m} u_{k-m}+\ldots+b_{1} u_{k-1}+b_{0} u_{k}
\end{array}
$$

beschreiben. Dabei werden die Koeffizienten $a_{i}$ und $b_{j}$ als bekannt vorausgesetzt, sie beschreiben das nominale Systemverhalten im fehlerfreien Fall. Aus (2.9) lässt sich die Darstellung

$$
y_{k}=\boldsymbol{\Psi}^{\top} \cdot \boldsymbol{\Theta}
$$

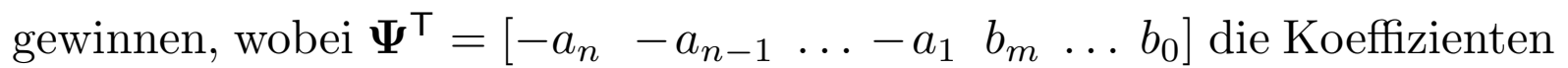

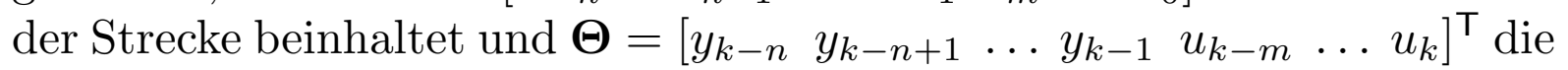
bekannten Werte von $y_{k}$ und $u_{k}$ zu den jeweiligen Zeitschritten. Auf Basis dieser Beschreibung wird eine Online-Parameterschätzung durchgeführt, indem die euklidische Norm von $y_{k}-\hat{\mathbf{\Psi}}^{\top} \boldsymbol{\Theta}$ minimiert wird. Daraus ergibt sich der Schätzwert für die Streckenkoeffizienten zu

$$
\hat{\mathbf{\Psi}}^{\top}=y_{k} \cdot \boldsymbol{\Theta}^{+} .
$$

Bei diesem einfachen Schema ist in jedem Zeitschritt die Pseudoinverse von $\Theta \mathrm{zu}$ berechnen. Es existieren jedoch auch rekursive Implementierungen, die dieses Problem umgehen [107]. 
Tritt eine Diskrepanz zwischen dem nominalen Vektor $\boldsymbol{\Psi}^{\top}$ und dem Schätzwert $\hat{\mathbf{\Psi}}^{\top}$ auf, so deutet dies auf Fehler im Prozess hin. Es ist also eine Fehlerdetektion möglich. Anzumerken ist, dass sich das Vorgehen mithilfe von entsprechenden Parameterschätzverfahren auch auf nichtlineare Systeme erweitern lässt [107]. Einen umfassenden Überblick hierzu bieten [108, 111]. Für die Fehlerisolation ist es darüber hinaus nötig, die physikalischen Prozessparameter dem Vektor $\boldsymbol{\Psi}^{\top}$ der Streckenkoeffizienten zuzuordnen. Da diese Abbildung häufig nichtlinear ist, kann ein Rückschluss auf einzelne physikalische Parameter schwierig sein [204].

\section{Fehlerdiagnose mittels Paritätsgleichungen}

Die Idee der paritätsbasierten Verfahren lässt sich ebenfalls anschaulich für lineare zeitdiskrete Systeme beschreiben. Für ein fehler- und störungsfreies System der Form

$$
\begin{aligned}
\boldsymbol{x}_{k+1} & =\boldsymbol{A} \boldsymbol{x}_{k}+\boldsymbol{B} \boldsymbol{u}_{k} \\
\boldsymbol{y}_{k} & =\boldsymbol{C} \boldsymbol{x}_{k}+\boldsymbol{D} \boldsymbol{u}_{k}
\end{aligned}
$$

ist der Ausgangsvektor vor $l$ Abtastschritten gegeben durch $\boldsymbol{y}_{k-l}$. Kennt man den Wert dieses Vektors sowie die Eingangsvektoren von diesem Abtastschritt an, das heißt $\boldsymbol{u}_{k-l}, \boldsymbol{u}_{k-l+1}, \ldots, \boldsymbol{u}_{k}$, so lassen sich die Ausgangsvektoren zu jedem Abtastschritt durch

$$
\begin{aligned}
\boldsymbol{y}_{k-l}= & \boldsymbol{C} \boldsymbol{x}_{k-l}+\boldsymbol{D} \boldsymbol{u}_{k-l}, \\
\boldsymbol{y}_{k-l+1}= & \boldsymbol{C} \boldsymbol{x}_{k-l+1}+\boldsymbol{D} \boldsymbol{u}_{k-l+1} \\
= & \boldsymbol{C} \boldsymbol{A} \boldsymbol{x}_{k-l}+\boldsymbol{C} \boldsymbol{B} \boldsymbol{u}_{k-l+1}+\boldsymbol{D} \boldsymbol{u}_{k-l+1}, \\
\boldsymbol{y}_{k-l+2}= & \boldsymbol{C} \boldsymbol{A}^{2} \boldsymbol{x}_{k-l}+\boldsymbol{C} \boldsymbol{A} \boldsymbol{B} \boldsymbol{u}_{k-l}+\boldsymbol{C} \boldsymbol{B} \boldsymbol{u}_{k-l+1}+\boldsymbol{D} \boldsymbol{u}_{k-l+2}, \\
& \vdots \\
\boldsymbol{y}_{k}= & \boldsymbol{C} \boldsymbol{A}^{l} \boldsymbol{x}_{k-l}+\boldsymbol{C} \boldsymbol{A}^{l-1} \boldsymbol{B} \boldsymbol{u}_{k-l}+\boldsymbol{C} \boldsymbol{A}^{l-2} \boldsymbol{B} \boldsymbol{u}_{k-l+1}+\ldots \\
& +\boldsymbol{C} \boldsymbol{B} \boldsymbol{u}_{k-1}+\boldsymbol{D} \boldsymbol{u}_{k}
\end{aligned}
$$

berechnen. Führt man die Vektoren $\boldsymbol{y}_{\mathrm{ges}, k}=\left[\begin{array}{llll}\boldsymbol{y}_{k-l}^{\top} & \boldsymbol{y}_{k-l+1}^{\top} & \ldots & \boldsymbol{y}_{k}^{\top}\end{array}\right]^{\top}$ und $\boldsymbol{u}_{\text {ges }, k}=\left[\begin{array}{lllll}\boldsymbol{u}_{k-l}^{\top} & \boldsymbol{u}_{k-l+1}^{\top} & \ldots & \boldsymbol{u}_{k}^{\top}\end{array}\right]^{\top}$ ein, so lässt sich zusammenfassend 


$$
\boldsymbol{y}_{\mathrm{ges}, k}=\underbrace{\left[\begin{array}{c}
C \\
C A \\
C A^{2} \\
\vdots \\
C A^{l}
\end{array}\right]}_{M_{B, l}} \boldsymbol{x}_{k-l}+\underbrace{\left[\begin{array}{ccccc}
\boldsymbol{D} & 0 & \cdots & \cdots & 0 \\
C B & D & \ddots & & \vdots \\
C A B & C B & D & \ddots & \vdots \\
\vdots & & \ddots & \ddots & 0 \\
C A^{l-1} \boldsymbol{B} & \cdots & \cdots & C B & D
\end{array}\right]}_{M_{u, l}} \boldsymbol{u}_{\mathrm{ges}, k}
$$

schreiben. Für ein vollständig beobachtbares System ist mit $l \geq n-1$ die Beziehung rang $\left(\boldsymbol{M}_{B, l}\right)=n$ erfüllt, wobei für $l>n / n_{y}$ die Matrix $\boldsymbol{M}_{B, l}$ mehr als $n$ Zeilen aufweist. Somit existiert nach dem Rangsatz A.1 eine Matrix ${ }^{\perp} \boldsymbol{M}_{B, l} \in \mathbb{R}^{\left(\ln _{y}-n\right) \times l n_{y}}$, für die ${ }^{\perp} \boldsymbol{M}_{B, l} \boldsymbol{M}_{B, l}=\mathbf{0}$ gilt. ${ }^{6)}$ Für jede Matrix $\boldsymbol{Z}$ gilt somit $\boldsymbol{Z} \cdot{ }^{\perp} \boldsymbol{M}_{B, l} \boldsymbol{M}_{B, l}=\mathbf{0}$. Auf Grundlage dieser Überlegungen wird ein Residuenvektor gemäß

$$
\boldsymbol{r}_{k}=\boldsymbol{Z} \cdot{ }^{\perp} \boldsymbol{M}_{B, l}\left(\boldsymbol{y}_{\mathrm{ges}, k}-\boldsymbol{M}_{u, l} \boldsymbol{u}_{\mathrm{ges}, k}\right)
$$

berechnet. Wegen (2.11) gilt hierfür $\boldsymbol{r}_{k}=\boldsymbol{Z} \cdot{ }^{\perp} \boldsymbol{M}_{B, l} \boldsymbol{M}_{B, l} \boldsymbol{x}_{k-l}=\mathbf{0}$, solange die Systemdynamik der Darstellung (2.10) entspricht. Ergibt sich bei der Auswertung von (2.12) zur Laufzeit, dass $\boldsymbol{r}_{k} \neq \mathbf{0}$ ist, so kann davon auf die Einwirkung eines oder mehrerer Fehler auf die Systemdynamik geschlossen werden. Neben der Matrix $\boldsymbol{Z}$ ist auch der Parameter $l$ zu entwerfen, der beschreibt, wie viele Abtastschritte in die Residuengenerierung mit einbezogen werden. Der Grundgedanke der Methode geht zurück auf [39], in [222] wurde er auf zeitkontinuierliche Systeme übertragen. Weiterentwicklungen hat das Konzept unter anderem in [84, 86, 142] erfahren, insbesondere hinsichtlich der Robustheit der Residuen und deren Strukturierung zur Fehlerisolation. Auch die Bücher [58] und [87] widmen sich intensiv der Thematik. In [56, 102] und [58, Abschnitt 5.7] werden wesentliche Beziehungen zwischen Ansätzen mit Paritätsgleichungen und beobachterbasierten Verfahren diskutiert. Unter gewissen Voraussetzungen ergibt sich für paritäts- und beobachterbasierter Residuengeneratoren die gleiche Dynamik, und die Parametrierungen von Paritätsgleichungen und Beobachtern zur Fehlerdiagnose lassen sich ineinander überführen. Es ist somit möglich, einen Residuengenerator durch Paritätsgleichungen zu entwerfen und ihn dann als Beobachter zu implementieren.

${ }^{6)}$ Die Zeilen von ${ }^{\perp} \boldsymbol{M}_{B, l}$ bilden eine Basis des Linksnullraumes von $\boldsymbol{M}_{B, l}$. Somit ist ${ }^{\perp} \boldsymbol{M}_{B, l}$ offensichtlich nicht eindeutig. In der Literatur wird die Matrix auch als (Links-)Annihilator von $\boldsymbol{M}_{B, l}$ bezeichnet. 


\subsection{Existierende Ansätze für modellbasierte Fehlerisolationsfilter}

Existierende Resultate im Bereich der modellbasierten Fehlerisolationsfilter beziehungsweise der beobachterbasierten Fehlerisolation lassen sich gemäß verschiedener Kriterien kategorisieren. Im Rahmen dieser Arbeit orientiert sich die Einteilung an dem hierarchischen Aufbau der Teilaufgaben der Fehlerdiagnose gemäß Abbildung 2.5. Die erste Gruppe von Verfahren basiert darauf, das Fehlerisolationsproblem auf mehrere Fehlerdetektionsprobleme zurückzuführen. Die zweite Kategorie umfasst Ansätze, welche auf eine Fehleridentifikation abzielen und die damit inhärent auch eine Isolation der Fehler erreichen. Die dritte Gruppe schließlich, der auch die im Fokus dieser Arbeit stehenden Verfahren zuzuordnen sind, beinhaltet Ansätze, die unmittelbar eine Fehlerisolation zum Ziel haben.

Anzumerken ist, dass die erwähnten Ansätze nicht immer eindeutig einer der Kategorien zugeordnet werden können. So eignen sich einige Verfahren sowohl für eine direkte Fehlerisolation als auch für eine Isolation durch Rückführung auf eine Reihe von Detektionsproblemen.

\subsubsection{Fehlerisolation durch Rückführung auf Detektionsprobleme}

Ein klassischer Ansatz zur beobachterbasierten Fehlerisolation ist es, das Isolationsproblem auf mehrere Detektionsprobleme zurückzuführen. Dies gelingt mittels sogenannter Beobachterbänke. Dabei werden mehrere Beobachter parallel implementiert und die jeweils erzeugten Residuen einer Auswertelogik zugeführt. Die Auslegung jedes Beobachters innerhalb der Beobachterbank stellt lediglich ein (robustes) Fehlerdetektionsproblem dar.

Für die Auslegung der Beobachter bestehen verschiedene Möglichkeiten, die unter anderem abhängig von der Art der Fehler sind. Ausführlich diskutiert wird die Rückführung von Isolations- auf Detektionsprobleme in [78], sie soll hier exemplarisch für die Isolation von Sensorfehlern in einem System mit drei Ausgängen beschrieben werden.

Der naheliegendste Ansatz hierfür sind sogenannte dedizierte Beobachter [43]. Dabei werden wie in Abbildung 2.16 für das Beispiel dargestellt drei Beobachter implementiert, die jeweils auf einen der Ausgänge zurückgreifen. Als Residuum eines Beobachters wird jeweils die Differenz zwischen gemessenem und geschätztem Ausgangssignal herangezogen, es ist also $r_{i}=y_{i}-\hat{y}_{i}$. Die Auswertelogik gestaltet sich bei dieser Anordnung 


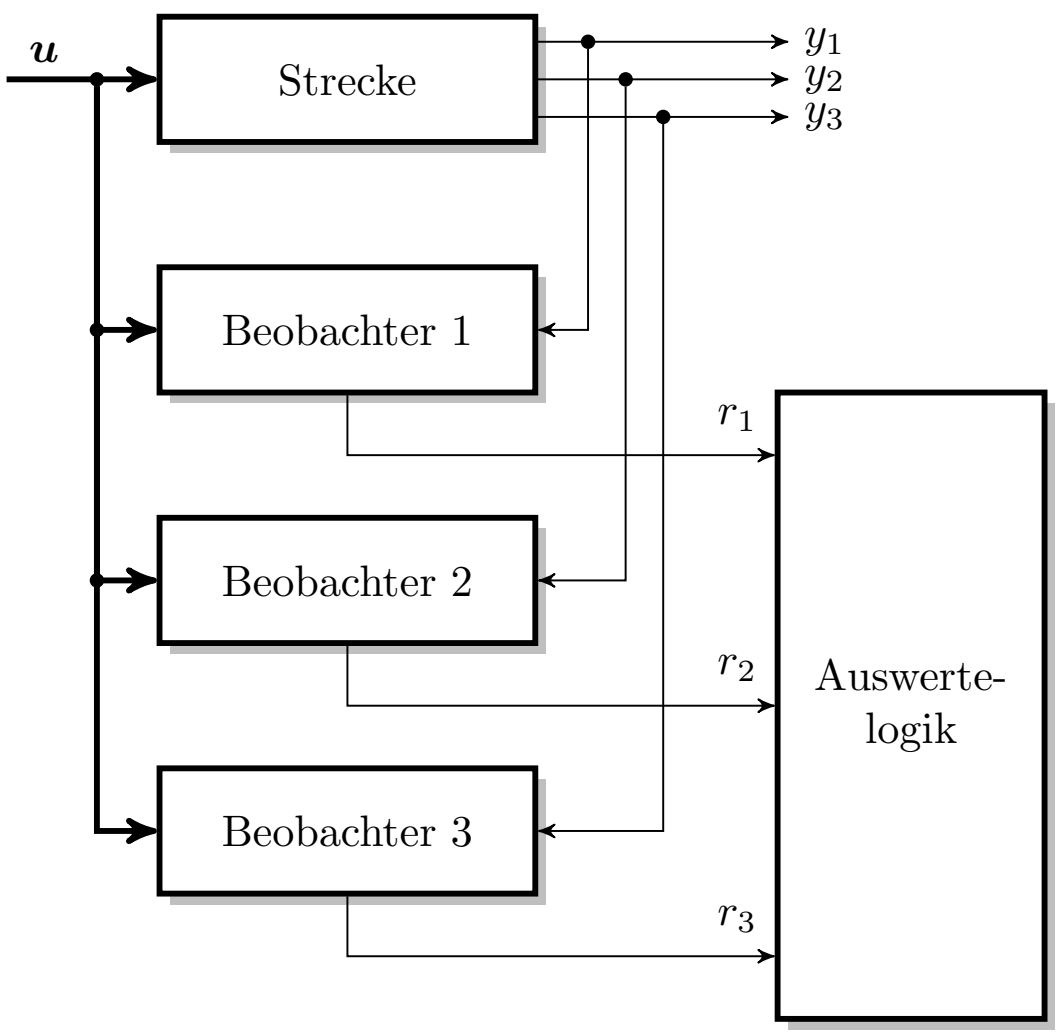

Abbildung 2.16: Beobachterbank mit dedizierten Beobachtern

äußerst einfach und ist vergleichbar mit dem Schema aus Abbildung 2.12. Ist ein Residuum $r_{i}$ betragsmäßig größer als ein bestimmter Schwellenwert, so wird der $i$-te Fehler erkannt und isoliert. Neben der einfachen Struktur und Auswertung ist ein Vorteil der dedizierten Beobachter darin zu sehen, dass gleichzeitig auftretende Fehler isoliert werden können. Nachteilig ist, dass zur Anwendung des Prinzips das System anhand der einzelnen Ausgangsgrößen $y_{i}$ jeweils vollständig beobachtbar sein muss. Bezeichnet man die Zeilen der Ausgangsmatrix mit $\boldsymbol{c}_{i}^{\top}$, so müssen also alle Paare $\left(\boldsymbol{A}, \boldsymbol{c}_{i}^{\top}\right)$ vollständig beobachtbar sein. Diese restriktive Forderung schränkt die Anwendbarkeit ein. Da jeweils nur eine Ausgangsgröße für die Beobachter zur Verfügung steht, existieren darüber hinaus nur wenige Freiheitsgrade, es können lediglich die Beobachtereigenwerte platziert werden.

Ein alternativer Ansatz wurde in [74] vorgeschlagen. Das dort eingeführte verallgemeinerte Beobachterschema ist für das betrachtete Beispiel in Abbildung 2.17 skizziert. Im Gegensatz zu den dedizierten Beobachtern werden hier dem $i$-ten Beobachter alle bis auf die $i$-te Messgröße zugeführt. Im Beispiel arbeitet also der erste Beobachter mit den Ausgangsgrößen $y_{2}$ und $y_{3}$. Für diesen Beobachter ergibt sich der Residuenvektor $\boldsymbol{r}_{1}=\left[\begin{array}{ll}y_{2} & y_{3}\end{array}\right]^{\top}-\left[\begin{array}{ll}\hat{y}_{2} & \hat{y}_{3}\end{array}\right]^{\top}$. Aufgrund der gewählten Struktur führt 


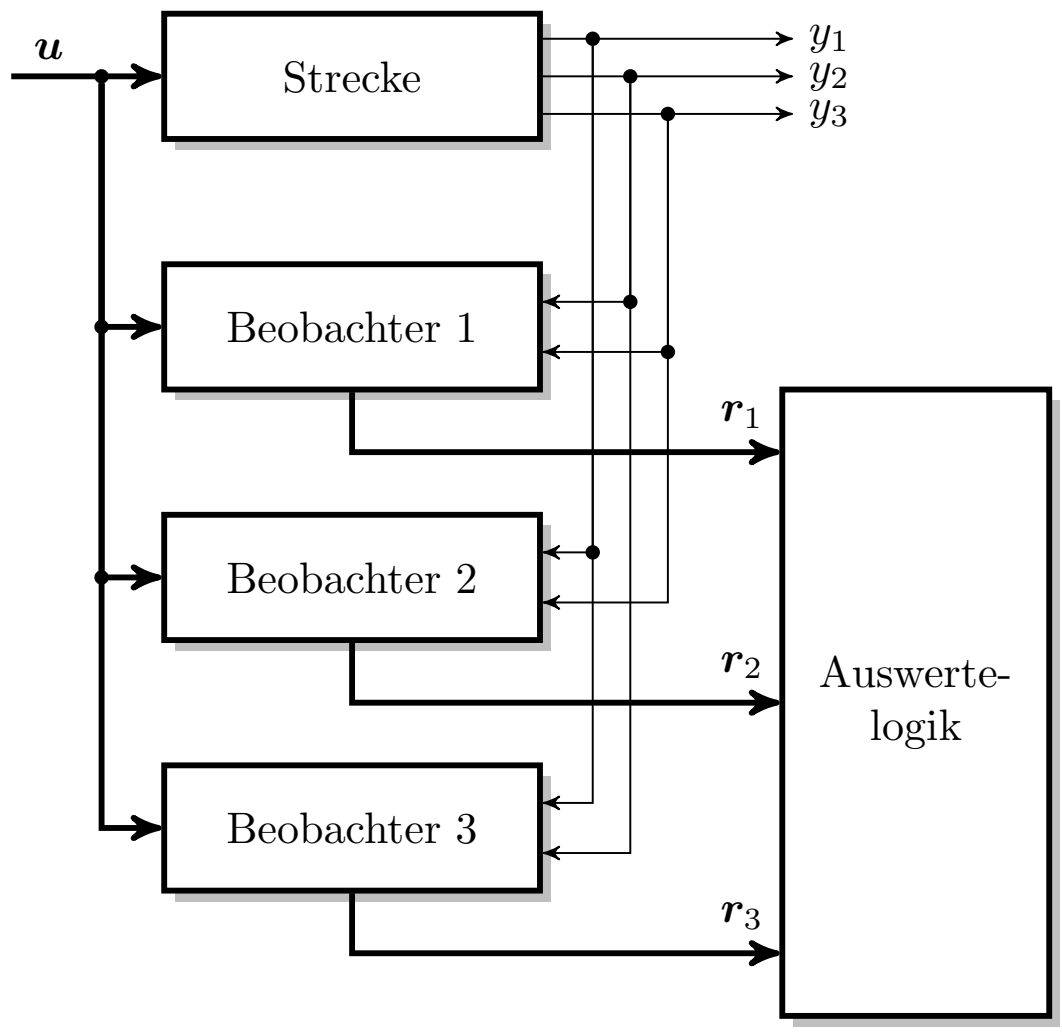

Abbildung 2.17: Beobachterbank mit verallgemeinerten Beobachtern

ein Fehler in Sensor 1 dazu, dass die Residuen $\boldsymbol{r}_{2}$ und $\boldsymbol{r}_{3}$ ausschlagen. Dementsprechend arbeitet die Auswertung bei einem einfachen statischen Grenzwert $J_{\text {th }}$ mit der Logik

$$
\begin{aligned}
& \left|\boldsymbol{r}_{1}\right|<J_{\mathrm{th}} \wedge\left|\boldsymbol{r}_{2}\right| \geq J_{\mathrm{th}} \wedge\left|\boldsymbol{r}_{3}\right| \geq J_{\mathrm{th}} \Rightarrow \text { Fehler } 1 \text { erkannt, } \\
& \left|\boldsymbol{r}_{1}\right| \geq J_{\mathrm{th}} \wedge\left|\boldsymbol{r}_{2}\right|<J_{\mathrm{th}} \wedge\left|\boldsymbol{r}_{3}\right| \geq J_{\mathrm{th}} \Rightarrow \text { Fehler } 2 \text { erkannt, } \\
& \left|\boldsymbol{r}_{1}\right| \geq J_{\mathrm{th}} \wedge\left|\boldsymbol{r}_{2}\right| \geq J_{\mathrm{th}} \wedge\left|\boldsymbol{r}_{3}\right|<J_{\mathrm{th}} \Rightarrow \text { Fehler } 3 \text { erkannt. }
\end{aligned}
$$

Nachteilig gegenüber dedizierten Beobachtern ist je nach Anforderungen an das Diagnosesystem die Tatsache, dass gleichzeitig auftretende Fehler nicht isoliert werden können. Da die Beobachter bei $n_{y}$ Messgrößen jedoch stets mit $n_{y}-1$ Ausgangsgrößen arbeiten, sind die Anforderungen hinsichtlich der Beobachtbarkeit wesentlich weniger restriktiv. Hinzu kommt, dass wegen des mehrdimensionalen Ausgangsvektors beim Entwurf der einzelnen Beobachter zusätzliche, über die Polvorgabe hinausgehende Freiheitsgrade zur Verfügung stehen.

Die beiden umrissenen Ansätze lassen sich für beliebige Typen von Fehlern verallgemeinern. Dazu wird jeder Beobachter der Beobachterbank mit dem gesamten Ausgangsvektor gespeist. Die Auslegung des $i$-ten Beobach- 
ters kann dann entweder so erfolgen, dass der Residuenvektor $\boldsymbol{r}_{i}$ sensitiv bezüglich des Fehlers $f_{i}$ ist, von allen anderen Fehlern $f_{j}$ jedoch unbeeinflusst bleibt. In diesem Fall ist eine Isolation gleichzeitig auftretender Fehler möglich, jedoch ist durch die Unterdrückung von $n_{f}-1$ Fehlern in jedem Beobachter eine strenge Forderung gegeben, wobei $n_{f}$ die Anzahl der einwirkenden Fehler beschreibt. Andererseits kann die Auslegung dergestalt erfolgen, dass der Residuenvektor $\boldsymbol{r}_{i}$ sensitiv bezüglich aller Fehler außer $f_{i}$ ist. Dann ist zwar keine Isolation gleichzeitiger Fehler mehr möglich, dafür verbleiben aufgrund der weniger restriktiven strukturellen Anforderung jedoch mehr Entwurfsfreiheitsgrade.

Ein Beispiel für eine Methode zur konkreten Auslegung der Beobachter einer Beobachterbank ist in [218] gegeben. Weitere Ansätze werden im Folgenden betrachtet.

\section{Unknown Input Observers (UIOs)}

Ein bedeutender und intensiv untersuchter Ansatz für den Entwurf von Beobachtern für Beobachterbänke sind die sogenannten Unknown Input Observers (UIOs). Die Grundidee geht auf das Problem zurück, die Zustände eines Systems

$$
\begin{aligned}
& \dot{\boldsymbol{x}}=\boldsymbol{A} \boldsymbol{x}+\boldsymbol{B} \boldsymbol{u}+\boldsymbol{B}_{d} \boldsymbol{d}, \\
& \boldsymbol{y}=\boldsymbol{C x}
\end{aligned}
$$

trotz der einwirkenden Störungen $\boldsymbol{d}$ asymptotisch zu schätzen, wobei die Störungen als unbekannte Eingänge aufgefasst werden. Die Problemstellung geht zurück auf [251], wo sie im Zusammenhang mit der Erkennung und Isolation von Sensorfehlern bei vorhandenen parametrischen Unsicherheiten im Prozess behandelt wurde. Erweiterungen zur gleichzeitigen Betrachtung von Sensor- und Aktorfehlern sind in [77, 259-261] zu finden. Ein weiterer wesentlicher Schritt ist in der systematischen Betrachtung der Robustheit von UIOs zu sehen, die unter anderem in [33, 76, 100-102] untersucht wurde. Zur Lösung des Problems wird in [30] die Struktur

$$
\begin{aligned}
& \dot{\boldsymbol{z}}=\boldsymbol{F} \boldsymbol{z}+\boldsymbol{T} \boldsymbol{B} \boldsymbol{u}+\boldsymbol{K} \boldsymbol{y} \\
& \hat{\boldsymbol{x}}=\boldsymbol{z}+\boldsymbol{H y}
\end{aligned}
$$

mit $\boldsymbol{z} \in \mathbb{R}^{n}$ herangezogen. In [32, Abschnitt 3.2] werden die Existenzbedingungen für UIOs obiger Struktur ausführlich diskutiert. Ebenso werden dort Entwurfsverfahren zur Auslegung der Matrizen $\boldsymbol{F}, \boldsymbol{T}, \boldsymbol{K}$ und $\boldsymbol{H}$ angegeben, die dazu führen, dass die Störungen $\boldsymbol{d}$ keinerlei Einfluss auf die 
geschätzten Zustände haben und dass der geschätzte Zustand $\hat{\boldsymbol{x}}$ asymptotisch gegen den tatsächlichen Zustandsvektor $\boldsymbol{x}$ konvergiert. Diese werden weiterhin für unterschiedliche Problemstellungen (zum Beispiel Isolation von Sensorfehlern, Isolation von Aktorfehlern etc.) genutzt. Alternative Ansätze für den Entwurf von UIOs finden sich in [50, 101, 124, 268].

Darüber hinaus existieren vielfältige Erweiterungen des UIO-Ansatzes. In [104] wird beispielsweise der Entwurf von UIOs reduzierter Dimension, das heißt mit $\boldsymbol{z} \in \mathbb{R}^{n_{z}}, n_{z}<n$, diskutiert, während sich [36] mit UIOs für nichtlineare Systeme befasst. Weiterhin lässt sich der Ansatz wie in [18, 23] gezeigt auf lineare, parametervariable (LPV) Systeme übertragen. Sogenannte Funktionalbeobachter [53, 69] zielen lediglich auf die asymptotische Schätzung einer Linearkombination der Zustände ab und ermöglichen somit weniger restriktive Entwurfsanforderungen.

\section{Ansätze mit Berücksichtigung beschränkter Frequenzbereiche}

Wie bereits erörtert existiert eine Vielzahl von Arbeiten, welche sich mit der Unterdrückung des Störgrößeneinflusses auf die geschätzten Zustände beziehungsweise der generierten Residuen befasst. Die Minimierung der $\mathcal{H}_{\infty}$-Norm liefert diesbezüglich eine Optimierung des Worst-Case, also eine Minimierung des größten Einflusses, der auftreten kann. Oftmals ist dadurch jedoch eine sehr konservative Abschätzung gegeben. Dies ist insbesondere dann der Fall, wenn Kenntnisse über die relevanten Frequenzbereiche der Störungen (beziehungsweise Stellgrößen und Fehler) vorhanden sind. Liegt dann die größte auftretende Verstärkung außerhalb dieses Frequenzbereiches, so ist die Minimierung der $\mathcal{H}_{\infty}$-Norm offensichtlich ein wenig geeignetes Optimierungskriterium, da sie an den tatsächlichen Anforderungen vorbei geht.

Ein klassischer Weg, diese Problematik zu beheben, sind Gewichtungsfilter. Ist beispielsweise bekannt, dass eine bestimmte Störung $d_{i}$ lediglich hochfrequente Anteile enthält, so wird das System für den Entwurf virtuell um einen Hochpass $g_{\mathrm{HP}}(s)$ erweitert. Für den Entwurf wird angenommen, dass $d_{i}(s)=g_{\mathrm{HP}}(s) \cdot d_{i}^{*}(s)$ gilt. Die Minimierung der $\mathcal{H}_{\infty}$-Norm bezüglich der virtuellen Störung $d_{i}^{*}$ bewirkt dann eine Minimierung des Einflusses der tatsächlichen Störung $d_{i}$ im relevanten Frequenzbereich. Ausführlich behandelt werden diese im Englischen frequency weighting filters genannten Ansätze in [25, 129, 135, 247]. Vorteilhaft ist die Tatsache, dass sich die bekannten Entwürfe zur Minimierung der $\mathcal{H}_{\infty}$-Norm prinzipiell unverändert einsetzen lassen. Neben der besseren Unterdrückung des Einflusses von Störungen auf die Residuen in den relevanten Frequenzberei- 
chen lässt sich darüber hinaus eine bessere Abschätzung für diesen Einfluss angeben. Dies lässt sich bei der Auslegung der Schwellenwerte in der Residuenauswertung ausnutzen (s. Abschnitt 2.2.2). Nachteilig anzumerken ist, dass die jeweiligen Gewichtungsfilter separat auszulegen sind, was einen zusätzlichen Entwurfsaufwand bedeutet. Weiterhin erhöht sich durch die Gewichtungsfilter die Ordnung des betrachteten Gesamtsystems. Bei Lösungsansätzen, die auf linearen Matrixungleichungen (LMIs) beruhen, sind außerdem oftmals strukturelle Beschränkungen der LMI-Variablen nötig, was den Lösungsraum einschränkt.

Aufgrund der genannten Probleme der Gewichtungsfilter wurde in jüngerer Vergangenheit ein Ansatz vorangetrieben, der das zusätzliche Wissen über die relevanten Frequenzbereiche von Störungen, Fehlern und Stellgrößen direkt in den Entwurf einfließen lässt. Dabei wird ausgenutzt, dass sich mithilfe des verallgemeinerten Kalman-YakubovichPopov-Lemmas [113] die $\mathcal{H}_{\infty}$-Norm eines Systems innerhalb eines beschränkten Frequenzbereichs durch entsprechende Matrixungleichungen direkt abschätzen lässt. Somit entfällt die separate Auslegung der Gewichtungsfilter und es ist keine Erhöhung der Dimension des betrachteten Systems nötig. Für die Fehlerdetektion in zeitkontinuierlichen Systemen wird dieses Konzept in [34, 48, 246, 254] eingesetzt. In [244, 245] wird das entsprechende Problem für zeitdiskrete Systeme betrachtet. Darüber hinaus finden sich in der Literatur Anwendungen des Konzeptes für die Diagnose in vernetzten Systemen [141] und in Takagi-Sugeno-Fuzzy-Systemen [269].

Anzumerken ist allerdings, dass die Matrixungleichungen zur Abschätzung der $\mathcal{H}_{\infty}$-Norm in beschränkten Frequenzbereichen eine kompliziertere Gestalt aufweisen als beim klassischen Ansatz mit unbeschränkten Frequenzbereichen.

\section{Mengenbasierte Ansätze}

Während die bisher vorgestellten Ansätze darauf abzielen, aufgetretene Fehler anhand eines zugeordneten Residuums zu detektieren und zu isolieren, verfolgen sogenannte mengenbasierte Ansätze ein grundsätzlich anderes Prinzip. Dabei kommen mengenbasierte Beobachter (engl. setvalued observers, SVOs) für zeitdiskrete Systeme zum Einsatz, die auf [197, 203, 258] zurückgehen. Bei diesem Ansatz wird basierend auf einer gegebenen Menge von möglichen Anfangszuständen $\mathcal{X}_{k, 0}$ sowie bekannten Eingangs- und Ausgangssignalen zu jedem Zeitschritt $k$ eine Menge von möglichen Zuständen $\mathcal{X}_{k}$ berechnet. Anschaulich gesprochen enthält diese Menge all jene Zustände, welche bei den gegebenen Anfangszuständen 
und Eingangssignalen die gemessenen Ausgangssignale erzeugen können. Vorteilhaft bei diesem Vorgehen ist, dass es sich ohne Weiteres auf Systeme mit parametrischen Unsicherheiten und Störungen sowie zeitvariante Systeme erweitern lässt. Dies schlägt sich lediglich in der Berechnung von $\mathcal{X}_{k}$ nieder. Da die Menge $\mathcal{X}_{k}$ im Allgemeinen nicht konvex ist, wird oftmals die konvexe Hülle $\tilde{\mathcal{X}}_{k}$ berechnet.

Der Einsatz von mengenbasierten Beobachtern zur Fehlerdetektion und -isolation ist in [44, 106, 188, 189] beschrieben. Dabei wird zum einen ein SVO $O_{\text {nom }}$ für den fehlerfreien Fall implementiert. Parallel dazu wird zum anderen für jeden möglichen Fehler ein SVO $O_{i}$ implementiert. Damit dies möglich ist, müssen sich die einzelnen Fehler modellieren und so in die dynamische Modellbeschreibung integrieren lassen. Tritt zu einem bestimmten Zeitpunkt $k$ der Fall $\tilde{\mathcal{X}}_{k}=\emptyset$ für den SVO $O_{\text {nom }}$ ein, so können die gemessenen Ein- und Ausgangssignale durch keinen Zustand des fehlerfreien Systems erklärt werden. Dementsprechend wird das fehlerfreie Modell falsifiziert und ein Fehler wird detektiert. Die Fehlerisolation erfolgt dann, indem sukzessive die den jeweiligen Fehlern zugeordneten SVOs $O_{i}$ falsifiziert werden.

Bei der beschriebenen Methode müssen keine Schwellenwerte für die Residuenauswertung festgelegt werden. Weiterhin ist auch die Erkennung nicht modellierter Fehlerfälle möglich. Dies ist dann der Fall, wenn für alle SVOs, also $O_{\text {nom }}$ und alle $O_{i}$, die Bedingung $\tilde{\mathcal{X}}_{k}=\emptyset$ erfüllt ist. Die gemessenen Ein- und Ausgangssignale können dann weder mit dem fehlerfreien Modell noch mit den angenommenen Fehlern erklärt werden.

Allerdings müssen wie bereits erwähnt die Fehler in ihrer Dynamik und ihrer möglichen Amplitude bekannt sein. Außerdem ist in jedem Zeitschritt die mögliche Menge der aktuellen Zustände $\mathcal{X}_{k}$ für alle möglichen Unsicherheiten, Störungen und Fehler zu berechnen, was einen hohen OnlineRechenaufwand bedingt.

\subsubsection{Fehlerisolation durch Lösung von Identifikationsproblemen}

Wie in Abbildung 2.5 dargestellt ist die Fehleridentifikation hierarchisch ein auf der Fehlerisolation aufbauender Schritt. Die Anwendung von Verfahren zur Fehleridentifikation impliziert daher inhärent eine Fehlerisolation. Im Folgenden werden mit Störgrößenbeobachtern und Sliding-ModeBeobachtern zwei wesentliche Konzepte zur direkten Fehleridentifikation näher erläutert. 


\section{Störgrößenbeobachter}

Mittels des aus der Zustandsschätzung bekannten Konzeptes eines Störgrößenbeobachters ist ebenfalls eine Fehlerisolation möglich. Dabei wird angenommen, dass sich die Fehler durch ein autonomes Differentialgleichungssystem (das sogenannte Fehlermodell) beschreiben lassen [143, Abschnitt 10.4.2]. Es ist also

$$
\begin{aligned}
\dot{\boldsymbol{\xi}} & =\boldsymbol{A}_{\boldsymbol{\xi}} \boldsymbol{\xi}, \boldsymbol{\xi}\left(t_{i}\right)=\boldsymbol{\xi}_{i} \in \mathbb{R}^{n_{\xi}}, \\
\boldsymbol{f} & =C_{\boldsymbol{\xi}} \boldsymbol{\xi} .
\end{aligned}
$$

Entwirft man nun einen Zustandsbeobachter für das um das Fehlermodell erweiterte Gesamtsystem mit dem Zustandsvektor $\left[\boldsymbol{x}^{\top} \boldsymbol{\xi}^{\top}\right]^{\top}$, so erhält man nicht nur eine Schätzung $\hat{\boldsymbol{x}}$ des Streckenzustandes, sondern ebenfalls eine Schätzung $\hat{f}$ des Fehlervektors. Somit kann eine Fehleridentifikation erreicht werden, die gemäß Abbildung 2.5 die Fehlerisolation beinhaltet.

Damit dies gelingt, muss jedoch ein Fehlermodell bekannt sein. Treten zum Beispiel unter der Annahme sinusförmiger Fehlerverläufe sprungförmige Fehler auf, so ist mit einem Störgrößenbeobachter keine ideale Fehleridentifikation (beziehungsweise -isolation) mehr möglich. Darüber hinaus konvergiert die Fehlerschätzung $\hat{f}$ selbst bei einem exakt bekannten Fehlermodell lediglich asymptotisch gegen die tatsächlichen Fehler $\boldsymbol{f}$. Tritt zum Beispiel ein Fehler $f_{i}$ auf, so werden im Allgemeinen auch die Fehlerschätzungen $\hat{f}_{j}$ ausschlagen, bevor sie asymptotisch gegen 0 konvergieren. Im Sinne von (2.7) ist also keine dynamische Fehlerisolation möglich, sondern lediglich eine stationäre (das heißt für $t \rightarrow \infty$ ). Abschließend ist anzumerken, dass der Entwurf eines Beobachters für das erweiterte Gesamtsystem die Implementierung eines dynamischen Systems der Ordnung $n+n_{\xi}$ erfordert. Dennoch finden sich in der Literatur erfolgreiche Anwendungen des Ansatzes (s. zum Beispiel [249]).

\section{Sliding-Mode-Beobachter}

Eine wesentliche Gruppe von Verfahren, die dem Ansatz der Störgrößenbeobachter zuzuordnen sind, stellen die Sliding-Mode-Beobachter dar. Dabei wird ebenfalls eine Schätzung $\hat{f}$ angestrebt, wobei durch die Fehleridentifikation wie bereits beschrieben eine Fehlerisolation erreicht wird.

Gegenüber klassischen Störgrößenbeobachtern kommen jedoch Beobachter zum Einsatz, die über diskontinuierliche Rückführungen verfügen. Eine allgemeine, in $[6,64]$ gegebene Struktur ist 


$$
\begin{aligned}
& \dot{\hat{\boldsymbol{x}}}=\boldsymbol{A} \hat{\boldsymbol{x}}+\boldsymbol{B u}+\boldsymbol{L}_{\mathrm{kont}}(\boldsymbol{y}-\hat{\boldsymbol{y}})+\boldsymbol{L}_{\text {diskont }} \boldsymbol{\nu}, \\
& \boldsymbol{\nu}=\left\{\begin{array}{cc}
-\rho(\boldsymbol{u}, \boldsymbol{y}) \frac{\boldsymbol{P}_{0} \cdot(\boldsymbol{y}-\hat{\boldsymbol{y}})}{\left\|\boldsymbol{P}_{0} \cdot(\boldsymbol{y}-\hat{\boldsymbol{y}})\right\|}, & \boldsymbol{y}-\hat{\boldsymbol{y}} \neq \mathbf{0}, \\
\mathbf{0} & , \boldsymbol{y}-\hat{\boldsymbol{y}}=\mathbf{0},
\end{array}\right. \\
& \hat{\boldsymbol{f}}=\boldsymbol{W} \boldsymbol{\nu},
\end{aligned}
$$

wobei die Matrizen $\boldsymbol{L}_{\text {kont }}, \boldsymbol{L}_{\text {diskont }}, \boldsymbol{P}_{0}$ und $\boldsymbol{W}$ und die Funktion $\rho(\boldsymbol{u}, \boldsymbol{y})$ auszulegen sind. Diese Art der Rückführung führt unter gewissen Voraussetzungen [64] dazu, dass der Schätzwert $\hat{f}$ mindestens asymptotisch gegen den tatsächlichen Fehlervektor $\boldsymbol{f}$ konvergiert.

Eine umfassende Übersicht zur Anwendung von Sliding-ModeBeobachtern für die Fehlerdetektion und -isolation findet sich in [65]. Die Beiträge $[6,214]$ konzentrieren sich auf die Diagnose von Sensorfehlern. Die explizite Berücksichtigung von Robustheitsaspekten erfolgt unter anderem in $[72,157,213,215]$. Weiterführende Ergebnisse erlauben darüber hinaus beispielsweise die Anwendung auf linear parameterveränderliche Systeme [5], Klassen nichtlinearer Systeme [37] sowie die Fehlerdiagnose in dezentralen [267] und stochastischen [82] Systemen und Erweiterungen auf fehlertolerante Regelungen [64].

Vorteile von Sliding-Mode-Ansätzen sind in ihren Robustheitseigenschaften zu sehen. Allerdings ist wie bei linearen Störgrößenbeobachtern nur eine stationäre Fehlerisolation und keine dynamische möglich.

\subsubsection{Direkte Ansätze zur Fehlerisolation}

Während mit den in Abschnitt 2.5.1 vorgestellten Verfahren das Isolationsproblem durch die Rückführung auf mehrere Detektionsprobleme zurückgeführt wird und in Abschnitt 2.5.2 die Isolation als "Nebenprodukt" der Fehleridentifikation erreicht wird, existieren verschiedene Ansätze, die direkt auf eine Fehlerisolation abzielen. Im Folgenden wird eine kurze Übersicht über die relevante Literatur gegeben. Eine detaillierte Betrachtung der existierenden Literatur wird zu Beginn der Kapitel 4 und 6 gegeben, da für die Abgrenzung und Einordnung der im Rahmen dieser Arbeit erzielten Ergebnisse noch verschiedene Grundbegriffe und Konzepte einzuführen sind.

\section{Fehlerisolationsbeobachter}

Im Gegensatz zu Beobachterbänken oder Störgrößenbeobachtern verfolgen Fehlerisolationsbeobachter das Ziel, mittels eines einzelnen, geeignet 
parametrierten Beobachters der Form

$$
\begin{aligned}
\dot{\hat{\boldsymbol{x}}} & =\boldsymbol{A} \hat{\boldsymbol{x}}+\boldsymbol{B} \boldsymbol{u}+\boldsymbol{L}(\boldsymbol{y}-\boldsymbol{C} \hat{\boldsymbol{x}}-\boldsymbol{D u}), \\
\boldsymbol{r} & =\boldsymbol{V}(\boldsymbol{y}-\boldsymbol{C} \hat{\boldsymbol{x}}-\boldsymbol{D} \boldsymbol{u})
\end{aligned}
$$

die in Abschnitt 2.3 beschriebenen Entwurfsziele zu erfüllen. Diesem Bereich sind auch die Ergebnisse der Kapitel 4 und 5 zuzuordnen. Der grundsätzliche Vorteil der Verfahren besteht darin, dass sie gegenüber Beobachterbänken einen geringeren Entwurfs- und Implementierungsaufwand aufweisen, da lediglich ein Beobachter beziehungsweise Filter zu parametrieren ist.

Der Ansatz geht zurück auf [13, 117], weiterentwickelt wurde er in $[42,59,133,147,167,168,255]$. Neuere Resultate auf Basis von Beobachtern finden sich in $[28,58,114,130]$. Diese stellen einerseits unterschiedliche Entwurfsverfahren zur Verfügung und erlauben andererseits die Behandlung größerer Systemklassen und die explizite Berücksichtigung beziehungsweise Optimierung der Robustheit.

\section{Fehlerisolationsfilter}

Neben Beobachtern lassen sich auch allgemeinere Filterstrukturen der Form

$$
\begin{aligned}
\dot{\boldsymbol{x}}_{f} & =\boldsymbol{A}_{f} \boldsymbol{x}_{f}+\boldsymbol{B}_{f, u} \boldsymbol{u}+\boldsymbol{B}_{f, y} \boldsymbol{y}, \\
\boldsymbol{r} & =\boldsymbol{C}_{f} \boldsymbol{x}_{f}+\boldsymbol{D}_{f, u} \boldsymbol{u}+\boldsymbol{D}_{f, y} \boldsymbol{y}
\end{aligned}
$$

zur Erfüllung der in Abschnitt 2.3 diskutierten Entwurfsziele ansetzen. Solche Filter werden in dieser Arbeit als allgemeine Fehlerisolationsfilter oder kurz Fehlerisolationsfilter bezeichnet, sie werden in Kapitel 6 ausführlich betrachtet. Anzumerken ist, dass die Begriffe Fehlerisolationsbeobachter und Fehlerisolationsfilter in der Literatur häufig synonym verwendet werden. In dieser Arbeit erfolgt jedoch eine explizite Unterscheidung, um den strukturellen Unterschied der Residuengeneratoren deutlich zu machen.

In [146] ist ein geometrisches Verfahren zur Parametrierung allgemeiner Fehlerisolationsfilter zu finden. In jüngerer Vergangenheit wurden neben einem algebraischen Entwurf [58, Abschnitt 13.4] optimierungsbasierte Verfahren entwickelt, welche die Einbeziehung von Robustheitsaspekten erlauben [148, 149]. Sie zielen jedoch im Wesentlichen auf eine Fehlerdetektion ab und erlauben keine systematische Behandlung von Fehlerisolationsproblemen. 


\section{Mathematische und regelungstechnische Grundlagen}

In diesem Kapitel werden die für das Verständnis der Arbeit wesentlichen Grundlagen zu Signal- und Systemnormen (Abschnitt 3.1), linearen Matrixungleichungen (Abschnitt 3.2), Nullstellen linearer Mehrgrößensysteme (Abschnitt 3.3) und linearen Entkopplungsreglern (Abschnitt 3.4) zusammengefasst. Es erfolgt jeweils nur eine kurze Einführung in die Grundbegriffe und -konzepte, für Details wird jeweils auf die einschlägige Literatur verwiesen.

\subsection{Signal- und Systemnormen}

In Abschnitt 2.3 wurden die Ziele beim Entwurf modellbasierter Fehlerisolationsfilter herausgearbeitet. Zwei wesentliche Aspekte sind dabei die Sensitivität bezüglich der Fehler $\boldsymbol{f}$ und die Robustheit bezüglich exogener Störungen $\boldsymbol{d}$ sowie Unsicherheiten im Systemmodell. Es ist einleuchtend, dass sich die Fehler möglichst stark und die Störungen und Stellgrößen möglichst wenig in den erzeugten Residuen niederschlagen sollen. Um die "Stärke" des Einflusses der verschiedenen anregenden Größen zu quantifizieren, ist eine geeignete mathematische Beschreibung nötig. Um die "Größe" von Signalen beziehungsweise Systemen zu beschreiben, existieren verschiedene Normen.

Ein intuitives Maß für die Norm eines vektoriellen Zeitsignals ist durch die $\mathcal{L}_{2}$-Norm gegeben [206].

Definition 3.1 $\left(\mathcal{L}_{2}\right.$-Norm eines Signals). Für ein Signal $\boldsymbol{u}(t) \in \mathbb{R}^{n_{u}}$ mit $u_{i}(t<0)=0$ für alle $i=1, \ldots, n_{u}$ ist die $\mathcal{L}_{2}$-Norm definiert als

$$
\|\boldsymbol{u}\|_{\mathcal{L}_{2}}=\sqrt{\int_{0}^{\infty} \boldsymbol{u}^{\top}(\tau) \cdot \boldsymbol{u}(\tau) d \tau .}
$$


Anschaulich gesprochen kann die $\mathcal{L}_{2}$-Norm als ein Maß aufgefasst werden, das proportional zur Gesamtenergie eines Signals ist.

Um die "Größe" eines Systems beziehungsweise einer Übertragungsmatrix zu quantifizieren, wird häufig auf induzierte Normen zurückgegriffen. Diese leiten sich aus Signalnormen ab, wobei

$$
\left\|\boldsymbol{G}_{\boldsymbol{y} \boldsymbol{u}}(s)\right\|=\sup _{\|\boldsymbol{u}\| \neq 0} \frac{\|\boldsymbol{y}\|}{\|\boldsymbol{u}\|}
$$

definiert wird. Indem unterschiedliche Normen von $\boldsymbol{u}$ beziehungsweise $\boldsymbol{y}$ herangezogen werden, lassen sich verschiedene Systemnormen charakterisieren.

Betrachtet man jeweils die $\mathcal{L}_{2}$-Norm von $\boldsymbol{u}$ und $\boldsymbol{y}$, so ergibt sich die sogenannte $\mathcal{H}_{\infty}$-Norm von $\boldsymbol{G}_{\boldsymbol{y} \boldsymbol{u}}(s)$.

Definition 3.2 $\left(\mathcal{H}_{\infty}\right.$-Norm). Für ein stabiles lineares System, welches durch die propere Übertragungsmatrix $\boldsymbol{G}_{\boldsymbol{y} \boldsymbol{u}}(s)$ beschrieben wird, ist die $\mathcal{H}_{\infty}$-Norm definiert als

$$
\left\|\boldsymbol{G}_{\boldsymbol{y} \boldsymbol{u}}(s)\right\|_{\infty}=\sup _{\|\boldsymbol{u}\|_{\mathcal{L}_{2}} \neq 0} \frac{\|\boldsymbol{y}\|_{\mathcal{L}_{2}}}{\|\boldsymbol{u}\|_{\mathcal{L}_{2}}}
$$

Bemerkung 3.1. Neben der $\mathcal{L}_{2}$-Norm existieren verschiedene andere Signalnormen, wie zum Beispiel die Maximumsnorm oder die 1-Norm. Aus diesen Normen beziehungsweise unterschiedlichen Normen für $\boldsymbol{u}$ und $\boldsymbol{y}$ in (3.1) ergeben sich jeweils andere induzierte Systemnormen, wie zum Beispiel die $\ell_{1}$ - oder peak-to-peak-Norm [206]. Weiterhin findet die $\mathcal{H}_{2^{-}}$ Norm häufig Anwendung, zum Beispiel in der Herleitung eines optimalen Kalman-Filters. Die $\mathcal{H}_{\infty}$-Norm ist für die Robustheitsoptimierung die naheliegendste Norm, da sie Worst-Case-Abschätzungen hinsichtlich der Verstärkung harmonischer Anregungen erlaubt. Prinzipiell lassen sich jedoch viele im Rahmen dieser Arbeit erzielten Ergebnisse auch auf andere Normen übertragen.

Für die $\mathcal{H}_{\infty}$-Norm gilt nach [206]

$$
\left\|\boldsymbol{G}_{\boldsymbol{y} \boldsymbol{u}}(s)\right\|_{\infty}=\sup _{\omega} \bar{\sigma}\left(\boldsymbol{G}_{\boldsymbol{y} \boldsymbol{u}}(j \omega)\right)
$$

das heißt die Norm beschreibt die kleinste obere Schranke für den größten Singulärwert $\bar{\sigma}$ von $\boldsymbol{G}_{\boldsymbol{y} \boldsymbol{u}}(j \omega)$ über alle Frequenzen. Sie stellt eine WorstCase-Abschätzung dar und beschreibt den größtmöglichen Einfluss, den 
das Eingangssignal $\boldsymbol{u}$ bezogen auf die Verstärkung harmonischer Anregungen auf die Ausgangsgrößen $\boldsymbol{y}$ nehmen kann. Dieser Zusammenhang begründet die große Bedeutung der $\mathcal{H}_{\infty}$-Norm in vielen Bereichen der Regelungstechnik, zum Beispiel beim Entwurf robuster Regelungen [126], in der Ordnungsreduktion [62] und insbesondere im Bereich der Fehlerdiagnose [32, 58]. Für Mehrgrößensysteme lässt sich nicht nur ermitteln, wie hoch die größte auftretende Verstärkung ist und bei welcher Frequenz $\omega_{\max }$ sie auftritt. Eine Singulärwertzerlegung von $\boldsymbol{G}_{\boldsymbol{y} \boldsymbol{u}}\left(j \omega_{\max }\right)$ liefert auch die Eingangsrichtung, in der das System für die größtmögliche Verstärkung anzuregen ist.

Im Bereich der robusten Fehlerisolation lässt sich die $\mathcal{H}_{\infty}$-Norm zur Quantifizierung der in Abschnitt 2.3 qualitativ genannten Robustheitsziele heranziehen. Um den Einfluss von Stell- und Störgrößen auf die generierten Residuen zu unterdrücken, wird eine Minimierung von $\left\|\boldsymbol{G}_{\boldsymbol{r u}}(s)\right\|_{\infty}$ beziehungsweise $\left\|\boldsymbol{G}_{\boldsymbol{r} \boldsymbol{d}}(s)\right\|_{\infty}$ angestrebt.

Gemäß (3.2) beschreibt die $\mathcal{H}_{\infty}$-Norm eine Schranke für den größten Singulärwert über alle Frequenzen. In vielen Anwendungsfällen beinhalten die betrachteten Eingangssignale jedoch nicht alle Frequenzen, die Anteile beschränken sich stattdessen auf bestimmte Frequenzbereiche. So sind beispielsweise Stellgrößen aufgrund physikalischer Limitierungen der Aktoren oftmals relativ niederfrequent und Störsignale können durch eine Spektralanalyse auf relevante Frequenzbereiche eingegrenzt werden. In solchen Fällen führt die Verwendung der $\mathcal{H}_{\infty}$-Norm unter Umständen zu sehr konservativen Abschätzungen. Es ist dann hilfreich, die kleinste obere Schranke für den größten Singulärwert von $\boldsymbol{G}_{\boldsymbol{y} \boldsymbol{u}}(j \omega)$ über den relevanten Frequenzbereich zu betrachten, was zu folgender Definition führt:

Definition 3.3 $\left(\mathcal{H}_{\infty}\right.$-Norm in begrenztem Frequenzbereich). Für ein stabiles lineares System, welches durch die Übertragungsmatrix $\boldsymbol{G}_{\boldsymbol{y} \boldsymbol{u}}(s)$ beschrieben wird, ist die $\mathcal{H}_{\infty}$-Norm im Frequenzbereich $\Omega$ definiert als

$$
\left\|\boldsymbol{G}_{\boldsymbol{y} \boldsymbol{u}}(s)\right\|_{\infty}^{\Omega}=\sup _{\omega \in \Omega} \bar{\sigma}\left(\boldsymbol{G}_{\boldsymbol{y} \boldsymbol{u}}(j \omega)\right) .
$$

Bemerkung 3.2. Wie bereits in Abschnitt 2.5.1 ausgeführt lässt sich die Berechnung der $\mathcal{H}_{\infty}$-Norm in einem beschränkten Frequenzintervall mithilfe von Gewichtungsfiltern näherungsweise auf die Berechnung der $\mathcal{H}_{\infty}$-Norm eines erweiterten Systems über alle Frequenzen zurückführen. Obige Definition liefert jedoch eine exakte Charakterisierung der Norm innerhalb eines beschränkten Frequenzbereiches.

Während die $\mathcal{H}_{\infty}$-Norm hilfreich bei der Beschreibung der Robustheitsanforderung ist, wird für die Quantifizierung der Sensitivität 
(s. Abschnitt 2.3) auf ein anderes Maß zurückgegriffen, welches die kleinstmögliche Verstärkung beschreibt. Im Sinne einer hohen Sensitivität bezüglich der Fehler wird angestrebt, die kleinste auftretende Verstärkung von $\boldsymbol{G}_{\boldsymbol{r} \boldsymbol{f}}(s) \mathrm{zu}$ maximieren. Um diese zu beschreiben, wird der $\mathcal{H}_{--}$ Index verwendet. In der Literatur existieren unterschiedliche Definitionen [32, 135, 243], hier wird der Index wie folgt verstanden:

Definition 3.4 ( $\mathcal{H}_{-}$-Index). Für ein stabiles lineares System, welches durch die Übertragungsmatrix $\boldsymbol{G}_{\boldsymbol{y} \boldsymbol{u}}(s)$ beschrieben wird, ist der $\mathcal{H}_{-}$-Index definiert als

$$
\left\|\boldsymbol{G}_{\boldsymbol{y} \boldsymbol{u}}(s)\right\|_{-}=\inf _{\omega} \underline{\sigma}\left(\boldsymbol{G}_{\boldsymbol{y} \boldsymbol{u}}(j \omega)\right)
$$

wobei $\underline{\sigma}$ den kleinsten Singulärwert von $\boldsymbol{G}_{\boldsymbol{y} \boldsymbol{u}}(j \omega)$ darstellt.

Offensichtlich ist für Systeme ohne Durchgriff der $\mathcal{H}_{-}$-Index nach obiger Definition stets 0 , da der kleinste Singulärwert von $\boldsymbol{G}_{\boldsymbol{y} \boldsymbol{u}}(j \omega)$ für $\omega \rightarrow \infty$ gegen 0 geht. Ebenso wie für die $\mathcal{H}_{\infty}$-Norm lassen sich die Betrachtungen auf einen bestimmten Frequenzbereich einschränken. Dies führt auf

Definition 3.5 ( $\mathcal{H}_{-}$-Index in begrenztem Frequenzbereich). Für ein stabiles lineares System, welches durch die Übertragungsmatrix $\boldsymbol{G}_{\boldsymbol{y} \boldsymbol{u}}(s)$ beschrieben wird, ist der $\mathcal{H}_{-}$-Index im Frequenzbereich $\Omega$ definiert als

$$
\left\|\boldsymbol{G}_{\boldsymbol{y} \boldsymbol{u}}(s)\right\|_{-}^{\Omega}=\inf _{\omega \in \Omega} \underline{\sigma}\left(\boldsymbol{G}_{\boldsymbol{y} \boldsymbol{u}}(j \omega)\right) .
$$

Für Eingrößensysteme lässt sich die $\mathcal{H}_{\infty}$-Norm anschaulich interpretieren als die kleinste obere Schranke des Amplitudenganges. In Abbildung 3.1 ist beispielhaft der Amplitudengang eines $\mathrm{PT}_{2}$-Gliedes mit den Eigenwerten $\lambda_{1 / 2}=-1 \pm 6 i$ und der stationären Verstärkung von $g_{y u}(s=0)=2$ betrachtet. Über alle Frequenzen ergibt sich die größte Verstärkung aus $\left\|g_{y u}(s)\right\|_{\infty}=6,16$. Geht man jedoch davon aus, dass das Eingangssignal lediglich Frequenzen aus dem blau eingefärbtem Bereich $\Omega_{1}=[10 ; \infty)$ enthält, so ist offensichtlich, dass im relevanten Frequenzbereich nur deutlich geringere Verstärkungen auftreten, als dies durch $\left\|g_{y u}(s)\right\|_{\infty}$ beschrieben wird. Im Beispiel aus Abbildung 3.1 ergibt sich ein Wert von $\left\|g_{y u}(s)\right\|_{\infty}^{\Omega_{1}}=1,12$. Betrachtet man weiterhin den Frequenzbereich $\Omega_{2}=[0 ; 0,1]$, so ergibt sich der $\mathcal{H}_{-}$-Index zu $\left\|g_{y u}(s)\right\|_{-}^{\Omega_{2}}=2,00$, was der stationären Verstärkung entspricht. Wegen $\left\|g_{y u}(s)\right\|_{-}=0$ lässt sich der $\mathcal{H}_{-}$-Index über alle Frequenzen in der logarithmischen Darstellung in Abbildung 3.1 nicht als Schranke darstellen. 


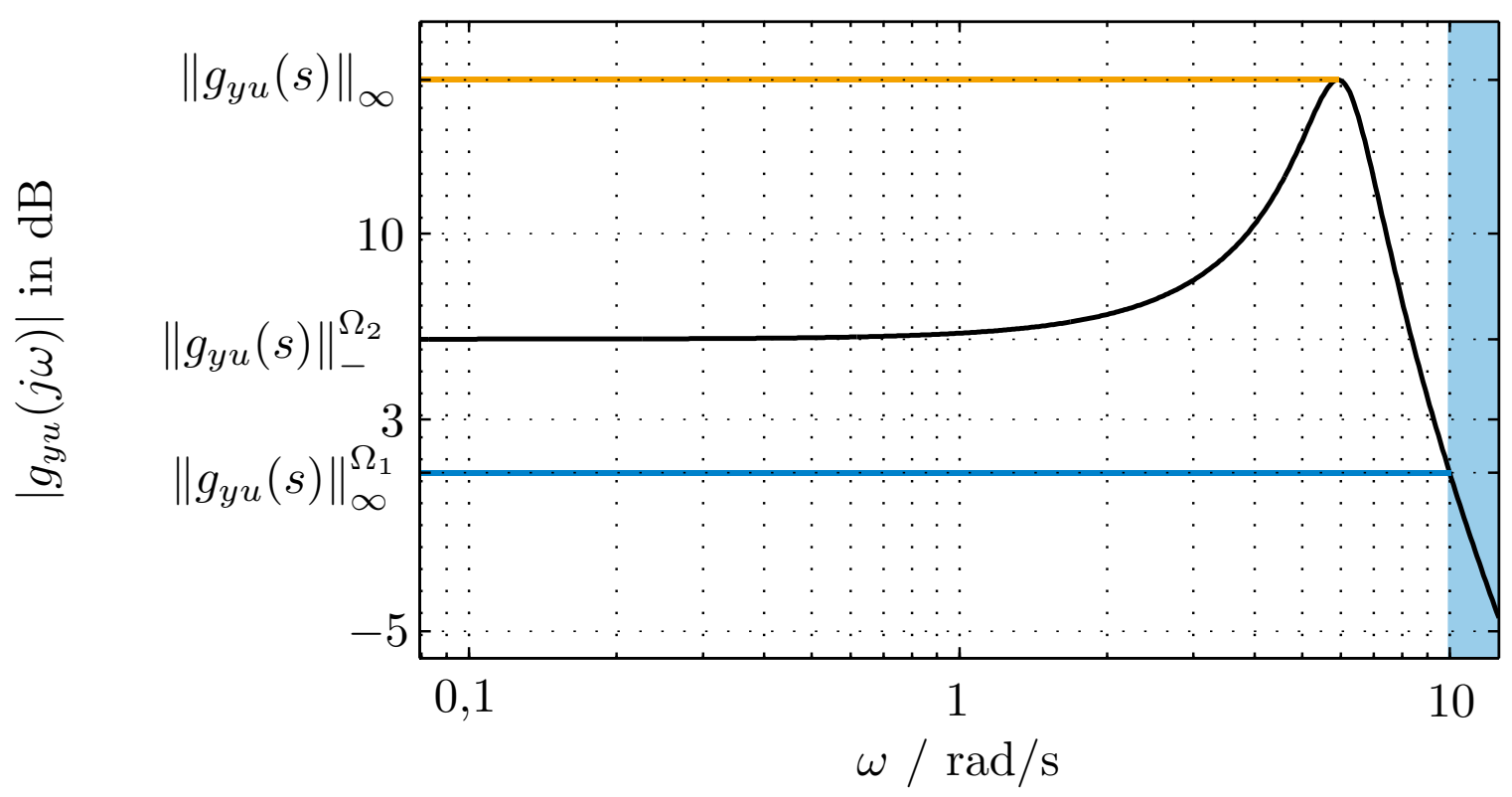

Abbildung 3.1: Veranschaulichung der $\mathcal{H}_{\infty}$-Norm und des $\mathcal{H}_{-}$-Index

\subsection{Lineare Matrixungleichungen}

Zahlreiche regelungstechnische Probleme lassen sich unter Rückgriff auf sogenannte lineare Matrixungleichungen (engl. linear matrix inequalities, LMIs) lösen. Dabei handelt es sich allgemein um eine matrixwertige Funktion $\boldsymbol{F}(\boldsymbol{x}) \in \mathbb{R}^{n \times n}$, die affin von den Elementen von $\boldsymbol{x} \in \mathbb{R}^{m}$ abhängt und die in der Form

$$
\boldsymbol{F}(\boldsymbol{x})=\boldsymbol{F}_{0}+\sum_{i=1}^{m} x_{i} \boldsymbol{F}_{i} \succ \mathbf{0}
$$

geschrieben werden kann. Dabei sind $\boldsymbol{F}_{0}=\boldsymbol{F}_{0}^{\boldsymbol{\top}}$ und $\boldsymbol{F}_{i}=\boldsymbol{F}_{i}^{\boldsymbol{\top}}, i=1, \ldots, m$, symmetrische Matrizen und das Symbol $\succ$ beschreibt die positive Definitheit $^{1)}$ von $\boldsymbol{F}(\boldsymbol{x})$. Es ist also $\boldsymbol{u}^{\top} \cdot \boldsymbol{F}(\boldsymbol{x}) \cdot \boldsymbol{u}>0$ für alle $\boldsymbol{u} \neq \mathbf{0} \in \mathbb{R}^{n}$ und alle Eigenwerte von $\boldsymbol{F}(\boldsymbol{x})$ sind positiv. Gleichung (3.3) beschreibt eine strikte LMI. Darüber hinaus existieren nichtstrikte LMIs, bei denen lediglich positive (beziehungsweise negative) Semidefinitheit gefordert wird, was durch das Symbol $\succeq(\preceq)$ beschrieben wird. Wie in [20] gezeigt lassen sich nichtstrikte LMIs stets in strikte LMIs überführen.

Die Quelle [20] stellt ein Standardwerk zu LMIs dar und bietet ebenso wie [193, 217] einen ausführlichen Überblick. An dieser Stelle werden lediglich die wesentlichen Grundlagen erörtert.

${ }^{1)}$ Die Symmetrie von $\boldsymbol{F}_{0}$ und $\boldsymbol{F}_{i}$ impliziert darüber hinaus die Symmetrie von $\boldsymbol{F}(\boldsymbol{x})$, die durch das Symbol $\succ$ ebenfalls zum Ausdruck gebracht wird. 
Ein wichtiges Merkmal von LMIs ist ihre Konvexität. Diese lässt sich für $\boldsymbol{F}(\boldsymbol{x}) \succ \mathbf{0}$ und $\boldsymbol{F}(\boldsymbol{y}) \succ \mathbf{0}$ mit einem Skalar $0 \leq \lambda \leq 1$ leicht durch

$$
\begin{aligned}
\boldsymbol{F}(\lambda \boldsymbol{x}+(1-\lambda) \boldsymbol{y}) & =\boldsymbol{F}_{0}+\sum_{i=1}^{m}\left(\lambda x_{i}+(1-\lambda) y_{i}\right) \boldsymbol{F}_{i} \\
& =\lambda \boldsymbol{F}_{0}+(1-\lambda) \boldsymbol{F}_{0}+\sum_{i=1}^{m} \lambda x_{i} \boldsymbol{F}_{i}+\sum_{i=1}^{m}(1-\lambda) y_{i} \boldsymbol{F}_{i} \\
& =\lambda\left(\boldsymbol{F}_{0}+\sum_{i=1}^{m} x_{i} \boldsymbol{F}_{i}\right)+(1-\lambda)\left(\boldsymbol{F}_{0}+\sum_{i=1}^{m} y_{i} \boldsymbol{F}_{i}\right) \\
& =\lambda \boldsymbol{F}(\boldsymbol{x})+(1-\lambda) \boldsymbol{F}(\boldsymbol{y}) \succ \mathbf{0}
\end{aligned}
$$

nachweisen. Diese Tatsache ist insofern bemerkenswert, als dass sich hinter (3.3) ein System polynomialer Ungleichungen verbirgt. Diese ergeben sich daraus, dass die positive Definitheit von $\boldsymbol{F}(\boldsymbol{x})$ genau dann gegeben ist, wenn alle Hauptabschnittsdeterminanten von $\boldsymbol{F}(\boldsymbol{x})$ positiv sind [14, Proposition 8.2.7].

Die Konvexität der LMIs bedingt, dass es sich bei Optimierungsproblemen der Form

$$
\begin{aligned}
& \underset{\boldsymbol{x}}{\operatorname{minimiere}} \boldsymbol{c}^{\top} \boldsymbol{x}, \text { sodass } \\
& \boldsymbol{F}(\boldsymbol{x}) \succ \mathbf{0}
\end{aligned}
$$

um konvexe Optimierungsprobleme handelt, da sowohl die Zielfunktion als auch die Nebenbedingungen konvex sind. Daneben werden häufig auch Lösbarkeitsprobleme der Form

$$
\begin{aligned}
& \text { finde } \boldsymbol{x}, \text { sodass } \\
& \boldsymbol{F}(\boldsymbol{x}) \succ \mathbf{0}
\end{aligned}
$$

betrachtet, die ebenfalls konvex sind.

Sowohl (3.4) als auch (3.5) lassen sich numerisch effizient lösen. Dies kann zuverlässig durch Ellipsoid-Algorithmen erfolgen. Diese gehen von einer Ellipse aus, welche die optimale Lösung $\boldsymbol{x}^{*}$ von (3.4) beziehungsweise eine gültige Lösung für (3.5) enthält. Anschließend werden iterativ Ellipsen mit kleinerem Volumen berechnet, welche jeweils die optimale beziehungsweise eine gültige Lösung enthalten. Auf diese Art und Weise wird $\boldsymbol{x}^{*}$ iterativ angenähert. Für Details wird auf $[15,20]$ verwiesen. Als numerisch effizienter haben sich Innere-Punkte-Verfahren erwiesen. 
Sie sind auch in den frei verfügbaren Solvern SEDuMi [210] und SDPT3 [216] umgesetzt. Innere-Punkte-Verfahren stellen fortgeschrittene Optimierungsverfahren dar, auf die Darstellung von Details wird an dieser Stelle verzichtet. Eine kurze Einführung findet sich in [119, Abschnitt 2.6.2], für tiefergehende Betrachtungen wird auf [19, 20, 263] verwiesen.

Da (3.3) lediglich skalare Variablen $x_{i}$ beinhaltet, erscheint die Struktur auf den ersten Blick restriktiv. Es lassen sich jedoch zahlreiche regelungstechnische Standardprobleme in der gegebenen Form beschreiben. Als klassisches Beispiel wird oftmals die Lyapunov-Ungleichung

$$
\boldsymbol{P} \boldsymbol{A}+\boldsymbol{A}^{\top} \boldsymbol{P}=\operatorname{He}(\boldsymbol{P} \boldsymbol{A}) \prec \mathbf{0}
$$

angeführt. ${ }^{2)}$ Bekanntlich ist die Ruhelage $\boldsymbol{x}_{R}=\mathbf{0}$ eines linearen Systems $\dot{\boldsymbol{x}}=\boldsymbol{A} \boldsymbol{x}$ genau dann asymptotisch stabil, wenn eine symmetrische Matrix $\boldsymbol{P} \succ \mathbf{0}$ existiert, sodass (3.6) erfüllt ist [1]. Für ein System 2. Ordnung mit

$$
\boldsymbol{P}=\left[\begin{array}{ll}
p_{11} & p_{12} \\
p_{12} & p_{22}
\end{array}\right]
$$

lässt sich (3.6) beispielhaft schreiben als

$$
\mathrm{He}\left(p_{11}\left[\begin{array}{ll}
1 & 0 \\
0 & 0
\end{array}\right] \boldsymbol{A}+p_{12}\left[\begin{array}{ll}
0 & 1 \\
1 & 0
\end{array}\right] \boldsymbol{A}+p_{22}\left[\begin{array}{ll}
0 & 0 \\
0 & 1
\end{array}\right] \boldsymbol{A}\right) \prec \mathbf{0} .
$$

Mit

$$
\begin{aligned}
\boldsymbol{x} & =\left[\begin{array}{lll}
p_{11} & p_{12} & p_{22}
\end{array}\right]^{\top}, \\
\boldsymbol{F}_{0} & =\mathbf{0} \\
\boldsymbol{F}_{1} & =\operatorname{He}\left(\left[\begin{array}{ll}
1 & 0 \\
0 & 0
\end{array}\right] \boldsymbol{A}\right), \boldsymbol{F}_{2}=\operatorname{He}\left(\left[\begin{array}{ll}
0 & 1 \\
1 & 0
\end{array}\right] \boldsymbol{A}\right), \boldsymbol{F}_{3}=\operatorname{He}\left(\left[\begin{array}{ll}
0 & 0 \\
0 & 1
\end{array}\right] \boldsymbol{A}\right)
\end{aligned}
$$

entspricht dies der Gleichung (3.3). Entsprechend lassen sich alle linearen beziehungsweise affinen Ungleichungen mit matrixwertigen Variablen in die Form (3.3) überführen. Gängige Schnittstellen wie YALMIP [128] oder Cvx [89, 90] erlauben es, LMIs unmittelbar mit matrixwertigen Variablen wie zum Beispiel in (3.6) einzugeben. Die Umwandlung in die Standardform (3.3) erfolgt dann automatisiert. Im weiteren Verlauf der Arbeit wird daher stets mit derartigen Matrixungleichungen gearbeitet.

Im Folgenden werden zunächst einige Rechenregeln für LMIs zusammengefasst, bevor in Abschnitt 3.2.2 für den weiteren Verlauf der Arbeit wesentliche LMIs dargestellt werden.

${ }^{2)}$ Im Sinne einer kompakten Notation wird in dieser Arbeit häufig die abkürzende Schreibweise $\operatorname{He}(\boldsymbol{X})=\boldsymbol{X}+\boldsymbol{X}^{\top}$ verwendet. 


\subsubsection{Rechenregeln für LMIs}

Da der Ausdruck $\boldsymbol{X} \succ \mathbf{0}$ die positive Definitheit von $\boldsymbol{X}$ beschreibt und somit gleichbedeutend damit ist, dass alle Eigenwerte von $\boldsymbol{X}$ positiv sind, lassen sich mehrere LMIs in einfacher Art und Weise zu einer LMI zusammenfassen.

Lemma 3.1 (Zusammenfassung von LMIs). Gegeben seien zwei symmetrische Matrizen $\boldsymbol{X}$ und $\boldsymbol{Y}$, welche $\boldsymbol{X} \succ \mathbf{0}$ und $\boldsymbol{Y} \succ \mathbf{0}$ erfüllen. Diese LMIs sind äquivalent $z u$

$$
\left[\begin{array}{cc}
\boldsymbol{X} & \mathbf{0} \\
\mathbf{0} & \boldsymbol{Y}
\end{array}\right] \succ \mathbf{0}
$$

Ein weiteres wesentliches Merkmal von LMIs ist, dass sie nicht eindeutig sind. Das heißt, dass die gleiche Lösungsmenge durch unendlich viele LMIs beschrieben werden kann. Formal ausgedrückt wird dies nach [20] durch

Lemma 3.2 (Kongruenztransformation von LMIs). Gegeben sei eine symmetrische Matrix $\boldsymbol{X} \in \mathbb{R}^{n \times n}$ und eine reguläre Matrix $\boldsymbol{T} \in \mathbb{R}^{n \times n}$. Dann sind die LMIs

1. $\boldsymbol{X} \succ \mathbf{0}$ und

2. $\boldsymbol{T}^{\top} \cdot \boldsymbol{X} \cdot \boldsymbol{T} \succ \mathbf{0}$

hinsichtlich der Lösungsmenge äquivalent.

Eine geschickte Wahl von $\boldsymbol{T}$ kann in Verbindung mit geeigneten Variablensubstitutionen oftmals dabei helfen, allgemeinere (zum Beispiel bilineare) Matrixungleichungen in LMIs zu überführen. Ein Beispiel hierfür ist das Auffinden einer stabilisierenden Zustandsrückführung $\boldsymbol{u}=-\boldsymbol{K} \boldsymbol{x}$ für ein lineares System der Form $\dot{\boldsymbol{x}}=\boldsymbol{A} \boldsymbol{x}+\boldsymbol{B} \boldsymbol{u}$. Der geschlossene Regelkreis ist asymptotisch stabil, wenn eine Matrix $\boldsymbol{P} \succ \mathbf{0}$ existiert, sodass

$$
\operatorname{He}(\boldsymbol{P}(\boldsymbol{A}-\boldsymbol{B} \boldsymbol{K})) \prec \mathbf{0}
$$

gilt. Da sowohl die Lyapunov-Matrix $\boldsymbol{P}$ als auch die Reglermatrix $\boldsymbol{K}$ gesucht werden, handelt es sich dabei um eine bilineare Matrixungleichung (BMI). Unter Rückgriff auf Lemma 3.2 ist (3.7) gleichbedeutend mit

$$
\boldsymbol{P}^{-1} \cdot \operatorname{He}(\boldsymbol{P}(\boldsymbol{A}-\boldsymbol{B} \boldsymbol{K})) \cdot \boldsymbol{P}^{-1}=\operatorname{He}\left((\boldsymbol{A}-\boldsymbol{B} \boldsymbol{K}) \cdot \boldsymbol{P}^{-1}\right) \prec \mathbf{0} .
$$

Führt man nun die Variablensubstitutionen $\boldsymbol{Q}=\boldsymbol{P}^{-1}$ und $\boldsymbol{Y}=\boldsymbol{K} \boldsymbol{Q}$ ein, so lässt sich

$$
\operatorname{He}(\boldsymbol{A} \boldsymbol{Q}-\boldsymbol{B} \boldsymbol{Y}) \prec \mathbf{0}
$$


schreiben. Bei (3.8) handelt es sich um eine LMI in den Variablen $\boldsymbol{Q}$ und $\boldsymbol{Y}$, welche die gleiche Lösungsmenge beschreibt wie die BMI (3.7). Nach dem Lösen von (3.8) erhält man die gesuchte Rückführmatrix aus der Rücksubstitution $\boldsymbol{K}=\boldsymbol{Y} \boldsymbol{Q}^{-1}$.

Von zentraler Bedeutung bei der Manipulation von Matrixungleichungen ist darüber hinaus das Schur-Komplement-Lemma (s. [20, Abschnitt 2.1]).

Lemma 3.3 (Schur-Komplement-Lemma). Gegeben seien die symmetrischen Matrizen $\boldsymbol{X} \in \mathbb{R}^{n \times n}$ und $\boldsymbol{Y} \in \mathbb{R}^{m \times m}$, sowie die Matrix $\boldsymbol{Z} \in \mathbb{R}^{n \times m}$. Dann gilt die Äquivalenz $z^{3)}$

$$
\left[\begin{array}{ll}
\boldsymbol{X} & \boldsymbol{Z} \\
\boldsymbol{Z}^{\top} & \boldsymbol{Y}
\end{array}\right]=\left[\begin{array}{cc}
\boldsymbol{X} & \boldsymbol{Z} \\
\star & \boldsymbol{Y}
\end{array}\right] \succ \mathbf{0} \quad \Leftrightarrow \quad \boldsymbol{Y} \succ \mathbf{0}, \boldsymbol{X}-\boldsymbol{Z} \boldsymbol{Y}^{-1} \boldsymbol{Z}^{\top} \succ \mathbf{0} .
$$

Beweis. Aus Lemma 3.2 folgt unmittelbar

$$
\left[\begin{array}{cc}
\boldsymbol{X} & \boldsymbol{Z} \\
\star & \boldsymbol{Y}
\end{array}\right] \succ \mathbf{0} \Leftrightarrow\left[\begin{array}{cc}
\boldsymbol{I}_{n} & -\boldsymbol{Z} \boldsymbol{Y}^{-1} \\
\mathbf{0} & \boldsymbol{I}_{m}
\end{array}\right]\left[\begin{array}{cc}
\boldsymbol{X} & \boldsymbol{Z} \\
\star & \boldsymbol{Y}
\end{array}\right]\left[\begin{array}{cc}
\boldsymbol{I}_{n} & \mathbf{0} \\
-\boldsymbol{Y}^{-1} \boldsymbol{Z}^{\top} & \boldsymbol{I}_{m}
\end{array}\right] \succ \mathbf{0}
$$

was gleichbedeutend ist mit

$$
\left[\begin{array}{cc}
\boldsymbol{X}-\boldsymbol{Z} \boldsymbol{Y}^{-1} \boldsymbol{Z}^{\top} & \mathbf{0} \\
\mathbf{0} & \boldsymbol{Y}
\end{array}\right] \succ \mathbf{0}
$$

Diese Blockdiagonalmatrix ist genau dann positiv definit, wenn ihre Diagonalblöcke positiv definit sind.

Aus dem Beweis ergibt sich weiterhin, dass das Schur-KomplementLemma auch für die negative Definitheit gültig ist. Es gilt demnach

$$
\left[\begin{array}{cc}
\boldsymbol{X} & \boldsymbol{Z} \\
\star & \boldsymbol{Y}
\end{array}\right] \prec \mathbf{0} \quad \Leftrightarrow \quad \boldsymbol{Y} \prec \mathbf{0}, \boldsymbol{X}-\boldsymbol{Z} \boldsymbol{Y}^{-1} \boldsymbol{Z}^{\top} \prec \mathbf{0} .
$$

Mit der Transformationsmatrix

$$
\boldsymbol{T}=\left[\begin{array}{cc}
\mathbf{0} & \boldsymbol{I}_{m} \\
\boldsymbol{I}_{n} & \mathbf{0}
\end{array}\right]
$$

ergibt sich aus Lemma 3.2 eine weitere Variante des Schur-KomplementLemmas zu

$$
\left[\begin{array}{cc}
\boldsymbol{X} & \boldsymbol{Z} \\
\star & \boldsymbol{Y}
\end{array}\right] \succ \mathbf{0} \Leftrightarrow\left[\begin{array}{cc}
\boldsymbol{Y} & \boldsymbol{Z}^{\top} \\
\star & \boldsymbol{X}
\end{array}\right] \succ \mathbf{0} \Leftrightarrow \boldsymbol{X} \succ \mathbf{0}, \boldsymbol{Y}-\boldsymbol{Z}^{\top} \boldsymbol{X}^{-1} \boldsymbol{Z} \succ \mathbf{0} .
$$

${ }^{3)}$ Das Symbol $\star$ steht dabei in dieser Arbeit stets für Elemente beziehungsweise Blöcke, die sich unmittelbar aus der Symmetrie der betrachteten Matrix ergeben. 
Auch diese Variante gilt ebenso für negative Definitheit. Mithilfe des SchurKomplement-Lemmas lassen sich häufig Matrixungleichungen, die quadratisch in einer Entscheidungsvariablen sind, in LMIs höherer Dimension überführen. Ein typisches Beispiel ist die Riccatiungleichung

$$
\mathrm{He}(\boldsymbol{P} \boldsymbol{A})+\boldsymbol{P} \boldsymbol{B} \boldsymbol{R}^{-1} \boldsymbol{B}^{\top} \boldsymbol{P}+\boldsymbol{Q} \prec \mathbf{0},
$$

die quadratisch in $\boldsymbol{P}$ ist. Durch Anwendung von Satz 3.3 mit $\boldsymbol{X}=\operatorname{He}(\boldsymbol{P} \boldsymbol{A})+\boldsymbol{Q}, \boldsymbol{Z}=\boldsymbol{P} \boldsymbol{B}$ und $\boldsymbol{Y}=-\boldsymbol{R}$ lässt sie sich bei positiv definitem $\boldsymbol{R}$ überführen in die LMI

$$
\left[\begin{array}{cc}
\mathrm{He}(\boldsymbol{P} \boldsymbol{A})+\boldsymbol{Q} & \boldsymbol{P} \boldsymbol{B} \\
\star & -\boldsymbol{R}
\end{array}\right] \prec \mathbf{0} .
$$

Neben Kongruenztransformationen, Variablensubstitutionen und dem Schur-Komplement-Lemma stellt das folgende Lemma (s. [205, Korollar 2.3.9]) ein weiteres Hilfsmittel zur Umformung beziehungsweise Vereinfachung von Matrixungleichungen dar.

Lemma 3.4. Gegeben seien zwei reelle Matrizen $\boldsymbol{Q}=\boldsymbol{Q}^{\top}$ und $\boldsymbol{Y}$. Dann sind die folgenden Aussagen äquivalent:

1. ${ }^{\perp} \boldsymbol{Y} \cdot \boldsymbol{Q} \cdot{ }^{\perp} \boldsymbol{Y}^{\top} \prec \mathbf{0}$.

2. Es existiert eine beliebige Matrix $\boldsymbol{X}$ passender Dimension, sodass $\boldsymbol{Q}+\operatorname{He}\left(\boldsymbol{X} \boldsymbol{Y}^{\boldsymbol{\top}}\right) \prec \mathbf{0}$ gilt.

Es handelt sich um einen Spezialfall des sogenannten EliminationsLemmas [20, Abschnitt 2.6.2], welches sich aus Finslers Lemma [70] herleitet. In der gezeigten Form erlaubt es, die in ${ }^{\perp} \boldsymbol{Y}$ quadratische Matrixungleichung in eine in $\boldsymbol{X}$ und $\boldsymbol{Y}$ bilineare Ungleichung zu überführen.

\subsubsection{Spezielle LMIs}

Die induzierte 2-Norm einer Matrix wird nach [14, Proposition 9.4.7] beschrieben durch ihren größten Singulärwert. Dies erlaubt es, die Norm durch LMIs wie folgt abzuschätzen, wobei sich das Lemma auch auf den Spezialfall von Vektoren anwenden lässt.

Lemma 3.5. Gegeben sei eine Matrix $\boldsymbol{Z} \in \mathbb{R}^{n \times m}$ und ein Skalar $\alpha>0$. Dann gilt $\|\boldsymbol{Z}\|_{2}<\alpha$ genau dann, wenn

$$
\left[\begin{array}{cc}
\alpha^{2} \boldsymbol{I}_{n} & \boldsymbol{Z} \\
\star & \boldsymbol{I}_{m}
\end{array}\right] \succ \mathbf{0} \quad \Leftrightarrow \quad \boldsymbol{Z} \boldsymbol{Z}^{\top} \prec \alpha^{2} \boldsymbol{I}_{n}
$$


Beweis. Nach [20] ist $\boldsymbol{Z} \boldsymbol{Z}^{\top} \prec \boldsymbol{I}_{n}$ äquivalent zu $\|\boldsymbol{Z}\|_{2}<1$. Aus dem SchurKomplement-Lemma (Lemma 3.3) folgt damit

$$
\|\boldsymbol{Z}\|_{2}<1 \Leftrightarrow \boldsymbol{Z} \boldsymbol{Z}^{\top} \prec \boldsymbol{I}_{n} \Leftrightarrow\left[\begin{array}{cc}
\boldsymbol{I}_{n} & \boldsymbol{Z} \\
\star & \boldsymbol{I}_{m}
\end{array}\right] \succ \mathbf{0} .
$$

Da $\|\boldsymbol{Z}\|_{2}<\alpha$ äquivalent zu $\frac{1}{\alpha}\|\boldsymbol{Z}\|_{2}=\left\|\frac{1}{\alpha} \boldsymbol{Z}\right\|_{2}<1$ ist, ist dies wegen (3.9) weiterhin gleichbedeutend mit

$$
\left[\begin{array}{cc}
\boldsymbol{I}_{n} & \frac{1}{\alpha} \boldsymbol{Z} \\
\star & \boldsymbol{I}_{m}
\end{array}\right] \succ \mathbf{0}
$$

Eine Kongruenztransformation mit $\boldsymbol{T}=\operatorname{diag}\left(\alpha \boldsymbol{I}_{n}, \boldsymbol{I}_{m}\right)$ führt schließlich auf

$$
\left[\begin{array}{cc}
\alpha^{2} \boldsymbol{I}_{n} & \boldsymbol{Z} \\
\star & \boldsymbol{I}_{m}
\end{array}\right] \succ \mathbf{0}
$$

Die Anwendung des Schur-Komplement-Lemmas auf (3.10) ergibt $\alpha^{2} \boldsymbol{I}_{n}-\boldsymbol{Z} \boldsymbol{Z}^{\top} \succ \mathbf{0}$, sodass $\boldsymbol{Z} \boldsymbol{Z}^{\top} \prec \alpha^{2} \boldsymbol{I}_{n}$ folgt.

Das Lemma lässt sich auch dazu nutzen, Gleichungsbedingungen durch Ungleichungsbedingungen anzunähern. Soll beispielsweise $\boldsymbol{X} \boldsymbol{A}=\boldsymbol{B}$ mit $\boldsymbol{X} \in \mathbb{R}^{n \times m}, \boldsymbol{A} \in \mathbb{R}^{m \times p}$ und $\boldsymbol{B} \in \mathbb{R}^{n \times p}$ erfüllt werden, so lässt sich dies annähern durch $\|\boldsymbol{X} \boldsymbol{A}-\boldsymbol{B}\|_{2}<\varepsilon$ mit einem beliebig kleinen Skalar $\varepsilon>0$. Mittels Lemma 3.5 kann hierfür äquivalent

$$
\left[\begin{array}{cc}
\varepsilon^{2} \boldsymbol{I}_{n} & \boldsymbol{X} \boldsymbol{A}-\boldsymbol{B} \\
\star & \boldsymbol{I}_{p}
\end{array}\right] \succ \mathbf{0}
$$

geschrieben werden. Gängige Schnittstellen wie YALmIP [128] oder Cvx $[89,90]$ nehmen eine solche Umwandlung automatisch vor, sodass lineare beziehungsweise affine Gleichungsbedingungen ohne Weiteres als Nebenbedingungen in LMI-Problemen berücksichtigt werden können.

\section{LMIs zur Abschätzung von Systemnormen}

Wie in Abschnitt 3.1 diskutiert lassen sich die Robustheitsanforderungen beim Entwurf von Fehlerisolationsbeobachtern mithilfe der $\mathcal{H}_{\infty}$-Norm von Übertragungsmatrizen quantifizieren. Das Bounded-Real-Lemma (BRL, s. [20, Abschnitt 2.7.3]) erlaubt es, die Beschränkung dieser Norm anhand linearer Matrixungleichungen zu charakterisieren. Daher ist es von zentraler Bedeutung für die vorliegende Arbeit. 
Satz 3.1 (Bounded-Real-Lemma). Gegeben sei ein stabiles lineares System $\dot{\boldsymbol{x}}=\boldsymbol{A} \boldsymbol{x}+\boldsymbol{B} \boldsymbol{u}, \boldsymbol{y}=\boldsymbol{C} \boldsymbol{x}+\boldsymbol{D} \boldsymbol{u}$ mit $\boldsymbol{x} \in \mathbb{R}^{n}, \boldsymbol{u} \in \mathbb{R}^{n_{u}}, \boldsymbol{y} \in \mathbb{R}^{n_{y}}$ und der Übertragungsmatrix

$$
\boldsymbol{G}_{\boldsymbol{y} \boldsymbol{u}}(s)=\boldsymbol{C}\left(s \boldsymbol{I}_{n}-\boldsymbol{A}\right)^{-1} \boldsymbol{B}+\boldsymbol{D} .
$$

Dann gilt $\left\|\boldsymbol{G}_{\boldsymbol{y u}}(s)\right\|_{\infty}<\gamma$ mit einem positiven Skalar $\gamma$ genau dann, wenn eine symmetrische, positiv definite Matrix $\boldsymbol{X}$ existiert, so dass

$$
\left[\begin{array}{ccc}
\operatorname{He}(\boldsymbol{X} \boldsymbol{A}) & \boldsymbol{X} \boldsymbol{B} & \boldsymbol{C}^{\top} \\
\star & -\gamma \boldsymbol{I}_{n_{u}} & \boldsymbol{D}^{\top} \\
\star & \star & -\gamma \boldsymbol{I}_{n_{y}}
\end{array}\right] \prec \mathbf{0} .
$$

Der Beweis ist in Anhang A auf Seite 346 skizziert. Wie in [192, Abschnitt 3.3] gezeigt lassen sich vergleichbare LMIs zur Abschätzung anderer Systemnormen herleiten. Dies ermöglicht es, die jeweils für die Optimierung der $\mathcal{H}_{\infty}$-Norm erzielten Ergebnisse auf die Minimierung anderer Normen zu übertragen.

Auch die $\mathcal{H}_{\infty}$-Norm in einem begrenzten Frequenzintervall lässt sich durch LMIs abschätzen. In [113, Abschnitt IV] beziehungsweise [176, Abschnitt IV] findet sich dazu der folgende Satz:

Satz 3.2 (Sonderfall des verallgemeinerten Kalman-Yakubovich-Popov-Lemmas). Gegeben sei ein stabiles lineares System $\dot{\boldsymbol{x}}=\boldsymbol{A} \boldsymbol{x}+\boldsymbol{B u}$, $\boldsymbol{y}=\boldsymbol{C} \boldsymbol{x}+\boldsymbol{D} \boldsymbol{u}$ mit $\boldsymbol{x} \in \mathbb{R}^{n}, \boldsymbol{u} \in \mathbb{R}^{n_{u}}, \boldsymbol{y} \in \mathbb{R}^{n_{y}}$ und der Übertragungsmatrix

$$
\boldsymbol{G}_{\boldsymbol{y} \boldsymbol{u}}(s)=\boldsymbol{C}\left(s \boldsymbol{I}_{n}-\boldsymbol{A}\right)^{-1} \boldsymbol{B}+\boldsymbol{D} .
$$

Weiterhin sei $\gamma$ ein positiver Skalar, $\Omega=\left[\omega_{l} ; \omega_{h}\right]$ ein abgeschlossener Frequenzbereich und $\omega_{c}=\frac{1}{2}\left(\omega_{l}+\omega_{h}\right)$. Dann sind die folgenden Aussagen äquivalent:

1. $\left\|\boldsymbol{G}_{\boldsymbol{y} \boldsymbol{u}}(s)\right\|_{\infty}^{\Omega}<\gamma$.

2. $\left[\begin{array}{c}\boldsymbol{G}_{\boldsymbol{y} \boldsymbol{u}}(j \omega) \\ \boldsymbol{I}_{n_{u}}\end{array}\right]^{\mathrm{H}} \boldsymbol{\Gamma}\left[\begin{array}{c}\boldsymbol{G}_{\boldsymbol{y} \boldsymbol{u}}(j \omega) \\ \boldsymbol{I}_{n_{u}}\end{array}\right] \prec \mathbf{0}, \quad \forall \omega \in \Omega$, mit $\boldsymbol{\Gamma}=\left[\begin{array}{cc}\boldsymbol{I}_{n_{y}} & \mathbf{0} \\ \mathbf{0} & -\gamma^{2} \boldsymbol{I}_{n_{u}}\end{array}\right]$.

3. Es existieren symmetrische Matrizen $\mathbb{R}^{n \times n} \ni \boldsymbol{X} \succ \mathbf{0}$ und $\boldsymbol{Y} \in \mathbb{R}^{n \times n}$, sodass

$$
\left[\begin{array}{cc}
\boldsymbol{A} & \boldsymbol{B} \\
\boldsymbol{I}_{n} & \mathbf{0}
\end{array}\right]^{\top} \boldsymbol{\Xi}\left[\begin{array}{cc}
\boldsymbol{A} & \boldsymbol{B} \\
\boldsymbol{I}_{n} & \mathbf{0}
\end{array}\right]+\left[\begin{array}{cc}
\boldsymbol{C} & \boldsymbol{D} \\
\mathbf{0} & \boldsymbol{I}_{n_{u}}
\end{array}\right]^{\top} \boldsymbol{\Gamma}\left[\begin{array}{cc}
\boldsymbol{C} & \boldsymbol{D} \\
\mathbf{0} & \boldsymbol{I}_{n_{u}}
\end{array}\right] \prec \mathbf{0}
$$




$$
\text { mit } \boldsymbol{\Xi}=\left[\begin{array}{cc}
-\boldsymbol{X} & \boldsymbol{Y}+j \omega_{c} \boldsymbol{X} \\
\boldsymbol{Y}-j \omega_{c} \boldsymbol{X} & -\omega_{l} \omega_{h} \boldsymbol{X}
\end{array}\right] \text { und } \boldsymbol{\Gamma}=\left[\begin{array}{cc}
\boldsymbol{I}_{n_{y}} & \mathbf{0} \\
\mathbf{0} & -\gamma^{2} \boldsymbol{I}_{n_{u}}
\end{array}\right] \text { gilt. }
$$

Bei Satz 3.2 handelt es sich um einen Spezialfall des verallgemeinerten Kalman-Yakubovich-Popov-Lemmas (engl. Generalized KYP Lemma, GKYP-Lemma). Seine große Bedeutung ergibt sich daraus, dass zur Überprüfung der ersten beiden Aussagen von Satz 3.2 unendlich viele Frequenzen $\omega$ zu betrachten sind, was praktisch im Allgemeinen nicht umsetzbar ist. Die dritte Aussage bietet einen rechnerischen Zugang, da lediglich eine einzige Matrixungleichung zu überprüfen ist. Wie in [113] ausführlich erörtert lassen sich mit dem GKYP-Lemma verschiedene Eigenschaften eines Systems in unterschiedlichen Frequenzbereichen charakterisieren. In der ursprünglichen Fassung aus [113] wird gefordert, dass die Matrizen $\boldsymbol{X}$ und $\boldsymbol{Y}$ hermitesch sind. In [176, Theorem 2] wird darauf aufbauend gezeigt, dass für $\boldsymbol{X}$ und $\boldsymbol{Y}$ ohne Einschränkung der Allgemeinheit reelle, symmetrische Matrizen angesetzt werden können. Dies verringert die Anzahl der Variablen des LMI-Problems signifikant und beschleunigt die Lösung.

Die Wahl von $\boldsymbol{\Gamma}$ in Satz 3.2 legt die zu untersuchende Eigenschaft fest. Wird $\boldsymbol{\Gamma}$ wie in Satz 3.2 gewählt, so wird durch $\gamma$ eine obere Schranke für die $\mathcal{H}_{\infty}$-Norm des Systems beschrieben. Für

$$
\boldsymbol{\Gamma}=\left[\begin{array}{cc}
-\boldsymbol{I}_{n_{y}} & \mathbf{0} \\
\mathbf{0} & \gamma^{2} \boldsymbol{I}_{n_{y}}
\end{array}\right]
$$

stellt der Skalar $\gamma$ eine untere Schranke für den $\mathcal{H}_{-}$-Index des Systems dar. Anstatt der ersten Aussage in Satz 3.2 ist in diesem Fall $\left\|\boldsymbol{G}_{\boldsymbol{y} \boldsymbol{u}}(s)\right\|_{-}^{\Omega}>\gamma$. Durch die Wahl von

$$
\boldsymbol{\Gamma}=\left[\begin{array}{cc}
\mathbf{0} & -\boldsymbol{I}_{n_{y}} \\
-\boldsymbol{I}_{n_{y}} & \mathbf{0}
\end{array}\right]
$$

ergibt sich anstelle der ersten Aussage in Satz 3.2 die Beziehung $\boldsymbol{G}_{\boldsymbol{u} \boldsymbol{y}}(j \omega)+\boldsymbol{G}_{\boldsymbol{u} \boldsymbol{y}}(j \omega)^{\mathrm{H}} \succ \mathbf{0}$, die zum Nachweis der positiven Reellheit der Übertragungsmatrix $\boldsymbol{G}_{\boldsymbol{y} \boldsymbol{u}}(j \omega)$ im gegebenen Frequenzbereich herangezogen werden kann.

Weiterhin lässt sich durch $\boldsymbol{\Xi}$ der betrachtete Frequenzbereich verändern. Für ein beidseitig abgeschlossenes Intervall ist $\boldsymbol{\Xi}$ wie in Satz 3.2 zu wählen. Ein nach oben beschränkter Frequenzbereich ergibt sich mit $\omega_{l}=0 \mathrm{zu}$ $\Omega=\left[0 ; \omega_{h}\right]$. Es ist dann die Matrix

$$
\boldsymbol{\Xi}=\left[\begin{array}{cc}
-\boldsymbol{X} & \boldsymbol{Y} \\
\boldsymbol{Y} & \omega_{h}^{2} \boldsymbol{X}
\end{array}\right]
$$


zu wählen. Ist der Frequenzbereich lediglich nach unten beschränkt, ist $\Omega=\left[\omega_{l} ; \infty\right)$ und man wählt

$$
\boldsymbol{\Xi}=\left[\begin{array}{cc}
\boldsymbol{X} & \boldsymbol{Y} \\
\boldsymbol{Y} & -\omega_{l}^{2} \boldsymbol{X}
\end{array}\right]
$$

Für einen unbeschränkten Frequenzbereich $\Omega=[0 ; \infty)$ ergibt sich aus Satz 3.2 das Bounded-Real-Lemma (Satz 3.1) als Spezialfall. Nähere Erläuterungen zum Kalman-Yakubovich-Popov-Lemma und seiner Beziehung zu den bekannten Kalman-Yakubovich-Popov-Gleichungen [125] sowie zur verallgemeinerten Variante des Kalman-Yakubovich-PopovLemmas und der Wahl der Matrizen $\boldsymbol{\Xi}$ finden sich in Anhang A auf Seite 347 .

\section{LMIs zur Vorgabe von Eigenwertbereichen}

Neben der erörterten Abschätzung von Matrizen- und Systemnormen eignen sich LMIs auch dafür, bestimmte Gebiete in der komplexen Ebene zu beschreiben. Einen Spezialfall hierfür stellt die Lyapunov-Ungleichung (3.6) dar, die erfüllt ist, wenn alle Eigenwerte der Matrix $\boldsymbol{A}$ in der offenen linken s-Halbebene liegen. Das in [38] ausführlich beschriebene Konzept der $\mathcal{D}$-Regionen erlaubt es, allgemeinere Gebiete durch LMIs zu charakterisieren. Die folgenden Lemmata erlauben die Beschreibung der im Rahmen dieser Arbeit verwendeten Regionen, die in Abbildung 3.2 dargestellt sind.

Lemma 3.6. Gegeben sei eine Matrix $\boldsymbol{A} \in \mathbb{R}^{n \times n}$ mit den Eigenwerten $\lambda_{i}, i=1, \ldots, n$, und ein Skalar $\alpha \in \mathbb{R}$. Dann gilt $\mathfrak{R e}\left(\lambda_{i}\right)<\alpha$ für alle $i=1, \ldots, n$, wenn eine positiv definite symmetrische Matrix $\boldsymbol{X} \in \mathbb{R}^{n \times n}$ existiert, sodass

$$
\mathrm{He}(\boldsymbol{X} \boldsymbol{A})-2 \alpha \boldsymbol{X} \prec \mathbf{0}
$$

erfüllt ist.

Lemma 3.7. Gegeben sei eine Matrix $\boldsymbol{A} \in \mathbb{R}^{n \times n}$ mit den Eigenwerten $\lambda_{i}$, $i=1, \ldots, n$, und ein Skalar $\varrho>0$. Dann gilt $\left|\lambda_{i}\right|<\varrho$ für alle $i=1, \ldots, n$, wenn eine positiv definite symmetrische Matrix $\boldsymbol{X} \in \mathbb{R}^{n \times n}$ existiert, sodass

$$
\left[\begin{array}{cc}
-\varrho \boldsymbol{X} & \boldsymbol{X} \boldsymbol{A} \\
\star & -\varrho \boldsymbol{X}
\end{array}\right] \prec \mathbf{0}
$$

erfüllt ist. 
Lemma 3.8. Gegeben sei eine Matrix $\boldsymbol{A} \in \mathbb{R}^{n \times n}$ mit den Eigenwerten $\lambda_{i}, i=1, \ldots, n$, und ein Skalar $0<\phi<\pi / 2$. Dann gilt $\left|\mathfrak{I m}\left(\lambda_{i}\right)\right|<\tan (\phi) \cdot\left|\mathfrak{R e}\left(\lambda_{i}\right)\right|$ für alle $i=1, \ldots, n$, wenn eine positiv definite symmetrische Matrix $\boldsymbol{X} \in \mathbb{R}^{n \times n}$ existiert, sodass

$$
\left[\begin{array}{cc}
\tan (\phi) \cdot \operatorname{He}(\boldsymbol{X} \boldsymbol{A}) & -\boldsymbol{X} \boldsymbol{A}+\boldsymbol{A}^{\top} \boldsymbol{X} \\
\star & \tan (\phi) \cdot \operatorname{He}(\boldsymbol{X} \boldsymbol{A})
\end{array}\right] \prec \mathbf{0}
$$

erfüllt ist.

Weitere LMIs zur Beschreibung von $\mathcal{D}$-Regionen finden sich zum Beispiel in [83]. Anzumerken ist, dass sich die LMIs aus Lemma 3.6 bis 3.8 kombinieren lassen. Dadurch werden wie in Abbildung 3.2 gezeigt Eigenwertbereiche beschrieben, die sich aus der Schnittmenge der jeweiligen Regionen ergeben, die also jeweils alle gestellten Bedingungen erfüllen.

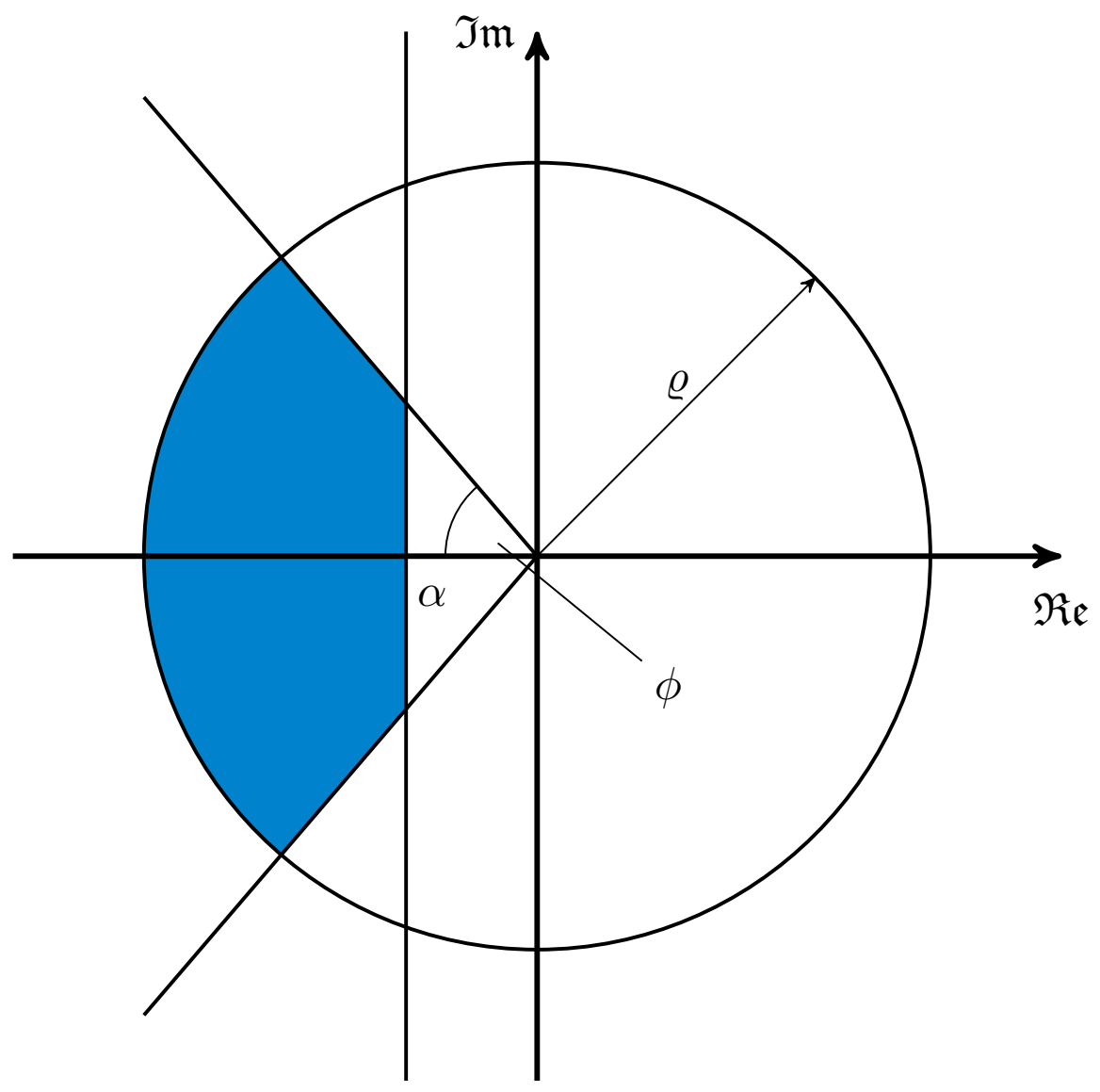

Abbildung 3.2: Eigenwertbereiche aus Lemma 3.6 bis 3.8 


\subsection{Nullstellen linearer Mehrgrößensysteme}

Für lineare Eingrößensystem ist der Nullstellenbegriff in einfacher Art und Weise durch die Nullstellen des Zählerpolynoms der Übertragungsfunktion $g(s)$ definiert. Für eine Nullstelle $\eta$ gilt also $g(\eta)=0$. Somit sorgt eine Nullstelle dafür, dass ein Eingangssignal der Form $u(t)=e^{\eta t}$ den Ausgang nicht anregt, die Übertragung wird somit blockiert. Weiterhin kann eine solche Nullstelle durch eine Regelung nicht verschoben werden. Fällt eine Nullstelle mit einem Pol der Übertragungsfunktion $g(s)$ zusammen, lassen sich die entsprechenden Faktoren im Zähler und Nenner kürzen. Dies wird durch nicht steuerbare beziehungsweise nicht beobachtbare Eigenwerte hervorgerufen.

Für Mehrgrößensysteme gestaltet sich die Situation ungleich vielschichtiger. Da sich im weiteren Verlauf der Arbeit zeigen wird, dass der Begriff der Nullstelle für den Entwurf von Fehlerisolationsbeobachtern von grundlegender Bedeutung ist, wird im Folgenden ein kurze Einführung in die Charakterisierung von Nullstellen linearer Mehrgrößensysteme gegeben. In der Literatur werden verschiedene Klassen von Nullstellen definiert, umfassende Ausführungen dazu finden sich in [98, 145, 186, 187, 190, 191, 196, 207, 211, 212, 264]. Da in der historischen Entwicklung der Begrifflichkeiten nicht immer konsistente Bezeichnungen verwendet wurden, dient der Abschnitt auch dazu, die im Rahmen dieser Arbeit verwendeten Begriffe zu definieren.

Den folgenden Ausführungen liegt stets ein lineares zeitinvariantes System der Form

$$
\begin{aligned}
& \dot{\boldsymbol{x}}=\boldsymbol{A} \boldsymbol{x}+\boldsymbol{B} \boldsymbol{u}, \boldsymbol{x}(t=0)=\boldsymbol{x}_{0}, \\
& \boldsymbol{y}=\boldsymbol{C} \boldsymbol{x}+\boldsymbol{D} \boldsymbol{u}
\end{aligned}
$$

mit $\boldsymbol{x} \in \mathbb{R}^{n}, \boldsymbol{u} \in \mathbb{R}^{n_{u}}, \boldsymbol{y} \in \mathbb{R}^{n_{y}}$ und dem Anfangszustand $\boldsymbol{x}_{0} \in \mathbb{R}^{n}$ zugrunde. Zunächst wird analysiert, unter welchen Voraussetzungen wie im Eingrößenfall eine Übertragungsblockierung auftritt. Dazu wird das System mittels $\boldsymbol{u}(t)=\boldsymbol{u}_{0} \cdot e^{\eta t}$ angeregt. Die Zeitlösung berechnet sich dann aus

$$
\boldsymbol{x}(t)=e^{\boldsymbol{A} t} \boldsymbol{x}_{0}+\int_{0}^{t} e^{\boldsymbol{A}(t-\tau)} \boldsymbol{B} \boldsymbol{u}_{0} e^{\eta \tau} d \tau .
$$

Unter der Voraussetzung, dass $\eta$ kein Eigenwert von $\boldsymbol{A}$ ist, lässt sich das darin auftretende Integral lösen und man erhält

$$
\boldsymbol{x}(t)=e^{\boldsymbol{A} t} \boldsymbol{x}_{0}-e^{\boldsymbol{A} t}\left(\eta \boldsymbol{I}_{n}-\boldsymbol{A}\right)^{-1} \boldsymbol{B} \boldsymbol{u}_{0}+\left(\eta \boldsymbol{I}_{n}-\boldsymbol{A}\right)^{-1} \boldsymbol{B} \boldsymbol{u}_{0} e^{\eta t} .
$$


Setzt man dies in (3.11b) ein, so ergibt sich als Zeitlösung für den Ausgangsvektor

$$
\begin{aligned}
\boldsymbol{y}(t) & =\boldsymbol{C} \boldsymbol{x}(t)+\boldsymbol{D} \boldsymbol{u}(t) \\
& =\boldsymbol{C} e^{\boldsymbol{A} t}\left(\boldsymbol{x}_{0}-\left(\eta \boldsymbol{I}_{n}-\boldsymbol{A}\right)^{-1} \boldsymbol{B} \boldsymbol{u}_{0}\right)+\left(\boldsymbol{C}\left(\eta \boldsymbol{I}_{n}-\boldsymbol{A}\right)^{-1} \boldsymbol{B}+\boldsymbol{D}\right) \boldsymbol{u}_{0} e^{\eta t}
\end{aligned}
$$

Für eine Übertragungsblockierung muss $\boldsymbol{y}(t)=\mathbf{0}$ für alle $t \geq 0$ gelten. Damit die komplexe Frequenz $\eta$ den Ausgang $\boldsymbol{y}$ nicht anregt, muss demnach

$$
\underbrace{\left(\boldsymbol{C}\left(\eta \boldsymbol{I}_{n}-\boldsymbol{A}\right)^{-1} \boldsymbol{B}+\boldsymbol{D}\right)}_{\boldsymbol{G}_{\boldsymbol{y} u}(\eta)} \boldsymbol{u}_{0}=\mathbf{0}
$$

gelten. Diese Gleichung hat eine nichttriviale Lösung, wenn $\boldsymbol{G}_{\boldsymbol{y} \boldsymbol{u}}(\eta)$ einen Rangabfall gegenüber dem Normalrang ${ }^{4)}$ aufweist. Erfüllt $\eta$ diese Bedingung, so spricht man von einer Übertragungsnullstelle. Offensichtlich sind jedoch im Gegensatz zum Eingrößenfall weitere Bedingungen für eine Übertragungsblockierung zu erfüllen. So muss das System nicht nur mit der Frequenz $e^{\eta t}$ angeregt werden, sondern auch in der zugehörigen Richtung, die durch $\boldsymbol{u}_{0}$ festgelegt wird. Man erhält sie als nichttriviale Lösung von $\boldsymbol{G}_{\boldsymbol{y} \boldsymbol{u}}(\eta) \cdot \boldsymbol{u}_{0}=\mathbf{0}$. Weiterhin geht aus (3.12) hervor, dass der Anfangszustand gemäß

$$
\boldsymbol{x}_{0}=\left(\eta \boldsymbol{I}_{n}-\boldsymbol{A}\right)^{-1} \boldsymbol{B} \boldsymbol{u}_{0}
$$

zu wählen ist, damit $\boldsymbol{y}(t)=\mathbf{0}$ für alle $t \geq 0$ gilt.

Neben den Übertragungsnullstellen existieren sogenannte Entkopplungsnullstellen. Diese beschreiben wie im Eingrößenfall nicht steuerbare beziehungsweise nicht beobachtbare Systemeigenwerte. Dementsprechend wird $\eta \in \mathbb{C}$ eine Eingangsentkopplungsnullstelle des Systems $(\boldsymbol{A}, \boldsymbol{B}, \boldsymbol{C}, \boldsymbol{D})$ genannt, wenn die Matrix

$$
\boldsymbol{P}_{o}(\eta)=\left[\eta \boldsymbol{I}_{n}-\boldsymbol{A} \quad-\boldsymbol{B}\right]
$$

einen Rangabfall gegenüber ihrem Normalrang aufweist [145]. Nach dem Hautus-Kriterium entspricht dies der Bedingung für einen nicht steuerbaren Eigenwert. Eingangsentkopplungsnullstellen beschreiben somit Eigenbewegungen $e^{\eta t}$, die durch den Eingang $\boldsymbol{u}$ nicht angeregt werden können.

${ }^{4)}$ Der Normalrang bezeichnet den Rang von $\boldsymbol{G}_{\boldsymbol{y} \boldsymbol{u}}(s)$, den die Matrix für fast alle $s \in \mathbb{C}$, das heißt für alle bis auf endlich viele $s \in \mathbb{C}$, aufweist. 
Analog dazu heißt $\eta$ Ausgangsentkopplungsnullstelle des Systems, wenn die Matrix

$$
\boldsymbol{P}_{l}(\eta)=\left[\begin{array}{c}
\eta \boldsymbol{I}_{n}-\boldsymbol{A} \\
\boldsymbol{C}
\end{array}\right]
$$

einen Rangabfall gegenüber ihrem Normalrang aufweist [145]. Sie beschreiben Eigenbewegungen $e^{\eta t}$, die am Systemausgang nicht beobachtet werden können. Ruft $\eta$ sowohl einen Rangabfall in (3.13) als auch in (3.14) hervor, so wird die Zahl als Ein-/Ausgangsentkopplungsnullstelle bezeichnet [145]. Als Überbegriff für Eingangs-, Ausgangs- sowie Ein/Ausgangsentkopplungsnullstellen spricht man von Entkopplungsnullstellen.

Die Gesamtheit der Nullstellen eines Systems setzt sich zusammen aus den Übertragungs- und Entkopplungsnullstellen [145], wie Abbildung 3.3 zeigt. Man spricht von den Systemnullstellen.

Für die weiteren Betrachtungen wird die Rosenbrock'sche Systemmatrix benötigt. Sie tritt auf, wenn man das System (3.11) gemäß

$$
\begin{aligned}
s \boldsymbol{x}(s)-\boldsymbol{x}_{0} & =\boldsymbol{A} \boldsymbol{x}(s)+\boldsymbol{B} \boldsymbol{u}(s), \\
\boldsymbol{y}(s) & =\boldsymbol{C} \boldsymbol{x}(s)+\boldsymbol{D} \boldsymbol{u}(s),
\end{aligned}
$$

in den Laplace-Bereich transformiert. Dies lässt sich kompakt darstellen als

$$
\underbrace{\left[\begin{array}{cc}
s \boldsymbol{I}_{n}-\boldsymbol{A} & -\boldsymbol{B} \\
\boldsymbol{C} & \boldsymbol{D}
\end{array}\right]}_{\boldsymbol{P}(s)}\left[\begin{array}{l}
\boldsymbol{x}(s) \\
\boldsymbol{u}(s)
\end{array}\right]=\left[\begin{array}{c}
\boldsymbol{x}_{0} \\
\boldsymbol{y}(s)
\end{array}\right] .
$$

Die darin eingeführte Matrix $\boldsymbol{P}(s)$ wird als Rosenbrock'sche Systemmatrix bezeichnet. Gegenüber der Übertragungsmatrix $\boldsymbol{G}_{\boldsymbol{y} \boldsymbol{u}}(s)=\boldsymbol{C}\left(s \boldsymbol{I}_{n}-\boldsymbol{A}\right)^{-1} \boldsymbol{B}+\boldsymbol{D}$ des Systems erlaubt $\boldsymbol{P}(s)$ Einblick in die innere Struktur des Systems. Außerdem werden in (3.15) auch die Anfangsbedingungen $\boldsymbol{x}_{0}$ und damit die Eigenbewegungen des Systems berücksichtigt. Weist die Matrix

$$
\boldsymbol{P}(\eta)=\left[\begin{array}{cc}
\eta \boldsymbol{I}_{n}-\boldsymbol{A} & -\boldsymbol{B} \\
\boldsymbol{C} & \boldsymbol{D}
\end{array}\right]
$$

für die komplexe Zahl $\eta \in \mathbb{C}$ einen Rangabfall gegenüber dem Normalrang von $\boldsymbol{P}(s)$ auf, so wird $\eta$ als invariante Nullstelle des Systems $(\boldsymbol{A}, \boldsymbol{B}, \boldsymbol{C}, \boldsymbol{D})$ bezeichnet $[196,264]$. Der Name der invarianten Nullstellen erklärt sich aus der Tatsache, dass sie durch eine Zustandsrückführung mit regulärem Vor- 
filter, $\boldsymbol{u}=-\boldsymbol{K} \boldsymbol{x}+\boldsymbol{F} \boldsymbol{w}$, nicht verändert werden können. Dies wird deutlich, wenn man den geschlossenen Regelkreis

$$
\begin{aligned}
& \dot{\boldsymbol{x}}=(\boldsymbol{A}-\boldsymbol{B} \boldsymbol{K}) \boldsymbol{x}+\boldsymbol{B} \boldsymbol{F} \boldsymbol{w} \\
& \boldsymbol{y}=(\boldsymbol{C}-\boldsymbol{D} \boldsymbol{K}) \boldsymbol{x}+\boldsymbol{D} \boldsymbol{F} \boldsymbol{w}
\end{aligned}
$$

betrachtet. Seine Rosenbrock'sche Systemmatrix lässt sich schreiben als

$$
\boldsymbol{P}_{g}(s)=\left[\begin{array}{cc}
s \boldsymbol{I}_{n}-(\boldsymbol{A}-\boldsymbol{B} \boldsymbol{K}) & -\boldsymbol{B} \boldsymbol{F} \\
\boldsymbol{C}-\boldsymbol{D} \boldsymbol{K} & \boldsymbol{D F}
\end{array}\right]=\underbrace{\left[\begin{array}{cc}
s \boldsymbol{I}_{n}-\boldsymbol{A} & -\boldsymbol{B} \\
\boldsymbol{C} & \boldsymbol{D}
\end{array}\right]}_{\boldsymbol{P}(s)}\left[\begin{array}{cc}
\boldsymbol{I}_{n} & \mathbf{0} \\
-\boldsymbol{K} & \boldsymbol{F}
\end{array}\right] .
$$

Sie ergibt sich also aus der Multiplikation der Rosenbrock'schen Systemmatrix des offenen Kreises mit einer regulären Matrix. Somit weist $\boldsymbol{P}_{g}(s)$ an den gleichen Stellen einen Rangabfall auf wie $\boldsymbol{P}(s)$ und die invarianten Nullstellen verändern sich durch die Regelung nicht.

Für quadratische Systeme mit $n_{u}=n_{y}$, die nicht degeneriert ${ }^{5)}$ sind, lassen sich die invarianten Nullstellen auch durch Lösen der Gleichung

$$
\operatorname{det}(\boldsymbol{P}(\eta))=\left|\begin{array}{cc}
\eta \boldsymbol{I}_{n}-\boldsymbol{A} & -\boldsymbol{B} \\
\boldsymbol{C} & \boldsymbol{D}
\end{array}\right|=0
$$

bestimmen. Nach [150, Abschnitt 6.2] lässt sich dies schreiben als

$$
\begin{aligned}
\operatorname{det}(\boldsymbol{P}(\eta)) & =\operatorname{det}\left(\eta \boldsymbol{I}_{n}-\boldsymbol{A}\right) \cdot \operatorname{det}\left(\boldsymbol{C}\left(\eta \boldsymbol{I}_{n}-\boldsymbol{A}\right)^{-1} \boldsymbol{B}+\boldsymbol{D}\right) \\
& =\operatorname{det}\left(\eta \boldsymbol{I}_{n}-\boldsymbol{A}\right) \cdot \operatorname{det}\left(\boldsymbol{G}_{\boldsymbol{y} \boldsymbol{u}}(\eta)\right) .
\end{aligned}
$$

Demnach setzen sich die invarianten Nullstellen in diesem Fall zusammen aus den Übertragungsnullstellen und denjenigen Eigenwerten von $\boldsymbol{A}$, die nicht als Pole in $\boldsymbol{G}_{\boldsymbol{y} \boldsymbol{u}}(s)$ auftreten. Dabei handelt es sich um die nicht steuerbaren beziehungsweise nicht beobachtbaren Eigenwerte, also um die Entkopplungsnullstellen. Folglich setzen sich für quadratische, nicht degenerierte Systeme die invarianten Nullstellen aus den Übertragungsnullstellen und den Entkopplungsnullstellen zusammen. Sie entsprechen damit den Systemnullstellen [212]. In Anlehnung an [145] und als Zusammenfassung der Resultate aus [145, 196, 212] veranschaulicht Abbildung 3.3a die Zusammenhänge. Ist das System darüber hinaus

5) Entspricht der Normalrang der Rosenbrock'schen Systemmatrix $n+\min \left\{n_{u}, n_{y}\right\}$, so wird das System als nicht degeneriert bezeichnet, andernfalls als degeneriert. 


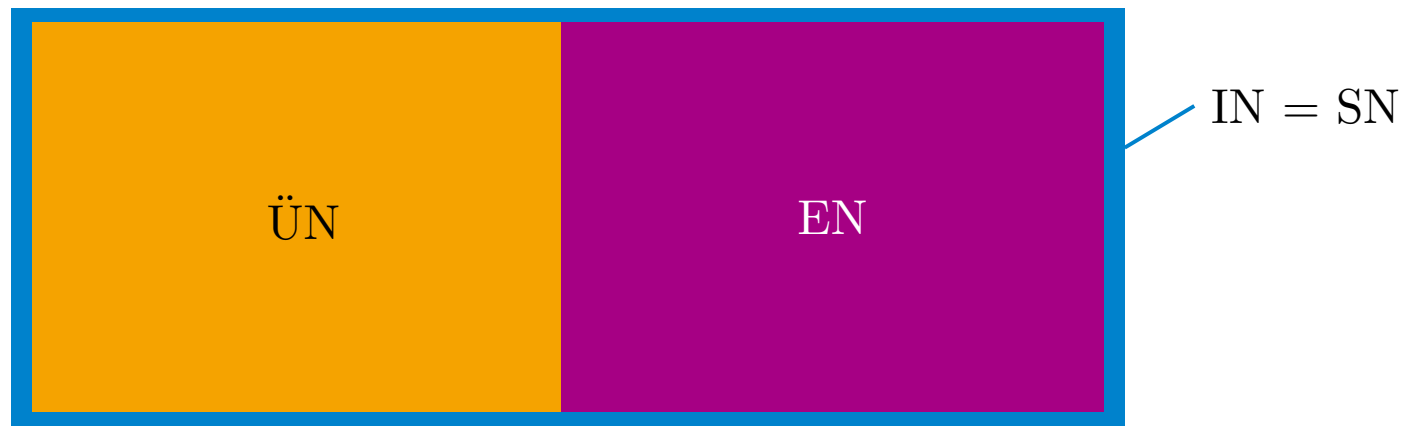

(a) quadratische, nicht degenerierte Systeme

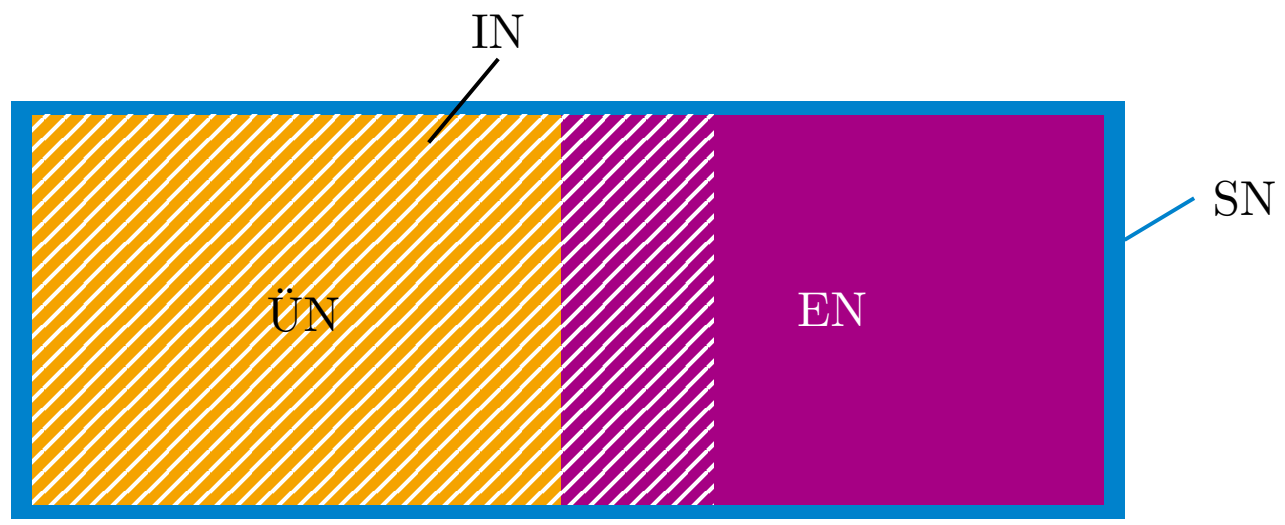

(b) nichtquadratische oder degenerierte Systeme

Abbildung 3.3: Zusammenhang zwischen Übertragungsnullstellen (ÜN), Entkopplungsnullstellen (EN), invarianten Nullstellen (IN) und Systemnullstellen $(\mathrm{SN})$

vollständig steuer- und beobachtbar, so existieren keine Entkopplungsnullstellen und die Übertragungsnullstellen entsprechen den invarianten Nullstellen beziehungsweise den Systemnullstellen.

In einem nichtquadratischen und/oder degenerierten System stellt sich die Situation wie in Abbildung 3.3b dar. Die Übertragungsnullstellen sind auch hier eine Untermenge der invarianten Nullstellen. Diese beinhalten jedoch nicht zwangsweise alle Entkopplungsnullstellen, wie dies in quadratischen, nicht degenerierten Systemen der Fall ist. Es können also Entkopplungsnullstellen existieren, die nicht invariant sind. Dieser Sachverhalt ist für die vorliegende Arbeit von grundlegender Bedeutung, er wird in Abschnitt 4.9 aufgegriffen. Die Systemnullstellen setzen sich wie im quadratischen, nicht degenerierten Fall aus den Übertragungsnullstellen und den Entkopplungsnullstellen zusammen [212].

Der Übersichtlichkeit halber fasst Tabelle 3.1 die unterschiedlichen Klassen von Nullstellen zusammen. 
Tabelle 3.1: Arten von Nullstellen

\begin{tabular}{|c|c|c|}
\hline Art & Berechnung & Bedeutung \\
\hline $\begin{array}{c}\text { Übertragungs- } \\
\text { nullstellen }\end{array}$ & $\begin{array}{l}\text { Rangabfall } \\
\text { in } \boldsymbol{G}_{\boldsymbol{y} \boldsymbol{u}}(s)\end{array}$ & $\begin{array}{c}\text { Blockierung der } \\
\text { Anregung mit } e^{\eta t}\end{array}$ \\
\hline $\begin{array}{c}\text { Entkopplungs- } \\
\text { nullstellen }\end{array}$ & $\begin{array}{c}\text { Rangabfall in } \\
{\left[s \boldsymbol{I}_{n}-\boldsymbol{A}-\boldsymbol{B}\right] \text { bzw. }} \\
{\left[\begin{array}{c}s \boldsymbol{I}_{n}-\boldsymbol{A} \\
\boldsymbol{C}\end{array}\right]}\end{array}$ & $\begin{array}{c}\text { nicht steuerbare } \\
\text { bzw. nicht beob- } \\
\text { achtbare Eigenwerte }\end{array}$ \\
\hline $\begin{array}{l}\text { Invariante } \\
\text { Nullstellen }\end{array}$ & $\begin{array}{l}\text { Rangabfall } \\
\quad \text { in } \boldsymbol{P}(s)\end{array}$ & $\begin{array}{c}\text { durch Rückführung } \\
\boldsymbol{u}=-\boldsymbol{K} \boldsymbol{x}+\boldsymbol{F} \boldsymbol{w} \text { nicht } \\
\text { verschiebbar }\end{array}$ \\
\hline $\begin{array}{c}\text { System- } \\
\text { nullstellen }\end{array}$ & $\begin{array}{c}\text { siehe Übertragungs- } \\
\text { bzw. Entkopplungs- } \\
\text { nullstellen }\end{array}$ & $\begin{array}{l}\text { Gesamtheit der } \\
\text { Nullstellen } \\
\text { eines Systems }\end{array}$ \\
\hline
\end{tabular}

Bemerkung 3.3. Alternativ zu den hier verwendeten Definitionen lassen sich invariante Nullstellen und Übertragungsnullstellen auch über die Smith-McMillan-Normalform von $\boldsymbol{P}(s)$ beziehungsweise $\boldsymbol{G}_{\boldsymbol{y} \boldsymbol{u}}(s)$ definieren [145, 186, 187, 212]. Dabei handelt es sich um eine Zerlegung der jeweiligen Matrix gemäß $\boldsymbol{S}_{\boldsymbol{P}}(s)=\boldsymbol{L}(s) \cdot \boldsymbol{P}(s) \cdot \boldsymbol{R}(s)$ beziehungsweise $\boldsymbol{S}_{\boldsymbol{G}}(s)=\boldsymbol{L}(s) \cdot \boldsymbol{G}_{\boldsymbol{y} \boldsymbol{u}}(s) \cdot \boldsymbol{R}(s)$ mittels unimodularen ${ }^{6)}$ Transformationsmatrizen $\boldsymbol{L}(s)$ und $\boldsymbol{R}(s)$.

Im weiteren Verlauf der Arbeit wird sich zeigen, dass die Lage der invarianten Nullstellen eines Systems von zentraler Bedeutung ist. In diesem Zusammenhang wird der Begriff der Minimalphasigkeit wie folgt definiert.

Definition 3.6 (Minimalphasigkeit). Ein lineares zeitinvariantes System der Form (3.11) heißt minimalphasig, wenn alle seine invarianten Nullstellen in der offenen linken s-Halbebene liegen.

Anzumerken ist, dass der Begriff der Minimalphasigkeit in der Literatur nicht eindeutig definiert ist. Insbesondere im Bereich der Signalverarbeitung [161, Abschnitt 5.6] wird der Begriff häufig verwendet, um Systeme

${ }^{6)}$ Eine Matrix heißt unimodular, wenn ihre Determinante von null verschieden und konstant, das heißt unabhängig von $s$, ist. 
zu beschreiben, die ausschließlich Pole und Nullstellen in der offenen linken s-Halbebene aufweisen. Dann lässt sich für SISO-Systeme ein Bezug zur grafischen Interpretation eines phasenminimalen Systems herstellen, ebenso kann die Stabilität der Nulldynamik nachgewiesen werden [105]. In der Entkopplungs- beziehungsweise Fehlerisolationsliteratur ist es jedoch üblich, den Begriff der Minimalphasigkeit ausschließlich über die Lage der invarianten Nullstellen zu definieren [140] und die Stabilität dabei nicht zu betrachten. Daher wird diese Definition auch in der vorliegenden Arbeit verwendet.

\subsection{Lineare Entkopplungsregler}

Viele der im Rahmen dieser Arbeit erzielten Ergebnisse weisen eine enge Verwandtschaft zu Resultaten aus dem Bereich der linearen Entkopplungsregelungen auf, einige Ergebnisse leiten sich unmittelbar aus dieser Beziehung ab. Daher wird im Folgenden ein kurzer Überblick über die Problemstellung linearer Entkopplungsregler gegeben, sowie eine Übersicht über die wesentlichen Publikationen hierzu.

Betrachtet werden lineare zeitinvariante Regelstrecken der Form

$$
\begin{aligned}
& \dot{\boldsymbol{x}}=\boldsymbol{A} \boldsymbol{x}+\boldsymbol{B u}, \quad \boldsymbol{x}(t=0)=\mathbf{0}, \\
& \boldsymbol{y}=\boldsymbol{C} \boldsymbol{x}
\end{aligned}
$$

mit $\boldsymbol{x} \in \mathbb{R}^{n}, \boldsymbol{u} \in \mathbb{R}^{n_{u}}$ und $\boldsymbol{y} \in \mathbb{R}^{n_{y}}$. Im Allgemeinen wirkt eine Stellgröße $u_{i}$ auf den gesamten Ausgangsgrößenvektor $\boldsymbol{y}$, sodass eine gezielte Beeinflussung einer einzelnen Ausgangsgröße $y_{j}$ schwierig ist. Ziel der Entkopplungsregelung ist es daher, eine lineare Zustandsrückführung mit Vorfilter

$$
\boldsymbol{u}=-\boldsymbol{K} \boldsymbol{x}+\boldsymbol{F} \boldsymbol{w}
$$

zu finden, sodass die sich ergebende Führungsübertragungsmatrix $\boldsymbol{G}_{\boldsymbol{y} \boldsymbol{w}}(s)$ Diagonalgestalt aufweist. Dann ist

$$
\boldsymbol{G}_{\boldsymbol{y} \boldsymbol{w}}(s)=\left[\begin{array}{cccc}
g_{1,1}(s) & 0 & \cdots & 0 \\
0 & g_{2,2}(s) & \ddots & \vdots \\
\vdots & \ddots & \ddots & 0 \\
0 & \cdots & 0 & g_{n_{y}, n_{y}}(s)
\end{array}\right]
$$

und demnach wird eine Ausgangsgröße $y_{i}$ ausschließlich von der ihr zugeordneten Führungsgröße $w_{i}$ beeinflusst. Darüber hinaus ist die Stabilität 
des geschlossenen Regelkreises sicherzustellen, das heißt $\boldsymbol{A}-\boldsymbol{B} \boldsymbol{K}$ muss eine Hurwitz-Matrix sein. Neben dem Entwurfsproblem in $\boldsymbol{K}$ und $\boldsymbol{F}$ ist dabei die Frage der Existenz eines stabilisierenden, entkoppelnden Reglers von besonderer Bedeutung.

Das Problem geht zurück auf Morgan [155] und wurde in [67] für quadratische Systeme mit ebenso vielen Eingangs- wie Ausgangsgrößen $\left(n_{u}=n_{y}\right)$ im Zeitbereich gelöst. Während in [266] ein geometrischer Ansatz vorgeschlagen wurde, findet sich in [265] eine Lösung im Frequenzbereich. Darüber hinaus bietet der sogenannte parametrische Entwurf die Möglichkeit, die Vollständige Modale Synthese [184] für den Entkopplungsreglerentwurf einzusetzen [185].

Durch den Einsatz dynamischer Regler lässt sich darüber hinaus eine Vergrößerung der handhabbaren Systemklasse erreichen. Frequenzbereichsansätze dazu finden sich in [46, 47] und [151]. Eine Zustandsraummethodik wurde in $[137,140]$ vorgestellt.

Ein nach wie vor nicht vollständig gelöstes Problem stellen Entkopplungsregelungen für nichtquadratische lineare Systeme dar, die über mehr Eingangs- als zu regelnde Ausgangsgrößen verfügen $\left(n_{u}>n_{y}\right)$. Zwar vereinfacht sich die Problemstellung intuitiv, wenn eine größere Anzahl an linear unabhängigen Eingangsgrößen vorhanden ist, die mathematische Charakterisierung wird jedoch wesentlich komplizierter. So existieren bisher keine allgemeingültigen notwendigen und hinreichenden Bedingungen, welche die Lösbarkeit des Problems garantieren. Schien das Problem 1988 in [54] gelöst, wurden für die dort angegebenen Bedingungen Gegenbeispiele gefunden [97]. Auch später erschienene Publikationen wie [208] arbeiten entweder mit eingeschränkten Systemklassen oder stützen sich auf hinreichende Bedingungen, was ebenso für die kürzlich erschienenen Beiträge $[26,252,253]$ der Fall ist. 


\section{Entwurf von Fehlerisolationsbeobachtern für nominale Systeme}

Gegenstand dieses Kapitels ist die Entwicklung von Entwurfsverfahren für Fehlerisolationsbeobachter (FIOs) für Systeme mit exakt bekannter Dynamik. Für solche nominalen Systeme liegt der Schwerpunkt auf Existenzbedingungen für FIOs sowie der Charakterisierung der Entwurfsfreiheitsgrade. Dabei ist das Kapitel entsprechend Abbildung 4.1 aufgebaut.

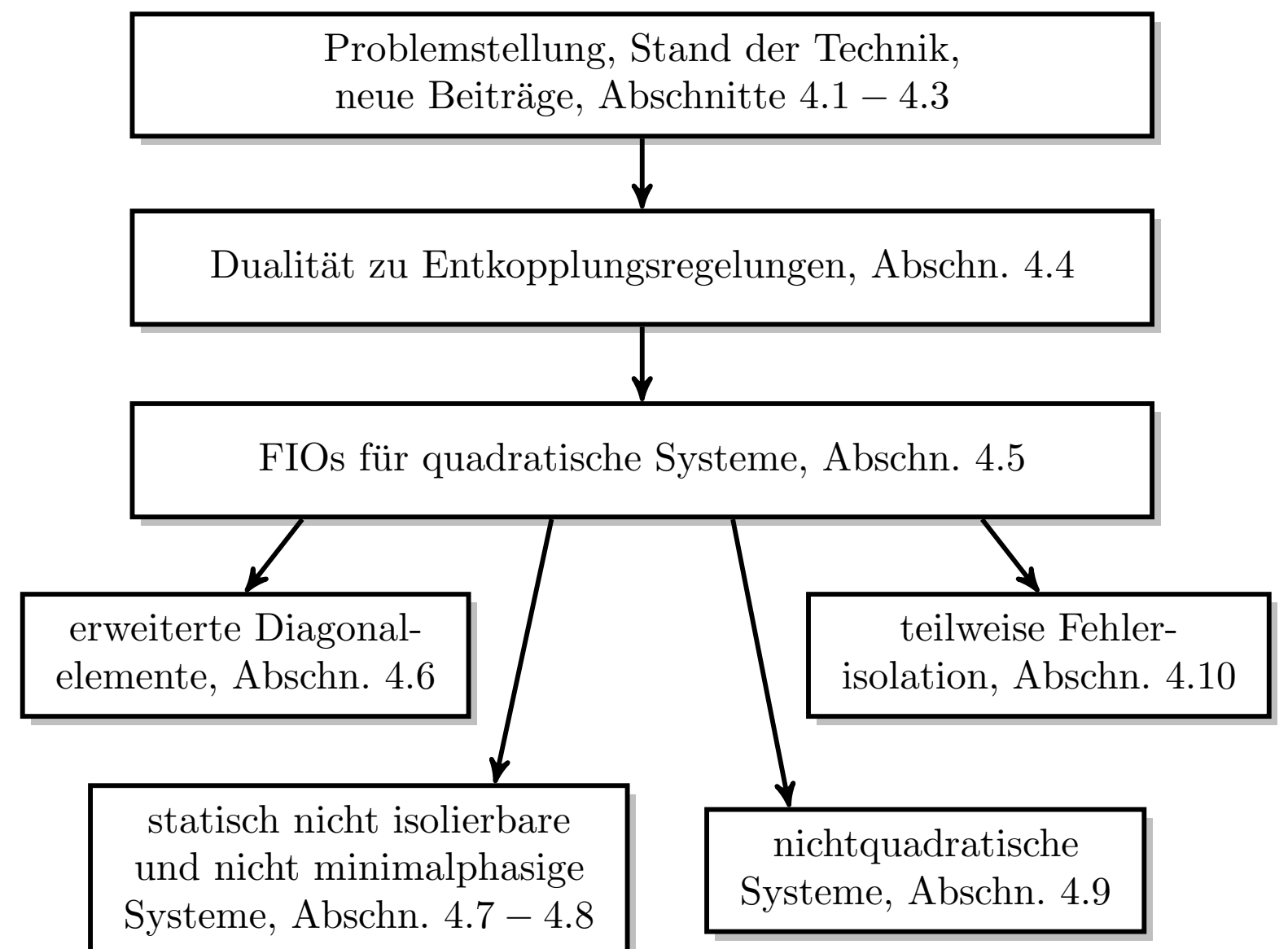

Abbildung 4.1: Aufbau des 4. Kapitels 
Die detaillierte Formulierung der Problemstellung in Abschnitt 4.1 erlaubt es, eine Verbindung zu den in Abschnitt 2.3 erarbeiteten Entwurfszielen herzustellen. Auf Basis der eingeführten Grundbegriffe und Definitionen wird in Abschnitt 4.2 ein Überblick über existierende Ergebnisse zum Entwurf von FIOs in der Literatur gegeben, sodass in Abschnitt 4.3 die im Rahmen dieser Arbeit neu entwickelten Ergebnisse herausgestellt werden können.

Grundlegend sind die Betrachtungen zu FIOs für quadratische Systeme (Abschnitt 4.5). Diese werden anschließend in verschiedener Hinsicht erweitert, wobei die Ergebnisse wie in Abbildung 4.1 dargestellt nicht hierarchisch zu verstehen sind. Abschnitt 4.6 liefert Methoden zur Erweiterung der Dynamik der Diagonalelemente in quadratischen Systemen. Die Abschnitte 4.7 und 4.8 erweitern die Ergebnisse auf statisch nicht isolierbare (sni) und nicht minimalphasige (nmph) Systeme. Nichtquadratische Systeme mit zusätzlichen Messgrößen werden in Abschnitt 4.9 behandelt, während sich Abschnitt 4.10 mit der teilweisen Fehlerisolation beschäftigt. Neben Existenzbedingungen und Entwurfsmethoden werden jeweils auch die verbleibenden Entwurfsfreiheitsgrade beschrieben. Auf Grundlage der Ergebnisse dieses Kapitels werden die herausgearbeiteten Freiheitsgrade in Kapitel 5 zur Robustheitsoptimierung genutzt.

\subsection{Problemstellung}

Behandelt werden lineare zeitinvariante Systeme der Form

$$
\begin{aligned}
& \dot{\boldsymbol{x}}=\boldsymbol{A} \boldsymbol{x}+\boldsymbol{B} \boldsymbol{u}+\boldsymbol{E}_{a} \boldsymbol{f}, \quad \boldsymbol{x}(t=0)=\boldsymbol{x}_{0}, \\
& \boldsymbol{y}=\boldsymbol{C} \boldsymbol{x}+\boldsymbol{D} \boldsymbol{u}+\boldsymbol{E}_{s} \boldsymbol{f} .
\end{aligned}
$$

Dabei ist $\boldsymbol{x} \in \mathbb{R}^{n}$ der Zustandsvektor des Systems, $\boldsymbol{u} \in \mathbb{R}^{n_{u}}$ beschreibt die Stellgrößen, $\boldsymbol{f} \in \mathbb{R}^{n_{f}}$ enthält die auf das System einwirkenden Fehler und $\boldsymbol{y} \in \mathbb{R}^{n_{y}}$ stellt die messbaren Ausgangsgrößen dar. Soweit nicht anders angemerkt wird im Rahmen dieser Arbeit stets $\boldsymbol{x}_{0}=\mathbf{0}$ angenommen, sodass im Weiteren auf die Angabe des Anfangszustandes von Zustandsraummodellen verzichtet wird.

Um die einwirkenden Fehler zu detektieren und zu isolieren, werden Beobachter der Form

$$
\begin{aligned}
\dot{\hat{\boldsymbol{x}}} & =\boldsymbol{A} \hat{\boldsymbol{x}}+\boldsymbol{B} \boldsymbol{u}+\boldsymbol{L}(\boldsymbol{y}-\boldsymbol{C} \hat{\boldsymbol{x}}-\boldsymbol{D u}), \quad \hat{\boldsymbol{x}}(t=0)=\hat{\boldsymbol{x}}_{0}, \\
\boldsymbol{r} & =\boldsymbol{V}(\boldsymbol{y}-\boldsymbol{C} \hat{\boldsymbol{x}}-\boldsymbol{D u})
\end{aligned}
$$


herangezogen. Für den Beobachteranfangszustand wird stets $\hat{\boldsymbol{x}}_{0}=\mathbf{0}$ angenommen, auf die Angabe wird daher im weiteren Verlauf ebenso wie bei Streckenmodellen verzichtet. Neben der klassischen Beobachtergleichung (4.2a) wird in (4.2b) die Differenz zwischen gemessenen und geschätzten Ausgangsgrößen mit einer Nachfiltermatrix $\boldsymbol{V} \in \mathbb{R}^{n_{f} \times n_{y}}$ multipliziert. Der auf diese Weise generierte Vektor $\boldsymbol{r} \in \mathbb{R}^{n_{f}}$ stellt den Residuenvektor dar.

Da letztendlich von den generierten Residuen auf die Fehler zurückgeschlossen werden soll, wird das Gesamtsystem bestehend aus Strecke (4.1) und Beobachter (4.2) betrachtet. Als Zustandsvektor wird $\tilde{\boldsymbol{x}}=\left[\begin{array}{ll}\boldsymbol{x}^{\top} & \hat{\boldsymbol{x}}^{\top}\end{array}\right]^{\top} \in \mathbb{R}^{2 n}$ eingeführt. Anhand von (4.1) und (4.2) erhält man

$$
\begin{aligned}
& \dot{\tilde{\boldsymbol{x}}}=\left[\begin{array}{c}
\dot{\boldsymbol{x}} \\
\dot{\hat{\boldsymbol{x}}}
\end{array}\right]=\left[\begin{array}{cc}
\boldsymbol{A} & \mathbf{0} \\
\boldsymbol{L} C & \boldsymbol{A}-\boldsymbol{L} C
\end{array}\right]\left[\begin{array}{l}
\boldsymbol{x} \\
\hat{\boldsymbol{x}}
\end{array}\right]+\left[\begin{array}{c}
\boldsymbol{B} \\
\boldsymbol{B}-\boldsymbol{L} \boldsymbol{D}
\end{array}\right] \boldsymbol{u}+\left[\begin{array}{c}
\boldsymbol{E}_{a} \\
\boldsymbol{L} \boldsymbol{E}_{s}
\end{array}\right] \boldsymbol{f}, \\
& \boldsymbol{r}=\left[\begin{array}{ll}
\boldsymbol{V} \boldsymbol{C} & -\boldsymbol{V} \boldsymbol{C}
\end{array}\right]\left[\begin{array}{l}
\boldsymbol{x} \\
\hat{\boldsymbol{x}}
\end{array}\right]+\boldsymbol{V} \boldsymbol{E}_{s} \boldsymbol{f} .
\end{aligned}
$$

$\mathrm{Zu}$ beachten ist dabei, dass (4.3) nur deshalb gilt, weil die Systemmatrizen der Strecke (4.1) als exakt bekannt vorausgesetzt wurden und denen des Beobachters (4.2) entsprechen. Betrachtet man unter dieser Annahme den Beobachterfehler $\boldsymbol{\xi}=\boldsymbol{x}-\hat{\boldsymbol{x}}$, so ergibt sich für dessen Dynamik beziehungsweise für die Dynamik der Residuenerzeugung aus (4.1) und (4.2) das virtuelle System

$$
\begin{aligned}
& \dot{\boldsymbol{\xi}}=(\boldsymbol{A}-\boldsymbol{L} \boldsymbol{C}) \boldsymbol{\xi}+\left(\boldsymbol{E}_{a}-\boldsymbol{L} \boldsymbol{E}_{s}\right) \boldsymbol{f}, \\
& \boldsymbol{r}=\boldsymbol{V} \boldsymbol{C} \boldsymbol{\xi}+\boldsymbol{V} \boldsymbol{E}_{s} \boldsymbol{f} .
\end{aligned}
$$

Ziel ist es im Folgenden, die Beobachtermatrizen $\boldsymbol{L}$ und $\boldsymbol{V}$ so zu parametrieren, dass die Übertragungsmatrix $\boldsymbol{G}_{\boldsymbol{r} \boldsymbol{f}}(s)$, welche das Übertragungsverhalten von Fehlern $\boldsymbol{f}$ zu Residuen $\boldsymbol{r}$ beschreibt, Diagonalgestalt aufweist. Es soll also

$$
\begin{aligned}
\boldsymbol{G}_{\boldsymbol{r} \boldsymbol{f}}(s)= & \frac{\boldsymbol{r}(s)}{\boldsymbol{f}(s)}=\boldsymbol{V} \boldsymbol{C}\left(s \boldsymbol{I}_{n}-(\boldsymbol{A}-\boldsymbol{L} \boldsymbol{C})\right)^{-1}\left(\boldsymbol{E}_{a}-\boldsymbol{L} \boldsymbol{E}_{s}\right)+\boldsymbol{V} \boldsymbol{E}_{s} \\
= & {\left[\begin{array}{cccc}
g_{1,1}(s) & 0 & \cdots & 0 \\
0 & \ddots & \ddots & \vdots \\
\vdots & \ddots & \ddots & 0 \\
0 & \cdots & 0 & g_{n_{f}, n_{f}}(s)
\end{array}\right]=\operatorname{diag}\left(g_{1,1}(s), \ldots, g_{n_{f}, n_{f}}(s)\right) }
\end{aligned}
$$

erreicht werden. Wie aus (4.5) hervorgeht, beeinflusst dann der $i$-te Fehler ausschließlich das ihm zugeordnete $i$-te Residuum. Folglich kann wie in 
Abschnitt 2.3 gefordert von dem entsprechenden Residuum eindeutig auf den zugehörigen Fehler geschlossen werden. Dieser kann somit nicht nur detektiert, sondern von allen anderen Fehlern isoliert werden, selbst wenn diese gleichzeitig auftreten sollten beziehungsweise zur gleichen Zeit aktiv sind.

Definition 4.1 (Fehlerisolationsbeobachter). Ein durch $(\boldsymbol{L}, \boldsymbol{V})$ parametrierter Beobachter der Form (4.2), der $\mathrm{zu}$ einer diagonalen Übertragungsmatrix $\boldsymbol{G}_{\boldsymbol{r} \boldsymbol{f}}(s)$ führt, wird als Fehlerisolationsbeobachter (engl. fault isolation observer, FIO) bezeichnet.

Durch einen FIO wird somit das erste Entwurfsziel (Strukturierung der Residuen, s. Abschnitt 2.3) gewährleistet. Weiterhin wird in diesem Kapitel die Stabilität von FIOs berücksichtigt, wozu entsprechend (4.4a) die Matrix $\boldsymbol{A}-\boldsymbol{L} \boldsymbol{C}$ zu betrachten ist. Die Forderungen nach ausreichender Detektionsgeschwindigkeit und Fehlersensitivität werden durch eine entsprechende Auslegung der Diagonalelemente $g_{i, i}(s)$ berücksichtigt. Robustheitsaspekte werden dagegen an dieser Stelle nicht betrachtet, sie werden ausführlich in Kapitel 5 behandelt.

Für die weiteren Betrachtungen sind einige Begriffsdefinitionen nötig. Zunächst werden in Erweiterung von [133] die Fehlerdetektionsindizes $\delta_{i}$ wie folgt eingeführt:

Definition 4.2 (Fehlerdetektionsindizes). Für ein System der Form (4.1) werden die Fehlerdetektionsindizes $\delta_{i}, i=1, \ldots, n_{f}$, definiert als

$$
\delta_{i}=\left\{\begin{array}{cl}
0 & , \boldsymbol{e}_{s_{i}} \neq \mathbf{0} \\
\min _{k}\left\{k \geq 1: \boldsymbol{C A}^{k-1} \boldsymbol{e}_{a_{i}} \neq \mathbf{0}\right\}, & \boldsymbol{e}_{s_{i}}=\mathbf{0} .
\end{array}\right.
$$

Dabei ist $\boldsymbol{e}_{a_{i}}$ beziehungsweise $\boldsymbol{e}_{s_{i}}$ die $i$-te Spalte der Aktorfehlermatrix $\boldsymbol{E}_{a}$ beziehungsweise der Sensorfehlermatrix $\boldsymbol{E}_{s}$. Sie modellieren, wie sich die jeweiligen Fehler auf die Dynamik des Systems beziehungsweise den Ausgangsvektor auswirken. Gemäß obiger Definition beschreibt der Fehlerindex $\delta_{i}$ also, wie oft der Ausgangsvektor $\boldsymbol{y}$ zeitlich abgeleitet werden muss, bis der $i$-te Fehler explizit in dem sich ergebenden Ausdruck auftaucht. Ist die entsprechende Spalte der Sensorfehlermatrix $\boldsymbol{E}_{s}$ keine Nullspalte, so besitzt der Fehler $f_{i}$ einen Durchgriff auf den Ausgang. Demnach ist keine zeitliche Ableitung von $\boldsymbol{y}$ nötig und für den entsprechenden Fehlerindex gilt $\delta_{i}=0$.

Weiterhin bestimmen die Fehlerindizes den Gesamtfehlerindex eines Systems. 
Definition 4.3 (Gesamtfehlerindex). Für ein System der Form (4.1) mit den Fehlerindizes $\delta_{i}$ ist der Gesamtfehlerindex $\delta$ definiert als

$$
\delta=\sum_{i=1}^{n_{f}} \delta_{i} .
$$

Ferner bestimmen die Fehlerindizes die sogenannte Fehlerdetektionsmatrix mit.

Definition 4.4 (Fehlerdetektionsmatrix). Für ein System der Form (4.1) mit den Fehlerindizes $\delta_{i}$ wird die Matrix

$$
\begin{aligned}
& \mathbb{R}^{n_{y} \times n_{f}} \ni \boldsymbol{D}^{*}=\left[\begin{array}{lll}
\boldsymbol{d}_{1}^{*} & \cdots & \boldsymbol{d}_{n_{f}}^{*}
\end{array}\right], \\
& \boldsymbol{d}_{i}^{*}=\left\{\begin{array}{cc}
\boldsymbol{e}_{s_{i}} & , \delta_{i}=0, \\
\boldsymbol{C} \boldsymbol{A}^{\delta_{i}-1} \boldsymbol{e}_{a_{i}}, & \delta_{i} \geq 1,
\end{array}\right.
\end{aligned}
$$

als Fehlerdetektionsmatrix bezeichnet.

Auffällig an diesen Definitionen ist ihre Ähnlichkeit zu den aus dem Bereich der Entkopplungsregelungen bekannten Begriffen der Differenzenordnungen (auch relative Grade genannt) und der Entkoppelbarkeitsmatrix. Darauf wird in Abschnitt 4.4 näher eingegangen.

\subsection{Stand der Technik}

Wie bereits in Abschnitt 2.5.3 erwähnt geht die Idee, dem Residuenvektor für unterschiedliche Fehler durch eine entsprechende Parametrierung eines einzelnen Beobachters jeweils eine definierte Richtung aufzuprägen, zurück auf $[13,117]$. Seitdem hat die Idee verschiedene Erweiterungen erfahren. In [147] wird eine konstruktive Lösung zur Parametrierung von FIOs auf der Grundlage geometrischer Überlegungen angegeben. Es wird erstmals die Verbindung zu Entkopplungsregelungen erwähnt, wobei sich die Ergebnisse auf den geometrischen Entwurf aus [266] stützen. Im Gegensatz dazu liefert [255] ein Entwurfsverfahren, das auf der Vorgabe der Eigenstruktur des Beobachters beruht. Dabei kommen die Ergebnisse zur Eigenstrukturvorgabe aus [154] zum Einsatz. Es besteht jedoch die Voraussetzung rang $\left(\boldsymbol{C} \boldsymbol{E}_{a}\right)=n_{y}$, sodass das Verfahren restriktiv hinsichtlich der handhabbaren Systeme ist. In $[167,168]$ werden diese Ergebnisse um ein einfacheres, konstruktives Entwurfsverfahren erweitert. Es besteht jedoch die gleiche, restriktive Entwurfsvoraussetzung. Eine zentrale Rolle nehmen 
die Ergebnisse aus [133] ein. Die Verbindung zu Entkopplungsregelungen wird erneut aufgegriffen, wobei ein Bezug zum klassischen Reglerentwurf nach [67] hergestellt wird. Unter anderem wird erstmals der Begriff der Fehlerdetektionsmatrix definiert, außerdem werden auch nichtquadratische Systeme mit $n_{y}>n_{f}$ berücksichtigt. Die Dualität zwischen Entkopplungsreglern und FIOs wird jedoch nicht vollständig erkannt, daher bleiben die Ergebnisse auf Systeme mit $\delta_{i}=1$ für alle $i=1, \ldots, n_{f}$ beschränkt. Wie man anhand von Definition 4.4 erkennt, ist diese Voraussetzung für quadratische Systeme mit $n_{y}=n_{f}$ und $\boldsymbol{E}_{s}=\mathbf{0}$ gleichbedeutend mit der Forderung rang $\left(\boldsymbol{C} \boldsymbol{E}_{a}\right)=n_{y}$.

Darüber hinaus kann [133] als Grundlage für die jüngeren Ergebnisse zur Steigerung der Robustheit von FIOs angesehen werden. Zwar widmete sich bereits [59] auf Basis der Eigenstrukturvorgabe dieser Thematik, lieferte jedoch keinen systematischen Entwurf. Mit der Unterdrückung des Störgrößeneinflusses auf die erzeugten Residuen bei gleichzeitiger Fehlerisolation beschäftigte sich parallel zu [133] auch [42]. Allerdings erfolgt die Beobachterparametrierung auf Grundlage von spieltheoretischen Überlegungen, was in einem komplizierten Entwurf resultiert.

Erst mit der zunehmenden Verbreitung LMI-basierter Verfahren und entsprechender Solver haben sich neuere Entwicklungen hinsichtlich der Robustheit von FIOs ergeben, die eine systematische Behandlung auf Grundlage von [133] bieten. So werden in [114] die in nichtquadratischen Systemen mit $n_{y}>n_{f}$ existierenden zusätzlichen Freiheitsgrade genutzt, um eine LMI-basierte Störunterdrückung zu erreichen. Der Entwurf findet dabei im Frequenzbereich statt, er ist jedoch weiterhin auf Systeme mit $\delta_{i}=1$ für alle $i=1, \ldots, n_{f}$ beschränkt. Diese Einschränkung kann in [28] durch einen Eigenstrukturvorgabe-Ansatz fallen gelassen werden. Interessanterweise ergibt sich für die Störunterdrückung das gleiche Optimierungsproblem wie in [114]. In [130] wird der Frequenzbereichsentwurf aus [114] dahingehend erweitert, dass auch mit dieser Methodik Systeme mit beliebigen $\delta_{i} \geq 1$ handhabbar sind und in ihrer Robustheit bezüglich exogener Störungen optimiert werden können.

Allen bisher genannten Resultaten ist gemeinsam, dass sie unmittelbar nur eine Isolation von Aktorfehlern erlauben. Für die Behandlung von Sensorfehlern müssen diese jeweils gemäß [169] in Pseudoaktorfehler umgewandelt werden (s. Abschnitt 2.1.1). Neben einem erhöhten Entwurfs- und Implementierungsaufwand setzt dies voraus, dass die Dynamik der Sensorfehler modelliert werden kann. Diese Nachteile werden in [58, Abschnitt 13.3] umgangen. Die Dualität zu Entkopplungsregelungen wird systematisch ausgenutzt, sodass Sensorfehler direkt berücksichtigt werden können 
und beliebige $\delta_{i} \geq 0$ erlaubt sind. Der Entwurf erfolgt dabei auf Grundlage geometrischer Überlegungen.

\subsection{Beiträge dieses Kapitels}

Die im folgenden Abschnitt näher beleuchtete Dualität zu Entkopplungsregelungen wird auch im Rahmen dieser Arbeit intensiv genutzt. Der Entwurf fußt jedoch im Gegensatz zu [58] nicht auf geometrischen Überlegungen, sondern basiert zum einen auf dem klassischen Entwurf nach Falb und Wolovich [67] und zum anderen auf der Vollständigen Modalen Synthese von Entkopplungsregelungen [185]. Auch auf diesem Weg lassen sich Sensorfehler direkt berücksichtigen und es bestehen keinerlei Einschränkungen hinsichtlich der Fehlerindizes. Insofern können die Ergebnisse aus Abschnitt 4.5 zunächst als alternative Entwurfsverfahren beziehungsweise Herleitungen für die Resultate aus [58, Abschnitt 13.3] angesehen werden. Sie bieten jedoch darüber hinaus eine Reihe von Vorteilen und Erweiterungsmöglichkeiten, die im Folgenden zusammengefasst sind.

1. Erweiterte Dynamik der Diagonalelemente. Die neuen Entwurfsverfahren erlauben es, die Dynamik der Diagonalelemente zu erweitern (s. Abschnitt 4.6). Daraus ergeben sich zusätzliche Freiheitsgrade, die sich einerseits zum integrierten Entwurf von Residuengenerierung und -auswertung ausnutzen lassen. So kann zum Beispiel eine näherungsweise Differentiation der Residuen zur Trendanalyse erfolgen. Andererseits lassen sich die Freiheitsgrade zur Unterdrückung hochfrequenter Störungen nutzen.

2. Erweiterung der handhabbaren Systemklasse. Die Vollständige Modale Synthese erlaubt es in Verbindung mit dynamischen Erweiterungen, die Entwurfsvoraussetzungen gegenüber [58] weiter abzuschwächen. Dazu lassen sich Ergebnisse aus dem Bereich der dynamischen Entkopplungsregelungen [137, 140] übertragen (s. Abschnitte 4.7 und 4.8).

3. Systematische Behandlung nichtquadratischer Systeme. Beide Entwurfsverfahren werden systematisch auf nichtquadratische Systeme erweitert (Abschnitt 4.9). Insbesondere die Vollständige Modale Synthese erlaubt eine transparente Charakterisierung der sich ergebenden zusätzlichen Freiheitsgrade. Dies liefert die Grundlage für die Optimierung der Robustheit in Kapitel 5 und erlaubt 
es erstmals, nicht nur Fragen der Störunterdrückung zu behandeln, sondern auch unsichere Systemparameter direkt zu berücksichtigen.

4. Ausnutzung schwächerer struktureller Anforderungen. Werden weniger restriktive Anforderungen an die Struktur der Matrix $\boldsymbol{G}_{\boldsymbol{r} \boldsymbol{f}}(s)$ gestellt (zum Beispiel bei der teilweisen Fehlerisolation, s. Abschnitt 4.10), so erlaubt die Vollständige Modale Synthese ebenfalls die Beschreibung und Ausnutzung der zusätzlichen Freiheitsgrade.

5. Grundlage für Fehlerisolationsfilter. Nicht zuletzt sind die systematischen Betrachtungen zum beobachterbasierten Ansatz die Grundlage für die in Kapitel 6 vorgestellten Ergebnisse zu robusten Fehlerisolationsfiltern allgemeinerer Struktur.

\subsection{Dualität zu Entkopplungsregelungen}

Bereits an der beschriebenen Problemstellung der Diagonalisierung von $\boldsymbol{G}_{\boldsymbol{r} \boldsymbol{f}}(s)$ lässt sich die Verwandtschaft zum Entwurf von linearen Entkopplungsregelungen erahnen. Wie in Abschnitt 3.4 beschrieben haben diese zum Ziel, der Führungsübertragungsmatrix eines geschlossenen Regelkreises Diagonalform aufzuprägen. Dies bedeutet wie in Abbildung 4.2a dargestellt, dass jede Führungsgröße $w_{i}$ nur eine ihr zugeordnete Ausgangsgröße $y_{i}$ beeinflusst, dass also $\boldsymbol{G}_{\boldsymbol{y} \boldsymbol{w}}(s)=\operatorname{diag}\left(g_{1,1}(s), \ldots, g_{n_{y}, n_{y}}(s)\right)$ gilt. Während die betrachteten Ausgangsgrößen unverändert bleiben, stellen die Führungsgrößen $w_{i}$ die neuen Eingangsgrößen des Gesamtsystems aus Strecke und Entkopplungsregler dar. Für den geschlossenen Regelkreis gilt durch Einsetzen von (3.17) in (3.16) die bekannte Beziehung

$$
\begin{aligned}
& \dot{\boldsymbol{x}}=(\boldsymbol{A}-\boldsymbol{B} \boldsymbol{K}) \boldsymbol{x}+\boldsymbol{B} \boldsymbol{F} \boldsymbol{w}, \\
& \boldsymbol{y}=(\boldsymbol{C}-\boldsymbol{D} \boldsymbol{K}) \boldsymbol{x}+\boldsymbol{D F} \boldsymbol{w},
\end{aligned}
$$

wobei in (3.16b) zusätzlich ein Durchgriff berücksichtigt wurde, das heißt $\boldsymbol{y}=\boldsymbol{C} \boldsymbol{x}+\boldsymbol{D} \boldsymbol{u}$. Die Entwurfsaufgabe besteht in der Auslegung von $\boldsymbol{K}$ und $\boldsymbol{F}$, sodass $\boldsymbol{G}_{\boldsymbol{y} \boldsymbol{w}}(s)$ diagonalisiert und der geschlossene Regelkreis stabilisiert wird.

Ist nun $\boldsymbol{G}_{\boldsymbol{y} \boldsymbol{w}}(s)$ eine Diagonalmatrix, so gilt offensichtlich

$$
\begin{aligned}
& \boldsymbol{G}_{\boldsymbol{y} \boldsymbol{w}}(s)=(\boldsymbol{C}-\boldsymbol{D} \boldsymbol{K})\left(s \boldsymbol{I}_{n}-(\boldsymbol{A}-\boldsymbol{B} \boldsymbol{K})\right)^{-1} \boldsymbol{B} \boldsymbol{F}+\boldsymbol{D} \boldsymbol{F} \\
= & \boldsymbol{G}_{\boldsymbol{y} \boldsymbol{w}}^{\top}(s)=\boldsymbol{F}^{\top} \boldsymbol{B}^{\top}\left(s \boldsymbol{I}_{n}-\left(\boldsymbol{A}^{\top}-\boldsymbol{K}^{\top} \boldsymbol{B}^{\top}\right)\right)^{-1}\left(\boldsymbol{C}^{\top}-\boldsymbol{K}^{\top} \boldsymbol{D}^{\top}\right)+\boldsymbol{F}^{\top} \boldsymbol{D}^{\top}
\end{aligned}
$$



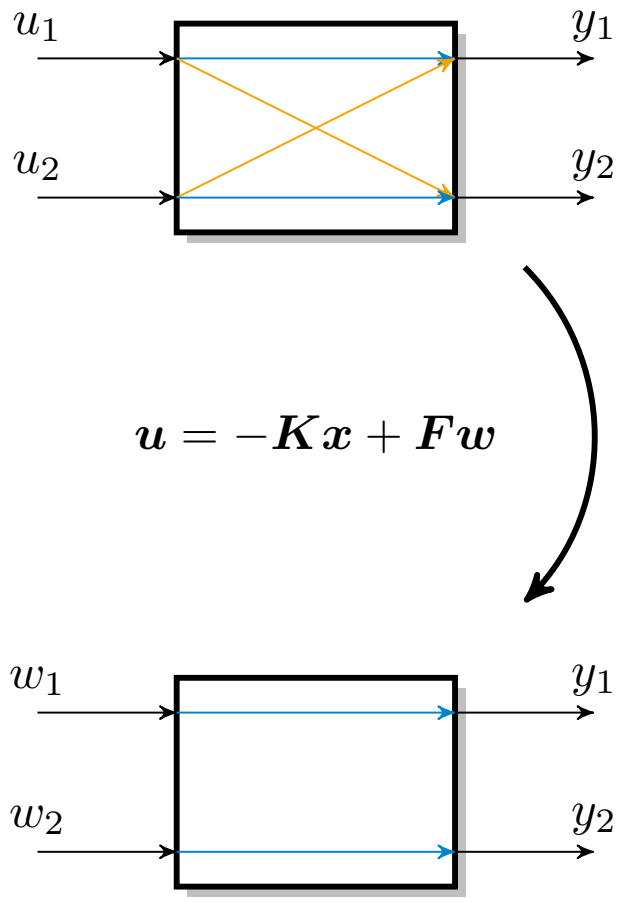

(a) Entkopplungsregler
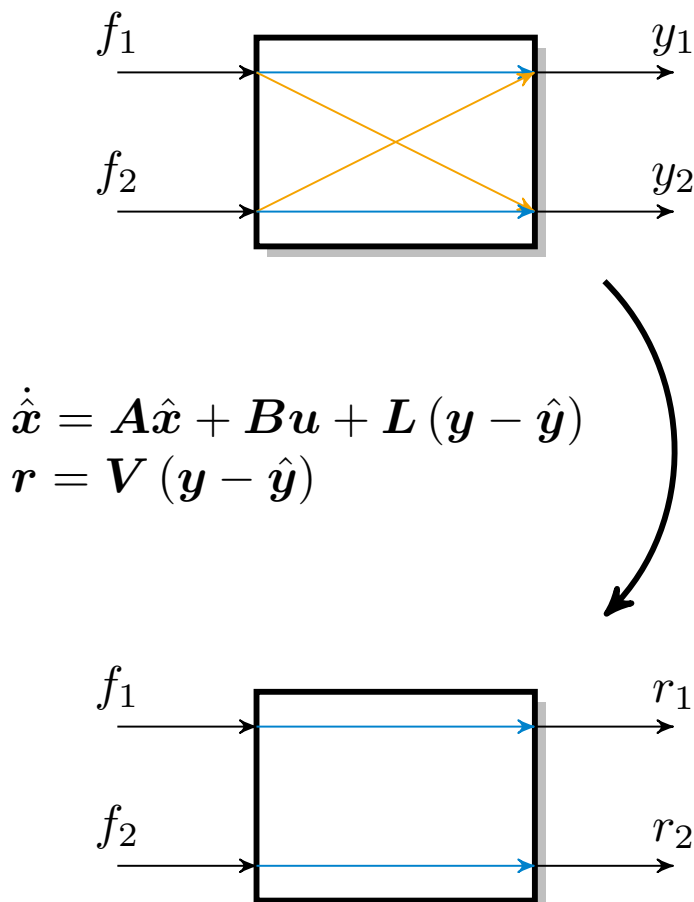

(b) Fehlerisolationsbeobachter

Abbildung 4.2: Prinzip von linearen Entkopplungsreglern und Fehlerisolationsbeobachtern

und das Ein-/Ausgangsverhalten des geschlossenen Regelkreises kann äquivalent beschrieben werden durch

$$
\begin{aligned}
\dot{\overline{\boldsymbol{x}}} & =\left(\boldsymbol{A}^{\top}-\boldsymbol{K}^{\top} \boldsymbol{B}^{\top}\right) \overline{\boldsymbol{x}}+\left(\boldsymbol{C}^{\top}-\boldsymbol{K}^{\top} \boldsymbol{D}^{\top}\right) \boldsymbol{w}, \\
\boldsymbol{y} & =\boldsymbol{F}^{\top} \boldsymbol{B}^{\top} \overline{\boldsymbol{x}}+\boldsymbol{F}^{\top} \boldsymbol{D}^{\top} \boldsymbol{w} .
\end{aligned}
$$

Vergleicht man diese Darstellung mit (4.4), so wird deutlich, dass es sich beim Entwurf von Entkopplungsregelungen mit Durchgriff und Fehlerisolationsbeobachtern für nominale Systemdynamiken um duale Probleme handelt. Dieser Sachverhalt geht ebenso aus Abbildung 4.2b hervor. Im Gegensatz zum Entkopplungsreglerentwurf bleiben jedoch beim Fehlerisolationsbeobachterentwurf die Eingangsgrößen des Systems gleich, es handelt sich dabei um die Fehler $\boldsymbol{f}$. Für das Gesamtsystem aus Strecke und Fehlerisolationsbeobachter stellen die Residuen $r_{i}$ die neuen Ausgangsgrößen dar.

Beim Reglerentwurf wird ein Durchgriff der Stellgröße $\boldsymbol{u}$ auf die Ausgangsgröße $\boldsymbol{y}$ oft nicht berücksichtigt $(\boldsymbol{D}=\mathbf{0})$, da ein solcher Durchgriff in technischen Systemen praktisch nicht auftritt. Beim dualen Problem 


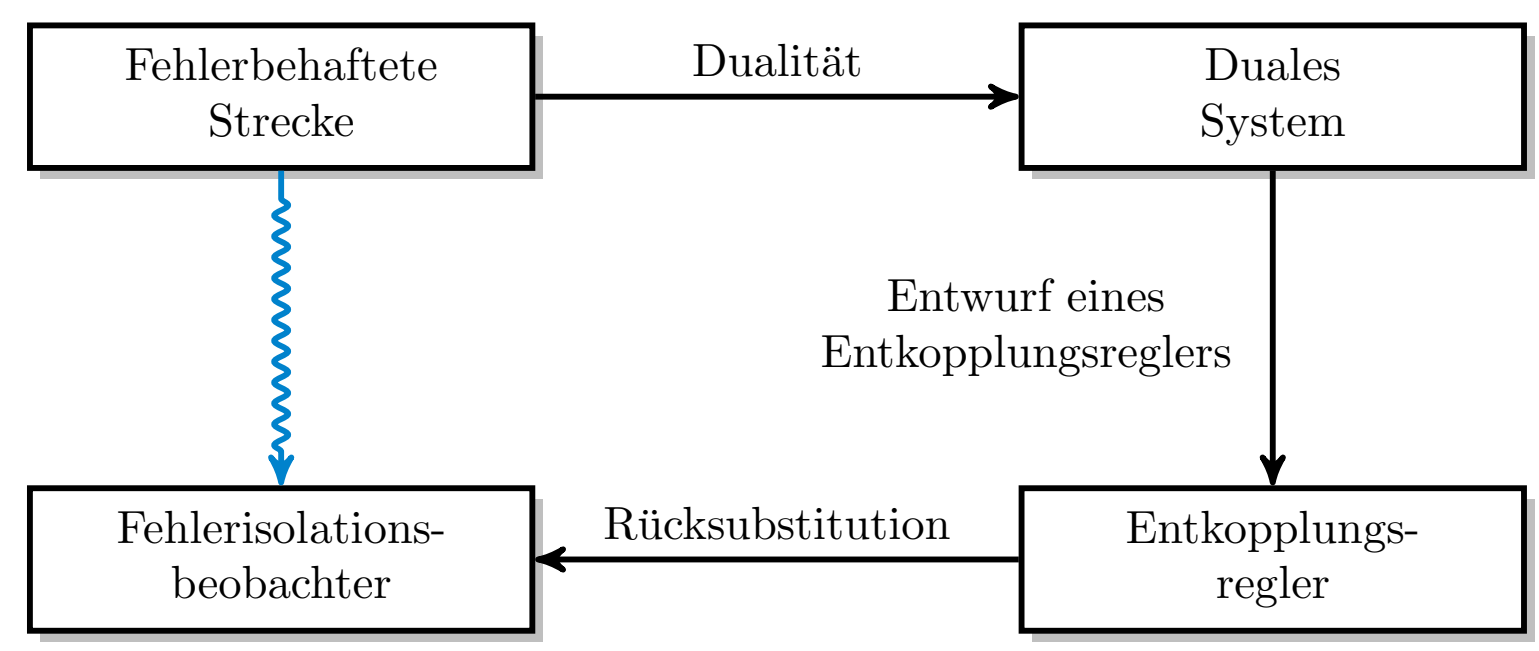

Abbildung 4.3: Dualitätsbasierter FIO-Entwurf

der Fehlerisolation entspricht ein solcher Term jedoch dem Auftreten von Sensorfehlern und gewinnt dadurch an praktischer Bedeutung.

Prinzipiell kann der Entwurf von Fehlerisolationsbeobachtern für eine nominale Systemdynamik also erfolgen, indem zunächst die Substitutionen

$$
\boldsymbol{A}^{\top} \rightarrow \boldsymbol{A}, \boldsymbol{C}^{\top} \rightarrow \boldsymbol{B}, \boldsymbol{E}_{a}^{\top} \rightarrow \boldsymbol{C}, \boldsymbol{E}_{s}^{\top} \rightarrow \boldsymbol{D}
$$

vorgenommen werden. Für das so generierte System kann dann zum Beispiel nach [67] oder [185] ein Entkopplungsregler entworfen werden, da sich die entsprechenden Verfahren geradlinig auf Systeme mit Durchgriff erweitern lassen. Nach erfolgtem Entkopplungsreglerentwurf muss die Parametrierung des Entkopplungsreglers $(\boldsymbol{F}, \boldsymbol{K})$ rücksubstituiert werden, indem

$$
\boldsymbol{L} \leftarrow \boldsymbol{K}^{\top}, \boldsymbol{V} \leftarrow \boldsymbol{F}^{\top}
$$

gebildet wird. Das beschriebene Vorgehen ist in Abbildung 4.3 skizziert und kann als Erweiterung der bekannten Dualität von linearen Zustandsrückführungen und Zustandsbeobachtern [144, Abschnitt 8.2.3] aufgefasst werden. Diese Dualität begründet auch die enge Verwandtschaft der in den Definitionen 4.2 bis 4.4 eingeführten Bezeichnungen zu den Begriffen der Differenzenordnung(en) und der Entkoppelbarkeitsmatrix aus dem Bereich der Entkopplungsregler.

Die erörterte Beziehung des Fehlerisolationsproblems zu Entkopplungsregelungen stellt die Basis für einen großen Teil der in diesem Kapitel vorgestellten Ergebnisse dar. In Abschnitt 4.5 werden Entwurfsverfahren für $\boldsymbol{L}$ und $\boldsymbol{V}$ angegeben, die als duale Resultate zu [67] beziehungsweise [185] angesehen werden können. Sie erlauben die Parametrierung eines FIOs 
ohne den in Abbildung 4.3 skizzierten "Umweg". Mithilfe des in Abbildung 4.3 skizzierten Vorgehens lassen sich prinzipiell auch die Ergebnisse zu dynamischen Entkopplungsreglern aus [137, 140] auf die Fehlerisolation übertragen. Die Abschnitte 4.7 und 4.8 erlauben durch die Verwendung von dynamisch erweiterten Beobachtern erneut einen direkten Entwurf von FIOs ohne den Umweg über das duale System. In Abschnitt 4.9 wird schließlich der Frage nachgegangen, welche zusätzlichen Freiheitsgrade sich durch den Einsatz zusätzlicher Sensoren ergeben. Aufgrund der beschriebenen Dualität lassen sich daraus Rückschlüsse für Entkopplungsregelungen mit zusätzlichen Aktoren ziehen [240]. Abschließend wird in Abschnitt 4.10 die Verwandtschaft zwischen teilweiser Fehlerisolation und teilweise entkoppelnden Zustandsrückführungen ausgenutzt.

Zusammenfassend lässt sich also sagen, dass die beschriebene Dualität der Ausgangspunkt für zahlreiche im Rahmen dieser Arbeit erzielten Ergebnisse ist. Es ist jedoch wichtig zu betonen, dass die herausgearbeitete Dualität nur mathematischer Natur ist. Dies liegt darin begründet, dass sich für den Zusammenhang zwischen Fehlern und generierten Residuen nur genau dann ein lineares System $n$-ter Ordnung wie in (4.4) ergibt, wenn die Systemmatrizen exakt bekannt sind. Das Ersatzsystem (4.4) basiert auf der Einführung des Beobachterzustandsfehlers $\boldsymbol{\xi}$, der eine virtuelle Größe darstellt. Der reale Zusammenhang zwischen Fehlern und Residuen wird durch (4.3) beschrieben, also durch ein System der Ordnung 2n. Dazu gibt es im Bereich der Entkopplungsregelungen mit statischem Regler keine Entsprechung, bei (4.6) handelt es sich immer um ein System $n$-ter Ordnung. Dieser wesentliche Unterschied wird bereits aus Abbildung 4.2 ersichtlich: Während zur Entkopplung auf der Reglerseite ein statisches System zum Einsatz kommt, wird für die Isolation auf Beobachterseite ein dynamisches System $n$-ter Ordnung benötigt. In Abschnitt 5.4.4 wird gezeigt, wie sich diese Erkenntnis nutzbringend für den Entwurf robuster Fehlerisolationsbeobachter für Strecken mit unsicheren Parametern einsetzen lässt.

\subsection{Vollständige Fehlerisolation in quadratischen Systemen}

In diesem Abschnitt werden Systeme der Form (4.1) betrachtet, bei denen die Anzahl der linear unabhängigen Messgrößen der Anzahl der Fehler entspricht. Es ist also $n_{y}=n_{f}$, weswegen von quadratischen Systemen 
gesprochen wird. In Auszügen sind die Ergebnisse dieses Abschnittes in [228, 238] veröffentlicht worden.

Um die Annahmen bezüglich des Systems (4.1) spezifizieren zu können ist anzumerken, dass sich der in Abschnitt 3.3 eingeführte Begriff der invarianten Nullstelle beziehungsweise der Minimalphasigkeit eines Systems im Rahmen dieser Arbeit stets auf das Übertragungsverhalten zwischen den Fehlern $\boldsymbol{f}$ und den Ausgängen $\boldsymbol{y}$ bezieht. Dies ist nicht zu verwechseln mit dem in der Literatur üblicherweise betrachteten Zusammenhang zwischen Stellgrößen $\boldsymbol{u}$ und Ausgängen $\boldsymbol{y}$. Folglich spielt die Matrix

$$
\boldsymbol{\Pi}(\eta)=\boldsymbol{\Pi}_{\eta}=\left[\begin{array}{cc}
\eta \boldsymbol{I}_{n}-\boldsymbol{A} & \boldsymbol{E}_{a} \\
-\boldsymbol{C} & \boldsymbol{E}_{s}
\end{array}\right]
$$

eine zentrale Rolle. Sie wird im Weiteren stets als Rosenbrock'sche Systemmatrix bezeichnet. Diese ist in der Literatur üblicherweise als

$$
\boldsymbol{\Pi}_{\eta}^{*}=\left[\begin{array}{cc}
\eta \boldsymbol{I}_{n}-\boldsymbol{A} & -\boldsymbol{E}_{a} \\
\boldsymbol{C} & \boldsymbol{E}_{s}
\end{array}\right]
$$

definiert (vgl. Abschnitt 3.3). Es gilt jedoch die Beziehung

$$
\boldsymbol{\Pi}_{\eta}=\left[\begin{array}{cc}
\boldsymbol{I}_{n} & \mathbf{0} \\
\mathbf{0} & -\boldsymbol{I}_{n}
\end{array}\right] \boldsymbol{\Pi}_{\eta}^{*}\left[\begin{array}{cc}
\boldsymbol{I}_{n} & \mathbf{0} \\
\mathbf{0} & -\boldsymbol{I}_{n}
\end{array}\right]
$$

und damit offensichtlich $\operatorname{rang}\left(\boldsymbol{\Pi}_{\eta}\right)=\operatorname{rang}\left(\boldsymbol{\Pi}_{\eta}^{*}\right)$. Da invariante Nullstellen über den Rangabfall von $\boldsymbol{\Pi}_{\eta}^{*}$ gegenüber dem Normalrang definiert sind (s. Abschnitt 3.3), gilt bei einem Rangabfall von $\Pi(\eta)$ somit, dass $\eta$ eine invariante Nullstelle des Systems $\left(\boldsymbol{A}, \boldsymbol{E}_{a}, \boldsymbol{C}, \boldsymbol{E}_{s}\right)$ ist.

Mit diesen Vorüberlegungen lassen sich die folgenden Annahmen bezüglich des Systems (4.1) treffen.

Annahme 4.1. Das Paar $(\boldsymbol{A}, \boldsymbol{C})$ ist vollständig beobachtbar.

Annahme 4.2. Die Fehlerdetektionsmatrix hat vollen Rang, das heißt es gilt $\operatorname{rang}\left(\boldsymbol{D}^{*}\right)=n_{f}$.

Annahme 4.3. Das System $\left(\boldsymbol{A}, \boldsymbol{E}_{a}, \boldsymbol{C}, \boldsymbol{E}_{s}\right)$ ist minimalphasig.

Während Annahme 4.1 sicherstellt, dass alle Eigenwerte des Beobachters beliebig platziert werden können, ist Annahme 4.2 für quadratische Systeme gleichbedeutend mit $\operatorname{det}\left(\boldsymbol{D}^{*}\right) \neq 0$. Annahme 4.3 stellt sicher, dass das System keine invarianten Nullstellen in der geschlossenen rechten s-Halbebene aufweist. Wie im Folgenden gezeigt wird, ist die Erfüllung 
von Annahme 4.2 und Annahme 4.3 hinreichend und bis auf die später diskutierte Ausnahme nicht verkoppelnder Nullstellen notwendig für die Existenz eines stabilen Fehlerisolationsbeobachters. Daher wird die folgende Definition verwendet.

Definition 4.5 (Statisch stabil isolierbare Systeme). Ein System der Form (4.1), welches die Annahmen 4.1-4.3 erfüllt, heißt statisch stabil (fehler-) isolierbar.

\subsubsection{Entwurf im Zeitbereich}

Basierend auf der in Abschnitt 4.4 herausgearbeiteten Dualität zu Entkopplungsregelungen lässt sich unter Rückgriff auf den klassischen Entkopplungsreglerentwurf nach [67] ein Entwurfsverfahren für FIOs herleiten. Dieses zeichnet sich dadurch aus, dass zwar für die Herleitung der in Abbildung 4.3 dargestellte Umweg beschritten wird, der Entwurf der FIOParametrierung $(\boldsymbol{L}, \boldsymbol{V})$ jedoch auf direktem Wege, das heißt ohne Verwendung des dualen Systems, möglich ist.

Für eine diagonale Übertragungsmatrix $\boldsymbol{G}_{\boldsymbol{r} \boldsymbol{f}}(s)=\boldsymbol{G}_{\boldsymbol{r} \boldsymbol{f}}^{\top}(s)$ lässt sich die Dynamik der Residuengenerierung (4.4) schreiben als

$$
\begin{aligned}
& \dot{\overline{\boldsymbol{\xi}}}=\left(\boldsymbol{A}^{\top}-\boldsymbol{C}^{\top} \boldsymbol{L}^{\top}\right) \overline{\boldsymbol{\xi}}+\boldsymbol{C}^{\top} \boldsymbol{V}^{\top} \boldsymbol{f}, \\
& \boldsymbol{r}=\left(\boldsymbol{E}_{a}^{\top}-\boldsymbol{E}_{s}^{\top} \boldsymbol{L}^{\top}\right) \overline{\boldsymbol{\xi}}+\boldsymbol{E}_{s}^{\top} \boldsymbol{V}^{\top} \boldsymbol{f},
\end{aligned}
$$

woraus sich mit den Substitutionen

$$
\begin{aligned}
& \overline{\boldsymbol{A}}=\boldsymbol{A}^{\top}, \overline{\boldsymbol{B}}=\boldsymbol{C}^{\top}, \overline{\boldsymbol{C}}=\boldsymbol{E}_{a}^{\top}, \overline{\boldsymbol{D}}=\boldsymbol{E}_{s}^{\top}, \\
& \overline{\boldsymbol{K}}=\boldsymbol{L}^{\top}, \overline{\boldsymbol{F}}=\boldsymbol{V}^{\top}
\end{aligned}
$$

die Darstellung

$$
\begin{aligned}
\dot{\bar{\xi}} & =(\overline{\boldsymbol{A}}-\overline{\boldsymbol{B}} \overline{\boldsymbol{K}}) \bar{\xi}+\overline{\boldsymbol{B}} \overline{\boldsymbol{F}} \boldsymbol{f}, \\
\boldsymbol{r} & =(\overline{\boldsymbol{C}}-\overline{\boldsymbol{D}} \overline{\boldsymbol{K}}) \bar{\xi}+\overline{\boldsymbol{D}} \overline{\boldsymbol{F}} \boldsymbol{f}
\end{aligned}
$$

ergibt. Diese beiden Schritte (Transponieren der Übertragungsmatrix und Variablensubstitution) entsprechen in Abbildung 4.3 dem Übergang auf das duale System.

Durch die Dynamik (4.8) wird eine virtuelle Regelstrecke $\dot{\overline{\boldsymbol{\xi}}}=\overline{\boldsymbol{A}} \overline{\boldsymbol{\xi}}+\overline{\boldsymbol{B}} \overline{\boldsymbol{u}}$, $\boldsymbol{r}=\overline{\boldsymbol{C}} \overline{\boldsymbol{\xi}}+\overline{\boldsymbol{D}} \overline{\boldsymbol{u}}$ beschrieben, die mittels $\overline{\boldsymbol{u}}=-\overline{\boldsymbol{K}} \overline{\boldsymbol{\xi}}+\overline{\boldsymbol{F}} \boldsymbol{f}$ geregelt wird. 
Für dieses virtuelle System wird im Weiteren gemäß [67] eine Reglerparametrierung $(\overline{\boldsymbol{K}}, \overline{\boldsymbol{F}})$ gesucht, welche der Führungsübertragungsmatrix Diagonalgestalt aufprägt.

Da für Fehler mit einem Fehlerindex $\delta_{i}=0$ definitionsgemäß $\boldsymbol{e}_{s_{i}} \neq \mathbf{0}$ gilt, ist $\overline{\boldsymbol{d}}_{i}^{\top} \neq \mathbf{0}^{\top}$ und somit

$$
r_{i}=\overline{\boldsymbol{c}}_{i}^{\top} \overline{\boldsymbol{\xi}}+\overline{\boldsymbol{d}}_{i}^{\top} \overline{\boldsymbol{u}}
$$

Für die übrigen Fehler ergibt sich gemäß der Definition der Fehlerindizes (s. Definition 4.2) durch sukzessives zeitliches Ableiten eines Elementes des Residuenvektors

$$
\begin{aligned}
& r_{i}=\overline{\boldsymbol{c}}_{i}^{\top} \overline{\boldsymbol{\xi}}, \\
& \dot{r}_{i}=\overline{\boldsymbol{c}}_{i}^{\top} \overline{\boldsymbol{A}} \overline{\boldsymbol{\xi}}, \\
& \vdots \\
& \stackrel{\left(\delta_{i}-1\right)}{r_{i}}=\overline{\boldsymbol{c}}_{i}^{\top} \overline{\boldsymbol{A}}^{\delta_{i}-1} \overline{\boldsymbol{\xi}}, \\
&{\stackrel{\left(\delta_{i}\right)}{r_{i}}}=\overline{\boldsymbol{c}}_{i}^{\top} \overline{\boldsymbol{A}}^{\delta_{i}} \overline{\boldsymbol{\xi}}+\overline{\boldsymbol{c}}_{i}^{\top} \overline{\boldsymbol{A}}^{\delta_{i}-1} \overline{\boldsymbol{B}} \overline{\boldsymbol{u}} .
\end{aligned}
$$

Setzt man nun wie in [67] für die Diagonalelemente der Führungsübertragungsmatrix $\mathrm{PT}_{n}$-Glieder der Form

$$
g_{i, i}(s)=\frac{z_{i, 0}}{s^{\delta_{i}}+q_{i, \delta_{i}-1} s^{\delta_{i}-1}+\ldots+q_{i, 1} s+q_{i, 0}}
$$

an, so wird im Zeitbereich die Dynamik eines Residuums durch

$$
\stackrel{\left(\delta_{i}\right)}{r_{i}}+q_{i, \delta_{i}-1} \stackrel{\left(\delta_{i}-1\right)}{r_{i}}+\ldots+q_{i, 1} \dot{r}_{i}+q_{i, 0} r_{i}=z_{i, 0} f_{i}
$$

beschrieben. Setzt man (4.10) in diese Beziehung ein, lässt sich kompakt

$$
\overline{\boldsymbol{c}}_{i}^{\top}\left(\overline{\boldsymbol{A}}^{\delta_{i}}+\sum_{k=0}^{\delta_{i}-1} q_{i, k} \overline{\boldsymbol{A}}^{k}\right) \overline{\boldsymbol{\xi}}+\underbrace{\overline{\boldsymbol{c}}_{i}^{\top} \overline{\boldsymbol{A}}^{\delta_{i}-1} \overline{\boldsymbol{B}}}_{\overline{\boldsymbol{d}}_{i}^{* \top}} \overline{\boldsymbol{u}}=z_{i, 0} f_{i}
$$

schreiben, wobei $\overline{\boldsymbol{d}}_{i}^{* \top}$ die $i$-te Zeile der Entkoppelbarkeitsmatrix $\overline{\boldsymbol{D}}^{*}$ des dualen Systems $(\overline{\boldsymbol{A}}, \overline{\boldsymbol{B}}, \overline{\boldsymbol{C}}, \overline{\boldsymbol{D}})$ ist. Dual zur Definition 4.4 der Fehlerdetektionsmatrix entspricht die $i$-te Zeile von $\overline{\boldsymbol{D}}^{*}$ der $i$-ten Zeile der Durchgriffsmatrix $\overline{\boldsymbol{D}}$, falls der Stellgrößenvektor $\overline{\boldsymbol{u}}$ eine direkte Wirkung auf die $i$-te 
Ausgangsgröße hat. Die Bedingungen (4.9) und (4.11) lassen sich kompakt zusammenfassen zu

$$
\bar{M} \bar{\xi}+\bar{D}^{*} \bar{u}=\bar{N} f
$$

wobei die Matrizen

$$
\overline{\boldsymbol{M}}=\left[\begin{array}{c}
\overline{\boldsymbol{c}}_{1}^{\mathrm{\top}}\left(\overline{\boldsymbol{A}}^{\delta_{1}}+\sum_{k=1}^{\delta_{1}-1} q_{1, k} \overline{\boldsymbol{A}}^{k}\right) \\
\vdots \\
\overline{\boldsymbol{c}}_{n_{f}}^{\top}\left(\overline{\boldsymbol{A}}^{\delta_{n_{f}}+\sum_{k=1}^{\delta_{n_{f}}-1}} q_{n_{f}, k} \overline{\boldsymbol{A}}^{k}\right)
\end{array}\right], \overline{\boldsymbol{N}}=\operatorname{diag}\left(z_{1,0}, \ldots, z_{n_{f}, 0}\right)
$$

eingeführt werden. Unter der Voraussetzung der Invertierbarkeit von $\overline{\boldsymbol{D}}^{*}$ ergibt sich aus (4.12) das entkoppelnde Regelgesetz für das duale System $(\overline{\boldsymbol{A}}, \overline{\boldsymbol{B}}, \overline{\boldsymbol{C}}, \overline{\boldsymbol{D}}) \mathrm{zu}$

$$
\boldsymbol{u}=-\underbrace{\bar{D}^{*-1} \bar{M}}_{\bar{K}} \bar{\xi}+\underbrace{\bar{D}^{*-1} \bar{N}}_{\overline{\boldsymbol{F}}} \boldsymbol{f} .
$$

Damit ist der zweite Schritt in Abbildung 4.3 abgeschlossen. Durch Rücksubstitution (vgl. (4.7)) ergeben sich daraus letztendlich die gesuchten Beobachtermatrizen $(\boldsymbol{L}, \boldsymbol{V})$ und es gilt

Satz 4.1 (FIO-Entwurf für quadratische Systeme im Zeitbereich). Gegeben sei ein System der Form

$$
\begin{aligned}
& \dot{\boldsymbol{x}}=\boldsymbol{A} \boldsymbol{x}+\boldsymbol{B} \boldsymbol{u}+\boldsymbol{E}_{a} \boldsymbol{f} \\
& \boldsymbol{y}=\boldsymbol{C} \boldsymbol{x}+\boldsymbol{D} \boldsymbol{u}+\boldsymbol{E}_{s} \boldsymbol{f}
\end{aligned}
$$

welches die Annahmen 4.1 und 4.2 erfüllt. Dann resultiert eine Beobachterparametrierung $(\boldsymbol{L}, \boldsymbol{V})$ mit $\boldsymbol{L}=\boldsymbol{M} \boldsymbol{D}^{*-1}, \boldsymbol{V}=\boldsymbol{N} \boldsymbol{D}^{*-1}$ und

$$
\boldsymbol{M}=\left[\begin{array}{c}
\left(\boldsymbol{A}^{\delta_{1}} \boldsymbol{e}_{a_{1}}+\sum_{k=0}^{\delta_{1}-1} q_{1, k} \boldsymbol{A}^{k} \boldsymbol{e}_{a_{1}}\right)^{\top} \\
\vdots \\
\left(\boldsymbol{A}^{\delta_{n_{f}}} \boldsymbol{e}_{a_{n_{f}}}+\sum_{k=0}^{\delta_{n_{f}}-1} q_{n_{f}, k} \boldsymbol{A}^{k} \boldsymbol{e}_{a_{n_{f}}}\right)^{\top}
\end{array}\right]^{\top}, \boldsymbol{N}=\operatorname{diag}\left(z_{1,0}, \ldots, z_{n_{f}, 0}\right)
$$

beim Einsatz eines FIOs der Form

$$
\begin{aligned}
\dot{\hat{x}} & =\boldsymbol{A} \hat{\boldsymbol{x}}+\boldsymbol{B u}+\boldsymbol{L}(\boldsymbol{y}-\boldsymbol{C} \hat{\boldsymbol{x}}-\boldsymbol{D u}), \\
\boldsymbol{r} & =\boldsymbol{V}(\boldsymbol{y}-\boldsymbol{C} \hat{\boldsymbol{x}}-\boldsymbol{D u})
\end{aligned}
$$


in einer diagonalisierten Übertragungsmatrix $\boldsymbol{G}_{\boldsymbol{r} \boldsymbol{f}}(s)$ mit

$$
g_{i, i}(s)=\frac{z_{i, 0}}{s^{\delta_{i}}+q_{i, \delta_{i}-1} s^{\delta_{i}-1}+\ldots+q_{i, 1} s+q_{i, 0}}
$$

und frei wählbaren Koeffizienten $q_{i, j}$ und $z_{i, 0}$, wenn $q_{i, 0}=1$ für Fehler mit $\delta_{i}=0$ gewählt wird.

Mithilfe dieses Satzes ist es also möglich, eine FIO-Parametrierung $(\boldsymbol{L}, \boldsymbol{V})$ direkt auf Basis des ursprünglichen Systems anzugeben, wie dies in Abbildung 4.3 durch den blau eingezeichneten Pfad angedeutet ist.

Satz 4.1 stellt ein konstruktives Verfahren zum Entwurf eines FIOs dar, die Fehlerisolation ist also sichergestellt. Neben diesem ersten Entwurfsziel kann die Detektionsgeschwindigkeit durch eine entsprechende Wahl der Koeffizienten $q_{i, j}$ beliebig festgelegt werden. Auch die Fehlersensitivität lässt sich frei einstellen. Bei Vorgabe einer gewünschten stationären Verstärkung $g_{i, i}(s=0)$ ist dafür der entsprechende Zählerkoeffizient als $z_{i, 0}=g_{i, i}(s=0) \cdot q_{i, 0}$ zu wählen.

Bemerkung 4.1. Streng genommen wird dadurch nur die Sensitivität bezüglich sprungförmiger Fehler eingestellt. Für den allgemeineren Fall von Fehlern beliebiger Frequenzen ist für die Auslegung von $z_{i, 0}$ der $\mathcal{H}_{--}$ Index von $g_{i, i}(s)$ zu betrachten. Da dieser wie in Abschnitt 3.1 für Systeme ohne Durchgriff stets 0 ist, ist für $\delta_{i} \geq 1$ der $\mathcal{H}_{-}$-Index in einem gewünschten Frequenzbereich $\Omega \mathrm{zu}$ betrachten. Der Koeffizient $z_{i, 0}$ ist dann so zu wählen, dass $\left\|g_{i, i}(s)\right\|_{-}^{\Omega}>\beta$ ist, wobei $\beta$ eine gewählte Schranke für die Fehlersensitivität ist. Der Übersichtlichkeit halber wird im weiteren Verlauf der Arbeit die Fehlersensitivität jedoch stets durch die Vorgabe einer stationären Verstärkung $g_{i, i}(s=0)$ berücksichtigt.

Bemerkung 4.2. Wählt man den Zähler in $g_{i, i}(s)$ entsprechend $z_{i, 0}=q_{i, 0}$, so gilt $g_{i, i}(s=0)=1$. Dies impliziert, dass im stationären Zustand das Residuum $r_{i}$ dem $i$-ten Fehler entspricht. Somit kann durch $r_{i}$ die Amplitude des Fehlers abgeschätzt werden und es wird über die Fehlerisolation hinaus auch eine Fehleridentifikation geleistet (s. Abschnitt 2.1.2).

Zur Veranschaulichung von Satz 4.1 wird das Entwurfsverfahren beispielhaft für das akademische System

$$
\begin{aligned}
& \boldsymbol{A}=\left[\begin{array}{rrr}
-10 & -16 & -20 \\
6 & 14 & 24 \\
-2 & -8 & -16
\end{array}\right], \boldsymbol{E}_{a}=\left[\begin{array}{cc}
0 & 8 \\
0,5 & -20 \\
0 & 16
\end{array}\right], \\
& \boldsymbol{C}=\left[\begin{array}{rrr}
3 & 2 & 1 \\
-9 & 0 & 5
\end{array}\right], \quad \boldsymbol{E}_{s}=\mathbf{0}
\end{aligned}
$$


angewendet. Die Beobachtbarkeitsmatrix des Systems lautet

$$
\boldsymbol{M}_{\mathrm{obsv}}=\left[\begin{array}{c}
\boldsymbol{C} \\
\boldsymbol{C A} \\
\boldsymbol{C A}^{2}
\end{array}\right]=\left[\begin{array}{rrr}
3 & 2 & 1 \\
-9 & 0 & 0 \\
-20 & -28 & -28 \\
80 & 104 & 100 \\
88 & 152 & 176 \\
-376 & -624 & -704
\end{array}\right]
$$

Sie weist offensichtlich vollen Rang auf, sodass Annahme 4.1 erfüllt ist. Im nächsten Schritt werden die Fehlerdetektionsindizes $\delta_{i}$ entsprechend Definition 4.2 bestimmt. Wegen

$$
\boldsymbol{C} \boldsymbol{e}_{a_{1}}=\left[\begin{array}{l}
1 \\
0
\end{array}\right], \quad \boldsymbol{C} \boldsymbol{e}_{a_{2}}=\left[\begin{array}{l}
0 \\
8
\end{array}\right]
$$

gilt $\delta_{1}=\delta_{2}=1$ und gemäß Definition 4.4 ergibt sich die Fehlerdetektionsmatrix zu

$$
\boldsymbol{D}^{*}=\left[\begin{array}{ll}
\boldsymbol{C} \boldsymbol{e}_{a_{1}} & \boldsymbol{C} \boldsymbol{e}_{a_{2}}
\end{array}\right]=\left[\begin{array}{ll}
1 & 0 \\
0 & 8
\end{array}\right] .
$$

Da $\boldsymbol{D}^{*}$ invertierbar ist, ist auch Annahme 4.2 erfüllt und es kann ein FIO entworfen werden. Der erste Übertragungskanal soll dabei einen Pol bei $\lambda_{B_{1,1}}=-3$ aufweisen, dementsprechend wird $q_{1,0}=3$ gewählt. Im zweiten Übertragungskanal wird ein Pol bei $\lambda_{B_{2,1}}=-5$ vorgegeben, es ist also $q_{2,0}=5$. Um für beide Kanäle eine stationäre Verstärkung von 1 einzustellen, werden die Zählerkoeffizienten $z_{1,0}=3$ und $z_{2,0}=5$ gewählt. Nun werden die Matrizen $\boldsymbol{M}$ und $\boldsymbol{N}$ berechnet, die sich für das Beispielsystem $\mathrm{zu}$

$$
\begin{aligned}
& \boldsymbol{M}=\left[\begin{array}{cc}
\boldsymbol{A} \boldsymbol{e}_{a_{1}}+q_{1,0} \boldsymbol{e}_{a_{1}} & \boldsymbol{A} \boldsymbol{e}_{a_{2}}+q_{2,0} \boldsymbol{e}_{a_{2}}
\end{array}\right]=\left[\begin{array}{cr}
-8 & -40 \\
8,5 & 52 \\
-4 & -32
\end{array}\right], \\
& \boldsymbol{N}=\left[\begin{array}{cc}
z_{1,0} & 0 \\
0 & z_{2,0}
\end{array}\right]=\left[\begin{array}{ll}
3 & 0 \\
0 & 5
\end{array}\right]
\end{aligned}
$$

ergeben. Daraus erhält man schließlich die FIO-Parametrierung

$$
\begin{aligned}
& \boldsymbol{L}=\boldsymbol{M} \boldsymbol{D}^{*-1}=\left[\begin{array}{cr}
-8 & -40 \\
8,5 & 52 \\
-4 & -32
\end{array}\right]\left[\begin{array}{ll}
1 & 0 \\
0 & \frac{1}{8}
\end{array}\right]=\left[\begin{array}{cc}
-8 & -5 \\
8,5 & 6,5 \\
-4 & -4
\end{array}\right], \\
& \boldsymbol{V}=\boldsymbol{N} \boldsymbol{D}^{*-1}=\left[\begin{array}{ll}
3 & 0 \\
0 & 5
\end{array}\right]\left[\begin{array}{ll}
1 & 0 \\
0 & \frac{1}{8}
\end{array}\right]=\left[\begin{array}{cc}
3 & 0 \\
0 & 0,625
\end{array}\right] .
\end{aligned}
$$


Es ergibt sich die diagonale Fehler-Residuen-Übertragungsmatrix

$$
\boldsymbol{G}_{\boldsymbol{r} \boldsymbol{f}}(s)=\left[\begin{array}{cc}
\frac{3}{s+3} & 0 \\
0 & \frac{5}{s+5}
\end{array}\right]
$$

Während die Strukturierung der Residuen durch den Entwurf nach Satz 4.1 ebenso erreicht wird wie eine gewünschte Detektionsgeschwindigkeit und Fehlersensitivität, lässt sich über die Stabilität des Fehlerisolationsbeobachters keine Aussage machen. Zwar können die $\delta$ Eigenwerte von $\boldsymbol{A}-\boldsymbol{L} \boldsymbol{C}$, die als Pole der Diagonalelemente $g_{i, i}(s)$ auftreten, beliebig vorgegeben werden. Im Fall $\delta<n$ ist jedoch nicht ersichtlich, was mit den verbleibenden $n-\delta$ Beobachtereigenwerten geschieht. In obigem Beispiel ergeben sich die Beobachtereigenwerte ${ }^{1)} \mathrm{zu}$

$$
\operatorname{spec}(\boldsymbol{A}-\boldsymbol{L} \boldsymbol{C})=\{-3 ;-5 ;-18\} .
$$

Der Eigenwert $\lambda_{B_{3}}=-18$ tauchte im Entwurf des FIOs nach Satz 4.1 nicht auf. Transparenter diesbezüglich ist das Verfahren mittels Vollständiger Modaler Synthese, welches im folgenden Abschnitt vorgestellt wird. Dieses erlaubt es wie beim Entkopplungsreglerentwurf [140] zu zeigen, dass die Stabilität des resultierenden Beobachters garantiert ist, falls zusätzlich Annahme 4.3 gilt.

\subsubsection{Entwurf mittels Vollständiger Modaler Synthese}

Ein alternatives Entwurfsverfahren für Entkopplungsregelungen wird in [185] vorgeschlagen. Es basiert auf der Vollständigen Modalen Synthese [184], also auf der Vorgabe der Eigenstruktur des geschlossenen Regelkreises.

Um das Verfahren in dualer Weise für den Entwurf von FIOs anwenden zu können, werden die Beobachtereigenwerte $\lambda_{B_{i}} \in \operatorname{spec}(\boldsymbol{A}-\boldsymbol{L} \boldsymbol{C})$, $i=1, \ldots, n$, analysiert. Ihnen zugeordnet sind jeweils die Rechtseigenvektoren $\boldsymbol{v}_{B_{i}}$, aus denen sich spaltenweise die Rechtseigenvektormatrix $\boldsymbol{V}_{B}$ zusammensetzt. Analog dazu beschreibt $\boldsymbol{W}_{B}$ die Linkseigenvektormatrix, deren Zeilen die Linkseigenvektoren $\boldsymbol{w}_{B_{i}}^{\top}$ sind. Bezüglich der Beobachtereigenwerte wird die folgende zusätzliche Annahme getroffen:

Annahme 4.4. Die Beobachtereigenwerte sind paarweise verschieden, das heißt, es gilt $\lambda_{B_{i}} \neq \lambda_{B_{j}} \forall i, j=1, \ldots, n, i \neq j$.

${ }^{1)} \operatorname{Mit} \operatorname{spec}(\boldsymbol{A})$ wird die Menge der Eigenwerte einer Matrix $\boldsymbol{A}$ bezeichnet. 
Wie in [134, 184] für das duale Problem des Entkopplungsreglerentwurfs gezeigt, stellt obige Annahme keine Einschränkung der Allgemeinheit dar. Sie stellt lediglich sicher, dass die Matrix $\boldsymbol{A}-\boldsymbol{L} \boldsymbol{C}$ durch eine Ähnlichkeitstransformation diagonalisiert werden kann, was die weiteren Betrachtungen vereinfacht. Durch die Verwendung von Hauptvektoren beziehungsweise einer Jordanform [134, 184] lässt sich das im Folgenden beschriebene Verfahren auch für Systeme mit mehrfachen Beobachtereigenwerten und linear abhängigen Eigenvektoren anwenden. Im Sinne einer übersichtlichen Notation wird darauf jedoch verzichtet. Weiterhin ist anzumerken, dass die Beobachtereigenwerte aufgrund von Annahme 4.1 beliebig platzierbar sind und somit Annahme 4.4 stets erfüllbar ist.

Die weiteren Überlegungen stellen die unmittelbare Übertragung des Entwurfsverfahrens aus [185] auf die Fehlerisolation dar, wobei zusätzlich Sensorfehler berücksichtigt werden, die wie bereits erörtert einem Durchgriffsterm entsprechen.

Aufgrund von Annahme 4.4 ist

$$
\boldsymbol{W}_{B}(\boldsymbol{A}-\boldsymbol{L} \boldsymbol{C}) \boldsymbol{V}_{B}=\operatorname{diag}\left(\lambda_{B_{1}}, \ldots, \lambda_{B_{n}}\right),
$$

sodass sich mit $\boldsymbol{W}_{B} \boldsymbol{V}_{B}=\boldsymbol{I}_{n}$ der Zusammenhang

$$
\boldsymbol{W}_{B}\left(s \boldsymbol{I}_{n}-(\boldsymbol{A}-\boldsymbol{L} \boldsymbol{C})\right) \boldsymbol{V}_{B}=\operatorname{diag}\left(s-\lambda_{B_{1}}, \ldots, s-\lambda_{B_{n}}\right)
$$

ergibt. Durch Inversion folgt

$$
\begin{aligned}
\operatorname{diag}\left(\frac{1}{s-\lambda_{B_{1}}}, \ldots, \frac{1}{s-\lambda_{B_{n}}}\right) & =\left(\operatorname{diag}\left(s-\lambda_{B_{1}}, \ldots, s-\lambda_{B_{n}}\right)\right)^{-1} \\
& =\left(\boldsymbol{W}_{B}\left(s \boldsymbol{I}_{n}-(\boldsymbol{A}-\boldsymbol{L} \boldsymbol{C})\right) \boldsymbol{V}_{B}\right)^{-1} \\
& =\boldsymbol{V}_{B}^{-1}\left(s \boldsymbol{I}_{n}-(\boldsymbol{A}-\boldsymbol{L} \boldsymbol{C})\right)^{-1} \boldsymbol{W}_{B}^{-1} \\
& =\boldsymbol{W}_{B}\left(s \boldsymbol{I}_{n}-(\boldsymbol{A}-\boldsymbol{L} \boldsymbol{C})\right)^{-1} \boldsymbol{V}_{B} .
\end{aligned}
$$

Damit lässt sich die in (4.5) beschriebene Übertragungsmatrix $\boldsymbol{G}_{\boldsymbol{r} \boldsymbol{f}}(s)$ als Partialbruchzerlegung

$$
\begin{aligned}
\boldsymbol{G}_{\boldsymbol{r} \boldsymbol{f}}(s) & =\boldsymbol{V} \boldsymbol{C} \underbrace{\boldsymbol{V}_{B} \boldsymbol{W}_{B}}_{\boldsymbol{I}_{n}}\left(s \boldsymbol{I}_{n}-(\boldsymbol{A}-\boldsymbol{L} \boldsymbol{C})\right)^{-1} \underbrace{\boldsymbol{V}_{B} \boldsymbol{W}_{B}}_{\boldsymbol{I}_{n}}\left(\boldsymbol{E}_{a}-\boldsymbol{L} \boldsymbol{E}_{s}\right)+\boldsymbol{V} \boldsymbol{E}_{s} \\
& =\boldsymbol{V} \boldsymbol{C} \boldsymbol{V}_{B} \operatorname{diag}\left(\frac{1}{s-\lambda_{B_{1}}}, \ldots, \frac{1}{s-\lambda_{B_{n}}}\right) \boldsymbol{W}_{B}\left(\boldsymbol{E}_{a}-\boldsymbol{L} \boldsymbol{E}_{s}\right)+\boldsymbol{V} \boldsymbol{E}_{s} \\
& =\sum_{k=1}^{n} \frac{\boldsymbol{V} \boldsymbol{C} \boldsymbol{v}_{B_{k}} \cdot \boldsymbol{w}_{B_{k}}^{\top}\left(\boldsymbol{E}_{a}-\boldsymbol{L} \boldsymbol{E}_{s}\right)}{s-\lambda_{B_{k}}}+\boldsymbol{V} \boldsymbol{E}_{s}
\end{aligned}
$$


schreiben. Im Folgenden wird für die ersten $\delta$ Beobachtereigenwerte eine Doppelindizierung $\lambda_{B_{i j}}$ eingeführt. Dabei beschreibt $i=1, \ldots, n_{f}$ den Übertragungskanal, dem der Eigenwert $\lambda_{B_{i j}}$ zugeordnet wird, und $j=1, \ldots, \delta_{i}$ nummeriert die Eigenwerte im $i$-ten Kanal. Wegen des dyadischen Produktes im Zähler von (4.15) müssen für einen Eigenwert $\lambda_{B_{i j}}$ die Bedingungen

$$
\begin{aligned}
\boldsymbol{V} \boldsymbol{C} \boldsymbol{v}_{B_{i j}} & =\alpha_{i j} \cdot \phi_{i}, \alpha_{i j} \neq 0, \\
\boldsymbol{w}_{B_{i j}}^{\top}\left(\boldsymbol{E}_{a}-\boldsymbol{L} \boldsymbol{E}_{s}\right) & =\boldsymbol{\phi}_{i}^{\top}
\end{aligned}
$$

gelten, damit er ausschließlich im $i$-ten Diagonalelement von $\boldsymbol{G}_{\boldsymbol{r} \boldsymbol{f}}(s)$ als Pol auftaucht. Dabei ist

$$
\phi_{i}^{\top}=\left[\begin{array}{lllllll}
0 & \cdots & 0 & 1 & 0 & \cdots & 0
\end{array}\right]
$$

ein Einheitsvektor, der in der $i$-ten Spalte das Element 1 enthält. Während (4.16a) sicherstellt, dass der Eigenwert $\lambda_{B_{i j}}$ der $i$-ten Zeile von $\boldsymbol{G}_{\boldsymbol{r} \boldsymbol{f}}(s)$ zugeordnet wird, bedingt (4.16b), dass der entsprechende Eigenwert an die $i$-te Spalte der Übertragungsmatrix $\boldsymbol{G}_{\boldsymbol{r} \boldsymbol{f}}(s)$ gebunden ist.

Falls der Gesamtfehlerindex $\delta$ der Systemordnung $n$ entspricht, sind durch (4.16) die Vorgaben an die Eigenstruktur von $\boldsymbol{G}_{\boldsymbol{r} \boldsymbol{f}}(s)$ vollständig beschrieben. Tritt jedoch der Fall $\delta<n$ auf, so sind die verbleibenden Beobachtereigenwerte $\lambda_{B_{k}}, k=\delta+1, \ldots, n$, im Beobachter nichtsteuerbar zu machen. Dies ist dadurch begründet, dass dann durch eine geschickte Wahl der Nachfiltermatrix $\boldsymbol{V}$ eine vollständige Diagonalisierung von $\boldsymbol{G}_{\boldsymbol{r} \boldsymbol{f}}(s)$ erreicht werden kann, wie am Ende dieses Abschnittes gezeigt wird. Sind die Eigenwerte $\lambda_{B_{k}}$ bezüglich des Paares $\left(\boldsymbol{A}-\boldsymbol{L} \boldsymbol{C}, \boldsymbol{E}_{a}-\boldsymbol{L} \boldsymbol{E}_{s}\right)$ nichtsteuerbar, so tauchen sie nicht als Pole der Übertragungsmatrix $\boldsymbol{G}_{\boldsymbol{r} \boldsymbol{f}}(s)$ auf. Nach dem Gilbert-Kriterium wird dies erreicht, wenn für den zugehörigen Linkseigenvektor

$$
\boldsymbol{w}_{B_{k}}^{\top}\left(\boldsymbol{E}_{a}-\boldsymbol{L} \boldsymbol{E}_{s}\right)=\mathbf{0}^{\top}
$$

gilt. Anstatt nun die Eigenstruktur des Beobachters direkt mittels der Rechts- beziehungsweise Linkseigenvektoren zu beeinflussen, werden $P a$ rametervektoren

$$
\boldsymbol{p}_{i j}^{\top}=-\boldsymbol{w}_{B_{i j}}^{\top} \boldsymbol{L}, \quad \boldsymbol{p}_{k}^{\top}=-\boldsymbol{w}_{B_{k}}^{\top} \boldsymbol{L}
$$

eingeführt, um (4.16b) und (4.17) zu erfüllen. Neben der bekannten Anwendung für den Reglerentwurf $[184,185]$ wurden diese auch schon für den modalen Beobachterentwurf vorgeschlagen [183]. 
Zunächst wird (4.16b) betrachtet. Aus der Definition der Linkseigenvektoren ergibt sich

$$
\boldsymbol{w}_{B_{i j}}^{\top}\left(\lambda_{B_{i j}} \boldsymbol{I}_{n}-(\boldsymbol{A}-\boldsymbol{L} \boldsymbol{C})\right)=\mathbf{0}^{\top}
$$

und nach Einsetzen von (4.18) somit

$$
\boldsymbol{w}_{B_{i j}}^{\top}\left(\lambda_{B_{i j}} \boldsymbol{I}_{n}-\boldsymbol{A}\right)-\boldsymbol{p}_{i j}^{\top} \boldsymbol{C}=\mathbf{0}^{\top} .
$$

Aus (4.16b) folgt mit (4.18) unmittelbar

$$
\boldsymbol{w}_{B_{i j}}^{\top} \boldsymbol{E}_{a}+\boldsymbol{p}_{i j}^{\top} \boldsymbol{E}_{s}=\phi_{i}^{\top}
$$

sodass sich zusammengefasst die Bedingung

$$
\left[\begin{array}{ll}
\boldsymbol{w}_{B_{i j}}^{\top} & \boldsymbol{p}_{i j}^{\top}
\end{array}\right]\left[\begin{array}{cc}
\lambda_{B_{i j}} \boldsymbol{I}_{n}-\boldsymbol{A} & \boldsymbol{E}_{a} \\
-\boldsymbol{C} & \boldsymbol{E}_{s}
\end{array}\right]=\left[\begin{array}{ll}
\boldsymbol{w}_{B_{i j}}^{\top} & \boldsymbol{p}_{i j}^{\top}
\end{array}\right] \boldsymbol{\Pi}_{i j}=\left[\begin{array}{ll}
\mathbf{0}^{\top} & \boldsymbol{\phi}_{i}^{\top}
\end{array}\right]
$$

ergibt. Darin bezeichnet $\boldsymbol{\Pi}_{i j} \in \mathbb{R}^{\left(n+n_{y}\right) \times\left(n+n_{f}\right)}$ die Rosenbrock'sche Systemmatrix des Systems $\left(\boldsymbol{A}, \boldsymbol{E}_{a}, \boldsymbol{C}, \boldsymbol{E}_{s}\right)$ an der Stelle $\lambda_{B_{i j}}$. Wenn der Eigenwert $\lambda_{B_{i j}}$ so gewählt wird, dass er nicht mit einer invarianten Nullstelle des Systems zusammenfällt, ist $\boldsymbol{\Pi}_{i j}$ invertierbar und der Parameter- beziehungsweise Linkseigenvektor ist eindeutig bestimmt durch

$$
\left[\begin{array}{ll}
\boldsymbol{w}_{B_{i j}}^{\top} & \boldsymbol{p}_{i j}^{\top}
\end{array}\right]=\left[\begin{array}{ll}
\mathbf{0}^{\top} & \boldsymbol{\phi}_{i}^{\top}
\end{array}\right] \boldsymbol{\Pi}_{i j}^{-1} .
$$

Für die nichtsteuerbar zu machenden Eigenwerte $\lambda_{B_{k}}$ folgt aus (4.17) und (4.18) analog zu (4.21) die Bedingung

$$
\left[\begin{array}{ll}
\boldsymbol{w}_{B_{k}}^{\top} & \boldsymbol{p}_{k}^{\top}
\end{array}\right]\left[\begin{array}{cc}
\lambda_{B_{k}} \boldsymbol{I}_{n}-\boldsymbol{A} & \boldsymbol{E}_{a} \\
-\boldsymbol{C} & \boldsymbol{E}_{s}
\end{array}\right]=\left[\begin{array}{ll}
\boldsymbol{w}_{B_{k}}^{\top} & \boldsymbol{p}_{k}^{\top}
\end{array}\right] \boldsymbol{\Pi}_{k}=\mathbf{0}^{\top} .
$$

Offensichtlich hat diese Gleichung nur dann eine nicht triviale Lösung, wenn die Matrix $\boldsymbol{\Pi}_{k}$ einen Linksnullraum ${ }^{2}$ ) besitzt. Dies ist nach Abschnitt 3.3 genau dann der Fall, wenn $\boldsymbol{\Pi}_{k}$ einen Rangabfall aufweist, wenn $\lambda_{B_{k}}$ also einer invarianten Nullstelle des Systems entspricht. In gleicher Weise wie beim Entkopplungsreglerentwurf (vgl. [184]) zeigt sich an dieser Stelle ein wesentlicher Vorteil der Vollständigen Modalen Synthese gegenüber der in Abschnitt 4.5.1 präsentierten Methode: Der Entwurf ist bezüglich der nichtsteuerbaren Eigenwerte transparenter und liefert eine Erklärung dafür, warum die Minimalphasigkeit des Systems für den hier beschriebenen Entwurf eine unablässige Anforderung ist.

${ }^{2)}$ Der Linksnullraum einer Matrix $\boldsymbol{Q} \in \mathbb{R}^{n \times m}$ ist definiert als Menge $\operatorname{lker}(\boldsymbol{Q})=\left\{\boldsymbol{x}^{\top} \in \mathbb{R}^{n} \mid \boldsymbol{x}^{\top} \boldsymbol{Q}=\mathbf{0}^{\top}\right\}$. 
Bemerkung 4.3. Eine Ausnahme stellen lediglich die sogenannten nicht verkoppelnden Nullstellen (engl. non-interconnecting zeros) dar [123, 136, 137]. Diese spezielle Unterklasse der invarianten Nullstellen kann beim Entwurf unkompensiert bleiben, ohne dass es zu Kopplungen in den NichtDiagonalelementen von $\boldsymbol{G}_{\boldsymbol{r} \boldsymbol{f}}(s)$ kommt. Gleichung (4.17) muss also für nicht verkoppelnde Nullstellen nicht erfüllt sein. Auf diesen Spezialfall wird in Abschnitt 4.8 näher eingegangen, an dieser Stelle wird zunächst der Fall allgemeiner invarianter Nullstellen weiter verfolgt.

Bemerkung 4.4. Aus (4.23) geht außerdem hervor, dass sich die Annahme der Beobachtbarkeit (s. Annahme 4.1 auf S. 79) abschwächen lässt. Der Entwurf ist auch für Systeme möglich, die lediglich detektierbar sind, die also unbeobachtbare Eigenwerte in der offenen linken s-Halbebene aufweisen. Wählt man in diesem Fall einen Beobachtereigenwert entsprechend einem unbeobachtbaren Eigenwert des Systems, so ergibt sich ein Rangabfall in $\boldsymbol{\Pi}_{k}$ und (4.23) kann gelöst werden.

Als Lösung für (4.23) ergibt sich

$$
\left[\begin{array}{ll}
\boldsymbol{w}_{B_{k}}^{\top} & \boldsymbol{p}_{k}^{\top}
\end{array}\right]=\boldsymbol{z}_{k}^{\top} \cdot{ }^{\perp} \boldsymbol{\Pi}_{k}, \boldsymbol{z}_{k}^{\top} \neq \mathbf{0}^{\top} .
$$

Nachdem alle Parameter- beziehungsweise Linkseigenvektoren bestimmt sind, lassen sich damit zeilenweise die Linkseigenvektormatrix $\boldsymbol{W}_{B}$ und die Parametervektormatrix $\boldsymbol{P}$ erzeugen. Gemäß der Definition der Parametervektoren (4.18) ergibt sich die Beobachtermatrix zu $\boldsymbol{L}=-\boldsymbol{W}_{B}^{-1} \boldsymbol{P}=-\boldsymbol{V}_{B} \boldsymbol{P}$.

Bemerkung 4.5. Anzumerken ist, dass Systeme mit mehrfachen invarianten Nullstellen wegen Annahme 4.4 von den Betrachtungen ausgeschlossen werden müssen. Wie zum Beispiel in [182, Abschnitt 2.4] für den Entkopplungsreglerentwurf gezeigt, kann die Annahme unter Verwendung von Hauptvektoren fallen gelassen werden, was die Darstellung jedoch verkompliziert. Daher wird im Rahmen dieser Arbeit darauf verzichtet.

Da die Betrachtungen gemäß obiger Bemerkung auf Systeme mit einfachen invarianten Nullstellen beschränkt sind, entartet der Vektor $\boldsymbol{z}_{k}^{\top}$ in (4.24) zu einem Skalar. Da die Parameter- beziehungsweise Linkseigenvektoren skalierungsinvariant sind (vgl. [184]), kann ohne Beschränkung der Allgemeinheit $\boldsymbol{z}_{k}^{\top}=z_{k}=1$ gewählt werden. Zusammenfassend ergibt sich

Lemma 4.1. Gegeben sei ein System der Form (4.1), welches die Annahmen 4.1-4.4 erfüllt. Dann ist durch die Wahl einer Beobachtermatrix

$$
\boldsymbol{L}=-\boldsymbol{W}_{B}^{-1} \boldsymbol{P}=-\boldsymbol{V}_{B} \boldsymbol{P}
$$


mit

$$
\begin{aligned}
& {\left[\begin{array}{rr}
\boldsymbol{w}_{B_{i j}}^{\top} & \boldsymbol{p}_{i j}^{\top}
\end{array}\right]=\left[\begin{array}{ll}
\mathbf{0}^{\top} & \boldsymbol{\phi}_{i}^{\top}
\end{array}\right] \boldsymbol{\Pi}_{i j}^{-1}, \quad i=1, \ldots, n_{f}, j=1, \ldots, \delta_{i}, } \\
& {\left[\begin{array}{ll}
\boldsymbol{w}_{B_{k}}^{\top} & \boldsymbol{p}_{k}^{\top}
\end{array}\right]={ }^{\perp} \boldsymbol{\Pi}_{k}, } k=\delta+1, \ldots, n,
\end{aligned}
$$

sichergestellt, dass (4.16b) und (4.17) gelten.

Wie bereits erwähnt ist durch die Wahl von $\boldsymbol{L}$ gemäß Lemma 4.1 sichergestellt, dass ein Eigenwert $\lambda_{B_{i j}}$ nur als Pol in der $i$-ten Spalte von $\boldsymbol{G}_{\boldsymbol{r} \boldsymbol{f}}(s)$ auftritt und dass die verbleibenden Eigenwerte $\lambda_{B_{k}}$ nichtsteuerbar gemacht werden. Damit ist jedoch noch keine vollständige Diagonalisierung von $\boldsymbol{G}_{\boldsymbol{r} \boldsymbol{f}}(s)$ erreicht. Diese ist durch die Wahl eines geeigneten Nachfilters $\boldsymbol{V}$ zu bewerkstelligen, was sich auf verschiedenen Wegen erreichen lässt. Da die Rechtseigenvektormatrix $\boldsymbol{V}_{B}$ durch Lemma 4.1 eindeutig bestimmt ist, kann die Bestimmung von $\boldsymbol{V}$ einerseits anhand der Beziehung (4.16a) erfolgen, worauf hier nicht näher eingegangen wird.

Andererseits lässt sich die Tatsache ausnutzen, dass die vollständige Diagonalisierung von $\boldsymbol{G}_{\boldsymbol{r} \boldsymbol{f}}(s)$ den Spezialfall $\boldsymbol{G}_{\boldsymbol{r} \boldsymbol{f}}(s=0)$ einschließt. Für diesen Fall ist

$$
\begin{aligned}
\boldsymbol{G}_{\boldsymbol{r} \boldsymbol{f}}(s=0) & =-\boldsymbol{V} \boldsymbol{C}(\boldsymbol{A}-\boldsymbol{L} \boldsymbol{C})^{-1}\left(\boldsymbol{E}_{a}-\boldsymbol{L} \boldsymbol{E}_{s}\right)+\boldsymbol{V} \boldsymbol{E}_{s} \\
& =-\boldsymbol{V} \underbrace{\left(\boldsymbol{C}(\boldsymbol{A}-\boldsymbol{L} \boldsymbol{C})^{-1}\left(\boldsymbol{E}_{a}-\boldsymbol{L} \boldsymbol{E}_{s}\right)-\boldsymbol{E}_{s}\right)}_{\Theta} .
\end{aligned}
$$

Gibt man für die stationäre Fehlerisolation eine Diagonalmatrix $\boldsymbol{S}=\operatorname{diag}\left(\tilde{z}_{1}, \ldots, \tilde{z}_{n_{f}}\right)$ vor, so ergibt sich für das Nachfilter die Forderung

$$
-V \Theta=S .
$$

Zur weiteren Berechnung wird das folgende Lemma benötigt:

Lemma 4.2. Gegeben sei ein System der Form (4.1), welches die Annahmen 4.1-4.4 erfüllt und für das eine Beobachtermatrix $\boldsymbol{L}$ gemäß Lemma 4.1 entworfen wurde, welche alle Eigenwerte von $\boldsymbol{A}-\boldsymbol{L C}$ in der offenen linken s-Halbebene platziert. Dann ist die Matrix $\boldsymbol{\Theta}=\boldsymbol{C}(\boldsymbol{A}-\boldsymbol{L} \boldsymbol{C})^{-1}\left(\boldsymbol{E}_{a}-\boldsymbol{L} \boldsymbol{E}_{s}\right)-\boldsymbol{E}_{s}$ invertierbar.

Beweis. Da $\eta=0$ gemäß Annahme 4.3 keine invariante Nullstelle des Systems ist, hat die Rosenbrock'sche Systemmatrix an dieser Stelle,

$$
\boldsymbol{\Pi}(0)=\left[\begin{array}{ll}
-\boldsymbol{A} & \boldsymbol{E}_{a} \\
-\boldsymbol{C} & \boldsymbol{E}_{s}
\end{array}\right]
$$


vollen Rang. Diese Matrix wird nun von links mit den zwei regulären Matrizen

$$
\mathbf{\Upsilon}_{1}=\left[\begin{array}{cc}
\boldsymbol{I}_{n} & \mathbf{0} \\
-\boldsymbol{C}(\boldsymbol{A}-\boldsymbol{L} \boldsymbol{C})^{-1} & \boldsymbol{I}_{n}
\end{array}\right], \mathbf{\Upsilon}_{2}=\left[\begin{array}{cc}
\boldsymbol{I}_{n} & -\boldsymbol{L} \\
\mathbf{0} & \boldsymbol{I}_{n_{y}}
\end{array}\right]
$$

multipliziert. Es ergibt sich

$$
\begin{gathered}
{\left[\begin{array}{cc}
\boldsymbol{I}_{n} & \mathbf{0} \\
-\boldsymbol{C}(\boldsymbol{A}-\boldsymbol{L} \boldsymbol{C})^{-1} & \boldsymbol{I}_{n}
\end{array}\right] \cdot\left[\begin{array}{cc}
\boldsymbol{I}_{n} & -\boldsymbol{L} \\
\mathbf{0} & \boldsymbol{I}_{n_{y}}
\end{array}\right] \cdot\left[\begin{array}{ll}
-\boldsymbol{A} & \boldsymbol{E}_{a} \\
-\boldsymbol{C} & \boldsymbol{E}_{s}
\end{array}\right]} \\
=\left[\begin{array}{cc}
-(\boldsymbol{A}-\boldsymbol{L} \boldsymbol{C}) & \boldsymbol{E}_{a}-\boldsymbol{L} \boldsymbol{E}_{s} \\
\mathbf{0} & -\boldsymbol{C}(\boldsymbol{A}-\boldsymbol{L} \boldsymbol{C})^{-1}\left(\boldsymbol{E}_{a}-\boldsymbol{L} \boldsymbol{E}_{s}\right)+\boldsymbol{E}_{s}
\end{array}\right] \\
=\left[\begin{array}{cc}
-(\boldsymbol{A}-\boldsymbol{L} \boldsymbol{C}) & \boldsymbol{E}_{a}-\boldsymbol{L} \boldsymbol{E}_{s} \\
\mathbf{0} & -\boldsymbol{\Theta}
\end{array}\right] .
\end{gathered}
$$

Da die beiden eingeführten Blockdreiecksmatrizen $\boldsymbol{\Upsilon}_{1}$ und $\boldsymbol{\Upsilon}_{2}$ in den Diagonalblöcken Einheitsmatrizen beinhalten, sind sie regulär. Weiterhin existiert $(\boldsymbol{A}-\boldsymbol{L} \boldsymbol{C})^{-1}$, da alle Beobachtereigenwerte in der offenen linken sHalbebene platziert wurden und somit $\lambda=0$ kein Eigenwert von $\boldsymbol{A}-\boldsymbol{L} \boldsymbol{C}$ ist. Die durch die Multiplikation mit den beiden regulären Matrizen entstandene Blockdreiecksmatrix ist folglich ebenfalls regulär. Dies impliziert, dass der untere rechte Block regulär ist, also dass $\Theta$ invertierbar ist.

Bemerkung 4.6. Ähnlich zum Beweis von Lemma 4.2 folgt, dass für eine invariante Nullstelle bei $\eta=0$ keine Diagonalisierung von $\boldsymbol{G}_{\boldsymbol{r} \boldsymbol{f}}(s)$ möglich ist. Da die Rosenbrock'sche Systemmatrix in diesem Fall ein Rangdefizit aufweist, hat auch die resultierende Matrix in (4.26) nicht mehr vollen Rang. Es existiert dann keine Matrix $\boldsymbol{V}$, welche die Beziehung (4.25) erfüllt, da $\boldsymbol{S}$ eine beliebige Diagonalmatrix mit Diagonalelementen ungleich 0 ist und sie somit regulär ist.

Aufgrund von Lemma 4.2 lässt sich für das Nachfilter

$$
\boldsymbol{V}=-\boldsymbol{S \Theta}^{-1}
$$

schreiben. Bemerkenswerterweise stellt diese Wahl von $\boldsymbol{V}$ die Diagonalisierung von $\boldsymbol{G}_{\boldsymbol{r} \boldsymbol{f}}(s)$ für alle $s \in \mathbb{C}$ sicher. Dies ergibt sich daraus, dass sich die Elemente von $\boldsymbol{G}_{\boldsymbol{r} \boldsymbol{f}}(s)$ entsprechend der Wahl von $\boldsymbol{L}$ nach Lemma 4.1 allgemein als

$$
g_{i, j}(s)=\frac{z_{i, j}(s)}{\left(s-\lambda_{B_{j 1}}\right) \cdot \ldots \cdot\left(s-\lambda_{B_{j \delta_{j}}}\right)}
$$


schreiben lassen. Da jedes Residuum $r_{i}$ durch eine Linearkombination von Elementen des Vektors $\boldsymbol{y}-\hat{\boldsymbol{y}}$ entsteht und die Fehlerindizes $\delta_{i}$ aussagen, wie oft der Ausgangsvektor zeitlich abgeleitet werden muss, bis ein Fehler $f_{i}$ explizit auftritt, ist die Differenzenordnung von (4.27) nach unten beschränkt durch $\delta_{j}$. Da weiterhin $\delta_{j}$ Eigenwerte als Pole in der $j$-ten Spalte von $\boldsymbol{G}_{\boldsymbol{r} \boldsymbol{f}}(s)$ zugewiesen werden, lässt sich folgern, dass die Differenzenordnung von (4.27) genau $\delta_{j}$ ist. Somit vereinfacht sich (4.27) zu

$$
g_{i, j}(s)=\frac{z_{i, j}}{\left(s-\lambda_{B_{j 1}}\right) \cdot \ldots \cdot\left(s-\lambda_{B_{j \delta_{j}}}\right)}
$$

mit einem konstanten Zähler. Da durch die Wahl des Nachfilters $\boldsymbol{V}$ die Einhaltung von $\boldsymbol{G}_{\boldsymbol{r} \boldsymbol{f}}(s=0)=\boldsymbol{S}$ sichergestellt ist, folgt für die einzelnen Elemente

$$
g_{i, j}(s=0)=\frac{z_{i, j}}{\left(-\lambda_{B_{j 1}}\right) \cdot \ldots \cdot\left(-\lambda_{B_{j \delta_{j}}}\right)}=0, i \neq j .
$$

Aus dieser Beziehung folgt unmittelbar $z_{i, j}=0$, sodass Nebendiagonalelemente von $\boldsymbol{G}_{\boldsymbol{r} \boldsymbol{f}}(s)$ für alle $s \in \mathbb{C}$ verschwinden. Es gilt also

Lemma 4.3. Gegeben sei ein System der Form (4.1), welches die Annahmen 4.1-4.4 erfüllt und für das eine Beobachtermatrix gemäß Lemma 4.1 entworfen wurde. Dann gewährleisten alle Nachfiltermatrizen

$$
\boldsymbol{V}=-\boldsymbol{S} \Theta^{-1}
$$

mit einer beliebigen Diagonalmatrix $\boldsymbol{S} \in \mathbb{R}^{n_{f} \times n_{f}}$ die Diagonalisierung von $\boldsymbol{G}_{\boldsymbol{r} \boldsymbol{f}}(s)$ für alle $s \in \mathbb{C}$.

Zusammengefasst ergibt sich aus Lemma 4.1 bis 4.3 der folgende Satz, der das parametrische Entwurfsverfahren für Fehlerisolationsbeobachter beschreibt:

Satz 4.2 (FIO-Entwurf für quadratische Systeme mittels Vollständiger Modaler Synthese). Gegeben sei ein System der Form

$$
\begin{aligned}
& \dot{\boldsymbol{x}}=\boldsymbol{A} \boldsymbol{x}+\boldsymbol{B} \boldsymbol{u}+\boldsymbol{E}_{a} \boldsymbol{f}, \\
& \boldsymbol{y}=\boldsymbol{C} \boldsymbol{x}+\boldsymbol{D} \boldsymbol{u}+\boldsymbol{E}_{s} \boldsymbol{f},
\end{aligned}
$$

welches die Annahmen 4.1-4.4 erfüllt. Dann erfüllt die gemäß

$$
\boldsymbol{L}=-\boldsymbol{W}_{B}^{-1} \boldsymbol{P}=-\boldsymbol{V}_{B} \boldsymbol{P}
$$


mit

$$
\begin{aligned}
& {\left[\begin{array}{ll}
\boldsymbol{w}_{B_{i j}}^{\top} & \boldsymbol{p}_{i j}^{\top}
\end{array}\right]=\left[\begin{array}{ll}
\mathbf{0}^{\top} & \boldsymbol{\phi}_{i}^{\top}
\end{array}\right] \boldsymbol{\Pi}_{i j}^{-1}, i=1, \ldots, n_{f}, j=1, \ldots, \delta_{i},} \\
& {\left[\begin{array}{ll}
\boldsymbol{w}_{B_{k}}^{\top} & \boldsymbol{p}_{k}^{\top}
\end{array}\right]={ }^{\perp} \boldsymbol{\Pi}_{k}, \quad k=\delta+1, \ldots, n,}
\end{aligned}
$$

parametrierte Beobachtermatrix die Gleichungen (4.16b) und (4.17). Wird weiterhin die Nachfiltermatrix durch

$$
\boldsymbol{V}=-\boldsymbol{S \Theta}^{-1}
$$

mit $\boldsymbol{\Theta}=\boldsymbol{C}(\boldsymbol{A}-\boldsymbol{L} \boldsymbol{C})^{-1}\left(\boldsymbol{E}_{a}-\boldsymbol{L} \boldsymbol{E}_{s}\right)-\boldsymbol{E}_{s}$ und einer beliebigen Diagonalmatrix $\boldsymbol{S} \in \mathbb{R}^{n_{f} \times n_{f}}$ berechnet, so ergibt sich beim Einsatz eines FIOs der Form

$$
\begin{aligned}
\dot{\hat{x}} & =\boldsymbol{A} \hat{\boldsymbol{x}}+\boldsymbol{B} \boldsymbol{u}+\boldsymbol{L}(\boldsymbol{y}-\boldsymbol{C} \hat{\boldsymbol{x}}-\boldsymbol{D u}), \\
\boldsymbol{r} & =\boldsymbol{V}(\boldsymbol{y}-\boldsymbol{C} \hat{\boldsymbol{x}}-\boldsymbol{D u})
\end{aligned}
$$

eine diagonale Übertragungsmatrix $\boldsymbol{G}_{\boldsymbol{r f}}(s)$, wobei die Stabilität des FIOs sichergestellt ist.

Das Entwurfsverfahren wird zunächst zur Verdeutlichung der einzelnen Schritte auf das Beispielsystem (4.14) von Seite 83 angewendet. Um Annahme 4.3 zu überprüfen, werden die invarianten Nullstellen des Systems ermittelt. Da es sich um ein quadratisches System handelt, lassen sich diese durch Lösen der Gleichung $\operatorname{det}(\boldsymbol{\Pi}(s))=0$ bestimmen. Durch sukzessive Entwicklung nach einzelnen Zeilen beziehungsweise Spalten ergibt sich im Beispielsystem für die Determinante der Rosenbrock'schen Systemmatrix

$$
\begin{aligned}
\operatorname{det}(\boldsymbol{\Pi}(s)) & =\left|\begin{array}{ccccc}
s \boldsymbol{I}_{3}-\boldsymbol{A} & \boldsymbol{E}_{a} \\
-\boldsymbol{C} & \boldsymbol{E}_{s}
\end{array}\right| \\
& =\left|\begin{array}{ccccc}
s+10 & 16 & 20 & 0 & 8 \\
-6 & s-14 & -24 & 0,5 & -20 \\
2 & 8 & s+16 & 0 & 16 \\
-3 & -2 & -1 & 0 & 0 \\
9 & 0 & -5 & 0 & 0
\end{array}\right|=8 s+144 .
\end{aligned}
$$

Demnach weist das System eine invariante Nullstelle bei $\eta=-18$ auf. Es ist damit minimalphasig und Annahme 4.3 ist erfüllt. Werden wie im Beispiel auf Seite 83 die Pole $\lambda_{B_{1,1}}=-3$ und $\lambda_{B_{2,1}}=-5$ vorgegeben, sind alle Beobachtereigenwerte paarweise verschieden und Annahme 4.4 
ist ebenfalls erfüllt. Für den ersten Beobachtereigenwert ergibt sich der Parameter- beziehungsweise Linkseigenvektor aus

$$
\begin{aligned}
{\left[\begin{array}{ll}
\boldsymbol{w}_{B_{11}}^{\top} & \boldsymbol{p}_{11}^{\top}
\end{array}\right] } & =\left[\begin{array}{lllll}
0 & 0 & 0 & 1 & 0
\end{array}\right] \cdot \boldsymbol{\Pi}(-3)^{-1} \\
& =\left[\begin{array}{lllll}
0,8667 & 2 & 2,0667 & -1,8 & -0,4
\end{array}\right] .
\end{aligned}
$$

Für den zweiten Beobachtereigenwert berechnet man entsprechend

$$
\begin{aligned}
{\left[\begin{array}{ll}
\boldsymbol{w}_{B_{21}}^{\top} & \boldsymbol{p}_{21}^{\top}
\end{array}\right] } & =\left[\begin{array}{lllll}
0 & 0 & 0 & 0 & 1
\end{array}\right] \cdot \boldsymbol{\Pi}(-5)^{-1} \\
& =\left[\begin{array}{lllll}
-0,125 & 0 & 0,125 & -0,5 & -0,125
\end{array}\right] .
\end{aligned}
$$

Setzt man gemäß Satz 4.2 den dritten Beobachtereigenwert als $\lambda_{B_{3}}=\eta=-18$ an, so weist die Rosenbrock'sche Systemmatrix $\Pi\left(\lambda_{B_{3}}\right)$ einen Rangabfall auf, wobei eine mögliche Beschreibung des Linksnullraumes durch

$$
\left[\begin{array}{ll}
\boldsymbol{w}_{B_{3}}^{\top} & \boldsymbol{p}_{3}^{\top}
\end{array}\right]={ }^{\perp} \boldsymbol{\Pi}(-18)=\left[\begin{array}{lllll}
-2 & 0 & 1 & -12 & -6
\end{array}\right]
$$

gegeben ist. Die Beobachtermatrix berechnet sich dann aus

$$
\begin{aligned}
\boldsymbol{L} & =-\boldsymbol{W}_{B}^{-1} \boldsymbol{P}=\left[\begin{array}{ccc}
0,8667 & 2 & 2,0667 \\
-0,125 & 0 & 0,125 \\
-2 & 0 & 1
\end{array}\right]^{-1} \cdot\left[\begin{array}{cc}
-1,8 & -0,4 \\
-0,5 & -0,125 \\
-12 & -6
\end{array}\right] \\
& =\left[\begin{array}{cc}
-8 & -5 \\
8,5 & 6,5 \\
-4 & -4
\end{array}\right] .
\end{aligned}
$$

Sie entspricht der Parametrierung, die auch mittels Satz 4.1 ermittelt wurde. Auf ihrer Grundlage findet man für die Matrix $\Theta$ im Beispiel

$$
\boldsymbol{\Theta}=\boldsymbol{C}(\boldsymbol{A}-\boldsymbol{L} \boldsymbol{C})^{-1}\left(\boldsymbol{E}_{a}-\boldsymbol{L} \boldsymbol{E}_{s}\right)-\boldsymbol{E}_{s}=\left[\begin{array}{cc}
-\frac{1}{3} & 0 \\
0 & -\frac{8}{5}
\end{array}\right] .
$$

Fordert man weiterhin für beide Übertragungskanäle eine stationäre Verstärkung von 1, so ergibt sich die Nachfiltermatrix zu

$$
\begin{aligned}
\boldsymbol{V}=-\boldsymbol{S} \boldsymbol{\Theta}^{-1} & =-\left[\begin{array}{ll}
1 & 0 \\
0 & 1
\end{array}\right] \cdot\left[\begin{array}{cc}
-\frac{1}{3} & 0 \\
0 & -\frac{8}{5}
\end{array}\right]^{-1} \\
& =\left[\begin{array}{cc}
3 & 0 \\
0 & 0,625
\end{array}\right] .
\end{aligned}
$$

Auch dieser Wert entspricht der gemäß Satz 4.1 bestimmten Parametrierung.

Der folgende Abschnitt demonstriert die Anwendung des FIO-Entwurfes für ein praktisches System. 


\subsubsection{Beispiel CE 150 Modellhubschrauber}

Betrachtet wird ein Modellhubschrauber der Marke Humusoft (Typ CE 150, [99]). Wie in Abbildung $4.4 \mathrm{zu}$ sehen ist der Hubschrauber an einem Stab befestigt und so mehrerer Freiheitsgrade beraubt. Es verbleiben lediglich die Gierbewegung (Gierwinkel $\psi$ ) und die Nickbewegung (Nickwinkel $\theta$ ). Diese können über eine Änderung der Drehzahl von Haupt- und Heckrotor beeinflusst werden. Da zusätzlich die Dynamik der Aktoren modelliert wird, ergibt sich nach der Linearisierung um den Arbeitspunkt ${ }^{3}$ ) $\psi_{\mathrm{AP}}=0, \dot{\psi}_{\mathrm{AP}}=0, \theta_{\mathrm{AP}}=-0,4$ und $\dot{\theta}_{\mathrm{AP}}=0$ das durch

$$
\boldsymbol{A}=\left[\begin{array}{cccccc}
0 & 1 & 0 & 0 & 0 & 0 \\
-5,7504 & -0,5 & 208,6646 & 0 & -1,6875 & 0 \\
0 & 0 & -5,3579 & 0 & 0 & 0 \\
0 & 0 & 0 & 0 & 1 & 0 \\
0 & 0 & -4,5455 & 0 & -0,2766 & 180,2153 \\
0 & 0 & 0 & 0 & 0 & -8,5442
\end{array}\right]
$$

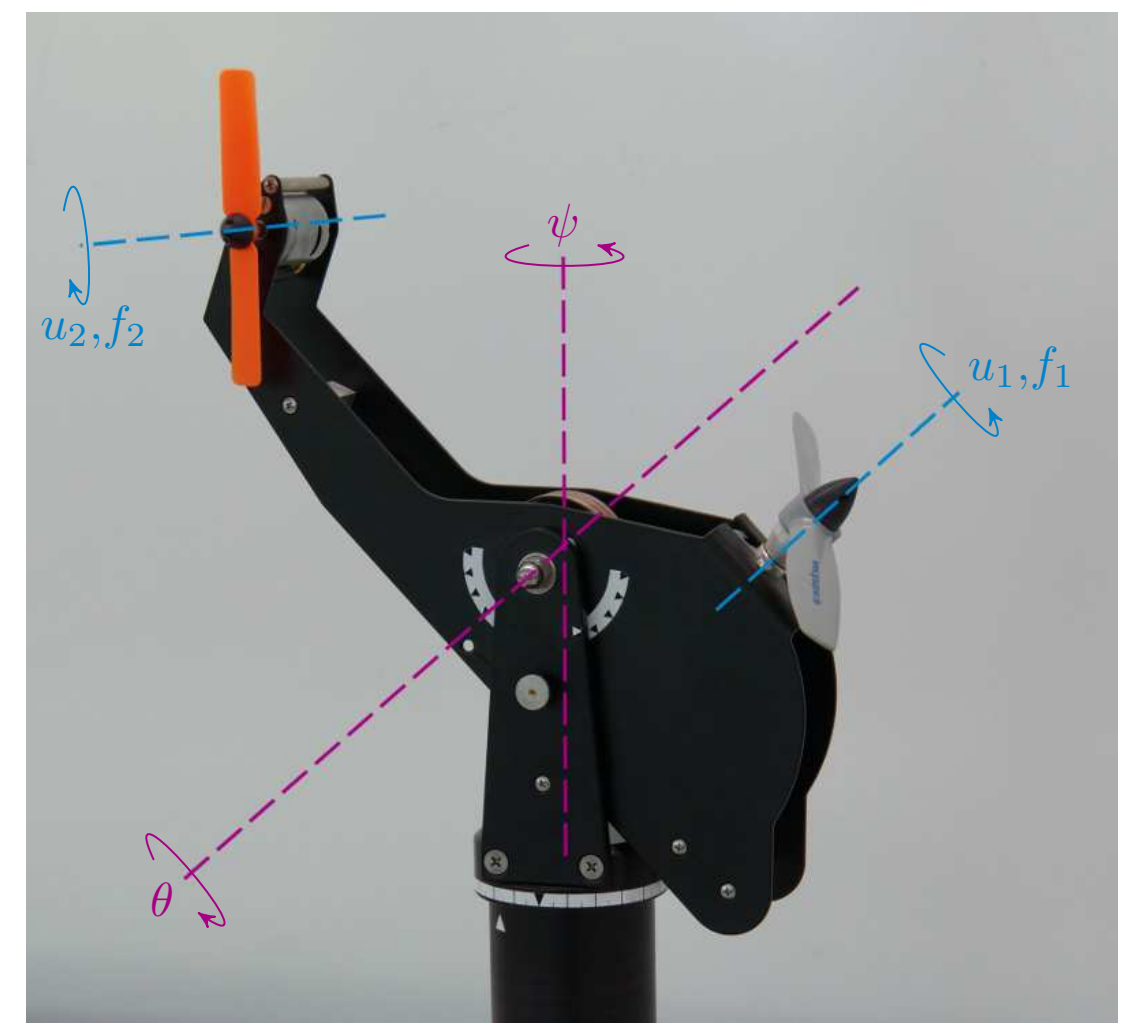

Abbildung 4.4: CE 150 Modellhubschrauber

${ }^{3)}$ Dieser entspricht einem um etwa $23^{\circ}$ nach unten geneigten Helikopter. 


$$
\boldsymbol{B}=\left[\begin{array}{ll}
0 & 0 \\
0 & 0 \\
1 & 0 \\
0 & 0 \\
0 & 0 \\
0 & 1
\end{array}\right], \boldsymbol{C}=\left[\begin{array}{llllll}
1 & 0 & 0 & 0 & 0 & 0 \\
0 & 0 & 0 & 1 & 0 & 0
\end{array}\right]
$$

beschriebene lineare Zustandsraummodell 6. Ordnung. Betrachtet werden Fehler in Haupt- und Heckrotor, es gilt $\boldsymbol{E}_{a}=\boldsymbol{B}, \boldsymbol{E}_{s}=\mathbf{0}$. Die Fehler können zum Beispiel durch Beschädigungen der Motoren hervorgerufen werden, die dazu führen, dass sich bei einer vom Regler vorgegebenen Eingangsspannung $u_{i}$ nicht die gewünschte Drehzahl des jeweiligen Rotors einstellt. Das System wird mittels einer dynamischen Ausgangsrückführung mit I-Anteil geregelt. Dies gewährleistet zwar stationäre Genauigkeit bezüglich sprungförmiger Führungsgrößen $\boldsymbol{w}$, maskiert jedoch die einwirkenden Fehler. Um diese dennoch zuverlässig detektieren und isolieren zu können, wird gemäß des Strukturbildes in Abbildung 4.5 ein FIO entworfen.

Die Systemanalyse liefert $\delta_{1}=\delta_{2}=3$. Somit ist $\delta=n$ und das System weist keine invarianten Nullstellen auf. Weiterhin gilt $\operatorname{det}\left(\boldsymbol{D}^{*}\right) \neq 0$, sodass ein stabiler FIO entworfen werden kann. Gibt man für den ersten Übertragungskanal die Eigenwerte $\lambda_{B_{1,1}}=-4, \lambda_{B_{1,2}}=-5$ und $\lambda_{B_{1,3}}=-6$ und für den zweiten Kanal die Eigenwerte $\lambda_{B_{2,1}}=-4,5, \lambda_{B_{2,2}}=-5,5$ und $\lambda_{B_{2,3}}=-6,5$ vor, so ergibt sowohl der Entwurf nach Satz 4.1 als auch nach Satz 4.2 die FIO-Parametrierung $(\boldsymbol{L}, \boldsymbol{V})$, die in Anhang C.1.1 angegeben ist. Dabei wird die stationäre Verstärkung für die Kanäle jeweils zu 1 gewählt.

Abbildung 4.6 zeigt den Verlauf der am Prüfstand in Echtzeit generier-

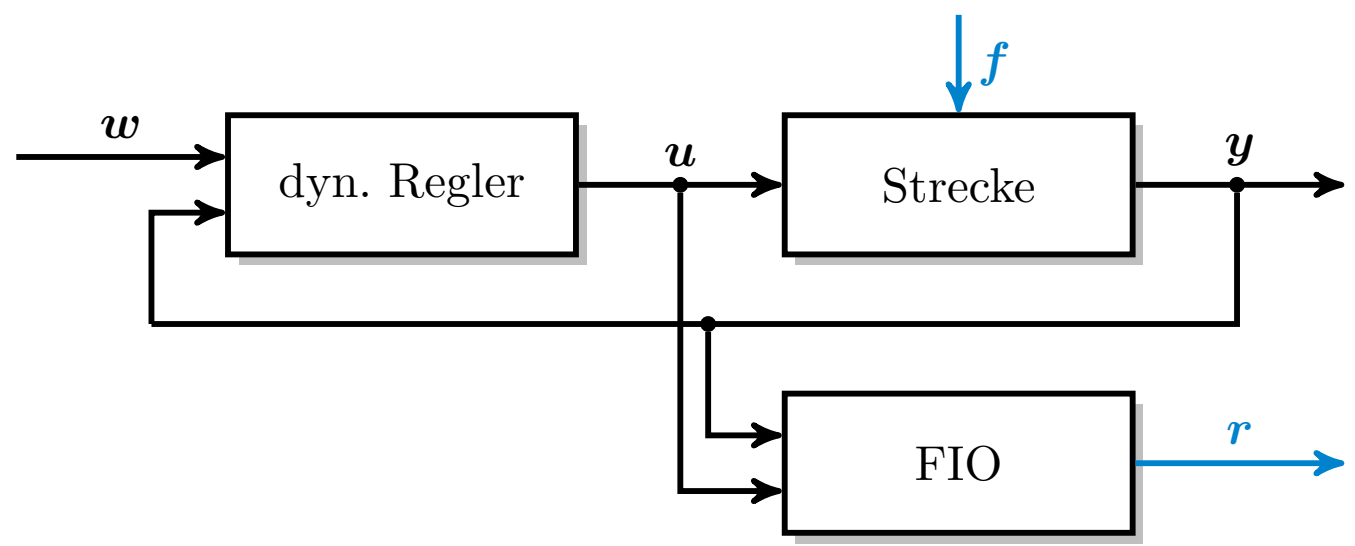

Abbildung 4.5: FIO für geschlossenen Regelkreis 

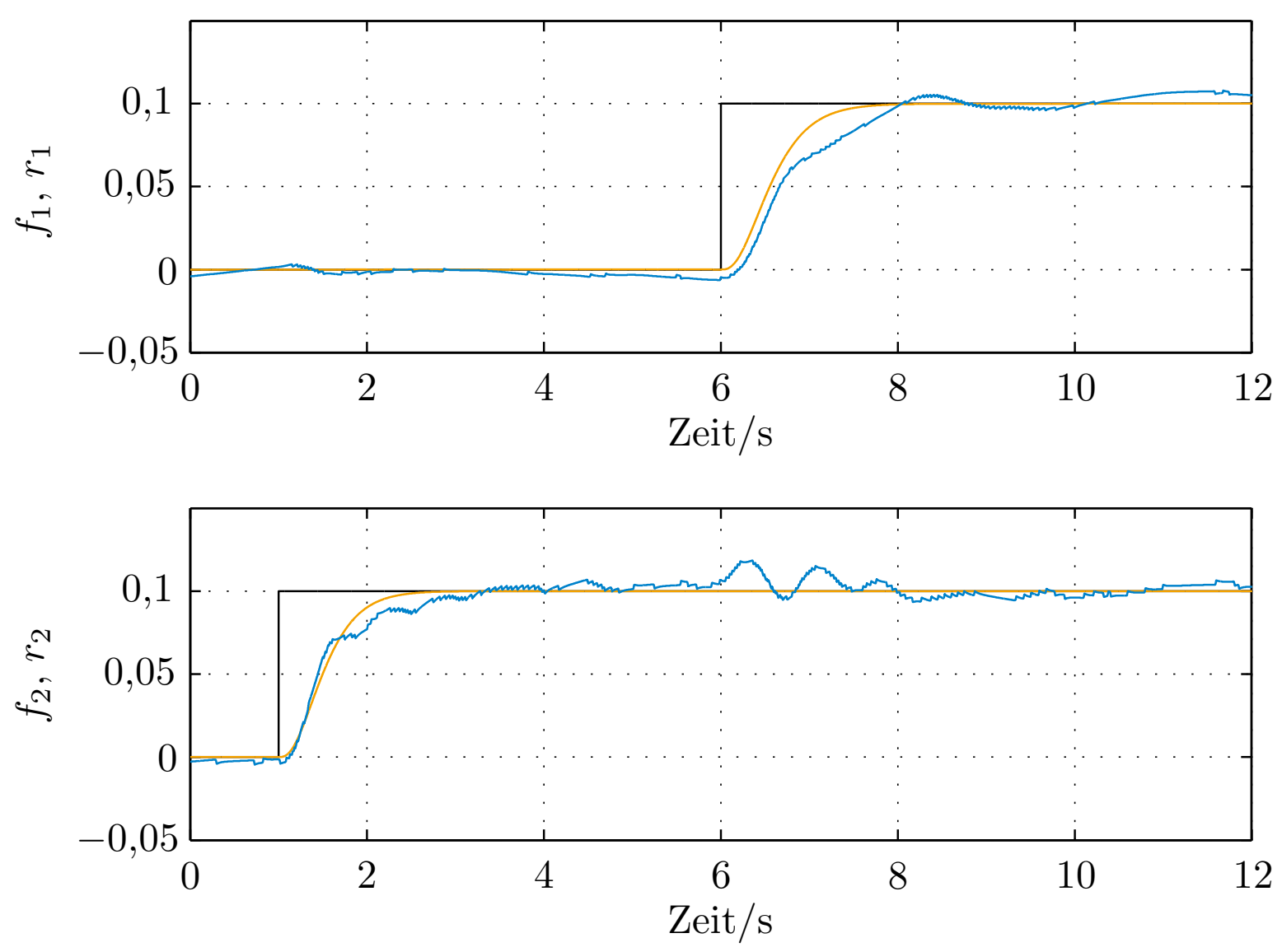

Abbildung 4.6: Fehler (-) und simulierte (-) beziehungsweise anhand von Messdaten generierte Residuen (-) für Aktorfehler des CE 150 Modellhubschraubers

ten Residuen. Es zeigt sich, dass die sprungförmigen Fehler $f_{1}(t)=0,1$ für $t>6 \mathrm{~s}$ und $f_{2}(t)=0,1$ für $t>1$ s sehr gut isoliert werden können. Der Einfluss des ersten Fehlers auf das zweite Residuum bleibt ebenso vernachlässigbar wie der Einfluss des zweiten Fehlers auf das erste Residuum. Zur Bewertung der Ergebnisse sind zusätzlich die Verläufe der simulierten Residuen gezeigt, wobei sich eine gute Übereinstimmung mit den gemessenen Residuen zeigt. Die Abweichungen, insbesondere im Verlauf von $r_{1}$, sind dadurch zu erklären, dass sich das System durch die einwirkenden Fehler zeitweise vom Arbeitspunkt entfernt, bevor es die dynamische Ausgangsrückführung wieder in diesen überführt.

\subsubsection{Fazit}

Auf Basis der in Abschnitt 4.4 dargestellten Dualität lassen sich bekannte Methoden zum Entkopplungsreglerentwurf auf den Entwurf von FIOs 
übertragen. In abgewandelter Form ist es wie in den Sätzen 4.1 und 4.2 gezeigt möglich, eine FIO-Parametrierung unmittelbar anhand der Matrizen $\boldsymbol{A}, \boldsymbol{E}_{a}, \boldsymbol{C}$ und $\boldsymbol{E}_{s}$ anzugeben, ohne den in Abbildung 4.3 skizzierten "Umweg" gehen zu müssen. Dabei lassen sich Sensorfehler direkt berücksichtigten und es besteht keine Beschränkung hinsichtlich der Fehlerindizes $\delta_{i}$.

\subsection{Erweiterte Dynamik der Diagonalelemente}

In Abschnitt 4.5 wurden Entwurfsverfahren für Fehlerisolationsbeobachter vorgestellt, welche auf eine diagonalisierte Übertragungsmatrix $\boldsymbol{G}_{\boldsymbol{r} \boldsymbol{f}}(s)$ mit Diagonalelementen der Form

$$
g_{i, i}(s)=\frac{z_{i, 0}}{s^{\delta_{i}}+q_{i, \delta_{i}-1} s^{\delta_{i}-1}+\ldots+q_{i, 1} s+q_{i, 0}}
$$

führen. Gegenstand dieses Abschnittes ist es, Methoden für den Entwurf von FIOs herzuleiten, welche gegenüber (4.28) eine erweiterte Dynamik der Diagonalelemente erlauben. Dies lässt sich im weiteren Verlauf der Arbeit unter anderem für die Erweiterung der handhabbaren Systemklasse einsetzen (s. Abschnitt 4.7). Darüber hinaus erlauben die Ansätze die Steigerung der Robustheit bezüglich hochfrequenter Störungen (s. Abschnitt 5.3.3).

\subsubsection{Idee des Verfahrens}

Die Fehlerdetektionsindizes geben nach Definition 4.2 an, wie oft der Ausgangsvektor $\boldsymbol{y}$ zeitlich abgeleitet werden muss, bis der Fehler $f_{i}$ explizit im sich ergebenden Ausdruck auftaucht. In Abschnitt 4.5 wurde gezeigt, dass sich daraus mit (4.28) ein zulässiger und naheliegender Ansatz für die Diagonalelemente von $\boldsymbol{G}_{\boldsymbol{r} \boldsymbol{f}}(s)$ ergibt. Taucht jedoch in der $\delta_{i}$-ten zeitlichen Ableitung von $\boldsymbol{y}$ der Fehler $f_{i}$ explizit auf, so ist dies ebenso für alle höheren Ableitungen der Fall, da

$$
\begin{aligned}
\stackrel{(k)}{\boldsymbol{y}} & =\boldsymbol{C} \boldsymbol{A}^{k} \boldsymbol{x}, \forall k=0, \ldots, \delta_{i}-1, \\
\stackrel{\left(\delta_{i}\right)}{\boldsymbol{y}} & =\boldsymbol{C} \boldsymbol{A}^{\delta_{i}} \boldsymbol{x}+\boldsymbol{C} \boldsymbol{A}^{\delta_{i}-1} \boldsymbol{e}_{a_{i}} f_{i}, \\
\stackrel{\left(\delta_{i}+1\right)}{\boldsymbol{y}} & =\boldsymbol{C} \boldsymbol{A}^{\delta_{i}+1} \boldsymbol{x}+\boldsymbol{C} \boldsymbol{A}^{\delta_{i}} \boldsymbol{e}_{a_{i}} f_{i}+\boldsymbol{C} \boldsymbol{A}^{\delta_{i}-1} \boldsymbol{e}_{a_{i}} \dot{f}_{i}
\end{aligned}
$$


für alle Fehler mit $\delta_{i}>0$ gilt. Für Sensorfehler gilt entsprechend $\boldsymbol{y}=\boldsymbol{C} \boldsymbol{x}+\boldsymbol{e}_{s_{i}} f_{i}$ und $\dot{\boldsymbol{y}}=\boldsymbol{C} \boldsymbol{A} \boldsymbol{x}+\boldsymbol{C} \boldsymbol{e}_{a_{i}} f_{i}+\boldsymbol{e}_{s_{i}} \dot{f}_{i}$.

Demzufolge stellen die Fehlerindizes $\delta_{i}$ jeweils eine untere Schranke für die Differenzenordnung der Diagonalelemente $g_{i, i}(s)$ dar. Diese Betrachtung motiviert die Erweiterung der Dynamik der Diagonalelemente, die in diesem Abschnitt ausführlich behandelt wird. In Auszügen sind die entsprechenden Ergebnisse in den Publikationen [232, 234, 239] veröffentlicht.

Im Gegensatz zu Abschnitt 4.5 wird für die Diagonalelemente

$$
g_{i, i}(s)=\frac{\sum_{j=0}^{\rho} z_{i, j} s^{j}}{s^{\delta_{i}+\rho}+\sum_{k=0}^{\delta_{i}+\rho-1} q_{i, k} s^{k}}
$$

angesetzt. Im Allgemeinen werden somit die Ordnungen der Zähler- und Nennerpolynome um $\rho$ erhöht. Je nach Wahl der Zählerkoeffizienten $z_{i, j}$ kann damit eine Vergrößerung der Differenzenordnung von $g_{i, i}(s)$ einhergehen. Dies ist der Fall, wenn in einem Kanal $z_{i, j}=0$ für alle $j=c, c+1, \ldots, \rho$ mit $c>0$ angesetzt wird. Falls $z_{i, j} \neq 0$ für alle $j=0, \ldots, \rho$ gilt, bleibt die Differenzenordnung von $\delta_{i}$ erhalten.

Bemerkung 4.7. Prinzipiell ist es mit den entwickelten Verfahren ebenso möglich, die Nennerordnungen in den einzelnen Übertragungsfunktionen $g_{i, i}(s)$ in unterschiedlichem Maße zu vergrößern. In diesem Fall ist anstatt einer gemeinsamen Größe $\rho$ jeweils ein individuelles $\rho_{i}$ für jeden Übertragungskanal anzusetzen.

Ein naheliegender Ansatz zur Realisierung von (4.29) ist es, zunächst einen FIO-Entwurf nach Abschnitt 4.5 durchzuführen und die erzeugten Residuensignale in einer nachgeschalteten Stufe zu filtern, wie es in Abbildung 4.7 dargestellt ist. Der FIO erzeugt die Residuensignale

$$
r_{i}^{*}(s)=\frac{z_{i, 0}}{s^{\delta_{i}}+q_{i, \delta_{i}-1} s^{\delta_{i}-1}+\ldots+q_{i, 1} s+q_{i, 0}} f_{i}(s),
$$

mithilfe derer die Residuen durch geeignete Auslegung eines kausalen Filters $g_{\text {filt }, i}(s)$ gemäß (4.29) erzeugt werden können. Dazu ist das entsprechende Filter so auszulegen, dass

$$
\frac{z_{i, 0}}{s^{\delta_{i}}+q_{i, \delta_{i}-1} s^{\delta_{i}-1}+\ldots+q_{i, 1} s+q_{i, 0}} \cdot g_{\text {filt }, i}(s)=\frac{\sum_{j=0}^{\rho} z_{i, j} s^{j}}{s^{\delta_{i}+\rho}+\sum_{k=0}^{\delta_{i}+\rho-1} q_{i, k} s^{k}}
$$

gilt.

In diesem Abschnitt wird jedoch ein anderer Ansatz zur Realisierung von (4.29) verfolgt. Ziel ist es, ein dynamisches System zu entwerfen, welches auf Basis der verfügbaren Signale $\boldsymbol{u}$ und $\boldsymbol{y}$ direkt die Residuen $r_{i}$ 


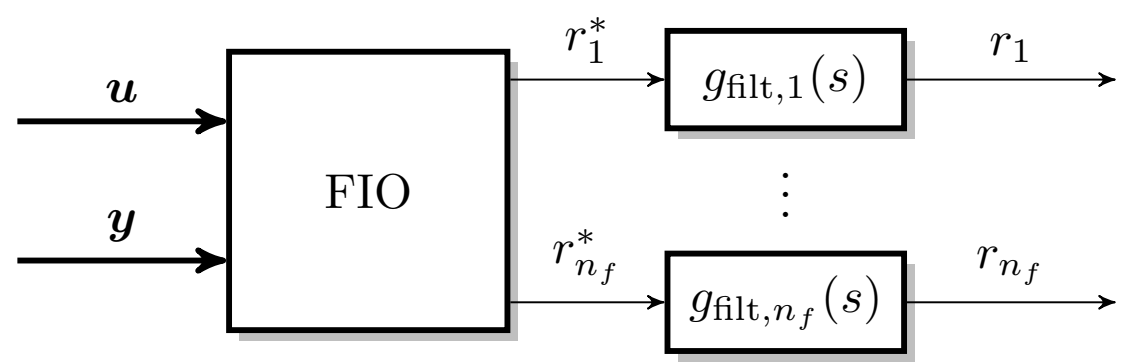

Abbildung 4.7: Dynamische Filterung der generierten Residuen

aus Abbildung 4.7 erzeugt. Dies bietet verschiedene Vorteile. Zunächst erlaubt es einen integrierten Entwurf und es ist kein separates Design von FIO und dynamischen Nachfiltern $g_{\text {filt, } i}(s)$ mehr nötig. Darüber hinaus werden durch einen FIO bei rauschbehafteten Messsignalen je nach geforderter Detektionsgeschwindigkeit unter Umständen sehr stark verrauschte Signale $r_{i}^{*}$ erzeugt. Auch wenn diese wie in Abbildung 4.7 durch die dynamischen Nachfilter zur Generierung von $r_{i}$ wieder geglättet werden können, kann es durch Signalsättigungen in $r_{i}^{*}$ zu Implementierungsproblemen kommen. Wie sich in Abschnitt 5.3.3 zeigt, kann das Auftreten stark verrauschter Signale durch den integrierten Entwurf unterbunden werden und eine nachträgliche Glättung ist nicht mehr notwendig. Ein weiterer Vorteil ist vorrangig regelungstheoretischer Natur. Soll für Kanäle mit ungeradem Fehlerindex eine erweiterte Dynamik (4.29) mit einem konjugiert komplexen Polpaar erzeugt werden, so erfordert dies ein dynamisches Nachfilter $g_{\text {filt }, i}(s) 2$. Ordnung. Ein reeller Pol, der dem Kanal durch den FIO zugewiesen wurde, muss durch das Nachfilter mittels einer Nullstelle kompensiert werden. Für den beschrieben Fall ergibt sich folglich beim integrierten Entwurf insgesamt ein Fehlerisolationssystem geringerer Ordnung.

\subsubsection{Dualität zwischen dynamischen Entkopplungsregelungen und dynamischen Fehlerisolationsbeobachtern}

Für den integrierten Entwurf wird auf dynamisch erweiterte Beobachter zurückgegriffen. Diese weisen prinzipiell die gleiche Struktur wie Luenberger-Beobachter auf, jedoch wird die Differenz aus gemessenem und geschätztem Ausgangsvektor nicht proportional, das heißt statisch, zurückgeführt, sondern dynamisch. Die Beobachtergleichungen lauten also 


$$
\begin{aligned}
\dot{\hat{\boldsymbol{x}}} & =\boldsymbol{A} \hat{\boldsymbol{x}}+\boldsymbol{B} \boldsymbol{u}+\boldsymbol{L}_{p}(\boldsymbol{y}-\boldsymbol{C} \hat{\boldsymbol{x}}-\boldsymbol{D} \boldsymbol{u})+\boldsymbol{L}_{i} \boldsymbol{\zeta}, \\
\dot{\boldsymbol{\zeta}} & =\boldsymbol{\Phi} \boldsymbol{\zeta}+\boldsymbol{\Gamma}(\boldsymbol{y}-\boldsymbol{C} \hat{\boldsymbol{x}}-\boldsymbol{D} \boldsymbol{u}) .
\end{aligned}
$$

Dabei stellt $\boldsymbol{\zeta} \in \mathbb{R}^{n_{\zeta}}$ den internen Beobachterzustand dar. Ein Beobachter der Struktur (4.30) wurde in [170] unter dem Namen dynamischer Beobachter vorgestellt. Dieser Name ist in der dynamischen Rückführung des Ausgangsfehlers begründet. Selbstverständlich handelt es sich auch beim Luenberger-Beobachter um ein dynamisches System, jedoch wird er in Abgrenzung zur Struktur (4.30) wegen der statischen Rückführung des Ausgangsfehlers im Weiteren als statischer Beobachter bezeichnet. Etwa zur gleichen Zeit wie [170] wurde der dynamische Beobachter in [202] eingeführt, dort unter dem Namen proportional integral fading observer. Dabei geht die Grundidee einer zusätzlichen Dynamik im Beobachter zurück auf [262], wo die Idee erstmals für SISO-Systeme realisiert wurde. Eine Verallgemeinerung auf MIMO-Systeme erfolgte in [12, 201], jedoch nicht in der allgemeinen Form (4.30). Anwendungen hat die Struktur bisher vorwiegend im Bereich der Zustands- und Störgrößenschätzung [27], der Fehlerdetektion [49, 81] und der fehlertoleranten Regelung [51] gefunden.

In dieser Arbeit wird die Struktur eines dynamischen Beobachters um eine zusätzliche Gleichung zur Residuengenerierung auf Basis der Ausgangsdifferenz und des internen Beobachterzustandes erweitert. Es ist also

$$
\begin{aligned}
\dot{\hat{\boldsymbol{x}}} & =\boldsymbol{A} \hat{\boldsymbol{x}}+\boldsymbol{B} \boldsymbol{u}+\boldsymbol{L}_{p}(\boldsymbol{y}-\boldsymbol{C} \hat{\boldsymbol{x}}-\boldsymbol{D} \boldsymbol{u})+\boldsymbol{L}_{i} \boldsymbol{\zeta}, \\
\dot{\boldsymbol{\zeta}} & =\boldsymbol{\Phi} \boldsymbol{\zeta}+\boldsymbol{\Gamma}(\boldsymbol{y}-\boldsymbol{C} \hat{\boldsymbol{x}}-\boldsymbol{D u}) \\
\boldsymbol{r} & =\boldsymbol{V}_{\boldsymbol{y}}(\boldsymbol{y}-\boldsymbol{C} \hat{\boldsymbol{x}}-\boldsymbol{D} \boldsymbol{u})+\boldsymbol{V}_{\boldsymbol{\zeta}} \boldsymbol{\zeta} .
\end{aligned}
$$

Für diese als dynamisch erweiterter Fehlerisolationsbeobachter (kurz: dynamischer Fehlerisolationsbeobachter, engl. dynamically extended fault isolation observer, DFIO) bezeichnete Struktur ergibt sich mit der Strecke (4.1) und unter Rückgriff auf den Beobachterfehler

$$
\boldsymbol{\xi}=\boldsymbol{x}-\hat{\boldsymbol{x}}
$$

ein Ersatzsystem der Ordnung $n+n_{\zeta}$. Es wird beschrieben durch

$$
\begin{aligned}
{\left[\begin{array}{c}
\dot{\boldsymbol{\xi}} \\
\dot{\boldsymbol{\zeta}}
\end{array}\right] } & =\left[\begin{array}{cc}
\boldsymbol{A}-\boldsymbol{L}_{p} \boldsymbol{C} & -\boldsymbol{L}_{i} \\
\boldsymbol{\Gamma} \boldsymbol{C} & \boldsymbol{\Phi}
\end{array}\right]\left[\begin{array}{l}
\boldsymbol{\xi} \\
\boldsymbol{\zeta}
\end{array}\right]+\left[\begin{array}{c}
\boldsymbol{E}_{a}-\boldsymbol{L}_{p} \boldsymbol{E}_{s} \\
\boldsymbol{\Gamma} \boldsymbol{E}_{s}
\end{array}\right] \boldsymbol{f}, \\
\boldsymbol{r} & =\left[\begin{array}{ll}
\boldsymbol{V}_{\boldsymbol{y}} \boldsymbol{C} & \boldsymbol{V}_{\boldsymbol{\zeta}}
\end{array}\right]\left[\begin{array}{l}
\boldsymbol{\xi} \\
\boldsymbol{\zeta}
\end{array}\right]+\boldsymbol{V}_{\boldsymbol{y}} \boldsymbol{E}_{s} \boldsymbol{f}
\end{aligned}
$$


und lässt sich äquivalent darstellen als

$$
\begin{aligned}
& {\left[\begin{array}{c}
\dot{\boldsymbol{\xi}} \\
\dot{\boldsymbol{\zeta}}
\end{array}\right]=(\underbrace{\left[\begin{array}{cc}
\boldsymbol{A} & \mathbf{0} \\
\mathbf{0} & \mathbf{0}
\end{array}\right]}_{\tilde{\boldsymbol{A}}}-\underbrace{\left[\begin{array}{cc}
\boldsymbol{L}_{p} & \boldsymbol{L}_{i} \\
-\boldsymbol{\Gamma} & -\boldsymbol{\Phi}
\end{array}\right]}_{\tilde{\boldsymbol{L}}} \underbrace{\left[\begin{array}{cc}
\boldsymbol{C} & \mathbf{0} \\
\mathbf{0} & \boldsymbol{I}_{n_{\zeta}}
\end{array}\right]}_{\tilde{\boldsymbol{C}}})\left[\begin{array}{l}
\boldsymbol{\xi} \\
\boldsymbol{\zeta}
\end{array}\right]+\ldots} \\
& +(\underbrace{\left[\begin{array}{c}
\boldsymbol{E}_{a} \\
\mathbf{0}
\end{array}\right]}_{\tilde{\boldsymbol{E}}_{a}}-\underbrace{\left[\begin{array}{cc}
\boldsymbol{L}_{p} & \boldsymbol{L}_{i} \\
-\boldsymbol{\Gamma} & -\boldsymbol{\Phi}
\end{array}\right]}_{\tilde{\boldsymbol{L}}} \underbrace{\left[\begin{array}{c}
\boldsymbol{E}_{s} \\
\mathbf{0}
\end{array}\right]}_{\tilde{\boldsymbol{E}}_{s}}) \boldsymbol{f} \\
& r=\underbrace{\left[\begin{array}{cc}
\boldsymbol{V}_{\boldsymbol{y}} & \boldsymbol{V}_{\zeta}
\end{array}\right]}_{\tilde{\boldsymbol{V}}} \underbrace{\left[\begin{array}{cc}
\boldsymbol{C} & \mathbf{0} \\
\mathbf{0} & \boldsymbol{I}_{n_{\zeta}}
\end{array}\right]}_{\tilde{\boldsymbol{C}}}\left[\begin{array}{c}
\boldsymbol{\xi} \\
\boldsymbol{\zeta}
\end{array}\right]+\underbrace{\left[\begin{array}{cc}
\boldsymbol{V}_{\boldsymbol{y}} & \boldsymbol{V}_{\zeta}
\end{array}\right]}_{\tilde{\boldsymbol{V}}} \underbrace{\left[\begin{array}{c}
\boldsymbol{E}_{s} \\
\mathbf{0}
\end{array}\right]}_{\tilde{\boldsymbol{E}}_{s}} \boldsymbol{f} .
\end{aligned}
$$

Für den Entwurf von dynamisch erweiterten Fehlerisolationsbeobachtern wird zunächst die in Abschnitt 4.4 herausgearbeitete Dualität zwischen Entkopplungsregelungen und Fehlerisolationsbeobachtern vertieft. Dazu werden erneut Systeme der Form

$$
\begin{aligned}
& \dot{x}=A \boldsymbol{x}+\boldsymbol{B u}, \\
& \boldsymbol{y}=\boldsymbol{C} \boldsymbol{x}+\boldsymbol{D} \boldsymbol{u}
\end{aligned}
$$

betrachtet. Neben der statischen Zustandsrückführung mit Vorfilter, $\boldsymbol{u}=-\boldsymbol{K} \boldsymbol{x}+\boldsymbol{F} \boldsymbol{w}$, können derartige Systeme auch mittels einer dynamischen Zustandsrückführung der Form

$$
\begin{aligned}
& \dot{\boldsymbol{\psi}}=\boldsymbol{A}_{u} \boldsymbol{\psi}+\boldsymbol{B}_{u, x} \boldsymbol{x}+\boldsymbol{B}_{u, w} \boldsymbol{w} \\
& \boldsymbol{u}=\boldsymbol{C}_{u} \boldsymbol{\psi}+\boldsymbol{D}_{u, x} \boldsymbol{x}+\boldsymbol{D}_{u, w} \boldsymbol{w}
\end{aligned}
$$

mit $\boldsymbol{\psi} \in \mathbb{R}^{n_{c}}$ geregelt werden (s. Anhang B).

Das sich ergebende Gesamtsystem aus (4.34) und (4.35) lautet

$$
\begin{aligned}
{\left[\begin{array}{c}
\dot{\boldsymbol{x}} \\
\dot{\boldsymbol{\psi}}
\end{array}\right]=} & \left(\left[\begin{array}{cc}
\boldsymbol{A} & \mathbf{0} \\
\mathbf{0} & \mathbf{0}
\end{array}\right]+\left[\begin{array}{cc}
\boldsymbol{B} & \mathbf{0} \\
\mathbf{0} & \boldsymbol{I}_{n_{c}}
\end{array}\right]\left[\begin{array}{ll}
\boldsymbol{D}_{u, x} & \boldsymbol{C}_{u} \\
\boldsymbol{B}_{u, x} & \boldsymbol{A}_{u}
\end{array}\right]\right)\left[\begin{array}{l}
\boldsymbol{x} \\
\boldsymbol{\psi}
\end{array}\right]+\ldots \\
& +\left[\begin{array}{cc}
\boldsymbol{B} & \mathbf{0} \\
\mathbf{0} & \boldsymbol{I}_{n_{c}}
\end{array}\right]\left[\begin{array}{l}
\boldsymbol{D}_{u, w} \\
\boldsymbol{B}_{u, w}
\end{array}\right] \boldsymbol{w} \\
\boldsymbol{y} & =\left(\left[\begin{array}{ll}
\boldsymbol{C} & \mathbf{0}
\end{array}\right]+\left[\begin{array}{ll}
\boldsymbol{D} & \mathbf{0}
\end{array}\right]\left[\begin{array}{ll}
\boldsymbol{D}_{u, x} & \boldsymbol{C}_{u} \\
\boldsymbol{B}_{u, x} & \boldsymbol{A}_{u}
\end{array}\right]\right)\left[\begin{array}{l}
\boldsymbol{x} \\
\boldsymbol{\psi}
\end{array}\right]+\left[\begin{array}{ll}
\boldsymbol{D} & \mathbf{0}
\end{array}\right]\left[\begin{array}{l}
\boldsymbol{D}_{u, w} \\
\boldsymbol{B}_{u, w}
\end{array}\right] \boldsymbol{w} .
\end{aligned}
$$


Gelingt es, die Übertragungsmatrix durch eine entsprechende Auslegung des dynamischen Reglers (4.35) zu diagonalisieren, so gilt wie im Fall eines mittels statischem Entkopplungsregler geregelten Systems $\boldsymbol{G}_{\boldsymbol{y} \boldsymbol{w}}(s)=\boldsymbol{G}_{\boldsymbol{y} \boldsymbol{w}}^{\boldsymbol{\top}}(s)$. Folglich beschreibt

$$
\begin{aligned}
{\left[\begin{array}{c}
\dot{\overline{\boldsymbol{x}}} \\
\dot{\boldsymbol{\psi}}
\end{array}\right]=\left(\left[\begin{array}{cc}
\boldsymbol{A}^{\top} & \mathbf{0} \\
\mathbf{0} & \mathbf{0}
\end{array}\right]-\left[\begin{array}{cc}
-\boldsymbol{D}_{u, x}^{\top} & -\boldsymbol{B}_{u, x}^{\top} \\
-\boldsymbol{C}_{u}^{\dagger} & -\boldsymbol{A}_{u}^{\top}
\end{array}\right]\left[\begin{array}{cc}
\boldsymbol{B}^{\top} & \mathbf{0} \\
\mathbf{0} & \boldsymbol{I}_{n_{\zeta}}
\end{array}\right]\right)\left[\begin{array}{c}
\overline{\boldsymbol{x}} \\
\overline{\boldsymbol{\psi}}
\end{array}\right]+\ldots } \\
+\left(\left[\begin{array}{c}
\boldsymbol{C}^{\top} \\
\mathbf{0}
\end{array}\right]-\left[\begin{array}{cc}
-\boldsymbol{D}_{u, x}^{\top} & -\boldsymbol{B}_{u, x}^{\top} \\
-\boldsymbol{C}_{u}^{\dagger} & -\boldsymbol{A}_{u}^{\dagger}
\end{array}\right]\left[\begin{array}{c}
\boldsymbol{D}^{\top} \\
\mathbf{0}
\end{array}\right]\right) \boldsymbol{w}, \\
\boldsymbol{y}=\left[\begin{array}{ll}
\boldsymbol{D}_{u, w}^{\top} & \boldsymbol{B}_{u, w}^{\top}
\end{array}\right]\left[\begin{array}{cc}
\boldsymbol{B}^{\top} & \mathbf{0} \\
\mathbf{0} & \boldsymbol{I}_{n_{\zeta}}
\end{array}\right]\left[\begin{array}{c}
\overline{\boldsymbol{x}} \\
\overline{\boldsymbol{\psi}}
\end{array}\right]+\left[\begin{array}{ll}
\boldsymbol{D}_{u, w}^{\top} & \boldsymbol{B}_{u, w}^{\top}
\end{array}\right]\left[\begin{array}{c}
\boldsymbol{D}^{\top} \\
\mathbf{0}
\end{array}\right] \boldsymbol{w}
\end{aligned}
$$

das gleiche Ein-/Ausgangsverhalten wie (4.36). Der Vergleich von (4.37) mit (4.33) offenbart wie bereits im statischen Fall die Dualität zwischen beiden Problemen. Der Entwurf eines dynamisch erweiterten Fehlerisolationsbeobachters kann also durch den Entwurf eines dynamischen Entkopplungsreglers für das duale System erfolgen. Dabei sind wie im statischen Fall die Substitutionen

$$
\boldsymbol{A}^{\top} \rightarrow \boldsymbol{A}, \boldsymbol{C}^{\top} \rightarrow \boldsymbol{B}, \boldsymbol{E}_{a}^{\top} \rightarrow \boldsymbol{C}, \boldsymbol{E}_{s}^{\top} \rightarrow \boldsymbol{D}
$$

vorzunehmen. Die gesuchten Beobachtermatrizen $\boldsymbol{L}_{p}, \boldsymbol{L}_{i}, \boldsymbol{\Phi}, \boldsymbol{\Gamma}, \boldsymbol{V}_{\boldsymbol{y}}$ und $\boldsymbol{V}_{\boldsymbol{\zeta}}$ ergeben sich dann durch die Rücksubstitutionen

$$
\boldsymbol{\Phi} \leftarrow \boldsymbol{A}_{u}^{\top}, \boldsymbol{\Gamma} \leftarrow \boldsymbol{C}_{u}^{\top}, \boldsymbol{L}_{p} \leftarrow-\boldsymbol{D}_{u, x}^{\top}, \boldsymbol{L}_{i} \leftarrow-\boldsymbol{B}_{u, x}^{\top}, \boldsymbol{V}_{\boldsymbol{y}} \leftarrow \boldsymbol{D}_{u, w}^{\top}, \boldsymbol{V}_{\boldsymbol{\zeta}} \leftarrow \boldsymbol{B}_{u, w}^{\top} .
$$

Dieses Vorgehen entspricht Abbildung 4.3. Es wird in den Abschnitten 4.6.3 und 4.6.4 genutzt, um auf Basis eines Entwurfsverfahrens für dynamische Entkopplungsregler dynamische Fehlerisolationsbeobachter zu entwerfen.

Alternativ lässt sich auch der Entwurf von DFIOs mittels der Vollständigen Modalen Synthese ohne den "Umweg" über das duale System und die entsprechenden Substitutionen durchführen. Dazu ist der Entwurf mittels der Vollständigen Modalen Synthese jedoch auf nichtquadratische Systeme mit mehr Messgrößen als Fehlern, also $n_{y}>n_{f}$, zu erweitern. Daher folgt eine detaillierte Beschreibung des Entwurfes auf Basis der Vollständigen Modalen Synthese erst in Abschnitt 4.9.5. 


\subsubsection{Entwurf im Laplace-Bereich}

Der Frequenzbereichsentwurf dynamischer Fehlerisolationsbeobachter basiert wie bereits angedeutet auf der Dualität zum Entwurf dynamischer Entkopplungsregler. Der Entwurf solcher Regler der Form (4.35) für Strecken der Form (4.34) erfolgt durch eine Verallgemeinerung des bekannten Entwurfsverfahrens von Falb und Wolovich [67]. Details zum Entwurfsverfahren finden sich im Anhang in Abschnitt B.1.

Für eine Strecke der Form (4.1), welche die Annahmen 4.1 bis 4.3 erfüllt, wird zunächst das duale System gebildet. Es werden also die Substitutionen (4.38) vorgenommen. Definitionsgemäß erfüllt das duale System die Annahmen B.1 bis B.3, wenn für das ursprüngliche System die Annahmen 4.1 bis 4.3 erfüllt sind. Somit lässt sich für das duale System gemäß Satz B.1 ein dynamischer Entkopplungsregler im Frequenzbereich berechnen. Dazu ist in (4.29) anhand von $\rho$ die Erhöhung des Nenner- beziehungsweise Zählergrades von $g_{i, i}(s)$ festzulegen. Die Koeffizienten sind prinzipiell frei wählbar, wobei für eine stationäre Fehlerisolation offensichtlich stets $z_{i, 0} \neq 0$ anzusetzen ist. Die Koeffizienten der Nennerpolynome können wie im statischen Fall beispielsweise durch Polvorgabe berechnet werden. Nachdem eine Zustandsraumrealisierung des dynamischen Entkopplungsreglers berechnet wurde, lässt sich eine Parametrierung des gesuchten dynamischen Fehlerisolationsbeobachters durch die Rücksubstitutionen (4.39) gewinnen.

Das Entwurfsschema folgt somit Abbildung 4.3, zusammengefasst ergibt sich der folgende Ablauf:

1. Gegeben sei eine Strecke der Form (4.1), welche die Annahmen 4.14.3 erfüllt. Substituiere $\boldsymbol{A}^{\top} \rightarrow \boldsymbol{A}, \boldsymbol{C}^{\top} \rightarrow \boldsymbol{B}, \boldsymbol{E}_{a}^{\top} \rightarrow \boldsymbol{C}$ und $\boldsymbol{E}_{s}^{\top} \rightarrow \boldsymbol{D}$, um das duale System zu erhalten.

2. Wähle $\rho$ sowie Koeffizienten $z_{i, j}$ und $p_{i, j}$ und entwirf für das duale System mittels Satz B.1 einen dynamischen Entkopplungsregler im Frequenzbereich.

3. Berechne eine Zustandsraumrealisierung des dynamischen Entkopplungsreglers.

4. Berechne die Parametrierung des DFIOs durch die Rücksubstitutionen $\boldsymbol{\Phi} \leftarrow \boldsymbol{A}_{u}^{\top}, \boldsymbol{\Gamma} \leftarrow \boldsymbol{C}_{u}^{\top}, \boldsymbol{L}_{p} \leftarrow-\boldsymbol{D}_{u, x}^{\top}, \boldsymbol{L}_{i} \leftarrow-\boldsymbol{B}_{u, x}^{\top}$, $\boldsymbol{V}_{\boldsymbol{y}} \leftarrow \boldsymbol{D}_{u, w}^{\top}, \boldsymbol{V}_{\boldsymbol{\zeta}} \leftarrow \boldsymbol{B}_{u, w}^{\top}$.

Vorteilhaft an diesem Entwurfsverfahren ist, dass sowohl die Zähler- als auch die Nennerdynamik von $g_{i, i}(s)$ erweitert werden können. Allerdings 
stellt das dualitätsbasierte Vorgehen einen Umweg dar, der den Entwurf verkompliziert. Darüber hinaus ist die Gewinnung der Zustandsraumrealisierung des Frequenzbereichsreglers in Schritt 3 mit zusätzlichem Aufwand verbunden.

\subsubsection{Entwurf im Zeitbereich}

Für den Fall, dass lediglich eine Erhöhung der Nennerordnung der Diagonalelemente von $g_{i, i}(s)$ angestrebt wird, wird in diesem Abschnitt ein deutlich vereinfachter Entwurf vorgestellt. Das Entwurfsziel lässt sich als Spezialfall von (4.29) mit $z_{i, j}=0$ für alle $i=1, \ldots, n_{f}$ und $j=1, \ldots, \rho$ auffassen. Es ergibt sich

$$
g_{i, i}(s)=\frac{z_{i, 0}}{s^{\delta_{i}+\rho}+\sum_{k=0}^{\delta_{i}+\rho-1} q_{i, k} s^{k}} .
$$

Die Entwurfsidee basiert auch hier auf der Dualität zu dynamischen Entkopplungsregelungen, ein entsprechender Reglerentwurf im Zeitbereich ist im Anhang in Abschnitt B.2 angegeben. Die Dualität wird jedoch nur für die Herleitung des Entwurfs benötigt. Für die konkrete Durchführung der DFIO-Parametrierung ist der Umweg über das duale System ebenso wenig nötig wie die Berechnung einer Zustandsraumrealisierung des dynamischen Reglers. Das Verfahren liefert unmittelbar die Parametrierung eines dynamischen Fehlerisolationsbeobachters, worin ein wesentlicher Vorteil gegenüber dem in Abschnitt 4.6.3 vorgestellten Ansatz zu sehen ist. Allerdings ist wie erwähnt die Dynamikerweiterung der Diagonalelemente auf die Nenner beschränkt.

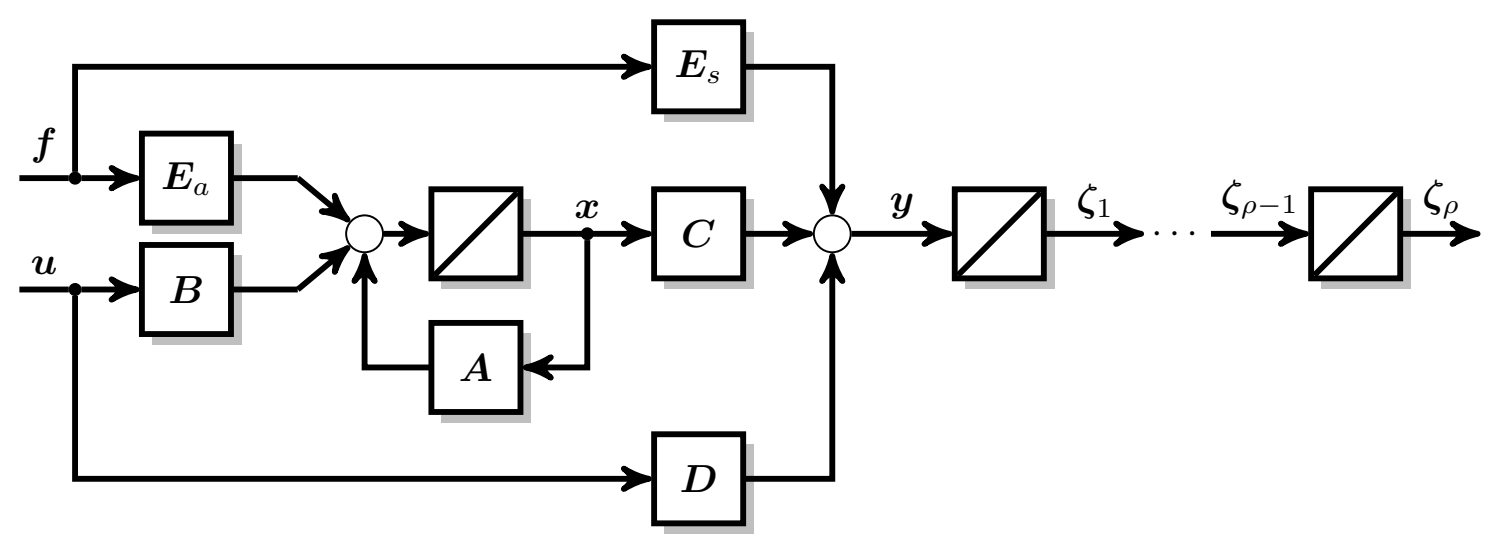

Abbildung 4.8: Virtuelle Systemerweiterung für erweiterte Dynamik der Diagonalelemente 
Das System (4.1) wird durch $\rho$-fache Integration des Ausgangsvektors $\boldsymbol{y}$ wie in Abbildung 4.8 gezeigt dynamisch erweitert. Mit dem neuen $\mathrm{Zu}-$ standsvektor $\tilde{\boldsymbol{x}}=\left[\begin{array}{llll}\boldsymbol{x}^{\top} & \boldsymbol{\zeta}_{1}^{\top} & \ldots & \boldsymbol{\zeta}_{\rho}^{\top}\end{array}\right]^{\top} \in \mathbb{R}^{n+\rho n_{y}}$ ergibt sich das virtuelle Gesamtsystem

$$
\begin{aligned}
& \dot{\tilde{\boldsymbol{x}}}=\underbrace{\left[\begin{array}{ccccc}
\boldsymbol{A} & \mathbf{0} & \cdots & \cdots & \mathbf{0} \\
\boldsymbol{C} & \mathbf{0} & \ddots & & \vdots \\
\mathbf{0} & \boldsymbol{I}_{n_{y}} & \ddots & \ddots & \vdots \\
\vdots & \ddots & \ddots & \ddots & \mathbf{0} \\
\mathbf{0} & \cdots & \mathbf{0} & \boldsymbol{I}_{n_{y}} & \mathbf{0}
\end{array}\right]}_{\tilde{\boldsymbol{A}}} \tilde{\boldsymbol{x}}+\underbrace{\left[\begin{array}{c}
\boldsymbol{B} \\
\boldsymbol{D} \\
\mathbf{0} \\
\vdots \\
\vdots \\
\mathbf{0}
\end{array}\right]}_{\tilde{\boldsymbol{B}}} \boldsymbol{u}+\underbrace{\left[\begin{array}{c}
\boldsymbol{E}_{a} \\
\boldsymbol{E}_{s} \\
\mathbf{0} \\
\vdots \\
\vdots \\
\mathbf{0}
\end{array}\right]}_{\tilde{\tilde{\boldsymbol{E}}}} \boldsymbol{f}, \\
& \tilde{\boldsymbol{y}}=\boldsymbol{\zeta}_{\rho}^{\left[\begin{array}{llll}
\mathbf{0} & \cdots & \mathbf{0} & \boldsymbol{I}_{n_{y}}
\end{array}\right]} \tilde{\boldsymbol{x}} .
\end{aligned}
$$

Bezeichnet man dabei entsprechend der eingeführten Nomenklatur die Fehlerindizes des virtuellen, dynamisch erweiterten Systems mit $\tilde{\delta}_{i}$, so gilt das folgende Lemma:

Lemma 4.4. Gegeben sei ein System der Form (4.1), welches die Annahmen 4.1-4.3 erfüllt. Wird das System gemäß (4.41) durch die $\rho$-fache Integration des Ausgangsvektors dynamisch erweitert, so gilt für die Fehlerindizes des resultierenden Gesamtsystems

$$
\tilde{\delta}_{i}=\delta_{i}+\rho .
$$

Weiterhin stimmt die Fehlerdetektionsmatrix des erweiterten Systems mit der des ursprünglichen Systems überein, das heißt es gilt $\tilde{\boldsymbol{D}}^{*}=\boldsymbol{D}^{*}$. Ebenso bleiben die invarianten Nullstellen des Systems durch die Erweiterungen unverändert.

Beweis. Da die Fehlerindizes nach Definition 4.2 angeben, wie oft der Ausgangsvektor zeitlich abgeleitet werden muss, bis der $i$-te Fehler explizit auftaucht, ist bereits anhand von Abbildung 4.8 ersichtlich, dass sich die Fehlerindizes durch die Integratorblöcke jeweils um $\rho$ erhöhen.

Die Beziehung $\tilde{\boldsymbol{D}}^{*}=\boldsymbol{D}^{*}$ ergibt sich durch die Analyse der Ausdrücke $\tilde{\boldsymbol{C}} \tilde{\boldsymbol{A}}^{\delta_{i}+\rho-1} \tilde{\boldsymbol{e}}_{a_{i}}$. Die Analyse erfolgt analog zum Vorgehen im Beweis zu Lemma B.2 im Anhang. Dabei zeigt sich, dass $\tilde{\boldsymbol{C}} \tilde{\boldsymbol{A}}^{\delta_{i}+\rho-1} \tilde{\boldsymbol{e}}_{a_{i}}=\boldsymbol{C} \boldsymbol{A}^{\delta_{i}-1} \boldsymbol{e}_{a_{i}}$ für Ausgänge mit $\delta_{i} \geq 1$ gilt und $\tilde{\boldsymbol{C}} \tilde{\boldsymbol{A}}^{\delta_{i}+\rho-1} \tilde{\boldsymbol{e}}_{a_{i}}=\boldsymbol{e}_{s_{i}}$, falls $\delta_{i}=0$ ist. Aufgrund der Dualität zu Lemma B.2 wird an dieser Stelle auf eine detaillierte Herleitung verzichtet. 
Weiterhin kann analog zu Lemma B.3 durch Entwicklung der Determinante der Rosenbrock'schen Systemmatrix des erweiterten Systems gezeigt werden, dass sich diese gegenüber dem ursprünglichen System nicht verändert. Damit bleiben auch die invarianten Nullstellen gleich.

Prinzipiell lässt sich also eine Fehlerisolation mit Diagonalelementen (4.40) erreichen, indem die $\rho$ Integratoren der Streckenerweiterung implementiert werden und anschließend ein statischer FIO für die erweiterte Strecke entworfen wird. Da jedoch für die Streckenerweiterung $n_{y} \rho$ Zustände nötig sind, ergibt sich daraus insgesamt ein Fehlerisolationssystem der Ordnung

$$
n_{\mathrm{ges}}=n_{y} \rho+n+n_{y} \rho=n+2 n_{y} \rho .
$$

Wie der folgende Satz zeigt, lässt sich das gleiche Übertragungsverhalten jedoch auch mit einem dynamischen FIO der Ordnung $n+n_{y} \rho$ erreichen.

Satz 4.3 (DFIO-Entwurf für erweiterte Nennerdynamiken im Zeitbereich). Gegeben sei ein System der Form

$$
\begin{aligned}
& \dot{\boldsymbol{x}}=\boldsymbol{A} \boldsymbol{x}+\boldsymbol{B} \boldsymbol{u}+\boldsymbol{E}_{a} \boldsymbol{f}, \\
& \boldsymbol{y}=\boldsymbol{C} \boldsymbol{x}+\boldsymbol{D} \boldsymbol{u}+\boldsymbol{E}_{s} \boldsymbol{f},
\end{aligned}
$$

welches die Annahmen 4.1-4.3 erfüllt und welches gemäß (4.41) dynamisch erweitert wurde. Weiterhin werden die Matrizen

$$
\begin{aligned}
\tilde{\boldsymbol{M}} & =\left[\begin{array}{lll}
\tilde{\boldsymbol{m}}_{1} & \cdots & \tilde{\boldsymbol{m}}_{n_{f}}
\end{array}\right], \\
\tilde{\boldsymbol{m}}_{i} & =\left(\tilde{\boldsymbol{A}}^{\delta_{i}+\rho}+\sum_{k=0}^{\delta_{i}+\rho-1} q_{i, k} \tilde{\boldsymbol{A}}^{k}\right) \tilde{\boldsymbol{e}}_{a_{i}}, \\
\tilde{\boldsymbol{N}} & =\operatorname{diag}\left(z_{1,0}, \ldots, z_{n_{f}, 0}\right)
\end{aligned}
$$

mit beliebigen Koeffizienten $q_{i, j}, i=1, \ldots, n_{f}, j=1, \ldots, \delta_{i}+\rho$, und $z_{i, 0}$, $i=1, \ldots, n_{f}$, berechnet. Durch $\tilde{\boldsymbol{L}}=\tilde{\boldsymbol{M}} \boldsymbol{D}^{*-1}$ und $\tilde{\boldsymbol{V}}=\tilde{\boldsymbol{N}} \boldsymbol{D}^{*-1}$ ist dann ein statischer FIO für das erweiterte System gegeben, welcher auf Diagonalelemente der Form

$$
g_{i, i}(s)=\frac{z_{i, 0}}{s^{\delta_{i}+\rho}+\sum_{k=0}^{\delta_{i}+\rho-1} q_{i, k} s^{k}} .
$$

führt. Partitioniert man

$$
\tilde{\boldsymbol{L}}=\left[\begin{array}{llll}
\boldsymbol{L}_{\boldsymbol{y}}^{\top} & \boldsymbol{L}_{\zeta_{1}}^{\top} & \cdots & \boldsymbol{L}_{\zeta_{\rho}}^{\top}
\end{array}\right]^{\top}
$$


so ist durch

$$
\begin{aligned}
\dot{\hat{\boldsymbol{x}}} & =\boldsymbol{A} \hat{\boldsymbol{x}}+\boldsymbol{B} \boldsymbol{u}+\boldsymbol{L}_{p}(\boldsymbol{y}-\boldsymbol{C} \hat{\boldsymbol{x}}-\boldsymbol{D} \boldsymbol{u})+\boldsymbol{L}_{i} \boldsymbol{\zeta} \\
\dot{\boldsymbol{\zeta}} & =\boldsymbol{\Phi} \boldsymbol{\zeta}+\boldsymbol{\Gamma}(\boldsymbol{y}-\boldsymbol{C} \hat{\boldsymbol{x}}-\boldsymbol{D} \boldsymbol{u}) \\
\boldsymbol{r} & =\boldsymbol{V}_{\boldsymbol{y}}(\boldsymbol{y}-\boldsymbol{C} \hat{\boldsymbol{x}}-\boldsymbol{D} \boldsymbol{u})+\boldsymbol{V}_{\boldsymbol{\zeta}} \boldsymbol{\zeta}
\end{aligned}
$$

mit

$$
\begin{array}{rlrl}
\boldsymbol{\Phi} & =\left[\begin{array}{ccccc}
\mathbf{0} & \cdots & \cdots & \mathbf{0} & -\boldsymbol{L}_{\zeta_{1}} \\
\boldsymbol{I}_{n_{y}} & \ddots & & \vdots & \vdots \\
\mathbf{0} & \ddots & \ddots & \vdots & \vdots \\
\vdots & \ddots & \ddots & \mathbf{0} & \vdots \\
\mathbf{0} & \cdots & \mathbf{0} & \boldsymbol{I}_{n_{y}} & -\boldsymbol{L}_{\zeta_{\rho}}
\end{array}\right], \quad \boldsymbol{\Gamma}=\left[\begin{array}{c}
\boldsymbol{I}_{n_{y}} \\
\mathbf{0} \\
\vdots \\
\vdots \\
\mathbf{0}
\end{array}\right], \\
\boldsymbol{L}_{i} & =\left[\begin{array}{llll}
\mathbf{0} & \cdots & \mathbf{0} & \boldsymbol{L}_{\boldsymbol{y}}
\end{array}\right], & & \boldsymbol{L}_{p}=\mathbf{0} \\
\boldsymbol{V}_{\boldsymbol{\zeta}} & =\left[\begin{array}{llll}
\mathbf{0} & \cdots & \mathbf{0} & \tilde{\boldsymbol{V}}
\end{array}\right], & & \boldsymbol{V}_{\boldsymbol{y}}=\mathbf{0}
\end{array}
$$

ein dynamischer FIO der Ordnung $n_{f} \rho$ für das ursprüngliche Systeme parametriert, welcher auf das gleiche Übertragungsverhalten führt.

Beweis. Der Satz ergibt sich unmittelbar aus der in Abschnitt 4.6.2 hergeleiteten Dualität zwischen dynamischen Zustandsreglern und dynamisch erweiterten Fehlerisolationsbeobachtern sowie Satz B.2 in Anhang B.

Es sei nochmals darauf hingewiesen, dass die dynamische Erweiterung der Strecke in Satz 4.3 lediglich für die Herleitung des Entwurfes benötigt wird. Sie muss nicht implementiert werden.

\subsubsection{Beispiel CE 150 Modellhubschrauber}

Beispielhaft wird für den in Abschnitt 4.5.3 vorgestellten CE 150 Modellhubschrauber ein DFIO entworfen. Erweitert man lediglich die Ordnung der Nenner der beiden Diagonalelemente $g_{1,1}(s)$ und $g_{2,2}(s)$ um $\rho=1$, so kann mittels Satz 4.3 unmittelbar eine DFIO-Parametrierung angegeben werden. Bei erneuter Vorgabe von $g_{1,1}(0)=g_{2,2}(0)=1$ ergeben sich für die Beobachtereigenwerte $\lambda_{B_{1,1}}=-4, \lambda_{B_{1,2}}=-5, \lambda_{B_{1,3}}=-6$ und $\lambda_{B_{1,4}}=-7$ sowie $\lambda_{B_{2,1}}=-4,5, \lambda_{B_{2,2}}=-5,5$ und $\lambda_{B_{2,3 / 4}}=-6,5 \pm 3 j$ die Koeffizienten

$$
\begin{aligned}
& q_{1,3}=22, q_{1,2}=179, q_{1,1}=638, \quad q_{1,0}=z_{1,0}=840 \\
& q_{2,3}=23, q_{2,2}=206, q_{2,1}=834,25, q_{2,0}=z_{2,0}=1268,4
\end{aligned}
$$


mit denen die Matrizen $\tilde{M}$ und $\tilde{N}$ berechnet werden. Über $\tilde{L}=\tilde{M} D^{*-1}$, $\tilde{\boldsymbol{V}}=\tilde{\boldsymbol{N}} \boldsymbol{D}^{*-1}$ und die in Satz 4.3 angegebene Partitionierung von $\tilde{\boldsymbol{L}}$ und $\tilde{\boldsymbol{V}}$ erhält man schließlich die in Anhang C.1.2 angegebene DFIOParametrierung. Auf die grafische Darstellung der Residuenverläufe wird an dieser Stelle verzichtet, da das Beispiel in Abschnitt 5.3.3 im Zuge der Unterdrückung hochfrequenter Störungen erneut aufgegriffen wird.

\subsubsection{Fazit}

In diesem Abschnitt wurden verschiedene Verfahren entwickelt, die den integrierten Entwurf dynamischer Fehlerisolationsbeobachter ermöglichen. Diese resultieren jeweils in einer erweiterten Dynamik der Diagonalelemente der Fehler-Residuen-Übertragungsmatrix. Der Frequenzbereichsentwurf ermöglicht eine Erweiterung sowohl der Zähler- als auch der Nennerpolynome, er bedarf jedoch des Umweges über das duale System und einer Zustandsraumrealisierung. Beide Nachteile werden vom vorgestellten Zeitbereichsentwurf umgangen, der allerdings in der vorgestellten Form lediglich eine Erweiterung der Nennerdynamik erlaubt. Ein auf der Vollständigen Modalen Synthese beruhendes Entwurfsverfahren kombiniert die Vorteile beider Entwürfe, es wird in Abschnitt 4.9.5 vorgestellt.

\subsection{Vollständige Fehlerisolation für statisch nicht isolierbare Systeme}

In den Abschnitten 4.5 und 4.6 wurde gemäß Annahme 4.2 vorausgesetzt, dass die Fehlerdetektionsmatrix $\boldsymbol{D}^{*}$ (vgl. Definition 4.4 auf Seite 72) vollen Rang aufweist. Für Systeme, welche diese Bedingung nicht erfüllen, wird die folgende Bezeichnung eingeführt:

Definition 4.6 (Statisch nicht isolierbare Systeme). Systeme der Form (4.1), für die rang $\left(\boldsymbol{D}^{*}\right)<n_{f}$ ist, werden statisch nicht (fehler-) isolierbar, kurz SNI, genannt.

Der Begriff ist dadurch motiviert, dass bei $\operatorname{rang}\left(\boldsymbol{D}^{*}\right)<n_{f}$ kein FIO der Form (4.2) entworfen werden kann. In diesem Abschnitt wird gezeigt, wie sich mithilfe von dynamischen Erweiterungen des Beobachters auch im Falle einer singulären Fehlerdetektionsmatrix eine vollständige Diagonalisierung von $\boldsymbol{G}_{\boldsymbol{r} \boldsymbol{f}}(s)$ und damit Fehlerisolation erreichen lässt. Die Entwurfsidee ist eng an das Vorgehen in [140] angelehnt und die Ergebnisse nutzen dabei erneut die Dualität zu Entkopplungsregelungen, was die 
Übertragung der Resultate von Lohmann [137, 140] auf den Bereich der Fehlerisolation ermöglicht. Hierin ist neben der Realisierung des erweiterten Gesamtsystems als DFIO der wesentliche Beitrag dieses Abschnittes zu sehen.

Es wird gezeigt, dass sich die Annahmen hinsichtlich des Systems gegenüber Abschnitt 4.5 abschwächen lassen. Vorausgesetzt wird im Folgenden lediglich

Annahme 4.5. Das Paar $(\boldsymbol{A}, \boldsymbol{C})$ ist vollständig beobachtbar.

Annahme 4.6. Das System $\left(\boldsymbol{A}, \boldsymbol{E}_{a}, \boldsymbol{C}, \boldsymbol{E}_{s}\right)$ weist eine endliche Anzahl von $\mu$ invarianten Nullstellen auf.

Annahme 4.7. Die Fehlerdetektionsmatrix weist ein Rangdefizit von $\kappa>0$ auf, das heißt es gilt rang $\left(\boldsymbol{D}^{*}\right)=n_{f}-\kappa$.

Annahme 4.8. Das System $\left(\boldsymbol{A}, \boldsymbol{E}_{a}, \boldsymbol{C}, \boldsymbol{E}_{s}\right)$ ist minimalphasig.

Der Unterschied besteht darin, dass die Forderung nach einer invertierbaren Fehlerdetektionsmatrix $\boldsymbol{D}^{*}$ ersetzt wird durch die weniger restriktiven Annahmen 4.6 und 4.7.

\subsubsection{Entwurf mittels virtueller Systemerweiterungen}

Grundgedanke ist es, ein statisch nicht isolierbares System derart zu erweitern, dass für das sich ergebende Gesamtsystem ein FIO entworfen werden kann. Die Erweiterungen sind dabei virtuell, sie werden also softwaretechnisch umgesetzt und setzen keine Erweiterung der real vorhandenen Strecke voraus. Zum Entwurf eines Fehlerisolationsbeobachters wird entsprechend dem Vorgehen in [137] die Strecke (4.1) wie in Abbildung 4.9 dynamisch erweitert. Die Permutationsmatrix $\boldsymbol{F} \in \mathbb{R}^{n_{f} \times n_{f}}$ dient dazu, die Fehler umzusortieren, wodurch sich im weiteren Verlauf einige Beweise ebenso vereinfachen wie die konkrete Umsetzung des Entwurfsverfahrens. Die Ausgangsgrößen $\boldsymbol{y}$ werden zunächst mit einer quadratischen, invertierbaren Matrix $\boldsymbol{H} \in \mathbb{R}^{n_{y} \times n_{y}}$ multipliziert, bevor die ersten $\alpha$ Elemente des resultierenden Vektors jeweils einem Integratorblock zugeführt werden. Partitioniert man die Matrix $\boldsymbol{H}=\left[\begin{array}{ll}\boldsymbol{H}_{1}^{\top} & \boldsymbol{H}_{2}^{\top}\end{array}\right]^{\top}$ mit $\boldsymbol{H}_{1} \in \mathbb{R}^{\alpha \times n_{y}}$ und $\boldsymbol{H}_{2} \in \mathbb{R}^{\left(n_{y}-\alpha\right) \times n_{y}}$, so gilt für den virtuellen Ausgangsvektor $\dot{\tilde{\boldsymbol{y}}}_{1}=\boldsymbol{H}_{1} \boldsymbol{y}$ und $\tilde{\boldsymbol{y}}_{2}=\boldsymbol{H}_{2} \boldsymbol{y}$ mit $\tilde{\boldsymbol{y}}=\left[\begin{array}{ll}\tilde{\boldsymbol{y}}_{1}^{\top} & \tilde{\boldsymbol{y}}_{2}^{\top}\end{array}\right]^{\top}$. Die Dynamik des resultierenden Gesamtsystems lässt sich mit dem Zustandsvektor $\boldsymbol{z}=\left[\begin{array}{ll}\boldsymbol{x}^{\top} & \tilde{\boldsymbol{y}}_{1}^{\top}\end{array}\right]^{\top} \in \mathbb{R}^{\tilde{n}}$, 


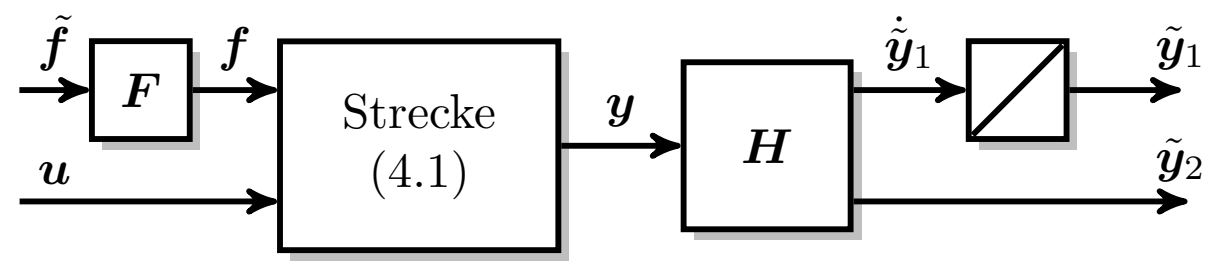

Abbildung 4.9: Virtuelle Systemerweiterung für statisch nicht isolierbare Systeme

$\tilde{n}=n+\alpha$, schreiben als

$$
\begin{gathered}
\dot{\boldsymbol{z}}=\underbrace{\left[\begin{array}{cc}
\boldsymbol{A} & \mathbf{0} \\
\boldsymbol{H}_{1} \boldsymbol{C} & \mathbf{0}
\end{array}\right]}_{\tilde{\boldsymbol{A}}} \boldsymbol{z}+\underbrace{\left[\begin{array}{c}
\boldsymbol{B} \\
\boldsymbol{H}_{1} \boldsymbol{D}
\end{array}\right]}_{\tilde{\boldsymbol{B}}} \boldsymbol{u}+\underbrace{\left[\begin{array}{c}
\boldsymbol{E}_{a} \boldsymbol{F} \\
\boldsymbol{H}_{1} \boldsymbol{E}_{s} \boldsymbol{F}
\end{array}\right]}_{\tilde{\boldsymbol{E}}_{a}} \boldsymbol{f}, \\
\tilde{\boldsymbol{y}}=\underbrace{\left[\begin{array}{cc}
\mathbf{0} & \boldsymbol{I}_{\alpha} \\
\boldsymbol{H}_{2} \boldsymbol{C} & \mathbf{0}
\end{array}\right]}_{\tilde{\boldsymbol{C}}} \boldsymbol{z}+\underbrace{\left[\begin{array}{c}
\mathbf{0} \\
\boldsymbol{H}_{2} \boldsymbol{D}
\end{array}\right]}_{\tilde{\boldsymbol{D}}} \boldsymbol{u}+\underbrace{\left[\begin{array}{c}
\mathbf{0} \\
\boldsymbol{H}_{2} \boldsymbol{E}_{s} \boldsymbol{F}
\end{array}\right]}_{\tilde{\boldsymbol{E}}_{s}} \boldsymbol{f} .
\end{gathered}
$$

Ziel des Verfahrens ist es, die Entwurfsparameter $\alpha, \boldsymbol{F}$ und $\boldsymbol{H}$ so zu wählen, dass das resultierende virtuelle Gesamtsystem (4.42) die Anforderungen aus Satz 4.1 für den Entwurf eines statischen FIOs erfüllt.

Besondere Beachtung verdient dabei Annahme 4.2, also die Invertierbarkeit der Matrix $\boldsymbol{D}^{*}$. Um zu überprüfen, ob ein System diese Annahme erfüllt, muss entweder der Rang von $\boldsymbol{D}^{*}$ oder die Determinante der Matrix bestimmt werden. Das folgende Lemma stellt ein alternatives Kriterium zur Überprüfung der Invertierbarkeit von $\boldsymbol{D}^{*}$ und damit zur Erfüllung von Annahme 4.2 und zur Existenz eines FIOs zur Verfügung, welches einfacher überprüfbar ist. Es ist damit grundlegend für den Entwurf und wird hier gegenüber [137] nicht nur auf das Problem der Fehlerisolation übertragen, sondern auch für Systeme mit Sensorfehlern erweitert und bewiesen.

Lemma 4.5. Gegeben sei ein System der Form (4.1), welches die Annahmen 4.5 und 4.6 erfüllt. Dann sind die folgenden Aussagen äquivalent:

1. Das System ist statisch fehlerisolierbar mittels eines FIOs der Form (4.2).

2. Die Fehlerdetektionsmatrix $\boldsymbol{D}^{*}$ ist invertierbar.

3. Die Differenz zwischen Systemordnung und Gesamtfehlerindex entspricht der Anzahl der invarianten Nullstellen des Systems, das heißt $n-\delta=\mu$. 
Die Idee zum Beweis der Implikation 2. $\Rightarrow 3$. ist es, die Rosenbrock'sche Systemmatrix mit einer geeigneten regulären Matrix zu multiplizieren, deren Determinante ein Polynom der Ordnung $\delta$ ist. Durch die Analyse der Determinante der resultierenden Matrix lässt sich dann zeigen, dass die Invertierbarkeit von $\boldsymbol{D}^{*}$ für die Anzahl der invarianten Nullstellen $\mu=n-\delta$ bedingt. Umgekehrt lässt sich durch Widerspruch zeigen, dass $n-\delta=\mu$ die Invertierbarkeit von $\boldsymbol{D}^{*}$ impliziert und somit $3 . \Rightarrow 2$. gilt. Der ausführliche Beweis findet sich in Anhang A auf Seite 350.

Das Ziel bei der Auslegung von $\alpha, \boldsymbol{F}$ und $\boldsymbol{H}$ ist es wie bereits erwähnt, ein statisch fehlerisolierbares Gesamtsystem zu erzeugen. Insbesondere muss dazu die Matrix $\tilde{\boldsymbol{D}}^{*}$ des erweiterten Systems invertierbar sein. Lemma 4.5 erlaubt es nun, dieses Entwurfsziel hinsichtlich $\alpha, \boldsymbol{F}$ und $\boldsymbol{H}$ äquivalent zu charakterisieren durch $\tilde{n}-\tilde{\delta}=\tilde{\mu}$. Im erweiterten System muss also die Differenz aus Systemordnung und Gesamtfehlerindex der Anzahl der invarianten Nullstellen entsprechen. Für die Anzahl der invarianten Nullstellen gilt dabei

Lemma 4.6. Gegeben sei ein System der Form (4.1), welches die Annahmen 4.5-4.8 erfüllt und reguläre Matrizen $\boldsymbol{F}$ und $\boldsymbol{H}$. Dann entspricht die Anzahl der invarianten Nullstellen des dynamisch erweiterten Systems (4.42) der Anzahl der invarianten Nullstellen von $\left(\boldsymbol{A}, \boldsymbol{E}_{a}, \boldsymbol{C}, \boldsymbol{E}_{s}\right)$. Es gilt also $\tilde{\mu}=\mu$.

Beweis. Die Rosenbrock'sche Systemmatrix des erweiterten Systems lautet gemäß der Definition auf Seite 79

$$
\tilde{\boldsymbol{\Pi}}(\eta)=\left[\begin{array}{cc}
\eta \boldsymbol{I}_{n+\alpha}-\tilde{\boldsymbol{A}} & \tilde{\boldsymbol{E}}_{a} \\
-\tilde{\boldsymbol{C}} & \tilde{\boldsymbol{E}}_{s}
\end{array}\right] .
$$

Da die invarianten Nullstellen den Nullstellen von $\operatorname{det}(\tilde{\boldsymbol{\Pi}}(\eta))$ entsprechen, wird

$$
\operatorname{det}(\tilde{\boldsymbol{\Pi}}(\eta))=\left|\begin{array}{cc:c}
\eta \boldsymbol{I}_{n}-\boldsymbol{A} & \mathbf{0} & \boldsymbol{E}_{a} \boldsymbol{F} \\
-\boldsymbol{H}_{1} \boldsymbol{C} & \eta \boldsymbol{I}_{\alpha} & \boldsymbol{H}_{1} \boldsymbol{E}_{s} \boldsymbol{F} \\
\hdashline \mathbf{0}^{-} & -\overline{\boldsymbol{I}}_{\alpha} & \mathbf{0} \\
-\boldsymbol{H}_{2} \boldsymbol{C} & \mathbf{0} & \boldsymbol{H}_{2} \boldsymbol{E}_{s} \boldsymbol{F}
\end{array}\right|
$$

betrachtet, wobei die Matrizen $\tilde{\boldsymbol{A}}, \tilde{\boldsymbol{E}}_{a}, \tilde{\boldsymbol{C}}$ und $\tilde{\boldsymbol{E}}_{s}$ aus (4.42) eingesetzt wurden. Die Entwicklung der Determinante nach den Zeilen $n+\alpha+1$ bis 
$n+2 \alpha$ führt auf

$$
\begin{aligned}
\operatorname{det}(\tilde{\boldsymbol{\Pi}}(\eta)) & =\left|\begin{array}{cc}
\eta \boldsymbol{I}_{n}-\boldsymbol{A} & \boldsymbol{E}_{a} \boldsymbol{F} \\
-\boldsymbol{H}_{1} \boldsymbol{C} & \boldsymbol{H}_{1} \boldsymbol{E}_{s} \boldsymbol{F} \\
-\boldsymbol{H}_{2} \boldsymbol{C} & \boldsymbol{H}_{2} \boldsymbol{E}_{s} \boldsymbol{F}
\end{array}\right| \\
& =\left|\begin{array}{cc}
\boldsymbol{I}_{n} & \mathbf{0} \\
\mathbf{0} & \boldsymbol{H}
\end{array}\right| \cdot \underbrace{\left|\begin{array}{cc}
\eta \boldsymbol{I}_{n}-\boldsymbol{A} & \boldsymbol{E}_{a} \\
-\boldsymbol{C} & \boldsymbol{E}_{s}
\end{array}\right|}_{\operatorname{det}(\boldsymbol{\Pi}(\eta))} \cdot\left|\begin{array}{cc}
\boldsymbol{I}_{n} & \mathbf{0} \\
\mathbf{0} & \boldsymbol{F}
\end{array}\right|
\end{aligned}
$$

Dementsprechend stimmen die invarianten Nullstellen des erweiterten Systems für reguläre Matrizen $\boldsymbol{F}$ und $\boldsymbol{H}$ mit denen des ursprünglichen Systems überein und es gilt $\tilde{\mu}=\mu$.

Da sich die Anzahl der invarianten Nullstellen gemäß Lemma 4.6 bei regulären Matrizen $\boldsymbol{F}$ und $\boldsymbol{H}$ nicht ändert, vereinfacht sich das Entwurfsziel für $\alpha, \boldsymbol{F}$ und $\boldsymbol{H}$ von $\tilde{n}-\tilde{\delta}=\tilde{\mu}$ zu $\tilde{n}-\tilde{\delta}=\mu$. Aus den Annahmen 4.6 und 4.7 ergibt sich mit den Überlegungen aus dem Beweis von Lemma 4.5, dass für das System (4.1)

$$
n-\delta>\mu
$$

gilt. Demnach ist der Gesamtfehlerindex $\delta$ durch die dynamischen Erweiterungen stärker anzuheben als die Systemordnung $n$, um die Differenz zwischen beiden Größen zu reduzieren und letztendlich $\tilde{n}-\tilde{\delta}=\mu$ zu erreichen.

Zur Auslegung der Permutationsmatrix $\boldsymbol{F}$ wird zunächst das rechte orthogonale Komplement $\boldsymbol{D}^{* \perp}$ der Fehlerdetektionsmatrix betrachtet, für das $\boldsymbol{D}^{*} \cdot \boldsymbol{D}^{* \perp}=\mathbf{0}$ gilt. ${ }^{4)}$ Aufgrund von Annahme 4.7 ist nach dem Rangsatz A.1 $\boldsymbol{D}^{* \perp} \in \mathbb{R}^{n_{f} \times \kappa}$. Aus der Dynamik des Gesamtsystems (4.42) geht hervor, dass $\boldsymbol{F}$ jeweils von rechts an die Fehlereingangsmatrizen $\boldsymbol{E}_{a}$ und $\boldsymbol{E}_{s}$ heranmultipliziert wird. Somit vertauscht die Matrix die Spalten der Fehlereingangsmatrizen und damit auch von $\boldsymbol{D}^{*}$. Dementsprechend werden durch $\boldsymbol{F}$ die Zeilen von $\boldsymbol{D}^{* \perp}$ umsortiert. Die Permutationsmatrix wird so gewählt, dass

$$
\left(\boldsymbol{D}^{*} \boldsymbol{F}\right)^{\perp}=\overline{\boldsymbol{D}}^{* \perp}=\left[\begin{array}{c}
\boldsymbol{Q}_{o} \\
\boldsymbol{Q}_{u} \\
\mathbf{0}_{\gamma \times \kappa}
\end{array}\right]
$$

gilt, wobei $\boldsymbol{Q}_{o} \in \mathbb{R}^{\kappa \times \kappa}$ eine invertierbare Matrix ist und $\boldsymbol{Q}_{u} \in \mathbb{R}^{\left(n_{f}-\kappa-\gamma\right) \times \kappa}$ eine beliebige Matrix. In (4.43) bezeichnet $\gamma$ die

${ }^{4)}$ Die Spalten von $\boldsymbol{D}^{* \perp}$ spannen eine Basis des Rechtsnullraumes von $\boldsymbol{D}^{*}$ auf. Dieser ist definiert als Menge $\operatorname{ker}\left(\boldsymbol{D}^{*}\right)=\left\{\boldsymbol{x} \mid \boldsymbol{D}^{*} \boldsymbol{x}=\mathbf{0}\right\}$. Damit ist $\boldsymbol{D}^{* \perp}$ nicht eindeutig. Die Matrix $\boldsymbol{D}^{* \perp}$ wird auch als (Rechts-)Annihilatormatrix von $\boldsymbol{D}^{*}$ bezeichnet. 
Anzahl der Nullzeilen in $\overline{\boldsymbol{D}}^{* \perp}$. An dieser Stelle wird die Bedeutung der Permutationsmatrix $\boldsymbol{F}$ deutlich: Sie strukturiert das rechte orthogonale Komplement der Fehlerdetektionsmatrix, sodass ich ein kompakter Block von $\gamma$ Nullzeilen ergibt. Dies vereinfacht die weiteren Betrachtungen.

Nachdem $\boldsymbol{F}$ bestimmt ist, wird ähnlich wie in [137] die zu wählende Matrix $\boldsymbol{H}$ partitioniert. Es ist

$$
\boldsymbol{H}=\left[\begin{array}{l}
\boldsymbol{H}_{o} \\
\boldsymbol{H}_{u}
\end{array}\right]
$$

mit $\boldsymbol{H}_{o} \in \mathbb{R}^{\left(n_{f}-\kappa\right) \times n_{f}}$ und $\boldsymbol{H}_{u} \in \mathbb{R}^{\kappa \times n_{f}}$. Zu beachten ist dabei, dass die Partitionierung (4.44) lediglich für den Entwurf und nicht für die spätere Implementierung des Fehlerisolationsbeobachters benötigt wird. Sie entspricht nicht notwendigerweise der zu implementierenden Partitionierung

$$
\boldsymbol{H}=\left[\begin{array}{l}
\boldsymbol{H}_{1} \\
\boldsymbol{H}_{2}
\end{array}\right] \text {. }
$$

Vielmehr wird sich im weiteren Verlauf der Herleitung des Entwurfsverfahrens zeigen, dass die Partitionierung $\boldsymbol{H}_{1}=\boldsymbol{H}_{o}$ und $\boldsymbol{H}_{2}=\boldsymbol{H}_{u}$ lediglich einen Spezialfall darstellt. Die Auslegung von $\boldsymbol{H}$ erfolgt anhand von

$$
\begin{aligned}
& \boldsymbol{H}_{o}=\boldsymbol{Z}^{+}+\boldsymbol{\Lambda}_{o} \cdot{ }^{\perp} \boldsymbol{Z}, \quad \boldsymbol{Z}=\overline{\boldsymbol{D}}^{*}\left[\begin{array}{c}
\mathbf{0}_{\kappa \times\left(n_{f}-\kappa\right)} \\
\boldsymbol{I}_{n_{f}-\kappa}
\end{array}\right] \in \mathbb{R}^{n_{f} \times\left(n_{f}-\kappa\right)}, \\
& \boldsymbol{H}_{u}=\boldsymbol{\Lambda}_{u} \cdot{ }^{\perp} \overline{\boldsymbol{D}}^{*}
\end{aligned}
$$

wobei $\boldsymbol{\Lambda}_{o}$ und $\boldsymbol{\Lambda}_{u} \neq \mathbf{0}$ frei wählbar sind. Es gilt das folgende Lemma, welches in Anhang A auf Seite 353 bewiesen wird:

Lemma 4.7. Gegeben sei ein System der Form (4.1), welches die Annahmen 4.5-4.8 erfüllt. Dann existiert eine Partitionierung der regulären Matrix $\boldsymbol{H}$ gemäß (4.45) mit beliebigen Matrizen $\boldsymbol{\Lambda}_{o} \in \mathbb{R}^{\left(n_{f}-\kappa\right) \times \kappa}$ und $\boldsymbol{\Lambda}_{u} \neq \mathbf{0} \in \mathbb{R}^{\kappa \times \kappa}$.

Nach der Parametrierung von $\boldsymbol{F}$ und $\boldsymbol{H}$ verbleibt noch die Festlegung der Anzahl der Integratoren, also die Wahl von $\alpha$. Der folgende Satz liefert nicht nur ein konstruktives Verfahren zur Auslegung von $\alpha$, sondern beschreibt auch die Auswirkung der Wahl von $\boldsymbol{F}$ und $\boldsymbol{H}$ gemäß (4.43) beziehungsweise (4.45) hinsichtlich der Differenz $\tilde{n}-\tilde{\delta}$. Es gilt

Satz 4.4. Gegeben sei ein System der Form (4.1), welches die Annahmen 4.5-4.8 erfüllt und für das eine dynamische Erweiterung nach (4.42) mit $\alpha=n_{f}-\kappa-\gamma$ und $\boldsymbol{F}$ und $\boldsymbol{H}$ gemäß (4.43) beziehungsweise (4.45) vorgenommen wurde. Dann gilt $\tilde{n}-\tilde{\delta}=n-\delta-\kappa$. 
Beweis. Durch Einsetzen in (4.45a) wird deutlich, dass

$$
\boldsymbol{H}_{o} \overline{\boldsymbol{D}}^{*}=\left[\begin{array}{ll}
\boldsymbol{\Phi} & \boldsymbol{I}_{n_{f}-\kappa}
\end{array}\right]
$$

gilt, wobei die Matrix $\boldsymbol{\Phi} \in \mathbb{R}^{\left(n_{f}-\kappa\right) \times \kappa}$ zunächst nicht näher spezifiziert ist. Aus (4.45b) folgt durch Multiplikation von rechts mit $\overline{\boldsymbol{D}}^{*}$ die Beziehung

$$
\boldsymbol{H}_{u} \overline{\boldsymbol{D}}^{*}=\mathbf{0}_{\kappa \times n_{f}} .
$$

Fasst man dies mit (4.46) zusammen und multipliziert von rechts mit $\overline{\boldsymbol{D}}^{* \perp}$, so folgt wegen $(4.43)$

$$
\boldsymbol{H} \overline{\boldsymbol{D}}^{*} \overline{\boldsymbol{D}}^{* \perp}=\left[\begin{array}{cc}
\boldsymbol{\Phi} & \boldsymbol{I}_{n_{f}-\kappa} \\
\mathbf{0}_{\kappa \times \kappa} & \mathbf{0}_{\kappa \times\left(n_{f}-\kappa\right)}
\end{array}\right]\left[\begin{array}{c}
\boldsymbol{Q}_{o} \\
\boldsymbol{Q}_{u} \\
\mathbf{0}_{\gamma \times \kappa}
\end{array}\right]=\mathbf{0} .
$$

Somit gilt

$$
\boldsymbol{\Phi} \boldsymbol{Q}_{o}+\boldsymbol{I}_{n_{f}-\kappa}\left[\begin{array}{c}
\boldsymbol{Q}_{u} \\
\mathbf{0}_{\gamma \times \kappa}
\end{array}\right]=\mathbf{0}
$$

und wegen der Invertierbarkeit von $\boldsymbol{Q}_{o}$ folgt

$$
\boldsymbol{\Phi}=-\boldsymbol{I}_{n_{f}-\kappa}\left[\begin{array}{c}
\boldsymbol{Q}_{u} \\
\mathbf{0}_{\gamma \times \kappa}
\end{array}\right] \boldsymbol{Q}_{o}^{-1}=\left[\begin{array}{c}
-\boldsymbol{Q}_{u} \boldsymbol{Q}_{o}^{-1} \\
\mathbf{0}_{\gamma \times \kappa}
\end{array}\right]=\left[\begin{array}{c}
\boldsymbol{\Phi}_{o} \\
\mathbf{0}_{\gamma \times \kappa}
\end{array}\right] .
$$

Für die Matrix $\boldsymbol{\Phi}_{o}=-\boldsymbol{Q}_{u} \boldsymbol{Q}_{o}^{-1} \in \mathbb{R}^{\left(n_{f}-\kappa-\gamma\right) \times \kappa}$ lässt sich darüber hinaus aussagen, dass sie keine Nullspalten besitzt. Um dies zu zeigen wird unter Berücksichtigung von (4.43)

$$
\overline{\boldsymbol{D}}^{*} \cdot \overline{\boldsymbol{D}}^{* \perp} \cdot \boldsymbol{Q}_{o}^{-1}=\overline{\boldsymbol{D}}^{*}\left[\begin{array}{c}
\boldsymbol{Q}_{o} \\
\boldsymbol{Q}_{u} \\
\mathbf{0}_{\gamma \times \kappa}
\end{array}\right] \boldsymbol{Q}_{o}^{-1}=\overline{\boldsymbol{D}}^{*}\left[\begin{array}{c}
\boldsymbol{I}_{\kappa} \\
-\boldsymbol{\Phi}_{o} \\
\mathbf{0}_{\gamma \times \kappa}
\end{array}\right]=\mathbf{0}
$$

betrachtet. Partitioniert man $\overline{\boldsymbol{D}}^{*}$ in drei Spaltenblöcke, so folgt weiter

$$
\overline{\boldsymbol{D}}^{*}\left[\begin{array}{c}
\boldsymbol{I}_{\kappa} \\
-\boldsymbol{\Phi}_{o} \\
\mathbf{0}_{\gamma \times \kappa}
\end{array}\right]=\left[\begin{array}{lll}
\overline{\boldsymbol{D}}_{1}^{*} & \overline{\boldsymbol{D}}_{2}^{*} & \overline{\boldsymbol{D}}_{3}^{*}
\end{array}\right]\left[\begin{array}{c}
\boldsymbol{I}_{\kappa} \\
-\boldsymbol{\Phi}_{o} \\
\mathbf{0}_{\gamma \times \kappa}
\end{array}\right]=\mathbf{0} .
$$

Also gilt $\overline{\boldsymbol{D}}_{1}^{*}-\overline{\boldsymbol{D}}_{2}^{*} \boldsymbol{\Phi}_{o}=\mathbf{0}$. Da $\overline{\boldsymbol{D}}^{*}$ ebenso wie $\boldsymbol{D}^{*}$ nach Definition 4.2 keine Nullspalten hat, impliziert dies, dass $\boldsymbol{\Phi}_{o}$ keine Nullspalten hat. 
Setzt man nun $\alpha=n_{f}-\kappa-\gamma$ an und berücksichtigt (4.46), (4.47) und (4.48), so folgt

$$
\left[\begin{array}{l}
\boldsymbol{H}_{1} \\
\boldsymbol{H}_{2}
\end{array}\right] \overline{\boldsymbol{D}}^{*}=\left[\begin{array}{l}
\boldsymbol{H}_{o} \\
\boldsymbol{H}_{u}
\end{array}\right] \overline{\boldsymbol{D}}^{*}=\left[\begin{array}{ccc}
\boldsymbol{\Phi}_{o} & \boldsymbol{I}_{n_{f}-\kappa-\gamma} & \mathbf{0} \\
\mathbf{0} & \mathbf{0} & \boldsymbol{I}_{\gamma} \\
\mathbf{0} & \mathbf{0} & \mathbf{0}
\end{array}\right] .
$$

Mithilfe dieser Beziehung werden die Fehlerindizes des erweiterten Systems analysiert. Dabei werden im Sinne einer kompakten Notation $\overline{\boldsymbol{E}}_{a}=\boldsymbol{E}_{a} \boldsymbol{F}$ und $\overline{\boldsymbol{E}}_{s}=\boldsymbol{E}_{s} \boldsymbol{F}$ eingeführt. Entsprechend Definition 4.4 sind die Spalten der Fehlerdetektionsmatrix $\tilde{\boldsymbol{D}}^{*}$ des erweiterten Systems gegeben durch

$$
\tilde{\boldsymbol{d}}_{i}^{*}=\left\{\begin{array}{cc}
\tilde{\boldsymbol{e}}_{s_{i}} & , \tilde{\delta}_{i}=0, \\
\tilde{\boldsymbol{C}} \tilde{\boldsymbol{A}}^{\tilde{\delta}_{i}-1} \tilde{\boldsymbol{e}}_{a_{i}}, & \tilde{\delta}_{i} \geq 1 .
\end{array}\right.
$$

Dabei gilt im erweiterten System mit permutierten Fehlern

$$
\begin{aligned}
\tilde{\boldsymbol{e}}_{s_{i}} & =\left[\begin{array}{c}
\mathbf{0} \\
\boldsymbol{H}_{2} \overline{\boldsymbol{e}}_{s_{i}}
\end{array}\right], \tilde{\delta}_{i}=0, \\
\tilde{\boldsymbol{C}} \tilde{\boldsymbol{e}}_{a_{i}} & =\left[\begin{array}{cc}
\mathbf{0} & \boldsymbol{I}_{\alpha} \\
\boldsymbol{H}_{2} \boldsymbol{C} & \mathbf{0}
\end{array}\right]\left[\begin{array}{c}
\overline{\boldsymbol{e}}_{a_{i}} \\
\boldsymbol{H}_{1} \overline{\boldsymbol{e}}_{s_{i}}
\end{array}\right]=\left[\begin{array}{c}
\boldsymbol{H}_{1} \overline{\boldsymbol{e}}_{s_{i}} \\
\boldsymbol{H}_{2} \boldsymbol{C} \overline{\boldsymbol{e}}_{a_{i}}
\end{array}\right], \tilde{\delta}_{i}=1, \\
\tilde{\boldsymbol{C}} \tilde{\boldsymbol{A}}^{\tilde{\delta}_{i}-1} \tilde{\boldsymbol{e}}_{a_{i}} & =\left[\begin{array}{cc}
\mathbf{0} & \boldsymbol{I}_{\alpha} \\
\boldsymbol{H}_{2} \boldsymbol{C} & \mathbf{0}
\end{array}\right]\left[\begin{array}{cc}
\boldsymbol{A} & \mathbf{0} \\
\boldsymbol{H}_{1} \boldsymbol{C} & \mathbf{0}
\end{array}\right]^{\tilde{\delta}_{i}-1}\left[\begin{array}{c}
\overline{\boldsymbol{e}}_{a_{i}} \\
\boldsymbol{H}_{1} \overline{\boldsymbol{e}}_{s_{i}}
\end{array}\right] \\
& =\left[\begin{array}{cc}
\mathbf{0} & \boldsymbol{I}_{\alpha} \\
\boldsymbol{H}_{2} \boldsymbol{C} & \mathbf{0}
\end{array}\right]\left[\begin{array}{cc}
\boldsymbol{A}^{\tilde{\delta}_{i}-1} & \mathbf{0} \\
\boldsymbol{H}_{1} \boldsymbol{C} \boldsymbol{A}^{\tilde{\delta}_{i}-2} & \mathbf{0}
\end{array}\right]\left[\begin{array}{c}
\overline{\boldsymbol{e}}_{a_{i}} \\
\boldsymbol{H}_{1} \overline{\boldsymbol{e}}_{s_{i}}
\end{array}\right] \\
& =\left[\begin{array}{cc}
\boldsymbol{H}_{1} \boldsymbol{C} \boldsymbol{A}^{\tilde{\delta}_{i}-2} \overline{\boldsymbol{e}}_{a_{i}} \\
\boldsymbol{H}_{2} \boldsymbol{C} \boldsymbol{A}^{\tilde{\delta}_{i}-1} \overline{\boldsymbol{e}}_{a_{i}}
\end{array}\right], \tilde{\delta}_{i} \geq 2 .
\end{aligned}
$$

Betrachtet man (4.50b), so wird deutlich, dass für permutierte Fehler mit $\bar{\delta}_{i}=0 \mathrm{im}$ erweiterten System die Beziehung $\tilde{\delta}_{i}=\bar{\delta}_{i}+1$ gilt, wenn $\boldsymbol{H}_{1} \overline{\boldsymbol{e}}_{s_{i}} \neq \mathbf{0}$ ist. Für Fehler mit $\bar{\delta}_{i} \geq 1$ ergibt sich dementsprechend aus (4.50c), dass der Fehlerindex im erweiterten System um 1 erhöht wird, falls $\boldsymbol{H}_{1} \boldsymbol{C} \boldsymbol{A}^{\bar{\delta}_{i}-1} \overline{\boldsymbol{e}}_{a_{i}} \neq \mathbf{0}$ gilt. Zusammenfassend führt also $\boldsymbol{H}_{1} \overline{\boldsymbol{d}}_{i}^{*} \neq \mathbf{0} \mathrm{zu}$ $\tilde{\delta}_{i}=\bar{\delta}_{i}+1$. Aus dem ersten Zeilenblock von (4.49) ergibt sich

$$
\boldsymbol{H}_{1} \overline{\boldsymbol{D}}^{*}=\left[\begin{array}{lll}
\boldsymbol{\Phi}_{o} & \boldsymbol{I}_{n_{f}-\kappa-\gamma} & \mathbf{0}_{\left(n_{f}-\kappa-\gamma\right) \times \kappa}
\end{array}\right] .
$$

Da $\boldsymbol{\Phi}_{o}$ wie gezeigt keine Nullspalten besitzt, folgt daraus $\boldsymbol{H}_{1} \overline{\boldsymbol{d}}_{i}^{*} \neq \mathbf{0}$ für alle $i=1, \ldots, n_{f}-\gamma$. 
Weiterhin ergibt sich aus (4.50a), dass $\tilde{\delta}_{i}=\bar{\delta}_{i}=0$ gilt, falls $\boldsymbol{H}_{2} \overline{\boldsymbol{e}}_{s_{i}} \neq \mathbf{0}$ erfüllt ist. Aus (4.50b) und (4.50c) ergibt sich als entsprechende Bedingung $\boldsymbol{H}_{2} \boldsymbol{C} \boldsymbol{A}^{\bar{\delta}_{i}-1} \overline{\boldsymbol{e}}_{a_{i}} \neq \mathbf{0}$. Wegen (4.50) muss also zusammenfassend $\boldsymbol{H}_{2} \overline{\boldsymbol{d}}_{i}^{*} \neq \mathbf{0}$ gelten, damit $\tilde{\delta}_{i}=\bar{\delta}_{i}$ ist. Unter Berücksichtigung der unteren beiden Spaltenblöcke von (4.49),

$$
\boldsymbol{H}_{2} \overline{\boldsymbol{D}}^{*}=\left[\begin{array}{ccc}
\mathbf{0} & \mathbf{0} & \boldsymbol{I}_{\gamma} \\
\mathbf{0} & \mathbf{0} & \mathbf{0}
\end{array}\right]
$$

ist dies für die letzten $\gamma$ Fehler $\left(i=n_{f}-\gamma+1, \ldots, n_{f}\right)$ in $\overline{\boldsymbol{D}}^{*}$ der Fall.

Somit gilt schließlich für den Gesamtfehlerindex des erweiterten Systems

$$
\begin{aligned}
\tilde{\delta} & =\sum_{i=1}^{n_{f}} \tilde{\delta}_{i}=\sum_{i=1}^{n_{f}-\gamma}\left(\bar{\delta}_{i}+1\right)+\sum_{i=n_{f}-\gamma+1}^{n_{f}} \bar{\delta}_{i}=\sum_{i=1}^{n_{f}} \bar{\delta}_{i}+\sum_{i=1}^{n_{f}-\gamma} 1 \\
& =\delta+n_{f}-\gamma .
\end{aligned}
$$

Die Differenz zwischen Systemordnung und Gesamtfehlerindex beträgt damit

$$
\tilde{n}-\tilde{\delta}=n+\alpha-\left(\delta+n_{f}-\gamma\right)=n-\delta-\kappa<n-\delta,
$$

was den Beweis abschließt.

Durch die Wahl von $\alpha, \boldsymbol{F}$ und $\boldsymbol{H}$ nach Satz 4.4 wird die Differenz zwischen Systemordnung und Gesamtfehlerindex um das Rangdefizit von $\boldsymbol{D}^{*}$, also um $\kappa>0$, verringert. Gilt nach der Erweiterung $\tilde{n}-\tilde{\delta}=n-\delta-\kappa=\mu$, so kann aufgrund von Lemma 4.5 ein statischer FIO für das entstandene Gesamtsystem entworfen werden. $\mathrm{Zu}$ beachten ist, dass dieser FIO für die permutierten Fehler $\tilde{\boldsymbol{f}}$ gilt. Das folgende, für statische FIOs allgemeingültige Lemma erlaubt es, die ursprünglichen Fehler $\boldsymbol{f} \mathrm{zu}$ isolieren, indem die Permutation der Fehler rückgängig gemacht wird.

Lemma 4.8. Sei $(\boldsymbol{L}, \boldsymbol{V})$ mit $\boldsymbol{M}$ und $\boldsymbol{N}$ nach Satz 4.1 eine FIOParametrierung für ein System der Form (4.1) und $\boldsymbol{F} \in \mathbb{R}^{n_{f} \times n_{f}}$ eine Permutationsmatrix. Dann ist $(\overline{\boldsymbol{L}}, \overline{\boldsymbol{V}})=\left(\boldsymbol{L}, \boldsymbol{N} \boldsymbol{F}^{\top} \boldsymbol{N}^{-1} \boldsymbol{V}\right)$ eine FIOParametrierung für das System (4.1) mit permutierten Fehlern $\overline{\boldsymbol{f}}=\boldsymbol{F}^{-1} \boldsymbol{f}$.

Beweis. Für das System mit permutierten Fehlern gilt $\overline{\boldsymbol{D}}^{*}=\boldsymbol{D}^{*} \boldsymbol{F}$ und $\overline{\boldsymbol{M}}=\boldsymbol{M} \boldsymbol{F}$. Eingesetzt in das Ergebnis von Satz $4.1 \mathrm{folgt}$

$$
\overline{\boldsymbol{L}}=\overline{\boldsymbol{M D}}^{*-1}=\boldsymbol{M F}\left(\boldsymbol{D}^{*} \boldsymbol{F}\right)^{-1}=\boldsymbol{M} \boldsymbol{F} \boldsymbol{F}^{-1} \boldsymbol{D}^{*-1}=\boldsymbol{M} \boldsymbol{D}^{*-1}=\boldsymbol{L} .
$$


Da $\overline{\boldsymbol{N}}=\boldsymbol{N}$ ist und für Permutationsmatrizen $\boldsymbol{F}^{-1}=\boldsymbol{F}^{\top}$ gilt, lautet das Nachfilter bei permutierten Fehlern

$$
\overline{\boldsymbol{V}}=\overline{\boldsymbol{N}}^{*-1}=\boldsymbol{N} \boldsymbol{F}^{\top} \boldsymbol{D}^{*-1}=\boldsymbol{N} \boldsymbol{F}^{\top} \underbrace{\boldsymbol{N}^{-1} \boldsymbol{N}}_{\boldsymbol{I}_{n_{f}}} \boldsymbol{D}^{*-1}=\boldsymbol{N} \boldsymbol{F}^{\top} \boldsymbol{N}^{-1} \boldsymbol{V},
$$

womit das Lemma bewiesen ist.

Mittels Lemma 4.8 ist es somit möglich, einen FIO $(\tilde{\boldsymbol{L}}, \tilde{\boldsymbol{V}})$ für das erweiterte System mit permutierten Fehlern zu entwerfen. Um eine FIOParametrierung $\left(\tilde{\boldsymbol{L}}, \boldsymbol{V}^{\prime}\right)$ für das ursprüngliche System zu erhalten, muss dann lediglich für das Nachfilter die Rücktransformation $\boldsymbol{V}^{\prime}=\boldsymbol{N} \boldsymbol{F} \boldsymbol{N}^{-1} \tilde{\boldsymbol{V}}$ durchgeführt werden.

Ist die 3. Bedingung aus Lemma 4.5 auch nach der Systemerweiterung nicht erfüllt, so wird das System erneut erweitert. Bezeichnet $(\kappa)_{i}$ das Rangdefizit von $\boldsymbol{D}^{*}$ vor der $i$-ten Systemerweiterung, so gilt nach $l$ Erweiterungen

$$
(\tilde{n})_{l}-(\tilde{\delta})_{l}=n-\delta-\sum_{i=1}^{l}(\kappa)_{l} \leq n-\delta-l,
$$

da $(\kappa)_{i} \geq 1$ ist. Damit ergibt sich

Korollar 4.1. Gegeben sei ein System der Form (4.1), welches die Annahmen 4.5-4.8 erfüllt. Dann sind höchstens $n-\delta-\mu$ dynamische Systemerweiterungen nach Satz 4.4 durchzuführen, um eine invertierbare Fehlerdetektionsmatrix $\tilde{\boldsymbol{D}}^{*}$ für das resultierende Gesamtsystem zu erhalten.

Aufgrund von Lemma 4.5, Lemma 4.6, Satz 4.4 und Korollar 4.1 ergibt sich, dass man nach endlich vielen Systemerweiterungen ein Gesamtsystem erhält, für das ein (statischer) FIO $(\tilde{\boldsymbol{L}}, \tilde{\boldsymbol{V}})$ entworfen werden kann. Dazu kann sowohl der Zeitbereichsentwurf aus Abschnitt 4.5.1 als auch der Entwurf mittels Vollständiger Modaler Synthese nach Abschnitt 4.5.2 herangezogen werden. Für die unmittelbare Implementierung wäre jedoch (bei einfacher Systemerweiterung) ein dynamisches System der Ordnung $n+2 \alpha$ nötig. Neben dem dynamisch erweiterten Beobachter für das System der Ordnung $n+\alpha$ müssten auch die $\alpha$ Integratoren für einen Teil des Ausgangsvektors realisiert werden. Im folgenden Abschnitt wird gezeigt, wie sich mithilfe der bereits vorgestellten dynamisch erweiterten Fehlerisolationsbeobachter eine Realisierung geringerer Ordnung erzielen lässt. 


\subsubsection{Realisierung mithilfe dynamisch erweiterter Beobachter}

Wie bereits erwähnt lässt sich für das Gesamtsystem (4.42) ein statischer FIO der Form

$$
\begin{aligned}
\dot{\hat{\boldsymbol{z}}} & =(\tilde{\boldsymbol{A}}-\tilde{\boldsymbol{L}} \tilde{\boldsymbol{C}}) \hat{\boldsymbol{z}}+(\tilde{\boldsymbol{B}}-\tilde{\boldsymbol{L}} \tilde{\boldsymbol{D}}) \boldsymbol{u}+\tilde{\boldsymbol{L}} \tilde{\boldsymbol{y}} \\
\boldsymbol{r} & =\boldsymbol{V}^{\prime}(\tilde{\boldsymbol{y}}-\hat{\tilde{\boldsymbol{y}}})
\end{aligned}
$$

entwerfen. Die Parametrierung $\left(\tilde{\boldsymbol{L}}, \boldsymbol{V}^{\prime}\right)$ macht dabei gegenüber $(\tilde{\boldsymbol{L}}, \tilde{\boldsymbol{V}})$ die Permutationen der Fehler rückgängig. Falls das System mehrfach erweitert wurde, ist dabei die Verkettung der Fehlerpermutationen rückgängig $\mathrm{zu}$ machen. Wurden $l$ Erweiterungen vorgenommen, wobei die Permutationsmatrix jeweils mit $\boldsymbol{F}_{i}$ bezeichnet wird, so ist demnach bei der Rücktransformation der Nachfiltermatrix die Permutationsmatrix

$$
\boldsymbol{F}_{\mathrm{ges}}=\boldsymbol{F}_{1} \cdot \boldsymbol{F}_{2} \cdot \ldots \cdot \boldsymbol{F}_{l}
$$

zu verwenden.

Der folgende Satz zeigt, wie sich durch eine Partitionierung der Beobachterparametrierung $\left(\tilde{\boldsymbol{L}}, \boldsymbol{V}^{\prime}\right)$ ein dynamischer FIO der Form (4.31) für das ursprüngliche System (4.1) berechnen lässt. Dabei ist hervorzuheben, dass zur Realisierung (bei einfacher Systemerweiterung) lediglich ein dynamisches System der Ordnung $n+\alpha$ implementiert werden muss.

Satz 4.5 (Realisierung von DFIOs zur Fehlerisolation in statisch nicht isolierbaren Systemen). Gegeben sei ein System der Form

$$
\begin{aligned}
& \dot{\boldsymbol{x}}=\boldsymbol{A} \boldsymbol{x}+\boldsymbol{B} \boldsymbol{u}+\boldsymbol{E}_{a} \boldsymbol{f}, \\
& \boldsymbol{y}=\boldsymbol{C} \boldsymbol{x}+\boldsymbol{D} \boldsymbol{u}+\boldsymbol{E}_{s} \boldsymbol{f},
\end{aligned}
$$

welches die Annahmen 4.5-4.8 erfüllt, und für das gemäß Satz 4.4 dynamische Erweiterungen der Ordnung $\alpha$ vorgenommen wurden, sodass das resultierende Gesamtsystem statisch fehlerisolierbar ist. Weiterhin sei durch (4.51) ein stabiler Fehlerisolationsbeobachter für das resultierende Gesamtsystem gegeben. Partitioniert man

$$
\tilde{\boldsymbol{L}}=\left[\begin{array}{ll}
\tilde{\boldsymbol{L}}_{11} & \tilde{\boldsymbol{L}}_{12} \\
\tilde{\boldsymbol{L}}_{21} & \tilde{\boldsymbol{L}}_{22}
\end{array}\right], \quad \boldsymbol{V}^{\prime}=\left[\begin{array}{ll}
\boldsymbol{V}_{1}^{\prime} & \boldsymbol{V}_{2}^{\prime}
\end{array}\right]
$$


mit $\tilde{\boldsymbol{L}}_{11} \in \mathbb{R}^{n \times \alpha}, \tilde{\boldsymbol{L}}_{12} \in \mathbb{R}^{n \times\left(n_{y}-\alpha\right)}, \tilde{\boldsymbol{L}}_{21} \in \mathbb{R}^{\alpha \times \alpha}, \tilde{\boldsymbol{L}}_{22} \in \mathbb{R}^{\alpha \times\left(n_{y}-\alpha\right)}$, $\boldsymbol{V}_{1}^{\prime} \in \mathbb{R}^{n_{f} \times \alpha}$ und $\boldsymbol{V}_{2}^{\prime} \in \mathbb{R}^{n_{f} \times\left(n_{y}-\alpha\right)}$, so ist durch

$$
\begin{aligned}
\dot{\hat{\boldsymbol{x}}} & =\boldsymbol{A} \hat{\boldsymbol{x}}+\boldsymbol{B} \boldsymbol{u}+\boldsymbol{L}_{p}(\boldsymbol{y}-\boldsymbol{C} \hat{\boldsymbol{x}}-\boldsymbol{D} \boldsymbol{u})+\boldsymbol{L}_{i} \boldsymbol{\zeta} \\
\dot{\boldsymbol{\zeta}} & =\boldsymbol{\Phi} \boldsymbol{\zeta}+\boldsymbol{\Gamma}(\boldsymbol{y}-\boldsymbol{C} \hat{\boldsymbol{x}}-\boldsymbol{D} \boldsymbol{u}) \\
\boldsymbol{r} & =\boldsymbol{V}_{\boldsymbol{y}}(\boldsymbol{y}-\boldsymbol{C} \hat{\boldsymbol{x}}-\boldsymbol{D} \boldsymbol{u})+\boldsymbol{V}_{\boldsymbol{\zeta}} \boldsymbol{\zeta}
\end{aligned}
$$

mit

$$
\begin{array}{rlrl}
\boldsymbol{L}_{p} & =\tilde{\boldsymbol{L}}_{12} \boldsymbol{H}_{2}, & \boldsymbol{L}_{i} & =\tilde{\boldsymbol{L}}_{11}, \\
\mathbb{R}^{\alpha \times \alpha} \ni \boldsymbol{\Phi} & =-\tilde{\boldsymbol{L}}_{21}, \quad \boldsymbol{\Gamma}=\boldsymbol{H}_{1}-\tilde{\boldsymbol{L}}_{22} \boldsymbol{H}_{2}, \\
\boldsymbol{V}_{\boldsymbol{y}} & =\boldsymbol{V}_{2}^{\prime} \boldsymbol{H}_{2}, \quad \boldsymbol{V}_{\boldsymbol{\zeta}}=\boldsymbol{V}_{1}^{\prime}
\end{array}
$$

ein stabiler dynamischer Fehlerisolationsbeobachter für das System (4.52) parametriert.

Beweis. Die Dynamik des Beobachters (4.51) lautet ausführlicher geschrieben

$$
\begin{aligned}
& {\left[\begin{array}{c}
\dot{\hat{\boldsymbol{x}}} \\
\dot{\hat{\tilde{\boldsymbol{y}}}}
\end{array}\right]=\left(\left[\begin{array}{cc}
\boldsymbol{A} & \mathbf{0} \\
\boldsymbol{H}_{1} \boldsymbol{C} & \mathbf{0}
\end{array}\right]-\left[\begin{array}{cc}
\tilde{\boldsymbol{L}}_{11} & \tilde{\boldsymbol{L}}_{12} \\
\tilde{\boldsymbol{L}}_{21} & \tilde{\boldsymbol{L}}_{22}
\end{array}\right]\left[\begin{array}{cc}
\mathbf{0} & \boldsymbol{I}_{\alpha} \\
\boldsymbol{H}_{2} \boldsymbol{C} & \mathbf{0}
\end{array}\right]\right)\left[\begin{array}{c}
\hat{\boldsymbol{x}} \\
\hat{\tilde{\boldsymbol{y}}}_{1}
\end{array}\right]+\ldots} \\
& +\left[\begin{array}{c}
\boldsymbol{B}-\tilde{\boldsymbol{L}}_{12} \boldsymbol{H}_{2} \boldsymbol{D} \\
\left(\boldsymbol{H}_{1}-\tilde{\boldsymbol{L}}_{22} \boldsymbol{H}_{2}\right) \boldsymbol{D}
\end{array}\right] \boldsymbol{u}+\left[\begin{array}{ll}
\tilde{\boldsymbol{L}}_{11} & \tilde{\boldsymbol{L}}_{12} \\
\tilde{\boldsymbol{L}}_{21} & \tilde{\boldsymbol{L}}_{22}
\end{array}\right]\left[\begin{array}{c}
\tilde{\boldsymbol{y}}_{1} \\
\tilde{\boldsymbol{y}}_{2}
\end{array}\right]
\end{aligned}
$$

woraus sich

$$
\dot{\hat{\boldsymbol{x}}}=\left(\boldsymbol{A}-\tilde{\boldsymbol{L}}_{12} \boldsymbol{H}_{2} \boldsymbol{C}\right) \hat{\boldsymbol{x}}-\tilde{\boldsymbol{L}}_{11} \hat{\tilde{\boldsymbol{y}}}_{1}+\left(\boldsymbol{B}-\tilde{\boldsymbol{L}}_{12} \boldsymbol{H}_{2} \boldsymbol{D}\right) \boldsymbol{u}+\tilde{\boldsymbol{L}}_{11} \tilde{\boldsymbol{y}}_{1}+\tilde{\boldsymbol{L}}_{12} \tilde{\boldsymbol{y}}_{2}
$$

ergibt. Durch Einführung einer neuen Zustandsvariablen $\boldsymbol{\zeta}=\tilde{\boldsymbol{y}}_{1}-\hat{\tilde{\boldsymbol{y}}}_{1} \in \mathbb{R}^{\alpha}$ vereinfacht sich dies aufgrund von $\tilde{\boldsymbol{y}}_{2}=\boldsymbol{H}_{2} \boldsymbol{y} \mathrm{zu}$

$$
\dot{\hat{\boldsymbol{x}}}=\boldsymbol{A} \hat{\boldsymbol{x}}+\boldsymbol{B u}+\tilde{\boldsymbol{L}}_{12} \boldsymbol{H}_{2}(\boldsymbol{y}-\boldsymbol{C} \hat{\boldsymbol{x}}-\boldsymbol{D} \boldsymbol{u})+\tilde{\boldsymbol{L}}_{11} \boldsymbol{\zeta} .
$$

Für die Dynamik von $\zeta$ ergibt sich anhand von (4.42) und (4.51) die Beziehung

$$
\begin{aligned}
& \dot{\boldsymbol{\zeta}}= \boldsymbol{H}_{1} \boldsymbol{C} \boldsymbol{x}+\boldsymbol{H}_{1} \boldsymbol{D} \boldsymbol{u}+\boldsymbol{H}_{1} \boldsymbol{E}_{s} \boldsymbol{F} \boldsymbol{f}-\left(\boldsymbol{H}_{1} \boldsymbol{C}-\tilde{\boldsymbol{L}}_{22} \boldsymbol{H}_{2} \boldsymbol{C}\right) \hat{\boldsymbol{x}}+\ldots \\
&+\tilde{\boldsymbol{L}}_{21} \hat{\tilde{\boldsymbol{y}}}_{1}-\left(\boldsymbol{H}_{1} \boldsymbol{D}-\tilde{\boldsymbol{L}}_{22} \boldsymbol{H}_{2} \boldsymbol{D}\right) \boldsymbol{u}-\tilde{\boldsymbol{L}}_{21} \tilde{\boldsymbol{y}}_{1}-\tilde{\boldsymbol{L}}_{22} \tilde{\boldsymbol{y}}_{2} \\
&=-\tilde{\boldsymbol{L}}_{21} \boldsymbol{\zeta}+\left(\boldsymbol{H}_{1}-\tilde{\boldsymbol{L}}_{22} \boldsymbol{H}_{2}\right)(\boldsymbol{y}-\boldsymbol{C} \hat{\boldsymbol{x}}-\boldsymbol{D} \boldsymbol{u}) .
\end{aligned}
$$


Die generierten Residuen können mit der eingeführten Variablensubstitution geschrieben werden als

$$
\begin{aligned}
\boldsymbol{r}=\boldsymbol{V}^{\prime}(\tilde{\boldsymbol{y}}-\hat{\tilde{\boldsymbol{y}}}) & =\left[\begin{array}{ll}
\boldsymbol{V}_{1}^{\prime} & \boldsymbol{V}_{2}^{\prime}
\end{array}\right]\left[\begin{array}{c}
\tilde{\boldsymbol{y}}_{1}-\hat{\tilde{\boldsymbol{y}}}_{1} \\
\boldsymbol{H}_{2} \boldsymbol{y}-\boldsymbol{H}_{2} \boldsymbol{C} \hat{\boldsymbol{x}}-\boldsymbol{H}_{2} \boldsymbol{D} \boldsymbol{u}
\end{array}\right] \\
& =\boldsymbol{V}_{1}^{\prime} \boldsymbol{\zeta}+\boldsymbol{V}_{2}^{\prime} \boldsymbol{H}_{2}(\boldsymbol{y}-\boldsymbol{C} \hat{\boldsymbol{x}}-\boldsymbol{D u}) .
\end{aligned}
$$

Die Gleichungen (4.53), (4.54) und (4.55) lassen sich zusammenfassen zu

$$
\begin{aligned}
& \dot{\hat{\boldsymbol{x}}}=\boldsymbol{A} \hat{\boldsymbol{x}}+\boldsymbol{B u}+\boldsymbol{v}, \\
& \boldsymbol{v}=\underbrace{\tilde{\boldsymbol{L}}_{12} \boldsymbol{H}_{2}}_{\boldsymbol{L}_{p}}(\boldsymbol{y}-\boldsymbol{C} \hat{\boldsymbol{x}}-\boldsymbol{D} \boldsymbol{u})+\underbrace{\tilde{\boldsymbol{L}}_{11}}_{\boldsymbol{L}_{i}} \boldsymbol{\zeta}, \\
& \dot{\boldsymbol{\zeta}}=\underbrace{-\tilde{\boldsymbol{L}}_{21}}_{\boldsymbol{\Phi}} \boldsymbol{\zeta}+\underbrace{\left(\boldsymbol{H}_{1}-\tilde{\boldsymbol{L}}_{22} \boldsymbol{H}_{2}\right)}_{\Gamma}(\boldsymbol{y}-\boldsymbol{C} \hat{\boldsymbol{x}}-\boldsymbol{D u}), \\
& \boldsymbol{r}=\underbrace{\boldsymbol{V}_{1}^{\prime}}_{\boldsymbol{V}_{\boldsymbol{\zeta}}} \boldsymbol{\zeta}+\underbrace{\boldsymbol{V}_{2}^{\prime} \boldsymbol{H}_{2}}_{\boldsymbol{V}_{\boldsymbol{y}}}(\boldsymbol{y}-\boldsymbol{C} \hat{\boldsymbol{x}}-\boldsymbol{D u}) .
\end{aligned}
$$

Obige Gleichungen stellen einem dynamisch erweiterten Beobachter dar.

Durch den dynamisch erweiterten FIO (4.56) werden also sowohl die notwendigen dynamischen Erweiterungen realisiert, als auch die Isolation der Fehler erreicht. Da für das virtuelle Gesamtsystem ein stabiler Fehlerisolationsbeobachter (4.51) entworfen wurde, ist die Stabilität des DFIOs sichergestellt.

\subsubsection{Zusammenfassung des Entwurfsverfahrens}

Der Übersichtlichkeit halber wird das in den Abschnitten 4.7.1 und 4.7.2 entworfene Verfahren auf der folgenden Seite und in Abbildung 4.10 kompakt zusammengefasst. 
Gegeben sei ein System der Form

$$
\begin{aligned}
& \dot{\boldsymbol{x}}=\boldsymbol{A} \boldsymbol{x}+\boldsymbol{B} \boldsymbol{u}+\boldsymbol{E}_{a} \boldsymbol{f}, \\
& \boldsymbol{y}=\boldsymbol{C} \boldsymbol{x}+\boldsymbol{D} \boldsymbol{u}+\boldsymbol{E}_{s} \boldsymbol{f},
\end{aligned}
$$

welches die Annahmen 4.5-4.8 erfüllt. Initialisiere die Permutationsmatrix mit $\boldsymbol{F}_{\text {ges }}=\boldsymbol{I}_{n_{f}}$ und durchlaufe das folgende Schema:

1. a) Bestimme das Rangdefizit $\kappa$ der Fehlerdetektionsmatrix $\boldsymbol{D}^{*}$ mittels $\kappa=n_{f}-\operatorname{rang}\left(\boldsymbol{D}^{*}\right)$ sowie die Anzahl $\gamma$ der Nullzeilen in $\boldsymbol{D}^{* \perp}$ und wähle $\boldsymbol{F}$ so, dass

$$
\left(\boldsymbol{D}^{*} \boldsymbol{F}\right)^{\perp}=\overline{\boldsymbol{D}}^{* \perp}=\left[\begin{array}{c}
\boldsymbol{Q}_{o} \\
\boldsymbol{Q}_{u} \\
\mathbf{0}_{\gamma \times \kappa}
\end{array}\right]
$$

gilt. Berechne damit $\boldsymbol{F}_{\text {ges }}:=\boldsymbol{F}_{\text {ges }} \boldsymbol{F}$.

b) Setze $\alpha=n_{f}-\kappa-\gamma$ und wähle $\boldsymbol{H}$ gemäß

$$
\begin{aligned}
& \boldsymbol{H}_{o}=\boldsymbol{Z}^{+}+\boldsymbol{\Lambda}_{o} \cdot{ }^{\perp} \boldsymbol{Z}, \quad \boldsymbol{Z}=\overline{\boldsymbol{D}}^{*}\left[\begin{array}{c}
\boldsymbol{0}_{\kappa \times\left(n_{f}-\kappa\right)} \\
\boldsymbol{I}_{n_{f}-\kappa}
\end{array}\right], \\
& \boldsymbol{H}_{u}=\boldsymbol{\Lambda}_{u} \cdot{ }^{\perp} \overline{\boldsymbol{D}}^{*}
\end{aligned}
$$

mit beliebigen Matrizen $\boldsymbol{\Lambda}_{o} \in \mathbb{R}^{\left(n_{f}-\kappa\right) \times \kappa}$ und $\boldsymbol{\Lambda}_{u} \neq \mathbf{0} \in \mathbb{R}^{\kappa \times \kappa}$.

c) Überprüfe, ob $\tilde{n}-\tilde{\delta}=\mu$ gilt. Falls nein, gehe zu 1.a). Falls ja, gehe zu 2.

2. a) Entwirf mittels Satz 4.1 oder Satz 4.2 einen statischen FIO $(\tilde{\boldsymbol{L}}, \tilde{\boldsymbol{V}})$ für das resultierende Gesamtsystem.

b) Berechne daraus mittels $\boldsymbol{V}^{\prime}=\boldsymbol{N} \boldsymbol{F}_{\text {ges }}^{\top} \boldsymbol{N}^{-1} \tilde{\boldsymbol{V}}$ eine FIO-Parametrierung $\left(\tilde{\boldsymbol{L}}, \boldsymbol{V}^{\prime}\right)$ für das Gesamtsystem mit unpermutierten Fehlern.

c) Berechne mithilfe von

$$
\begin{aligned}
\tilde{\boldsymbol{L}} & =\left[\begin{array}{ll}
\tilde{\boldsymbol{L}}_{11} & \tilde{\boldsymbol{L}}_{12} \\
\tilde{\boldsymbol{L}}_{21} & \tilde{\boldsymbol{L}}_{22}
\end{array}\right], & \boldsymbol{V}^{\prime} & =\left[\begin{array}{ll}
\boldsymbol{V}_{1}^{\prime} & \boldsymbol{V}_{2}^{\prime}
\end{array}\right], \\
\boldsymbol{L}_{p} & =\tilde{\boldsymbol{L}}_{12} \boldsymbol{H}_{2}, & \boldsymbol{L}_{i} & =\tilde{\boldsymbol{L}}_{11}, \\
\boldsymbol{\Phi} & =-\tilde{\boldsymbol{L}}_{21}, & \boldsymbol{\Gamma} & =\boldsymbol{H}_{1}-\tilde{\boldsymbol{L}}_{22} \boldsymbol{H}_{2}, \\
\boldsymbol{V}_{\boldsymbol{y}} & =\boldsymbol{V}_{2}^{\prime} \boldsymbol{H}_{2}, & \boldsymbol{V}_{\boldsymbol{\zeta}} & =\boldsymbol{V}_{1}^{\prime}
\end{aligned}
$$

aus $\left(\tilde{\boldsymbol{L}}, \boldsymbol{V}^{\prime}\right)$ die Parametrierung eines dynamisch erweiterten Fehlerisolationsbeobachters für das ursprüngliche System. 


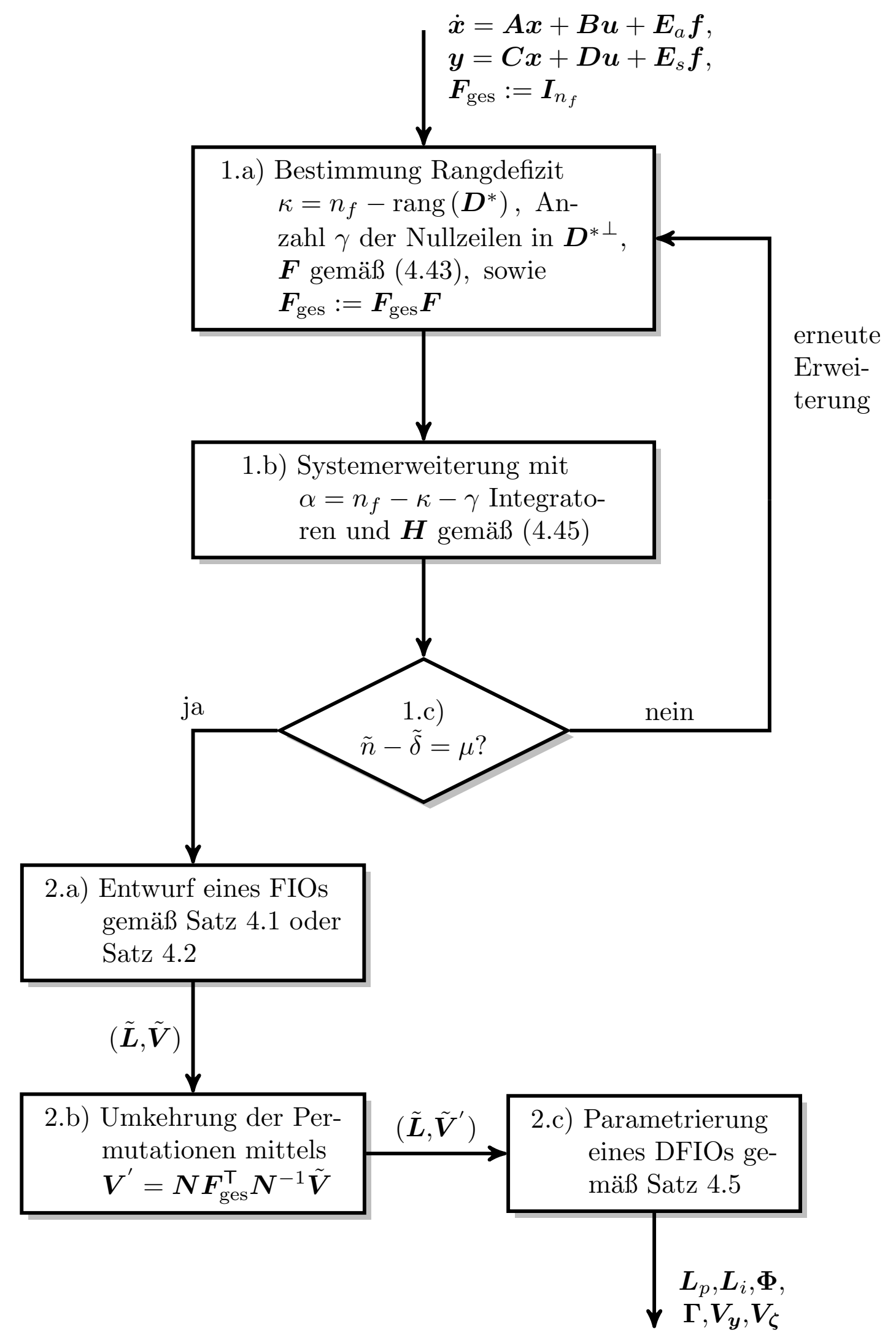

Abbildung 4.10: Ablaufplan des Entwurfs von DFIOs für statisch nicht isolierbare Systeme 


\subsubsection{Alternatives Entwurfsverfahren}

Da das in Abschnitt 4.6 vorgestellte Verfahren die Vorgabe von erweiterten Dynamiken für die Diagonalelemente von $\boldsymbol{G}_{\boldsymbol{r} \boldsymbol{f}}(s)$ ermöglicht, ergibt sich ein alternatives Entwurfsverfahren zu dem in diesem Abschnitt präsentierten Vorgehen für statisch nicht isolierbare Systeme. Das präsentierte Verfahren garantiert unter den Annahmen 4.5 bis 4.8 die Existenz eines DFIOs für das statisch nicht isolierbare System, wobei sich die Übertragungsmatrix $\boldsymbol{G}_{\boldsymbol{r} \boldsymbol{f}}(s)$ durch Diagonalelemente auszeichnet, deren Differenzenordnung gegenüber den Fehlerindizes $\delta_{i}$ erhöht sind. Welche Elemente $g_{i, i}(s)$ sich dabei ändern und wie stark ihre Differenzenordnung ansteigt, lässt sich wie im vorangegangenen Abschnitt durch eine Analyse der Matrix $\boldsymbol{D}^{*}$ ermitteln. So folgt aus dem Beweis von Satz 4.4, dass sich bei der vorgestellten Systemerweiterung die Fehlerindizes der ersten $n_{f}-\gamma$ (permutierten) Fehler um 1 erhöhen.

Nachdem auf diese Art und Weise die Struktur einer mittels eines DFIOs realisierbaren Übertragungsmatrix $\boldsymbol{G}_{\boldsymbol{r} \boldsymbol{f}}(s)$ ermittelt wurde, kann mit dem in Abschnitt 4.6.3 vorgestellten Entwurf im Laplace-Bereich ein DFIO parametriert werden. Die Existenz der auftretenden Inversen $\boldsymbol{D}^{*-1}(s)$ ist dabei aufgrund der im vorangegangenen Abschnitt präsentierten Ergebnisse für fast alle $s \in \mathbb{C}$ garantiert.

\subsubsection{Beispiel DHC-2 Beaver}

Zur Veranschaulichung wird das Entwurfsverfahren zur Fehlerisolation in der Lateralbewegung eines Flugzeuges angewendet. Betrachtet wird die Dynamik einer DHC-2 Beaver (s. Abbildung 4.11).

Bei stationärer Longitudinalbewegung lässt sich aus dem in [180, Abschnitt 3.2] gegebenen nichtlinearen Modell für die Lateraldynamik ein linearisiertes Modell 4. Ordnung generieren. Zustandsgrößen des Systems sind der Schiebewinkel ${ }^{5)} \beta$, die Gierrate $r$ um die körperfeste $z$-Achse, die Rollrate $p$ um die körperfeste $x$-Achse, sowie der Hängewinkel ${ }^{6)} \Phi$. Beeinflusst werden kann die Bewegung durch den Querruderwinkel $u_{1}$ und die Auslenkung des Seitenruders $u_{2}$, als messbar angenommen werden der erste und der letzte Zustand. Bei einer Reisegeschwindigkeit von $v=45 \mathrm{~m} / \mathrm{s}$ und einer Flughöhe von $H=1828,8 \mathrm{~m}$ wird das Modell beschrieben durch

\footnotetext{
${ }^{5)}$ Der Schiebewinkel ist die Winkel zwischen der anströmenden Luft und der körperfesten $z$-Achse.

${ }^{6)}$ Der Hängewinkel ist der ins erdfeste Koordinatensystem transformierte Rollwinkel.
} 


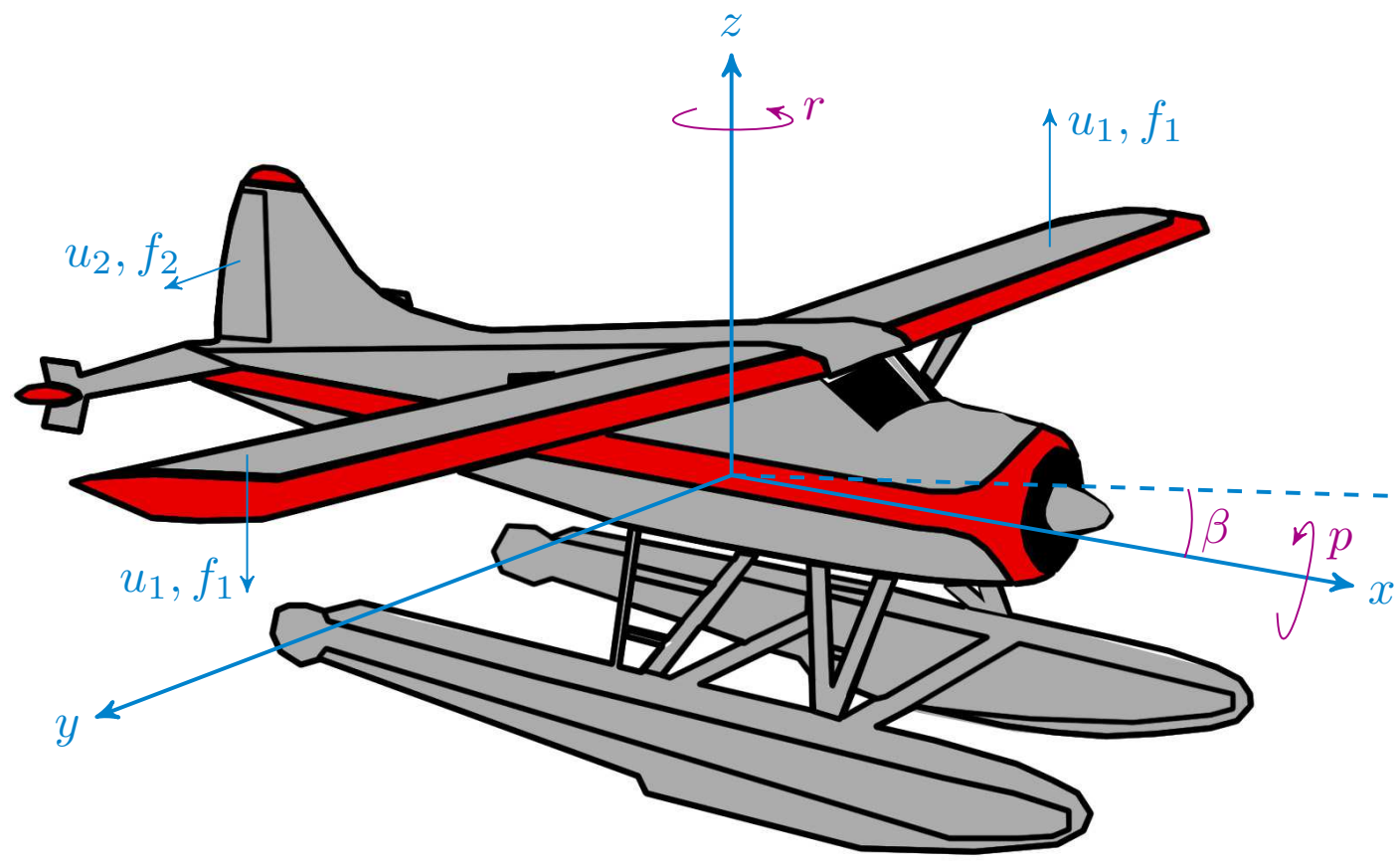

Abbildung 4.11: DHC-2 Beaver

$$
\begin{aligned}
& \boldsymbol{A}=\left[\begin{array}{rrrc}
-0,179 & -0,976 & 0,139 & 0,216 \\
0,172 & -0,552 & -0,871 & 0 \\
-4,053 & 1,796 & -5,402 & 0 \\
0 & 0,145 & 1 & 0
\end{array}\right], \boldsymbol{B}=\boldsymbol{E}_{a}=\left[\begin{array}{rc}
-0,007 & 0,045 \\
-0,199 & -2,606 \\
-7,298 & 0,398 \\
0 & 0
\end{array}\right], \\
& \boldsymbol{C}=\left[\begin{array}{llll}
1 & 0 & 0 & 0 \\
0 & 0 & 0 & 1
\end{array}\right], \quad \boldsymbol{E}_{s}=\mathbf{0} .
\end{aligned}
$$

Wie aus (4.57) hervorgeht, werden Fehler in beiden Aktoren modelliert.

Die Analyse des Systems ergibt die Fehlerindizes $\delta_{1}=\delta_{2}=1$ und eine invariante Nullstelle bei $\eta=-58,48$. Die Fehlerdetektionsmatrix $\boldsymbol{D}^{*}$ ist nicht invertierbar, sodass kein statischer FIO entworfen werden kann. Die in den Annahmen 4.5 bis 4.8 formulierten Bedingungen sind jedoch erfüllt, wobei für das Rangdefizit der Fehlerdetektionsmatrix $\kappa=1$ gilt. Für den Rechtsnullraum von $\boldsymbol{D}^{*}$ findet man die Basis

$$
\boldsymbol{D}^{* \perp}=\left[\begin{array}{l}
0,988 \\
0,152
\end{array}\right] \text {. }
$$

Damit liegt $\boldsymbol{D}^{* \perp}$ bereits in der in (4.43) geforderten Form vor und die Permutation der Fehler entfällt. Dies wird durch $\boldsymbol{F}=\boldsymbol{I}_{n_{f}}$ beschrieben. Des Weiteren geht aus (4.58) hervor, dass $\boldsymbol{D}^{* \perp}$ keine Nullzeilen aufweist, 
sodass $\gamma=0$ gilt. Daraus folgt gemäß Satz 4.4 für die Ordnung der Systemerweiterung $\alpha=n_{f}-\kappa-\gamma=1$. Für die frei wählbaren Matrizen in (4.45), die im Beispiel zu Skalaren entarten, wird $\boldsymbol{\Lambda}_{o}=-4$ und $\boldsymbol{\Lambda}_{u}=2$ gewählt. Die Wahl der Matrizen ändert lediglich die Parametrierung des DFIO, auf das Übertragungsverhalten $\boldsymbol{G}_{\boldsymbol{r} \boldsymbol{f}}(s)$ hat sie keinen Einfluss. Bereits nach einer Systemerweiterung gilt $\tilde{n}-\tilde{\delta}=5-4=1=\mu$, für das erweiterte System kann also ein statischer FIO gemäß Satz 4.1 oder Satz 4.2 entworfen werden. Dabei werden die Pole im ersten Übertragungskanal bei $\lambda_{B_{1,1}}=-2$ und $\lambda_{B_{1,2}}=-7$ und im zweiten Kanal bei $\lambda_{B_{2,1}}=-3,2$ und $\lambda_{B_{2,2}}=-3,5$ platziert und die Zählerkoeffizienten jeweils so vorgegeben, dass sich eine stationäre Verstärkung von 1 ergibt. Mithilfe von Satz 4.5 ergibt sich schließlich die gesuchte Parametrierung eines dynamisch erweiterten Fehlerisolationsbeobachters. Die Zahlenwerte der Parametrierung finden sich in Anhang C.1.3.

Abbildung 4.12 zeigt den zeitlichen Verlauf der simulierten Fehler und der generierten Residuen. Nach $t=1$ s tritt ein sprungförmiger Fehler im Querruderwinkel auf. Es ist $f_{1}=0,087$, was in etwa einem Fehler von $5^{\circ}$ in
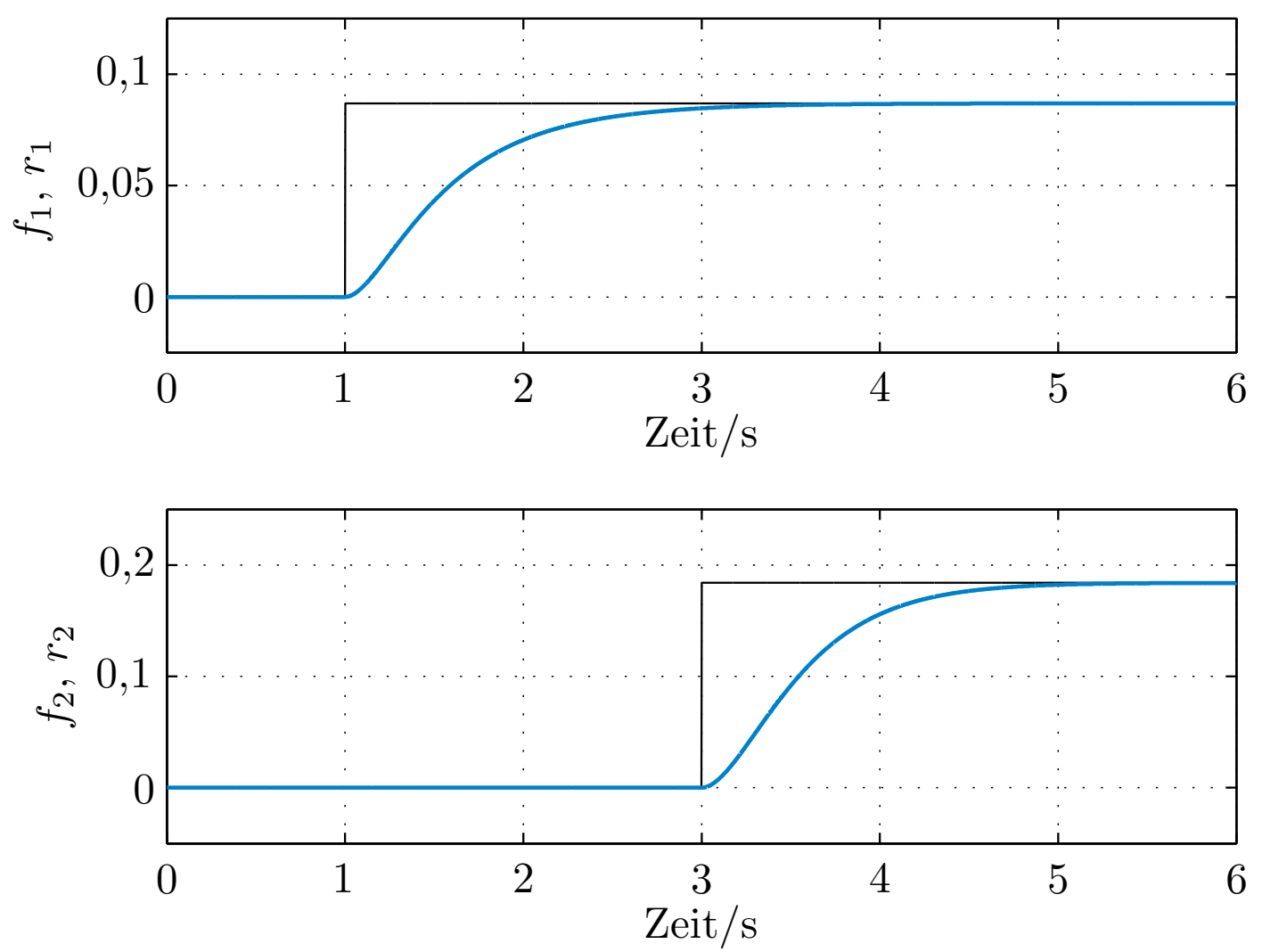

Abbildung 4.12: Fehler und Residuen für die Lateraldynamik der DHC-2 Beaver 
der Ruderstellung entspricht, da die Winkel im Modell in rad angenommen werden. Nach $t=3 \mathrm{~s}$ tritt zusätzlich der zweite Fehler auf $\left(f_{2}=0,174\right.$, ca. $10^{\circ}$ Fehler in Stellung des Seitenruders). Wie anhand von Abbildung 4.12 ersichtlich ist, beeinflusst der Fehler $f_{1}$ ausschließlich das ihm zugeordnete Residuum $r_{1}$. Entsprechendes gilt für das zweite Residuum, sodass eine Fehlerisolation möglich ist.

\subsubsection{Fazit}

Durch den vorgestellten systematischen Entwurf ist es möglich, dynamisch erweiterte Fehlerisolationsbeobachter für Systeme zu entwerfen, für die mittels eines statischen FIOs keine Fehlerisolation möglich ist. Somit wird die Klasse behandelbarer linearer Systeme erweitert. Mit der entwickelten Parametrierung der DFIOs werden sowohl die Systemerweiterungen als auch die Fehlerisolationseigenschaft des Beobachters realisiert. Es ergeben sich daraus stabile dynamische Systeme niedriger Ordnung für die Fehlerisolation. Offensichtlich ist jedoch, dass der Entwurfsaufwand gegenüber den statischen FIOs aus Abschnitt 4.5 ansteigt.

\subsection{Vollständige Fehlerisolation für nicht minimalphasige Systeme}

Neben der Invertierbarkeit der Fehlerdetektionsmatrix schränkt die Annahme der Minimalphasigkeit den Entwurf von Fehlerisolationsbeobachtern auf Systeme ein, deren invariante Nullstellen in der offenen linken s-Halbebene liegen (vgl. Annahme 4.3 auf S. 79). Ähnlich wie in Abschnitt 4.7 lässt sich diese Einschränkung mithilfe von virtuellen Systemerweiterungen umgehen. Das Vorgehen und die Beweisideen sind an die Resultate aus $[137,140]$ angelehnt, und das Verfahren kann als duale Methode zum Entwurf von dynamischen Entkopplungsreglern für nicht minimalphasige Systeme verstanden werden. Darüber hinaus erfolgt eine Realisierung durch dynamisch erweiterte Fehlerisolationsbeobachter.

Es wird gezeigt, dass beim Einsatz solcher DFIOs gegenüber Abschnitt 4.5 weniger restriktive Anforderungen an das betrachtete System zu stellen sind. Vorausgesetzt werden lediglich

Annahme 4.9. Das Paar $(\boldsymbol{A}, \boldsymbol{C})$ ist vollständig beobachtbar.

Annahme 4.10. Die Matrix $D^{*}$ hat vollen Rang. 
Annahme 4.11. Das System $\left(\boldsymbol{A}, \boldsymbol{E}_{a}, \boldsymbol{C}, \boldsymbol{E}_{s}\right)$ weist eine endliche Anzahl invarianter Nullstellen auf.

Annahme 4.12. Das System $\left(\boldsymbol{A}, \boldsymbol{E}_{a}, \boldsymbol{C}, \boldsymbol{E}_{s}\right)$ weist keine invariante Nullstelle bei $\eta=0$ auf.

Annahme 4.13. Keine invariante Nullstelle von $\left(\boldsymbol{A}, \boldsymbol{E}_{a}, \boldsymbol{C}, \boldsymbol{E}_{s}\right)$ fällt mit einem Eigenwert von $\boldsymbol{A}$ zusammen.

Der wesentliche Unterschied gegenüber Abschnitt 4.5 besteht darin, dass die invarianten Nullstellen des Systems nicht mehr sämtlich in der offenen linken s-Halbebene liegen müssen, wie in Annahme 4.3 gefordert. Hinreichend sind bereits die Annahmen 4.11 bis 4.13. Die Annahme 4.13 stellt dabei keine Einschränkung der Allgemeinheit dar, da aufgrund von Annahme 4.9 die Eigenwerte von $\boldsymbol{A}$ vor dem Entwurf eines Fehlerisolationsbeobachters beliebig verschoben werden können. Wie in Abschnitt 4.5.2 erörtert ist Annahme 4.12 notwendig für die Diagonalisierung von $\boldsymbol{G}_{\boldsymbol{r} \boldsymbol{f}}(s)$.

\subsubsection{Entwurf mittels virtueller Systemerweiterungen}

Gemäß den Ausführungen in Abschnitt 4.5.2 müssen invariante Nullstellen im Allgemeinen durch Beobachtereigenwerte kompensiert werden, um $\boldsymbol{G}_{\boldsymbol{r} \boldsymbol{f}}(s)$ zu diagonalisieren. Dadurch werden $n-\delta$ Beobachtereigenwerte nichtsteuerbar gemacht und der Nachfilterentwurf nach Lemma 4.3 stellt die Diagonalisierung der entsprechenden Übertragungsmatrix für alle $s \in \mathbb{C}$ sicher. Wie in [123] für das duale Problem des Entkopplungsreglerentwurfs herausgearbeitet, gibt es jedoch eine Ausnahme von dieser Regel, die auf einer speziellen Klasse von invarianten Nullstellen fußt. Diese sogenannten nicht verkoppelnden Nullstellen (engl. non-interconnecting zeros), die im Weiteren näher beleuchtet werden, müssen nicht durch einen nichtsteuerbaren Eigenwert kompensiert werden. Vielmehr können für derartige Nullstellen zusätzliche Pole bestimmten Spalten von $\boldsymbol{G}_{\boldsymbol{r} \boldsymbol{f}}(s)$ zugewiesen werden. Während im Allgemeinen dann der Nachfilterentwurf nach Lemma 4.3 keine Diagonalisierung für alle $s \in \mathbb{C}$ mehr gewährleistet, ist dies bei nicht verkoppelnden Nullstellen der Fall, woraus sich ihr Name erklärt.

Da invariante Nullstellen jedoch selten nicht verkoppelnde Nullstellen sind, wird wie in [137] im Weiteren der Ansatz verfolgt, das System (4.1) dynamisch zu erweitern. Durch die in Abbildung 4.13 gezeigten Erweiterungen soll erreicht werden, dass invariante Nullstellen, die sich in der geschlossenen rechten s-Halbebene befinden, für das resultierende, virtuelle 


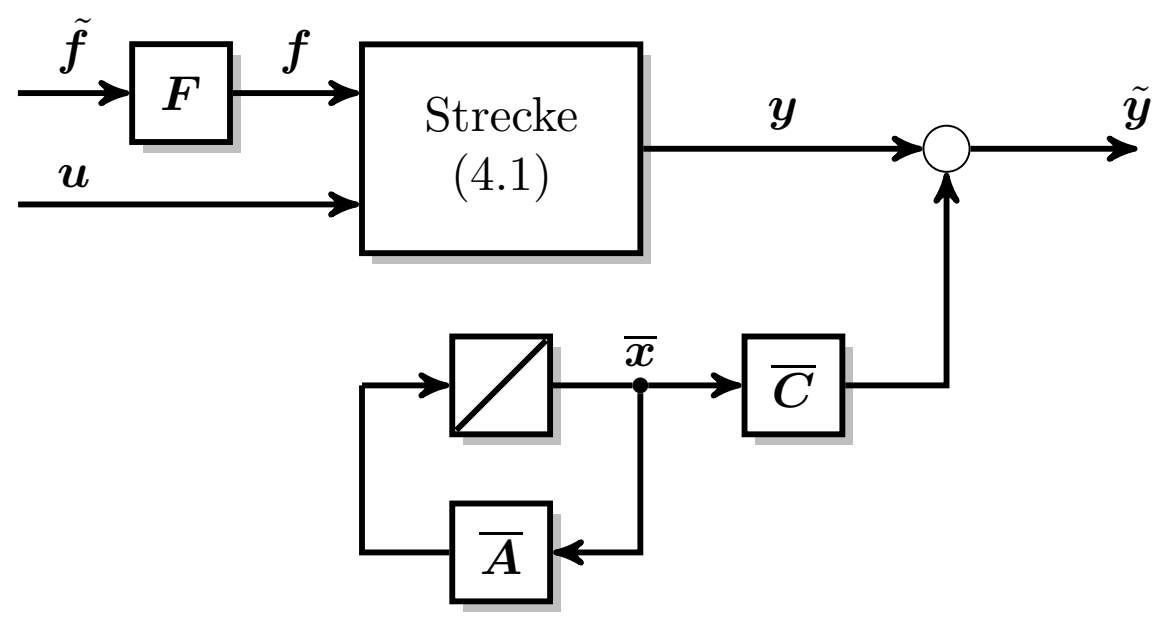

Abbildung 4.13: Virtuelle Systemerweiterung für nicht minimalphasige Systeme

Gesamtsystem in nicht verkoppelnde Nullstellen umgewandelt werden. Dazu ist neben den Matrizen $\boldsymbol{F} \in \mathbb{R}^{n_{f} \times n_{f}}, \overline{\boldsymbol{A}} \in \mathbb{R}^{\nu \times \nu}$ und $\overline{\boldsymbol{C}} \in \mathbb{R}^{n_{y} \times \nu}$ auch die Ordnung $\nu$ der hinzugefügten Dynamik zu wählen. Die Matrix $\boldsymbol{F}$ ist dabei wie in Abschnitt 4.7 lediglich eine Permutationsmatrix, welche die Herleitung des Entwurfsverfahrens vereinfacht. Anhand von Abbildung 4.13 wird deutlich, dass die zusätzliche Dynamik eine interne, nichtsteuerbare Dynamik ist. Sie darf dabei instabil sein, da sie lediglich virtuell ist und nur für den Entwurf benötigt wird. In der in Abbildung 4.13 gezeigten Form muss sie nicht implementiert werden.

Da nicht verkoppelnde Nullstellen der Schlüssel zur Auslegung der virtuellen Systemerweiterungen sind, werden sie im Folgenden zunächst definiert. In Anlehnung an die in [137] eingeführte Charakterisierung nicht verkoppelnder Nullstellen im Zustandsraum wird hier die folgende Definition verwendet:

Definition 4.7 (Nicht verkoppelnde Nullstellen). Gegeben sei ein System der Form (4.1), welches die Annahmen 4.9-4.13 erfüllt. Weiterhin sei $\eta$ eine invariante Nullstelle des Systems. Dann wird $\eta$ als nicht verkoppelnde Nullstelle bezeichnet, wenn

$$
\begin{aligned}
\boldsymbol{\Pi}(\eta) \cdot\left[\begin{array}{c}
\boldsymbol{z}_{x} \\
\boldsymbol{z}_{f}
\end{array}\right] & =\mathbf{0}, \\
\boldsymbol{z}_{f} & =k \boldsymbol{\phi}_{i}=\left[\begin{array}{lllllll}
0 & \cdots & 0 & k & 0 & \cdots & 0
\end{array}\right]^{\top}, k \neq 0
\end{aligned}
$$

gilt, wobei $\boldsymbol{z}_{x} \in \mathbb{R}^{n}$ ein beliebiger Vektor ist und der Vektor $\boldsymbol{z}_{f} \in \mathbb{R}^{n_{f}}$ genau ein Nichtnullelement in einer beliebigen Zeile $i$ besitzt. 
Mit dieser Definition lässt sich nun in Anlehnung an die Ergebnisse aus $[46,47,136,137,140]$ das folgende Lemma angeben, das zeigt, dass nicht verkoppelnde Nullstellen beim FIO-Entwurf nicht kompensiert werden müssen. Es überträgt die existierenden Resultate auf das Problem der beobachterbasierten Fehlerisolation und erweitert sie auf Systeme mit Aktor- und Sensorfehlern.

Lemma 4.9. Gegeben sei ein System der Form (4.1), welches die Annahmen 4.9-4.13 erfüllt. Wenn $\eta$ eine nicht verkoppelnde Nullstelle des Systems ist, muss sie nicht durch einen Beobachtereigenwert $\lambda_{B_{k}}$ kompensiert werden, um der Übertragungsmatrix $\boldsymbol{G}_{\boldsymbol{r} \boldsymbol{f}}(s)$ beim Entwurf der Nachfiltermatrix nach $\boldsymbol{V}=-\boldsymbol{S \Theta}^{-1}$ mit einer Diagonalmatrix $\boldsymbol{S}$ und $\boldsymbol{\Theta}=\boldsymbol{C}(\boldsymbol{A}-\boldsymbol{L} \boldsymbol{C})^{-1}\left(\boldsymbol{E}_{a}-\boldsymbol{L} \boldsymbol{E}_{s}\right)-\boldsymbol{E}_{s}$ Diagonalgestalt aufzuprägen. Enthält $\boldsymbol{z}_{f}$ gemäß (4.59b) in der $i$-ten Zeile ein Nichtnullelement, so ist beim Entwurf mittels Vollständiger Modaler Synthese nach Abschnitt 4.5.2 in der $i$-ten Spalte von $\boldsymbol{G}_{\boldsymbol{r} \boldsymbol{f}}(s)$ ein zusätzlicher Pol zu platzieren.

Beweis. Zunächst werden zwei reguläre Matrizen $\boldsymbol{\Phi}(\eta)$ und $\boldsymbol{\Psi}$ eingeführt, welche durch

$$
\boldsymbol{\Phi}(\eta)=\left[\begin{array}{cc}
\boldsymbol{I}_{n} & \mathbf{0} \\
\boldsymbol{V} \boldsymbol{C}\left(\eta \boldsymbol{I}_{n}-(\boldsymbol{A}-\boldsymbol{L} \boldsymbol{C})\right)^{-1} & \boldsymbol{I}_{n}
\end{array}\right], \quad \boldsymbol{\Psi}=\left[\begin{array}{cc}
\boldsymbol{I}_{n} & -\boldsymbol{L} \\
\mathbf{0} & \boldsymbol{V}
\end{array}\right]
$$

gegeben sind. Dabei impliziert die Regularität von $\boldsymbol{V}$ die Regularität von $\boldsymbol{\Psi}$, und $\boldsymbol{\Phi}(\eta)$ existiert, da nach Annahme 4.9 die Beobachtereigenwerte stets so platziert werden können, dass keiner von ihnen mit der invarianten Nullstelle $\eta$ zusammenfällt. Durch die linksseitige Multiplikation von (4.59a) mit $\boldsymbol{\Psi}$ und $\boldsymbol{\Phi}(\eta)$ ergibt sich

$$
\begin{aligned}
& \boldsymbol{\Phi}(\eta) \cdot \boldsymbol{\Psi} \cdot \boldsymbol{\Pi}(\eta) \cdot\left[\begin{array}{l}
\boldsymbol{z}_{x} \\
\boldsymbol{z}_{f}
\end{array}\right]= \\
& {\left[\begin{array}{cc}
\eta \boldsymbol{I}_{n}-(\boldsymbol{A}-\boldsymbol{L} \boldsymbol{C}) & \\
\mathbf{0} & \underbrace{\boldsymbol{V} \boldsymbol{C}\left(\eta \boldsymbol{I}_{n}-(\boldsymbol{A}-\boldsymbol{L} \boldsymbol{C})\right)^{-1}\left(\boldsymbol{E}_{a}-\boldsymbol{L} \boldsymbol{E}_{s}\right)+\boldsymbol{V} \boldsymbol{E}_{s}}_{\boldsymbol{G}_{r f}(\eta)}
\end{array}\right]\left[\begin{array}{l}
\boldsymbol{z}_{x} \\
\boldsymbol{z}_{f}
\end{array}\right]} \\
& =\mathbf{0} .
\end{aligned}
$$

Daraus folgt $\boldsymbol{G}_{\boldsymbol{r} \boldsymbol{f}}(\eta) \cdot \boldsymbol{z}_{f}=\mathbf{0}$. Berücksichtigt man, dass $\boldsymbol{z}_{f}$ definitionsgemäß lediglich in der $i$-ten Zeile ein Nichtnullelement beinhaltet, so ist 


$$
\boldsymbol{G}_{\boldsymbol{r} \boldsymbol{f}}(\eta) \cdot \boldsymbol{z}_{f}=\left[\begin{array}{ccc}
g_{1,1}(\eta) & \cdots & g_{1, n_{f}}(\eta) \\
\vdots & \ddots & \vdots \\
g_{n_{f}, 1}(\eta) & \cdots & g_{n_{f}, n_{f}}(\eta)
\end{array}\right]\left[\begin{array}{c}
0 \\
\vdots \\
0 \\
k \\
0 \\
\vdots \\
0
\end{array}\right]=\mathbf{0}
$$

und folglich $g_{j, i}(\eta)=0$ für alle $j=1, \ldots, n_{f}$. Das Diagonalelement $g_{i, i}(s)$ weist demnach die Nullstelle $\eta$ auf. Nimmt man nun an, dass entsprechend dem in Abschnitt 4.5.2 vorgestellten Verfahren $\delta_{i}+1$ Eigenwerte als Pole für die $i$-te Spalte zugewiesen wurden, so ergibt sich für $g_{i, i}(s)$ die allgemeine Form

$$
g_{i, i}(s)=\frac{z_{i, 0}(s-\eta)}{\left(s-\lambda_{i, 1}\right) \cdot \ldots \cdot\left(s-\lambda_{i, \delta_{i}+1}\right)}=\frac{z_{i, i}(s)}{n_{i, i}(s)}
$$

wobei die Nullstelle $\eta$ unkompensiert bleibt. Weiterhin ist aus Abschnitt 4.5.2 bekannt, dass das Nachfilter $\boldsymbol{V}$ so entworfen wird, dass im stationären Zustand für die Nichtdiagonalelemente von $\boldsymbol{G}_{\boldsymbol{r} \boldsymbol{f}}(s)$ die Beziehung $g_{i, j}(0)=0$ für alle $i \neq j$ gilt.

Durch Widerspruch kann nun gezeigt werden, dass die beiden Bedingungen $g_{j, i}(\eta)=0$ und $g_{j, i}(0)=0$ nur dann erfüllt sein können, wenn auch $g_{j, i}(s)=0 \forall s \in \mathbb{C}$ gilt. Dazu sei angenommen, dass beide Bedingungen erfüllt seien. Dann ist allgemein

$$
g_{j, i}(s)=\frac{z_{j, i}(s)}{n_{j, i}(s)}=\frac{s \cdot(s-\eta) \cdot z_{j, i, R}(s)}{n_{j, i}(s)},
$$

wobei $z_{j, i, R}(s)$ ein beliebiges Polynom ist. Die Differenzenordnung von $g_{j, i}(s)$ ist wie in Abschnitt 4.5.2 gezeigt durch $\delta_{i}$ nach unten beschränkt. Es gilt also

$$
\operatorname{deg}\left(n_{j, i}(s)\right)-\operatorname{deg}\left(z_{j, i}(s)\right) \geq \delta_{i} .
$$

Da der Entwurf mittels der Vollständigen Modalen Synthese nach Abschnitt 4.5.2 allen Übertragungsfunktionen der $i$-ten Spalte von $\boldsymbol{G}_{\boldsymbol{r} \boldsymbol{f}}(s)$ die gleichen Nennerpolynome zuweist, gilt $n_{j, i}(s)=n_{i, i}(s)$ und somit

$$
\begin{aligned}
\operatorname{deg}\left(n_{i, i}(s)\right)-\operatorname{deg}\left(z_{j, i}(s)\right) & \geq \delta_{i}, \\
\delta_{i}+1-\operatorname{deg}\left(z_{j, i}(s)\right) & \geq \delta_{i} .
\end{aligned}
$$


Es muss dementsprechend $\operatorname{deg}\left(z_{j, i}(s)\right) \leq 1$ gelten. Damit $g_{j, i}(s)$ jedoch wie in (4.60) zwei Nullstellen aufweist, muss $\operatorname{deg}\left(z_{j, i}(s)\right) \geq 2$ gelten. Dieser Widerspruch kann nur dadurch gelöst werden, dass für das Restpolynom $z_{j, i, R}(s)=0$ gilt. Damit ist $g_{j, i}(s)=0$ für alle $s \in \mathbb{C}$ gezeigt.

Die in Abschnitt 4.5 eingeführte Definition von statisch stabil fehlerisolierbaren Systemen (s. Seite 80) lässt sich auf dieser Grundlage erweitern $\mathrm{zu}$

Definition 4.8 (Statisch stabil isolierbare Systeme). Ein System der Form (4.1), welches die Annahmen 4.9 und 4.10 erfüllt und das mit Ausnahme seiner nicht verkoppelnden Nullstellen ausschließlich endlich viele invariante Nullstellen in der offenen linken s-Halbebene aufweist, heißt statisch stabil (fehler-) isolierbar.

Für die Auslegung der zusätzlichen internen Dynamik wird zunächst das Gesamtsystem mit dem Zustandsvektor $\boldsymbol{z}=\left[\boldsymbol{x}^{\top} \overline{\boldsymbol{x}}^{\top}\right]^{\top} \in \mathbb{R}^{n+\nu}$ betrachtet. Es ergibt sich anhand von (4.1) und Abbildung $4.13 \mathrm{zu}$

$$
\begin{aligned}
& \dot{z}=\underbrace{\left[\begin{array}{cc}
\boldsymbol{A} & \mathbf{0} \\
\mathbf{0} & \overline{\boldsymbol{A}}
\end{array}\right]}_{\tilde{\boldsymbol{A}}} \boldsymbol{z}+\underbrace{\left[\begin{array}{c}
\boldsymbol{B} \\
\mathbf{0}
\end{array}\right]}_{\tilde{\boldsymbol{B}}} \boldsymbol{u}+\underbrace{\left[\begin{array}{c}
\boldsymbol{E}_{a} \boldsymbol{F} \\
\mathbf{0}
\end{array}\right]}_{\tilde{\boldsymbol{E}}_{a}} \tilde{\boldsymbol{f}}, \\
& \tilde{\boldsymbol{y}}=\underbrace{\left[\begin{array}{cc}
C & \bar{C}
\end{array}\right]}_{\tilde{C}} \boldsymbol{z}+\underbrace{\boldsymbol{D}}_{\tilde{\boldsymbol{D}}} \boldsymbol{u}+\underbrace{\boldsymbol{E}_{s} \boldsymbol{F}}_{\tilde{\boldsymbol{E}}_{s}} \tilde{\boldsymbol{f}} .
\end{aligned}
$$

Gemäß der Definition auf Seite 79 lautet die Rosenbrock'sche Systemmatrix des Gesamtsystems (4.61)

$$
\tilde{\boldsymbol{\Pi}}(\eta)=\left[\begin{array}{cc}
\eta \boldsymbol{I}_{n+\nu}-\tilde{\boldsymbol{A}} & \tilde{\boldsymbol{E}}_{a} \\
-\tilde{\boldsymbol{C}} & \tilde{\boldsymbol{E}}_{s}
\end{array}\right] .
$$

Dementsprechend berechnet sich ihre Determinante zu

$$
\operatorname{det}(\tilde{\boldsymbol{\Pi}}(\eta))=\left|\begin{array}{cc:c}
\eta \boldsymbol{I}_{n}-\boldsymbol{A} & \mathbf{0} & \boldsymbol{E}_{a} \boldsymbol{F} \\
\hdashline-\boldsymbol{0} & \eta \boldsymbol{I}_{\nu}-\overline{\boldsymbol{A}} & \mathbf{0} \\
\hdashline-\bar{C} & -\overline{\boldsymbol{C}} & \boldsymbol{E}_{s} \boldsymbol{F}
\end{array}\right|
$$

und durch Zeilen- und Spaltenvertauschungen folgt

$$
\operatorname{det}(\tilde{\boldsymbol{\Pi}}(\eta))=\left|\begin{array}{cc:c}
\eta \boldsymbol{I}_{n}-\boldsymbol{A} & \boldsymbol{E}_{a} \boldsymbol{F} & \mathbf{0} \\
\hdashline \boldsymbol{C} & \boldsymbol{E}_{s} \boldsymbol{F} & -\overline{\boldsymbol{C}} \\
\hdashline \mathbf{0} & \mathbf{0} & \eta \boldsymbol{I}_{\nu}-\overline{\boldsymbol{A}}
\end{array}\right|
$$


Zwar ändert sich bei der Vertauschung von Zeilen und Spalten einer Matrix jeweils das Vorzeichen der Determinante [150, Abschnitt 6.1]. Da jedoch für die Umformung in (4.62) $2 n_{y}$ solcher Operationen notwendig sind, findet eine gerade Anzahl von Vertauschungen statt und das Vorzeichen ändert sich nicht. Aufgrund der Blockdreiecksstruktur der Determinante in (4.62) folgt weiter

$$
\begin{aligned}
\operatorname{det}(\tilde{\boldsymbol{\Pi}}(\eta)) & =\operatorname{det}\left(\eta \boldsymbol{I}_{\nu}-\overline{\boldsymbol{A}}\right) \cdot\left|\begin{array}{cc}
\eta \boldsymbol{I}_{n}-\boldsymbol{A} & \boldsymbol{E}_{a} \boldsymbol{F} \\
-\boldsymbol{C} & \boldsymbol{E}_{s} \boldsymbol{F}
\end{array}\right| \\
& =\operatorname{det}\left(\eta \boldsymbol{I}_{\nu}-\overline{\boldsymbol{A}}\right) \cdot \operatorname{det}(\boldsymbol{\Pi}(\eta)) \cdot\left|\begin{array}{cc}
\boldsymbol{I}_{n} & \mathbf{0} \\
\mathbf{0} & \boldsymbol{F}
\end{array}\right|
\end{aligned}
$$

Wird $\boldsymbol{F}$ als Permutationsmatrix angesetzt, so ist die Matrix regulär. Somit ergibt sich aus (4.63), dass sich die invarianten Nullstellen des Gesamtsystems (4.61) aus den invarianten Nullstellen des ursprünglichen Systems (4.1) und den Eigenwerten der hinzugefügten Matrix $\overline{\boldsymbol{A}}$ zusammensetzen. Auf Grundlage dieser Erkenntnis werden im Folgenden die Ordnung $\nu$ der zusätzlichen Dynamik sowie die Matrizen $\boldsymbol{F}, \overline{\boldsymbol{A}}$ und $\overline{\boldsymbol{C}}$ ausgelegt.

Dazu wird eine invariante Nullstelle $\eta$ des Systems (4.1) betrachtet. Da eine solche Nullstelle definitionsgemäß ein Rangdefizit in der Rosenbrock'schen Systemmatrix hervorruft, hat (4.59a) eine nichttriviale Lösung. Dabei sei $\alpha$ die Anzahl der Nichtnullelemente in $\boldsymbol{z}_{f}$ in (4.59b). Aufgrund von (4.63) bleibt $\alpha$ unter einer beliebigen Permutationsmatrix $\boldsymbol{F}$ unverändert. Es lässt sich folglich immer eine Matrix $\boldsymbol{F}$ finden, sodass sich die ersten $\alpha$ Elemente des Vektors $\tilde{\boldsymbol{z}}_{f}=\boldsymbol{F}^{-1} \boldsymbol{z}_{f}$ von null unterscheiden. Die Matrix $\boldsymbol{F}$ wird also bestimmt über

$$
\boldsymbol{z}_{f}=\boldsymbol{F} \cdot \tilde{\boldsymbol{z}}_{f}=\boldsymbol{F} \cdot\left[\begin{array}{llllll}
\tilde{z}_{f, 1} & \cdots & \tilde{z}_{f, \alpha} & 0 & \cdots & 0
\end{array}\right]^{\top} .
$$

Nachdem $\boldsymbol{F}$ gewählt ist, wird für die Ordnung der internen Dynamik $\nu=\alpha-1$ angesetzt und

$$
\overline{\boldsymbol{A}}=\eta \boldsymbol{I}_{\alpha-1}
$$

gewählt. Daraus ergibt sich mit (4.63), dass $\eta$ eine invariante Nullstelle des virtuellen Gesamtsystems (4.61) der Vielfachheit $\alpha$ ist. Es verbleibt nun, $\bar{C}$ so zu wählen, dass $\eta$ eine nicht verkoppelnde Nullstelle des Gesamtsystems ist.

Dazu wird Gleichung (4.59a) für das Gesamtsystem (4.1) ausgewertet. Da $\eta$ eine invariante Nullstelle der Vielfachheit $\alpha$ ist, werden die Vektoren $\boldsymbol{z}_{x, k}=\left[\begin{array}{ll}\boldsymbol{z}_{x, o, k}^{\top} & \boldsymbol{z}_{x, u, k}^{\top}\end{array}\right]^{\top}, k=1, \ldots, \alpha$, eingeführt. Dann muss gemäß (4.59b) 
die Beziehung

$$
\begin{gathered}
{\left[\begin{array}{ccc}
\eta \boldsymbol{I}_{n}-\boldsymbol{A} & \mathbf{0} & \boldsymbol{E}_{a} \boldsymbol{F} \\
\mathbf{0} & \eta \boldsymbol{I}_{\alpha-1}-\overline{\boldsymbol{A}} & \mathbf{0} \\
-\boldsymbol{C} & -\overline{\boldsymbol{C}} & \boldsymbol{E}_{s} \boldsymbol{F}
\end{array}\right]\left[\begin{array}{c}
\boldsymbol{z}_{x, o, k} \\
\boldsymbol{z}_{x, u, k} \\
\boldsymbol{z}_{f}
\end{array}\right]} \\
\quad=\left[\begin{array}{ccc}
\eta \boldsymbol{I}_{n}-\boldsymbol{A} & \mathbf{0} & \boldsymbol{E}_{a} \boldsymbol{F} \\
\mathbf{0} & \mathbf{0} & \mathbf{0} \\
-\boldsymbol{C} & -\overline{\boldsymbol{C}} & \boldsymbol{E}_{s} \boldsymbol{F}
\end{array}\right]\left[\begin{array}{c}
\boldsymbol{z}_{x, o, k} \\
\boldsymbol{z}_{x, u, k} \\
\boldsymbol{\phi}_{k}
\end{array}\right]=\mathbf{0}
\end{gathered}
$$

für alle $k=1, \ldots, \alpha$ gelten, damit $\eta$ eine nicht verkoppelnde Nullstelle ist. Diese $\alpha$ Bedingungen werden zusammengefasst $\mathrm{zu}$

$$
\begin{gathered}
{\left[\begin{array}{ccc}
\eta \boldsymbol{I}_{n}-\boldsymbol{A} & \mathbf{0} & \boldsymbol{E}_{a} \boldsymbol{F} \\
\mathbf{0} & \eta \boldsymbol{I}_{\alpha-1}-\overline{\boldsymbol{A}} & \mathbf{0} \\
-\boldsymbol{C} & -\overline{\boldsymbol{C}} & \boldsymbol{E}_{s} \boldsymbol{F}
\end{array}\right]\left[\begin{array}{ccc}
\boldsymbol{z}_{x, o, 1} & \cdots & \boldsymbol{z}_{x, o, \alpha} \\
\boldsymbol{z}_{x, u, 1} & \cdots & \boldsymbol{z}_{x, u, \alpha} \\
\boldsymbol{\phi}_{1} & \cdots & \boldsymbol{\phi}_{\alpha}
\end{array}\right]} \\
\quad=\left[\begin{array}{ccc}
\eta \boldsymbol{I}_{n}-\boldsymbol{A} & \mathbf{0} & \boldsymbol{E}_{a} \boldsymbol{F} \\
\mathbf{0} & \mathbf{0} & \mathbf{0} \\
-\boldsymbol{C} & -\overline{\boldsymbol{C}} & \boldsymbol{E}_{s} \boldsymbol{F}
\end{array}\right]\left[\begin{array}{c}
\boldsymbol{Z}_{x, o} \\
\boldsymbol{Z}_{x, u} \\
\boldsymbol{I}_{\alpha} \\
\mathbf{0}_{\left(n_{f}-\alpha\right) \times \alpha}
\end{array}\right]=\mathbf{0} .
\end{gathered}
$$

Dabei ist $\boldsymbol{Z}_{x, o} \in \mathbb{R}^{n \times \alpha}$ und $\boldsymbol{Z}_{x, u} \in \mathbb{R}^{(\alpha-1) \times \alpha}$. Weiterhin wird abkürzend $\tilde{\boldsymbol{Z}}_{f}=\left[\begin{array}{ll}\boldsymbol{I}_{\alpha} & \mathbf{0}_{\alpha \times\left(n_{f}-\alpha\right)}\end{array}\right]^{\top}$ eingeführt. Damit leiten sich aus (4.66) zunächst die Bedingungen

$$
\begin{aligned}
\left(\eta \boldsymbol{I}_{n}-\boldsymbol{A}\right) \boldsymbol{Z}_{x, o}+\boldsymbol{E}_{a} \boldsymbol{F} \tilde{\boldsymbol{Z}}_{f} & =\mathbf{0}_{n \times \alpha} \\
-\boldsymbol{C} \boldsymbol{Z}_{x, o}-\overline{\boldsymbol{C}} \boldsymbol{Z}_{x, u}+\boldsymbol{E}_{s} \boldsymbol{F} \tilde{\boldsymbol{Z}}_{f} & =\mathbf{0}_{n_{y} \times \alpha}
\end{aligned}
$$

ab. Da nach Annahme 4.13 die invariante Nullstelle $\eta$ nicht mit einem Eigenwert von $\boldsymbol{A}$ zusammenfällt, lässt sich aus (4.67a) für die Matrix $\boldsymbol{Z}_{x, o}$ die Beziehung

$$
\boldsymbol{Z}_{x, o}=-\left(\eta \boldsymbol{I}_{\alpha}-\boldsymbol{A}\right)^{-1} \boldsymbol{E}_{a} \boldsymbol{F} \tilde{\boldsymbol{Z}}_{f}
$$

ableiten. Durch Einsetzen in (4.67b) folgt damit für die beiden verbleibenden unbekannten Matrizen $\bar{C}$ und $\boldsymbol{Z}_{x, u}$ die Gleichung

$$
\overline{\boldsymbol{C}} \boldsymbol{Z}_{x, u}=\left(\boldsymbol{C}\left(\eta \boldsymbol{I}_{n}-\boldsymbol{A}\right)^{-1} \boldsymbol{E}_{a}+\boldsymbol{E}_{s}\right) \boldsymbol{F} \tilde{\boldsymbol{Z}}_{f}=\boldsymbol{\Theta}(\eta) .
$$

Diese ist nach [14, Proposition 6.1.7] genau dann lösbar, wenn

$$
\operatorname{rang}(\bar{C})=\operatorname{rang}\left(\left[\begin{array}{ll}
\bar{C} & \boldsymbol{\Theta}(\eta)
\end{array}\right]\right)
$$


gilt. Dementsprechend wird $\bar{C}$ als Linearkombination der linear unabhängigen Spalten von $\boldsymbol{\Theta}$ gewählt, das heißt

$$
\bar{C}=\boldsymbol{\Theta}(\eta) \cdot \Lambda,
$$

mit einer beliebigen Matrix $\boldsymbol{\Lambda} \in \mathbb{R}^{\alpha \times \vartheta}$ vollen Ranges. Aus der Definition der invarianten Nullstellen und Annahme 4.13 folgt, dass $\left(\boldsymbol{C}\left(\eta \boldsymbol{I}_{n}-\boldsymbol{A}\right)^{-1} \boldsymbol{E}_{a}+\boldsymbol{E}_{s}\right) \boldsymbol{z}_{f}=\mathbf{0}$ gilt. Mit $\boldsymbol{z}_{f}=\boldsymbol{F} \tilde{\boldsymbol{z}}_{f}$ folgt unter Berücksichtigung der Tatsache, dass $\eta$ eine invariante Nullstelle des Systems (4.1) ist, dass $\operatorname{rang}(\boldsymbol{\Theta}(\eta))<\alpha$ gilt. Somit ist auch $\vartheta<\alpha$.

Damit sind $\boldsymbol{F}, \nu, \overline{\boldsymbol{A}}$ und $\overline{\boldsymbol{C}}$ festgelegt und $\eta$ ist eine nicht verkoppelnde Nullstelle für das resultierende virtuelle Gesamtsystem. Für dieses kann dann mittels der Vollständigen Modalen Synthese wie in Abschnitt 4.5.2 beziehungsweise in Lemma 4.9 ein Fehlerisolationsbeobachter entworfen werden. Zusammenfassend gilt

Satz 4.6. Gegeben sei ein System der Form (4.1), welches die Annahmen 4.9-4.13 erfüllt. Dann lässt sich für das resultierende Gesamtsystem (4.61) ein stabiler Fehlerisolationsbeobachter entwerfen, wenn die Auslegung von $\nu, \boldsymbol{F}, \overline{\boldsymbol{A}}$ und $\overline{\boldsymbol{C}}$ gemäß (4.64), (4.65) und (4.69) erfolgt.

Auf den Entwurf beziehungsweise die Realisierung eines solchen Fehlerisolationsbeobachters wird im folgenden Abschnitt näher eingegangen.

\subsubsection{Realisierung mithilfe dynamisch erweiterter Beobachter}

Prinzipiell lässt sich ein Fehlerisolationsbeobachter für nicht minimalphasige Systeme entwerfen, indem zunächst die im vorigen Abschnitt eingeführten dynamischen Erweiterungen realisiert werden und anschließend für das resultierende Gesamtsystem der Entwurf durchgeführt wird. Dies ist jedoch mit Problemen verbunden. Zum einen zieht das Vorgehen wie auch bei den in den Abschnitten 4.6.4 und 4.7.1 beschriebenen Verfahren einen erhöhten Implementierungsaufwand nach sich, da beispielsweise für ein System mit einer nicht zu kompensierenden Nullstelle ein dynamisches System der Ordnung $n+2(\alpha-1)$ zu implementieren ist. Zum anderen ist die interne Dynamik instabil, da die Eigenwerte von $\overline{\boldsymbol{A}}$ invarianten Nullstellen in der rechten s-Halbebene entsprechen. Ein drittes Problemfeld sind konjugiert komplexe Nullstellenpaare, die zu komplexen Matrizen $\overline{\boldsymbol{A}}$ und $\bar{C}$ führen. In diesem Abschnitt wird gezeigt, wie sich mithilfe von dynamisch erweiterten Fehlerisolationsbeobachtern eine rein reelle, stabile 
Parametrierung mit gleichzeitig geringem Implementierungsaufwand realisieren lässt.

Zunächst wird das Problem konjugiert komplexer Nullstellenpaare betrachtet. In [140] wurde für das duale Problem des Entkopplungsreglerentwurfs eine Ähnlichkeitstransformation vorgeschlagen, die auf rein reelle Systemmatrizen führt. Im Folgenden werden die entsprechenden Resultate verallgemeinert und für den Entwurf von FIOs bewiesen. Den konstruktiven Entwurf der Transformationsmatrizen fasst das folgende Lemma zusammen, welches in Anhang A auf Seite 353 bewiesen wird.

Lemma 4.10. Gegeben sei ein System der Form (4.1), welches die Annahmen 4.9-4.13 erfüllt. Weiterhin sei durch $\eta_{1 / 2}=a \pm j b$ ein konjugiert komplexes Paar invarianter Nullstellen mit beliebigem $\alpha \geq 2$ gegeben. Für jede der invarianten Nullstellen sei jeweils eine dynamische Erweiterung des Systems nach (4.64), (4.65), (4.69) durchgeführt worden, wobei für beide Erweiterungen die gleiche Matrix $\boldsymbol{\Lambda}$ in (4.69) gewählt wurde. Dann führt eine Ähnlichkeitstransformation mit

$$
\boldsymbol{T}^{-1}=\left[\begin{array}{cc}
\boldsymbol{I}_{n} & \mathbf{0} \\
\mathbf{0} & \boldsymbol{T}_{m r}^{-1}
\end{array}\right], \quad \boldsymbol{T}_{m r}^{-1}=\left[\begin{array}{cc}
1 & -j \\
1 & j
\end{array}\right] \otimes \boldsymbol{I}_{\alpha-1}
$$

auf rein reelle Matrizen $\quad \boldsymbol{A}^{\prime}=\boldsymbol{T} \tilde{\boldsymbol{A}} \boldsymbol{T}^{-1}, \quad \boldsymbol{B}^{\prime}=\boldsymbol{T} \tilde{\boldsymbol{B}}, \quad \boldsymbol{E}_{a}^{\prime}=\boldsymbol{T} \tilde{\boldsymbol{E}}_{a}$ $\boldsymbol{C}^{\prime}=\tilde{\boldsymbol{C}} \boldsymbol{T}^{-1}, \boldsymbol{D}^{\prime}=\tilde{\boldsymbol{D}}$ und $\boldsymbol{E}_{s}^{\prime}=\tilde{\boldsymbol{E}}_{s}$.

Für das dynamisch erweiterte und transformierte Gesamtsystem

$$
\begin{aligned}
& \dot{\boldsymbol{z}}^{\prime}=\boldsymbol{A}^{\prime} \boldsymbol{z}^{\prime}+\boldsymbol{B}^{\prime} \boldsymbol{u}+\boldsymbol{E}_{a}^{\prime} \boldsymbol{f} \text { mit } \boldsymbol{z}^{\prime}=\left[\begin{array}{l}
\boldsymbol{x}^{\prime} \\
\overline{\boldsymbol{x}}^{\prime}
\end{array}\right] \\
& \boldsymbol{y}=\boldsymbol{C}^{\prime} \boldsymbol{z}^{\prime}+\boldsymbol{D}^{\prime} \boldsymbol{u}+\boldsymbol{E}_{s}^{\prime} \boldsymbol{f}
\end{aligned}
$$

kann somit eine stabile, reelle FIO-Parametrierung $\left(\boldsymbol{L}^{\prime}, \boldsymbol{V}^{\prime}\right)$ gefunden werden. Dazu wird der Entwurf mittels Vollständiger Modaler Synthese nach Satz 4.2 durchgeführt, wobei nur invariante Nullstellen in der offenen linken s-Halbebene gemäß (4.24) durch nichtsteuerbare Beobachtereigenwerte kompensiert werden. Alle übrigen Beobachtereigenwerte werden entsprechenden Spalten von $\boldsymbol{G}_{\boldsymbol{r} \boldsymbol{f}}(s)$ als Pole zugewiesen. Um die Anzahl der an eine Spalte gebundenen Pole festzulegen, sei $\kappa_{i}$ die Anzahl der invarianten Nullstellen in der abgeschlossenen rechten s-Halbebene, für die das $i$-te Element von $\boldsymbol{z}_{f}$ in (4.59b) ungleich null ist. Dann ist jedem Übertragungskanal $g_{i, i}(s)$ der Matrix $\boldsymbol{G}_{\boldsymbol{r} \boldsymbol{f}}(s)$ mittels (4.22) eine Anzahl von $\delta_{i}+\kappa_{i}$ Polen zuzuweisen. 
Der resultierende FIO für das Gesamtsystem mit reellen Matrizen ist durch

$$
\begin{aligned}
\dot{\hat{z}}^{\prime} & =\left(\boldsymbol{A}^{\prime}-\boldsymbol{L}^{\prime} \boldsymbol{C}^{\prime}\right) \hat{\boldsymbol{z}}^{\prime}+\left(\boldsymbol{B}^{\prime}-\boldsymbol{L}^{\prime} \boldsymbol{D}^{\prime}\right) \boldsymbol{u}+\boldsymbol{L}^{\prime} \tilde{\boldsymbol{y}} \\
\boldsymbol{r} & =\boldsymbol{V}^{\prime}(\tilde{\boldsymbol{y}}-\hat{\tilde{\boldsymbol{y}}})
\end{aligned}
$$

gegeben. Problematisch dabei ist jedoch, dass neben obigem Beobachter auch noch die instabile zusätzliche Dynamik implementiert werden müsste. Dies lässt sich durch die Anwendung des folgenden Satzes umgehen.

Satz 4.7 (Realisierung von DFIOs zur Fehlerisolation in nicht minimalphasigen Systemen). Gegeben sei ein System der Form

$$
\begin{aligned}
& \dot{\boldsymbol{x}}=\boldsymbol{A} \boldsymbol{x}+\boldsymbol{B} \boldsymbol{u}+\boldsymbol{E}_{a} \boldsymbol{f}, \\
& \boldsymbol{y}=\boldsymbol{C} \boldsymbol{x}+\boldsymbol{D} \boldsymbol{u}+\boldsymbol{E}_{s} \boldsymbol{f},
\end{aligned}
$$

welches die Annahmen 4.9-4.13 erfüllt und für das gemäß Satz 4.6 dynamische Erweiterungen vorgenommen wurde. Weiterhin sei das resultierende Gesamtsystem gegebenenfalls mittels Lemma 4.10 in ein System mit rein reellen Matrizen transformiert, für das durch (4.71) sei ein stabiler Fehlerisolationsbeobachter gegeben sei. Partitioniert man

$$
\boldsymbol{A}^{\prime}=\left[\begin{array}{cc}
\boldsymbol{A} & \mathbf{0} \\
\mathbf{0} & \boldsymbol{A}_{22}^{\prime}
\end{array}\right], \boldsymbol{C}^{\prime}=\left[\begin{array}{ll}
\boldsymbol{C} & \boldsymbol{C}_{2}^{\prime}
\end{array}\right], \boldsymbol{L}^{\prime}=\left[\begin{array}{c}
\boldsymbol{L}_{1}^{\prime} \\
\boldsymbol{L}_{2}^{\prime}
\end{array}\right]
$$

mit $\boldsymbol{L}_{1}^{\prime} \in \mathbb{R}^{n \times n_{y}}$ und $\boldsymbol{L}_{2}^{\prime} \in \mathbb{R}^{\nu \times n_{y}}$, so ist durch

$$
\begin{aligned}
\dot{\hat{\boldsymbol{x}}} & =\boldsymbol{A} \hat{\boldsymbol{x}}+\boldsymbol{B} \boldsymbol{u}+\boldsymbol{L}_{p}(\boldsymbol{y}-\boldsymbol{C} \hat{\boldsymbol{x}}-\boldsymbol{D} \boldsymbol{u})+\boldsymbol{L}_{i} \boldsymbol{\zeta}, \\
\dot{\boldsymbol{\zeta}} & =\boldsymbol{\Phi} \boldsymbol{\zeta}+\boldsymbol{\Gamma}(\boldsymbol{y}-\boldsymbol{C} \hat{\boldsymbol{x}}-\boldsymbol{D} \boldsymbol{u}) \\
\boldsymbol{r} & =\boldsymbol{V}_{\boldsymbol{y}}(\boldsymbol{y}-\boldsymbol{C} \hat{\boldsymbol{x}}-\boldsymbol{D} \boldsymbol{u})+\boldsymbol{V}_{\boldsymbol{\zeta}} \boldsymbol{\zeta} .
\end{aligned}
$$

mit

$$
\begin{aligned}
\boldsymbol{L}_{p} & =\boldsymbol{L}_{1}^{\prime}, & \boldsymbol{L}_{i} & =\boldsymbol{L}_{1}^{\prime} \boldsymbol{C}_{2}^{\prime}, \\
\mathbb{R}^{\nu \times \nu} \ni \boldsymbol{\Phi} & =\boldsymbol{A}_{22}^{\prime}-\boldsymbol{L}_{2}^{\prime} \boldsymbol{C}_{2}^{\prime}, & \boldsymbol{\Gamma} & =-\boldsymbol{L}_{2}^{\prime}, \\
\boldsymbol{V}_{\boldsymbol{y}} & =\boldsymbol{V}^{\prime}, & \boldsymbol{V}_{\boldsymbol{\zeta}} & =\boldsymbol{V}^{\prime} \boldsymbol{C}_{2}^{\prime}
\end{aligned}
$$

und $\nu=\sum_{k=1}^{\mu_{g r H E}}\left(\alpha_{k}-1\right)$ ein stabiler dynamischer Fehlerisolationsbeobachter für das System (4.72) parametriert. Die Größen $\alpha_{k}$ bezeichnen dabei die Anzahl der Nichtnullelemente von $\boldsymbol{z}_{f}$ in (4.59b) und $\mu_{\text {grHE }}$ ist die Anzahl der nicht zu kompensierenden invarianten Nullstellen. 
Beweis. Für die Matrix $\boldsymbol{A}^{\prime}$ gilt nach (A.8)

$$
\boldsymbol{A}^{\prime}=\left[\begin{array}{cc}
\boldsymbol{A} & \mathbf{0} \\
\mathbf{0} & \boldsymbol{T}_{\mathrm{mr}} \overline{\boldsymbol{A}} \boldsymbol{T}_{\mathrm{mr}}^{-1}
\end{array}\right]=\left[\begin{array}{cc}
\boldsymbol{A} & \mathbf{0} \\
\mathbf{0} & \boldsymbol{A}_{22}^{\prime}
\end{array}\right] .
$$

Ebenso ist nach (A.12)

$$
\boldsymbol{C}^{\prime}=\left[\begin{array}{lll}
\boldsymbol{C} & \boldsymbol{\Theta}_{1}\left(\eta_{1}\right) \boldsymbol{\Lambda} & \boldsymbol{\Theta}_{2}\left(\eta_{2}\right) \boldsymbol{\Lambda}
\end{array}\right]\left[\begin{array}{cc}
\boldsymbol{I}_{n} & \mathbf{0} \\
\mathbf{0} & \boldsymbol{T}_{\mathrm{mr}}^{-1}
\end{array}\right]=\left[\begin{array}{ll}
\boldsymbol{C} & \boldsymbol{C}_{2}^{\prime}
\end{array}\right]
$$

Aufgrund der Struktur der Transformationsmatrix $\boldsymbol{T}$ ist weiterhin $\boldsymbol{x}^{\prime}=\boldsymbol{x}$ und die Dynamik des Beobachters (4.71) für das Gesamtsystem lässt sich schreiben als

$$
\begin{aligned}
{\left[\begin{array}{c}
\dot{\hat{\boldsymbol{x}}} \\
\dot{\hat{\boldsymbol{x}}}^{\prime}
\end{array}\right]=} & \left(\left[\begin{array}{cc}
\boldsymbol{A} & \mathbf{0} \\
\mathbf{0} & \boldsymbol{A}_{22}^{\prime}
\end{array}\right]-\left[\begin{array}{c}
\boldsymbol{L}_{1}^{\prime} \\
\boldsymbol{L}_{2}^{\prime}
\end{array}\right]\left[\begin{array}{ll}
\boldsymbol{C} & \boldsymbol{C}_{2}^{\prime}
\end{array}\right]\right)\left[\begin{array}{c}
\hat{\boldsymbol{x}} \\
\hat{\boldsymbol{x}}^{\prime}
\end{array}\right]+\ldots \\
& +\left(\left[\begin{array}{c}
\boldsymbol{B} \\
\mathbf{0}
\end{array}\right]-\left[\begin{array}{c}
\boldsymbol{L}_{1}^{\prime} \\
\boldsymbol{L}_{2}^{\prime}
\end{array}\right] \boldsymbol{D}\right) \boldsymbol{u}+\left[\begin{array}{c}
\boldsymbol{L}_{1}^{\prime} \\
\boldsymbol{L}_{2}^{\prime}
\end{array}\right]\left(\boldsymbol{y}+\boldsymbol{C}_{2}^{\prime} \overline{\boldsymbol{x}}^{\prime}\right) .
\end{aligned}
$$

Für die Dynamik von $\hat{\boldsymbol{x}}$ ergibt sich daraus

$$
\dot{\hat{\boldsymbol{x}}}=\left(\boldsymbol{A}-\boldsymbol{L}_{1}^{\prime} \boldsymbol{C}\right) \hat{\boldsymbol{x}}-\boldsymbol{L}_{1}^{\prime} \boldsymbol{C}_{2}^{\prime} \hat{\overline{\boldsymbol{x}}}^{\prime}+\left(\boldsymbol{B}-\boldsymbol{L}_{1}^{\prime} \boldsymbol{D}\right) \boldsymbol{u}+\boldsymbol{L}_{1}^{\prime} \boldsymbol{y}+\boldsymbol{L}_{1}^{\prime} C_{2}^{\prime} \overline{\boldsymbol{x}}^{\prime}
$$

Mithilfe der Variablentransformation $\boldsymbol{\zeta}=\overline{\boldsymbol{x}}^{\prime}-\hat{\overline{\boldsymbol{x}}}^{\prime}$ lässt sich weiter schreiben

$$
\begin{aligned}
\dot{\hat{\boldsymbol{x}}} & =\left(\boldsymbol{A}-\boldsymbol{L}_{1}^{\prime} \boldsymbol{C}\right) \hat{\boldsymbol{x}}+\left(\boldsymbol{B}-\boldsymbol{L}_{1}^{\prime} \boldsymbol{D}\right) \boldsymbol{u}+\boldsymbol{L}_{1}^{\prime} \boldsymbol{y}+\boldsymbol{L}_{1}^{\prime} \boldsymbol{C}_{2}^{\prime} \boldsymbol{\zeta} \\
& =\boldsymbol{A} \hat{\boldsymbol{x}}+\boldsymbol{B} \boldsymbol{u}+\boldsymbol{L}_{1}^{\prime}(\boldsymbol{y}-\boldsymbol{C} \hat{\boldsymbol{x}}-\boldsymbol{D} \boldsymbol{u})+\boldsymbol{L}_{1}^{\prime} \boldsymbol{C}_{2}^{\prime} \boldsymbol{\zeta} .
\end{aligned}
$$

Für die Dynamik der eingeführten Zustandsvariable $\boldsymbol{\zeta}$ ergibt sich anhand von (4.70) und (4.73)

$$
\begin{aligned}
& \dot{\boldsymbol{\zeta}}=\dot{\overline{\boldsymbol{x}}}^{\prime}-\dot{\overline{\boldsymbol{x}}}^{\prime}= \boldsymbol{A}_{22}^{\prime} \overline{\boldsymbol{x}}^{\prime}+\boldsymbol{L}_{2}^{\prime} \boldsymbol{C} \hat{\boldsymbol{x}}-\left(\boldsymbol{A}_{22}^{\prime}-\boldsymbol{L}_{2}^{\prime} \boldsymbol{C}_{2}^{\prime}\right) \hat{\overline{\boldsymbol{x}}}^{\prime}+\boldsymbol{L}_{2}^{\prime} \boldsymbol{D} \boldsymbol{u}+\ldots \\
& \quad-\boldsymbol{L}_{2}^{\prime} \boldsymbol{y}-\boldsymbol{L}_{2}^{\prime} \boldsymbol{C}_{2}^{\prime} \overline{\boldsymbol{x}}^{\prime} \\
&=\left(\boldsymbol{A}_{22}^{\prime}-\boldsymbol{L}_{2}^{\prime} \boldsymbol{C}_{2}^{\prime}\right) \boldsymbol{\zeta}-\boldsymbol{L}_{2}^{\prime}(\boldsymbol{y}-\boldsymbol{C} \hat{\boldsymbol{x}}-\boldsymbol{D u}) .
\end{aligned}
$$

Ebenfalls durch Einsetzen von (4.70) und (4.73) kann man die generierten Residuen schreiben als

$$
\begin{aligned}
\boldsymbol{r}=\boldsymbol{V}^{\prime}(\tilde{\boldsymbol{y}}-\hat{\tilde{\boldsymbol{y}}}) & =\boldsymbol{V}^{\prime}\left(\boldsymbol{y}+\boldsymbol{C}_{2}^{\prime} \overline{\boldsymbol{x}}^{\prime}-\boldsymbol{C} \hat{\boldsymbol{x}}-\boldsymbol{D u}-C_{2}^{\prime} \hat{\overline{\boldsymbol{x}}}^{\prime}\right) \\
& =\boldsymbol{V}^{\prime}(\boldsymbol{y}-\boldsymbol{C} \hat{\boldsymbol{x}}-\boldsymbol{D} \boldsymbol{u})+\boldsymbol{V}^{\prime} C_{2}^{\prime} \boldsymbol{\zeta}
\end{aligned}
$$


Fasst man (4.74), (4.75) und (4.76) zusammen, so ergibt sich

$$
\begin{aligned}
& \dot{\hat{\boldsymbol{x}}}=\boldsymbol{A} \hat{\boldsymbol{x}}+\boldsymbol{B u}+\boldsymbol{v}, \\
& \boldsymbol{v}=\underbrace{\boldsymbol{L}_{1}^{\prime}}_{\boldsymbol{L}_{p}}(\boldsymbol{y}-\boldsymbol{C} \hat{\boldsymbol{x}}-\boldsymbol{D} \boldsymbol{u})+\underbrace{\boldsymbol{L}_{1}^{\prime} \boldsymbol{C}_{2}^{\prime}}_{\boldsymbol{L}_{i}} \boldsymbol{\zeta}, \\
& \dot{\boldsymbol{\zeta}}=\underbrace{\left(\boldsymbol{A}_{22}^{\prime}-\boldsymbol{L}_{2}^{\prime} \boldsymbol{C}_{2}^{\prime}\right)}_{\boldsymbol{\Phi}} \boldsymbol{\zeta}+\underbrace{\left(-\boldsymbol{L}_{2}^{\prime}\right)}_{\boldsymbol{\Gamma}}(\boldsymbol{y}-\boldsymbol{C} \hat{\boldsymbol{x}}-\boldsymbol{D} \boldsymbol{u}), \\
& \boldsymbol{r}=\underbrace{\boldsymbol{V}^{\prime} C_{2}^{\prime}}_{\boldsymbol{V}_{\boldsymbol{\zeta}}} \boldsymbol{\zeta}+\underbrace{\boldsymbol{V}^{\prime}}_{\boldsymbol{V}_{\boldsymbol{y}}}(\boldsymbol{y}-\boldsymbol{C} \hat{\boldsymbol{x}}-\boldsymbol{D u}) .
\end{aligned}
$$

Dies stellt einen dynamisch erweiterten Beobachter dar.

Bemerkenswert ist, dass durch (4.77) die dynamischen Erweiterungen und die Fehlerisolationseigenschaft des Beobachters gleichzeitig realisiert werden. Da alle Eigenwerte des statischen Beobachters für das erweiterte System in der offenen linken s-Halbebene platziert werden können, ist die Stabilität des DFIOs gewährleistet.

\subsubsection{Zusammenfassung des Entwurfsverfahrens}

Nach der ausführlichen Herleitung wird das Entwurfsverfahren auf den folgenden Seiten zusammengefasst. Eine grafische Übersicht über den Entwurf zeigt Abbildung 4.14. 
Gegeben sei ein System der Form

$$
\begin{aligned}
& \dot{\boldsymbol{x}}=\boldsymbol{A} \boldsymbol{x}+\boldsymbol{B} \boldsymbol{u}+\boldsymbol{E}_{a} \boldsymbol{f}, \\
& \boldsymbol{y}=\boldsymbol{C} \boldsymbol{x}+\boldsymbol{D} \boldsymbol{u}+\boldsymbol{E}_{s} \boldsymbol{f},
\end{aligned}
$$

welches die Annahmen 4.9-4.13 erfüllt und das über $\mu_{\mathrm{grHE}}$ invariante Nullstellen in der abgeschlossenen rechten s-Halbebene verfügt. Setze $\kappa_{i}:=0$ für alle $i=1, \ldots, n_{f}$ und durchlaufe das folgende Schema:

1. Überprüfe anhand von

$$
\boldsymbol{\Pi}(\eta) \cdot\left[\begin{array}{c}
\boldsymbol{z}_{x} \\
\boldsymbol{z}_{f}
\end{array}\right]=\mathbf{0}
$$

ob es sich bei den invarianten Nullstellen in der geschlossenen rechten s-Halbebene um nicht verkoppelnde Nullstellen handelt, das heißt, ob $\boldsymbol{z}_{f}$ genau ein Nichtnullelement enthält. Ist dies für das $i$-te Element von $\boldsymbol{z}_{f}$ der Fall, setze $\kappa_{i}:=\kappa_{i}+1$.

2. Für alle $\mu_{\mathrm{grHE}}$ Nullstellen des Systems in der geschlossenen rechten s-Halbebene, die keine nicht verkoppelnden Nullstellen sind:

a) Erweitere das System dynamisch gemäß Abbildung 4.13 mit

$$
\begin{aligned}
& \overline{\boldsymbol{A}}=\eta \boldsymbol{I}_{\alpha-1}, \\
& \overline{\boldsymbol{C}}=\left(\boldsymbol{C}\left(\eta \boldsymbol{I}_{n}-\boldsymbol{A}\right)^{-1} \boldsymbol{E}_{a}+\boldsymbol{E}_{s}\right) \boldsymbol{F}\left[\begin{array}{ll}
\boldsymbol{I}_{\alpha} & \mathbf{0}_{\alpha,\left(n_{f}-\alpha\right)}
\end{array}\right]^{\top} \cdot \boldsymbol{\Lambda},
\end{aligned}
$$

wobei die Matrix $\boldsymbol{F}$ so gewählt wird, dass die ersten $\alpha$ Elemente von $\boldsymbol{z}_{f}$ in (4.78) Nichtnullelemente sind und $\boldsymbol{\Lambda}$ eine beliebige Matrix vollen Ranges ist.

b) Setze $\kappa_{i}:=\kappa_{i}+1$ für alle Kanäle $i$, in denen das $i$-te Element von $\boldsymbol{z}_{f}$ in $(4.59 \mathrm{~b})$ ungleich 0 ist.

c) Führe gegebenenfalls eine Ähnlichkeitstransformation nach Lemma 4.10 durch, falls die Matrizen $\tilde{\boldsymbol{A}}, \tilde{\boldsymbol{B}}, \tilde{\boldsymbol{E}}_{a}, \tilde{\boldsymbol{C}}, \tilde{\boldsymbol{D}}$ und $\tilde{\boldsymbol{E}}_{s}$ des erweiterten Gesamtsystems nicht rein reell sind.

3. Entwirf mittels Satz 4.2 einen statischen FIO für das resultierende Gesamtsystem. Dabei werden mittels (4.22) für den $i$-ten Übertragungskanal $\delta_{i}+\kappa_{i}$ Pole vorgegeben. Invariante Nullstellen in der offenen linken s-Halbebene werden gemäß (4.24) mittels nichtsteuerbarer Beobachtereigenwerte kompensiert.

4. Realisiere den statischen FIO für das virtuelle Gesamtsystem mittels Satz 4.7 durch einen dynamischen FIO für das reale System. 


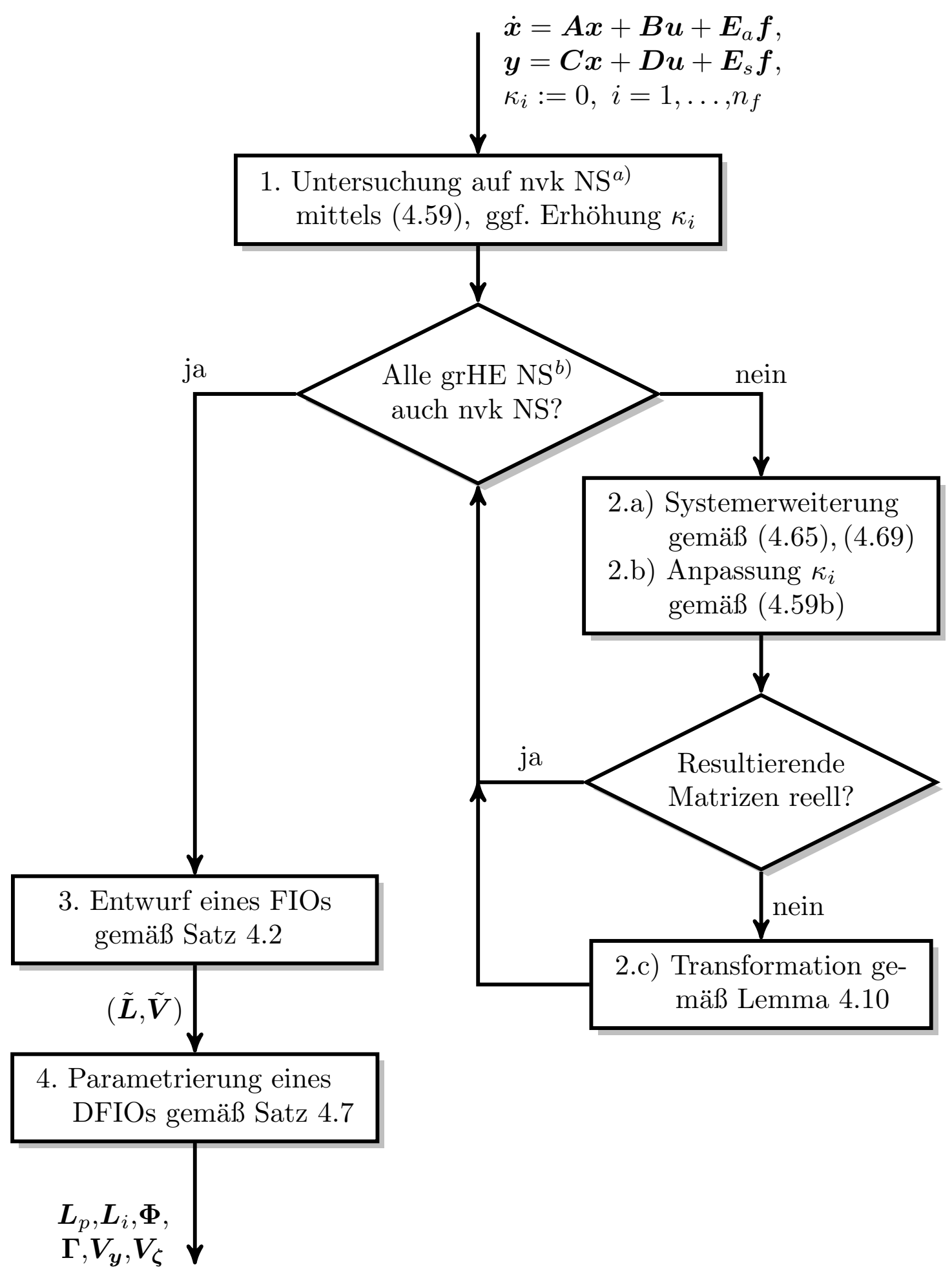

Abbildung 4.14: Ablaufplan des Entwurfs von DFIOs für nicht minimalphasige Systeme

${ }^{a)}$ Die Abkürzung $n v k N S$ steht für nicht verkoppelnde Nullstellen.

${ }^{b)}$ Die Abkürzung grHE NS steht für Nullstellen in der geschlossenen rechten sHalbebene. 
Bemerkung 4.8. Wie zuvor ausführlich dargestellt, sind die dynamischen Erweiterungen notwendig, um stabile FIOs für nicht minimalphasige Systeme entwerfen zu können (mit Ausnahme des Spezialfalles nicht verkoppelnder Nullstellen in der geschlossenen rechten s-Halbebene). Es kann jedoch auch nützlich sein, das Verfahren in minimalphasigen Systemen anzuwenden. Dabei bleiben invariante Nullstellen, welche in der offenen linken s-Halbebene liegen, unkompensiert.

Dies kann verschiedene Vorteile haben. Weist ein System zum Beispiel invariante Nullstellen auf, die zwar einen negativen Realteil haben, jedoch nah an der imaginären Achse liegen, so wird ein entsprechender statischer FIO mindestens einen sehr langsamen Eigenwert besitzen. Dies führt zu einem langsamen Abklingen eventuell vorhandener Anfangsfehler $\boldsymbol{\xi}(0)=\boldsymbol{x}(0)-\hat{\boldsymbol{x}}(0) \neq \mathbf{0}$ im Beobachter. Ein dynamischer FIO, welcher die entsprechende Nullstelle unkompensiert lässt, kann durch die frei wählbaren Eigenwerte auf schnelleres Abklingen von Anfangsauslenkungen ausgelegt werden.

Weiterhin führen invariante Nullstellen, die weit links in der linken s-Halbebene liegen, beim Entwurf von statischen FIOs zu betragsmäßig großen Eigenwerten. Diese können sowohl hinsichtlich der Rauschverstärkung als auch in Bezug auf die Implementierung Probleme bereiten, weswegen es vorteilhaft sein kann, sie nicht zu kompensieren.

Bemerkung 4.9. Verfügt das System nicht nur über invariante Nullstellen in der abgeschlossenen rechten s-Halbebene, sondern ist darüber hinaus auch die Fehlerdetektionsmatrix $\boldsymbol{D}^{*}$ singulär, so lassen sich die Ergebnisse aus diesem Abschnitt und Abschnitt 4.7 kombinieren. In einem ersten Schritt wird dabei das System derart erweitert, dass für das resultierende Gesamtsystem die Fehlerdetektionsmatrix invertierbar ist. Wie in Abschnitt 4.7 erörtert bleiben dadurch die invarianten Nullstellen unverändert. Im zweiten Schritt wird das System dann zusätzlich erweitert, um die invarianten Nullstellen in der rechten s-Halbebene in nicht verkoppelnde Nullstellen zu verwandeln. Für das letztendlich resultierende Gesamtsystem lässt sich dann wieder ein statischer FIO entwerfen, der als dynamischer FIO für das ursprüngliche System implementiert werden kann.

\subsubsection{Beispiel Verladebrücke}

Als Beispielsystem für die Fehlerisolation in einem nicht minimalphasigen System dient der Verladebrückenprüfstand, der in Abbildung 4.15 gezeigt 


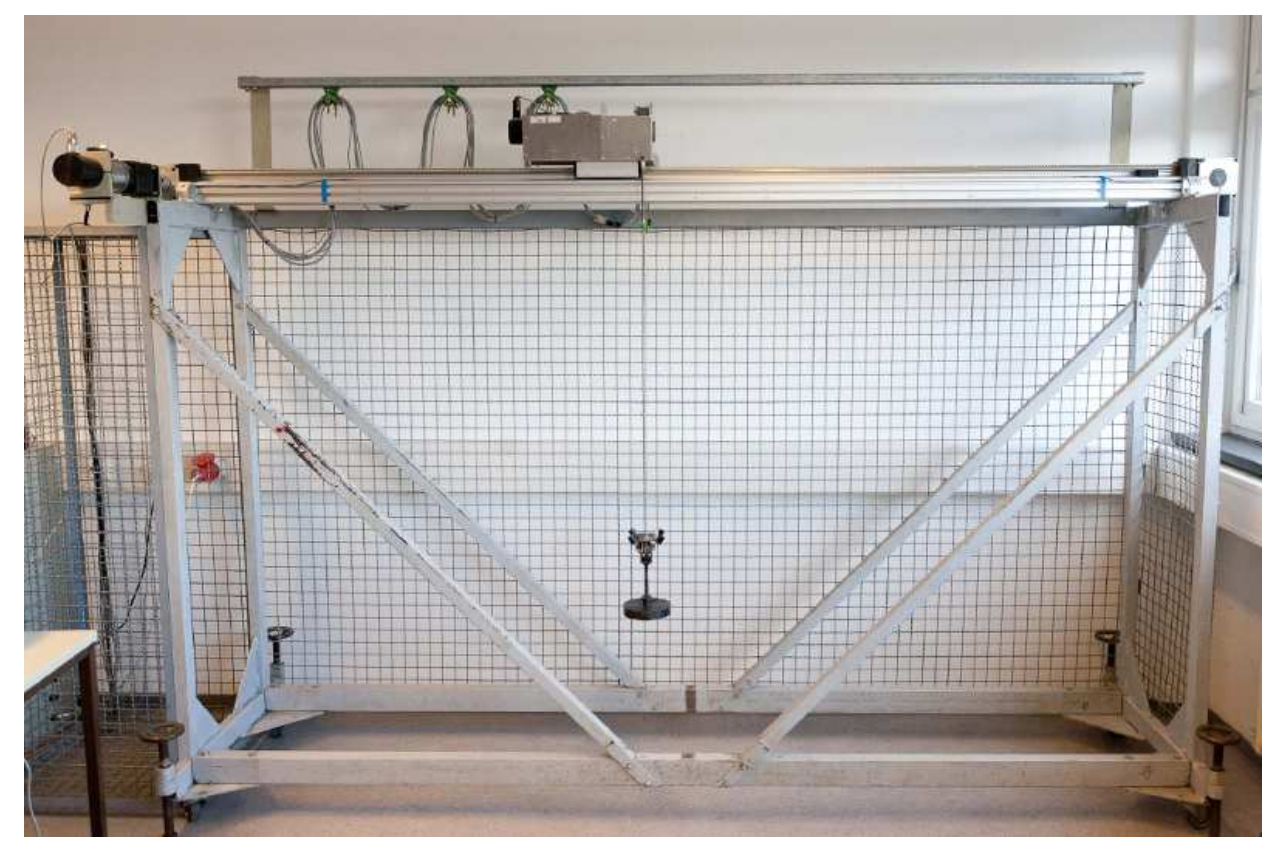

Abbildung 4.15: Verladebrücke

ist. Die Laufkatze wird von einem Gleichstrommotor angetrieben und kann somit in horizontaler Richtung verfahren werden. An einem Seil ist eine Lastmasse angebracht, die es typischerweise zu positionieren gilt. In guter Näherung kann die Verladebrücke als ein lineares System 4. Ordnung modelliert werden, wobei sich der Zustandsvektor aus Position und Geschwindigkeit der Laufkatze sowie Pendelwinkel und -winkelgeschwindigkeit zusammensetzt. Die Eingangsspannung $u$ des Gleichstrommotors stellt die einzige Stellgröße des Systems dar $\left(n_{u}=1\right)$. Das lineare Zustandsraummodell ist in Abhängigkeit von den Systemparametern wie Seillänge, Lastmasse usw. in [93] angegeben. Mit den Prüfstandsparametern ergeben sich folgende Matrizen zur Beschreibung der Systemdynamik:

$$
\boldsymbol{A}=\left[\begin{array}{cccc}
0 & 1 & 0 & 0 \\
0 & -70,31 & -0,97 & 0 \\
0 & 0 & 0 & 1 \\
0 & -30,31 & -11,75 & 0
\end{array}\right], \quad \boldsymbol{B}=\left[\begin{array}{c}
0 \\
5,89 \\
0 \\
5,89
\end{array}\right]
$$

Gemessen werden können lediglich die Position der Laufkatze und der Pendelwinkel $\left(n_{y}=2\right)$. Folglich ist die Ausgangsmatrix gegeben durch

$$
\boldsymbol{C}=\left[\begin{array}{llll}
0 & 0 & 1 & 0 \\
1 & 0 & 0 & 0
\end{array}\right]
$$


Es werden zwei potentielle Fehler im System modelliert $\left(n_{f}=2\right)$. Der erste Fehler repräsentiert einen Fehler in der Messung des Pendelwinkels, es handelt sich also um einen Sensorfehler. Der zweiten Fehler ist ein klassischer Aktorfehler in der auf die Laufkatze wirkende Kraft. Somit sind die Fehlereingangsmatrizen gegeben durch

$$
\boldsymbol{E}_{a}=\left[\begin{array}{cc}
0 & 0 \\
0 & 5,89 \\
0 & 0 \\
0 & 5,89
\end{array}\right], \quad \boldsymbol{E}_{s}=\left[\begin{array}{ll}
0 & 0 \\
1 & 0
\end{array}\right]
$$

Da $f_{1}$ ein Sensorfehler ist, ergibt sich $\delta_{1}=0$, nach Definition 4.2 gilt für den zweiten Fehler $\delta_{2}=2$. Die weitere Analyse zeigt, dass $(\boldsymbol{A}, \boldsymbol{C})$ vollständig beobachtbar ist und dass die Fehlerdetektionsmatrix $\boldsymbol{D}^{*}$ invertierbar ist. Des Weiteren verfügt das System über ein konjugiert komplexes Paar invarianter Nullstellen $\eta_{1 / 2}= \pm 3,28 j$. Da sich dieses Nullstellenpaar auf der imaginären Achse befindet, ist der Entwurf eines stabilen FIOs gemäß Abschnitt 4.5 nicht möglich. Die Anzahl der invarianten Nullstellen ist jedoch endlich, es liegt keine Nullstelle bei $\eta=0$ vor und darüber hinaus fällt keine invariante Nullstelle mit den Eigenwerten der Strecke zusammen, die bei $\lambda_{1}=0, \lambda_{2}=-70,30$ und $\lambda_{3 / 4}=-0,007 \pm 3,284 j$ liegen. Somit sind die Annahmen 4.9 bis 4.13 erfüllt und es kann ein stabiler DFIO entworfen werden.

Der Entwurf erfolgt gemäß des Schemas aus Abschnitt 4.8.3 beziehungsweise Abbildung 4.14. Zunächst ergibt die Auswertung von (4.59) für $\eta_{1}$ und $\eta_{2}$, dass keine der beiden Nullstellen zur Klasse der nicht verkoppelnden Nullstellen gehört. Dementsprechend muss das System zweimal dynamisch erweitert werden. Für $\eta_{1}$ sind alle Elemente von $\boldsymbol{z}_{f}$ in (4.59b) ungleich 0, sodass in beiden Übertragungskanälen ein zusätzlicher Pol vorgegeben werden kann. Weiterhin ist $\alpha=2$, sodass $\overline{\boldsymbol{A}}_{1}=3,28 \mathrm{j}$ angesetzt wird. Zur Bestimmung von $\overline{\boldsymbol{C}}_{1}$ wird $\boldsymbol{\Lambda}=\left[\begin{array}{ll}1 & 5\end{array}\right]^{\top}$ gewählt, es ergibt sich $\overline{\boldsymbol{C}}_{1}=\left[\begin{array}{ll}0 & 31,34\end{array}\right]^{\top}$. Die Wahl von $\boldsymbol{\Lambda}$ beeinflusst ausschließlich die Parametrierung des resultierenden DFIOs und nicht das Übertragungsverhalten $\boldsymbol{G}_{\boldsymbol{r} \boldsymbol{f}}(s)$. Für die zweite invariante Nullstelle erfolgt die Systemerweiterung analog, auch hier kann für jeden Kanal ein zusätzlicher Pol vorgegeben werden. Aus den beiden Systemerweiterungen ergibt sich insgesamt

$$
\overline{\boldsymbol{A}}=\left[\begin{array}{cc}
3,28 j & 0 \\
0 & -3,28 j
\end{array}\right], \quad \overline{\boldsymbol{C}}=\left[\begin{array}{cc}
0 & 0 \\
31,34 & 31,34
\end{array}\right] .
$$

Da es sich bei $\overline{\boldsymbol{A}}$ um eine komplexe Matrix handelt, wird das Gesamtsystem 
mithilfe von Lemma 4.10 transformiert, woraus sich

$$
\boldsymbol{A}^{\prime}=\left[\begin{array}{cc}
0 & 3,28 \\
-3,28 & 0
\end{array}\right], \quad \boldsymbol{C}^{\prime}=\left[\begin{array}{cc}
0 & 0 \\
62,68 & 0
\end{array}\right]
$$

ergibt. Für das derart erweiterte System wird nun mittels Satz 4.2 ein FIO entworfen, wobei die Pole des ersten Übertragungskanals zu $\lambda_{B_{1,1}}=-3,2$ und $\lambda_{B_{1,2}}=-3,3$ gewählt werden. Für den zweiten Kanal werden $\lambda_{B_{2,1}}=-8,0, \lambda_{B_{2,2}}=-8,2, \lambda_{B_{2,3}}=-8,5$ und $\lambda_{B_{2,4}}=-9,0$ vorgegeben. $\mathrm{Zu}$ beachten ist, dass die invarianten Nullstellen des Systems unkompensiert bleiben. Die statische Verstärkung wird in beiden Kanälen zu $g_{i, i}(s=0)=1$ gewählt. Im letzten Schritt wird anhand von Satz 4.7 eine DFIO-Parametrierung ermittelt, die genauen Zahlenwerte finden sich in Anhang C.1.4.

Der DFIO wird am Prüfstand in einer SIMULINK ${ }^{\circledR}$-Umgebung realisiert, welche über entsprechende Schnittstellen auch das Einlesen von Sensorwerten und die Ausgabe von Stellsignalen übernimmt. Getestet wird ein Fehlerszenario, bei dem nach 1 s ein sprungförmiger Sensorfehler $f_{1}=2$ auftritt und bei dem zusätzlich nach $2 \mathrm{~s}$ ein Aktorfehler $f_{2}=1$ aktiv ist. Beide Fehler lassen sich am Prüfstand definiert einbringen, indem jeweils
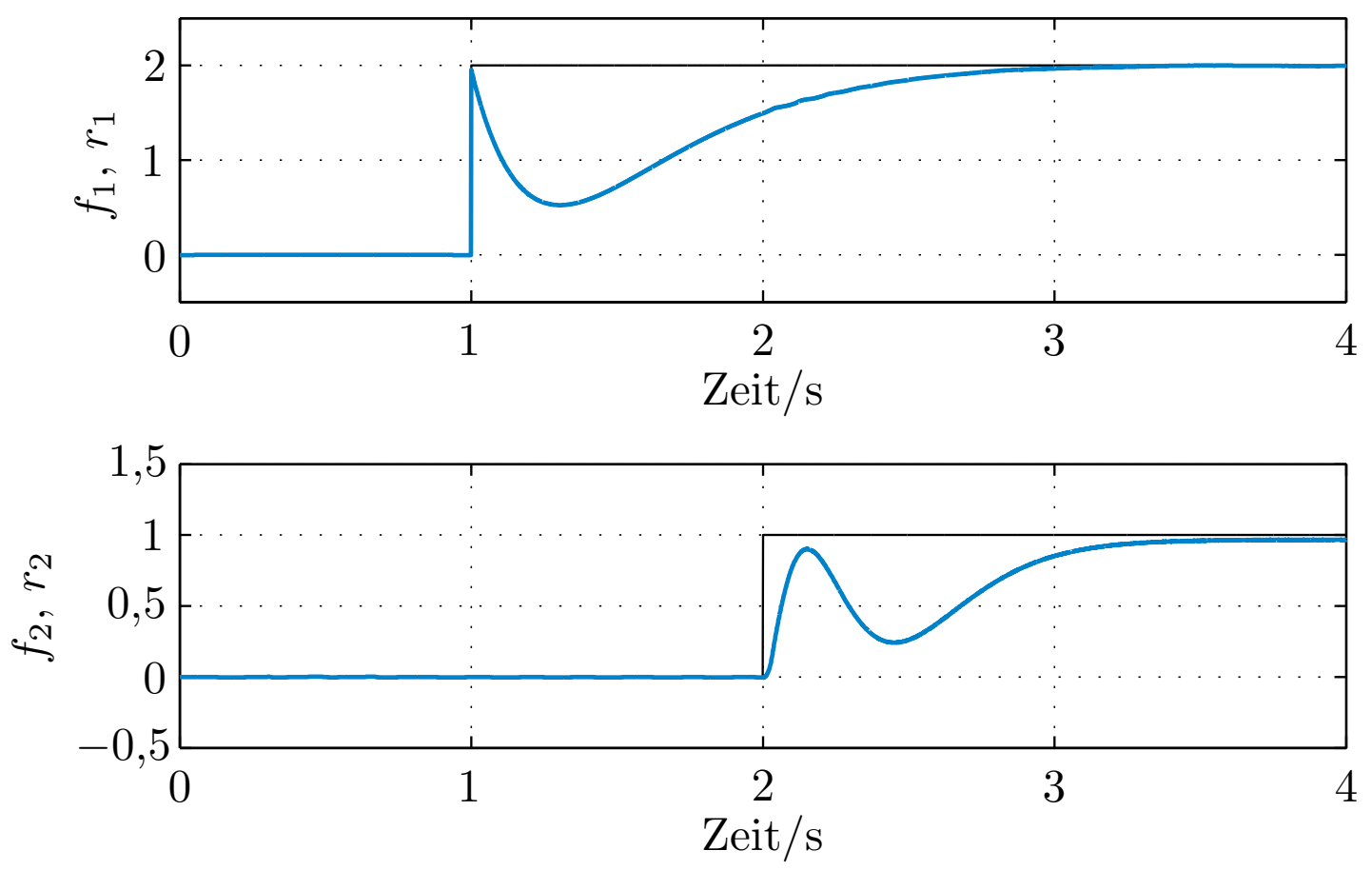

Abbildung 4.16: Eingebrachte Fehler (-) und generierte Residuen (-) an der Verladebrücke mit Sensorfehler 
ein künstlicher Offset auf das Messsignal des Pendelwinkelsensors beziehungsweise das Stellsignal $u$ addiert wird. Abbildung 4.16 zeigt den zeitlichen Verlauf der eingebrachten Fehler und der generierten Residuen. Zu betonen ist, dass es sich dabei um Residuenverläufe handelt, die online und unter Verwendung realer Messdaten generiert wurden. Die Residuenverläufe weisen das für nicht minimalphasige Systeme typische Überbeziehungsweise Unterschwingen auf, wobei eine nahezu ideale Fehlerisolation erreicht wird. Beim Auftreten des ersten Fehlers schlägt das zweite Residuum nicht erkennbar aus, ebenso beeinflusst der zweite Fehler den Verlauf des ersten Residuums nicht. Der erste Fehler wird durch das entsprechende Residuum stationär genau abgebildet, beim zweiten Fehler tritt lediglich ein geringer Offset auf.

\subsubsection{Beispiel DHC-2 Beaver}

An dieser Stelle wird das Beispiel der DHC-2 Beaver aus Abschnitt 4.7.5 aufgegriffen, um die Bemerkungen 4.8 und $4.9 \mathrm{zu}$ veranschaulichen. Wie aus Abschnitt 4.7.5 bekannt ist, kann für das Modell der Lateraldynamik der DHC-2 Beaver kein statischer FIO entworfen werden, was sich jedoch durch eine entsprechende Systemerweiterung beheben lässt. Allerdings verfügt das System über eine invariante Nullstelle bei $\eta=-58,48$, die beim Entwurf eines FIOs für das erweiterte System kompensiert wird. Wie in Bemerkung 4.8 erörtert kann eine derart weit links in der s-Halbebene gelegene Nullstelle bei verrauschten Messsignalen zu Problemen führen. Um dies zu demonstrieren, wird die Ausgangsgleichung des Systems erweitert $\mathrm{zu}$

$$
\boldsymbol{y}=\boldsymbol{C x}+\boldsymbol{v},
$$

wobei $\boldsymbol{v}$ mittelwertfreies Gauß'sches Rauschen repräsentiert. In Abbildung 4.17 wird das gleiche Fehlerszenario simuliert wie in Abschnitt 4.7.5. Allerdings ist aufgrund der stark verrauschten Residuensignale (blaue Kurven) keine Fehlerisolation möglich, wie insbesondere der Verlauf des ersten Residuums zeigt. Im fehlerfreien Fall erreicht das Residuum Werte von $r_{1}>0,5$, die aufgrund des Rauschens im fehlerbehafteten Fall zum Teil unterschritten werden. Es lässt sich also kein Schwellenwert finden, der eine Erkennung beziehungsweise Isolation des Fehlers ermöglicht.

Entsprechend Bemerkung 4.9 wird daher eine zusätzliche Systemerweiterung vorgenommen, die es ermöglicht, die invariante Nullstelle des Systems unkompensiert zu lassen. Dazu wird in einem ersten Schritt die gleiche Systemerweiterung durchgeführt wie in Abschnitt 4.7.5. Diese führt auf das 
erweiterte System $\left(\tilde{\boldsymbol{A}}, \tilde{\boldsymbol{E}}_{a}, \tilde{\boldsymbol{C}}, \tilde{\boldsymbol{E}}_{s}\right)$ der Form (4.42), für das sich ein stabiler, statischer FIO entwerfen lässt. Auch das erweiterte System weist die invariante Nullstelle $\eta=-58,48$ auf, für die das System erneut erweitert wird. Es ergibt sich gemäß (4.42) und (4.61) ein Gesamtsystem der Form

$$
\begin{aligned}
& \dot{z}=\left[\begin{array}{ccc}
A & \mathbf{0} & \mathbf{0} \\
\boldsymbol{H}_{1} \boldsymbol{C} & \mathbf{0} & \mathbf{0} \\
\mathbf{0} & \mathbf{0} & \overline{\boldsymbol{A}}
\end{array}\right] \boldsymbol{z}+\left[\begin{array}{c}
\boldsymbol{B} \\
\boldsymbol{H}_{1} \boldsymbol{D} \\
\mathbf{0}
\end{array}\right] \boldsymbol{u}+\left[\begin{array}{c}
\boldsymbol{E}_{a} \boldsymbol{F} \\
\boldsymbol{H}_{1} \boldsymbol{E}_{s} \boldsymbol{F} \\
\mathbf{0}
\end{array}\right] \boldsymbol{f}, \\
& \tilde{\boldsymbol{y}}=\left[\begin{array}{ccc}
\mathbf{0} & \boldsymbol{I}_{\alpha} & \bar{C} \\
\boldsymbol{H}_{2} \boldsymbol{C} & \mathbf{0}
\end{array}\right] \boldsymbol{z}+\left[\begin{array}{c}
\mathbf{0} \\
\boldsymbol{H}_{2} \boldsymbol{D}
\end{array}\right] \boldsymbol{u}+\left[\begin{array}{c}
\mathbf{0} \\
\boldsymbol{H}_{2} \boldsymbol{E}_{s} \boldsymbol{F}
\end{array}\right] \boldsymbol{f} .
\end{aligned}
$$

Dabei entsprechen $\boldsymbol{H}$ und $\boldsymbol{F}$ den Werten aus Abschnitt 4.7.5 und die Analyse der invarianten Nullstelle nach (4.59) ergibt, dass alle Elemente von $\boldsymbol{z}_{f} \in \mathbb{R}^{2}$ in (4.59b) ungleich 0 sind. Daher ist $\overline{\boldsymbol{A}}=-58,48$ und mit der Wahl von $\boldsymbol{\Lambda}=\left[\begin{array}{ll}50 & 1\end{array}\right]^{\top}$ ergibt sich weiterhin $\overline{\boldsymbol{C}}=\left[\begin{array}{ll}-0,006 & -0,236\end{array}\right]^{\top}$, wodurch das erweiterte Gesamtsystem eindeutig festgelegt ist. Gemäß (4.59)
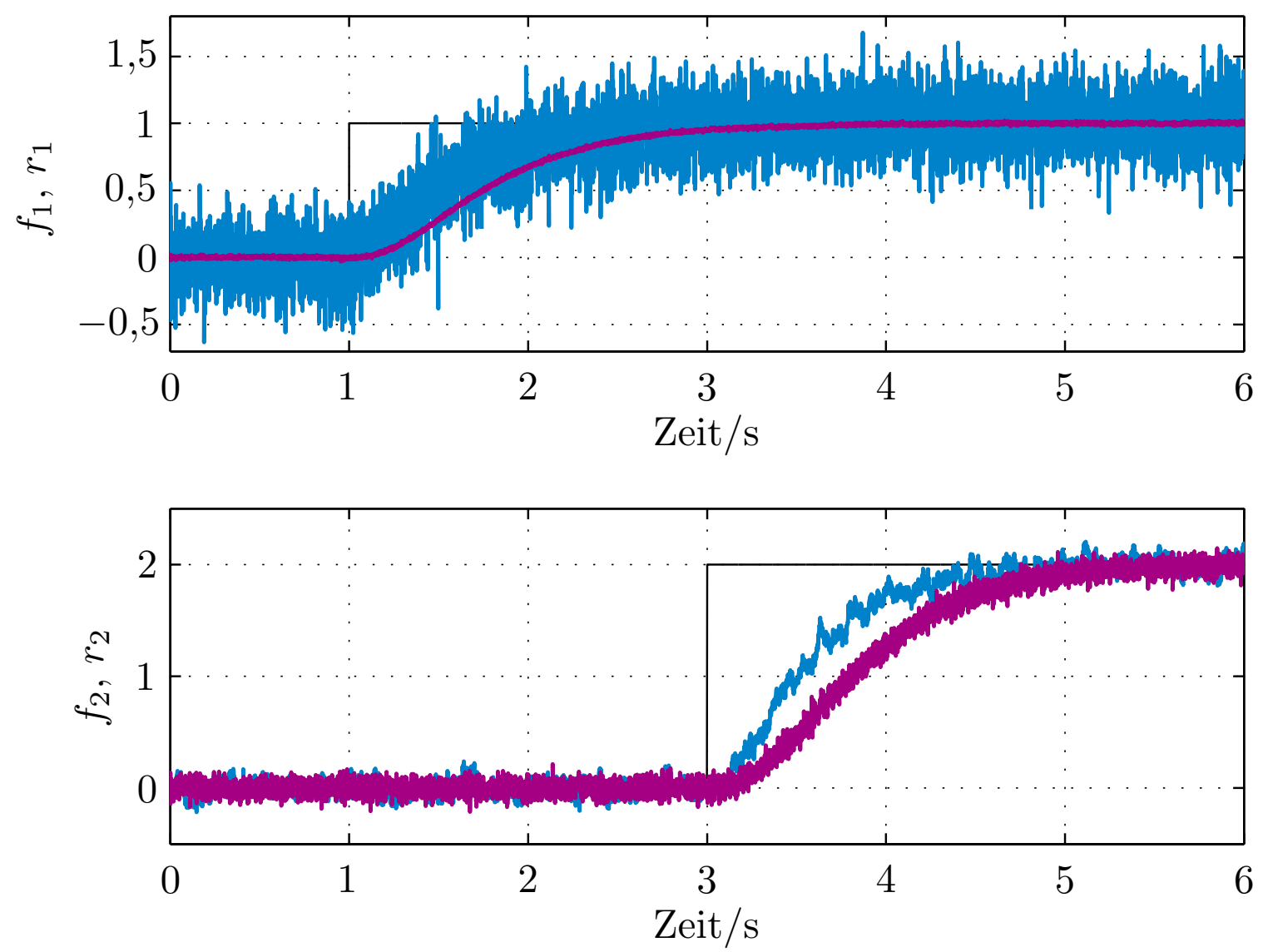

Abbildung 4.17: Fehler (-) und generierte Residuen der Lateraldynamik der DHC-2 Beaver bei einfacher (-) und doppelter (-) Systemerweiterung 
wird für jeden Übertragungskanal ein zusätzlicher Pol vorgegeben, es werden $\lambda_{B_{1,1}}=-2, \lambda_{B_{1,2}}=-4, \lambda_{B_{1,3}}=-7$ und $\lambda_{B_{2,1}}=-3, \lambda_{B_{2,2}}=-3,2$ und $\lambda_{B_{2,3}}=-3,5$ gewählt. Die Beobachterparametrierung für das zweifach erweiterte Gesamtsystem findet sich in Anhang C.1.5.

In Abbildung 4.17 sind in magenta die Residuen des FIOs für das doppelt erweiterte System gezeigt. Es ist ersichtlich, dass sich mittels des so entworfenen DFIOs wesentlich weniger verrauschte Residuen ergeben, mithilfe derer eine Fehlerisolation möglich ist. Die Ordnung des Fehlerisolationssystems wird lediglich um 1 erhöht, wobei sich die Qualität der generierten Residuen erheblich verbessert. Grundsätzlich ließe sich ein vergleichbares Übertragungsverhalten zwischen Fehlern und Residuen auch durch eine Tiefpassfilterung der vom einfach erweiterten DFIO generierten Residuen (blaue Kurven in Abbildung 4.17) erreichen. Abgesehen von möglichen Implementierungsproblemen durch die stark verrauschten, ungefilterten Residuensignale wäre hierfür jedoch für jedes Residuum ein eigener Tiefpass nötig. Dies bedingt ein Fehlerisolationssystem der Ordnung $(n+1)+2=n+3$, wohingegen durch die zusätzliche Systemerweiterung, welche die invariante Nullstelle $\eta=-58,48$ unkompensiert lässt, lediglich ein Fehlerisolationssystem der Ordnung $n+2 \mathrm{zu}$ implementieren ist. Der Implementierungsaufwand ist somit geringer als bei einer klassischen Tiefpassfilterung der einzelnen Residuensignale.

Eine detailliertere Betrachtung der Robustheitsproblematik von FIOs erfolgt in Kapitel 5.

\subsubsection{Fazit}

Mittels der vorgestellten dynamischen Erweiterungen ist es möglich, die Anforderungen an das System für den Entwurf von Fehlerisolationsbeobachtern weiter abzuschwächen. Dies erlaubt den Entwurf auch für nicht minimalphasige Systeme. Grundlage dafür stellen die Ergebnisse zu nicht minimalphasigen Systemen im Bereich der linearen Entkopplungsregelungen in Verbindung mit der in Abschnitt 4.4 herausgearbeiteten Dualität dar. Auch eine direkte Berücksichtigung von Sensorfehlern ist möglich, wobei diese häufig invariante Nullstellen des Systems mit sich bringen. Das vorgestellte Verfahren zur systematischen Parametrierung von dynamisch erweiterten Fehlerisolationsbeobachtern erlaubt die Realisierung der Systemerweiterungen durch rein reelle dynamische Systeme niedriger Ordnung. Festzustellen ist aber auch, dass sich der Entwurf ebenso wie für statisch nicht isolierbare Systeme (s. Abschnitt 4.7) gegenüber den statischen FIOs aus Abschnitt 4.5 in nicht unerheblichem Maße verkompliziert. 


\subsection{Vollständige Fehlerisolation in nichtquadratischen Systemen}

In den Abschnitten 4.5 bis 4.8 wurden quadratische Systeme betrachtet. Im Weiteren konzentrieren sich die Betrachtungen auf nichtquadratische Systeme, in denen $n_{y}>n_{f}$ gilt. Es stehen also mehr Messgrößen zur Verfügung, als nach den Ergebnissen von Abschnitt 4.5 für eine vollständige Fehlerisolation nötig sind. ${ }^{7)}$ Daraus ergibt sich intuitiv, dass im Allgemeinen gegenüber dem quadratischen Fall zusätzliche Freiheitsgrade vorhanden sein sollten.

Beachtenswert ist dabei der Rang der Ausgangsmatrix $C$. Gilt rang $(\boldsymbol{C})=n_{f}$, so sind $n_{y}-n_{f}$ Messgrößen redundant, wenn sich auch die jeweiligen Zeilen von $\boldsymbol{E}_{s}$ durch entsprechende Linearkombinationen erzeugen lassen. Es ist dann $\operatorname{rang}\left(\left[\begin{array}{ll}\boldsymbol{C} & \boldsymbol{E}_{s}\end{array}\right]\right)=n_{f}$ und die zusätzlichen Ausgänge stellen strukturell keine zusätzliche Information für die Fehlerisolation zur Verfügung. Hinsichtlich der Robustheit bezüglich exogener Störungen lassen sich die zusätzlichen messbaren Ausgangssignale jedoch gewinnbringend einsetzen, wie in Abschnitt 5.4 gezeigt wird. Für den Fall $\operatorname{rang}(\boldsymbol{C})>n_{f}$ beziehungsweise sogar $\operatorname{rang}(\boldsymbol{C})=n_{y}$ wird durch die zusätzlichen, linear unabhängigen Messungen strukturell neue Information für die Fehlerisolation gewonnen. Obwohl sich aufgrund dieser zusätzlichen Information das Fehlerisolationsproblem intuitiv einfacher gestalten sollte, ist die umfassende theoretische Behandlung weitaus komplizierter. Wie bereits in Abschnitt 3.4 für das duale Problem des Entkopplungsreglerentwurfs diskutiert, ist die Charakterisierung von stabil fehlerisolierbaren Systemen mit rang $(\boldsymbol{C})>n_{f}$ anhand von notwendigen und hinreichenden Bedingungen ein bisher ungelöstes Problem. Im Rahmen dieser Arbeit wird daher auf hinreichende Bedingungen zurückgegriffen, welche die Existenz eines stabilen FIOs garantieren. Diese entsprechen weitgehend den Annahmen 4.1 bis 4.3 , sie werden der Übersichtlichkeit halber hier nochmals aufgeführt:

Annahme 4.14. Das Paar $(\boldsymbol{A}, \boldsymbol{C})$ ist vollständig beobachtbar.

Annahme 4.15. Die Fehlerdetektionsmatrix hat vollen Rang, das heißt es gilt rang $\left(\boldsymbol{D}^{*}\right)=n_{f}$.

Annahme 4.16. Das System $\left(\boldsymbol{A}, \boldsymbol{E}_{a}, \boldsymbol{C}, \boldsymbol{E}_{s}\right)$ ist minimalphasig und weist genau $\mu$ invariante Nullstellen auf.

${ }^{7)}$ Solche Systeme werden in der englischsprachigen Literatur auch als fat systems bezeichnet, um sie explizit von nichtquadratischen Systemen mit $n_{y}<n_{f}$ abzugrenzen. 
Im Falle redundanter Messungen entsprechen die Annahmen den Voraussetzungen 4.1 bis 4.3 aus Abschnitt 4.5. Im allgemeineren Fall rang $(\boldsymbol{C})>n_{f}$ sind die Annahmen für nichtquadratische Systeme wenig restriktiv. Ist insbesondere ein quadratisches, statisch stabil fehlerisolierbares System gegeben und wird es um eine zusätzliche, linear unabhängige Messgröße erweitert, so sind die Annahmen 4.14 bis 4.16 offensichtlich erfüllt. Darüber hinaus weisen nichtquadratische Systeme nur in seltenen Fällen invariante Nullstellen auf [130], sodass Minimalphasigkeit meist sichergestellt ist.

Ziel dieses Abschnittes ist es, die sich in nichtquadratischen Systemen ergebenden Freiheitsgrade herauszuarbeiten und die Beobachtermatrizen $(\boldsymbol{L}, \boldsymbol{V})$ in Abhängigkeit von den Freiheitsgraden zu parametrieren. Dies erlaubt im weiteren Verlauf die gezielte Ausnutzung der Freiheitsgrade für sekundäre Entwurfsziele, insbesondere zur Robustheitssteigerung.

\subsubsection{Entwurf im Zeitbereich}

Für den Entwurf eines FIOs für ein nichtquadratisches System im Zeitbereich ergeben sich weitgehend analoge Überlegungen wie im quadratischen Fall (s. Abschnitt 4.5.1). Nach Übergang auf das duale System $(\overline{\boldsymbol{A}}, \overline{\boldsymbol{B}}, \overline{\boldsymbol{C}}, \overline{\boldsymbol{D}})$ ist auch hier die Bedingung

$$
\bar{M} \bar{\xi}+\bar{D}^{*} \bar{u}=\bar{N} f
$$

mit $\overline{\boldsymbol{D}}^{*}, \overline{\boldsymbol{M}}$ und $\overline{\boldsymbol{N}}$ aus (4.11) beziehungsweise (4.13). Allerdings handelt es sich nun bei der Entkoppelbarkeitsmatrix für das duale System, $\overline{\boldsymbol{D}}^{*} \in \mathbb{R}^{n_{f} \times n_{y}}$, um eine nichtquadratische Matrix. Da sie nach Annahme 4.15 ebenso wie $\boldsymbol{D}^{*}$ vollen Rang hat, lösen alle durch

$$
\begin{aligned}
& \overline{\boldsymbol{K}}=\overline{\boldsymbol{D}}^{*+} \overline{\boldsymbol{M}}+\overline{\boldsymbol{D}}^{* \perp} \cdot \overline{\boldsymbol{R}}_{K}, \\
& \overline{\boldsymbol{F}}=\overline{\boldsymbol{D}}^{*+} \overline{\boldsymbol{N}}+\overline{\boldsymbol{D}}^{* \perp} \cdot \overline{\boldsymbol{R}}_{F}
\end{aligned}
$$

gegebenen Matrizen mit $\overline{\boldsymbol{u}}=-\overline{\boldsymbol{K}} \overline{\boldsymbol{\xi}}+\overline{\boldsymbol{F}} \boldsymbol{f}$ die Gleichung (4.79). Dabei sind $\overline{\boldsymbol{R}}_{K}$ und $\overline{\boldsymbol{R}}_{F}$ beliebige Matrizen. Die Beobachterparametrierung für das ursprüngliche System ergibt sich dann nach der Rücksubstitution $\boldsymbol{R}_{L} \leftarrow \overline{\boldsymbol{R}}_{K}$, $\boldsymbol{R}_{V} \leftarrow \overline{\boldsymbol{R}}_{F}$ gemäß

Satz 4.8 (FIO-Entwurf für nichtquadratische Systeme im Zeitbereich). Gegeben sei ein System der Form

$$
\begin{aligned}
& \dot{\boldsymbol{x}}=\boldsymbol{A} \boldsymbol{x}+\boldsymbol{B} \boldsymbol{u}+\boldsymbol{E}_{a} \boldsymbol{f}, \\
& \boldsymbol{y}=\boldsymbol{C} \boldsymbol{x}+\boldsymbol{D} \boldsymbol{u}+\boldsymbol{E}_{s} \boldsymbol{f},
\end{aligned}
$$


welches die Annahmen 4.14 und 4.15 erfült, und beliebige Matrizen $\boldsymbol{R}_{L} \in \mathbb{R}^{n \times\left(n_{y}-n_{f}\right)}$ und $\boldsymbol{R}_{V} \in \mathbb{R}^{n_{f} \times\left(n_{y}-n_{f}\right)}$. Dann resultieren alle Beobachterparametrierungen

$$
\begin{aligned}
\boldsymbol{L} & =\boldsymbol{L}_{0}+\boldsymbol{R}_{L} \cdot{ }^{\perp} \boldsymbol{D}^{*}, \\
\boldsymbol{V} & =\boldsymbol{V}_{0}+\boldsymbol{R}_{V} \cdot{ }^{\perp} \boldsymbol{D}^{*}
\end{aligned}
$$

mit $\boldsymbol{L}_{0}=\boldsymbol{M} \boldsymbol{D}^{*+}, \boldsymbol{V}_{0}=\boldsymbol{N} \boldsymbol{D}^{*+}$ und

$$
\boldsymbol{M}=\left[\begin{array}{c}
\left(\boldsymbol{A}^{\delta_{1}} \boldsymbol{e}_{a_{1}}+\sum_{k=0}^{\delta_{1}-1} q_{1, k} \boldsymbol{A}^{k} \boldsymbol{e}_{a_{1}}\right)^{\top} \\
\vdots \\
\left(\boldsymbol{A}^{\delta_{n_{f}}} \boldsymbol{e}_{a_{n_{f}}}+\sum_{k=0}^{\delta_{n_{f}}-1} q_{n_{f}, k} \boldsymbol{A}^{k} \boldsymbol{e}_{a_{n_{f}}}\right)^{\top}
\end{array}\right]^{\top}, \boldsymbol{N}=\operatorname{diag}\left(z_{1,0}, \ldots, z_{n_{f}, 0}\right)
$$

beim Einsatz eines FIOs der Form

$$
\begin{aligned}
\dot{\hat{\boldsymbol{x}}} & =\boldsymbol{A} \hat{\boldsymbol{x}}+\boldsymbol{B} \boldsymbol{u}+\boldsymbol{L}(\boldsymbol{y}-\boldsymbol{C} \hat{\boldsymbol{x}}-\boldsymbol{D} \boldsymbol{u}), \\
\boldsymbol{r} & =\boldsymbol{V}(\boldsymbol{y}-\boldsymbol{C} \hat{\boldsymbol{x}}-\boldsymbol{D u})
\end{aligned}
$$

in einer diagonalisierten Übertragungsmatrix $\boldsymbol{G}_{\boldsymbol{r} \boldsymbol{f}}(s)$ mit

$$
g_{i, i}(s)=\frac{z_{i, 0}}{s^{\delta_{i}}+q_{i, \delta_{i}-1} s^{\delta_{i}-1}+\ldots+q_{i, 1} s+q_{i, 0}}
$$

und frei wählbaren Koeffizienten $q_{i, j}$ und $z_{i, 0}$, wenn $q_{i, 0}=1$ für Fehler mit $\delta_{i}=0$ gewählt wird.

Bemerkung 4.10. Ähnlich wie Satz 4.1 beinhaltet Satz 4.8 keine Aussage über die Stabilität des resultierenden FIOs. In der Tat zeigt sich in Beispielen mit rang $(\boldsymbol{C})>n_{f}$, dass durch unterschiedliche Matrizen $\boldsymbol{R}_{L}$ die nichtsteuerbaren Eigenwerte von $\boldsymbol{G}_{\boldsymbol{r} \boldsymbol{f}}(s)$ zum Teil beeinflusst werden können. Es können jedoch Beobachtereigenwerte auftreten, die invariant bezüglich $\boldsymbol{R}_{L}$ sind [228]. Es ist hier keine unmittelbare Systematik zu erkennen und eine ungeeignete Wahl von $\boldsymbol{R}_{L}$ kann ebenso zur Instabilität des Beobachters führen [58, Theorem 13.4] wie invariante Nullstellen in der geschlossenen rechten s-Halbebene. Für nichtquadratische Systeme wird somit der Vorteil der größeren Transparenz bezüglich der internen Systemdynamik bei Anwendung der Vollständigen Modalen Synthese noch deutlicher.

Satz 4.8 stellt eine Verallgemeinerung der Ergebnisse aus [114,133] dar, da er für Systeme mit beliebigen Fehlerdetektionsindizes gilt und nicht 
auf Systeme mit $\delta_{i}=1$ für alle $i=1, \ldots, n_{f}$ beschränkt ist. Ein ähnliches Ergebnis wird in [130] durch Frequenzbereichsbetrachtungen erzielt, es ist allerdings auf Systeme ohne Sensorfehler beschränkt. In [58, Abschnitt 13.3.3] wird ein auf geometrischen Methoden beruhender Entwurf angegeben, welcher auf das gleiche Ergebnis wie Satz 4.8 führt.

Zur Veranschaulichung wird das akademische Beispielsystem von Seite 83 wieder aufgegriffen. Es wird um einen zusätzlichen messbaren Ausgang erweitert und beschrieben durch

$$
\begin{aligned}
& \boldsymbol{A}=\left[\begin{array}{rrr}
-10 & -16 & -20 \\
6 & 14 & 24 \\
-2 & -8 & -16
\end{array}\right], \quad \boldsymbol{E}_{a}=\left[\begin{array}{cc}
0 & 8 \\
0,5 & -20 \\
0 & 16
\end{array}\right] \\
& \boldsymbol{C}=\left[\begin{array}{crc}
3 & 2 & 1 \\
-9 & 0 & 5 \\
-0,5 & 0 & 0,5
\end{array}\right], \boldsymbol{E}_{s}=\mathbf{0}
\end{aligned}
$$

Da $\boldsymbol{C}$ vollen Rang aufweist, ist das Paar $(\boldsymbol{A}, \boldsymbol{C})$ vollständig beobachtbar und Annahme 4.14 ist erfüllt. Wegen

$$
\boldsymbol{C} \boldsymbol{e}_{a_{1}}=\left[\begin{array}{l}
1 \\
0 \\
0
\end{array}\right] \neq \mathbf{0}, \quad \boldsymbol{C e}_{a_{2}}=\left[\begin{array}{l}
0 \\
8 \\
4
\end{array}\right] \neq \mathbf{0}
$$

ist $\delta_{1}=\delta_{2}=1$. Weiterhin ergibt sich

$$
\boldsymbol{D}^{*}=\left[\begin{array}{ll}
1 & 0 \\
0 & 8 \\
0 & 4
\end{array}\right] \text {. }
$$

Folglich hat $\boldsymbol{D}^{*}$ vollen Rang und Annahme 4.15 ist erfüllt. Für den FIO-Entwurf werden wie im quadratischen Fall (vgl. Beispiel ab Seite 83) die Koeffizienten $q_{1,0}=3$ und $q_{2,0}=5$ vorgegeben, wodurch die Pole $\lambda_{B_{1,1}}=-3$ und $\lambda_{B_{2,1}}=-5$ festgelegt werden. Die Zählerkoeffizienten lauten auch hier $z_{1,0}=3$ und $z_{2,0}=5$, um jeweils eine stationäre Verstärkung von 1 sicherzustellen. Für die weitere Berechnung wird zunächst die Pseudo-Inverse von $\boldsymbol{D}^{*}$ bestimmt. Sie lautet

$$
\boldsymbol{D}^{*+}=\left[\begin{array}{ccc}
1 & 0 & 0 \\
0 & 0,1 & 0,05
\end{array}\right]
$$

und erlaubt die Berechnung von $\boldsymbol{L}_{0}$ und $\boldsymbol{V}_{0}$. 
Diese ergeben sich zu

$$
\begin{aligned}
\boldsymbol{L}_{0}=\boldsymbol{M} \boldsymbol{D}^{*+} & =\left[\begin{array}{cr}
-8 & -40 \\
8,5 & 52 \\
-4 & -32
\end{array}\right]\left[\begin{array}{ccc}
1 & 0 & 0 \\
0 & 0,1 & 0,05
\end{array}\right] \\
& =\left[\begin{array}{ccc}
-8 & -4 & -2 \\
8,5 & 5,2 & 2,6 \\
-4 & -3,2 & -1,6
\end{array}\right] \\
\boldsymbol{V}_{0}=\boldsymbol{N} \boldsymbol{D}^{*+} & =\left[\begin{array}{cc}
3 & 0 \\
0 & 5
\end{array}\right]\left[\begin{array}{ccc}
1 & 0 & 0 \\
0 & 0,1 & 0,05
\end{array}\right] \\
& =\left[\begin{array}{ccc}
3 & 0 & 0 \\
0 & 0,5 & 0,25
\end{array}\right] .
\end{aligned}
$$

Bei der Betrachtung von $\boldsymbol{D}^{*}$ wird außerdem deutlich, dass eine mögliche Wahl für ${ }^{\perp} \boldsymbol{D}^{*}$ gegeben ist durch

$$
{ }^{\perp} \boldsymbol{D}^{*}=\left[\begin{array}{lll}
0 & 1 & -2
\end{array}\right] .
$$

Mithilfe dieser Matrix werden nun zwei unterschiedliche FIOParametrierungen ermittelt. Für

$$
\boldsymbol{R}_{L, 1}=\left[\begin{array}{c}
-2,3 \\
-2 \\
-4,65
\end{array}\right], \quad \boldsymbol{R}_{V, 1}=\left[\begin{array}{l}
1 \\
2
\end{array}\right]
$$

erhält man

$$
\begin{aligned}
\boldsymbol{L}_{1}=\boldsymbol{L}_{0}+\boldsymbol{R}_{L, 1} \cdot{ }^{\perp} \boldsymbol{D}^{*} & =\left[\begin{array}{ccc}
-8 & -4 & -2 \\
8,5 & 5,2 & 2,6 \\
-4 & -3,2 & -1,6
\end{array}\right]+\left[\begin{array}{c}
-2,3 \\
-2 \\
-4,65
\end{array}\right]\left[\begin{array}{lll}
0 & 1 & -2
\end{array}\right] \\
& =\left[\begin{array}{ccc}
-8 & -6,3 & 2,6 \\
8,5 & 3,2 & 6,6 \\
-4 & 7,85 & 7,7
\end{array}\right] \\
\boldsymbol{V}_{1}=\boldsymbol{V}_{0}+\boldsymbol{R}_{V, 1} \cdot{ }^{\perp} \boldsymbol{D}^{*} & =\left[\begin{array}{ccc}
3 & 0 & 0 \\
0 & 0,5 & 0,25
\end{array}\right]+\left[\begin{array}{l}
1 \\
2
\end{array}\right]\left[\begin{array}{lll}
0 & 1 & -2
\end{array}\right] \\
& =\left[\begin{array}{ccc}
3 & 1 & -2 \\
0 & 2,5 & -3,75
\end{array}\right] .
\end{aligned}
$$

Eine alternative FIO-Parametrierung ergibt sich für

$$
\boldsymbol{R}_{L, 2}=\left[\begin{array}{c}
6,2 \\
0 \\
5,35
\end{array}\right], \quad \boldsymbol{R}_{V, 2}=\left[\begin{array}{l}
0 \\
7
\end{array}\right]
$$


Sie ist durch die Matrizen

$$
\begin{aligned}
\boldsymbol{L}_{2}=\boldsymbol{L}_{0}+\boldsymbol{R}_{L, 2} \cdot{ }^{\perp} \boldsymbol{D}^{*} & =\left[\begin{array}{ccc}
-8 & -4 & -2 \\
8,5 & 5,2 & 2,6 \\
-4 & -3,2 & -1,6
\end{array}\right]+\left[\begin{array}{c}
6,2 \\
0 \\
5,35
\end{array}\right]\left[\begin{array}{lll}
0 & 1 & -2
\end{array}\right] \\
& =\left[\begin{array}{ccc}
-8 & 2,2 & -14,4 \\
8,5 & 5,2 & 2,6 \\
-4 & 2,15 & -12,3
\end{array}\right] \\
\boldsymbol{V}_{2}=\boldsymbol{V}_{0}+\boldsymbol{R}_{V, 2} \cdot{ }^{\perp} \boldsymbol{D}^{*} & =\left[\begin{array}{ccc}
3 & 0 & 0 \\
0 & 0,5 & 0,25
\end{array}\right]+\left[\begin{array}{l}
0 \\
7
\end{array}\right]\left[\begin{array}{lll}
0 & 1 & -2
\end{array}\right] \\
& =\left[\begin{array}{ccc}
3 & 0 & 0 \\
0 & 7,5 & -13,75
\end{array}\right]
\end{aligned}
$$

gegeben. Während die Parametrierungen $\left(\boldsymbol{L}_{1}, \boldsymbol{V}_{1}\right)$ und $\left(\boldsymbol{L}_{2}, \boldsymbol{V}_{2}\right)$ beide auf die Übertragungsmatrix

$$
\boldsymbol{G}_{\boldsymbol{r} \boldsymbol{f}}(s)=\left[\begin{array}{cc}
\frac{3}{s+3} & 0 \\
0 & \frac{5}{s+5}
\end{array}\right]
$$

führen, zeigt sich bei der Analyse der Beobachtereigenwerte ein gravierender Unterschied: Für sie gilt

$$
\begin{aligned}
& \operatorname{spec}\left(\boldsymbol{A}-\boldsymbol{L}_{1} \boldsymbol{C}\right)=\{-3 ;-5 ;-13\}, \\
& \operatorname{spec}\left(\boldsymbol{A}-\boldsymbol{L}_{2} \boldsymbol{C}\right)=\{-3 ;-5 ; 15\} .
\end{aligned}
$$

Die Parametrierung $\left(\boldsymbol{L}_{2}, \boldsymbol{V}_{2}\right)$ führt also im Gegensatz zu $\left(\boldsymbol{L}_{1}, \boldsymbol{V}_{1}\right)$ auf einen instabilen FIO. Mittels der im folgenden Abschnitt vorgestellten Vollständigen Modalen Synthese für nichtquadratische Systeme lässt sich die Stabilität des Beobachters systematisch sicherstellen.

\subsubsection{Entwurf mittels Vollständiger Modaler Synthese}

Gegenüber dem quadratischen Fall (vgl. Abschnitt 4.5.2) ändert sich die Zielsetzung beim Entwurf der Beobachtermatrix $\boldsymbol{L}$ mittels Vollständiger Modaler Synthese nicht. Die Beobachtereigenwerte $\lambda_{B_{i j}}$ mit $i=1, \ldots, n_{f}$ und $j=1, \ldots, \delta_{i}$, die als Pole der Übertragungsmatrix $\boldsymbol{G}_{\boldsymbol{r} \boldsymbol{f}}(s)$ auftreten, werden gemäß der Entwurfsbedingung

$$
\boldsymbol{w}_{B_{i j}}^{\top}\left(\boldsymbol{E}_{a}-\boldsymbol{L} \boldsymbol{E}_{s}\right)=\boldsymbol{\phi}_{i}^{\top}
$$


jeweils der $i$-ten Spalte von $\boldsymbol{G}_{\boldsymbol{r} \boldsymbol{f}}(s)$ zugewiesen. Die verbleibenden Eigenwerte $\lambda_{B_{k}}, k=\delta+1, \ldots, n$, sind durch

$$
\boldsymbol{w}_{B_{k}}^{\top}\left(\boldsymbol{E}_{a}-\boldsymbol{L} \boldsymbol{E}_{s}\right)=\mathbf{0}^{\top}
$$

nichtsteuerbar zu machen. Analog zu den Überlegungen für quadratische Systeme in Abschnitt 4.5.2 lassen sich die Entwurfsbedingungen mithilfe der Parametervektoren $\boldsymbol{p}_{i j}^{\top}=-\boldsymbol{w}_{B_{i j}}^{\top}$ beziehungsweise $\boldsymbol{p}_{k}^{\top}=-\boldsymbol{w}_{B_{k}}^{\top} \boldsymbol{L}$ und der Rosenbrock'schen Systemmatrix $\boldsymbol{\Pi}_{i j}$ beziehungsweise $\boldsymbol{\Pi}_{k}$ schreiben als

$$
\begin{gathered}
{\left[\begin{array}{ll}
\boldsymbol{w}_{B_{i j}}^{\top} & \boldsymbol{p}_{i j}^{\top}
\end{array}\right]\left[\begin{array}{cc}
\lambda_{B_{i j}} \boldsymbol{I}_{n}-\boldsymbol{A} & \boldsymbol{E}_{a} \\
-\boldsymbol{C} & \boldsymbol{E}_{s}
\end{array}\right]=\left[\begin{array}{ll}
\boldsymbol{w}_{B_{i j}}^{\top} & \boldsymbol{p}_{i j}^{\top}
\end{array}\right] \boldsymbol{\Pi}_{i j}=\left[\begin{array}{ll}
\mathbf{0}^{\boldsymbol{\top}} & \boldsymbol{\phi}_{i}^{\top}
\end{array}\right]} \\
{\left[\begin{array}{ll}
\boldsymbol{w}_{B_{k}}^{\top} & \boldsymbol{p}_{k}^{\top}
\end{array}\right]\left[\begin{array}{cc}
\lambda_{B_{k}} \boldsymbol{I}_{n}-\boldsymbol{A} & \boldsymbol{E}_{a} \\
-\boldsymbol{C} & \boldsymbol{E}_{s}
\end{array}\right]=\left[\begin{array}{ll}
\boldsymbol{w}_{B_{k}}^{\top} & \boldsymbol{p}_{k}^{\top}
\end{array}\right] \boldsymbol{\Pi}_{k}=\mathbf{0}^{\top} .}
\end{gathered}
$$

Die Parametrierung von $\boldsymbol{L}$ erfolgt nun durch Lösen von (4.83).

Dabei ist die Rosenbrock'sche Systemmatrix $\boldsymbol{\Pi}_{i j} \in \mathbb{R}^{\left(n+n_{y}\right) \times\left(n+n_{f}\right)}$ aufgrund der zusätzlichen Messgrößen nichtquadratisch, sie besitzt mehr Zeilen als Spalten. Wird nun der Eigenwert $\lambda_{B_{i j}}$ so gewählt, dass er nicht mit einer invarianten Nullstelle von $\left(\boldsymbol{A}, \boldsymbol{E}_{a}, \boldsymbol{C}, \boldsymbol{E}_{s}\right)$ zusammenfällt, so folgt aus dem Rangsatz (Satz A.1), dass ein linkes orthogonales Komplement ${ }^{\perp} \boldsymbol{\Pi}_{i j} \in \mathbb{R}^{\left(n_{y}-n_{f}\right) \times\left(n+n_{y}\right)}$ existiert. Demnach ist $\boldsymbol{\Pi}_{i j}^{+} \cdot \boldsymbol{\Pi}_{i j}=\boldsymbol{I}_{n+n_{f}}$ und ${ }^{\perp} \boldsymbol{\Pi}_{i j} \cdot \boldsymbol{\Pi}_{i j}=\mathbf{0}$, und alle durch

$$
\left[\begin{array}{ll}
\boldsymbol{w}_{B_{i j}}^{\top} & \boldsymbol{p}_{i j}^{\top}
\end{array}\right]=\left[\begin{array}{ll}
\mathbf{0}^{\top} & \boldsymbol{\phi}_{i}^{\top}
\end{array}\right] \boldsymbol{\Pi}_{i j}^{+}+\boldsymbol{z}_{i j}^{\top} \cdot{ }^{\perp} \boldsymbol{\Pi}_{i j}
$$

beschriebenen Parameter- und Linkseigenvektoren erfüllen (4.83a). Dabei ist $\boldsymbol{z}_{i j}^{\top} \in \mathbb{R}^{n_{y}-n_{f}}$ ein beliebiger Vektor, welcher gegenüber dem quadratischen Fall $n_{y}-n_{f}$ zusätzliche Freiheitsgrade zur Verfügung stellt (vgl. (4.22) auf Seite 88). Diese erlauben es, die Richtung von $\left[\begin{array}{ll}\boldsymbol{w}_{B_{i j}}^{\top} & \boldsymbol{p}_{i j}^{\top}\end{array}\right]$ in dem von den Zeilen von ${ }^{\perp} \boldsymbol{\Pi}_{i j}$ aufgespannten Unterraum zu verändern. Es gibt also unendlich viele Lösungen für die Bedingung (4.83a). Quadratische Systeme mit $n_{y}=n_{f}$ sind in obiger Darstellung als Spezialfall enthalten: Der Unterraum möglicher Lösungen entartet zu einem Punkt und die Lösung der Bedingung (4.83a) ist nach (4.22) eindeutig bestimmt.

In ähnlicher Weise können die Eigenwerte $\lambda_{B_{k}}$ betrachtet werden, die nichtsteuerbar zu machen sind. Entscheidend ist, dass auch für diese immer ein linkes orthogonales Komplement ${ }^{\perp} \boldsymbol{\Pi}_{k}$ existiert. Im Gegensatz zum 
quadratischen Fall müssen die nichtsteuerbaren Eigenwerte also nicht mit invarianten Nullstellen des Systems zusammenfallen, damit (4.83b) eine Lösung hat. Dies liegt darin begründet, dass in nichtquadratischen Systemen die Eingangsentkopplungsnullstellen nicht notwendigerweise zur Klasse der invarianten Nullstellen gehören (s. Abschnitt 3.3). Um (4.83b) zu lösen, können die Eigenwerte $\lambda_{B_{k}}$ demnach prinzipiell beliebig gewählt werden. Weist das System jedoch invariante Nullstellen auf, so ergeben sich bei beliebiger Wahl von $\lambda_{B_{k}}$ linear abhängige Linkseigenvektoren, sodass die Linkseigenvektormatrix nicht invertierbar ist. Werden aber Eigenwerte $\lambda_{B_{k}}$ verwendet, um invariante Nullstellen wie im quadratischen Fall zu kompensieren, so vergrößert sich die Dimension von ${ }^{\perp} \boldsymbol{\Pi}_{k}$ und es lassen sich linear unabhängige Linkseigenvektoren finden.

Für diejenigen Beobachtereigenwerte $\lambda_{B_{\mathrm{in}, m}}$, die zur Kompensation von invarianten Nullstellen herangezogen werden, ergibt sich die Gesamtheit der Lösungen von (4.83b) zu

$$
\left[\begin{array}{ll}
\boldsymbol{w}_{B_{m}}^{\top} & \boldsymbol{p}_{m}^{\top}
\end{array}\right]=\boldsymbol{z}_{m}^{\top} \cdot{ }^{\perp} \boldsymbol{\Pi}_{m}, m=\delta+1, \ldots, \delta+\mu,
$$

mit beliebigem $\boldsymbol{z}_{m}^{\top} \neq \mathbf{0}^{\top} \in \mathbb{R}^{n_{y}-n_{f}+1}$. Da die Parameter- und Linkseigenvektoren skalierungsinvariant sind, ergeben sich durch $\boldsymbol{z}_{m}^{\top}$ wie für die steuerbaren Beobachtereigenwerte $n_{y}-n_{f}$ Freiheitsgrade.

Die übrigen nichtsteuerbaren Beobachtereigenwerte können beliebig platziert werden, die entsprechenden Parameter- und Linkseigenvektoren folgen aus

$$
\left[\begin{array}{ll}
\boldsymbol{w}_{B_{k}}^{\top} & \boldsymbol{p}_{k}^{\top}
\end{array}\right]=\boldsymbol{z}_{k}^{\top} \cdot{ }^{\perp} \boldsymbol{\Pi}_{k}, k=\delta+\mu+1, \ldots, n,
$$

mit $\boldsymbol{z}_{k}^{\top} \neq \mathbf{0}^{\top} \in \mathbb{R}^{n_{y}-n_{f}}$. Aufgrund der Skalierungsinvarianz stellt obige Gleichung lediglich $n_{y}-n_{f}-1$ Freiheitsgrade zur Verfügung. Da jedoch $\lambda_{B_{k}}$ beliebig platziert werden kann, stehen auch für die nichtsteuerbaren Eigenwerte, welche nicht zur Kompensation von Nullstellen herangezogen werden müssen, $n_{y}-n_{f}$ Freiheitsgrade zur Verfügung.

Die vorangegangenen Betrachtungen begründen die in Bemerkung 4.10 erörterten Effekte bezüglich der Matrix $\boldsymbol{R}_{L}$. Die Matrix erlaubt es für rang $(\boldsymbol{C})>n_{f}$, einen Teil der nichtsteuerbaren Beobachtereigenwerte zu beeinflussen. Dies sind die frei platzierbaren Eigenwerte $\lambda_{B_{k}}$, welche offensichtlich auch in der geschlossenen rechten s-Halbebene platziert werden können. Daneben können invariante Nullstellen existieren, welche durch Beobachtereigenwerte kompensiert werden müssen. Diese können dementsprechend auch durch $\boldsymbol{R}_{L}$ nicht beeinflusst werden. 
Nachdem die Beobachtermatrix $\boldsymbol{L}$ bestimmt ist, lässt sich mittels analoger Überlegungen wie in Abschnitt 4.5.2 eine Nachfiltermatrix $\boldsymbol{V}$ herleiten, welche eine vollständige Diagonalisierung von $\boldsymbol{G}_{\boldsymbol{r} \boldsymbol{f}}(s)$ gewährleistet. Es ist dafür die Gleichung

$$
\boldsymbol{G}_{\boldsymbol{r} \boldsymbol{f}}(s=0)=-\boldsymbol{V} \boldsymbol{\Theta}=\boldsymbol{S}
$$

mit der Matrix $\boldsymbol{\Theta}=\boldsymbol{C}(\boldsymbol{A}-\boldsymbol{L} \boldsymbol{C})^{-1}\left(\boldsymbol{E}_{a}-\boldsymbol{L} \boldsymbol{E}_{s}\right)-\boldsymbol{E}_{s}$ zu erfüllen. Die stationären Verstärkungen der Diagonalelemente von $\boldsymbol{G}_{\boldsymbol{r} \boldsymbol{f}}(s)$ werden dabei durch die beliebig wählbare Diagonalmatrix $\boldsymbol{S}$ vorgegeben. Da die Matrix $\boldsymbol{\Theta} \in \mathbb{R}^{n_{y} \times n_{f}}$ in (4.84) jetzt ebenfalls nichtquadratisch ist, ergeben sich auch für das Nachfilter zusätzliche Freiheitsgrade. Alle Lösungen werden parametriert durch

$$
\boldsymbol{V}=-\boldsymbol{S} \boldsymbol{\Theta}^{+}-\boldsymbol{Z}_{V} \cdot{ }^{\perp} \boldsymbol{\Theta},
$$

mit einer beliebigen Matrix $\boldsymbol{Z}_{V} \in \mathbb{R}^{n_{f} \times\left(n_{y}-n_{f}\right)}$. Dass diese Wahl hinreichend für die Diagonalisierung von $\boldsymbol{G}_{\boldsymbol{r} \boldsymbol{f}}(s)$ für alle $s \in \mathbb{C}$ ist, lässt sich in analoger Weise wie in Abschnitt 4.5.2 zeigen.

Zusammenfassend gilt somit

Satz 4.9 (FIO-Entwurf für nichtquadratische Systeme mittels Vollständiger Modaler Synthese). Gegeben sei ein System der Form

$$
\begin{aligned}
& \dot{\boldsymbol{x}}=\boldsymbol{A} \boldsymbol{x}+\boldsymbol{B u}+\boldsymbol{E}_{a} \boldsymbol{f}, \\
& \boldsymbol{y}=\boldsymbol{C} \boldsymbol{x}+\boldsymbol{D} \boldsymbol{u}+\boldsymbol{E}_{s} \boldsymbol{f}
\end{aligned}
$$

welches die Annahmen 4.14-4.16 erfüllt, und beliebige Vektoren $\boldsymbol{z}_{i j}^{\top} \in \mathbb{R}^{n_{y}-n_{f}}, \boldsymbol{z}_{m}^{\top} \neq \mathbf{0}^{\top} \in \mathbb{R}^{n_{y}-n_{f}+1}$ und $\boldsymbol{z}_{k}^{\top} \neq \mathbf{0}^{\top} \in \mathbb{R}^{n_{y}-n_{f}}$. Weiterhin seien alle Beobachtereigenwerte paarweise verschieden. Werden die $\mu \mathrm{Be}$ obachtereigenwerte $\lambda_{B_{\delta+1}}, \ldots, \lambda_{B_{\delta+\mu}}$ entsprechend den $\mu$ invarianten Nullstellen des Systems gewählt, dann erfüllen alle Beobachtermatrizen $\boldsymbol{L}$, welche durch

$$
\boldsymbol{L}=-\boldsymbol{W}_{B}^{-1} \boldsymbol{P}=-\boldsymbol{V}_{B} \boldsymbol{P}
$$

mit

$$
\begin{aligned}
& {\left[\begin{array}{ll}
\boldsymbol{w}_{B_{i j}}^{\top} & \boldsymbol{p}_{i j}^{\top}
\end{array}\right]=\left[\begin{array}{ll}
\mathbf{0}^{\top} & \boldsymbol{\phi}_{i}^{\top}
\end{array}\right] \boldsymbol{\Pi}_{i j}^{+}+\boldsymbol{z}_{i j}^{\top} \cdot{ }^{\perp} \boldsymbol{\Pi}_{i j}, \quad i=1, \ldots, n_{f}, j=1, \ldots, \delta_{i},} \\
& {\left[\begin{array}{ll}
\boldsymbol{w}_{B_{m}}^{\top} & \boldsymbol{p}_{m}^{\top}
\end{array}\right]=\boldsymbol{z}_{m}^{\top} \cdot{ }^{\perp} \boldsymbol{\Pi}_{m}, \quad m=\delta+1, \ldots, \delta+\mu,} \\
& {\left[\begin{array}{ll}
\boldsymbol{w}_{B_{k}}^{\top} & \boldsymbol{p}_{k}^{\top}
\end{array}\right]=\boldsymbol{z}_{k}^{\top} \cdot{ }^{\perp} \boldsymbol{\Pi}_{k}, \quad k=\delta+\mu+1, \ldots, n,}
\end{aligned}
$$


parametriert sind, die Beziehungen (4.81) und (4.82). Sei weiterhin $\boldsymbol{Z}_{V} \in \mathbb{R}^{n_{f} \times\left(n_{y}-n_{f}\right)}$, so gewährleisten alle Nachfiltermatrizen

$$
\boldsymbol{V}=-\boldsymbol{S} \boldsymbol{\Theta}^{+}-\boldsymbol{Z}_{V} \cdot{ }^{\perp} \Theta
$$

mit $\boldsymbol{\Theta}=\boldsymbol{C}(\boldsymbol{A}-\boldsymbol{L} \boldsymbol{C})^{-1}\left(\boldsymbol{E}_{a}-\boldsymbol{L} \boldsymbol{E}_{s}\right)-\boldsymbol{E}_{s}$ und einer beliebigen Diagonalmatrix $\boldsymbol{S} \in \mathbb{R}^{n_{f} \times n_{f}}$ beim Einsatz eines FIOs der Form

$$
\begin{aligned}
\dot{\hat{x}} & =\boldsymbol{A} \hat{\boldsymbol{x}}+\boldsymbol{B u}+\boldsymbol{L}(\boldsymbol{y}-\boldsymbol{C} \hat{\boldsymbol{x}}-\boldsymbol{D} \boldsymbol{u}), \\
\boldsymbol{r} & =\boldsymbol{V}(\boldsymbol{y}-\boldsymbol{C} \hat{\boldsymbol{x}}-\boldsymbol{D u})
\end{aligned}
$$

eine diagonale Übertragungsmatrix $\boldsymbol{G}_{\boldsymbol{r} \boldsymbol{f}}(s)$.

Wie zuvor erläutert bietet jeder als Pol zugewiesene Eigenwert $\lambda_{B_{i j}}$ gegenüber dem quadratischen Fall $n_{y}-n_{f}$ zusätzliche Freiheitsgrade, ebenso wie die nichtsteuerbaren Eigenwerte. Insgesamt ergeben sich in nichtquadratischen Systemen also $n\left(n_{y}-n_{f}\right)$ Freiheitsgrade gegenüber dem quadratischen Fall. Es sei erneut angemerkt, dass diese zusätzlichen Freiheitsgrade auch im Fall $n_{f}<\operatorname{rang}(\boldsymbol{C})<n_{y}$ existieren. Allerdings treten dann tendenziell öfter invariante Nullstellen auf, die durch entsprechende Beobachtereigenwerte zu kompensieren sind. Bei der Wahl des Nachfilters ergeben sich entsprechend der Dimensionen von $\boldsymbol{Z}_{V}$ weitere $n_{f}\left(n_{y}-n_{f}\right)$ Freiheitsgrade. Beim Zeitbereichsentwurf (s. Satz 4.8) sind die zusätzlichen Freiheitsgrade in den Matrizen $\boldsymbol{R}_{L} \in \mathbb{R}^{n \times\left(n_{y}-n_{f}\right)}$ und $\boldsymbol{R}_{V} \in \mathbb{R}^{n_{f} \times\left(n_{y}-n_{f}\right)}$ konzentriert. Die Betrachtung der Dimensionen von $\boldsymbol{R}_{L}$ und $\boldsymbol{R}_{V}$ zeigt, dass bei beiden Entwurfsverfahren jedoch die gleiche Anzahl von freien Parametern zur Verfügung steht.

Weiterhin ist anzumerken, dass eine durch Vollständige Modale Synthese bestimmte Beobachtermatrix $\boldsymbol{L}_{\mathrm{VMS}}$ immer durch eine Matrix $\tilde{\boldsymbol{R}}_{L}$ in eine durch den Zeitbereichsentwurf gewonnene Matrix $\boldsymbol{L}_{\mathrm{ZB}}$ überführt werden kann. Es existiert also immer ein $\tilde{\boldsymbol{R}}_{L}$, so dass $\boldsymbol{L}_{\mathrm{ZB}}=\boldsymbol{L}_{\mathrm{VMS}}+\tilde{\boldsymbol{R}}_{L} \cdot{ }^{\perp} \boldsymbol{D}^{*}$ gilt. Dies ist dadurch zu begründen, dass $\boldsymbol{L}_{\mathrm{VMS}} \boldsymbol{D}^{*}=\boldsymbol{L}_{\mathrm{ZB}} \boldsymbol{D}^{*}=\boldsymbol{M}$ gilt, wodurch impliziert wird, dass $\boldsymbol{L}_{\mathrm{VMS}}-\boldsymbol{L}_{\mathrm{ZB}}$ durch eine Linearkombination der Zeilenvektoren von ${ }^{\perp} \boldsymbol{D}^{*}$ dargestellt werden kann. Diese Beobachtung ist von Bedeutung, da entsprechend Satz 4.8 lediglich $\delta$ Pole für die Übertragungsmatrix $\boldsymbol{G}_{\boldsymbol{r} \boldsymbol{f}}(s)$ zugewiesen werden. Die $n-\delta$ nichtsteuerbaren Eigenwerte von $\left(\boldsymbol{A}-\boldsymbol{L} \boldsymbol{C}, \boldsymbol{E}_{a}-\boldsymbol{L} \boldsymbol{E}_{s}\right)$ können wie bereits erwähnt bei $\operatorname{rang}(\boldsymbol{C})>n_{f}$ je nach Wahl von $\boldsymbol{R}_{L}$ instabil werden. Basierend auf obigen Überlegungen gilt jedoch

Korollar 4.2. Gegeben sei ein System der Form (4.1), welches die Annahmen 4.14-4.16 erfüllt und für das nach Satz 4.8 ein FIO entworfen 
wurde. Dann existiert immer eine Matrix $\boldsymbol{R}_{L}$, sodass der sich ergebende FIO stabil ist.

Auch der Entwurf mittels Vollständiger Modaler Synthese soll anhand des Beispielsystems (4.80) exemplarisch durchgeführt werden. Bereits auf Seite 152 wurde ermittelt, dass das System vollständig beobachtbar ist und dass $\boldsymbol{D}^{*}$ vollen Rang besitzt. Die Annahmen 4.14 und 4.15 sind also erfüllt. Ferner wird bei der Betrachtung der Rosenbrock'schen Systemmatrix,

$$
\Pi(s)=\left[\begin{array}{ccc:cc}
s+10 & 16 & 20 & 0 & 8 \\
-6 & s-14 & -24 & 0,5 & -20 \\
2 & 8 & s+16 & 0 & 16 \\
\hdashline-3 & -2 & -1 & 0 & 0 \\
9 & 0 & -5 & 0 & 0 \\
0,5 & 0 & -0,5 & 0 & 0
\end{array}\right]
$$

deutlich, dass das System keine invarianten Nullstellen aufweist. Dies liegt darin begründet, dass $\boldsymbol{\Pi}(s)$ im unteren linken Block durch die Matrix $-\boldsymbol{C}$ drei linear unabhängige Zeilen aufweist und im oberen rechten Block durch $\boldsymbol{E}_{a}$ zwei weitere linear unabhängige Zeilen vorkommen. Somit ist für alle $s \in \mathbb{C}$ die Gleichung rang $(\boldsymbol{\Pi}(s))=5$ erfüllt und es treten keine invarianten Nullstellen auf.

Für den ersten Parameter- beziehungsweise Linkseigenvektor erhält man für die Wahl $\lambda_{B_{1,1}}=-3$

$$
\left[\begin{array}{ll}
\boldsymbol{w}_{B_{11}}^{\top} & \boldsymbol{p}_{11}^{\top}
\end{array}\right]=\left[\begin{array}{lllll}
0 & 0 & 0 & 1 & 0
\end{array}\right] \boldsymbol{\Pi}(-3)^{+}+\boldsymbol{z}_{11}^{\top} \cdot{ }^{\perp} \boldsymbol{\Pi}(-3) .
$$

Der Vektor $\boldsymbol{z}_{11}^{\top}$ entartet hier wegen $n_{y}-n_{f}=1 \mathrm{zu}$ einem Skalar. Der Einfachheit halber wird $\boldsymbol{z}_{11}^{\top}=z_{11}=0$ gewählt und es ergibt sich

$$
\boldsymbol{w}_{B_{11}}^{\top}=\left[\begin{array}{lll}
1,0838 & 2 & 1,9581
\end{array}\right], \quad \boldsymbol{p}_{11}^{\top}=\left[\begin{array}{lll}
-0,4972 & -0,1557 & 0,8143
\end{array}\right] .
$$

Auf die gleiche Art und Weise erhält man bezüglich des Eigenwertes $\lambda_{B_{2,1}}=-5$, der dem zweiten Übertragungskanal zugewiesen wird, mit $\boldsymbol{z}_{21}^{\top}=z_{21}=0$ die Vektoren

$$
\boldsymbol{w}_{B_{21}}^{\top}=\left[\begin{array}{lll}
-0,0574 & 0 & 0,0912
\end{array}\right], \quad \boldsymbol{p}_{21}^{\top}=\left[\begin{array}{lll}
-0,0945 & -0,0321 & 0,2197
\end{array}\right] .
$$

Da das System keine invarianten Nullstellen aufweist, kann der dritte Beobachtereigenwert beliebig platziert werden. Setzt man ihn zu $\lambda_{B_{3}}=-15$ und wählt für den entarteten Vektor $z_{3}^{\top}=z_{3}=1$, so erhält man

$$
\boldsymbol{w}_{B_{3}}^{\top}=\left[\begin{array}{lll}
-0,1496 & 0 & 0,0748
\end{array}\right], \quad \boldsymbol{p}_{3}^{\top}=\left[\begin{array}{lll}
-0,8974 & -0,3926 & -0,1122
\end{array}\right] .
$$


Daraus ergibt sich schließlich die Beobachtermatrix

$$
\begin{aligned}
\boldsymbol{L}=-\boldsymbol{W}_{B}^{-1} \boldsymbol{P} & =\left[\begin{array}{rrr}
1,0838 & 2 & 1,9581 \\
-0,0574 & 0 & 0,0912 \\
-0,1496 & 0 & 0,0748
\end{array}\right]^{-1}\left[\begin{array}{rrr}
-0,4972 & -0,1557 & 0,8143 \\
-0,0945 & -0,0321 & 0,2197 \\
-0,8974 & -0,3926 & -0,1122
\end{array}\right] \\
& =\left[\begin{array}{rrr}
-8 & -3,5741 & -2,8518 \\
8,5 & 3,8731 & 5,2537 \\
-4 & -1,8982 & -4,2036
\end{array}\right] .
\end{aligned}
$$

Hiermit kann man weiterhin die Matrix $\Theta \mathrm{zu}$

$$
\boldsymbol{\Theta}=\boldsymbol{C}(\boldsymbol{A}-\boldsymbol{L} \boldsymbol{C})^{-1}\left(\boldsymbol{E}_{a}-\boldsymbol{L} \boldsymbol{E}_{s}\right)-\boldsymbol{E}_{s}=\left[\begin{array}{cc}
-\frac{1}{3} & 0 \\
0 & -\frac{8}{5} \\
0 & -\frac{4}{5}
\end{array}\right]
$$

berechnen. Ihr Linksnullraum lässt sich wie derjenige der Matrix $\boldsymbol{D}^{*}$ beschreiben mittels

$$
{ }^{\perp_{\Theta}}=\left[\begin{array}{lll}
0 & 1 & -2
\end{array}\right] .
$$

Wählt man den Freiheitsgrad $\boldsymbol{Z}_{V}=\left[\begin{array}{ll}5 & -3\end{array}\right]^{\top}$ und fordert eine stationäre Verstärkung von 1 für beide Übertragungskanäle, so ergibt sich für das Nachfilter

$$
\begin{aligned}
\boldsymbol{V}=-\boldsymbol{S}^{+}-\boldsymbol{Z}_{V} \cdot{ }^{\perp} \boldsymbol{\Theta}= & -\left[\begin{array}{cc}
1 & 0 \\
0 & 1
\end{array}\right]\left[\begin{array}{ccc}
-3 & 0 & 0 \\
0 & -0,5 & -0,25
\end{array}\right]+\ldots \\
& -\left[\begin{array}{c}
5 \\
-3
\end{array}\right]\left[\begin{array}{ccc}
0 & 1 & -2
\end{array}\right] \\
= & {\left[\begin{array}{ccc}
3 & 5 & -10 \\
0 & -2,5 & 6,25
\end{array}\right] . }
\end{aligned}
$$

Die ermittelte Parametrierung $(\boldsymbol{L}, \boldsymbol{V})$ führt wie gewünscht auf

$$
\boldsymbol{G}_{\boldsymbol{r} \boldsymbol{f}}(s)=\left[\begin{array}{cc}
\frac{3}{s+3} & 0 \\
0 & \frac{5}{s+5}
\end{array}\right]
$$

und $\operatorname{spec}(\boldsymbol{A}-\boldsymbol{L} \boldsymbol{C})=\{-3 ;-5 ;-15\}$, also zu einem stabilen FIO.

Bemerkung 4.11. Aufgrund der in Abschnitt 4.4 erörterten Dualität zum Entkopplungsreglerentwurf lässt sich das vorgestellte Verfahren in abgewandelter Form auch für den Entwurf von Entkopplungsregelungen für Systeme mit mehr Eingangs- als zu regelnden Ausgangsgrößen anwenden, wie in [240] gezeigt wird. 
Bemerkung 4.12. Prinzipiell lassen sich die in den Abschnitten 4.7 und 4.8 vorgestellten dynamischen Systemerweiterungen auch für nichtquadratische Systeme anwenden. Insbesondere für $\operatorname{rang}(\boldsymbol{C})=n_{y}$ weisen solche Systeme jedoch selten ein Rangdefizit in der Entkoppelbarkeitsmatrix oder invariante Nullstellen auf.

\subsubsection{Beispiel Hydrauliksystem}

Als Beispielsystem für den Entwurf von FIOs für nichtquadratische Systeme wird ein hydraulisch-mechanisches System betrachtet. Es ist an das hydraulische Positioniersystem aus [127] angelehnt, wird jedoch hier um eine zusätzliche Masse erweitert, die über ein Feder-Dämpfer-Element an die erste Masse gekoppelt ist. Abbildung 4.18 skizziert das System. Eingangsgröße des Systems ist eine Spannung $u$, über die der Druck $p_{1}$ beeinflusst werden kann. Die Dynamik der Druckerzeugung kann näherungsweise beschrieben werden durch $T \dot{p}_{1}+p_{1}=K_{1} u$, wobei $T$ und $K_{1}$ die Dynamik und die Verstärkung des Druckaufbaus beschreiben. Durch eine Leckage im Zylinder (Fehler $f_{1}$ ) verringert sich der Druck auf den Kolben. Dieser ist wiederum mit der Masse $m_{1}$ verbunden, die über eine Feder (Federkonstante $k_{1}$ ) am Zylinder abgestützt ist. Das durch die Federkonstante $k_{2}$ und die Dämpferkonstante $d$ beschriebene Feder-Dämpfer-Element verbindet die Masse $m_{1}$ mit der Masse $m_{2}$. Ein Zusammenstoß der beiden Massen bewirkt eine Kraft gleichen Betrages mit unterschiedlichem Vorzeichen, die auf die beiden Massen wirkt. Sie beschreibt den zweiten modellierten Fehler $f_{2}$ im System. Zustände des Systems sind der Druck $p_{1}$ sowie Positionen und Geschwindigkeiten der beiden Massen. Die Zustandsraumdarstellung

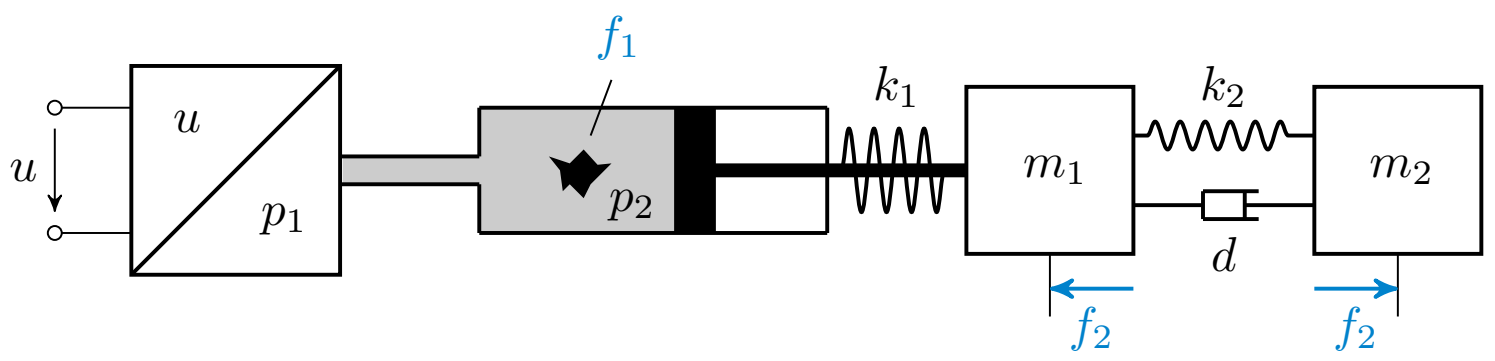

Abbildung 4.18: Fehlerbehaftetes Hydrauliksystem 
lautet

$$
\begin{aligned}
& \boldsymbol{A}=\left[\begin{array}{ccccc}
-\frac{1}{T} & 0 & 0 & 0 & 0 \\
0 & 0 & 1 & 0 & 0 \\
\frac{A_{k}}{m_{1}} & -\frac{k_{1}+k_{2}}{m_{1}} & -\frac{R+d}{m_{1}}-\frac{A_{k}^{2}}{m_{1} K_{2}} & \frac{k_{2}}{m_{1}} & \frac{d}{m_{1}} \\
0 & 0 & 0 & 0 & 1 \\
0 & \frac{k_{2}}{m_{2}} & \frac{d}{m_{2}} & -\frac{k_{2}}{m_{2}} & -\frac{R+d}{m_{2}}
\end{array}\right], \boldsymbol{B}=\left[\begin{array}{c}
-\frac{K_{1}}{T} \\
0 \\
0 \\
0 \\
0
\end{array}\right] \\
& \boldsymbol{C}=\left[\begin{array}{lllll}
0 & 1 & 0 & 0 & 0 \\
0 & 0 & 0 & 1 & 0 \\
0 & 0 & 0 & 0 & 1
\end{array}\right], \boldsymbol{E}_{a}=\left[\begin{array}{cc}
-\frac{K_{1}}{T} & 0 \\
0 & 0 \\
0 & \frac{1}{m_{1}} \\
0 & 0 \\
0 & -\frac{1}{m_{2}}
\end{array}\right] .
\end{aligned}
$$

Daraus geht hervor, dass die Position der ersten Masse sowie Position und Geschwindigkeit der zweiten Masse gemessen werden können. Der Parameter $A_{k}$ beschreibt die Kolbenfläche, $R$ ist eine Reibkonstante und $K_{2}$ ist der Übertragungsbeiwert des Rohres. Die Zahlenwerte der Parameter finden sich in Anhang C.1.6.

Mittels der Vollständigen Modalen Synthese können $\delta_{1}=3$ Beobachtereigenwerte dem ersten Übertragungskanal zugewiesen werden. Gewählt werden hierfür $\lambda_{B_{1,1}}=-1,5, \lambda_{B_{1,2}}=-2$ und $\lambda_{B_{1,3}}=-2,5$. Die Systemanalyse ergibt weiterhin $\delta_{2}=1$, sodass ein Eigenwert (hier $\lambda_{B_{2,1}}=-3$ ) dem zweiten Übertragungskanal von $\boldsymbol{G}_{\boldsymbol{r} \boldsymbol{f}}(s)$ zugewiesen werden kann. Da das System über keine invarianten Nullstellen bezüglich der Fehler verfügt, kann der verbleibende Beobachtereigenwert beliebig platziert werden. Hier wird $\lambda_{B_{5}}=-10$ gewählt und als stationäre Verstärkung jeweils $g_{i, i}(s=0)=1$ vorgegeben. Wählt man die verbleibenden Freiheitsgrade zu $\boldsymbol{z}_{11}^{\top}=\boldsymbol{z}_{12}^{\top}=\boldsymbol{z}_{13}^{\top}=\boldsymbol{z}_{21}^{\top}=\mathbf{0}^{\top}, \boldsymbol{z}_{5}^{\top}=1$ und $\boldsymbol{Z}_{V}=\mathbf{0}$, so ergibt sich die in Anhang C.1.6 angegebene FIO-Parametrierung.

\subsubsection{Dualität zur Control Allocation}

Für den Spezialfall rang $(\boldsymbol{C})=n_{f}$ ergibt sich eine interessante Verbindung zu einem weiteren regelungstechnischen Gebiet, die in diesem Abschnitt erörtert wird. Zur Vereinfachung der Notation wird dabei im Folgenden $\boldsymbol{E}_{s}=\mathbf{0}$ und $\boldsymbol{D}=\mathbf{0}$ vorausgesetzt.

Für rang $(\boldsymbol{C})=n_{f}$ sind wie bereits erwähnt $n_{y}-n_{f}$ Messgrößen redundant und es lässt sich ohne Beschränkung der Allgemeinheit folgende Annahme treffen: 
Annahme 4.17. Es ist

$$
\boldsymbol{C}=\left[\begin{array}{l}
\boldsymbol{C}_{o} \\
\boldsymbol{C}_{u}
\end{array}\right]
$$

mit rang $\left(\boldsymbol{C}_{o}\right)=n_{f}, \boldsymbol{C}_{o} \in \mathbb{R}^{n_{f} \times n_{f}}$ und $\boldsymbol{C}_{u} \in \mathbb{R}^{\left(n_{y}-n_{f}\right) \times n_{f}}$.

Liegt die Ausgangsmatrix eines Systems nicht in einer Form vor, die Annahme 4.17 genügt, so lässt sich das System durch eine Ähnlichkeitstransformation stets dahingehend transformieren.

Damit lässt sich bereits die Dualität zu sogenannten Control Allocation-Problemen erkennen. Dabei wird die Regelung von Systemen mit redundanten Eingängen, also linear abhängigen Spalten in der Eingangsmatrix, betrachtet (s. zum Beispiel [60, 115, 270]). Die sich ergebenden zusätzlichen Freiheitsgrade werden zu verschiedenen Zwecken genutzt, unter anderem zur fehlertoleranten Regelung [24] und zur Berücksichtigung von Stellgrößenbeschränkungen [270].

Bei dem System $\left(\boldsymbol{A}, \boldsymbol{E}_{a}, \boldsymbol{C}_{o}\right)$ handelt sich es um ein quadratisches System, für das wie in Abschnitt 4.5 beschrieben ein FIO $\left(\boldsymbol{L}_{l}, \boldsymbol{V}_{l}\right)$ entworfen werden kann, wenn die Annahmen 4.1 bis 4.3 jeweils mit der Matrix $\boldsymbol{C}_{o}$ erfüllt sind. Das folgende Lemma charakterisiert die zusätzlichen Freiheitsgrade, die sich bei Verwendung der redundanten Messgrößen ergeben.

Lemma 4.11. Gegeben sei ein System der Form (4.1) mit $\boldsymbol{D}=\mathbf{0}, \boldsymbol{E}_{s}=\mathbf{0}$, wobei das Teilsystem $\left(\boldsymbol{A}, \boldsymbol{E}_{a}, \boldsymbol{C}_{o}\right)$ die Bedingungen 4.1-4.3 erfüllt. Sei weiterhin durch $\left(\boldsymbol{L}_{l}, \boldsymbol{V}_{l}\right)$ eine FIO-Parametrierung für $\left(\boldsymbol{A}, \boldsymbol{E}_{a}, \boldsymbol{C}_{o}\right)$ gegeben, dann ist $(\boldsymbol{L}(t), \boldsymbol{V}(t))$ mit zeitvarianten Matrizen $\boldsymbol{L}(t)$ und $\boldsymbol{V}(t)$ eine FIOParametrierung für $\left(\boldsymbol{A}, \boldsymbol{E}_{a}, \boldsymbol{C}\right)$, wenn

$$
\begin{aligned}
\boldsymbol{L}_{l} \boldsymbol{C}_{o} & =\boldsymbol{L}(t) \cdot \boldsymbol{C}, \\
\boldsymbol{V}_{l} \boldsymbol{C}_{o} & =\boldsymbol{V}(t) \cdot \boldsymbol{C}
\end{aligned}
$$

gilt.

Beweis. Die Residuen werden unter Verwendung von $\left(\boldsymbol{L}_{l}, \boldsymbol{V}_{l}\right)$ durch

$$
\begin{aligned}
\dot{\hat{\boldsymbol{x}}} & =\boldsymbol{A} \hat{\boldsymbol{x}}+\boldsymbol{B} \boldsymbol{u}+\boldsymbol{L}_{l}\left(\boldsymbol{y}_{o}-\hat{\boldsymbol{y}}_{o}\right), \\
\boldsymbol{r} & =\boldsymbol{V}_{l}\left(\boldsymbol{y}_{o}-\hat{\boldsymbol{y}}_{o}\right)
\end{aligned}
$$

erzeugt, wobei $\boldsymbol{y}_{o}$ die ersten $n_{f}$ Elemente von $\boldsymbol{y}$ enthält. Offensichtlich wird die Differentialgleichung (4.86a) bei Rückgriff auf die redundanten Messgrößen auf dieselbe Art und Weise angeregt, wenn

$$
\begin{aligned}
\boldsymbol{L}_{l}\left(\boldsymbol{y}_{o}-\hat{\boldsymbol{y}}_{o}\right) & =\boldsymbol{L}(t)(\boldsymbol{y}-\hat{\boldsymbol{y}}), \\
\boldsymbol{L}_{l} \boldsymbol{C}_{o}(\boldsymbol{x}-\hat{\boldsymbol{x}}) & =\boldsymbol{L}(t) \boldsymbol{C}(\boldsymbol{x}-\hat{\boldsymbol{x}})
\end{aligned}
$$


gilt. Dafür ist die Bedingung (4.85a) hinreichend. Durch analoge Überlegungen ergibt sich aus (4.86b), dass (4.85) hinreichend dafür ist, dass mittels eines durch $(\boldsymbol{L}(t), \boldsymbol{V}(t))$ parametrierten FIOs die gleichen Residuen erzeugt werden wie durch (4.86).

Beachtenswert ist insbesondere die Tatsache, dass sich ebenso wie bei der dynamischen Control Allocation nach [270] die Matrizen $\boldsymbol{L}(t)$ und $\boldsymbol{V}(t)$ beliebig schnell zeitlich ändern dürfen, solange (4.85) eingehalten ist.

Lemma 4.11 sagt aus, dass in den betrachteten nichtquadratischen Systemen zusätzliche Freiheitsgrade existieren. Der folgende Satz liefert eine explizite Charakterisierung dieser Freiheitsgrade.

Satz 4.10. Die Freiheitsgrade, die durch (4.85) in Lemma 4.11 gegeben sind, lassen sich mittels zweier beliebiger zeitvarianter Matrizen $\boldsymbol{Z}_{L}(t) \in \mathbb{R}^{n \times\left(n_{y}-n_{f}\right)} \quad$ und $\boldsymbol{Z}_{V}(t) \in \mathbb{R}^{n_{f} \times\left(n_{y}-n_{f}\right)}$ explizit charakterisieren durch

$$
\begin{aligned}
\boldsymbol{L}(t) & =\boldsymbol{L}_{l} \boldsymbol{C}_{o} \boldsymbol{C}^{+}+\boldsymbol{Z}_{L}(t) \cdot{ }^{\perp} \boldsymbol{C}, \\
\boldsymbol{V}(t) & =\boldsymbol{V}_{l} \boldsymbol{C}_{o} \boldsymbol{C}^{+}+\boldsymbol{Z}_{V}(t) \cdot{ }^{\perp} \boldsymbol{C} .
\end{aligned}
$$

Beweis. Durch Einsetzen von (4.87a) in (4.85a) folgt

$$
\begin{aligned}
& \boldsymbol{L} C=\left(\boldsymbol{L}_{l} \boldsymbol{C}_{o} \boldsymbol{C}^{+}+\boldsymbol{Z}_{L}{ }^{\perp} \boldsymbol{C}\right) \boldsymbol{C}=\boldsymbol{L}_{l} \boldsymbol{C}_{o} \boldsymbol{C}^{+} \boldsymbol{C}+\boldsymbol{Z}_{l} \cdot \underbrace{{ }^{\perp} \boldsymbol{C} C}_{\mathbf{0}} \\
& =\boldsymbol{L}_{l} \underbrace{\left[\begin{array}{ll}
\boldsymbol{I}_{n_{f}} & \mathbf{0}
\end{array}\right] \boldsymbol{C}}_{\boldsymbol{C}_{o}} \boldsymbol{C}^{+} \boldsymbol{C}=\boldsymbol{L}_{l}\left[\begin{array}{ll}
\boldsymbol{I}_{n_{f}} & \mathbf{0}
\end{array}\right]\left[\begin{array}{l}
\boldsymbol{C}_{o} \\
\boldsymbol{C}_{u}
\end{array}\right] \\
& =\boldsymbol{L}_{l} \boldsymbol{C}_{o},
\end{aligned}
$$

wobei zur Vereinfachung der Notation $\boldsymbol{L}=\boldsymbol{L}(t)$ und $\boldsymbol{Z}_{L}=\boldsymbol{Z}_{L}(t)$ geschrieben wurde. In analoger Weise lässt sich zeigen, dass $(4.87 \mathrm{~b})$ die Bedingung (4.85b) erfüllt.

Beachtenswert ist die Ähnlichkeit von (4.87) zu der Struktur der Beobachter- beziehungsweise Nachfiltermatrix gemäß Satz 4.8. Da $\boldsymbol{Z}_{L}(t)$ in (4.87a) im Linksnullraum von $\boldsymbol{C}$ liegt, beeinflusst die Matrix die Eigenwerte von $\boldsymbol{A}-\boldsymbol{L} \boldsymbol{C}$ im Gegensatz zu $\boldsymbol{R}_{L}$ in Satz 4.8 nicht. Weiterhin darf $\boldsymbol{Z}_{L}(t)$ wie erwähnt zeitabhängig sein, wohingegen $\boldsymbol{R}_{L}$ eine konstante Matrix ist.

Die Zeitabhängigkeit eröffnet Möglichkeiten zur Online-Adaption von $\boldsymbol{Z}_{L}(t)$ und $\boldsymbol{Z}_{V}(t)$. Auf dieses Themenfeld wird im Rahmen dieser Arbeit jedoch nicht näher eingegangen, für zeitdiskrete Systeme wird es in [231] eingehender untersucht. 


\subsubsection{Entwurf für erweiterte Diagonalelemente mittels Vollständiger Modaler Synthese}

Wie bereits in Abschnitt 4.6.1 angedeutet lässt sich der Entwurf dynamischer Fehlerisolationsbeobachter mit erweiterter Dynamik der Diagonalelemente $g_{i, i}(s)$ auch mittels der Vollständigen Modalen Synthese durchführen. Dafür wird zunächst die Gesamtdynamik eines aus der Strecke und einem DFIO bestehenden Systems wiederholt (vgl. (4.33) auf Seite 102). Das Ein-/Ausgangsverhalten zwischen Fehlern und Residuen wird mit dem Beobachterfehler $\boldsymbol{\xi}=\boldsymbol{x}-\hat{\boldsymbol{x}}$ und dem internen Zustand $\boldsymbol{\zeta}$ des dynamisch erweiterten Beobachters beschrieben durch

$$
\begin{aligned}
{\left[\begin{array}{c}
\dot{\boldsymbol{\xi}} \\
\dot{\boldsymbol{\zeta}}
\end{array}\right] } & =(\tilde{\boldsymbol{A}}-\tilde{\boldsymbol{L}} \tilde{\boldsymbol{C}})\left[\begin{array}{l}
\boldsymbol{\xi} \\
\boldsymbol{\zeta}
\end{array}\right]+\left(\tilde{\boldsymbol{E}}_{a}-\tilde{\boldsymbol{L}} \tilde{\boldsymbol{E}}_{s}\right) \boldsymbol{f} \\
\boldsymbol{r} & =\tilde{\boldsymbol{V}} \tilde{\boldsymbol{C}}\left[\begin{array}{l}
\boldsymbol{\xi} \\
\boldsymbol{\zeta}
\end{array}\right]+\tilde{\boldsymbol{V}} \tilde{\boldsymbol{E}}_{s} \boldsymbol{f} .
\end{aligned}
$$

Dabei ist das erweiterte System gegeben durch

$$
\begin{aligned}
& \tilde{\boldsymbol{A}}=\left[\begin{array}{cc}
\boldsymbol{A} & \mathbf{0} \\
\mathbf{0} & \mathbf{0}
\end{array}\right], \quad \tilde{\boldsymbol{E}}_{a}=\left[\begin{array}{c}
\boldsymbol{E}_{a} \\
\mathbf{0}
\end{array}\right], \\
& \tilde{\boldsymbol{C}}=\left[\begin{array}{cc}
\boldsymbol{C} & \mathbf{0} \\
\mathbf{0} & \boldsymbol{I}_{n_{\zeta}}
\end{array}\right], \tilde{\boldsymbol{E}}_{s}=\left[\begin{array}{c}
\boldsymbol{E}_{s} \\
\mathbf{0}
\end{array}\right]
\end{aligned}
$$

und die Matrizen $\tilde{\boldsymbol{L}}$ und $\tilde{\boldsymbol{V}}$ beinhalten gemäß

$$
\tilde{\boldsymbol{L}}=\left[\begin{array}{cc}
\boldsymbol{L}_{p} & \boldsymbol{L}_{i} \\
-\boldsymbol{\Gamma} & -\boldsymbol{\Phi}
\end{array}\right], \quad \tilde{\boldsymbol{V}}=\left[\begin{array}{ll}
\boldsymbol{V}_{\boldsymbol{y}} & \boldsymbol{V}_{\boldsymbol{\zeta}}
\end{array}\right]
$$

die Parametrierung des DFIOs. Aus (4.88) ergibt sich die Übertragungsmatrix $\boldsymbol{G}_{\boldsymbol{r} \boldsymbol{f}}(s)$, sie lautet

$$
\boldsymbol{G}_{\boldsymbol{r} \boldsymbol{f}}(s)=\tilde{\boldsymbol{V}} \tilde{\boldsymbol{C}}\left(s \boldsymbol{I}_{n+n_{\zeta}}-(\tilde{\boldsymbol{A}}-\tilde{\boldsymbol{L}} \tilde{\boldsymbol{C}})\right)^{-1}\left(\tilde{\boldsymbol{E}}_{a}-\tilde{\boldsymbol{L}} \tilde{\boldsymbol{E}}_{s}\right)+\tilde{\boldsymbol{V}} \tilde{\boldsymbol{E}}_{s}
$$

Da die dynamischen Erweiterungen des Beobachters als Teil der Strecke angenommen werden, lässt sich der Ansatz der Vollständigen Modalen Synthese aus Abschnitt 4.5.2 auf die erweiterte Strecke $\left(\tilde{\boldsymbol{A}}, \tilde{\boldsymbol{E}}_{a}, \tilde{\boldsymbol{C}}, \tilde{\boldsymbol{E}}_{s}\right)$ übertragen. Offensichtlich verfügt das erweiterte System über ebenso viele Fehler wie das ursprüngliche, reale System. Es ist also $\tilde{n}_{f}=n_{f}$. Da jedoch die internen Beobachterzustände $\boldsymbol{\zeta}$ rein virtuell und damit im Sinne des 
erweiterten Systems messbar sind, gilt $\tilde{n}_{y}=n_{y}+n_{\zeta}$. Wegen $\tilde{n}_{y}>\tilde{n}_{f}$ ist das erweiterte System nichtquadratisch, obwohl das ursprüngliche System quadratisch ist $\left(n_{y}=n_{f}\right)$.

Der Entwurf der Beobachtermatrix $\tilde{\boldsymbol{L}}$ erfolgt für das erweiterte System weitgehend analog zu Satz 4.9. Die invarianten Nullstellen der ursprünglichen Strecke $\left(\boldsymbol{A}, \boldsymbol{E}_{a}, \boldsymbol{C}, \boldsymbol{E}_{s}\right)$ müssen dabei weiterhin kompensiert werden. Dies ergibt sich aus der Tatsache, dass sie ebenfalls invariante Nullstellen von $\left(\tilde{\boldsymbol{A}}, \tilde{\boldsymbol{E}}_{a}, \tilde{\boldsymbol{C}}, \tilde{\boldsymbol{E}}_{s}\right)$ sind, da für das erweiterte System (4.88) die Beziehung

$$
\begin{aligned}
& \operatorname{rang}\left(\left[\begin{array}{cc}
s \boldsymbol{I}_{n+n_{\zeta}}-\tilde{\boldsymbol{A}} & \tilde{\boldsymbol{E}}_{a} \\
-\tilde{\boldsymbol{C}} & \tilde{\boldsymbol{E}}_{s}
\end{array}\right]\right)=\operatorname{rang}\left(\left[\begin{array}{ccc}
s \boldsymbol{I}_{n}-\boldsymbol{A} & \mathbf{0} & \boldsymbol{E}_{a} \\
\mathbf{0} & s \boldsymbol{I}_{n_{\zeta}} & \mathbf{0} \\
-\boldsymbol{C} & \mathbf{0} & \boldsymbol{E}_{s} \\
\mathbf{0} & -\boldsymbol{I}_{n_{\zeta}} & \mathbf{0}
\end{array}\right]\right) \\
& =\operatorname{rang}\left(\left[\begin{array}{cc:c}
s \boldsymbol{I}_{n}-\boldsymbol{A} & \boldsymbol{E}_{a} & \mathbf{0} \\
-\boldsymbol{C} & \boldsymbol{E}_{s} & \mathbf{0} \\
\hdashline \mathbf{0} & \mathbf{0} & s \overline{\boldsymbol{I}}_{n_{\zeta}} \\
\mathbf{0} & \mathbf{0} & -\boldsymbol{I}_{n_{\zeta}}
\end{array}\right]\right)
\end{aligned}
$$

gilt. Im Unterschied zu Satz 4.9 werden jedoch jeder Spalte von $\boldsymbol{G}_{\boldsymbol{r} \boldsymbol{f}}(s)$ genau $\delta_{i}+\rho$ Eigenwerte zugewiesen. Daraus ergibt sich unmittelbar die Ordnung der internen Dynamik des Beobachters zu $n_{\zeta}=\rho \cdot n_{f}$.

Lemma 4.12. Gegeben sei ein System der Form (4.1), welches die Annahmen 4.14-4.16 und 4.4 erfüllt. Für das zugehörige dynamisch erweiterte System $\left(\tilde{\boldsymbol{A}}, \tilde{\boldsymbol{E}}_{a}, \tilde{\boldsymbol{C}}, \tilde{\boldsymbol{E}}_{s}\right)$ seien die Parameter- und Linkseigenvektoren gemäß

$$
\begin{aligned}
& {\left[\begin{array}{ll}
\tilde{\boldsymbol{w}}_{B_{i j}}^{\top} & \tilde{\boldsymbol{p}}_{i j}^{\top}
\end{array}\right]=\left[\begin{array}{ll}
\mathbf{0}^{\top} & \boldsymbol{\phi}_{i}^{\top}
\end{array}\right] \tilde{\boldsymbol{\Pi}}_{i j}^{+}+\tilde{\boldsymbol{z}}_{i j}^{\top} \cdot{ }^{\perp} \tilde{\boldsymbol{\Pi}}_{i j}, \quad i=1, \ldots, n_{f}, j=1, \ldots, \delta_{i}+\rho,} \\
& {\left[\begin{array}{ll}
\tilde{\boldsymbol{w}}_{B_{k}}^{\top} & \tilde{\boldsymbol{p}}_{k}^{\top}
\end{array}\right]=\tilde{\boldsymbol{z}}_{k}^{\top} \cdot{ }^{\perp} \tilde{\boldsymbol{\Pi}}_{k}, \quad k=\delta+\rho \cdot n_{f}+1, \ldots, n+\rho \cdot n_{f},}
\end{aligned}
$$

festgelegt, wobei die vorgegebenen Eigenwerte $\lambda_{B_{i j}}$ beliebig platziert werden können und die verbleibenden Eigenwerte $\lambda_{B_{k}}$ entsprechend den invarianten Nullstellen des Systems (4.1) gesetzt werden. Ferner seien die Vektoren $\tilde{\boldsymbol{z}}_{i j}^{\top} \in \mathbb{R}^{\rho n_{f}}$ und $\tilde{\boldsymbol{z}}_{k} \neq \mathbf{0}^{\top} \in \mathbb{R}^{\rho n_{f}+1}$ so gewählt, dass sich linear unabhängige Linkseigenvektoren ergeben. Dann bewirkt die Beobachtermatrix

$$
\tilde{\boldsymbol{L}}=-\tilde{\boldsymbol{W}}_{B}^{-1} \tilde{\boldsymbol{P}}
$$

dass die Eigenwerte $\lambda_{B_{i j}}$ ausschließlich in der $i$-ten Spalte von $\boldsymbol{G}_{\boldsymbol{r} \boldsymbol{f}}(s)$ als Pole auftreten und die verbleibenden Eigenwerte $\lambda_{B_{k}}$ bezüglich $\left(\tilde{\boldsymbol{A}}-\tilde{\boldsymbol{L}} \tilde{\boldsymbol{C}}, \tilde{\boldsymbol{E}}_{a}-\tilde{\boldsymbol{L}} \tilde{\boldsymbol{E}}_{s}\right)$ nichtsteuerbar sind. 
Beweis. Werden die Beobachtereigenwerte $\lambda_{B_{i j}}$ so gewählt, dass sie nicht mit invarianten Nullstellen von $\left(\boldsymbol{A}, \boldsymbol{E}_{a}, \boldsymbol{C}, \boldsymbol{E}_{s}\right)$ zusammenfallen, ergibt sich aus dem Rangsatz (Satz A.1) für die Vektoren $\tilde{\boldsymbol{z}}_{i j}^{\top}$ die Dimension $\rho \cdot n_{f}$. Da die Eigenwerte $\lambda_{B_{k}}$ invariante Nullstellen des Systems kompensieren, handelt es sich bei $\tilde{\boldsymbol{z}}_{k}^{\mathrm{T}}$ um $\left(\rho \cdot n_{f}+1\right)$-dimensionale Vektoren. Der Beweis der Zuweisung zu einer Spalte beziehungsweise der Nichtsteuerbarkeit erfolgt analog zu Abschnitt 4.9.2.

Nach dem Entwurf von $\tilde{\boldsymbol{L}}$ verbleibt wie in den Abschnitten 4.5.2 und 4.9.2 die Auslegung der Nachfiltermatrix $\tilde{\boldsymbol{V}}$, sodass $\boldsymbol{G}_{\boldsymbol{r} \boldsymbol{f}}(s)$ die gewünschte Form annimmt. Das dort angewandte Vorgehen, die Nachfiltermatrix lediglich anhand der gewünschten stationären Verstärkung, das heißt für $s=0$, auszulegen, führt jedoch hier nicht zum Erfolg. Aufgrund der erweiterten Dynamik der Diagonalelemente versagt die Argumentation, die zu Lemma 4.3 führte. Aus der Vorgabe $g_{i, j}(s=0)=0$ kann nicht mehr $g_{i, j}(s)=0$ für alle $s \in \mathbb{C}$ gefolgert werden, da $g_{i, j}(s)$ Nullstellen aufweisen kann.

Der Fehlerindex $\delta_{j}$ stellt wie in Abschnitt 4.5.2 erörtert eine untere Schranke für die Differenzenordnung von $g_{i, j}(s)$ dar. Aus der Zuweisung von $\delta_{j}+\rho$ Polen zur $j$-ten Spalte folgt, dass der Zählergrad von $g_{i, j}(s)$ höchstens $\rho$ sein kann. Diese Erkenntnis erlaubt es, das Vorgehen aus Abschnitt 4.5.2 in abgewandelter Form auch für eine erweiterte Dynamik der Diagonalelemente anzuwenden.

Lemma 4.13. Gegeben sei ein System der Form (4.1), welches die Annahmen 4.14-4.16 erfüllt. Für das zugehörige dynamisch erweiterte System $\left(\tilde{\boldsymbol{A}}, \tilde{\boldsymbol{E}}_{a}, \tilde{\boldsymbol{C}}, \tilde{\boldsymbol{E}}_{s}\right)$ sei eine Beobachtermatrix $\tilde{\boldsymbol{L}}$ gemäß Lemma 4.12 entworfen. Gilt dann für die Nichtdiagonalelemente $g_{i, j}(s)$ von $\boldsymbol{G}_{\boldsymbol{r f}}(s)$ durch eine entsprechende Wahl der Nachfiltermatrix $\tilde{\boldsymbol{V}}$ die Beziehung

$$
g_{i, j}\left(s_{l}\right)=0, i, j=1, \ldots, n_{f}, i \neq j, l=1, \ldots, \rho+1,
$$

für $\rho+1$ Frequenzen $s_{l}$, so folgt

$$
g_{i, j}(s)=0, i, j=1, \ldots, n_{f}, i \neq j, \forall s \in \mathbb{C} .
$$

Beweis. Es gibt nur zwei Möglichkeiten, die Gleichung (4.89) zu erfüllen: Entweder muss (4.90) gelten, oder $g_{i, j}(s)$ muss für jede Frequenz $s_{l}$ eine Nullstelle aufweisen. Der Beweis von Lemma 4.13 erfolgt durch Widerspruch der zweiten Möglichkeit. Um sie zu erfüllen, müsste $g_{i, j}(s)$ die Struktur

$$
g_{i, j}(s)=\frac{k_{i, j}\left(s-s_{1}\right) \cdot \ldots \cdot\left(s-s_{\rho+1}\right)}{\left(s-\lambda_{B_{j 1}}\right) \cdot \ldots \cdot\left(s-\lambda_{B_{j \delta_{j}+\rho}}\right)}
$$


aufweisen. Dazu sind jedoch $\rho+1$ Nullstellen nötig, was im Widerspruch zum maximalen Zählergrad von $\rho$ steht.

Aus Lemma 4.13 ergibt sich ein konstruktives Entwurfsverfahren für die Nachfiltermatrix $\tilde{\boldsymbol{V}}$. Dabei wird die gewünschte Matrix $\boldsymbol{G}_{\boldsymbol{r} \boldsymbol{f}}(s)$ mit den angestrebten Diagonalelementen an $\rho+1$ Frequenzen $s_{l}, l=1, \ldots, \rho+1$, ausgewertet. Mit der gemäß Lemma 4.12 berechneten Beobachtermatrix ergibt sich jeweils die Gleichung

$$
\boldsymbol{G}_{\boldsymbol{r} \boldsymbol{f}}\left(s_{l}\right)=\tilde{\boldsymbol{V}}[\underbrace{\tilde{\boldsymbol{C}}\left(s_{l} \boldsymbol{I}_{n+\rho n_{f}}-(\tilde{\boldsymbol{A}}-\tilde{\boldsymbol{L}} \tilde{\boldsymbol{C}})\right)^{-1}\left(\tilde{\boldsymbol{E}}_{a}-\tilde{\boldsymbol{L}} \tilde{\boldsymbol{E}}_{s}\right)+\tilde{\boldsymbol{E}}_{s}}_{\tilde{\boldsymbol{M}}_{s_{l}}}] .
$$

Darin ist $\boldsymbol{G}_{\boldsymbol{r} \boldsymbol{f}}\left(s_{l}\right)$ eine $\left(n_{f} \times n_{f}\right)$-dimensionale Matrix, während $\tilde{\boldsymbol{M}}_{s_{l}}$ die Dimension $\left(n_{y}+\rho n_{f}\right) \times n_{f}$ hat. Da die Nachfiltermatrix $\tilde{\boldsymbol{V}}$ die Beziehung (4.91) für $\rho+1$ Frequenzen erfüllen muss, ergibt sich zusammengefasst das Gleichungssystem

$$
\tilde{\boldsymbol{V}}\left[\begin{array}{lll}
\tilde{\boldsymbol{M}}_{s_{1}} & \cdots & \tilde{\boldsymbol{M}}_{s_{\rho+1}}
\end{array}\right]=\tilde{\boldsymbol{V}} \tilde{\boldsymbol{M}}=\left[\begin{array}{lll}
\boldsymbol{G}_{\boldsymbol{r} \boldsymbol{f}}\left(s_{1}\right) & \cdots & \boldsymbol{G}_{\boldsymbol{r} \boldsymbol{f}}\left(s_{\rho+1}\right)
\end{array}\right] .
$$

Für ein System, für das ein statischer FIO entworfen werden kann, ist gemäß Abschnitt 4.6 die Existenz eines dynamischen FIOs garantiert. Da beim Entwurf von $\tilde{\boldsymbol{L}}$ nach Lemma 4.12 jeder Spalte der FehlerResiduen-Übertragungsmatrix $\rho$ zusätzliche Pole zugewiesen wurden, ist (4.92) lösbar. Bei $\tilde{\boldsymbol{M}}$ handelt es sich um eine $\left(n_{y}+\rho n_{f}\right) \times\left((\rho+1) n_{f}\right)$ dimensionale Matrix und da das ursprüngliche System $\left(\boldsymbol{A}, \boldsymbol{E}_{a}, \boldsymbol{C}, \boldsymbol{E}_{s}\right)$ quadratisch ist und daher $n_{y}=n_{f}$ gilt, ist $\tilde{\boldsymbol{M}}$ quadratisch. Folglich ergibt $\operatorname{sich} \tilde{\boldsymbol{V}}$ aus

Satz 4.11 (Entwurf von FIOs mit erweiterter Dynamik der Diagonalelemente mittels Vollständiger Modaler Synthese). Gegeben sei ein System der Form

$$
\begin{aligned}
& \dot{\boldsymbol{x}}=\boldsymbol{A} \boldsymbol{x}+\boldsymbol{B} \boldsymbol{u}+\boldsymbol{E}_{a} \boldsymbol{f}, \\
& \boldsymbol{y}=\boldsymbol{C} \boldsymbol{x}+\boldsymbol{D} \boldsymbol{u}+\boldsymbol{E}_{s} \boldsymbol{f},
\end{aligned}
$$

welches die Annahmen 4.14-4.16 erfüllt. Für das zugehörige dynamisch erweiterte System $\left(\tilde{\boldsymbol{A}}, \tilde{\boldsymbol{E}}_{a}, \tilde{\boldsymbol{C}}, \tilde{\boldsymbol{E}}_{s}\right)$ sei ein Beobachter $\tilde{\boldsymbol{L}}$ gemäß Lemma 4.12 entworfen. Wertet man die Matrix

$$
\boldsymbol{G}_{\boldsymbol{r} \boldsymbol{f}}(s)=\operatorname{diag}\left(\frac{\sum_{j=0}^{\rho} z_{1, j} s^{j}}{s^{\delta_{1}+\rho}+\sum_{k=0}^{\delta_{1}+\rho-1} q_{1, k} s^{k}}, \ldots, \frac{\sum_{j=0}^{\rho} z_{n_{f}, j} s^{j}}{s^{\delta_{n_{f}}+\rho}+\sum_{k=0}^{\delta_{n_{f}}+\rho-1} q_{n_{f}, k} s^{k}}\right)
$$


an $\rho+1$ paarweise verschiedenen Frequenzen $s_{l}$ aus, so führt die Nachfiltermatrix

$$
\tilde{\boldsymbol{V}}=\left[\begin{array}{lll}
\boldsymbol{G}_{\boldsymbol{r} \boldsymbol{f}}\left(s_{1}\right) & \cdots & \boldsymbol{G}_{\boldsymbol{r} \boldsymbol{f}}\left(s_{\rho+1}\right)
\end{array}\right]\left[\begin{array}{lll}
\tilde{\boldsymbol{M}}_{s_{1}} & \cdots & \tilde{\boldsymbol{M}}_{s_{\rho+1}}
\end{array}\right]^{-1}
$$

auf die vorgegebene Übertragungsmatrix $\boldsymbol{G}_{\boldsymbol{r} \boldsymbol{f}}(s)$.

Die Matrizen $\tilde{\boldsymbol{L}}$ und $\tilde{\boldsymbol{V}}$ parametrieren einen statischen FIO für das erweiterte System. Die Beobachtermatrizen des dynamisch erweiterten FIOs für das ursprüngliche System ergeben sich durch die Partitionierung

$$
\left[\begin{array}{cc}
\boldsymbol{L}_{p} & \boldsymbol{L}_{i} \\
-\boldsymbol{\Gamma} & -\boldsymbol{\Phi}
\end{array}\right]=\tilde{\boldsymbol{L}}, \quad\left[\begin{array}{ll}
\boldsymbol{V}_{\boldsymbol{y}} & \boldsymbol{V}_{\boldsymbol{\zeta}}
\end{array}\right]=\tilde{\boldsymbol{V}}
$$

Gegenüber den in Abschnitt 4.6 vorgestellten Verfahren weist der Entwurf mittels Vollständiger Modaler Synthese verschiedene Vorteile auf. Im Vergleich zur Methode aus Abschnitt 4.6.3 ist es nicht notwendig, zunächst Substitutionen durchzuführen um dann das duale Regelungsproblem lösen zu können. Außerdem erhält man aus Satz 4.11 unmittelbar eine Parametrierung der Matrizen des DFIOs, es muss keine Zustandsraumrealisierung eines im Laplace-Bereich beschriebenen Systems gefunden werden. Darüber hinaus ist im Gegensatz zum Entwurf nach Abschnitt 4.6.4 die Dynamikerweiterung der Diagonalelemente nicht auf den Nenner beschränkt. Tabelle 4.1 stellt die drei Verfahren zusammenfassend gegenüber. Sie verdeutlicht, dass der Ansatz mittels der Vollständigen Modalen Synthese die Vorteile der anderen beiden Verfahren vereint.

Bemerkung 4.13. Betrachtet man ein quadratisches System mit den Fehlerindizes $\delta_{i}$ und erweitert es um einen zusätzlichen, nicht redundanten Ausgang, so ergeben sich neue Fehlerindizes $\tilde{\delta}_{i} \leq \delta_{i}$. Bisher wurden stets FIOs entworfen, welche $\tilde{\delta}_{i}$ Pole in den Diagonalelementen von $g_{i, i}(s)$ vorgeben. Dies erlaubt bei einer entsprechenden Parametrierung der Nachfiltermatrix $\boldsymbol{V}$ wie in Abschnitt 4.5.2 den Schluss $\boldsymbol{G}_{\boldsymbol{r} \boldsymbol{f}}(s=0)=\boldsymbol{S} \Rightarrow \boldsymbol{G}_{\boldsymbol{r} \boldsymbol{f}}(s)=\operatorname{diag}\left(g_{1,1}(s), \ldots, g_{n_{f}, n_{f}}(s)\right)$ für alle $s \in \mathbb{C}$. Offensichtlich ist es jedoch auch möglich, die Fehlerindizes $\delta_{i}$ des quadratischen Systems für das nichtquadratische System vorzugeben (eine triviale Lösung ist es, die mit dem neuen Ausgang assoziierten Spalten von $\boldsymbol{L}$ und $\boldsymbol{V}$ zu null zu setzen). Um die Diagonalisierung von $\boldsymbol{G}_{\boldsymbol{r} \boldsymbol{f}}(s)$ für alle $s \in \mathbb{C}$ zu gewährleisten, kann dann zum Entwurf von $\boldsymbol{V}$ wie in Lemma 4.13 für mehr als eine Frequenz $s_{l}$ eine Vorgabe von $\boldsymbol{G}_{\boldsymbol{r} \boldsymbol{f}}\left(s_{l}\right)=\boldsymbol{S}_{l}$ erfolgen.

Bemerkung 4.14. In dualer Art und Weise lässt sich das vorgestellte Verfahren auch zum Entwurf der in Anhang B behandelten dynamischen Entkopplungsregler einsetzen. 
Tabelle 4.1: Vergleich der Entwurfsverfahren für erweiterte Dynamik der Diagonalelemente

\begin{tabular}{l||c|c|c} 
& $\begin{array}{l}\text { Frequenzbe- } \\
\text { reichsentwurf, } \\
\text { Abschnitt 4.6.3 }\end{array}$ & $\begin{array}{l}\text { Zeitbereichs- } \\
\text { entwurf, } \\
\text { Abschnitt 4.6.4 }\end{array}$ & $\begin{array}{l}\text { VMS, } \\
\text { Abschnitt } \\
4.9 .5\end{array}$ \\
\hline \hline $\begin{array}{l}\text { Lösung des dua- } \\
\text { len Regelungspro- } \\
\text { blems nötig }\end{array}$ & ja & nein & nein \\
\hline $\begin{array}{l}\text { Zustandsraum- } \\
\text { realisierung nötig }\end{array}$ & ja & nein & nein \\
\hline $\begin{array}{l}\text { Struktur der Dia- } \\
\text { gonalelemente }\end{array}$ & $\begin{array}{l}\text { Zähler und } \\
\text { Nenner } \\
\text { erweitert }\end{array}$ & $\begin{array}{l}\text { Nenner } \\
\text { erweitert }\end{array}$ & $\begin{array}{l}\text { Zähler und } \\
\text { Nenner } \\
\text { erweitert }\end{array}$
\end{tabular}

\subsubsection{Fazit}

In nichtquadratischen Systemen mit mehr messbaren Ausgangsgrößen als Fehlern existieren gegenüber quadratischen Systemen mit $n_{y}=n_{f}$ zusätzliche Freiheitsgrade beim Entwurf von Fehlerisolationsbeobachtern. Diese lassen sich sowohl durch eine Erweiterung des auf dem Verfahren von Falb und Wolovich basierenden Entwurfes charakterisieren als auch mittels der Vollständigen Modalen Synthese. Während der erstgenannte Ansatz den Vorteil bietet, dass er die zusätzlichen Freiheitsgrade in beliebig wählbaren Matrizen konzentriert, bietet letzterer eine bessere Transparenz hinsichtlich der inneren Systemstruktur und somit eine bessere Interpretierbarkeit der Freiheitsgrade.

\subsection{Partielle Fehlerisolation}

Wie in Abschnitt 4.1 erörtert bedingt die Forderung nach vollständiger Fehlerisolation strenge strukturelle Beschränkungen in $\boldsymbol{G}_{\boldsymbol{r} \boldsymbol{f}}(s)$. Dadurch werden die Freiheitsgrade beim FIO-Entwurf stark reduziert. Häufig ist jedoch eine weniger restriktive Struktur von $\boldsymbol{G}_{\boldsymbol{r} \boldsymbol{f}}(s)$ für die Isolation von Fehlern ausreichend. Ist beispielsweise bekannt, dass Fehler in einem System nicht gleichzeitig aktiv sind, so ist eine eindeutige Fehlerisolation wie 
in Abschnitt 2.3 erörtert auch dann möglich, wenn $\boldsymbol{G}_{\boldsymbol{r} \boldsymbol{f}}(s)$ die Form

$$
\boldsymbol{G}_{\boldsymbol{r} \boldsymbol{f}}(s)=\left[\begin{array}{cccc}
g_{1,1}(s) & g_{1,2}(s) & \cdots & g_{1, n_{f}}(s) \\
0 & g_{2,2}(s) & \ddots & \vdots \\
\vdots & \ddots & \ddots & g_{n_{f}-1, n_{f}}(s) \\
0 & \cdots & 0 & g_{n_{f}, n_{f}}(s)
\end{array}\right]
$$

aufweist, also eine obere Dreiecksstruktur besitzt.

Die vollständige Diagonalisierung von $\boldsymbol{G}_{\boldsymbol{r} \boldsymbol{f}}(s)$ wie in (4.5) und die Dreiecksstruktur aus (4.93) stellen dabei zwei Extreme dar. Während (4.5) zur Isolation notwendig ist, falls alle Fehler im System gleichzeitig auftreten können, ist (4.93) hinreichend, falls jeweils nur ein Fehler zu einem Zeitpunkt aktiv sein kann. Selbstverständlich sind Zwischenstufen möglich. Falls zum Beispiel in einem System mit drei Fehlern die ersten beiden Fehler nicht simultan auftreten können, der dritte Fehler jedoch zeitgleich zu einem der ersten beiden wirken kann, so ist

$$
\boldsymbol{G}_{\boldsymbol{r} \boldsymbol{f}}(s)=\left[\begin{array}{cc:c}
g_{1,1}(s) & g_{1,2}(s) & 0 \\
\hdashline 0 & g_{2,2}(s) & 0 \\
\hdashline 0 & 0 & g_{3,3}(s)
\end{array}\right]
$$

ausreichend für eine eindeutige Isolation der Fehler. Dies wird deutlich, wenn man die Wirkung der Fehler auf die einzelnen Residuen betrachtet: Wegen

$$
\begin{aligned}
& r_{3}(s)=g_{3,3}(s) \cdot f_{3}(s) \\
& r_{2}(s)=g_{2,2}(s) \cdot f_{2}(s) \\
& r_{1}(s)=g_{1,1}(s) \cdot f_{1}(s)+g_{1,2}(s) \cdot f_{2}(s)
\end{aligned}
$$

beeinflusst der dritte Fehler ausschließlich das Residuum $r_{3}$. Anhand dieses Residuums ist also eine eindeutige Isolation des Fehlers $f_{3}$ möglich, unabhängig davon, ob die anderen Fehler aktiv sind. Das Residuum $r_{2}$ wiederum wird ausschließlich vom Fehler $f_{2}$ beeinflusst und kann daher zur Isolation dieses Fehlers herangezogen werden. Da der Fehler $f_{2}$ ebenso wie $f_{1}$ auf das erste Residuum wirkt, ist eine eindeutige Isolation des ersten Fehlers nur dann möglich, wenn $f_{2}=0$ gilt, wenn also der zweite Fehler nicht aktiv ist.

Strukturen der Form (4.94) lassen sich mit dem hier vorgestellten Verfahren leicht realisieren. Ihr Entwurf lässt sich als Spezialfall der teilweisen Fehlerisolation auffassen, auf den in Abschnitt 5.5 näher eingegangen wird. 
Für die vorgestellte Entwurfsmethodik werden die gleichen Annahmen getroffen wie in Abschnitt 4.5. Bei Betrachtung der Struktur von $\boldsymbol{G}_{\boldsymbol{r} \boldsymbol{f}}(s)$ in (4.93) wird deutlich, dass die teilweise Fehlerisolation eng verwandt ist mit dem Problem der Dreiecksentkopplung $\left.{ }^{8}\right)[138,139,156]$. Daraus ist ersichtlich, dass die Annahmen 4.2 und 4.3, also rang $\left(\boldsymbol{D}^{*}\right)=\boldsymbol{n}_{f}$ und die Minimalphasigkeit des Systems $\left(\boldsymbol{A}, \boldsymbol{E}_{a}, \boldsymbol{C}, \boldsymbol{E}_{s}\right)$, für den Entwurf eines FIOs zur teilweisen Fehlerisolation nicht notwendig sind. Ein solcher FIO wird im Weiteren abkürzend als PFIO (engl. partial fault isolation observer) bezeichnet, in Abgrenzung zu einem FFIO (engl. full fault isolation obser$v e r)$. Hinreichend für die Existenz eines stabilen PFIOs für quadratische Systeme mit $n_{y}=n_{f}$ ist

$$
\operatorname{rang}\left(\boldsymbol{G}_{\boldsymbol{y} \boldsymbol{f}}(s)\right)=n_{y} \text { für fast alle } s \in \mathbb{C} .
$$

Dies ergibt sich unmittelbar aus der in Abschnitt 4.4 herausgearbeiteten Dualität zu Entkopplungsregelungen und der in [80] angegebenen Existenzbedingung für statische Zustandsrückführungen zur Dreiecksentkopplung. Hier werden jedoch die gleichen Annahmen getroffen wie für die vollständige Fehlerisolation, um später die resultierenden FIOs hinsichtlich ihrer Robustheit vergleichen zu können. Da die strukturellen Anforderungen von (4.93) schwächer sind als in (4.5), müssen sich bei gleichen Annahmen an das System zusätzliche Entwurfsfreiheitsgrade ergeben. Diese zu charakterisieren ist Gegenstand des folgenden Abschnittes, wobei die Ergebnisse in Auszügen bereits in [229] veröffentlicht wurden.

\subsubsection{Eine alternative Formulierung des Entwurfsverfahrens für FFIOs}

Zunächst wird eine alternative Formulierung des Entwurfsverfahrens für Beobachter zur vollständigen Fehlerisolation mittels der Vollständigen Modalen Synthese angegeben. Vorteil des beschriebenen Verfahrens ist, dass die Bedeutung der Parametervektoren anschaulicher ist als in Satz 4.2, was die Herleitung des Entwurfsverfahrens für Beobachter zur teilweisen Fehlerisolation übersichtlicher gestaltet. Ähnlich wie bei Satz 4.2 gelten dabei die Annahmen

Annahme 4.18. Das Paar $(\boldsymbol{A}, \boldsymbol{C})$ ist vollständig beobachtbar.

8) Ziel der Dreiecksentkopplung ist es, für ein System der Form $\dot{\boldsymbol{x}}=\boldsymbol{A} \boldsymbol{x}+\boldsymbol{B u}, \boldsymbol{y}=\boldsymbol{C} \boldsymbol{x}$ eine statische Zustandsrückführung mit Vorfilter $\boldsymbol{u}=-\boldsymbol{K} \boldsymbol{x}+\boldsymbol{F} \boldsymbol{w}$ dergestalt auszulegen, dass sich für die Übertragungsmatrix $\boldsymbol{G}_{\boldsymbol{y} \boldsymbol{w}}(s)$ des geschlossenen Regelkreises eine obere beziehungsweise untere Dreiecksstruktur ergibt. 
Annahme 4.19. Die Fehlerdetektionsmatrix hat vollen Rang, das heißt es gilt rang $\left(\boldsymbol{D}^{*}\right)=n_{f}$.

Annahme 4.20. Das System $\left(\boldsymbol{A}, \boldsymbol{E}_{a}, \boldsymbol{C}, \boldsymbol{E}_{s}\right)$ ist minimalphasig.

Annahme 4.21. Die Beobachtereigenwerte sind paarweise verschieden, das heißt, es gilt $\lambda_{B_{i}} \neq \lambda_{B_{j}} \forall i, j=1, \ldots, n, i \neq j$.

Weiterhin ist für das alternative Entwurfsverfahren die folgende zusätzliche Annahme zu treffen:

Annahme 4.22. Kein Beobachtereigenwert $\lambda_{B_{i}}, i=1, \ldots, n$, fällt mit einem Streckeneigenwert zusammen.

Da die steuerbaren Beobachtereigenwerte frei platziert werden können, stellt diese Annahme keine wesentliche Einschränkung dar. Es gilt der folgende Satz, der im Anschluss bewiesen wird:

Satz 4.12 (Alternatives Verfahren zum Entwurf von FIOs mittels Vollständiger Modaler Synthese). Gegeben sei ein System der Form

$$
\begin{aligned}
& \dot{\boldsymbol{x}}=\boldsymbol{A} \boldsymbol{x}+\boldsymbol{B} \boldsymbol{u}+\boldsymbol{E}_{a} \boldsymbol{f}, \\
& \boldsymbol{y}=\boldsymbol{C} \boldsymbol{x}+\boldsymbol{D} \boldsymbol{u}+\boldsymbol{E}_{s} \boldsymbol{f},
\end{aligned}
$$

welches die Annahmen 4.18-4.22 erfüllt. Dann führt eine durch

$$
\begin{aligned}
\boldsymbol{L}_{\mathrm{ffi}} & =-\boldsymbol{W}_{B, \mathrm{ffi}}^{-1} \boldsymbol{P}_{\mathrm{ffi}}, \\
\boldsymbol{p}_{\mathrm{ff}, i j}^{\top} & =\boldsymbol{\phi}_{i}^{\top}\left(\boldsymbol{C}\left(\lambda_{B_{i j}} \boldsymbol{I}_{n}-\boldsymbol{A}\right)^{-1} \boldsymbol{E}_{a}+\boldsymbol{E}_{s}\right)^{-1} \\
& =\boldsymbol{\phi}_{i}^{\top} \boldsymbol{G}_{\boldsymbol{y} \boldsymbol{f}}^{-1}\left(\lambda_{B_{i j}}\right), i=1, \ldots, n_{f}, j=1, \ldots, \delta_{i}, \\
\boldsymbol{w}_{B, \mathrm{ffi}, i j}^{\top} & =\boldsymbol{p}_{\mathrm{ffi}, i j}^{\top} \boldsymbol{C}\left(\lambda_{B_{i j}} \boldsymbol{I}_{n}-\boldsymbol{A}\right)^{-1}, \\
{\left[\boldsymbol{w}_{B, \mathrm{ff}, k}^{\top} \quad \boldsymbol{p}_{\mathrm{ffi}, k}^{\top}\right] } & ={ }^{\perp}\left(\boldsymbol{\Pi}\left(\lambda_{B_{k}}\right)\right)={ }^{\perp} \boldsymbol{\Pi}_{k}, k=\delta+1, \ldots, n
\end{aligned}
$$

parametrierte Beobachtermatrix $\boldsymbol{L}_{\mathrm{ffi}}$ mit einer Nachfiltermatrix

$$
\boldsymbol{V}_{\mathrm{ffi}}=-\boldsymbol{S}_{\mathrm{ffi}}\left(\boldsymbol{C}\left(\boldsymbol{A}-\boldsymbol{L}_{\mathrm{ffi}} \boldsymbol{C}\right)^{-1}\left(\boldsymbol{E}_{a}-\boldsymbol{L}_{\mathrm{ffi}} \boldsymbol{E}_{s}\right)-\boldsymbol{E}_{s}\right)^{-1}
$$

mit einer beliebigen Diagonalmatrix $\boldsymbol{S}_{\mathrm{ffi}} \in \mathbb{R}^{n_{f} \times n_{f}}=\operatorname{diag}\left(\tilde{z}_{1}, \ldots, \tilde{z}_{n_{f}}\right)$ beim Einsatz eines FIOs der Form

$$
\begin{aligned}
\dot{\hat{\boldsymbol{x}}} & =\boldsymbol{A} \hat{\boldsymbol{x}}+\boldsymbol{B} \boldsymbol{u}+\boldsymbol{L}_{\mathrm{ffi}}(\boldsymbol{y}-\boldsymbol{C} \hat{\boldsymbol{x}}-\boldsymbol{D} \boldsymbol{u}), \\
\boldsymbol{r} & =\boldsymbol{V}_{\mathrm{ffi}}(\boldsymbol{y}-\boldsymbol{C} \hat{\boldsymbol{x}}-\boldsymbol{D} \boldsymbol{u})
\end{aligned}
$$


auf eine diagonale Übertragungsmatrix $\boldsymbol{G}_{\boldsymbol{r} \boldsymbol{f}}(s)$. Die Diagonalelemente dieser Matrix lauten

$$
g_{i, i}(s)=\frac{\tilde{z}_{i} \cdot \prod_{j=1}^{\delta_{i}}\left(-\lambda_{B_{i j}}\right)}{\left(s-\lambda_{B_{i 1}}\right) \cdot \ldots \cdot\left(s-\lambda_{B_{i \delta_{i}}}\right)} .
$$

Beweis. Der Beweis stimmt im Wesentlichen mit dem Vorgehen aus Abschnitt 4.5.2 überein. Ein Unterschied ergibt sich lediglich in der Behandlung steuerbarer Beobachtereigenwerte. Da Annahme 4.22 die Existenz der Inversen von $\left(\lambda_{B_{i j}} \boldsymbol{I}_{n}-\boldsymbol{A}\right)$ sicherstellt, lässt sich (4.19) umschreiben zu

$$
\boldsymbol{w}_{B, \mathrm{ffi}, i j}^{\top}=\boldsymbol{p}_{\mathrm{ffi}, i j}^{\top} \boldsymbol{C}\left(\lambda_{B_{i j}} \boldsymbol{I}_{n}-\boldsymbol{A}\right)^{-1} .
$$

Setzt man dies in (4.20) ein, so ergibt sich

$$
\boldsymbol{p}_{\mathrm{ffi}, i j}^{\top}\left(\boldsymbol{C}\left(\lambda_{B_{i j}} \boldsymbol{I}_{n}-\boldsymbol{A}\right)^{-1} \boldsymbol{E}_{a}+\boldsymbol{E}_{s}\right)=\boldsymbol{p}_{\mathrm{ffi}, i j}^{\top} \boldsymbol{G}_{\boldsymbol{y} \boldsymbol{f}}\left(\lambda_{B_{i j}}\right)=\boldsymbol{\phi}_{i}^{\top} .
$$

Die Gleichungen (4.95b) und (4.95c) sind somit unter Annahme 4.22 äquivalent zu (4.22), und (4.95) entspricht der Wahl von $\boldsymbol{L}_{\text {ffi }}$ nach Lemma 4.1.

\subsubsection{Entwurf vollständiger Beobachter zur teilweisen Fehlerisolation}

Auf Basis von Satz 4.12 werden im Weiteren teilweise isolierende Fehlerisolationsbeobachter entworfen. Das Vorgehen weist dabei Ähnlichkeiten zum Entwurf von teilweise entkoppelnden Reglern im Zustandsraum [136, 138-140] auf. Dort wird für das duale Problem des Entkopplungsreglerentwurfs (s. Abschnitt 3.4) Verkopplung in lediglich einer Zeile der Führungsübertragungsmatrix erreicht. Während beim Entwurf teilweise entkoppelnder Regler jedoch möglichst geringe Kopplungen angestrebt werden, sind bei dem hier betrachteten Problem der teilweisen Fehlerisolation wie zu Beginn von Abschnitt 4.10 erörtert schwächere strukturelle Anforderungen an die Fehler-Residuen-Übertragungsmatrix $\boldsymbol{G}_{\boldsymbol{r} \boldsymbol{f}}(s) \mathrm{zu}$ stellen. Daraus ergibt sich eine vergrößerte Anzahl von Entwurfsfreiheitsgraden.

Kernüberlegung des Verfahrens ist es, einen Beobachtereigenwert $\lambda_{B_{i j}}$ nicht mehr ausschließlich als Pol an die $i$-te Spalte der Übertragungsmatrix $\boldsymbol{G}_{\boldsymbol{r} \boldsymbol{f}}(s)$ zu binden, sondern ihn auch in allen Spalten $l$ mit $l>i$ zuzulassen. 
Mithilfe der Parametervektoren und der Bedingung (4.97) lässt sich dies einfach realisieren, indem der entsprechende Parametervektor als

$$
\begin{aligned}
\boldsymbol{p}_{\mathrm{pf}, i j}^{\top} & =\left(\boldsymbol{\phi}_{i}^{\top}+\sum_{l=i+1}^{n_{f}} k_{d, i j l} \boldsymbol{\phi}_{l}^{\top}\right) \boldsymbol{G}_{\boldsymbol{y} \boldsymbol{f}}^{-1}\left(\lambda_{B_{i j}}\right) \\
& =\boldsymbol{p}_{\mathrm{ff}, i j}^{\top}+\sum_{l=i+1}^{n_{f}} k_{d, i j l} \underbrace{\boldsymbol{\phi}_{l}^{\top} \boldsymbol{G}_{\boldsymbol{y} \boldsymbol{f}}^{-1}\left(\lambda_{B_{i j}}\right)}_{\boldsymbol{p}_{c, i j l}^{\top}}
\end{aligned}
$$

gewählt wird. Dabei werden die Skalare $k_{d, i j l}$ als dynamische Kopplungszahlen bezeichnet, da sie die Eigenstruktur von $\boldsymbol{A}-\boldsymbol{L} \boldsymbol{C}$ gemäß

$$
\boldsymbol{G}_{\boldsymbol{r} \boldsymbol{f}}(s)=\left[\begin{array}{cccccc}
g_{1,1}(s) & \cdots & g_{1, i}(s) & g_{1, i+1}(s) & \cdots & g_{1, n_{f}}(s) \\
\vdots & & \vdots & \vdots & & \vdots \\
g_{n_{f}, 1}(s) & \cdots & g_{n_{f}, i}(s) & g_{n_{f}, i+1}(s) & \cdots & g_{n_{f}, n_{f}}(s)
\end{array}\right]
$$

dahingehend verändern, dass der $j$-te Pol des $i$-ten Diagonalelementes auch in der $l$-ten Spalte von $\boldsymbol{G}_{\boldsymbol{r} \boldsymbol{f}}(s)$ auftaucht.

Sie stellen gegenüber der vollständigen Fehlerisolation zusätzliche Entwurfsfreiheitsgrade dar. Die Anzahl der dynamischen Kopplungszahlen wird mit $n_{k_{d}}$ bezeichnet, sie ergibt sich aus

$$
n_{k_{d}}=\sum_{i=1}^{n_{f}-1} \sum_{l=i+1}^{n_{f}} \delta_{i}=\sum_{i=1}^{n_{f}-1} \delta_{i}\left(n_{f}-i\right) .
$$

Fasst man die dynamischen Kopplungszahlen im Vektor $\boldsymbol{k}_{d} \in \mathbb{R}^{n_{k_{d}}}$ zusammen und berechnet alle Parametervektoren nach (4.98), so ergibt sich für die Parametervektormatrix

$$
\begin{aligned}
\boldsymbol{P}_{\mathrm{pfi}}\left(\boldsymbol{k}_{d}\right) & =\boldsymbol{P}_{\mathrm{ffi}}+\sum_{i=1}^{n_{f}-1} \sum_{l=i+1}^{n_{f}} \sum_{j=1}^{\delta_{i}} k_{d, i j l} \boldsymbol{P}_{c, i j l} \\
& =\boldsymbol{P}_{\mathrm{ffi}}+\sum_{\nu=1}^{n_{k_{d}}} k_{d, \nu} \boldsymbol{P}_{c, \nu} .
\end{aligned}
$$


Dabei enthält die Matrix $\boldsymbol{P}_{c, i j l}$ außer in $\operatorname{der}\left(j+\sum_{m=1}^{i-1} \delta_{m}\right)$-ten Zeile nur Nullzeilen, das heißt

$$
\boldsymbol{P}_{c, i j l}=\left[\begin{array}{c}
\mathbf{0}^{\top} \\
\vdots \\
\mathbf{0}^{\top} \\
\boldsymbol{p}_{c, i j l}^{\top} \\
\mathbf{0}^{\top} \\
\vdots \\
\mathbf{0}^{\top}
\end{array}\right] .
$$

Im Sinne einer kompakten Notation werden im Weiteren die Laufindizes $i$, $j$ und $l$ durch einen einzelnen Index $\nu=1, \ldots, n_{k_{d}}$ ersetzt. Die zugehörigen Linkseigenvektoren werden entsprechend $(4.95 \mathrm{c})$ mittels

$$
\begin{aligned}
\boldsymbol{w}_{B, \mathrm{pfi}, i j}^{\top}\left(\boldsymbol{k}_{d}\right) & =\boldsymbol{p}_{\mathrm{pfi}, i j}^{\top}\left(\boldsymbol{k}_{d}\right) \cdot \boldsymbol{C}\left(\lambda_{B_{i j}} \boldsymbol{I}_{n}-\boldsymbol{A}\right)^{-1} \\
& =\boldsymbol{w}_{B, \mathrm{ff}, i j}^{\top}+\sum_{l=i+1}^{n_{f}} k_{d, i j l} \cdot \underbrace{\boldsymbol{p}_{c, i j l}^{\top} \boldsymbol{C}\left(\lambda_{B_{i j}} \boldsymbol{I}_{n}-\boldsymbol{A}\right)^{-1}}_{\boldsymbol{w}_{B c, i j l}^{\top}}
\end{aligned}
$$

berechnet.

Die verbleibenden $n-\delta$ Beobachtereigenwerte werden gegenüber der vollständigen Fehlerisolation unverändert nichtsteuerbar gemacht, indem sie gemäß (4.95d) gewählt werden. Dies ist für eine teilweise Fehlerisolation wie bereits erörtert nicht notwendig, da hier grundsätzlich ebenso schwächere Anforderungen gelten wie für die teilweise Führungsentkopplung [45, 80, 138, 139, 156, 158]. Prinzipiell lässt sich für eine invariante Nullstelle $\eta$ ein zusätzlicher Pole in der letzten Spalte von $\boldsymbol{G}_{\boldsymbol{r} \boldsymbol{f}}(s)$ vorgeben, ohne dass die Dreiecksstruktur verloren geht, wenn in der nichttrivialen Lösung von

$$
\left[\begin{array}{cc}
\eta \boldsymbol{I}_{n}-\boldsymbol{A} & \boldsymbol{E}_{a} \\
-\boldsymbol{C} & \boldsymbol{E}_{s}
\end{array}\right] \cdot\left[\begin{array}{l}
\boldsymbol{z}_{x} \\
\boldsymbol{z}_{f}
\end{array}\right]=\mathbf{0}
$$

das $n_{f}$-te Element von $\boldsymbol{z}_{f}$ ungleich 0 ist. Dies ergibt sich unmittelbar als duales Resultat aus den Betrachtungen in [140, Abschnitt 3.3]. Dennoch werden invariante Nullstellen im Rahmen dieser Arbeit wie bei der vollständigen Fehlerisolation kompensiert. Hintergrund ist, dass dadurch im weiteren Verlauf der Arbeit (s. Abschnitt 5.5) der Vergleich von vollständiger und teilweiser Fehlerisolation hinsichtlich der Robustheit der Residuengenerierung möglich wird. 
Aus den Linkseigenvektoren $\boldsymbol{w}_{B, \mathrm{pfi}, i j}^{\top}$ und $\boldsymbol{w}_{B, \mathrm{pfi}, k}^{\top}$ erhält man für die Linkseigenvektormatrix analog zu (4.100) die Beziehung

$$
\begin{aligned}
\boldsymbol{W}_{B, \mathrm{pfi}}\left(\boldsymbol{k}_{d}\right) & =\boldsymbol{W}_{B, \mathrm{ffi}}+\sum_{i=1}^{n_{f}-1} \sum_{l=i+1}^{n_{f}} \sum_{j=1}^{\delta_{i}} k_{d, i j l} \boldsymbol{W}_{B c, i j l} \\
& =\boldsymbol{W}_{B, \mathrm{ffi}}+\sum_{\nu=1}^{n_{k_{d}}} k_{d, \nu} \boldsymbol{W}_{B c, \nu} .
\end{aligned}
$$

Dabei sind ebenso wie in $\boldsymbol{P}_{c, \nu}$ alle Zeilen von $\boldsymbol{W}_{B c, \nu}$ bis auf die $\left(j+\sum_{m=1}^{i-1} \delta_{m}\right)$-te Zeile Nullzeilen. Die Beobachtermatrix zur teilweisen Fehlerisolation lässt sich damit entsprechend der Definition der Parametervektoren in (4.18) schreiben als

$$
\boldsymbol{L}_{\mathrm{pfi}}\left(\boldsymbol{k}_{d}\right)=\boldsymbol{W}_{B, \mathrm{pfi}}^{-1}\left(\boldsymbol{k}_{d}\right) \cdot \boldsymbol{P}_{\mathrm{pfi}}\left(\boldsymbol{k}_{d}\right) .
$$

Darüber hinaus ist zur Erfüllung von (4.93) für die stationäre Verstärkung zwischen Fehlern und Residuen eine Struktur der Form

$$
\boldsymbol{G}_{\boldsymbol{r} \boldsymbol{f}}(s=0)=\left[\begin{array}{cccc}
\tilde{z}_{1} & k_{s, 12} & \cdots & k_{s, 1 n_{f}} \\
0 & \tilde{z}_{2} & \ddots & \vdots \\
\vdots & \ddots & \ddots & k_{s, n_{f}-1 n_{f}} \\
0 & \cdots & 0 & \tilde{z}_{n_{f}}
\end{array}\right]=\boldsymbol{S}_{\mathrm{pfi}}\left(\boldsymbol{k}_{s}\right)
$$

hinreichend. Gegenüber der vollständigen Fehlerisolation, bei der im stationären Fall $\boldsymbol{G}_{\boldsymbol{r} \boldsymbol{f}}(s=0)=\boldsymbol{S}_{\mathrm{ffi}}=\operatorname{diag}\left(\tilde{z}_{1}, \ldots, \tilde{z}_{n_{f}}\right)$ gelten muss, ergeben sich folglich durch die eingeführten statischen Kopplungszahlen $k_{s, i j}$ weitere Freiheitsgrade. Diese werden zum Vektor $\boldsymbol{k}_{s} \in \mathbb{R}^{n_{k_{s}}}$ zusammengefasst. Dabei bewirkt $k_{s, i j} \neq 0$, dass der $j$-te Fehler im stationären Fall das $i$-te Residuum beeinflusst. Im Sinne einer kompakten Notation werden dazu Matrizen $\boldsymbol{S}_{c, i j}$ eingeführt. Die Elemente dieser Matrizen sind überall 0, bis auf den Eintrag in der $i$-ten Zeile und der $j$-ten Spalte. Das entsprechende Element ist 1. Damit lässt sich

$$
\begin{aligned}
\boldsymbol{G}_{\boldsymbol{r} \boldsymbol{f}}(s=0) & =\boldsymbol{S}_{\mathrm{ffi}}+\sum_{i=1}^{n_{f}-1} \sum_{j=i+1}^{n_{f}} k_{s, i j} \boldsymbol{S}_{c, i j} \\
& =\boldsymbol{S}_{\mathrm{ffi}}+\sum_{\mu=1}^{n_{k_{s}}} k_{s, \mu} \boldsymbol{S}_{c, \mu}=\boldsymbol{S}_{\mathrm{pfi}}\left(\boldsymbol{k}_{s}\right)
\end{aligned}
$$


schreiben. In dieser Darstellung wird ebenfalls auf einen einzelnen Laufindex $\mu=1, \ldots, n_{k_{s}}$ zurückgegriffen, wobei $n_{k_{s}}$ die Gesamtanzahl der statischen Kopplungszahlen beschreibt. Mithilfe der Gauß'schen Summenformel [21, Abschnitt 1.2.4], das heißt $\sum_{k=1}^{n} k=0,5 n(n+1)$, berechnet sie sich zu

$$
\begin{aligned}
n_{k_{s}} & =\sum_{i=1}^{n_{f}-1} \sum_{j=i+1}^{n_{f}} 1=\sum_{i=1}^{n_{f}-1}\left(n_{f}-i\right)=\sum_{i=1}^{n_{f}-1} n_{f}-\sum_{i=1}^{n_{f}-1} i \\
& =n_{f}\left(n_{f}-1\right)-\frac{\left(n_{f}-1\right) n_{f}}{2}=\frac{n_{f}\left(n_{f}-1\right)}{2} .
\end{aligned}
$$

Ähnlich wie in Satz 4.12 erhält man die Nachfiltermatrix $\boldsymbol{V}_{\text {pfi }}$ mittels

$$
\begin{aligned}
\boldsymbol{V}_{\mathrm{pfi}}\left(\boldsymbol{k}_{d}, \boldsymbol{k}_{s}\right)=-\boldsymbol{S}_{\mathrm{pfi}}\left(\boldsymbol{k}_{s}\right) \cdot \ldots \\
\cdot(\underbrace{\boldsymbol{C}\left(\boldsymbol{A}-\boldsymbol{L}_{\mathrm{pfi}}\left(\boldsymbol{k}_{d}\right) \boldsymbol{C}\right)^{-1}\left(\boldsymbol{E}_{a}-\boldsymbol{L}_{\mathrm{pfi}}\left(\boldsymbol{k}_{d}\right) \boldsymbol{E}_{s}\right)-\boldsymbol{E}_{s}}_{\Theta_{\mathrm{pfi}}\left(\boldsymbol{k}_{d}\right)})^{-1} \cdot
\end{aligned}
$$

Beachtenswert ist, dass $\boldsymbol{V}_{\text {pfi }}$ wie im Falle der vollständigen Fehlerisolation in Abschnitt 4.5.2 lediglich anhand der stationären Betrachtung $s=0$ entworfen wird. Wie der folgende Satz zeigt, ergibt sich daraus die in (4.93) geforderte Dreiecksstruktur von $\boldsymbol{G}_{\boldsymbol{r} \boldsymbol{f}}(s)$ für alle $s \in \mathbb{C}$.

Satz 4.13 (PFIO-Entwurf mittels Vollständiger Modaler Synthese). Gegeben sei ein System der Form

$$
\begin{aligned}
& \dot{\boldsymbol{x}}=\boldsymbol{A} \boldsymbol{x}+\boldsymbol{B} \boldsymbol{u}+\boldsymbol{E}_{a} \boldsymbol{f}, \\
& \boldsymbol{y}=\boldsymbol{C} \boldsymbol{x}+\boldsymbol{D} \boldsymbol{u}+\boldsymbol{E}_{s} \boldsymbol{f},
\end{aligned}
$$

welches die Annahmen 4.18-4.21 erfüllt. Weiterhin sei durch $\left(\boldsymbol{L}_{\mathrm{ff}}, \boldsymbol{V}_{\mathrm{ffi}}\right)$ ein FFIO gemäß Satz 4.12 parametriert. Wird die Beobachtermatrix $\boldsymbol{L}_{\mathrm{pfi}}$ mittels

$$
\begin{aligned}
\boldsymbol{L}_{\mathrm{pfi}} & =-\boldsymbol{W}_{B, \mathrm{pfi}}^{-1} \boldsymbol{P}_{\mathrm{pfi}}, \\
\boldsymbol{p}_{\mathrm{pfi}, i j}^{\top} & =\boldsymbol{p}_{\mathrm{ffi}, i j}^{\top}+\sum_{l=i+1}^{n_{f}} k_{d, i j l} \boldsymbol{p}_{c, i j l}^{\top}, i=1, \ldots, n_{f}, j=1, \ldots, \delta_{i}, \\
\boldsymbol{w}_{B, \mathrm{pfi}, i j}^{\top} & =\boldsymbol{w}_{B, \mathrm{ffi}, i j}^{\top}+\sum_{l=i+1}^{n_{f}} k_{d, i j l} \boldsymbol{w}_{B c, i j l}^{\top}, \\
{\left[\boldsymbol{w}_{B, \mathrm{pf}, k}^{\top} \quad \boldsymbol{p}_{\mathrm{pfi}, k}^{\top}\right] } & ={ }^{\perp}\left(\boldsymbol{\Pi}\left(\lambda_{B_{k}}\right)\right)={ }^{\perp} \boldsymbol{\Pi}_{k}, k=\delta+1, \ldots, n
\end{aligned}
$$


mit einem beliebigen Vektor $\boldsymbol{k}_{d} \in \mathbb{R}^{n_{k_{d}}}$ berechnet und ist die Nachfiltermatrix durch

$$
\boldsymbol{V}_{\mathrm{pfi}}=-\boldsymbol{S}_{\mathrm{pfi}} \cdot\left(\boldsymbol{C}\left(\boldsymbol{A}-\boldsymbol{L}_{\mathrm{pfi}} \boldsymbol{C}\right)^{-1}\left(\boldsymbol{E}_{a}-\boldsymbol{L}_{\mathrm{pfi}} \boldsymbol{E}_{s}\right)-\boldsymbol{E}_{s}\right)^{-1}
$$

mit einem beliebigen Vektor $\boldsymbol{k}_{s} \in \mathbb{R}^{n_{k_{s}}}$ und der daraus folgenden oberen Dreiecksmatrix $\boldsymbol{S}_{\mathrm{pfi}}\left(\boldsymbol{k}_{s}\right) \in \mathbb{R}^{n_{f} \times n_{f}}$ gegeben, so weist die sich ergebende Übertragungsmatrix $\boldsymbol{G}_{\boldsymbol{r} \boldsymbol{f}}(s)$ beim Einsatz eines FIOs der Form

$$
\begin{aligned}
\dot{\hat{\boldsymbol{x}}} & =\boldsymbol{A} \hat{\boldsymbol{x}}+\boldsymbol{B} \boldsymbol{u}+\boldsymbol{L}_{\mathrm{pfi}}(\boldsymbol{y}-\boldsymbol{C} \hat{\boldsymbol{x}}-\boldsymbol{D} \boldsymbol{u}), \\
\boldsymbol{r} & =\boldsymbol{V}_{\mathrm{pfi}}(\boldsymbol{y}-\boldsymbol{C} \hat{\boldsymbol{x}}-\boldsymbol{D} \boldsymbol{u})
\end{aligned}
$$

eine obere Dreiecksstruktur für alle $s \in \mathbb{C}$ auf. Die Diagonalelemente ändern sich gegenüber der vollständigen Fehlerisolation (vgl. Satz 4.12) nicht.

Beweis. Zunächst wird die erste Spalte von $\boldsymbol{G}_{\boldsymbol{r} \boldsymbol{f}}(s)$ betrachtet. Da in (4.98) die Beziehung $l>i$ gilt, werden der ersten Spalte ausschließlich die Eigenwerte $\lambda_{B_{1 j}}$ mit $j=1, \ldots, n_{f}$ zugewiesen. Diesbezüglich ändert sich also beim Übergang zur teilweisen Fehlerisolation nichts. Dementsprechend ergibt sich mit der gleichen Argumentation wie in Abschnitt 4.5.2, dass

$$
\begin{aligned}
g_{1,1}(s) & =\frac{\tilde{z}_{1} \cdot \prod_{j=1}^{\delta_{1}}\left(-\lambda_{B_{1 j}}\right)}{\left(s-\lambda_{B_{11}}\right) \cdot \ldots \cdot\left(s-\lambda_{B_{1 \delta_{1}}}\right)}, \\
g_{j, 1}(s) & =0, j=2, \ldots, n_{f}
\end{aligned}
$$

gilt. Dies impliziert aufgrund des dyadischen Produktes in (4.15) auf Seite 86, dass (4.16a) erfüllt ist. Es ist also

$$
\boldsymbol{V} \boldsymbol{C} \boldsymbol{v}_{B, \mathrm{pfi}, 1 j}=\alpha_{i j} \cdot \phi_{1}, \alpha_{i j} \neq 0 .
$$

Zwar werden durch die dynamischen Kopplungszahlen $k_{d, 1 j l}$ die Eigenwerte $\lambda_{B_{1 j}}$ auch für alle Spalten $l$ mit $l>1$ vorgegeben. Aus (4.105) geht jedoch hervor, dass die Eigenwerte $\lambda_{B_{1 j}}$ nur in der ersten Zeile von $\boldsymbol{G}_{\boldsymbol{r} \boldsymbol{f}}(s)$ auftreten. Somit gelten für die Elemente der zweiten Spalte von $\boldsymbol{G}_{\boldsymbol{r} \boldsymbol{f}}(s)$ die Gleichungen

$$
\begin{aligned}
g_{1,2}(s) & =\frac{z_{1,2}(s)}{\left(s-\lambda_{B_{11}}\right) \cdot \ldots \cdot\left(s-\lambda_{B_{1 \delta_{1}}}\right) \cdot\left(s-\lambda_{B_{21}}\right) \cdot \ldots \cdot\left(s-\lambda_{B_{2 \delta_{2}}}\right)}, \\
g_{2,2}(s) & =\frac{\tilde{z}_{2} \cdot \prod_{j=1}^{\delta_{2}}\left(-\lambda_{B_{2 j}}\right)}{\left(s-\lambda_{B_{21}}\right) \cdot \ldots \cdot\left(s-\lambda_{B_{2 \delta_{2}}}\right)}, \\
g_{j, 2}(s) & =0, j=3, \ldots, n_{f} .
\end{aligned}
$$


Aus obigen Überlegungen ergibt sich rekursiv, dass die Diagonalelemente von $\boldsymbol{G}_{\boldsymbol{r} \boldsymbol{f}}(s)$ denen der vollständigen Fehlerisolation, also (4.96) entsprechen. Weiterhin gilt für alle Elemente unterhalb der Diagonalen, das heißt mit $i>j$, die Beziehung $g_{i, j}(s)=0$.

Die Anwendung von Satz 4.13 entspricht weitgehend dem Entwurf eines FFIOs mittels Vollständiger Modaler Synthese. Für das bereits mehrfach herangezogene akademisch Beispielsystem

$$
\begin{aligned}
& \boldsymbol{A}=\left[\begin{array}{rrr}
-10 & -16 & -20 \\
6 & 14 & 24 \\
-2 & -8 & -16
\end{array}\right], \quad \boldsymbol{E}_{a}=\left[\begin{array}{cc}
0 & 8 \\
0,5 & -20 \\
0 & 16
\end{array}\right], \\
& \boldsymbol{C}=\left[\begin{array}{rrr}
3 & 2 & 1 \\
-9 & 0 & 5
\end{array}\right], \quad \boldsymbol{E}_{s}=\mathbf{0}
\end{aligned}
$$

wird wie bei der vollständigen Fehlerisolation im Beispiel auf Seite 93 die invariante Nullstelle $\eta=-18$ berechnet und es werden die Beobachtereigenwerte $\lambda_{B_{1,1}}=-3$ und $\lambda_{B_{2,1}}=-5$ vorgegeben. Im Beispiel gibt es lediglich eine dynamische Kopplungszahl $k_{d, 112}$, welche den einzigen Pol des ersten Übertragungskanals in den zweiten Übertragungskanal koppelt. Wählt man diesen Entwurfsparameter als $k_{d, 112}=5$, so erhält man für den entsprechenden Parameter- beziehungsweise Linkseigenvektor

$$
\begin{aligned}
\boldsymbol{p}_{\mathrm{pfi}, 11}^{\top}= & \boldsymbol{p}_{\mathrm{ffi}, 11}^{\top}+k_{d, 112} \cdot \boldsymbol{\phi}_{2}^{\top} \cdot \boldsymbol{G}_{\boldsymbol{y} \boldsymbol{f}}^{-1}\left(\lambda_{B_{1,1}}\right) \\
= & {\left[\begin{array}{ll}
-1,8 & -0,4
\end{array}\right]+5\left[\begin{array}{ll}
0 & 1
\end{array}\right]\left[\begin{array}{cc}
-1,8 & -0,4 \\
-1,3 & -0,275
\end{array}\right] } \\
= & {\left[\begin{array}{ll}
-8,3 & -1,775
\end{array}\right], } \\
\boldsymbol{w}_{\mathrm{pfi}, 11}^{\top}= & \boldsymbol{w}_{\mathrm{ff}, 11}^{\top}+k_{d, 112} \cdot \boldsymbol{\phi}_{2}^{\top} \cdot \boldsymbol{G}_{\boldsymbol{y} \boldsymbol{f}}^{-1}\left(\lambda_{B_{1,1}}\right) \cdot \boldsymbol{C} \cdot\left(\lambda_{B_{1,1}} \boldsymbol{I}_{3}-\boldsymbol{A}\right)^{-1} \\
= & {\left[\begin{array}{lll}
0,8667 & 2 & 2,0667
\end{array}\right]+\ldots } \\
& +5\left[\begin{array}{ll}
0 & 1
\end{array}\right]\left[\begin{array}{ll}
-1,8 & -0,4 \\
-1,3 & -0,275
\end{array}\right]\left[\begin{array}{ccc}
3 & 2 & 1 \\
-9 & 0 & 5
\end{array}\right]\left[\begin{array}{ccc}
9,6667 & 16 & 14,6667 \\
-10 & -17 & -16 \\
4,6667 & 8 & 7,6667
\end{array}\right] \\
= & {\left[\begin{array}{lll}
-0,425 & 2 & 3,025
\end{array}\right] . }
\end{aligned}
$$

Die weiteren Parameter- und Linkseigenvektoren bleiben gegenüber der 
vollständigen Fehlerisolation unverändert und es ergibt sich

$$
\begin{aligned}
\boldsymbol{L}_{\mathrm{pfi}}=-\boldsymbol{W}_{B, \mathrm{pfi}}^{-1} \cdot \boldsymbol{P}_{\mathrm{pfi}} & =-\left[\begin{array}{ccc}
-0,425 & 2 & 3,025 \\
-0,125 & 0 & 0,125 \\
-2 & 0 & 1
\end{array}\right]^{-1} \cdot\left[\begin{array}{cc}
-8,3 & -1,775 \\
-0,5 & -0,125 \\
-12 & -6
\end{array}\right] \\
& =\left[\begin{array}{cc}
-8 & -5 \\
8,5 & 5,875 \\
-4 & -4
\end{array}\right] .
\end{aligned}
$$

Weiterhin berechnet man mit dieser Beobachtermatrix

$$
\boldsymbol{\Theta}_{\mathrm{pfi}}=\boldsymbol{C}\left(\boldsymbol{A}-\boldsymbol{L}_{\mathrm{pfi}} \boldsymbol{C}\right)^{-1}\left(\boldsymbol{E}_{a}-\boldsymbol{L}_{\mathrm{pfi}} \boldsymbol{E}_{s}\right)-\boldsymbol{E}_{s}=\left[\begin{array}{cc}
-\frac{1}{3} & -\frac{2}{3} \\
0 & -\frac{8}{5}
\end{array}\right] .
$$

Da das System $n_{f}=2$ Fehler aufweist, verbleibt die Wahl der Kopplungszahl $k_{s, 12}$, welche hier zu $k_{s, 12}=-3$ gesetzt wird. Daraus ergibt sich die Nachfiltermatrix

$$
\begin{aligned}
\boldsymbol{V}_{\mathrm{pfi}}=-\boldsymbol{S}_{\mathrm{pfi}} \boldsymbol{\Theta}_{\mathrm{pfi}}^{-1} & =-\left[\begin{array}{cc}
1 & -3 \\
0 & 1
\end{array}\right] \cdot\left[\begin{array}{cc}
-\frac{1}{3} & -\frac{2}{3} \\
0 & -\frac{8}{5}
\end{array}\right]^{-1} \\
& =\left[\begin{array}{rr}
-3 & -3,125 \\
0 & 0,625
\end{array}\right] .
\end{aligned}
$$

Aus der Parametrierung $\left(\boldsymbol{L}_{\mathrm{pfi}}, \boldsymbol{V}_{\mathrm{pfi}}\right)$ ergibt sich die Übertragungsmatrix

$$
\boldsymbol{G}_{\boldsymbol{r} \boldsymbol{f}}(s)=\left[\begin{array}{cc}
\frac{3}{s+3} & \frac{-25(s+1,8)}{(s+3)(s+5)} \\
0 & \frac{5}{s+5}
\end{array}\right]
$$

welche die gewünschte obere Dreiecksstruktur aufweist. Darüber hinaus ist die Stabilität des PFIOs sichergestellt.

Anzumerken ist, dass sich die Methode auch für die teilweise Fehlerisolation in nichtquadratischen Systemen erweitern lässt. Dann stehen ähnlich wie in Abschnitt 4.9 gegenüber dem in Satz 4.13 beschriebenen Entwurf für quadratische Systeme zusätzliche Freiheitsgrade zur Verfügung.

\subsubsection{Beispiel Verladebrücke}

Zur weiteren Veranschaulichung der partiellen Fehlerisolation wird erneut die Verladebrücke betrachtet, welche bereits bei der Fehlerisolation für nicht minimalphasige Systeme in Abschnitt 4.8.4 untersucht wurde. 
Für die partielle Fehlerisolation wird ein anderes Fehlerszenario betrachtet. Der Aktorfehler $f_{2}$, der einen Fehler im Stellsignal beschreibt, bleibt erhalten. Er stellt einen Fehler in der auf die Katze ausgeübten Kraft dar. Anstelle des Sensorfehlers wird hier nun als Fehler $f_{1}$ ein zweiter, auf die Streckendynamik wirkender Fehler angenommen. Dabei handelt es sich um eine horizontale, an der Lastmasse angreifende Kraft. Diese kann unter anderem durch eine Kollision der Lastmasse mit einem Hindernis hervorgerufen werden. Die Fehlereingangsmatrizen lauten für dieses Szenario

$$
\boldsymbol{E}_{a}=\left[\begin{array}{cc}
0 & 0 \\
0 & 5,89 \\
0 & 0 \\
-0,10 & 5,89
\end{array}\right], \quad \boldsymbol{E}_{s}=\mathbf{0}
$$

Das System erfüllt die Annahmen 4.18 bis 4.20 und für die Fehlerindizes gilt $\delta_{1}=\delta_{2}=2$. Gegenüber der vollständigen Fehlerisolation können somit die beiden dem ersten Fehler zugeordneten Beobachtereigenwerte $\lambda_{B_{11}}$ und $\lambda_{B_{12}}$ zusätzlich in die zweite Spalte von $\boldsymbol{G}_{\boldsymbol{r} \boldsymbol{f}}(s)$ gekoppelt wer-
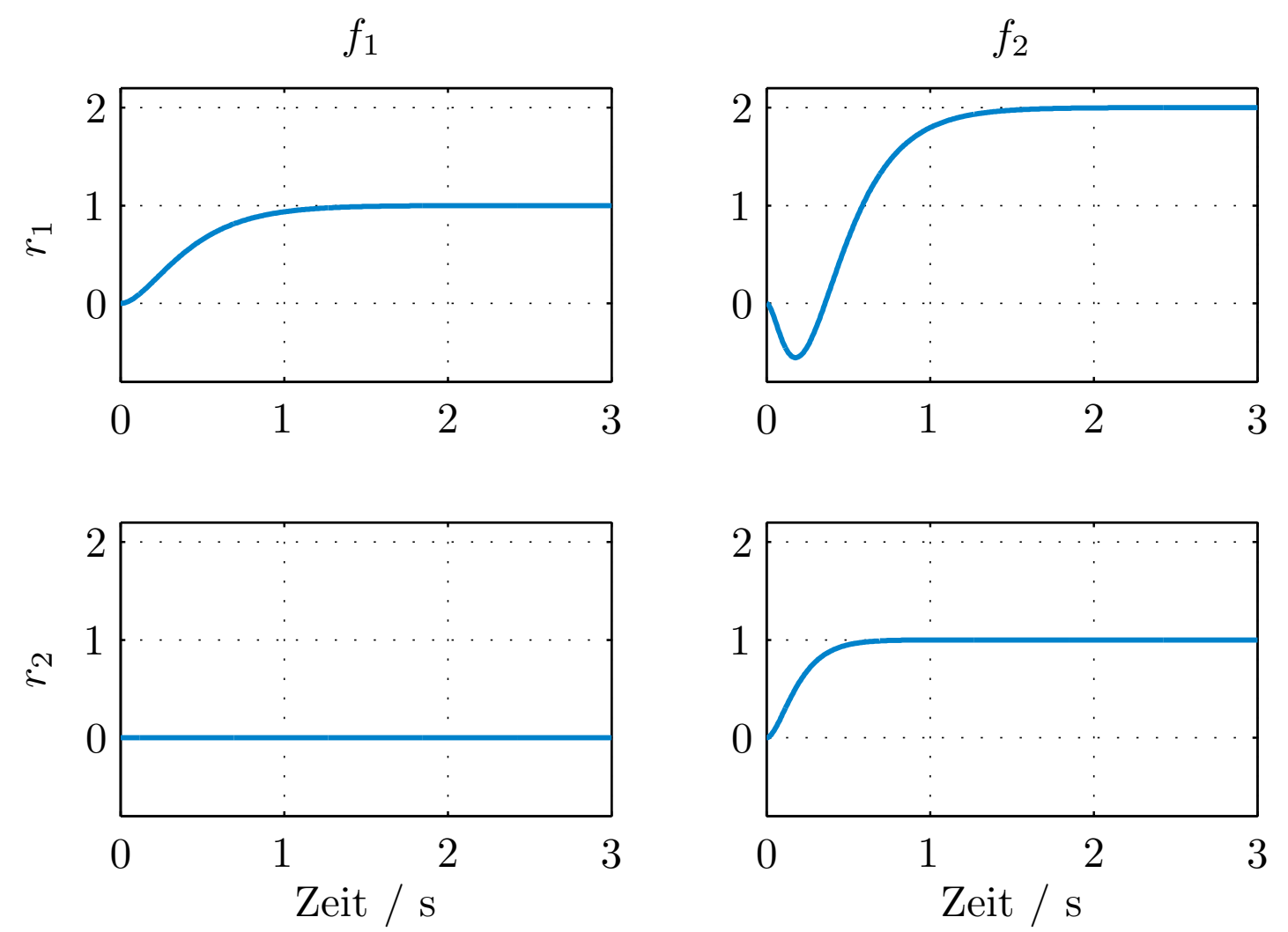

Abbildung 4.19: Sprungantwort von $\boldsymbol{G}_{\boldsymbol{r} \boldsymbol{f}}(s)$ bei teilweiser Fehlerisolation an der Verladebrücke 
den. Dies geschieht gemäß (4.98) durch die dynamischen Kopplungszahlen $k_{d, 112}$ und $k_{d, 122}$. Überdies kann nach (4.103) durch die statische Kopplungszahl $k_{s, 12}$ die stationäre Verstärkung des oberen rechten Elements von $\boldsymbol{G}_{\boldsymbol{r} \boldsymbol{f}}(s)$ eingestellt werden.

Für eine schnelle Fehlererkennung werden die Beobachtereigenwerte im Beispiel zu $\lambda_{B_{11}}=-4, \lambda_{B_{12}}=-5, \lambda_{B_{21}}=-9$ und $\lambda_{B_{22}}=-10$ gewählt. Die stationäre Verstärkung der Diagonalelemente von $\boldsymbol{G}_{\boldsymbol{r} \boldsymbol{f}}(s)$ wird jeweils mit 1 vorgegeben und die Kopplungszahlen werden mit $k_{d, 112}=2$, $k_{d, 122}=-1$ und $k_{s, 12}=2$ angesetzt. Da an dieser Stelle noch kein Optimierungskriterium formuliert wurde, ist diese Wahl willkürlich. Das Beispiel dient lediglich der Demonstration der Einhaltung der strukturellen Beschränkungen bezüglich der Fehler-Residuen-Übertragungsmatrix. Es wird im Abschnitt zur Robustheitssteigerung bei partieller Fehlerisolation (Abschnitt 5.5) erneut aufgegriffen. Die resultierende Beobachterparametrierung $\left(\boldsymbol{L}_{\mathrm{pfi}}, \boldsymbol{V}_{\mathrm{pfi}}\right)$ ist in Anhang C.1.7 angegeben. Abbildung 4.19 zeigt die Sprungantwort von $\boldsymbol{G}_{\boldsymbol{r} \boldsymbol{f}}(s)$. Offensichtlich wird das zweite Residuum ausschließlich vom Fehler $f_{2}$ beeinflusst, das Residuum $r_{1}$ jedoch von beiden Fehlern.

\subsubsection{Fazit}

Die Vollständige Modale Synthese erlaubt es in einfacher Art und Weise, die durch die strukturell schwächeren Anforderungen der teilweisen Fehlerisolation auftretenden Freiheitsgrade zu charakterisieren. Sowohl für die Beobachtermatrix $\boldsymbol{L}_{\mathrm{pfi}}$ als auch die Nachfiltermatrix $\boldsymbol{V}_{\text {pfi }}$ ergeben sich gegenüber der vollständigen Fehlerisolation Freiheiten, die im weiteren Verlauf der Arbeit zur Robustheitssteigerung genutzt werden.

\subsection{Zusammenfassung des Kapitels}

Der beobachterbasierte Ansatz zur Fehlerisolation stellt ein intuitives Konzept dar, da er auf der Diskrepanz von gemessenen und geschätzten Systemausgängen beruht. Auf Basis der Dualität zwischen Fehlerisolationsbeobachtern für nominale Systeme und linearen Entkopplungsreglern lässt sich der Entwurf für eine große Klasse linearer Systeme durchführen. Mit dem dualen Verfahren zum Entkopplungsreglerentwurf nach Falb und Wolovich und der Vollständigen Modalen Synthese sind zwei Ansätze gegeben, die einen geschlossenen, analytischen Entwurf liefern. Während die erstgenannte Methode einer intuitiven Entwurfsidee im Zeitbereich folgt, bietet 
letztere einen genaueren Einblick in die interne Struktur des Systems beziehungsweise des Beobachters.

Darauf aufbauend erlaubt die Vollständige Modale Synthese die Erweiterung bestehender Resultate sowohl auf statisch nicht isolierbare als auch auf nicht minimalphasige Systeme, wobei die benötigten dynamischen Erweiterungen durch dynamisch erweiterte Fehlerisolationsbeobachter realisiert werden können. Somit wurde eine Erweiterung der Klasse handhabbarer Systeme erreicht.

Durch eine Erweiterung der Dynamik der Diagonalelemente von $\boldsymbol{G}_{\boldsymbol{r} \boldsymbol{f}}(s)$ lassen sich zusätzliche Entwurfsfreiheitsgrade gewinnen, die mit beiden vorgestellten Entwurfsverfahren beschrieben werden können. Die Methode basierend auf dem Ansatz von Falb und Wolovich verkompliziert sich jedoch im Allgemeinen, da der Entwurf für das duale Regelungsproblem im Frequenzbereich durchgeführt wird. Die Vollständige Modale Synthese lässt sich hingegen ohne strukturelle Änderungen zur Parametrierung dynamisch erweiterter Fehlerisolationsbeobachter heranziehen.

In nichtquadratischen Systemen mit zusätzlichen Messgrößen ergeben sich ebenfalls zusätzliche Entwurfsfreiheitsgrade. Diese lassen sich mit beiden Verfahren auf unterschiedliche Art und Weise charakterisieren. Der auf dem Ansatz von Falb und Wolovich basierende Entwurf konzentriert die zusätzlichen Freiheitsgrade auf frei wählbare Matrizen. Die Vollständige Modale Synthese liefert demgegenüber eine anschaulichere Interpretation der Freiheitsgrade. Wie sich in Kapitel 5 zeigt lassen sich die Vorteile der beiden Methoden je nach betrachtetem Robustheitsaspekt gezielt auszunutzen.

Eine weitere Möglichkeit, Entwurfsfreiheitsgrade zu generieren, besteht in der Abschwächung der strukturellen Anforderungen. Dies ist Gegenstand der teilweisen Fehlerisolation, für die sich die Vollständige Modale Synthese in geradliniger Art und Weise erweitern lässt.

Zusammenfassend stellen die vorgestellten Entwurfsverfahren für Fehlerisolationsbeobachter sehr flexible Methoden dar, die gegenüber existierenden Ergebnissen vielfältig erweitert wurden. Festzuhalten ist jedoch auch, dass für die unterschiedlichen Problemstellungen jeweils verschiedene Anpassungen der Entwurfsverfahren nötig sind und dass somit kein einheitlicher Entwurf gegeben ist. 


\section{Optimierung der Robustheit von}

\section{Fehlerisolationsbeobachtern}

Die Ergebnisse aus dem vorangegangenen Kapitel liefern Existenzbedingungen und Entwurfsverfahren für störungsfreie Systeme mit exakt bekannter Systemdynamik. In der praktischen Anwendung ist jedoch stets davon auszugehen, dass das betrachtete System Störeinflüssen und/oder parametrischen Unsicherheiten unterliegt. Wie in Abschnitt 2.3 diskutiert wirft diese Problematik die Frage nach der Robustheit der Residuengenerierung auf. Sinnvoll einsetzbar sind Fehlerisolationsbeobachter nur dann, wenn sie trotz des Einflusses von Störungen und Unsicherheiten die wesentlichen Entwurfsziele (Isolation, Stabilität, Detektionsgeschwindigkeit und Sensitivität) noch hinreichend gut erfüllen.

Gegenstand dieses Kapitels ist es daher, die in Kapitel 4 herausgearbeiteten verbleibenden Freiheitsgrade im FIO-Entwurf dergestalt zu nutzen, den Einfluss von Störungen und Unsicherheiten bestmöglich zu reduzieren. In Erweiterung von (4.1) werden Systeme der Form

$$
\begin{aligned}
& \dot{\boldsymbol{x}}=\left(\boldsymbol{A}_{0}+\Delta \boldsymbol{A}\right) \boldsymbol{x}+\left(\boldsymbol{B}_{0}+\Delta \boldsymbol{B}\right) \boldsymbol{u}+\left(\boldsymbol{E}_{a, 0}+\Delta \boldsymbol{E}_{a}\right) \boldsymbol{f}+\left(\boldsymbol{B}_{d, 0}+\Delta \boldsymbol{B}_{d}\right) \boldsymbol{d}, \\
& \boldsymbol{y}=\left(\boldsymbol{C}_{0}+\Delta \boldsymbol{C}\right) \boldsymbol{x}+\left(\boldsymbol{D}_{0}+\Delta \boldsymbol{D}\right) \boldsymbol{u}+\left(\boldsymbol{E}_{s, 0}+\Delta \boldsymbol{E}_{s}\right) \boldsymbol{f}+\left(\boldsymbol{D}_{d, 0}+\Delta \boldsymbol{D}_{d}\right) \boldsymbol{d}
\end{aligned}
$$

betrachtet. Die Matrizen $\boldsymbol{A}_{0}, \boldsymbol{B}_{0}$ usw. beschreiben die angenommene nominale Systemdynamik. Die Abweichungen $\Delta \boldsymbol{A}, \Delta \boldsymbol{B}$ usw. stellen Abweichungen dar, die verschiedenste Ursachen haben können. Neben nicht exakt bekannten Systemparametern können sie durch Alterungseffekte hervorgerufen werden. Ergibt sich die lineare Systemdynamik durch die Linearisierung eines nichtlinearen Systems um einen Arbeitspunkt, so können die Abweichungen in den Systemmatrizen in (5.1) auch daher rühren, dass sich das System nicht exakt im Arbeitspunkt befindet. Die Abweichungen $\Delta \boldsymbol{A}$, $\Delta \boldsymbol{B}$ usw. werden im Rahmen dieser Arbeit nicht als gänzlich unbekannt 
angenommen, sondern es werden Kenntnisse über ihre Struktur vorausgesetzt. Auf die Ausprägung der Unsicherheiten wird in Abschnitt 5.4.4 näher eingegangen.

$\mathrm{Zu}$ betonen ist, dass an dieser Stelle die Abweichungen in den beschreibenden Matrizen als Unsicherheiten aufgefasst werden, deren Einfluss auf die generierten Residuen zu unterdrücken ist. Wie in Abschnitt 2.1.1 beschrieben können derartige Abweichungen in den Systemmatrizen auch als multiplikative Fehler verstanden werden, die es zu detektieren und isolieren gilt. Auf welche der beiden Interpretationen zurückgegriffen wird, ist jeweils von den konkreten Anforderungen an das Fehlerdiagnosesystem abhängig. Diese müssen in einem vorgelagerten Schritt definiert werden.

Die Störungen $\boldsymbol{d}$ beschreiben exogene Einflüsse auf das System. Als klassisches Beispiel hierfür ist Sensorrauschen zu nennen, auch die Dynamikgleichung (5.1a) kann jedoch von Störungen beeinflusst werden. Neben exogenen Einflüssen wie zum Beispiel Reibkräften in mechanischen Systemen kann der Term $\boldsymbol{B}_{d} \boldsymbol{d}$ auch nicht berücksichtigte Nichtlinearitäten beschreiben. Oftmals lassen sich dabei die Störeingangsmatrizen $\boldsymbol{B}_{d}$ und $\boldsymbol{D}_{d}$ nicht ohne Weiteres exakt angeben. Dies ist unter anderem dann der Fall, wenn durch die Störungen vernachlässigte Nichtlinearitäten beschrieben werden. Offensichtlich ist jedoch die Kenntnis der Matrizen für den Entwurf von FIOs von elementarer Bedeutung. Da das Problem auch für den Entwurf von Unknown Input Observers wesentlich ist, existieren zahlreiche Ergebnisse, die sich mit der Abschätzung der Matrizen $\boldsymbol{B}_{d}$ und $\boldsymbol{D}_{d}$ beschäftigen [29, 63, 85, 172-174]. Die praktische Umsetzung der Ansätze ist unter anderem in $[249,250]$ dokumentiert. Im Rahmen dieser Arbeit wird stets angenommen, dass die Matrizen $\boldsymbol{B}_{d}$ und $\boldsymbol{D}_{d}$ bekannt sind beziehungsweise durch entsprechende Verfahren hinreichend genau abgeschätzt wurden.

Die Ergebnisse dieses Kapitels fußen wie in Abbildung 5.1 dargestellt auf den Resultaten des 4. Kapitels. Die Gliederung wird in Abschnitt 5.2 im Zuge der Diskussion der Beiträge des Kapitels näher erläutert.

\subsection{Stand der Technik}

Während wie in Abschnitt 2.5 erörtert im Bereich der robusten beobachterbasierten Fehlerdetektion zahlreiche Ergebnisse existieren, ist die Robustheit von FIOs bisher nicht umfassend untersucht worden. Neben den frühen Ansätzen [42, 59] sind die LMI-basierten Methoden aus $[28,114,130] \mathrm{zu}$ nennen. Wie in Abschnitt 4.2 beschrieben führen diese 


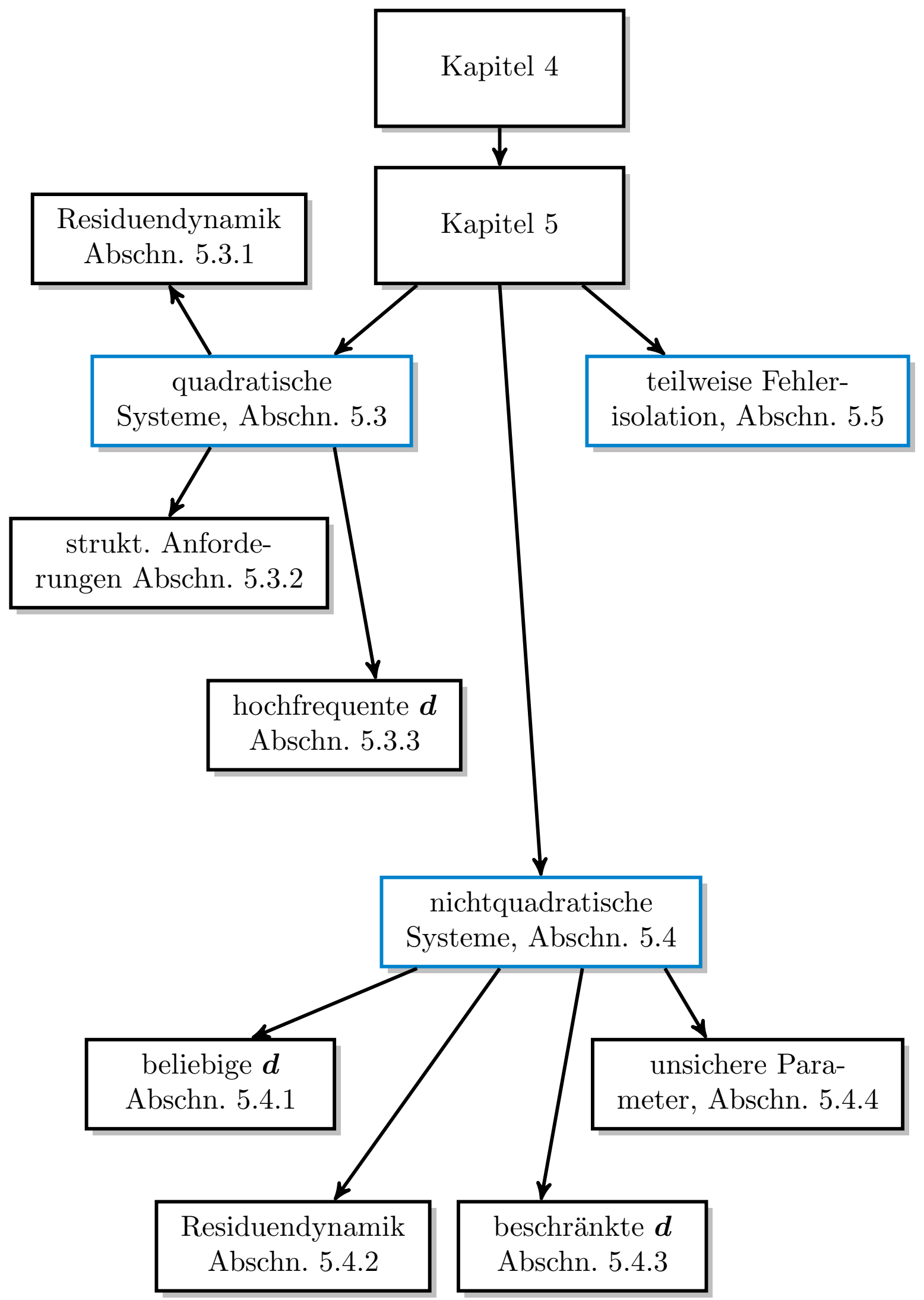

Abbildung 5.1: Aufbau des 5. Kapitels 
auf unterschiedlichen Wegen jeweils auf das gleiche LMI-Problem. Dabei werden ausschließlich nichtquadratische Systeme mit $n_{y}>n_{f}$ betrachtet, die keinerlei unsichere Parameter aufweisen, sondern lediglich exogenen Störungen unterliegen. Es wird also das Problem

$$
\begin{aligned}
& \underset{\boldsymbol{L}, \boldsymbol{V}}{\operatorname{minimiere}}\left\|\boldsymbol{G}_{\boldsymbol{r d}}(s)\right\|_{\infty}, \text { sodass } \\
& \boldsymbol{G}_{\boldsymbol{r} \boldsymbol{f}}(s)=\operatorname{diag}\left(g_{1,1}(s), \ldots, g_{n_{f}, n_{f}}(s)\right)
\end{aligned}
$$

gelöst. Dies wird damit begründet, dass sich parametrische Unsicherheiten nach $[58,175]$ als exogene Störungen formulieren lassen. Betrachtet man beispielsweise Unsicherheiten in der Matrix $\boldsymbol{A}$ mit einer normbegrenzten Matrix $\Delta \boldsymbol{A}$, so lässt sich

$$
\dot{\boldsymbol{x}}=\left(\boldsymbol{A}_{0}+\Delta \boldsymbol{A}\right) \boldsymbol{x}=\boldsymbol{A}_{0} \boldsymbol{x}+\underbrace{\boldsymbol{I}_{n}}_{\boldsymbol{B}_{d}} \cdot \underbrace{\Delta \boldsymbol{A} \cdot \boldsymbol{x}}_{\boldsymbol{d}}
$$

schreiben, solange $\boldsymbol{\Delta} \boldsymbol{A}$ die Stabilität des Systems nicht beeinträchtigt. In $[31,88,175]$ werden weitere Arten von Unsicherheitsbeschreibungen in exogene Störungen umformuliert. Bei dieser Methodik ist $\boldsymbol{A}=\boldsymbol{A}_{0}, \boldsymbol{B}=\boldsymbol{B}_{0}$ usw. und es lässt sich kompakt

$$
\begin{aligned}
& \dot{\boldsymbol{x}}=\boldsymbol{A} \boldsymbol{x}+\boldsymbol{B u}+\boldsymbol{E}_{a} \boldsymbol{f}+\boldsymbol{B}_{d} \boldsymbol{d}, \\
& \boldsymbol{y}=\boldsymbol{C} \boldsymbol{x}+\boldsymbol{D} \boldsymbol{u}+\boldsymbol{E}_{s} \boldsymbol{f}+\boldsymbol{D}_{d} \boldsymbol{d}
\end{aligned}
$$

betrachten. Die Robustheitsuntersuchung beziehungsweise -steigerung reduziert sich dann auf die Betrachtung der exogenen Störungen $\boldsymbol{d}$.

Zwar vereinfacht die Rückführung des Einflusses unsicherer Parameter auf virtuelle exogene Störungen die Robustheitsbetrachtungen. Allerdings ist offensichtlich, dass durch den Ansatz $\boldsymbol{B}_{d}=\boldsymbol{I}_{n}, \boldsymbol{d}=\Delta \boldsymbol{A} \boldsymbol{x}$ in (5.2) strukturelles Wissen verloren geht beziehungsweise ungenutzt bleibt. Dies ist insbesondere bei mechanischen Systemen der Fall, bei denen die Matrix $\Delta \boldsymbol{A}$ oft spärlich besetzt ist. Wie in [58, Abschnitt 8.2] angemerkt kann dies zu konservativen Ergebnissen bei der Robustheitsoptimierung führen.

\subsection{Beiträge dieses Kapitels}

Im Rahmen dieses Kapitels werden wie in Abbildung 5.1 dargestellt die existierenden Ergebnisse im Bereich der robusten FIOs in wesentlichen Punkten erweitert und verbessert. Dies umfasst 
1. Optimierung der Störunterdrückung in quadratischen Systemen. Während in $[28,114,130]$ ausschließlich nichtquadratische Systeme behandelt werden, wird in dieser Arbeit gezeigt, wie sich auch in quadratischen Systemen die Robustheit von FIOs steigern lässt. Dazu erfolgt unter anderem die Formulierung als StandardRegelungsproblem unter Strukturbeschränkungen (Abschnitt 5.3.1). Diese wurde bisher lediglich für die Fehlerdetektion [35] vorgeschlagen, wobei keine Strukturbeschränkungen zu berücksichtigen sind. Weiterhin wird auf Basis der Vollständigen Modalen Synthese in Abschnitt 5.3.2 ein Entwurfsverfahren entwickelt, bei dem sich aus der Abschwächung der strukturellen Anforderungen Freiheitsgrade zur Robustheitsoptimierung ergeben. Außerdem wird ein Verfahren entwickelt, bei dem durch die Erweiterung der Dynamik der Diagonalelemente eine verbesserte Unterdrückung hochfrequenter Störungen ermöglicht wird (Abschnitt 5.3.3).

2. Erweiterung der Resultate aus [28, 114, 130]. Die Optimierung bezüglich exogener Störungen in nichtquadratischen Systemen wird in Abschnitt 5.4.1 dahingehend erweitert, dass eine direkte Berücksichtigung von Sensorfehlern ohne dynamische Erweiterungen [169] möglich ist. Darüber hinaus können Eigenwertbereiche für die nichtsteuerbaren Beobachtereigenwerte vorgegeben werden.

3. Einbeziehung von zusätzlichem Wissen über die Störsignale. Ein weiterer wesentlicher Beitrag ist in einem Entwurfsverfahren $\mathrm{zu}$ sehen, welches es ermöglicht, zusätzliches Wissen über relevante Frequenzbereiche von Störungen einzubeziehen (Abschnitt 5.4.3). Dies erlaubt es, gegenüber existierenden Resultaten wesentlich weniger konservative Ergebnisse und eine deutlich verbesserte Störunterdrückung in der Fehlerisolation zu erreichen.

4. Explizite Berücksichtigung unsicherer Parameter. In Abschnitt 5.4.4 wird ein Verfahren vorgestellt, welches parametrische Unsicherheiten direkt berücksichtigt, ohne sie in der gängigen Art und Weise in virtuelle exogene Störungen umzuwandeln. Da somit die Kenntnis der Struktur der Matrizen $\Delta \boldsymbol{A}, \Delta \boldsymbol{B}$ usw. ausgenutzt wird, erlaubt dieses auf der Vollständigen Modalen Synthese basierende Verfahren weniger konservative Ergebnisse als existierende Resultate.

5. Optimierung der Robustheit bei abgeschwächten strukturellen Anforderungen. Ist lediglich eine teilweise Fehlerisola- 
tion gefordert, so lassen sich die in Abschnitt 4.10 erarbeiteten zusätzlichen Freiheitsgrade ebenfalls zur Robustheitssteigerung heranziehen. Dazu werden in Abschnitt 5.5 zwei unterschiedliche Entwurfsverfahren vorgestellt.

Das Kapitel ist entsprechend des Aufbaus von Kapitel 4 gegliedert: Zunächst wird in Abschnitt 5.3 die Robustheit in quadratischen Systemen mit $n_{y}=n_{f}$ betrachtet. Abschnitt 5.4 befasst sich mit der Ausnutzung zusätzlicher Freiheitsgrade zur Robustheitssteigerung in nichtquadratischen Systemen mit $n_{y}>n_{f}$. Die Optimierung der Robustheit bei partieller Fehlerisolation wird in Abschnitt 5.5 behandelt.

\subsection{Steigerung der Robustheit in quadratischen Systemen}

Durch die strukturelle Vorgabe einer diagonalen Übertragungsmatrix $\boldsymbol{G}_{\boldsymbol{r} \boldsymbol{f}}(s)$ steht in quadratischen Systemen mit ebenso vielen messbaren Ausgangsgrößen wie potentiellen Fehlern $\left(n_{y}=n_{f}\right)$ nur eine sehr begrenzte Anzahl von Freiheitsgraden zur Verfügung. Aus Abschnitt 4.5 geht hervor, dass bei Vorgabe einer exakten Diagonalstruktur von $\boldsymbol{G}_{\boldsymbol{r} \boldsymbol{f}}(s)$ sowie der Festlegung der Diagonalelemente der FIO eindeutig bestimmt ist und somit keinerlei Freiheitsgrade zur Verfügung stehen.

In diesem Abschnitt werden drei Verfahren untersucht, mithilfe derer sich dennoch die Robustheit von FIOs optimieren lässt. Grundgedanke in Abschnitt 5.3.1 ist es, die Diagonalelemente von $\boldsymbol{G}_{\boldsymbol{r} \boldsymbol{f}}(s)$ nicht fest vorzugeben, sondern deren Koeffizienten $q_{i, j}$ und $z_{i, 0}$ für die Robustheitssteigerung zu nutzen. Eine andere Möglichkeit zur Gewinnung von Freiheitsgraden für die Robustheitsoptimierung ist es, keine exakte Diagonalstruktur von $\boldsymbol{G}_{\boldsymbol{r} \boldsymbol{f}}(s)$ zu fordern, sondern nur eine näherungsweise diagonalisierte Übertragungsmatrix. Dieser Ansatz wird in Abschnitt 5.3.2 verfolgt. Schließlich wird in Abschnitt 5.3.3 die Erweiterung der Dynamik der Diagonalelemente (s. Abschnitt 4.6) zur Verbesserung der Robustheit bezüglich hochfrequenter Störungen genutzt.

\subsubsection{Optimierung durch Anpassung der vorgegebenen Residuendynamik}

In quadratischen Systemen verbleiben bei vollständiger Fehlerisolation lediglich die Koeffizienten der Zähler- und Nennerpolynome der Diagonalele- 
mente $g_{i, i}(s)$ der Übertragungsmatrix $\boldsymbol{G}_{\boldsymbol{r} \boldsymbol{f}}(s)$ als Freiheitsgrade. Diese lassen sich wie in Abschnitt 4.5 beschrieben zum Einstellen einer gewünschten Residuendynamik sowie der Fehlersensitivität heranziehen. Aus den entsprechenden Anforderungen ergibt sich jedoch kein exaktes Verfahren zur Wahl der Koeffizienten $q_{i, j}$ und $z_{i, 0}$. Vielmehr lassen sich lediglich Bereiche für die Parameter angeben. Eine gewünschte Residuendynamik lässt sich beispielsweise durch einen Bereich für die entsprechenden Beobachtereigenwerte darstellen - wie die Eigenwerte in diesem Bereich genau zu wählen sind, ist zunächst nicht klar.

Durch die Strukturbeschränkungen in $\boldsymbol{L}\left(\boldsymbol{q}_{i, j}\right)$ und $\boldsymbol{V}\left(\boldsymbol{z}_{i, 0}\right)$ ist die Diagonalisierung von $\boldsymbol{G}_{\boldsymbol{r} \boldsymbol{f}}(s)$ für nominale Systemmatrizen sichergestellt. ${ }^{1)}$ Es verleibt also, den Einfluss exogener Störungen $\boldsymbol{d}$ auf die Residuengenerierung im Sinne der $\mathcal{H}_{\infty}$-Norm zu minimieren. Dies lässt sich formal durch

$$
\begin{aligned}
& \underset{\boldsymbol{q}_{i, j}, \boldsymbol{z}_{i, 0}}{\operatorname{minimiere}} \gamma, \text { sodass } \\
& \boldsymbol{L}\left(\boldsymbol{q}_{i, j}\right)=\boldsymbol{M}\left(\boldsymbol{q}_{i, j}\right) \boldsymbol{D}^{*-1}, \\
& \boldsymbol{V}\left(\boldsymbol{z}_{i, 0}\right)=\boldsymbol{N}\left(\boldsymbol{z}_{i, 0}\right) \boldsymbol{D}^{*-1}, \\
& \left\|\boldsymbol{G}_{\boldsymbol{r d}}(s)\right\|_{\infty}<\gamma
\end{aligned}
$$

beschreiben. Anzumerken ist, dass bei nominaler Systemdynamik der Beobachteransatz hinsichtlich der Wirkung der Stellgrößen auf die Residuen unmittelbar $\boldsymbol{G}_{\boldsymbol{r u}}(s)=\mathbf{0}$ sicherstellt und sich der Einfluss von Fehlern und Störungen auf die erzeugten Residuen aufgrund des Superpositionsprinzips für lineare Systeme unabhängig voneinander analysieren lässt. Analog zu (4.4) wird die Wirkung der Störungen auf die Residuen, also $\boldsymbol{G}_{\boldsymbol{r} \boldsymbol{d}}(s)$, durch

$$
\begin{aligned}
\dot{\boldsymbol{\xi}} & =(\boldsymbol{A}-\boldsymbol{L} \boldsymbol{C}) \boldsymbol{\xi}+\left(\boldsymbol{B}_{d}-\boldsymbol{L} \boldsymbol{D}_{d}\right) \boldsymbol{d} \\
\boldsymbol{r} & =\boldsymbol{V} \boldsymbol{C} \boldsymbol{\xi}+\boldsymbol{V} \boldsymbol{D}_{d} \boldsymbol{d}
\end{aligned}
$$

beschrieben, wobei $\boldsymbol{\xi}=\boldsymbol{x}-\hat{\boldsymbol{x}}$ die Differenz zwischen Strecken- und Beobachterzustand ist. Aufgrund der Isolationseigenschaft des durch $\left(\boldsymbol{L}\left(\boldsymbol{q}_{i, j}\right), \boldsymbol{V}\left(\boldsymbol{z}_{i, 0}\right)\right)$ parametrierten FIOs sind die Beobachtermatrizen strukturell beschränkt. Daraus ergibt sich, dass das Optimierungsproblem (5.3) nichtkonvex ist.

${ }^{1)}$ Die Schreibweise $\boldsymbol{q}_{i, j}, \boldsymbol{z}_{i, 0}$ deutet an, dass die freien Koeffizienten $q_{i, j}$ und $z_{i, 0}$ in Vektoren zusammengefasst werden. 


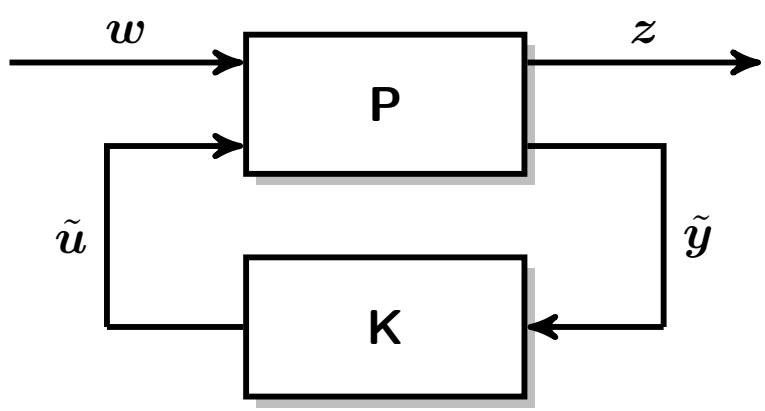

Abbildung 5.2: Blockschaltbild des Standard-Regelungsproblems

Die in Auszügen bereits in [241] veröffentlichte Grundidee zur Lösung ist es, das Problem (5.3) umzuformulieren und es als ein sogenanntes Standard-Regelungsproblem darzustellen. Zwar wird auch in [35] ein Fehlerdiagnoseproblem auf ein Standard-Regelungsproblem zurückgeführt, allerdings wird dort lediglich der einfachere Fall der Fehlerdetektion betrachtet, bei dem die Beobachtermatrix keinerlei strukturellen Beschränkungen unterliegt.

Im Framework des Standard-Regelungsproblems wird eine verallgemeinerte Strecke $\mathbf{P}$ betrachtet, welche mit einem verallgemeinerten Regler $\mathbf{K}$ geregelt wird. Auf $\mathbf{P}$ wirken exogene Eingangssignale $\boldsymbol{w}$ und vom Regler $\mathbf{K}$ generierte Eingangsgrößen $\tilde{\boldsymbol{u}}$. Diese werden von $\mathbf{K}$ auf Grundlage der messbaren Ausgangssignale $\tilde{\boldsymbol{y}}$ erzeugt, weiterhin existieren sogenannte Performance-Ausgänge $\boldsymbol{z}$ der verallgemeinerten Strecke. In Abbildung 5.2 ist die allgemeine Struktur dargestellt. Zahlreiche regelungstechnische Probleme lassen sich in dieser Weise darstellen (s. zum Beispiel [206]) und es stehen verschiedene ausgereifte Solver-Toolboxen (zum Beispiel Hifoo [91], Hinfstruct [9] oder Systune [8]) zur Verfügung, um PerformanceMaße (zum Beispiel $\left\|\boldsymbol{G}_{\boldsymbol{z} \boldsymbol{w}}(s)\right\|_{\infty}$ ) im Standard-Regelungsproblem zu optimieren. Im Weiteren wird eine Darstellung von (5.3) hergeleitet, die es erlaubt, solche Standard-Tools auf die spezielle Problemstellung des Entwurfs eines robusten FIOs anzuwenden.

Darüber hinaus ist ein weiterer wesentlicher Vorteil des Verfahrens zu nennen. Wie bei allen Polvorgabeverfahren stellt sich auch bei den in Kapitel 4 vorgestellten FIO-Entwürfen die Frage, wie die Pole zu platzieren sind. Meist sind die Anforderungen an das Regelungs- beziehungsweise Diagnosesystem zum Beispiel in Ausregelzeiten oder Überschwingweiten formuliert, die nicht in geradliniger Art und Weise mit der Lage der Pole verknüpft sind. Wesentlich intuitiver als die exakte Platzierung der Pole ist die Vorgabe von Polbereichen, die ein gewünschtes dynamisches Verhalten 
gewährleisten [38, 122, 181]. Mit dem in diesem Abschnitt erarbeiteten Verfahren ist es möglich, einen solchen Bereich durch eine obere Schranke für die Realteile der Beobachtereigenwerte vorzugeben, anstatt die Pole exakt zu platzieren.

Im Allgemeinen lässt sich das Standard-Regelungsproblem mit statischer Ausgangsrückführung in Zustandsraumdarstellung schreiben als

$$
\begin{aligned}
& \underset{\tilde{\boldsymbol{K}}}{\operatorname{minimiere}}\left\|\boldsymbol{G}_{\boldsymbol{z w}}(s)\right\|_{\infty} \operatorname{mit} \\
& \mathbf{P}:\left\{\begin{array}{l}
\dot{\tilde{\boldsymbol{x}}}=\tilde{\boldsymbol{A}} \tilde{\boldsymbol{x}}+\tilde{\boldsymbol{B}}_{1} \boldsymbol{w}+\tilde{\boldsymbol{B}}_{2} \tilde{\boldsymbol{u}} \\
\boldsymbol{z}=\tilde{\boldsymbol{C}}_{1} \tilde{\boldsymbol{x}}+\tilde{\boldsymbol{D}}_{11} \boldsymbol{w}+\tilde{\boldsymbol{D}}_{12} \tilde{\boldsymbol{u}} \\
\tilde{\boldsymbol{y}}=\tilde{\boldsymbol{C}}_{2} \tilde{\boldsymbol{x}}+\tilde{\boldsymbol{D}}_{21} \boldsymbol{w}+\tilde{\boldsymbol{D}}_{22} \tilde{\boldsymbol{u}},
\end{array}\right. \\
& \mathbf{K}: \tilde{\boldsymbol{u}}=\tilde{\boldsymbol{K}} \tilde{\boldsymbol{y}}
\end{aligned}
$$

Für den FIO-Entwurf wird $\tilde{\boldsymbol{x}}=\boldsymbol{\xi}=\boldsymbol{x}-\hat{\boldsymbol{x}}$ angesetzt und exogene Störungen werden als verallgemeinerte Eingangsgröße $\boldsymbol{w}=\boldsymbol{d}$ aufgefasst. Der Performance-Ausgang ist gleichbedeutend mit dem generierten Residuenvektor, also ist $\boldsymbol{z}=\boldsymbol{r}$. Definiert man die Differenz zwischen gemessenem und geschätztem Ausgangsvektor, $\tilde{\boldsymbol{y}}=\boldsymbol{y}-\hat{\boldsymbol{y}}$, als messbares Signal, so kann (5.4) bezüglich des Ein-/Ausgangsverhaltens äquivalent in der Form $(5.5 \mathrm{~b}),(5.5 \mathrm{c}) \mathrm{mit}$

$$
\begin{aligned}
& \tilde{\boldsymbol{A}}=\boldsymbol{A}, \quad \tilde{\boldsymbol{B}}_{1}=\boldsymbol{B}_{d}, \quad \tilde{\boldsymbol{B}}_{2}=\left[\begin{array}{ll}
\mathbf{0} & -\boldsymbol{I}_{n}
\end{array}\right], \\
& \tilde{C}_{1}=\mathbf{0}, \quad \tilde{\boldsymbol{D}}_{11}=\mathbf{0}, \quad \tilde{\boldsymbol{D}}_{12}=\left[\begin{array}{ll}
\boldsymbol{I}_{n_{f}} & \mathbf{0}
\end{array}\right], \\
& \tilde{\boldsymbol{C}}_{2}=\boldsymbol{C}, \quad \tilde{\boldsymbol{D}}_{21}=\boldsymbol{D}_{d}, \quad \tilde{\boldsymbol{D}}_{22}=\mathbf{0}
\end{aligned}
$$

geschrieben werden, wenn der verallgemeinerte Regler angesetzt wird als

$$
\tilde{\boldsymbol{K}}=\left[\begin{array}{l}
\boldsymbol{V}\left(\boldsymbol{z}_{i, 0}\right) \\
\boldsymbol{L}\left(\boldsymbol{q}_{i, j}\right)
\end{array}\right] .
$$

In obiger Form sind die strukturellen Beschränkungen in $\boldsymbol{L}\left(\boldsymbol{q}_{i, j}\right)$ und $\boldsymbol{V}\left(\boldsymbol{z}_{i, 0}\right)$ noch nicht offensichtlich. In den verfügbaren Standard-Solvern zur Optimierung von Performancemaßen in Standard-Regelungsproblemen werden strukturelle Beschränkungen meist durch Nullelemente im verallgemeinerten Regler realisiert. Eine solche Darstellung lässt sich auch für den robusten FIO-Entwurf erreichen. Zunächst wird die Matrix $\boldsymbol{M}\left(\boldsymbol{q}_{i, j}\right)$ aus Satz 4.1 auf Seite 82 aufgeteilt in

$$
\boldsymbol{M}\left(\boldsymbol{q}_{i, j}\right)=\boldsymbol{M}_{\alpha}+\boldsymbol{M}_{\beta} \boldsymbol{Q}_{L}\left(\boldsymbol{q}_{i, j}\right) .
$$


Die Matrizen $\boldsymbol{M}_{\alpha} \in \mathbb{R}^{n \times n_{f}}$ und $\boldsymbol{M}_{\beta} \in \mathbb{R}^{n \times \delta}$ sind dabei gegeben durch

$$
\begin{aligned}
\boldsymbol{M}_{\alpha} & =\left[\begin{array}{lllllll}
\boldsymbol{A}^{\delta_{1}} \boldsymbol{e}_{a_{1}} & \cdots & \boldsymbol{A}^{\delta_{n_{f}}} \boldsymbol{e}_{a_{n_{f}}}
\end{array}\right] \\
\boldsymbol{M}_{\beta} & =\left[\begin{array}{llllllll}
\boldsymbol{A}^{\delta_{1}-1} \boldsymbol{e}_{a_{1}} & \boldsymbol{A}^{\delta_{1}-2} \boldsymbol{e}_{a_{1}} & \cdots & \boldsymbol{A} \boldsymbol{e}_{a_{1}} & \boldsymbol{e}_{a_{1}} & \boldsymbol{A}^{\delta_{2}-1} \boldsymbol{e}_{a_{2}} & \cdots & \boldsymbol{e}_{a_{n_{f}}}
\end{array}\right] .
\end{aligned}
$$

Sie hängen also ausdrücklich nicht von den zu wählenden Koeffizienten $q_{i, j}$ ab, sondern sind ausschließlich durch die Strecke selbst bestimmt. Im Gegensatz dazu enthält die Matrix $\boldsymbol{Q}_{L}\left(\boldsymbol{q}_{i, j}\right) \in \mathbb{R}^{\delta \times n_{f}}$ nur Nullelemente oder freie Koeffizienten, sie lautet

$$
\boldsymbol{Q}_{L}\left(\boldsymbol{q}_{i, j}\right)=\left[\begin{array}{cccccc}
q_{1, \delta_{1}-1} & 0 & \cdots & \cdots & \cdots & 0 \\
\vdots & \vdots & & & & \vdots \\
q_{1,0} & 0 & \cdots & \cdots & \cdots & 0 \\
0 & q_{2, \delta_{2}-1} & 0 & \cdots & \cdots & 0 \\
\vdots & \vdots & \vdots & & & \vdots \\
0 & q_{2,0} & 0 & \ldots & \cdots & 0 \\
& & \ddots & & & \\
0 & \ldots & \cdots & \cdots & 0 & q_{n_{f}, \delta_{n_{f}}-1} \\
\vdots & & & & \vdots & \vdots \\
0 & \ldots & \cdots & \cdots & 0 & q_{n_{f}, 0}
\end{array}\right] .
$$

Ebenso wird die Nachfiltermatrix $\boldsymbol{V}\left(\boldsymbol{z}_{i, 0}\right)$ zerlegt in

$$
\boldsymbol{V}\left(\boldsymbol{z}_{i, 0}\right)=\boldsymbol{Q}_{V}\left(\boldsymbol{z}_{i, 0}\right) \boldsymbol{D}^{*-1}=\operatorname{diag}\left(z_{1,0}, \ldots, z_{n_{f}, 0}\right) \boldsymbol{D}^{*-1} .
$$

Im Folgenden wird aus Gründen einer kompakten Notation $\boldsymbol{Q}_{L}=\boldsymbol{Q}_{L}\left(\boldsymbol{q}_{i, j}\right)$ und $\boldsymbol{Q}_{V}=\boldsymbol{Q}_{V}\left(\boldsymbol{z}_{i, 0}\right) \in \mathbb{R}^{n_{f} \times n_{y}}$ geschrieben. Setzt man nun die partitionierte Beobachtermatrix $\boldsymbol{L}=\left(\boldsymbol{M}_{\alpha}+\boldsymbol{M}_{\beta} \boldsymbol{Q}_{L}\right) \boldsymbol{D}^{*-1}$ in die Beobachterfehlerdynamik (5.4a) ein, so ergibt sich

$$
\dot{\boldsymbol{\xi}}=\left(\boldsymbol{A}-\boldsymbol{M}_{\alpha} \boldsymbol{D}^{*-1} \boldsymbol{C}\right) \boldsymbol{\xi}-\boldsymbol{M}_{\beta} \boldsymbol{Q}_{L} \boldsymbol{D}^{*-1}(\boldsymbol{y}-\hat{\boldsymbol{y}})+\left(\boldsymbol{B}_{d}-\boldsymbol{M}_{\alpha} \boldsymbol{D}^{*-1} \boldsymbol{D}_{d}\right) \boldsymbol{d} .
$$

Der erste Schlüsselgedanke ist es nun, den Term $\boldsymbol{M}_{\alpha} \boldsymbol{D}^{*-1}$, welcher im realen System Teil der Beobachterdynamik ist, der verallgemeinerten Strecke zuzuschlagen. Daraus ergibt sich als neue Parametrierung $\mathbf{P}^{\prime}$ mit

$$
\tilde{\boldsymbol{A}}^{\prime}=\boldsymbol{A}-\boldsymbol{M}_{\alpha} \boldsymbol{D}^{*-1} \boldsymbol{C}, \quad \tilde{\boldsymbol{B}}_{1}^{\prime}=\boldsymbol{B}_{d}-\boldsymbol{M}_{\alpha} \boldsymbol{D}^{*-1} \boldsymbol{D}_{d}, \quad \tilde{\boldsymbol{B}}_{2}^{\prime}=\left[\begin{array}{ll}
\mathbf{0} & -\boldsymbol{I}_{n}
\end{array}\right]
$$




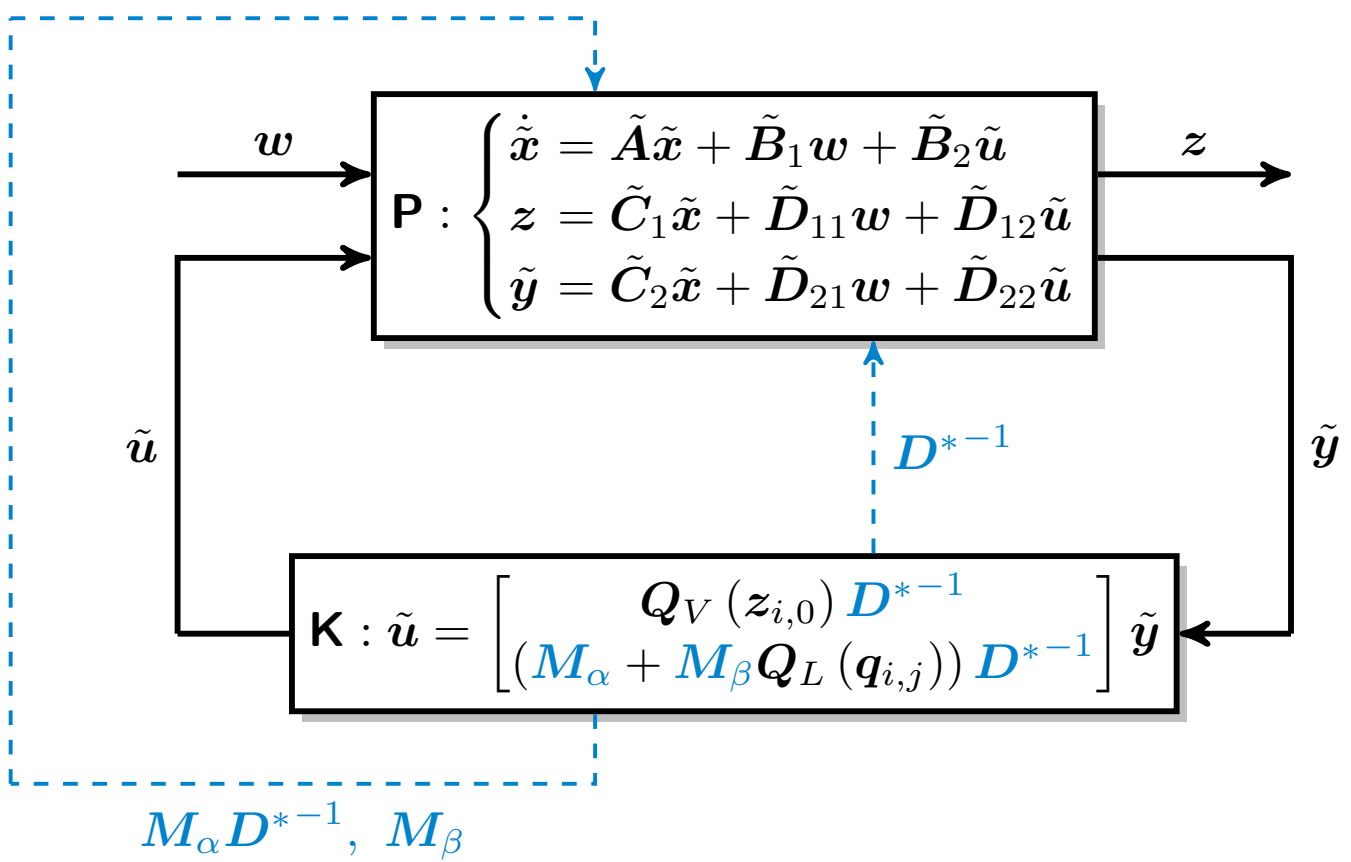

Abbildung 5.3: Zur Umformulierung des robusten FIO-Problems als Standard-Regelungsproblem durch den Übergang von $\mathbf{P}, \mathbf{K}$ auf $\mathbf{P}^{\prime \prime}, \mathbf{K}^{\prime \prime}$

und dem zugehörigen verallgemeinerten Regler

$$
\tilde{\boldsymbol{K}}^{\prime}=\left[\begin{array}{c}
\boldsymbol{Q}_{V} \boldsymbol{D}^{*-1} \\
\boldsymbol{M}_{\beta} \boldsymbol{Q}_{L} \boldsymbol{D}^{*-1}
\end{array}\right]
$$

Im nächsten Schritt wird die Matrix $\boldsymbol{M}_{\beta}$ ebenfalls in die virtuelle, verallgemeinerte Strecke verschoben und der Term $\boldsymbol{D}^{*-1}$ als virtuelle Modifikation der Messung aufgefasst. Abbildung 5.3 fasst die Schritte grafisch zusammen.

Als Resultat der erläuterten Modifikationen von verallgemeinerter Strecke und verallgemeinertem Regler ergibt sich die Darstellung

$$
\begin{aligned}
& \mathbf{P}^{\prime \prime}:\left\{\begin{array}{l}
\tilde{\boldsymbol{A}}^{\prime \prime}=\boldsymbol{A}-\boldsymbol{M}_{\alpha} \boldsymbol{D}^{*-1} \boldsymbol{C}, \\
\tilde{\boldsymbol{B}}_{1}^{\prime \prime}=\boldsymbol{B}_{d}-\boldsymbol{M}_{\alpha} \boldsymbol{D}^{*-1} \boldsymbol{D}_{d}, \quad \tilde{\boldsymbol{B}}_{2}^{\prime \prime}=\left[\begin{array}{ll}
\mathbf{0} & -\boldsymbol{M}_{\beta}
\end{array}\right], \\
\tilde{\boldsymbol{C}}_{1}^{\prime \prime}=\mathbf{0}, \quad \tilde{\boldsymbol{D}}_{11}^{\prime \prime}=\mathbf{0}, \quad \tilde{\boldsymbol{D}}_{12}^{\prime \prime}=\left[\begin{array}{ll}
\boldsymbol{I}_{n_{f}} & \mathbf{0}
\end{array}\right], \\
\tilde{\boldsymbol{C}}_{2}^{\prime \prime}=\boldsymbol{D}^{*-1} \boldsymbol{C}, \quad \tilde{\boldsymbol{D}}_{21}^{\prime \prime}=\boldsymbol{D}^{*-1} \boldsymbol{D}_{d}, \quad \tilde{\boldsymbol{D}}_{22}^{\prime \prime}=\mathbf{0},
\end{array}\right. \\
& \mathbf{K}^{\prime \prime}: \tilde{\boldsymbol{K}}^{\prime \prime}=\left[\begin{array}{l}
\boldsymbol{Q}_{V}\left(\boldsymbol{z}_{i, 0}\right) \\
\boldsymbol{Q}_{L}\left(\boldsymbol{q}_{i, j}\right)
\end{array}\right] .
\end{aligned}
$$

Diese liefert eine $\mathrm{zu}$ (5.5b), (5.5c) und (5.6) beziehungsweise (5.4) äquivalente Formulierung des Übertragungsverhaltens $\boldsymbol{G}_{\boldsymbol{z} \boldsymbol{w}}(s)=\boldsymbol{G}_{\boldsymbol{r} \boldsymbol{d}}(s)$. 
Sie weist dabei den großen Vorteil auf, dass der verallgemeinerte Regler $\tilde{\boldsymbol{K}}^{\prime \prime}$ in (5.7) ausschließlich freie Koeffizienten oder Nullelemente enthält. In dieser Standardform lassen sich nun generische Solver wie Hifoo [91], Hinfstruct [9] oder Systune [8] anwenden, um das Optimierungsproblem

$$
\begin{aligned}
& \underset{\boldsymbol{q}_{i, j}, \boldsymbol{z}_{i, 0}}{\operatorname{minimiere}} \gamma, \text { sodass } \\
& \left\|\boldsymbol{G}_{\boldsymbol{z} \boldsymbol{w}}(s)\right\|_{\infty}<\gamma, \quad(5.7)
\end{aligned}
$$

zu lösen.

$\mathrm{Zu}$ beachten ist dabei, dass die Optimierung zu sehr kleinen Werten für die Zählerkoeffizienten $z_{i, 0}$ führen kann, da die Fehlersensitivität in (5.8) nicht berücksichtigt wird. Um diesem Problem zu begegnen lässt sich für die einzelnen Kanäle eine statische Verstärkung, das heißt $g_{i, i}(s=0)=k_{s, i}$, vorgeben, indem $z_{i, 0}=k_{s, i} q_{i, 0}$ gefordert wird. Dadurch wird eine ausreichende Fehlersensitivität bei niedrigen Frequenzen gewährleistet. Zu diesem Zweck werden die Matrizen

$$
\boldsymbol{\Phi}=\left[\begin{array}{c}
k_{s, 1} \tilde{\boldsymbol{\phi}}_{1}^{\top} \\
\vdots \\
k_{s, n_{f}} \tilde{\boldsymbol{\phi}}_{n_{f}}^{\top}
\end{array}\right], \mathbf{\Upsilon}=\left[\begin{array}{c}
\boldsymbol{\Phi} \\
\boldsymbol{I}_{\delta}
\end{array}\right]
$$

eingeführt, wobei $\tilde{\phi}_{i}^{\top}=\left[\begin{array}{llllll}0 & \cdots & 0 & 1 & 0 & \cdots\end{array}\right]$ ein Einheitsvektor ist, welcher in der $\sum_{k=1}^{i} \delta_{k}$-ten Spalte das Element 1 enthält. ${ }^{2)}$ Damit lässt sich der verallgemeinerte Regler erneut umschreiben zu $\tilde{\boldsymbol{K}}^{\prime \prime}=\boldsymbol{\Upsilon} \boldsymbol{Q}_{L}$. Entsprechend dem bisherigen Vorgehen kann nun die Matrix $\boldsymbol{\Upsilon}$ wiederum als ein Teil der verallgemeinerten Strecke aufgefasst werden, was zu den Matrizen

$$
\tilde{\boldsymbol{B}}_{2}^{\prime \prime \prime}=\left[\begin{array}{ll}
\mathbf{0} & -\boldsymbol{M}_{\beta}
\end{array}\right] \boldsymbol{\Upsilon}, \tilde{\boldsymbol{D}}_{12}^{\prime \prime \prime}=\left[\begin{array}{ll}
\boldsymbol{I}_{n_{f}} & \mathbf{0}
\end{array}\right] \boldsymbol{\Upsilon}, \tilde{\boldsymbol{K}}^{\prime \prime \prime}=\boldsymbol{Q}_{L}
$$

führt. Für den Entwurf verleiben somit nur noch die Nennerkoeffizienten von $g_{i, i}(s)$ als Freiheitsgrade. Zusammenfassend gilt demnach

Satz 5.1 (Entwurf robuster FIOs durch Lösung eines strukturbeschränkten Standard-Regelungsproblems). Das Optimierungsproblem

$$
\begin{aligned}
& \underset{\boldsymbol{q}_{i, j}, \boldsymbol{z}_{i, 0}}{\operatorname{minimiere}} \gamma, \text { sodass } \\
& \left\|\boldsymbol{G}_{\boldsymbol{r} \boldsymbol{d}}\right\|_{\infty}<\gamma \\
& \boldsymbol{G}_{\boldsymbol{r} \boldsymbol{f}}(s)=\operatorname{diag}\left(g_{1,1}(s), \ldots, g_{n_{f}, n_{f}}(s)\right), g_{i, i}(s=0)=k_{s, i} \forall i=1, \ldots, n_{f}
\end{aligned}
$$

${ }^{2)}$ Dieser Vektor ist nicht zu verwechseln mit dem bereits eingeführten Vektor $\phi_{i}^{\top}$, der in der $i$-ten Spalte das Element 1 beinhaltet. 
lässt sich äquivalent formulieren als strukturbeschränktes StandardRegelungsproblem der Form (5.5) mit

$$
\begin{aligned}
& \tilde{\boldsymbol{A}}^{\prime \prime \prime}=\boldsymbol{A}-\boldsymbol{M}_{\alpha} \boldsymbol{D}^{*-1} \boldsymbol{C}, \\
& \tilde{\boldsymbol{B}}_{1}^{\prime \prime \prime}=\boldsymbol{B}_{d}-\boldsymbol{M}_{\alpha} \boldsymbol{D}^{*-1} \boldsymbol{D}_{d}, \quad \tilde{\boldsymbol{B}}_{2}^{\prime \prime \prime}=\left[\begin{array}{ll}
\mathbf{0} & -\boldsymbol{M}_{\beta}
\end{array}\right] \mathbf{\Upsilon}, \\
& \tilde{\boldsymbol{C}}_{1}^{\prime \prime \prime}=\mathbf{0}, \quad \tilde{\boldsymbol{D}}_{11}^{\prime \prime \prime}=\mathbf{0}, \quad \tilde{\boldsymbol{D}}_{12}^{\prime \prime \prime}=\left[\begin{array}{ll}
\boldsymbol{I}_{n_{f}} & \mathbf{0}
\end{array}\right] \mathbf{\Upsilon}, \\
& \tilde{\boldsymbol{C}}_{2}^{\prime \prime \prime}=\boldsymbol{D}^{*-1} \boldsymbol{C}, \quad \tilde{\boldsymbol{D}}_{21}^{\prime \prime \prime}=\boldsymbol{D}^{*-1} \boldsymbol{D}_{d}, \quad \tilde{\boldsymbol{D}}_{22}^{\prime \prime \prime}=\mathbf{0}
\end{aligned}
$$

und dem verallgemeinerten Regler

$$
\tilde{\boldsymbol{K}}^{\prime \prime \prime}=\boldsymbol{Q}_{L}\left(\boldsymbol{q}_{i, j}\right) .
$$

Im Folgenden werden zwei Erweiterungen des Verfahrens diskutiert.

\section{Erweiterung zur Vorgabe einer}

\section{Mindestdetektionsgeschwindigkeit}

Ein Punkt, der bisher außer Acht gelassen wurde, ist die Detektionsgeschwindigkeit, das heißt die Dynamik der generierten Residuen. Um eine hinreichende Geschwindigkeit bei der Erkennung von Fehlern zu gewährleisten, sollten alle Beobachtereigenwerte $\lambda_{B_{i}} \in \operatorname{spec}\left(\boldsymbol{A}-\boldsymbol{L}\left(\boldsymbol{q}_{i, j}\right) \boldsymbol{C}\right)$ das Kriterium $\mathfrak{R e}\left(\lambda_{B_{i}}\right)<\alpha<0$ erfüllen. Es wird eine zweite, virtuelle Strecke betrachtet, um die Eigenwerte von $\boldsymbol{A}-\boldsymbol{L}\left(\boldsymbol{q}_{i, j}\right) \boldsymbol{C}$ zu beschränken. Sie wird durch

$$
\begin{aligned}
\overline{\boldsymbol{A}} & =\boldsymbol{A}-\boldsymbol{M}_{\alpha} \boldsymbol{D}^{*-1} \boldsymbol{C}, \\
\overline{\boldsymbol{B}}_{2} & =-\boldsymbol{M}_{\beta}, \\
\overline{\boldsymbol{C}}_{2} & =\boldsymbol{D}^{*-1} \boldsymbol{C}
\end{aligned}
$$

parametriert, wobei alle anderen Matrizen der verallgemeinerten Strecke nicht benötigt und als Nullmatrix angesetzt werden. Die verallgemeinerte Strecke (5.9) wird dabei vom gleichen Regler $\mathbf{K}$ beeinflusst wie (5.7a). In der Optimierung wird dann die $\mathcal{H}_{\infty}$-Norm der ersten verallgemeinerten Strecke minimiert, wobei die Beschränkung des größten Realteils eines Eigenwertes der zweiten Strecke als Nebenbedingung einzuhalten ist. ${ }^{3)}$

Bemerkung 5.1. Die Bedingung $\mathfrak{R e}\left(\lambda_{B_{i}}\right)<\alpha$ betrifft alle Eigenwerte von $\boldsymbol{A}-\boldsymbol{L}\left(\boldsymbol{q}_{i, j}\right) \boldsymbol{C}$. Demnach müssen auch eventuell zu kompensierende invariante Nullstellen des Systems die Anforderung erfüllen.

3) Diese Anforderung lässt sich zum Beispiel im Solver Hifoo [91] in einfacher Art und Weise berücksichtigen, da der Solver für die multikriterielle Optimierung von Streckenensembles ausgelegt ist. 


\section{Gezielte Optimierung der Störunterdrückung in vorgegebenen Frequenzbereichen}

Oftmals ist bekannt, welche Frequenzanteile Störsignale enthalten. Dann kann die Optimierung der $\mathcal{H}_{\infty}$-Norm von $\boldsymbol{G}_{\boldsymbol{r} \boldsymbol{d}}(s)$ über alle Frequenzen zu konservativen Ergebnissen führen. Die Standard-Solver lassen sich bei Verwendung von den in Abschnitt 2.5.1 diskutierten Gewichtungsfiltern weiterhin einsetzen. Dazu wird nicht die Auswirkung der Störungen $\boldsymbol{d}$ auf die Residuen untersucht, sondern der Einfluss virtueller, gefilterter Störungen

$$
\tilde{\boldsymbol{d}}(s)=\boldsymbol{G}_{\mathrm{filt}}(s) \cdot \boldsymbol{d}(s)=\operatorname{diag}\left(g_{\mathrm{filt}, 1}(s), \ldots, g_{\mathrm{filt}, n_{d}}(s)\right) \cdot \boldsymbol{d}(s) .
$$

Dabei kann für jede Störung ein eigener Frequenzbereich vorgegeben werden, indem jeweils eine separate Gewichtungsübertragungsfunktion $g_{\text {filt }, i}(s)$ angesetzt wird. Typischerweise sind die Gewichtungsfunktionen Tief-, Band- oder Hochpässe. Die Filterung der Störungen kann im Zustandsraum geschrieben werden als

$$
\begin{array}{r}
\dot{\boldsymbol{x}}_{f}=\boldsymbol{A}_{f} \boldsymbol{x}_{f}+\boldsymbol{B}_{f} \boldsymbol{d}, \\
\tilde{\boldsymbol{d}}=\boldsymbol{C}_{f} \boldsymbol{x}_{f}+\boldsymbol{D}_{f} \boldsymbol{d} .
\end{array}
$$

Schlägt man die Filterung der verallgemeinerten Strecke zu, so gilt

Satz 5.2 (Entwurf robuster FIOs in beschränkten Frequenzbereichen durch Lösung eines strukturbeschränkten Standard-Regelungsproblems). Das Optimierungsproblem

$$
\begin{aligned}
& \underset{\boldsymbol{q}_{i, j}, \boldsymbol{z}_{i, 0}}{\operatorname{minimiere}} \gamma, \text { sodass } \\
& \left\|\boldsymbol{G}_{\boldsymbol{r} \boldsymbol{d}} \cdot \boldsymbol{G}_{f i t}(s)\right\|_{\infty}<\gamma \\
& \boldsymbol{G}_{\boldsymbol{r} \boldsymbol{f}}(s)=\operatorname{diag}\left(g_{1,1}(s), \ldots, g_{n_{f}, n_{f}}(s)\right), g_{i, i}(s=0)=k_{s, i} \forall i=1, \ldots, n_{f}
\end{aligned}
$$

lässt sich äquivalent formulieren als strukturbeschränktes StandardRegelungsproblem der Form (5.5) mit

$$
\begin{aligned}
& \tilde{\boldsymbol{A}}=\left[\begin{array}{cc}
\boldsymbol{A}-\boldsymbol{M}_{\alpha} \boldsymbol{D}^{*-1} \boldsymbol{C} & \boldsymbol{B}_{d} \boldsymbol{C}_{f}-\boldsymbol{M}_{\alpha} \boldsymbol{D}^{*-1} \boldsymbol{D}_{d} \boldsymbol{C}_{f} \\
\mathbf{0} & \boldsymbol{A}_{f}
\end{array}\right],
\end{aligned}
$$

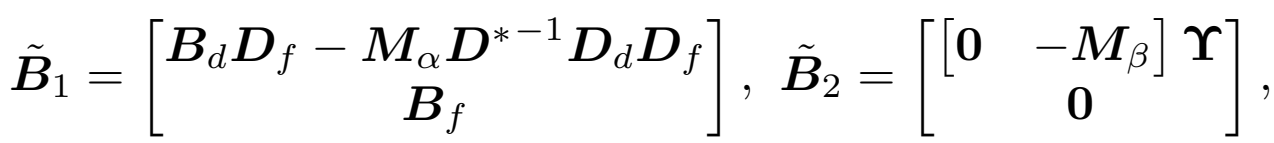

$$
\begin{aligned}
& \tilde{C}_{1}=\mathbf{0}, \tilde{\boldsymbol{D}}_{11}=\mathbf{0}, \quad \tilde{\boldsymbol{D}}_{12}=\left[\begin{array}{ll}
\boldsymbol{I}_{n_{f}} & \mathbf{0}
\end{array}\right] \mathbf{\Upsilon}, \\
& \tilde{\boldsymbol{C}}_{2}=\left[\begin{array}{ll}
\boldsymbol{D}^{*-1} \boldsymbol{C} & \boldsymbol{D}^{*-1} \boldsymbol{D}_{d} \boldsymbol{C}_{f}
\end{array}\right], \tilde{\boldsymbol{D}}_{21}=\boldsymbol{D}^{*-1} \boldsymbol{D}_{d} \boldsymbol{D}_{f}, \tilde{\boldsymbol{D}}_{22}=\mathbf{0}
\end{aligned}
$$


und dem verallgemeinerten Regler

$$
\tilde{\boldsymbol{K}}=\boldsymbol{Q}_{L}\left(\boldsymbol{q}_{i, j}\right) .
$$

Beweis. Setzt man die Parametrierung der verallgemeinerten Strecke und des verallgemeinerten Reglers ein, so ergibt sich

$$
\begin{aligned}
\dot{\boldsymbol{\xi}} & =(\boldsymbol{A}-\boldsymbol{L} \boldsymbol{C}) \boldsymbol{\xi}+\left(\boldsymbol{B}_{d}-\boldsymbol{L} \boldsymbol{D}_{d}\right) \tilde{\boldsymbol{d}}, \\
\dot{\boldsymbol{x}}_{f} & =\boldsymbol{A}_{f} \boldsymbol{x}_{f}+\boldsymbol{B}_{f} \boldsymbol{d}, \\
\tilde{\boldsymbol{d}} & =\boldsymbol{C}_{f} \boldsymbol{x}_{f}+\boldsymbol{D}_{f} \boldsymbol{d} \\
\boldsymbol{r} & =\boldsymbol{z}=\boldsymbol{V} \boldsymbol{C} \boldsymbol{\xi}+\boldsymbol{V} \boldsymbol{D}_{d} \tilde{\boldsymbol{d}} .
\end{aligned}
$$

Das Ein-/Ausgangsverhalten ist also äquivalent zu einer mit den gefilterten Störungen beaufschlagten Strecke.

Als Vorteil ist die Anwendbarkeit der Standard-Solver herauszuheben. Nachteilig ist jedoch, dass die Gewichtungsfilter separat entworfen werden müssen und darüber hinaus für den Entwurf die Systemordnung erhöhen. In Abschnitt 5.4.3 wird für nichtquadratische Systeme ein Verfahren vorgestellt, was eine Optimierung ohne Gewichtungsfilter ermöglicht.

\section{Beispiel CE 150 Modellhubschrauber}

In Abschnitt 4.5.3 wurden für den Entwurf eines FIOs für den CE 150 Modellhubschrauber die Beobachtereigenwerte $\lambda_{B_{1,1}}=-4, \lambda_{B_{1,2}}=-5$ und $\lambda_{B_{1,3}}=-6$ und für den zweiten Kanal $\lambda_{B_{2,1}}=-4,5, \lambda_{B_{2,2}}=-5,5$ und $\lambda_{B_{2,3}}=-6,5$ vorgegeben. Werden nun die Ausgangsgrößen $\boldsymbol{y}$ zusätzlich mit Störsignalen überlagert, so kann es zu Problemen kommen, da sich die Störungen auf die erzeugten Residuen auswirken. Bei Störeingangsmatrizen

$$
\boldsymbol{B}_{d}=\mathbf{0}, \quad \boldsymbol{D}_{d}=\left[\begin{array}{cc}
1 & 0 \\
0 & 0,1
\end{array}\right]
$$

zeigt Abbildung 5.4 den Verlauf des größten Singulärwertes von $\boldsymbol{G}_{\boldsymbol{r} \boldsymbol{d}}(s)$. Offensichtlich werden insbesondere hochfrequente Störungen verstärkt. Mittels des vorgestellten Verfahrens sollen nun die Beobachtereigenwerte derart angepasst werden, dass Störungen mit Frequenzen $\omega \geq \omega_{l}=3 \mathrm{rad} / \mathrm{s}$ möglichst gut unterdrückt werden und die Realteile aller Beobachtereigenwerte kleiner als $\alpha=-2,5$ sind. Zur Gewichtung der Störungen wird jeweils ein Hochpass

$$
g_{\mathrm{filt}, i}(s)=\frac{s}{s+\omega_{l}}
$$




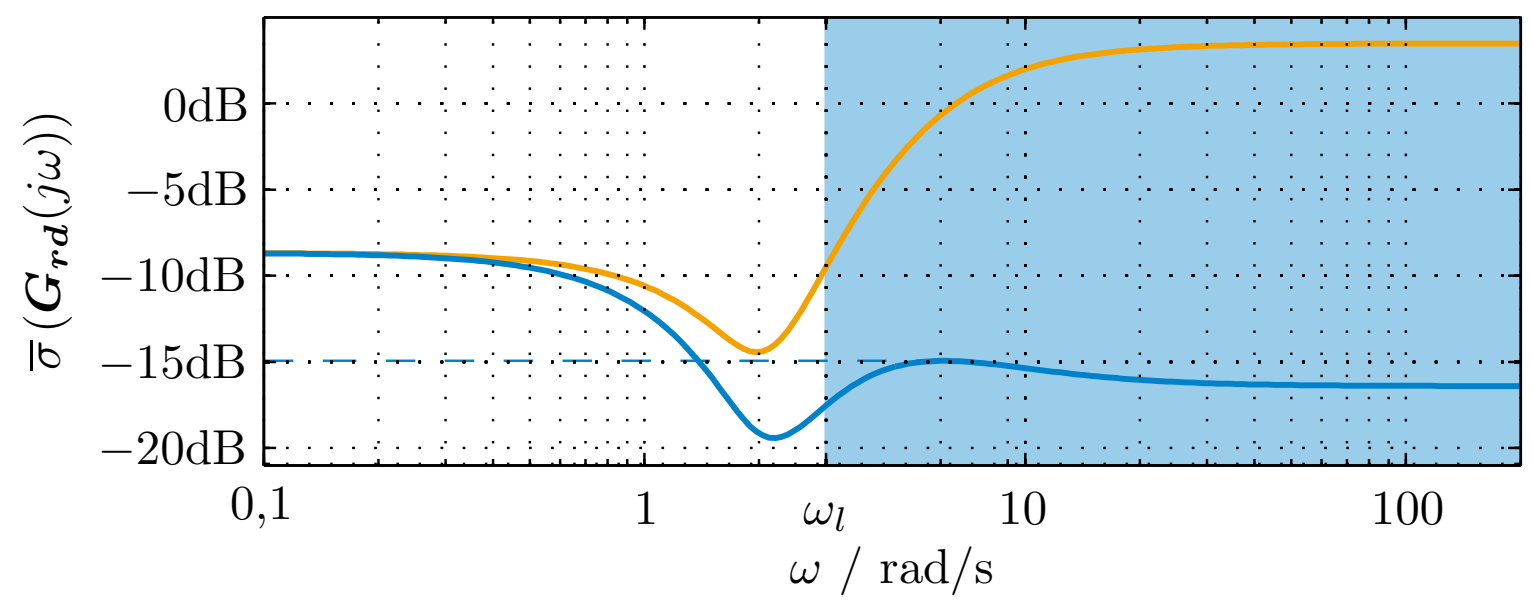

Abbildung 5.4: Verlauf des größten Singulärwertes von $\boldsymbol{G}_{\boldsymbol{r} \boldsymbol{d}}(s)$ für initiale (-) sowie hinsichtlich der Beobachtereigenwerte optimierte (-) FIO-Parametrierung

angesetzt. Im Zustandsraum lässt sich die Filterung der Störungen somit beschreiben durch

$$
\begin{array}{ll}
\boldsymbol{A}_{f}=\left[\begin{array}{cc}
-3 & 0 \\
0 & -3
\end{array}\right], & \boldsymbol{B}_{f}=\left[\begin{array}{ll}
2 & 0 \\
0 & 2
\end{array}\right], \\
\boldsymbol{C}_{f}=\left[\begin{array}{cc}
-1,5 & 0 \\
0 & -1,5
\end{array}\right], & \boldsymbol{D}_{f}=\left[\begin{array}{ll}
1 & 0 \\
0 & 1
\end{array}\right] .
\end{array}
$$

Ausgehend von der FIO-Parametrierung aus Abschnitt 4.5.3 liefert der Entwurf nach Satz 5.2 die in Anhang C.2.1 angegebene Parametrierung $\left(\boldsymbol{L}_{\text {opt,ew }}, \boldsymbol{V}_{\text {opt,ew }}\right)$. Sie liefert eine Schranke von $\gamma=0,179$ und platziert die Beobachtereigenwerte bei

$$
\begin{aligned}
& \lambda_{B_{1,1}}=-2,502, \quad \lambda_{B_{1,2 / 3}}=-2,50 \pm 0,131 j, \\
& \lambda_{B_{2,1}}=-2,505, \quad \lambda_{B_{2,2 / 3}}=-2,50 \pm 0,025 j .
\end{aligned}
$$

Dieses Ergebnis ist insofern einleuchtend, als dass die Eigenwerte so wenig wie möglich nach links verschoben werden. Dies führt zu betragsmäßig kleinen Einträgen in $\boldsymbol{L}$ und $\boldsymbol{V}$, die eine geringere Verstärkung hochfrequenter Störungen bedingen. Diese Tatsache ist auch dem Singulärwertverlauf in Abbildung $5.4 \mathrm{zu}$ entnehmen.

\subsubsection{Optimierung durch Relaxierung der strukturellen Anforderungen}

Wie im vorangegangenen Abschnitt erörtert müssen zur vollständigen Diagonalisierung von $\boldsymbol{G}_{\boldsymbol{r} \boldsymbol{f}}(s)$ bei gleichzeitiger Festlegung der Diagonalele- 
mente alle Entwurfsfreiheitsgrade herangezogen werden. Nimmt man die Dynamik der Diagonalelemente als fest an, so verbleiben dementsprechend keine Freiheitsgrade zur Steigerung der Robustheit.

Oftmals ist jedoch eine exakte Diagonalstruktur von $\boldsymbol{G}_{\boldsymbol{r} \boldsymbol{f}}(s)$ nicht unbedingt notwendig. Ausreichend ist es, wenn sich ein bestimmter Fehler deutlich stärker auf ein ihm zugeordnetes Residuum auswirkt als auf alle übrigen. Anschaulich gesprochen sollten also die Diagonalelemente von $\boldsymbol{G}_{\boldsymbol{r} \boldsymbol{f}}(s)$ groß gegenüber den Nebendiagonalelementen sein. Als ein Maß für die Größe der Elemente der Übertragungsmatrix kann entweder die statische Verstärkung oder aber die $\mathcal{H}_{\infty}$-Norm herangezogen werden, beide Varianten werden im Folgenden erörtert. Die im Vergleich zur exakten Diagonalisierung von $\boldsymbol{G}_{\boldsymbol{r} \boldsymbol{f}}(s)$ durch diese strukturelle Relaxierung gewonnenen Freiheitsgrade werden in diesem Abschnitt zur Steigerung der Robustheit bezüglich exogener Störungen herangezogen. Die präsentierten Ergebnisse wurden in Auszügen bereits in [227] veröffentlicht. Sie basieren auf der Vollständigen Modalen Synthese und sind ein Beispiel für die Flexibilität der Methode.

Aus Abschnitt 4.5.2 ist bekannt, dass für eine exakte Diagonalisierung von $\boldsymbol{G}_{\boldsymbol{r} \boldsymbol{f}}(s)$ die Bedingungen

$$
\begin{aligned}
\boldsymbol{w}_{B_{i j}}^{\top}\left(\boldsymbol{E}_{a}-\boldsymbol{L} \boldsymbol{E}_{s}\right) & =\boldsymbol{\phi}_{i}^{\top}, i=1, \ldots, n_{f}, j=1, \ldots, \delta_{i}, \\
\boldsymbol{w}_{B_{k}}^{\top}\left(\boldsymbol{E}_{a}-\boldsymbol{L} \boldsymbol{E}_{s}\right) & =\mathbf{0}^{\top}, k=\delta+1, \ldots, n, \\
-\boldsymbol{V} \boldsymbol{\Theta} & =\operatorname{diag}\left(\tilde{z}_{1}, \ldots, \tilde{z}_{n_{f}}\right)=\boldsymbol{S}
\end{aligned}
$$

mit der Matrix $\boldsymbol{\Theta}=\boldsymbol{C}(\boldsymbol{A}-\boldsymbol{L} \boldsymbol{C})^{-1}\left(\boldsymbol{E}_{a}-\boldsymbol{L} \boldsymbol{E}_{s}\right)-\boldsymbol{E}_{s}$ erfüllt sein müssen. Die Gleichung (5.10a) stellt die Zuordnung der steuerbaren Beobachtereigenwerte zur jeweiligen Spalte von $\boldsymbol{G}_{\boldsymbol{r} \boldsymbol{f}}(s)$ sicher, während (5.10b) dafür sorgt, dass die entsprechenden Beobachtereigenwerte $\lambda_{B_{k}}$ nichtsteuerbar gemacht werden. Die Beziehung (5.10c) stellt schließlich die Diagonalstruktur von $\boldsymbol{G}_{\boldsymbol{r} \boldsymbol{f}}(s)$ für alle $s \in \mathbb{C}$ sicher. Somit sind in quadratischen Systemen bei Vorgabe der Eigenwerte und der stationären Verstärkungen der Diagonalelemente die Beobachtermatrizen $\boldsymbol{L}$ und $\boldsymbol{V}$ eindeutig festgelegt.

Um Freiheitsgrade für die Robustheitsoptimierung zu gewinnen, ist nun die Grundidee, die Gleichungen (5.10) nicht exakt, sondern nur näherungsweise zu erfüllen. Zunächst wird bezüglich der Beobachtermatrix $\boldsymbol{L}$ erneut die strukturelle Anforderung (5.10a) betrachtet. In der relaxierten Form ergibt sie sich zu

$$
\left\|\boldsymbol{w}_{B_{i j}}^{\top}\left(\boldsymbol{E}_{a}-\boldsymbol{L} \boldsymbol{E}_{s}\right)-\boldsymbol{\phi}_{i}^{\top}\right\|_{2}<\beta_{\boldsymbol{L}, i j}, i=1, \ldots, n_{f}, j=1, \ldots, \delta_{i} .
$$


Dabei ist $\beta_{\boldsymbol{L}, i j}$ ein positiver Skalar, welcher die zulässige Abweichung von der für eine ideale Diagonalisierung von $\boldsymbol{G}_{\boldsymbol{r} \boldsymbol{f}}(s)$ zu erfüllenden Anforderungen beschreibt. Je größer diese Schranke gewählt wird, desto größer ist die Freiheit in der Wahl des entsprechenden Parameter- beziehungsweise Linkseigenvektors. Gleichzeitig wird der entsprechende Eigenwert jedoch umso stärker in andere Spalten von $\boldsymbol{G}_{\boldsymbol{r} \boldsymbol{f}}(s)$ eingekoppelt. Analog zur Übertragungsfunktion $\boldsymbol{G}_{\boldsymbol{r} \boldsymbol{f}}(s)$ in (4.15) auf Seite 86 wird die Wirkung der Störungen auf die generierten Residuen anhand der Partialbruchzerlegung von $\boldsymbol{G}_{\boldsymbol{r} \boldsymbol{d}}(s)$ betrachtet. Sie lautet

$$
\boldsymbol{G}_{\boldsymbol{r} \boldsymbol{d}}(s)=\sum_{k=1}^{n} \frac{\boldsymbol{V} \boldsymbol{C} \boldsymbol{v}_{B_{k}} \cdot \boldsymbol{w}_{B_{k}}^{\top}\left(\boldsymbol{B}_{d}-\boldsymbol{L} \boldsymbol{D}_{d}\right)}{s-\lambda_{B_{k}}}+\boldsymbol{V} \boldsymbol{D}_{d}
$$

Um eingangsseitig den Effekt der Störungen möglichst gering zu halten, ist in (5.12) jeweils $\left\|\boldsymbol{w}_{B_{i j}}^{\top}\left(\boldsymbol{B}_{d}-\boldsymbol{L} \boldsymbol{D}_{d}\right)\right\|_{2}$ zu minimieren. Berücksichtigt man weiterhin die Gleichungsbedingung $\boldsymbol{w}_{B_{i j}}^{\top}\left(\lambda_{B_{i j}} \boldsymbol{I}_{n}-\boldsymbol{A}\right)=\boldsymbol{p}_{i j}^{\top} \boldsymbol{C}$, welche Parameter- und Linkseigenvektoren verknüpft, ergibt sich unter Rückgriff auf Lemma 3.5 für die steuerbaren Eigenwerte $\lambda_{B_{i j}}$ das LMI-Problem

$$
\begin{aligned}
& \underset{\boldsymbol{p}_{i j}^{\top}, \boldsymbol{w}_{B_{i j}^{\top}}^{\top}}{\operatorname{minimiere}} \gamma_{\boldsymbol{L}, i j}, \text { sodass } \\
& \boldsymbol{w}_{B_{i j}}^{\top}\left(\lambda_{B_{i j}} \boldsymbol{I}_{n}-\boldsymbol{A}\right)-\boldsymbol{p}_{i j}^{\top} \boldsymbol{C}=\mathbf{0}^{\top}, \\
& {\left[\begin{array}{cc}
\beta_{\boldsymbol{L}, i j} & \boldsymbol{w}_{B_{i j}}^{\top} \boldsymbol{E}_{a}+\boldsymbol{p}_{i j}^{\top} \boldsymbol{E}_{s}-\boldsymbol{\phi}_{i}^{\top} \\
\star & \boldsymbol{I}_{n_{f}}
\end{array}\right] \succ \mathbf{0},} \\
& {\left[\begin{array}{cc}
\gamma_{\boldsymbol{L}, i j} & \boldsymbol{w}_{B_{i j}}^{\top} \boldsymbol{B}_{d}+\boldsymbol{p}_{i j}^{\top} \boldsymbol{D}_{d} \\
\star & \boldsymbol{I}_{n_{d}}
\end{array}\right] \succ \mathbf{0 .}}
\end{aligned}
$$

Dabei wird durch die LMI (5.13c) die Einhaltung von (5.11) gewährleistet, während (5.13d) die Beschränkung $\left\|\boldsymbol{w}_{B_{i j}}^{\top}\left(\boldsymbol{B}_{d}-\boldsymbol{L} \boldsymbol{D}_{d}\right)\right\|_{2}<\gamma_{\boldsymbol{L}, i j}$ impliziert. Offensichtlich hat die Optimierung umso mehr Spielraum für die Minimierung von $\gamma_{\boldsymbol{L}, i j}$, je größer $\beta_{\boldsymbol{L}, i j}$ gewählt wird. Für $\beta_{\boldsymbol{L}, i j} \rightarrow 0$ entartet das Optimierungsproblem (5.13) zur exakten Lösung von (5.10a).

Für die nichtsteuerbaren Beobachtereigenwerte $\lambda_{B_{k}}$ ist das Vorgehen prinzipiell gleich. Anstelle von (5.10b) ist die Gleichung

$$
\left\|\boldsymbol{w}_{B_{k}}^{\top}\left(\boldsymbol{E}_{a}-\boldsymbol{L} \boldsymbol{E}_{s}\right)\right\|_{2}<\beta_{\boldsymbol{L}, k}, k=\delta+1, \ldots, n
$$

für eine gegebene Schranke $\beta_{\boldsymbol{L}, k}$ zu erfüllen. Innerhalb der dadurch beschriebenen Menge der Parameter- und Linkseigenvektoren werden diejenigen Elemente gesucht, welche die Anregung durch die Störungen minimieren. Dabei ist zu beachten, dass zusätzlich $\boldsymbol{p}_{k}^{\top} \neq \mathbf{0}^{\top}$ gelten muss, da 
andernfalls die Lösung des Optimierungsproblems zu $\gamma_{\boldsymbol{L}, k}=0, \boldsymbol{p}_{k}^{\top}=\mathbf{0}^{\top}$ und $\boldsymbol{w}_{B, k}^{\top}=\mathbf{0}^{\top}$ degenerieren würde. Dementsprechend würde sich eine singuläre Linkseigenvektormatrix ergeben. Aufgrund der Skalierungsinvarianz der Parametervektoren kann äquivalent auch die Bedingung $\left\|\boldsymbol{p}_{k}^{\top}\right\|_{2} \geq 1$ herangezogen werden. Zusammengefasst ergibt sich für die nichtsteuerbaren Eigenwerte das Optimierungsproblem

$$
\begin{aligned}
& \underset{\boldsymbol{p}_{k}^{\top}, \boldsymbol{w}_{B_{k}}^{\top}}{\operatorname{minimiere}} \gamma_{\boldsymbol{L}, k}, \text { sodass } \\
& \boldsymbol{w}_{B_{k}}^{\top}\left(\lambda_{B_{k}} \boldsymbol{I}_{n}-\boldsymbol{A}\right)-\boldsymbol{p}_{k}^{\top} \boldsymbol{C}=\mathbf{0}^{\boldsymbol{\top}}, \\
& {\left[\begin{array}{cc}
\beta_{\boldsymbol{L}, k} & \boldsymbol{w}_{B_{k}}^{\top} \boldsymbol{E}_{a}+\boldsymbol{p}_{k}^{\top} \boldsymbol{E}_{s} \\
\star & \boldsymbol{I}_{n_{f}}
\end{array}\right] \succ \mathbf{0},} \\
& {\left[\begin{array}{cc}
\gamma_{\boldsymbol{L}, k} & \boldsymbol{w}_{B_{k}}^{\top} \boldsymbol{B}_{d}+\boldsymbol{p}_{k}^{\top} \boldsymbol{D}_{d} \\
\star & \boldsymbol{I}_{n_{d}}
\end{array}\right] \succ \mathbf{0},} \\
& \left\|\boldsymbol{p}_{k}^{\top}\right\|_{2} \geq 1 .
\end{aligned}
$$

Wegen der Normbeschränkung (5.14e) handelt es sich bei (5.14) im Gegensatz zu (5.13) um ein nichtkonvexes Optimierungsproblem. Neben der Anwendung spezieller Lösungsverfahren (s. zum Beispiel [73]) bietet sich hierfür eine konvexe Approximation an. Das durch $\left\|\boldsymbol{p}_{k}^{\top}\right\|_{2} \geq 1$ beschriebene nichtkonvexe Gebiet wird dazu durch mehrere konvexe Gebiete angenähert. Für ein System mit $n_{y}=n_{f}=2$ Ausgängen beziehungsweise Fehlern ist eine einfache Approximation in Abbildung 5.5a skizziert. Es

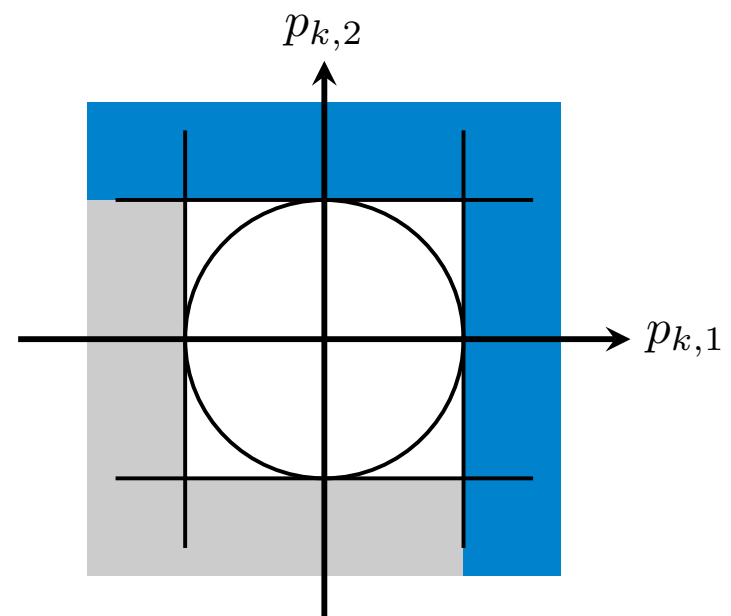

(a) Approximation mit vier Tangenten

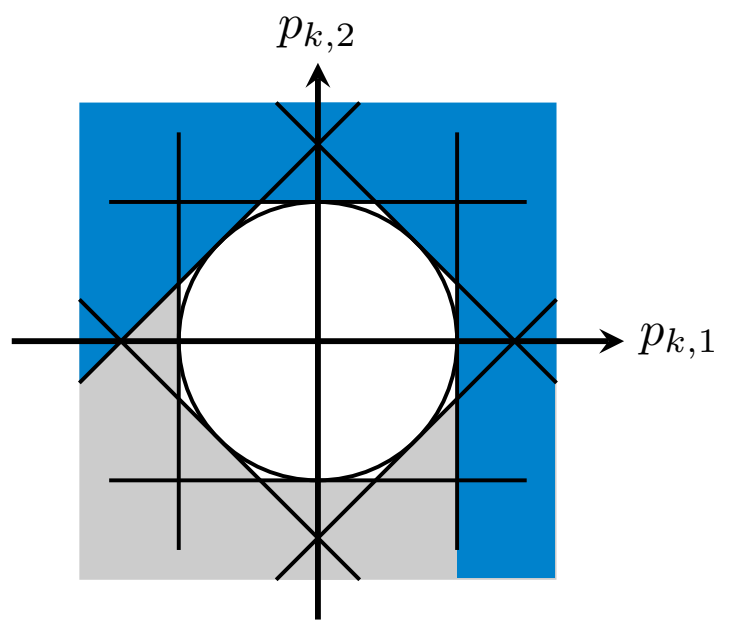

(b) Approximation mit acht Tangenten

Abbildung 5.5: Äußere Approximation einer Hyperkugel durch Tangenten 
werden jeweils Tangenten an den durch $\left\|\boldsymbol{p}_{k}^{\top}\right\|_{2}=1$ definierten Kreis gelegt. In der Abbildung ist das dadurch beschriebene Gebiet in grau beziehungsweise blau dargestellt, es nähert das Äußere des Kreises an.

Bezeichnet man mit $p_{k, l}$ die Komponenten des $k$-ten Parametervektors, so ist es aufgrund der Skalierungsinvarianz der Parametervektoren ausreichend, jeweils Tangenten mit $p_{k, l} \geq 1$ zu betrachten. Die zu untersuchenden Bereiche sind in Abbildung 5.5a in blau eingefärbt.

Für jede der Tangenten ergibt sich ein LMI-Problem der Form

$$
\begin{aligned}
& \underset{\boldsymbol{p}_{k}^{\top}, \boldsymbol{w}_{B_{k}}^{\top}}{\operatorname{minimiere}} \gamma_{\boldsymbol{L}, k}, \text { sodass } \\
& \boldsymbol{w}_{B_{k}}^{\top}\left(\lambda_{B_{k}} \boldsymbol{I}_{n}-\boldsymbol{A}\right)-\boldsymbol{p}_{k}^{\top} \boldsymbol{C}=\mathbf{0}^{\boldsymbol{\top}}, \\
& {\left[\begin{array}{cc}
\beta_{\boldsymbol{L}, k} & \boldsymbol{w}_{B_{k}}^{\top} \boldsymbol{E}_{a}+\boldsymbol{p}_{k}^{\top} \boldsymbol{E}_{s} \\
\star & \boldsymbol{I}_{n_{f}}
\end{array}\right] \succ \mathbf{0},} \\
& {\left[\begin{array}{cc}
\gamma_{\boldsymbol{L}, k} & \boldsymbol{w}_{B_{k}}^{\top} \boldsymbol{B}_{d}+\boldsymbol{p}_{k}^{\top} \boldsymbol{D}_{d} \\
\star & \boldsymbol{I}_{n_{d}}
\end{array}\right] \succ \mathbf{0 ,}} \\
& p_{k, l} \geq 1 .
\end{aligned}
$$

Im gezeigten Beispiel sind also zwei LMI-Probleme der Gestalt (5.15) zu lösen. Anschließend wird unter den Parametrierungen $\left(\boldsymbol{p}_{k}^{\top}, \boldsymbol{w}_{B, k}^{\top}\right)$, welche eine gültige Lösung für (5.15d) liefern, diejenige ausgewählt, welche die kleinste Schranke $\gamma_{\boldsymbol{L}, k}$ liefert. Die Konservativität der Approximation kann wie in Abbildung 5.5b gezeigt durch weitere Tangenten an die durch $\left\|\boldsymbol{p}_{k}^{\top}\right\|_{2} \geq 1$ gegebene Hyperkugel verbessert werden. Dadurch steigt jedoch auch die Anzahl der zu lösenden LMI-Probleme.

Nachdem durch Lösung der Optimierungsprobleme (5.13) und (5.14) beziehungsweise (5.15) alle Parameter- und Linkseigenvektoren bestimmt wurden, ergibt sich wie in Lemma 4.1 die Beobachtermatrix zu $\boldsymbol{L}=-\boldsymbol{W}_{B}^{-1} \boldsymbol{P}$. Zur Bestimmung der Nachfiltermatrix $\boldsymbol{V}$ ist bei Relaxierung der Strukturanforderungen die Gleichung (5.10c) nicht mehr exakt zu erfüllen, stattdessen wird

$$
\|-\boldsymbol{V} \boldsymbol{\Theta}-\boldsymbol{S}\|_{2}<\beta_{\boldsymbol{V}}
$$

betrachtet. Unter dieser Bedingung kann dann mithilfe des Bounded-RealLemmas (Satz 3.1) die $\mathcal{H}_{\infty}$-Norm von $\boldsymbol{G}_{\boldsymbol{r} \boldsymbol{d}}(s)$ minimiert werden, um eine möglichst gute Störunterdrückung zu gewährleisten. Es ergibt sich das 
LMI-Problem

$$
\begin{aligned}
& \underset{\boldsymbol{X}, \boldsymbol{V}}{\operatorname{minimiere}} \gamma_{\boldsymbol{V}}, \text { sodass } \\
& {\left[\begin{array}{cc}
\beta_{\boldsymbol{V}} \boldsymbol{I}_{n_{f}} & -\boldsymbol{V} \boldsymbol{\Theta}-\boldsymbol{S} \\
\star & \boldsymbol{I}_{n_{f}}
\end{array}\right] \succ \mathbf{0},} \\
& \boldsymbol{X} \succ \mathbf{0}, \\
& {\left[\begin{array}{ccc}
\operatorname{He}(\boldsymbol{X}(\boldsymbol{A}-\boldsymbol{L} \boldsymbol{C})) & \boldsymbol{X}\left(\boldsymbol{B}_{d}-\boldsymbol{L} \boldsymbol{D}_{d}\right) & (\boldsymbol{V} \boldsymbol{C})^{\top} \\
\star & -\gamma_{\boldsymbol{V}} \boldsymbol{I}_{n_{d}} & \left(\boldsymbol{V} \boldsymbol{D}_{d}\right)^{\top} \\
\star & \star & -\gamma_{\boldsymbol{V}} \boldsymbol{I}_{n_{f}}
\end{array}\right] \prec \mathbf{0 .}}
\end{aligned}
$$

Durch (5.16b) wird die Abweichung von $-\boldsymbol{V} \boldsymbol{\Theta}$ von der Diagonalmatrix $\boldsymbol{S}$ beschränkt, während (5.16c) und (5.16d) die $\mathcal{H}_{\infty}$-Norm von $\boldsymbol{G}_{\boldsymbol{r} \boldsymbol{d}}(s)$ beschränken. Es zeigt sich jedoch, dass in obigem Optimierungsproblem die Lösung dazu tendiert, die stationäre Verstärkung von $\boldsymbol{G}_{\boldsymbol{r} \boldsymbol{f}}(s)$ gegenüber der exakten Fehlerisolation zu verringern. Dabei nimmt zwar auch $\left\|\boldsymbol{G}_{\boldsymbol{r} \boldsymbol{d}}(s)\right\|_{\infty}$ ab, das Verhältnis zwischen Störempfindlichkeit und Fehlersensitivität bleibt jedoch weitgehend gleich. Eine einfache Modifikation von (5.16) ermöglicht diesbezüglich wesentlich bessere Ergebnisse. Dabei wird die Nebenbedingung (5.16b) aufgeteilt in mehrere Bedingungen. Für die Diagonalelemente wird eine geringe Abweichung $\beta_{\boldsymbol{V}}$ von der vorgegebenen stationären Verstärkung gefordert. Für die Nichtdiagonalelemente gilt eine modifizierte Schranke $\beta_{\boldsymbol{V}}^{*}>\beta_{\boldsymbol{V}}$. Das entsprechende LMI-Problem lautet

$$
\begin{aligned}
& \underset{\boldsymbol{V}, \boldsymbol{X}}{\operatorname{minimiere}} \gamma_{\boldsymbol{V}} \text {, sodass }
\end{aligned}
$$

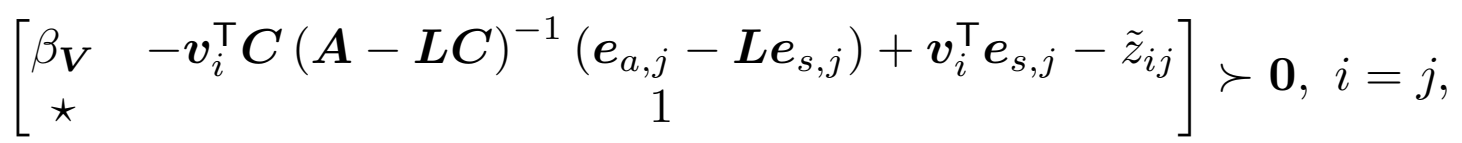

$$
\begin{aligned}
& {\left[\begin{array}{cc}
\beta_{\boldsymbol{V}}^{*} & -\boldsymbol{v}_{i}^{\top} \boldsymbol{C}(\boldsymbol{A}-\boldsymbol{L} \boldsymbol{C})^{-1}\left(\boldsymbol{e}_{a, j}-\boldsymbol{L} \boldsymbol{e}_{s, j}\right)+\boldsymbol{v}_{i}^{\top} \boldsymbol{e}_{s, j} \\
\star & 1
\end{array}\right] \succ \mathbf{0}, i \neq j,} \\
& \boldsymbol{X} \succ \mathbf{0}, \\
& {\left[\begin{array}{ccc}
\operatorname{He}(\boldsymbol{X}(\boldsymbol{A}-\boldsymbol{L} \boldsymbol{C})) & \boldsymbol{X}\left(\boldsymbol{B}_{d}-\boldsymbol{L} \boldsymbol{D}_{d}\right) & \left(\boldsymbol{V}^{\top} \boldsymbol{C}\right)^{\top} \\
\star & -\gamma_{\boldsymbol{V}} \boldsymbol{I}_{n_{d}} & \left(\boldsymbol{V} \boldsymbol{D}_{d}\right)^{\top} \\
\star & \star & -\gamma_{\boldsymbol{V}} \boldsymbol{I}_{n_{f}}
\end{array}\right] \prec \mathbf{0},}
\end{aligned}
$$

wobei jeweils $i=1, \ldots, n_{f}$ und $j=1, \ldots, n_{f}$ gilt. Durch die Bedingungen (5.17b) beziehungsweise (5.17c) wird sichergestellt, dass sich die stati- 
onären Verstärkungen in $\boldsymbol{G}_{\boldsymbol{r} \boldsymbol{f}}(s)$ nur in einem durch $\beta_{\boldsymbol{V}}$ beziehungsweise $\beta_{V}^{*}$ definierten Maß vom Fall der exakten Fehlerisolation unterscheiden.

Wie zu Beginn des Abschnittes erwähnt, kann zur Quantifizierung der Größe der Nichtdiagonalelemente von $\boldsymbol{G}_{\boldsymbol{r} \boldsymbol{f}}(s)$ alternativ auch die $\mathcal{H}_{\infty^{-}}$ Norm herangezogen werden. In diesem Fall ist (5.17c) zu ersetzen durch

$$
\begin{aligned}
& \boldsymbol{X}_{i j} \succ \mathbf{0}, i \neq j, \\
& {\left[\begin{array}{ccc}
\operatorname{He}\left(\boldsymbol{X}_{i j}(\boldsymbol{A}-\boldsymbol{L} \boldsymbol{C})\right) & \boldsymbol{X}_{i j}\left(\boldsymbol{e}_{a, j}-\boldsymbol{L} \boldsymbol{e}_{s, j}\right) & \left(\boldsymbol{v}_{i}^{\top} \boldsymbol{C}\right)^{\top} \\
\star & -\beta_{\boldsymbol{V}}^{*} & \left(\boldsymbol{v}_{i}^{\top} \boldsymbol{E}_{s}\right)^{\top} \\
\star & \star & -\beta_{\boldsymbol{V}}^{*}
\end{array}\right] \prec \mathbf{0 .}}
\end{aligned}
$$

Im Gegensatz zu (5.17c) wird dadurch die Verstärkung von $g_{i, j}(s)$ über alle Frequenzen beschränkt. Dadurch können beispielsweise starkes Überoder Unterschwingen ausgeschlossen werden. Bemerkenswert ist, dass für jedes Nichtdiagonalelement eine eigene Matrix $\boldsymbol{X}_{i j}$ angesetzt werden kann, sodass die Lösung keine Konservativität erfährt. Dies ist dadurch zu begründen, dass die Matrix $\boldsymbol{L}$ bereits durch (5.13) und (5.14) beziehungsweise (5.15) eindeutig festgelegt ist.

Zusammengefasst erfolgt der Entwurf also wie bei der Vollständigen Modalen Synthese zur Parametrierung von FIOs in zwei Schritten:

1. Entwurf der Beobachtermatrix $\boldsymbol{L}$ durch Lösen von (5.13) und (5.14) beziehungsweise (5.15) und anschließender Berechnung von $\boldsymbol{L}=-\boldsymbol{W}_{B}^{-1} \boldsymbol{P}$.

2. Entwurf der Nachfiltermatrix $\boldsymbol{V}$ durch Lösen von (5.17) (ggf. mit (5.18)).

Bemerkung 5.2. Da die Matrizen $\boldsymbol{L}$ und $\boldsymbol{V}$ sequentiell optimiert werden, ist nicht garantiert, dass das globale Optimum hinsichtlich $\left\|\boldsymbol{G}_{\boldsymbol{r d}}(s)\right\|_{\infty}$ gefunden wird.

Bemerkung 5.3. Das Verfahren kann auch zur Steigerung der Robustheit bezüglich exogener Störungen in statisch nicht isolierbaren Systemen oder nicht minimalphasigen Systemen angewendet werden. Dazu wird zunächst wie in den Abschnitten 4.7 und 4.8 beschrieben das jeweilige System dynamisch erweitert. Beim Entwurf eines statischen Fehlerisolationsbeobachters für das erweiterte Gesamtsystem können dann die relaxierten Strukturanforderungen berücksichtigt werden. Die Realisierung des entsprechenden DFIOs für das ursprüngliche System ergibt sich unverändert gemäß Satz 4.5 beziehungsweise Satz 4.7 . 


\section{Beispiel Verladebrücke}

Zur Demonstration der Methodik wird auf das Beispiel der Verladebrücke zurückgegriffen. Wie in Abschnitt 4.10.3 wird ein Szenario betrachtet, in dem zum einen eine horizontale Kraft auf die Lastmasse wirken kann (Fehler $f_{1}$ ), zum anderen wird ein Aktorfehler $f_{2}$ in der auf die Laufkatze wirkenden Kraft betrachtet. Im Gegensatz zu Abschnitt 4.10.3 wird an dieser Stelle jedoch eine vollständige Fehlerisolation angestrebt.

Wie bereits diskutiert weist das System keine invarianten Nullstellen auf und es ist $\delta_{1}=\delta_{2}=2$. Für eine schnelle Erkennung des Aktorfehlers werden die Beobachtereigenwerte $\mathrm{zu} \lambda_{B_{1,1}}=-3, \lambda_{B_{1,2}}=-4$, $\lambda_{B_{2,1}}=-14$ und $\lambda_{B_{2,2}}=-15$ gewählt. Als stationäre Verstärkung wird jeweils $g_{i, i}(s=0)=1$ gefordert. Betrachtet werden Störungen, welche gemäß

$$
\boldsymbol{B}_{d}=\mathbf{0}, \quad \boldsymbol{D}_{d}=\left[\begin{array}{cc}
0,01 & 0 \\
0 & 0,05
\end{array}\right]
$$

die gemessenen Ausgangssignale überlagern. Die Positionsmessung unterliegt also größeren Störungen als die Winkelmessung. Entwirft man einen FIO $\left(\boldsymbol{L}_{\text {exakt }}, \boldsymbol{V}_{\text {exakt }}\right)$ zur exakten Fehlerisolation, so ergibt sich der in Abbildung 5.6 gezeigte Singulärwertverlauf von $\boldsymbol{G}_{\boldsymbol{r} \boldsymbol{d}}(s)$. Daraus geht hervor, dass insbesondere Frequenzen oberhalb $\omega=1 \mathrm{rad} / \mathrm{s}$ erheblich verstärkt werden. Die $\mathcal{H}_{\infty}$-Norm von $\boldsymbol{G}_{\boldsymbol{r} \boldsymbol{d}}(s)$ wird durch $\gamma_{\text {exakt }}=7,10$ begrenzt.

Relaxiert man die strukturellen Entwurfsanforderungen durch $\gamma_{L, i j}=0,1, \beta_{\boldsymbol{V}}=0,003$ und $\beta_{\boldsymbol{V}}^{*}=0,25$, so ergibt sich die in Anhang C.2.2 angegebene FIO-Parametrierung $\left(\boldsymbol{L}_{\text {relax }}, \boldsymbol{V}_{\text {relax }}\right)$, wobei zur Quantifizie-

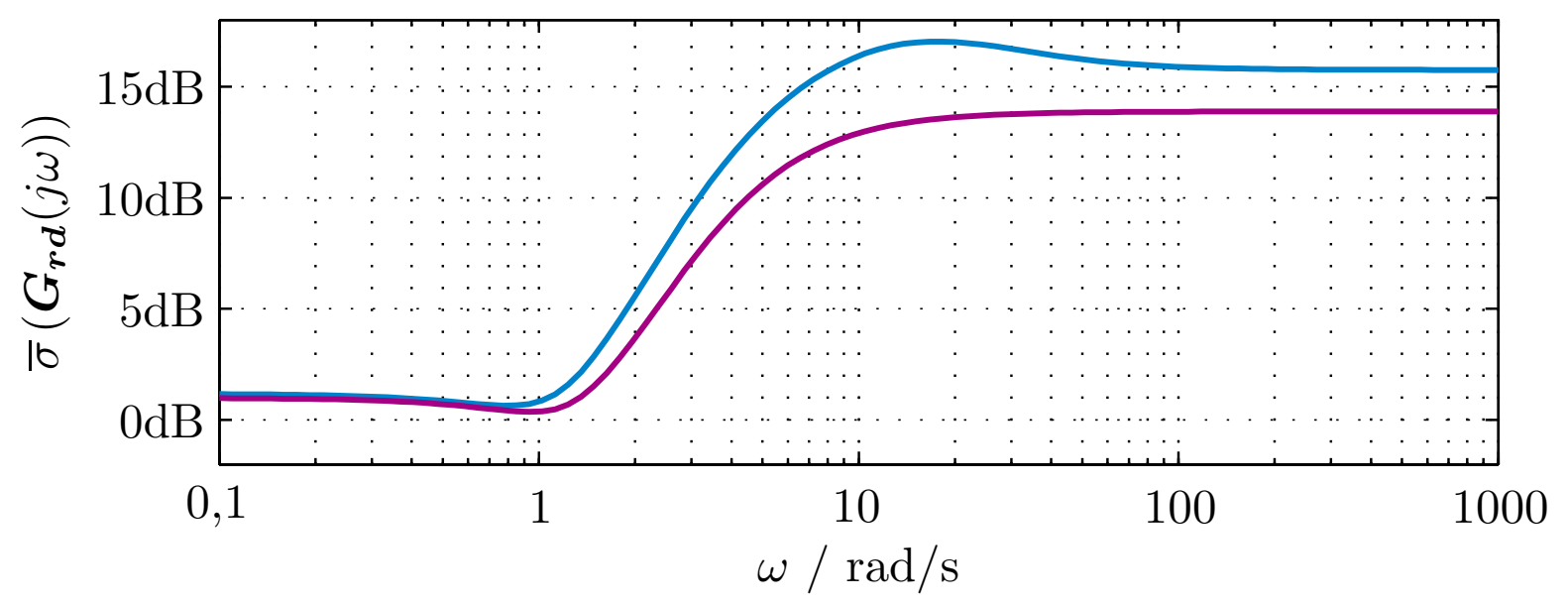

Abbildung 5.6: Verlauf des größten Singulärwertes von $\boldsymbol{G}_{\boldsymbol{r} \boldsymbol{d}}(s)$ für exakten FIO (-) sowie FIO mit relaxierten strukturellen Anforderungen $(-)$ 

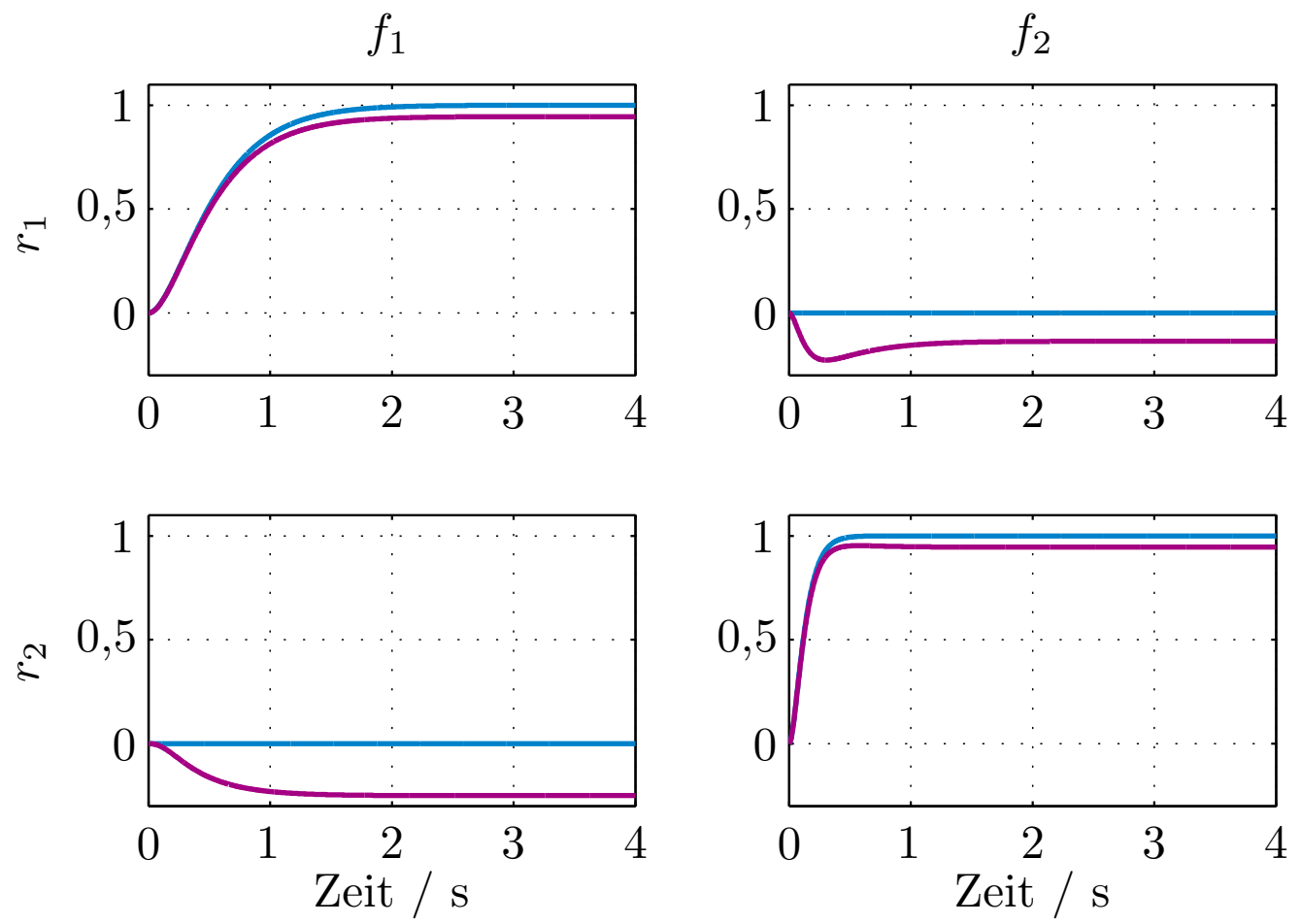

Abbildung 5.7: Sprungantwort von $\boldsymbol{G}_{\boldsymbol{r} \boldsymbol{f}}(s)$ des exakten FIOs (-) sowie des FIOs mit relaxierten strukturellen Anforderungen (-)

rung der Nebendiagonalelemente von $\boldsymbol{G}_{\boldsymbol{r} \boldsymbol{f}}(s)$ auf (5.18) zurückgegriffen wurde. Auffällig ist, dass die Elemente der ersten Spalte von $\boldsymbol{L}_{\text {relax }}$, die dem weniger gestörten Sensor zugeordnet sind, im Gegensatz zu $\boldsymbol{L}_{\text {exakt }}$ betragsmäßig größer sind als die der zweiten Spalte. Daraus ergibt sich eine verringerte Schranke für $\left\|\boldsymbol{G}_{\boldsymbol{r} \boldsymbol{d}}(s)\right\|_{\infty}$ von $\gamma_{\text {relax }}=4,94$, die auch in Abbildung 5.6 abzulesen ist.

In Abbildung 5.7 sind darüber hinaus die Sprungantworten von $\boldsymbol{G}_{\boldsymbol{r} \boldsymbol{f}}(s)$ dargestellt. Es ist deutlich zu erkennen, dass $\left(\boldsymbol{L}_{\text {relax }}, \boldsymbol{V}_{\text {relax }}\right)$ zwar keine exakte Diagonalstruktur liefert. Eine Fehlerisolation ist dennoch ohne Weiteres möglich, da sich die Fehler jeweils vorwiegend auf das ihnen zugeordnete Residuum auswirken.

\subsubsection{Unterdrückung hochfrequenter Störungen}

Häufig treten in Systemen hochfrequente Störungen auf, die insbesondere durch Messrauschen hervorgerufen werden. Zwar ist es stets möglich, diese Tatsache beim Entwurf von FIOs zunächst unberücksichtigt zu lassen und die generierten Residuen anhand von Tiefpässen zu filtern. Dieses Vorgehen weist jedoch wie in Abschnitt 4.6 angedeutet verschiedene 
Nachteile auf. Zum einen müssen die Tiefpässe separat entworfen werden, zum anderen steigt der Implementierungsaufwand. Weiterhin kann es bei der Implementierung zu Problemen kommen, wenn die Filterzustände beziehungsweise die generierten Residuen aufgrund des Rauschens zu große Amplituden annehmen. Sind die zulässigen Bereiche für diese Größen softwaretechnisch begrenzt, kann es zu Signalsättigungen und damit einer fehlerhaften Diagnose kommen. In diesem Abschnitt wird daher ein Verfahren präsentiert, welches die Entstehung von zu stark verrauschten Filterzuständen beziehungsweise Residuensignalen schon im Entwurf verhindert. Die Idee dazu wurde bereits in $[232,234,239]$ veröffentlicht.

Das Verfahren basiert auf der Tatsache, dass gemäß dem Entwurfsziel einer hinreichenden Erkennungsgeschwindigkeit die Dynamik des zu entwerfenden FIOs schneller ist als die Dynamik der (ggf. geregelten) Strecke. Dies ist dadurch gewährleistet, dass die Beobachtereigenwerte in der s-Halbebene links der Streckeneigenwerte platziert werden. Bekanntermaßen führen zu weit links liegende Beobachtereigenwerte jedoch zu differenzierendem Charakter, sodass hochfrequente Störsignale verstärkt werden [144, Abschnitt 8.3.3], [195]. Die Idee des Verfahrens ist es nun, die zulässige Verstärkung hochfrequenter Störsignale mittels $\left\|\boldsymbol{G}_{\boldsymbol{r} \boldsymbol{d}}(s)\right\|_{\infty}^{\Omega}$, das heißt mittels der $\mathcal{H}_{\infty}$-Norm der Störübertragungsmatrix im Frequenzbereich $\Omega$, zu beschreiben. Dabei ist $\Omega=\left[\omega_{l} ; \infty\right)$ ein nach oben unbeschränktes, nach unten jedoch durch $\omega_{l}$ beschränktes Frequenzintervall. Für die Störverstärkung wird eine zulässige Schranke $\gamma_{\mathrm{HF}}$ festgelegt, es ist also die Bedingung

$$
\left\|\boldsymbol{G}_{\boldsymbol{r} \boldsymbol{d}}(s)\right\|_{\infty}^{\Omega}<\gamma_{\mathrm{HF}}
$$

einzuhalten. Dazu wird zunächst mittels Satz 4.1 oder Satz 4.2 ein FIO entworfen, der die Beobachterpole $\lambda_{B_{i j}}$ vorgibt. Dabei wird vorausgesetzt, dass für die gewählten Pole die Bedingung (5.19) eingehalten wird. Dies lässt sich bei gegebener FIO-Parametrierung $(\boldsymbol{L}, \boldsymbol{V})$ in einfacher Art und Weise mittels des verallgemeinerten Kalman-Yakubovich-Popov-Lemmas überprüfen. Dazu wird gemäß Satz 3.2 mit zwei Matrizen $\mathbb{R}^{n \times n} \ni \boldsymbol{X} \succ \mathbf{0}$ und $\boldsymbol{Y}=\boldsymbol{Y}^{\top} \in \mathbb{R}^{n \times n}$ das LMI-Lösbarkeitsproblem

finde $\boldsymbol{X}, \boldsymbol{Y}$, sodass

$$
\left[\begin{array}{cc}
\boldsymbol{A}-\boldsymbol{L C} & \boldsymbol{B}_{d}-\boldsymbol{L} \boldsymbol{D}_{d} \\
\boldsymbol{I}_{n} & \mathbf{0} \\
\boldsymbol{V} \boldsymbol{C} & \boldsymbol{V} \boldsymbol{D}_{d} \\
\mathbf{0} & \boldsymbol{I}_{n_{d}}
\end{array}\right]^{\top} \cdot\left[\begin{array}{cc}
\boldsymbol{\Xi} & \mathbf{0} \\
\mathbf{0} & \boldsymbol{\Gamma}
\end{array}\right] \cdot\left[\begin{array}{cc}
\boldsymbol{A}-\boldsymbol{L C} & \boldsymbol{B}_{d}-\boldsymbol{L} \boldsymbol{D}_{d} \\
\boldsymbol{I}_{n} & \mathbf{0} \\
\boldsymbol{V} \boldsymbol{C} & \boldsymbol{V} \boldsymbol{D}_{d} \\
\mathbf{0} & \boldsymbol{I}_{n_{d}}
\end{array}\right] \prec \mathbf{0}
$$




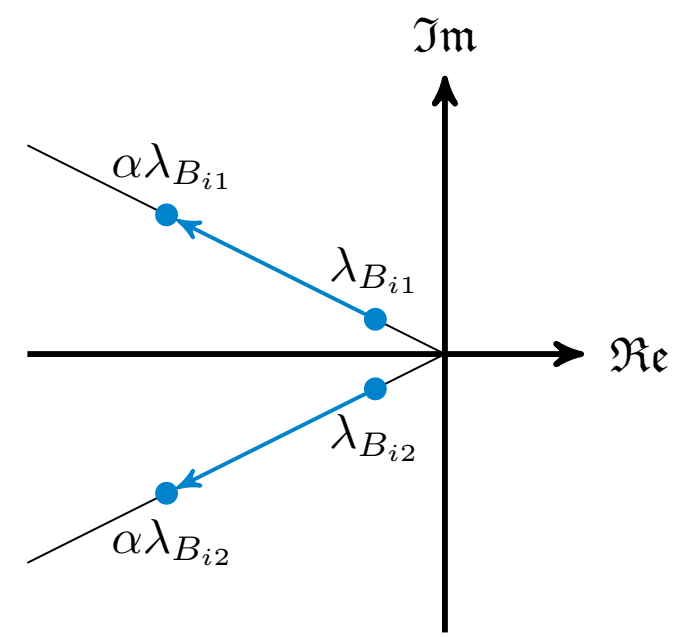

Abbildung 5.8: Skalierung vorgegebener Pole

mit den Matrizen

$$
\boldsymbol{\Xi}=\left[\begin{array}{cc}
\boldsymbol{X} & \boldsymbol{Y} \\
\star & -\omega_{l}^{2} \boldsymbol{X}
\end{array}\right], \boldsymbol{\Gamma}=\left[\begin{array}{cc}
\boldsymbol{I}_{n_{f}} & \mathbf{0} \\
\mathbf{0} & -\gamma_{\mathrm{HF}}^{2} \boldsymbol{I}_{n_{d}}
\end{array}\right]
$$

betrachtet. Ist dieses Problem nicht lösbar, müssen langsamere Eigenwerte vorgegeben werden. Wird jedoch eine Lösung für (5.20) gefunden, so kann die Detektionsgeschwindigkeit offensichtlich noch erhöht werden. Dazu werden die vorgegebenen Beobachterpole auf Strahlen in der s-Halbebene nach links verschoben. Dieses Vorgehen ist in Abbildung 5.8 skizziert und kann durch eine Skalierung der Pole mittels $\lambda_{B_{i j}}^{*}=\alpha \lambda_{B_{i j}}$ mit $\alpha>1$ realisiert werden. Für die skalierten Pole $\lambda_{B_{i j}}^{*}$ wird die FIO-Parametrierung $\left(\boldsymbol{L}^{*}, \boldsymbol{V}^{*}\right)$ berechnet, für die erneut (5.20) überprüft wird. Auf diese Art und Weise lässt sich der maximale Skalierungsfaktor $\alpha_{\max }$ berechnen, für den $\gamma_{\mathrm{HF}}$ noch eingehalten wird.

Mit dem beschriebenen Vorgehen wird bei einer gegebenen Schranke $\gamma_{\mathrm{HF}}$ die Detektionsgeschwindigkeit maximiert. Wählt man für die initiale FIOParametrierung Pole, welche (5.20) nicht erfüllen, und skaliert die Pole dann mittels eines Faktors $0<\alpha<1$, so wird die Detektionsgeschwindigkeit zur Einhaltung einer gegebenen Schranke für die Störverstärkung reduziert.

\section{Erweiterung auf DFIOs}

Das Verfahren lässt sich ohne Weiteres auf dynamisch erweiterte Fehlerisolationsbeobachter ausdehnen. Insbesondere das in Abschnitt 4.6.4 be- 
schriebene Verfahren, bei dem gemäß (4.40) lediglich die Nenner der Diagonalelemente erweitert werden, eignet sich hierfür. Analog zu (4.32) lässt sich die Wirkung der Störungen auf die generierten Residuen durch

$$
\begin{aligned}
{\left[\begin{array}{c}
\dot{\boldsymbol{\xi}} \\
\dot{\boldsymbol{\zeta}}
\end{array}\right] } & =\left[\begin{array}{cc}
\boldsymbol{A}-\boldsymbol{L}_{p} \boldsymbol{C} & -\boldsymbol{L}_{i} \\
\boldsymbol{\Gamma} \boldsymbol{C} & \boldsymbol{\Phi}
\end{array}\right]\left[\begin{array}{l}
\boldsymbol{\xi} \\
\boldsymbol{\zeta}
\end{array}\right]+\left[\begin{array}{c}
\boldsymbol{B}_{d}-\boldsymbol{L}_{p} \boldsymbol{D}_{d} \\
\boldsymbol{\Gamma} \boldsymbol{D}_{d}
\end{array}\right] \boldsymbol{d}, \\
\boldsymbol{r} & =\left[\begin{array}{ll}
\boldsymbol{V}_{\boldsymbol{y}} \boldsymbol{C} & \boldsymbol{V}_{\boldsymbol{\zeta}}
\end{array}\right]\left[\begin{array}{l}
\boldsymbol{\xi} \\
\boldsymbol{\zeta}
\end{array}\right]+\boldsymbol{V}_{\boldsymbol{y}} \boldsymbol{D}_{d} \boldsymbol{d}
\end{aligned}
$$

beschreiben. Da hierin die gleiche Dynamikmatrix wie in (4.32) auftritt, führt die Erweiterung der Nenner in (4.40) zu zusätzlichen Polen in der Störübertragungsmatrix $\boldsymbol{G}_{\boldsymbol{r} \boldsymbol{d}}(s)$. Daraus ergibt sich wiederum ein Abfall der Verstärkung bei hohen Frequenzen und damit eine bessere Unterdrückung hochfrequenter Störungen. Wie bereits erörtert lässt sich hinsichtlich des Ein-/Ausgangsverhaltens der gleiche Effekt durch eine Tiefpassfilterung der Einzelresiduen erreichen. Das beschriebene Vorgehen weist jedoch den Vorteil eines integrierten Entwurfs auf. Das weitere Vorgehen bleibt gegenüber der Verwendung statischer FIOs unverändert: Es werden Pole für die Diagonalelemente (4.40) vorgegeben, diese werden anschließend derart skaliert, dass die gewählte Schranke $\gamma_{\mathrm{HF}}$ eingehalten wird. Formal zusammengefasst ist das Vorgehen im nichtkonvexen Optimierungsproblem

$$
\begin{aligned}
& \text { maximiere } \alpha, \text { sodass } \\
& \boldsymbol{G}_{\boldsymbol{r} \boldsymbol{f}}(s)=\operatorname{diag}\left(g_{1,1}(s), \ldots, g_{n_{f}, n_{f}}(s)\right), \\
& g_{i, i}(s)=\frac{(-\alpha)^{\delta_{i}+\rho} z_{i, 0}}{\left(s-\alpha \lambda_{B_{i 1}}\right) \cdot \ldots \cdot\left(s-\alpha \lambda_{B_{i \delta_{i}+\rho}}\right)}, \\
& \left\|\boldsymbol{G}_{\boldsymbol{r d}}(s)\right\|_{\infty}^{\left[\omega_{l}, \infty\right)}<\gamma_{\mathrm{HF}} .
\end{aligned}
$$

Die Lösung des Optimierungsproblems erfolgt durch eine Bisektion in $\alpha$. Dabei wird zunächst ein Intervall $\alpha \in\left[\alpha_{\mathrm{lo}} ; \alpha_{\text {up }}\right]$ angesetzt, welches sich dadurch auszeichnet, dass $\left\|\boldsymbol{G}_{\boldsymbol{r d}}(s)\right\|_{\infty}^{\left[\omega_{l}, \infty\right)}<\gamma_{\mathrm{HF}}$ für $\alpha_{\text {lo }}$ erfüllt ist, für $\alpha_{\text {up }}$ jedoch nicht. Ausgehend von dieser Initialisierung wird für die Intervallmitte, also für $\left(\alpha_{\text {lo }}+\alpha_{\text {up }}\right) / 2$ untersucht, ob $\left\|\boldsymbol{G}_{\boldsymbol{r} \boldsymbol{d}}(s)\right\|_{\infty}^{\left[\omega_{l}, \infty\right)}<\gamma_{\mathrm{HF}}$ erfüllt ist. Falls ja, so wird als neues Intervall $\left[\left(\alpha_{\text {lo }}+\alpha_{\text {up }}\right) / 2 ; \alpha_{\text {up }}\right]$ angesetzt. Falls nein wird mit $\left[\alpha_{\text {lo }} ;\left(\alpha_{\text {lo }}+\alpha_{\text {up }}\right) / 2\right]$ fortgefahren. Auf diese Art und Weise halbiert sich in jedem Schritt die Intervallbreite und die untere Intervallgrenze stellt jeweils eine untere Schranke für den größten zulässigen Skalierungsfaktor $\alpha$ dar. 

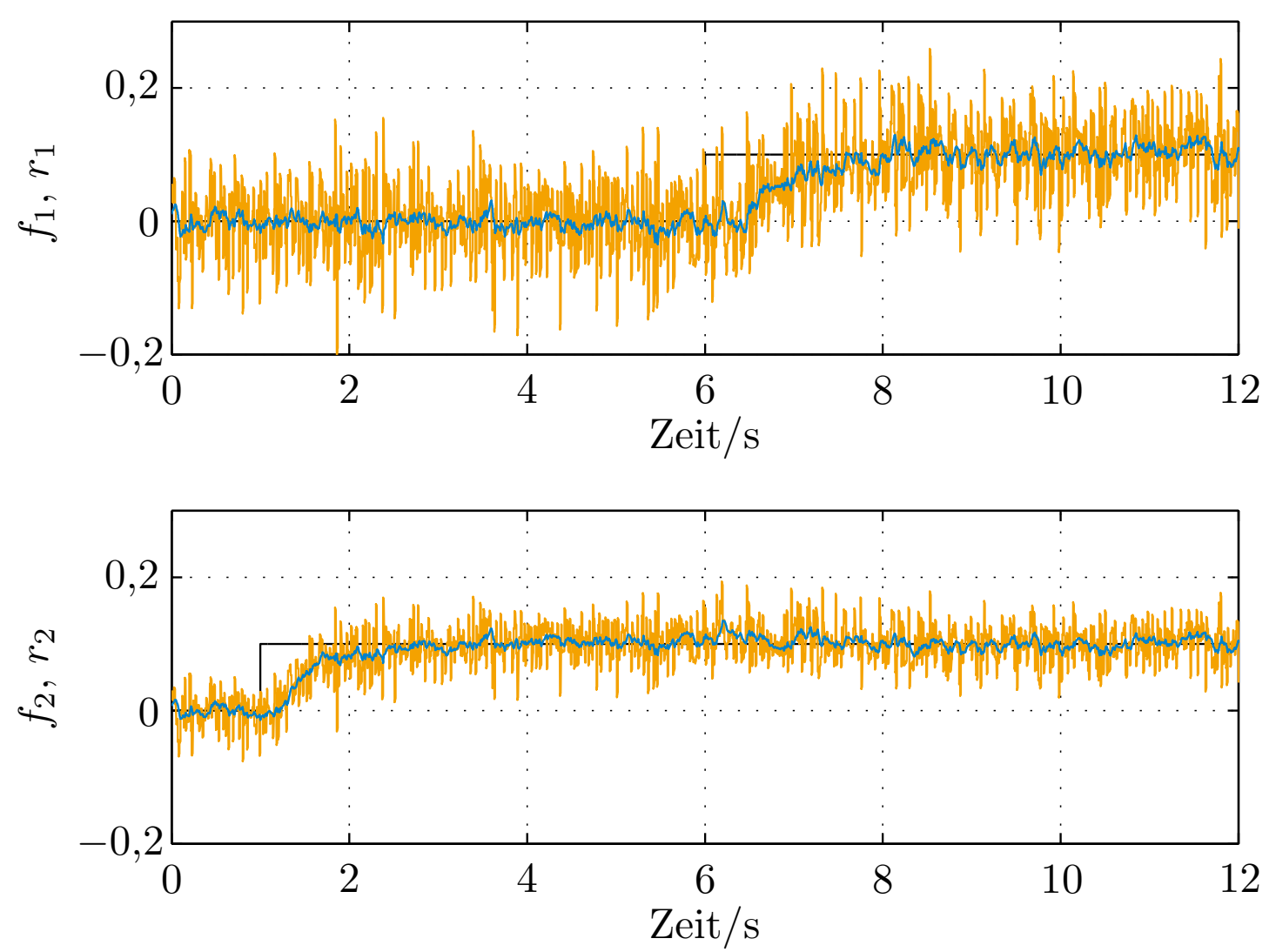

Abbildung 5.9: Fehler (-) und generierte Residuen (-: FIO, -: DFIO) für Aktorfehler des CE 150 Modellhubschraubers bei verrauschten Messsignalen

\section{Beispiel CE 150 Modellhubschrauber}

Bereits in den Abschnitten 4.5.3 und 4.6.5 wurden ein FIO beziehungsweise ein DFIO für den CE 150 Modellhubschrauber entworfen. Um das Verfahren zur Unterdrückung hochfrequenter Störungen zu demonstrieren, werden die gemessenen Ausgangsgrößen des Helikopters (Nick- und Gierwinkel) für die Fehlerisolation mit einem zusätzlichen, hochfrequenten Störsignal überlagert. Abbildung 5.9 zeigt, dass mit der ursprünglichen FIO-Parametrierung aus Abschnitt 4.5.3 ohne weitere Filterungsmaßnahmen keine Fehlerisolation möglich ist, da die generierten Residuen zu stark rauschbehaftet sind. Im fehlerfreien Fall treten zum Teil größere Residuenamplituden auf als im fehlerbehafteten Fall.

Für den Entwurf eines robusten DFIOs werden die Störeingangsmatrizen entsprechend den Rauschamplituden zu

$$
\boldsymbol{B}_{d}=\mathbf{0}, \boldsymbol{D}_{d}=\left[\begin{array}{cc}
1 & 0 \\
0 & 0,1
\end{array}\right]
$$


gewählt. Als zulässige Schranke für die Störverstärkung wird $\gamma_{\mathrm{HF}}=0,3$ angesetzt, der betrachtete Frequenzbereich wird durch $\omega_{l}=20 \mathrm{rad} / \mathrm{s}$ beschrieben. Ausgehend von dem in Abschnitt 4.6.5 entworfenen DFIO liefert das vorgestellte Verfahren einen Skalierungsfaktor von $\alpha=1,154$. Dies führt auf die Beobachtereigenwerte $\lambda_{B_{1,1}}=-4,616, \lambda_{B_{1,2}}=-5,770$, $\lambda_{B_{1,3}}=-6,924$ und $\lambda_{B_{1,4}}=-8,078$ sowie für den zweiten Pfad $\lambda_{B_{2,1}}=-5,193, \lambda_{B_{2,2}}=-6,347$ und $\lambda_{B_{2,3 / 4}}=-7,501 \pm 3,462 i$. Die resultierende DFIO-Parametrierung ist in Anhang C.2.1 gegeben. Aus Abbildung 5.9 ist ersichtlich, dass die hochfrequenten Störsignale sehr gut unterdrückt werden, sodass eine Fehlerisolation mittels einfacher Schwellenwerte ohne Weiteres möglich ist.

Prinzipiell lässt sich eine Unterdrückung der hochfrequenten Störungen auch durch zusätzliche Tiefpassfilter für die einzelnen Residuen erreichen, wie zu Beginn dieses Abschnittes und in Abschnitt 4.6.1 diskutiert wurde. Es sei an dieser Stelle nochmals darauf hingewiesen, dass sich mit DFIOs jedoch nicht nur eine Unterdrückung hochfrequenter Störungen erreichen lässt. Vielmehr ermöglichen sie erst die Isolation von Fehlern in statisch nicht isolierbaren und nicht minimalphasigen Systemen (vgl. Abschnitt 4.7 beziehungsweise 4.8).

\subsubsection{Fazit}

In quadratischen Systemen mit $n_{y}=n_{f}$ sind die Entwurfsfreiheitsgrade aufgrund der strukturellen Beschränkungen von $\boldsymbol{L}$ und $\boldsymbol{V}$ stark eingeschränkt. Dennoch lässt sich die Robustheit der generierten Residuen steigern, indem die verbleibenden Freiheitsgrade (die Koeffizienten der Diagonalelemente von $\boldsymbol{G}_{\boldsymbol{r} \boldsymbol{f}}(s)$ ) angepasst werden (s. Abschnitt 5.3.1). Weiterhin können die strukturellen Anforderungen abgeschwächt werden, um zusätzliche Freiheitsgrade zur Robustheitsoptimierung zu gewinnen (s. Abschnitt 5.3.2). Selbiges wird durch die Erweiterung der Dynamik der Diagonalelemente (s. Abschnitt 5.3.3) erreicht. Zur weiteren Verbesserung lassen sich die vorgestellten Methoden beziehungsweise Maßnahmen auch kombinieren.

\subsection{Steigerung der Robustheit in nichtquadratischen Systemen}

Wie in Abschnitt 4.9 herausgearbeitet lässt sich eine vorgegebene Dyna$\operatorname{mik} \boldsymbol{G}_{\boldsymbol{r} \boldsymbol{f}}(s)=\operatorname{diag}\left(g_{1,1}(s), \ldots, g_{n_{f}, n_{f}}(s)\right)$ in nichtquadratischen Systemen 
durch unendlich viele FIO-Parametrierungen $(\boldsymbol{L}, \boldsymbol{V})$ erreichen. Gegenstand dieses Abschnittes ist es, diese zusätzlichen Freiheitsgrade zur Steigerung der Robustheit zu nutzen. Betrachtet werden dabei erneut lineare zeitinvariante Systeme, die exogenen Störungen $\boldsymbol{d} \in \mathbb{R}^{n_{d}}$ unterliegen. Die Dynamik wird also beschrieben durch

$$
\begin{aligned}
& \dot{\boldsymbol{x}}=\boldsymbol{A} \boldsymbol{x}+\boldsymbol{B} \boldsymbol{u}+\boldsymbol{E}_{a} \boldsymbol{f}+\boldsymbol{B}_{d} \boldsymbol{d}, \\
& \boldsymbol{y}=\boldsymbol{C} \boldsymbol{x}+\boldsymbol{D} \boldsymbol{u}+\boldsymbol{E}_{s} \boldsymbol{f}+\boldsymbol{D}_{d} \boldsymbol{d} .
\end{aligned}
$$

Dabei gelten hinsichtlich der Matrizen $\boldsymbol{A}, \boldsymbol{E}_{a}, \boldsymbol{C}$ und $\boldsymbol{E}_{s}$ die gleichen Annahmen wie in Abschnitt 4.9, also

Annahme 5.1. Das Paar $(\boldsymbol{A}, \boldsymbol{C})$ ist vollständig beobachtbar.

Annahme 5.2. Die Fehlerdetektionsmatrix hat vollen Rang, das heißt es gilt $\operatorname{rang}\left(\boldsymbol{D}^{*}\right)=n_{f}$.

Annahme 5.3. Das System $\left(\boldsymbol{A}, \boldsymbol{E}_{a}, \boldsymbol{C}, \boldsymbol{E}_{s}\right)$ ist minimalphasig und weist genau $\mu$ invariante Nullstellen auf.

Ziel ist es im Weiteren stets, den Einfluss der Störungen auf die generierten Residuen zu minimieren, wobei die Diagonalisierung von $\boldsymbol{G}_{\boldsymbol{r} \boldsymbol{f}}(s)$ zu gewährleisten ist.

\subsubsection{Robustheit bezüglich beliebiger exogener Störungen}

Wie im quadratischen Fall lässt sich der Einfluss der Störungen auf die generierten Residuen beschreiben durch (5.4). Geht man von Störungen mit beliebigen Frequenzen aus, so ist das bekannte Optimierungsproblem

$$
\begin{aligned}
& \underset{\boldsymbol{L}, \boldsymbol{V}}{\operatorname{minimiere}}\left\|\boldsymbol{G}_{\boldsymbol{r d}}(s)\right\|_{\infty}, \text { sodass } \\
& \boldsymbol{G}_{\boldsymbol{r} \boldsymbol{f}}(s)=\operatorname{diag}\left(g_{1,1}(s), \ldots, g_{n_{f}, n_{f}}(s)\right)
\end{aligned}
$$

zu lösen. Da beim Entwurf eines FIOs nach Satz 4.8 die Einhaltung der strukturbeschränkenden Nebenbedingung (5.22b) inhärent gewährleistet ist, lässt sich (5.22) mit $\boldsymbol{L}=\boldsymbol{L}_{0}+\boldsymbol{R}_{L} \cdot{ }^{\perp} \boldsymbol{D}^{*}$ und $\boldsymbol{V}=\boldsymbol{V}_{0}+\boldsymbol{R}_{V} \cdot{ }^{\perp} \boldsymbol{D}^{*}$ äquivalent formulieren als

$$
\underset{\boldsymbol{R}_{L}, \boldsymbol{R}_{V}}{\operatorname{minimiere}}\left\|\boldsymbol{G}_{\boldsymbol{r} \boldsymbol{d}}(s)\right\|_{\infty} .
$$


Bemerkenswert ist dabei, dass die Matrizen $\boldsymbol{R}_{L}$ und $\boldsymbol{R}_{V}$ im Gegensatz zu $\boldsymbol{L}$ und $\boldsymbol{V}$ keinerlei Strukturbeschränkungen unterliegen, sondern voll besetzt und frei wählbar sind. Mithilfe des Bounded-Real-Lemmas (Satz 3.1) wird nun die Minimierung von $\left\|\boldsymbol{G}_{\boldsymbol{r} \boldsymbol{d}}(s)\right\|_{\infty}$ als Optimierungsproblem mit Matrixungleichungen als Nebenbedingungen formuliert. Es ergibt sich

$$
\begin{aligned}
& \underset{\boldsymbol{R}_{L}, \boldsymbol{R}_{V}, \boldsymbol{X}}{\operatorname{minimiere}} \gamma \text {, sodass } \\
& \mathbb{R}^{n \times n} \ni \boldsymbol{X}=\boldsymbol{X}^{\boldsymbol{\top}} \succ \mathbf{0}, \\
& {\left[\begin{array}{ccc}
\operatorname{He}(\boldsymbol{X}(\boldsymbol{A}-\boldsymbol{L} \boldsymbol{C})) & \boldsymbol{X}\left(\boldsymbol{B}_{d}-\boldsymbol{L} \boldsymbol{D}_{d}\right) & (\boldsymbol{V} \boldsymbol{C})^{\top} \\
\star & -\gamma \boldsymbol{I}_{n_{d}} & \left(\boldsymbol{V} \boldsymbol{D}_{d}\right)^{\top} \\
\star & \star & -\gamma \boldsymbol{I}_{n_{f}}
\end{array}\right] \prec \mathbf{0},} \\
& \boldsymbol{L}=\boldsymbol{L}_{0}+\boldsymbol{R}_{L} \cdot{ }^{\perp} \boldsymbol{D}^{*}, \quad \boldsymbol{V}=\boldsymbol{V}_{0}+\boldsymbol{R}_{V} \cdot{ }^{\perp} \boldsymbol{D}^{*} .
\end{aligned}
$$

Dabei stellt (5.23) aufgrund der multiplikativen Terme $\boldsymbol{X} \boldsymbol{R}_{L}$ in (5.23c) ein Problem mit bilinearen Matrixungleichungen (BMIs) als Nebenbedingungen dar. Durch die Substitution $\boldsymbol{Y}=\boldsymbol{X} \boldsymbol{R}_{L} \in \mathbb{R}^{n \times\left(n_{y}-n_{f}\right)}$ lässt sich (5.23) jedoch überführen in

$$
\begin{aligned}
& \underset{\boldsymbol{X}, \boldsymbol{Y}, \boldsymbol{R}_{V}}{\operatorname{minimiere}} \gamma, \text { sodass } \\
& \mathbb{R}^{n \times n} \ni \boldsymbol{X}=\boldsymbol{X}^{\top} \succ \mathbf{0}, \\
& {\left[\begin{array}{ccc}
\boldsymbol{\Sigma}_{11} & \boldsymbol{\Sigma}_{12} & \boldsymbol{\Sigma}_{13} \\
\star & -\gamma \boldsymbol{I}_{n_{d}} & \boldsymbol{\Sigma}_{23} \\
\star & \star & -\gamma \boldsymbol{I}_{n_{f}}
\end{array}\right] \prec \mathbf{0},} \\
& \boldsymbol{\Sigma}_{11}=\operatorname{He}\left(\boldsymbol{X}\left(\boldsymbol{A}-\boldsymbol{L}_{0} \boldsymbol{C}\right)-\boldsymbol{Y} \cdot{ }^{\perp} \boldsymbol{D}^{*} \cdot \boldsymbol{C}\right), \\
& \boldsymbol{\Sigma}_{12}=\boldsymbol{X}\left(\boldsymbol{B}_{d}-\boldsymbol{L}_{0} \boldsymbol{D}_{d}\right)-\boldsymbol{Y} \cdot{ }^{\perp} \boldsymbol{D}^{*} \cdot \boldsymbol{D}_{d}, \\
& \boldsymbol{\Sigma}_{13}=\left(\boldsymbol{V}_{0} \boldsymbol{C}+\boldsymbol{R}_{V} \cdot{ }^{\perp} \boldsymbol{D}^{*} \cdot \boldsymbol{C}\right)^{\top}, \\
& \boldsymbol{\Sigma}_{23}=\left(\boldsymbol{V}_{0} \boldsymbol{D}_{d}+\boldsymbol{R}_{V} \cdot{ }^{\perp} \boldsymbol{D}^{*} \cdot \boldsymbol{D}_{d}\right)^{\top} .
\end{aligned}
$$

Dabei handelt es sich um ein LMI-Optimierungsproblem, das sich wegen seiner Konvexität effizient lösen lässt (s. Abschnitt 3.2). Nach Lösung des Optimierungsproblems ergeben sich die Beobachtermatrizen aus $\boldsymbol{L}=\boldsymbol{L}_{0}+\boldsymbol{X}^{-1} \boldsymbol{Y} \cdot{ }^{\perp} \boldsymbol{D}^{*}$ und $\boldsymbol{V}=\boldsymbol{V}_{0}+\boldsymbol{R}_{V} \cdot{ }^{\perp} \boldsymbol{D}^{*}$. Ähnliche Resultate sind in $[28,114]$ gegeben, wobei diese wie erwähnt zum Teil hinsichtlich der erlaubten Fehlerindizes $\delta_{i}$ eingeschränkt sind und darüber hinaus lediglich Aktorfehler berücksichtigen.

$\mathrm{Zu}$ bemerken ist, dass nach [150, Proposition 8.2.7] durch (5.24c) auch He $(\boldsymbol{X}(\boldsymbol{A}-\boldsymbol{L} \boldsymbol{C})) \prec \mathbf{0}$ sichergestellt ist und somit die Stabilität des Be- 
obachters gewährleistet ist. Darüber hinaus bestehen jedoch keine Beschränkungen für die bezüglich $\left(\boldsymbol{A}-\boldsymbol{L} \boldsymbol{C}, \boldsymbol{E}_{a}-\boldsymbol{L} \boldsymbol{E}_{s}\right)$ nichtsteuerbaren Eigenwerte $\lambda_{B_{k}}$. Bei der Lösung von (5.24) können sich daher einerseits Eigenwerte mit $\mathfrak{R e}\left(\lambda_{B_{k}}\right) \approx 0$ ergeben, die zu einem sehr langsamen Abklingen von Beobachteranfangsfehlern $\boldsymbol{\xi}(t=0) \neq \mathbf{0}$ führen. Andererseits können auch Eigenwerte $\lambda_{B_{k}}$ mit betragsmäßig sehr großen Realteilen auftreten, die sowohl numerische Probleme bei der Lösung von (5.24) als auch Probleme bei der Implementierung hervorrufen können. Um diese Phänomene zu unterbinden lässt sich das Optimierungsproblem (5.24) wie in Abschnitt 3.2.2 beschrieben um zusätzliche LMI-Nebenbedingungen erweitern. Durch Anwendung von Lemma 3.6 auf die Matrix $\boldsymbol{A}-\boldsymbol{L} \boldsymbol{C}$ lassen sich die Beobachtereigenwerte beispielsweise auf $\mathfrak{R e}\left(\lambda_{B_{k}}\right)<\alpha$ beschränken, indem

$$
\operatorname{He}\left(\boldsymbol{X}\left(\boldsymbol{A}-\boldsymbol{L}_{0} \boldsymbol{C}\right)-\boldsymbol{X} \boldsymbol{R}_{L} \cdot{ }^{\perp} \boldsymbol{D}^{*} \boldsymbol{C}\right)-2 \alpha \boldsymbol{X} \prec \mathbf{0}
$$

gefordert wird. Mittels der Variablensubstitution $\boldsymbol{Y}=\boldsymbol{X} \boldsymbol{R}_{L}$ ergibt sich daraus

$$
\mathrm{He}\left(\boldsymbol{X}\left(\boldsymbol{A}-\boldsymbol{L}_{0} \boldsymbol{C}\right)-\boldsymbol{Y} \cdot{ }^{\perp} \boldsymbol{D}^{*} \boldsymbol{C}\right)-2 \alpha \boldsymbol{X} \prec \mathbf{0} .
$$

Analog dazu lassen sich Lemma 3.7 und 3.8 auf die Matrix $\boldsymbol{A}-\boldsymbol{L} \boldsymbol{C}$ anwenden, sodass sich zusammengefasst die ergänzenden Nebenbedingungen

$$
\begin{aligned}
& \mathrm{He}\left(\boldsymbol{X}\left(\boldsymbol{A}-\boldsymbol{L}_{0} \boldsymbol{C}\right)-\boldsymbol{Y} \cdot{ }^{\perp} \boldsymbol{D}^{*} \boldsymbol{C}\right)-2 \alpha \boldsymbol{X} \prec \mathbf{0}, \\
& {\left[\begin{array}{cc}
-\varrho \boldsymbol{X} & \boldsymbol{X}\left(\boldsymbol{A}-\boldsymbol{L}_{0} \boldsymbol{C}\right)-\boldsymbol{Y} \cdot{ }^{\perp} \boldsymbol{D}^{*} \boldsymbol{C} \\
\star & -\varrho \boldsymbol{X}
\end{array} \quad \mathbf{0}\right.} \\
& {\left[\begin{array}{cc}
\boldsymbol{\Phi}_{11} & \boldsymbol{\Phi}_{12} \\
\star & \boldsymbol{\Phi}_{22}
\end{array}\right] \prec \mathbf{0},} \\
& \boldsymbol{\Phi}_{11}=\boldsymbol{\Phi}_{22}=\tan (\phi) \cdot \operatorname{He}\left(\boldsymbol{X}\left(\boldsymbol{A}-\boldsymbol{L}_{0} \boldsymbol{C}\right)-\boldsymbol{Y} \cdot{ }^{\perp} \boldsymbol{D}^{*} \boldsymbol{C}\right), \\
& \boldsymbol{\Phi}_{12}=-\boldsymbol{X}\left(\boldsymbol{A}-\boldsymbol{L}_{0} \boldsymbol{C}\right)+\left(\boldsymbol{A}-\boldsymbol{L}_{0} \boldsymbol{C}\right)^{\boldsymbol{\top}} \boldsymbol{X}+\boldsymbol{Y} \cdot{ }^{\perp} \boldsymbol{D}^{*} \boldsymbol{C}-\left(\boldsymbol{Y} \cdot{ }^{\perp} \boldsymbol{D}^{*} \boldsymbol{C}\right)^{\top}
\end{aligned}
$$

ergeben. Durch (5.25a) ist wie bereits erörtert sichergestellt, dass alle Beobachtereigenwerte links der Schranke $\alpha$ liegen. Die Bedingung (5.25b) beschränkt den Betrag der Eigenwerte durch $\varrho$, während mittels (5.25c)-(5.25e) eine durch $\left|\mathfrak{I m}\left(\lambda_{B_{k}}\right)\right|<\tan (\phi) \cdot\left|\mathfrak{R e}\left(\lambda_{B_{k}}\right)\right|$ definierte Mindestdämpfung sichergestellt ist. Dabei ist darauf zu achten, dass die zur Festlegung der Fehlererkennungsdynamik platzierten Pole $\lambda_{B_{i j}}$ ebenso in der durch $\alpha$, $\varrho$ und $\phi$ definierten $\mathcal{D}$-Region liegen wie eventuelle zu kompensierende invariante Nullstellen, da andernfalls die LMIs (5.25) keine Lösung haben. Zusammenfassend gilt 
Satz 5.3 (LMI-basierter Entwurf robuster FIOs für nichtquadratische Systeme mit Beschränkung der Beobachtereigenwerte). Gegeben sei ein System der Form

$$
\begin{aligned}
& \dot{\boldsymbol{x}}=\boldsymbol{A} \boldsymbol{x}+\boldsymbol{B u}+\boldsymbol{E}_{a} \boldsymbol{f}+\boldsymbol{B}_{d} \boldsymbol{d}, \\
& \boldsymbol{y}=\boldsymbol{C} \boldsymbol{x}+\boldsymbol{D} \boldsymbol{u}+\boldsymbol{E}_{s} \boldsymbol{f}+\boldsymbol{D}_{d} \boldsymbol{d},
\end{aligned}
$$

welches die Annahmen 5.1-5.3 erfüllt, und eine FIO-Parametrierung $\left(\boldsymbol{L}_{0}, \boldsymbol{V}_{0}\right)$ gemäß Satz 4.8. Dann wird $\left\|\boldsymbol{G}_{\boldsymbol{r d}}(s)\right\|_{\infty}$ minimiert durch die Lösung des LMI-Problems

$$
\begin{aligned}
& \underset{\boldsymbol{X}, \boldsymbol{Y}, \boldsymbol{R}_{V}}{\operatorname{minimiere}} \gamma, \text { sodass } \\
& (5.24 \mathrm{~b})-(5.24 \mathrm{~g}), \quad(5.25),
\end{aligned}
$$

wobei $\mathfrak{R e}\left(\lambda_{B_{i}}\right)<-\alpha,\left|\lambda_{B_{i}}\right|<\varrho$ und $\left|\mathfrak{I m}\left(\lambda_{B_{i}}\right)\right|<\tan (\phi) \cdot\left|\mathfrak{R e}\left(\lambda_{B_{i}}\right)\right|$ für alle $i=1, \ldots, n$ sichergestellt ist. Die gesuchte Beobachterparametrierung ergibt sich aus

$$
\begin{aligned}
\boldsymbol{L} & =\boldsymbol{L}_{0}+\boldsymbol{X}^{-1} \boldsymbol{Y} \cdot{ }^{\perp} \boldsymbol{D}^{*} \\
\boldsymbol{V} & =\boldsymbol{V}_{0}+\boldsymbol{R}_{V} \cdot{ }^{\perp} \boldsymbol{D}^{*}
\end{aligned}
$$

Bemerkung 5.4. Wie bereits angemerkt kann die Parametrierung $\left(\boldsymbol{L}_{0}, \boldsymbol{V}_{0}\right)$ auch mittels Vollständiger Modaler Synthese, das heißt mittels Satz 4.9 gewonnen werden. Da in Satz 5.3 ein konvexes Optimierungsproblem gelöst wird, ergibt sich für unterschiedliche Parametrierungen $\left(\boldsymbol{L}_{0}, \boldsymbol{V}_{0}\right)$ jeweils die gleiche optimale Schranke $\gamma$, die jedoch mit unterschiedlichen Werten für $\boldsymbol{R}_{L}$ und $\boldsymbol{R}_{V}$ erreicht wird.

Bemerkung 5.5. Für nichtquadratische Systeme lassen sich darüber hinaus ebenso wie in Abschnitt 5.3.2 für quadratische Systeme gezeigt durch Abschwächung der strukturellen Anforderungen weitere Freiheitsgrade zur Störunterdrückung gewinnen. Das Verfahren aus Abschnitt 5.3.2 ist geradlinig auch für nichtquadratische Systeme anwendbar, wobei die nichtsteuerbaren Eigenwerte, welche nicht zur Kompensation invarianter Nullstellen herangezogen werden müssen, zusätzliche Freiheitsgrade bieten.

\section{Beispiel Hydrauliksystem}

Ausgehend von der FIO-Parametrierung aus Abschnitt 4.9.3 wird Satz 5.3 angewendet, um die Robustheit bezüglich exogener Störungen zu optimieren. Als Störeingangsmatrizen werden dabei 


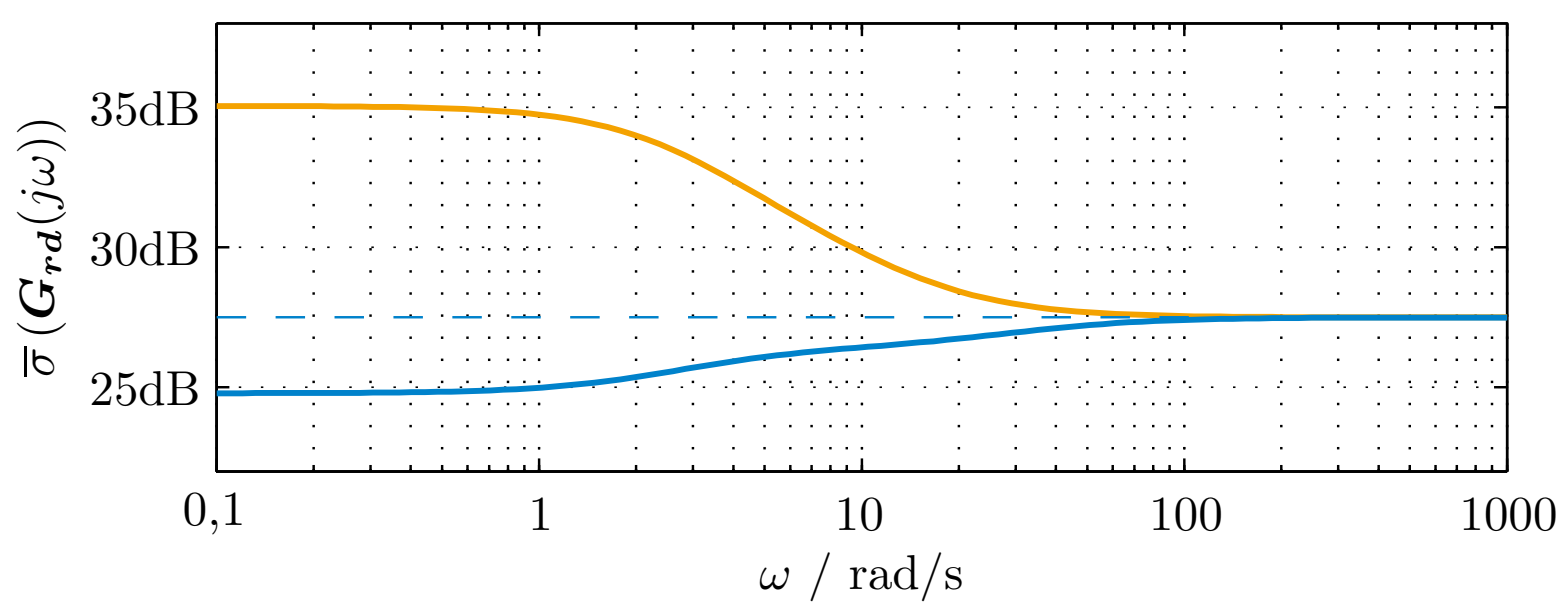

Abbildung 5.10: Verlauf des größten Singulärwertes von $\boldsymbol{G}_{\boldsymbol{r} \boldsymbol{d}}(s)$ für initiale $(-)$ und optimierte (-) FIO-Parametrierung

$$
\boldsymbol{B}_{d}=\left[\begin{array}{ccc}
0,5 & 0 & 0 \\
0 & 0 & 0 \\
-0,1 & 0 & 0 \\
0 & 0 & 0 \\
0 & 0 & 0
\end{array}\right], \boldsymbol{D}_{d}=\left[\begin{array}{ccc}
0 & 0,2 & 0 \\
0 & 0 & 0,1 \\
0 & 2 & 0
\end{array}\right]
$$

angenommen. Begrenzt man den zulässigen Bereich für die Beobachtereigenwerte durch $\alpha=1$ und $\varrho=50$, so liefert das Optimierungsproblem aus Satz 5.3 die in Anhang C.2.3 angegebene FIO-Parametrierung. Eine Vorgabe des Winkels $\phi$ ist nicht notwendig, da wegen $n_{y}-n_{f}=1$ lediglich ein nichtsteuerbarer Beobachtereigenwert existiert, der stets reell ist. Der FIO verschiebt den nichtsteuerbaren Beobachtereigenwert auf $\lambda_{B_{5}}=-31,39$ und führt auf eine Schranke von $\gamma_{\text {opt }}=23,70$. Dies stellt eine deutliche Verbesserung gegenüber der ursprünglichen Parametrierung aus Abschnitt 4.9.3 dar, für die sich $\gamma_{\text {init }}=56,56$ ergibt. Abbildung 5.10 verdeutlicht dies anhand des Verlaufes der größten Singulärwerte von $\boldsymbol{G}_{\boldsymbol{r} \boldsymbol{d}}(s)$ für beide FIOs.

$\mathrm{Zu}$ betonen ist, dass die Störunterdrückung des optimierten Beobachters nicht für alle Frequenzen besser ist als die der initialen Parametrierung. Für hochfrequente Störungen ist praktisch keine Veränderung festzustellen. Dies steht jedoch nicht im Widerspruch zur angestrebten Optimierung, deren Ziel es war, die größtmögliche Verstärkung zu minimieren. Diese tritt bei der initialen Parametrierung bei niedrigen Frequenzen auf und beim optimierten Beobachter bei hohen Frequenzen (s. Abbildung 5.10). An dieser Stelle zeigt sich deutlich, dass durch die Minimierung der $\mathcal{H}_{\infty}$-Norm 
der Worst-Case optimiert wird.

Der Vergleich der beiden Beobachterparametrierungen zeigt, dass sich die Matrizen $\boldsymbol{L}$ und $\boldsymbol{V}$ jeweils in der zweiten Spalte von der initialen Parametrierung unterscheiden. Dies erklärt sich durch die Basis des Linksnullraumes der Matrix $\boldsymbol{D}^{*}$, die durch ${ }^{\perp} \boldsymbol{D}^{*}=\left[\begin{array}{lll}0 & 1 & 0\end{array}\right]$ gegeben ist.

\subsubsection{Erweiterung durch Nutzung aller Freiheitsgrade}

Im vorangegangenen Abschnitt wurden die zusätzlichen Freiheitsgrade, die sich durch zusätzliche Messgrößen ergeben, für die Robustheitsoptimierung genutzt. Dabei ist jedoch die Dynamik der Diagonalelemente von $\boldsymbol{G}_{\boldsymbol{r} \boldsymbol{f}(s)}$ fest vorgegeben. Im Folgenden wird gezeigt, wie sich das Verfahren zur Optimierung der Diagonalelemente aus Abschnitt 5.3.1 auf nichtquadratische Systeme erweitern lässt.

Grundidee ist es erneut, die Koeffizienten der Diagonalelemente für die Optimierung mit zu nutzen, es ist also

$$
\begin{aligned}
& \underset{\boldsymbol{q}_{i, j}, \boldsymbol{z}_{i, 0}, \boldsymbol{R}_{L}, \boldsymbol{R}_{V}}{\operatorname{minimiere}} \gamma, \text { sodass } \\
& \left\|\boldsymbol{G}_{\boldsymbol{r} \boldsymbol{d}}(s)\right\|_{\infty}<\gamma, \\
& \boldsymbol{G}_{\boldsymbol{r} \boldsymbol{f}}(s)=\operatorname{diag}\left(g_{1,1}(s), \ldots, g_{n_{f}, n_{f}}(s)\right)
\end{aligned}
$$

zu lösen. Dies lässt sich analog zu (5.5b), (5.5c) und (5.6) als StandardRegelungsproblem

$$
\begin{aligned}
& \underset{\tilde{\tilde{\boldsymbol{K}}}}{\operatorname{minimiere}}\left\|\boldsymbol{G}_{\boldsymbol{z} \boldsymbol{w}}(s)\right\|_{\infty} \operatorname{mit} \\
& \mathbf{P}:\left\{\begin{array}{l}
\dot{\tilde{\boldsymbol{x}}}=\tilde{\boldsymbol{A}} \tilde{\boldsymbol{x}}+\tilde{\boldsymbol{B}}_{1} \boldsymbol{w}+\tilde{\boldsymbol{B}}_{2} \tilde{\boldsymbol{u}} \\
\boldsymbol{z}=\tilde{\boldsymbol{C}}_{1} \tilde{\boldsymbol{x}}+\tilde{\boldsymbol{D}}_{11} \boldsymbol{w}+\tilde{\boldsymbol{D}}_{12} \tilde{\boldsymbol{u}} \\
\tilde{\boldsymbol{y}}=\tilde{\boldsymbol{C}}_{2} \tilde{\boldsymbol{x}}+\tilde{\boldsymbol{D}}_{21} \boldsymbol{w}+\tilde{\boldsymbol{D}}_{22} \tilde{\boldsymbol{u}},
\end{array}\right. \\
& \mathbf{K}: \tilde{\boldsymbol{u}}=\tilde{\boldsymbol{K}} \tilde{\boldsymbol{y}}
\end{aligned}
$$

mit den Matrizen

$$
\begin{aligned}
& \tilde{\boldsymbol{A}}=\boldsymbol{A}, \quad \tilde{\boldsymbol{B}}_{1}=\boldsymbol{B}_{d}, \quad \tilde{\boldsymbol{B}}_{2}=\left[\begin{array}{ll}
\mathbf{0} & -\boldsymbol{I}_{n}
\end{array}\right], \\
& \tilde{\boldsymbol{C}}_{1}=\mathbf{0}, \quad \tilde{\boldsymbol{D}}_{11}=\mathbf{0}, \quad \tilde{\boldsymbol{D}}_{12}=\left[\begin{array}{ll}
\boldsymbol{I}_{n_{f}} & \mathbf{0}
\end{array}\right], \\
& \tilde{\boldsymbol{C}}_{2}=\boldsymbol{C}, \quad \tilde{\boldsymbol{D}}_{21}=\boldsymbol{D}_{d}, \quad \tilde{\boldsymbol{D}}_{22}=\mathbf{0}
\end{aligned}
$$

und dem verallgemeinerten Regler

$$
\tilde{\boldsymbol{K}}=\left[\begin{array}{l}
\boldsymbol{V}\left(\boldsymbol{z}_{i, 0}, \boldsymbol{R}_{V}\right) \\
\boldsymbol{L}\left(\boldsymbol{q}_{i, j}, \boldsymbol{R}_{L}\right)
\end{array}\right]
$$


schreiben. Die Struktur der Reglermatrix $\tilde{\boldsymbol{K}}$ stellt die Diagonalisierung von $\boldsymbol{G}_{\boldsymbol{r} \boldsymbol{f}}(s)$ sicher und das Ein-/Ausgangsverhalten des StandardRegelungsproblems entspricht der Übertragung der Störungen auf die Residuen. Es ist somit $\boldsymbol{G}_{\boldsymbol{z} \boldsymbol{w}}(s)=\boldsymbol{G}_{\boldsymbol{r} \boldsymbol{d}}(s)$. Beachtenswert ist, dass im Gegensatz zum quadratischen Fall aus Abschnitt 5.3.1 die verallgemeinerte Reglermatrix $\tilde{\boldsymbol{K}}$ nicht nur von den Koeffizienten $\boldsymbol{z}_{i, 0}$ und $\boldsymbol{q}_{i, j}$ abhängt, sondern auch von den frei wählbaren Matrizen $\boldsymbol{R}_{V}$ und $\boldsymbol{R}_{L}$. Wie bereits erörtert beschreiben diese gemäß

$$
\begin{aligned}
\boldsymbol{L}\left(\boldsymbol{q}_{i, j}, \boldsymbol{R}_{L}\right) & =\boldsymbol{M}\left(\boldsymbol{q}_{i, j}\right) \boldsymbol{D}^{*+}+\boldsymbol{R}_{L} \cdot{ }^{\perp} \boldsymbol{D}^{*} \\
\boldsymbol{V}\left(\boldsymbol{z}_{i, 0}, \boldsymbol{R}_{L}\right) & =\boldsymbol{N}\left(\boldsymbol{z}_{i, 0}\right) \boldsymbol{D}^{*+}+\boldsymbol{R}_{V} \cdot{ }^{\perp} \boldsymbol{D}^{*}
\end{aligned}
$$

die zusätzlichen Freiheitsgrade gegenüber dem quadratischen Fall, wobei die Matrizen $\boldsymbol{M}\left(\boldsymbol{q}_{i, j}\right)$ und $\boldsymbol{N}\left(\boldsymbol{z}_{i, 0}\right)$ in Satz 4.8 eingeführt wurden.

Es erfolgt nun wiederum eine Umformung in ein StandardRegelungsproblem, bei dem die Elemente der verallgemeinerten Reglermatrix entweder auf null fixiert sind oder aber freie Parameter der Optimierung darstellen. Greift man auf die Matrizen

$$
\begin{aligned}
& \boldsymbol{Q}_{L}\left(\boldsymbol{q}_{i, j}\right)=\left[\begin{array}{cccccc}
q_{1, \delta_{1}-1} & 0 & \cdots & \cdots & \cdots & 0 \\
\vdots & \vdots & & & & \vdots \\
q_{1,0} & 0 & \cdots & \cdots & \ldots & 0 \\
0 & q_{2, \delta_{2}-1} & 0 & \cdots & \cdots & 0 \\
\vdots & \vdots & \vdots & & & \vdots \\
0 & q_{2,0} & 0 & \cdots & \cdots & 0 \\
& & \ddots & & & \\
0 & \ldots & \cdots & \cdots & 0 & q_{n_{f}, \delta_{n_{f}-1}} \\
\vdots & & & & \vdots & \vdots \\
0 & \cdots & \cdots & \cdots & 0 & q_{n_{f}, 0}
\end{array}\right] \text {, } \\
& \boldsymbol{Q}_{V}\left(\boldsymbol{z}_{i, 0}\right)=\operatorname{diag}\left(z_{1,0}, \ldots, z_{n_{f}, 0}\right) \text {, } \\
& \boldsymbol{M}_{\alpha}=\left[\begin{array}{lll}
\boldsymbol{A}^{\delta_{1}} \boldsymbol{e}_{a_{1}} & \cdots & \boldsymbol{A}^{\delta_{n_{f}}} \boldsymbol{e}_{a_{n_{f}}}
\end{array}\right], \\
& \boldsymbol{M}_{\beta}=\left[\begin{array}{llllllll}
\boldsymbol{A}^{\delta_{1}-1} \boldsymbol{e}_{a_{1}} & \boldsymbol{A}^{\delta_{1}-2} \boldsymbol{e}_{a_{1}} & \cdots & \boldsymbol{A} \boldsymbol{e}_{a_{1}} & \boldsymbol{e}_{a_{1}} & \boldsymbol{A}^{\delta_{2}-1} \boldsymbol{e}_{a_{2}} & \cdots & \boldsymbol{e}_{a_{n_{f}}}
\end{array}\right]
\end{aligned}
$$

zurück und betrachtet das Ein-/Ausgangsverhalten bezüglich $\boldsymbol{w}$ und $\boldsymbol{z}$ (also die Übertragungsmatrix $\boldsymbol{G}_{\boldsymbol{z} \boldsymbol{w}}(s)$ ), so wird deutlich, dass das Standard- 
Regelungsproblem mit den Matrizen

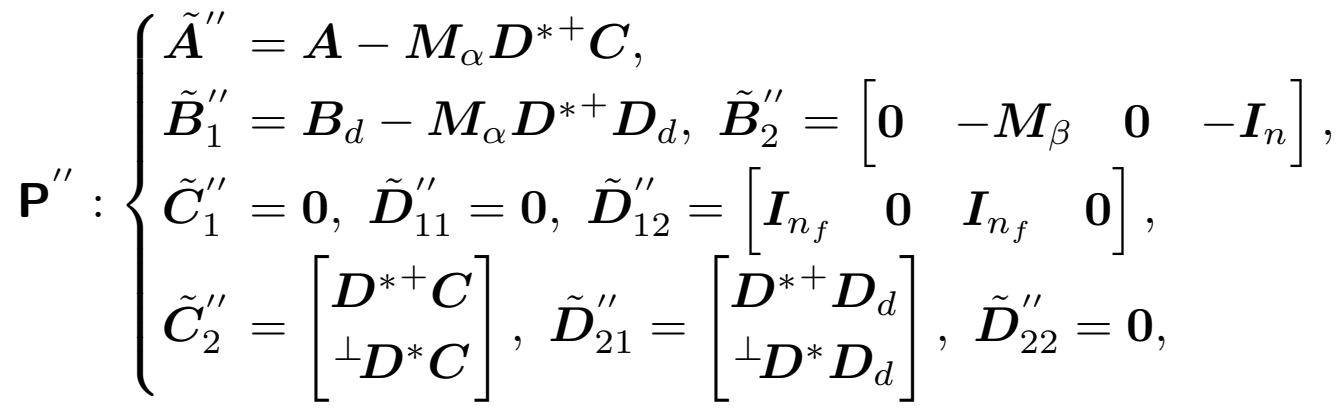

$$
\begin{aligned}
& \mathbf{K}^{\prime \prime}: \tilde{\boldsymbol{K}}^{\prime \prime}=\left[\begin{array}{cc}
\boldsymbol{Q}_{V}\left(\boldsymbol{z}_{i, 0}\right) & \mathbf{0} \\
\boldsymbol{Q}_{L}\left(\boldsymbol{q}_{i, j}\right) & \mathbf{0} \\
\mathbf{0} & \boldsymbol{R}_{V} \\
\mathbf{0} & \boldsymbol{R}_{L}
\end{array}\right]
\end{aligned}
$$

das gleiche Übertragungsverhalten realisiert wie $(5.26 \mathrm{~b}),(5.26 \mathrm{c}) \mathrm{mit}$ (5.27). Dazu wird jeweils $\tilde{\boldsymbol{y}}=\tilde{\boldsymbol{C}}_{2} \tilde{\boldsymbol{x}}+\tilde{\boldsymbol{D}}_{21} \boldsymbol{w}+\tilde{\boldsymbol{D}}_{22} \tilde{\boldsymbol{u}}$ in $\tilde{\boldsymbol{u}}=\tilde{\boldsymbol{K}} \tilde{\boldsymbol{y}}$ eingesetzt. Setzt man das sich ergebende $\tilde{\boldsymbol{u}}$ in die Dynamikgleichung $\dot{\tilde{\boldsymbol{x}}}$ beziehungsweise die Ausgangsgleichung von $\boldsymbol{z}$ ein, so erhält man das jeweilige dynamische System, welches $\boldsymbol{w}$ und $\boldsymbol{z}$ verknüpft und daraus die Übertragungsmatrix $\boldsymbol{G}_{\boldsymbol{z} \boldsymbol{w}}(s)$. Dabei weist $\tilde{\boldsymbol{K}}^{\prime \prime}$ die geforderte Struktur auf, die es erlaubt, Standard-Solver wie Hifoo [91], Hinfstruct [9] oder SysTUNE [8] zur Lösung des Problems zu verwenden. Die Ideen, die den Umformungen von $(\mathbf{P}, \mathbf{K}) \mathrm{zu}\left(\mathbf{P}^{\prime \prime}, \mathbf{K}^{\prime \prime}\right)$ zu Grunde liegen, entsprechen dabei denen aus Abschnitt 5.3.1: Es werden Teile des verallgemeinerten Reglers der verallgemeinerten Strecke zugeschlagen, sodass in $\tilde{\boldsymbol{K}}^{\prime \prime}$ lediglich Elemente verbleiben, die entweder Nullelemente sind oder aber frei wählbare Parameter darstellen.

Um die degenerierte Lösung $\boldsymbol{z}_{i, 0}=\mathbf{0}$ zu vermeiden und eine stationäre Verstärkung der Fehler-Residuen-Übertragungskanäle von $g_{i, i}(s=0)=k_{s, i}$ sicherzustellen, wird analog zu Abschnitt 5.3.1 verfahren. Um jeweils $z_{i, 0}=k_{s, i} q_{i, 0}$ zu gewährleisten, wird auf die Matrix

$$
\mathbf{\Upsilon}=\left[\begin{array}{c}
\boldsymbol{\Phi} \\
\boldsymbol{I}_{\delta}
\end{array}\right] \operatorname{mit} \mathbf{\Phi}=\left[\begin{array}{c}
k_{s, 1} \tilde{\boldsymbol{\phi}}_{1}^{\top} \\
\vdots \\
k_{s, n_{f}} \tilde{\boldsymbol{\phi}}_{n_{f}}^{\top}
\end{array}\right]
$$

zurückgegriffen. Sie beinhaltet mit $\tilde{\phi}_{i}^{\top}=\left[\begin{array}{lllllll}0 & \cdots & 0 & 1 & 0 & \cdots & 0\end{array}\right]$ Einheitsvektoren, welche in der $\sum_{k=1}^{i} \delta_{k}$-ten Spalte das Element 1 enthalten. ${ }^{4)}$

${ }^{4)}$ Diese Vektoren sind nicht zu verwechseln mit dem bereits eingeführten Vektor $\phi_{i}^{\top}$, der in der $i$-ten Spalte das Element 1 beinhaltet. 
Damit lässt sich das Standard-Regelungsproblem bei unverändertem Übertragungsverhalten $\boldsymbol{G}_{\boldsymbol{z} \boldsymbol{w}}(s)=\boldsymbol{G}_{\boldsymbol{r} \boldsymbol{d}}(s)$ gemäß

$$
\begin{aligned}
\tilde{\boldsymbol{B}}_{2}^{\prime \prime \prime} & =\left[\begin{array}{llll}
\mathbf{0} & -\boldsymbol{M}_{\beta} & \mathbf{0} & -\boldsymbol{I}_{n}
\end{array}\right] \cdot \operatorname{diag}\left(\boldsymbol{\Upsilon}, \boldsymbol{I}_{n_{f}}, \boldsymbol{I}_{n}\right), \\
\tilde{\boldsymbol{D}}_{12}^{\prime \prime \prime} & =\left[\begin{array}{llll}
\boldsymbol{I}_{n_{f}} & \mathbf{0} & \boldsymbol{I}_{n_{f}} & \mathbf{0}
\end{array}\right] \cdot \operatorname{diag}\left(\boldsymbol{\Upsilon}, \boldsymbol{I}_{n_{f}}, \boldsymbol{I}_{n}\right), \\
\tilde{\boldsymbol{K}}^{\prime \prime \prime} & =\left[\begin{array}{cc}
\boldsymbol{Q}_{L}\left(\boldsymbol{q}_{i, j}\right) & \mathbf{0} \\
\mathbf{0} & \boldsymbol{R}_{V} \\
\mathbf{0} & \boldsymbol{R}_{L}
\end{array}\right]
\end{aligned}
$$

modifizieren. Zusammenfassend gilt

Satz 5.4 (Entwurf robuster FIOs für nichtquadratische Systeme durch Lösung eines strukturbeschränkten Standard-Regelungsproblems). Das Optimierungsproblem

$$
\begin{aligned}
& \underset{\boldsymbol{q}_{i, j}, \boldsymbol{z}_{i, 0}, \boldsymbol{R}_{L}, \boldsymbol{R}_{V}}{\operatorname{minimiere}} \gamma, \text { sodass } \\
& \left\|\boldsymbol{G}_{\boldsymbol{r} \boldsymbol{d}}\right\|_{\infty}<\gamma \\
& \boldsymbol{G}_{\boldsymbol{r} \boldsymbol{f}}(s)=\operatorname{diag}\left(g_{1,1}(s), \ldots, g_{n_{f}, n_{f}}(s)\right), g_{i, i}(s=0)=k_{s, i} \forall i=1, \ldots, n_{f}
\end{aligned}
$$

lässt sich äquivalent formulieren als strukturbeschränktes StandardRegelungsproblem der Form (5.26) mit

$$
\begin{aligned}
& \tilde{\boldsymbol{A}}^{\prime \prime \prime}=\boldsymbol{A}-\boldsymbol{M}_{\alpha} \boldsymbol{D}^{*+} \boldsymbol{C}, \\
& \tilde{\boldsymbol{B}}_{1}^{\prime \prime \prime}=\boldsymbol{B}_{d}-\boldsymbol{M}_{\alpha} \boldsymbol{D}^{*+} \boldsymbol{D}_{d}, \tilde{\boldsymbol{B}}_{2}^{\prime \prime \prime}=\left[\begin{array}{llll}
\mathbf{0} & -\boldsymbol{M}_{\beta} & \mathbf{0} & -\boldsymbol{I}_{n}
\end{array}\right] \cdot \operatorname{diag}\left(\boldsymbol{\Upsilon}, \boldsymbol{I}_{n_{f}}, \boldsymbol{I}_{n}\right), \\
& \tilde{\boldsymbol{C}}_{1}^{\prime \prime \prime}=\mathbf{0}, \tilde{\boldsymbol{D}}_{11}^{\prime \prime \prime}=\mathbf{0}, \tilde{\boldsymbol{D}}_{12}^{\prime \prime \prime}=\left[\begin{array}{llll}
\boldsymbol{I}_{n_{f}} & \mathbf{0} & \boldsymbol{I}_{n_{f}} & \mathbf{0}
\end{array}\right] \cdot \operatorname{diag}\left(\boldsymbol{\Upsilon}, \boldsymbol{I}_{n_{f}}, \boldsymbol{I}_{n}\right), \\
& \tilde{\boldsymbol{C}}_{2}^{\prime \prime \prime}=\left[\begin{array}{l}
\boldsymbol{D}^{*+} \boldsymbol{C} \\
{ }^{\perp} \boldsymbol{D}^{*} \boldsymbol{C}
\end{array}\right], \tilde{\boldsymbol{D}}_{21}^{\prime \prime \prime}=\left[\begin{array}{l}
\boldsymbol{D}^{*+} \boldsymbol{D}_{d} \\
{ }^{\perp} \boldsymbol{D}^{*} \boldsymbol{D}_{d}
\end{array}\right], \tilde{\boldsymbol{D}}_{22}^{\prime \prime \prime}=\mathbf{0}
\end{aligned}
$$

und dem verallgemeinerten Regler

$$
\tilde{\boldsymbol{K}}^{\prime \prime \prime}=\left[\begin{array}{cc}
\boldsymbol{Q}_{L}\left(\boldsymbol{q}_{i, j}\right) & \mathbf{0} \\
\mathbf{0} & \boldsymbol{R}_{V} \\
\mathbf{0} & \boldsymbol{R}_{L}
\end{array}\right] .
$$

Um den Realteil der Beobachtereigenwerte zu beschränken, kann auch bei nichtquadratischen Systemen eine zusätzliche verallgemeinerte Strecke definiert werden, für welche die Beschränkung einzuhalten ist. Diese 
virtuelle Strecke wird beschrieben durch

$$
\begin{aligned}
\overline{\boldsymbol{A}} & =\boldsymbol{A}-\boldsymbol{M}_{\alpha} \boldsymbol{D}^{*+} \boldsymbol{C}, \\
\overline{\boldsymbol{B}}_{2} & =\left[\begin{array}{llll}
\mathbf{0} & -\boldsymbol{M}_{\beta} & \mathbf{0} & -\boldsymbol{I}_{n}
\end{array}\right] \cdot \operatorname{diag}\left(\boldsymbol{\Upsilon}, \boldsymbol{I}_{n_{f}}, \boldsymbol{I}_{n}\right), \\
\overline{\boldsymbol{C}}_{2} & =\left[\begin{array}{l}
\boldsymbol{D}^{*+} \boldsymbol{C} \\
{ }^{\perp} \boldsymbol{D}^{*} \boldsymbol{C}
\end{array}\right],
\end{aligned}
$$

wobei die übrigen Matrizen der Strecke identisch 0 sind.

\section{Beispiel Hydrauliksystem}

In Abschnitt 5.4.1 ließ sich für das Hydrauliksystem durch die zusätzlichen Freiheitsgrade im nichtquadratischen System eine Schranke von $\gamma_{\mathrm{opt}}=23,70$ für den maximalen Singulärwert von $\boldsymbol{G}_{\boldsymbol{r} \boldsymbol{d}}(s)$ erzielen. Bezieht man die Diagonalelemente von $\boldsymbol{G}_{\boldsymbol{r} \boldsymbol{f}}(s)$ in die Optimierung mit ein, so lässt sich diese Schranke weiter senken. Mit der Parametrierung aus Abschnitt 5.4.1 als Startwert und einer Beschränkung der Beobachtereigenwerte von $\mathfrak{R e}\left(\lambda_{B_{i}}\right)<-1,5$ durch (5.28) findet man mittels Satz 5.4 die FIO-Parametrierung $\left(\boldsymbol{L}_{\text {opt,ew }}, \boldsymbol{V}_{\text {opt,ew }}\right)$, die in Anhang C.2.3 angegeben ist. Sie platziert die Beobachtereigenwerte bei

$$
\begin{aligned}
& \lambda_{B_{1,1}}=-2,196, \quad \lambda_{B_{1,2 / 3}}=-1,705 \pm 2,927 j, \\
& \lambda_{B_{2,1}}=-1,500, \quad \lambda_{B_{5}}=-44,632
\end{aligned}
$$

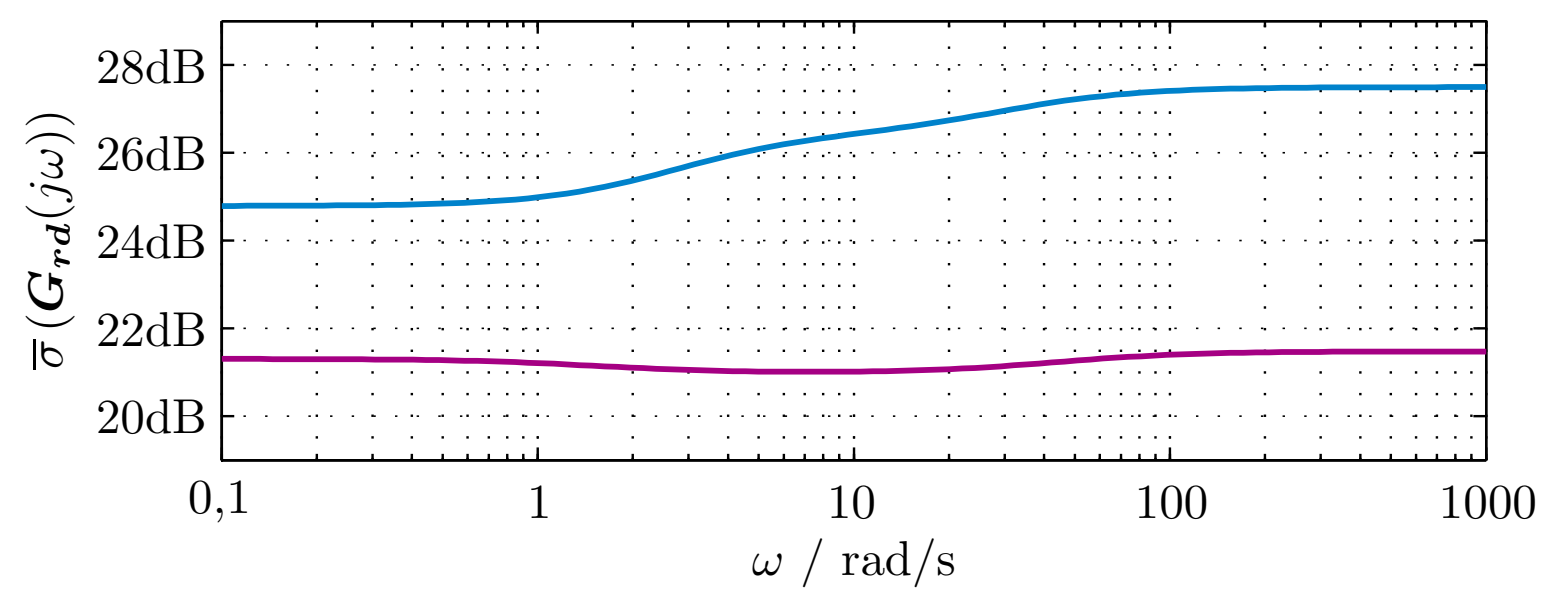

Abbildung 5.11: Verlauf des größten Singulärwertes von $\boldsymbol{G}_{\boldsymbol{r} \boldsymbol{d}}(s)$ für mittels $\boldsymbol{R}_{L}$ und $\boldsymbol{R}_{V}$ optimierten FIO (-) und für mittels $\boldsymbol{R}_{L}, \boldsymbol{R}_{V}$ und $\boldsymbol{q}_{i, j}$ optimierten FIO (-) 
und erreicht damit eine Schranke von $\gamma_{\text {opt,ew }}=11,85$. Diese signifikante Verbesserung geht auch aus dem Singulärwertverlauf von $\boldsymbol{G}_{\boldsymbol{r} \boldsymbol{d}}(s)$ in Abbildung 5.11 hervor. Dieser zeigt in verändertem Maßstab und in blauer Farbe nochmals den Verlauf für die FIO-Parametrierung aus Abschnitt 5.4.1 (vgl. Abbildung 5.10). Zusätzlich ist der Verlauf für die Parametrierung $\left(\boldsymbol{L}_{\text {opt,ew }}, \boldsymbol{V}_{\text {opt,ew }}\right)$ zu sehen, der über alle Frequenzen deutlich verbessert ist. $\mathrm{Zu}$ bemerken ist allerdings auch, dass dies zum Teil mit einer verringerten Detektionsgeschwindigkeit im zweiten Übertragungskanal erkauft wird.

\subsubsection{Robustheit bezüglich exogener Störungen in beschränkten Frequenzbereichen}

In Abschnitt 5.4.1 wurde die Robustheit der Residuengenerierung bezüglich beliebiger exogener Störungen optimiert. Durch die Verwendung von $\left\|\boldsymbol{G}_{\boldsymbol{r} \boldsymbol{d}}(s)\right\|_{\infty}$ als Robustheitsmaß wird dabei der Worst-Case abgedeckt, das heißt, es wird die größtmögliche Störverstärkung minimiert. Diese Herangehensweise bietet sich an, wenn keinerlei weitere Kenntnisse über die Störungen vorhanden sind. Häufig ist beim Entwurf jedoch zusätzliches Wissen vorhanden, da oftmals relevante Frequenzbereiche von Störungen bekannt sind. Nimmt man an, dass Störungen nur in einem Frequenzbereich $\Omega=\left[\omega_{l} ; \omega_{h}\right]$ auftreten können, so ist wie in den Abschnitten 2.5 und 3.1 erörtert durch

$$
\left\|\boldsymbol{G}_{\boldsymbol{r d}}(s)\right\|_{\infty}^{\Omega}
$$

ein intuitives Maß für die Robustheit gegeben. Man spricht von beschränkten Frequenzbereichen (engl. finite frequency ranges).

Abbildung 3.1 auf Seite 49 veranschaulicht den Unterschied zwischen den beiden verwendeten Robustheitsmaßen. Daraus geht unmittelbar hervor, dass die Verwendung von $\left\|\boldsymbol{G}_{\boldsymbol{r} \boldsymbol{d}}(s)\right\|_{\infty}$ eine sehr konservative Abschätzung für die relevante Störverstärkung darstellen kann, falls die Störungen nur im Frequenzbereich $\Omega$ liegen. Diese schlechte Abschätzung impliziert auch konservative Resultate beim Entwurf eines robusten FIOs nach Satz 5.3. Durch die Verwendung von $\left\|\boldsymbol{G}_{\boldsymbol{r} \boldsymbol{d}}(s)\right\|_{\infty}^{\Omega}$ lässt sich also Prozesswissen über die relevanten Frequenzbereiche von Störungen in den Entwurf einbringen, um so die Konservativität zu senken.

Dabei wird ähnlich wie in Abschnitt 5.4.1 das ursprüngliche Optimierungsproblem

$$
\begin{aligned}
& \underset{\boldsymbol{L}, \boldsymbol{V}}{\operatorname{minimiere}}\left\|\boldsymbol{G}_{\boldsymbol{r d}}(s)\right\|_{\infty}^{\Omega}, \text { sodass } \\
& \boldsymbol{G}_{\boldsymbol{r} \boldsymbol{f}}(s)=\operatorname{diag}\left(g_{1,1}(s), \ldots, g_{n_{f}, n_{f}}(s)\right)
\end{aligned}
$$


durch Rückgriff auf Satz 4.8 mit $\quad \boldsymbol{L}=\boldsymbol{L}_{0}+\boldsymbol{R}_{L} \cdot{ }^{\perp} \boldsymbol{D}^{*} \quad$ und $\boldsymbol{V}=\boldsymbol{V}_{0}+\boldsymbol{R}_{V} \cdot{ }^{\perp} \boldsymbol{D}^{*}$ als

$$
\underset{\boldsymbol{R}_{L}, \boldsymbol{R}_{V}}{\operatorname{minimiere}}\left\|\boldsymbol{G}_{\boldsymbol{r d}}(s)\right\|_{\infty}^{\Omega}
$$

geschrieben. Verwandte Problemstellungen sind aus dem Bereich der Fehlerdetektion bekannt, sie werden wie in Abschnitt 2.5 erörtert typischerweise durch den Einsatz von Gewichtungsfiltern gelöst [25, 129, 135, 247]. Dabei wird $\left\|\boldsymbol{G}_{\boldsymbol{r} \boldsymbol{d}}(s)\right\|_{\infty}^{\Omega} \approx\left\|\boldsymbol{G}_{\boldsymbol{r} \boldsymbol{d}}(s) \cdot \boldsymbol{G}_{\mathrm{filt}}(s)\right\|_{\infty}$ angenähert (vgl. Abschnitt 5.3.1), um mit der bekannten $\mathcal{H}_{\infty}$-Norm über alle Frequenzen arbeiten zu können. $\mathrm{Zu}$ diesem Zweck ist jedoch das Gewichtungsfilter $\boldsymbol{G}_{\text {filt }}(s)$ separat auszulegen und die Ordnung des betrachteten Systems erhöht sich.

Eine alternative Herangehensweise wird durch das verallgemeinerte Kalman-Yakubovich-Popov-Lemma (GKYP-Lemma $[113,176]$, s. Satz 3.2) eröffnet. Es ermöglicht eine direkte Charakterisierung der $\mathcal{H}_{\infty^{-}}$ Norm in beschränkten Frequenzbereichen. Sowohl im Bereich diskreter Filter [245] als auch der Fehlerdetektion in kontinuierlichen [48, 246, 254], diskreten [244] und vernetzten Systemen [141] hat das Lemma in jüngerer Vergangenheit vielfach Anwendung gefunden.

Auch für das vorliegende Fehlerisolationsproblem ist es mittels Satz 3.2 möglich, das Optimierungsproblem (5.29) umzuformulieren zu

$$
\begin{aligned}
& \operatorname{minimiere}_{\boldsymbol{X}, \boldsymbol{Y}, \boldsymbol{R}_{L}, \boldsymbol{R}_{V}} \gamma, \text { sodass } \\
& \boldsymbol{X} \succ \mathbf{0}, \omega_{c}=\frac{1}{2}\left(\omega_{l}+\omega_{h}\right), \\
& \boldsymbol{\Xi}=\left[\begin{array}{cc}
-\boldsymbol{X} & \boldsymbol{Y}+j \omega_{c} \boldsymbol{X} \\
\boldsymbol{Y}-j \omega_{c} \boldsymbol{X} & -\omega_{l} \omega_{h} \boldsymbol{X}
\end{array}\right], \boldsymbol{\Gamma}=\left[\begin{array}{cc}
\boldsymbol{I}_{n_{f}} & \mathbf{0} \\
\mathbf{0} & -\gamma^{2} \boldsymbol{I}_{n_{d}}
\end{array}\right], \\
& \underbrace{\left[\begin{array}{cc}
\boldsymbol{A}-\boldsymbol{L C} & \boldsymbol{B}_{d}-\boldsymbol{L} \boldsymbol{D}_{d} \\
\boldsymbol{I}_{n} & \mathbf{0} \\
\boldsymbol{V} \boldsymbol{C} & \boldsymbol{V} \boldsymbol{D}_{d} \\
\mathbf{0} & \boldsymbol{I}_{n_{d}}
\end{array}\right]^{\top}}_{\perp_{\boldsymbol{N}}} \cdot \underbrace{\left[\begin{array}{cc}
\boldsymbol{\Xi} & \mathbf{0} \\
\mathbf{0} & \boldsymbol{\Gamma}
\end{array}\right]}_{\boldsymbol{Z}} \cdot \underbrace{\left[\begin{array}{cc}
\boldsymbol{A}-\boldsymbol{L C} & \boldsymbol{B}_{d}-\boldsymbol{L} \boldsymbol{D}_{d} \\
\boldsymbol{I}_{n} & \mathbf{0} \\
\boldsymbol{V} \boldsymbol{C} & \boldsymbol{V} \boldsymbol{D}_{d} \\
\mathbf{0} & \boldsymbol{I}_{n_{d}}
\end{array}\right]}_{\perp_{\boldsymbol{N}^{\top}}} \prec \mathbf{0} \\
& \boldsymbol{L}=\boldsymbol{L}_{0}+\boldsymbol{R}_{L} \cdot{ }^{\perp} \boldsymbol{D}^{*}, \quad \boldsymbol{V}=\boldsymbol{V}_{0}+\boldsymbol{R}_{V} \cdot{ }^{\perp} \boldsymbol{D}^{*} .
\end{aligned}
$$

Darin ist ${ }^{\perp} \boldsymbol{N} \in \mathbb{R}^{\left(n+n_{d}\right) \times\left(2 n+n_{f}+n_{d}\right)}$, sodass aus Satz A.1 die Beziehung $\operatorname{rang}(\boldsymbol{N})=n+n_{f}$ folgt. Dementsprechend muss die zugehörige Matrix 
$\boldsymbol{N} \in \mathbb{R}^{\left(2 n+n_{f}+n_{d}\right) \times \chi}$ sein, wobei $\chi \geq n+n_{f}$ ist. Mithilfe von Lemma 3.4, welches sich aus Finslers Lemma [205] ergibt, lässt sich die Matrixungleichung (5.30d) schreiben als

$$
\boldsymbol{Z}+\mathrm{He}\left(\boldsymbol{\Lambda} \boldsymbol{N}^{\top}\right) \prec \mathbf{0},
$$

wobei $\boldsymbol{\Lambda} \in \mathbb{R}^{\left(2 n+n_{f}+n_{d}\right) \times \chi}$ eine beliebige Matrix mit mindestens $n+n_{f}$ Spalten ist.

Da ${ }^{\perp} \boldsymbol{N} \cdot \boldsymbol{N}=\mathbf{0}$ gilt, gilt auch $\boldsymbol{N}^{\top} \cdot{ }^{\perp} \boldsymbol{N}^{\top}=\mathbf{0}$ und mit einer beliebigen Matrix $\boldsymbol{\Psi} \in \mathbb{R}^{\chi \times\left(n+n_{f}\right)}$ vollen Ranges lässt sich die Matrix $\boldsymbol{N}^{\top}$ parametrieren als

$$
\boldsymbol{N}^{\top}=\boldsymbol{\Psi}\left[\begin{array}{cccc}
\boldsymbol{I}_{n} & -(\boldsymbol{A}-\boldsymbol{L} \boldsymbol{C}) & \mathbf{0} & -\left(\boldsymbol{B}_{d}-\boldsymbol{L} \boldsymbol{D}_{d}\right) \\
\mathbf{0} & -\boldsymbol{V} \boldsymbol{C} & \boldsymbol{I}_{n_{f}} & -\boldsymbol{V} \boldsymbol{D}_{d}
\end{array}\right]
$$

Im Weiteren wird (5.30e) eingesetzt und es werden die abkürzenden Schreibweisen

$$
\begin{aligned}
& \tilde{\Lambda}=\Lambda \Psi, \quad \tilde{\boldsymbol{C}}=\left[\begin{array}{llll}
\mathbf{0} & -{ }^{\perp} \boldsymbol{D}^{*} \cdot \boldsymbol{C} & \mathbf{0} & -{ }^{\perp} \boldsymbol{D}^{*} \cdot \boldsymbol{D}_{d}
\end{array}\right], \\
& \tilde{\boldsymbol{L}}=\left[\begin{array}{c}
\boldsymbol{R}_{L} \\
-\boldsymbol{R}_{V}
\end{array}\right], \tilde{\boldsymbol{A}}=\left[\begin{array}{cccc}
\boldsymbol{I}_{n} & -\left(\boldsymbol{A}-\boldsymbol{L}_{0} \boldsymbol{C}\right) & \mathbf{0} & -\left(\boldsymbol{B}_{d}-\boldsymbol{L}_{0} \boldsymbol{D}_{d}\right) \\
\mathbf{0} & -\boldsymbol{V}_{0} \boldsymbol{C} & \boldsymbol{I}_{n_{f}} & -\boldsymbol{V}_{0} \boldsymbol{D}_{d}
\end{array}\right]
\end{aligned}
$$

eingeführt. Zu beachten ist dabei, dass $\tilde{\Lambda} \in \mathbb{R}^{\left(2 n+n_{f}+n_{d}\right) \times\left(n+n_{f}\right)}$ gilt und die unbestimmte Anzahl $\chi \geq n+n_{f}$ der Spalten von $\boldsymbol{\Lambda}$ keine Rolle spielt. Damit lässt sich (5.31) weiter vereinfachen und das Optimierungsproblem (5.30) lässt sich äquivalent formulieren als

$$
\begin{aligned}
& \underset{\boldsymbol{X}, \boldsymbol{Y}, \tilde{\boldsymbol{\Lambda}}, \boldsymbol{R}_{L}, \boldsymbol{R}_{V}}{\operatorname{minimiere}} \gamma, \text { sodass } \\
& \boldsymbol{X} \succ \mathbf{0} \\
& \boldsymbol{Z}+\operatorname{He}(\tilde{\boldsymbol{\Lambda}}(\tilde{\boldsymbol{A}}-\tilde{\boldsymbol{L}} \tilde{\boldsymbol{C}})) \prec \mathbf{0} .
\end{aligned}
$$

Dabei stellt (5.32c) im Gegensatz zu (5.30d) eine BMI dar. Ähnlich zum Vorgehen in [48, 245] lässt sich diese in eine LMI überführen, wenn $\boldsymbol{R}_{V}=\mathbf{0}$ gewählt wird. Da dies jedoch die Anzahl der zur Verfügung stehenden Freiheitsgrade verringert, wird dieser Ansatz hier nicht weiter verfolgt.

Wie bei der Optimierung bezüglich exogener Störungen mit beliebigen Frequenzen in Abschnitt 5.4.1 erörtert ist es weiterhin sinnvoll, in (5.32) die Beobachtereigenwerte bei der Optimierung einzuschränken. Dazu wird eine weitere symmetrische Matrix $\boldsymbol{X}_{\mathrm{EW}} \in \mathbb{R}^{n \times n}$ eingeführt. Die Beschränkung 
der nichtsteuerbaren Beobachtereigenwerte auf die in Abbildung 3.2 auf Seite 59 gezeigte $\mathcal{D}$-Region ist gewährleistet, wenn

$$
\begin{aligned}
& \boldsymbol{X}_{\mathrm{EW}} \succ \mathbf{0}, \\
& \mathrm{He}\left(\boldsymbol{X}_{\mathrm{EW}}\left(\boldsymbol{A}-\boldsymbol{L}_{0} \boldsymbol{C}\right)-\boldsymbol{X}_{\mathrm{EW}} \boldsymbol{R}_{L} \cdot{ }^{\perp} \boldsymbol{D}^{*} \cdot \boldsymbol{C}\right)-2 \alpha \boldsymbol{X}_{\mathrm{EW}} \prec \mathbf{0}, \\
& {\left[\begin{array}{cc}
-\varrho \boldsymbol{X}_{\mathrm{EW}} & \boldsymbol{X}_{\mathrm{EW}}\left(\boldsymbol{A}-\boldsymbol{L}_{0} \boldsymbol{C}\right)-\boldsymbol{X}_{\mathrm{EW}} \boldsymbol{R}_{L} \cdot{ }^{\perp} \boldsymbol{D}^{*} \cdot \boldsymbol{C} \\
\star & -\varrho \boldsymbol{X}_{\mathrm{EW}}
\end{array}\right] \prec \mathbf{0},} \\
& {\left[\begin{array}{cc}
\boldsymbol{\Phi}_{11} & \boldsymbol{\Phi}_{12} \\
\star & \boldsymbol{\Phi}_{11}
\end{array}\right] \prec \mathbf{0}} \\
& \boldsymbol{\Phi}_{11}=\tan (\phi) \cdot \operatorname{He}\left(\boldsymbol{X}_{\mathrm{EW}}\left(\boldsymbol{A}-\boldsymbol{L}_{0} \boldsymbol{C}\right)-\boldsymbol{X}_{\mathrm{EW}} \boldsymbol{R}_{L} \cdot{ }^{\perp} \boldsymbol{D}^{*} \boldsymbol{C}\right), \\
& \boldsymbol{\Phi}_{12}=-\boldsymbol{X}_{\mathrm{EW}}\left(\boldsymbol{A}-\boldsymbol{L}_{0} \boldsymbol{C}\right)+\left(\boldsymbol{A}-\boldsymbol{L}_{0} \boldsymbol{C}\right)^{\top} \boldsymbol{X}_{\mathrm{EW}}+\ldots \\
& +\boldsymbol{X}_{\mathrm{EW}} \boldsymbol{R}_{L} \cdot{ }^{\perp} \boldsymbol{D}^{*} \boldsymbol{C}-\left(\boldsymbol{X}_{\mathrm{EW}} \boldsymbol{R}_{L} \cdot{ }^{\perp} \boldsymbol{D}^{*} \boldsymbol{C}\right)^{\top}
\end{aligned}
$$

gilt. Zusammenfassend folgt

Satz 5.5 (BMI-basierter Entwurf robuster FIOs in beschränkten Frequenzbereichen für nichtquadratische Systeme mit Beschränkung der Beobachtereigenwerte). Gegeben sei ein System der Form

$$
\begin{aligned}
& \dot{\boldsymbol{x}}=\boldsymbol{A} \boldsymbol{x}+\boldsymbol{B} \boldsymbol{u}+\boldsymbol{E}_{a} \boldsymbol{f}+\boldsymbol{B}_{d} \boldsymbol{d}, \\
& \boldsymbol{y}=\boldsymbol{C} \boldsymbol{x}+\boldsymbol{D} \boldsymbol{u}+\boldsymbol{E}_{s} \boldsymbol{f}+\boldsymbol{D}_{d} \boldsymbol{d},
\end{aligned}
$$

welches die Annahmen 5.1-5.3 erfüllt, und eine FIO-Parametrierung $\left(\boldsymbol{L}_{0}, \boldsymbol{V}_{0}\right)$ gemäß Satz 4.8. Dann liefert das Optimierungsproblem

$$
\begin{aligned}
& \underset{\boldsymbol{X}, \boldsymbol{Y}, \tilde{\boldsymbol{\Lambda}}, \boldsymbol{R}_{L}, \boldsymbol{R}_{V}, \boldsymbol{X}_{E W}}{\operatorname{minimi}} \gamma, \text { sodass } \\
& (5.32 \mathrm{~b}), \quad(5.32 \mathrm{c}), \quad(5.33)
\end{aligned}
$$

eine obere Schranke für $\left\|\boldsymbol{G}_{\boldsymbol{r d}}(s)\right\|_{\infty}^{\Omega}$, wobei $\mathfrak{R e}\left(\lambda_{B_{i}}\right)<-\alpha,\left|\lambda_{B_{i}}\right|<\varrho$ und $\left|\mathfrak{I m}\left(\lambda_{B_{i}}\right)\right|<\tan (\phi) \cdot\left|\mathfrak{R e}\left(\lambda_{B_{i}}\right)\right|$ für alle $i=1, \ldots, n$ sichergestellt ist.

Bemerkung 5.6. In (5.33b), (5.33c) und (5.33d)-(5.33f) wird eine gemeinsame Matrix $\boldsymbol{X}_{\mathrm{EW}}$ für die durch $\alpha, \varrho$ und $\phi$ beschriebenen $\mathcal{D}$-Regionen angesetzt. In der Umsetzung der beiden in diesem Abschnitt beschriebenen Lösungsverfahren zeigt sich, dass die dadurch eingeführte Konservativität vernachlässigbar ist. Theoretisch lässt sie sich eliminieren, indem für jede der durch $\alpha, \varrho$ und $\phi$ beschriebenen $\mathcal{D}$-Regionen eine individuelle Matrix $\boldsymbol{X}_{\mathrm{EW}, \alpha}$ beziehungsweise $\boldsymbol{X}_{\mathrm{EW}, \varrho}$ und $\boldsymbol{X}_{\mathrm{EW}, \phi}$ angesetzt wird. Dadurch erhöht sich jedoch die Dimension des Lösungsraumes, wodurch die zur Lösung benötigte Rechenzeit ansteigt. 
Ziel ist es im Weiteren, das in Satz 5.5 formulierte Optimierungsproblem zu lösen. Es handelt sich um ein nichtkonvexes Problem, sodass nicht gewährleistet ist, dass das globale Optimum gefunden wird. Weiterhin hängt die gefundene Lösung von den gewählten Startwerten $\left(\boldsymbol{L}_{\text {init }}, \boldsymbol{V}_{\text {init }}\right)$ ab. Eine mögliche Wahl von $\left(\boldsymbol{L}_{\text {init }}, \boldsymbol{V}_{\text {init }}\right)$ ergibt sich durch $\boldsymbol{R}_{L}=\mathbf{0}, \boldsymbol{R}_{V}=\mathbf{0}$. Alternativ kann die Lösung des Optimierungsproblems aus Satz 5.3 als initiale Parametrierung verwendet werden.

Ausgehend von dieser initialen Lösung wird das BMI-Problem aus Satz 5.5 iterativ gelöst. Es existieren verschiedene Ansätze, um für BMIProbleme zumindest lokale Optima zu finden (s. zum Beispiel [121, 165, 200]). An dieser Stelle werden zwei unterschiedliche Ansätze verfolgt: Zum einen das Path-Following-Verfahren und zum anderen die Rückführung auf ein rangbeschränktes LMI-Problem. Bei beiden Ansätzen werden zum Teil Umformungen vorgenommen, die mathematisch betrachtet gegenüber dem Optimierungsproblem aus Satz 5.5 keine Vereinfachung darstellen, sondern lediglich auf eine äquivalente Formulierung führen. Diese Umformungen dienen dazu, das Problem mittels gängiger verfügbarer Solver für LMI-Probleme mit oder ohne Rangbeschränkungen handhabbar zu machen.

\section{Lösung mittels Path-Following}

Das Path-Following-Verfahren geht auf [95] zurück, es wurde in [165] um eine Schrittweitensteuerung erweitert. Es beruht darauf, eine BMI in eine Taylorreihe zu entwickeln und das resultierende LMI-Problem zu lösen. Die Validität der erzielten Lösung für die ursprüngliche BMI wird überprüft und das Verfahren wird für den erhaltenen neuen Linearisierungspunkt fortgesetzt. Ist die neue Lösung gültig, wird für den nächsten Iterationsschritt die Umgebung, in der eine Lösung gesucht wird, vergrößert. Ist die neue Lösung dagegen ungültig, wird der Suchbereich für die nächste Iteration verkleinert.

Dieses schrittweise Vorgehen wird in Abbildung 5.12 veranschaulicht und gibt dem Verfahren seinen Namen. Im ersten Iterationsschritt (Abbildung 5.12a) wird ausgehend von einer initialen Lösung in einer Umgebung des Linearisierungspunktes das resultierende LMI-Problem gelöst. Die Lösung ist mit einem Dreieck gekennzeichnet, sie erweist sich für das ursprüngliche Problem als ungültig. Dementsprechend wird in der zweiten Iteration (Abbildung 5.12b) der Suchbereich um den Linearisierungspunkt verkleinert. Nun wird eine gültige Lösung gefunden, diese wird als neuer Linearisierungspunkt für die dritte Iteration verwendet und der Suchbe- 


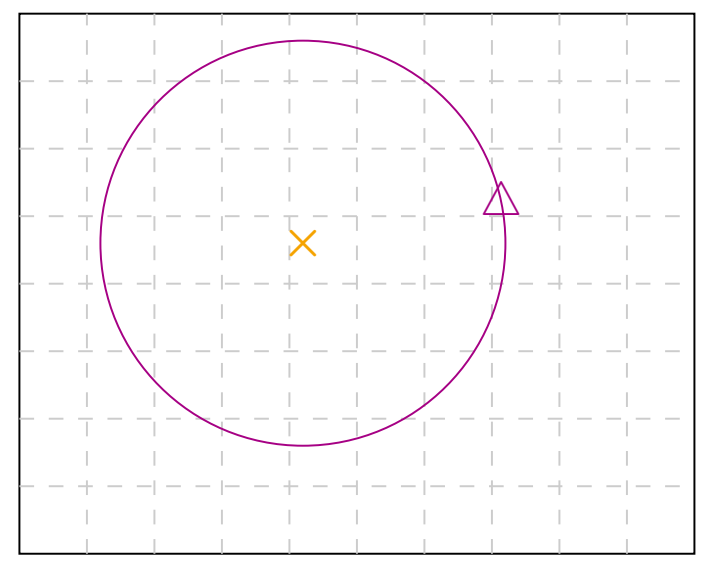

(a) 1. Iteration

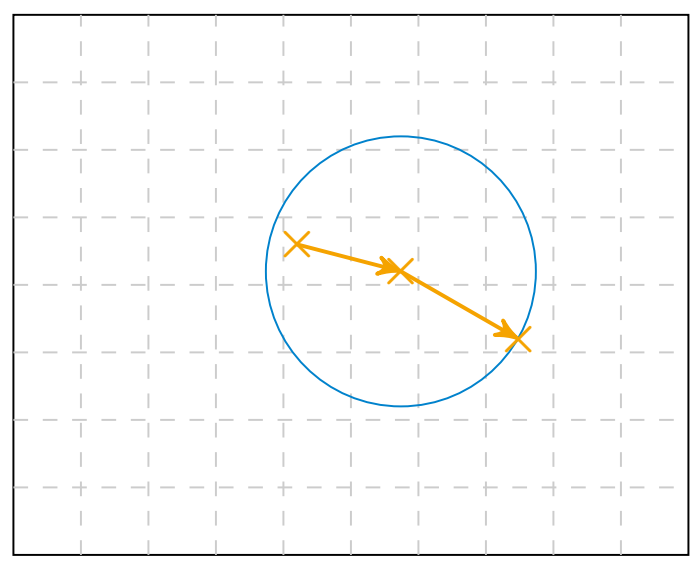

(c) 3. Iteration

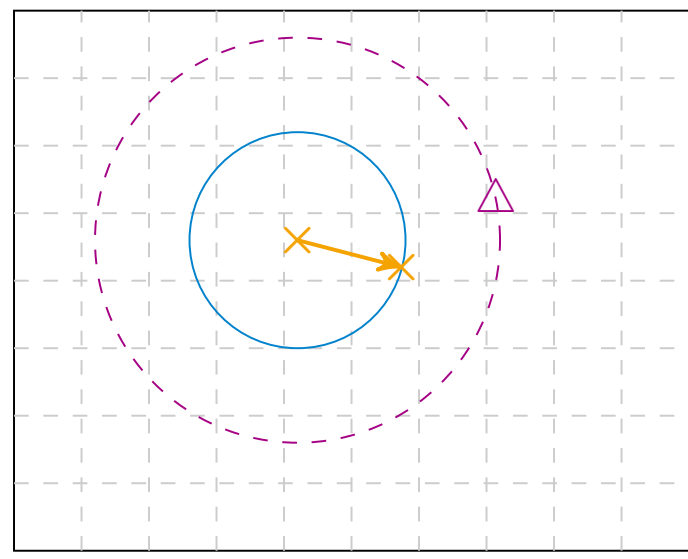

(b) 2. Iteration

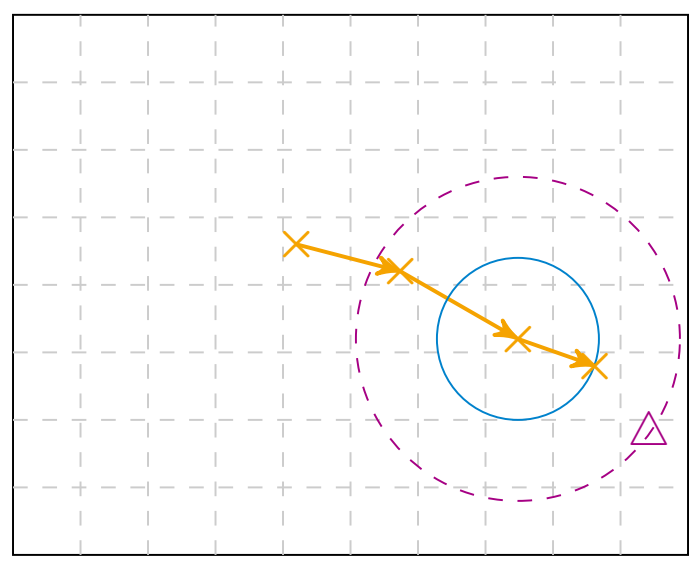

(d) 4. und 5. Iteration

Abbildung 5.12: Prinzip des Path-Following-Verfahrens

reich wird wieder leicht vergrößert. In der dritten Iteration wird erneut eine gültige Lösung gefunden. Der weiter vergrößerte Suchbereich in der vierten Iteration führt auf eine ungültige Lösung (vgl. Abbildung 5.12d). Erst in der fünften Iteration wird mit verkleinertem Suchbereich wieder eine gültige Lösung gefunden.

Bei dem in Satz 5.5 formulierten Optimierungsproblem stellen die Gleichungen (5.32c) beziehungsweise (5.33b) und (5.33c) aufgrund der Produktterme $\tilde{\boldsymbol{\Lambda}} \tilde{\boldsymbol{L}}$ und $\boldsymbol{X}_{\mathrm{EW}} \boldsymbol{R}_{L}$ zu linearisierende BMIs dar. Durch Entwicklung in eine Taylorreihe um den Punkt $\left(\boldsymbol{Z}_{f}, \tilde{\boldsymbol{\Lambda}}_{f}, \tilde{\boldsymbol{L}}_{f}\right)$ beziehungsweise $\left(\boldsymbol{X}_{f}, \boldsymbol{Y}_{f}, \gamma_{f}, \tilde{\boldsymbol{\Lambda}}_{f}, \tilde{\boldsymbol{L}}_{f}\right)$ ergibt sich für (5.32c) die Approximation

$$
\begin{aligned}
& \boldsymbol{Z}+\operatorname{He}(\tilde{\boldsymbol{\Lambda}}(\tilde{\boldsymbol{A}}-\tilde{\boldsymbol{L}} \tilde{\boldsymbol{C}})) \approx \\
& \quad \boldsymbol{Z}_{f}+\Delta \boldsymbol{Z}+\operatorname{He}\left(\left(\tilde{\boldsymbol{\Lambda}}_{f}+\Delta \tilde{\boldsymbol{\Lambda}}\right)\left(\tilde{\boldsymbol{A}}-\tilde{\boldsymbol{L}}_{f} \tilde{\boldsymbol{C}}\right)-\tilde{\boldsymbol{\Lambda}}_{f} \Delta \tilde{\boldsymbol{L}} \tilde{\boldsymbol{C}}\right) \prec \mathbf{0}
\end{aligned}
$$


mit

$$
\begin{aligned}
& \Delta \boldsymbol{Z}=\operatorname{diag}(\Delta \boldsymbol{\Xi}, \Delta \boldsymbol{\Gamma}), \Delta \boldsymbol{\Gamma}=\left[\begin{array}{cc}
\mathbf{0} & \mathbf{0} \\
\mathbf{0} & -2 \gamma_{f} \Delta \gamma \boldsymbol{I}_{n_{d}}
\end{array}\right], \\
& \Delta \boldsymbol{\Xi}=\left[\begin{array}{cc}
-\Delta \boldsymbol{X} & \Delta \boldsymbol{Y}+j \omega_{c} \Delta \boldsymbol{X} \\
\Delta \boldsymbol{Y}-j \omega_{c} \Delta \boldsymbol{X} & -\omega_{l} \omega_{h} \Delta \boldsymbol{X}
\end{array}\right]
\end{aligned}
$$

wenn lediglich Terme erster Ordnung betrachtet werden. Dabei ergeben sich die Matrizen in (5.35) jeweils aus den partiellen Ableitungen von $\boldsymbol{Z}+\operatorname{He}(\tilde{\boldsymbol{\Lambda}}(\tilde{\boldsymbol{A}}-\tilde{\boldsymbol{L}} \tilde{\boldsymbol{C}}))$ nach den Variablen $\Delta \boldsymbol{X}, \Delta \boldsymbol{Y}$ und $\Delta \gamma$. Ebenso kann (5.33b) um den Punkt $\left(\boldsymbol{X}_{\mathrm{EW}, f}, \tilde{\boldsymbol{\Lambda}}_{f}\right)$ beziehungsweise $\left(\boldsymbol{X}_{\mathrm{EW}, f}, \boldsymbol{R}_{L, f}\right)$ angenähert werden als

$$
\begin{aligned}
& \mathrm{He}\left(\boldsymbol{X}_{\mathrm{EW}}\left(\boldsymbol{A}-\left(\boldsymbol{L}_{0}+\boldsymbol{R}_{L} \cdot{ }^{\perp} \boldsymbol{D}^{*}\right) \boldsymbol{C}\right)\right)-2 \alpha \boldsymbol{X}_{\mathrm{EW}} \approx \\
& \quad \mathrm{He}\left(\left(\boldsymbol{X}_{\mathrm{EW}, f}+\Delta \boldsymbol{X}_{\mathrm{EW}}\right)\left(\boldsymbol{A}-\left(\boldsymbol{L}_{0} \boldsymbol{C}+\boldsymbol{R}_{L, f} \cdot{ }^{\perp} \boldsymbol{D}^{*}\right) \boldsymbol{C}\right)\right)+\ldots \\
& \quad-\mathrm{He}\left(\boldsymbol{X}_{\mathrm{EW}, f} \Delta \boldsymbol{R}_{L} \cdot{ }^{\perp} \boldsymbol{D}^{*} \boldsymbol{C}\right)-2 \alpha\left(\boldsymbol{X}_{\mathrm{EW}, f}+\Delta \boldsymbol{X}_{\mathrm{EW}}\right) \prec \mathbf{0} .
\end{aligned}
$$

Für (5.33c) ergibt sich entsprechend

$$
\begin{aligned}
& {\left[\begin{array}{cc}
-\varrho \boldsymbol{X}_{\mathrm{EW}} & \boldsymbol{X}_{\mathrm{EW}}\left(\boldsymbol{A}-\left(\boldsymbol{L}_{0}+\boldsymbol{R}_{L} \cdot{ }^{\perp} \boldsymbol{D}^{*}\right) \boldsymbol{C}\right) \\
\star & -\varrho \boldsymbol{X}_{\mathrm{EW}}
\end{array}\right] \approx} \\
& {\left[\begin{array}{cc}
-\varrho\left(\boldsymbol{X}_{\mathrm{EW}, f}+\Delta \boldsymbol{X}_{\mathrm{EW}}\right) & \left(\boldsymbol{X}_{\mathrm{EW}, f}+\Delta \boldsymbol{X}_{\mathrm{EW}}\right)\left(\boldsymbol{A}-\left(\boldsymbol{L}_{0}+\boldsymbol{R}_{L, f} \cdot{ }^{\perp} \boldsymbol{D}^{*}\right) \boldsymbol{C}\right) \\
\star & -\varrho\left(\boldsymbol{X}_{\mathrm{EW}, f}+\Delta \boldsymbol{X}_{\mathrm{EW}}\right)
\end{array}\right]} \\
& +\left[\begin{array}{cc}
\mathbf{0} & -\boldsymbol{X}_{\mathrm{EW}, f} \Delta \boldsymbol{R}_{L} \cdot{ }^{\perp} \boldsymbol{D}^{*} \boldsymbol{C} \\
\star & \mathbf{0}
\end{array}\right] \prec \mathbf{0}
\end{aligned}
$$

und die Taylorentwicklung der Matrizen $\boldsymbol{\Phi}_{11}$ und $\boldsymbol{\Phi}_{12}$ zur Beschreibung des Winkelsektors aus (5.33d) bis (5.33f) führt auf

$$
\begin{aligned}
& \boldsymbol{\Phi}_{11} \approx \tan (\phi) \cdot \mathrm{He}\left(\left(\boldsymbol{X}_{\mathrm{EW}, f}+\Delta \boldsymbol{X}_{\mathrm{EW}}\right)\left(\boldsymbol{A}-\left(\boldsymbol{L}_{0}+\boldsymbol{R}_{L, f} \cdot{ }^{\perp} \boldsymbol{D}^{*}\right) \boldsymbol{C}\right)\right. \\
&\left.-\boldsymbol{X}_{\mathrm{EW}, f} \Delta \boldsymbol{R}_{L} \cdot{ }^{\perp} \boldsymbol{D}^{*} \boldsymbol{C}\right) \\
& \boldsymbol{\Phi}_{12} \approx \\
&-\left(\boldsymbol{X}_{\mathrm{EW}, f}+\Delta \boldsymbol{X}_{\mathrm{EW}}\right)\left(\boldsymbol{A}-\left(\boldsymbol{L}_{0}+\boldsymbol{R}_{L, f} \cdot{ }^{\perp} \boldsymbol{D}^{*}\right) \boldsymbol{C}\right)+\ldots \\
&+\left(\left(\boldsymbol{X}_{\mathrm{EW}, f}+\Delta \boldsymbol{X}_{\mathrm{EW}}\right)\left(\boldsymbol{A}-\left(\boldsymbol{L}_{0}+\boldsymbol{R}_{L, f} \cdot{ }^{\perp} \boldsymbol{D}^{*}\right) \boldsymbol{C}\right)\right)^{\top}+\ldots \\
&+\boldsymbol{X}_{\mathrm{EW}, f} \Delta \boldsymbol{R}_{L} \cdot{ }^{\perp} \boldsymbol{D}^{*} \boldsymbol{C}-\left(\boldsymbol{X}_{\mathrm{EW}, f} \Delta \boldsymbol{R}_{L} \cdot{ }^{\perp} \boldsymbol{D}^{*} \boldsymbol{C}\right)^{\top} .
\end{aligned}
$$

Damit bei der Lösung des sich aus der Linearisierung ergebenden LMIProblems der Suchraum auf eine Umgebung des Linearisierungspunktes 
eingeschränkt wird, wird analog zu [165]

$$
\|\Delta \boldsymbol{Z}\|_{2}<\zeta\left\|\boldsymbol{Z}_{f}\right\|_{2}, \quad\left\|\Delta \boldsymbol{X}_{\mathrm{EW}}\right\|_{2}<\zeta\left\|\boldsymbol{X}_{\mathrm{EW}, f}\right\|_{2}
$$

gefordert. Somit kann $\zeta$ als ein Schrittweitenparameter aufgefasst werden, er wird typischerweise zu Beginn des Algorithmus auf $\zeta=0,2$ gesetzt. Mithilfe von Lemma 3.5 können die Bedingungen (5.37) durch die LMIs

$$
\left[\begin{array}{cc}
\zeta^{2}\left\|\boldsymbol{Z}_{f}\right\|_{2} \boldsymbol{I}_{2 n} & \Delta \boldsymbol{Z} \\
\star & \boldsymbol{I}_{2 n}
\end{array}\right] \succ \mathbf{0}, \quad\left[\begin{array}{cc}
\zeta^{2}\left\|\boldsymbol{X}_{\mathrm{EW}, f}\right\|_{2} \boldsymbol{I}_{n} & \Delta \boldsymbol{X}_{\mathrm{EW}} \\
\star & \boldsymbol{I}_{n}
\end{array}\right] \succ \mathbf{0}
$$

ausgedrückt werden. Alternativ dazu sind wie im Anhang in Lemma A.6 bewiesen die LMIs

$$
\left[\begin{array}{cc}
\zeta \boldsymbol{Z}_{f} & \Delta \boldsymbol{Z} \\
\star & \zeta \boldsymbol{Z}_{f}
\end{array}\right] \succ \mathbf{0}, \quad\left[\begin{array}{cc}
\zeta \boldsymbol{X}_{\mathrm{EW}, f} & \Delta \boldsymbol{X}_{\mathrm{EW}} \\
\star & \zeta \boldsymbol{X}_{\mathrm{EW}, f}
\end{array}\right] \succ \mathbf{0}
$$

hinreichend für (5.37). Im Allgemeinen erweist sich diese Formulierung als numerisch günstiger für die Beschränkung der Normabweichung und führt zu einer schnelleren Konvergenz des Verfahrens. Ein alternativer Beweis zur auch in [165] verwendeten Beziehung findet sich in [164].

Mit den durchgeführten Linearisierungen der auftretenden BMIs lässt sich das Path-Following-Verfahren anwenden. Dabei wird die Tatsache ausgenutzt, dass für eine beliebige initiale Parametrierung $\left(\boldsymbol{L}_{\text {init }}, \boldsymbol{V}_{\text {init }}\right)$ ein LMI-Problem vorliegt. Es wird dann lediglich eine obere Schranke für $\left\|\boldsymbol{G}_{\boldsymbol{r d}}(s)\right\|_{\infty}^{\Omega}$ bei gegebener FIO-Parametrierung abgeschätzt. Ausgehend von diesem Initialisierungsschritt wird solange iteriert, bis entweder keine signifikante Verbesserung mehr erreicht wird oder eine maximale Anzahl von Iterationsschritten absolviert wurde. Abbildung 5.13 zeigt einen Ablaufplan des Vorgehens, detailliert dargestellt ist dieses in

\section{Algorithmus 5.1.}

1. Initialisierung

Setze

$$
\tilde{\boldsymbol{L}}=\tilde{\boldsymbol{L}}_{f}=\tilde{\boldsymbol{L}}_{\text {init }}=\left[\begin{array}{c}
\boldsymbol{R}_{L, \text { init }} \\
-\boldsymbol{R}_{V, \text { init }}
\end{array}\right]
$$

und löse das sich ergebende LMI-Problem

$$
\begin{aligned}
& \underset{\boldsymbol{X}, \boldsymbol{Y}, \tilde{\boldsymbol{\Lambda}}, \boldsymbol{X}_{\mathrm{EW}}}{\operatorname{minimiere}} \bar{\gamma}, \text { sodass } \\
& \boldsymbol{X} \succ \mathbf{0}, \boldsymbol{X}_{\mathrm{EW}} \succ \mathbf{0}, \\
& \boldsymbol{Z}+\operatorname{He}\left(\tilde{\boldsymbol{\Lambda}}\left(\tilde{\boldsymbol{A}}-\tilde{\boldsymbol{L}}_{\text {init }} \tilde{\boldsymbol{C}}\right)\right) \prec \mathbf{0}
\end{aligned}
$$


mit den zusätzlichen Nebenbedingungen

$$
\begin{aligned}
& \mathrm{He}\left(\boldsymbol{X}_{\mathrm{EW}}\left(\boldsymbol{A}-\left(\boldsymbol{L}_{0}+\boldsymbol{R}_{L, \text { init }} \cdot{ }^{\perp} \boldsymbol{D}^{*}\right) \boldsymbol{C}\right)\right)-2 \alpha \boldsymbol{X}_{\mathrm{EW}} \prec \mathbf{0}, \\
& {\left[\begin{array}{cc}
-\varrho \boldsymbol{X}_{\mathrm{EW}} & \boldsymbol{X}_{\mathrm{EW}}\left(\boldsymbol{A}-\left(\boldsymbol{L}_{0}+\boldsymbol{R}_{L, \text { init }} \cdot{ }^{\perp} \boldsymbol{D}^{*}\right) \boldsymbol{C}\right) \\
\star & -\varrho \mathbf{X},
\end{array}\right.} \\
& {\left[\begin{array}{cc}
\boldsymbol{\Phi}_{11} & \boldsymbol{\Phi}_{12} \\
\star & \boldsymbol{\Phi}_{11}
\end{array}\right] \prec \mathbf{0},} \\
& \boldsymbol{\Phi}_{11}=\tan (\phi) \cdot \mathrm{He}\left(\boldsymbol{X}_{\mathrm{EW}}\left(\boldsymbol{A}-\left(\boldsymbol{L}_{0}+\boldsymbol{R}_{L, \text { init }} \cdot{ }^{\perp} \boldsymbol{D}^{*}\right) \boldsymbol{C}\right)\right), \\
& \boldsymbol{\Phi}_{12}=-\boldsymbol{X}_{\mathrm{EW}}\left(\boldsymbol{A}-\left(\boldsymbol{L}_{0}+\boldsymbol{R}_{L, \text { init }} \cdot{ }^{\perp} \boldsymbol{D}^{*}\right) \boldsymbol{C}\right)+\ldots \\
& \quad+\left(\boldsymbol{X}_{\mathrm{EW}}\left(\boldsymbol{A}-\left(\boldsymbol{L}_{0}+\boldsymbol{R}_{L, \text { init }} \cdot{ }^{\perp} \boldsymbol{D}^{*}\right) \boldsymbol{C}\right)\right)^{\top}
\end{aligned}
$$

und mit $\bar{\gamma}=\gamma^{2}>0$. Definiere die gefundene Lösung als $\gamma_{f}=\sqrt{\bar{\gamma}}$, $\boldsymbol{X}_{f}, \boldsymbol{Y}_{f}, \tilde{\Lambda}_{f}, \boldsymbol{X}_{\mathrm{EW}, f}$.

2. Path-Following-Schritt

a) Update-Schritt

Löse das LMI-Problem

$$
\begin{aligned}
& \underset{\Delta \tilde{\boldsymbol{L}}, \Delta \boldsymbol{X}, \Delta \boldsymbol{Y}, \Delta \tilde{\boldsymbol{\Lambda}}, \Delta \boldsymbol{X}_{\mathrm{EW}}}{\operatorname{minimiere}} \quad \Delta \gamma, \text { sodass } \\
& \boldsymbol{X}_{f}+\Delta \boldsymbol{X} \succ \mathbf{0}, \boldsymbol{X}_{\mathrm{EW}, f}+\Delta \boldsymbol{X}_{\mathrm{EW}} \succ \mathbf{0}, \\
& \Delta \gamma<0, \gamma_{f}^{2}+2 \gamma_{f} \Delta \gamma>0 \\
& (5.34)-(5.36),(5.38) .
\end{aligned}
$$

b) Verifikationsschritt

Setze $\gamma=\gamma_{f}+\Delta \gamma$ und $\tilde{\boldsymbol{L}}=\tilde{\boldsymbol{L}}_{f}+\Delta \tilde{\boldsymbol{L}}$ und überprüfe die Lösbarkeit von

$$
\begin{aligned}
& \text { finde } \boldsymbol{X}, \boldsymbol{Y}, \tilde{\boldsymbol{\Lambda}}, \boldsymbol{X}_{\mathrm{EW}}, \text { sodass } \\
& \boldsymbol{X} \succ \mathbf{0}, \boldsymbol{X}_{\mathrm{EW}} \succ \mathbf{0}, \\
& \boldsymbol{Z}+\operatorname{He}(\tilde{\boldsymbol{\Lambda}}(\tilde{\boldsymbol{A}}-\tilde{\boldsymbol{L}} \tilde{\boldsymbol{C}})) \prec \mathbf{0}
\end{aligned}
$$


mit den zusätzlichen Nebenbedingungen

$$
\begin{aligned}
& \mathrm{He}\left(\boldsymbol{X}_{\mathrm{EW}}\left(\boldsymbol{A}-\left(\boldsymbol{L}_{0}+\boldsymbol{R}_{L} \cdot{ }^{\perp} \boldsymbol{D}^{*}\right) \boldsymbol{C}\right)\right)-2 \alpha \boldsymbol{X}_{\mathrm{EW}} \prec \mathbf{0}, \\
& {\left[\begin{array}{cc}
-\varrho \boldsymbol{X}_{\mathrm{EW}} & \boldsymbol{X}_{\mathrm{EW}}\left(\boldsymbol{A}-\left(\boldsymbol{L}_{0}+\boldsymbol{R}_{L} \cdot{ }^{\perp} \boldsymbol{D}^{*}\right) \boldsymbol{C}\right) \\
\star & -\varrho \boldsymbol{X}_{\mathrm{EW}}
\end{array}\right] \prec \mathbf{0},} \\
& \left.\begin{array}{cc}
\boldsymbol{\Phi}_{11} & \boldsymbol{\Phi}_{12} \\
\star & \boldsymbol{\Phi}_{11}
\end{array}\right] \prec \mathbf{0}, \\
& \boldsymbol{\Phi}_{11}=\tan (\phi) \cdot \mathrm{He}\left(\boldsymbol{X}_{\mathrm{EW}}\left(\boldsymbol{A}-\left(\boldsymbol{L}_{0}+\boldsymbol{R}_{L} \cdot{ }^{\perp} \boldsymbol{D}^{*}\right) \boldsymbol{C}\right)\right), \\
& \boldsymbol{\Phi}_{12}=-\boldsymbol{X}_{\mathrm{EW}}\left(\boldsymbol{A}-\left(\boldsymbol{L}_{0}+\boldsymbol{R}_{L} \cdot{ }^{\perp} \boldsymbol{D}^{*}\right) \boldsymbol{C}\right)+\ldots \\
& \quad+\left(\boldsymbol{X}_{\mathrm{EW}}\left(\boldsymbol{A}-\left(\boldsymbol{L}_{0}+\boldsymbol{R}_{L} \cdot{ }^{\perp} \boldsymbol{D}^{*}\right) \boldsymbol{C}\right)\right)^{\top} .
\end{aligned}
$$

Falls das Problem lösbar ist, definiere die gefundene Lösung als $\left(\gamma_{f}, \tilde{\boldsymbol{L}}_{f}, \boldsymbol{X}_{f}, \boldsymbol{Y}_{f}, \tilde{\boldsymbol{\Lambda}}_{f}, \boldsymbol{X}_{\mathrm{EW}, f}\right)$. Falls die Abbruchbedingung 3. nicht erfüllt ist, setze die neue Schrittweite auf $\zeta:=\eta^{+} \zeta$ und gehe zu 2.a). Falls das Problem nicht lösbar ist und die Abbruchbedingung 3 . nicht erfüllt ist, setze $\zeta:=\eta^{-} \zeta$ und gehe zu 2.a) ohne die Variablen $\gamma_{f}, \tilde{\boldsymbol{L}}_{f}, \boldsymbol{X}_{f}, \boldsymbol{Y}_{f}, \tilde{\boldsymbol{\Lambda}}_{f}$ und $\boldsymbol{X}_{\mathrm{EW}, f} \mathrm{zu}$ verändern.

3. Abbruchkriterium

Beende den Algorithmus, falls eine gegebene Anzahl von Iterationen durchlaufen wurde oder die relative Verbesserung von $\gamma$ in zwei aufeinander folgenden Iterationen eine gegebene Schranke $\varepsilon$ unterschreitet.

Durch die Bedingungen $\boldsymbol{X}_{f}+\Delta \boldsymbol{X} \succ \mathbf{0}$ und $\boldsymbol{X}_{\mathrm{EW}, f}+\Delta \boldsymbol{X}_{\mathrm{EW}} \succ \mathbf{0}$ in Schritt 2.a) wird sichergestellt, dass die sich ergebenden neuen Matrizen $\boldsymbol{X}$ und $\boldsymbol{X}_{\mathrm{EW}}$ positiv definit sind. Die Bedingungen $\Delta \gamma<0$ und $\gamma_{f}^{2}+2 \gamma_{f} \Delta \gamma>0$ sind theoretisch redundant. Bei den verwendeten Solvern beschleunigen sie jedoch die Konvergenz des Verfahrens. Mit den Parametern $\eta^{+}>1$ und $0<\eta^{-}<1$ erfolgt in Schritt 2.b) eine Schrittweitenadaption. Diese beschleunigt ebenfalls die Konvergenz des Verfahrens [165].

Da im Path-Following-Algorithmus die Minimierung von $\gamma$ in jeder Iteration auf Basis eines linearisierten BMI-Problems erfolgt, ist die Konvergenz auf das globale Optimum nicht sichergestellt. Jedoch ist gewährleistet, dass das Verfahren auf ein lokales Minimum von $\gamma$ konvergiert [165]. Dies impliziert auch, dass gegenüber der gewählten initialen FIO-Parametrierung $\left(\boldsymbol{L}_{\text {init }}, \boldsymbol{V}_{\text {init }}\right)$ keine Verschlechterung hinsichtlich $\gamma$ auftreten kann. Für 


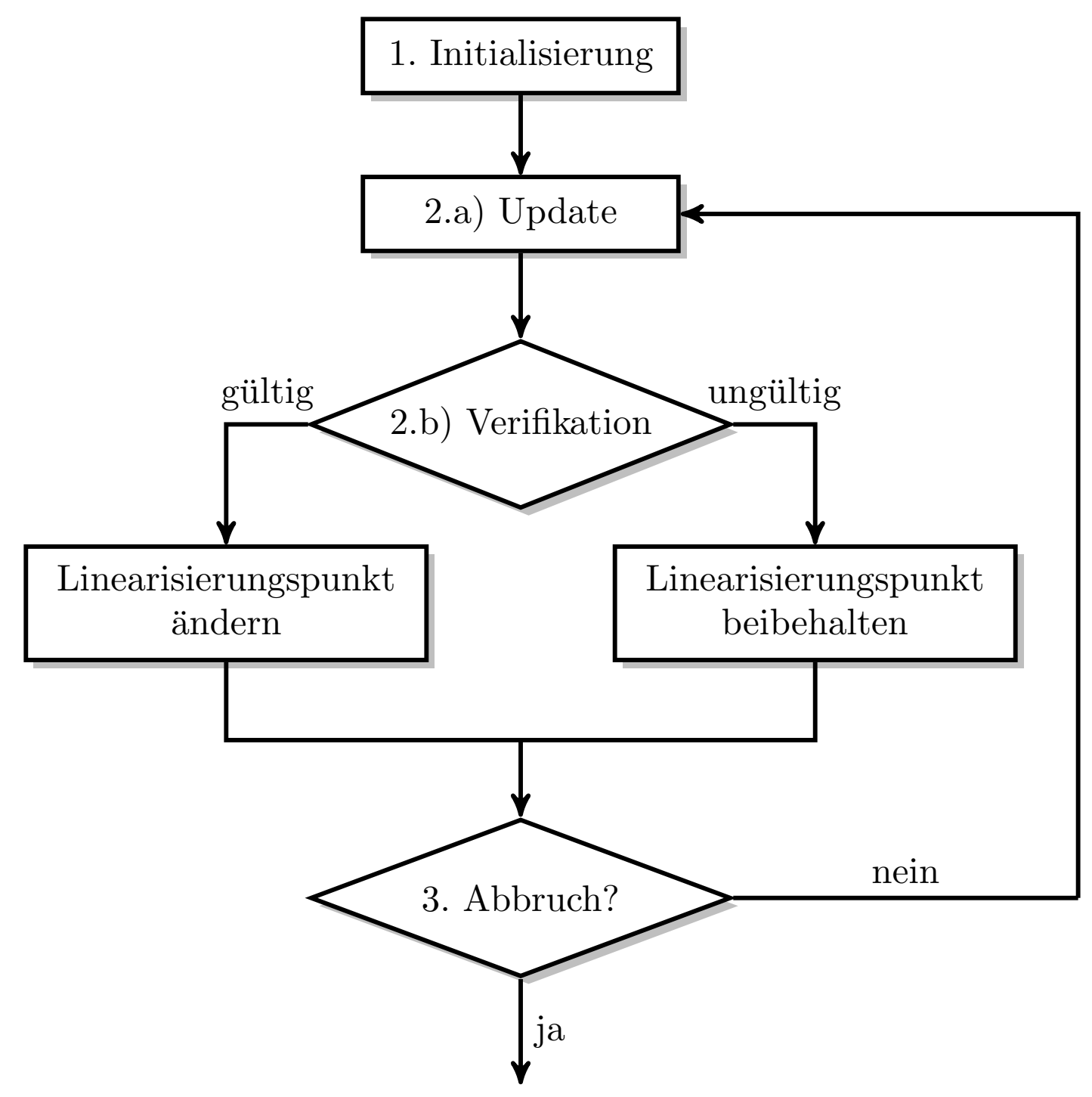

Abbildung 5.13: Schematischer Ablauf des Path-Following-Algorithmus

die mittels des Path-Following-Algorithmus gefundene Schranke gilt stets $\gamma_{\mathrm{PF}} \leq \gamma_{\text {init }}$.

Bemerkung 5.7. Selbstverständlich kann auch die Ausgangsgleichung (5.30d) linearisiert werden, um das Path-Following-Verfahren anzuwenden. Jedoch führt die Umformulierung zu (5.32c) wie bereits erörtert auf ein allgemeines BMI-Problem. Diese Darstellung als Standardproblem ermöglicht somit die Anwendung beliebiger Lösungsverfahren für BMIProbleme, wie zum Beispiel der im Folgenden dargestellten Formulierung als rangbeschränktes LMI-Problem. Überdies ist der Einsatz anderer BMILösungsansätze, wie sie unter anderem in [121, 200] beschrieben sind, möglich. 


\section{Lösung durch Rückführung auf ein rangbeschränktes LMI-Problem}

Die Umformung von (5.30d) in die BMI (5.32c) eröffnet einen alternativen Lösungsansatz. Wie im Folgenden gezeigt wird, lässt sich das BMIProblem (5.32c) umformulieren in ein rangbeschränktes LMI-Problem. Für diese Klasse von Optimierungsproblemen existieren leistungsfähige Solver wie etwa LMIRANK [162] basierend auf [163].

Zunächst wird in (5.32c) die Variablensubstitution

$$
\tilde{\boldsymbol{W}}=\tilde{\boldsymbol{\Lambda}} \tilde{\boldsymbol{L}} \in \mathbb{R}^{\left(2 n+n_{f}+n_{d}\right) \times\left(n_{y}-n_{f}\right)}
$$

durchgeführt, wodurch sich die LMI

$$
\boldsymbol{Z}+\operatorname{He}(\tilde{\boldsymbol{\Lambda}} \tilde{\boldsymbol{A}}-\tilde{\boldsymbol{W}} \tilde{\boldsymbol{C}}) \prec \mathbf{0}
$$

ergibt. Aus (5.32) erhält man damit zwar das LMI-Problem

$$
\begin{aligned}
& \underset{\boldsymbol{X}, \boldsymbol{Y}, \tilde{\boldsymbol{\Lambda}}, \tilde{\boldsymbol{W}}}{\operatorname{minimiere}} \gamma, \text { sodass } \\
& \boldsymbol{X} \succ \mathbf{0}, \\
& \boldsymbol{Z}+\operatorname{He}(\tilde{\boldsymbol{\Lambda}} \tilde{\boldsymbol{A}}-\tilde{\boldsymbol{W}} \tilde{\boldsymbol{C}}) \prec \mathbf{0},
\end{aligned}
$$

jedoch weist die Matrix $\tilde{\boldsymbol{\Lambda}} \in \mathbb{R}^{\left(2 n+n_{f}+n_{d}\right) \times\left(n+n_{f}\right)}$ mehr Zeilen als Spalten auf, sie ist somit im Allgemeinen nicht rechtsinvertierbar.

Im Gegensatz zur Variablensubstitution in (5.24) auf Seite 215 kann $\tilde{\boldsymbol{L}}$ nicht ohne Weiteres aus den Matrizen $\tilde{\boldsymbol{W}}$ und $\tilde{\boldsymbol{\Lambda}}$ berechnet werden. Dies ist nur möglich, wenn

$$
\operatorname{rang}(\tilde{\Lambda})=\operatorname{rang}\left(\left[\begin{array}{ll}
\tilde{\Lambda} & \tilde{\boldsymbol{W}}
\end{array}\right]\right)
$$

gilt, da dann $\tilde{\boldsymbol{L}}=\tilde{\boldsymbol{\Lambda}}^{+} \cdot \tilde{\boldsymbol{W}}$ ist [14, Proposition 6.1.7].

Aus Lemma A.1 folgt für den Rang von $\tilde{\Lambda}$ die Ungleichung

$$
\operatorname{rang}(\tilde{\boldsymbol{\Lambda}})=\operatorname{rang}(\boldsymbol{\Lambda} \boldsymbol{\Psi}) \geq \operatorname{rang}(\boldsymbol{\Lambda})+\operatorname{rang}(\boldsymbol{\Psi})-\chi=2\left(n+n_{f}\right)-\chi .
$$

Wie anhand von (5.30) herausgearbeitet, gilt $\chi \geq n+n_{f}$ und es folgt die Abschätzung $\operatorname{rang}(\tilde{\boldsymbol{\Lambda}}) \geq n+n_{f}$. Aufgrund der Dimension von $\tilde{\boldsymbol{\Lambda}}$ muss gleichzeitig $\operatorname{rang}(\tilde{\boldsymbol{\Lambda}}) \leq n+n_{f}$ sein, sodass $\operatorname{sich} \operatorname{rang}(\tilde{\boldsymbol{\Lambda}})=n+n_{f}$ ergibt. 
Somit lässt sich (5.32) äquivalent ausdrücken durch das rangbeschränkte LMI-Problem

$$
\begin{aligned}
& \underset{\boldsymbol{X}, \boldsymbol{Y}, \tilde{\boldsymbol{\Lambda}}, \tilde{\boldsymbol{W}}}{\operatorname{minimiere}} \gamma, \text { sodass } \\
& \boldsymbol{X} \succ \mathbf{0}, \\
& \boldsymbol{Z}+\operatorname{He}(\tilde{\boldsymbol{\Lambda}} \tilde{\boldsymbol{A}}-\tilde{\boldsymbol{W}} \tilde{\boldsymbol{C}}) \prec \mathbf{0}, \\
& \operatorname{rang}\left(\left[\begin{array}{ll}
\tilde{\boldsymbol{\Lambda}} & \tilde{\boldsymbol{W}}
\end{array}\right]\right) \leq n+n_{f} .
\end{aligned}
$$

Um weiterhin die Beschränkung der nichtsteuerbaren Beobachtereigenwerte auf die durch (5.33) beschriebene $\mathcal{D}$-Region zu berücksichtigen, wird $\boldsymbol{Y}_{\mathrm{EW}}=\boldsymbol{X}_{\mathrm{EW}} \boldsymbol{R}_{L}$ eingeführt. Partitioniert man $\tilde{\boldsymbol{\Lambda}}=\left[\begin{array}{ll}\tilde{\boldsymbol{\Lambda}}_{1} & \tilde{\boldsymbol{\Lambda}}_{2}\end{array}\right]$, so ergibt sich aus $\tilde{\boldsymbol{\Lambda}} \tilde{\boldsymbol{L}}=\tilde{\boldsymbol{W}}$ und $\boldsymbol{Y}_{\mathrm{EW}}=\boldsymbol{X}_{\mathrm{EW}} \boldsymbol{R}_{L}$ die Bedingung

$$
\left[\begin{array}{cc}
\tilde{\boldsymbol{\Lambda}}_{1} & \tilde{\boldsymbol{\Lambda}}_{2} \\
\boldsymbol{X}_{\mathrm{EW}} & \mathbf{0}
\end{array}\right]\left[\begin{array}{c}
\boldsymbol{R}_{L} \\
-\boldsymbol{R}_{V}
\end{array}\right]=\left[\begin{array}{c}
\tilde{\boldsymbol{W}} \\
\boldsymbol{Y}_{\mathrm{EW}}
\end{array}\right]
$$

Da bereits $\tilde{\Lambda}$ mehr Zeilen als Spalten aufweist und vollen Rang hat, besitzt auch die Matrix

$$
\left[\begin{array}{cc}
\tilde{\boldsymbol{\Lambda}}_{1} & \tilde{\boldsymbol{\Lambda}}_{2} \\
\boldsymbol{X}_{\mathrm{EW}} & \mathbf{0}
\end{array}\right]
$$

vollen Rang. Analog zu den Überlegungen zu (5.39) ergibt sich für die Lösbarkeit von (5.40) die Bedingung

$$
\operatorname{rang}\left(\left[\begin{array}{ccc}
\tilde{\boldsymbol{\Lambda}}_{1} & \tilde{\boldsymbol{\Lambda}}_{2} & \tilde{\boldsymbol{W}} \\
\boldsymbol{X}_{\mathrm{EW}} & \mathbf{0} & \boldsymbol{Y}_{\mathrm{EW}}
\end{array}\right]\right) \leq n+n_{f}
$$

Die rangbeschränkte Matrix in obigem Ausdruck ist $\left(3 n+n_{f}+n_{d}\right) \times\left(n+n_{y}\right)$-dimensional und damit nichtquadratisch, weshalb sich die gängigen Solver nicht unmittelbar anwenden lassen. Diese unterstützen lediglich Rangbeschränkungen für quadratische Matrizen. Das im Anhang angegebene Lemma A.5 ermöglicht es, die Rangbeschränkung einer nichtquadratischen Matrix durch die Einführung zusätzlicher Hilfsvariablen auf die Rangbeschränkung einer quadratischen Matrix höherer Dimension zurückzuführen. Dazu werden die quadratischen Hilfsvariablen $\boldsymbol{\Upsilon}_{1} \in \mathbb{R}^{\left(3 n+n_{f}+n_{d}\right) \times\left(3 n+n_{f}+n_{d}\right)}$ und $\boldsymbol{\Upsilon}_{2} \in \mathbb{R}^{\left(n+n_{y}\right) \times\left(n+n_{y}\right)}$ benötigt. Das in Satz 5.5 formulierte Optimierungs- 
problem lässt sich damit alternativ beschreiben durch

$$
\begin{aligned}
& \underset{\boldsymbol{X}, \boldsymbol{Y}, \tilde{\boldsymbol{\Lambda}}, \tilde{\boldsymbol{W}}, \boldsymbol{X}_{\mathrm{EW}}, \boldsymbol{Y}_{\mathrm{EW}}, \boldsymbol{\Upsilon}_{1}, \boldsymbol{\Upsilon}_{2},}{\operatorname{minimiere}}, \text { sodass } \\
& \boldsymbol{X} \succ \mathbf{0}, \quad \boldsymbol{X}_{\mathrm{EW}} \succ \mathbf{0} \\
& \boldsymbol{Z}+\operatorname{He}(\tilde{\boldsymbol{\Lambda}} \tilde{\boldsymbol{A}}-\tilde{\boldsymbol{W}} \tilde{\boldsymbol{C}}) \prec \mathbf{0}, \\
& \text { He }\left(\boldsymbol{X}_{\mathrm{EW}}\left(\boldsymbol{A}-\boldsymbol{L}_{0} \boldsymbol{C}\right)-\boldsymbol{Y}_{\mathrm{EW}} \cdot{ }^{\perp} \boldsymbol{D}^{*} \cdot \boldsymbol{C}\right)-2 \alpha \boldsymbol{X}_{\mathrm{EW}} \prec \mathbf{0} \text {, } \\
& {\left[\begin{array}{cc}
-\varrho \boldsymbol{X}_{\mathrm{EW}} & \boldsymbol{X}_{\mathrm{EW}}\left(\boldsymbol{A}-\boldsymbol{L}_{0} \boldsymbol{C}\right)-\boldsymbol{Y}_{\mathrm{EW}} \cdot{ }^{\perp} \boldsymbol{D}^{*} \cdot \boldsymbol{C} \\
\star & -\varrho \boldsymbol{X}_{\mathrm{EW}}
\end{array}\right] \prec \mathbf{0},} \\
& {\left[\begin{array}{cc}
\boldsymbol{\Phi}_{11} & \mathbf{\Phi}_{12} \\
\star & \boldsymbol{\Phi}_{11}
\end{array}\right] \prec \mathbf{0}} \\
& \boldsymbol{\Phi}_{11}=\tan (\phi) \cdot \operatorname{He}\left(\boldsymbol{X}_{\mathrm{EW}}\left(\boldsymbol{A}-\boldsymbol{L}_{0} \boldsymbol{C}\right)-\boldsymbol{Y}_{\mathrm{EW}} \cdot{ }^{\perp} \boldsymbol{D}^{*} \boldsymbol{C}\right) \text {, } \\
& \boldsymbol{\Phi}_{12}=-\boldsymbol{X}_{\mathrm{EW}}\left(\boldsymbol{A}-\boldsymbol{L}_{0} \boldsymbol{C}\right)+\left(\boldsymbol{A}-\boldsymbol{L}_{0} \boldsymbol{C}\right)^{\top} \boldsymbol{X}_{\mathrm{EW}}+\ldots \\
& +\boldsymbol{Y}_{\mathrm{EW}} \cdot{ }^{\perp} \boldsymbol{D}^{*} \boldsymbol{C}-\left(\boldsymbol{Y}_{\mathrm{EW}} \cdot{ }^{\perp} \boldsymbol{D}^{*} \boldsymbol{C}\right)^{\top},
\end{aligned}
$$

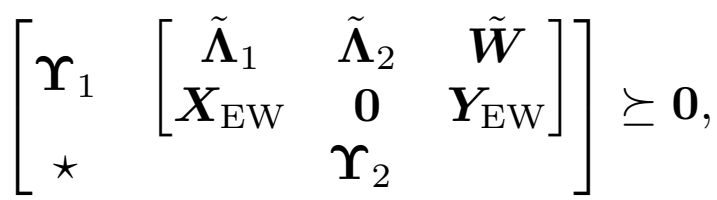

$$
\begin{aligned}
& \operatorname{rang}\left(\left[\begin{array}{cc}
\mathbf{\Upsilon}_{1} & \mathbf{0} \\
\mathbf{0} & \mathbf{\Upsilon}_{2}
\end{array}\right]\right) \leq 2\left(n+n_{f}\right) \text {. }
\end{aligned}
$$

Eine weitere Einschränkung des spezialisierten Solvers LMIRANK [162] ist, dass er lediglich Lösbarkeitsprobleme der Form

$$
\begin{aligned}
& \text { finde } \boldsymbol{X}, \boldsymbol{Y}, \tilde{\boldsymbol{\Lambda}}, \tilde{\boldsymbol{W}}, \boldsymbol{X}_{\mathrm{EW}}, \boldsymbol{Y}_{\mathrm{EW}}, \boldsymbol{\Upsilon}_{1}, \boldsymbol{\Upsilon}_{2} \text {, sodass } \\
& (5.41 \mathrm{~b})-(5.41 \mathrm{k}),
\end{aligned}
$$

unterstützt und keine Optimierungsprobleme. Da die Gütefunktion in (5.41a) ein Skalar ist, bietet sich wie in Abschnitt 5.3.3 zur Lösung ein Bisektionsverfahren an. Dabei wird zunächst ein Intervall $\left[\gamma_{\mathrm{lo}} ; \gamma_{\mathrm{up}}\right]$ bestimmt. Dieses zeichnet sich dadurch aus, dass (5.42) für $\gamma_{\text {up }}$ lösbar ist, für $\gamma_{\text {lo }}$ jedoch nicht. Ein solches Intervall ist in Abbildung 5.14 beispielhaft dargestellt. Die gesuchte untere Schranke für $\gamma$ in (5.41) liegt also innerhalb dieses Intervalls. Ausgehend davon wird die Lösbarkeit von (5.42) für die Mitte des Intervalls untersucht. Dies führt auf ein neues Intervall mit halber Breite, welches die gesuchte Schranke $\gamma$ enthält. Im Beispiel, das in Abbildung 5.14 gezeigt ist, stellt sich in der ersten Iteration heraus, 
1. Iteration

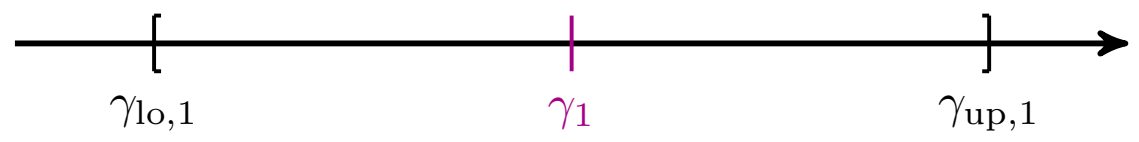

2. Iteration

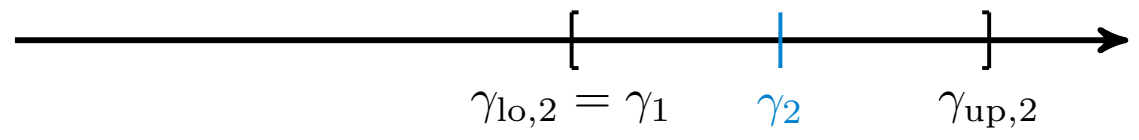

3. Iteration

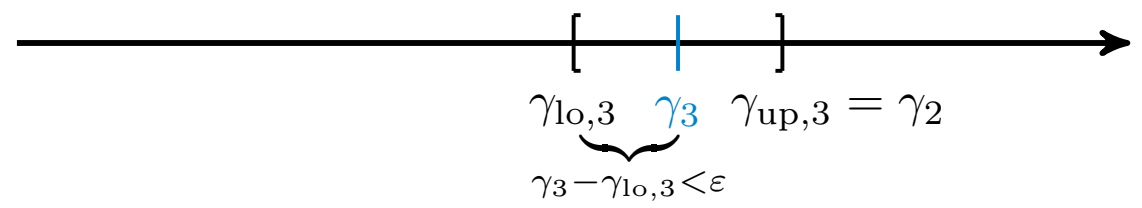

Abbildung 5.14: Prinzip des Bisektionsverfahrens

dass das Problem (5.42) für die Intervallmitte $\gamma_{1}$ nicht lösbar ist. Dementsprechend wird für die nächste Iteration die untere Intervallgrenze auf $\gamma_{1}$ gesetzt. Für die neue Intervallmitte $\gamma_{2}$ stellt sich (5.42) als lösbar heraus, sodass nun die obere Intervallgrenze angepasst wird. Dieses Vorgehen wird wiederholt, bis die Breite des Intervalls einen vorgegebenen Wert $\varepsilon$ unterschreitet.

Zusammengefasst ergibt sich zur Lösung des Optimierungsproblems aus Satz 5.5 der

\section{Algorithmus 5.2.}

1. Initialisierung

a) Wähle ein $\gamma_{\mathrm{lo}}>0$, mit dem (5.42) nicht lösbar ist und setze $\gamma_{\text {up }}=2 \gamma_{\text {lo }}$.

b) Falls (5.42) mit $\gamma_{\text {up }}$ lösbar ist, gehe zu 2 .

Andernfalls, setze $\gamma_{\text {up }}:=2 \gamma_{\text {up }}$ und wiederhole 1.b).

2. Bisektionsschritt

Setze $\gamma=\frac{1}{2}\left(\gamma_{\text {up }}+\gamma_{\text {lo }}\right)$ und überprüfe die Lösbarkeit von (5.41).

Falls (5.42) lösbar ist, setze $\gamma_{\text {up }}=\gamma$, überprüfe das Abbruchkriterium und gehe zu 2.

Falls (5.42) nicht lösbar ist, setze $\gamma_{\mathrm{lo}}=\gamma$, überprüfe das Abbruchkriterium und gehe zu 2.

3. Abbruchkriterium

Terminiere den Algorithmus falls $\gamma_{\text {up }}-\gamma_{\text {lo }}<\varepsilon$ mit einer gegebenen Schranke $\varepsilon$ ist. 
Es sei erneut darauf hingewiesen, dass es sich bei (5.42) aufgrund der Rangbeschränkung um ein nichtkonvexes Problem handelt. Somit kann nicht garantiert werden, dass es sich bei dem ermittelten Wert $\gamma$ um eine globale Lösung für (5.32) handelt. Die gefundene Lösung ist abhängig von den gewählten Startwerten, also unter anderem $\boldsymbol{R}_{L}$ und $\boldsymbol{R}_{V}$.

\section{Erweiterungen des Verfahrens}

Die vorgestellte Methode lässt sich leicht erweitern, wenn verschiedene Störungen auf das System wirken, die jeweils in unterschiedlichen Frequenzbereichen liegen. Betrachtet man $N$ Störungen, für die jeweils $\boldsymbol{d}_{i} \in \Omega_{i}$ mit einem eigenen Frequenzbereich $\Omega_{i}$ gilt, so kann das Optimierungsproblem aus Satz 5.5 abgewandelt werden. Anstatt der dort verwendeten Schranke $\gamma$ wird das Maß

$$
J=\sum_{i=1}^{N} \theta_{i} \gamma_{i}
$$

als Optimierungskriterium herangezogen. Dabei sind $\theta_{i}$ frei wählbare, positive Gewichtungsfaktoren, mit denen die Unterdrückung der Störungen $\boldsymbol{d}_{i}$ priorisiert werden können. Einzuhalten sind die Nebenbedingungen

$$
\left\|\boldsymbol{G}_{\boldsymbol{r} \boldsymbol{d}_{i}}(s)\right\|_{\infty}^{\Omega_{i}}<\gamma_{i}, \quad \forall i=1, \ldots, N .
$$

Da beim Path-Following-Verfahren eine Linearisierung des Problems stattfindet, kann man für jedes Stör-Residuen-Übertragungssystem in (5.32) separate Matrizen $\boldsymbol{X}_{i}, \boldsymbol{Y}_{i}$ und $\tilde{\boldsymbol{\Lambda}}_{i}$ ansetzen. Somit bedingen die $N$ Nebenbedingungen keine Konservativität der erzielten Lösung. Gleiches gilt für den Ansatz des rangbeschränkten LMI-Problems.

Wird in quadratischen Systemen wie in Abschnitt 5.3.2 die Störunterdrückung durch eine Relaxierung der Strukturanforderungen optimiert, so lassen sich ebenfalls beschränkte Frequenzbereiche betrachten. Im Optimierungsproblem (5.17) sind dabei die letzten beiden Bedingungen durch entsprechende Matrixungleichungen zur Einhaltung von $\left\|\boldsymbol{G}_{\boldsymbol{r} \boldsymbol{d}}(s)\right\|_{\infty}^{\Omega}$ zu ersetzen. Das weitere Vorgehen entspricht mit der Umwandlung in ein BMIProblem und der Anwendung des Path-Following-Algorithmus dem in diesem Abschnitt beschriebenen Verfahren.

In diesem Abschnitt wurde stets eine gegebene Dynamik der Diagonalelemente vorausgesetzt. Prinzipiell ist es möglich, auch bei der Optimierung von $\left\|\boldsymbol{G}_{\boldsymbol{r} \boldsymbol{d}}(s)\right\|_{\infty}^{\Omega}$ die Diagonalelemente in die Optimierung mit einzubeziehen und damit alle vorhandenen Freiheitsgrade auszunutzen. Dies 
kann einerseits ähnlich wie in den Abschnitten 5.3.1 und 5.4.2 durch Umformung in ein Standard-Regelungsproblem erfolgen. Die Umwandlung in die Struktur gemäß Abbildung 5.2 gelingt durch Rückgriff auf Gewichtungsfilter, die separat auszulegen sind. Damit können die bereits mehrfach erwähnten Solver eingesetzt werden. Da die Matrixungleichungen des GKYP-Lemmas ohnehin beim Path-Following-Verfahren linearisiert werden, ist es alternativ auch möglich, die Koeffizienten $q_{i, j}$ und $z_{i, 0}$ als zusätzliche Freiheitsgrade in (5.30) zu verwenden.

\section{Beispiel Hydrauliksystem}

Zur Demonstration der Leistungsfähigkeit der vorgestellten Verfahren wird das Beispiel aus Abschnitt 5.4.1 erweitert. Dabei wird davon ausgegangen, dass zusätzliches Wissen über die Störungen vorhanden ist. Diese sollen lediglich Frequenzen im Bereich $\Omega=\left[\omega_{l} ; \omega_{h}\right]$ mit $\omega_{l}=4 \mathrm{rad} / \mathrm{s}$ und $\omega_{h}=8 \mathrm{rad} / \mathrm{s}$ enthalten.

Für die initiale Beobachterparametrierung ergibt sich in diesem Frequenzbereich eine maximale Störverstärkung von $\gamma_{\text {init }}=41,57$. Der FIO, der aus der Optimierung nach Satz 5.3 (Robustheit bezüglich exogener Störungen beliebiger Frequenzen) gewonnen wurde, liefert in diesem Frequenzbereich $\gamma_{\mathrm{opt}}=20,73$. In Abbildung 5.15 sind diese Schranken strichliert in den Verlauf der größten Singulärwerte von $\boldsymbol{G}_{\boldsymbol{r} \boldsymbol{d}}(s)$ eingetragen.

Nun wird ausgehend von der Lösung $\left(\boldsymbol{L}_{\text {opt }}, \boldsymbol{V}_{\text {opt }}\right)$, die zur Unterdrückung von Störungen beliebiger Frequenzen in Abschnitt 5.4.1 ermittelt wurde, der Path-Following-Algorithmus angewendet, um die Störunterdrückung im relevanten Frequenzbereich gezielt zu verbessern. Als Parameter des Algorithmus werden $\zeta=0,2, \eta^{+}=1,1$ und $\eta^{-}=0,5$ gesetzt. Die resultierende FIO-Parametrierung $\left(\boldsymbol{L}_{\mathrm{opt}, \mathrm{ff}, \mathrm{pf}}, \boldsymbol{V}_{\mathrm{opt}, \mathrm{ff}, \mathrm{pf}}\right)$ befindet sich in Anhang C.2.4. Sie platziert den nichtsteuerbaren Beobachtereigenwert bei $\lambda_{B_{5}}=-36,79$, was gegenüber der Optimierung bezüglich beliebiger Störfrequenzen keine große Veränderung darstellt (s. Abschnitt 5.4.1). Allerdings ändern sich die Beobachtermatrix und insbesondere die Nachfiltermatrix in erheblichem Maße. Dies führt zu einer deutlich reduzierten Schranke für die Störverstärkung von $\gamma_{\mathrm{opt}, \mathrm{ff}, \mathrm{pf}}=9,56$. Durch die Lösung des rangbeschränkten LMI-Problems lässt sich im Beispiel ein nochmals leicht verbessertes Ergebnis von $\gamma_{\text {opt,ff,rc }}=8,18$ erreichen, was auch in Abbildung 5.15 abzulesen ist. Der nichtsteuerbare Beobachtereigenwert wird dabei bei $\lambda_{B_{5}}=-43,85$ platziert, die FIO-Parametrierung $\left(\boldsymbol{L}_{\mathrm{opt}, \mathrm{ff}, \mathrm{rc}}, \boldsymbol{V}_{\mathrm{opt}, \mathrm{ff}, \mathrm{rc}}\right)$ ist ebenfalls in Anhang C.2.4 angegeben. Auch bei anderen Beispielsystemen zeigen die beiden Lösungsansätze vergleichbare Ergebnisse. 


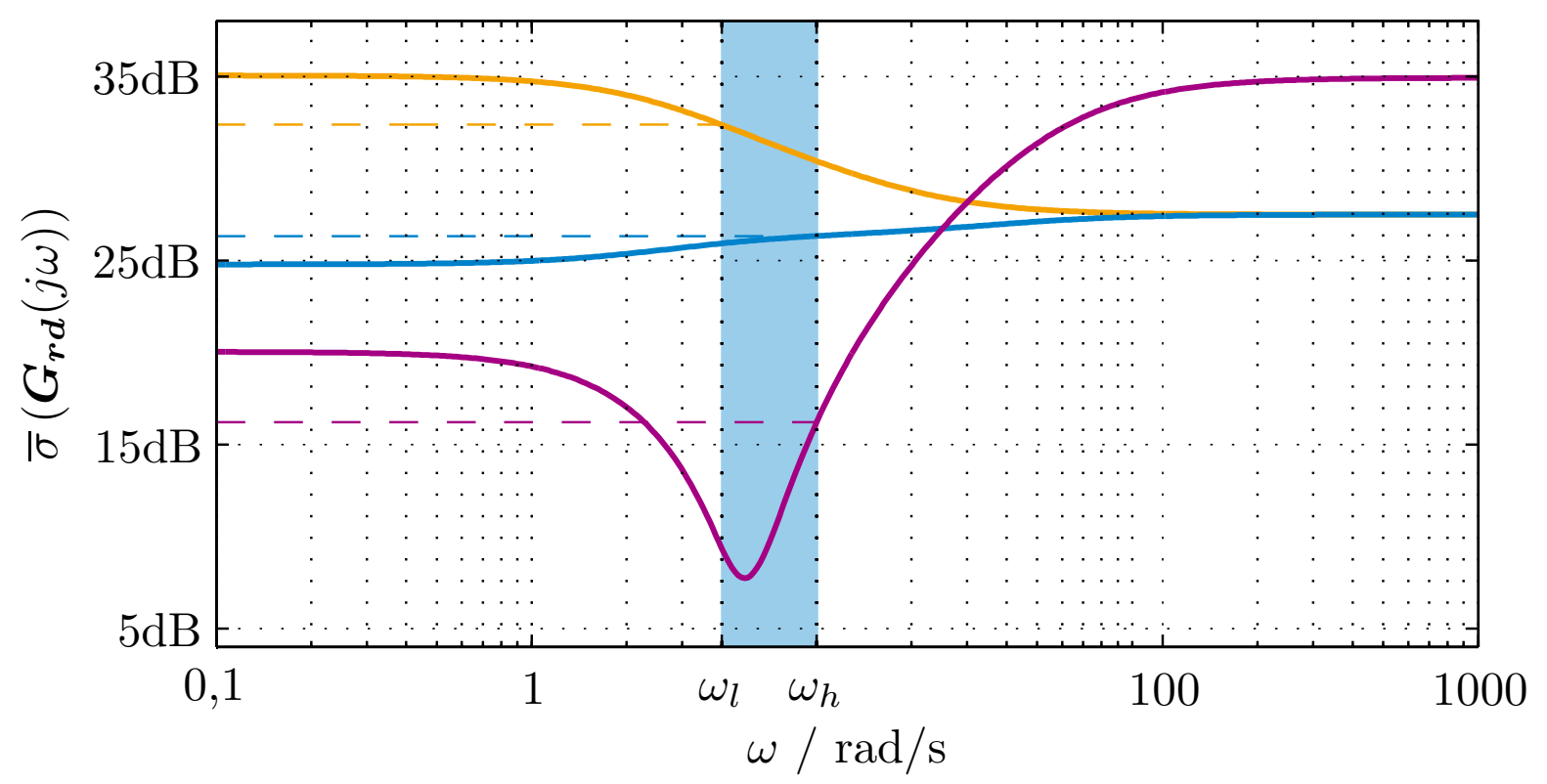

Abbildung 5.15: Verlauf des größten Singulärwertes von $\boldsymbol{G}_{\boldsymbol{r} \boldsymbol{d}}(s)$ für initiale $(-)$, auf beliebige Frequenzen optimierte $(-)$ und auf beschränkte Frequenzen optimierte (-) FIO-Parametrierung

Anhand von Abbildung 5.15 wird deutlich, dass nun gezielt auf den relevanten, blau hinterlegten Frequenzbereich optimiert wird. Zwischen $\omega_{l}$ und $\omega_{h}$ ist die Störverstärkung wesentlich geringer. Zwar ist der größte Singulärwert von $\boldsymbol{G}_{\boldsymbol{r} \boldsymbol{d}}(s)$ für hohe Frequenzen wesentlich größer als für die initiale Parametrierung. Dieser Frequenzbereich spielt jedoch im Beispiel keine Rolle, da keine Störungen auftreten, die diese Frequenzen enthalten. $\mathrm{Zu}$ betonen ist überdies, dass im Gegensatz zum Verfahren aus Abschnitt 5.4.2 die Pole von $\boldsymbol{G}_{\boldsymbol{r} \boldsymbol{f}}(s)$ nicht verändert werden. Die verbesserte Störunterdrückung wird also bei gleichbleibender Detektionsgeschwindigkeit erreicht.

Die Wirksamkeit der Störunterdrückung wird im Zeitverlauf der Residuen in Abbildung 5.16 ebenfalls deutlich. In der Simulation tritt nach 2s der sprungförmige Fehler $f_{1}=3$ auf, nach $5 \mathrm{~s}$ ist $f_{2}=2$ aktiv. Außerdem wird das System beeinflusst vom Störvektor

$$
\boldsymbol{d}(t)=\left[\begin{array}{c}
3 \cdot \sin (4 t) \\
0,06 \cdot \sin (6 t+\pi / 3) \\
0,02 \cdot \sin (8 t+\pi / 2)
\end{array}\right] .
$$

Das Residuum $r_{1}$ ist für beide FIOs nahezu deckungsgleich. Während sich in dem durch $\left(\boldsymbol{L}_{\text {opt }}, \boldsymbol{V}_{\text {opt }}\right)$ generierten Residuum $r_{2}$ die Störungen sehr stark widerspiegeln, ist anhand des durch $\left(\boldsymbol{L}_{\mathrm{opt}, \mathrm{ff}}, \boldsymbol{V}_{\mathrm{opt}, \mathrm{ff}}\right)$ erzeugten Residuums 

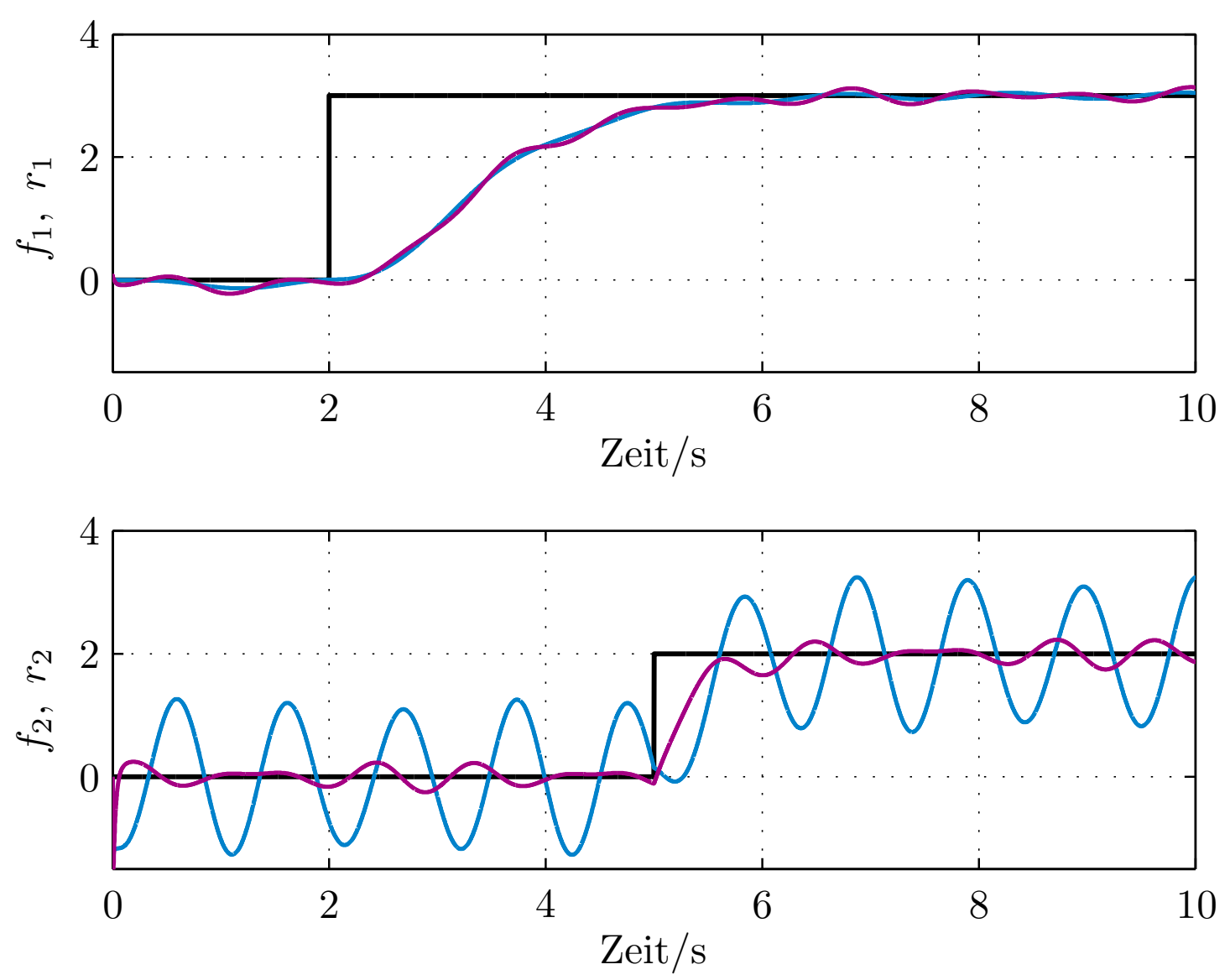

Abbildung 5.16: Zeitverlauf der Residuen bei auf beliebige Frequenzen optimiertem FIO (-) und auf beschränkte Frequenzen optimiertem FIO (-)

ohne Weiteres eine Fehlerisolation möglich. Der Einfluss der Störungen wird hier deutlich besser unterdrückt.

\subsubsection{Robustheit bezüglich unsicherer Systemparameter}

Die bisherigen Betrachtungen dieses Kapitels waren auf Systeme beschränkt, deren Systemmatrizen exakt bekannt sind und die lediglich exogenen Störungen unterliegen. In der Praxis unterliegen Systeme jedoch häufig unsicheren Systemparametern. Diese können zum Beispiel durch Alterungseffekte oder nicht exakt bekannte Lastzustände hervorgerufen werden. Sie können ihren Ursprung aber auch darin haben, dass ein linearisiertes System außerhalb des Arbeitspunktes betrieben wird. Wie in Abschnitt 5.1 beschrieben können derartige parametrische Unsicherheiten als exogene Störungen umformuliert werden, solange sie die Systemstabi- 
lität nicht beeinträchtigen. Wie in [58, Abschnitt 8.2] diskutiert kann dies jedoch zu konservativen Ergebnissen führen, da die vorhandene Information über die Struktur der Unsicherheiten nicht vollständig ausgenutzt wird. In diesem Abschnitt wird ein neuer Ansatz vorgestellt, der parametrische Unsicherheiten direkt berücksichtigt. Er wurde bereits in [224, 230, 235] präsentiert beziehungsweise veröffentlicht.

Kerngedanke ist es, zunächst mittels Satz 4.9 einen FIO zu entwerfen, der für das nominale System eine ideale Fehlerisolation gewährleistet. Bekanntlich existiert in nichtquadratischen Systemen eine unendliche Anzahl derartiger Parametrierungen, deren Beobachtermatrizen $\boldsymbol{L}=-\boldsymbol{W}_{B}^{-1} \boldsymbol{P}$ sich dadurch auszeichnen, dass sie

$$
\begin{array}{rlrl}
{\left[\begin{array}{ll}
\boldsymbol{w}_{B_{i j}}^{\top} & \boldsymbol{p}_{i j}^{\top}
\end{array}\right]} & =\left[\begin{array}{ll}
\mathbf{0}^{\top} & \boldsymbol{\phi}_{i}^{\top}
\end{array}\right] \boldsymbol{\Pi}_{i j}^{+}+\boldsymbol{z}_{i j}^{\top} \cdot{ }^{\perp} \boldsymbol{\Pi}_{i j}, & i=1, \ldots, n_{f}, j=1, \ldots, \delta_{i}, \\
{\left[\begin{array}{ll}
\boldsymbol{w}_{B_{m}}^{\top} & \boldsymbol{p}_{m}^{\top}
\end{array}\right]} & =\boldsymbol{z}_{m}^{\top} \cdot{ }^{\perp} \boldsymbol{\Pi}_{m}, & m & =\delta+1, \ldots, \delta+\mu, \\
{\left[\begin{array}{ll}
\boldsymbol{w}_{B_{k}}^{\top} & \boldsymbol{p}_{k}^{\top}
\end{array}\right]} & =\boldsymbol{z}_{k}^{\top} \cdot{ }^{\perp} \boldsymbol{\Pi}_{k}, & k & =\delta+\mu+1, \ldots, n,
\end{array}
$$

erfüllen. Die erste Beziehung beschreibt die Parameter- beziehungsweise Linkseigenvektoren für steuerbare Beobachtereigenwerte. Die zweite Gleichung behandelt zu kompensierende invariante Nullstellen, während die dritte Gleichung die Freiheitsgrade hinsichtlich frei platzierbarer nichtsteuerbarer Beobachtereigenwerte beschreibt. Sind alle Bedingungen aus (5.43) durch $\boldsymbol{L}$ erfüllt, so führen gemäß Satz 4.9 alle Nachfiltermatrizen

$$
\boldsymbol{V}=-\boldsymbol{S} \Theta^{+}-Z_{V} \cdot{ }^{\perp} \Theta
$$

mit $\boldsymbol{\Theta}=\boldsymbol{C}(\boldsymbol{A}-\boldsymbol{L} \boldsymbol{C})^{-1}\left(\boldsymbol{E}_{a}-\boldsymbol{L} \boldsymbol{E}_{s}\right)-\boldsymbol{E}_{s}$, einer beliebigen Diagonalmatrix $\boldsymbol{S}$ und einer frei wählbaren Matrix $\boldsymbol{Z}_{V}$ auf einer diagonalisierte Übertragungsmatrix $\boldsymbol{G}_{\boldsymbol{r} \boldsymbol{f}}(s)$. Die Menge aller FIO-Parametrierungen kann also durch

$$
\begin{aligned}
& \mathcal{L}=\left\{\boldsymbol{L} \mid \boldsymbol{L}=-\boldsymbol{W}_{B}^{-1} \boldsymbol{P},(5.43)\right\}, \\
& \mathcal{V}=\{\boldsymbol{V} \mid \boldsymbol{L} \in \mathcal{L},(5.44)\}
\end{aligned}
$$

beschrieben werden. Es gilt dann, aus diesen Mengen diejenige Parametrierung $\boldsymbol{L} \in \mathcal{L}, \boldsymbol{V} \in \mathcal{V}$ auszuwählen, welche die bestmögliche Robustheit bezüglich der unsicheren Parameter gewährleistet. Bezogen auf die Übertragungsmatrizen zwischen Fehlern, Stellgrößen und Störgrößen und den erzeugten Residuen bedeutet dies entsprechend den in Abschnitt 2.3 
definierten Zielen im Idealfall

$$
\begin{aligned}
& \boldsymbol{G}_{\boldsymbol{r} \boldsymbol{f}}(s)=\operatorname{diag}\left(g_{1,1}(s), \ldots, g_{n_{f}, n_{f}}(s)\right), \\
& \boldsymbol{G}_{\boldsymbol{r u}}(s)=\mathbf{0}, \\
& \boldsymbol{G}_{\boldsymbol{r} \boldsymbol{d}}(s)=\mathbf{0}
\end{aligned}
$$

trotz vorhandener parametrischer Unsicherheiten. Diese Anforderungen sollen durch eine entsprechende Wahl der verbleibenden Entwurfsfreiheitsgrade bestmöglich erfüllt werden. Der Übersichtlichkeit halber werden dabei zunächst fehlerbehaftete Systeme betrachtet, die autonom $(\boldsymbol{u}=\mathbf{0})$ und störungsfrei $(\boldsymbol{d}=\mathbf{0})$ sind. Es wird also das erste Ziel aus (5.45) betrachtet, im weiteren Verlauf wird das Verfahren dann auf Systeme mit Stellgrößen $\boldsymbol{u}$ und exogenen Störungen $\boldsymbol{d}$ erweitert.

Um ein unsicherheitsbehaftetes System zu beschreiben, wird die Zustandsraumdarstellung

$$
\begin{aligned}
& \dot{\boldsymbol{x}}=\boldsymbol{A}_{\chi} \boldsymbol{x}+\boldsymbol{E}_{a, \chi} \boldsymbol{f}, \\
& \boldsymbol{y}=\boldsymbol{C}_{\chi} \boldsymbol{x}+\boldsymbol{E}_{s, \chi} \boldsymbol{f}
\end{aligned}
$$

herangezogen. Dabei gilt für die Systemmatrizen

$$
\begin{aligned}
{\left[\begin{array}{ll}
\boldsymbol{A}_{\boldsymbol{\chi}} & \boldsymbol{E}_{a, \boldsymbol{\chi}} \\
\boldsymbol{C}_{\boldsymbol{\chi}} & \boldsymbol{E}_{s, \boldsymbol{\chi}}
\end{array}\right] \in \mathcal{M} } & =\left\{\left[\begin{array}{ll}
\boldsymbol{A}_{\boldsymbol{\chi}} & \boldsymbol{E}_{a, \boldsymbol{\chi}} \\
\boldsymbol{C}_{\boldsymbol{\chi}} & \boldsymbol{E}_{s, \boldsymbol{\chi}}
\end{array}\right]=\sum_{l=1}^{N} \chi_{l}\left[\begin{array}{ll}
\boldsymbol{A}_{l} & \boldsymbol{E}_{a, l} \\
\boldsymbol{C}_{l} & \boldsymbol{E}_{s, l}
\end{array}\right]\right\}, \\
\sum_{l=1}^{N} \chi_{l} & =1, \quad \chi_{l} \geq 0 .
\end{aligned}
$$

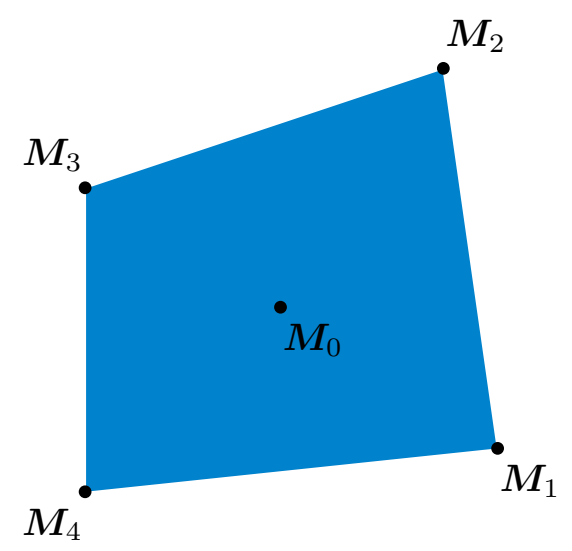

Abbildung 5.17: Gebiet polytopischer Unsicherheiten 
Die Matrizen $\boldsymbol{A}_{\boldsymbol{\chi}}, \boldsymbol{E}_{a, \boldsymbol{\chi}}, \boldsymbol{C}_{\boldsymbol{\chi}}$ und $\boldsymbol{E}_{s, \boldsymbol{\chi}}$ lassen sich also als konvexe Kombinationen von Eckpunkten eines Polytops beschreiben. Durch diese in Abbildung 5.17 veranschaulichte Beschreibung lässt sich eine große Klasse von unsicherheitsbehafteten Systemen darstellen. Sie umfasst beispielsweise Systeme mit Matrizen der Form

$$
\boldsymbol{A}=\boldsymbol{A}_{0}+\sum_{i=1}^{K} p_{i} \cdot \Delta \boldsymbol{A}_{i},
$$

die affin von $K$ unsicheren Parametern $p_{i}$ abhängen. Durch sogenanntes polytopic covering [7, Abschnitt 3.2.2] lässt sich auch für Systeme, deren Matrizen polynomial von unsicheren Parametern abhängen, eine Beschreibung der Form (5.47) angeben. Dabei werden fiktive Parameter eingeführt, die es erlauben, die nichtlinearen Parameterabhängigkeiten in multilineare Abhängigkeiten zu überführen. Mittels einer Variablentransformation erhält man dann eine affine Darstellung. Auf Details wird an dieser Stelle nicht näher eingegangen, es sei jedoch angemerkt, dass bei diesem Ansatz einhüllende Funktionen für die nichtlinearen Abhängigkeiten gefunden werden müssen. Dabei ist eine Abwägung zwischen der eingeführten Konservativität (bei grober Approximation der nichtlinearen Abhängigkeiten) und des rechnerischen Aufwandes (bei feiner Approximation der nichtlinearen Abhängigkeiten) zu treffen.

Durch (5.46) und (5.47) wird also die Menge der möglichen Systemdynamiken beschrieben. Für den Entwurf wird nun davon ausgegangen, dass ein nominales Modell existiert, für das offensichtlich

$$
\left[\begin{array}{ll}
\boldsymbol{A}_{0} & \boldsymbol{E}_{a, 0} \\
\boldsymbol{C}_{0} & \boldsymbol{E}_{s, 0}
\end{array}\right] \in \mathcal{M}
$$

gilt. Fasst man nun wie in (4.3) beziehungsweise (4.4) die Strecken- und die Beobachterdynamik zu einem dynamischen Gesamtsystem zusammen, so ergibt sich für die $N$ Eckpunkte des Polytops $\mathcal{M}$ jeweils die Beschreibung

$$
\begin{aligned}
{\left[\begin{array}{c}
\dot{\boldsymbol{x}} \\
\dot{\hat{\boldsymbol{x}}}
\end{array}\right] } & =\left(\left[\begin{array}{cc}
\boldsymbol{A}_{l} & \mathbf{0} \\
\mathbf{0} & \boldsymbol{A}_{0}
\end{array}\right]-\left[\begin{array}{c}
\mathbf{0} \\
-\boldsymbol{L}
\end{array}\right]\left[\begin{array}{ll}
\boldsymbol{C}_{l} & -\boldsymbol{C}_{0}
\end{array}\right]\right)\left[\begin{array}{l}
\boldsymbol{x} \\
\hat{\boldsymbol{x}}
\end{array}\right]+\left(\left[\begin{array}{c}
\boldsymbol{E}_{a, l} \\
\mathbf{0}
\end{array}\right]-\left[\begin{array}{c}
\mathbf{0} \\
-\boldsymbol{L}
\end{array}\right] \boldsymbol{E}_{s, l}\right) \boldsymbol{f} \\
& =\left(\tilde{\boldsymbol{A}}_{l}-\tilde{\boldsymbol{L}} \tilde{\boldsymbol{C}}_{l}\right) \tilde{\boldsymbol{x}}+\left(\tilde{\boldsymbol{E}}_{a, l}-\tilde{\boldsymbol{L}} \tilde{\boldsymbol{E}}_{s, l}\right) \boldsymbol{f}, \\
\boldsymbol{r} & =\boldsymbol{V}\left[\begin{array}{ll}
\boldsymbol{C}_{l} & \boldsymbol{C}_{0}
\end{array}\right]\left[\begin{array}{c}
\boldsymbol{x} \\
\hat{\boldsymbol{x}}
\end{array}\right]+\boldsymbol{V} \boldsymbol{E}_{s, l} \boldsymbol{f} \\
& =\boldsymbol{V} \tilde{\boldsymbol{C}}_{l} \tilde{\boldsymbol{x}}+\boldsymbol{V} \tilde{\boldsymbol{E}}_{s, l} \boldsymbol{f} .
\end{aligned}
$$


Entscheidend ist an dieser Stelle, dass die Systemmatrizen nun im Allgemeinen nicht mehr den nominalen Matrizen entsprechen. Dies hat zur Folge, dass man nicht mehr wie im Falle exakt bekannter Systemmatrizen durch eine Variablensubstitution der Form $\boldsymbol{\xi}=\boldsymbol{x}-\hat{\boldsymbol{x}}$ auf ein $n$-dimensionales Ersatzsystem transformieren kann. Dies war in Abschnitt 4.4 jedoch die Voraussetzung, um die Dualität zu Entkopplungsregelungen für den Entwurf von FIOs auszunutzen. Die Betrachtung unsicherer Systeme verdeutlicht somit, dass die Dualität lediglich mathematischer Natur ist. Wie in den bisherigen Abschnitten gezeigt lässt sie sich zwar sehr nutzbringend anwenden, es darf dabei jedoch nicht außer Acht gelassen werden, dass es sich beim Entwurf von Entkopplungsregelungen und der Auslegung von FIOs um grundsätzlich unterschiedliche Problemstellungen handelt.

Ziel ist es zunächst, für den gesamten durch $\mathcal{M}$ beschriebenen Bereich die Übertragungsmatrix zwischen Fehlern und Residuen möglichst gut zu diagonalisieren. Für die $N$ Eckpunkte des Polytops $\mathcal{M}$ ergibt sich für die entsprechenden Übertragungsmatrizen die Darstellung

$$
\begin{aligned}
\boldsymbol{G}_{\boldsymbol{r f}, l}(s) & =\boldsymbol{V} \tilde{\boldsymbol{C}}_{l}\left(s \boldsymbol{I}_{2 n}-\left(\tilde{\boldsymbol{A}}_{l}-\tilde{\boldsymbol{L}} \tilde{\boldsymbol{C}}_{l}\right)\right)^{-1}\left(\tilde{\boldsymbol{E}}_{a, l}-\tilde{\boldsymbol{L}} \tilde{\boldsymbol{E}}_{s, l}\right)+\boldsymbol{V} \tilde{\boldsymbol{E}}_{s, l} \\
& =\sum_{k=1}^{2 n} \frac{\boldsymbol{V} \tilde{\boldsymbol{C}}_{l} \tilde{\boldsymbol{v}}_{B_{k}} \cdot \tilde{\boldsymbol{w}}_{B_{k}}^{\top}\left(\tilde{\boldsymbol{E}}_{a, l}-\tilde{\boldsymbol{L}} \tilde{\boldsymbol{E}}_{s, l}\right)}{s-\lambda_{B_{k}}}+\boldsymbol{V} \tilde{\boldsymbol{E}}_{s, l}, l=1, \ldots, N
\end{aligned}
$$

wenn wie in Annahme 4.4 von paarweise verschiedenen Beobachtereigenwerten ausgegangen wird. Diese Formulierung setzt also auch voraus, dass die Streckeneigenwerte paarweise verschieden sind, was sich jedoch gegebenenfalls durch den Entwurf einer Regelung erreichen lässt, wenn das System vollständig steuerbar ist. Das Ziel der Diagonalisierung von $\boldsymbol{G}_{\boldsymbol{r f}, l}(s)$ wird nach den Überlegungen von Abschnitt 4.5.2 ideal erreicht, wenn

1. die steuerbaren Beobachtereigenwerte $(k=n+1, \ldots, n+\delta)$ nur in der jeweils vorgegebenen Spalte von $\boldsymbol{G}_{\boldsymbol{r} \boldsymbol{f}, l}(s)$ als Pole auftauchen,

2. die nichtsteuerbaren Beobachtereigenwerte $(k=n+\delta+1, \ldots, 2 n)$ nicht als Pole von $\boldsymbol{G}_{\boldsymbol{r} \boldsymbol{f}, l}(s)$ auftauchen,

3. die Beziehung $\boldsymbol{G}_{\boldsymbol{r} \boldsymbol{f}, l}(s=0)=\boldsymbol{S}$ mit einer beliebigen Diagonalmatrix $\boldsymbol{S}$ gilt und

4. die Streckeneigenwerte $(k=1, \ldots, n)$ nicht als Pole von $\boldsymbol{G}_{\boldsymbol{r f}, l}(s)$ auftauchen. 
Die vierte Bedingung tauchte dabei in Abschnitt 4.9.1 nicht auf, da lediglich das virtuelle Ersatzsystem der Ordnung $n$ betrachtet wurde. Mit der gleichen Argumentation wie in der Herleitung von Lemma 4.3 (s. Seite 92) lässt sich jedoch nachweisen, dass die 4. Bedingung dazu führt, dass die Einhaltung der 3. Bedingung die Erfüllung von $\boldsymbol{G}_{\boldsymbol{r} \boldsymbol{f}}(s)=$ $\operatorname{diag}\left(g_{1,1}(s), \ldots, g_{n_{f}, n_{f}}(s)\right)$ für alle $s \in \mathbb{C}$ sicherstellt.

Für alle $\boldsymbol{L} \in \mathcal{L}$ und $\boldsymbol{V} \in \mathcal{V}$ sind die angeführten Bedingungen für das nominale System erfüllt. Die Mengen $\mathcal{L}$ und $\mathcal{V}$ werden nach Satz 4.9 durch die verbleibenden Freiheitsgrade $\boldsymbol{z}_{i j}^{\top}, \boldsymbol{z}_{m}^{\top}, \boldsymbol{z}_{k}^{\top}, \lambda_{B_{k}}$ und $\boldsymbol{Z}_{V}$ charakterisiert. Diese werden im Weiteren ausgenutzt, um die Anforderungen 1 bis 4 trotz vorhandener parametrischer Unsicherheiten möglichst gut zu erfüllen.

\section{Steuerbare Beobachtereigenwerte}

Um die Bedingung 1 für den gesamten Bereich möglichst gut zu erfüllen, wird die größte sich ergebende Abweichung betrachtet. Das Entwurfsziel lautet also für alle $i=1, \ldots, n_{f}$ und $j=1, \ldots, \delta_{i}$

$$
\underset{\boldsymbol{z}_{i j}^{\top}}{\operatorname{minimiere}} \max _{\chi}\left\|\tilde{\boldsymbol{w}}_{B_{i j}, \boldsymbol{\chi}}^{\top}\left(\tilde{\boldsymbol{E}}_{a, \boldsymbol{\chi}}-\tilde{\boldsymbol{L}} \tilde{\boldsymbol{E}}_{s, \boldsymbol{\chi}}\right) \pm \boldsymbol{\phi}_{i}^{\top}\right\|_{2}
$$

Das unbestimmte Vorzeichen im Optimierungsproblem ist dadurch zu erklären, dass lediglich die Richtung von $\phi_{i}^{\top}$ entscheidend dafür ist, dass ein Eigenwert an eine Spalte gebunden ist. Die Orientierung, das heißt das Vorzeichen von $\phi_{i}^{\top}$, ist dabei nicht entscheidend. Das Optimierungsproblem (5.49) wird daher für beide Vorzeichen gelöst und die Lösung mit dem geringeren Gütemaß wird ausgewählt. Allerdings ist (5.49) in der vorliegenden Form nicht handhabbar, da es für eine unendlich große Anzahl von Systemen zu lösen ist. Es werden daher lediglich die Eckpunkte des Polytops $\mathcal{M}$ betrachtet, das heißt

$$
\underset{\boldsymbol{z}_{i j}^{\top}}{\operatorname{minimiere}} \max _{l=1, \ldots, N}\left\|\tilde{\boldsymbol{w}}_{B_{i j}, l}^{\top}\left(\tilde{\boldsymbol{E}}_{a, l}-\tilde{\boldsymbol{L}} \tilde{\boldsymbol{E}}_{s, l}\right) \pm \boldsymbol{\phi}_{i}^{\top}\right\|_{2} .
$$

Problematisch an dieser Darstellung ist, dass sowohl der Linkseigenvektor $\tilde{\boldsymbol{w}}_{B_{i j}, l}^{\top}$ als auch die Beobachtermatrix $\tilde{\boldsymbol{L}}$ des Gesamtsystems von den Freiheitsgraden $\boldsymbol{z}_{i j}^{\top}$ abhängen. Im Folgenden wird gezeigt, wie sich (5.50) in ein konvexes Optimierungsproblem mit LMI-Nebenbedingungen überführen lässt. 
Dazu werden zunächst die Linkseigenvektoren des Gesamtsystems analysiert, für die nach Definition

$$
\begin{aligned}
\tilde{\boldsymbol{w}}_{B_{i j}, l}^{\top}\left(\tilde{\boldsymbol{A}}_{l}-\tilde{\boldsymbol{L}} \tilde{\boldsymbol{C}}_{l}\right) & =\lambda_{B_{i j}} \tilde{\boldsymbol{w}}_{B_{i j}, l}^{\top}, \\
\Rightarrow \tilde{\boldsymbol{w}}_{B_{i j}, l}^{\top}\left(\lambda_{B_{i j}} \boldsymbol{I}_{2 n}-\tilde{\boldsymbol{A}}_{l}\right) & =-\tilde{\boldsymbol{w}}_{B_{i j}, l}^{\top} \tilde{\boldsymbol{L}} \tilde{\boldsymbol{C}}_{l}
\end{aligned}
$$

gilt. Zerlegt man nun den Linkseigenvektor des Gesamtsystems in die Komponenten

$$
\tilde{\boldsymbol{w}}_{B_{i j}, l}^{\top}=\left[\begin{array}{ll}
\boldsymbol{w}_{B_{i j}, l, s}^{\top} & \boldsymbol{w}_{B_{i j}, l, b}^{\top}
\end{array}\right] \in \mathbb{R}^{2 n}
$$

so korrespondiert $\boldsymbol{w}_{B_{i j}, l, s}^{\top} \in \mathbb{R}^{n}$ mit den Zuständen der Strecke und $\boldsymbol{w}_{B_{i j}, l, b}^{\top} \in \mathbb{R}^{n}$ ist den Beobachterzuständen zugeordnet. Damit lässt sich für (5.51) schreiben

$$
\begin{gathered}
{\left[\begin{array}{ll}
\boldsymbol{w}_{B_{i j}, l, s}^{\top} & \boldsymbol{w}_{i j, l, b}^{\top}
\end{array}\right]\left[\begin{array}{cc}
\lambda_{B_{i j}} \boldsymbol{I}_{n}-\boldsymbol{A}_{l} & \mathbf{0} \\
\mathbf{0} & \lambda_{B_{i j}} \boldsymbol{I}_{n}-\boldsymbol{A}_{0}
\end{array}\right]} \\
=-\left[\begin{array}{ll}
\boldsymbol{w}_{B_{i j}, l, s}^{\top} & \boldsymbol{w}_{B_{i j}, l, b}^{\top}
\end{array}\right]\left[\begin{array}{c}
\mathbf{0} \\
-\boldsymbol{L}
\end{array}\right]\left[\begin{array}{ll}
\boldsymbol{C}_{l} & -\boldsymbol{C}_{0}
\end{array}\right] \\
=\boldsymbol{w}_{B_{i j}, l, b}^{\top} \boldsymbol{L}\left[\begin{array}{ll}
\boldsymbol{C}_{l} & -\boldsymbol{C}_{0}
\end{array}\right] .
\end{gathered}
$$

Aus dem zweiten Spaltenblock folgt die Beziehung

$$
\boldsymbol{w}_{B_{i j}, l, b}^{\top}\left(\lambda_{B_{i j}} \boldsymbol{I}_{n}-\boldsymbol{A}_{0}\right)=-\boldsymbol{w}_{B_{i j}, l, b}^{\top} \boldsymbol{L} \boldsymbol{C}_{0}=\boldsymbol{p}_{i j}^{\top} \boldsymbol{C}_{0} .
$$

Dies entspricht der Bedingung (4.19) von Seite 88, deren Einhaltung beim Entwurf für das nominale System sichergestellt wurde. Somit lässt sich festhalten, dass für den zweiten Teil des Linkseigenvektors des Gesamtsystems die einfache Gleichung

$$
\boldsymbol{w}_{B_{i j}, l, b}^{\top}=\boldsymbol{w}_{B_{i j}, 0}^{\top}
$$

gilt. Aus dem ersten Spaltenblock von (5.52) ergibt sich damit

$$
\boldsymbol{w}_{B_{i j}, l, s}^{\top}\left(\lambda_{B_{i j}} \boldsymbol{I}_{n}-\boldsymbol{A}_{l}\right)=\boldsymbol{w}_{B_{i j}, l, b}^{\top} \boldsymbol{L} \boldsymbol{C}_{l}=-\boldsymbol{p}_{i j}^{\top} \boldsymbol{C}_{l} .
$$

Für den zu minimierenden Term in (5.50) ergibt sich mit der gleichen 
Zerlegung von $\tilde{\boldsymbol{w}}_{B_{i j}, l}^{\top}$ die Beziehung

$$
\begin{aligned}
\tilde{\boldsymbol{w}}_{B_{i j}, l}^{\top}\left(\tilde{\boldsymbol{E}}_{a, l}-\tilde{\boldsymbol{L}} \tilde{\boldsymbol{E}}_{s, l}\right) & =\left[\begin{array}{ll}
\boldsymbol{w}_{B_{i j}, l, s}^{\top} & \boldsymbol{w}_{B_{i j}, l, b}^{\top}
\end{array}\right]\left(\left[\begin{array}{c}
\boldsymbol{E}_{a, l} \\
\mathbf{0}
\end{array}\right]-\left[\begin{array}{c}
\mathbf{0} \\
-\boldsymbol{L}
\end{array}\right] \boldsymbol{E}_{s, l}\right) \\
& =\left[\begin{array}{ll}
\boldsymbol{w}_{B_{i j}, l, s}^{\top} & \boldsymbol{w}_{B_{i j}, l, b}^{\top}
\end{array}\right]\left[\begin{array}{c}
\boldsymbol{E}_{a, l} \\
\boldsymbol{L} \boldsymbol{E}_{s, l}
\end{array}\right] \\
& =\boldsymbol{w}_{B_{i j}, l, s}^{\top} \boldsymbol{E}_{a, l}+\boldsymbol{w}_{B_{i j}, l, b}^{\top} \boldsymbol{L} \boldsymbol{E}_{s, l} \\
& =\boldsymbol{w}_{B_{i j}, l, s}^{\top} \boldsymbol{E}_{a, l}-\boldsymbol{p}_{i j}^{\top} \boldsymbol{E}_{s, l} .
\end{aligned}
$$

Zwar taucht hierin neben $\boldsymbol{p}_{i j}^{\top}$ noch der davon abhängende Ausdruck $\boldsymbol{w}_{B_{i j}, l, s}^{\top}$ auf, jedoch ist durch (5.53) eine Gleichungsbedingung gegeben, die $\boldsymbol{w}_{B_{i j}, l, s}^{\top}$ und $\boldsymbol{p}_{i j}^{\top}$ verknüpft.

Um nun noch explizit die Abhängigkeit vom gesuchten Freiheitsgrad $\boldsymbol{z}_{i j}^{\top}$ $\mathrm{zu}$ berücksichtigen, wird die aus dem Entwurf für das nominale System herrührende Beziehung (5.43a) verwendet. Sie impliziert

$$
\boldsymbol{p}_{i j}^{\top}=\left(\left[\begin{array}{ll}
\mathbf{0}^{\top} & \boldsymbol{\phi}_{i}^{\top}
\end{array}\right] \boldsymbol{\Pi}_{i j, 0}^{+}+\boldsymbol{z}_{i j}^{\top} \cdot{ }^{\perp} \boldsymbol{\Pi}_{i j, 0}\right)\left[\begin{array}{c}
\mathbf{0} \\
\boldsymbol{I}_{n_{y}}
\end{array}\right] .
$$

Setzt man dies in (5.53) und (5.54) ein, so lässt sich das Optimierungsproblem (5.50) mithilfe von Lemma 3.5 schreiben als

$$
\begin{aligned}
& \underset{\boldsymbol{z}_{i j}^{\top}, \boldsymbol{w}_{B_{i j}, l, s}^{\top}}{\operatorname{minimiere}} \gamma_{f, i j}, \text { sodass } \forall l=1, \ldots, N \\
& {\left[\begin{array}{ccc}
\gamma_{f, i j} & \left.\boldsymbol{w}_{B_{i j}, l, s}^{\top} \boldsymbol{E}_{a, l}-\left(\left[\begin{array}{ll}
\mathbf{0}^{\top} & \boldsymbol{\phi}_{i}^{\top}
\end{array}\right] \boldsymbol{\Pi}_{i j, 0}^{+}+\boldsymbol{z}_{i j}^{\top} \cdot{ }^{\perp} \boldsymbol{\Pi}_{i j, 0}\right)\left[\begin{array}{c}
\mathbf{0} \\
\boldsymbol{I}_{n_{y}}
\end{array}\right] \boldsymbol{E}_{s, l} \pm \boldsymbol{\phi}_{i}^{\top}\right] \succ \mathbf{0}, \\
\star & \boldsymbol{I}_{n_{f}}
\end{array}\right]} \\
& \boldsymbol{w}_{B_{i j}, l, s}^{\top}\left(\lambda_{B_{i j}} \boldsymbol{I}_{n}-\boldsymbol{A}_{l}\right)=-\left(\left[\begin{array}{ll}
\mathbf{0}^{\top} & \left.\boldsymbol{\phi}_{i}^{\top}\right]
\end{array}\right] \boldsymbol{\Pi}_{i j, 0}^{+}+\boldsymbol{z}_{i j}^{\top} \cdot{ }^{\perp} \boldsymbol{\Pi}_{i j, 0}\right)\left[\begin{array}{c}
\mathbf{0} \\
\boldsymbol{I}_{n_{y}}
\end{array}\right] \boldsymbol{C}_{l} .
\end{aligned}
$$

Die Tatsache, dass in (5.55) Gleichungsbedingungen enthalten sind, stellt wie in Abschnitt 3.2.2 diskutiert kein Hindernis dar. Die Gleichungsbedingung lässt sich wie dort beschrieben in eine Ungleichungsbedingung mit beliebig kleinem $\varepsilon>0$ umwandeln. Mithilfe von Schnittstellen wie YALMIP [128] ist auch die direkte Eingabe von Gleichungsbedingungen möglich, eine Umwandlung in Ungleichungsbedingungen erfolgt dann automatisiert.

Es ist jedoch zu betonen, dass die durch (5.55) ermittelte Schranke im Allgemeinen nicht garantiert, dass auch das Maximum über alle $\chi$ in (5.49) 
kleiner ist als $\gamma_{f, i j}$. Betrachtet man hinreichend viele Systeme $N$ innerhalb von $\mathcal{M}$, zum Beispiel indem man ein Gitter in das Gebiet legt, so ist zwar für die praktische Anwendung davon auszugehen, dass (5.55) zu sehr guten Ergebnissen hinsichtlich der Robustheit führt. Theoretisch ist dies jedoch bis auf Spezialfälle nicht abgesichert. Ist beispielsweise $\boldsymbol{A}_{\boldsymbol{\chi}}=\boldsymbol{A}_{0}$ und $\boldsymbol{C}_{\boldsymbol{\chi}}=\boldsymbol{C}_{0}$, so folgt aus (5.53) unmittelbar, dass der Linkseigenvektor $\boldsymbol{w}_{B_{i j}, l, s}^{\top}=\boldsymbol{w}_{B_{i j}, 0, s}^{\top}$ für alle Systeme in $\mathcal{M}$ gleich ist. Für den einfachen Fall eines unsicheren Parameters, von $\operatorname{dem} \boldsymbol{E}_{a, \chi}$ und $\boldsymbol{E}_{s, \boldsymbol{\chi}}$ affin abhängen, lässt sich $\boldsymbol{E}_{a, \boldsymbol{\chi}}=\chi \boldsymbol{E}_{a, 1}+(1-\chi) \boldsymbol{E}_{a, 2}$ und $\boldsymbol{E}_{s, \boldsymbol{\chi}}=\chi \boldsymbol{E}_{s, 1}+(1-\chi) \boldsymbol{E}_{s, 2}$ schreiben. Wegen (5.55b) gilt dann

$$
\begin{aligned}
{\left[\begin{array}{cc}
\gamma_{f, i j} & \boldsymbol{w}_{B_{i j}, 0, s}^{\top} \boldsymbol{E}_{a, l}-\boldsymbol{p}_{i j}^{\top} \boldsymbol{E}_{s, l} \\
\star & \boldsymbol{I}_{n_{f}}
\end{array}\right] } & \underbrace{\left[\begin{array}{cc}
\gamma_{f, i j} & \boldsymbol{w}_{B_{i j}, 0, s}^{\top} \boldsymbol{E}_{a, 1}-\boldsymbol{p}_{i j}^{\top} \boldsymbol{E}_{s, 1} \\
\star & \boldsymbol{I}_{n_{f}}
\end{array}\right]}_{\succ 0} \\
& +(1-\chi) \underbrace{\left[\begin{array}{cc}
\gamma_{f, i j} & \boldsymbol{w}_{B_{i j}, 0, s}^{\top} \boldsymbol{E}_{a, 2}-\boldsymbol{p}_{i j}^{\top} \boldsymbol{E}_{s, 2} \\
\star & \boldsymbol{I}_{n_{f}}
\end{array}\right]}_{\succ \mathbf{0}} \succ \mathbf{0}
\end{aligned}
$$

und es ist gewährleistet, dass $\gamma_{f, i j}$ für alle Systeme in $\mathcal{M}$ Gültigkeit besitzt.

\section{Nichtsteuerbare Beobachtereigenwerte}

Für die verbleibenden Beobachtereigenwerte (Bedingung 2) ist im Idealfall

$$
\tilde{\boldsymbol{w}}_{B_{k}, \boldsymbol{\chi}}^{\top}\left(\tilde{\boldsymbol{E}}_{a, \boldsymbol{\chi}}-\tilde{\boldsymbol{L}} \tilde{\boldsymbol{E}}_{s, \boldsymbol{\chi}}\right)=\mathbf{0}^{\top},
$$

da sie nach dem Gilbert-Kriterium dann nichtsteuerbar sind und somit nicht in $\boldsymbol{G}_{\boldsymbol{r} \boldsymbol{f}}(s)$ auftauchen. Betrachtet werden zunächst nur diejenigen nichtsteuerbaren Beobachtereigenwerte, die nicht zur Kompensation invarianter Nullstellen herangezogen werden müssen und die somit als Freiheitsgrade für die Optimierung zur Verfügung stehen (vgl. Abschnitt 4.9.2). Entsprechend (5.50) lässt sich die größte Abweichung von dieser Bedingung für die ideale Isolation ausdrücken durch

$$
\underset{\boldsymbol{z}_{k}^{\top}, \lambda_{B_{k}}}{\operatorname{minimiere}} \max _{l=1, \ldots, N}\left\|\tilde{\boldsymbol{w}}_{B_{k}, l}^{\top}\left(\tilde{\boldsymbol{E}}_{a, l}-\tilde{\boldsymbol{L}} \tilde{\boldsymbol{E}}_{s, l}\right)\right\|_{2} .
$$

Die Beziehung (5.54) gilt für die nichtsteuerbaren Eigenwerte analog, die Parametervektoren ergeben sich aus der Lösung (5.43c) für das nominale 
System zu

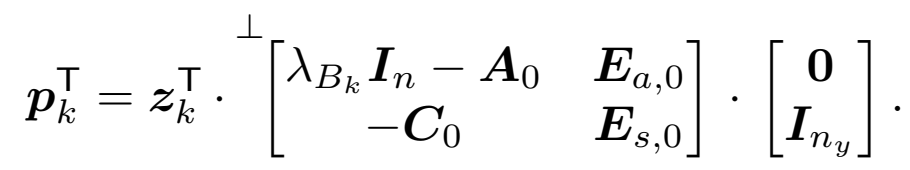

Das Optimierungsproblem lässt sich also äquivalent formulieren als

$$
\begin{aligned}
& \underset{\boldsymbol{z}_{k}^{\top}, \boldsymbol{w}_{B_{k}, l, s}^{\top}, \lambda_{B_{k}}}{\operatorname{minimiere}} \gamma_{f, k}, \text { sodass } \forall l=1, \ldots, N \\
& {\left[\begin{array}{cc}
\gamma_{f, k} & \boldsymbol{\chi}_{l}^{\top} \\
\star & \boldsymbol{I}_{n_{f}}^{\top}
\end{array}\right] \succ \mathbf{0},} \\
& \boldsymbol{\chi}_{l}^{\top}=\boldsymbol{w}_{B_{k}, l, s}^{\top} \boldsymbol{E}_{a, l}-\boldsymbol{z}_{k}^{\top} \cdot\left[\begin{array}{cr}
\lambda_{B_{k}} \boldsymbol{I}_{n}-\boldsymbol{A}_{0} & \boldsymbol{E}_{a, 0} \\
-\boldsymbol{C}_{0} & \boldsymbol{E}_{s, 0}
\end{array}\right]\left[\begin{array}{c}
\mathbf{0} \\
\boldsymbol{I}_{n_{y}}
\end{array}\right] \boldsymbol{E}_{s, l}, \\
& \boldsymbol{w}_{B_{k}, l, s}^{\top}\left(\lambda_{B_{k}} \boldsymbol{I}_{n}-\boldsymbol{A}_{l}\right)=-\boldsymbol{z}_{k}^{\top} \cdot\left[\begin{array}{ll}
\lambda_{B_{k}} \boldsymbol{I}_{n}-\boldsymbol{A}_{0} & \boldsymbol{E}_{a, 0} \\
-\boldsymbol{C}_{0} & \boldsymbol{E}_{s, 0}
\end{array}\right]\left[\begin{array}{c}
\mathbf{0} \\
\boldsymbol{I}_{n_{y}}
\end{array}\right] \boldsymbol{C}_{l}, \\
& \left\|\boldsymbol{z}_{k}^{\top}\right\|_{2} \geq 1, \\
& \lambda_{B_{k}} \in \mathcal{D} \subset \mathbb{C}^{-} .
\end{aligned}
$$

In dieser Formulierung wird durch (5.56e) der Tatsache Rechnung getragen, dass $\boldsymbol{z}_{k}^{\top} \neq \mathbf{0}^{\top}$ sein muss, da sich andernfalls eine singuläre Linkseigenvektormatrix $\boldsymbol{W}_{B}$ ergibt. Um eine degenerierte Lösung mit $\boldsymbol{z}^{\top}=\mathbf{0}^{\top}$ auszuschließen, wird (5.56e) als zusätzliche Nebenbedingung in das Optimierungsproblem eingefügt. Da die Linkseigenvektoren und Parametervektoren skalierungsinvariant sind, stellt die Beschränkung der Norm von $\boldsymbol{z}_{k}^{\top}$ auf einen Wert oberhalb der Schranke 1 wie bereits in Abschnitt 5.3.2 beschrieben keine Einschränkung der Allgemeinheit dar. Da durch $\lambda_{B_{k}}$ keine invariante Nullstelle zu kompensieren ist, kann der Beobachtereigenwert weiterhin prinzipiell frei gewählt werden. Um sehr langsame Eigenbewegungen ebenso auszuschließen wie zu große Beträge der Eigenwerte, bietet es sich an, den zulässigen Eigenwertbereich auf eine vorgegebene Region $\mathcal{D}$ zu beschränken, das heißt (5.56f) als Nebenbedingung zu berücksichtigen.

Aufgrund der multiplikativen Terme in (5.56c) und (5.56d) und der Normbeschränkung für $\boldsymbol{z}_{k}^{\top}$ handelt es sich bei (5.56) im Gegensatz zu (5.55) nicht um ein konvexes Optimierungsproblem. Die Lösung kann zum Beispiel auf Basis von zufällig gewählten Startwerten mithilfe iterativer Lösungsstrategien erfolgen (s. zum Beispiel [73]). Aufgrund der geringen Anzahl von freien Parametern lassen sich die Optimierungsprobleme effizient lösen, die Konvergenz auf das globale Optimum ist für dieses Teilproblem jedoch nicht sichergestellt. 
Für nichtsteuerbare Beobachtereigenwerte $\lambda_{B_{m}}$, die zur Kompensation invarianter Nullstellen herangezogen werden, vereinfacht sich das Problem. Da die Eigenwerte selbst nicht mehr als Entwurfsfreiheitsgrade zur Verfügung stehen, ist ${ }^{\perp} \boldsymbol{\Pi}_{m, 0}$ eine konstante Matrix und die multiplikativen Terme in den Optimierungsvariablen in (5.56c) und (5.56d) werden affin in den verbleibenden Freiheitsgraden $\boldsymbol{w}_{B_{m}, l, s}^{\top}$ und $\boldsymbol{z}_{m}^{\top}$. Es ergibt sich

$$
\begin{aligned}
& \underset{\boldsymbol{z}_{m}^{\top}, \boldsymbol{w}_{B_{m}, l, s}^{\top}}{\operatorname{minimiere}} \gamma_{f, m}, \text { sodass } \forall l=1, \ldots, N \\
& {\left[\begin{array}{cc}
\gamma_{f, m} & \left.\boldsymbol{w}_{B_{m}, l, s}^{\top} \boldsymbol{E}_{a, l}-\boldsymbol{z}_{m}^{\top} \cdot{ }^{\perp} \boldsymbol{\Pi}_{m, 0}\left[\begin{array}{c}
\mathbf{0} \\
\boldsymbol{I}_{n_{y}}
\end{array}\right] \boldsymbol{E}_{s, l}\right] \succ \mathbf{0}, \\
\star & \boldsymbol{I}_{n_{f}}
\end{array}\right]} \\
& \boldsymbol{w}_{B_{m}, l, s}^{\top}\left(\lambda_{B_{m}} \boldsymbol{I}_{n}-\boldsymbol{A}_{l}\right)=-\boldsymbol{z}_{m}^{\top} \cdot{ }^{\perp} \boldsymbol{\Pi}_{m, 0}\left[\begin{array}{c}
\mathbf{0} \\
\boldsymbol{I}_{n_{y}}
\end{array}\right] \boldsymbol{C}_{l}, \\
& \left\|\boldsymbol{z}_{m}^{\top}\right\|_{2} \geq 1 .
\end{aligned}
$$

In (5.57) verbleibt lediglich die nichtkonvexe Nebenbedingung (5.57d). Diese lässt sich entsprechend dem Vorgehen in Abschnitt 5.3.2 durch mehrere konvexe Nebenbedingungen annähern, sodass sich ein LMI-Problem ergibt. Bemerkung 5.8. Existieren mehrere beliebig platzierbare nichtsteuerbare Beobachtereigenwerte, so ist zu beachten, dass komplexe Beobachtereigenwerte stets in konjugiert komplexen Paaren auftreten müssen, um eine reelle Beobachtermatrix zu erhalten. In einfacher Art und Weise lässt sich dies sicherstellen, wenn man die zusätzliche Einschränkung rein reeller Beobachtereigenwerte einführt.

\section{Optimierung der stationären Isolation}

Um Bedingung 3 zu erfüllen, also stationär möglichst gut zu isolieren, wird

$$
\boldsymbol{G}_{\boldsymbol{r} \boldsymbol{f}, l}(s=0)=-\boldsymbol{V} \tilde{\boldsymbol{C}}_{l}\left(\tilde{\boldsymbol{A}}_{l}-\tilde{\boldsymbol{L}} \tilde{\boldsymbol{C}}_{l}\right)^{-1}\left(\tilde{\boldsymbol{E}}_{a, l}-\tilde{\boldsymbol{L}} \tilde{\boldsymbol{E}}_{s, l}\right)+\boldsymbol{V} \tilde{\boldsymbol{E}}_{s, l}
$$

betrachtet. Um im stationären Fall die Abweichung zur vorgegebenen Diagonalmatrix $\boldsymbol{S}$ zu minimieren, wird

$$
\underset{\boldsymbol{Z}_{V}}{\operatorname{minimiere}} \max _{\chi}\left\|\boldsymbol{G}_{\boldsymbol{r} \boldsymbol{f}, \boldsymbol{\chi}}(s=0)-\boldsymbol{S}\right\|_{2}
$$

angestrebt. Da $\boldsymbol{G}_{\boldsymbol{r} \boldsymbol{f}, l}(s=0)$ über (5.58) mit $\boldsymbol{V}=-\boldsymbol{S \Theta}^{+}-\boldsymbol{Z}_{V} \cdot{ }^{\perp} \boldsymbol{\Theta}$ von $\boldsymbol{Z}_{V}$ abhängt, kann (5.59) nun für die $N$ Eckpunkte von $\mathcal{M}$ ohne Weiteres mithilfe von Lemma 3.5 als LMI-Problem formuliert werden. Mit 
der Matrix $\boldsymbol{\Theta}=\boldsymbol{C}_{0}\left(\boldsymbol{A}_{0}-\boldsymbol{L} \boldsymbol{C}_{0}\right)^{-1}\left(\boldsymbol{E}_{a, 0}-\boldsymbol{L} \boldsymbol{E}_{s, 0}\right)-\boldsymbol{E}_{s, 0}$ ergibt sich unter Anwendung von Lemma 3.5

$$
\begin{aligned}
& \underset{\boldsymbol{Z}_{V}}{\operatorname{minimiere}} \gamma_{f, V}, \text { sodass } \forall l=1, \ldots, N \\
& {\left[\begin{array}{cc}
\gamma_{f, V} \boldsymbol{I}_{n_{f}} & \boldsymbol{G}_{\boldsymbol{r} \boldsymbol{f}, l}(s=0)-\boldsymbol{S} \\
\star & \boldsymbol{I}_{n_{f}}
\end{array}\right] \succ \mathbf{0}} \\
& \boldsymbol{G}_{\boldsymbol{r f}, l}(s=0)=-\boldsymbol{V} \tilde{\boldsymbol{C}}_{l}\left(\tilde{\boldsymbol{A}}_{l}-\tilde{\boldsymbol{L}} \tilde{\boldsymbol{C}}_{l}\right)^{-1}\left(\tilde{\boldsymbol{E}}_{a, l}-\tilde{\boldsymbol{L}} \tilde{\boldsymbol{E}}_{s, l}\right)+\boldsymbol{V} \tilde{\boldsymbol{E}}_{s, l}, \\
& \boldsymbol{V}=-\boldsymbol{S \Theta}^{+}-\boldsymbol{Z}_{V} \cdot{ }^{\perp} \boldsymbol{\Theta}
\end{aligned}
$$

Es handelt sich dabei wie bei (5.55) um ein LMI-Problem mit Gleichungsbedingungen. Auch die mittels (5.60) gefundene Schranke $\gamma_{f, V}$ gilt nur für die Eckpunkte von $\mathcal{M}$. Für Spezialfälle wie die im Zusammenhang mit (5.55) diskutierte Situation $\boldsymbol{A}_{\boldsymbol{\chi}}=\boldsymbol{A}_{0}$ und $\boldsymbol{C}_{\boldsymbol{\chi}}=\boldsymbol{C}_{0}$ lässt sich darüber hinaus die Gültigkeit für die gesamte konvexe Hülle von $\mathcal{M}$ zeigen.

Bemerkung 5.9. Die Parametrierung von $\boldsymbol{Z}_{V}$ (und damit $\boldsymbol{V}$ ) nach (5.60) berücksichtigt allein die stationäre Verstärkung von $\boldsymbol{G}_{\boldsymbol{r} f, l}(s)$, also die 3 . Bedingung von Seite 246. Ebenso ist es möglich, die 4. Bedingung, welche die Streckeneigenwerte betrifft, beim Entwurf von $\boldsymbol{V}$ mit einzubeziehen. Aufgrund der Struktur von $\tilde{\boldsymbol{L}}$ können diese Eigenwerte (und die zugehörigen Linkseigenvektoren) durch die Beobachtermatrix $\boldsymbol{L}$ nicht beeinflusst werden. Allerdings kann die Matrix $\boldsymbol{V}$ neben (5.60) auch dazu verwendet werden, in der Partialbruchzerlegung (5.48) die Norm der Zählerterme

$$
\left\|\boldsymbol{V} \tilde{\boldsymbol{C}}_{l} \tilde{\boldsymbol{v}}_{B_{k}, l} \cdot \tilde{\boldsymbol{w}}_{B_{k}, l}^{\top}\left(\tilde{\boldsymbol{E}}_{a, l}-\tilde{\boldsymbol{L}} \tilde{\boldsymbol{E}}_{s, l}\right)\right\|_{2} \forall k=1, \ldots, n
$$

zu minimieren. Damit wird der Einfluss der Streckeneigenwerte in $\boldsymbol{G}_{\boldsymbol{r} \boldsymbol{f}, l}(s)$ minimiert. Um sowohl Bedingung 3 als auch Bedingung $4 \mathrm{zu}$ berücksichtigen kann eine gewichtete Summe aus $\gamma_{f, V}$ und einer Schranke für (5.61) optimiert werden.

\section{Erweiterung auf Systeme mit Stellgrößen}

Mit dem bisher vorgestellten Verfahren lässt sich eine näherungsweise Diagonalisierung von $\boldsymbol{G}_{\boldsymbol{r} \boldsymbol{f}}(s)$ für Systeme mit unsicheren Parametern erreichen. Im praktischen Einsatz beeinflussen jedoch stets auch die Stellgrößen $\boldsymbol{u}$ und Störgrößen $\boldsymbol{d}$ die Strecke. Dieser Effekt muss beim Entwurf eines robusten FIOs mitberücksichtigt werden, da andernfalls die Optimierung 
hinsichtlich $\boldsymbol{G}_{\boldsymbol{r} \boldsymbol{f}}(s)$ zu einer Vergrößerung des Einflusses von $\boldsymbol{u}$ beziehungsweise $\boldsymbol{d}$ auf die Residuen führen kann.

Betrachtet werden im Folgenden Systeme der Form

$$
\begin{aligned}
& \dot{x}=A_{\chi} x+B_{\chi} u+E_{a, \chi} f+B_{d, \chi} d, \\
& y=C_{\chi} x+D_{\chi} u+E_{s, \chi} f+D_{d, \chi} d,
\end{aligned}
$$

bei denen zusätzlich zu (5.47)

$$
\begin{gathered}
{\left[\begin{array}{ll}
\boldsymbol{B}_{\boldsymbol{\chi}} & \boldsymbol{B}_{d, \boldsymbol{\chi}} \\
\boldsymbol{D}_{\boldsymbol{\chi}} & \boldsymbol{D}_{d, \boldsymbol{\chi}}
\end{array}\right] \in \mathcal{M}_{u d}=\left\{\left[\begin{array}{ll}
\boldsymbol{B}_{\boldsymbol{\chi}} & \boldsymbol{B}_{d, \boldsymbol{\chi}} \\
\boldsymbol{D}_{\boldsymbol{\chi}} & \boldsymbol{D}_{d, \boldsymbol{\chi}}
\end{array}\right]=\sum_{l=1}^{N} \chi_{l}\left[\begin{array}{ll}
\boldsymbol{B}_{l} & \boldsymbol{B}_{d, l} \\
\boldsymbol{D}_{l} & \boldsymbol{D}_{d, l}
\end{array}\right]\right\},} \\
\sum_{l=1}^{N} \chi_{l}=1, \quad \chi_{l} \geq 0
\end{gathered}
$$

gilt. Zunächst wird der Einfluss der Stellgrößen auf die generierten Residuen betrachtet, wobei im Idealfall $\boldsymbol{G}_{\boldsymbol{r u}, \boldsymbol{\chi}}(s)=0$ zu erreichen ist. Analog zu (5.48) lässt sich die Wirkung der Stellgrößen beschreiben durch

$$
\begin{aligned}
\boldsymbol{G}_{\boldsymbol{r u}, l}(s) & =\boldsymbol{V} \tilde{\boldsymbol{C}}_{l}\left(s \boldsymbol{I}_{2 n}-\left(\tilde{\boldsymbol{A}}_{l}-\tilde{\boldsymbol{L}} \tilde{\boldsymbol{C}}_{l}\right)\right)^{-1}\left(\tilde{\boldsymbol{B}}_{l}-\tilde{\boldsymbol{L}} \tilde{\boldsymbol{D}}_{l}\right)+\boldsymbol{V} \tilde{\boldsymbol{D}}_{l}, \\
\tilde{\boldsymbol{B}}_{l} & =\left[\begin{array}{c}
\boldsymbol{B}_{l} \\
\boldsymbol{B}_{0}
\end{array}\right], \quad \tilde{\boldsymbol{D}}_{l}=\boldsymbol{D}_{l}-\boldsymbol{D}_{0} .
\end{aligned}
$$

Aus (4.4) geht hervor, dass die Stellgrößen $\boldsymbol{u}$ die Dynamik des Beobachterfehlers im nominalen System nicht anregen. Dies motiviert das Entwurfsziel, die Beobachtereigenwerte bezüglich des Paares $\left(\tilde{\boldsymbol{A}}_{l}-\tilde{\boldsymbol{L}} \tilde{\boldsymbol{C}}_{l}, \tilde{\boldsymbol{B}}_{l}-\tilde{\boldsymbol{L}}_{\tilde{\boldsymbol{D}}_{l}}\right)$ nichtsteuerbar zu machen. Näherungsweise wird dies durch die Minimierung von $\left\|\tilde{\boldsymbol{w}}_{B_{i j}, l}^{\top}\left(\tilde{\boldsymbol{B}}_{l}-\tilde{\boldsymbol{L}} \tilde{\boldsymbol{D}}_{l}\right)\right\|_{2}$ erreicht. Um dieses Optimierungsproblem zu lösen, muss der zu minimierende Term als Funktion der verbleibenden Freiheitsgrade $\boldsymbol{z}_{i j}^{\top}$ (im Fall der steuerbaren Beobachtereigenwerte) ausgedrückt werden. Unter erneutem Rückgriff auf $\boldsymbol{w}_{B_{i j}, l, b}^{\top}=\boldsymbol{w}_{B_{i j}, 0}^{\top}$ und $\boldsymbol{p}_{i j}^{\top}=-\boldsymbol{w}_{B_{i j}, 0}^{\top} \boldsymbol{L}$ ergibt sich mit den in (5.64) eingeführten Abkürzungen

$$
\begin{aligned}
\tilde{\boldsymbol{w}}_{B_{i j}, l}^{\top}\left(\tilde{\boldsymbol{B}}_{l}-\tilde{\boldsymbol{L}} \tilde{\boldsymbol{D}}_{l}\right) & =\left[\begin{array}{ll}
\boldsymbol{w}_{B_{i j}, l, s}^{\top} & \boldsymbol{w}_{B_{i j}, l, b}^{\top}
\end{array}\right]\left[\begin{array}{c}
\boldsymbol{B}_{l} \\
\boldsymbol{B}_{0}-\boldsymbol{L}\left(\boldsymbol{D}_{0}-\boldsymbol{D}_{l}\right)
\end{array}\right] \\
& =\boldsymbol{w}_{B_{i j}, l, s}^{\top} \boldsymbol{B}_{l}+\boldsymbol{w}_{B_{i j}, l, b}^{\top} \boldsymbol{B}_{0}+\boldsymbol{p}_{i j}^{\top}\left(\boldsymbol{D}_{0}-\boldsymbol{D}_{l}\right) \\
& =\boldsymbol{w}_{B_{i j}, l, s}^{\top} \boldsymbol{B}_{l}+\left[\begin{array}{ll}
\boldsymbol{w}_{B_{i j}, 0}^{\top} & \boldsymbol{p}_{i j}^{\top}
\end{array}\right]\left[\begin{array}{c}
\boldsymbol{B}_{0} \\
\boldsymbol{D}_{0}-\boldsymbol{D}_{l}
\end{array}\right] .
\end{aligned}
$$


Somit ergibt sich das Optimierungsproblem

$$
\begin{aligned}
& \underset{\boldsymbol{z}_{i j}^{\top}, \boldsymbol{w}_{B_{i j}, l, s}^{\top}}{\operatorname{minimiere}} \gamma_{u, i j}, \text { sodass } \forall l=1, \ldots, N \\
& {\left[\begin{array}{cc}
\gamma_{u, i j} & \boldsymbol{w}_{B_{i j}, l, s}^{\top} \boldsymbol{B}_{l}+\left[\begin{array}{cc}
\boldsymbol{w}_{B_{i j}, 0}^{\top} & \boldsymbol{p}_{i j}^{\top}
\end{array}\right]\left[\begin{array}{c}
\boldsymbol{B}_{0} \\
\boldsymbol{D}_{0}-\boldsymbol{D}_{l}
\end{array}\right] \\
\star & \boldsymbol{I}_{n_{u}}
\end{array}\right] \succ \mathbf{0},} \\
& {\left[\begin{array}{ll}
\boldsymbol{w}_{B_{i j}, 0}^{\top} & \boldsymbol{p}_{i j}^{\top}
\end{array}\right]=\left[\begin{array}{ll}
\mathbf{0}^{\top} & \boldsymbol{\phi}_{i}^{\top}
\end{array}\right] \boldsymbol{\Pi}_{i j, 0}^{+}+\boldsymbol{z}_{i j}^{\top} \cdot{ }^{\perp} \boldsymbol{\Pi}_{i j, 0},} \\
& \boldsymbol{w}_{B_{i j}, l, s}^{\top}\left(\lambda_{B_{i j}} \boldsymbol{I}_{n}-\boldsymbol{A}_{l}\right)=-\boldsymbol{p}_{i j}^{\top} \boldsymbol{C}_{l} .
\end{aligned}
$$

Offensichtlich handelt es sich bei (5.55) und (5.65) um konkurrierende Probleme. Auf die Abwägung der Entwurfsziele (Minimierung von $\gamma_{f, i j}$ beziehungsweise $\gamma_{u, i j}$ ) wird in der Zusammenfassung des Entwurfsverfahrens näher eingegangen.

Die Behandlung der nichtsteuerbaren Beobachtereigenwerte $\lambda_{B_{k}}$ und $\lambda_{B_{m}}$ beziehungsweise der damit verknüpften Entwurfsfreiheitsgrade gestaltet sich weitgehend analog zu (5.65). Während die Bedingungen (5.65b) und (5.65d) erhalten bleiben, ist (5.65c) $\mathrm{zu}$ ersetzen durch $\left[\begin{array}{ll}\boldsymbol{w}_{B_{k}, 0}^{\top} & \boldsymbol{p}_{k}^{\top}\end{array}\right]=\boldsymbol{z}_{k}^{\top} \cdot{ }^{\perp} \boldsymbol{\Pi}_{k, 0} \quad$ beziehungsweise $\quad\left[\begin{array}{lll}\boldsymbol{w}_{B_{m}, 0}^{\top} & \boldsymbol{p}_{m}^{\top}\end{array}\right]=\boldsymbol{z}_{m}^{\top} \cdot{ }^{\perp} \boldsymbol{\Pi}_{m, 0}$. Zusätzlich ist die Normbeschränkung $\left\|z_{k}^{\top}\right\|_{2} \geq 1$ beziehungsweise $\left\|\boldsymbol{z}_{m}^{\top}\right\|_{2} \geq 1$ sowie die Vorgabe des Eigenwertbereiches $\lambda_{B_{k}} \in \mathcal{D} \quad \mathrm{zu}$ berücksichtigen.

Um den stationären Einfluss der Stellgrößen auf die erzeugten Residuen zu minimieren, kann beim Entwurf der Nachfiltermatrix das Optimierungskriterium

$$
\underset{\boldsymbol{Z}_{V}}{\operatorname{minimiere}} \max _{l=1, \ldots, N}\left\|\boldsymbol{G}_{\boldsymbol{r u}, l}(s=0)\right\|_{2}
$$

verfolgt werden. Dies lässt sich als LMI-Problem der Form

$$
\begin{aligned}
& \underset{\boldsymbol{Z}_{V}}{\operatorname{minimiere}} \gamma_{u, V}, \text { sodass } \forall l=1, \ldots, N \\
& {\left[\begin{array}{cc}
\gamma_{u, V} \boldsymbol{I}_{n_{f}} & \boldsymbol{G}_{\boldsymbol{r u}, l}(s=0) \\
\star & \boldsymbol{I}_{n_{u}}
\end{array}\right] \succ \mathbf{0}} \\
& \boldsymbol{G}_{\boldsymbol{r u}, l}(s=0)=-\boldsymbol{V} \tilde{\boldsymbol{C}}_{l}\left(\tilde{\boldsymbol{A}}_{l}-\tilde{\boldsymbol{L}} \tilde{\boldsymbol{C}}_{l}\right)^{-1}\left(\tilde{\boldsymbol{B}}_{l}-\tilde{\boldsymbol{L}} \tilde{\boldsymbol{D}}_{l}\right)+\boldsymbol{V} \tilde{\boldsymbol{D}}_{l}, \\
& \boldsymbol{V}=-\boldsymbol{S} \Theta^{+}-\boldsymbol{Z}_{V} \cdot{ }^{\perp} \Theta
\end{aligned}
$$

formulieren.

Bemerkung 5.10. Die Optimierung nach (5.66) bietet sich insbesondere dann an, wenn die Stellgrößen überwiegend niederfrequent sind. Anstelle 
der Minimierung von $\left\|\boldsymbol{G}_{\boldsymbol{r u}, l}(s=0)\right\|_{2}$ lässt sich unter Rückgriff auf das Bounded-Real-Lemma (Satz 3.1) alternativ die $\mathcal{H}_{\infty}$-Norm von $\boldsymbol{G}_{\boldsymbol{r u}, l}(s)$ minimieren, um den größten Einfluss der Stellgrößen über alle Frequenzen zu berücksichtigen.

\section{Erweiterung auf Systeme mit Störgrößen}

Unterliegt ein System zusätzlich zu den parametrischen Unsicherheiten noch exogenen Störungen, so wird deren Einfluss entsprechend (5.48) beziehungsweise (5.64) beschrieben durch

$$
\begin{aligned}
\boldsymbol{G}_{\boldsymbol{r d}, l}(s) & =\boldsymbol{V} \tilde{\boldsymbol{C}}_{l}\left(s \boldsymbol{I}_{2 n}-\left(\tilde{\boldsymbol{A}}_{l}-\tilde{\boldsymbol{L}} \tilde{\boldsymbol{C}}_{l}\right)\right)^{-1}\left(\tilde{\boldsymbol{B}}_{d, l}-\tilde{\boldsymbol{L}} \tilde{\boldsymbol{D}}_{d, l}\right)+\boldsymbol{V} \tilde{\boldsymbol{D}}_{d, l}, \\
\tilde{\boldsymbol{B}}_{d, l} & =\left[\begin{array}{c}
\boldsymbol{B}_{d, l} \\
\mathbf{0}
\end{array}\right], \quad \tilde{\boldsymbol{D}}_{d, l}=\boldsymbol{D}_{d, l} .
\end{aligned}
$$

Um eingangsseitig den Einfluss der Störgrößen $\boldsymbol{d}$ auf die Residuendynamik zu minimieren, wird bei der Optimierung der mit den Beobachtereigenwerten verknüpften Freiheitsgrade die Minimierung von $\left\|\tilde{\boldsymbol{w}}_{B, k}^{\top}\left(\tilde{\boldsymbol{B}}_{d, l}-\tilde{\boldsymbol{L}} \tilde{\boldsymbol{D}}_{d, l}\right)\right\|_{2}$ angestrebt. Strukturell ergeben sich folglich hinsichtlich $\boldsymbol{z}_{i j}^{\top}, \boldsymbol{z}_{m}^{\top}, \lambda_{B_{k}}$ und $\boldsymbol{z}_{k}^{\top}$ die gleichen Optimierungsprobleme wie bei der Unterdrückung des Einflusses der Stellgrößen, wobei anstelle von $\tilde{\boldsymbol{B}}_{l}$ und $\tilde{\boldsymbol{D}}_{l}$ jeweils $\tilde{\boldsymbol{B}}_{d, l}$ und $\tilde{\boldsymbol{D}}_{d, l}$ zu betrachten sind.

Sind keine zusätzlichen Informationen hinsichtlich der Störgrößen vorhanden, so ist die Minimierung von $\left\|\boldsymbol{G}_{\boldsymbol{r} \boldsymbol{d}, l}(s)\right\|_{\infty}$ ein naheliegendes Ziel bei der Auslegung von $\boldsymbol{Z}_{V}$ beziehungsweise $\boldsymbol{V}$. Anhand des Bounded-RealLemmas (Satz 3.1) ergibt sich

$$
\begin{array}{ccc}
\underset{\boldsymbol{Z}_{V}, \boldsymbol{X}}{\operatorname{minimiere}} \gamma_{d, V}, \text { sodass } & \forall l=1, \ldots, N & \\
\boldsymbol{X} \succ \mathbf{0}, & \\
{\left[\begin{array}{ccc}
\operatorname{He}\left(\boldsymbol{X}\left(\tilde{\boldsymbol{A}}_{l}-\tilde{\boldsymbol{L}} \tilde{\boldsymbol{C}}_{l}\right)\right) & \boldsymbol{X}\left(\tilde{\boldsymbol{B}}_{d, l}-\tilde{\boldsymbol{L}} \tilde{\boldsymbol{D}}_{d, l}\right) & \left(\boldsymbol{V} \tilde{\boldsymbol{C}}_{l}\right)^{\top} \\
\star & -\gamma_{d, V} \boldsymbol{I}_{n_{d}} & \left(\boldsymbol{V} \tilde{\boldsymbol{D}}_{d, l}\right)^{\top} \\
\star & \star & -\gamma_{d, V} \boldsymbol{I}_{n_{f}}
\end{array}\right] \prec \mathbf{0},} \\
\boldsymbol{V}=-\boldsymbol{S \Theta}^{+}-\boldsymbol{Z}_{V} \cdot{ }^{\perp} \boldsymbol{\Theta} . & &
\end{array}
$$

Da eine gemeinsame Matrix $\boldsymbol{X}$ für alle Eckpunkte von $\mathcal{M}$ verwendet wird, gilt die Schranke $\gamma_{d, V}$ in diesem Fall für alle Systeme in $\mathcal{M}$. 


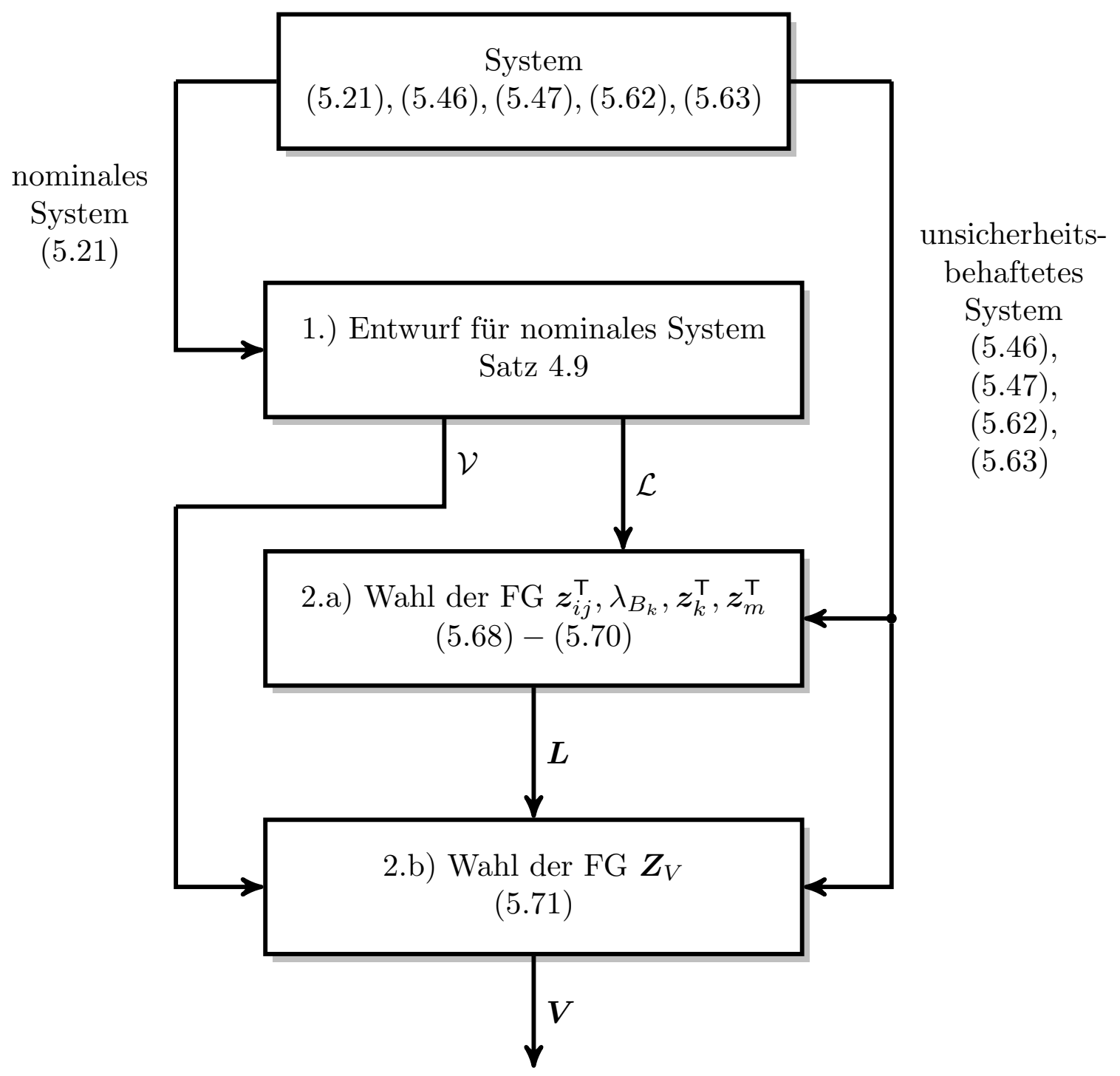

Abbildung 5.18: Entwurf robuster FIOs für Strecken mit unsicheren Parametern

\section{Zusammenfassung des Entwurfsverfahrens}

Da in diesem Abschnitt zum Entwurf von $\boldsymbol{L}$ und $\boldsymbol{V}$ unterschiedliche Optimierungsprobleme betrachtet wurden, wird die gesamte Entwurfsmethodik nochmals zusammengefasst, wobei der Ablauf in Abbildung 5.18 verdeutlicht ist.

Im ersten Schritt werden die Mengen $\mathcal{L}$ und $\mathcal{V}$ bestimmt, die alle FIOParametrierungen beschreiben, die im Nominalfall eine ideale Fehlerisolation hinsichtlich $\boldsymbol{f}$ und $\boldsymbol{u}$ erreichen. Wie aus Abbildung 5.18 hervorgeht, wird für diesen Schritt lediglich das nominale Systemmodell benötigt.

In Schritt 2.a) werden die hinsichtlich der Beobachtermatrix $\boldsymbol{L}$ ver- 
bleibenden Freiheitsgrade $\boldsymbol{z}_{i j}^{\top}, \lambda_{B_{k}}, \boldsymbol{z}_{k}^{\top}$ und $\boldsymbol{z}_{m}^{\top}$ ausgelegt, wobei auf die Beschreibung des unsicheren Systems zurückgegriffen wird. Um auf die unterschiedlichen Anforderungen hinsichtlich der Größen $\boldsymbol{f}, \boldsymbol{u}$ und $\boldsymbol{d}$ einzugehen, wird für die Freiheitsgrade $\boldsymbol{z}_{i j}^{\top}$ das Optimierungsproblem

$$
\begin{aligned}
& \underset{\boldsymbol{z}_{i j}^{\top}, \boldsymbol{w}_{B_{i j}, l, s}^{\top}}{\operatorname{minimiere}} \alpha_{f} \gamma_{f, i j}+\alpha_{u} \gamma_{u, i j}+\alpha_{d} \gamma_{d, i j}, \text { sodass } \forall l=1, \ldots, N \\
& (5.55 \mathrm{~b}),(5.55 \mathrm{c}),(5.65 \mathrm{~b})
\end{aligned}
$$

gelöst. Darin sind $\alpha_{f} \geq 0, \alpha_{u} \geq 0$ und $\alpha_{d} \geq 0$ Gewichtungsfaktoren, die eine Abwägung der Entwurfsziele erlauben. In ähnlicher Art und Weise ergibt sich für die nichtsteuerbaren Beobachtereigenwerte $\lambda_{B_{k}}$ das Optimierungsproblem

$$
\begin{aligned}
& \underset{\lambda_{B_{k}}, \boldsymbol{z}_{k}^{\top}, \boldsymbol{w}_{B_{k}, l, s}^{\top}}{\operatorname{minimiere}} \alpha_{f} \gamma_{f, k}+\alpha_{u} \gamma_{u, k}+\alpha_{d} \gamma_{d, k}, \text { sodass } \forall l=1, \ldots, N \\
& (5.56 \mathrm{c})-(5.56 \mathrm{f})
\end{aligned}
$$

und für die Eigenwerte zur Kompensation invarianter Nullstellen

$$
\begin{aligned}
& \underset{\boldsymbol{z}_{m}^{\top}, \boldsymbol{w}_{B_{m}, l, s}^{\top}}{\operatorname{minimiere}} \alpha_{f} \gamma_{f, m}+\alpha_{u} \gamma_{u, m}+\alpha_{d} \gamma_{d, m}, \text { sodass } \forall l=1, \ldots, N \\
& (5.57 \mathrm{~b})-(5.57 \mathrm{~d}) .
\end{aligned}
$$

Nachdem in Schritt 2.a) eine Beobachtermatrix $\boldsymbol{L} \in \mathcal{L}$ festgelegt wurde, widmet sich Schritt 2.b) der Auslegung einer Nachfiltermatrix $\boldsymbol{V} \in \mathcal{V}$. Durch die Kombination der Optimierungsprobleme (5.60), (5.66) und (5.67) ergibt sich

$$
\begin{aligned}
& \underset{Z_{V}}{\operatorname{minimiere}} \alpha_{f} \gamma_{f, V}+\alpha_{u} \gamma_{u, V}+\alpha_{d} \gamma_{d, V}, \text { sodass } \forall l=1, \ldots, N \\
& (5.60 \mathrm{~b})-(5.60 \mathrm{~d}),(5.66 \mathrm{~b}),(5.67 \mathrm{~b}),(5.67 \mathrm{c}) .
\end{aligned}
$$

Damit sind alle Entwurfsfreiheitsgrade ausgenutzt und die Parametrierung $(\boldsymbol{L}, \boldsymbol{V})$ ist gefunden.

Bemerkung 5.11. Da die durch (5.55), (5.56) usw. gefundenen Schranken wie diskutiert jeweils nur für die Eckpunkte von $\mathcal{M}$ gelten und nicht für die gesamte konvexe Hülle, lässt sich das Verfahren auch dann anwenden, wenn die Systemmatrizen nicht in affiner Art und Weise von den unsicheren Parametern abhängen. Die Voraussetzung wurde getroffen, um im weiteren Verlauf der Arbeit eine Vergleichbarkeit zu robusten allgemeinen Fehlerisolationsfiltern (s. Abschnitt 6.5.2) herzustellen. 


\section{Akademisches Beispiel}

Die Anwendung des Verfahrens wird anhand eines akademischen Beispielsystems verdeutlicht, welches durch das nominale System

$$
\begin{array}{ll}
\boldsymbol{A}_{0}=\left[\begin{array}{rrr}
-1 & 2 & 4 \\
0 & -2 & 3 \\
0 & 0 & -3
\end{array}\right], \quad \boldsymbol{B}_{0}=\left[\begin{array}{c}
0 \\
2 \\
-1
\end{array}\right], \quad \boldsymbol{E}_{a, 0}=\left[\begin{array}{ll}
0 & 0 \\
1 & 0 \\
0 & 4
\end{array}\right], \\
\boldsymbol{C}_{0}=\left[\begin{array}{rrr}
1 & 0 & -1 \\
0 & 1 & 0 \\
0 & 0 & 1
\end{array}\right], \quad \boldsymbol{D}_{0}=\mathbf{0}, \quad \boldsymbol{E}_{s, 0}=\mathbf{0}
\end{array}
$$

beschrieben wird. Zum anderen sind die Matrizen

$$
\begin{aligned}
\Delta \boldsymbol{A} & =\left[\begin{array}{ccc}
0 & -0,5 & 0,2 \\
0 & 0 & 0,2 \\
0 & 0 & 0
\end{array}\right], \Delta \boldsymbol{B}=\left[\begin{array}{c}
0 \\
0,04 \\
0,06
\end{array}\right], \Delta \boldsymbol{E}_{a}=\left[\begin{array}{cc}
0 & 0 \\
0,2 & 0 \\
0 & -0,1
\end{array}\right], \\
\Delta \boldsymbol{C} & =\left[\begin{array}{ccc}
0,01 & 0 & -0,03 \\
0 & 0 & 0 \\
0 & -0,05 & 0,02
\end{array}\right]
\end{aligned}
$$

gegeben, sodass mit dem Parameter $\rho \in[-1 ; 1]$ durch $\boldsymbol{A}_{0}+\rho \Delta \boldsymbol{A}$, $\boldsymbol{B}_{0}+\rho \Delta \boldsymbol{B}, \boldsymbol{E}_{a, 0}+\rho \Delta \boldsymbol{E}_{a}$ und $\boldsymbol{C}_{0}+\rho \Delta \boldsymbol{C}$ eine unsichere Dynamik der Form (5.47), (5.62), (5.63) gegeben ist. Exogene Störgrößen wirken im Beispiel nicht, es ist $\boldsymbol{B}_{d, \boldsymbol{\chi}}=\mathbf{0}, \boldsymbol{D}_{d, \boldsymbol{\chi}}=\mathbf{0}$.

Für das nominale System ist $\delta_{1}=\delta_{2}=1$ und es gilt $\operatorname{rang}\left(\boldsymbol{D}^{*}\right)=2$. Mittels Satz 4.9 wird zunächst ein FIO entworfen, der den Diagonalelementen von $\boldsymbol{G}_{\boldsymbol{r} \boldsymbol{f}}(s)$ die Pole $\lambda_{B_{1,1}}=-4$ und $\lambda_{B_{2,1}}=-6$ zuweist und eine stationäre Verstärkung von $g_{i, i}(s=0)=1$ einstellt. Die verbleibenden Freiheitsgrade werden dabei willkürlich zu $\boldsymbol{z}_{11}^{\top}=z_{11}=0$, $\boldsymbol{z}_{21}^{\top}=z_{21}=0, \lambda_{B_{3}}=-7$ und $\boldsymbol{Z}_{V}=\left[\begin{array}{ll}0 & 0\end{array}\right]^{\top}$ gewählt. Die resultierende FIO-Parametrierung $\left(\boldsymbol{L}_{\text {init }}, \boldsymbol{V}_{\text {init }}\right)$ ist in Anhang C.2.5 zu finden. In Abbildung 5.19 ist die Sprungantwort von $\boldsymbol{G}_{\boldsymbol{r} \boldsymbol{f}}(s)$ für unterschiedliche Werte von $\rho$ zu sehen. Während für $\rho=0$ erwartungsgemäß eine ideale Fehlerisolation erreicht wird, ist bei davon abweichenden Werten von $\rho$ eine deutliche Abweichung vom gewünschten Verhalten im ersten Residuum zu erkennen. Ebenso zeigt sich in Abbildung 5.20, dass die Eingangsgröße $u$ das erste Residuum zu einem gewissen Grad beeinflusst.

Zur Verbesserung des Resultates wird gemäß des Schemas aus Abbildung 5.18 ein robuster FIO entworfen. Die Lösung von (5.68) liefert mit den Gewichtungsfaktoren $\alpha_{f}=\alpha_{u}=1$ für die entsprechenden Freiheitsgrade Werte von $\boldsymbol{z}_{11}^{\top}=z_{11}=-4,83$ und $\boldsymbol{z}_{21}^{\top}=z_{21}=-1,86$. Mittels (5.69) 

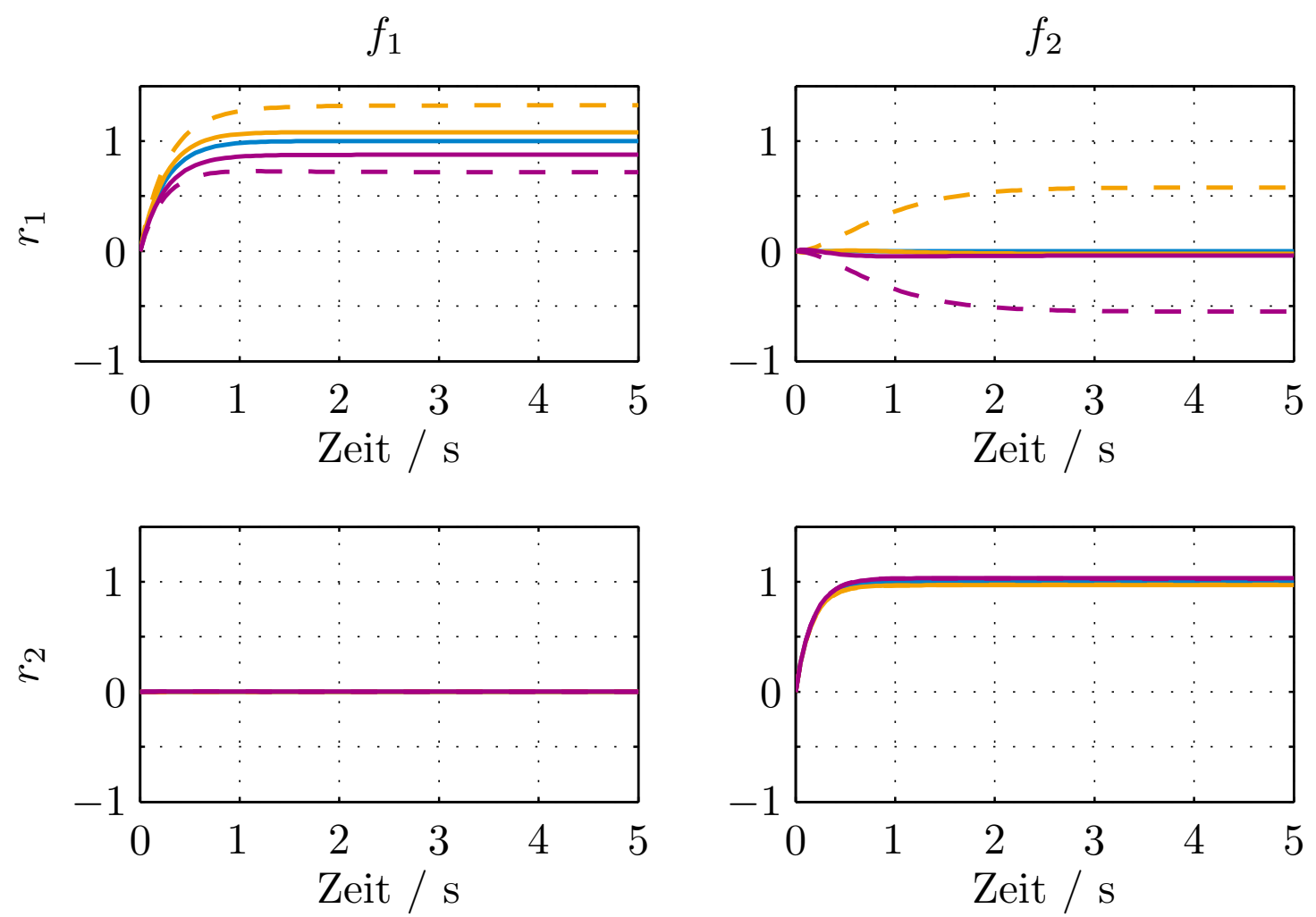

Abbildung 5.19: Sprungantwort von $\boldsymbol{G}_{\boldsymbol{r} \boldsymbol{f}}(s)$ des initialen FIOs $(--)$ sowie des optimierten FIOs $(-)$ für $\rho=0(-), \rho=-1(-)$ und $\rho=1(-)$

wird der nichtsteuerbare Beobachtereigenwert bei $\lambda_{B_{3}}=-10,18$ platziert. Schließlich liefert (5.71) den Wert $\boldsymbol{Z}_{V}=\left[\begin{array}{lll}-13,13 & 1,14\end{array}\right]^{\top}$. Daraus berechnet sich die ebenfalls in Anhang C.2.5 aufgeführte Parametrierung $\left(\boldsymbol{L}_{\mathrm{opt}}, \boldsymbol{V}_{\mathrm{opt}}\right)$. Wie aus Abbildung 5.20 hervorgeht, resultiert sie hinsichtlich des Einflusses der Stellgröße auf das erste Residuum in einer geringfügigen Verschlechterung gegenüber der initialen Parametrierung. Insgesamt verbessert sich die Residuengenerierung jedoch deutlich, da in Abbildung 5.19 nun trotz der unsicheren Parameter eine nahezu ideale Diagonalisierung von $\boldsymbol{G}_{\boldsymbol{r} \boldsymbol{f}}(s)$ erkennbar ist.

\subsubsection{Fazit}

In diesem Abschnitt hat sich gezeigt, dass in nichtquadratischen Systemen mit $n_{y}>n_{f}$ aufgrund der in Kapitel 4 herausgearbeiteten zusätzlichen Freiheitsgrade vielfältige Möglichkeiten zur Steigerung der Robustheit von FIOs existieren. Die einfachste Variante stellt dabei die Minimierung der $\mathcal{H}_{\infty}$-Norm von $\boldsymbol{G}_{\boldsymbol{r} \boldsymbol{d}}(s)$ dar (s. Abschnitt 5.4.1), die sich auch auf die Anpas- 

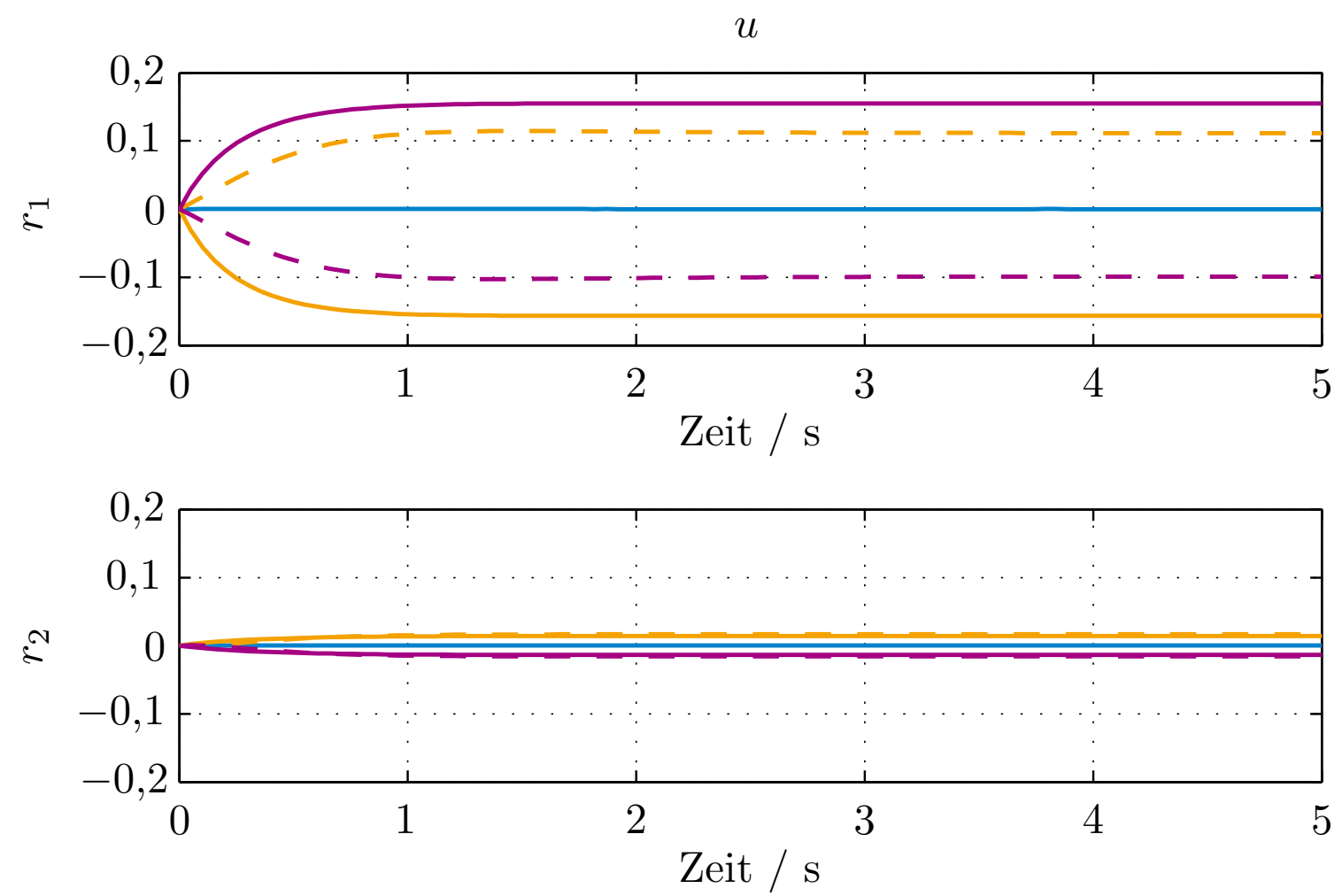

Abbildung 5.20: Sprungantwort von $\boldsymbol{G}_{\boldsymbol{r u}}(s)$ des initialen FIOs $(--)$ sowie des optimierten FIOs $(-)$ für $\rho=0(-), \rho=-1(-)$ und $\rho=1(-)$

sung der Diagonalelemente von $\boldsymbol{G}_{\boldsymbol{r} \boldsymbol{f}}(s)$ erweitern lässt (s. Abschnitt 5.4.2). Es lässt sich aber auch zusätzliches Wissen über relevante Störfrequenzen (s. Abschnitt 5.4.3) gezielt ausnutzen. Ebenso ist die direkte Behandlung von parametrischen Unsicherheiten ohne eine Umformulierung in virtuelle exogene Störungen möglich (s. Abschnitt 5.4.4). Hierbei lässt sich der Einblick, den die Vollständige Modale Synthese hinsichtlich der Struktur der Systemdynamik bietet, ausnutzen.

Allerdings ist auch festzustellen, dass die Entwurfsverfahren zum Teil mit einigem Aufwand verbunden sind und dass darüber hinaus kein einheitliches Entwurfsverfahren für die unterschiedlichen Teilproblemstellungen gegeben ist. 


\subsection{Steigerung der Robustheit bei partieller Fehlerisolation}

Wie in Abschnitt 4.10 herausgearbeitet ergeben sich im Falle der partiellen Fehlerisolation gegenüber der vollständigen Isolation zusätzliche Freiheitsgrade. Diese werden durch die dynamischen und statischen Kopplungszahlen beschrieben, welche aus der weniger strengen Anforderung einer Dreiecksstruktur der Matrix $\boldsymbol{G}_{\boldsymbol{r} \boldsymbol{f}}(s)$ resultieren.

Gegenstand dieses Abschnittes ist es, die zusätzlichen Freiheitsgrade zu nutzen, um die Robustheit der generierten Residuen bezüglich exogener Störungen zu erhöhen. Dieses Ziel lässt sich formal als

$$
\underset{\boldsymbol{k}_{d}, \boldsymbol{k}_{s}}{\operatorname{minimiere}}\left\|\boldsymbol{G}_{\boldsymbol{r d}}\left(s, \boldsymbol{k}_{d}, \boldsymbol{k}_{s}\right)\right\|_{\infty}
$$

beschreiben, wobei die Dreiecksstruktur von $\boldsymbol{G}_{\boldsymbol{r} \boldsymbol{f}}(s)$ als Nebenbedingung einzuhalten ist. Dies ist inhärent der Fall, wenn die Beobachterparametrierung $\left(\boldsymbol{L}_{\mathrm{pfi}}, \boldsymbol{V}_{\mathrm{pfi}}\right)$ gemäß Satz 4.13 erfolgt. Es wird also zunächst ein FIO zur vollständigen Fehlerisolation (FFIO) entworfen, auf dessen Basis mithilfe von $\boldsymbol{k}_{d}$ und $\boldsymbol{k}_{s}$ die Robustheit eines FIOs zur teilweisen Fehlerisolation (PFIO) optimiert wird. Um möglichst viele Freiheitsgrade zur Verfügung zu stellen, werden die Fehler so sortiert, dass sich eine absteigende Reihenfolge in $\delta_{i}$ ergibt. Dies lässt sich wie in den Abschnitten 4.7 und 4.8 gezeigt durch eine Permutationsmatrix erreichen. Aus Gründen der Übersichtlichkeit wird an dieser Stelle darauf verzichtet und davon ausgegangen, dass $\delta_{1} \geq \delta_{2} \geq \ldots \geq \delta_{n_{f}}$ ist, wodurch nach (4.99) die Anzahl $n_{k_{d}}$ der dynamischen Kopplungszahlen maximiert wird.

Wie bereits in Abschnitt 4.10 erwähnt weist die partielle Fehlerisolation eine enge Verwandtschaft zur teilweisen Entkopplungsregelung auf. Die Nutzung der gegenüber der vollständigen Fehlerisolation beziehungsweise Entkopplung auftretenden zusätzlichen Freiheitsgrade unterscheidet sich jedoch wesentlich. In [138-140] wird beim Entwurf teilweise entkoppelnder Zustandsrückführungen angestrebt, die auftretenden Kopplungen möglichst gering zu halten. Dazu wird die quadratische Fläche der Sprungantworten der Nichtdiagonalelemente minimiert. Im Gegensatz dazu ist die Größe der zusätzlichen Kopplungen in $\boldsymbol{G}_{\boldsymbol{r} \boldsymbol{f}}(s)$ für die partielle Fehlerisolation unerheblich. Aufgrund der Auswertelogik, die in Abbildung 2.13 auf Seite 26 dargestellt ist, sind lediglich die Diagonalelemente von $\boldsymbol{G}_{\boldsymbol{r} \boldsymbol{f}}(s)$ von Belang. Diese entsprechen gemäß Satz 4.13 den Diagonalelementen, die sich auch im Falle der vollständigen Fehlerisolation ergeben. Die Koeffizienten $\boldsymbol{k}_{d}$ und $\boldsymbol{k}_{s}$ sind somit hinsichtlich der Übertragungsmatrix 
$\boldsymbol{G}_{\boldsymbol{r} \boldsymbol{f}}(s)$ frei wählbar und können entsprechend (5.74) zur Optimierung der Störunterdrückung herangezogen werden. Wird angenommen, dass bestimmte Fehlerkombinationen gleichzeitig auftreten können, andere Fehler jedoch nicht, so ergeben sich wie in Abschnitt 4.10 erläutert strukturelle Anforderungen der Form (4.94). Diese lassen sich mit den im Folgenden erarbeiteten Entwurfsverfahren ohne Weiteres als Spezialfall realisieren, indem die entsprechenden Kopplungszahlen $k_{d, i j l}=0$ und $k_{s, i j}=0$ gesetzt werden.

Zunächst wird in diesem Abschnitt ein Verfahren vorgestellt, welches eine bestimmte Systematik in der Abhängigkeit der sich ergebenden Beobachtermatrizen $\boldsymbol{L}_{\mathrm{pfi}}$ und $\boldsymbol{V}_{\text {pi }}$ von den Kopplungszahlen ausnutzt (Abschnitte 5.5.1 und 5.5.2). Vorläufige Ergebnisse dazu wurden bereits in [229] veröffentlicht. Darüber hinaus wird in Abschnitt 5.5.3 ein zweites Verfahren beschrieben, welches direkt auf Basis der Vollständigen Modalen Synthese arbeitet und dadurch nicht nur wesentlich einfacher durchzuführen, sondern auch hinsichtlich der zu erfüllenden Annahmen weniger restriktiv ist.

\subsubsection{Abhängigkeit der FIO-Parametrierung von den Kopplungszahlen}

Um die durch die dynamischen Kopplungszahlen $\boldsymbol{k}_{d}$ gegebenen zusätzlichen Freiheitsgrade zur Robustheitsoptimierung zu nutzen, wird zunächst eine kompakte Darstellung der Abhängigkeit der Matrix $\boldsymbol{L}_{\mathrm{pfi}}\left(\boldsymbol{k}_{d}\right)$ von ebendiesen hergeleitet. Wie im Folgenden bewiesen wird, gilt auf Grundlage von Satz 4.13 aus Abschnitt 4.10 zunächst

Lemma 5.1. Gegeben sei ein System der Form

$$
\begin{aligned}
& \dot{\boldsymbol{x}}=\boldsymbol{A} \boldsymbol{x}+\boldsymbol{B u}+\boldsymbol{E}_{a} \boldsymbol{f}, \\
& \boldsymbol{y}=\boldsymbol{C} \boldsymbol{x}+\boldsymbol{D} \boldsymbol{u}+\boldsymbol{E}_{s} \boldsymbol{f}
\end{aligned}
$$

welches die Annahmen 5.1-5.3 erfüllt. Weiterhin seien $\boldsymbol{L}_{\mathrm{ffi}}$ die Beobachtermatrix eines FFIOs gemäß Satz 4.12 und $\boldsymbol{k}_{d}$ dynamische Kopplungszahlen. Dann lässt sich die resultierende Beobachtermatrix $\boldsymbol{L}_{\mathrm{pfi}}$ schreiben als

$$
\begin{aligned}
\boldsymbol{L}_{\mathrm{pfi}}\left(\boldsymbol{k}_{d}\right) & =\boldsymbol{L}_{\mathrm{ffi}}+\boldsymbol{L}_{c}\left(\boldsymbol{k}_{d}\right) \\
\boldsymbol{L}_{c}\left(\boldsymbol{k}_{d}\right) & =-\left(\boldsymbol{W}_{B, \mathrm{ffi}}+\sum_{\nu=1}^{n_{k_{d}}} k_{d_{\nu}} \boldsymbol{W}_{B c, \nu}\right)^{-1} \cdot \sum_{\nu=1}^{n_{k_{d}}} k_{d_{v}}\left(\boldsymbol{P}_{c, \nu}+\boldsymbol{W}_{B c, \nu} \boldsymbol{L}_{\mathrm{ffi}}\right) .
\end{aligned}
$$


Beweis. Definitionsgemäß ist $\quad \boldsymbol{P}_{\mathrm{pfi}}\left(\boldsymbol{k}_{d}\right)=\boldsymbol{W}_{B, \mathrm{pfi}}\left(\boldsymbol{k}_{d}\right) \cdot \boldsymbol{L}_{\mathrm{pfi}}\left(\boldsymbol{k}_{d}\right) \quad$ und $\boldsymbol{P}_{\mathrm{ffi}}=-\boldsymbol{W}_{B, \text { ffi }} \cdot \boldsymbol{L}_{\mathrm{ffi}}$. Setzt man (4.98), (4.101) und (4.102) ein, so ergibt sich

$$
\begin{aligned}
\boldsymbol{P}_{\mathrm{pfi}}\left(\boldsymbol{k}_{d}\right)-\boldsymbol{P}_{\mathrm{ffi}} & =-\left(\boldsymbol{W}_{B, \mathrm{ffi}}+\sum_{\nu=1}^{n_{k_{d}}} k_{d_{\nu}} \boldsymbol{W}_{B c, \nu}\right) \cdot\left(\boldsymbol{L}_{\mathrm{ffi}}+\boldsymbol{L}_{c}\left(\boldsymbol{k}_{d}\right)\right)+\boldsymbol{W}_{B, \mathrm{ffi}} \boldsymbol{L}_{\mathrm{ffi}} \\
& =\sum_{\nu=1}^{n_{k_{d}}} k_{d_{\nu}} \boldsymbol{P}_{c, \nu} .
\end{aligned}
$$

Durch Aufösen nach $\boldsymbol{L}_{c}\left(\boldsymbol{k}_{d}\right)$ ist das Lemma bewiesen.

Lemma 5.1 liefert zwar eine kompakte Darstellung der Abhängigkeit $\boldsymbol{L}_{\mathrm{pfi}}\left(\boldsymbol{k}_{d}\right)$, jedoch hängt der Term $\boldsymbol{L}_{c}\left(\boldsymbol{k}_{d}\right)$ offensichtlich nichtlinear von $\boldsymbol{k}_{d}$ ab. Der folgende Satz liefert eine einfach überprüfbare, hinreichende Bedingung dafür, dass $\boldsymbol{L}_{c}\left(\boldsymbol{k}_{d}\right)$ linear von $\boldsymbol{k}_{d}$ abhängt. Dies bedingt wiederum eine affine Abhängigkeit der Matrix $\boldsymbol{L}_{\mathrm{pfi}}\left(\boldsymbol{k}_{d}\right)$ von $\boldsymbol{k}_{d}$.

Satz 5.6 (Affine Abhängigkeit der Beobachtermatrix von den dynamischen Kopplungszahlen). Gegeben sei ein System der Form

$$
\begin{aligned}
& \dot{\boldsymbol{x}}=\boldsymbol{A} \boldsymbol{x}+\boldsymbol{B} \boldsymbol{u}+\boldsymbol{E}_{a} \boldsymbol{f}, \\
& \boldsymbol{y}=\boldsymbol{C} \boldsymbol{x}+\boldsymbol{D} \boldsymbol{u}+\boldsymbol{E}_{s} \boldsymbol{f},
\end{aligned}
$$

welches die Annahmen 5.1-5.3 erfüllt. Weiterhin seien $\boldsymbol{L}_{\mathrm{ffi}}$ die Beobachtermatrix eines FFIOs gemäß Satz 4.12, $\boldsymbol{k}_{d}$ dynamische Kopplungszahlen und $\boldsymbol{v}_{B, f f i, q r}$ die $\left(r+\sum_{m=1}^{q-1} \delta_{m}\right)$-te Spalte der Rechtseigenvektormatrix $\boldsymbol{V}_{B, \text { ffi }}$ von $\boldsymbol{A}-\boldsymbol{L}_{\mathrm{ffi}} \boldsymbol{C}$. Dann hängt die Matrix $\boldsymbol{L}_{\mathrm{pfi}}\left(\boldsymbol{k}_{d}\right)=\boldsymbol{L}_{\mathrm{ffi}}+\boldsymbol{L}_{c}\left(\boldsymbol{k}_{d}\right)$ affin von $\boldsymbol{k}_{d} a b$, wenn

$$
\phi_{l}^{\top} \boldsymbol{G}_{\boldsymbol{y} \boldsymbol{f}}^{-1}\left(\lambda_{B_{i j}}\right) \cdot \boldsymbol{C}\left(\lambda_{B_{i j}} \boldsymbol{I}_{n}-\boldsymbol{A}\right)^{-1} \boldsymbol{v}_{B, f f, q r}=0
$$

für alle $i, q=1, \ldots, n_{f}-1, j=1, \ldots, \delta_{i}, r=1, \ldots, \delta_{q}$ und $l=i+1, \ldots, n_{f}$ gilt. Es ist dann

$$
\boldsymbol{L}_{c}\left(\boldsymbol{k}_{d}\right)=-\sum_{\nu=1}^{n_{k_{d}}} k_{d_{\nu}} \boldsymbol{W}_{B, \mathrm{ffi}}^{-1}\left(\boldsymbol{P}_{c, \nu}+\boldsymbol{W}_{B c, \nu} \boldsymbol{L}_{\mathrm{ffi}}\right)=\sum_{\nu=1}^{n_{k_{d}}} k_{d_{\nu}} \boldsymbol{L}_{c, \nu} .
$$

Beweis. Ist (5.75) erfüllt, so gilt

$$
\boldsymbol{\Phi}_{i j l} \boldsymbol{G}_{\boldsymbol{y} \boldsymbol{f}}^{-1}\left(\lambda_{B_{i j}} \boldsymbol{I}_{n}-\boldsymbol{A}\right)^{-1} \boldsymbol{V}_{B, \mathrm{ffi}} \boldsymbol{\Phi}_{q r l}=\mathbf{0},
$$


wobei die Matrix

$$
\boldsymbol{\Phi}_{i j l}=\left[\begin{array}{lllllll}
\mathbf{0} & \cdots & \mathbf{0} & \boldsymbol{\phi}_{l} & \mathbf{0} & \cdots & \mathbf{0}
\end{array}\right]^{\top}
$$

in der $\left(j+\sum_{m=1}^{i-1} \delta_{m}\right)$-ten Zeile den Vektor $\boldsymbol{\phi}_{l}^{\top}$ enthält und $\boldsymbol{\Phi}_{q r l}$ in der $\left(r+\sum_{m=1}^{q-1} \delta_{m}\right)$-ten Zeile den Vektor $\phi_{l}^{\top}$ beinhaltet. Weiterhin lässt sich mit (4.98) und (4.101) kompakt

$$
\begin{aligned}
\boldsymbol{W}_{B c, i j l} & =\boldsymbol{\Phi}_{i j l} \boldsymbol{G}_{\boldsymbol{y} \boldsymbol{f}}^{-1}\left(\lambda_{B_{i j}}\right) \cdot \boldsymbol{C}\left(\lambda_{B_{i j}} \boldsymbol{I}_{n}-\boldsymbol{A}\right)^{-1}, \\
\boldsymbol{P}_{c, q r l} & =\boldsymbol{\Phi}_{q r l} \boldsymbol{G}_{\boldsymbol{y} \boldsymbol{f}}^{-1}\left(\lambda_{B_{q r}}\right), \\
\boldsymbol{W}_{B c, q r l} & =\boldsymbol{\Phi}_{q r l} \boldsymbol{G}_{\boldsymbol{y} \boldsymbol{f}}^{-1}\left(\lambda_{B_{q r}}\right) \cdot \boldsymbol{C}\left(\lambda_{B_{q r}} \boldsymbol{I}_{n}-\boldsymbol{A}\right)^{-1}
\end{aligned}
$$

schreiben. Berücksichtigt man zusätzlich $\boldsymbol{V}_{B, \text { ffi }}=\boldsymbol{W}_{B \text {,ffi }}^{-1}$, so lässt sich die Beziehung

$$
\begin{aligned}
& \boldsymbol{W}_{B c, i j l} \boldsymbol{W}_{B, \mathrm{ffi}}^{-1}\left(\boldsymbol{P}_{c, q r l}+\boldsymbol{W}_{B c, q r l} \boldsymbol{L}_{\mathrm{ffi}}\right) \\
&=\underbrace{\boldsymbol{\Phi}_{i j l} \boldsymbol{G}_{\boldsymbol{y} \boldsymbol{f}}^{-1}\left(\lambda_{B_{i j}}\right) \cdot \boldsymbol{C}\left(\lambda_{B_{i j}} \boldsymbol{I}_{n}-\boldsymbol{A}\right)^{-1}}_{\boldsymbol{W}_{B c, i j l}} \cdot \underbrace{\boldsymbol{V}_{B, \mathrm{ffi}}}_{\boldsymbol{W}_{B, \mathrm{ffi}}^{-1}} \cdots \\
& \underbrace{\boldsymbol{\Phi}_{q r l} \boldsymbol{G}_{\boldsymbol{y} \boldsymbol{f}}^{-1}\left(\lambda_{B_{q r}}\right)\left(\boldsymbol{I}_{n_{y}}+\boldsymbol{C}\left(\lambda_{B_{q r}} \boldsymbol{I}_{n}-\boldsymbol{A}\right)^{-1} \boldsymbol{L}_{\mathrm{ffi}}\right)}_{\boldsymbol{P}_{c, q r l}+\boldsymbol{W}_{B c, q r l} \boldsymbol{L}_{\mathrm{ffi}}}
\end{aligned}
$$

ableiten. Dementsprechend ist (5.77) hinreichend dafür, dass

$$
\boldsymbol{W}_{B c, i j l} \boldsymbol{W}_{B, f f i}^{-1}\left(\boldsymbol{P}_{c, q r l}+\boldsymbol{W}_{B c, q r l} \boldsymbol{L}_{\mathrm{ffi}}\right)=\mathbf{0}
$$

ist. Dies wiederum impliziert

$$
\begin{aligned}
& \sum_{\nu=1}^{n_{k_{d}}} k_{d_{\nu}}\left(\boldsymbol{P}_{c, \nu}+\boldsymbol{W}_{B c, \nu} \boldsymbol{L}_{\mathrm{ffi}}\right) \\
&=\left(\boldsymbol{I}_{n}+\sum_{\kappa=1}^{n_{k_{d}}} k_{d_{\kappa}} \boldsymbol{W}_{B c, \kappa} \boldsymbol{W}_{B, \mathrm{ffi}}^{-1}\right) \sum_{\nu=1}^{n_{k_{d}}} k_{d_{\nu}}\left(\boldsymbol{P}_{c, \nu}+\boldsymbol{W}_{B c, \nu} \boldsymbol{L}_{\mathrm{ffi}}\right) \\
&=\left(\boldsymbol{W}_{B, \mathrm{ffi}}+\sum_{\kappa=1}^{n_{k_{d}}} k_{d_{\kappa}} \boldsymbol{W}_{B c, \kappa}\right) \boldsymbol{W}_{B, \mathrm{ffi}}^{-1} \cdot \ldots \\
& \cdot \sum_{\nu=1}^{n_{k_{d}}} k_{d_{\nu}}\left(\boldsymbol{P}_{c, \nu}+\boldsymbol{W}_{B c, \nu} \boldsymbol{L}_{\mathrm{ffi}}\right)
\end{aligned}
$$


und damit

$$
\begin{aligned}
\sum_{\nu=1}^{n_{k_{d}}} k_{d_{\nu}} & \left(\boldsymbol{P}_{c, \nu}+\boldsymbol{W}_{B c, \nu} \boldsymbol{L}_{\mathrm{ffi}}\right) \\
& =\boldsymbol{W}_{B, \mathrm{pfi}}\left(\boldsymbol{k}_{d}\right) \cdot \boldsymbol{W}_{B, \text { ffi }}^{-1} \sum_{\nu=1}^{n_{k_{d}}} k_{d_{\nu}}\left(\boldsymbol{P}_{c, \nu}+\boldsymbol{W}_{B c, \nu} \boldsymbol{L}_{\mathrm{ffi}}\right) .
\end{aligned}
$$

Die Multiplikation dieser Gleichung von links mit $\boldsymbol{W}_{B, \mathrm{pfi}}^{-1}\left(\boldsymbol{k}_{d}\right)$ führt auf

$$
\begin{aligned}
\boldsymbol{W}_{B, \mathrm{pfi}}^{-1}\left(\boldsymbol{k}_{d}\right) \sum_{\nu=1}^{n_{k_{d}}} k_{d_{\nu}}\left(\boldsymbol{P}_{c, \nu}+\boldsymbol{W}_{B c, \nu} \boldsymbol{L}_{\mathrm{ffi}}\right) \\
=\boldsymbol{W}_{B, \mathrm{ffi}}^{-1} \sum_{\nu=1}^{n_{k_{d}}} k_{d_{\nu}}\left(\boldsymbol{P}_{c, \nu}+\boldsymbol{W}_{B c, \nu} \boldsymbol{L}_{\mathrm{ffi}}\right) .
\end{aligned}
$$

Da nach Lemma 5.1 stets

$$
\boldsymbol{L}_{c}\left(\boldsymbol{k}_{d}\right)=-\boldsymbol{W}_{B, \mathrm{pfi}}^{-1}\left(\boldsymbol{k}_{d}\right) \cdot \sum_{\nu=1}^{n_{k_{d}}} k_{d_{v}}\left(\boldsymbol{P}_{c, \nu}+\boldsymbol{W}_{B c, \nu} \boldsymbol{L}_{\mathrm{ffi}}\right)
$$

gilt, lässt sich mit (5.78)

$$
\begin{aligned}
\boldsymbol{L}_{c}\left(\boldsymbol{k}_{d}\right) & =-\boldsymbol{W}_{B, \mathrm{ffi}}^{-1} \cdot \sum_{\nu=1}^{n_{k_{d}}} k_{d_{v}}\left(\boldsymbol{P}_{c, \nu}+\boldsymbol{W}_{B c, \nu} \boldsymbol{L}_{\mathrm{ffi}}\right) \\
& =\sum_{\nu=1}^{n_{k_{d}}} k_{d_{\nu}}\left(-\boldsymbol{W}_{B, \mathrm{ffi}}^{-1}\left(\boldsymbol{P}_{c, \nu}+\boldsymbol{W}_{B c, \nu} \boldsymbol{L}_{\mathrm{ffi}}\right)\right) \\
& =\sum_{\nu=1}^{n_{k_{d}}} k_{d_{\nu}} \boldsymbol{L}_{c, \nu}
\end{aligned}
$$

schreiben.

Ist die Bedingung (5.75) aus Satz 5.6 erfüllt, ergibt sich gegenüber Lemma 5.1 also eine wesentliche Vereinfachung hinsichtlich der Abhängigkeit der Matrix $\boldsymbol{L}_{\text {pfi }}$ von $\boldsymbol{k}_{d}$. Nachteilig ist jedoch, dass zur Überprüfung von (5.75) zunächst ein FFIO entworfen werden muss. Dann ist gemäß (5.75) für alle Pole $\lambda_{B_{i j}}$ von $\boldsymbol{G}_{\boldsymbol{r} \boldsymbol{f}}(s)$, die in Spalten $l=i+1, \ldots, n_{f}$ gekoppelt werden, zu überprüfen, ob das entsprechende Produkt in dem durch $q$ und 
$r$ definierten Rechtseigenvektor von $\boldsymbol{A}-\boldsymbol{L}_{\mathrm{ff}} \boldsymbol{C}$ null ergibt. Lediglich anhand der Systemmatrizen oder Systemeigenschaften wie $n$ und $\delta_{i}$ erlaubt Satz 5.6 keine Aussage darüber, ob eine affine Beziehung $\boldsymbol{L}_{\mathrm{pfi}}\left(\boldsymbol{k}_{d}\right)$ gegeben ist. Die folgenden beiden Lemmata betrachten diesbezüglich jeweils Spezialfälle.

Lemma 5.2. Für ein System mit $n=2, n_{f}=2, \delta_{1}=\delta_{2}=1$ ist stets eine affine Abhängigkeit $\boldsymbol{L}_{\mathrm{pfi}}\left(\boldsymbol{k}_{d}\right)$ gegeben.

Beweis. Der Eigenwert $\lambda_{B_{11}}$ tritt sowohl in der ersten Spalte von $\boldsymbol{G}_{\boldsymbol{r} \boldsymbol{f}}(s)$ als auch mittels $k_{d, 112}$ in der zweiten Spalte auf. Der Eigenwert $\lambda_{B_{21}}$ taucht ausschließlich in der zweiten Spalte auf. Da $\delta_{1}=\delta_{2}=1$ ist, muss $\boldsymbol{E}_{s}=\mathbf{0}$ sein und es gilt

$$
\boldsymbol{W}_{B, \mathrm{pfi}} \boldsymbol{E}_{a}=\left[\begin{array}{cc}
1 & k_{d, 112} \\
0 & 1
\end{array}\right]
$$

Bei vollständiger Isolation ist

$$
\boldsymbol{W}_{B, \mathrm{ff}} \boldsymbol{E}_{a}=\left[\begin{array}{ll}
1 & 0 \\
0 & 1
\end{array}\right]
$$

Löst man (5.79) und (5.80) nach $\boldsymbol{E}_{a}$ auf und setzt die Ergebnisse gleich, so erhält man

$$
\boldsymbol{V}_{B, \text { ffi }}=\boldsymbol{V}_{B, \text { pfi }}\left[\begin{array}{cc}
1 & k_{d, 112} \\
0 & 1
\end{array}\right]
$$

woraus sich

$$
\boldsymbol{V}_{B, \mathrm{pfi}}=\boldsymbol{V}_{B, \mathrm{ffi}}-k_{d, 112} \boldsymbol{V}_{B, \text { ffi }}\left[\begin{array}{ll}
0 & 1 \\
0 & 0
\end{array}\right]
$$

ergibt. Setzt man dies in $\boldsymbol{L}_{\mathrm{pfi}}=-\boldsymbol{V}_{B, \mathrm{pfi}} \boldsymbol{P}_{\mathrm{pfi}}$ ein und berücksichtigt weiterhin (4.98), so folgt

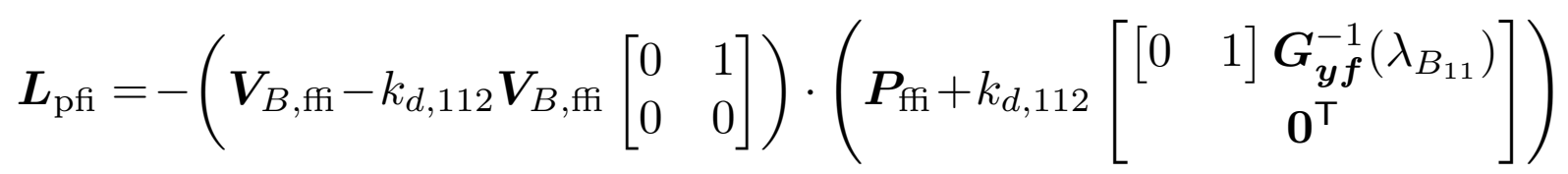

$$
\begin{aligned}
& =\boldsymbol{L}_{\mathrm{ffi}}+k_{d, 112} \boldsymbol{V}_{B, \text { ffi }}\left(\left[\begin{array}{ll}
0 & 1 \\
0 & 0
\end{array}\right] \boldsymbol{P}_{\mathrm{ffi}}-\left[\begin{array}{c}
{\left[\begin{array}{cc}
0 & 1
\end{array}\right] \boldsymbol{G}_{\boldsymbol{y} \boldsymbol{f}}^{-1}\left(\lambda_{B_{11}}\right)} \\
\mathbf{0}^{\top}
\end{array}\right]\right)+\ldots \\
& -k_{d, 112}^{2} \boldsymbol{V}_{B, f f i} \underbrace{\left[\begin{array}{ll}
0 & 1 \\
0 & 0
\end{array}\right]\left[\begin{array}{cc}
{\left[\begin{array}{cc}
0 & 1
\end{array}\right] \boldsymbol{G}_{\boldsymbol{y} \boldsymbol{f}}^{-1}\left(\lambda_{B_{11}}\right)} \\
\mathbf{0}^{\top}
\end{array}\right]}_{\mathbf{0}} \text {. }
\end{aligned}
$$


Offensichtlich entfällt der in $k_{d, 112}$ quadratische Term und es besteht eine affine Abhängigkeit $\boldsymbol{L}_{\mathrm{pfi}}\left(k_{d, 112}\right)=\boldsymbol{L}_{\mathrm{ffi}}+\boldsymbol{L}_{c}\left(k_{d, 112}\right)$.

Lemma 5.3. Für ein System mit $n_{f}=2, \delta_{\max }=\max \left\{\delta_{i}\right\} \geq 2$ ist eine affine Abhängigkeit $\boldsymbol{L}_{\mathrm{pfi}}\left(\boldsymbol{k}_{d}\right)$ gegeben, wenn $\boldsymbol{V}_{B \text {,pfi }}$ affin von $\boldsymbol{k}_{d}$ abhängt und die ersten $\delta_{\max }$ Rechtseigenvektoren von $\boldsymbol{A}-\boldsymbol{L}_{\mathrm{pfi}} \boldsymbol{C}$ denen von $\boldsymbol{A}-\boldsymbol{L}_{\mathrm{ffi}} \boldsymbol{C}$ entsprechen.

Beweis. Betrachtet man erneut $\boldsymbol{L}_{\mathrm{pfi}}=-\boldsymbol{V}_{B, \mathrm{pfi}} \boldsymbol{P}_{\mathrm{pfi}}$, so ergibt sich mit (4.100)

$$
\boldsymbol{L}_{\mathrm{pfi}}=-\boldsymbol{V}_{B, \mathrm{pfi}}\left(\boldsymbol{P}_{\mathrm{ffi}}+\sum_{\nu=1}^{n_{k_{d}}} k_{d, \nu} \boldsymbol{P}_{c, \nu}\right),
$$

wobei der Term $\sum_{\nu=1}^{n_{k_{d}}} k_{d, \nu} \boldsymbol{P}_{c, \nu}$ gemäß (4.98) lediglich in den ersten $\delta_{\max }$ Zeilen Elemente enthält, die von 0 verschieden sind. Wenn nun $\boldsymbol{V}_{B \text {,pfi }}$ affin von den Kopplungszahlen $k_{d, 1 j 2}$ abhängt, ist eine affine Abhängigkeit $\boldsymbol{L}_{\mathrm{pfi}}\left(\boldsymbol{k}_{d}\right)$ demnach gegeben, wenn sich gegenüber $\boldsymbol{V}_{B, \text { ffi }}$ die ersten $\delta_{\max }$

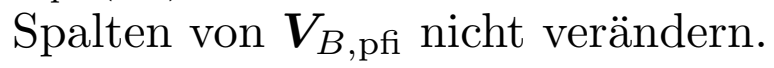

Für Systeme mit $n_{f} \geq 3$ lässt sich darüber hinaus bereits für den einfachen Fall $\delta_{i}=1$ für alle $i=1, \ldots, n_{f}$ plausibilisieren, dass keine affine Abhängigkeit $\boldsymbol{L}_{\mathrm{pfi}}\left(\boldsymbol{k}_{d}\right)$ besteht. Es ist dann

$$
\boldsymbol{W}_{B, \mathrm{ff}} \boldsymbol{E}_{a}=\left[\begin{array}{ccc}
1 & 0 & 0 \\
0 & 1 & 0 \\
0 & 0 & 1
\end{array}\right], \boldsymbol{W}_{B, \mathrm{pfi}} \boldsymbol{E}_{a}=\left[\begin{array}{ccc}
1 & k_{d, 112} & k_{d, 113} \\
0 & 1 & k_{d, 213} \\
0 & 0 & 1
\end{array}\right]
$$

woraus sich

$$
\boldsymbol{V}_{B, \mathrm{pfi}}=\boldsymbol{V}_{B, \mathrm{ffi}}\left[\begin{array}{ccc}
1 & -k_{d, 112} & k_{d, 112} k_{d, 213}-k_{d, 113} \\
0 & 1 & -k_{d, 213} \\
0 & 0 & 1
\end{array}\right]
$$

ergibt. Setzt man dies in $\boldsymbol{L}_{\mathrm{pfi}}=-\boldsymbol{V}_{B, \mathrm{pfi}} \boldsymbol{P}_{\mathrm{pfi}}$ ein, so treten offensichtlich bilineare Terme der Form $k_{d, 112} \cdot k_{d, 213}$ auf.

Nachdem der Zusammenhang zwischen der Beobachtermatrix $\boldsymbol{L}_{\mathrm{pfi}}$ und den dynamischen Kopplungszahlen eingehend untersucht wurde, verbleibt die Analyse der Nachfiltermatrix $\boldsymbol{V}_{\text {pfi }}\left(\boldsymbol{k}_{d}, \boldsymbol{k}_{s}\right)$. Während sie im Allgemeinen gemäß Gleichung (4.104) auf Seite 178 nichtlinear von $\boldsymbol{k}_{d}$ und $\boldsymbol{k}_{s}$ abhängt, liefert der folgende Satz eine wesentliche Vereinfachung unter der Bedingung, dass $\boldsymbol{L}_{\mathrm{pfi}}\left(\boldsymbol{k}_{d}\right)$ affin von $\boldsymbol{k}_{d}$ abhängt. 
Satz 5.7 (Affin bilineare Abhängigkeit der PFIO-Parametrierung von den Kopplungszahlen). Gegeben sei ein System der Form

$$
\begin{aligned}
& \dot{\boldsymbol{x}}=\boldsymbol{A} \boldsymbol{x}+\boldsymbol{B} \boldsymbol{u}+\boldsymbol{E}_{a} \boldsymbol{f}, \\
& \boldsymbol{y}=\boldsymbol{C} \boldsymbol{x}+\boldsymbol{D} \boldsymbol{u}+\boldsymbol{E}_{s} \boldsymbol{f}
\end{aligned}
$$

welches die Annahmen 5.1-5.3 erfüllt und für das durch $\left(\boldsymbol{L}_{\mathrm{ff}}, \boldsymbol{V}_{\mathrm{ffi}}\right)$ ein FFIO parametriert ist. Hängt die Beobachtermatrix $\boldsymbol{L}_{\mathrm{pfi}}$ für die partielle Fehlerisolation affin von den dynamischen Kopplungszahlen $\boldsymbol{k}_{d}$ ab, so ergibt sich für die Nachfiltermatrix $\boldsymbol{V}_{\text {pfi }}$ eine affin bilineare Abhängigkeit von den Kopplungszahlen $\boldsymbol{k}_{d}$ und $\boldsymbol{k}_{s}$ der Form

$$
\begin{aligned}
\boldsymbol{V}_{\mathrm{pfi}}\left(\boldsymbol{k}_{d}, \boldsymbol{k}_{s}\right) & =\boldsymbol{V}_{\mathrm{ffi}}+\boldsymbol{V}_{c}\left(\boldsymbol{k}_{d}, \boldsymbol{k}_{s}\right) \\
& =\boldsymbol{V}_{\mathrm{ffi}}+\sum_{\nu=1}^{n_{k_{d}}} k_{d, \nu} \boldsymbol{V}_{d, \nu}+\sum_{\mu=1}^{n_{k_{s}}} k_{s, \mu} \boldsymbol{V}_{s, \mu}+\sum_{\mu=1}^{n_{k_{s}}} \sum_{\nu=1}^{n_{k_{d}}} k_{s, \mu} k_{d, \nu} \boldsymbol{V}_{s d, \mu \nu} .
\end{aligned}
$$

Dabei gilt

$$
\begin{aligned}
\boldsymbol{V}_{d, \nu} & =-\boldsymbol{V}_{\mathrm{ffi}} \boldsymbol{C}\left(\boldsymbol{A}-\boldsymbol{L}_{\mathrm{ffi}} \boldsymbol{C}\right)^{-1} \boldsymbol{L}_{c, \nu}, \\
\boldsymbol{V}_{s, \mu} & =-\boldsymbol{S}_{c, \mu} \boldsymbol{\Theta}_{\mathrm{ffi}}^{-1} \\
\boldsymbol{V}_{s d, \mu \nu} & =\boldsymbol{S}_{c, \mu} \boldsymbol{\Theta}_{\mathrm{ffi}}^{-1} \boldsymbol{C}\left(\boldsymbol{A}-\boldsymbol{L}_{\mathrm{ffi}} \boldsymbol{C}\right)^{-1} \boldsymbol{L}_{c, \nu}
\end{aligned}
$$

und $\boldsymbol{S}_{c, \mu}$ und $\boldsymbol{L}_{c, \nu}$ sind in (4.103) beziehungsweise (5.76) gegeben.

Beweis. Analog zu $\boldsymbol{\Theta}_{\mathrm{ffi}}$ wird

$$
\boldsymbol{\Theta}_{\mathrm{pfi}}\left(\boldsymbol{k}_{d}\right)=\boldsymbol{C}\left(\boldsymbol{A}-\boldsymbol{L}_{\mathrm{pfi}}\left(\boldsymbol{k}_{d}\right) \boldsymbol{C}\right)^{-1}\left(\boldsymbol{E}_{a}-\boldsymbol{L}_{\mathrm{pfi}}\left(\boldsymbol{k}_{d}\right) \boldsymbol{E}_{s}\right)-\boldsymbol{E}_{s}
$$

eingeführt. Nach Lemma 4.2 ist $\boldsymbol{\Theta}_{\text {pfi }}\left(\boldsymbol{k}_{d}\right)$ ebenso invertierbar wie $\boldsymbol{\Theta}_{\text {ffi. }}$. Entsprechend (4.103) beziehungsweise Satz 4.13 ist

$$
\boldsymbol{V}_{\mathrm{pfi}}\left(\boldsymbol{k}_{d}, \boldsymbol{k}_{s}\right)=-\left(\boldsymbol{S}_{\mathrm{ffi}}+\boldsymbol{S}_{c}\left(\boldsymbol{k}_{s}\right)\right) \cdot \boldsymbol{\Theta}_{\mathrm{pfi}}^{-1}\left(\boldsymbol{k}_{d}\right)=\boldsymbol{V}_{\mathrm{ffi}}+\boldsymbol{V}_{c}\left(\boldsymbol{k}_{d}, \boldsymbol{k}_{s}\right) .
$$

Dementsprechend gilt für die von den Kopplungszahlen abhängige Differenz zwischen den Nachfiltermatrizen

$$
\begin{aligned}
\boldsymbol{V}_{c}\left(\boldsymbol{k}_{d}, \boldsymbol{k}_{s}\right) & =-\boldsymbol{S}_{\mathrm{ffi}} \cdot \boldsymbol{\Theta}_{\mathrm{pfi}}^{-1}\left(\boldsymbol{k}_{d}\right)-\boldsymbol{S}_{c}\left(\boldsymbol{k}_{s}\right) \cdot \boldsymbol{\Theta}_{\mathrm{pfi}}^{-1}\left(\boldsymbol{k}_{d}\right) \underbrace{+\boldsymbol{S}_{\mathrm{ffi}} \boldsymbol{\Theta}_{\mathrm{ffi}}^{-1}}_{-\boldsymbol{V}_{\mathrm{ffi}}} \\
& =-\boldsymbol{S}_{\mathrm{ffi}}\left(\boldsymbol{\Theta}_{\mathrm{pfi}}^{-1}\left(\boldsymbol{k}_{d}\right)-\boldsymbol{\Theta}_{\mathrm{ffi}}^{-1}\right)-\boldsymbol{S}_{c}\left(\boldsymbol{k}_{s}\right) \cdot \boldsymbol{\Theta}_{\mathrm{pfi}}^{-1}\left(\boldsymbol{k}_{d}\right) .
\end{aligned}
$$


In dieser Beziehung wird zunächst der Ausdruck $\boldsymbol{\Theta}_{\mathrm{pfi}}^{-1}\left(\boldsymbol{k}_{d}\right)-\boldsymbol{\Theta}_{\mathrm{ffi}}^{-1}$ analysiert. Setzt man im ersten Teil von Lemma A.2 $\boldsymbol{A}=\boldsymbol{\Theta}_{\mathrm{pfi}}\left(\boldsymbol{k}_{d}\right)$ und $\boldsymbol{B}=\boldsymbol{\Theta}_{\mathrm{ffi}}$, so ergibt sich

$$
\boldsymbol{\Theta}_{\mathrm{pfi}}^{-1}\left(\boldsymbol{k}_{d}\right)-\boldsymbol{\Theta}_{\mathrm{ffi}}^{-1}=\boldsymbol{\Theta}_{\mathrm{ffi}}^{-1} \cdot\left(\boldsymbol{\Theta}_{\mathrm{ffi}}-\boldsymbol{\Theta}_{\mathrm{pfi}}\left(\boldsymbol{k}_{d}\right)\right) \cdot \boldsymbol{\Theta}_{\mathrm{pfi}}^{-1}\left(\boldsymbol{k}_{d}\right) .
$$

Schreibt man die Beobachtermatrix für die vollständige Fehlerisolation als $\boldsymbol{L}_{\mathrm{ffi}}=\boldsymbol{L}_{\mathrm{pfi}}\left(\boldsymbol{k}_{d}\right)-\boldsymbol{L}_{c}\left(\boldsymbol{k}_{d}\right)$, erhält man für die Differenz $\boldsymbol{\Theta}_{\mathrm{ffi}}-\boldsymbol{\Theta}_{\mathrm{pfi}}\left(\boldsymbol{k}_{d}\right)$ den Ausdruck

$$
\begin{aligned}
\Theta_{\mathrm{ffi}}-\Theta_{\mathrm{pfi}}\left(\boldsymbol{k}_{d}\right)= & \boldsymbol{C}\left(\boldsymbol{A}-\boldsymbol{L}_{\mathrm{ffi}} \boldsymbol{C}\right)^{-1}\left(\boldsymbol{E}_{a}-\boldsymbol{L}_{\mathrm{ffi}} \boldsymbol{E}_{s}\right)-\boldsymbol{E}_{s}+\ldots \\
& -\left(\boldsymbol{C}\left(\boldsymbol{A}-\boldsymbol{L}_{\mathrm{pfi}}\left(\boldsymbol{k}_{d}\right) \boldsymbol{C}\right)^{-1}\left(\boldsymbol{E}_{a}-\boldsymbol{L}_{\mathrm{pfi}}\left(\boldsymbol{k}_{d}\right) \boldsymbol{E}_{s}\right)-\boldsymbol{E}_{s}\right) \\
= & \boldsymbol{C}\left(\boldsymbol{A}-\boldsymbol{L}_{\mathrm{ffi}} \boldsymbol{C}\right)^{-1}\left(\boldsymbol{E}_{a}-\boldsymbol{L}_{\mathrm{pfi}}\left(\boldsymbol{k}_{d}\right) \boldsymbol{E}_{s}\right)+\ldots \\
& +\boldsymbol{C}\left(\boldsymbol{A}-\boldsymbol{L}_{\mathrm{ffi}} \boldsymbol{C}\right)^{-1} \boldsymbol{L}_{c}\left(\boldsymbol{k}_{d}\right) \boldsymbol{E}_{s}+\ldots \\
& -\boldsymbol{C}\left(\boldsymbol{A}-\boldsymbol{L}_{\mathrm{pfi}}\left(\boldsymbol{k}_{d}\right) \boldsymbol{C}\right)^{-1}\left(\boldsymbol{E}_{a}-\boldsymbol{L}_{\mathrm{pfi}}\left(\boldsymbol{k}_{d}\right) \boldsymbol{E}_{s}\right) \\
= & \boldsymbol{C}\left[\left(\boldsymbol{A}-\boldsymbol{L}_{\mathrm{ffi}} \boldsymbol{C}\right)^{-1}-\left(\boldsymbol{A}-\boldsymbol{L}_{\mathrm{pfi}}\left(\boldsymbol{k}_{d}\right) \boldsymbol{C}\right)^{-1}\right] \cdot \ldots \\
& \cdot\left(\boldsymbol{E}_{a}-\boldsymbol{L}_{\mathrm{pfi}}\left(\boldsymbol{k}_{d}\right) \boldsymbol{E}_{s}\right)+\boldsymbol{C}\left(\boldsymbol{A}-\boldsymbol{L}_{\mathrm{ffi}} \boldsymbol{C}\right)^{-1} \boldsymbol{L}_{c}\left(\boldsymbol{k}_{d}\right) \boldsymbol{E}_{s} .
\end{aligned}
$$

Auf die Differenz der beiden invertierbaren Matrizen innerhalb der eckigen Klammern in obiger Gleichung wird nun der zweite Teil von Lemma A.2 angewendet. Wegen $\boldsymbol{L}_{\mathrm{pfi}}\left(\boldsymbol{k}_{d}\right)-\boldsymbol{L}_{\mathrm{ffi}}=\boldsymbol{L}_{c}\left(\boldsymbol{k}_{d}\right)$ ergibt sich daraus

$$
\begin{aligned}
\left(\boldsymbol{A}-\boldsymbol{L}_{\mathrm{ffi}} \boldsymbol{C}\right)^{-1}-\left(\boldsymbol{A}-\boldsymbol{L}_{\mathrm{pfi}}\left(\boldsymbol{k}_{d}\right) \boldsymbol{C}\right)^{-1}= & -\left(\boldsymbol{A}-\boldsymbol{L}_{\mathrm{ffi}} \boldsymbol{C}\right)^{-1} \boldsymbol{L}_{c}\left(\boldsymbol{k}_{d}\right) \cdot \ldots \\
& \cdot \boldsymbol{C}\left(\boldsymbol{A}-\boldsymbol{L}_{\mathrm{pfi}}\left(\boldsymbol{k}_{d}\right) \boldsymbol{C}\right)^{-1} .
\end{aligned}
$$

Setzt man dies in (5.84) ein, vereinfacht sich der Ausdruck zu

$$
\begin{aligned}
\Theta_{\mathrm{ffi}}-\Theta_{\mathrm{pfi}}\left(\boldsymbol{k}_{d}\right)= & -\boldsymbol{C}\left(\boldsymbol{A}-\boldsymbol{L}_{\mathrm{ffi}} \boldsymbol{C}\right)^{-1} \boldsymbol{L}_{c}\left(\boldsymbol{k}_{d}\right) \boldsymbol{C}\left(\boldsymbol{A}-\boldsymbol{L}_{\mathrm{pfi}}\left(\boldsymbol{k}_{d}\right) \boldsymbol{C}\right)^{-1} \cdot \ldots \\
& \cdot\left(\boldsymbol{E}_{a}-\boldsymbol{L}_{\mathrm{pfi}}\left(\boldsymbol{k}_{d}\right) \boldsymbol{E}_{s}\right)+\boldsymbol{C}\left(\boldsymbol{A}-\boldsymbol{L}_{\mathrm{ffi}} \boldsymbol{C}\right)^{-1} \boldsymbol{L}_{c}\left(\boldsymbol{k}_{d}\right) \boldsymbol{E}_{s} \\
= & -\boldsymbol{C}\left(\boldsymbol{A}-\boldsymbol{L}_{\mathrm{ffi}} \boldsymbol{C}\right)^{-1} \cdot \boldsymbol{L}_{c}\left(\boldsymbol{k}_{d}\right) \cdot \boldsymbol{\Theta}_{\mathrm{pfi}}\left(\boldsymbol{k}_{d}\right) .
\end{aligned}
$$

Damit folgt aus (5.83)

$$
\boldsymbol{\Theta}_{\mathrm{pfi}}^{-1}\left(\boldsymbol{k}_{d}\right)-\boldsymbol{\Theta}_{\mathrm{ffi}}^{-1}=-\boldsymbol{\Theta}_{\mathrm{ffi}}^{-1} \cdot \boldsymbol{C}\left(\boldsymbol{A}-\boldsymbol{L}_{\mathrm{ffi}} \boldsymbol{C}\right)^{-1} \cdot \boldsymbol{L}_{c}\left(\boldsymbol{k}_{d}\right),
$$

woraus sich weitergehend

$$
\boldsymbol{\Theta}_{\mathrm{pfi}}^{-1}\left(\boldsymbol{k}_{d}\right)=\boldsymbol{\Theta}_{\mathrm{ffi}}^{-1}\left(\boldsymbol{I}_{n_{y}}-\boldsymbol{C}\left(\boldsymbol{A}-\boldsymbol{L}_{\mathrm{ffi}} \boldsymbol{C}\right)^{-1} \cdot \boldsymbol{L}_{c}\left(\boldsymbol{k}_{d}\right)\right)
$$


ergibt. Damit lässt sich für (5.82) schließlich

$$
\begin{aligned}
\boldsymbol{V}_{c}\left(\boldsymbol{k}_{d}, \boldsymbol{k}_{s}\right)= & \underbrace{\boldsymbol{S}_{\mathrm{ffi}} \boldsymbol{\Theta}_{\mathrm{ffi}}^{-1}}_{-\boldsymbol{V}_{\mathrm{ffi}}} \boldsymbol{C}\left(\boldsymbol{A}-\boldsymbol{L}_{\mathrm{ffi}} \boldsymbol{C}\right)^{-1} \boldsymbol{L}_{c}\left(\boldsymbol{k}_{d}\right)+\ldots \\
& -\boldsymbol{S}_{c}\left(\boldsymbol{k}_{s}\right) \boldsymbol{\Theta}_{\mathrm{ffi}}^{-1}+\boldsymbol{S}_{c}\left(\boldsymbol{k}_{s}\right) \boldsymbol{\Theta}_{\mathrm{ffi}}^{-1} \boldsymbol{C}\left(\boldsymbol{A}-\boldsymbol{L}_{\mathrm{ffi}} \boldsymbol{C}\right)^{-1} \boldsymbol{L}_{c}\left(\boldsymbol{k}_{d}\right)
\end{aligned}
$$

schreiben. Der Beweis wird abgeschlossen, indem die in (4.103) und (5.76) eingeführten Summenschreibweisen für $\boldsymbol{S}_{c}\left(\boldsymbol{k}_{s}\right)$ und $\boldsymbol{L}_{c}\left(\boldsymbol{k}_{d}\right)$ berücksichtigt werden, woraus (5.81) folgt.

Die Aussage von Satz 5.7 ist insofern bemerkenswert, als dass (4.104) einen komplizierteren nichtlinearen Zusammenhang zwischen den Kopplungszahlen und der sich ergebenden Nachfiltermatrix $\boldsymbol{V}_{\text {pfi vermuten lässt. }}$ Angemerkt sei, dass (5.85) auch dann gilt, wenn $\boldsymbol{L}_{\mathrm{pfi}}$ nichtlinear von den dynamischen Kopplungszahlen $\boldsymbol{k}_{d}$ abhängt. Es ändert sich dann lediglich $\boldsymbol{L}_{c}\left(\boldsymbol{k}_{d}\right)$.

Auf Basis der herausgearbeiteten linearen beziehungsweise bilinearen Abhängigkeit der Matrizen $\boldsymbol{L}_{c}\left(\boldsymbol{k}_{d}\right)$ und $\boldsymbol{V}_{c}\left(\boldsymbol{k}_{d}, \boldsymbol{k}_{s}\right)$ von den Kopplungszahlen wird im folgenden Abschnitt ein Verfahren zur Optimierung der Robustheit der Residuengenerierung erarbeitet.

\subsubsection{Entwurfsverfahren bei affiner Abhängigkeit der Beobachtermatrix von den dynamischen Kopplungszahlen}

Ziel dieses Abschnittes ist es, ein effizientes Lösungsverfahren für das Optimierungsproblem

$$
\underset{\boldsymbol{k}_{d}, \boldsymbol{k}_{s}}{\operatorname{minimiere}}\left\|\boldsymbol{G}_{\boldsymbol{r d}}\left(s, \boldsymbol{k}_{d}, \boldsymbol{k}_{s}\right)\right\|_{\infty}
$$

zu finden, wenn die Beobachtermatrix affin von den dynamischen Kopplungszahlen abhängt. Dies ist der Fall, wenn die Bedingung (5.75) aus Satz 5.6 erfüllt ist. Zur Lösung wird (5.86) auf ein BMI-Problem zurückgeführt, welches sich mithilfe des Path-Following-Verfahrens ähnlich wie in Abschnitt 5.4.3 durch die iterative Lösung von LMI-Problemen approximativ lösen lässt.

Unter Rückgriff auf das Bounded-Real-Lemma (Satz 3.1 auf Seite 56) 
lässt sich (5.86) äquivalent formulieren als

$$
\underset{\boldsymbol{X}, \boldsymbol{k}_{d}, \boldsymbol{k}_{s}}{\operatorname{minimiere}} \gamma, \text { sodass }
$$

$\boldsymbol{X} \succ \mathbf{0}$,

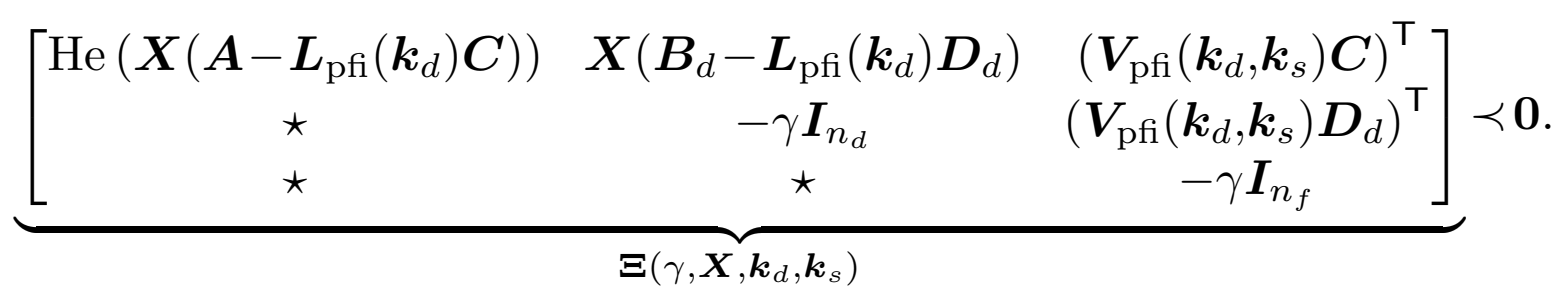

Bei (5.87c) handelt es sich um eine bilineare Matrixungleichung (BMI). Dies liegt zum einen darin begründet, dass bei einer affinen Abhängigkeit der Beobachtermatrix $\boldsymbol{L}_{\mathrm{pfi}}$ von den dynamischen Kopplungszahlen $\boldsymbol{k}_{d}$ in den Termen $\boldsymbol{X} \boldsymbol{L}_{\mathrm{pfi}}$ multiplikative Ausdrücke in $k_{d, \nu}$ und $\boldsymbol{X}$ auftreten. Zum anderen besteht in diesem Fall wie in Satz 5.7 gezeigt eine bilineare Abhängigkeit der Matrix $\boldsymbol{V}_{\text {pfi }}$ von den statischen und dynamischen Kopplungszahlen $\boldsymbol{k}_{s}$ und $\boldsymbol{k}_{d}$.

Da die Kopplungszahlen $k_{d, \nu}$ und $k_{s, \mu}$ in Gleichung (5.87c) sowohl linear als auch in Form von Produkttermen wie $k_{d, \nu} k_{s, \mu}$ auftreten, lässt sich die BMI nicht ohne zusätzliche Rangbeschränkungen durch eine Variablentransformation in eine LMI überführen. Daher wird zur Lösung des BMIProblems (5.87) wie schon in Abschnitt 5.4.3 der Path-Following-Ansatz angewendet.

Ist die Bedingung aus Satz 5.7 erfüllt, so treten in (5.87c) lediglich bilineare Terme auf, und die Matrix $\boldsymbol{\Xi}$ lässt sich in einfacher Art und Weise in eine Taylorreihe entwickeln. Berücksichtigt man dabei nur Terme erster Ordnung, so ergibt sich für den Entwicklungspunkt $\left(\gamma_{f}, \boldsymbol{X}_{f}, \boldsymbol{k}_{d, f}, \boldsymbol{k}_{s, f}\right)$ die Approximation

$$
\begin{aligned}
\boldsymbol{\Xi}\left(\gamma, \boldsymbol{X}, \boldsymbol{k}_{d}, \boldsymbol{k}_{s}\right) \approx & \boldsymbol{\Xi}\left(\gamma_{f}, \boldsymbol{X}_{f}, \boldsymbol{k}_{d, f}, \boldsymbol{k}_{s, f}\right)+\ldots \\
& +\boldsymbol{\Xi}_{\Delta \gamma}\left(\Delta \gamma, \boldsymbol{X}_{f}, \boldsymbol{k}_{d, f}, \boldsymbol{k}_{s, f}\right)+\ldots \\
& +\boldsymbol{\Xi}_{\Delta \boldsymbol{X}}\left(\gamma_{f}, \Delta \boldsymbol{X}, \boldsymbol{k}_{d, f}, \boldsymbol{k}_{s, f}\right)+\ldots \\
& +\boldsymbol{\Xi}_{\Delta \boldsymbol{k}_{d}}\left(\gamma_{f}, \boldsymbol{X}_{f}, \Delta \boldsymbol{k}_{d}, \boldsymbol{k}_{s, f}\right)+\ldots \\
& +\boldsymbol{\Xi}_{\Delta \boldsymbol{k}_{s}}\left(\gamma_{f}, \boldsymbol{X}_{f}, \boldsymbol{k}_{d, f}, \Delta \boldsymbol{k}_{s}\right) .
\end{aligned}
$$

Die darin auftauchenden Matrizen, die sich aus der partiellen Ableitung 
von $\boldsymbol{\Xi}$ nach den jeweiligen Variablen ergeben, lauten

$$
\begin{aligned}
& \boldsymbol{\Xi}_{\Delta \gamma}=\left[\begin{array}{ccc}
\mathbf{0} & \mathbf{0} & \mathbf{0} \\
\star & -\Delta \gamma \boldsymbol{I}_{n_{d}} & \mathbf{0} \\
\star & \star & -\Delta \gamma \boldsymbol{I}_{n_{f}}
\end{array}\right] \\
& \boldsymbol{\Xi}_{\Delta \boldsymbol{X}}=\left[\begin{array}{ccc}
\operatorname{He}\left(\Delta \boldsymbol{X}\left(\boldsymbol{A}-\boldsymbol{L}_{\mathrm{pfi}}\left(\boldsymbol{k}_{d, f}\right) \boldsymbol{C}\right)\right) & \Delta \boldsymbol{X}\left(\boldsymbol{B}_{d}-\boldsymbol{L}_{\mathrm{pfi}}\left(\boldsymbol{k}_{d, f}\right) \boldsymbol{D}_{d}\right) & \mathbf{0} \\
\star & \mathbf{0} & \mathbf{0} \\
\star & \star & \mathbf{0}
\end{array}\right] \text {, } \\
& \boldsymbol{\Xi}_{\Delta \boldsymbol{k}_{d}}=\left[\begin{array}{ccc}
-\mathrm{He}\left(\boldsymbol{X}_{f} \sum_{\nu=1}^{n_{k_{d}}} \Delta k_{d, \nu} \boldsymbol{L}_{c, \nu} \boldsymbol{C}\right) & \boldsymbol{\Xi}_{\Delta \boldsymbol{k}_{d}, 12} & \boldsymbol{\Xi}_{\Delta \boldsymbol{k}_{d}, 13}^{\top} \\
\star & \mathbf{0} & \boldsymbol{\Xi}_{\Delta \boldsymbol{k}_{d}, 23}^{\top} \\
\star & \star & \mathbf{0}
\end{array}\right], \\
& \boldsymbol{\Xi}_{\Delta \boldsymbol{k}_{d}, 12}=-\boldsymbol{X}_{f} \sum_{\nu=1}^{n_{k_{d}}} \Delta k_{d, \nu} \boldsymbol{L}_{c, \nu} \boldsymbol{D}_{d} \\
& \boldsymbol{\Xi}_{\Delta \boldsymbol{k}_{d}, 13}^{\top}=\sum_{\nu=1}^{n_{k_{d}}} \Delta k_{d, \nu}\left(\boldsymbol{V}_{d, \nu}+\sum_{\mu=1}^{n_{k_{s}}} k_{s, \mu, f} \boldsymbol{V}_{s d, \mu \nu}\right) \boldsymbol{C} \\
& \boldsymbol{\Xi}_{\Delta \boldsymbol{k}_{d}, 23}^{\mathrm{T}}=\sum_{\nu=1}^{n_{k_{d}}} \Delta k_{d, \nu}\left(\boldsymbol{V}_{d, \nu}+\sum_{\mu=1}^{n_{k_{s}}} k_{s, \mu, f} \boldsymbol{V}_{s d, \mu \nu}\right) \boldsymbol{D}_{d} \\
& \boldsymbol{\Xi}_{\Delta \boldsymbol{k}_{s}}=\left[\begin{array}{ccc}
\mathbf{0} & \mathbf{0} & \left(\sum_{\mu=1}^{n_{k_{s}}} \Delta k_{s, \mu}\left(\boldsymbol{V}_{s, \mu} \sum_{\nu=1}^{n_{k_{d}}} k_{d, \nu, f} \boldsymbol{V}_{s d, \mu \nu}\right) \boldsymbol{C}\right)^{\top} \\
\star & \mathbf{0} & \left(\sum_{\mu=1}^{n_{k_{s}}} \Delta k_{s, \mu}\left(\boldsymbol{V}_{s, \mu} \sum_{\nu=1}^{n_{k_{d}}} k_{d, \nu, f} \boldsymbol{V}_{s d, \mu \nu}\right) \boldsymbol{D}_{d}\right)^{\top} \\
\star & \star & \mathbf{0}
\end{array}\right] .
\end{aligned}
$$

Die Approximation der Matrix $\boldsymbol{\Xi}$, die in (5.88) angegeben ist, hängt folglich linear von den Variablen $\Delta \gamma, \Delta \boldsymbol{X}, \Delta \boldsymbol{k}_{d}$ und $\Delta \boldsymbol{k}_{s}$ ab und (5.87c) lässt sich durch die LMI

$$
\boldsymbol{\Xi}_{f}+\boldsymbol{\Xi}_{\Delta \gamma}+\boldsymbol{\Xi}_{\Delta \boldsymbol{X}}+\boldsymbol{\Xi}_{\Delta \boldsymbol{k}_{d}}+\boldsymbol{\Xi}_{\Delta \boldsymbol{k}_{s}} \prec \mathbf{0}
$$

annähern. Für das Path-Following-Verfahren wird zunächst für eine initiale Parametrierung $\left(\boldsymbol{L}_{\mathrm{pfi}}\left(\boldsymbol{k}_{d, \text { init }}\right), \boldsymbol{V}_{\mathrm{pfi}}\left(\boldsymbol{k}_{d, \text { init }}, \boldsymbol{k}_{s, \text { init }}\right)\right)$ eine Schranke $\gamma_{\text {init }}$ mit einer zugehörigen Matrix $\boldsymbol{X}_{\text {init }}$ berechnet. Anschließend wird das sich aus der Approximation von (5.87) ergebende LMI-Problem gelöst, wobei eine Minimierung von $\gamma$ angestrebt wird und (5.89) als Nebenbedingung einzuhalten ist. Nach dem Verifikationsschritt wird dies solange wiederholt, bis das Abbruchkriterium erfüllt ist. Zusammengefasst ergibt sich 


\section{Algorithmus 5.3.}

1. Initialisierung

Setze $\boldsymbol{k}_{d}=\boldsymbol{k}_{d, f}=\boldsymbol{k}_{d \text {,init }}$ und $\boldsymbol{k}_{s}=\boldsymbol{k}_{s, f}=\boldsymbol{k}_{s, \text { init }}$ und löse das sich ergebende LMI-Problem

$$
\underset{\boldsymbol{X}}{\operatorname{minimiere}} \gamma \text {, sodass } \boldsymbol{X} \succ \mathbf{0},(5.87 \mathrm{c}) .
$$

Definiere die gefundene Lösung als $\gamma_{f}, \boldsymbol{X}_{f}$.

2. Path-Following-Schritt

a) Update-Schritt

Löse das LMI-Problem

$$
\begin{aligned}
& \underset{\Delta \boldsymbol{X}, \Delta \boldsymbol{k}_{d}, \Delta \boldsymbol{k}_{s}}{\operatorname{minimiere}} \Delta \gamma, \text { sodass } \\
& \boldsymbol{X}_{f}+\Delta \boldsymbol{X} \succ \mathbf{0}, \Delta \gamma<0, \gamma_{f}+\Delta \gamma>0, \\
& {\left[\begin{array}{cc}
\zeta \boldsymbol{X}_{f} & \Delta \boldsymbol{X} \\
\star & \zeta \boldsymbol{X}_{f}
\end{array}\right] \succ \mathbf{0},} \\
& \boldsymbol{\Xi}_{f}+\boldsymbol{\Xi}_{\Delta \gamma}+\boldsymbol{\Xi}_{\Delta \boldsymbol{X}}+\boldsymbol{\Xi}_{\Delta \boldsymbol{k}_{d}}+\boldsymbol{\Xi}_{\Delta \boldsymbol{k}_{s}} \prec \mathbf{0} .
\end{aligned}
$$

b) Verifikationsschritt

Setze $\gamma=\gamma_{f}+\Delta \gamma, \boldsymbol{k}_{d}=\boldsymbol{k}_{d, f}+\Delta \boldsymbol{k}_{d}$ und $\boldsymbol{k}_{s}=\boldsymbol{k}_{s, f}+\Delta \boldsymbol{k}_{s}$ und überprüfe die Lösbarkeit von

$$
\text { finde } \boldsymbol{X} \text {, sodass } \boldsymbol{X} \succ \mathbf{0},(5.87 \mathrm{c}) \text {. }
$$

Falls das Problem lösbar ist, definiere die gefundene Lösung als $\left(\gamma_{f}, \boldsymbol{X}_{f}, \boldsymbol{k}_{d, f}, \boldsymbol{k}_{s, f}\right)$. Falls die Abbruchbedingung 3. nicht erfüllt ist, setze die neue Schrittweite auf $\zeta:=\eta^{+} \zeta$ und gehe zu 2.a). Falls das Problem nicht lösbar ist und die Abbruchbedingung 3. nicht erfüllt ist, setze $\zeta:=\eta^{-} \zeta$ und gehe zu 2.a) ohne die Variablen $\gamma_{f}, \boldsymbol{X}_{f}, \boldsymbol{k}_{d, f}$ und $\boldsymbol{k}_{s, f}$ zu verändern.

3. Abbruchkriterium

Beende den Algorithmus, falls eine gegebene Anzahl von Iterationen durchlaufen wurde oder die relative Verbesserung von $\gamma$ in zwei aufeinander folgenden Iterationen eine gegebene Schranke $\varepsilon$ unterschreitet. 
Bei der Lösung von (5.90) im Update-Schritt begrenzt die LMI (5.90c) wie in Abschnitt 5.4.3 erörtert und im Anhang in Lemma A.6 bewiesen die Abweichung vom Linearisierungspunkt. Ein tpyischer Wert für die Wahl des Parameters $\zeta$ zu Beginn des Algorithmus ist $\zeta=0,2$, für die Schrittweitenadaption wird häufig $\eta^{+}=1,1$ und $\eta^{-}=0,5$ gesetzt. Es sei erneut betont, dass es sich bei (5.87) um ein nichtkonvexes Optimierungsproblem handelt und somit die Konvergenz auf das globale Optimum nicht garantiert ist. Der Path-Following-Algorithmus gewährleistet jedoch die Konvergenz auf ein lokales Optimum [165]. Damit hängt die gefundene Lösung von den Startwerten $\boldsymbol{k}_{d \text {,init }}$ und $\boldsymbol{k}_{s, \text { init }}$ ab. Diese können zum Beispiel zu $\boldsymbol{k}_{d, \text { init }}=\mathbf{0}, \boldsymbol{k}_{s, \text { init }}=\mathbf{0}$ gewählt werden, wodurch die Störunterdrückung ausgehend vom vollständigen Fehlerisolationsbeobachter verbessert wird.

Bemerkung 5.12. Da für das Path-Following-Verfahren prinzipbedingt eine Taylorentwicklung von $\boldsymbol{\Xi}\left(\gamma, \boldsymbol{X}, \boldsymbol{k}_{d}, \boldsymbol{k}_{s}\right)$ in (5.88) berechnet werden muss, lässt sich der verfolgte Ansatz prinzipiell auch dann anwenden, wenn die Bedingung (5.75) nicht erfüllt ist und somit die Beobachtermatrix nach Lemma 5.1 nichtlinear von den dynamischen Kopplungszahlen abhängt. Dies wird jedoch hier nicht weiter verfolgt. Darüber hinaus sei angemerkt, dass sich im allgemeinen Fall nichtlineare Optimierungsverfahren wie zum Beispiel evolutionäre Algorithmen anwenden lassen, um die Robustheit durch direktes Lösen von (5.86) zu optimieren.

Bemerkung 5.13. Weiterhin lässt sich das beschriebene Verfahren auch dann anwenden, wenn auf das System Störungen wirken, die in einem beschränkten Frequenzbereich liegen. Dazu ist die Beobachterparametrierung $\left(\boldsymbol{L}_{\mathrm{pfi}}, \boldsymbol{V}_{\mathrm{pfi}}\right)$, die von den Kopplungszahlen $\boldsymbol{k}_{d}$ und $\boldsymbol{k}_{s}$ abhängt, in das Optimierungsproblem (5.30) einzusetzen. Mit dem gleichen Vorgehen wie in Abschnitt 5.4.3 lässt sich dann mithilfe des Path-Following-Verfahrens das Optimierungsproblem

$$
\underset{\boldsymbol{k}_{d}, \boldsymbol{k}_{s}}{\operatorname{minimiere}}\left\|\boldsymbol{G}_{\boldsymbol{r d}}\left(s, \boldsymbol{k}_{d}, \boldsymbol{k}_{s}\right)\right\|_{\infty}^{\Omega}
$$

iterativ lösen.

\subsubsection{Entwurfsverfahren auf Basis der Vollständigen Modalen Synthese}

Alternativ zum im vorangegangenen Abschnitt vorgestellten Verfahren lässt sich eine Robustheitsoptimierung auch mittels der Vollständigen Modalen Synthese durchführen. Das sich daraus ergebende Verfahren unter- 
liegt somit nicht der Einschränkung, dass die Beobachtermatrix linear von den dynamischen Kopplungszahlen abhängen muss.

Das Vorgehen weist große Gemeinsamkeiten zu Abschnitt 5.3.2 auf. Beim Entwurf der Beobachtermatrix $\boldsymbol{L}_{\text {pfi }}$ werden die sich aus der partiellen Fehlerisolation ergebenden zusätzlichen Freiheitsgrade dahingehend genutzt, die Anregung der Eigenbewegungen des Beobachters durch exogene Störungen zu minimieren. Bei Betrachtung der Partialbruchzerlegung von $\boldsymbol{G}_{\boldsymbol{r} \boldsymbol{d}}(s)$, die in (5.12) auf Seite 202 gegeben ist, lässt sich dies formulieren als Minimierung von $\left\|\boldsymbol{w}_{B_{i j}}^{\top}\left(\boldsymbol{B}_{d}-\boldsymbol{L}_{\mathrm{pfi}} \boldsymbol{D}_{d}\right)\right\|_{2}$.

Für alle Beobachtereigenwerte $\lambda_{B_{i j}}$, die mittels der dynamischen Kopplungszahlen $k_{d, i j l}$ in andere Spalten von $\boldsymbol{G}_{\boldsymbol{r} \boldsymbol{f}}(s)$ eingekoppelt werden, ist beim Entwurf folglich das Optimierungsproblem

$$
\begin{aligned}
& \underset{k_{d, i j l}, \boldsymbol{w}_{B_{i j}}^{\top} \boldsymbol{p}_{i j}^{\boldsymbol{\top}}}{\operatorname{minimiere}} \gamma_{i j}, \text { sodass } \\
& {\left[\begin{array}{cc}
\gamma_{i j} & \boldsymbol{w}_{B_{i j}}^{\top} \boldsymbol{B}_{d}+\boldsymbol{p}_{i j}^{\top} \boldsymbol{D}_{d} \\
\star & \boldsymbol{I}_{n_{d}}
\end{array}\right] \succ \mathbf{0},} \\
& {\left[\begin{array}{ll}
\boldsymbol{w}_{B_{i j}}^{\top} & \boldsymbol{p}_{i j}^{\top}
\end{array}\right]=\left[\begin{array}{ll}
\mathbf{0}^{\top} & \boldsymbol{\phi}_{i}^{\top}+\sum_{l=i+1}^{n_{f}} k_{d, i j l} \boldsymbol{\phi}_{l}^{\top}
\end{array}\right] \mathbf{\Pi}_{i j}^{-1}}
\end{aligned}
$$

zu lösen. Dabei handelt es sich um ein einfaches LMI-Problem. Während die erste Nebenbedingung die Norm von $\boldsymbol{w}_{B_{i j}}^{\top}\left(\boldsymbol{B}_{d}-\boldsymbol{L}_{\mathrm{pfi}} \boldsymbol{D}_{d}\right)$ beschränkt, gewährleistet die Gleichungsbedingung wie in Abschnitt 4.10 erörtert die Einhaltung der strukturellen Anforderungen bezüglich $\boldsymbol{G}_{\boldsymbol{r} \boldsymbol{f}}(s)$. Nachdem alle Parameter- und Linkseigenvektoren berechnet wurden, ergibt sich mit der bekannten Beziehung $\boldsymbol{L}_{\mathrm{pfi}}=-\boldsymbol{W}_{B, \mathrm{pfi}}^{-1} \boldsymbol{P}_{\mathrm{pfi}}$ die Beobachtermatrix $\boldsymbol{L}_{\mathrm{pfi}}$.

Anschließend erfolgt der Entwurf der Nachfiltermatrix. Wie schon in Abschnitt 5.3.2 lässt sich diese mithilfe des Bounded-Real-Lemmas berechnen. Es ergibt sich ein LMI-Problem, da die Matrix $\boldsymbol{L}_{\text {pfi }}$ bereits berechnet wurde und somit alle Variablen linear in das Problem eingehen. Zur Minimierung der $\mathcal{H}_{\infty}$-Norm von $\boldsymbol{G}_{\boldsymbol{r} \boldsymbol{d}}(s)$ ergibt sich das Optimierungsproblem

$$
\begin{aligned}
& \underset{\boldsymbol{X}, \boldsymbol{k}_{s}}{\operatorname{minimiere}} \gamma, \text { sodass } \\
& \boldsymbol{X} \succ \mathbf{0}, \\
& {\left[\begin{array}{ccc}
\mathrm{He}\left(\boldsymbol{X}\left(\boldsymbol{A}-\boldsymbol{L}_{\mathrm{pfi}} \boldsymbol{C}\right)\right) & \boldsymbol{X}\left(\boldsymbol{B}_{d}-\boldsymbol{L}_{\mathrm{pfi}} \boldsymbol{D}_{d}\right) & \left(\boldsymbol{V}_{\mathrm{pfi}} \boldsymbol{C}\right)^{\top} \\
\star & -\gamma \boldsymbol{I}_{n_{d}} & \left(\boldsymbol{V}_{\mathrm{pfi}} \boldsymbol{D}_{d}\right)^{\top} \\
\star & \star & -\gamma \boldsymbol{I}_{n_{f}}
\end{array}\right] \prec \mathbf{0},} \\
& -\boldsymbol{V}_{\mathrm{pfi}} \boldsymbol{\Theta}_{\mathrm{pfi}}=\boldsymbol{S}_{\mathrm{pfi}}\left(\boldsymbol{k}_{s}\right) .
\end{aligned}
$$


Während die ersten beiden Bedingungen vom Bounded-Real-Lemma (Satz 3.1) herrühren, wird durch die Gleichungsbedingung wie in Abschnitt 4.10 beschrieben die obere Dreiecksstruktur von $\boldsymbol{G}_{\boldsymbol{r} \boldsymbol{f}}(s)$ sichergestellt. Dabei ist $\boldsymbol{\Theta}_{\mathrm{pfi}}=\boldsymbol{C}\left(\boldsymbol{A}-\boldsymbol{L}_{\mathrm{pfi}} \boldsymbol{C}\right)^{-1}\left(\boldsymbol{E}_{a}-\boldsymbol{L}_{\mathrm{pfi}} \boldsymbol{E}_{s}\right)-\boldsymbol{E}_{s}$ und die Matrix $\boldsymbol{S}_{\mathrm{pfi}}\left(\boldsymbol{k}_{s}\right)$ hängt wie in (4.103) eingeführt linear von den statischen Kopplungszahlen $k_{s, i j}$ ab.

Im Vergleich zum Verfahren aus Abschnitt 5.5.2 lässt sich mithilfe der einfachen LMI-Probleme (5.91) und (5.92) die Robustheit der Residuengenerierung hinsichtlich exogener Störungen wesentlich einfacher optimieren. Der zweite wesentliche Vorteil des Verfahrens ist wie bereits erwähnt, dass keine Einschränkung bezüglich der Abhängigkeit der Beobachtermatrix von den dynamischen Kopplungszahlen besteht.

Als Nachteil gegenüber dem Path-Following-Verfahren aus Abschnitt 5.5.2 ist die sequentielle Optimierung von $\boldsymbol{L}_{\mathrm{pfi}}$ und $\boldsymbol{V}_{\mathrm{pfi}} \mathrm{zu}$ erwähnen. Diese führt dazu, dass das Lösen von (5.91) und (5.92) nicht notwendigerweise auf ein lokales Optimum hinsichtlich (5.86) führt. Die nachfolgenden Beispiele zeigen jedoch, dass sich mit dem Verfahren trotz des einfachen, sequentiellen Entwurfs gute Ergebnisse erzielen lassen.

Bemerkung 5.14. Wie bereits erwähnt sind die Fehler derart zu sortieren, dass sich eine absteigende Reihenfolge der Fehlerindizes ergibt, da somit die Anzahl der verfügbaren Freiheitsgrade maximiert wird. Weist ein System Indizes $\delta_{i}=\delta_{j}$ für $i \neq j$ auf, so ist mit den beiden vorgestellten Verfahren keine Aussage darüber möglich, welche Sortierung der Fehler zu einem besseren Ergebnis hinsichtlich der $\mathcal{H}_{\infty}$-Norm von $\boldsymbol{G}_{\boldsymbol{r} \boldsymbol{d}}(s)$ führt. Es bietet sich an, die entsprechenden Optimierungsprobleme jeweils für die unterschiedlichen Fehlerpermutationen zu lösen und diejenige PFIOParametrierung auszuwählen, welche auf das beste Ergebnis führt.

Bemerkung 5.15. Das Verfahren auf Basis der Vollständigen Modalen Synthese lässt sich mit dem Vorgehen bei der Relaxierung der strukturellen Anforderungen aus Abschnitt 5.3.2 kombinieren. Fordert man ähnlich wie dort nicht die exakte Erfüllung von (5.91c) und (5.92d), so ergeben sich zusätzliche Freiheitsgrade für die Robustheitssteigerung. Diese wird dann auf Kosten einer nicht mehr exakten Dreiecksstruktur von $\boldsymbol{G}_{\boldsymbol{r} \boldsymbol{f}}(s)$ erreicht.

\subsubsection{Akademisches Beispiel}

Zunächst wird ein akademisches Beispielsystem betrachtet. Hintergrund ist, dass dieses System lediglich über eine dynamische und eine statische Kopplungszahl verfügt. Dadurch lässt sich $\left\|\boldsymbol{G}_{\boldsymbol{r} \boldsymbol{d}}\left(s, \boldsymbol{k}_{d}, \boldsymbol{k}_{s}\right)\right\|_{\infty}$ grafisch dar- 
stellen, sodass die Qualität der von den beiden Optimierungsverfahren gefundenen Lösungen hinsichtlich der Störunterdrückung beurteilt werden kann.

Das System wird beschrieben durch

$$
\begin{aligned}
\boldsymbol{A} & =\left[\begin{array}{ll}
-1 & -4 \\
0,5 & -2
\end{array}\right], & \boldsymbol{E}_{a}=\left[\begin{array}{rr}
1 & -10 \\
-1 & 3
\end{array}\right], \\
\boldsymbol{C} & =\left[\begin{array}{rr}
5 & 1 \\
-1 & 2
\end{array}\right], & \boldsymbol{E}_{s}=\mathbf{0} \\
\boldsymbol{B}_{d} & =\left[\begin{array}{rr}
0,2 & 0,3 \\
-0,7 & 0,4
\end{array}\right], & \boldsymbol{D}_{d}=\left[\begin{array}{rr}
-0,3 & -0,1 \\
-0,45 & 0,6
\end{array}\right] .
\end{aligned}
$$

Es ist stabil und erfüllt bei $\delta_{1}=\delta_{2}=1$ die Annahmen 5.1 bis 5.3. Somit lässt sich nach Abschnitt 4.5 ein FIO zur vollständigen Fehlerisolation entwerfen, der für die beiden Übertragungskanäle die Pole $\lambda_{B_{1,1}}=-8$ und $\lambda_{B_{2,1}}=-9$ vorgibt, sowie $\boldsymbol{G}_{\boldsymbol{r} \boldsymbol{f}}(s=0)=\boldsymbol{I}_{2}$ gewährleistet. Die Analyse der $\mathcal{H}_{\infty}$-Norm von $\boldsymbol{G}_{\boldsymbol{r} \boldsymbol{d}}(s)$ für den ermittelten FFIO ergibt einen Wert von $\left\|\boldsymbol{G}_{\boldsymbol{r} \boldsymbol{d}}(s)\right\|_{\infty}<3,881$.

Soll lediglich eine partielle Fehlerisolation durchgeführt werden, so kann mittels der dynamischen Kopplungszahl $k_{d, 112}$ der Pol des ersten Übertragungskanals auch in die zweite Spalte von $\boldsymbol{G}_{\boldsymbol{r} \boldsymbol{f}}(s)$ eingekoppelt werden. Darüber hinaus kann über die statische Kopplungszahl $k_{s, 12}$ Einfluss auf die stationäre Verstärkung von $\boldsymbol{G}_{\boldsymbol{r} \boldsymbol{f}}(s)$ genommen werden.

Beim auf der Vollständigen Modalen Synthese basierenden Verfahren aus Abschnitt 5.5.3 ist das Optimierungsproblem (5.91) lediglich ein Mal (für den Eigenwert $\lambda_{B_{1,1}}$ ) zu lösen, um die Beobachtermatrix $\boldsymbol{L}_{\mathrm{pfi}, \mathrm{VMS}} \mathrm{zu}$ bestimmen. Es ergibt sich ein Wert von $k_{d, 112}=31,171$. Anschließend wird durch Lösen von (5.92) die statische Kopplungszahl $k_{s, 12}=-6,314$ ermittelt. Für die PFIO-Parametrierung $\left(\boldsymbol{L}_{\mathrm{pfi}, \mathrm{VMS}}, \boldsymbol{V}_{\mathrm{pfi}, \mathrm{VMS}}\right)$ ergibt sich eine obere Schranke für $\left\|\boldsymbol{G}_{\boldsymbol{r} \boldsymbol{d}}(s)\right\|_{\infty}$ von 0,544 , was eine signifikante Ver-

Tabelle 5.1: Vergleich der Lösungen zur partiellen Fehlerisolation

\begin{tabular}{c||c|c|c} 
& $k_{d, 112}$ & $k_{s, 12}$ & $\left\|\boldsymbol{G}_{\boldsymbol{r} \boldsymbol{d}}(s)\right\|_{\infty}$ \\
\hline \hline FFIO & 0 & 0 & 3,881 \\
\hline PFIO VMS, Abschnitt 5.5.3 & 31,171 & $-6,314$ & 0,544 \\
\hline PFIO PF, Abschnitt 5.5.2 & 21,321 & $-7,198$ & 0,526
\end{tabular}


besserung gegenüber dem Fall der vollständigen Fehlerisolation darstellt.

Weiterhin erfüllt das System offensichtlich die Bedingungen aus Lemma 5.2. Somit kann die ermittelte PFIO-Parametrierung als initiale Parametrierung zur weiteren Verbesserung des Ergebnisses mithilfe des PathFollowing-Ansatzes aus Abschnitt 5.5.2 herangezogen werden. Mit einer initialen Schrittweite von $\zeta=0,2$ und den Schrittweitensteuerungsparametern $\eta^{+}=1,1$ und $\eta^{-}=0,5$ liefert der Algorithmus $k_{d, 112}=21,321$ und $k_{s, 12}=-7,198$, woraus sich $\left\|\boldsymbol{G}_{\boldsymbol{r} \boldsymbol{d}}(s)\right\|_{\infty}<0,526$ ergibt. Zum besseren Vergleich sind die Zahlenwerte in Tabelle 5.1 zusammengefasst, die jeweiligen Beobachterparametrierungen finden sich in Anhang C.2.6.

Obwohl anhand der Abschätzungen für die $\mathcal{H}_{\infty}$-Norm von $\boldsymbol{G}_{\boldsymbol{r} \boldsymbol{d}}(s)$ ersichtlich ist, dass die Störunterdrückung bei teilweiser Fehlerisolation sehr viel besser ist als bei vollständiger Fehlerisolation, ist aufgrund der Nichtkonvexität des Problems keine Aussage darüber möglich, ob das globale Optimum hinsichtlich $k_{d, 112}$ und $k_{s, 12}$ gefunden wurde. An dieser Stelle

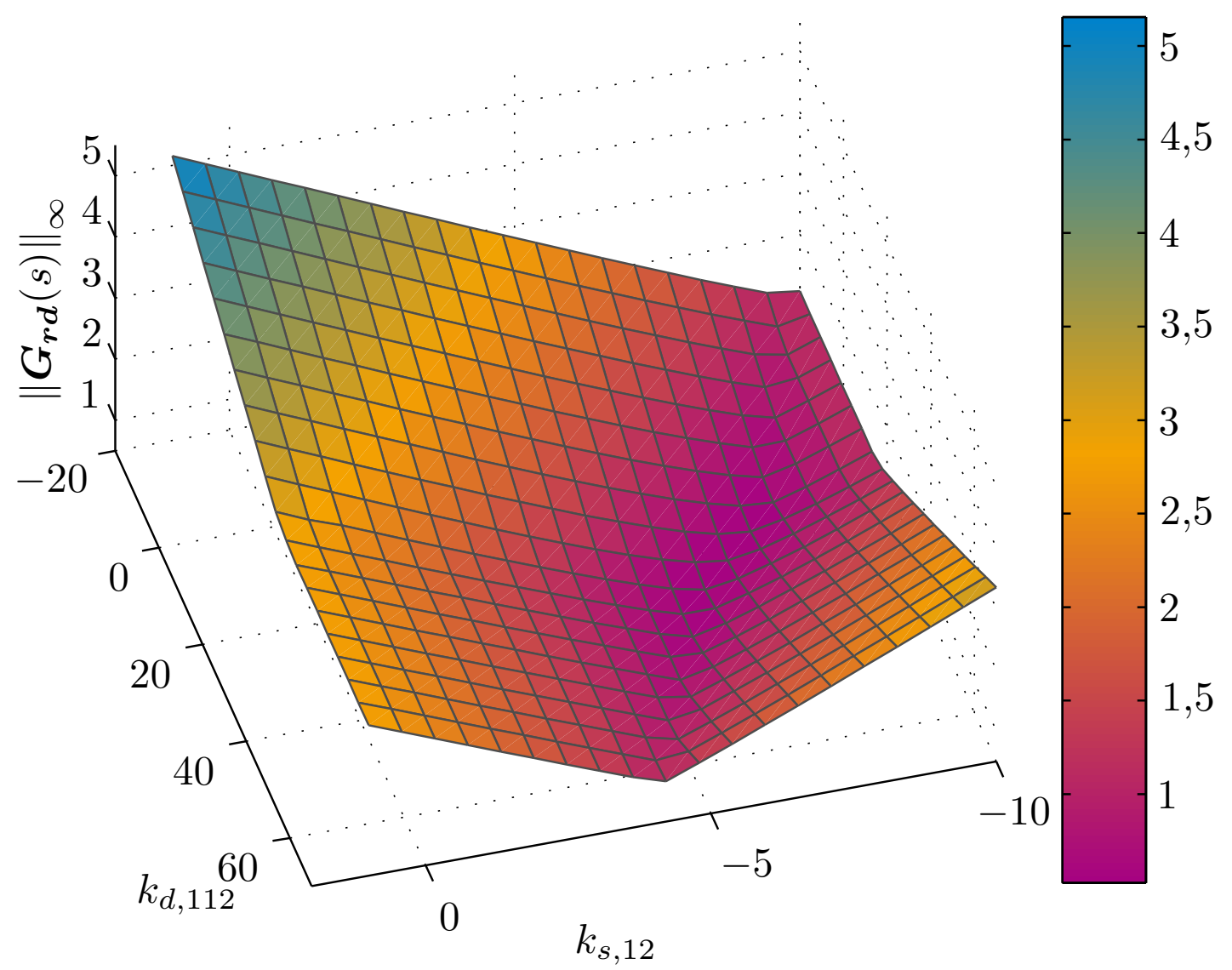

Abbildung 5.21: Abhängigkeit von $\left\|\boldsymbol{G}_{\boldsymbol{r} \boldsymbol{d}}(s)\right\|_{\infty}$ von $k_{d, 112}$ und $k_{s, 12}$ 
lässt sich die Qualität der Ergebnisse jedoch anhand von Abbildung 5.21 einordnen.

Sie zeigt die Abschätzung von $\left\|\boldsymbol{G}_{\boldsymbol{r} \boldsymbol{d}}(s)\right\|_{\infty}$ grafisch über den beiden Kopplungszahlen. Dazu wurde die Norm über ein hinreichend feines Gitter abgeschätzt. Offensichtlich findet der Path-Following-Algorithmus in diesem Beispiel eine Lösung, die dem globalen Optimum sehr nahe kommt. Auch die in der Umsetzung deutlich einfachere und schnellere Optimierung auf Basis der Vollständigen Modalen Synthese findet eine Parametrierung, die kaum schlechtere Ergebnisse liefert.

\subsubsection{Beispiel Verladebrücke}

Um die Umsetzung der entwickelten Verfahren zur robusten partiellen Fehlerisolation zu demonstrieren, wird das Beispiel der Verladebrücke aus Abschnitt 4.10.3 (s. Seite 181) fortgeführt.

Die Störeingangsmatrizen $\boldsymbol{B}_{d}$ und $\boldsymbol{D}_{d}$ entsprechen denen aus Abschnitt 4.10.3. Am Prüfstand werden die unterschiedlichen Rauschamplituden der beiden Sensoren emuliert, indem das Messsignal des Positionssensors mit einem stärkeren Rauschsignal überlagert wird als das des Seilwinkelsensors.

Mit dem Ansatz auf Basis der Vollständigen Modalen Synthese aus Abschnitt 5.5.3 ergibt sich für die optimierten dynamischen Kopplungsparameter $k_{d, 112}=3,410$ und $k_{d, 122}=4,327$ und für die statische Kopplungszahl $k_{s, 12}=-1,610$. Dies führt auf eine obere Schranke für die Störverstärkung von $\gamma_{\text {pfi,VMS }}=7,300$. Gegenüber der vollständigen Fehlerisolation $\left(\left\|\boldsymbol{G}_{\boldsymbol{r} \boldsymbol{d}}(s)\right\|_{\infty}<9,819\right)$ stellt dies bereits eine deutliche Verbesserung von $25,7 \%$ dar, was auch aus dem Verlauf des größten Singulärwertes (vgl. Abbildung 5.22) hervorgeht.

Auch der Path-Following-Ansatz lässt sich in der vorgestellten Form anwenden. Da $n_{f}=2$ und $\delta_{1}=\delta_{2}=2$ sind, sind gemäß Satz 5.6 die Fälle

$$
\begin{array}{llllrl}
i=1, & q=1, & j=1, & r=1, & l=2, \\
i=1, & q=1, & j=1, & r=2, & l=2, \\
i=1, & q=1, & j=2, & r=1, & l=2, \\
i=1, & q=1, & j=2, & r=2, & l=2
\end{array}
$$

für (5.75) zu überprüfen. Es ergibt sich jeweils

$$
\phi_{l}^{\top} \boldsymbol{G}_{\boldsymbol{y} \boldsymbol{f}}^{-1}\left(\lambda_{B_{i j}}\right) \cdot \boldsymbol{C}\left(\lambda_{B_{i j}} \boldsymbol{I}_{n}-\boldsymbol{A}\right)^{-1} \boldsymbol{v}_{B, \mathrm{ffi}, q r}=0,
$$




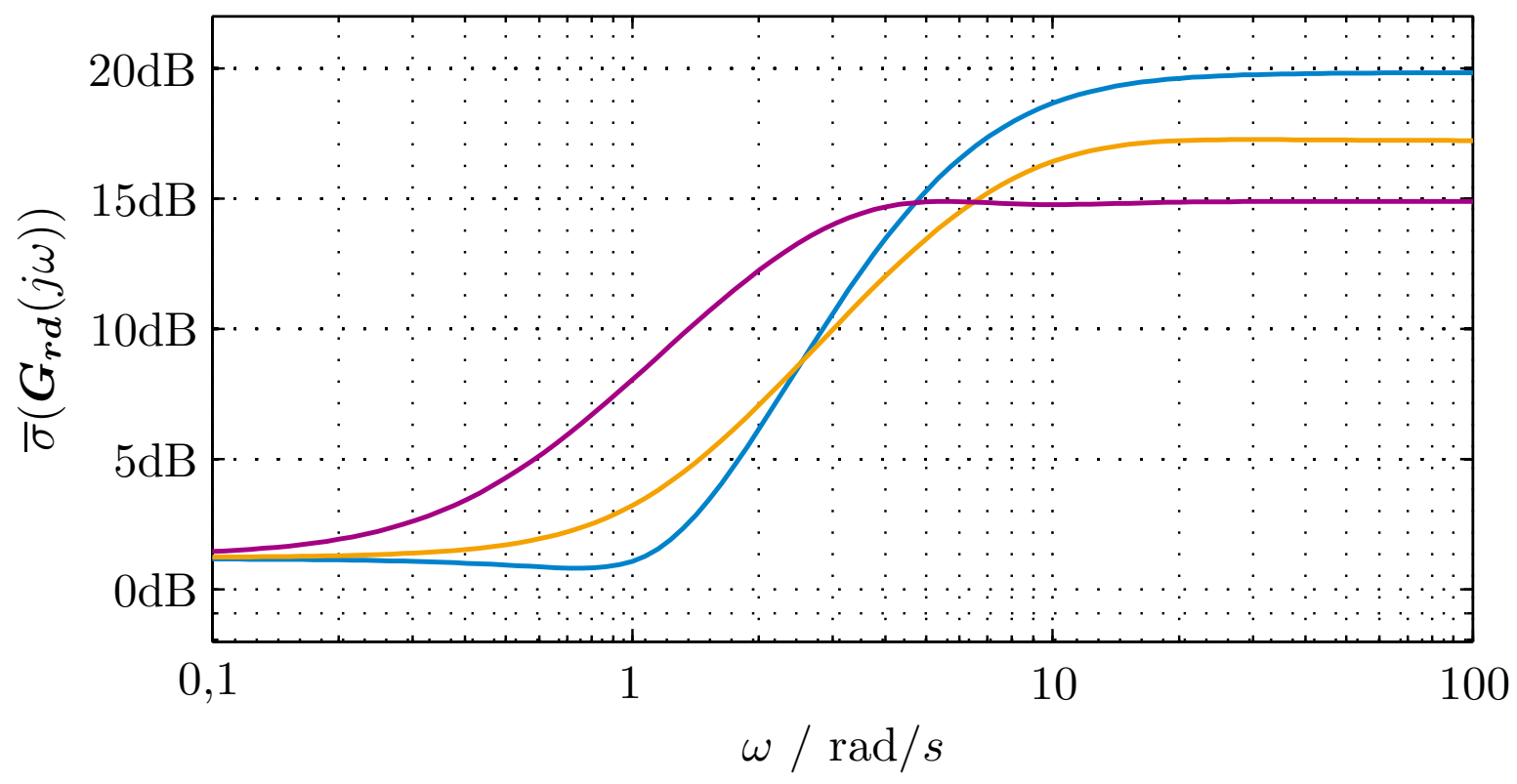

Abbildung 5.22: Verlauf des größten Singulärwertes von $\boldsymbol{G}_{\boldsymbol{r} \boldsymbol{d}}(s)$ für FFIO (-), PFIO mittels VMS (-) und PFIO mittels Path-Following (-)

sodass die affine Abhängigkeit der Matrix $\boldsymbol{L}_{\mathrm{pfi}}$ von den dynamischen Kopplungszahlen $\boldsymbol{k}_{d}$ gewährleistet ist.

Mithilfe des Path-Following-Ansatzes lässt sich nun die obere Schranke für die Störverstärkung weiter senken. In der Initialisierung wird mithilfe des Bounded-Real-Lemmas für die initiale Beobachterparametrierung eine Schranke $\gamma_{\mathrm{pfi}, \mathrm{PF}}$ sowie eine zugehörige Matrix $\boldsymbol{X}$ berechnet. Ausgehend von der zuvor auf Basis der Vollständigen Modalen Synthese ermittelten Parametrierung lässt sich die Störunterdrückung innerhalb von 13 Iterationen auf $\gamma_{\mathrm{pfi}, \mathrm{PF}}=5,554$ verbessern (vgl. Abbildung 5.22). Dies stellt gegenüber der vollständigen Fehlerisolation eine Verbesserung von 43,4\% dar. Dabei wurden als Parameter des Algorithmus erneut $\zeta=0,2$, $\eta^{+}=1,1$ und $\eta^{-}=0,5$ gewählt und es ergeben sich die Parameter $k_{d, 112}=-29,695, k_{d, 122}=-57,735$ und $k_{s, 12}=-3,980$. Die Tatsache, dass sich die Kopplungszahlen der beiden Lösungsansätze deutlich unterscheiden, stellt keinen Widerspruch dar. Wie im vorangegangenen Beispiel gezeigt wurde, kann es durchaus stark differierende Parametrierungen geben, die auf ähnlich gute Lösungen hinsichtlich $\left\|\boldsymbol{G}_{\boldsymbol{r} \boldsymbol{d}}(s)\right\|_{\infty}$ führen. Dies wird auch anhand der Zahlenwerte, die sich für die einzelnen Beobachterparametrierungen ergeben, deutlich. Sie finden sich in Anhang C.2.6 und unterscheiden sich deutlich weniger, als es die unterschiedlichen Kopplungszahlen vermuten lassen. 


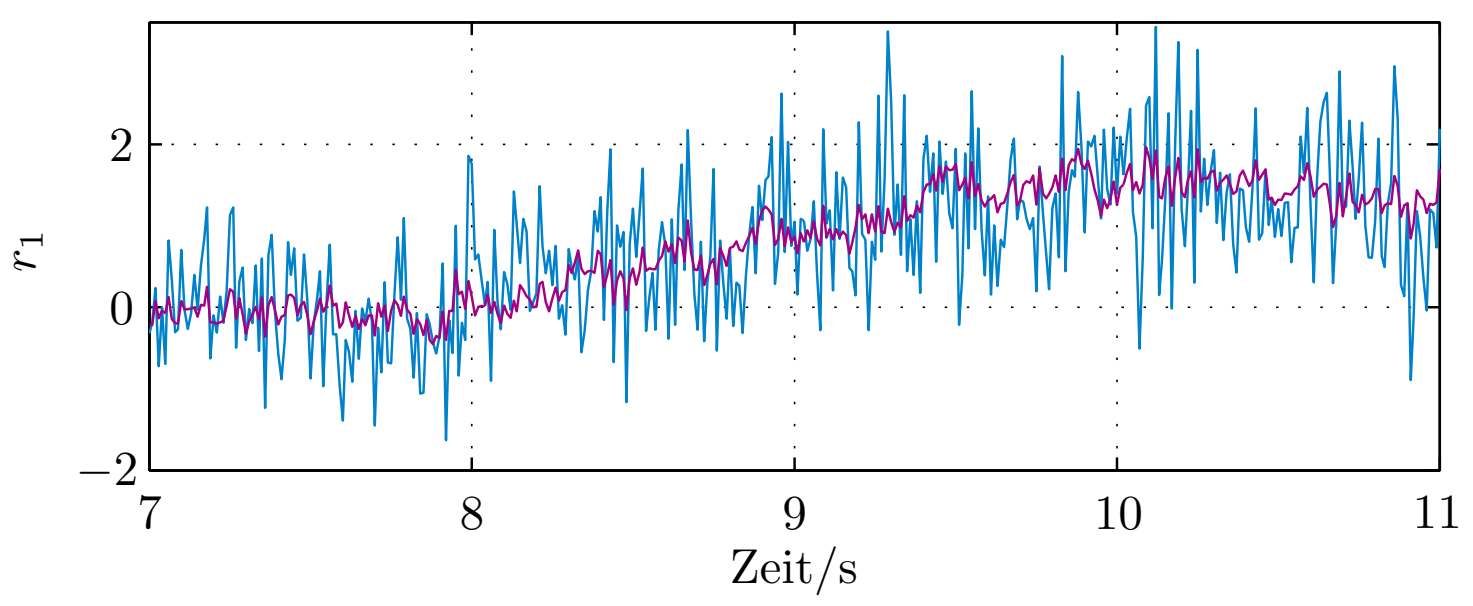

(a) Residuen des 1. Fehlers bei partieller Fehlerisolation

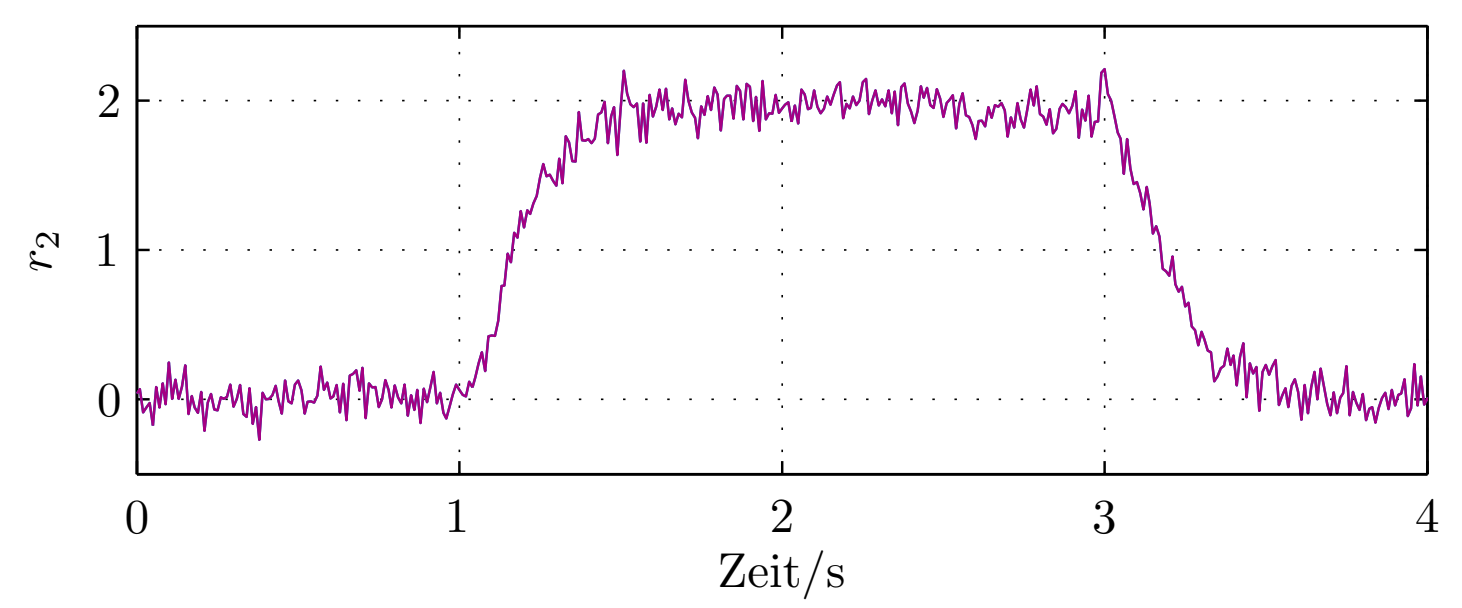

(b) Residuen des 2. Fehlers bei partieller Fehlerisolation

Abbildung 5.23: Vergleich der Residuen bei vollständiger (-) und teilweiser (-) Fehlerisolation

Am Prüfstand wird nach $t=1$ s ein Fehler in die Aktorik eingebracht. Zu diesem Zeitpunkt ändert sich der zweite Fehler sprungförmig auf $f_{1}=2$. Nach etwa 8s wird manuell eine rampenförmige horizontale Kraft auf die Lastmasse ausgeübt. Abbildung 5.23 zeigt die generierten Residuensignale der unterschiedlichen Beobachter, die in Echtzeit aus den gemessen Daten am Prüfstand erzeugt wurde. Der Übersichtlichkeit halber sind lediglich der Residuenverläufe des Beobachters zur vollständigen Fehlerisolation und der mittels des Path-Following-Verfahrens optimierten Lösung gezeigt. $\mathrm{Zu}$ beachten sind die unterschiedlichen Zeitskalen von Abbildung 5.23a und 5.23b, aus denen hervorgeht, dass die beiden Fehler nicht gleichzeitig auftreten. 
Das Residuum $r_{2}$ in Abbildung 5.23b weist für beide Beobachter einen nahezu deckungsgleichen Verlauf auf. Jedoch schlägt das Residuum $r_{1}$ des PFIOs ebenfalls aus (dies ist aufgrund der angepassten Zeitachse in Abbildung 5.23a nicht zu sehen). Ein wesentlicher Unterschied zeigt sich im Residuum $r_{1}$. Wie aus Abbildung 5.23a hervorgeht, ist es mittels des FFIOs ohne zusätzliche Filterungsmaßnahmen nicht möglich, den Fehler $f_{1}$ zu erkennen beziehungsweise zu isolieren, da das erzeugte Residuum zu stark verrauscht ist. Mit dem PFIO ergibt sich ein wesentlich weniger verrauschtes Residuensignal, mit dessen Hilfe eine Fehlerisolation möglich ist.

\subsubsection{Fazit}

In diesem Abschnitt wurde gezeigt, wie sich die gegenüber der vollständigen Fehlerisolation weniger restriktiven strukturellen Anforderungen der partiellen Fehlerisolation zur Steigerung der Robustheit der Residuengenerierung nutzen lassen. Dazu wurden zwei unterschiedliche Verfahren vorgestellt. Der Path-Following-Ansatz (Abschnitt 5.5.2) optimiert $\boldsymbol{L}_{\mathrm{pfi}}$ und $\boldsymbol{V}_{\mathrm{pfi}}$ simultan und garantiert die Konvergenz auf ein lokales Minimum bezüglich $\left\|\boldsymbol{G}_{\boldsymbol{r} \boldsymbol{d}}(s)\right\|_{\infty}$. In der vorgestellten Form ist er auf Systeme beschränkt, deren Matrix $\boldsymbol{L}_{\text {pfi }}$ affin von den dynamischen Kopplungszahlen $d_{d, i j l}$ abhängt, prinzipiell lässt er sich jedoch allgemeiner anwenden. Dann ergeben sich allerdings komplizierte Formeln aus der Linearisierung, was die Anwendung erschwert. Wesentlich einfacher gestaltet sich in dieser Hinsicht der Ansatz auf Basis der Vollständigen Modalen Synthese (Abschnitt 5.5.3). Er unterliegt hinsichtlich der Abhängigkeit der Matrix $\boldsymbol{L}_{\mathrm{pfi}}$ von den dynamischen Kopplungszahlen keinerlei Einschränkungen und führt auf einfache LMI-Probleme. Allerdings werden $\boldsymbol{L}_{\mathrm{pfi}}$ und $\boldsymbol{V}_{\mathrm{pfi}}$ sequentiell optimiert, wodurch nicht gewährleistet ist, dass ein Optimum hinsichtlich $\left\|\boldsymbol{G}_{\boldsymbol{r d}}(s)\right\|_{\infty}$ erreicht wird.

\subsection{Zusammenfassung des Kapitels}

In diesem Kapitel wurden verschiedene Möglichkeiten zur Steigerung der Robustheit von Fehlerisolationsbeobachtern vorgestellt. Die behandelten Aspekte sind in Tabelle 5.2 zusammengefasst. Sie betreffen sowohl die vollständige Fehlerisolation in quadratischen und nichtquadratischen Systemen sowie die teilweise Fehlerisolation. Dabei wurden Verfahren zur Unterdrückung beliebiger exogener Störungen, hochfrequenter Störungen, 
Tabelle 5.2: Übersicht der behandelten Robustheitsaspekte (FFI q: vollständige Fehlerisolation in quadratischen Systemen, FFI nq: vollständige Fehlerisolation in nichtquadratischen Systemen, PFI q: teilweise Fehlerisolation in quadratischen Systemen)

\begin{tabular}{c||c|c|c} 
& FFI q & FFI nq & PFI q \\
\hline \hline $\boldsymbol{d}$ beliebig & 5.3 .1 & 5.4 .1 & 5.5 \\
& 5.3 .2 & 5.4 .2 & \\
\hline $\boldsymbol{d}$ hochfrequent & 5.3 .3 & & \\
\hline $\boldsymbol{d}$ beschränkt & & 5.4 .3 & \\
\hline unsichere & & 5.4 .4 & \\
Parameter & & &
\end{tabular}

Störungen in allgemeinen beschränkten Frequenzbereichen sowie zur Steigerung der Robustheit bezüglich unsicherer Parameter erarbeitet.

Wie bereits in den jeweiligen Abschnitten angedeutet lassen sich die Resultate zum Teil miteinander verbinden. So ist zum Beispiel eine Relaxierung der strukturellen Anforderungen in nichtquadratischen Systemen ebenso denkbar wie die partielle Fehlerisolation für nichtquadratische Systeme.

In Verbindung mit den Ergebnissen aus Kapitel 4 lässt sich daher festhalten, dass mit FIOs ein vielseitiger und flexibler Ansatz gegeben ist, der nicht nur den Entwurf von Fehlerisolationssystemen niedriger Ordnung für eine große Klasse linearer Systeme ermöglicht, sondern auch auf vielfältige Art und Weise die Steigerung der Robustheit der generierten Residuen erlaubt. Dies geschieht jedoch durch unterschiedliche Entwürfe, ein einheitliches Vorgehen ist nicht gegeben. 


\section{Allgemeine Fehlerisolationsfilter}

In den Kapiteln 4 und 5 wurden zur Generierung der Residuen stets Beobachterstrukturen herangezogen. Die im Bereich der Fehlerdiagnose am häufigsten verwendete Struktur eines vollständigen, statischen Beobachters,

$$
\begin{aligned}
\dot{\hat{\boldsymbol{x}}} & =\boldsymbol{A} \hat{\boldsymbol{x}}+\boldsymbol{B} \boldsymbol{u}+\boldsymbol{L}(\boldsymbol{y}-\boldsymbol{C} \hat{\boldsymbol{x}}-\boldsymbol{D} \boldsymbol{u}), \\
\boldsymbol{r} & =\boldsymbol{V}(\boldsymbol{y}-\boldsymbol{C} \hat{\boldsymbol{x}}-\boldsymbol{D u}),
\end{aligned}
$$

ermöglicht dabei zahlreiche Resultate, die überwiegend auf der in Abschnitt 4.4 herausgearbeiteten Dualität zu Entkopplungsregelungen basieren. Jedoch zeigen die Betrachtungen in Abschnitt 5.4.4, dass diese Dualität lediglich mathematischer Natur ist und nur für die nominale Systemdynamik Gültigkeit besitzt. Darüber hinaus ist anzumerken, dass die Wahl eines Beobachters strukturelle Einschränkungen mit sich bringt. Zwar lassen sich die Eigenwerte der Beobachterdynamik - vollständige Beobachtbarkeit vorausgesetzt - beliebig platzieren, die Dynamikmatrix des Beobachters ergibt sich jedoch stets zu $\boldsymbol{A}-\boldsymbol{L} \boldsymbol{C}$.

In diesem Kapitel wird eine alternative Struktur herangezogen. Grundgedanke ist es, für die Erzeugung der Residuen eine allgemeine Filterstruktur der Form

$$
\begin{aligned}
\dot{\boldsymbol{x}}_{f} & =\boldsymbol{A}_{f} \boldsymbol{x}_{f}+\boldsymbol{B}_{f, u} \boldsymbol{u}+\boldsymbol{B}_{f, y} \boldsymbol{y}, \\
\boldsymbol{r} & =\boldsymbol{C}_{f} \boldsymbol{x}_{f}+\boldsymbol{D}_{f, u} \boldsymbol{u}+\boldsymbol{D}_{f, y} \boldsymbol{y}
\end{aligned}
$$

zu verwenden. Dabei sind die Matrizen $\boldsymbol{A}_{f}, \boldsymbol{B}_{f, u}, \boldsymbol{B}_{f, y}, \boldsymbol{C}_{f}, \boldsymbol{D}_{f, u}$ und $\boldsymbol{D}_{f, y}$ prinzipiell frei wählbar, ebenso wie die Ordnung des Filters, die sich aus $\boldsymbol{x}_{f} \in \mathbb{R}^{n_{\text {filt }}}$ ergibt. Die Struktur von (6.1) erklärt nun, warum in Definition 2.3 Residuen ganz allgemein als Fehlerindikatoren eingeführt wurden: In (6.1) werden sie offensichtlich nicht wie bei FIOs aus der Differenz gemessener und geschätzter Ausgangssignale erzeugt, dennoch werden sie Fehlerindikatoren herangezogen. 
Die Gleichungen (6.1) stellen die allgemeinste mögliche Struktur eines linearen dynamischen Filters dar. $\mathrm{Zu}$ beachten ist, dass nicht nur die Filtereigenwerte beliebig platziert werden können, sondern dass die gesamte Matrix $\boldsymbol{A}_{f}$ ebenso frei vorgebbar ist wie die übrigen Filtermatrizen. Zwar sind aus der Beobachterstruktur zahlreiche interessante Ergebnisse hervorgegangen, allerdings besteht intuitiv keine Notwendigkeit, sich durch die Verwendung eines Beobachters strukturellen Beschränkungen zu unterwerfen. In Abgrenzung zu Fehlerisolationsbeobachtern werden allgemeine Fehlerisolationsfilter wie folgt definiert.

Definition 6.1 (Allgemeine Fehlerisolationsfilter). Ein allgemeines dynamisches Filter der Form (6.1), welches in Verbindung mit der Strecke

$$
\begin{aligned}
& \dot{\boldsymbol{x}}=\boldsymbol{A} \boldsymbol{x}+\boldsymbol{B} \boldsymbol{u}+\boldsymbol{E}_{a} \boldsymbol{f} \\
& \boldsymbol{y}=\boldsymbol{C} \boldsymbol{x}+\boldsymbol{D} \boldsymbol{u}+\boldsymbol{E}_{s} \boldsymbol{f}
\end{aligned}
$$

zu einer diagonalen Übertragungsmatrix $\boldsymbol{G}_{\boldsymbol{r} \boldsymbol{f}}(s)$ und darüber hinaus zu $\boldsymbol{G}_{\boldsymbol{r u}}(s)=\mathbf{0}$ führt, wird als allgemeines Fehlerisolationsfilter (abkürzend Fehlerisolationsfilter, FIF) bezeichnet.

Dabei ist zu beachten, dass bei FIFs der Einfluss der Stellgrößen über die Übertragungsmatrix $\boldsymbol{G}_{\boldsymbol{r} \boldsymbol{u}}(s)$ berücksichtigt werden muss. Dies ist bei FIOs nicht der Fall, da hier gemäß der Beobachterfehlerdynamik (4.4) von Seite 70 die Forderung $\boldsymbol{G}_{\boldsymbol{r u}}(s)=\mathbf{0}$ für die nominale Systemdynamik strukturbedingt immer erfüllt ist.

Weiterhin sei angemerkt, dass der Begriff des Fehlerisolationsfilters in der Literatur nicht einheitlich definiert ist. In [133] werden beispielsweise Beobachter, die zur Fehlerisolation herangezogen werden, als Fehlerisolationsfilter bezeichnet. Demgegenüber erfolgt in dieser Arbeit anhand der Struktur eine klare Trennung zwischen FIOs (Definition 4.1, s. Seite 71) und FIFs (Definition 6.1).

\subsection{Stand der Technik und Beiträge dieses Kapitels}

Zunächst ist zu erwähnen, dass sich alle Arten von linearen modellbasierten Fehlerisolationssystemen im Frequenzbereich in der Form

$$
\boldsymbol{r}(s)=\left[\begin{array}{ll}
\boldsymbol{R}_{\boldsymbol{u}}(s) & \boldsymbol{R}_{\boldsymbol{y}}(s)
\end{array}\right] \cdot\left[\begin{array}{l}
\boldsymbol{u}(s) \\
\boldsymbol{y}(s)
\end{array}\right]
$$


darstellen lassen [58]. In diese Darstellung lassen sich neben FIOs auch klassische Ansätze wie Bänke von UIOs (vgl. Abschnitt 2.4) und paritätsbasierte Ansätze [58, Abschnitt 13.3] überführen. Für (6.3) lässt sich dann stets eine Zustandsraumrealisierung der Form (6.1) finden. Betrachtet werden an dieser Stelle jedoch nur Ansätze, welche von Filtern der Form (6.1) ausgehen und die sich mit der Filterparametrierung im Sinne der Fehlerisolation befassen.

In der Literatur finden sich nur wenige Resultate zu derartigen Fehlerisolationsfiltern. Zwar wurde bereits in [146] die Struktur eines allgemeinen Filters für die Fehlerisolation vorgeschlagen und in Anlehnung an [147] wird ein geometrisches Entwurfsverfahren zur Parametrierung der Filtermatrizen angegeben. Dabei werden jedoch keine Robustheitsaspekte behandelt. Die Idee des LMI-basierten Entwurfs allgemeiner Filter in der Fehlerdiagnose, die auch in dieser Arbeit verfolgt wird, geht zurück auf [148, 149]. Die Ergebnisse erlauben zwar die Berücksichtigung der Robustheit sowohl hinsichtlich exogener Störungen als auch unsicherer Parameter, sie beschränken sich jedoch im Wesentlichen auf eine reine Fehlerdetektion. Fehlerisolationsprobleme werden nicht systematisch behandelt.

In einer solchen systematischen Betrachtung des Fehlerisolationsproblems mittels allgemeiner dynamischer Filter ist der Hauptbeitrag dieses Kapitels zu sehen. Die Neuerungen, die in Auszügen bereits in [225, 226] veröffentlicht wurden, unterteilen sich in die folgenden Punkte.

1. Systematisches Entwurfsverfahren für FIFs niedriger Ordnung. Es wird eine Verbindung zwischen FIOs und FIFs hergestellt. Die Ergebnisse zu FIOs aus Kapitel 4 erlauben dann den systematischen Entwurf von FIFs niedriger Ordnung. Hervorzuheben ist, dass ein vereinheitlichendes Entwurfsverfahren sowohl für quadratische und nichtquadratische als auch für statisch nicht isolierbare und nicht minimalphasige Systeme erarbeitet wird. Darüber hinaus lassen sich die Filtereigenwerte auf vorgegebene Bereiche einschränken.

2. Entwurf robuster FIFs. Die Ergebnisse lassen sich unmittelbar auf Systeme übertragen, die exogenen Störungen und parametrischen Unsicherheiten unterliegen. Es wird ein einheitliches Entwurfsverfahren vorgestellt, welches eine einfache Gewichtung unterschiedlicher Entwurfsziele ermöglicht.

In diesem Kapitel wird zunächst in Abschnitt 6.2 die Grundidee des Entwurfs solcher allgemeiner dynamischer Filter für die Fehlerdetektion beschrieben. Dabei wird der Ansatz aus [149] erweitert und es wird her- 
ausgearbeitet, warum dieser in der Literatur bisher im Wesentlichen nur zur Fehlerdetektion herangezogen wurde. Unter Rückgriff auf die Ergebnisse aus Kapitel 4 wird dann gezeigt, dass sich allgemeine dynamische Filter auch sehr flexibel zur Fehlerisolation einsetzen lassen. Während sich bei den beobachterbasierten Verfahren jeweils eigenständige Entwurfsverfahren für unterschiedliche Fehlerisolationsprobleme (quadratische, nichtquadratische Systeme, statisch nicht isolierbare Systeme, nicht minimalphasige Systeme etc.) ergeben, wird in diesem Kapitel gezeigt, dass mit Fehlerisolationsfiltern ein einheitlicher Entwurf möglich ist, bei dem lediglich in einem ersten Schritt die Entwurfsziele spezifiziert werden müssen.

\subsection{Grundidee des Entwurfs}

Zur Beschreibung der Grundidee des Entwurfsverfahrens wird das in Abbildung 6.1 dargestellte Gesamtsystem aus Strecke

$$
\begin{aligned}
& \dot{\boldsymbol{x}}=\boldsymbol{A} \boldsymbol{x}+\boldsymbol{B} \boldsymbol{u}+\boldsymbol{E}_{a} \boldsymbol{f}+\boldsymbol{B}_{d} \boldsymbol{d}, \\
& \boldsymbol{y}=\boldsymbol{C} \boldsymbol{x}+\boldsymbol{D} \boldsymbol{u}+\boldsymbol{E}_{s} \boldsymbol{f}+\boldsymbol{D}_{d} \boldsymbol{d}
\end{aligned}
$$

und allgemeinem dynamischen Filter (6.1) betrachtet. Zunächst beschränken sich die Betrachtungen dabei wie in Kapitel 4 auf Systeme mit exakt bekannter Systemdynamik. Eine Erweiterung hinsichtlich unsicherer Systemparameter folgt in Abschnitt 6.5. Für die nominale Systemdynamik ergibt sich das Gesamtsystem zu

$$
\begin{aligned}
& \dot{\tilde{\boldsymbol{x}}}=\left[\begin{array}{c}
\dot{\boldsymbol{x}} \\
\dot{\boldsymbol{x}}_{f}
\end{array}\right]=\left[\begin{array}{cc}
\boldsymbol{A} & \mathbf{0} \\
\boldsymbol{B}_{f, y} \boldsymbol{C} & \boldsymbol{A}_{f}
\end{array}\right]\left[\begin{array}{c}
\boldsymbol{x} \\
\boldsymbol{x}_{f}
\end{array}\right]+\left[\begin{array}{c}
\boldsymbol{B} \\
\boldsymbol{B}_{f, u}+\boldsymbol{B}_{f, y} \boldsymbol{D}
\end{array}\right] \boldsymbol{u}+\ldots \\
& +\left[\begin{array}{c}
\boldsymbol{E}_{a} \\
\boldsymbol{B}_{f, y} \boldsymbol{E}_{s}
\end{array}\right] \boldsymbol{f}+\left[\begin{array}{c}
\boldsymbol{B}_{d} \\
\boldsymbol{B}_{f, y} \boldsymbol{D}_{d}
\end{array}\right] \boldsymbol{d}, \\
& \boldsymbol{r}=\left[\begin{array}{ll}
\boldsymbol{D}_{f, y} \boldsymbol{C} & \boldsymbol{C}_{f}
\end{array}\right]\left[\begin{array}{c}
\boldsymbol{x} \\
\boldsymbol{x}_{f}
\end{array}\right]+\left(\boldsymbol{D}_{f, u}+\boldsymbol{D}_{f, y} \boldsymbol{D}\right) \boldsymbol{u}+\ldots \\
& +\left(\boldsymbol{D}_{f, y} \boldsymbol{E}_{s}\right) \boldsymbol{f}+\left(\boldsymbol{D}_{f, y} \boldsymbol{D}_{d}\right) \boldsymbol{d} \text {. }
\end{aligned}
$$

Dadurch wird die Wirkung von Stellgrößen $\boldsymbol{u}$, Fehlern $\boldsymbol{f}$ und Störgrößen $\boldsymbol{d}$ auf die generierten Residuen beschrieben.

Weiterhin wird ein virtuelles Referenzmodell der Form

$$
\begin{aligned}
\dot{\boldsymbol{x}}_{\mathrm{ref}} & =\boldsymbol{A}_{\mathrm{ref}} \boldsymbol{x}_{\mathrm{ref}}+\boldsymbol{B}_{\mathrm{ref}, u} \boldsymbol{u}+\boldsymbol{B}_{\mathrm{ref}, f} \boldsymbol{f}+\boldsymbol{B}_{\mathrm{ref}, d} \boldsymbol{d}, \\
\boldsymbol{r}_{\mathrm{ref}} & =\boldsymbol{C}_{\mathrm{ref}} \boldsymbol{x}_{\mathrm{ref}}+\boldsymbol{D}_{\mathrm{ref}, u} \boldsymbol{u}+\boldsymbol{D}_{\mathrm{ref}, f} \boldsymbol{f}+\boldsymbol{D}_{\mathrm{ref}, d} \boldsymbol{d}
\end{aligned}
$$




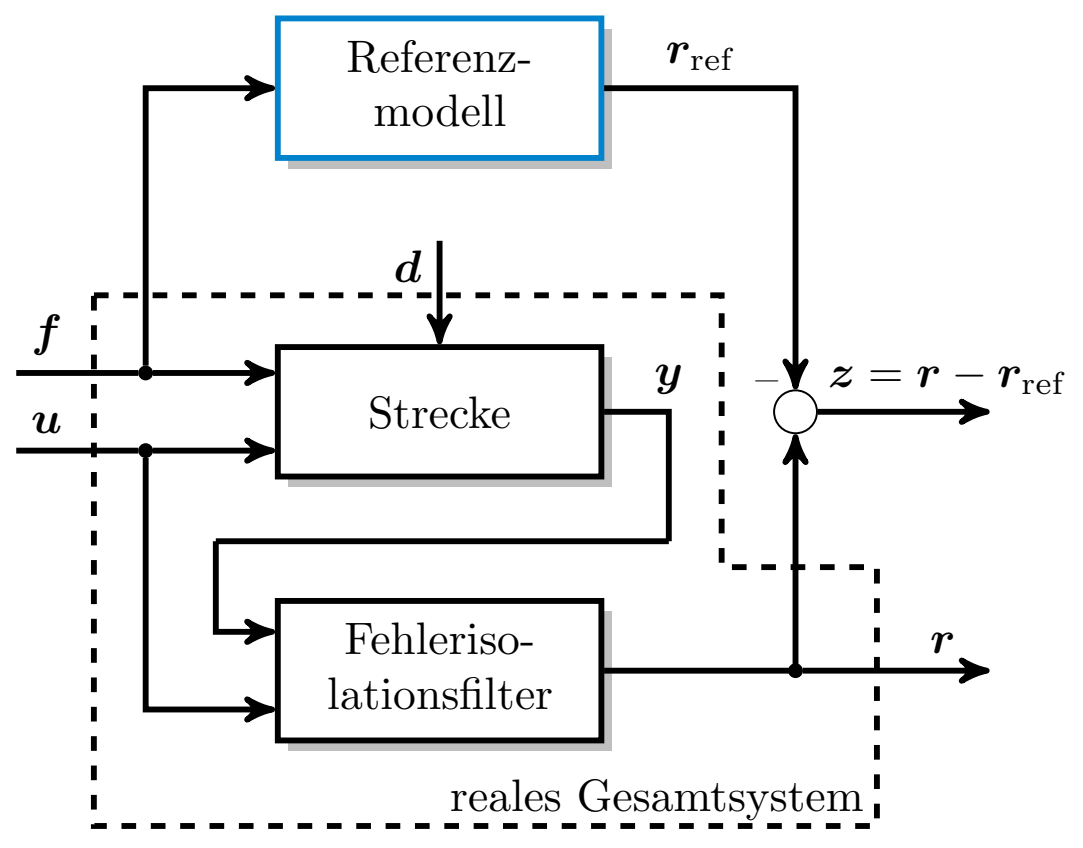

Abbildung 6.1: Zum Entwurf eines allgemeinen dynamischen Filters mittels virtuellem Referenzmodell und Performance-Ausgang $\boldsymbol{z}$

angesetzt. Dieses Modell dient lediglich dem Entwurf des Filters und muss nicht implementiert werden. Durch das Referenzmodell wird beschrieben, wie sich die erzeugten Residuen bei Anregung durch Fehler, Stellgrößen und Störungen idealerweise verhalten sollten. Ihm kommt daher eine zentrale Bedeutung zu.

Ziel ist es dann, die Filtermatrizen derart zu parametrieren, dass das Übertragungsverhalten des realen Gesamtsystems (6.4) dem des virtuellen Referenzmodells (6.5) entspricht, wie dies in Abbildung 6.1 angedeutet ist. Fasst man Stellgrößen, Fehler und Störungen zum verallgemeinerten Eingangsvektor

$$
\tilde{\boldsymbol{u}}=\left[\begin{array}{l}
\boldsymbol{u} \\
\boldsymbol{f} \\
\boldsymbol{d}
\end{array}\right] \in \mathbb{R}^{n_{u}+n_{f}+n_{d}}
$$

zusammen, so lässt sich das Problem formalisieren als

$$
\underset{\boldsymbol{A}_{f}, \boldsymbol{B}_{f, u}, \boldsymbol{B}_{f, y}, \boldsymbol{C}_{f}, \boldsymbol{D}_{f, u}, \boldsymbol{D}_{f, y}}{\operatorname{minimiere}}\left\|\boldsymbol{G}_{\boldsymbol{r} \tilde{\boldsymbol{u}}}(s)-\boldsymbol{G}_{\mathrm{ref}}(s)\right\|_{\infty} .
$$

Diese Idee wird in der Literatur als Model-Matching-Ansatz bezeichnet. ${ }^{1)}$

1) Zur Quantifizierung der Diskrepanz zwischen den beiden Übertragungsmatrizen in (6.6) lassen sich auch andere Normen heranziehen. Üblich ist jedoch die Verwendung der $\mathcal{H}_{\infty}$-Norm, die auch in dieser Arbeit verwendet wird. 
Im Bereich der beobachterbasierten Fehlerdetektion wird sie unter anderem in [271] angewendet, um die Robustheit bezüglich unsicherer Systemparameter zu verbessern. Um die Minimierung von $\left\|\boldsymbol{G}_{\boldsymbol{r} \tilde{\boldsymbol{u}}}(s)-\boldsymbol{G}_{\mathrm{ref}}(s)\right\|_{\infty}$ in ein LMI-Problem überführen zu können, sind jedoch zum Teil strukturelle Einschränkungen in den auftretenden LMI-Variablen vorzunehmen. Dies führt zu einer Einschränkung des Lösungsraumes und zu konservativen Ergebnissen. Wie in $[148,149]$ gezeigt lässt sich dieses Problem durch die Anwendung allgemeiner dynamischer Filter umgehen. Für diese lässt sich äquivalent zu (6.6) ohne Einschränkung der Lösungsmenge ein konvexes Optimierungsproblem zur Filterparametrierung formulieren.

Das zentrale Problem stellt dabei die Wahl eines geeigneten Referenzmodells dar. Wie bereits in [79] diskutiert führt die Wahl eines unpassenden Referenzmodells zu sehr schlechten Ergebnissen der Optimierung (6.6). Dies ist immer dann der Fall, wenn das Gesamtsystem bestehend aus Strecke und Filter das vom Referenzmodell vorgegebene Verhalten strukturell nicht abbilden kann. Darin ist auch der Grund zu sehen, warum die Ergebnisse in [149] auf die Fehlerdetektion beschränkt bleiben.

Um den Model-Matching-Entwurf auch für die Parametrierung von FIFs anzuwenden, sind folglich zwei wesentliche Teilprobleme zu lösen:

1. Wahl eines geeigneten Referenzmodells (6.5).

2. Auslegen der Filtermatrizen (6.1) für das gewählte Referenzmodell.

Die beiden Entwurfsschritte werden in den folgenden Abschnitten ausführlich behandelt, wobei insbesondere die Verbindung zu den beobachterbasierten Resultaten aus Kapitel 4 aufgezeigt wird.

\subsection{Wahl eines zulässigen Referenzmodells}

In [79] wird für das Problem der robusten Fehlerdetektion erörtert, dass die Wahl des Referenzmodells entscheidenden Einfluss auf die Robustheit des resultierenden Fehlerdetektors hat. Da beim Entwurf von Fehlerisolationsfiltern der Übertragungsmatrix $\boldsymbol{G}_{\boldsymbol{r} \boldsymbol{f}}(s)$ Diagonalstruktur aufgeprägt werden soll und somit Strukturbeschränkungen einzuhalten sind, spielt das Referenzmodell bereits für die nominale Systemdynamik eine wichtige Rolle. In [149] wird die Auslegung eines Referenzmodells lediglich angerissen und es wird darauf verwiesen, dass es für die Fehlerisolation eine Diagonalstruktur aufweisen muss. An dieser Stelle wird vertiefend auf die Auslegung eines Referenzmodells eingegangen, wobei nicht nur die Struktur 
von $\boldsymbol{G}_{\mathrm{ref}, \boldsymbol{r} \boldsymbol{f}}(s)$, sondern auch die Gestalt der einzelnen Diagonalelemente dieser Übertragungsmatrix beleuchtet wird.

Um den Begriff eines geeigneten Referenzmodells präziser zu fassen, wird in Anlehnung an [79] die folgende Definition verwendet:

Definition 6.2 (Zulässiges Referenzmodell). Für eine nominale Systemdynamik der Form (6.2) wird ein durch

$$
\begin{aligned}
& \boldsymbol{G}_{\mathrm{ref}, \boldsymbol{r} \boldsymbol{f}}(s)=\boldsymbol{C}_{\mathrm{ref}}\left(s \boldsymbol{I}_{n_{\mathrm{ref}}}-\boldsymbol{A}_{\mathrm{ref}}\right)^{-1} \boldsymbol{B}_{\mathrm{ref}, f}+\boldsymbol{D}_{\mathrm{ref}, f}, \\
& \boldsymbol{G}_{\mathrm{ref}, \boldsymbol{r u}}(s)=\boldsymbol{C}_{\mathrm{ref}}\left(s \boldsymbol{I}_{n_{\mathrm{ref}}}-\boldsymbol{A}_{\mathrm{ref}}\right)^{-1} \boldsymbol{B}_{\mathrm{ref}, u}+\boldsymbol{D}_{\mathrm{ref}, u}
\end{aligned}
$$

beschriebenes Referenzmodell zulässig genannt, falls ein Residuengenerator der Form (6.1) existiert, sodass die Gleichungen

$$
\begin{gathered}
\left\|\boldsymbol{G}_{\boldsymbol{r} \boldsymbol{f}}(s)-\boldsymbol{G}_{\mathrm{ref}, \boldsymbol{r} \boldsymbol{f}}(s)\right\|_{\infty}<\varepsilon_{1}, \\
\left\|\boldsymbol{G}_{\boldsymbol{r u}}(s)-\boldsymbol{G}_{\mathrm{ref}, \boldsymbol{r u}}(s)\right\|_{\infty}<\varepsilon_{2}
\end{gathered}
$$

für beliebig kleine Skalare $\varepsilon_{1}>0, \varepsilon_{2}>0$ erfüllbar sind.

Die Zulässigkeit eines Referenzmodells wird demnach ausschließlich über den Einfluss von Fehlern und Stellgrößen definiert, Störgrößen spielen an dieser Stelle keine Rolle.

Da nach den in Abschnitt 2.3 definierten Entwurfszielen eine ideale Fehlerisolation gegeben ist, wenn sich Störungen und Stellgrößen nicht auf die generierten Residuen auswirken, wird im Rahmen dieser Arbeit stets

$$
\begin{array}{ll}
\boldsymbol{B}_{\mathrm{ref}, u}=\mathbf{0}, & \boldsymbol{D}_{\mathrm{ref}, u}=\mathbf{0}, \\
\boldsymbol{B}_{\mathrm{ref}, d}=\mathbf{0}, & \boldsymbol{D}_{\mathrm{ref}, d}=\mathbf{0}
\end{array}
$$

gewählt. Anschaulich gesprochen muss also eine Filterparametrierung $\left(\boldsymbol{A}_{f}, \boldsymbol{B}_{f, u}, \boldsymbol{B}_{f, y}, \boldsymbol{C}_{f}, \boldsymbol{D}_{f, u}, \boldsymbol{D}_{f, y}\right)$ existieren, die dazu führt, dass bezüglich der Fehler $\boldsymbol{f}$ das Übertragungsverhalten des realen Gesamtsystems (6.4) mit dem des virtuellen Referenzmodells (6.5) beliebig genau übereinstimmt.

Entscheidend ist an dieser Stelle, dass die Ergebnisse aus dem Bereich der beobachterbasierten Fehlerisolation aus Kapitel 4 die Grundlage für die konstruktive Auslegung eines zulässigen Referenzmodells darstellen. Die Gleichungen eines FIOs lassen sich umschreiben zu

$$
\begin{aligned}
\dot{\hat{\boldsymbol{x}}} & =(\boldsymbol{A}-\boldsymbol{L} \boldsymbol{C}) \hat{\boldsymbol{x}}+(\boldsymbol{B}-\boldsymbol{L} \boldsymbol{D}) \boldsymbol{u}+\boldsymbol{L} \boldsymbol{y}, \\
\boldsymbol{r} & =-\boldsymbol{V} \boldsymbol{C} \hat{\boldsymbol{x}}+-\boldsymbol{V} \boldsymbol{D} \boldsymbol{u}+\boldsymbol{V} \boldsymbol{y} .
\end{aligned}
$$


Aus dem Vergleich von (6.8) mit (6.1) ergibt sich, dass ein FIO als Spezialfall eines allgemeinen dynamischen Filters mit $n_{\text {filt }}=n$ und

$$
\begin{array}{lll}
\boldsymbol{A}_{f}=\boldsymbol{A}-\boldsymbol{L} \boldsymbol{C}, & \boldsymbol{B}_{f, u}=\boldsymbol{B}-\boldsymbol{L} \boldsymbol{D}, & \boldsymbol{B}_{f, y}=\boldsymbol{L}, \\
\boldsymbol{C}_{f}=-\boldsymbol{V} \boldsymbol{C}, & \boldsymbol{D}_{f, u}=-\boldsymbol{V} \boldsymbol{D}, & \boldsymbol{D}_{f, y}=\boldsymbol{V}
\end{array}
$$

aufgefasst werden kann. Daraus ergibt sich unmittelbar, dass die in Kapitel 4 eingeführten Bedingungen für die Existenz von FIOs (beziehungsweise DFIOs) hinreichend für die Existenz eines stabilen allgemeinen dynamischen Fehlerisolationsfilters sind. Für statisch stabil fehlerisolierbare Systeme sind die folgenden Annahmen hinreichend:

Annahme 6.1. Das Paar $(\boldsymbol{A}, \boldsymbol{C})$ ist vollständig beobachtbar.

Annahme 6.2. Die Fehlerdetektionsmatrix hat vollen Rang, das heißt es gilt $\operatorname{rang}\left(\boldsymbol{D}^{*}\right)=n_{f}$.

Annahme 6.3. Das System $\left(\boldsymbol{A}, \boldsymbol{E}_{a}, \boldsymbol{C}, \boldsymbol{E}_{s}\right)$ ist minimalphasig.

Wie in Abschnitt 4.7 beschrieben, lassen sich diese für statisch nicht isolierbare Systeme abschwächen zu

Annahme 6.4. Das Paar $(\boldsymbol{A}, \boldsymbol{C})$ ist vollständig beobachtbar.

Annahme 6.5. Das System $\left(\boldsymbol{A}, \boldsymbol{E}_{a}, \boldsymbol{C}, \boldsymbol{E}_{s}\right)$ weist eine endliche Anzahl von $\mu$ invarianten Nullstellen auf.

Annahme 6.6. Die Fehlerdetektionsmatrix weist ein Rangdefizit von $\kappa>0$ auf, das heißt es gilt $\operatorname{rang}\left(\boldsymbol{D}^{*}\right)=n_{f}-\kappa$.

Annahme 6.7. Das System $\left(\boldsymbol{A}, \boldsymbol{E}_{a}, \boldsymbol{C}, \boldsymbol{E}_{s}\right)$ ist minimalphasig.

In diesem Fall wird ebenso wie für nicht minimalphasige Systeme ein DFIO benötigt. Ein solcher existiert nach Abschnitt 4.8, wenn das System die folgenden Annahmen erfüllt:

Annahme 6.8. Das Paar $(\boldsymbol{A}, \boldsymbol{C})$ ist vollständig beobachtbar.

Annahme 6.9. Die Fehlerdetektionsmatrix hat vollen Rang, das heißt es gilt $\operatorname{rang}\left(\boldsymbol{D}^{*}\right)=n_{f}$.

Annahme 6.10. Das System $\left(\boldsymbol{A}, \boldsymbol{E}_{a}, \boldsymbol{C}, \boldsymbol{E}_{s}\right)$ weist eine endliche Anzahl invarianter Nullstellen auf.

Annahme 6.11. Das System $\left(\boldsymbol{A}, \boldsymbol{E}_{a}, \boldsymbol{C}, \boldsymbol{E}_{s}\right)$ weist keine invariante Nullstelle bei $\eta=0$ auf. 
Annahme 6.12. Keine invariante Nullstelle von $\left(\boldsymbol{A}, \boldsymbol{E}_{a}, \boldsymbol{C}, \boldsymbol{E}_{s}\right)$ fällt mit einem Eigenwert von $\boldsymbol{A}$ zusammen.

Gemäß obigen Ausführungen gilt

Satz 6.1 (Existenz eines FIFs). Gegeben sei ein System der Form

$$
\begin{aligned}
& \dot{\boldsymbol{x}}=\boldsymbol{A} \boldsymbol{x}+\boldsymbol{B} \boldsymbol{u}+\boldsymbol{E}_{a} \boldsymbol{f} \\
& \boldsymbol{y}=\boldsymbol{C} \boldsymbol{x}+\boldsymbol{D} \boldsymbol{u}+\boldsymbol{E}_{s} \boldsymbol{f}
\end{aligned}
$$

für das ein stabiler statischer FIO (dynamischer FIO der Ordnung $n_{\zeta}$ ) entworfen werden kann. Dann existiert ein stabiles FIF der Ordnung $n_{\text {filt }} \geq n$ $\left(n_{\text {filt }} \geq n+n_{\zeta}\right)$.

Neben diesem Existenzsatz lässt sich auch ein konstruktives Verfahren zur Auslegung eines zulässigen Referenzmodells angeben. Dabei wird ausgenutzt, dass beim FIO-Entwurf für nominale Systeme die Struktur der sich ergebenden Übertragungsmatrix $\boldsymbol{G}_{\boldsymbol{r} \boldsymbol{f}}(s)$ bereits allein durch eine Systemanalyse angegeben werden kann. Ohne den FIO-Entwurf durchführen zu müssen, ist nach den Ergebnissen aus den Abschnitten 4.5 beziehungsweise 4.9 für eine statisch stabil fehlerisolierbare Strecke klar, dass sich zwischen Fehlern und Residuen eine diagonale Übertragungsmatrix mit den Diagonalelementen

$$
g_{i, i}(s)=\frac{z_{i, 0}}{s^{\delta_{i}}+q_{i, \delta_{i}-1} s^{\delta_{i}-1}+\ldots+q_{i, 1} s+q_{i, 0}}
$$

ergibt. Setzt man diese Übertragungsmatrix mit beliebigen Koeffizienten $z_{i, 0}, q_{i, j}$ an, so ergibt sich mit $\boldsymbol{G}_{\mathrm{ref}, \boldsymbol{r u}}(s)=\mathbf{0}$ ein zulässiges Referenzmodell $\boldsymbol{G}_{\mathrm{ref}, \boldsymbol{r} \boldsymbol{f}}(s)$.

Aufgrund der Diagonalgestalt von $\boldsymbol{G}_{\mathrm{ref}, \boldsymbol{r} \boldsymbol{f}}(s)$ lassen sich die das Referenzmodell beschreibenden Matrizen $\boldsymbol{A}_{\mathrm{ref}}, \boldsymbol{B}_{\mathrm{ref}, f}, \boldsymbol{C}_{\mathrm{ref}}$ und $\boldsymbol{D}_{\mathrm{ref}, f}$ schreiben als

$$
\begin{aligned}
\boldsymbol{A}_{\mathrm{ref}} & =\operatorname{diag}\left(\boldsymbol{A}_{\mathrm{ref}, 1}, \ldots, \boldsymbol{A}_{\mathrm{ref}, n_{f}}\right), \\
\boldsymbol{B}_{\mathrm{ref}, f} & =\operatorname{diag}\left(\boldsymbol{B}_{\mathrm{ref}, f, 1}, \ldots, \boldsymbol{B}_{\mathrm{ref}, f, n_{f}}\right), \\
\boldsymbol{C}_{\mathrm{ref}} & =\operatorname{diag}\left(\boldsymbol{C}_{\mathrm{ref}, 1}, \ldots, \boldsymbol{C}_{\mathrm{ref}, n_{f}}\right), \\
\boldsymbol{D}_{\mathrm{ref}, f} & =\operatorname{diag}\left(\boldsymbol{D}_{\mathrm{ref}, f, 1}, \ldots, \boldsymbol{D}_{\mathrm{ref}, f, n_{f}}\right) .
\end{aligned}
$$

Dabei stellen die Matrizen $\left(\boldsymbol{A}_{\mathrm{ref}, i}, \boldsymbol{B}_{\mathrm{ref}, f, i}, \boldsymbol{C}_{\mathrm{ref}, i}, \boldsymbol{D}_{\mathrm{ref}, f, i}\right)$ jeweils Zustandsraumrealisierungen der SISO-Übertragungsfunktionen $g_{i, i}(s)$ dar. Durch 
(6.7) und (6.9) ist also ein zulässiges Referenzmodell festgelegt, welches die Entwurfsziele der Diagonalisierung von $\boldsymbol{G}_{\boldsymbol{r} \boldsymbol{f}}(s)$ sowie der Unterdrückung des Einflusses von Stell- und Störgrößen auf die generierten Residuen in idealer Weise abbildet. Durch die Wahl der Koeffizienten $q_{i, j}$ und $z_{i, 0}$ lassen sich darüber hinaus Anforderungen an die Detektionsgeschwindigkeit und die Fehlersensitivität umsetzen.

Bemerkenswert ist, dass dieses Vorgehen zur Ermittlung eines zulässigen Referenzmodells nicht auf quadratische Systeme, welche die Annahmen 6.1 bis 6.3 erfüllen, beschränkt ist. Für nichtquadratische Systeme bleibt das Vorgehen unverändert. Angemerkt sei, dass hinsichtlich der Ordnung der Diagonalelemente von $\boldsymbol{G}_{\mathrm{ref}, \boldsymbol{r} \boldsymbol{f}}(s)$ zwei Möglichkeiten existieren, wenn ein nichtquadratisches System aus einem um einen zusätzlichen Sensor ergänzten quadratischen System hervorgeht (s. Bemerkung 4.13 auf Seite 169). Zum einen kann die Ordnung jeweils entsprechend den Entwürfen aus Abschnitt $4.9 \mathrm{zu} \delta_{i}$ gewählt werden. Weist das ursprüngliche, quadratische System die Fehlerindizes $\delta_{i, \text { quad }} \geq \delta_{i}$ auf, so ist es zum anderen offensichtlich auch möglich, diese als Ordnung vorzugeben.

Weiterhin können auch für statisch nicht isolierbare Systeme (vgl. Abschnitt 4.7) und nicht minimalphasige Systeme (vgl. Abschnitt 4.8) auf Grundlage der Systemanalyse zulässige Referenzmodelle gefunden werden. Für erstere ist entsprechend den Ergebnissen aus Abschnitt 4.7 im Allgemeinen die Ordnung der Nennerpolynome der Diagonalelemente zu erhöhen. Details ergeben sich aus der Analyse des Rechtsnullraumes der Matrix $\boldsymbol{D}^{*}$. Für nicht minimalphasige Systeme sind diejenigen invarianten Nullstellen, die unkompensiert bleiben sollen, als Nullstellen bestimmter Zählerpolynome auf der Diagonalen von $\boldsymbol{G}_{\mathrm{ref}, \boldsymbol{r} \boldsymbol{f}}(s)$ vorzugeben, wobei die Ordnung der Nennerpolynome zu erhöhen ist. Details sind in Abschnitt 4.8 beschrieben.

Es sei an dieser Stelle ausdrücklich darauf hingewiesen, dass die Struktur von $\boldsymbol{G}_{\boldsymbol{r} \boldsymbol{f}}(s)$ feststeht, ohne dass der FIO-Entwurf durchgeführt werden muss. Der Entwurf erfolgt stets nach dem in Abbildung 6.2 skizzierten Schema. Zunächst wird das System in Schritt 1.a) also hinsichtlich der Fehlerindizes $\delta_{i}$, der Fehlerdetektionsmatrix $\boldsymbol{D}^{*}$ und eventueller invarianter Nullstellen analysiert. Daraus ergibt sich wie erwähnt eine zulässige Struktur für die Matrix $\boldsymbol{G}_{\text {ref, } \boldsymbol{r} \boldsymbol{f}}(s)$. In Schritt 1.b) sind die Koeffizienten der Diagonalelemente festzulegen und es muss eine Zustandsraumrealisierung für das Referenzmodell ermittelt werden, wie es in diesem Abschnitt beschrieben wurde. Der zweite Schritt ist dann die eigentliche Parametrierung der Filtermatrizen auf Grundlage des konstruierten Referenzmodells, auf die in den folgenden Abschnitten eingegangen wird. Ein wesentlicher 


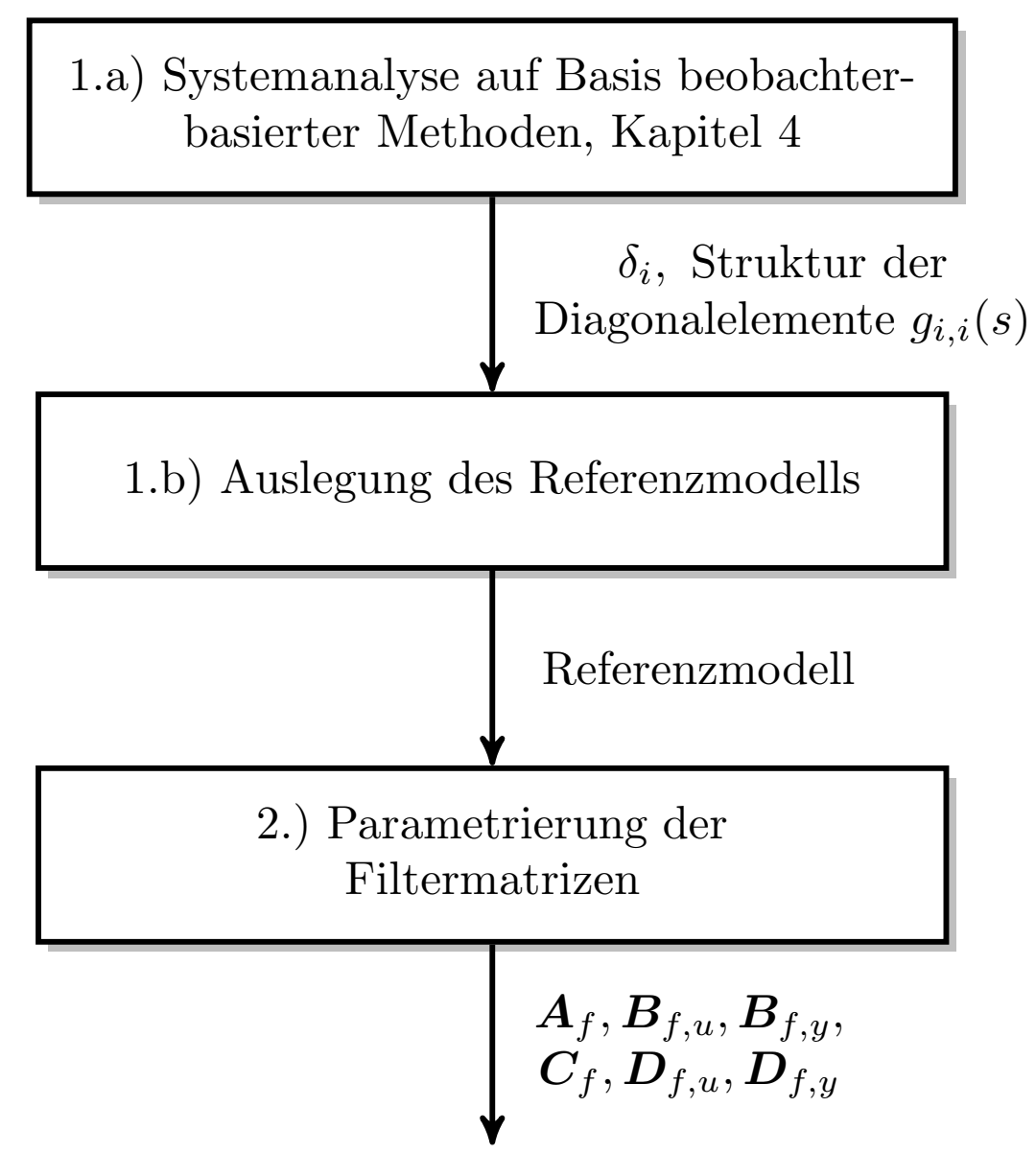

Abbildung 6.2: Ablauf des Entwurfs von Fehlerisolationsfiltern

Vorteil gegenüber dem Entwurf von FIOs ist im einheitlichen Vorgehen zu sehen. Während bei FIOs beispielsweise für nicht minimalphasige Systeme ein erheblicher Aufwand nötig ist (dynamische Erweiterungen, Realisierung als dynamisch erweiterte FIOs), ändert sich beim Entwurf von FIFs das generelle Vorgehen gegenüber minimalphasigen Systemen nicht. Es ist lediglich das Referenzmodell entsprechend anzupassen.

Der Vollständigkeit halber sei erwähnt, dass es wie in [271] diskutiert alternativ auch möglich ist, $\boldsymbol{B}_{\text {ref, } d} \neq \mathbf{0}, \boldsymbol{D}_{\text {ref }, d} \neq \mathbf{0}$ anzusetzen. Dies trägt der Tatsache Rechnung, dass im Allgemeinen bereits für ein System ohne parametrische Unsicherheiten keine ideale Fehlerisolation im Sinne von $\boldsymbol{G}_{\boldsymbol{r} \boldsymbol{d}}(s)=\mathbf{0}$ möglich ist, wenn exogene Störungen auf das System wirken. In [271] wird zunächst für die nominale Systemdynamik ein Beobachter $(\boldsymbol{L}, \boldsymbol{V})$ parametriert, welcher hinsichtlich der Fehlerdetektion einen bestmöglichen Kompromiss zwischen Fehlersensitivität und Störunterdrückung bietet. In einem zweiten Schritt wird mittels eines Model-Matching-Ansatzes die Robustheit bezüglich unsicherer Systempa- 
rameter gesteigert. Dabei wird für das Referenzmodell

$$
\boldsymbol{B}_{\mathrm{ref}, d}=\boldsymbol{B}_{d}-\boldsymbol{L} \boldsymbol{D}_{d}, \quad \boldsymbol{D}_{\mathrm{ref}, d}=\boldsymbol{V} \boldsymbol{D}_{d}
$$

gewählt. Dadurch zielt der Model-Matching-Ansatz nicht auf das ideale Entwurfsziel $\boldsymbol{G}_{\boldsymbol{r} \boldsymbol{d}}(s)=\mathbf{0}$ ab, sondern versucht, das Systemverhalten zu rekonstruieren, welches sich für ein System ohne parametrische Unsicherheiten ergibt.

Dieses Vorgehen lässt sich auf den Entwurf von allgemeinen Fehlerisolationsfiltern übertragen. Dazu wird zunächst mittels der Ergebnisse aus Kapitel 5 eine Beobachterparametrierung $(\boldsymbol{L}, \boldsymbol{V})$ ermittelt, welche die Störunterdrückung optimiert. Mittels (6.7a), (6.9) und (6.10) ist das Referenzmodell eindeutig bestimmt. Allerdings muss für diese Wahl der Beobachterentwurf zur Gewinnung von $(\boldsymbol{L}, \boldsymbol{V})$ durchgeführt werden. Bei der Wahl nach (6.7b) ist wie bereits erörtert lediglich die Analyse des Systems hinsichtlich der Fehlerindizes notwendig.

\subsection{LMI-basierte Lösung des Entwurfsproblems bei nominaler Systemdynamik}

Nachdem auf Basis der Systemanalyse wie im vorangegangenen Abschnitt beschrieben ein zulässiges Referenzmodell aufgestellt wurde, verbleibt die Auslegung der Filtermatrizen derart, dass das gewünschte Übertragungsverhalten bestmöglich abgebildet wird. Dieses Problem wird im Folgenden zunächst für nominale, störungsfreie Strecken betrachtet, bevor Abschnitt 6.5 Robustheitsaspekte beleuchtet.

Um das Ein-/Ausgangsverhalten des in Abbildung 6.1 dargestellten Gesamtsystems zu beschreiben, werden der Zustandsvektor

$$
\boldsymbol{x}_{g}=\left[\begin{array}{c}
\boldsymbol{x}_{\mathrm{ref}} \\
\boldsymbol{x} \\
\boldsymbol{x}_{f}
\end{array}\right] \in \mathbb{R}^{n_{\mathrm{ref}}+n+n_{\mathrm{filt}}}
$$

und der Performance-Ausgang $\boldsymbol{z}=\boldsymbol{r}-\boldsymbol{r}_{\text {ref }} \in \mathbb{R}^{n_{f}}$ eingeführt. Mithilfe dieser Größen sowie (6.1), (6.2) und (6.5) lässt sich das Übertragungsverhalten von Fehlern und Stellgrößen auf den Performance- 
ausgang wie folgt beschreiben. Für die Wirkung der Fehler gilt

$$
\begin{aligned}
\boldsymbol{G}_{\boldsymbol{z} \boldsymbol{f}}(s) & =\boldsymbol{C}_{\boldsymbol{z} \boldsymbol{f}}\left(s \boldsymbol{I}_{n_{\mathrm{ref}}+n+n_{\mathrm{filt}}}-\boldsymbol{A}_{\boldsymbol{z} \boldsymbol{f}}\right)^{-1} \boldsymbol{B}_{\boldsymbol{z} \boldsymbol{f}}+\boldsymbol{D}_{\boldsymbol{z} \boldsymbol{f}}, \\
\boldsymbol{A}_{\boldsymbol{z} \boldsymbol{f}} & =\left[\begin{array}{ccc}
\boldsymbol{A}_{\mathrm{ref}} & \mathbf{0} & \mathbf{0} \\
\mathbf{0} & \boldsymbol{A} & \mathbf{0} \\
\mathbf{0} & \boldsymbol{B}_{f, y} \boldsymbol{C} & \boldsymbol{A}_{f}
\end{array}\right], \quad \boldsymbol{B}_{\boldsymbol{z f}}=\left[\begin{array}{c}
\boldsymbol{B}_{\mathrm{ref}, f} \\
\boldsymbol{E}_{a} \\
\boldsymbol{B}_{f, y} \boldsymbol{E}_{s}
\end{array}\right], \\
\boldsymbol{C}_{\boldsymbol{z} \boldsymbol{f}} & =\left[\begin{array}{lll}
-\boldsymbol{C}_{\mathrm{ref}} & \boldsymbol{D}_{f, y} \boldsymbol{C} & \boldsymbol{C}_{f}
\end{array}\right], \boldsymbol{D}_{\boldsymbol{z} \boldsymbol{f}}=\boldsymbol{D}_{f, y} \boldsymbol{E}_{s}-\boldsymbol{D}_{\mathrm{ref}, f} .
\end{aligned}
$$

Für den Einfluss der Stellgrößen auf $\boldsymbol{z}$ folgt

$$
\begin{aligned}
\boldsymbol{G}_{\boldsymbol{z u}}(s) & =\boldsymbol{C}_{\boldsymbol{z} \boldsymbol{u}}\left(s \boldsymbol{I}_{n_{\mathrm{ref}}+n+n_{\mathrm{filt}}}-\boldsymbol{A}_{\boldsymbol{z u}}\right)^{-1} \boldsymbol{B}_{\boldsymbol{z u}}+\boldsymbol{D}_{\boldsymbol{z u}}, \\
\boldsymbol{A}_{\boldsymbol{z} \boldsymbol{u}} & =\boldsymbol{A}_{\boldsymbol{z} \boldsymbol{f}}, \quad \boldsymbol{B}_{\boldsymbol{z} \boldsymbol{u}}=\left[\begin{array}{c}
\boldsymbol{B}_{\mathrm{ref}, u} \\
\boldsymbol{B} \\
\boldsymbol{B}_{f, u}+\boldsymbol{B}_{f, y} \boldsymbol{D}
\end{array}\right] \\
\boldsymbol{C}_{\boldsymbol{z} \boldsymbol{u}} & =\boldsymbol{C}_{\boldsymbol{z} \boldsymbol{f}}, \quad \boldsymbol{D}_{\boldsymbol{z} \boldsymbol{u}}=\boldsymbol{D}_{f, u}+\boldsymbol{D}_{f, y} \boldsymbol{D}-\boldsymbol{D}_{\mathrm{ref}, u} .
\end{aligned}
$$

Die Systeme (6.11) und (6.12) erlauben es, die Anforderungen an die Filtermatrizen zu formalisieren. Aus den Überlegungen in Abschnitt 6.3 folgt unmittelbar

Lemma 6.1. Gegeben sei eine stabile Strecke der Form (6.2), welche entweder die Annahmen 6.1-6.3 oder die Annahmen 6.4-6.7 beziehungsweise 6.8-6.12 erfüllt. Dann existiert ein zulässiges Referenzmodell $\boldsymbol{G}_{r e f, \boldsymbol{r} \boldsymbol{f}}(s)$ und das Problem

$$
\begin{aligned}
& \text { finde } \boldsymbol{A}_{f}, \boldsymbol{B}_{f, u}, \boldsymbol{B}_{f, y}, \boldsymbol{C}_{f}, \boldsymbol{D}_{f, u}, \boldsymbol{D}_{f, y} \text {, sodass } \\
& \left\|\boldsymbol{G}_{\boldsymbol{z} \boldsymbol{f}}(s)\right\|_{\infty}<\varepsilon_{f}, \\
& \left\|\boldsymbol{G}_{\boldsymbol{z} \boldsymbol{u}}(s)\right\|_{\infty}<\varepsilon_{u}
\end{aligned}
$$

ist lösbar für beliebig kleine $\varepsilon_{f}$ und $\varepsilon_{u}$, wobei $\boldsymbol{A}_{f}$ eine Hurwitz-Matrix ist.

Beweis. Für ein System, welches die Annahmen 6.1 bis 6.3 erfüllt, kann mittels Satz 4.1 und Satz 4.2 beziehungsweise Satz 4.8 und Satz 4.9 ein stabiler FIO entworfen werden, der $\boldsymbol{G}_{\boldsymbol{z} \boldsymbol{f}}(s)=\mathbf{0}$ und $\boldsymbol{G}_{\boldsymbol{z} \boldsymbol{u}}(s)=\mathbf{0}$ gewährleistet und der einen Spezialfall eines allgemeinen Fehlerisolationsfilters darstellt. Ebenso kann mit den Ergebnissen aus Abschnitt 4.7 und 4.8 für statisch nicht isolierbare und nicht minimalphasige Systeme verfahren werden.

Die Einschränkung auf stabile Systeme ist nötig, da $\boldsymbol{A}_{\boldsymbol{z} \boldsymbol{f}}$ eine Blockdreiecksstruktur aufweist und auf der Diagonalen den Block $\boldsymbol{A}$ beinhaltet. 
Die Eigenwerte von $\boldsymbol{A}$ tauchen also als Eigenwerte von $\boldsymbol{A}_{\boldsymbol{z} \boldsymbol{f}}$ auf. Durch das FIF werden die Eigenwerte von $\boldsymbol{A}$ nicht beeinflusst, sodass $\boldsymbol{A}$ eine Hurwitz-Matrix sein muss, damit alle Eigenwerte von $\boldsymbol{A}_{\boldsymbol{z} \boldsymbol{f}}$ negativen Realteil haben können.

Zur Lösung des Problems (6.13) aus Lemma 6.1 wird zunächst der verallgemeinerte Eingangsvektor

$$
\tilde{\boldsymbol{u}}=\left[\begin{array}{l}
\boldsymbol{u} \\
\boldsymbol{f}
\end{array}\right] \in \mathbb{R}^{n_{u}+n_{f}}
$$

eingeführt. Damit lassen sich (6.11) und (6.12) kompakt im Zustandsraum darstellen als

$$
\begin{aligned}
& \dot{\boldsymbol{x}}_{g}=\underbrace{\left[\begin{array}{ccc}
\boldsymbol{A}_{\mathrm{ref}} & \mathbf{0} & \mathbf{0} \\
\mathbf{0} & \boldsymbol{A} & \mathbf{0} \\
\mathbf{0} & \boldsymbol{B}_{f, y} \boldsymbol{C} & \boldsymbol{A}_{f}
\end{array}\right]}_{\boldsymbol{A}_{\boldsymbol{z} \tilde{u}}} \boldsymbol{x}_{g}+\underbrace{\left[\begin{array}{cc}
\boldsymbol{B}_{\mathrm{ref}, u} & \boldsymbol{B}_{\mathrm{ref}, f} \\
\boldsymbol{B} & \boldsymbol{E}_{a} \\
\boldsymbol{B}_{f, u}+\boldsymbol{B}_{f, y} \boldsymbol{D} & \boldsymbol{B}_{f, y} \boldsymbol{E}_{s}
\end{array}\right]}_{\boldsymbol{B}_{\boldsymbol{z} \tilde{u}}} \tilde{\boldsymbol{u}}, \\
& \boldsymbol{z}=\underbrace{\left[\begin{array}{lll}
-C_{\mathrm{ref}} & \boldsymbol{D}_{f, y} \boldsymbol{C} & \boldsymbol{C}_{f}
\end{array}\right]}_{\boldsymbol{C}_{\boldsymbol{z} \tilde{u}}} \boldsymbol{x}_{g}+\ldots \\
& +\underbrace{\left[\begin{array}{cc}
\boldsymbol{D}_{f, u}+\boldsymbol{D}_{f, y} \boldsymbol{D}-\boldsymbol{D}_{\mathrm{ref}, u} & \boldsymbol{D}_{f, y} \boldsymbol{E}_{s}-\boldsymbol{D}_{\mathrm{ref}, f}
\end{array}\right]}_{\boldsymbol{D}_{z \tilde{u}}} \tilde{\boldsymbol{u}} .
\end{aligned}
$$

Unter Rückgriff auf das Bounded-Real-Lemma (Satz 3.1 auf Seite 56) ergibt sich damit als alternative Darstellung für (6.13) die Formulierung

$$
\begin{aligned}
& \text { finde } \boldsymbol{P}, \boldsymbol{A}_{f}, \boldsymbol{B}_{f, u}, \boldsymbol{B}_{f, y}, \boldsymbol{C}_{f}, \boldsymbol{D}_{f, u}, \boldsymbol{D}_{f, y}, \text { sodass } \\
& \mathbb{R}^{n_{\mathrm{ref}}+n+n_{\mathrm{filt}}} \ni \boldsymbol{P} \succ \mathbf{0}, \\
& {\left[\begin{array}{ccc}
\operatorname{He}\left(\boldsymbol{P} \boldsymbol{A}_{\boldsymbol{z} \tilde{\boldsymbol{u}}}\right) & \boldsymbol{P} \boldsymbol{B}_{\boldsymbol{z} \tilde{\boldsymbol{u}}} & \boldsymbol{C}_{\boldsymbol{z} \tilde{\boldsymbol{u}}}^{\boldsymbol{\top}} \\
\star & -\varepsilon \boldsymbol{I}_{n_{u}+n_{f}} & \boldsymbol{D}_{\boldsymbol{z} \tilde{\boldsymbol{u}}}^{\top} \\
\star & \star & -\varepsilon \boldsymbol{I}_{n_{f}}
\end{array}\right] \prec \mathbf{0 .}}
\end{aligned}
$$

Dabei wurde zusätzlich die Matrix $\boldsymbol{P}$ eingeführt und es wird lediglich eine einzige Schranke $\varepsilon$ benötigt. Des Weiteren ist die Filterordnung $n_{\text {filt }}$ festzulegen.

In der Form (6.16) ist das Entwurfsproblem schwierig zu lösen, da es sich bei (6.16c) um eine bilineare Matrixungleichung handelt. Dies rührt daher, dass die Matrizen $\boldsymbol{A}_{\boldsymbol{z} \tilde{\boldsymbol{u}}}$ und $\boldsymbol{B}_{\boldsymbol{z} \tilde{\boldsymbol{u}}}$ jeweils von den Filterparametern abhängig sind und somit multiplikative Terme mit der ebenfalls zu ermittelnden Matrix $\boldsymbol{P}$ auftreten. Es lässt sich jedoch eine Variablentransformation finden, welche (6.16) in ein LMI-Problem überführt, wie im Folgenden gezeigt wird. 
Es sei nochmals betont, dass die Garantie der Existenz einer Lösung von (6.16) mit beliebig kleinem $\varepsilon$ auf den Überlegungen zur beobachterbasierten Fehlerisolation beruht. Wie bereits in Abbildung 6.2 skizziert wird durch die Systemanalyse die Grundlage gelegt, um den Entwurf allgemeiner dynamischer Filter auch für die Fehlerisolation zu erweitern. Ein Fehlerisolationsbeobachter muss dazu nicht entworfen werden, entscheidend ist lediglich die Ermittlung und Analyse der Fehlerdetektionsindizes, der Fehlerdetektionsmatrix und der invarianten Nullstellen. Wie in Abschnitt 6.5 gezeigt wird, lässt sich das Optimierungsproblem (6.16) in geradliniger Art und Weise erweitern, wenn exogene Störungen und parametrische Unsicherheiten Berücksichtigung finden sollen.

\subsubsection{Linearisierende Variablentransformation}

Die Überführung von (6.16) in ein LMI-Problem wurde im Kontext der reinen Fehlerdetektion bereits in [149] vorgestellt. In [225] wurde der Ansatz für die Fehlerisolation angewendet und in zwei wesentlichen Punkten erweitert. Dies sind zum einen die Berücksichtigung von Eigenwertbereichen für die entworfene Filtermatrix $\boldsymbol{A}_{f}$ und zum anderen der Entwurf von Filtern reduzierter Ordnung. An dieser Stelle werden die Erweiterungen in ausführlicherer Form diskutiert und die Herleitung des Verfahrens um Beweise ergänzt, die weder in [149] noch [225] geliefert wurden. Die durchgeführte Variablentransformation geht auf eine Idee aus [194] zurück, wo sie zum Entwurf dynamischer Ausgangsrückführungen herangezogen wurde.

Zur Überführung in ein LMI-Problem wird die Filterordnung zunächst auf $n_{\text {filt }}=n+n_{\text {ref }}$ festgelegt, es wird dann von einem FIF voller Ordnung gesprochen. Diese Wahl wird im weiteren Verlauf der Herleitung begründet. In Abschnitt 6.4.2 wird eine Erweiterung des Verfahrens präsentiert, die den Entwurf von Fehlerisolationsfiltern reduzierter Ordnung erlaubt. Zunächst werden die Matrizen $\boldsymbol{P}$ und $\boldsymbol{P}^{-1}$ partitioniert und als

$$
\boldsymbol{P}=\left[\begin{array}{cc}
\boldsymbol{Y}_{11} & \boldsymbol{U} \\
\star & \boldsymbol{Y}_{22}
\end{array}\right], \boldsymbol{P}^{-1}=\left[\begin{array}{cc}
\boldsymbol{X}_{11} & \boldsymbol{W} \\
\star & \boldsymbol{X}_{22}
\end{array}\right]
$$

geschrieben. Dabei sind

$$
\begin{aligned}
\boldsymbol{Y}_{11}=\boldsymbol{Y}_{11}^{\top} & \in \mathbb{R}^{\left(n_{\mathrm{ref}}+n\right) \times\left(n_{\mathrm{ref}}+n\right)}, & \boldsymbol{Y}_{22}=\boldsymbol{Y}_{22}^{\top} & \in \mathbb{R}^{n_{\mathrm{fil}} \times n_{\mathrm{filt}}}, \\
\boldsymbol{X}_{11}=\boldsymbol{X}_{11}^{\top} & \in \mathbb{R}^{\left(n_{\mathrm{ref}}+n\right) \times\left(n_{\mathrm{ref}}+n\right)}, & \boldsymbol{X}_{22}=\boldsymbol{X}_{22}^{\top} & \in \mathbb{R}^{n_{\mathrm{filt}} \times n_{\mathrm{filt}}}, \\
\boldsymbol{U} & \in \mathbb{R}^{\left(n_{\mathrm{ref}}+n\right) \times n_{\mathrm{filt}}}, & \boldsymbol{W} & \in \mathbb{R}^{\left(n_{\mathrm{ref}}+n\right) \times n_{\mathrm{filt}}} .
\end{aligned}
$$


Grundlage für die Variablentransformation ist das folgende Lemma. Es liefert alternative LMI-Bedingungen für die Forderung $\boldsymbol{P} \succ \mathbf{0}$, also für die positive Definitheit der Matrix, bei $n_{\text {filt }}=n+n_{\text {ref }}$.

Lemma 6.2. Gegeben seien zwei symmetrische Matrizen $\boldsymbol{X}_{11}^{-1}=\boldsymbol{Z} \in \mathbb{R}^{\left(n+n_{\mathrm{ref}}\right) \times\left(n+n_{\mathrm{ref}}\right)} \quad$ und $\quad \boldsymbol{Y}_{11} \in \mathbb{R}^{\left(n+n_{\mathrm{ref}}\right) \times\left(n+n_{\mathrm{ref}}\right)}$, welche die Bedingungen

$$
\begin{aligned}
\boldsymbol{Z} & \succ \mathbf{0}, \\
\boldsymbol{Y}_{11}-\boldsymbol{Z} & \succ \mathbf{0}
\end{aligned}
$$

erfüllen. Weiterhin sei $\boldsymbol{P}$ eine invertierbare, gemäß (6.17) partitionierte Matrix und $n_{\mathrm{filt}}=n+n_{\mathrm{ref}}$. Dann ist $\boldsymbol{P}$ positiv definit.

Beweis. Durch rechtsseitige Multiplikation mit $\boldsymbol{Z}^{-1}$ folgt aus (6.18b) der Zusammenhang

$$
\boldsymbol{I}_{n+n_{\text {ref }}}-\boldsymbol{Y}_{11} \boldsymbol{Z}^{-1} \prec \mathbf{0} .
$$

Da $\boldsymbol{P} \boldsymbol{P}^{-1}=\boldsymbol{I}_{n_{\mathrm{filt}}+n+n_{\text {ref }}}$ gilt, folgen aus (6.17) die Gleichungen

$$
\begin{aligned}
\boldsymbol{Y}_{11} \boldsymbol{X}_{11}+\boldsymbol{U} \boldsymbol{W}^{\top} & =\boldsymbol{I}_{n+n_{\mathrm{ref}}}, \\
\boldsymbol{Y}_{11} \boldsymbol{W}+\boldsymbol{U} \boldsymbol{X}_{22} & =\mathbf{0}_{\left(n+n_{\mathrm{ref}}\right) \times n_{\mathrm{filt}}}, \\
\boldsymbol{U}^{\top} \boldsymbol{X}_{11}+\boldsymbol{Y}_{22} \boldsymbol{W}^{\top} & =\mathbf{0}_{n_{\mathrm{filt}} \times\left(n+n_{\mathrm{ref}}\right)}, \\
\boldsymbol{U}^{\top} \boldsymbol{W}+\boldsymbol{Y}_{22} \boldsymbol{X}_{22} & =\boldsymbol{I}_{n_{\mathrm{filt}}}
\end{aligned}
$$

und es ist

$$
\boldsymbol{U} \boldsymbol{W}^{\top}=\boldsymbol{I}_{n_{\mathrm{ref}}+n}-\boldsymbol{Y}_{11} \boldsymbol{X}_{11}=\boldsymbol{I}_{n+n_{\mathrm{ref}}}-\boldsymbol{Y}_{11} \boldsymbol{Z}^{-1} .
$$

Wegen (6.19) gilt somit $\boldsymbol{U} \boldsymbol{W}^{\top} \prec \mathbf{0}$, was rang $\left(\boldsymbol{U} \boldsymbol{W}^{\top}\right)=n+n_{\text {ref impliziert. }}$ Da es sich bei $\boldsymbol{U}$ und $\boldsymbol{W}^{\top}$ für $n_{\text {filt }}=n+n_{\text {ref }}$ um quadratische Matrizen handelt, ist damit $\operatorname{rang}(\boldsymbol{U})=\operatorname{rang}\left(\boldsymbol{W}^{\boldsymbol{\top}}\right)=n+n_{\text {ref }}$ gezeigt.

Aus (6.18b) folgt weiterhin $\boldsymbol{Y}_{11}-\boldsymbol{Z} \boldsymbol{Z}^{-1} \boldsymbol{Z} \succ \mathbf{0}$, sodass insgesamt aus (6.18) mithilfe des Schur-Komplement-Lemmas (Lemma 3.3) die LMI

$$
\left[\begin{array}{cc}
Z & Z \\
\star & Y_{11}
\end{array}\right] \succ \mathbf{0}
$$

folgt. Aus $\boldsymbol{P} \boldsymbol{P}^{-1}=\boldsymbol{I}_{n_{\mathrm{filt}}+n+n_{\mathrm{ref}}}$ und (6.20) beziehungsweise (6.21) ergeben sich die Beziehungen

$$
\begin{aligned}
\boldsymbol{Y}_{22} \boldsymbol{W}^{\top} & =-\boldsymbol{U}^{\top} \boldsymbol{X}_{11}=-\boldsymbol{U}^{\top} \boldsymbol{Z}^{-1}, \\
\boldsymbol{U} \boldsymbol{W}^{\top} \boldsymbol{Z} & =\left(\boldsymbol{U} \boldsymbol{W}^{\top} \boldsymbol{Z}\right)^{\top}=\boldsymbol{Z}-\boldsymbol{Y}_{11}, \\
\boldsymbol{Z} \boldsymbol{W} \boldsymbol{Y}_{22} \boldsymbol{W}^{\top} \boldsymbol{Z} & =\boldsymbol{Y}_{11}-\boldsymbol{Z},
\end{aligned}
$$


mit deren Hilfe sich (6.22) schreiben lässt als

$$
\begin{aligned}
& {\left[\begin{array}{cc}
\boldsymbol{Z} & \boldsymbol{Z} \\
\star & \boldsymbol{Y}_{11}
\end{array}\right]} \\
& =\left[\begin{array}{cc}
\boldsymbol{Y}_{11}+\boldsymbol{U} \boldsymbol{W}^{\top} \boldsymbol{Z}+\boldsymbol{Z} \boldsymbol{W} \boldsymbol{U}^{\top}+\boldsymbol{Z} \boldsymbol{W} \boldsymbol{Y}_{22} \boldsymbol{W}^{\top} \boldsymbol{Z} & \boldsymbol{Y}_{11}+\boldsymbol{Z} \boldsymbol{W} \boldsymbol{U}^{\top} \\
\star
\end{array}\right. \\
& =\left[\begin{array}{cc}
\boldsymbol{I}_{n+n_{\mathrm{ref}}} & \boldsymbol{Z} \boldsymbol{W} \\
\boldsymbol{I}_{n+n_{\mathrm{ref}}} & \mathbf{0}
\end{array}\right]\left[\begin{array}{cc}
\boldsymbol{Y}_{11} & \boldsymbol{U} \\
\star & \boldsymbol{Y}_{22}
\end{array}\right]\left[\begin{array}{cc}
\boldsymbol{I}_{n+n_{\mathrm{ref}}} & \boldsymbol{I}_{n+n_{\mathrm{ref}}} \\
\boldsymbol{W}^{\top} \boldsymbol{Z} & \mathbf{0}
\end{array}\right] .
\end{aligned}
$$

Führt man nun die Matrix

$$
\boldsymbol{\Psi}=\left[\begin{array}{cc}
\boldsymbol{I}_{n+n_{\mathrm{ref}}} & \boldsymbol{I}_{n+n_{\mathrm{ref}}} \\
\boldsymbol{W}^{\top} \boldsymbol{Z} & \mathbf{0}_{n_{\mathrm{filt}} \times\left(n_{\mathrm{ref}}+n\right)}
\end{array}\right]
$$

ein, so ergibt sich

$$
\left[\begin{array}{cc}
Z & Z \\
\star & Y_{11}
\end{array}\right]=\boldsymbol{\Psi}^{\top} \boldsymbol{P} \boldsymbol{\Psi} \succ \mathbf{0}
$$

Da $\boldsymbol{W}^{\top}$ wie gezeigt vollen Rang hat, handelt es sich bei der in (6.24) eingeführten Transformationsmatrix $\boldsymbol{\Psi}$ um eine reguläre Matrix, sodass $\boldsymbol{\Psi}^{\top} \boldsymbol{P} \boldsymbol{\Psi} \succ \mathbf{0}$ nach Lemma 3.2 gleichbedeutend ist mit $\boldsymbol{P} \succ \mathbf{0}$.

Aus obigen Überlegungen wird deutlich, dass die Wahl von $n_{\text {filt }}=n+n_{\text {ref }}$ entscheidend für die Äquivalenz

$$
\boldsymbol{P} \succ \mathbf{0} \Leftrightarrow\left\{\begin{array}{l}
\boldsymbol{Z} \succ \mathbf{0}, \\
\boldsymbol{Y}_{11}-\boldsymbol{Z} \succ \mathbf{0}
\end{array}\right.
$$

und damit für den weiteren Entwurf ist. Die Festlegung der Filterordnung garantiert, dass die eingeführte Transformationsmatrix $\boldsymbol{\Psi}$ regulär ist. Jedoch ist aus dem Entwurf von FIOs beziehungsweise Satz 6.1 bekannt, dass zum Beispiel für statisch isolierbare Strecken ein Filter der Ordnung $n_{\text {filt }}=n$ existiert, welches zu einer idealen Fehlerisolation führt. Dieser Fall wird in Abschnitt 6.4.2 näher beleuchtet, an dieser Stelle wird zunächst mit $n_{\text {filt }}=n+n_{\text {ref }}$ fortgefahren.

Durch Lemma 6.2 ist eine alternative Beschreibung für (6.16b) gefunden. Im Weiteren wird auch die Bedingung (6.16c) durch eine geeignete Kongruenztransformation in eine äquivalente LMI-Bedingung überführt. Dazu wird die Matrix $\boldsymbol{T}=\operatorname{diag}\left(\boldsymbol{\Psi}, \boldsymbol{I}_{n_{u}+n_{f}}, \boldsymbol{I}_{n_{f}}\right)$ herangezogen, die nach den Überlegungen aus Lemma 6.2 regulär ist. Nach Lemma 3.2 ist (6.16c) 
daher äquivalent $\mathrm{zu}$

$$
\begin{aligned}
& {\left[\begin{array}{ccc}
\boldsymbol{\Psi}^{\top} & \mathbf{0} & \mathbf{0} \\
\mathbf{0} & \boldsymbol{I} & \mathbf{0} \\
\mathbf{0} & \mathbf{0} & \boldsymbol{I}
\end{array}\right]\left[\begin{array}{ccc}
\mathrm{He}\left(\boldsymbol{P} \boldsymbol{A}_{\boldsymbol{z} \tilde{\boldsymbol{u}}}\right) & \boldsymbol{P} \boldsymbol{B}_{\boldsymbol{z} \tilde{\boldsymbol{u}}} & \boldsymbol{C}_{\boldsymbol{z} \tilde{\boldsymbol{u}}}^{\top} \\
\star & -\varepsilon \boldsymbol{I}_{n_{u}+n_{f}} & \boldsymbol{D}_{\boldsymbol{z} \tilde{\boldsymbol{u}}}^{\top} \\
\star & \star & -\varepsilon \boldsymbol{I}_{n_{f}}
\end{array}\right]\left[\begin{array}{ccc}
\boldsymbol{\Psi} & \mathbf{0} & \mathbf{0} \\
\mathbf{0} & \boldsymbol{I} & \mathbf{0} \\
\mathbf{0} & \mathbf{0} & \boldsymbol{I}
\end{array}\right]} \\
& =\left[\begin{array}{ccc}
\boldsymbol{\Psi}^{\top} \mathrm{He}\left(\boldsymbol{P} \boldsymbol{A}_{\boldsymbol{z} \tilde{\boldsymbol{u}}}\right) \boldsymbol{\Psi} & \boldsymbol{\Psi}^{\top} \boldsymbol{P} \boldsymbol{B}_{\boldsymbol{z} \tilde{\boldsymbol{u}}} & \boldsymbol{\Psi}^{\top} \boldsymbol{C}_{\boldsymbol{z} \tilde{\boldsymbol{u}}}^{\top} \\
\star & -\varepsilon \boldsymbol{I}_{n_{u}+n_{f}} & \boldsymbol{D}_{\boldsymbol{z} \tilde{\boldsymbol{u}}}^{\mathrm{T}} \\
\star & \star & -\varepsilon \boldsymbol{I}_{n_{f}}
\end{array}\right] \prec \mathbf{0} .
\end{aligned}
$$

Im Weiteren werden die einzelnen Blöcke der Blockmatrix in (6.26) näher analysiert, wozu abkürzend die konstanten Matrizen

$$
\begin{array}{ll}
\overline{\boldsymbol{A}}=\left[\begin{array}{cc}
\boldsymbol{A}_{\mathrm{ref}} & \mathbf{0} \\
\mathbf{0} & \boldsymbol{A}
\end{array}\right], \quad \overline{\boldsymbol{B}}=\left[\begin{array}{cc}
\boldsymbol{B}_{\mathrm{ref}, u} & \boldsymbol{B}_{\mathrm{ref}, f} \\
\boldsymbol{B} & \boldsymbol{E}_{a}
\end{array}\right], \\
\overline{\boldsymbol{C}}=\left[\begin{array}{ll}
\mathbf{0} & \boldsymbol{C}
\end{array}\right], & \overline{\boldsymbol{D}}=\left[\begin{array}{ll}
\boldsymbol{D} & \boldsymbol{E}_{s}
\end{array}\right]
\end{array}
$$

eingeführt werden.

Damit lässt sich für $\boldsymbol{P} \boldsymbol{A}_{\boldsymbol{z} \tilde{\boldsymbol{u}}}$ der Ausdruck

$$
\begin{aligned}
\boldsymbol{P} \boldsymbol{A}_{\boldsymbol{z} \tilde{\boldsymbol{u}}} & =\left[\begin{array}{cc}
\boldsymbol{Y}_{11} & \boldsymbol{U} \\
\boldsymbol{U}^{\top} & \boldsymbol{Y}_{22}
\end{array}\right]\left[\begin{array}{ccc}
\boldsymbol{A}_{\mathrm{ref}} & \mathbf{0} & \mathbf{0} \\
\mathbf{0} & \boldsymbol{A} & \mathbf{0} \\
\mathbf{0} & \boldsymbol{B}_{f, y} \boldsymbol{C} & \boldsymbol{A}_{f}
\end{array}\right] \\
& =\left[\begin{array}{cc}
\boldsymbol{Y}_{11} & \boldsymbol{U} \\
\boldsymbol{U}^{\top} & \boldsymbol{Y}_{22}
\end{array}\right]\left[\begin{array}{cc}
\overline{\boldsymbol{A}} & \mathbf{0} \\
\boldsymbol{B}_{f, y} \overline{\boldsymbol{C}} & \boldsymbol{A}_{f}
\end{array}\right] \\
& =\left[\begin{array}{cc}
\boldsymbol{Y}_{11} \overline{\boldsymbol{A}}+\boldsymbol{U} \boldsymbol{B}_{f, y} \overline{\boldsymbol{C}} & \boldsymbol{U} \boldsymbol{A}_{f} \\
\boldsymbol{U}^{\top} \overline{\boldsymbol{A}}+\boldsymbol{Y}_{22} \boldsymbol{B}_{f, y} \overline{\boldsymbol{C}} & \boldsymbol{Y}_{22} \boldsymbol{A}_{f}
\end{array}\right]
\end{aligned}
$$

herleiten, der beispielsweise in $\boldsymbol{Y}_{22} \boldsymbol{A}_{f}$ eine Bilinearität in den Variablen enthält. Wegen $\boldsymbol{A}^{\top} \operatorname{He}(\boldsymbol{B}) \boldsymbol{A}=\boldsymbol{A}^{\top} \boldsymbol{B} \boldsymbol{A}+\boldsymbol{A}^{\top} \boldsymbol{B}^{\top} \boldsymbol{A}=\operatorname{He}\left(\boldsymbol{A}^{\top} \boldsymbol{B} \boldsymbol{A}\right)$ ergibt sich aus (6.28)

$$
\begin{aligned}
& \boldsymbol{\Psi}^{\top} \operatorname{He}\left(\boldsymbol{P} \boldsymbol{A}_{\boldsymbol{z} \tilde{\boldsymbol{u}}}\right) \boldsymbol{\Psi} \\
& \quad=\operatorname{He}\left(\left[\begin{array}{cc}
\boldsymbol{I} & \boldsymbol{Z} \boldsymbol{W} \\
\boldsymbol{I} & \mathbf{0}
\end{array}\right]\left[\begin{array}{cc}
\boldsymbol{Y}_{11} \overline{\boldsymbol{A}}+\boldsymbol{U} \boldsymbol{B}_{f, y} \overline{\boldsymbol{C}} & \boldsymbol{U} \boldsymbol{A}_{f} \\
\boldsymbol{U}^{\top} \overline{\boldsymbol{A}}+\boldsymbol{Y}_{22} \boldsymbol{B}_{f, y} \overline{\boldsymbol{C}} & \boldsymbol{Y}_{22} \boldsymbol{A}_{f}
\end{array}\right]\left[\begin{array}{cc}
\boldsymbol{I} & \boldsymbol{I} \\
\boldsymbol{W}^{\top} \boldsymbol{Z} & \mathbf{0}
\end{array}\right]\right) .
\end{aligned}
$$

Unter Rückgriff auf (6.20) und (6.23) vereinfacht sich dies weiter zu

$$
\begin{aligned}
\boldsymbol{\Psi}^{\top} & \operatorname{He}\left(\boldsymbol{P} \boldsymbol{A}_{\boldsymbol{z} \tilde{\boldsymbol{u}}}\right) \boldsymbol{\Psi} \\
& =\mathrm{He}\left(\left[\begin{array}{cc}
\boldsymbol{Z} \overline{\boldsymbol{A}} & \mathbf{0} \\
\boldsymbol{Y}_{11} \overline{\boldsymbol{A}}+\boldsymbol{U} \boldsymbol{B}_{f, y} \overline{\boldsymbol{C}} & \boldsymbol{U} \boldsymbol{A}_{f}
\end{array}\right]\left[\begin{array}{cc}
\boldsymbol{I} & \boldsymbol{I} \\
\boldsymbol{W}^{\top} \boldsymbol{Z} & \mathbf{0}
\end{array}\right]\right) \\
& =\mathrm{He}\left(\left[\begin{array}{cc}
\boldsymbol{Z} \overline{\boldsymbol{A}} \\
\boldsymbol{Y}_{11} \overline{\boldsymbol{A}}+\boldsymbol{U} \boldsymbol{B}_{f, y} \overline{\boldsymbol{C}}+\boldsymbol{U} \boldsymbol{A}_{f} \boldsymbol{W}^{\top} \boldsymbol{Z} & \boldsymbol{Y}_{11} \overline{\boldsymbol{A}}+\boldsymbol{U} \boldsymbol{B}_{f, y} \overline{\boldsymbol{C}}
\end{array}\right]\right) .
\end{aligned}
$$


Führt man die Variablensubstitutionen

$$
\begin{aligned}
\hat{\boldsymbol{A}}_{f} & =\boldsymbol{U} \boldsymbol{A}_{f} \boldsymbol{W}^{\top} \boldsymbol{Z}, \\
\hat{\boldsymbol{B}}_{f, y} & =\boldsymbol{U} \boldsymbol{B}_{f, y}
\end{aligned}
$$

ein, so findet man schließlich für den oberen linken Block in (6.26) die Beziehung

$$
\boldsymbol{\Psi}^{\top} \operatorname{He}\left(\boldsymbol{P} \boldsymbol{A}_{\boldsymbol{z} \tilde{\boldsymbol{u}}}\right) \boldsymbol{\Psi}=\mathrm{He}\left(\left[\begin{array}{cc}
\boldsymbol{Z} \overline{\boldsymbol{A}} & \boldsymbol{Z} \overline{\boldsymbol{A}} \\
\boldsymbol{Y}_{11} \overline{\boldsymbol{A}}+\hat{\boldsymbol{B}}_{f, y} \overline{\boldsymbol{C}}+\hat{\boldsymbol{A}}_{f} & \boldsymbol{Y}_{11} \overline{\boldsymbol{A}}+\hat{\boldsymbol{B}}_{f, y} \overline{\boldsymbol{C}}
\end{array}\right]\right) .
$$

Bemerkenswert dabei ist, dass (6.30) linear in den Variablen $\boldsymbol{Z}, \boldsymbol{Y}_{11}, \hat{\boldsymbol{A}}_{f}$ und $\hat{\boldsymbol{B}}_{f, y}$ ist. Es treten also im Gegensatz zu (6.28) keine bilinearen Terme in den Entscheidungsvariablen mehr auf.

Für den mittleren oberen Block in (6.26) ergibt sich unter erneutem Rückgriff auf (6.20) und (6.27) der Zusammenhang

$$
\begin{aligned}
\boldsymbol{\Psi}^{\top} \boldsymbol{P} \boldsymbol{B}_{\boldsymbol{z} \tilde{\boldsymbol{u}}} & =\left[\begin{array}{cc}
\boldsymbol{I} & \boldsymbol{Z} \boldsymbol{W} \\
\boldsymbol{I} & \mathbf{0}
\end{array}\right]\left[\begin{array}{cc}
\boldsymbol{Y}_{11} & \boldsymbol{U} \\
\boldsymbol{U}^{\top} & \boldsymbol{Y}_{22}
\end{array}\right]\left[\begin{array}{cc}
\boldsymbol{B}_{\mathrm{ref}, u} & \boldsymbol{B}_{\mathrm{ref}, f} \\
\boldsymbol{B} & \boldsymbol{E}_{a} \\
\boldsymbol{B}_{f, u}+\boldsymbol{B}_{f, y} \boldsymbol{D} & \boldsymbol{B}_{f, y} \boldsymbol{E}_{s}
\end{array}\right] \\
& =\left[\begin{array}{cc}
\boldsymbol{Z} & \mathbf{0} \\
\boldsymbol{Y}_{11} & \boldsymbol{U}
\end{array}\right]\left[\begin{array}{cc}
\overline{\boldsymbol{B}} \\
\boldsymbol{B}_{f, u}\left[\begin{array}{ll}
\boldsymbol{I}_{n_{u}} & \mathbf{0}
\end{array}\right]+\boldsymbol{B}_{f, y} \overline{\boldsymbol{D}}
\end{array}\right] \\
& =\left[\begin{array}{cc}
\boldsymbol{Z} \overline{\boldsymbol{B}} \\
\left.\boldsymbol{Y}_{11} \overline{\boldsymbol{B}}+\hat{\boldsymbol{B}}_{f, u}\left[\begin{array}{ll}
\boldsymbol{I}_{n_{u}} & \mathbf{0}
\end{array}\right]+\hat{\boldsymbol{B}}_{f, y} \overline{\boldsymbol{D}}\right]
\end{array}\right]
\end{aligned}
$$

wobei analog zu (6.29b) die Variablensubstitution

$$
\hat{\boldsymbol{B}}_{f, u}=\boldsymbol{U} \boldsymbol{B}_{f, u}
$$

vorgenommen wurde. Auch (6.31) ist linear in den Entscheidungsvariablen $\boldsymbol{Z}, \boldsymbol{Y}_{11}, \hat{\boldsymbol{B}}_{f, u}$ und $\hat{\boldsymbol{B}}_{f, y}$.

Für den rechten oberen Block in (6.26) wird als weitere Variablensubstitution

$$
\begin{aligned}
\hat{\boldsymbol{C}}_{f} & =\boldsymbol{C}_{f} \boldsymbol{W}^{\top} \boldsymbol{Z}, \\
\hat{\boldsymbol{D}}_{f, y} & =\boldsymbol{D}_{f, y}
\end{aligned}
$$

eingeführt. Die Matrix $\hat{\boldsymbol{D}}_{f, y}$ entspricht $\boldsymbol{D}_{f, y}$, sie wird aus Gründen einer 
einheitlichen Notation eingeführt. Damit ist

$$
\begin{aligned}
\boldsymbol{\Psi}^{\top} \boldsymbol{C}_{\boldsymbol{z} \tilde{\boldsymbol{u}}}^{\top} & =\left(\boldsymbol{C}_{\boldsymbol{z} \tilde{\boldsymbol{u}}} \boldsymbol{\Psi}\right)^{\top} \\
& =\left(\left[\begin{array}{lll}
-\boldsymbol{C}_{\mathrm{ref}} & \boldsymbol{D}_{f, y} \boldsymbol{C} & \boldsymbol{C}_{f}
\end{array}\right]\left[\begin{array}{cc}
\boldsymbol{I} & \boldsymbol{I} \\
\boldsymbol{W}^{\top} \boldsymbol{Z} & \mathbf{0}
\end{array}\right]\right)^{\top} \\
& =\left[\begin{array}{lll}
-\boldsymbol{C}_{\mathrm{ref}} & \hat{\boldsymbol{D}}_{f, y} \boldsymbol{C}
\end{array}\right]+\hat{\boldsymbol{C}}_{f}\left[\begin{array}{ll}
-\boldsymbol{C}_{\mathrm{ref}} & \hat{\boldsymbol{D}}_{f, y} \boldsymbol{C}
\end{array}\right]^{\top} .
\end{aligned}
$$

Auch für diesen Block ist eine lineare Abhängigkeit von den Entscheidungsvariablen $\left(\hat{\boldsymbol{C}}_{f}\right.$ und $\left.\hat{\boldsymbol{D}}_{f, y}\right)$ gegeben.

Schließlich wird noch $\hat{\boldsymbol{D}}_{f, u}=\boldsymbol{D}_{f, u}$ eingeführt. Fasst man damit (6.30), (6.31) und (6.34) zusammen, so lässt sich für (6.26) die LMI

$$
\begin{aligned}
& {\left[\begin{array}{ccc}
\boldsymbol{\Sigma}_{11} & \boldsymbol{\Sigma}_{12} & \boldsymbol{\Sigma}_{13} \\
\star & -\varepsilon \boldsymbol{I}_{n_{u}+n_{f}} & \boldsymbol{\Sigma}_{23} \\
\star & \star & -\varepsilon \boldsymbol{I}_{n_{f}}
\end{array}\right] \prec \mathbf{0},} \\
& \boldsymbol{\Sigma}_{11}=\mathrm{He}\left(\left[\begin{array}{cc}
\boldsymbol{Z} \overline{\boldsymbol{A}} & \boldsymbol{Z} \overline{\boldsymbol{A}} \\
\boldsymbol{Y}_{11} \overline{\boldsymbol{A}}+\hat{\boldsymbol{B}}_{f, y} \overline{\boldsymbol{C}}+\hat{\boldsymbol{A}}_{f} & \boldsymbol{Y}_{11} \overline{\boldsymbol{A}}+\hat{\boldsymbol{B}}_{f, y} \overline{\boldsymbol{C}}
\end{array}\right]\right), \\
& \boldsymbol{\Sigma}_{12}=\left[\begin{array}{c}
\boldsymbol{Z} \overline{\boldsymbol{B}} \\
\left.\boldsymbol{Y}_{11} \overline{\boldsymbol{B}}+\hat{\boldsymbol{B}}_{f, u}\left[\begin{array}{ll}
\boldsymbol{I}_{n_{u}} & \mathbf{0}
\end{array}\right]+\hat{\boldsymbol{B}}_{f, y} \overline{\boldsymbol{D}}\right]
\end{array}\right]
\end{aligned}
$$

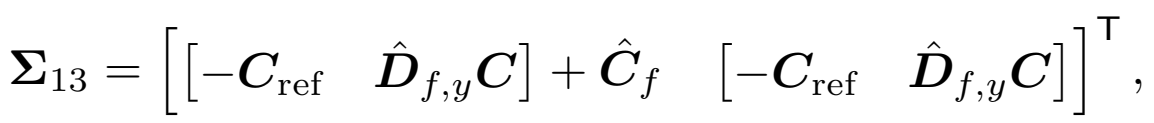

$$
\begin{aligned}
& \boldsymbol{\Sigma}_{23}=\left[\begin{array}{ll}
\hat{\boldsymbol{D}}_{f, u}+\hat{\boldsymbol{D}}_{f, y} \boldsymbol{D}-\boldsymbol{D}_{\mathrm{ref}, u} & \hat{\boldsymbol{D}}_{f, y} \boldsymbol{E}_{s}-\boldsymbol{D}_{\mathrm{ref}, f}
\end{array}\right]^{\top}
\end{aligned}
$$

schreiben. Durch (6.35) wird die gleiche Lösungsmenge beschrieben wie durch (6.16c). Entscheidend ist, dass es sich bei (6.35) um eine LMI in den Variablen $\boldsymbol{Z}, \boldsymbol{Y}_{11}, \hat{\boldsymbol{A}}_{f}, \hat{\boldsymbol{B}}_{f, u}, \hat{\boldsymbol{B}}_{f, y}, \hat{\boldsymbol{C}}_{f}, \hat{\boldsymbol{D}}_{f, u}$ und $\hat{\boldsymbol{D}}_{f, y}$ handelt. Im Sinne des Lösungsraumes wurde also die BMI (6.16c) verlustfrei in eine LMI überführt und das Lösbarkeitsproblem

finde $\boldsymbol{Z}, \boldsymbol{Y}_{11}, \hat{\boldsymbol{A}}_{f}, \hat{\boldsymbol{B}}_{f, u}, \hat{\boldsymbol{B}}_{f, y}, \hat{\boldsymbol{C}}_{f}, \hat{\boldsymbol{D}}_{f, u}, \hat{\boldsymbol{D}}_{f, y}$, sodass

stellt sicher, dass (6.13b) und (6.13c) eingehalten werden.

Durch (6.36) wird die Lösung des Entwurfsproblems in den transformierten Variablen beschrieben. Aus diesen Größen müssen für die Realisierung des Filters noch die Filtermatrizen durch eine Rücktransformation ermittelt werden. Entscheidend ist dabei Gleichung (6.21), also

$$
\boldsymbol{U} \boldsymbol{W}^{\top}=\boldsymbol{I}_{n_{\mathrm{ref}}+n}-\boldsymbol{Y}_{11} \boldsymbol{Z}^{-1}
$$


Wird wie zu Beginn des Abschnitts $n_{\text {filt }}=n_{\text {ref }}+n$ angesetzt, so sind $\boldsymbol{U}$ und $\boldsymbol{W}^{\top}$ quadratische Matrizen. Da $\boldsymbol{Z}$ und $\boldsymbol{Y}_{11}$ nach dem Lösen von (6.36) bekannt sind, ergeben sich $\boldsymbol{U}$ und $\boldsymbol{W}^{\top}$ durch eine Faktorisierung von $\boldsymbol{I}_{n_{\text {ref }+n}}-\boldsymbol{Y}_{11} \boldsymbol{Z}^{-1}$. Möglich sind hier zum Beispiel eine LU-Faktorisierung [150, Abschnitt 3.10] oder eine Singulärwertzerlegung. Da sowohl $\boldsymbol{U}$ als auch $\boldsymbol{W}^{\top}$ reguläre Matrizen sind, ist auch $\boldsymbol{I}_{n_{\mathrm{ref}}+n}-\boldsymbol{Y}_{11} \boldsymbol{Z}^{-1}$ regulär und die Zerlegung lässt sich schreiben als

$$
\boldsymbol{U} \boldsymbol{W}^{\top}=\boldsymbol{I}_{n_{\mathrm{ref}}+n}-\boldsymbol{Y}_{11} \boldsymbol{Z}^{-1}=\boldsymbol{\Gamma} \boldsymbol{\Xi} \boldsymbol{\Lambda}
$$

wobei $\boldsymbol{\Xi}$ eine Diagonalmatrix ist, die auf der Diagonalen die Singulärwerte von $\boldsymbol{I}_{n_{\text {ref }}+n}-\boldsymbol{Y}_{11} \boldsymbol{Z}^{-1}$ enthält. Da aufgrund von (6.19) keiner dieser Singulärwerte 0 ist, erhält man die gesuchten regulären Matrizen $\boldsymbol{U}$ und $\boldsymbol{W}^{\top}$ über

$$
\begin{aligned}
\boldsymbol{U} & =\boldsymbol{\Gamma} \boldsymbol{\Xi}^{1 / 2}, \\
\boldsymbol{W}^{\top} & =\boldsymbol{\Xi}^{1 / 2} \boldsymbol{\Lambda} .
\end{aligned}
$$

Anhand von (6.29), (6.32), (6.33) sowie $\boldsymbol{U}$ und $\boldsymbol{W}^{\boldsymbol{\top}}$ ergeben sich dann die gesuchten Filtermatrizen zu

$$
\begin{aligned}
\boldsymbol{A}_{f} & =\boldsymbol{U}^{-1} \hat{\boldsymbol{A}}_{f}\left(\boldsymbol{W}^{\top} \boldsymbol{Z}\right)^{-1}, \\
\boldsymbol{B}_{f, u} & =\boldsymbol{U}^{-1} \hat{\boldsymbol{B}}_{f, u}, \\
\boldsymbol{B}_{f, y} & =\boldsymbol{U}^{-1} \hat{\boldsymbol{B}}_{f, y} \\
\boldsymbol{C}_{f} & =\hat{\boldsymbol{C}}_{f}\left(\boldsymbol{W}^{\top} \boldsymbol{Z}\right)^{-1} \\
\boldsymbol{D}_{f, u} & =\hat{\boldsymbol{D}}_{f, u} \\
\boldsymbol{D}_{f, y} & =\hat{\boldsymbol{D}}_{f, y}
\end{aligned}
$$

Das Entwurfsverfahren zur Parametrierung der Filtermatrizen auf Basis eines zulässigen Referenzmodells ist in folgendem Satz zusammengefasst.

Satz 6.2 (LMI-basierter FIF-Entwurf für statisch stabil fehlerisolierbare Systeme). Gegeben sei eine Strecke der Form

$$
\begin{aligned}
& \dot{\boldsymbol{x}}=\boldsymbol{A} \boldsymbol{x}+\boldsymbol{B u}+\boldsymbol{E}_{a} \boldsymbol{f} \\
& \boldsymbol{y}=\boldsymbol{C} \boldsymbol{x}+\boldsymbol{D} \boldsymbol{u}+\boldsymbol{E}_{s} \boldsymbol{f}
\end{aligned}
$$

welche die Annahmen 6.1-6.3 erfüllt, sowie ein durch (6.9) und $\boldsymbol{B}_{\mathrm{ref}, u}=\mathbf{0}$, $\boldsymbol{D}_{\mathrm{ref}, u}=\mathbf{0}$ beschriebenes zulässiges Referenzmodell der Ordnung $n_{\mathrm{ref}}$. 
Dann gilt beim Einsatz eines FIFs der Form

$$
\begin{aligned}
\dot{\boldsymbol{x}}_{f} & =\boldsymbol{A}_{f} \boldsymbol{x}_{f}+\boldsymbol{B}_{f, u} \boldsymbol{u}+\boldsymbol{B}_{f, y} \boldsymbol{y}, \\
\boldsymbol{r} & =\boldsymbol{C}_{f} \boldsymbol{x}_{f}+\boldsymbol{D}_{f, u} \boldsymbol{u}+\boldsymbol{D}_{f, y} \boldsymbol{y}
\end{aligned}
$$

mit $n_{\mathrm{filt}}=n+n_{\mathrm{ref}}$ die Beziehung $\left\|\left[\boldsymbol{G}_{\boldsymbol{z} \boldsymbol{f}}(s) \boldsymbol{G}_{\boldsymbol{z u}}(s)\right]\right\|_{\infty}<\varepsilon$ für ein beliebig kleines $\varepsilon>0$, wenn die Filtermatrizen durch Lösen des LMI-Problems (6.36) und anschließende Rücktransformation gemäß (6.37)-(6.39) ermittelt werden.

Somit ist das Entwurfsproblem für statisch stabil fehlerisolierbare Systeme gelöst. Im Folgenden werden verschiedene Erweiterungen von Satz 6.2 vorgestellt.

\section{Erweiterungen durch Anpassung des Referenzmodells}

Während die Betrachtung statisch nicht isolierbarer und nicht minimalphasiger Systeme bei den beobachterbasierten Ansätzen mit einem erheblich vergrößerten Entwurfsaufwand verbunden ist (s. Abschnitte 4.7 und 4.8), ist dies beim Entwurf allgemeiner dynamischer Filter ohne Weiteres möglich. Dabei ist wie bereits erwähnt lediglich das Referenzmodell anzupassen, der Ansatz zur LMI-basierten Lösung des Model-MatchingProblems bleibt unverändert. Zu betonen ist, dass der Entwurf des dynamisch erweiterten Beobachters selbst nicht durchzuführen ist. Es muss lediglich eine Analyse hinsichtlich der Fehlerdetektionsmatrix $\boldsymbol{D}^{*}$ (beziehungsweise ihres Nullraumes) nach Abschnitt 4.7 und der invarianten Nullstellen des Systems nach Abschnitt 4.8 durchgeführt werden. Aus den Resultaten zu dynamisch erweiterten Fehlerisolationsbeobachtern ist dann bekannt, dass in jedem Fall ein stabiler DFIO für das System existiert. Somit ist auch sichergestellt, dass ein stabiles allgemeines Filter (mit entsprechend hoher Ordnung) existiert, welches die Fehlerisolation gewährleistet. Es gilt

Satz 6.3 (LMI-basierter FIF-Entwurf für statisch nicht isolierbare und nicht minimalphasige Systeme). Gegeben sei eine Strecke der Form

$$
\begin{aligned}
& \dot{\boldsymbol{x}}=\boldsymbol{A} \boldsymbol{x}+\boldsymbol{B u}+\boldsymbol{E}_{a} \boldsymbol{f} \\
& \boldsymbol{y}=\boldsymbol{C} \boldsymbol{x}+\boldsymbol{D} \boldsymbol{u}+\boldsymbol{E}_{s} \boldsymbol{f}
\end{aligned}
$$

welche die Annahmen 6.4-6.7 beziehungsweise 6.8-6.12 erfüllt, sowie ein durch (6.9) und $\boldsymbol{B}_{\mathrm{ref}, u}=\mathbf{0}, \boldsymbol{D}_{\mathrm{ref}, u}=\mathbf{0}$ beschriebenes zulässiges Referenz- 


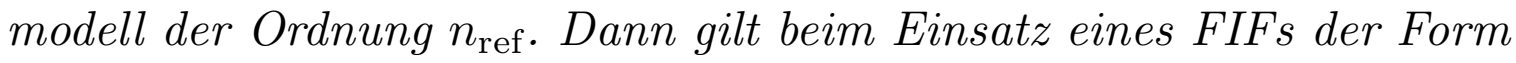

$$
\begin{aligned}
\dot{\boldsymbol{x}}_{f} & =\boldsymbol{A}_{f} \boldsymbol{x}_{f}+\boldsymbol{B}_{f, u} \boldsymbol{u}+\boldsymbol{B}_{f, y} \boldsymbol{y}, \\
\boldsymbol{r} & =\boldsymbol{C}_{f} \boldsymbol{x}_{f}+\boldsymbol{D}_{f, u} \boldsymbol{u}+\boldsymbol{D}_{f, y} \boldsymbol{y}
\end{aligned}
$$

mit $n_{\mathrm{filt}}=n+n_{\mathrm{ref}}$ die Beziehung $\left\|\left[\boldsymbol{G}_{\boldsymbol{z} \boldsymbol{f}}(s) \boldsymbol{G}_{\boldsymbol{z u}}(s)\right]\right\|_{\infty}<\varepsilon$ für ein beliebig kleines $\varepsilon>0$, wenn die Filtermatrizen durch Lösen des LMI-Problems (6.36) und anschließende Rücktransformation gemäß (6.37)-(6.39) ermittelt werden.

Bemerkenswert ist, dass (6.35a) impliziert, dass $\boldsymbol{A}_{f}$ eine Hurwitz-Matrix ist. Dies rührt daher, dass (6.35a) äquivalent zu (6.16c) ist. Demnach ist wegen [150, Proposition 8.2.7] die Matrix $\boldsymbol{A}_{\boldsymbol{z} \tilde{\boldsymbol{u}}}$ eine Hurwitz-Matrix. Da sie eine Blockdreiecksstrukturstruktur aufweist und $\boldsymbol{A}_{f}$ als Diagonalblock auftritt, ist dadurch die Stabilität des Filters auch für nicht minimalphasige Systeme sichergestellt.

Anzumerken ist überdies, dass sich Erweiterungen der Dynamik der Diagonalelemente von $\boldsymbol{G}_{\boldsymbol{r} \boldsymbol{f}}(s)$, wie sie in Abschnitt 4.6 für die beobachterbasierten Ansätze diskutiert wurden, mit der vorgestellten Methodik sehr einfach umsetzen lassen. Wird beispielsweise die näherungsweise Approximation der zeitlichen Ableitung eines Residuums zur Trendanalyse angestrebt, ist lediglich das Element $g_{i, i}(s)$ mit einem entsprechenden $\mathrm{DT}_{1}$ Glied zu ergänzen. Die erweiterte Dynamik des entsprechenden Diagonalelementes von $\boldsymbol{G}_{\mathrm{ref}, f}(s)$ führt dann zu einem Referenzmodell höherer Ordnung. Für dieses lässt sich das diskutierte LMI-basierte Model-MatchingVerfahren nach Satz 6.2 zum Auffinden der Filterparametrierung anwenden, wenn die Filterordnung entsprechend erhöht wird.

\section{Vorgabe von Eigenwertbereichen}

Wie beim Entwurf von FIOs für nichtquadratische Systeme weist ein mittels (6.36) entworfenes Fehlerisolationsfilter nichtsteuerbare Eigenwerte auf. Dies ist unmittelbar aus der Tatsache ersichtlich, dass das Referenzmodell die Ordnung $n_{\text {ref }}$ aufweist. Das entworfene Filter bildet bei einem zulässigen Referenzmodell dessen Ein-/Ausgangsverhalten beliebig genau ab, besitzt jedoch die Ordnung $n_{\text {filt }}=n+n_{\text {ref. }}$. Folglich müssen nichtsteuerbare Eigenwerte existieren. Auch im Falle von $\delta=n$ tritt im Gegensatz zu den beobachterbasierten Methoden eine interne Filterdynamik auf. Darüber hinaus treten nicht steuerbare Filtereigenwerte in nichtquadratischen Systemen mit $n_{y}>n_{f}$ auf. Das Entwurfsverfahren ist jedoch das gleiche wie für quadratische Systeme. 
Um nichtsteuerbare Eigenwerte nahe der imaginären Achse ebenso zu vermeiden wie betragsmäßig sehr große Filtereigenwerte oder Filtereigenwerte mit schlechter Dämpfung, werden wie bei den beobachterbasierten Methoden Eigenwertbereiche vorgegeben. Mithilfe des folgenden Lemmas lassen sich die Realteile der Filtereigenwerte nach oben beschränken, sodass alle Filtereigenwerte links einer Schranke $\alpha$ liegen.

Lemma 6.3. Beim Entwurf eines Fehlerisolationsfilters nach Satz 6.2 beziehungsweise 6.3 gilt für alle Filtereigenwerte $\lambda_{i}, i=1, \ldots, n_{\mathrm{filt}}$, die Beziehung $\mathfrak{R e}\left(\lambda_{i}\right)<\alpha$, wenn die zusätzliche $L M I$

$$
\mathrm{He}\left(\left[\begin{array}{cc}
\boldsymbol{Z} \overline{\boldsymbol{A}} & \boldsymbol{Z} \overline{\boldsymbol{A}} \\
\boldsymbol{Y}_{11} \overline{\boldsymbol{A}}+\hat{\boldsymbol{B}}_{f, y} \overline{\boldsymbol{C}}+\hat{\boldsymbol{A}}_{f} & \boldsymbol{Y}_{11} \overline{\boldsymbol{A}}+\hat{\boldsymbol{B}}_{f, y} \overline{\boldsymbol{C}}
\end{array}\right]\right)-2 \alpha\left[\begin{array}{cc}
\boldsymbol{Z} & \boldsymbol{Z} \\
\star & \boldsymbol{Y}_{11}
\end{array}\right] \prec \mathbf{0}
$$

erfüllt ist.

Beweis. Aus (6.25) und (6.30) folgen die Beziehungen

$$
\begin{aligned}
\operatorname{He}\left(\left[\begin{array}{cc}
\boldsymbol{Z} \overline{\boldsymbol{A}} & \boldsymbol{Z} \overline{\boldsymbol{A}} \\
\boldsymbol{Y}_{11} \overline{\boldsymbol{A}}+\hat{\boldsymbol{B}}_{f, y} \overline{\boldsymbol{C}}+\hat{\boldsymbol{A}}_{f} & \boldsymbol{Y}_{11} \overline{\boldsymbol{A}}+\hat{\boldsymbol{B}}_{f, y} \overline{\boldsymbol{C}}
\end{array}\right]\right) & =\boldsymbol{\Psi}^{\top} \operatorname{He}\left(\boldsymbol{P} \boldsymbol{A}_{\boldsymbol{z} \tilde{\boldsymbol{u}}}\right) \boldsymbol{\Psi} \\
{\left[\begin{array}{cc}
\boldsymbol{Z} & \boldsymbol{Z} \\
\star & \boldsymbol{Y}_{11}
\end{array}\right] } & =\boldsymbol{\Psi}^{\top} \boldsymbol{P} \boldsymbol{\Psi} .
\end{aligned}
$$

Somit lässt sich die LMI (6.40) schreiben als

$$
\boldsymbol{\Psi}^{\top}\left(\operatorname{He}\left(\boldsymbol{P} \boldsymbol{A}_{\boldsymbol{z} \tilde{\boldsymbol{u}}}\right)-2 \alpha \boldsymbol{P}\right) \boldsymbol{\Psi} \prec \mathbf{0} .
$$

Dies ist wegen der regulären Matrix $\boldsymbol{\Psi}$ nach Lemma 3.2 gleichbedeutend mit

$$
\mathrm{He}\left(\boldsymbol{P} \boldsymbol{A}_{\boldsymbol{z} \tilde{\boldsymbol{u}}}\right)-2 \alpha \boldsymbol{P} \prec \mathbf{0},
$$

was wiederum $\mathfrak{R e}\left(\lambda_{i}\right)<\alpha$ für alle $i=1, \ldots, n_{\text {filt }}$ sicherstellt.

Bei Verwendung von Lemma 6.3 ist zu beachten, dass die Matrix $\boldsymbol{A}_{\boldsymbol{z} \tilde{\boldsymbol{u}}}$ nicht nur das zu entwerfende Filter, sondern das Gesamtsystem bestehend aus Referenzmodell, Strecke und Filter beschreibt. Dementsprechend enthält sie die Eigenwerte aller drei Teilsysteme. Da die Eigenwerte von Referenzmodell und Strecke durch den Filterentwurf nicht verändert werden können, ist darauf zu achten, dass die entsprechenden Eigenwerte die Vorgabe $\mathfrak{R e}\left(\lambda_{i}\right)<\alpha$ erfüllen. Andernfalls ist (6.40) nicht lösbar. Im Sinne einer schnellen Fehlererkennung und -isolation wird man wie in Abschnitt 2.3 
erörtert die Dynamik des Referenzmodells schneller als die Streckendynamik auslegen. Somit stellt der langsamste Streckeneigenwert eine untere Schranke für den Parameter $\alpha$ in Lemma 6.3 dar.

In ähnlicher Art und Weise lassen sich betragsmäßig zu große Filtereigenwerte mithilfe des folgenden Lemmas verhindern.

Lemma 6.4. Beim Entwurf eines Fehlerisolationsfilters nach Satz 6.2 beziehungsweise 6.3 gilt für alle Filtereigenwerte $\lambda_{i}, i=1, \ldots, n_{\mathrm{filt}}$, die Beziehung $\left|\lambda_{i}\right|<\varrho$, wenn die zusätzliche LMI

$$
\left[\begin{array}{cccc}
-\varrho \boldsymbol{Z} & -\varrho \boldsymbol{Z} & \boldsymbol{Z} \overline{\boldsymbol{A}} & \boldsymbol{Z} \overline{\boldsymbol{A}} \\
\star & -\varrho \boldsymbol{Y}_{11} & \boldsymbol{Y}_{11} \overline{\boldsymbol{A}}+\hat{\boldsymbol{B}}_{f, y} \overline{\boldsymbol{C}}+\hat{\boldsymbol{A}}_{f} & \boldsymbol{Y}_{11} \overline{\boldsymbol{A}}+\hat{\boldsymbol{B}}_{f, y} \overline{\boldsymbol{C}} \\
\star & \star & -\varrho \boldsymbol{Z} & -\varrho \boldsymbol{Z} \\
\star & \star & \star & -\varrho \boldsymbol{Y}_{11}
\end{array}\right] \prec \mathbf{0}
$$

erfüllt ist.

Beweis. Aus (6.25) ist bekannt, dass

$$
\left[\begin{array}{cc}
Z & Z \\
\star & Y_{11}
\end{array}\right]=\Psi^{\top} P \Psi
$$

gilt. Weiterhin gilt nach (6.30) der Zusammenhang

$$
\left[\begin{array}{cc}
Z \bar{A} & Z \bar{A} \\
\boldsymbol{Y}_{11} \bar{A}+\hat{B}_{f, y} \bar{C}+\hat{A}_{f} & \boldsymbol{Y}_{11} \bar{A}+\hat{B}_{f, y} \bar{C}
\end{array}\right]=\Psi^{\top} \boldsymbol{P} \boldsymbol{A}_{z \tilde{u}} \boldsymbol{\Psi} .
$$

Dementsprechend lässt sich für die LMI (6.41) äquivalent

$$
\begin{aligned}
& {\left[\begin{array}{cc}
-\varrho \boldsymbol{\Psi}^{\top} \boldsymbol{P} \boldsymbol{\Psi} & \boldsymbol{\Psi}^{\top} \boldsymbol{P} \boldsymbol{A}_{\boldsymbol{z} \tilde{\boldsymbol{u}} \boldsymbol{\Psi}} \\
\star & -\varrho \boldsymbol{\Psi}^{\top} \boldsymbol{P} \boldsymbol{\Psi}
\end{array}\right]} \\
& \quad=\left[\begin{array}{cc}
\boldsymbol{\Psi}^{\top} & \mathbf{0} \\
\mathbf{0} & \boldsymbol{\Psi}^{\top}
\end{array}\right]\left[\begin{array}{cc}
-\varrho \boldsymbol{P} & \boldsymbol{P} \boldsymbol{A}_{\boldsymbol{z} \tilde{\boldsymbol{u}}} \\
\star & -\varrho \boldsymbol{P}
\end{array}\right]\left[\begin{array}{cc}
\boldsymbol{\Psi} & \mathbf{0} \\
\mathbf{0} & \boldsymbol{\Psi}
\end{array}\right] \prec \mathbf{0}
\end{aligned}
$$

schreiben. Da $\boldsymbol{\Psi}$ regulär ist, impliziert dies gemäß Lemma 3.2

$$
\left[\begin{array}{cc}
-\varrho \boldsymbol{P} & \boldsymbol{P} \boldsymbol{A}_{\boldsymbol{z} \tilde{\boldsymbol{u}}} \\
\star & -\varrho \boldsymbol{P}
\end{array}\right] \prec \mathbf{0}
$$

wodurch $\left|\lambda_{i}\right|<\varrho$ für alle $i=1, \ldots, n_{\text {filt }}$ sichergestellt ist.

Auch in der Anwendung dieses Lemmas ist darauf zu achten, dass die Eigenwerte der Strecke und des Referenzmodells die Bedingung $\left|\lambda_{i}\right|<\varrho$ 
erfüllen. Der betragsmäßig größte Eigenwert von Strecke und Referenzmodell stellt demnach eine untere Schranke für $\varrho$ dar, andernfalls ist (6.41) nicht lösbar.

Wie beim Beobachterentwurf lässt sich auch für Fehlerisolationsfilter eine Winkelbeschränkung für den zulässigen Sektor der Filtereigenwerte vorgeben. Dazu dient

Lemma 6.5. Beim Entwurf eines Fehlerisolationsfilters nach Satz 6.2 beziehungsweise 6.3 gilt für alle Filtereigenwerte $\lambda_{i}, i=1, \ldots, n_{\mathrm{filt}}$, die Beziehung $\left|\mathfrak{I m}\left(\lambda_{i}\right)\right|<\tan (\phi) \cdot\left|\mathfrak{R e}\left(\lambda_{i}\right)\right|$, wenn die zusätzliche $L M I$

$$
\begin{aligned}
& \boldsymbol{\Phi}=\left[\begin{array}{cc}
\boldsymbol{\Phi}_{11} & \boldsymbol{\Phi}_{12} \\
\star & \boldsymbol{\Phi}_{11}
\end{array}\right] \prec \mathbf{0}, \\
& \boldsymbol{\Phi}_{11}=\tan (\phi) \cdot \operatorname{He}\left(\left[\begin{array}{cc}
\boldsymbol{Z}_{11} \overline{\boldsymbol{A}}+\hat{\boldsymbol{B}}_{f, y} \overline{\boldsymbol{C}}+\hat{\boldsymbol{A}}_{f} & \boldsymbol{Y}_{11} \overline{\boldsymbol{A}}+\hat{\boldsymbol{B}}_{f, y} \overline{\boldsymbol{C}}
\end{array}\right]\right), \quad(6.42 \mathrm{~b}) \\
& \boldsymbol{\Phi}_{12}=\left[\begin{array}{cc}
-\boldsymbol{Z} \overline{\boldsymbol{A}}+\overline{\boldsymbol{A}}^{\top} \boldsymbol{Z} & -\boldsymbol{Z} \overline{\boldsymbol{A}}+\overline{\boldsymbol{A}}^{\top} \boldsymbol{Y}_{11}+\overline{\boldsymbol{C}}^{\top} \hat{\boldsymbol{B}}_{f, y}^{\top}+\hat{\boldsymbol{A}}_{f}^{\top} \\
-\boldsymbol{Y}_{11} \overline{\boldsymbol{A}}+\overline{\boldsymbol{A}}^{\top} \boldsymbol{Z}-\hat{\boldsymbol{B}}_{f, y} \overline{\boldsymbol{C}}-\hat{\boldsymbol{A}}_{f} & -\boldsymbol{Y}_{11} \overline{\boldsymbol{A}}+\overline{\boldsymbol{A}}^{\top} \boldsymbol{Y}_{11}-\hat{\boldsymbol{B}}_{f, y} \overline{\boldsymbol{C}}^{\mathrm{C}} \overline{\boldsymbol{C}}^{\mathrm{B}} \hat{\boldsymbol{B}}_{f, y}^{\mathrm{\top}}
\end{array}\right]
\end{aligned}
$$

erfüllt ist.

Beweis. Analog zu Lemma 6.4 ergibt sich unter Rückgriff auf die Beziehungen (6.20), dass

$$
\begin{aligned}
{\left[\begin{array}{cc}
\boldsymbol{\Phi}_{11} & \boldsymbol{\Phi}_{12} \\
\star & \boldsymbol{\Phi}_{11}
\end{array}\right]=} & \\
& {\left[\begin{array}{cc}
\boldsymbol{\Psi}^{\top} & \mathbf{0} \\
\mathbf{0} & \boldsymbol{\Psi}^{\top}
\end{array}\right]\left[\begin{array}{cc}
\tan (\phi) \cdot \operatorname{He}\left(\boldsymbol{P} \boldsymbol{A}_{\boldsymbol{z} \tilde{\boldsymbol{u}}}\right) & -\boldsymbol{P} \boldsymbol{A}_{\boldsymbol{z} \tilde{\boldsymbol{u}}}+\boldsymbol{A}_{\boldsymbol{z} \tilde{\boldsymbol{u}}}^{\top} \boldsymbol{P} \\
\star & \tan (\phi) \cdot \operatorname{He}\left(\boldsymbol{P} \boldsymbol{A}_{\boldsymbol{z} \tilde{\boldsymbol{u}}}\right)
\end{array}\right]\left[\begin{array}{cc}
\boldsymbol{\Psi} & \mathbf{0} \\
\mathbf{0} & \boldsymbol{\Psi}
\end{array}\right] }
\end{aligned}
$$

ist. Da $\boldsymbol{\Psi}$ regulär ist, impliziert $\boldsymbol{\Phi} \prec \mathbf{0}$ gemäß Lemma 3.2

$$
\left[\begin{array}{cc}
\tan (\phi) \cdot \operatorname{He}\left(\boldsymbol{P} \boldsymbol{A}_{\boldsymbol{z} \tilde{\boldsymbol{u}}}\right) & -\boldsymbol{P} \boldsymbol{A}_{\boldsymbol{z} \tilde{\boldsymbol{u}}}+\boldsymbol{A}_{\boldsymbol{z} \tilde{\boldsymbol{u}}}^{\top} \boldsymbol{P} \\
\star & \tan (\phi) \cdot \operatorname{He}\left(\boldsymbol{P} \boldsymbol{A}_{\boldsymbol{z} \tilde{\boldsymbol{u}}}\right)
\end{array}\right] \prec \mathbf{0}
$$

wodurch $\left|\mathfrak{I m}\left(\lambda_{i}\right)\right|<\tan (\phi) \cdot\left|\mathfrak{R e}\left(\lambda_{i}\right)\right|$ für alle $i=1, \ldots, n_{\text {filt }}$ sichergestellt ist.

Es sei auch hier darauf hingewiesen, dass die Streckeneigenwerte die Bedingung an die Dämpfung erfüllen müssen, da die LMI aus Lemma 6.5 sonst nicht lösbar ist. 
Zusammenfassend lassen sich also Satz 6.2 beziehungsweise 6.3 erweitern $\mathrm{Zu}$

Satz 6.4 (LMI-basierter FIF-Entwurf mit Einschränkung der Filtereigenwerte). Gegeben sei eine Strecke der Form

$$
\begin{aligned}
& \dot{\boldsymbol{x}}=\boldsymbol{A} \boldsymbol{x}+\boldsymbol{B u}+\boldsymbol{E}_{a} \boldsymbol{f} \\
& \boldsymbol{y}=\boldsymbol{C} \boldsymbol{x}+\boldsymbol{D} \boldsymbol{u}+\boldsymbol{E}_{s} \boldsymbol{f}
\end{aligned}
$$

welche die Annahmen 6.1-6.3 (6.4-6.7 beziehungsweise 6.8-6.12) erfüllt, sowie ein durch (6.9) und $\boldsymbol{B}_{\mathrm{ref}, u}=\mathbf{0}, \boldsymbol{D}_{\mathrm{ref}, u}=\mathbf{0}$ beschriebenes zulässiges Referenzmodell der Ordnung $n_{\mathrm{ref}}$. Dann gilt beim Einsatz eines FIFs der Form

$$
\begin{aligned}
\dot{\boldsymbol{x}}_{f} & =\boldsymbol{A}_{f} \boldsymbol{x}_{f}+\boldsymbol{B}_{f, u} \boldsymbol{u}+\boldsymbol{B}_{f, y} \boldsymbol{y}, \\
\boldsymbol{r} & =\boldsymbol{C}_{f} \boldsymbol{x}_{f}+\boldsymbol{D}_{f, u} \boldsymbol{u}+\boldsymbol{D}_{f, y} \boldsymbol{y}
\end{aligned}
$$

mit $n_{\mathrm{filt}}=n+n_{\mathrm{ref}}$ die Beziehung $\left\|\left[\boldsymbol{G}_{\boldsymbol{z} \boldsymbol{f}}(s) \boldsymbol{G}_{\boldsymbol{z u}}(s)\right]\right\|_{\infty}<\varepsilon$ für ein beliebig kleines $\varepsilon>0$, und für alle Filtereigenwerte $\lambda_{i}, i=1, \ldots, n_{\mathrm{filt}}$, gilt $\mathfrak{R e}\left(\lambda_{i}\right)<\alpha,\left|\lambda_{i}\right|<\varrho$ und $\left|\mathfrak{I m}\left(\lambda_{i}\right)\right|<\tan (\phi) \cdot\left|\mathfrak{R e}\left(\lambda_{i}\right)\right|$, wenn die Filtermatrizen durch Lösen des LMI-Problems

finde $\boldsymbol{Z}, \boldsymbol{Y}_{11}, \hat{\boldsymbol{A}}_{f}, \hat{\boldsymbol{B}}_{f, u}, \hat{\boldsymbol{B}}_{f, y}, \hat{\boldsymbol{C}}_{f}, \hat{\boldsymbol{D}}_{f, u}, \hat{\boldsymbol{D}}_{f, y}$, sodass (6.18), (6.35), (6.40)- (6.42)

und anschließende Rücktransformation gemäß (6.37)-(6.39) ermittelt werden.

$\mathrm{Zu}$ beachten ist, dass auch eventuell zu kompensierende invariante Nullstellen des Systems in dem durch $\alpha, \varrho$ und $\phi$ definierten Bereich liegen müssen, damit das Entwurfsproblem lösbar ist.

\section{Berechnung einer gut konditionierten Lösung}

Rein mathematisch betrachtet lässt sich mittels Satz 6.4 aufgrund von Lemma 6.1 stets eine Lösung für das Entwurfsproblem (6.13) finden. Bei der Rücktransformation kann es jedoch in (6.21) bei der Faktorisierung von $\boldsymbol{I}_{n+n_{\text {ref }}}-\boldsymbol{Y}_{11} \boldsymbol{Z}^{-1}$ in $\boldsymbol{U}$ und $\boldsymbol{W}^{\top}$ zu numerischen Problemen kommen. Dies ist dann der Fall, wenn $\boldsymbol{I}_{n+n_{\text {ref }}}-\boldsymbol{Y}_{11} \boldsymbol{Z}^{-1}$ schlecht konditioniert ist, das heißt wenn das Verhältnis des größten zum kleinsten Singulärwert der Matrix sehr groß ist. Dieses Phänomen tritt offensichtlich dann auf, wenn 
der größte Eigenwert der negativ definiten Matrix $\boldsymbol{I}_{n+n_{\text {ref }}}-\boldsymbol{Y}_{11} \boldsymbol{Z}^{-1}$ nahe der imaginären Achse liegt. Wegen

$$
\lambda_{\max }\left(\boldsymbol{I}_{n+n_{\text {ref }}}-\boldsymbol{Y}_{11} \boldsymbol{Z}^{-1}\right)=1-\lambda_{\min }\left(\boldsymbol{Y}_{11} \boldsymbol{Z}^{-1}\right)
$$

wird wie in [194] angestrebt, den kleinsten Eigenwert der positiv definiten Matrix $\boldsymbol{Y}_{11} \boldsymbol{Z}^{-1}$ zu maximieren. Zwar wird damit nicht die Konditionszahl von $\boldsymbol{I}_{n+n_{\text {ref }}}-\boldsymbol{Y}_{11} \boldsymbol{Z}^{-1}$ direkt minimiert, es ist jedoch plausibel, dass sehr schlechte Konditionen vermieden werden können. Um den kleinsten Eigenwert von $\boldsymbol{Y}_{11} \boldsymbol{Z}^{-1}$ mittels LMI-Bedingungen abschätzen zu können, wird das folgende Lemma verwendet.

Lemma 6.6. Gegeben seien die symmetrischen Matrizen $\boldsymbol{Y}_{11}$ und $\boldsymbol{Z} \succ \mathbf{0}$. Dann gilt $\lambda_{\min }\left(\boldsymbol{Y}_{11} \boldsymbol{Z}^{-1}\right)>\beta^{2}$ mit $\beta>0$ genau dann, wenn

$$
\left[\begin{array}{cc}
\boldsymbol{Y}_{11} & \beta \boldsymbol{Z} \\
\star & \boldsymbol{Z}
\end{array}\right] \succ \mathbf{0}
$$

Beweis. Nach [14, Lemma 8.4.1] gilt $\lambda_{\min }\left(\boldsymbol{Y}_{11} \boldsymbol{Z}^{-1}\right)>\beta^{2}$ genau dann, wenn $\beta^{2} \boldsymbol{I}_{n+n_{\text {ref }}} \prec \boldsymbol{Y}_{11} \boldsymbol{Z}^{-1}$. Da $\boldsymbol{Y}_{11} \boldsymbol{Z}^{-1}$ positiv definit ist, ist $\beta>0$ und es ergibt sich durch Äquivalenzumformungen

$$
\begin{aligned}
\lambda_{\min }\left(\boldsymbol{Y}_{11} \boldsymbol{Z}^{-1}\right)>\beta^{2} & \Leftrightarrow \beta^{2} \boldsymbol{I}_{n+n_{\mathrm{ref}}} \prec \boldsymbol{Y}_{11} \boldsymbol{Z}^{-1} \\
& \Leftrightarrow \boldsymbol{Y}_{11}-\beta^{2} \boldsymbol{Z} \succ \mathbf{0} \\
& \Leftrightarrow \boldsymbol{Y}_{11}-\beta \boldsymbol{Z} \cdot \boldsymbol{Z}^{-1} \cdot \beta \boldsymbol{Z} \succ \mathbf{0} \\
& \Leftrightarrow \boldsymbol{Z} \succ \mathbf{0},\left[\begin{array}{cc}
\boldsymbol{Y}_{11} & \beta \boldsymbol{Z} \\
\star & \boldsymbol{Z}
\end{array}\right] \succ \mathbf{0},
\end{aligned}
$$

wobei im letzten Schritt das Lemma 3.3 angewendet wurde und $\boldsymbol{Z}$ nach Voraussetzung positiv definit ist.

Das Lösbarkeitsproblem aus Satz 6.4 weist eine unendliche Anzahl von Lösungen auf. Dies ist offensichtlich, da man die Dynamik (6.1) beliebigen Koordinatentransformationen unterwerfen kann, ohne das Ein-/Ausgangsverhalten zu verändern. Entsprechend obiger Überlegungen wird nun aus dieser Menge diejenige Lösung ausgewählt, welche den kleinsten Eigenwert von $\boldsymbol{Y}_{11} \boldsymbol{Z}^{-1}$ maximiert. Dies führt auf das Optimierungsproblem

$$
\begin{aligned}
& \underset{\boldsymbol{Z}, \boldsymbol{Y}_{11}, \hat{\boldsymbol{A}}_{f}, \hat{\boldsymbol{B}}_{f, u}, \hat{\boldsymbol{B}}_{f, y}, \hat{\boldsymbol{C}}_{f}, \hat{\boldsymbol{D}}_{f, u}, \hat{\boldsymbol{D}}_{f, y}}{\operatorname{maximiere}} \beta, \text { sodass } \\
& (6.18),(6.35),(6.40)-(6.43)
\end{aligned}
$$


$\mathrm{Zu}$ beachten ist, dass es sich dabei aufgrund des Produkttermes $\beta \boldsymbol{Z}$ in (6.43) um ein Pseudo-LMI-Problem handelt. Es lässt sich jedoch durch eine Bisektion in $\beta$ effizient als LMI-Problem lösen. Für die Durchführung des Entwurfes wird also eine kleine Schranke $\varepsilon$ gewählt. ${ }^{2)}$ Die Lösung des LMI-Problems (6.44) führt dann auf Matrizen $\boldsymbol{Z}, \boldsymbol{Y}_{11}, \hat{\boldsymbol{A}}_{f}, \hat{\boldsymbol{B}}_{f, u}, \hat{\boldsymbol{B}}_{f, y}, \hat{\boldsymbol{C}}_{f}$, $\hat{\boldsymbol{D}}_{f, u}$ und $\hat{\boldsymbol{D}}_{f, y}$, aus denen sich mithilfe von (6.37) bis (6.39) die gesuchte Filterparametrierung $\boldsymbol{A}_{f}, \boldsymbol{B}_{f, u}, \boldsymbol{B}_{f, y}, \boldsymbol{C}_{f}, \boldsymbol{D}_{f, u}, \boldsymbol{D}_{f, y}$ ergibt.

\subsubsection{Fehlerisolationsfilter reduzierter Ordnung}

Während in [149] stets $n_{\text {filt }}=n_{\text {ref }}+n$ vorausgesetzt wird und dies auch bei den bisherigen Betrachtungen im Rahmen dieser Arbeit angesetzt wurde, motivieren die Resultate aus Kapitel 4 auch den Ansatz $n_{\text {filt }}<n_{\text {ref }}+n$. Aus den beobachterbasierten Ansätzen ist bekannt, dass für statisch stabil fehlerisolierbare Systeme ein allgemeines dynamisches Filter $n$-ter Ordnung besteht, welches für die nominale, störungsfreie Systemdynamik eine ideale Fehlerisolation gewährleistet. Es existiert in einem solchen Fall also für $n_{\text {filt }}=n<n_{\text {ref }}+n$ eine Parametrierung, welche das LMI-Problem aus Satz 6.4 mit einem beliebig kleinen $\varepsilon$ löst.

In der Tat lässt sich Lemma 6.2 auf den Fall $n_{\text {filt }} \leq n+n_{\text {ref }}$ erweitern. Es gilt

Lemma 6.7. Gegeben seien zwei symmetrische Matrizen $\boldsymbol{Z} \in \mathbb{R}^{\left(n+n_{\mathrm{ref}}\right) \times\left(n+n_{\mathrm{ref}}\right)} \quad$ und $\quad \boldsymbol{Y}_{11} \in \mathbb{R}^{\left(n+n_{\mathrm{ref}}\right) \times\left(n+n_{\mathrm{ref}}\right)}$, welche die $B e$ dingungen

$$
\begin{aligned}
\boldsymbol{Z} & \succ \mathbf{0}, \\
{\left[\begin{array}{cc}
\boldsymbol{Z} & \boldsymbol{Z} \\
\star & \boldsymbol{Y}_{11}
\end{array}\right] } & \succeq \mathbf{0}, \\
\operatorname{rang}\left(\left[\begin{array}{cc}
\boldsymbol{Z} & \boldsymbol{Z} \\
\star & \boldsymbol{Y}_{11}
\end{array}\right]\right) & =n_{\text {filt }}+n+n_{\text {ref }}
\end{aligned}
$$

erfüllen. Weiterhin sei $\boldsymbol{P}$ eine invertierbare, gemäß (6.17) partitionierte Matrix und $n_{\text {filt }} \leq n+n_{\text {ref. }}$ Dann ist $\boldsymbol{P}$ positiv definit.

Beweis. Die Bedingung (6.45a) impliziert $\operatorname{rang}(\boldsymbol{Z})=n+n_{\text {ref. }}$ Nach [14, Proposition 2.8.3] ist daher

$$
\operatorname{rang}\left(\left[\begin{array}{cc}
\boldsymbol{Z} & \boldsymbol{Z} \\
\star & \boldsymbol{Y}_{11}
\end{array}\right]\right)=n+n_{\mathrm{ref}}+\operatorname{rang}\left(\boldsymbol{Y}_{11}-\boldsymbol{Z} \boldsymbol{Z}^{-1} \boldsymbol{Z}\right)
$$

${ }^{2)}$ Je nach numerischer Konditionierung haben sich in untersuchten Beispielen Werte im Bereich $\varepsilon \in[0,0001 ; 0,01]$ bewährt. 
woraus mit $(6.45 \mathrm{c})$

$$
\operatorname{rang}\left(\boldsymbol{Y}_{11}-\boldsymbol{Z}\right)=n_{\text {filt }}
$$

folgt. Da die Multiplikation mit einer regulären Matrix den Rang nicht verändert, ergibt sich mit (6.21) weiterhin

$$
\begin{aligned}
\operatorname{rang}\left(\boldsymbol{Y}_{11}-\boldsymbol{Z}\right) & =\operatorname{rang}\left(\boldsymbol{Z}-\boldsymbol{Y}_{11}\right) \\
& =\operatorname{rang}\left(\boldsymbol{I}_{n+n_{\text {ref }}}-\boldsymbol{Y}_{11} \boldsymbol{Z}^{-1}\right) \\
& =\operatorname{rang}\left(\boldsymbol{U} \boldsymbol{W}^{\boldsymbol{\top}}\right)=n_{\text {filt }} .
\end{aligned}
$$

Da $\boldsymbol{U} \in \mathbb{R}^{\left(n+n_{\text {ref }}\right) \times n_{\text {filt }}}$ und $\boldsymbol{W}^{\top} \in \mathbb{R}^{n_{\text {filt }} \times\left(n+n_{\text {ref }}\right)}$ gilt und $n_{\text {filt }} \leq n+n_{\text {ref }}$ ist, $\operatorname{muss} \operatorname{rang}(\boldsymbol{U})=\operatorname{rang}\left(\boldsymbol{W}^{\top}\right)=n_{\text {filt }}$ gelten. Somit gilt für die Transformationsmatrix $\boldsymbol{\Psi} \in \mathbb{R}^{\left(n_{\text {filt }}+n+n_{\text {ref }}\right) \times 2\left(n+n_{\text {ref }}\right)}$ die Rangbeziehung $\operatorname{rang}(\boldsymbol{\Psi})=n_{\text {filt }}+n+n_{\text {ref. }}$ Analog zu Lemma 6.2 gilt wegen (6.45)

$$
\left[\begin{array}{cc}
Z & Z \\
\star & Y_{11}
\end{array}\right]=\Psi^{\top} \boldsymbol{P} \Psi \succeq \mathbf{0}
$$

wobei im Gegensatz zum Fall $n_{\text {filt }}=n+n_{\text {ref }}$ lediglich positive Semidefinitheit gilt.

Für die weitere Analyse wird eine Singulärwertzerlegung von $\boldsymbol{\Psi}$ durchgeführt. Aufgrund der Dimension der Matrix und $\operatorname{rang}(\boldsymbol{\Psi})=n_{\text {ref }}+n+n_{\text {filt }}$ ergibt sie sich zu

$$
\boldsymbol{\Psi}=\boldsymbol{\Gamma}\left[\boldsymbol{\Xi} \mathbf{0}_{\left(n_{\text {ref }}+n+n_{\text {filt }}\right) \times\left(n+n_{\text {ref }}-n_{\text {filt }}\right)}\right] \boldsymbol{\Lambda}
$$

mit unitären Matrizen $^{3)} \quad \boldsymbol{\Gamma} \in \mathbb{R}^{\left(n_{\text {ref }}+n+n_{\text {filt }}\right) \times\left(n_{\text {ref }}+n+n_{\text {filt }}\right)} \quad$ und $\boldsymbol{\Lambda} \in \mathbb{R}^{2\left(n+n_{\text {ref }}\right) \times 2\left(n+n_{\text {ref }}\right)}$. Die Singulärwerte von $\boldsymbol{\Psi}$ finden sich in der Matrix $\boldsymbol{\Xi}=\operatorname{diag}\left(\sigma_{1}(\boldsymbol{\Psi}), \ldots, \sigma_{n_{\text {ref }}+n+n_{\text {filt }}}(\boldsymbol{\Psi})\right)$. Damit ergibt sich

$$
\begin{aligned}
\boldsymbol{\Psi}^{\top} \boldsymbol{P} \boldsymbol{\Psi} & =\boldsymbol{\Lambda}^{\top}\left[\begin{array}{c}
\boldsymbol{\Xi}^{\top} \\
\mathbf{0}
\end{array}\right] \boldsymbol{\Gamma}^{\top} \boldsymbol{P} \boldsymbol{\Gamma}\left[\begin{array}{ll}
\boldsymbol{\Xi} & \mathbf{0}
\end{array}\right] \boldsymbol{\Lambda} \\
& =\boldsymbol{\Lambda}^{\top}\left[\begin{array}{cc}
\boldsymbol{\Xi}^{\top} \boldsymbol{\Gamma}^{\top} \boldsymbol{P} \boldsymbol{\Gamma} \boldsymbol{\Xi} & \mathbf{0} \\
\mathbf{0} & \mathbf{0}_{\left(n+n_{\mathrm{ref}}-n_{\mathrm{filt}}\right) \times\left(n+n_{\mathrm{ref}}-n_{\mathrm{filt}}\right)}
\end{array}\right] \boldsymbol{\Lambda} .
\end{aligned}
$$

Da es sich bei $\boldsymbol{\Lambda}$ und $\boldsymbol{\Gamma}$ um unitäre Matrizen handelt, lässt sich daraus

3) Eine quadratische Matrix heißt unitär, wenn ihre Spaltenvektoren orthonormal, das heißt orthogonal zueinander und normiert, sind. 


$$
\begin{aligned}
& \operatorname{rang}\left(\boldsymbol{\Psi}^{\top} \boldsymbol{P} \boldsymbol{\Psi}\right)=\operatorname{rang}\left(\boldsymbol{\Lambda}^{\top}\left[\begin{array}{cc}
\boldsymbol{\Xi}^{\top} \boldsymbol{\Gamma}^{\top} \boldsymbol{P} \boldsymbol{\Gamma} \boldsymbol{\Xi} & \mathbf{0} \\
\mathbf{0} & \mathbf{0}_{\left(n+n_{\mathrm{ref}}-n_{\mathrm{filt}}\right) \times\left(n+n_{\mathrm{ref}}-n_{\mathrm{filt}}\right)}
\end{array}\right] \boldsymbol{\Lambda}\right) \\
& =\operatorname{rang}\left(\left[\begin{array}{cc}
\boldsymbol{\Xi}^{\top} \boldsymbol{\Gamma}^{\top} \boldsymbol{P} \boldsymbol{\Gamma} \boldsymbol{\Xi} & \mathbf{0} \\
\mathbf{0} & \mathbf{0}_{\left(n+n_{\mathrm{ref}}-n_{\mathrm{filt}}\right) \times\left(n+n_{\mathrm{ref}}-n_{\mathrm{filt}}\right)}
\end{array}\right]\right) \\
& =\operatorname{rang}\left(\boldsymbol{\Xi}^{\top} \boldsymbol{\Gamma}^{\top} \boldsymbol{P} \boldsymbol{\Gamma} \boldsymbol{\Xi}\right) \\
& =\operatorname{rang}(\boldsymbol{P})
\end{aligned}
$$

ableiten. Aus (6.45c), (6.46) und $\boldsymbol{\Psi}^{\top} \boldsymbol{P} \boldsymbol{\Psi} \succeq \mathbf{0}$ folgt somit $\boldsymbol{P} \succ \mathbf{0}$.

Der weitere Entwurf, das heißt die Kongruenztransformation von (6.16c), bleibt gegenüber dem Entwurf von FIFs voller Ordnung unverändert. In Abwandlung von (6.36) ist also das Lösbarkeitsproblem

finde $\boldsymbol{Z}, \boldsymbol{Y}_{11}, \hat{\boldsymbol{A}}_{f}, \hat{\boldsymbol{B}}_{f, u}, \hat{\boldsymbol{B}}_{f, y}, \hat{\boldsymbol{C}}_{f}, \hat{\boldsymbol{D}}_{f, u}, \hat{\boldsymbol{D}}_{f, y}$, sodass

zu betrachten. Besondere Beachtung verdient dabei die Rangbeschränkung (6.45c). Sie bedingt, dass es sich bei (6.47) um ein nichtkonvexes Problem handelt. Da jedoch wie bereits ausführlich diskutiert für ein statisch stabil fehlerisolierbares System eine ideale Fehlerisolation für $n_{\text {filt }}=n$ möglich ist, ist die Lösbarkeit von (6.47) für beliebig kleine $\varepsilon$ sichergestellt. Dies rechtfertigt es, spezielle Solver für rangbeschränkte LMI-Probleme einzusetzen. Ein Beispiel hierfür ist LMIRANK [162], basierend auf [163].

Nach Lösen des rangbeschränkten Problems (6.47) verbleibt die Rücktransformation zur Gewinnung der Filtermatrizen. Es gilt also eine Faktorisierung von

$$
\boldsymbol{U} \boldsymbol{W}^{\top}=\boldsymbol{I}_{n+n_{\mathrm{ref}}}-\boldsymbol{Y}_{11} \boldsymbol{Z}^{-1}
$$

zu finden, wobei $\boldsymbol{U}, \boldsymbol{W} \in \mathbb{R}^{\left(n+n_{\text {ref }}\right) \times n_{\text {filt }}}$ nichtquadratische Matrizen sind und $\boldsymbol{I}_{n+n_{\text {ref }}}-\boldsymbol{Y}_{11} \boldsymbol{Z}^{-1}$ wegen der Rangbeschränkung eine singuläre Matrix ist. Zu diesem Zweck wird ähnlich wie in (6.37) eine Singulärwertzerlegung von $\boldsymbol{I}_{n+n_{\text {ref }}}-\boldsymbol{Y}_{11} \boldsymbol{Z}^{-1}$ vorgenommen. Wegen $\operatorname{rang}\left(\boldsymbol{I}_{n+n_{\text {ref }}}-\boldsymbol{Y}_{11} \boldsymbol{Z}^{-1}\right)=n_{\text {filt }}$ lässt sich diese Zerlegung für FIFs reduzierter Ordnung darstellen als

$$
\boldsymbol{I}_{n+n_{\mathrm{ref}}}-\boldsymbol{Y}_{11} \boldsymbol{Z}^{-1}=\boldsymbol{\Gamma}\left[\begin{array}{cc}
\boldsymbol{\Xi}_{\mathrm{lo}} & \mathbf{0} \\
\mathbf{0} & \mathbf{0}_{\left(n+n_{\mathrm{ref}}-n_{\mathrm{filt}}\right) \times\left(n+n_{\mathrm{ref}}-n_{\mathrm{filt}}\right)}
\end{array}\right] \boldsymbol{\Lambda} .
$$

Partitioniert man $\boldsymbol{\Gamma}=\left[\begin{array}{ll}\boldsymbol{\Gamma}_{l} & \boldsymbol{\Gamma}_{r}\end{array}\right]$ und $\boldsymbol{\Lambda}=\left[\begin{array}{ll}\boldsymbol{\Lambda}_{o}^{\top} & \boldsymbol{\Lambda}_{u}^{\top}\end{array}\right]^{\top}$, so ergibt sich

$$
\boldsymbol{U} \boldsymbol{W}^{\top}=\boldsymbol{I}_{n+n_{\mathrm{ref}}}-\boldsymbol{Y}_{11} \boldsymbol{X}_{11}=\boldsymbol{\Gamma}_{l} \boldsymbol{\Xi}_{\mathrm{lo}} \boldsymbol{\Lambda}_{o}=\boldsymbol{\Gamma}_{l} \boldsymbol{\Xi}_{\mathrm{lo}}^{1 / 2} \cdot \boldsymbol{\Xi}_{\mathrm{lo}}^{1 / 2} \boldsymbol{\Lambda}_{o}
$$


Der letzte Umformungsschritt in (6.49) ist dabei zulässig, da $\boldsymbol{\Xi}_{\text {lo }}$ definitionsgemäß eine invertierbare Matrix ist, sodass $\boldsymbol{\Xi}_{\text {lo }}^{1 / 2}$ existiert. Ähnlich wie für FIFs voller Ordnung (vgl. (6.38)) findet man die gesuchte Faktorisierung durch

$$
\begin{aligned}
\boldsymbol{U} & =\boldsymbol{\Gamma}_{l} \boldsymbol{\Xi}_{\mathrm{lo}}^{1 / 2}, \\
\boldsymbol{W}^{\top} & =\boldsymbol{\Xi}_{\mathrm{lo}}^{1 / 2} \boldsymbol{\Lambda}_{o} .
\end{aligned}
$$

Unter Rückgriff auf die Pseudoinversen lässt sich dann mit (6.50) die Rücktransformation

$$
\begin{aligned}
\boldsymbol{A}_{f} & =\boldsymbol{U}^{+} \hat{\boldsymbol{A}}_{f}\left(\boldsymbol{W}^{\top} \boldsymbol{Z}\right)^{+}, \\
\boldsymbol{B}_{f, u} & =\boldsymbol{U}^{+} \hat{\boldsymbol{B}}_{f, u}, \\
\boldsymbol{B}_{f, y} & =\boldsymbol{U}^{+} \hat{\boldsymbol{B}}_{f, y} \\
\boldsymbol{C}_{f} & =\hat{\boldsymbol{C}}_{f}\left(\boldsymbol{W}^{\top} \boldsymbol{Z}\right)^{+}, \\
\boldsymbol{D}_{f, u} & =\hat{\boldsymbol{D}}_{f, u} \\
\boldsymbol{D}_{f, y} & =\hat{\boldsymbol{D}}_{f, y}
\end{aligned}
$$

durchführen.

Die Erweiterungen zur Vorgabe von Eigenwertbereichen gelten gegenüber dem Entwurf von FIFs voller Ordnung unverändert, ebenso ist eine Betrachtung statisch nicht isolierbarer und nicht minimalphasiger Systeme möglich. Zusammenfassend gilt der folgende Entwurfssatz:

Satz 6.5 (LMI-basierter Entwurf von FIFs reduzierter Ordnung mit Einschränkung der Filtereigenwerte). Gegeben sei eine Strecke der Form

$$
\begin{aligned}
& \dot{\boldsymbol{x}}=\boldsymbol{A} \boldsymbol{x}+\boldsymbol{B} \boldsymbol{u}+\boldsymbol{E}_{a} \boldsymbol{f}, \\
& \boldsymbol{y}=\boldsymbol{C} \boldsymbol{x}+\boldsymbol{D} \boldsymbol{u}+\boldsymbol{E}_{s} \boldsymbol{f},
\end{aligned}
$$

welche die Annahmen 6.1-6.3 (6.4-6.7 beziehungsweise 6.8-6.12) erfüllt, sowie ein durch (6.9) und $\boldsymbol{B}_{\mathrm{ref}, u}=\mathbf{0}, \boldsymbol{D}_{\mathrm{ref}, u}=\mathbf{0}$ beschriebenes zulässiges Referenzmodell. Dann gilt beim Einsatz eines FIFs der Form

$$
\begin{aligned}
\dot{\boldsymbol{x}}_{f} & =\boldsymbol{A}_{f} \boldsymbol{x}_{f}+\boldsymbol{B}_{f, u} \boldsymbol{u}+\boldsymbol{B}_{f, y} \boldsymbol{y}, \\
\boldsymbol{r} & =\boldsymbol{C}_{f} \boldsymbol{x}_{f}+\boldsymbol{D}_{f, u} \boldsymbol{u}+\boldsymbol{D}_{f, y} \boldsymbol{y}
\end{aligned}
$$

mit $n_{\text {filt }}=n\left(n_{\text {filt }}=n+n_{\zeta}\right.$, wobei $n_{\zeta}$ die für einen stabilen DFIO benötigte Ordnung ist) die Beziehung $\left\|\left[\boldsymbol{G}_{\boldsymbol{z} \boldsymbol{f}}(s) \boldsymbol{G}_{\boldsymbol{z u}}(s)\right]\right\|_{\infty}<\varepsilon$ für ein beliebig 
kleines $\varepsilon>0$, und für alle Filtereigenwerte $\lambda_{i}, i=1, \ldots, n_{\text {filt }}$, gilt $\mathfrak{R e}\left(\lambda_{i}\right)<\alpha,\left|\lambda_{i}\right|<\varrho$ und $\left|\mathfrak{I m}\left(\lambda_{i}\right)\right|<\tan (\phi) \cdot\left|\mathfrak{R e}\left(\lambda_{i}\right)\right|$, wenn die Filtermatrizen durch Lösen des rangbeschränkten LMI-Problems

finde $\boldsymbol{Z}, \boldsymbol{Y}_{11}, \hat{\boldsymbol{A}}_{f}, \hat{\boldsymbol{B}}_{f, u}, \hat{\boldsymbol{B}}_{f, y}, \hat{\boldsymbol{C}}_{f}, \hat{\boldsymbol{D}}_{f, u}, \hat{\boldsymbol{D}}_{f, y}$, sodass (6.35), (6.40)-(6.42), (6.45)

und anschließende Rücktransformation gemäß (6.48)-(6.51) ermittelt werden.

\subsubsection{Beispiele}

Um die Anwendung des FIF-Entwurfs zu verdeutlichen, werden verschiedene Beispielsysteme betrachtet. Zunächst wird mit dem Hydrauliksystem aus Abschnitt 4.9.3 ein nichtquadratisches System betrachtet. Weiterhin wird ein FIF für die DHC-2 Beaver aus Abschnitt 4.7.5 entworfen. Dadurch wird die Anwendbarkeit des Entwurfs für statisch nicht isolierbare Systeme demonstriert.

Entscheidend ist, dass in beiden Beispielen im Gegensatz zu den beobachterbasierten Methoden das Vorgehen beim Entwurf gleich ist. Am Ende der Systemanalyse steht ein zulässiges Referenzmodell sowie die Filterordnung. Der Entwurf des Filters erfolgt dann stets mittels Satz 6.4 beziehungsweise Satz 6.5.

\section{Hydrauliksystem}

Das Hydrauliksystem aus Abschnitt 4.9.3 (s. Seite 161) ist ein nichtquadratisches System mit $n_{y}=3>2=n_{f}$ und $\delta_{1}=3, \delta_{2}=1$. Dementsprechend ist ein zulässiges Referenzmodell gegeben durch

$$
\boldsymbol{G}_{\mathrm{ref}, \boldsymbol{r} \boldsymbol{f}}(s)=\left[\begin{array}{cc}
\frac{7,5}{s^{3}+6 s^{2}+11,75 s+7,5} & 0 \\
0 & \frac{3}{s+3}
\end{array}\right],
$$

wodurch wie beim FIO-Entwurf aus Abschnitt 4.9.3 die Pole $\lambda_{B_{1,1}}=-1,5$, $\lambda_{B_{1,2}}=-2, \lambda_{B_{1,3}}=-2,5$ und $\lambda_{B_{2,1}}=-3$ vorgegeben werden und eine stationäre Verstärkung von 1 für beide Kanäle festgelegt wird. Die beiden Diagonalelemente von $\boldsymbol{G}_{\mathrm{ref}, \boldsymbol{r} \boldsymbol{f}}(s)$ können jeweils durch ein SISO-System in Steuerungsnormalform realisiert werden. Wie in (6.9) in Abschnitt 6.3 beschrieben werden die Systemmatrizen jeweils zu Blockdiagonalmatrizen zusammengefügt. Somit erhält man eine Zustandsraumrealisierung des Referenzmodells als 


$$
\begin{aligned}
& \boldsymbol{A}_{\mathrm{ref}}=\left[\begin{array}{ccc:c}
0 & 1 & 0 & 0 \\
0 & 0 & 1 & 0 \\
\hdashline-7,5 & -11,75 & -6 & 0 \\
\hdashline 0 & 0 & 0 & -3
\end{array}\right], \quad \boldsymbol{B}_{\mathrm{ref}, f}=\left[\begin{array}{c:c}
0 & 0 \\
0 & 0 \\
1 & 0 \\
\hdashline 0 & 1
\end{array}\right], \\
& \boldsymbol{C}_{\mathrm{ref}}=\left[\begin{array}{ccc:c}
7,5 & 0 & 0 & 0 \\
\hdashline 0 & 0 & 0 & 3
\end{array}\right], \quad \boldsymbol{D}_{\mathrm{ref}, f}=\left[\begin{array}{c:c}
0 & 0 \\
\hdashline 0 & 0
\end{array}\right] .
\end{aligned}
$$

Wegen $n=5$ und $n_{\text {ref }}=4$ ergibt sich die Filterordnung $n_{\text {filt }}=9$. Unter Rückgriff auf Satz 6.4 erhält man schließlich die in Anhang C.3.1 angegebene Filterparametrierung, wobei die Schranke $\varepsilon=0,0001$ und zur Beschränkung der Filtereigenwerte $\alpha=-0,1, \varrho=12$ und $\phi=45^{\circ}$ vorgegeben wurden. Die Filtereigenwerte ergeben sich zu $\lambda_{1}=-1,5, \lambda_{2}=-2$, $\lambda_{3}=-2,5, \lambda_{4}=-3, \lambda_{5}=-4,905, \lambda_{6 / 7}=-8,509 \pm 5,793 j, \lambda_{8}=-8,621$ und $\lambda_{9}=-10,225$, die Eigenwertbeschränkungen werden also eingehalten.

Um zu demonstrieren, dass sich auch die für FIOs in Abschnitt 4.6 beschriebene Erweiterung der Dynamik der Diagonalelemente bei FIFs sehr einfach umsetzen lässt, wird ein zusätzlicher Pol in den zweiten Übertragungskanal eingebracht. Gibt man ihn als $\lambda_{B_{2,2}}=-4$ vor, so ergibt sich das modifizierte Referenzmodell

$$
\boldsymbol{G}_{\mathrm{ref}, \boldsymbol{r} \boldsymbol{f}}(s)=\left[\begin{array}{cc}
\frac{7,5}{s^{3}+6 s^{2}+11,75 s+7,5} & 0 \\
0 & \frac{12}{s^{2}+7 s+12}
\end{array}\right] .
$$

Aus den Überlegungen zum FIO-Entwurf mit erweiterten Diagonalelementen ist bekannt, dass ein Filter der Ordnung $n_{\text {filt }}=n+1$ existiert, welches dieses Übertragungsverhalten abbilden kann. Die Anwendung von Satz 6.5 führt auf die in Anhang C.3.1 angegebene Parametrierung eines FIFs der Ordnung $n+1=6$, der eine Fehlerisolation mit dynamischer Erweiterung des zweiten Übertagungskanals gewährleistet.

\section{DHC-2 Beaver}

Die DHC-2 Beaver wurde bereits in Abschnitt 4.7.5 als Beispiel für ein statisch nicht isolierbares System eingeführt. Nun wird für das gleiche System ein FIF entworfen. Aus Abschnitt 4.7.5 ist bekannt, dass für das System $\delta_{1}=\delta_{2}=1$ gilt und $\boldsymbol{D}^{*}$ singulär ist. Weiterhin weist das System eine invariante Nullstelle bei $\eta=-58,48$ auf. Wie die Analysemethode aus Abschnitt 4.7 zeigt, kann ein dynamischer FIO der Ordnung 1 entworfen werden, wobei für die Diagonalelemente von $\boldsymbol{G}_{\boldsymbol{r} \boldsymbol{f}}(s)$ jeweils $\mathrm{PT}_{2}$-Glieder vorgegeben werden können. Mit den gleichen Polen wie in Abschnitt 4.7.5, 
das heißt $\lambda_{B_{1,1}}=-2, \lambda_{B_{1,2}}=-7, \lambda_{B_{2,1}}=-3,2$ und $\lambda_{B_{2,2}}=-3,5$, ist somit ein zulässiges Referenzmodell gegeben durch

$$
\boldsymbol{G}_{\mathrm{ref}, \boldsymbol{r} \boldsymbol{f}}(s)=\left[\begin{array}{cc}
\frac{14}{s^{2}+9 s+14} & 0 \\
0 & \frac{11,2}{s^{2}+6,7 s+11,2}
\end{array}\right] .
$$

Weiterhin wird die Filterordnung zu $n_{\text {filt }}=n+1$ festgelegt, da aus dem beobachterbasierten Ansatz bekannt ist, dass für diese Ordnung der Entwurf eines FIFs möglich ist. Es wird wie im zweiten Teil des vorangegangenen Beispiels des Hydrauliksystems ein FIF reduzierter Ordnung entworfen.

Mithilfe dieser Vorgaben lässt sich mittels Satz 6.5 unmittelbar die Parametrierung des FIFs berechnen. Wählt man als Entwurfsparameter die Schranke $\varepsilon=0,0001$, so ergibt sich die in Anhang C.3.1 aufgeführte Filterparametrierung. Die Analyse der Filtereigenwerte ergibt

$$
\operatorname{spec}\left(\boldsymbol{A}_{f}\right)=\{-2,00 ;-3,20 ;-3,50 ;-7,00 ;-58,48\} .
$$

Die Filtereigenwerte setzen sich also erwartungsgemäß zusammen aus den vorgegebenen Polen von $\boldsymbol{G}_{\mathrm{ref}, \boldsymbol{r} \boldsymbol{f}}(s)$ sowie der invarianten Nullstelle des Systems. Auf eine Darstellung des zeitlichen Verlaufs der generierten Residuen wird hier verzichtet, da er deckungsgleich zu Abbildung 4.12 ist.

Soll wie in Abschnitt 4.8.5 die invariante Nullstelle des Systems bei $\eta=-58,48$ unkompensiert bleiben, so kann das System wie in Bemerkung 4.9 beschrieben mehrfach erweitert werden. Für das resultierende Gesamtsystem kann dann ein FIO entworfen werden, ohne die Nullstelle zu kompensieren. Mittels eines FIFs lässt sich das gleiche Entwurfsziel mit wesentlich geringerem Aufwand erreichen. Es muss gemäß der Analyse in Abschnitt 4.8.5 lediglich für jeden Übertragungskanal ein zusätzlicher Pol im Referenzmodell vorgegeben werden, wobei die invariante Nullstelle des Systems dann als Nullstelle in beiden Übertragungskanälen auftritt. Um das gleiche Übertragungsverhalten zu erreichen wie in Abschnitt 4.8.5 werden erneut $\lambda_{B_{1,1}}=-2, \lambda_{B_{1,2}}=-4, \lambda_{B_{1,3}}=-7$ und $\lambda_{B_{2,1}}=-3$, $\lambda_{B_{2,2}}=-3,2$ und $\lambda_{B_{2,3}}=-3,5$ gewählt, woraus sich das Referenzmodell

$$
\boldsymbol{G}_{\mathrm{ref}, \boldsymbol{r} \boldsymbol{f}}(s)=\left[\begin{array}{cc}
\frac{0,9575(s+58,48)}{s^{3}+13 s^{2}+50 s+56} & 0 \\
0 & \frac{0,5745(s+58,48)}{s^{3}+9,7 s^{2}+31,3 s+33,6}
\end{array}\right] .
$$

ergibt. Der Entwurf eines FIFs der Ordnung $n_{\text {filt }}=n+2=6$, bei dem die Filtereigenwerte durch $\alpha=0,01$ und $\varrho=15$ beschränkt werden, führt auf die ebenfalls in Anhang C.3.1 angegebene Filterparametrierung. Sie liefert das gleiche Übertragungsverhalten wie der FIO für das doppelt erweiterte System und ist direkt implementierbar. 


\subsubsection{Fazit}

Fehlerisolationsfilter stellen eine strukturelle Verallgemeinerung von Fehlerisolationsbeobachtern dar. Ihr Entwurf lässt sich nach der Auslegung eines zulässigen Referenzmodells auf ein konvexes Optimierungsproblem mit LMI-Nebenbedingungen zurückführen. Die beobachterbasierten Methoden aus Kapitel 4 dienen der Konstruktion eines Referenzmodells, wobei der Beobachterentwurf nicht durchgeführt werden muss. Während auch FIOs wie gezeigt für verschiedene Fehlerisolationsprobleme verwendet werden können, ist der wesentliche Vorteil von FIFs gegenüber FIOs in dem einheitlichen Entwurfsschema zu sehen. Wie die Beispiele zeigen gestaltet sich der Entwurf darüber hinaus insbesondere für fortgeschrittene Problemstellungen wie die Fehlerisolation in statisch nicht isolierbaren Systemen gegenüber FIOs wesentlich einfacher.

\subsection{Robustheitsaspekte beim Entwurf von Fehlerisolationsfiltern}

Auf Basis der Ergebnisse des vorangegangenen Abschnittes werden im Weiteren Robustheitsaspekte beim Entwurf von Fehlerisolationsfiltern betrachtet. Entsprechend dem Aufbau des Kapitels 5 zur Robustheit von Fehlerisolationsbeobachtern wird dabei zunächst die Unterdrückung exogener Störungen untersucht, bevor der Einfluss parametrischer Unsicherheiten betrachtet wird.

\subsubsection{Robuste Fehlerisolationsfilter bei nominaler Systemdynamik}

Zunächst werden wie in Kapitel 5 störungsbehaftete Systeme betrachtet, deren Systemmatrizen exakt bekannt sind. Es gilt also

$$
\begin{aligned}
& \dot{\boldsymbol{x}}=\boldsymbol{A} \boldsymbol{x}+\boldsymbol{B u}+\boldsymbol{E}_{a} \boldsymbol{f}+\boldsymbol{B}_{d} \boldsymbol{d}, \\
& \boldsymbol{y}=\boldsymbol{C} \boldsymbol{x}+\boldsymbol{D} \boldsymbol{u}+\boldsymbol{E}_{s} \boldsymbol{f}+\boldsymbol{D}_{d} \boldsymbol{d} .
\end{aligned}
$$

Neben der strukturellen Anforderung $\boldsymbol{G}_{\boldsymbol{r u}}(s)=\mathbf{0}$ und $\boldsymbol{G}_{\boldsymbol{r} \boldsymbol{f}}(s)=\boldsymbol{G}_{\mathrm{ref}, \boldsymbol{r} \boldsymbol{f}}(s)$ aus dem vorigen Abschnitt gilt es nun zusätzlich, den Einfluss der Störungen auf die generierten Residuen zu minimieren. Dieser Einfluss lässt sich wie aus Kapitel 5 bekannt formal mittels $\left\|\boldsymbol{G}_{\boldsymbol{r} \boldsymbol{d}}(s)\right\|_{\infty}$ beschreiben. 
Einen intuitiven Ansatz dazu beschreibt das Optimierungsproblem

$$
\begin{array}{ll}
\operatorname{minimiere} & \left\|\boldsymbol{G}_{\boldsymbol{r} \boldsymbol{d}}(s)\right\|_{\infty}, \text { sodass } \\
\left\|\left[\begin{array}{ll}
\boldsymbol{G}_{\boldsymbol{r} \boldsymbol{u}} & \boldsymbol{G}_{\boldsymbol{r} \boldsymbol{f}}(s)-\boldsymbol{G}_{\mathrm{ref}, \boldsymbol{r} \boldsymbol{f}}(s)
\end{array}\right]\right\|_{\infty}<\varepsilon .
\end{array}
$$

Analog zu (6.11) und (6.12) lässt sich die Wirkung exogener Störungen $\boldsymbol{d}$ auf den Performance-Ausgang $\boldsymbol{z}=\boldsymbol{r}-\boldsymbol{r}_{\text {ref }}$ beschreiben durch

$$
\begin{aligned}
\boldsymbol{G}_{\boldsymbol{z d}}(s) & =\boldsymbol{C}_{\boldsymbol{z} \boldsymbol{d}}\left(s \boldsymbol{I}_{n_{\mathrm{ref}}+n+n_{\mathrm{filt}}}-\boldsymbol{A}_{\boldsymbol{z} \boldsymbol{d}}\right)^{-1} \boldsymbol{B}_{\boldsymbol{z} \boldsymbol{d}}+\boldsymbol{D}_{\boldsymbol{z} \boldsymbol{d}}, \\
\boldsymbol{A}_{\boldsymbol{z} \boldsymbol{d}} & =\boldsymbol{A}_{\boldsymbol{z} \boldsymbol{f}}, \quad \boldsymbol{B}_{\boldsymbol{z} \boldsymbol{d}}=\left[\begin{array}{c}
\boldsymbol{B}_{\mathrm{ref}, d} \\
\boldsymbol{B}_{d} \\
\boldsymbol{B}_{f, y} \boldsymbol{D}_{d}
\end{array}\right], \\
\boldsymbol{C}_{\boldsymbol{z d}} & =\boldsymbol{C}_{\boldsymbol{z} \boldsymbol{f}}, \quad \boldsymbol{D}_{\boldsymbol{z} \boldsymbol{d}}=\boldsymbol{D}_{f, y} \boldsymbol{D}_{d}-\boldsymbol{D}_{\mathrm{ref}, d} .
\end{aligned}
$$

Werden wie in Gleichung (6.7b) in Abschnitt 6.3 die Matrizen $\boldsymbol{B}_{\text {ref }, d}=\mathbf{0}$ und $\boldsymbol{D}_{\text {ref, } d}=\mathbf{0}$ für das Referenzmodell angesetzt, so gilt

$$
\left\|\boldsymbol{G}_{\boldsymbol{z} \boldsymbol{d}}(s)\right\|_{\infty}=\left\|\boldsymbol{G}_{\boldsymbol{r} \boldsymbol{d}}(s)-\boldsymbol{G}_{\mathrm{ref}, \boldsymbol{r} \boldsymbol{d}}(s)\right\|_{\infty}=\left\|\boldsymbol{G}_{\boldsymbol{r} \boldsymbol{d}}(s)\right\|_{\infty} .
$$

Mithilfe des Bounded-Real-Lemmas (Satz 3.1), Gleichung (6.15) und (6.53) lässt sich die Optimierung von (6.52) bewerkstelligen durch

$$
\begin{aligned}
& \underset{\boldsymbol{P}, \boldsymbol{A}_{f}, \boldsymbol{B}_{f, u}, \boldsymbol{B}_{f, y}, \boldsymbol{C}_{f}, \boldsymbol{D}_{f, u}, \boldsymbol{D}_{f, y}}{\operatorname{minimiere}}, \text { sodass } \\
& \mathbb{R}^{n_{\mathrm{ref}}+n+n_{\mathrm{filt}}} \ni \boldsymbol{P} \succ \mathbf{0}, \\
& {\left[\begin{array}{ccc}
\operatorname{He}\left(\boldsymbol{P} \boldsymbol{A}_{\boldsymbol{z} \tilde{\boldsymbol{u}}}\right) & \boldsymbol{P} \boldsymbol{B}_{\boldsymbol{z} \tilde{\boldsymbol{u}}} & \boldsymbol{C}_{\boldsymbol{z} \tilde{\boldsymbol{u}}}^{\boldsymbol{\top}} \\
\star & -\varepsilon \boldsymbol{I}_{n_{u}+n_{f}} & \boldsymbol{D}_{\boldsymbol{z} \tilde{\boldsymbol{u}}}^{\top} \\
\star & \star & -\varepsilon \boldsymbol{I}_{n_{f}}
\end{array}\right] \prec \mathbf{0},} \\
& {\left[\begin{array}{ccc}
\operatorname{He}\left(\boldsymbol{P} \boldsymbol{A}_{\boldsymbol{z} \boldsymbol{d}}\right) & \boldsymbol{P} \boldsymbol{B}_{\boldsymbol{z d}} & \boldsymbol{C}_{\boldsymbol{z} \boldsymbol{d}}^{\top} \\
\star & -\gamma \boldsymbol{I}_{n_{d}} & \boldsymbol{D}_{\boldsymbol{z} \boldsymbol{d}}^{\top} \\
\star & \star & -\gamma \boldsymbol{I}_{n_{f}}^{\top}
\end{array}\right] \prec \mathbf{0 .}}
\end{aligned}
$$

Durch (6.54d) ist sichergestellt, dass $\left\|\boldsymbol{G}_{\boldsymbol{r} \boldsymbol{d}}(s)\right\|_{\infty}<\gamma$ gilt. Ebenso wie bei $(6.54 \mathrm{c})$ handelt es sich bei der entsprechenden Bedingung um eine BMI. Mit der in Abschnitt 6.4.1 beschriebenen Variablentransformation lassen sie sich jedoch in äquivalente LMI-Bedingungen umformulieren. Somit lässt sich durch (6.54) das Optimierungsproblem (6.52) einfach lösen. Es ist allerdings anzumerken, dass die linearisierende Variablentransformation nur dann gelingt, wenn in (6.54c) und (6.54d) eine gemeinsame Matrix $\boldsymbol{P}$ angesetzt wird. Dies schränkt jedoch den Lösungsraum ein, sodass sich konservative Lösungen ergeben. Die Bedingungen (6.54b) bis 
(6.54d) sind somit lediglich hinreichend für (6.52). Setzt man für (6.54c) und (6.54d) jeweils eine eigene Matrix $\boldsymbol{P}_{1}$ beziehungsweise $\boldsymbol{P}_{2}$ an, so gelingt die Rücktransformation nur durch zusätzliche Rangbeschränkungen (s. Abschnitt 5.4.3), dass Problem ist dann nicht mehr konvex.

Um weniger konservative Lösungen zu erhalten, wird ein Ansatz aus [149] modifiziert. Dabei wird die Wirkung von Stellgrößen $\boldsymbol{u}$, Fehlern $\boldsymbol{f}$ und exogenen Störungen $\boldsymbol{d}$ auf den Performance-Ausgang $\boldsymbol{z}$ mittels eines einzigen Systems beschrieben, wozu der verallgemeinerte Eingangsvektor aus (6.14) erweitert wird zu

$$
\tilde{\boldsymbol{u}}=\left[\begin{array}{l}
\boldsymbol{u} \\
\boldsymbol{f} \\
\boldsymbol{d}
\end{array}\right] \in \mathbb{R}^{n_{u}+n_{f}+n_{d}} .
$$

Unter Verwendung des Zustandsvektors $\boldsymbol{x}_{g}=\left[\begin{array}{lll}\boldsymbol{x}_{\mathrm{ref}}^{\top} & \boldsymbol{x}^{\top} & \boldsymbol{x}_{f}^{\top}\end{array}\right]^{\top}$ ergibt sich entsprechend (6.4) das Gesamtsystem

$$
\begin{aligned}
& \dot{\boldsymbol{x}}_{g}=\underbrace{\left[\begin{array}{ccc}
\boldsymbol{A}_{\mathrm{ref}} & \mathbf{0} & \mathbf{0} \\
\mathbf{0} & \boldsymbol{A} & \mathbf{0} \\
\mathbf{0} & \boldsymbol{B}_{f, y} \boldsymbol{C} & \boldsymbol{A}_{f}
\end{array}\right]}_{\boldsymbol{A}_{\boldsymbol{z} \tilde{u}}} \boldsymbol{x}_{g}+\ldots \\
& +\underbrace{\left[\begin{array}{ccc}
\boldsymbol{B}_{\mathrm{ref}, u} & \boldsymbol{B}_{\mathrm{ref}, f} & \boldsymbol{B}_{\mathrm{ref}, d} \\
\boldsymbol{B} & \boldsymbol{E}_{a} & \boldsymbol{B}_{d} \\
\boldsymbol{B}_{f, u}+\boldsymbol{B}_{f, y} \boldsymbol{D} & \boldsymbol{B}_{f, y} \boldsymbol{E}_{s} & \boldsymbol{B}_{f, y} \boldsymbol{D}_{d}
\end{array}\right]}_{\boldsymbol{B}_{\boldsymbol{z} \tilde{u}}} \tilde{\boldsymbol{u}}, \\
& \boldsymbol{z}=\underbrace{\left[\begin{array}{lll}
-\boldsymbol{C}_{\mathrm{ref}} & \boldsymbol{D}_{f, y} \boldsymbol{C} & \boldsymbol{C}_{f}
\end{array}\right]}_{\boldsymbol{C}_{\boldsymbol{z} \tilde{u}}} \boldsymbol{x}_{g}+\ldots \\
& +\underbrace{\left[\begin{array}{ccc}
\boldsymbol{D}_{f, u}+\boldsymbol{D}_{f, y} \boldsymbol{D}-\boldsymbol{D}_{\mathrm{ref}, u} & \boldsymbol{D}_{f, y} \boldsymbol{E}_{s}-\boldsymbol{D}_{\mathrm{ref}, f} & \boldsymbol{D}_{f, y} \boldsymbol{D}_{d}-\boldsymbol{D}_{\mathrm{ref}, d}
\end{array}\right]}_{\boldsymbol{D}_{\boldsymbol{z} \tilde{u}}} \tilde{\boldsymbol{u}} .
\end{aligned}
$$

Mit $\boldsymbol{P} \succ \mathbf{0}$ und

$$
\left[\begin{array}{ccc}
\operatorname{He}\left(\boldsymbol{P} \boldsymbol{A}_{\boldsymbol{z} \tilde{\boldsymbol{u}}}\right) & \boldsymbol{P}_{\boldsymbol{z} \tilde{\boldsymbol{u}}} & \boldsymbol{C}_{\boldsymbol{z} \tilde{\boldsymbol{u}}}^{\top} \\
\star & -\gamma \boldsymbol{I}_{n_{u}+n_{f}+n_{d}} & \boldsymbol{D}_{\boldsymbol{z} \tilde{\boldsymbol{u}}}^{\top} \\
\star & \star & -\gamma \boldsymbol{I}_{n_{f}}
\end{array}\right] \prec \mathbf{0}
$$

lässt sich für $\boldsymbol{B}_{\mathrm{ref}, u}=\mathbf{0}, \boldsymbol{D}_{\mathrm{ref}, u}=\mathbf{0}, \boldsymbol{B}_{\mathrm{ref}, d}=\mathbf{0}$ und $\boldsymbol{D}_{\mathrm{ref}, d}=\mathbf{0}$ die Einhaltung von

$$
\left\|\left[\boldsymbol{G}_{\boldsymbol{r u}}(s) \quad \boldsymbol{G}_{\boldsymbol{r} \boldsymbol{f}}(s)-\boldsymbol{G}_{\mathrm{ref}, \boldsymbol{r} \boldsymbol{f}}(s) \quad \boldsymbol{G}_{\boldsymbol{r d}}(s)\right]\right\|_{\infty}<\gamma
$$


mittels einer einzigen Matrix $\boldsymbol{P}$ sicherstellen. Somit lässt sich ohne Weiteres die linearisierende Variablentransformation aus Abschnitt 6.4.1 anwenden.

Allerdings ist (6.57) hinsichtlich des ursprünglichen Optimierungsproblems (6.52) nur bedingt aussagekräftig. Verfügen beispielsweise die Störeingangsmatrizen $\boldsymbol{B}_{d}$ und $\boldsymbol{D}_{d}$ über betragsmäßig große Elemente, so kann es bei der Minimierung von $\gamma$ in (6.57) dazu kommen, dass der Term $\left\|\boldsymbol{G}_{\boldsymbol{r} \boldsymbol{f}}(s)-\boldsymbol{G}_{\mathrm{ref}, \boldsymbol{r} \boldsymbol{f}}(s)\right\|_{\infty}$ auf Kosten der Senkung von $\left\|\boldsymbol{G}_{\boldsymbol{r} \boldsymbol{d}}(s)\right\|_{\infty}$ derart große Werte annimmt, dass keine Fehlerisolation mehr möglich ist. Es geht also beim Entwurf die Möglichkeit verloren, zwischen den Entwurfszielen hinsichtlich der Fehler, der Stellgrößen und der Störgrößen Gewichtungen vorzunehmen. Dies lässt sich durch eine einfache Modifikation des Verfahrens erreichen. Dabei werden die auf das System wirkenden Eingangsgrößen $\boldsymbol{f}, \boldsymbol{u}$ und $\boldsymbol{d}$ für den Entwurf mittels der Skalare $\alpha_{u} \geq 0, \alpha_{f} \geq 0$ und $\alpha_{d} \geq 0$ skaliert. Es ist zu betonen, dass diese Skalierung rein virtueller Natur ist und nur für den Entwurf vorgenommen wird. Anschaulich betrachtet wird durch die Skalierung der Eingangsgrößen der jeweilige "Beitrag" in (6.57) gewichtet. Somit können die angestrebten Entwurfsziele

$$
\boldsymbol{G}_{\boldsymbol{r} \boldsymbol{f}}(s)=\boldsymbol{G}_{\mathrm{ref}, \boldsymbol{r} \boldsymbol{f}}(s), \quad \boldsymbol{G}_{\boldsymbol{r u}}(s)=\mathbf{0}, \quad \boldsymbol{G}_{\boldsymbol{r} \boldsymbol{d}}(s)=\mathbf{0}
$$

durch die Gewichtung beim Entwurf mittels (6.57) priorisiert werden.

Die Skalierung der Eingangsgrößen lässt sich äquivalent dadurch erreichen, dass die entsprechenden Eingangs- beziehungsweise Durchgriffsmatrizen mit den Faktoren skaliert werden. Es wird für den Entwurf also

$$
\begin{aligned}
& \boldsymbol{B}_{\mathrm{ref}, u}^{\prime}=\alpha_{u} \boldsymbol{B}_{\mathrm{ref}, u}, \quad \boldsymbol{D}_{\mathrm{ref}, u}^{\prime}=\alpha_{u} \boldsymbol{D}_{\mathrm{ref}, u}, \quad \boldsymbol{B}^{\prime}=\alpha_{u} \boldsymbol{B}, \quad \boldsymbol{D}^{\prime}=\alpha_{u} \boldsymbol{D}, \\
& \boldsymbol{B}_{\mathrm{ref}, f}^{\prime}=\alpha_{f} \boldsymbol{B}_{\mathrm{ref}, f}, \quad \boldsymbol{D}_{\mathrm{ref}, f}^{\prime}=\alpha_{f} \boldsymbol{D}_{\mathrm{ref}, f}, \quad \boldsymbol{E}_{a}^{\prime}=\alpha_{f} \boldsymbol{E}_{a}, \quad \boldsymbol{E}_{s}^{\prime}=\alpha_{f} \boldsymbol{E}_{s}, \\
& \boldsymbol{B}_{\mathrm{ref}, d}^{\prime}=\alpha_{d} \boldsymbol{B}_{\mathrm{ref}, d}, \quad \boldsymbol{D}_{\mathrm{ref}, d}^{\prime}=\alpha_{d} \boldsymbol{D}_{\mathrm{ref}, d}, \quad \boldsymbol{B}_{d}^{\prime}=\alpha_{d} \boldsymbol{B}_{d}, \quad \boldsymbol{D}_{d}^{\prime}=\alpha_{d} \boldsymbol{D}_{d}
\end{aligned}
$$

gesetzt. An dieser Stelle ist anzumerken, dass die Stellgröße $\boldsymbol{u}$ zusätzlich über die Filtermatrizen $\boldsymbol{B}_{f, u}$ und $\boldsymbol{D}_{f, u}$ auf die generierten Residuen wirkt. Daher werden abweichend zum störungsfreien Fall die Variablensubstitutionen

$$
\begin{aligned}
& \hat{\boldsymbol{B}}_{f, u}=\alpha_{u} \boldsymbol{U} \boldsymbol{B}_{f, u}, \\
& \hat{\boldsymbol{D}}_{f, u}=\alpha_{u} \boldsymbol{D}_{f, u}
\end{aligned}
$$


vorgenommen. Dies muss bei der Rücktransformation berücksichtigt werden. Die übrigen Variablensubstitutionen bleiben unverändert. Je größer ein Gewichtungsfaktor gewählt wird, desto stärker wird der Einfluss der zugeordneten Eingangsgröße in (6.57) bestraft. Somit wird die $\mathcal{H}_{\infty}$-Norm der entsprechenden Übertragungsmatrix, also $\boldsymbol{G}_{\boldsymbol{r} \boldsymbol{u}}(s), \boldsymbol{G}_{\boldsymbol{r} \boldsymbol{f}}(s)-\boldsymbol{G}_{\mathrm{ref}, \boldsymbol{r} \boldsymbol{f}}(s)$ oder $\boldsymbol{G}_{\boldsymbol{r} \boldsymbol{d}}(s)$, tendenziell stärker gesenkt. Dabei ist nicht der absolute Wert der Faktoren $\alpha_{u}, \alpha_{f}$ und $\alpha_{d}$ entscheidend, sondern deren Verhältnis.

Zur Durchführung der Variablentransformation werden in Anlehnung an (6.27) die Abkürzungen

$$
\begin{array}{ll}
\overline{\boldsymbol{A}}=\left[\begin{array}{cc}
\boldsymbol{A}_{\mathrm{ref}} & \mathbf{0} \\
\mathbf{0} & \boldsymbol{A}
\end{array}\right], & \overline{\boldsymbol{B}}_{1}=\left[\begin{array}{cc}
\boldsymbol{B}_{\mathrm{ref}, u}^{\prime} & \boldsymbol{B}_{\mathrm{ref}, f}^{\prime} \\
\boldsymbol{B}^{\prime} & \boldsymbol{E}_{a}^{\prime}
\end{array}\right], \overline{\boldsymbol{B}}_{2}=\left[\begin{array}{c}
\boldsymbol{B}_{\mathrm{ref}, d}^{\prime} \\
\boldsymbol{B}_{d}^{\prime}
\end{array}\right], \\
\overline{\boldsymbol{C}}=\left[\begin{array}{ll}
\mathbf{0} & \boldsymbol{C}
\end{array}\right], & \overline{\boldsymbol{D}}=\left[\begin{array}{ll}
\boldsymbol{D}^{\prime} & \boldsymbol{E}_{s}^{\prime}
\end{array}\right]
\end{array}
$$

eingeführt. Ähnlich wie in Abschnitt 6.4.1 wird damit eine Kongruenztransformation von (6.56) mit $\boldsymbol{T}=\operatorname{diag}\left(\boldsymbol{\Psi}, \boldsymbol{I}_{n_{u}+n_{f}+n_{d}}, \boldsymbol{I}_{n_{f}}\right)$ durchgeführt. Daraus ergibt sich das LMI-Problem

$$
\begin{aligned}
& \underset{\boldsymbol{Z}, \boldsymbol{Y}_{11}, \hat{\boldsymbol{A}}_{f}, \hat{\boldsymbol{B}}_{f, u}, \hat{\boldsymbol{B}}_{f, y}, \hat{\boldsymbol{C}}_{f}, \hat{\boldsymbol{D}}_{f, u}, \hat{\boldsymbol{D}}_{f, y}}{\operatorname{minimie}} \gamma, \text { sodass } \\
& \boldsymbol{Z} \succ \mathbf{0}, \quad \boldsymbol{Y}_{11}-\boldsymbol{Z} \succ \mathbf{0}, \\
& {\left[\begin{array}{ccc}
\boldsymbol{\Sigma}_{11} & \boldsymbol{\Sigma}_{12} & \boldsymbol{\Sigma}_{13} \\
\star & -\gamma \boldsymbol{I}_{n_{u}+n_{f}+n_{d}} & \boldsymbol{\Sigma}_{23} \\
\star & \star & -\gamma \boldsymbol{I}_{n_{f}}
\end{array}\right] \prec \mathbf{0},} \\
& \boldsymbol{\Sigma}_{11}=\operatorname{He}\left(\left[\begin{array}{cc}
\boldsymbol{Z} \overline{\boldsymbol{A}} & \boldsymbol{Z} \overline{\boldsymbol{A}} \\
\boldsymbol{Y}_{11} \overline{\boldsymbol{A}}+\hat{\boldsymbol{B}}_{f, y} \overline{\boldsymbol{C}}+\hat{\boldsymbol{A}}_{f} & \boldsymbol{Y}_{11} \overline{\boldsymbol{A}}+\hat{\boldsymbol{B}}_{f, y} \overline{\boldsymbol{C}}
\end{array}\right]\right), \\
& \boldsymbol{\Sigma}_{12}=\left[\begin{array}{cc}
\boldsymbol{Z} \overline{\boldsymbol{B}}_{1} & \\
\boldsymbol{Y}_{11} \overline{\boldsymbol{B}}_{1}+\hat{\boldsymbol{B}}_{f, u}\left[\begin{array}{ll}
\boldsymbol{I}_{n_{u}} & \mathbf{0}
\end{array}\right]+\hat{\boldsymbol{B}}_{f, y} \overline{\boldsymbol{D}} & \boldsymbol{Y}_{11} \overline{\boldsymbol{B}}_{2}+\hat{\boldsymbol{B}}_{f, y} \boldsymbol{D}_{d}^{\prime}
\end{array}\right], \\
& \boldsymbol{\Sigma}_{13}=\left[\begin{array}{ll}
-C_{\mathrm{ref}} & \hat{\boldsymbol{D}}_{f, y} \boldsymbol{C}
\end{array}\right]+\hat{\boldsymbol{C}}_{f} \quad\left[\begin{array}{ll}
-\boldsymbol{C}_{\mathrm{ref}} & \hat{\boldsymbol{D}}_{f, y} \boldsymbol{C}
\end{array}\right]^{\top}, \\
& \boldsymbol{\Sigma}_{23}=\left[\begin{array}{lll}
\hat{\boldsymbol{D}}_{f, u}+\hat{\boldsymbol{D}}_{f, y} \boldsymbol{D}^{\prime}-\boldsymbol{D}_{\mathrm{ref}, u}^{\prime} & \hat{\boldsymbol{D}}_{f, y} \boldsymbol{E}_{s}^{\prime}-\boldsymbol{D}_{\mathrm{ref}, f}^{\prime} & \hat{\boldsymbol{D}}_{f, y} \boldsymbol{D}_{d}^{\prime}-\boldsymbol{D}_{\mathrm{ref}, d}^{\prime}
\end{array}\right]^{\top} .
\end{aligned}
$$

Zur Vorgabe von Eigenwertbereichen können dabei wie in Lemma 6.3 bis 6.5 beschrieben zusätzliche LMIs herangezogen werden.

Nach dem Lösen von (6.61) erfolgt die Rücktransformation auf die Filterparameter $\boldsymbol{A}_{f}, \boldsymbol{B}_{f, u}, \boldsymbol{B}_{f, y}, \boldsymbol{C}_{f}, \boldsymbol{D}_{f, u}$ und $\boldsymbol{D}_{f, y}$ weitgehend analog 
zu (6.39) beziehungsweise bei reduzierter Filterordnung analog zu (6.51). Mittels einer Singulärwertzerlegung von $\boldsymbol{I}_{n+n_{\text {ref }}}-\boldsymbol{Y}_{11} \boldsymbol{Z}^{-1}$ und (6.50) werden die Matrizen $\boldsymbol{U}$ und $\boldsymbol{W}^{\top}$ berechnet. Die Filterparameter ergeben sich dann aus

$$
\begin{aligned}
\boldsymbol{A}_{f} & =\boldsymbol{U}^{+} \hat{\boldsymbol{A}}_{f}\left(\boldsymbol{W}^{\top} \boldsymbol{Z}\right)^{+}, \\
\boldsymbol{B}_{f, u} & =\frac{1}{\alpha_{u}} \boldsymbol{U}^{+} \hat{\boldsymbol{B}}_{f, u}, \\
\boldsymbol{B}_{f, y} & =\boldsymbol{U}^{+} \hat{\boldsymbol{B}}_{f, y}, \\
\boldsymbol{C}_{f} & =\hat{\boldsymbol{C}}_{f}\left(\boldsymbol{W}^{\top} \boldsymbol{Z}\right)^{+}, \\
\boldsymbol{D}_{f, u} & =\frac{1}{\alpha_{u}} \hat{\boldsymbol{D}}_{f, u}, \\
\boldsymbol{D}_{f, y} & =\hat{\boldsymbol{D}}_{f, y} .
\end{aligned}
$$

Dies schließt den Fall von Filtern voller Ordnung, das heißt $n_{\text {filt }}=n+n_{\text {ref }}$, mit ein, bei dem die Pseudoinversen den Inversen $\boldsymbol{U}^{-1}$ beziehungsweise $\left(\boldsymbol{W}^{\top} \boldsymbol{Z}\right)^{-1}$ entsprechen. Die Rücktransformation (6.62) unterscheidet sich von (6.39) beziehungsweise (6.51) lediglich in der Skalierung von $\boldsymbol{B}_{f, u}$ und $\boldsymbol{D}_{f, u}$ mittels des Faktors $1 / \alpha_{u}$. Diese rührt daher, dass die Matrizen $\boldsymbol{B}, \boldsymbol{D}$, $\boldsymbol{B}_{\text {ref, } u}$ und $\boldsymbol{D}_{\text {ref, } u}$ gemäß (6.58) modifiziert wurden und dementsprechend die abgewandelten Variablensubstitutionen (6.59) vorgenommen wurden.

Die Ergebnisse sind in den folgenden beiden Sätzen zusammengefasst. Zunächst gilt für den Entwurf von Fehlerisolationsfiltern voller Ordnung

Satz 6.6 (LMI-basierter Entwurf von bezüglich exogener Störungen robusten FIFs mit Einschränkung der Filtereigenwerte). Gegeben sei eine Strecke der Form

$$
\begin{aligned}
& \dot{\boldsymbol{x}}=\boldsymbol{A} \boldsymbol{x}+\boldsymbol{B u}+\boldsymbol{E}_{a} \boldsymbol{f}+\boldsymbol{B}_{d} \boldsymbol{d}, \\
& \boldsymbol{y}=\boldsymbol{C} \boldsymbol{x}+\boldsymbol{D} \boldsymbol{u}+\boldsymbol{E}_{s} \boldsymbol{f}+\boldsymbol{D}_{d} \boldsymbol{d},
\end{aligned}
$$

welche die Bedingungen 6.1-6.3 (6.4-6.7 beziehungsweise 6.8-6.12) erfüllt, sowie ein durch (6.7), (6.9) beschriebenes zulässiges Referenzmodell der

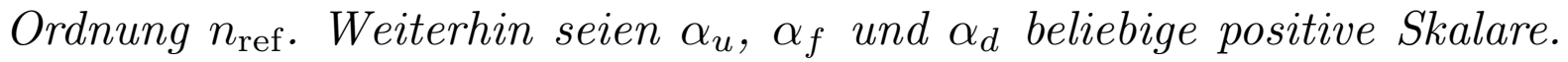
Dann gilt beim Einsatz eines FIFs der Form

$$
\begin{aligned}
\dot{\boldsymbol{x}}_{f} & =\boldsymbol{A}_{f} \boldsymbol{x}_{f}+\boldsymbol{B}_{f, u} \boldsymbol{u}+\boldsymbol{B}_{f, y} \boldsymbol{y}, \\
\boldsymbol{r} & =\boldsymbol{C}_{f} \boldsymbol{x}_{f}+\boldsymbol{D}_{f, u} \boldsymbol{u}+\boldsymbol{D}_{f, y} \boldsymbol{y}
\end{aligned}
$$


mit $n_{\mathrm{filt}}=n+n_{\mathrm{ref}}$ die Beziehung

$$
\left\|\left[\alpha_{u} \boldsymbol{G}_{\boldsymbol{r u}}(s) \quad \alpha_{f}\left(\boldsymbol{G}_{\boldsymbol{r} \boldsymbol{f}}(s)-\boldsymbol{G}_{r e f, \boldsymbol{r} \boldsymbol{f}}(s)\right) \quad \alpha_{d} \boldsymbol{G}_{\boldsymbol{r} \boldsymbol{d}}(s)\right]\right\|_{\infty}<\gamma
$$

und für alle Filtereigenwerte $\lambda_{i}, i=1, \ldots, n_{\text {filt }}$, gilt $\mathfrak{R e}\left(\lambda_{i}\right)<\alpha,\left|\lambda_{i}\right|<\varrho$ und $\left|\mathfrak{I m}\left(\lambda_{i}\right)\right|<\tan (\phi) \cdot\left|\mathfrak{R e}\left(\lambda_{i}\right)\right|$, wenn die Filtermatrizen durch Lösen des LMI-Problems

$$
\begin{aligned}
& \underset{\boldsymbol{Z}, \boldsymbol{Y}_{11}, \hat{\boldsymbol{A}}_{f}, \hat{\boldsymbol{B}}_{f, u}, \hat{\boldsymbol{B}}_{f, y}, \hat{\boldsymbol{C}}_{f}, \hat{\boldsymbol{D}}_{f, u}, \hat{\boldsymbol{D}}_{f, y}}{\operatorname{minimiere}} \boldsymbol{\gamma} \text { sodass } \\
& (6.40)-(6.42),(6.61 \mathrm{~b})-(6.61 \mathrm{~g})
\end{aligned}
$$

und anschließende Rücktransformation gemäß (6.37), (6.38), (6.62) ermittelt werden.

Der zweite Entwurfssatz gilt für den Entwurf robuster FIFs reduzierter Ordnung.

Satz 6.7 (LMI-basierter Entwurf von bezüglich exogener Störungen robusten FIFs reduzierter Ordnung mit Einschränkung der Filtereigenwerte). Gegeben sei eine Strecke der Form

$$
\begin{aligned}
& \dot{\boldsymbol{x}}=\boldsymbol{A} \boldsymbol{x}+\boldsymbol{B} \boldsymbol{u}+\boldsymbol{E}_{a} \boldsymbol{f}+\boldsymbol{B}_{d} \boldsymbol{d}, \\
& \boldsymbol{y}=\boldsymbol{C} \boldsymbol{x}+\boldsymbol{D} \boldsymbol{u}+\boldsymbol{E}_{s} \boldsymbol{f}+\boldsymbol{D}_{d} \boldsymbol{d},
\end{aligned}
$$

welche die Bedingungen 6.1-6.3 (6.4-6.7 beziehungsweise 6.8-6.12) erfüllt, sowie ein durch (6.7), (6.9) beschriebenes zulässiges Referenzmodell. Weiterhin seien $\alpha_{u}, \alpha_{f}$ und $\alpha_{d}$ beliebige positive Skalare. Dann gilt beim Einsatz eines FIFs der Form

$$
\begin{aligned}
\dot{\boldsymbol{x}}_{f} & =\boldsymbol{A}_{f} \boldsymbol{x}_{f}+\boldsymbol{B}_{f, u} \boldsymbol{u}+\boldsymbol{B}_{f, y} \boldsymbol{y}, \\
\boldsymbol{r} & =\boldsymbol{C}_{f} \boldsymbol{x}_{f}+\boldsymbol{D}_{f, u} \boldsymbol{u}+\boldsymbol{D}_{f, y} \boldsymbol{y}
\end{aligned}
$$

mit $n_{\mathrm{filt}}=n\left(n_{\mathrm{filt}}=n+n_{\zeta}\right.$, wobei $n_{\zeta}$ die für einen stabilen DFIO benötigte Ordnung ist) die Beziehung

$$
\left\|\left[\alpha_{u} \boldsymbol{G}_{\boldsymbol{r u}}(s) \quad \alpha_{f}\left(\boldsymbol{G}_{\boldsymbol{r} \boldsymbol{f}}(s)-\boldsymbol{G}_{r e f, \boldsymbol{r} \boldsymbol{f}}(s)\right) \quad \alpha_{d} \boldsymbol{G}_{\boldsymbol{r} \boldsymbol{d}}(s)\right]\right\|_{\infty}<\gamma
$$

und für alle Filtereigenwerte $\lambda_{i}, i=1, \ldots, n_{\text {filt }}$, gilt $\mathfrak{R e}\left(\lambda_{i}\right)<\alpha,\left|\lambda_{i}\right|<\varrho$ und $\left|\mathfrak{I m}\left(\lambda_{i}\right)\right|<\tan (\phi) \cdot\left|\mathfrak{R e}\left(\lambda_{i}\right)\right|$, wenn die Filtermatrizen durch Lösen des rangbeschränkten LMI-Problems

$$
\begin{gathered}
\underset{\boldsymbol{Z}, \boldsymbol{Y}_{11}, \hat{\boldsymbol{A}}_{f}, \hat{\boldsymbol{B}}_{f, u}, \hat{\boldsymbol{B}}_{f, y}, \hat{\boldsymbol{C}}_{f}, \hat{\boldsymbol{D}}_{f, u}, \hat{\boldsymbol{D}}_{f, y}}{\operatorname{minimiere}} \boldsymbol{\gamma} \text { sodass } \\
(6.40)-(6.42),(6.45),(6.61 \mathrm{c})-(6.61 \mathrm{~g})
\end{gathered}
$$


und anschließende Rücktransformation gemäß (6.48)-(6.50), (6.62) ermittelt werden.

$\mathrm{Zu}$ beachten ist, dass aktuelle Solver für rangbeschränkte LMI-Probleme wie LMIRANK [162] lediglich Lösbarkeitsprobleme beherrschen. Die Minimierung von $\gamma$ kann daher ähnlich wie in Abschnitt 5.4.3 beschrieben mittels einer Bisektion erfolgen.

Bemerkung 6.1. Wird wie in Abschnitt 5.5 lediglich eine teilweise Fehlerisolation angestrebt, so lässt sich die Optimierung der Robustheit prinzipiell ebenfalls leicht mit allgemeinen Fehlerisolationsfiltern erreichen. Hinsichtlich des Einflusses der Fehler auf die Residuen sind dann lediglich die Elemente

$$
g_{r_{i}, f_{j}}(s)-g_{\mathrm{ref}, r_{i}, f_{j}}(s), i \geq j
$$

von Belang, da die Elemente oberhalb der Hauptdiagonalen für die teilweise Isolation keine Rolle spielen. Somit sind zwar schwächere strukturelle Anforderungen gegeben, diese lassen sich jedoch nicht in kompakter Art und Weise als eine einzige LMI-Nebenbedingung wie zum Beispiel in Satz 6.6 formulieren. Für die Elemente (6.63) wird jeweils eine Nebenbedingung formuliert, wobei jedoch gemeinsame Matrizen $\boldsymbol{Z}$ und $\boldsymbol{Y}_{11}$ $\mathrm{zu}$ verwenden sind, damit nach Lösung des LMI-Problems die Filterparameter zurückgewonnen werden können. Dies führt zu konservativen Ergebnissen und wird daher an dieser Stelle nicht weiter verfolgt. Denkbar wäre die Einführung separater Matrizen $\boldsymbol{Z}$ und $\boldsymbol{Y}_{11}$ für jedes Element mit zusätzlichen Rangbeschränkungen oder ein Path-Following-Ansatz, um die Konservativität zu senken.

\section{Beispiel Hydrauliksystem}

Für das Hydrauliksystem wurde in Abschnitt 5.4.1 ein robuster FIO zur Unterdrückung exogener Störungen beliebiger Frequenzen entworfen. Für die gleichen Störeingangsmatrizen wird nun mittels Satz 6.6 ein robuster FIF voller Ordnung $n_{\text {filt }}=9$ entworfen.

Um dabei eine möglichst exakte Diagonalisierung von $\boldsymbol{G}_{\boldsymbol{r f}}(s)$ sowie eine (nahezu) vollständige Unterdrückung des Stellgrößeneinflusses auf die generierten Residuen zu erreichen, werden die Gewichtungsfaktoren zu $\alpha_{u}=\alpha_{f}=1$ und $\alpha_{d}=10^{-5}$ gewählt. Es ergibt sich die in Anhang C.3.2 aufgeführte FIF-Parametrierung, aus der für die $\mathcal{H}_{\infty}$-Norm von $\boldsymbol{G}_{\boldsymbol{r} \boldsymbol{d}}(s)$ eine Schranke von $\gamma_{r d}=23,70$ folgt. Diese geht auch aus dem Singulärwertverlauf von $\boldsymbol{G}_{\boldsymbol{r} \boldsymbol{d}}(s)$ in Abbildung 6.3 hervor und entspricht 


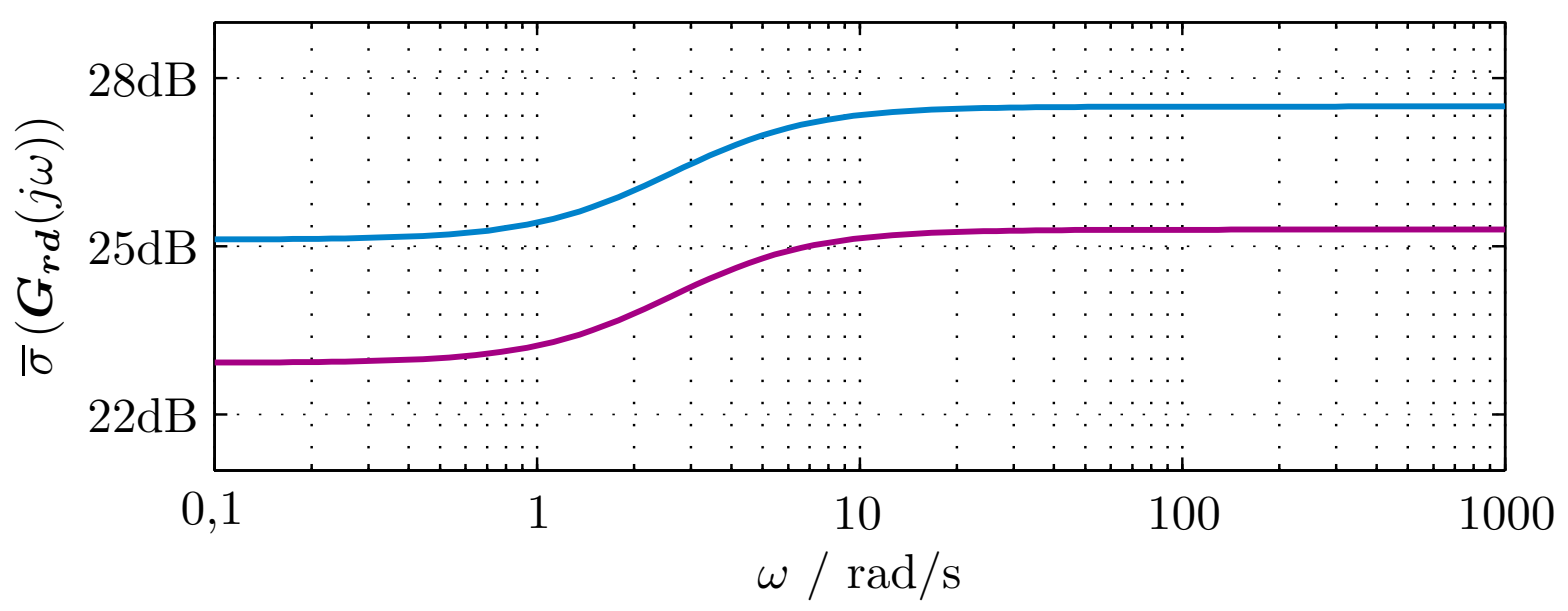

Abbildung 6.3: Verlauf des größten Singulärwertes von $\boldsymbol{G}_{\boldsymbol{r} \boldsymbol{d}}(s)$ für einen FIF mit $\alpha_{d}=10^{-5}(-)$ sowie einen FIF mit $\alpha_{d}=0,008(-)$

exakt dem Wert, der sich auch für den robusten FIO in Abschnitt 5.4.1 ergibt. Der Vorteil des FIFs ist also in diesem Fall nicht in einer verbesserten Störunterdrückung zu sehen, sondern in seinem einheitlichen Entwurf.

Darüber hinaus lässt sich durch die Anpassung der Gewichtungsfaktoren eine für FIOs in Abschnitt 5.3.2 diskutierte Relaxierung der strukturellen Anforderungen sehr einfach realisieren. Vergrößert man den Skalierungsfaktor für die Störungen auf $\alpha_{d}=0,008$, so wird die exakte Diagonalisierung von $\boldsymbol{G}_{\boldsymbol{r} \boldsymbol{f}}(s)$ bei der vorgegebenen stationären Verstärkung gegenüber der Störunterdrückung geringer gewichtet. Durch Satz 6.7 lässt sich damit für einen FIF reduzierter Ordnung $\left(n_{\text {filt }}=n=5\right.$, Parametrierung s. Anhang C.3.2) eine Schranke von $\gamma_{r d}=18,04$ erreichen (vgl. Abbildung 6.3). Der Verlauf der Sprungantwort von $\boldsymbol{G}_{\boldsymbol{r} \boldsymbol{f}}(s)$ in Abbildung 6.4 zeigt, dass diese Verbesserung vorwiegend durch eine Verringerung der stationären Verstärkung im zweiten Übertragungskanal erreicht wird.

Anzumerken ist, dass sich eine derart verringerte Schranke für $\gamma_{r d}$ auch mit beobachterbasierten Methoden erreichen lässt. Dazu sind einerseits die stationären Verstärkungen anzupassen und andererseits die strukturellen Anforderungen zu relaxieren. Es ist aber nicht offensichtlich, wie dies getan werden muss, um $\gamma_{r d}$ so weit wie möglich abzusenken. Die Auslegung der entsprechenden Freiheitsgrade führt zu einem relativ aufwändigen Verfahren, bei dem man auf Versuch und Irrtum angewiesen ist. Mittels der Fehlerisolationsfilter ist ein sehr viel intuitiverer und einfacherer Entwurf möglich, bei dem lediglich die Gewichtungsfaktoren entsprechend der angestrebten Entwurfsziele anzupassen sind. 

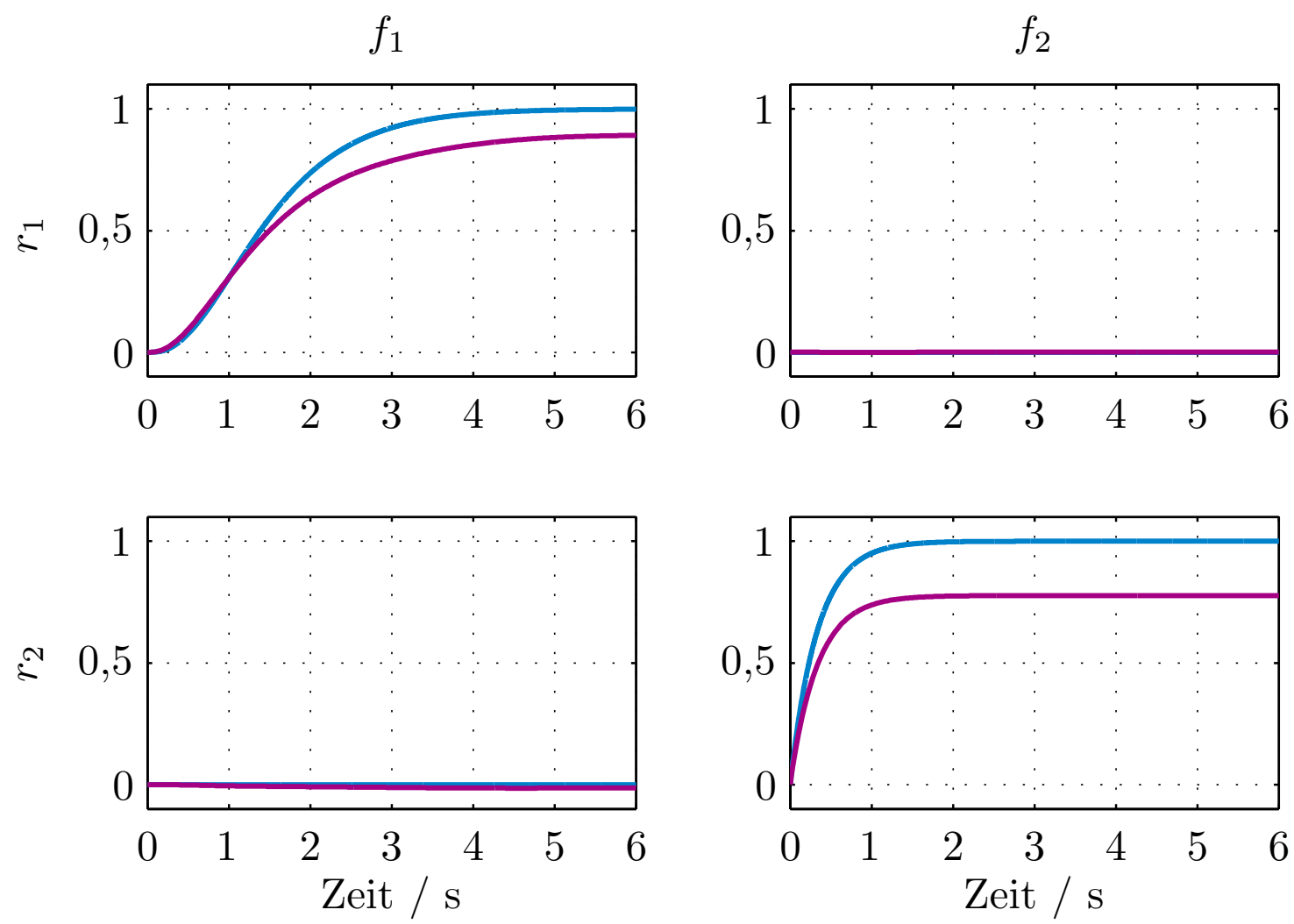

Abbildung 6.4: Sprungantwort von $\boldsymbol{G}_{\boldsymbol{r} \boldsymbol{f}}(s)$ des FIFs mit $\alpha_{d}=10^{-5}(-)$ sowie des FIFs mit $\alpha_{d}=0,008(-)$

\subsubsection{Robuste Fehlerisolationsfilter für unsichere Systeme}

Die Betrachtungen im vorangegangenen Abschnitt waren auf störungsbehaftete Systeme mit exakt bekannten Systemmatrizen beschränkt. In diesem Abschnitt wird der Entwurf von FIFs auf Systeme erweitert, die zusätzlich zu exogenen Störungen noch Unsicherheiten in den Systemmatrizen unterliegen.

Wie in Abschnitt 5.4.4 wird dies beschrieben durch die Systemdarstellung

$$
\begin{aligned}
& \dot{\boldsymbol{x}}=\boldsymbol{A}_{\chi} \boldsymbol{x}+\boldsymbol{B}_{\chi} \boldsymbol{u}+\boldsymbol{E}_{a, \chi} \boldsymbol{f}+\boldsymbol{B}_{d, \chi} \boldsymbol{d}, \\
& \boldsymbol{y}=\boldsymbol{C}_{\chi} \boldsymbol{x}+\boldsymbol{D}_{\chi} \boldsymbol{u}+\boldsymbol{E}_{s, \chi} \boldsymbol{f}+\boldsymbol{D}_{d, \chi} \boldsymbol{d} .
\end{aligned}
$$

Die Matrizen $\boldsymbol{A}_{\boldsymbol{\chi}}, \boldsymbol{B}_{\boldsymbol{\chi}}, \boldsymbol{E}_{a, \boldsymbol{\chi}}, \boldsymbol{B}_{d, \boldsymbol{\chi}}, \boldsymbol{C}_{\boldsymbol{\chi}}, \boldsymbol{D}_{\boldsymbol{\chi}}, \boldsymbol{E}_{s, \boldsymbol{\chi}}$ und $\boldsymbol{D}_{d, \boldsymbol{\chi}}$ sind dabei 
nicht mehr exakt bekannt, es wird lediglich vorausgesetzt, dass

$$
\begin{gathered}
{\left[\begin{array}{llll}
\boldsymbol{A}_{\boldsymbol{\chi}} & \boldsymbol{B}_{\boldsymbol{\chi}} & \boldsymbol{E}_{a, \boldsymbol{\chi}} & \boldsymbol{B}_{d, \boldsymbol{\chi}} \\
\boldsymbol{C}_{\boldsymbol{\chi}} & \boldsymbol{D}_{\boldsymbol{\chi}} & \boldsymbol{E}_{s, \boldsymbol{\chi}} & \boldsymbol{D}_{d, \boldsymbol{\chi}}
\end{array}\right] \in \mathcal{M},} \\
\mathcal{M}=\left\{\left[\begin{array}{llll}
\boldsymbol{A}_{\boldsymbol{\chi}} & \boldsymbol{B}_{\boldsymbol{\chi}} & \boldsymbol{E}_{a, \boldsymbol{\chi}} & \boldsymbol{B}_{d, \boldsymbol{\chi}} \\
\boldsymbol{C}_{\boldsymbol{\chi}} & \boldsymbol{D}_{\boldsymbol{\chi}} & \boldsymbol{E}_{s, \boldsymbol{\chi}} & \boldsymbol{D}_{d, \boldsymbol{\chi}}
\end{array}\right] \mid\right. \\
\left.\quad\left[\begin{array}{llll}
\boldsymbol{A}_{\boldsymbol{\chi}} & \boldsymbol{B}_{\boldsymbol{\chi}} & \boldsymbol{E}_{a, \boldsymbol{\chi}} & \boldsymbol{B}_{d, \boldsymbol{\chi}} \\
\boldsymbol{C}_{\boldsymbol{\chi}} & \boldsymbol{D}_{\boldsymbol{\chi}} & \boldsymbol{E}_{s, \boldsymbol{\chi}} & \boldsymbol{D}_{d, \boldsymbol{\chi}}
\end{array}\right]=\sum_{l=1}^{N}\left[\begin{array}{llll}
\boldsymbol{A}_{l} & \boldsymbol{B}_{l} & \boldsymbol{E}_{a, l} & \boldsymbol{B}_{d, l} \\
\boldsymbol{C}_{l} & \boldsymbol{D}_{l} & \boldsymbol{E}_{s, l} & \boldsymbol{D}_{d, l}
\end{array}\right]\right\} \\
\sum_{l=1}^{N} \chi_{l}=1, \quad \chi_{l} \geq 0
\end{gathered}
$$

gilt.

Wie im Fall exakt bekannter Systemmatrizen werden die auf das Gesamtsystem aus Referenzmodell, Strecke und Filter einwirkenden Eingangsgrößen $\boldsymbol{f}, \boldsymbol{u}$ und $\boldsymbol{d}$ skaliert, um ihre Wirkung zu gewichten. Es ergibt sich daraus das Optimierungsproblem

$$
\begin{aligned}
& \underset{\boldsymbol{A}_{f}, \boldsymbol{B}_{f, u}, \boldsymbol{B}_{f, y}, \boldsymbol{C}_{f}, \boldsymbol{D}_{f, u}, \boldsymbol{D}_{f, y}}{\operatorname{minimiere}} \gamma \text {, sodass } \\
& \max _{\boldsymbol{\chi}}\left\|\left[\begin{array}{l}
\alpha_{u}\left(\boldsymbol{G}_{\boldsymbol{r u}, \boldsymbol{\chi}}(s)-\boldsymbol{G}_{\mathrm{ref}, \boldsymbol{r u}}(s)\right)^{\top} \\
\alpha_{f}\left(\boldsymbol{G}_{\boldsymbol{r} \boldsymbol{f}, \boldsymbol{\chi}}(s)-\boldsymbol{G}_{\mathrm{ref}, \boldsymbol{r} \boldsymbol{f}}(s)\right)^{\top} \\
\alpha_{d}\left(\boldsymbol{G}_{\boldsymbol{r} \boldsymbol{d}, \boldsymbol{\chi}}(s)-\boldsymbol{G}_{\mathrm{ref}, \boldsymbol{r} \boldsymbol{d}}(s)\right)^{\top}
\end{array}\right]^{\top}\right\|_{\infty}<\gamma
\end{aligned}
$$

Es wird also über alle möglichen $\chi$ die größte gewichtete Abweichung vom Referenzmodell minimiert.

In gleicher Art und Weise wie in Gleichung (6.55) lässt sich die Wirkung von $\boldsymbol{u}, \boldsymbol{f}$ und $\boldsymbol{d}$ auf den Performance-Ausgang $\boldsymbol{z}=\boldsymbol{r}-\boldsymbol{r}_{\text {ref }}$ für jeden Eckpunkt des durch (6.64) gegebenen Polytops beschreiben durch

$$
\begin{aligned}
& \dot{\boldsymbol{x}}_{g}=\underbrace{\left[\begin{array}{ccc}
\boldsymbol{A}_{\mathrm{ref}} & \mathbf{0} & \mathbf{0} \\
\mathbf{0} & \boldsymbol{A}_{l} & \mathbf{0} \\
\mathbf{0} & \boldsymbol{B}_{f, y} \boldsymbol{C}_{l} & \boldsymbol{A}_{f}
\end{array}\right]}_{\boldsymbol{A}_{\boldsymbol{z} \tilde{u}, l}} \boldsymbol{x}_{g}+\ldots \\
& +\underbrace{\left[\begin{array}{ccc}
\boldsymbol{B}_{\mathrm{ref}, u} & \boldsymbol{B}_{\mathrm{ref}, f} & \boldsymbol{B}_{\mathrm{ref}, d} \\
\boldsymbol{B}_{l} & \boldsymbol{E}_{a, l} & \boldsymbol{B}_{d, l} \\
\boldsymbol{B}_{f, u}+\boldsymbol{B}_{f, y} \boldsymbol{D}_{l} & \boldsymbol{B}_{f, y} \boldsymbol{E}_{s, l} & \boldsymbol{B}_{f, y} \boldsymbol{D}_{d, l}
\end{array}\right]}_{\boldsymbol{B}_{\boldsymbol{z} \tilde{\boldsymbol{u}}, l}} \tilde{\boldsymbol{u}}
\end{aligned}
$$


und

$$
\begin{aligned}
\boldsymbol{z} & =\underbrace{\left[\begin{array}{lll}
-\boldsymbol{C}_{\mathrm{ref}} & \boldsymbol{D}_{f, y} \boldsymbol{C}_{l} & \boldsymbol{C}_{f}
\end{array}\right]}_{\boldsymbol{C}_{\boldsymbol{z} \tilde{\boldsymbol{u}}, l}} \boldsymbol{x}_{g}+\ldots \\
& +\underbrace{\left[\begin{array}{lll}
\boldsymbol{D}_{f, u}+\boldsymbol{D}_{f, y} \boldsymbol{D}_{l}-\boldsymbol{D}_{\mathrm{ref}, u} & \boldsymbol{D}_{f, y} \boldsymbol{E}_{s, l}-\boldsymbol{D}_{\mathrm{ref}, f} & \boldsymbol{D}_{f, y} \boldsymbol{D}_{d, l}-\boldsymbol{D}_{\mathrm{ref}, d}
\end{array}\right]}_{\boldsymbol{D}_{\boldsymbol{z} \tilde{\boldsymbol{u}}, l}} \tilde{\boldsymbol{u}} .
\end{aligned}
$$

Entscheidend ist für die weitere Herleitung des Entwurfsverfahrens das folgende Lemma.

Lemma 6.8. Gegeben sei ein Gesamtsystem der Form (6.66), (6.67) und eine positiv definite Matrix $\boldsymbol{P} \succ \mathbf{0}$, welche

$$
\left[\begin{array}{ccc}
\operatorname{He}\left(\boldsymbol{P} \boldsymbol{A}_{\boldsymbol{z} \tilde{\boldsymbol{u}}, l}\right) & \boldsymbol{P B}_{\boldsymbol{z} \tilde{\boldsymbol{u}}, l} & \boldsymbol{C}_{\boldsymbol{z} \tilde{\boldsymbol{u}}, l} \\
\star & -\gamma \boldsymbol{I}_{n_{u}+n_{f}+n_{d}} & \boldsymbol{D}_{\boldsymbol{z} \tilde{\boldsymbol{u}}, l} \\
\star & \star & -\gamma \boldsymbol{I}_{n_{f}}
\end{array}\right] \prec \mathbf{0}, \quad \forall l=1, \ldots, N
$$

erfüllt. Dann gilt

$$
\left\|\boldsymbol{C}_{\boldsymbol{z} \tilde{\boldsymbol{u}}, \boldsymbol{\chi}}\left(s \boldsymbol{I}_{n_{\mathrm{ref}}+n+n_{\mathrm{filt}}}-\boldsymbol{A}_{\boldsymbol{z} \tilde{\boldsymbol{u}}, \boldsymbol{\chi}}\right)^{-1} \boldsymbol{B}_{\boldsymbol{z} \tilde{\boldsymbol{u}}, \boldsymbol{\chi}}+\boldsymbol{D}_{\boldsymbol{z} \tilde{\boldsymbol{u}}, \boldsymbol{\chi}}\right\|_{\infty}<\gamma, \forall \boldsymbol{\chi} .
$$

Beweis. Aus dem Bounded-Real-Lemma (Satz 3.1) folgt unmittelbar, dass

$$
\left\|\boldsymbol{C}_{\boldsymbol{z} \tilde{\boldsymbol{u}}, l}\left(s \boldsymbol{I}_{n_{\mathrm{ref}}+n+n_{\mathrm{filt}}}-\boldsymbol{A}_{\boldsymbol{z} \tilde{\boldsymbol{u}}, l}\right)^{-1} \boldsymbol{B}_{\boldsymbol{z} \tilde{\boldsymbol{u}}, l}+\boldsymbol{D}_{\boldsymbol{z} \tilde{\boldsymbol{u}}, l}\right\|_{\infty}<\gamma, \forall l=1, \ldots, N
$$

gilt. Da für alle $N$ Eckpunkte des Polytops (6.64) eine gemeinsame Matrix $\boldsymbol{P}$ existiert und sich die Matrizen $\boldsymbol{A}_{\boldsymbol{\chi}}, \boldsymbol{B}_{\boldsymbol{\chi}}, \boldsymbol{E}_{a, \boldsymbol{\chi}}, \boldsymbol{B}_{d, \boldsymbol{\chi}}, \boldsymbol{C}_{\boldsymbol{\chi}}, \boldsymbol{D}_{\boldsymbol{\chi}}, \boldsymbol{E}_{s, \boldsymbol{\chi}}$ und $\boldsymbol{D}_{d, \boldsymbol{\chi}}$ als konvexe Kombinationen der Eckpunkte von $\mathcal{M}$ darstellen lassen, gilt die Schranke $\gamma$ nicht nur für die Eckpunkte, sondern für die gesamte konvexe Hülle des Polytops.

Analog zu (6.60) werden für jeden Eckpunkt des Polytops die abkürzenden Schreibweisen

$$
\begin{array}{ll}
\overline{\boldsymbol{A}}_{l}=\left[\begin{array}{cc}
\boldsymbol{A}_{\mathrm{ref}} & \mathbf{0} \\
\mathbf{0} & \boldsymbol{A}_{l}
\end{array}\right], & \overline{\boldsymbol{B}}_{1, l}=\left[\begin{array}{cc}
\boldsymbol{B}_{\mathrm{ref}, u}^{\prime} & \boldsymbol{B}_{\mathrm{ref}, f}^{\prime} \\
\boldsymbol{B}_{l}^{\prime} & \boldsymbol{E}_{a, l}^{\prime}
\end{array}\right], \overline{\boldsymbol{B}}_{2, l}=\left[\begin{array}{c}
\boldsymbol{B}_{\mathrm{ref}, d}^{\prime} \\
\boldsymbol{B}_{d, l}^{\prime}
\end{array}\right], \\
\overline{\boldsymbol{C}}_{l}=\left[\begin{array}{ll}
\mathbf{0} & \boldsymbol{C}_{l}
\end{array}\right], & \overline{\boldsymbol{D}}_{l}=\left[\begin{array}{ll}
\boldsymbol{D}_{l}^{\prime} & \boldsymbol{E}_{s, l}^{\prime}
\end{array}\right]
\end{array}
$$

eingeführt, wobei eine Skalierung der Eingangs- und Durchgriffsmatrizen gemäß (6.58) vorgenommen wurde. Die Lösung von (6.65) lässt sich wie in 
(6.61) mithilfe von Lemma 6.8 und mit der Variablentransformation aus Abschnitt 6.4.1 formulieren als

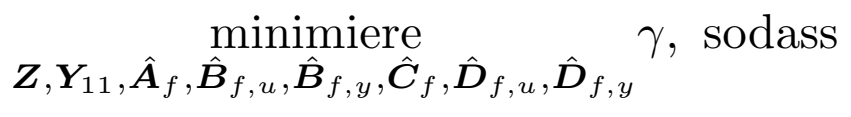

$$
\begin{aligned}
& \boldsymbol{Z} \succ \mathbf{0}, \quad \boldsymbol{Y}_{11}-\boldsymbol{Z} \succ \mathbf{0}, \\
& {\left[\begin{array}{ccc}
\boldsymbol{\Sigma}_{11, l} & \boldsymbol{\Sigma}_{12, l} & \boldsymbol{\Sigma}_{13, l} \\
\star & -\gamma \boldsymbol{I}_{n_{u}+n_{f}+n_{d}} & \boldsymbol{\Sigma}_{23, l} \\
\star & \star & -\gamma \boldsymbol{I}_{n_{f}}
\end{array}\right] \prec \mathbf{0}, \forall l=1, \ldots, N,} \\
& \boldsymbol{\Sigma}_{11, l}=\operatorname{He}\left(\left[\begin{array}{cc}
\boldsymbol{Z} \overline{\boldsymbol{A}}_{l} & \boldsymbol{Z} \overline{\boldsymbol{A}}_{l} \\
\boldsymbol{Y}_{11} \overline{\boldsymbol{A}}_{l}+\hat{\boldsymbol{B}}_{f, y} \overline{\boldsymbol{C}}_{l}+\hat{\boldsymbol{A}}_{f} & \boldsymbol{Y}_{11} \overline{\boldsymbol{A}}_{l}+\hat{\boldsymbol{B}}_{f, y} \overline{\boldsymbol{C}}_{l}
\end{array}\right]\right), \\
& \boldsymbol{\Sigma}_{12, l}=\left[\begin{array}{c}
\boldsymbol{Z} \overline{\boldsymbol{B}}_{1, l} \\
\boldsymbol{Y}_{11} \overline{\boldsymbol{B}}_{1, l}+\hat{\boldsymbol{B}}_{f, u}\left[\begin{array}{cc}
\boldsymbol{I}_{n_{u}} & \mathbf{0}
\end{array}\right]+\hat{\boldsymbol{B}}_{f, y} \overline{\boldsymbol{D}}_{l} \quad \boldsymbol{Y}_{11} \overline{\boldsymbol{B}}_{2, l}+\hat{\boldsymbol{B}}_{f, y} \boldsymbol{D}_{d, l}^{\prime}
\end{array}\right], \\
& \boldsymbol{\Sigma}_{13, l}=\left[\begin{array}{ll}
-C_{\mathrm{ref}} & \hat{\boldsymbol{D}}_{f, y} \boldsymbol{C}_{l}
\end{array}\right]+\hat{\boldsymbol{C}}_{f} \quad\left[\begin{array}{ll}
-\boldsymbol{C}_{\mathrm{ref}} & \hat{\boldsymbol{D}}_{f, y} \boldsymbol{C}_{l}
\end{array}\right]^{\top}, \\
& \boldsymbol{\Sigma}_{23, l}=\left[\begin{array}{lll}
\hat{\boldsymbol{D}}_{f, u}+\hat{\boldsymbol{D}}_{f, y} \boldsymbol{D}_{l}^{\prime}-\boldsymbol{D}_{\mathrm{ref}, u}^{\prime} & \hat{\boldsymbol{D}}_{f, y} \boldsymbol{E}_{s, l}^{\prime}-\boldsymbol{D}_{\mathrm{ref}, f}^{\prime} \quad \hat{\boldsymbol{D}}_{f, y} \boldsymbol{D}_{d, l}^{\prime}-\boldsymbol{D}_{\mathrm{ref}, d}^{\prime}
\end{array}\right]^{\top} \text {. }
\end{aligned}
$$

Auch hier können mittels (6.40) und (6.41) Eigenwertbereiche für die Filtereigenwerte vorgegeben werden. Die Rücktransformation auf die Filterparameter erfolgt wie bei exakt bekannten Systemmatrizen durch (6.62) beziehungsweise mithilfe der Pseudoinversen von $\boldsymbol{U}$ und $\boldsymbol{W}^{\top}$ bei Filtern reduzierter Ordnung. Zusammenfassend gilt

Satz 6.8 (LMI-basierter Entwurf robuster FIFs mit Einschränkung der Filtereigenwerte). Gegeben sei eine für alle $\boldsymbol{\chi}$ stabile Strecke der Form

$$
\begin{aligned}
& \dot{x}=A_{\chi} \boldsymbol{x}+\boldsymbol{B}_{\chi} \boldsymbol{u}+\boldsymbol{E}_{a, \chi} \boldsymbol{f}+\boldsymbol{B}_{d, \chi} \boldsymbol{d} \\
& \boldsymbol{y}=\boldsymbol{C}_{\chi} \boldsymbol{x}+\boldsymbol{D}_{\chi} \boldsymbol{u}+\boldsymbol{E}_{s, \chi} \boldsymbol{f}+\boldsymbol{D}_{d, \chi} \boldsymbol{d}
\end{aligned}
$$

mit (6.64), sowie ein durch (6.7), (6.9) beschriebenes Referenzmodell der

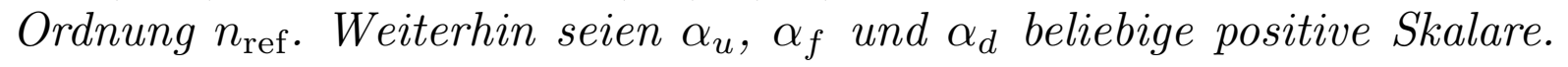
Dann gilt beim Einsatz eines FIFs der Form

$$
\begin{aligned}
\dot{\boldsymbol{x}}_{f} & =\boldsymbol{A}_{f} \boldsymbol{x}_{f}+\boldsymbol{B}_{f, u} \boldsymbol{u}+\boldsymbol{B}_{f, y} \boldsymbol{y}, \\
\boldsymbol{r} & =\boldsymbol{C}_{f} \boldsymbol{x}_{f}+\boldsymbol{D}_{f, u} \boldsymbol{u}+\boldsymbol{D}_{f, y} \boldsymbol{y}
\end{aligned}
$$


mit $n_{\mathrm{filt}}=n+n_{\mathrm{ref}}$ die Beziehung

$$
\left\|\left[\alpha_{u} \boldsymbol{G}_{\boldsymbol{r u}, \boldsymbol{\chi}}(s) \quad \alpha_{f}\left(\boldsymbol{G}_{\boldsymbol{r} \boldsymbol{f}, \boldsymbol{\chi}}(s)-\boldsymbol{G}_{r e f, \boldsymbol{r} \boldsymbol{f}}(s)\right) \quad \alpha_{d} \boldsymbol{G}_{\boldsymbol{r d}, \boldsymbol{\chi}}(s)\right]\right\|_{\infty}<\gamma, \quad \forall \boldsymbol{\chi},
$$

und für alle Filtereigenwerte $\lambda_{i}, i=1, \ldots, n_{\text {filt }}$, gilt $\mathfrak{R e}\left(\lambda_{i}\right)<\alpha,\left|\lambda_{i}\right|<\varrho$ und $\left|\mathfrak{I m}\left(\lambda_{i}\right)\right|<\tan (\phi) \cdot\left|\mathfrak{R e}\left(\lambda_{i}\right)\right|$, wenn die Filtermatrizen durch Lösen des LMI-Problems

$$
\begin{aligned}
& \underset{\boldsymbol{Z}, \boldsymbol{Y}_{11}, \hat{\boldsymbol{A}}_{f}, \hat{\boldsymbol{B}}_{f, u}, \hat{\boldsymbol{B}}_{f, y}, \hat{\boldsymbol{C}}_{f}, \hat{\boldsymbol{D}}_{f, u}, \hat{\boldsymbol{D}}_{f, y}}{\operatorname{minimier}} \boldsymbol{\gamma} \text { sodass } \\
& (6.68 \mathrm{~b}), \\
& (6.40)-(6.42),(6.68 \mathrm{c})-(6.68 \mathrm{~g}) \forall l=1, \ldots, N
\end{aligned}
$$

und anschließende Rücktransformation gemäß (6.37), (6.38), (6.62) ermittelt werden.

Für FIFs reduzierter Ordnung ändert sich der Entwurfssatz nur marginal und es gilt entsprechend

Satz 6.9 (LMI-basierter Entwurf robuster FIFs reduzierter Ordnung mit Einschränkung der Filtereigenwerte). Gegeben sei eine für alle $\chi$ stabile Strecke der Form

$$
\begin{aligned}
& \dot{\boldsymbol{x}}=\boldsymbol{A}_{\chi} \boldsymbol{x}+\boldsymbol{B}_{\chi} \boldsymbol{u}+\boldsymbol{E}_{a, \chi} \boldsymbol{f}+\boldsymbol{B}_{d, \chi} \boldsymbol{d}, \\
& \boldsymbol{y}=\boldsymbol{C}_{\chi} \boldsymbol{x}+\boldsymbol{D}_{\chi} \boldsymbol{u}+\boldsymbol{E}_{s, \chi} \boldsymbol{f}+\boldsymbol{D}_{d, \chi} \boldsymbol{d}
\end{aligned}
$$

mit (6.64), sowie ein durch (6.7), (6.9) beschriebenes Referenzmodell der

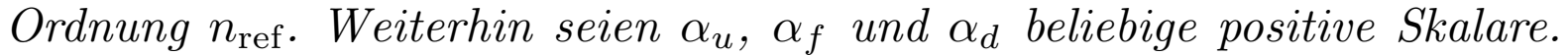
Dann gilt beim Einsatz eines FIFs der Form

$$
\begin{aligned}
\dot{\boldsymbol{x}}_{f} & =\boldsymbol{A}_{f} \boldsymbol{x}_{f}+\boldsymbol{B}_{f, u} \boldsymbol{u}+\boldsymbol{B}_{f, y} \boldsymbol{y}, \\
\boldsymbol{r} & =\boldsymbol{C}_{f} \boldsymbol{x}_{f}+\boldsymbol{D}_{f, u} \boldsymbol{u}+\boldsymbol{D}_{f, y} \boldsymbol{y}
\end{aligned}
$$

mit $n_{\mathrm{filt}}=n$ die Beziehung

$$
\left\|\left[\alpha_{u} \boldsymbol{G}_{\boldsymbol{r u}, \boldsymbol{\chi}}(s) \quad \alpha_{f}\left(\boldsymbol{G}_{\boldsymbol{r} \boldsymbol{f}, \boldsymbol{\chi}}(s)-\boldsymbol{G}_{r e f, \boldsymbol{r} \boldsymbol{f}}(s)\right) \quad \alpha_{d} \boldsymbol{G}_{\boldsymbol{r d}, \boldsymbol{\chi}}(s)\right]\right\|_{\infty}<\gamma, \quad \forall \boldsymbol{\chi},
$$

und für alle Filtereigenwerte $\lambda_{i}, i=1, \ldots, n_{\text {filt }}$, gilt $\mathfrak{R e}\left(\lambda_{i}\right)<\alpha,\left|\lambda_{i}\right|<\varrho$ und $\left|\mathfrak{I m}\left(\lambda_{i}\right)\right|<\tan (\phi) \cdot\left|\mathfrak{R e}\left(\lambda_{i}\right)\right|$, wenn die Filtermatrizen durch Lösen des rangbeschränkten LMI-Problems

$$
\begin{aligned}
& \underset{\boldsymbol{Z}, \boldsymbol{Y}_{11}, \hat{\boldsymbol{A}}_{f}, \hat{\boldsymbol{B}}_{f, u}, \hat{\boldsymbol{B}}_{f, y}, \hat{\boldsymbol{C}}_{f}, \hat{\boldsymbol{D}}_{f, u}, \hat{\boldsymbol{D}}_{f, y}}{\operatorname{minimie}} \gamma \text {, sodass } \\
& (6.40)-(6.42),(6.68 \mathrm{c})-(6.68 \mathrm{~g}) \forall l=1, \ldots, N
\end{aligned}
$$


und anschließende Rücktransformation gemäß (6.48)-(6.50), (6.62) ermittelt werden.

Verschiedene Aspekte der beiden Entwurfssätze verdienen besondere Beachtung. Zunächst ist auffällig, dass sich die LMI-Nebenbedingungen kaum von denen der Sätze 6.6 und 6.7 unterscheiden. Es sind lediglich die LMIs zur Beschränkung von $\gamma$ und zur Beschränkung der Eigenwerte für jeden Eckpunkt des Polytops $\mathcal{M}$ einzuhalten. Im Vergleich zum beobachterbasierten Ansatz (vgl. Abschnitt 5.4.4) ergibt sich also ein wesentlich einfacheres Entwurfsverfahren.

Darüber hinaus ist einerseits zu betonen, dass wegen Lemma 6.8 die gefundene Schranke $\gamma$ nicht nur wie beim beobachterbasierten Ansatz für die Eckpunkte des Polytops gilt, sondern für alle Systeme innerhalb von $\mathcal{M}$ garantiert ist. Andererseits ist auch festzuhalten, dass dafür (6.68c) bis (6.68g), (6.40), (6.41) und (6.42) für alle $l=1, \ldots, N$ Eckpunkte mit gemeinsamen Matrizen $\boldsymbol{Z}$ und $\boldsymbol{Y}_{11}$ zu erfüllen sind, wodurch die Lösung konservativ ist.

Ebenfalls auffällig ist, dass die beiden Entwurfssätze kein nominales Modell voraussetzen und dass somit als einzige Anforderung an die unsichere Strecke die Stabilität für alle möglichen Parameter zu stellen ist. Theoretisch betrachtet sind keine weiteren Anforderungen zu stellen und es kann ein beliebiges Referenzmodell angesetzt werden. Um beim Entwurf sinnvolle Ergebnisse zu erhalten, ist es jedoch nötig, ein nominales Modell $\boldsymbol{M}_{0} \in \mathcal{M}$ zu analysieren und auf dieser Grundlage (insbesondere der ermittelten Fehlerindizes und der Fehlerdetektionsmatrix) ein Referenzmodell auszulegen. Sollten dynamische Erweiterungen notwendig sein, ist in den Entwurfssätzen 6.8 und 6.9 entsprechend die Filterordnung zu erhöhen. Alternativ kann man auch fordern, dass alle Systeme aus $\mathcal{M}$ die gleichen Fehlerindizes aufweisen müssen und das Referenzmodell dann anhand dieser $\delta_{i}$ konstruieren. Dann ist kein ausgezeichnetes nominales Modell nötig. Bemerkung 6.2. In Anlehnung an das am Ende von Abschnitt 6.3 beschriebene Verfahren aus [271] ist für Systeme mit unsicheren Parametern auch ein Entwurf in zwei Schritten möglich. Dabei ist ein störungsbehaftetes, nominales Modell anzusetzen, für das wie in Abschnitt 6.5.1 ein robustes FIF der Ordnung $n_{\text {filt,1 }}$ entworfen wird. Das sich ergebende Übertragungsverhalten $\boldsymbol{G}_{\boldsymbol{r} \boldsymbol{d}}(s)$ beziehungsweise $\boldsymbol{G}_{\boldsymbol{r} \boldsymbol{u}}(s)$ wird dann für das neue Referenzmodell des zweiten Entwurfsschrittes herangezogen, in dem die unsicheren Parameter berücksichtigt werden. Nachteilig bei diesem Vorgehen ist jedoch, dass sich für den zweiten Entwurfsschritt ein Referenzmodell erhöhter Ordnung $n_{\text {ref,2 }}=n+n_{\text {filt, } 1}$ ergibt. 


\section{Berücksichtigung eines nominalen Modells}

Bei der Lösung von (6.65) wird die größte Abweichung vom gewichteten Referenzmodell im Sinne der $\mathcal{H}_{\infty}$-Norm minimiert. Für ein nominales Modell wird beim vorgestellten FIF-Ansatz im Gegensatz zur beobachterbasierten Methode aus Abschnitt 5.4.4 keine ideale Fehlerisolation gewährleistet. Dies lässt sich jedoch durch eine Erweiterung ebenfalls erreichen. Dazu wird (6.68) um die zusätzliche LMI-Bedingung

$$
\begin{aligned}
& {\left[\begin{array}{ccc}
\boldsymbol{\Sigma}_{11,0} & \boldsymbol{\Sigma}_{12,0} & \boldsymbol{\Sigma}_{13,0} \\
\star & -\varepsilon \boldsymbol{I}_{n_{u}+n_{f}} & \boldsymbol{\Sigma}_{23,0} \\
\star & \star & -\varepsilon \boldsymbol{I}_{n_{f}}
\end{array}\right] \prec \mathbf{0},} \\
& \boldsymbol{\Sigma}_{11,0}=\operatorname{He}\left(\left[\begin{array}{cc}
\boldsymbol{Z} \overline{\boldsymbol{A}}_{0} & \boldsymbol{Z} \overline{\boldsymbol{A}}_{0} \\
\boldsymbol{Y}_{11} \overline{\boldsymbol{A}}_{0}+\hat{\boldsymbol{B}}_{f, y} \overline{\boldsymbol{C}}_{0}+\hat{\boldsymbol{A}}_{f} & \boldsymbol{Y}_{11} \overline{\boldsymbol{A}}_{0}+\hat{\boldsymbol{B}}_{f, y} \overline{\boldsymbol{C}}_{0}
\end{array}\right]\right), \\
& \boldsymbol{\Sigma}_{12,0}=\left[\begin{array}{c}
\boldsymbol{Z} \overline{\boldsymbol{B}}_{0} \\
\boldsymbol{Y}_{11} \overline{\boldsymbol{B}}_{0}+\hat{\boldsymbol{B}}_{f, u}\left[\begin{array}{ll}
\boldsymbol{I}_{n_{u}} & \mathbf{0}
\end{array}\right]+\hat{\boldsymbol{B}}_{f, y} \overline{\boldsymbol{D}}_{0}
\end{array}\right], \\
& \boldsymbol{\Sigma}_{13,0}=\left[\begin{array}{ll}
-C_{\mathrm{ref}} & \hat{\boldsymbol{D}}_{f, y} \boldsymbol{C}_{0}
\end{array}\right]+\hat{\boldsymbol{C}}_{f} \quad\left[\begin{array}{ll}
-\boldsymbol{C}_{\mathrm{ref}} & \hat{\boldsymbol{D}}_{f, y} \boldsymbol{C}_{0}
\end{array}\right]^{\top}, \\
& \boldsymbol{\Sigma}_{23,0}=\left[\begin{array}{ll}
\hat{\boldsymbol{D}}_{f, u}+\hat{\boldsymbol{D}}_{f, y} \boldsymbol{D}_{0}-\boldsymbol{D}_{\mathrm{ref}, u} & \hat{\boldsymbol{D}}_{f, y} \boldsymbol{E}_{s, 0}-\boldsymbol{D}_{\mathrm{ref}, f}
\end{array}\right]^{\top}
\end{aligned}
$$

ergänzt. Diese entspricht (6.35) und stellt sicher, dass für das nominale System

$$
\left\|\left[\boldsymbol{G}_{\boldsymbol{r u}, 0}(s)-\boldsymbol{G}_{\mathrm{ref}, \boldsymbol{r u}}(s) \quad \boldsymbol{G}_{\boldsymbol{r f}, 0}(s)-\boldsymbol{G}_{\mathrm{ref}, \boldsymbol{r} \boldsymbol{f}}(s)\right]\right\|_{\infty}<\varepsilon
$$

mit einem beliebig kleinen, vorgegebenen Skalar $\varepsilon>0$ gilt. Damit wird für das nominale, störungsfreie System eine ideale Fehlerisolation sichergestellt.

\section{Akademisches Beispiel}

Um die Leistungsfähigkeit des Ansatzes zu veranschaulichen, wird erneut auf das durch (5.72) und (5.73) definierte Beispiel aus Abschnitt 5.4.4 zurückgegriffen (s. Seite 242). Um einen Vergleich zwischen dem beobachterbasierten Ansatz und dem FIF zu ermöglichen, werden für das Referenzmodell die gleichen Pole vorgegeben wie für den in Abschnitt 5.4.4 entworfenen FIO, es ist also

$$
\boldsymbol{A}_{\mathrm{ref}}=\left[\begin{array}{rr}
-4 & 0 \\
0 & -6
\end{array}\right], \quad \boldsymbol{B}_{\mathrm{ref}, f}=\boldsymbol{I}_{2}, \boldsymbol{C}_{\mathrm{ref}}=\left[\begin{array}{ll}
4 & 0 \\
0 & 6
\end{array}\right], \quad \boldsymbol{D}_{\mathrm{ref}, f}=\mathbf{0}
$$



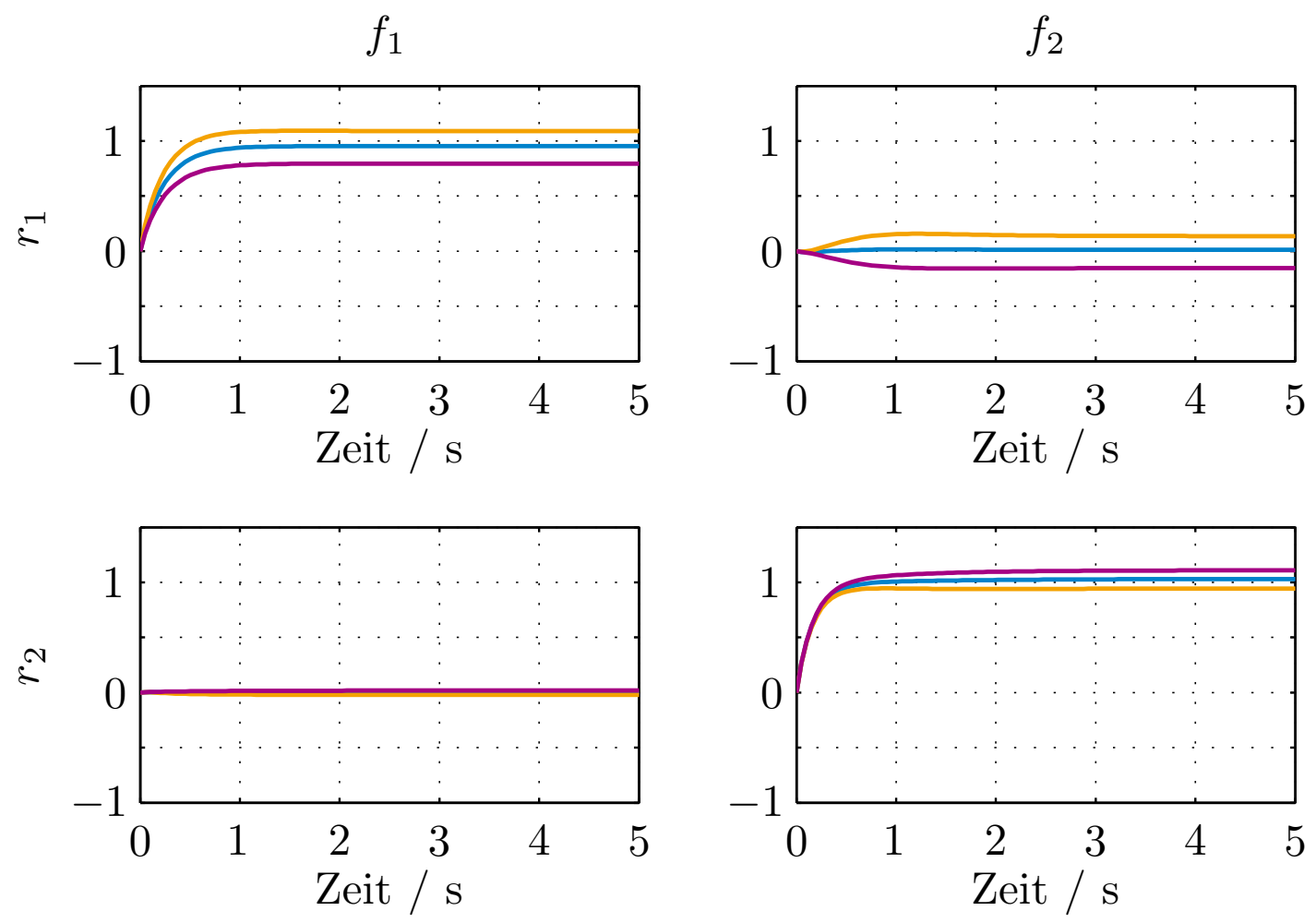

Abbildung 6.5: Sprungantwort von $\boldsymbol{G}_{\boldsymbol{r} \boldsymbol{f}}(s)$ des optimierten FIFs für $\rho=0$ $(-), \rho=-1(-)$ und $\rho=1(-)$

und $\boldsymbol{B}_{\mathrm{ref}, u}=\mathbf{0}, \boldsymbol{D}_{\mathrm{ref}, u}=\mathbf{0}$. Die Filtereigenwerte werden durch $\alpha=-0,1$, $\varrho=25$ und $\phi=\pi / 4$ beschränkt. Um eine nahezu ideale Fehlerisolation im nominalen Fall zu gewährleisten, wird (6.69) mit $\varepsilon=0,1$ angesetzt. Der Entwurf eines FIFs nach Satz 6.8 liefert dann die in Anhang C.3.3 angegebene Parametrierung. Die resultierenden Sprungantworten von $\boldsymbol{G}_{\boldsymbol{r} \boldsymbol{f}}(s)$ und $\boldsymbol{G}_{\boldsymbol{r} \boldsymbol{u}}(s)$ sind für verschiedene Werte des unsicheren Parameters $\rho$ in den Abbildungen 6.5 und $6.6 \mathrm{zu}$ sehen.

$\mathrm{Zu}$ erkennen ist zunächst, dass für die betrachteten Werte von $\rho$ sowohl eine gute Unterdrückung des Einflusses der Stellgröße $u$ auf die generierten Residuen erfolgt, als auch eine weitgehende Diagonalisierung von $\boldsymbol{G}_{\boldsymbol{r} \boldsymbol{f}}(s)$. Im Vergleich zum beobachterbasierten Ansatz aus Abschnitt 5.4.4 ergibt sich eine vergleichbare Qualität der Fehlerisolation (vgl. Abbildungen 5.19 und 5.20). Während der FIO bezüglich der Diagonalisierung von $\boldsymbol{G}_{\boldsymbol{r} \boldsymbol{f}}(s)$ ein etwas besseres Ergebnis erzielt, führt der FIF auf eine leicht verbesserte Unterdrückung der Stellgröße. Bei insgesamt gleicher Qualität der Ergebnisse ist erneut die Tatsache zu betonen, dass durch den FIF-Entwurf die gesamte konvexe Hülle der unsicheren Systeme abgedeckt ist und dass sich die Entwurfsmethodik wesentlich einfacher und transparenter gestaltet. 

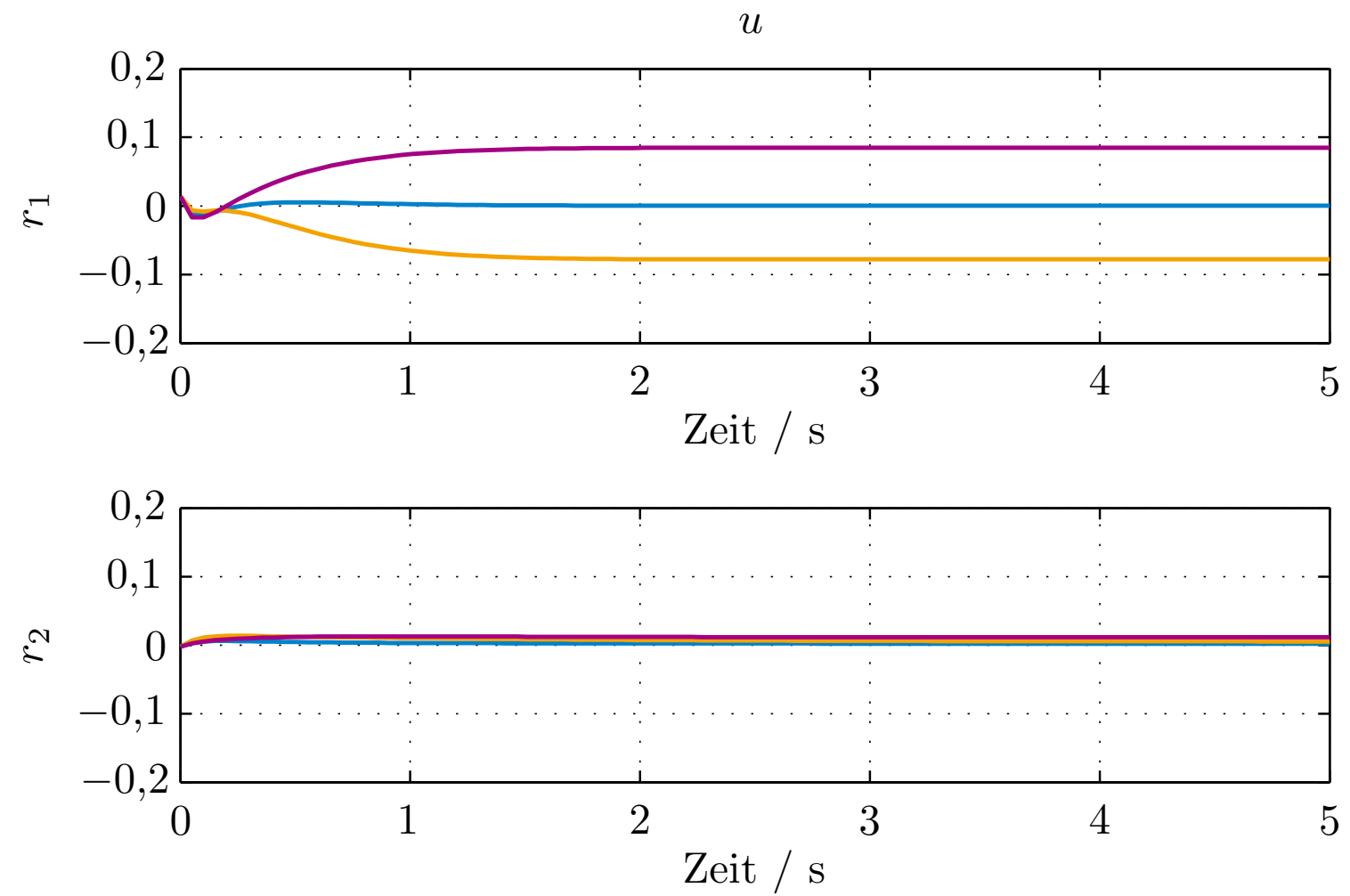

Abbildung 6.6: Sprungantwort von $\boldsymbol{G}_{\boldsymbol{r} \boldsymbol{u}}(s)$ des optimierten FIFs für $\rho=0$ $(-), \rho=-1(-)$ und $\rho=1(-)$

\subsubsection{Fazit}

Mit FIFs ist ebenso wie mit FIOs eine Optimierung der Robustheit bezüglich exogener Störungen möglich. Steht die gleiche Anzahl von Messgrößen zur Verfügung und werden die gleichen strukturellen Anforderungen gestellt (zum Beispiel ideale Diagonalisierung von $\boldsymbol{G}_{\boldsymbol{r} \boldsymbol{f}}(s)$ ), so wird mit beiden Konzepten das gleiche Störunterdrückungsniveau erreicht. Ein wesentlicher Vorteil der FIFs ist wie schon in Abschnitt 6.4 erwähnt im einfachen Entwurfsverfahren zu sehen. Stehen beispielsweise in einem nichtquadratischen System zusätzliche Messgrößen zur Verfügung, so werden diese inhärent genutzt, die Anwendung von Satz 6.6 beziehungsweise Satz 6.7 bleibt unverändert. Darüber hinaus weisen FIFs eine höhere Flexibilität auf. Durch eine Anpassung der Gewichtungsfaktoren kann in einfacher Art und Weise zwischen den konkurrierenden Entwurfszielen abgewogen werden. So ist es beispielsweise möglich, die ideale Diagonalisierung von $\boldsymbol{G}_{\boldsymbol{r} \boldsymbol{f}}(s)$ zu Gunsten einer weiter verbesserten Störunterdrückung abzuschwächen. Dies ist zwar wie gezeigt auch für FIOs möglich, jedoch ist für die Beobachter wiederum ein spezialisiertes Entwurfsverfahren nötig. 
Weiterhin lässt sich der FIF-Entwurf geradlinig auf Systeme mit unsicheren Parametern erweitern. Dem Entwurfsproblem sind lediglich endlich viele zusätzliche LMI-Nebenbedingungen hinzuzufügen. Da gemäß Lemma 6.8 eine gemeinsame Matrix $\boldsymbol{P}$ für alle Eckpunkte eines Polytops herangezogen wird, ergeben sich konservative Lösungen. Der Entwurf gestaltet sich jedoch wesentlich einfacher als mittels FIOs, wobei zusätzlich die Einhaltung der gefundenen Schranke für die Robustheit nicht nur für einige ausgezeichnete Systeme gilt, sondern für alle Systeme innerhalb des betrachteten Polytops garantiert ist.

\subsection{Zusammenfassung des Kapitels}

In diesem Kapitel wurde mit Fehlerisolationsfiltern ein gegenüber Fehlerisolationsbeobachtern strukturell allgemeinerer Ansatz vorgestellt. Der Entwurf erfolgt dabei bereits für nominale, störungsfreie Systeme nicht analytisch sondern optimierungsbasiert durch Lösen eines ModelMatching-Problems. Schlüssel für den zweistufigen Entwurf ist zum einen der konstruktive Entwurf des Referenzmodells auf Grundlage der Systemanalyse. Dabei wird auf die Resultate aus Kapitel 4 zurückgegriffen, ohne dass ein FIO entworfen werden muss. Weiterhin gelingt durch eine geeignete Variablentransformation die Umwandlung in ein LMI-Problem, somit ist ein effizient zu lösendes konvexes Problem gegeben.

Der wesentliche Vorteil gegenüber beobachterbasierten Konzepten ist im einheitlichen Entwurf zu sehen. Es spielt dabei keine Rolle, ob ein quadratisches oder nichtquadratisches System betrachtet wird, ob es beispielsweise statisch nicht isolierbar oder nicht minimalphasig ist oder ob eine erweiterte Dynamik der Diagonalelemente von $\boldsymbol{G}_{\boldsymbol{r} \boldsymbol{f}}(s)$ angestrebt wird. Nach einer entsprechenden Auslegung des Referenzmodells ändert sich bei der Parametrierung der Filtermatrizen nichts. Dies vereinfacht den Entwurf gegenüber FIOs wesentlich. Zwar wurde der optimierungsbasierte Ansatz zur Parametrierung allgemeiner Filter für die Fehlerdetektion bereits in [149] vorgeschlagen, das Potential des Ansatzes für die Fehlerisolation wurde jedoch erst durch die in diesem Kapitel geschaffene Verbindung mit den beobachterbasierten Methoden und den in Kapitel 4 erzielten neuen Ergebnissen aus diesem Bereich ausgenutzt. In ebenso einfacher Art und Weise lässt sich die Robustheit sowohl gegenüber exogenen Störungen als auch unsicheren Parametern steigern. Die Freiheitsgrade in nichtquadratischen Systemen werden dabei inhärent genutzt, auch strukturelle Relaxierungen lassen sich durch eine Anpassung der Gewichtungsfaktoren leicht einbin- 
den. Diese Gewichtungsfaktoren stellen die wesentlichen Entwurfsparameter dar, wobei ihre Wahl intuitiv ist: Sie beschreiben jeweils, wie viel Wert auf die Einhaltung der Anforderungen hinsichtlich der Fehler, der Stellgrößen und der Störgrößen gelegt wird. Für unsichere Systeme sind die gefundenen Ergebnisse außerdem nicht nur für eine endliche Anzahl von Systemen abgesichert, vielmehr besitzen sie für alle Systeme innerhalb einer konvexen Hülle Gültigkeit. Anzumerken ist, dass sich die Qualität der Fehlerisolation (insbesondere die Robustheit) mittels FIFs gegenüber FIOs grundsätzlich nicht steigern lässt, da auch ihnen nicht mehr Information zur Verfügung steht. Es lässt sich jedoch die gleiche Qualität mit höherer Flexibilität bei geringerem Aufwand mittels eines einheitlichen Entwurfes erreichen.

Denkbar ist es darüber hinaus auch, für FIFs ebenso wie in Kapitel 5 für FIOs vorgestellt Störungen in beschränkten Frequenzbereichen zu betrachten oder den Entwurf auf ein Standard-Regelungsproblem zurück zu führen. 


\section{Zusammenfassung und Ausblick}

Ziel der Arbeit war der Entwurf robuster Fehlerisolationsfilter niedriger Ordnung für eine möglichst große Klasse linearer zeitinvarianter Systeme.

Als ein Schlüssel zur Erreichung dieses Ziels hat sich die systematische Ausnutzung der Dualität von Fehlerisolationsbeobachtern zum linearen Entkopplungsreglerentwurf herausgestellt. Auf Basis dieser Erkenntnis lassen sich mit FIOs Fehlerisolationssysteme niedriger Ordnung entwerfen, wohingegen klassische Resultate meist eine Bank von Beobachtern zur Fehlerisolation benötigen. In Kapitel 4 wurde darüber hinaus gezeigt, wie sich unter Rückgriff auf Resultate aus dem Bereich der statisch nicht stabil entkoppelbaren Systeme die Klasse der mittels FIOs fehlerisolierbaren Systeme erweitern lässt. Durch entsprechende virtuelle Systemerweiterungen und deren Realisierung mittels dynamisch erweiterter Beobachter konnte so die Klasse der handhabbaren Systeme erweitert werden, wobei stets konstruktive Entwurfsverfahren entwickelt wurden. Wesentliches Hilfsmittel dabei ist die Vollständige Modale Synthese. Sie wurde im Rahmen dieser Arbeit erstmals systematisch dazu verwendet, der Fehler-ResiduenÜbertragungsmatrix eine gewünschte Struktur aufzuprägen. Neben den angesprochenen Erweiterungen der handhabbaren Systemklasse erlaubt sie die Charakterisierung der sich ergebenden Freiheitsgrade sowohl in nichtquadratischen Systemen als auch bei Abschwächung der strukturellen Anforderungen an die Fehler-Residuen-Übertragungsmatrix. Dies schließt die teilweise Fehlerisolation ebenso ein wie eine näherungsweise Diagonalisierung von $\boldsymbol{G}_{\boldsymbol{r} \boldsymbol{f}}(s)$.

Damit stellt die Vollständige Modale Synthese auch die Grundlage für verschiedene Verfahren zur Optimierung der Robustheit von FIOs dar (s. Kapitel 5). Zunächst wurde in Abschnitt 5.3 gezeigt, wie sich in quadratischen Systemen die verbleibenden Freiheitsgrade zur Steigerung der Robustheit bezüglich exogener Störungen nutzen lassen. Dies umfasst die Optimierung der einzelnen Elemente von $\boldsymbol{G}_{\boldsymbol{r} \boldsymbol{f}}(s)$, die Relaxierung der strukturellen Anforderungen an ebendiese Übertragungsmatrix sowie eine Erweiterung der Dynamik ihrer Diagonalelemente und deren 
Realisierung mittels dynamisch erweiterter Fehlerisolationsbeobachter. Die zusätzlichen Freiheitsgrade in nichtquadratischen Systemen bieten weitergehende Möglichkeiten zur Robustheitssteigerung (s. Abschnitt 5.4). Dabei wurden im Rahmen dieser Arbeit erstmals im Bereich der FIOs nicht nur allgemeine exogene Störungen berücksichtigt, sondern sowohl Störungen in beschränkten Frequenzbereichen als auch parametrische Unsicherheiten. Während Ersteres die Einbindung von zusätzlichem Prozesswissen in den Entwurf erlaubt, lässt sich mit Letzterem die strukturelle Information über die unsicheren Parameter direkt im Entwurf ausnutzen. Auch bei der teilweisen Fehlerisolation lassen sich wie gezeigt die zusätzlichen Freiheitsgrade zur Robustheitsoptimierung nutzen. Alle dabei entwickelten Verfahren lassen sich automatisiert umsetzen, es müssen lediglich intuitive Entwurfsparameter wie zum Beispiel Gewichtungsfaktoren für den Einfluss von Stell- und Störgrößen oder Eigenwertbereiche angepasst werden. Bei aller Flexibilität und Vielseitigkeit des beobachterbasierten Ansatzes zeigt sich jedoch, dass für spezifische Problemstellungen jeweils eigene Entwurfsverfahren herangezogen werden müssen.

Einen wesentlichen Fortschritt stellen in dieser Hinsicht allgemeine Fehlerisolationsfilter dar (s. Kapitel 6). Während die grundsätzliche Struktur bereits bekannt war, lässt sich ihr ganzes Potential erst auf Basis der im Rahmen dieser Arbeit erzielten Ergebnisse aus dem Bereich der beobachterbasierten Fehlerisolation ausnutzen. Der Entwurf erfolgt optimierungsbasiert, wobei ein Referenzmodell auszulegen ist. Für diese Auslegung bieten die Ergebnisse aus Kapitel 4 die Grundlage. Ein wesentliches Merkmal von FIFs ist, dass dynamische Erweiterungen, wie sie zum Beispiel für nicht minimalphasige Systeme nötig sind, beim optimierungsbasierten Entwurf automatisch erfolgen. Es ist lediglich das Referenzmodell auf Basis der Systemanalyse anzupassen, der Entwurf des Filters selbst bleibt unverändert. Es zeigt sich weiterhin, dass die Optimierung der Robustheit sowohl hinsichtlich exogener Störungen als auch bezüglich unsicherer Parameter in geradliniger Art und Weise erfolgen kann. Im Gegensatz zum beobachterbasierten Ansatz sind keine spezialisierten Entwurfsverfahren nötig. Damit ermöglichen FIFs den einfachen und einheitlichen Entwurf robuster modellbasierter Fehlerisolationsfilter niedriger Ordnung.

Aufbauend auf den erzielten Ergebnissen bietet sich eine Vielzahl von Anknüpfungspunkten für weitere Untersuchungen.

Zeitdiskrete Systeme. Naheliegend ist die Übertragung der Entwurfsverfahren auf zeitdiskrete Systeme. Da sowohl für das Bounded-RealLemma als auch für das Verallgemeinerte Kalman-Yakubovich-PopovLemma entsprechende zeitdiskrete Varianten existieren [58, 113], ist die 
Behandlung solcher Systeme ohne Weiteres möglich. Dies erlaubt beispielsweise die Betrachtung digital vernetzter Regelungssysteme. Darüber hinaus ergeben sich neue Möglichkeiten, zum Beispiel hinsichtlich der Optimierung der $\mathcal{H}_{2}$-Norm der Störübertragungsmatrix. Diese Norm, die ein Maß für die mittlere Störverstärkung darstellt, wurde im Rahmen der vorliegenden Arbeit nicht näher betrachtet, da sie für Systeme mit $\boldsymbol{D}_{d} \neq \mathbf{0}$ nicht definiert ist. Dies ist für zeitdiskrete Systeme jedoch der Fall, sodass ein weiteres Optimierungskriterium herangezogen werden kann.

Erweiterungen der entwickelten Verfahren. Auch für zeitkontinuierliche Systeme können andere Normen bezüglich der Störübertragungsmatrix (zum Beispiel die peak-to-peak-Norm) optimiert werden, für die ebenfalls hinreichende LMI-Bedingungen existieren.

Darüber hinaus bietet der Entwurf zeitvarianter Matrizen $\boldsymbol{L}(t)$ und $\boldsymbol{V}(t)$, der im Rahmen dieser Arbeit nur angerissen wurde, erhebliches Potential. Dieses ist insbesondere dann gegeben, wenn sich die Charakteristik der auf das System einwirkenden Störungen mit der Zeit ändert. Denkbar ist beispielsweise, dass das Rauschen eines Sensors durch Temperatureinflüsse mit der Zeit ansteigt. In Verbindung mit einer Online-Schätzung von $\boldsymbol{D}_{d}$ könnte dann eine Anpassung der Beobachterparametrierung erfolgen.

In dieser Arbeit wurden stets Systeme untersucht, für die $n_{y} \geq n_{f}$ gilt. Insbesondere wenn Sensorfehler mit betrachtet werden ist es jedoch denkbar, dass die Anzahl der möglichen Fehler die Anzahl der Messgrößen übersteigt, das heißt, dass $n_{y}<n_{f}$ gilt. In solchen Fällen ist es prinzipiell möglich, mehrere Fehler zu Fehlergruppen zusammenzufassen. Wählt man die Anzahl der Fehlergruppen entsprechend der Anzahl der Messgrößen, so kann angestrebt werden, der Matrix $\boldsymbol{G}_{\boldsymbol{r} \boldsymbol{f}}(s)$ eine Blockdiagonalstruktur aufzuprägen. Dann ist zumindest eine blockweise Fehlerisolation möglich. Es kann also ermittelt werden, aus welcher Fehlergruppe ein Fehler aufgetreten ist. Neben der Frage nach Existenzbedingungen stellt sich die Entwurfsfrage für eine nominale Systemdynamik ebenso wie die Frage nach der Optimierung der Robustheit. Darüber hinaus ist zu untersuchen, wie die Fehler am günstigsten zu gruppieren sind.

Ebenso wie für die beobachterbasierten Methoden ist es auch für allgemeine Fehlerisolationsfilter möglich, Störungen gezielt in eingeschränkten Frequenzbereichen zu betrachten und somit zusätzliches Prozesswissen für eine Verbesserung der Störunterdrückung zu nutzen. Weiterhin ist es auch denkbar, das bisher fest vorgegebene Referenzmodell simultan zu optimieren. Dies ist einerseits durch eine Formulierung als StandardRegelungsproblem realisierbar oder mittels eines Path-Following-Ansatzes. 
Erweiterung auf größere Systemklassen. Die Betrachtungen im Rahmen dieser Arbeit waren stets auf lineare zeitinvariante Systeme konzentriert. Die Ergebnisse können als Grundlage dienen, auch Klassen nichtlinearer Systeme, lineare zeitvariante oder lineare parametervariable (LPV) Systeme zu betrachten. Insbesondere letztere stellen im Bereich der Fehlerdiagnose ein aktives und viel beachtetes Forschungsfeld dar (s. zum Beispiel $[92,152])$. Grund hierfür ist unter anderem, dass sich viele nichtlineare Systeme in einem relevanten Arbeitsbereich als Quasi-LPV Systeme modellieren lassen. Somit könnte das Problem der Fehlerisolation für nichtlineare Systeme auf Methoden zum Entwurf von FIOs oder FIFs für lineare (parametervariable) Systeme zurückgeführt werden.

Erweiterung auf umfassendere Problemstellungen. Neben den genannten Punkten bieten sich Anknüpfungen zu umfassenderen Problemstellungen. Ein zur Zeit intensiv erforschtes Gebiet stellen sogenannte Multi-Agenten-Systeme dar, die aus einer Vielzahl kooperierender oder konkurrierender Einzelsysteme bestehen. In jüngerer Vergangenheit wurden Fragen der Fehlerdiagnose innerhalb solcher Systeme aufgeworfen. Dabei ist einerseits eine kooperative Fehlerisolation denkbar, bei der die einzelnen Agenten den Informationsaustausch untereinander dazu nutzen, Fehler im Gesamtsystem schneller oder zuverlässiger zu isolieren oder dies durch die Kooperation erst zu ermöglichen [17]. Andererseits kann auch das bewusste Stören der Kommunikation seitens eines Agenten als Fehler aufgefasst werden [171]. Dann kann es mittels einer Fehlerisolation gelingen, den entsprechenden Agenten zu identifizieren und aus dem Netzwerk auszuschließen. In beiden Fällen ist ein Einsatz der im Rahmen dieser Arbeit vorgestellten Methoden denkbar.

Für den zuverlässigen Betrieb technischer Systeme stellen Methoden der Fehlerisolation beziehungsweise der Fehlerdiagnose im Allgemeinen lediglich eine Zwischenstufe dar. Ziel ist letztendlich die Schaffung fehlertoleranter Systeme, um die Verfügbarkeit zu erhöhen [16]. In dieser Hinsicht ist zu untersuchen, inwiefern sich die erarbeiteten Methoden zur Fehlerisolation beispielsweise zur Rekonfiguration der Regelung nutzen lassen.

Nicht zuletzt werfen die Ergebnisse der Arbeit Fragen bezüglich der praktischen Anwendbarkeit in realen Systemen auf. Zwar erfolgte bereits im Rahmen dieser Arbeit sowohl die Erprobung anhand von Simulationen als auch anhand von Messungen an Laborprüfständen. Reale industrielle Problemstellungen bringen jedoch wesentlich komplexere Fragestellungen mit sich und es ist zu untersuchen, inwieweit sich die erzielten Ergebnisse in diesem Umfeld anwenden lassen. 


\section{A Mathematische Sätze und Lemmata}

Satz A.1 (Rangsatz). Gegeben sei eine Matrix $\boldsymbol{P} \in \mathbb{R}^{n \times m}$. Dann gelten nach [150, Abschnitt 4.4] die Beziehungen

$$
\begin{aligned}
\operatorname{rang}(\boldsymbol{P})+\operatorname{dim}(\operatorname{ker}(\boldsymbol{P})) & =m, \\
\operatorname{rang}(\boldsymbol{P})+\operatorname{dim}(\operatorname{lker}(\boldsymbol{P})) & =n,
\end{aligned}
$$

wobei $\operatorname{ker}(\boldsymbol{P})$ der Rechtsnullraum und $1 \mathrm{ker}(\boldsymbol{P})$ der Linksnullraum von $\boldsymbol{P}$ ist.

Lemma A.1. Gegeben seien zwei Matrizen $\boldsymbol{A} \in \mathbb{R}^{n \times m}$ und $\boldsymbol{B} \in \mathbb{R}^{m \times p}$. Dann gilt nach [14, Proposition 2.5.8] die Ungleichung

$$
\operatorname{rang}(\boldsymbol{A})+\operatorname{rang}(\boldsymbol{B}) \leq m+\operatorname{rang}(\boldsymbol{A} \boldsymbol{B}) .
$$

Lemma A.2. Gegeben seien zwei invertierbare Matrizen $\boldsymbol{A} \in \mathbb{R}^{n \times n}$ und $\boldsymbol{B} \in \mathbb{R}^{n \times n}$. Dann gilt

$$
A^{-1}-B^{-1}=B^{-1}(B-A) A^{-1}=-A^{-1}(A-B) B^{-1} .
$$

Beweis. Der Ausdruck $\boldsymbol{A}^{-1}-\boldsymbol{B}^{-1}$ kann geschrieben werden als

$$
\boldsymbol{B}^{-1}\left(\boldsymbol{B} \boldsymbol{A}^{-1}-\boldsymbol{B} \boldsymbol{B}^{-1}\right)=\boldsymbol{B}^{-1}\left(\boldsymbol{B} \boldsymbol{A}^{-1}-\boldsymbol{I}_{n}\right) .
$$

Demnach gilt

$$
A^{-1}-B^{-1}=B^{-1}\left((B-A) A^{-1}\right)=B^{-1}(B-A) A^{-1} .
$$

Der zweite Teil des Lemmas folgt analog.

Lemma A.3. Gegeben seien zwei invertierbare Matrizen $\boldsymbol{P} \in \mathbb{R}^{n \times n}$ und $\boldsymbol{Q} \in \mathbb{R}^{m \times m}$. Dann gilt nach [223, Abschnitt 1.2.5]

$$
\boldsymbol{P}^{-1} \otimes \boldsymbol{Q}^{-1}=(\boldsymbol{P} \otimes \boldsymbol{Q})^{-1} .
$$


Lemma A.4. Gegeben seien zwei Matrizen $\boldsymbol{P} \in \mathbb{R}^{n \times r}$ und $\boldsymbol{Q} \in \mathbb{R}^{r \times m}$. Dann gilt nach [223, Abschnitt 1.2.5]

$$
\left(\boldsymbol{P} \otimes \boldsymbol{I}_{s}\right)\left(\boldsymbol{Q} \otimes \boldsymbol{I}_{s}\right)=(\boldsymbol{P} \boldsymbol{Q}) \otimes \boldsymbol{I}_{s} .
$$

Lemma A.5. Gegeben sei eine Matrix $\boldsymbol{X} \in \mathbb{R}^{n \times m}$. Dann gilt $\operatorname{rang}(\boldsymbol{X}) \leq r$ nach [68, Abschnitt 3.2] genau dann, wenn Matrizen $\boldsymbol{Y}=\boldsymbol{Y}^{\top} \in \mathbb{R}^{n \times n}$ und $\boldsymbol{Z}=\boldsymbol{Z}^{\top} \in \mathbb{R}^{m \times m}$ existieren, sodass

$$
\begin{aligned}
& {\left[\begin{array}{ll}
\boldsymbol{Y} & \boldsymbol{X} \\
\star & \boldsymbol{Z}
\end{array}\right] \succeq \mathbf{0},} \\
& \operatorname{rang}(\boldsymbol{Y})+\operatorname{rang}(\boldsymbol{Z}) \leq 2 r
\end{aligned}
$$

gilt.

Lemma A.6. Gegeben seien eine positiv definite, symmetrische Matrix $\boldsymbol{X}_{0} \in \mathbb{R}^{n \times n}$, eine symmetrische Matrix $\Delta \boldsymbol{X} \in \mathbb{R}^{n \times n}$ und ein positiver Skalar $\alpha$. Dann gilt $\|\Delta \boldsymbol{X}\|_{2}<\alpha\left\|\boldsymbol{X}_{0}\right\|_{2}$, wenn

$$
\left[\begin{array}{cc}
\alpha \boldsymbol{X}_{0} & \Delta \boldsymbol{X} \\
\star & \alpha \boldsymbol{X}_{0}
\end{array}\right] \succ \mathbf{0}
$$

Beweis. Aus (A.1) folgt mittels des Schur-Komplement-Lemmas

$$
\alpha \boldsymbol{X}_{0}-\Delta \boldsymbol{X} \cdot\left(\alpha \boldsymbol{X}_{0}\right)^{-1} \cdot \Delta \boldsymbol{X} \succ \mathbf{0} .
$$

Da $\boldsymbol{X}_{0}$ positiv definit ist, existiert nach [14, S. 278] eine eindeutige, positiv definite Matrix $\boldsymbol{X}_{0}^{1 / 2}$ und somit auch $\boldsymbol{X}_{0}^{-1 / 2}$. Multipliziert man (A.2) mit $\alpha$ sowie beidseitig mit $\boldsymbol{X}_{0}^{-1 / 2}$, so ergibt sich

$$
\alpha^{2} \boldsymbol{I}_{n}-\left(\boldsymbol{X}_{0}^{-1 / 2} \cdot \Delta \boldsymbol{X} \cdot \boldsymbol{X}_{0}^{-1 / 2}\right) \cdot\left(\boldsymbol{X}_{0}^{-1 / 2} \cdot \Delta \boldsymbol{X} \cdot \boldsymbol{X}_{0}^{-1 / 2}\right) \succ \mathbf{0} .
$$

Es ist also

$$
\left(\boldsymbol{X}_{0}^{-1 / 2} \cdot \Delta \boldsymbol{X} \cdot \boldsymbol{X}_{0}^{-1 / 2}\right)^{2} \prec \alpha^{2} \boldsymbol{I}_{n}
$$

was nach Lemma 3.5 gleichbedeutend mit

$$
\left\|\boldsymbol{X}_{0}^{-1 / 2} \cdot \Delta \boldsymbol{X} \cdot \boldsymbol{X}_{0}^{-1 / 2}\right\|_{2}<\alpha
$$

ist. Nach [14, Korollar 9.4.9] gilt für die induzierte 2-Norm $\|\boldsymbol{A} \boldsymbol{B}\|_{2} \leq\|\boldsymbol{A}\|_{2} \cdot\|\boldsymbol{B}\|_{2}$. Außerdem gilt nach [14, Fakt 9.11.13] die Beziehung

$$
\left\|\boldsymbol{X}_{0}\right\|_{2}=\left\|\boldsymbol{X}_{0}^{1 / 2} \cdot \boldsymbol{X}_{0}^{1 / 2}\right\|_{2}=\left\|\boldsymbol{X}_{0}^{1 / 2}\right\|_{2} \cdot\left\|\boldsymbol{X}_{0}^{1 / 2}\right\|_{2} .
$$


Damit folgt

$$
\begin{aligned}
& \left\|\boldsymbol{X}_{0}^{-1 / 2} \cdot \Delta \boldsymbol{X} \cdot \boldsymbol{X}_{0}^{-1 / 2}\right\|_{2} \cdot\left\|\boldsymbol{X}_{0}\right\|_{2}= \\
& \left\|\boldsymbol{X}_{0}^{1 / 2}\right\|_{2} \cdot\left\|\boldsymbol{X}_{0}^{-1 / 2} \cdot \boldsymbol{X}_{0} \cdot \boldsymbol{X}_{0}^{-1 / 2}\right\|_{2} \cdot\left\|\boldsymbol{X}_{0}^{1 / 2}\right\|_{2} \\
& \geq\|\underbrace{\boldsymbol{X}_{0}^{1 / 2} \cdot \boldsymbol{X}_{0}^{-1 / 2}}_{\boldsymbol{I}_{n}} \cdot \Delta \boldsymbol{X} \cdot \underbrace{\boldsymbol{X}_{0}^{-1 / 2} \cdot \boldsymbol{X}_{0}^{1 / 2}}_{\boldsymbol{I}_{n}}\|_{2}=\|\Delta \boldsymbol{X}\|_{2} .
\end{aligned}
$$

Fasst man (A.3) und (A.4) zusammen, ergibt sich

$$
\frac{\|\Delta \boldsymbol{X}\|_{2}}{\left\|\boldsymbol{X}_{0}\right\|_{2}} \leq\left\|\boldsymbol{X}_{0}^{-1 / 2} \cdot \Delta \boldsymbol{X} \cdot \boldsymbol{X}_{0}^{-1 / 2}\right\|_{2}<\alpha
$$

sodass (A.1) die Einhaltung von $\|\Delta \boldsymbol{X}\|_{2}<\alpha\left\|\boldsymbol{X}_{0}\right\|_{2}$ impliziert.

Beweis des Bounded-Real-Lemmas. Es wird gezeigt, dass die LMI in Satz 3.1 hinreichend ist für $\left\|\boldsymbol{G}_{\boldsymbol{y} \boldsymbol{u}}(s)\right\|_{\infty}<\gamma$. Zunächst wird eine Kongruenztransformation mit $\boldsymbol{T}=\operatorname{diag}\left(\sqrt{\gamma} \boldsymbol{I}_{n}, \sqrt{\gamma} \boldsymbol{I}_{n_{n}}, 1 / \sqrt{\gamma} \boldsymbol{I}_{n_{y}}\right)$ durchgeführt, woraus mit der Variablentransformation $\boldsymbol{P}:=\gamma \boldsymbol{P}$

$$
\left[\begin{array}{ccc}
\mathrm{He}(\boldsymbol{P} \boldsymbol{A}) & \boldsymbol{P} \boldsymbol{B} & \boldsymbol{C}^{\top} \\
\star & -\gamma^{2} \boldsymbol{I}_{n_{u}} & \boldsymbol{D}^{\top} \\
\star & \star & -\boldsymbol{I}_{n_{y}}
\end{array}\right] \prec \mathbf{0}
$$

folgt. Die Anwendung des Schur-Komplement-Lemmas (Lemma 3.3) liefert dann

$$
\left[\begin{array}{cc}
\mathrm{He}(\boldsymbol{P} \boldsymbol{A}) & \boldsymbol{P} \boldsymbol{B} \\
\star & \mathbf{0}
\end{array}\right]+\left[\begin{array}{cc}
\boldsymbol{C}^{\top} \boldsymbol{C} & \boldsymbol{C}^{\top} \\
\star & -\gamma^{2} \boldsymbol{I}_{n_{u}}+\boldsymbol{D}^{\top} \boldsymbol{D}
\end{array}\right] \prec \mathbf{0} .
$$

Somit gilt für beliebige $\left[\boldsymbol{x}^{\top} \boldsymbol{u}^{\top}\right]^{\top} \neq \mathbf{0}$ die Beziehung

$$
\begin{aligned}
{\left[\begin{array}{ll}
\boldsymbol{x}^{\top} & \boldsymbol{u}^{\top}
\end{array}\right]\left[\begin{array}{cc}
\operatorname{He}(\boldsymbol{P} \boldsymbol{A}) & \boldsymbol{P} \boldsymbol{B} \\
\star & \mathbf{0}
\end{array}\right]\left[\begin{array}{l}
\boldsymbol{x} \\
\boldsymbol{u}
\end{array}\right] } \\
<\underbrace{\left[\begin{array}{ll}
\boldsymbol{x}^{\top} & \boldsymbol{u}^{\top}
\end{array}\right]\left[\begin{array}{cc}
-\boldsymbol{C}^{\top} \boldsymbol{C} & -\boldsymbol{C}^{\top} \boldsymbol{D} \\
\star & \gamma^{2} \boldsymbol{I}_{n_{u}}-\boldsymbol{D}^{\top} \boldsymbol{D}
\end{array}\right]\left[\begin{array}{l}
\boldsymbol{x} \\
\boldsymbol{u}
\end{array}\right]}_{-\boldsymbol{y}^{\top} \boldsymbol{y}+\gamma^{2} \boldsymbol{u}^{\top} \boldsymbol{u}} .
\end{aligned}
$$

Setzt man nun eine Speicherfunktion ${ }^{1)} V(\boldsymbol{x})=\boldsymbol{x}^{\top} \boldsymbol{P} \boldsymbol{x}$ an, so ist

$$
\dot{V}(\boldsymbol{x}, \boldsymbol{u})=\dot{\boldsymbol{x}}^{\top} \boldsymbol{P} \boldsymbol{x}+\boldsymbol{x}^{\top} \boldsymbol{P} \dot{\boldsymbol{x}}=\left[\begin{array}{ll}
\boldsymbol{x}^{\top} & \boldsymbol{u}^{\top}
\end{array}\right]\left[\begin{array}{cc}
\mathrm{He}(\boldsymbol{P} \boldsymbol{A}) & \boldsymbol{P} \boldsymbol{B} \\
\star & \mathbf{0}
\end{array}\right]\left[\begin{array}{l}
\boldsymbol{x} \\
\boldsymbol{u}
\end{array}\right] .
$$

1) Der Begriff stammt aus der Dissipativitätstheorie, auf die an dieser Stelle nicht näher eingegangen wird. 
Mit (A.5) folgt daraus

$$
\dot{V}<\gamma^{2} \boldsymbol{u}^{\top} \boldsymbol{u}-\boldsymbol{y}^{\top} \boldsymbol{y} .
$$

Da das System stabil ist, ist $\lim _{t \rightarrow \infty} V(\boldsymbol{x})=\boldsymbol{x}_{\infty}=\mathbf{0}$. Nimmt man weiterhin den Anfangszustand $\boldsymbol{x}_{0}=\mathbf{0}$ an, so ergibt die zeitliche Integration von (A.6)

$$
\int_{0}^{\infty} \dot{V}(\boldsymbol{x}, \boldsymbol{u}) d \tau=V\left(\boldsymbol{x}_{\infty}\right)-V\left(\boldsymbol{x}_{0}\right)=0<\gamma^{2} \int_{0}^{\infty} \boldsymbol{u}^{\top}(\tau) \boldsymbol{u}(\tau) d \tau-\int_{0}^{\infty} \boldsymbol{y}^{\top}(\tau) \boldsymbol{y}(\tau) d \tau .
$$

Demnach ist

$$
\frac{\sqrt{\int_{0}^{\infty} \boldsymbol{y}^{\top}(\tau) \boldsymbol{y}(\tau) d \tau}}{\sqrt{\int_{0}^{\infty} \boldsymbol{u}^{\top}(\tau) \boldsymbol{u}(\tau) d \tau}}=\left\|\boldsymbol{G}_{\boldsymbol{u y}}(s)\right\|_{\infty}<\gamma
$$

Der Beweis, dass die LMI aus Satz 3.1 auch notwendig ist, findet sich zum Beispiel in [272].

Bemerkung A.1 (Erläuterungen zum verallgemeinerten Kalman-Yakubovich-Popov-Lemma). In seiner allgemeinen Form besagt das KalmanYakubovich-Popov-Lemma (KYP-Lemma, [179]), dass die Aussagen

$$
\begin{aligned}
\text { 1a. } & {\left[\begin{array}{c}
(j \omega \boldsymbol{I}-\boldsymbol{A})^{-1} \boldsymbol{B} \\
\boldsymbol{I}
\end{array}\right]^{\mathrm{H}} \boldsymbol{\Theta}\left[\begin{array}{c}
(j \omega \boldsymbol{I}-\boldsymbol{A})^{-1} \boldsymbol{B} \\
\boldsymbol{I}
\end{array}\right] \prec \mathbf{0} \text { für alle } \omega \text { und } } \\
\text { 1b. } & {\left[\begin{array}{cc}
\boldsymbol{A} & \boldsymbol{B} \\
\boldsymbol{I} & \mathbf{0}
\end{array}\right]^{\mathrm{H}}\left[\begin{array}{cc}
\mathbf{0} & \boldsymbol{P} \\
\boldsymbol{P} & \mathbf{0}
\end{array}\right]\left[\begin{array}{cc}
\boldsymbol{A} & \boldsymbol{B} \\
\boldsymbol{I} & \mathbf{0}
\end{array}\right]+\boldsymbol{\Theta} \prec \mathbf{0} \text { mit einer hermiteschen Matrix } }
\end{aligned}
$$

für steuerbare Paare $(\boldsymbol{A}, \boldsymbol{B})$ äquivalent sind. Da für die erste Aussage unendlich viele Frequenzen $\omega$ zu überprüfen sind, lässt sie sich lediglich grafisch auswerten. Für die zweite Aussage ist dagegen lediglich eine Matrixungleichung zu lösen, was eine numerische Analyse erlaubt.

Durch die Wahl von

$$
\Theta=\left[\begin{array}{cc}
C & D \\
\mathbf{0} & I
\end{array}\right]^{\mathrm{H}} \Gamma\left[\begin{array}{cc}
C & D \\
\mathbf{0} & I
\end{array}\right]
$$

lassen sich auf Grundlage der allgemeinen Form des KYP-Lemmas unterschiedliche Eigenschaften von Übertragungsmatrizen untersuchen, denn es ist

$$
\left[\begin{array}{cc}
\boldsymbol{C} & \boldsymbol{D} \\
\mathbf{0} & \boldsymbol{I}
\end{array}\right]\left[\begin{array}{c}
(j \omega \boldsymbol{I}-\boldsymbol{A})^{-1} \boldsymbol{B} \\
\boldsymbol{I}
\end{array}\right]=\left[\begin{array}{c}
\boldsymbol{C}(j \omega \boldsymbol{I}-\boldsymbol{A})^{-1} \boldsymbol{B}+\boldsymbol{D} \\
\boldsymbol{I}
\end{array}\right]=\left[\begin{array}{c}
\boldsymbol{G}(j \omega) \\
\boldsymbol{I}
\end{array}\right] .
$$


Eingesetzt in die Aussagen 1a und 1b ergibt sich die Äquivalenz von

$$
\begin{aligned}
& \text { 2a. }\left[\begin{array}{c}
\boldsymbol{G}(j \omega) \\
\boldsymbol{I}
\end{array}\right]^{\mathrm{H}} \boldsymbol{\Gamma}\left[\begin{array}{c}
\boldsymbol{G}(j \omega) \\
\boldsymbol{I}
\end{array}\right] \prec \mathbf{0} \text { und } \\
& \text { 2b. }\left[\begin{array}{cc}
\boldsymbol{A} & \boldsymbol{B} \\
\boldsymbol{I} & \mathbf{0}
\end{array}\right]^{\mathrm{H}}\left[\begin{array}{cc}
\mathbf{0} & \boldsymbol{P} \\
\boldsymbol{P} & \mathbf{0}
\end{array}\right]\left[\begin{array}{cc}
\boldsymbol{A} & \boldsymbol{B} \\
\boldsymbol{I} & \mathbf{0}
\end{array}\right]+\left[\begin{array}{cc}
\boldsymbol{C} & \boldsymbol{D} \\
\mathbf{0} & \boldsymbol{I}
\end{array}\right]^{\mathrm{H}} \boldsymbol{\Gamma}\left[\begin{array}{cc}
\boldsymbol{C} & \boldsymbol{D} \\
\mathbf{0} & \boldsymbol{I}
\end{array}\right] \prec \mathbf{0 .}
\end{aligned}
$$

Wie in Abschnitt 3.2.2 angegeben lassen sich durch eine entsprechende Wahl der Matrix $\boldsymbol{\Gamma}$ unterschiedliche Eigenschaften des Systems untersuchen. Für

$$
\boldsymbol{\Gamma}=\left[\begin{array}{cc}
\boldsymbol{I} & \mathbf{0} \\
\mathbf{0} & -\gamma^{2} \boldsymbol{I}
\end{array}\right]
$$

ergibt sich einerseits aus $2 a$

$$
\begin{aligned}
& {\left[\begin{array}{c}
\boldsymbol{G}(j \omega) \\
\boldsymbol{I}
\end{array}\right]^{\mathrm{H}}\left[\begin{array}{cc}
\boldsymbol{I} & \mathbf{0} \\
\mathbf{0} & -\gamma^{2} \boldsymbol{I}
\end{array}\right]\left[\begin{array}{c}
\boldsymbol{G}(j \omega) \\
\boldsymbol{I}
\end{array}\right]=\boldsymbol{G}(j \omega)^{\mathrm{H}} \cdot \boldsymbol{G}(j \omega)-\gamma^{2} \boldsymbol{I} \prec \mathbf{0}} \\
& \Rightarrow \boldsymbol{G}(j \omega)^{\mathrm{H}} \cdot \boldsymbol{G}(j \omega) \prec \gamma^{2} \boldsymbol{I} \\
& \Rightarrow \bar{\sigma}(\boldsymbol{G}(j \omega))<\gamma \Rightarrow\|\boldsymbol{G}(j \omega)\|_{\infty}<\gamma
\end{aligned}
$$

und andererseits aus $2 \mathrm{~b}$

$$
\left[\begin{array}{cc}
\mathrm{He}(\boldsymbol{P} \boldsymbol{A})+\boldsymbol{C}^{\top} \boldsymbol{C} & \boldsymbol{P} \boldsymbol{B}+\boldsymbol{C}^{\top} \boldsymbol{D} \\
\star & \boldsymbol{D}^{\top} \boldsymbol{D}-\gamma^{2} \boldsymbol{I}
\end{array}\right] \prec \mathbf{0} .
$$

Mit einer positiv definiten Matrix $\boldsymbol{P}$ folgt daraus durch Anwendung des Schur-Komplement-Lemmas (Satz 3.3) das Bounded-Real-Lemma (Satz 3.1). In analoger Art und Weise lässt sich mit

$$
\boldsymbol{\Gamma}=\left[\begin{array}{cc}
-\boldsymbol{I} & \mathbf{0} \\
\mathbf{0} & \gamma^{2} \boldsymbol{I}
\end{array}\right]
$$

ein Zusammenhang für die größte untere Schranke des kleinsten Singulärwertes von $\boldsymbol{G}(j \omega)$, also für $\|\boldsymbol{G}(j \omega)\|_{-}$, herleiten. Für

$$
\Gamma=\left[\begin{array}{cc}
0 & -\boldsymbol{I} \\
-\boldsymbol{I} & 0
\end{array}\right]
$$

wiederum folgt aus 1a und 1b die Äquivalenz

$$
\boldsymbol{G}(j \omega)^{\mathrm{H}}+\boldsymbol{G}(j \omega) \succeq \mathbf{0} \Leftrightarrow\left[\begin{array}{cc}
\operatorname{He}(\boldsymbol{P} \boldsymbol{A}) & \boldsymbol{P} \boldsymbol{B}-\boldsymbol{C}^{\top} \\
\star & -\boldsymbol{D}^{\top}-\boldsymbol{D}
\end{array}\right] \preceq \mathbf{0} .
$$


Führt man zwei beliebige Matrizen $\Upsilon \Upsilon$ und $\boldsymbol{\Lambda}$ ein, so ist die Matrix

$$
-\left[\begin{array}{l}
\mathbf{\Upsilon}^{\top} \\
\boldsymbol{\Lambda}^{\top}
\end{array}\right]\left[\begin{array}{ll}
\mathbf{\Upsilon} & \boldsymbol{\Lambda}
\end{array}\right]
$$

negativ semidefinit. Dementsprechend ist $\boldsymbol{G}(j \omega)^{\mathrm{H}}+\boldsymbol{G}(j \omega) \succeq \mathbf{0}$ erfüllt, wenn es Matrizen $\boldsymbol{P} \succ \mathbf{0}, \boldsymbol{\Upsilon}$ und $\boldsymbol{\Lambda}$ gibt, sodass die Gleichungen

$$
\begin{aligned}
\operatorname{He}(\boldsymbol{P} \boldsymbol{A}) & =-\boldsymbol{\Upsilon}^{\top} \boldsymbol{\Upsilon}, \\
\boldsymbol{P} \boldsymbol{B}-\boldsymbol{C}^{\top} & =-\boldsymbol{\Upsilon}^{\top} \boldsymbol{\Lambda}, \\
\boldsymbol{D}+\boldsymbol{D}^{\top} & =\boldsymbol{\Lambda}^{\top} \boldsymbol{\Lambda}
\end{aligned}
$$

erfüllt sind. Diese werden in der Literatur häufig als Kalman-YakubovichPopov-Gleichungen [125] bezeichnet.

Das verallgemeinerte Kalman-Yakubovich-Popov-Lemma (GKYPLemma, [112, 113]) erweitert obige Betrachtungen auf beschränkte beziehungsweise halboffene Frequenzintervalle. Anstelle von

$$
\left[\begin{array}{ll}
0 & P \\
P & 0
\end{array}\right]
$$

wird dazu gemäß [112] in 1b die Matrix

$$
\boldsymbol{\Xi}=\boldsymbol{\Phi} \otimes \boldsymbol{Y}+\boldsymbol{\Psi} \otimes \boldsymbol{X}
$$

angesetzt. Die Matrizen $\boldsymbol{\Phi}$ und $\boldsymbol{\Psi}$ definieren dabei jeweils quadratische Formen

$$
f_{q}(j \omega, \boldsymbol{\Phi})=\left[\begin{array}{c}
j \omega \\
1
\end{array}\right]^{\mathrm{H}} \boldsymbol{\Phi}\left[\begin{array}{c}
j \omega \\
1
\end{array}\right], f_{q}(j \omega, \boldsymbol{\Psi})=\left[\begin{array}{c}
j \omega \\
1
\end{array}\right]^{\mathrm{H}} \boldsymbol{\Psi}\left[\begin{array}{c}
j \omega \\
1
\end{array}\right] .
$$

Diese beschreiben wiederum den Frequenzbereich $\Omega$, wobei

$$
\Omega=\left\{\omega \mid f_{q}(j \omega, \boldsymbol{\Phi})=0, \quad f_{q}(j \omega, \mathbf{\Psi}) \geq 0\right\}
$$

gilt. Die Forderung $f_{q}(j \omega, \mathbf{\Phi})=0$ ist offensichtlich durch die Wahl von

$$
\boldsymbol{\Phi}=\left[\begin{array}{ll}
0 & 1 \\
1 & 0
\end{array}\right]
$$

erfüllt, denn es gilt

$$
f_{q}(j \omega, \mathbf{\Phi})=\left[\begin{array}{ll}
-j \omega & 1
\end{array}\right]\left[\begin{array}{ll}
0 & 1 \\
1 & 0
\end{array}\right]\left[\begin{array}{c}
j \omega \\
1
\end{array}\right]=-j \omega+j \omega=0 .
$$


Für nach oben durch $\omega_{h}$ begrenzte Frequenzbereiche gilt nun beispielsweise mit der Wahl von

$$
\boldsymbol{\Psi}=\left[\begin{array}{cc}
-1 & 0 \\
0 & \omega_{h}^{2}
\end{array}\right]
$$

die Beziehung

$$
\begin{aligned}
f_{q}(j \omega, \boldsymbol{\Psi}) & =\left[\begin{array}{ll}
-j \omega & 1
\end{array}\right]\left[\begin{array}{cc}
-1 & 0 \\
0 & \omega_{h}^{2}
\end{array}\right]\left[\begin{array}{c}
j \omega \\
1
\end{array}\right] \\
& =-\omega^{2}+\omega_{h}^{2} \geq 0 \\
& \Rightarrow \omega \leq \omega_{h} .
\end{aligned}
$$

Ebenso lassen sich Matrizen $\boldsymbol{\Psi}$ für nach unten beziehungsweise beidseitig beschränkte Frequenzintervalle finden und zusammenfassend gilt

$$
\begin{aligned}
\boldsymbol{\Psi}_{\text {low }}=\left[\begin{array}{cc}
-1 & 0 \\
0 & \omega_{h}^{2}
\end{array}\right] & \Rightarrow \omega \leq \omega_{h}, \\
\boldsymbol{\Psi}_{\text {mid }}=\left[\begin{array}{cc}
-1 & j \omega_{c} \\
-j \omega_{c} & -\omega_{l} \omega_{h}
\end{array}\right] & \Rightarrow \omega_{l} \leq \omega \leq \omega_{h} \\
\boldsymbol{\Psi}_{\text {high }}=\left[\begin{array}{cc}
1 & 0 \\
0 & -\omega_{l}^{2}
\end{array}\right] & \Rightarrow \omega \geq \omega_{l} .
\end{aligned}
$$

Durch Auswertung der Kroneckerprodukte ergeben sich dann die in Satz 3.2 in Abschnitt 3.2.2 angesetzten Matrizen $\boldsymbol{\Xi}$ in ihrer kompakten Form. Das Kalman-Yakubovich-Popov-Lemma für alle Frequenzen ergibt sich als Spezialfall, bei dem keine Einschränkungen hinsichtlich $\omega$ gelten. Dazu ist lediglich $\boldsymbol{\Psi}=\mathbf{0}$ zu setzen. Dann ist $\boldsymbol{f}_{q}(j \omega, \boldsymbol{\Psi}) \geq 0$ für alle $\omega$ erfüllt und $\Omega$ beschreibt einen unbeschränkten Frequenzbereich.

Beweis von Lemma 4.5. Die Äquivalenz 1. $\Leftrightarrow 2$. ist in [67] für das duale Problem des Entkopplungsreglerentwurfs gezeigt.

Um die Implikation 2. $\Rightarrow 3$. zu zeigen, wird ein ähnlicher Beweis wie in [137] vollzogen. Dazu werden die Matrizen

$$
\begin{aligned}
\check{\boldsymbol{E}}_{a}(s) & =\left[\begin{array}{lll}
\check{\boldsymbol{e}}_{a_{1}}(s) & \cdots & \check{\boldsymbol{e}}_{a_{n_{f}}}(s)
\end{array}\right] \\
\check{\boldsymbol{e}}_{a_{i}}(s) & =\left\{\begin{array}{cc}
\mathbf{0} & , \delta_{i}=0 \\
\sum_{k=0}^{\delta_{i}-1} s^{\delta_{i}-k-1} \boldsymbol{A}^{k} \boldsymbol{e}_{a_{i}}, & \delta_{i} \geq 1
\end{array}\right. \\
\check{\boldsymbol{E}}_{s}(s) & =\operatorname{diag}\left(s^{\delta_{1}}, \ldots, s^{\delta_{n_{f}}}\right)
\end{aligned}
$$


eingeführt. Mit diesen Matrizen wird die Matrix $\check{\boldsymbol{M}}=-\left(s \boldsymbol{I}_{n}-\boldsymbol{A}\right) \check{\boldsymbol{E}}_{a}(s)+\boldsymbol{E}_{a} \check{\boldsymbol{E}}_{s}(s)$ berechnet, deren Spalten durch

$$
\check{\boldsymbol{m}}_{i}=-\left(\sum_{k=0}^{\delta_{i}-1}\left(s^{\delta_{i}-k} \boldsymbol{A}^{k}-s^{\delta_{i}-k-1} \boldsymbol{A}^{k+1}\right) \boldsymbol{e}_{a_{i}}\right)+s^{\delta_{i}} \boldsymbol{e}_{a_{i}}=\boldsymbol{A}^{\delta_{i}} \boldsymbol{e}_{a_{i}}
$$

gegeben sind. Zu beachten ist dabei, dass $\check{M}$ unabhängig von $s$ ist. Weiterhin entsprechen die Spalten von $\boldsymbol{C \check { \boldsymbol { E } }}_{a}(s)+\boldsymbol{E}_{s} \check{\boldsymbol{E}}_{s}(s)$ für $\delta_{i} \geq 1 \mathrm{dem}$ Ausdruck $\boldsymbol{C} \boldsymbol{A}^{\delta_{i}-1} \boldsymbol{e}_{a_{i}}$ und für $\delta_{i}=0$ der $i$-ten Spalte der Sensorfehlermatrix $\boldsymbol{E}_{s}$. Somit lässt sich

$$
\underbrace{\left[\begin{array}{cc}
s \boldsymbol{I}_{n}-\boldsymbol{A} & \boldsymbol{E}_{a} \\
-\boldsymbol{C} & \boldsymbol{E}_{s}
\end{array}\right]}_{\Pi(s)}\left[\begin{array}{cc}
\boldsymbol{I}_{n} & -\check{\boldsymbol{E}}_{a}(s) \\
\mathbf{0} & \check{\boldsymbol{E}}_{s}(s)
\end{array}\right]=\underbrace{\left[\begin{array}{cc}
s \boldsymbol{I}_{n}-\boldsymbol{A} & \check{\boldsymbol{M}} \\
-\boldsymbol{C} & \boldsymbol{D}^{*}
\end{array}\right]}_{\check{\boldsymbol{\Pi}}(s)}
$$

schreiben. Die Berechnung der Determinanten führt dann auf

$$
\operatorname{det}(\boldsymbol{\Pi}(s)) \cdot s^{\delta}=\operatorname{det}(\check{\mathbf{\Pi}}(s)) .
$$

Wenn nach 2. die Matrix $\boldsymbol{D}^{*}$ invertierbar ist, lässt sich nach [150, Abschnitt 6.2] die entsprechende Determinante umformen zu

$$
\operatorname{det}(\check{\boldsymbol{\Pi}}(s))=\operatorname{det}\left(\boldsymbol{D}^{*}\right) \cdot \operatorname{det}\left(\left(s \boldsymbol{I}_{n}-\boldsymbol{A}\right)+\boldsymbol{C} \boldsymbol{D}^{*-1} \check{\boldsymbol{M}}\right),
$$

was ein Polynom vom Grad $n$ ist. Dies impliziert, $\operatorname{dass} \operatorname{det}(\boldsymbol{\Pi}(s))$ in (A.7) ein Polynom vom Grad $n-\delta$ ist. Da der Grad von $\operatorname{det}(\boldsymbol{\Pi}(s))$ der Anzahl der invarianten Nullstellen des Systems $\left(\boldsymbol{A}, \boldsymbol{E}_{a}, \boldsymbol{C}, \boldsymbol{E}_{s}\right)$ entspricht, folgt daraus, dass $n-\delta=\mu$ gilt, wenn $\boldsymbol{D}^{*}$ invertierbar ist.

Abschließend ist $2 . \Leftarrow 3$. zu zeigen. Dazu wird erneut von (A.7) ausgegangen. Da der Grad von det $(\boldsymbol{\Pi}(s))$ der Anzahl der invarianten Nullstellen des Systems entspricht, ergibt sich aus 3., dass det $(\boldsymbol{\Pi}(s))$ ein Polynom vom Grad $n-\delta$ ist. Wegen (A.7) ist also $\operatorname{det}(\check{\mathbf{\Pi}}(s))$ ein Polynom vom Grad $n$. Der Beweis, dass daraus die Invertierbarkeit von $\boldsymbol{D}^{*}$ folgt, wird durch Widerspruch geführt.

Angenommen sei, dass $\boldsymbol{D}^{*}$ nicht invertierbar ist, also mindestens einen Eigenwert in null besitzt. Dann existiert eine Ähnlichkeitstransformation mit einer Matrix $\boldsymbol{R}$, die $\boldsymbol{D}^{*}$ in die Jordanform

$$
\boldsymbol{R}^{-1} \boldsymbol{D}^{*} \boldsymbol{R}=\left[\begin{array}{cc}
\boldsymbol{J}_{0} & \mathbf{0} \\
\mathbf{0} & \boldsymbol{P}_{u}
\end{array}\right]
$$


überführt. Dabei ist $\boldsymbol{J}_{0}$ ein Jordanblock zu einem Eigenwert 0. Der Rang der Matrix $\check{\Pi}(s)$ ändert sich durch die Ähnlichkeitstransformation

$$
\left[\begin{array}{cc}
\boldsymbol{I}_{n} & \mathbf{0} \\
\mathbf{0} & \boldsymbol{R}^{-1}
\end{array}\right]\left[\begin{array}{cc}
s \boldsymbol{I}_{n}-\boldsymbol{A} & \check{\boldsymbol{M}} \\
-\boldsymbol{C} & \boldsymbol{D}^{*}
\end{array}\right]\left[\begin{array}{cc}
\boldsymbol{I}_{n} & \mathbf{0} \\
\mathbf{0} & \boldsymbol{R}
\end{array}\right]=\left[\begin{array}{cc}
s \boldsymbol{I}_{n}-\boldsymbol{A} & \check{\boldsymbol{M}} \boldsymbol{R} \\
-\boldsymbol{R}^{-1} \boldsymbol{C} & \boldsymbol{R}^{-1} \boldsymbol{D}^{*} \boldsymbol{R}
\end{array}\right]
$$

nicht. Partitioniert man nun die resultierende Matrix, so erhält man

$$
\left[\begin{array}{cc}
s \boldsymbol{I}_{n}-\boldsymbol{A} & \check{\boldsymbol{M} R} \\
-\boldsymbol{R}^{-1} \boldsymbol{C} & \boldsymbol{R}^{-1} \boldsymbol{D}^{*} \boldsymbol{R}
\end{array}\right]=\left[\begin{array}{ccc}
s \boldsymbol{I}_{n}-\boldsymbol{A} & \check{\boldsymbol{M}} \boldsymbol{R}_{l} & \check{\boldsymbol{M}} \boldsymbol{R}_{r} \\
-\left(\boldsymbol{R}^{-1}\right)_{o} \boldsymbol{C} & \boldsymbol{J}_{0} & \mathbf{0} \\
-\left(\boldsymbol{R}^{-1}\right)_{u} \boldsymbol{C} & \mathbf{0} & \boldsymbol{P}_{u}
\end{array}\right]
$$

Diese Matrix wird von links mit einer weiteren regulären Matrix multipliziert, sodass der Rang weiterhin unverändert bleibt. Es ergibt sich

$$
\begin{aligned}
& {\left[\begin{array}{ccc}
\boldsymbol{I}_{n} & \mathbf{0} & -\check{\boldsymbol{M}} \boldsymbol{R}_{r} \boldsymbol{P}_{u}^{-1} \\
\mathbf{0} & \boldsymbol{I} & \mathbf{0} \\
\mathbf{0} & \mathbf{0} & \boldsymbol{I}
\end{array}\right]\left[\begin{array}{ccc}
s \boldsymbol{I}_{n}-\boldsymbol{A} & \check{\boldsymbol{M}} \boldsymbol{R}_{l} & \check{\boldsymbol{M}} \boldsymbol{R}_{r} \\
-\left(\boldsymbol{R}^{-1}\right)_{o} \boldsymbol{C} & \boldsymbol{J}_{0} & \mathbf{0} \\
-\left(\boldsymbol{R}^{-1}\right)_{u} \boldsymbol{C} & \mathbf{0} & \boldsymbol{P}_{u}
\end{array}\right]} \\
& =\left[\begin{array}{cccc}
s \boldsymbol{I}_{n}-\boldsymbol{A}+\check{\boldsymbol{M}} \boldsymbol{R}_{r} \boldsymbol{P}_{u}^{-1}\left(\boldsymbol{R}^{-1}\right)_{u} \boldsymbol{C} & \check{\boldsymbol{M}} \boldsymbol{R}_{l} & \check{M} \boldsymbol{R}_{r}-\check{\boldsymbol{M}} \boldsymbol{R}_{r} \boldsymbol{P}_{u}^{-1} \boldsymbol{P}_{u} \\
-\left(\boldsymbol{R}^{-1}\right)_{o} \boldsymbol{C} & \boldsymbol{J}_{0} & \mathbf{0} \\
-\left(\boldsymbol{R}^{-1}\right)_{u} \boldsymbol{C} & \mathbf{0} & \boldsymbol{P}_{u}
\end{array}\right] \\
& =\left[\begin{array}{ccc:}
s \boldsymbol{I}_{n}-\boldsymbol{A}+\check{\boldsymbol{M}} \boldsymbol{R}_{r} \boldsymbol{P}_{u}^{-1}\left(\boldsymbol{R}^{-1}\right)_{u} \boldsymbol{C} & \check{\boldsymbol{M}} \boldsymbol{R}_{l} & \mathbf{0} \\
-\left(\boldsymbol{R}^{-1}\right)_{o} \boldsymbol{C} & \boldsymbol{J}_{0} & \mathbf{0} \\
\hdashline-\left(\boldsymbol{R}^{-1}\right)_{u} \boldsymbol{C} & \mathbf{0} & \boldsymbol{P}_{u}
\end{array}\right] .
\end{aligned}
$$

Aufgrund der sich ergebenden Blockdreiecksstruktur folgt, dass der Grad von $\operatorname{det}(\check{\mathbf{\Pi}}(s))$ dem Grad von

$$
\operatorname{det}\left(\left[\begin{array}{cc}
s \boldsymbol{I}_{n}-\boldsymbol{A}+\check{\boldsymbol{M}} \boldsymbol{R}_{r} \boldsymbol{P}_{u}^{-1}\left(\boldsymbol{R}^{-1}\right)_{u} \boldsymbol{C} & \check{\boldsymbol{M}} \boldsymbol{R}_{l} \\
-\left(\boldsymbol{R}^{-1}\right)_{o} \boldsymbol{C} & \boldsymbol{J}_{0}
\end{array}\right]\right)=\operatorname{det}(\boldsymbol{\Upsilon}(s))
$$

entspricht. Da im unteren rechten Block von $\mathbf{\Upsilon}(s)$ die Matrix

$$
\boldsymbol{J}_{0}=\left[\begin{array}{ccccc}
0 & 1 & 0 & \cdots & 0 \\
\vdots & \ddots & \ddots & \ddots & \vdots \\
\vdots & & \ddots & \ddots & 0 \\
\vdots & & & \ddots & 1 \\
0 & \cdots & \cdots & \cdots & 0
\end{array}\right]
$$


auftaucht, die eine Nullspalte enthält, muss der Grad von $\operatorname{det}(\mathbf{\Upsilon}(s))$ kleiner als $n$ sein und (A.7) kann nicht gelten. Demnach ist für $n-\delta=\mu$ die Nichtinvertierbarkeit von $\boldsymbol{D}^{*}$ ausgeschlossen.

Beweis von Lemma 4.7. Die Dimension von $\boldsymbol{\Lambda}_{u}$ ergibt sich unmittelbar aus dem Rangsatz A.1 unter Annahme 4.7. Für die Matrix $\boldsymbol{\Lambda}_{o}$ erklärt sich die Dimension wie folgt: Die Matrix

$$
\boldsymbol{\Psi}=\left[\overline{\boldsymbol{D}}^{* \perp}\left[\begin{array}{c}
\mathbf{0}_{\kappa \times\left(n_{f}-\kappa\right)} \\
\boldsymbol{I}_{n_{f}-\kappa}
\end{array}\right]\right]=\left[\begin{array}{ccc}
\boldsymbol{Q}_{o} & \mathbf{0} & \mathbf{0} \\
\boldsymbol{Q}_{u} & \boldsymbol{I}_{n_{f}-\kappa-\gamma} & \mathbf{0} \\
\mathbf{0} & \mathbf{0} & \boldsymbol{I}_{\gamma}
\end{array}\right] \in \mathbb{R}^{n_{f} \times n_{f}}
$$

hat aufgrund ihrer Blockdreiecksstruktur offensichtlich vollen Rang, da $\boldsymbol{Q}_{o}$ invertierbar ist. Dementsprechend gilt

$$
\begin{aligned}
n_{f}-\kappa=\operatorname{rang}\left(\overline{\boldsymbol{D}}^{*}\right) & =\operatorname{rang}\left(\overline{\boldsymbol{D}}^{*} \cdot \boldsymbol{\Psi}\right) \\
& =\operatorname{rang}\left(\overline{\boldsymbol{D}}^{*} \cdot \overline{\boldsymbol{D}}^{* \perp}+\overline{\boldsymbol{D}}^{*}\left[\begin{array}{c}
\mathbf{0}_{\kappa \times\left(n_{f}-\kappa\right)} \\
\boldsymbol{I}_{n_{f}-\kappa}
\end{array}\right]\right) \\
& =\operatorname{rang}(\boldsymbol{Z}) .
\end{aligned}
$$

Es ergibt sich somit $\boldsymbol{Z} \boldsymbol{Z}^{+}=\boldsymbol{I}_{n_{f}-\kappa}$ und aus dem Rangsatz A.1 folgt schließlich ${ }^{\perp} \boldsymbol{Z} \in \mathbb{R}^{\kappa \times n_{f}}$, was die Dimension von $\boldsymbol{\Lambda}_{o}$ begründet.

Weiterhin ist die aus (4.45) resultierende Matrix $\boldsymbol{H}$ invertierbar. Um dies zu zeigen, werden (4.45a) und (4.45b) von rechts mit der Matrix $\boldsymbol{Z}$ multipliziert. Es ergibt sich wegen $n_{y}=n_{f}$

$$
\left[\begin{array}{c}
\boldsymbol{H}_{o} \\
\boldsymbol{H}_{u}
\end{array}\right] \boldsymbol{Z}=\boldsymbol{H} \boldsymbol{Z}=\left[\begin{array}{c}
\boldsymbol{I}_{n_{f}-\kappa} \\
\mathbf{0}_{\kappa \times\left(n_{f}-\kappa\right)}
\end{array}\right] \text {. }
$$

Damit ist $\operatorname{rang}(\boldsymbol{H} \boldsymbol{Z})=\operatorname{rang}(\boldsymbol{Z})=n_{f}-\kappa$ und folglich hat $\boldsymbol{H}$ vollen Rang.

Beweis von Lemma 4.10. Für die Matrix $\boldsymbol{A}^{\prime}$ ergibt sich

$$
\boldsymbol{A}^{\prime}=\left[\begin{array}{cc}
\boldsymbol{I}_{n} & \mathbf{0} \\
\mathbf{0} & \boldsymbol{T}_{\mathrm{mr}}
\end{array}\right]\left[\begin{array}{cc}
\boldsymbol{A} & \mathbf{0} \\
\mathbf{0} & \overline{\boldsymbol{A}}
\end{array}\right]\left[\begin{array}{cc}
\boldsymbol{I}_{n} & \mathbf{0} \\
\mathbf{0} & \boldsymbol{T}_{\mathrm{mr}}^{-1}
\end{array}\right]=\left[\begin{array}{cc}
\boldsymbol{A} & \mathbf{0} \\
\mathbf{0} & \boldsymbol{T}_{\mathrm{mr}} \overline{\boldsymbol{A}} \boldsymbol{T}_{\mathrm{mr}}^{-1}
\end{array}\right] .
$$

Entsprechend (4.65) ist die Matrix $\overline{\boldsymbol{A}}$ darin gegeben durch

$$
\overline{\boldsymbol{A}}=\left[\begin{array}{cc}
a+j b & 0 \\
0 & a-j b
\end{array}\right] \otimes \boldsymbol{I}_{\alpha-1} .
$$


Gemäß den Rechenregeln für Kroneckerprodukte (s. Lemma A.3 in Anhang A) gilt für $\boldsymbol{T}_{\mathrm{mr}}$ die Beziehung

$\boldsymbol{T}_{\mathrm{mr}}=\left(\left[\begin{array}{rr}1 & -j \\ 1 & j\end{array}\right] \otimes \boldsymbol{I}_{\alpha-1}\right)^{-1}=\left[\begin{array}{rr}1 & -j \\ 1 & j\end{array}\right]^{-1} \otimes \boldsymbol{I}_{\alpha-1}=\left[\begin{array}{cc}0,5 & 0,5 \\ 0,5 j & -0,5 j\end{array}\right] \otimes \boldsymbol{I}_{\alpha-1}$.

Unter Anwendung einer weiteren Rechenregel für Kroneckerprodukte (s. Lemma A.4 in Anhang A) folgt damit

$$
\begin{aligned}
\boldsymbol{T}_{\mathrm{mr}} \overline{\boldsymbol{A}} \boldsymbol{T}_{\mathrm{mr}}^{-1} & =\left(\left[\begin{array}{ll}
0,5 & 0,5 \\
0,5 j & -0,5 j
\end{array}\right] \otimes \boldsymbol{I}_{\alpha-1}\right) \cdot\left(\left[\begin{array}{cc}
a+j b & 0 \\
0 & a-j b
\end{array}\right] \otimes \boldsymbol{I}_{\alpha-1}\right) \\
\cdot\left(\left[\begin{array}{cc}
1 & -j \\
1 & j
\end{array}\right] \otimes \boldsymbol{I}_{\alpha-1}\right) & \\
& =\left(\left[\begin{array}{cc}
0,5 & 0,5 \\
0,5 j & -0,5 j
\end{array}\right]\left[\begin{array}{cc}
a+j b & 0 \\
0 & a-j b
\end{array}\right]\left[\begin{array}{cc}
1 & -j \\
1 & j
\end{array}\right]\right) \otimes \boldsymbol{I}_{\alpha-1} \\
& =\left[\begin{array}{rr}
a & b \\
-b & a
\end{array}\right] \otimes \boldsymbol{I}_{\alpha-1} .
\end{aligned}
$$

Damit ist bewiesen, dass die durch (A.8) gegebene Matrix reell ist.

Für die Aktorfehlermatrix $\boldsymbol{E}_{a}^{\prime}$ gilt mit der eingeführten Ähnlichkeitstransformation

$$
\boldsymbol{E}_{a}^{\prime}=\left[\begin{array}{cc}
\boldsymbol{I}_{n} & \mathbf{0} \\
\mathbf{0} & \boldsymbol{T}_{\mathrm{mr}}
\end{array}\right]\left[\begin{array}{c}
\boldsymbol{E}_{a} \boldsymbol{F} \\
\mathbf{0}
\end{array}\right]=\tilde{\boldsymbol{E}}_{a}
$$

Die Matrix bleibt also unverändert und ist ebenso wie $\tilde{\boldsymbol{E}}_{a}$ reell. Gleiches gilt für $\boldsymbol{B}^{\prime}=\tilde{\boldsymbol{B}}, \quad \boldsymbol{D}^{\prime}=\tilde{\boldsymbol{D}}=\boldsymbol{D}$ und die Sensorfehlermatrix $\boldsymbol{E}_{s}^{\prime}=\tilde{\boldsymbol{E}}_{s}=\boldsymbol{E}_{s} \boldsymbol{F}$.

Um darüber hinaus zu zeigen, dass auch $C^{\prime}$ reell ist, wird die Ausgangsmatrix $\tilde{\boldsymbol{C}}$ des Gesamtsystems nach den beiden Systemerweiterungen aufgrund von $\eta_{1}$ und $\eta_{2}$ betrachtet. Mit einer gemeinsamen Matrix $\boldsymbol{\Lambda}$ für beide Erweiterungen lautet sie gemäß (4.69)

$$
\tilde{\boldsymbol{C}}=\left[\begin{array}{lll}
\boldsymbol{C} & \boldsymbol{\Theta}_{1}\left(\eta_{1}\right) \boldsymbol{\Lambda} & \boldsymbol{\Theta}_{2}\left(\eta_{2}\right) \boldsymbol{\Lambda}
\end{array}\right] .
$$

Nach (4.68) ist $\boldsymbol{\Theta}_{1}\left(\eta_{1}\right)$ gegeben durch

$$
\boldsymbol{\Theta}_{1}\left(\eta_{1}\right)=\left(\boldsymbol{C}\left(\eta_{1} \boldsymbol{I}_{n}-\boldsymbol{A}\right)^{-1} \boldsymbol{E}_{a}+\boldsymbol{E}_{s}\right) \boldsymbol{F} \tilde{\boldsymbol{Z}}_{f}
$$

Bezeichnet man mit $\overline{\boldsymbol{A}}_{1}$ und $\overline{\boldsymbol{C}}_{1}$ die System- beziehungsweise Ausgangsmatrix des Gesamtsystems nach der ersten Systemerweiterung, so lässt 
sich $\boldsymbol{\Theta}_{2}\left(\eta_{2}\right)$ schreiben als

$$
\begin{aligned}
\boldsymbol{\Theta}_{2}\left(\eta_{2}\right) & =\left(\left[\begin{array}{ll}
\boldsymbol{C} & \overline{\boldsymbol{C}}_{1}
\end{array}\right]\left(\eta_{2} \boldsymbol{I}_{n+\alpha-1}-\left[\begin{array}{cc}
\boldsymbol{A} & \mathbf{0} \\
\mathbf{0} & \overline{\boldsymbol{A}}_{1}
\end{array}\right]\right)^{-1}\left[\begin{array}{c}
\boldsymbol{E}_{a} \\
\mathbf{0}
\end{array}\right]+\boldsymbol{E}_{s}\right) \boldsymbol{F} \tilde{\boldsymbol{Z}}_{f} \\
& =\left(\left[\begin{array}{ll}
\boldsymbol{C} & \overline{\boldsymbol{C}}_{1}
\end{array}\right]\left[\begin{array}{cc}
\eta_{2} \boldsymbol{I}_{n}-\boldsymbol{A} & \mathbf{0} \\
\mathbf{0} & \eta_{2} \boldsymbol{I}_{\alpha-1}-\overline{\boldsymbol{A}}_{1}
\end{array}\right]^{-1}\left[\begin{array}{c}
\boldsymbol{E}_{a} \\
\mathbf{0}
\end{array}\right]+\boldsymbol{E}_{s}\right) \boldsymbol{F} \tilde{\boldsymbol{Z}}_{f} \\
& =\left(\boldsymbol{C}\left(\eta_{2} \boldsymbol{I}_{n}-\boldsymbol{A}\right)^{-1} \boldsymbol{E}_{a}+\boldsymbol{E}_{s}\right) \boldsymbol{F} \tilde{\boldsymbol{Z}}_{f}
\end{aligned}
$$

Da $\eta_{1}$ und $\eta_{2}$ ein konjugiert komplexes Nullstellenpaar bilden, zeigt der Vergleich von (A.10) und (A.11), dass der dritte Spaltenblock in (A.9) das konjugiert Komplexe des zweiten Spaltenblocks ist. Weiterhin ist in

$$
\left[\begin{array}{ll}
\boldsymbol{\Theta}_{1}\left(\eta_{1}\right) \boldsymbol{\Lambda} & \boldsymbol{\Theta}_{2}\left(\eta_{2}\right) \boldsymbol{\Lambda}
\end{array}\right]=\left[\begin{array}{ll}
\boldsymbol{\Theta}_{1}\left(\eta_{1}\right) \boldsymbol{\Lambda} & \overline{\left(\boldsymbol{\Theta}_{1}\left(\eta_{1}\right) \boldsymbol{\Lambda}\right)}
\end{array}\right]
$$

die $(i+\alpha-1)$-te Spalte jeweils das konjugiert Komplexe der $i$-ten Spalte. Daraus folgt, dass

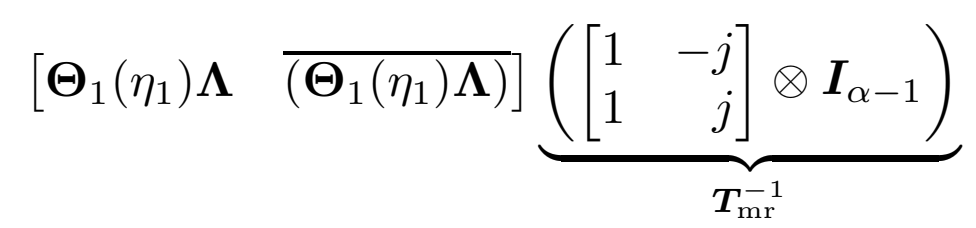

eine reelle Matrix ist. Dies impliziert, dass

$$
\boldsymbol{C}^{\prime}=\left[\begin{array}{lll}
\boldsymbol{C} & \boldsymbol{\Theta}_{1}\left(\eta_{1}\right) \boldsymbol{\Lambda} & \boldsymbol{\Theta}_{2}\left(\eta_{2}\right) \boldsymbol{\Lambda}
\end{array}\right] \underbrace{\left[\begin{array}{cc}
\boldsymbol{I}_{n} & \mathbf{0} \\
\mathbf{0} & \boldsymbol{T}_{\mathrm{mr}}^{-1}
\end{array}\right]}_{\boldsymbol{T}^{-1}}
$$

reell ist. 


\section{B Dynamische Entkopplungsregler für lineare Systeme}

Betrachtet werden lineare zeitinvariante Systeme der Form

$$
\begin{aligned}
& \dot{\boldsymbol{x}}=\boldsymbol{A} \boldsymbol{x}+\boldsymbol{B u}, \\
& \boldsymbol{y}=\boldsymbol{C} \boldsymbol{x}+\boldsymbol{D u}
\end{aligned}
$$

mit $\boldsymbol{x} \in \mathbb{R}^{n}, \boldsymbol{u} \in \mathbb{R}^{n_{u}}, \boldsymbol{y} \in \mathbb{R}^{n_{y}}$ und Matrizen entsprechender Dimensionen. Angenommen wird dabei $n_{u}=n_{y}$. Für derartige Systeme werden zwei Verfahren für den Entwurf dynamischer Entkopplungsregler für Systeme der Form (B.1) vorgestellt. In Auszügen wurden diese bereits in [232, 234, 239] publiziert.

Den bekannten Definitionen [67, 185] folgend werden die Begriffe der Differenzenordnungen und der Entkoppelbarkeitsmatrix eingeführt.

Definition B.1 (Differenzenordnungen). Für ein System der Form (B.1) sind die Differenzenordnungen der Ausgänge definiert als

$$
\delta_{i}=\left\{\begin{array}{cc}
0 & , d_{i}^{\top} \neq \mathbf{0}^{\top}, \\
\min _{k}\left\{k: \boldsymbol{c}_{i}^{\top} \boldsymbol{A}^{k-1} \boldsymbol{B} \neq \mathbf{0}^{\top}\right\}, & \boldsymbol{d}_{i}^{\top}=\mathbf{0}^{\top} .
\end{array}\right.
$$

Durch Summation aller Differenzenordnungen ergibt sich die Differenzordnung $\delta$ des Systems zu

$$
\delta=\sum_{i=0}^{n_{y}} \delta_{i}
$$

Definition B.2 (Entkoppelbarkeitsmatrix). Für ein System der Form (B.1) mit den Differenzenordnungen $\delta_{i}$ ist die Entkoppelbarkeitsmatrix 
definiert als

$$
\begin{aligned}
& \boldsymbol{D}^{*}=\left[\begin{array}{c}
\boldsymbol{d}_{1}^{* \top} \\
\vdots \\
\boldsymbol{d}_{n_{y}}^{*} \mathrm{~T}
\end{array}\right], \\
& \boldsymbol{d}_{i}^{* \top}=\left\{\begin{array}{cc}
\boldsymbol{d}_{i}^{\top}, & \delta_{i}=0, \\
\boldsymbol{c}_{i}^{\top} \boldsymbol{A}^{\delta_{i}-1} \boldsymbol{B}, & \delta_{i} \geq 1 .
\end{array}\right.
\end{aligned}
$$

Auf dieser Grundlage werden hinsichtlich der Strecke (B.1) die folgenden Annahmen getroffen.

Annahme B.1. Das Paar $(\boldsymbol{A}, \boldsymbol{B})$ ist vollständig steuerbar.

Annahme B.2. Die Entkoppelbarkeitsmatrix $\boldsymbol{D}^{*}$ hat vollen Rang.

Annahme B.3. Das System $(\boldsymbol{A}, \boldsymbol{B}, \boldsymbol{C}, \boldsymbol{D})$ ist minimalphasig.

Wie unter anderem in $[67,185]$ gezeigt wird, sind die Annahmen B.1 bis B.3 hinreichend für den Entwurf statisch stabiler Entkopplungsregler der Form $\boldsymbol{u}=-\boldsymbol{K} \boldsymbol{x}+\boldsymbol{F} \boldsymbol{w}$. Es ergeben sich für die Übertragungsmatrix $\boldsymbol{G}_{\boldsymbol{y} \boldsymbol{w}}(s)$ die Diagonalelemente

$$
g_{i, i}(s)=\frac{z_{i, 0}}{s^{\delta_{i}}+q_{i, \delta_{i}-1} s^{\delta_{i}-1}+\ldots+q_{i, 1} s+q_{i, 0}} .
$$

Mit Ausnahme nicht verkoppelnder Nullstellen in der abgeschlossenen rechten s-Halbebene [123, 136, 138, 139] ist die Bedingung B.3 auch notwendig. Dieser Sonderfall wird hier jedoch nicht näher betrachtet.

In den folgenden Abschnitten werden dynamische Entkopplungsregler der Form

$$
\begin{aligned}
& \dot{\boldsymbol{\psi}}=\boldsymbol{A}_{u} \boldsymbol{\psi}+\boldsymbol{B}_{u, x} \boldsymbol{x}+\boldsymbol{B}_{u, w} \boldsymbol{w} \\
& \boldsymbol{u}=\boldsymbol{C}_{u} \boldsymbol{\psi}+\boldsymbol{D}_{u, x} \boldsymbol{x}+\boldsymbol{D}_{u, w} \boldsymbol{w}
\end{aligned}
$$

mit $\boldsymbol{\psi} \in \mathbb{R}^{n_{c}}$ herangezogen. Üblicherweise kommen solche dynamischen Regler zum Einsatz, wenn die Annahmen B.2 und/oder B.3 nicht erfüllt sind. Wie zum Beispiel in $[137,140]$ gezeigt, lassen sich unter schwächeren Annahmen dynamische Regler zur Diagonalisierung von $\boldsymbol{G}_{\boldsymbol{y} \boldsymbol{w}}(s)$ entwerfen. In diesem Kapitel werden dynamische Regler der Form (B.3) für Systeme angewendet, welche die Annahmen B.1 bis B.3 erfüllen. Dadurch ist es 
möglich, eine gegenüber (B.2) erweiterte Dynamik der Diagonalelemente von $\boldsymbol{G}_{\boldsymbol{y} \boldsymbol{w}}(s)$ der Form

$$
g_{i, i}(s)=\frac{\sum_{j=0}^{\rho} z_{i, j} s^{j}}{s^{\delta_{i}+\rho}+\sum_{k=0}^{\delta_{i}+\rho-1} q_{i, k} s^{k}}
$$

zu realisieren.

\section{B.1 Entwurf dynamischer Entkopplungsregler im Laplace-Bereich}

Für den Entwurf wird die verallgemeinerte Entkoppelbarkeitsmatrix $\boldsymbol{D}^{*}(s)$ eingeführt. Sie ist im Gegensatz zur in Definition B.2 eingeführten Entkoppelbarkeitsmatrix abhängig von den vorgegebenen Nennerkoeffizienten der Diagonalelemente $g_{i, i}(s)$ und definiert durch

Definition B.3 (Verallgemeinerte Entkoppelbarkeitsmatrix). Für ein System der Form (B.1) mit den Differenzenordnungen $\delta_{i}, i=1, \ldots, n_{y}$, ist die verallgemeinerte Entkoppelbarkeitsmatrix $\boldsymbol{D}^{*}(s)$ definiert als

$$
\boldsymbol{D}^{*}(s)=\left[\begin{array}{c}
\boldsymbol{d}_{1}^{* \mathrm{~T}}(s) \\
\vdots \\
\boldsymbol{d}_{n_{y}}^{*}{ }^{\mathrm{T}}(s)
\end{array}\right]
$$

mit

$$
\begin{gathered}
\boldsymbol{d}_{i}^{* \top}(s)=\boldsymbol{c}_{i}^{\top} \sum_{l=0}^{\rho-1} s^{l}\left(A^{\rho-1-l}+\sum_{k=1}^{\rho-1-l} q_{i, k+l}\right) \boldsymbol{B}+\ldots \\
+\boldsymbol{d}_{i}^{\top}\left(s^{\rho}+\sum_{l=0}^{\rho-1} s^{l} q_{i, l}\right)
\end{gathered}
$$

für $\delta_{i}=0$ beziehungsweise

$$
\boldsymbol{d}_{i}^{* \top}(s)=\boldsymbol{c}_{i}^{\top} \sum_{l=0}^{\rho} s^{l}\left(\boldsymbol{A}^{\delta_{i}+\rho-1-l}+\sum_{k=0}^{\rho-1-l} q_{i, \delta_{i}+k+l} \boldsymbol{A}^{\delta_{i}+k-1}\right) \boldsymbol{B}
$$

für $\delta_{i} \geq 1$. 
Zwar hängt die Matrix von den Nennerkoeffizienten von $g_{i, i}(s)$ ab, jedoch gilt

Lemma B.1. Erfüllt ein System der Form (B.1) die Annahme B.2, so ist die verallgemeinerte Entkoppelbarkeitsmatrix unabhängig von der Wahl von $q_{i, j}$ für fast alle $s \in \mathbb{C}$ invertierbar.

Beweis. Die verallgemeinerte Entkoppelbarkeitsmatrix $\boldsymbol{D}^{*}(s)$ lässt sich zerlegen in

$$
\boldsymbol{D}^{*}(s)=\boldsymbol{D}_{s^{0}}^{*}+s \cdot \boldsymbol{D}_{s^{1}}^{*}+\ldots+s^{\rho} \cdot \boldsymbol{D}_{s^{\rho}}^{*} .
$$

Dabei zeigt sich durch Auswertung der Gleichungen (B.5) beziehungsweise (B.6) für $l=\rho$, dass

$$
\left.\boldsymbol{d}_{i}^{* \top}(s)\right|_{l=\rho}=s^{\rho} \cdot\left\{\begin{array}{cc}
\boldsymbol{d}_{i}^{\top} \quad, & \delta_{i}=0, \\
\boldsymbol{c}_{i}^{\top} \boldsymbol{A}^{\delta_{i}-1} \boldsymbol{B}, & \delta_{i} \geq 1
\end{array}\right.
$$

gilt. Daher entspricht $\boldsymbol{D}_{s^{\rho}}^{*}$ der Entkoppelbarkeitsmatrix des Systems (B.1) und ist nach Annahme B.2 invertierbar. Damit ist $\boldsymbol{D}^{*}(s)$ für fast alle $s \in \mathbb{C}$ invertierbar, da Terme in geringeren Potenzen von $s$ den Normalrang von $\boldsymbol{D}^{*}(s)$ nicht reduzieren können, wenn $\boldsymbol{D}_{s^{\rho}}^{*}$ vollen Rang hat.

Der folgende Satz liefert das auf Definition B.3 basierende Entwurfsverfahren für dynamische Entkopplungsregler. Es kann als unmittelbare Erweiterung des klassischen Ergebnisses aus [67] aufgefasst werden.

Satz B.1 (Entwurf dynamischer Entkopplungsregler mit erweiterter Dynamik der Diagonalelemente im Frequenzbereich). Gegeben sei ein System der Form (B.1), welches die Annahmen B.1-B.3 erfüllt, und ein Skalar $\rho \in \mathbb{N}$. Sei $\boldsymbol{D}^{*}(s)$ die verallgemeinerte Entkoppelbarkeitsmatrix nach Definition B.3, dann führt die dynamische Zustandsrückführung

$$
\boldsymbol{u}(s)=-\boldsymbol{D}^{*-1}(s) \cdot \boldsymbol{M} \cdot \boldsymbol{x}(s)+\boldsymbol{D}^{*-1}(s) \cdot \boldsymbol{N}(s) \cdot \boldsymbol{w}(s)
$$

mit

$$
\begin{aligned}
& \boldsymbol{m}_{i}^{\top}=\boldsymbol{c}_{i}^{\top}\left(\boldsymbol{A}^{\delta_{i}+\rho}+\sum_{k=0}^{\delta_{i}+\rho-1} q_{i, k} \boldsymbol{A}^{k}\right)
\end{aligned}
$$

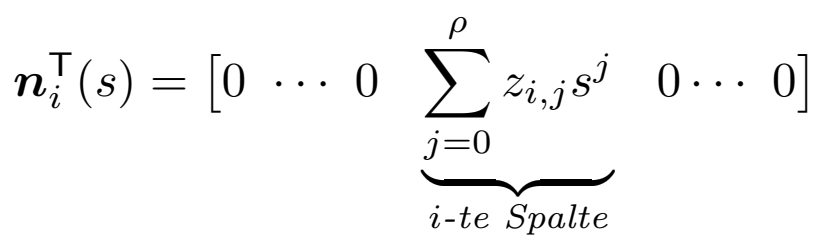


auf eine diagonale Übertragungsmatrix $\boldsymbol{G}_{\boldsymbol{y} \boldsymbol{w}}(s)$ mit den Diagonalelementen (B.4).

Beweis. Entsprechend (B.4) muss für den $i$-ten Übertragungskanal im Laplace-Bereich

$$
s^{\delta_{i}+\rho} y_{i}+\ldots+s q_{i, 1} y_{i}+q_{i, 0} y_{i}=s^{\rho} z_{i, \rho} w_{i}+\ldots+z_{i, 0} w_{i}
$$

gelten. Für Ausgänge mit $\delta_{i}=0$ ist nach Definition B.1

$$
\begin{aligned}
y_{i}(s) & =\boldsymbol{c}_{i}^{\top} \boldsymbol{x}(s)+\boldsymbol{d}_{i}^{\top} \boldsymbol{u}(s), \\
s \cdot y_{i}(s) & =\boldsymbol{c}_{i}^{\top} \boldsymbol{A} \boldsymbol{x}(s)+\boldsymbol{c}_{i}^{\top} \boldsymbol{B} \boldsymbol{u}(s)+s \cdot \boldsymbol{d}_{i}^{\top} \boldsymbol{u}(s), \\
s^{2} \cdot y_{i}(s) & =\boldsymbol{c}_{i}^{\top} \boldsymbol{A}^{2} \boldsymbol{x}(s)+\boldsymbol{c}_{i}^{\top} \boldsymbol{A} \boldsymbol{B} \boldsymbol{u}(s)+s \cdot \boldsymbol{c}_{i}^{\top} \boldsymbol{B} \boldsymbol{u}(s)+s^{2} \cdot \boldsymbol{d}_{i}^{\top} \boldsymbol{u}(s), \\
& \vdots \\
s^{l} \cdot y_{i}(s) & =\boldsymbol{c}_{i}^{\top}\left(\boldsymbol{A}^{l} \boldsymbol{x}(s)+\sum_{k=0}^{\rho-1} s^{k} \cdot \boldsymbol{A}^{l-1-k} \boldsymbol{B} \boldsymbol{u}(s)\right)+s^{l} \cdot \boldsymbol{d}_{i}^{\top} \boldsymbol{u}(s) .
\end{aligned}
$$

Ebenfalls anhand von Definition B.1 und der Systemdynamik (B.1) ergeben sich für Ausgänge mit $\delta_{i} \geq 1$ die in den Laplace-Bereich transformierten zeitlichen Ableitungen zu

$$
\begin{aligned}
s^{k} \cdot y_{i}(s) & =\boldsymbol{c}_{i}^{\top} \boldsymbol{A}^{k} \boldsymbol{x}(s), \forall k=0, \ldots, \delta_{i}-1, \\
s^{\delta_{i}} \cdot y_{i}(s) & =\boldsymbol{c}_{i}^{\top}\left(\boldsymbol{A}^{\delta_{i}} \boldsymbol{x}(s)+\boldsymbol{A}^{\delta_{i}-1} \boldsymbol{B} \boldsymbol{u}(s)\right), \\
s^{\delta_{i}+1} \cdot y_{i}(s) & =\boldsymbol{c}_{i}^{\boldsymbol{\top}}\left(\boldsymbol{A}^{\delta_{i}+1} \boldsymbol{x}(s)+\left(\boldsymbol{A}^{\delta_{i}}+s \cdot \boldsymbol{A}^{\delta_{i}-1}\right) \boldsymbol{B} \boldsymbol{u}(s)\right), \\
& \vdots \\
s^{\delta_{i}+l} y_{i}(s) & =\boldsymbol{c}_{i}^{\boldsymbol{\top}}\left(\boldsymbol{A}^{\delta_{i}+l} \boldsymbol{x}(s)+\sum_{k=0}^{\rho} s^{k} \cdot \boldsymbol{A}^{\delta_{i}+\rho-1-k} \boldsymbol{B} \boldsymbol{u}(s)\right) .
\end{aligned}
$$

Setzt man (B.8) und (B.9) in (B.7) ein, so ergibt sich die Bestimmungsgleichung

$$
\underbrace{\left[\begin{array}{c}
\boldsymbol{d}_{1}^{* \top}(s) \\
\vdots \\
\boldsymbol{d}_{n_{y}}^{*}(s)
\end{array}\right]}_{\boldsymbol{D}^{*}(s)} \cdot \boldsymbol{u}(s)=-\underbrace{\left[\begin{array}{c}
\boldsymbol{m}_{1}^{\top} \\
\vdots \\
\boldsymbol{m}_{n_{y}}^{\top}
\end{array}\right]}_{\boldsymbol{M}} \cdot \boldsymbol{x}(s)+\underbrace{\left[\begin{array}{c}
\boldsymbol{n}_{1}^{\top}(s) \\
\vdots \\
\boldsymbol{n}_{n_{y}}^{\top}(s)
\end{array}\right]}_{\boldsymbol{N}(s)} \cdot \boldsymbol{w}(s),
$$


wobei $\boldsymbol{D}^{*}$ der Definition B.2 entspricht und $\boldsymbol{M}$ und $\boldsymbol{N}(s)$ den in Satz B.1 angegebenen Matrizen. Da $\boldsymbol{D}^{*}(s)$ nach Lemma B.1 für fast alle $s \in \mathbb{C}$ invertierbar ist, handelt es sich bei

$$
\boldsymbol{u}(s)=-\boldsymbol{D}^{*-1}(s) \cdot \boldsymbol{M} \cdot \boldsymbol{x}(s)+\boldsymbol{D}^{*-1}(s) \cdot \boldsymbol{N}(s) \cdot \boldsymbol{w}(s)
$$

um einen entkoppelnden dynamischen Regler, welcher auf die Diagonalelemente (B.4) führt.

Der Entwurf nach Satz B.1 liefert den gesuchten dynamischen Regler im Frequenzbereich. Für eine Implementierung ist es abschließend notwendig, eine Zustandsraumrealisierung des Reglers zu berechnen. Dies kann beispielsweise wie in [52] gezeigt erfolgen.

\section{B.2 Entwurf dynamischer Entkopplungsregler im Zeitbereich}

Das im vorangegangenen Abschnitt erarbeitete Entwurfsverfahren für dynamische Entkopplungsregler liefert einen Regler im Frequenzbereich, der in eine Zustandsraumrealisierung überführt werden muss. Im Gegensatz dazu wird in diesem Abschnitt gezeigt, wie sich in einfacher Art und Weise eine entkoppelnde dynamische Zustandsrückführung parametrieren lässt, wenn lediglich die Nennerordnung der Diagonalelemente gegenüber (B.2) vergrößert wird.

Es soll also

$$
g_{i, i}(s)=\frac{z_{i, 0}}{s^{\delta_{i}+\rho}+\sum_{k=0}^{\delta_{i}+\rho-1} q_{i, k} s^{k}}
$$

erreicht werden. Dazu wird eine virtuelle dynamische Erweiterung der Regelstrecke vorgenommen, wie sie in Abbildung B.1 gezeigt ist. Es wird eine Kette von $\rho$ Integratorblöcken vor die Strecke geschaltet. Die Ausgänge der Integratorblöcke werden mit $\boldsymbol{\zeta}_{i}, i=1, \ldots, \rho-1$, bezeichnet, wobei jeweils $\zeta \in \mathbb{R}^{n_{u}}$ gilt. Der Ausgang des letzten Integratorblocks ist die reale Stellgröße $\boldsymbol{u}$. Für die virtuelle, erweiterte Regelstrecke ist $\boldsymbol{\zeta}_{\rho}$ die Stellgröße. Mit dem Zustandsvektor $\tilde{\boldsymbol{x}}=\left[\begin{array}{lllll}\boldsymbol{x}^{\top} & \boldsymbol{u}^{\top} & \boldsymbol{\zeta}_{1}^{\top} & \ldots & \boldsymbol{\zeta}_{\rho-1}^{\top}\end{array}\right]^{\top} \in \mathbb{R}^{n+\rho n_{u}}$ ergibt sich 


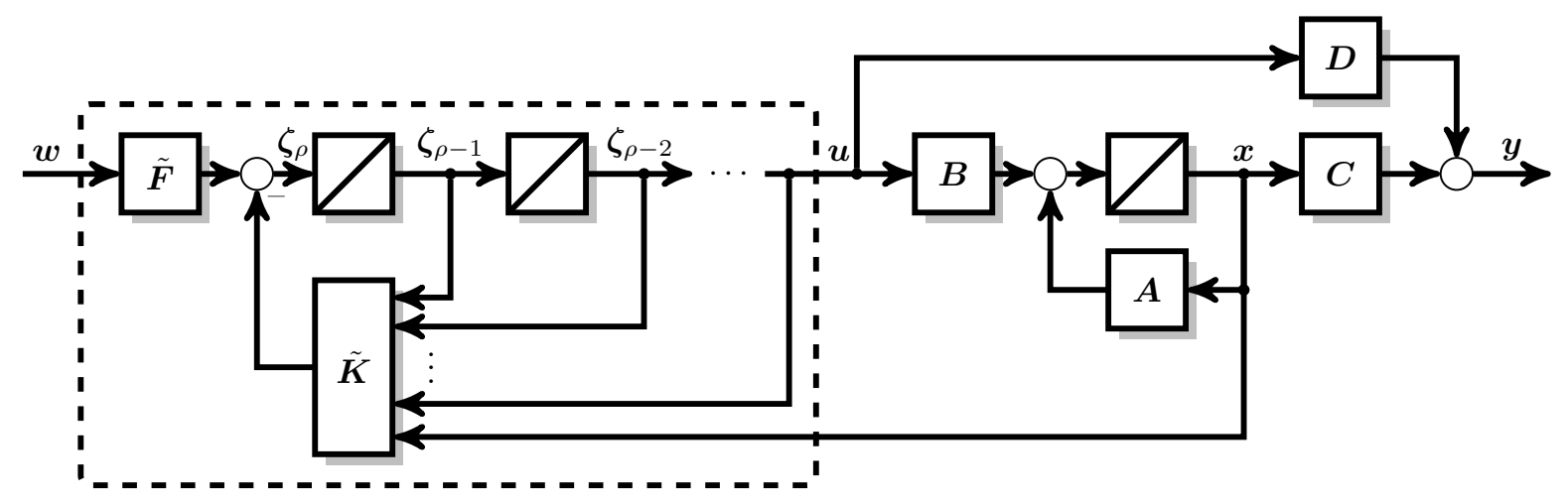

Abbildung B.1: Virtuelle Systemerweiterung für dynamische Entkopplungsregler

daraus die erweiterte Strecke zu

$$
\begin{aligned}
& \dot{\tilde{\boldsymbol{x}}}=\underbrace{\left[\begin{array}{ccccc}
\boldsymbol{A} & \boldsymbol{B} & \mathbf{0} & \cdots & \mathbf{0} \\
\mathbf{0} & \mathbf{0} & \boldsymbol{I}_{n_{u}} & \ddots & \vdots \\
\vdots & & \ddots & \ddots & \mathbf{0} \\
\mathbf{0} & \cdots & \ldots & \mathbf{0} & \boldsymbol{I}_{n_{u}} \\
\mathbf{0} & \cdots & \cdots & \cdots & \mathbf{0}
\end{array}\right]}_{\tilde{\boldsymbol{A}}} \tilde{\boldsymbol{x}}+\underbrace{\left[\begin{array}{c}
\mathbf{0} \\
\vdots \\
\vdots \\
\mathbf{0} \\
\boldsymbol{I}_{n_{u}}
\end{array}\right]}_{\tilde{\boldsymbol{B}}} \boldsymbol{\zeta}_{\rho}, \\
& \boldsymbol{y}=\underbrace{\left[\begin{array}{lllll}
\boldsymbol{C} & \boldsymbol{D} & \mathbf{0} & \cdots & \mathbf{0}
\end{array}\right]}_{\tilde{C}} \tilde{\boldsymbol{x}} .
\end{aligned}
$$

Anhand von Abbildung B.1 lässt sich bereits vermuten, dass die Differenzenordnungen $\tilde{\delta}_{i}$ gegenüber der realen Strecke durch die Integratorblöcke jeweils um $\rho$ erhöht werden. In der Tat gilt

Lemma B.2. Gegeben sei ein System der Form (B.1), welches die Annahmen B.1-B.3 erfüllt. Wird das System um $\rho$ dem Eingang vorgeschaltete Integratorblöcke dynamisch erweitert, so gilt für die Differenzenordnungen des erweiterten Systems $\tilde{\delta}_{i}=\delta_{i}+\rho$. Weiterhin ist das resultierende erweiterte System entkoppelbar und für seine Entkoppelbarkeitsmatrix gilt $\tilde{D}^{*}=D^{*}$.

Beweis. Der Beweis ergibt sich im Wesentlichen durch sukzessives Poten- 
zieren der Matrix $\tilde{\boldsymbol{A}}$, die durch

$$
\tilde{\boldsymbol{A}}=\left[\begin{array}{ccccc}
\boldsymbol{A} & \boldsymbol{B} & \mathbf{0} & \cdots & \mathbf{0} \\
\mathbf{0} & \mathbf{0} & \boldsymbol{I}_{n_{u}} & \ddots & \vdots \\
\vdots & & \ddots & \ddots & \mathbf{0} \\
\mathbf{0} & \cdots & \cdots & \mathbf{0} & \boldsymbol{I}_{n_{u}} \\
\mathbf{0} & \cdots & \cdots & \cdots & \mathbf{0}
\end{array}\right]
$$

gegeben ist. Für ihre Potenzen gilt dann

$$
\begin{aligned}
\tilde{\boldsymbol{A}}^{2}= & {\left[\begin{array}{cccccc}
\boldsymbol{A}^{2} & \boldsymbol{A} \boldsymbol{B} & \boldsymbol{B} & \mathbf{0} & \cdots & \mathbf{0} \\
\mathbf{0} & \mathbf{0} & \mathbf{0} & \boldsymbol{I}_{n_{u}} & \ddots & \vdots \\
\vdots & \vdots & \vdots & \ddots & \ddots & \mathbf{0} \\
\mathbf{0} & \mathbf{0} & \mathbf{0} & \cdots & \mathbf{0} & \boldsymbol{I}_{n_{u}} \\
\mathbf{0} & \mathbf{0} & \mathbf{0} & \cdots & \cdots & \mathbf{0} \\
\mathbf{0} & \mathbf{0} & \mathbf{0} & \cdots & \cdots & \mathbf{0}
\end{array}\right], } \\
\tilde{\boldsymbol{A}}^{\rho-1}= & {\left[\begin{array}{ccccc}
\boldsymbol{A}^{\rho-1} & \boldsymbol{A}^{\rho-2} \boldsymbol{B} & \cdots & \boldsymbol{B} & \mathbf{0} \\
\mathbf{0} & \mathbf{0} & \cdots & \mathbf{0} & \boldsymbol{I}_{n_{u}} \\
\mathbf{0} & \mathbf{0} & \ddots & & \mathbf{0} \\
\vdots & \vdots & \ddots & \ddots & \vdots \\
\mathbf{0} & \mathbf{0} & \cdots & \cdots & \mathbf{0}
\end{array}\right], } \\
\tilde{\boldsymbol{A}}^{\rho+k}= & {\left[\begin{array}{cccc}
\boldsymbol{A}^{\rho+k} & \boldsymbol{A}^{\rho+k-1} \boldsymbol{B} & \cdots & \boldsymbol{A}^{k} \boldsymbol{B} \\
\mathbf{0} & \mathbf{0} & \cdots & \mathbf{0} \\
\vdots & \vdots & & \ddots \\
\mathbf{0} & \mathbf{0} & \cdots & \mathbf{0}
\end{array}\right], \forall k \geq 0 . }
\end{aligned}
$$

Es ist ersichtlich, dass die letzten $k n_{u}$ Zeilen von $\tilde{\boldsymbol{A}}^{k}$ Nullzeilen sind. Für die Matrix $\tilde{\boldsymbol{A}}^{\rho-2}$ verbleiben somit $n+2 n_{u}$ Zeilen, die Nichtnullelemente enthalten, wobei gemäß der Systematik in (B.12) der 3. Zeilenblock im letzten Spaltenblock die Einheitsmatrix $\boldsymbol{I}_{n_{u}}$ enthält. Daraus ergibt sich 
für Ausgänge mit $\delta_{i}=0$ die Beziehung

$$
\tilde{\boldsymbol{c}}_{i}^{\top} \tilde{\boldsymbol{A}}^{k} \tilde{\boldsymbol{B}}=\left[\begin{array}{lllll}
\boldsymbol{c}_{i}^{\top} & \boldsymbol{d}_{i}^{\top} & \mathbf{0}^{\top} & \ldots & \mathbf{0}^{\top}
\end{array}\right]\left[\begin{array}{c}
\mathbf{0} \\
\vdots \\
\mathbf{0} \\
\boldsymbol{I}_{n_{u}} \\
\mathbf{0} \\
\vdots \\
\mathbf{0}
\end{array}\right]=\mathbf{0}^{\top}, k=1, \ldots, \rho-2 .
$$

Für $k=\rho-1$ folgt aus (B.12)

$$
\tilde{\boldsymbol{c}}_{i}^{\top} \tilde{\boldsymbol{A}}^{\rho-1} \tilde{\boldsymbol{B}}=\left[\begin{array}{lllll}
\boldsymbol{c}_{i}^{\top} & \boldsymbol{d}_{i}^{\top} & \mathbf{0}^{\top} & \cdots & \mathbf{0}^{\top}
\end{array}\right]\left[\begin{array}{c}
\mathbf{0} \\
\boldsymbol{I}_{n_{u}} \\
\mathbf{0} \\
\vdots \\
\mathbf{0}
\end{array}\right]=\boldsymbol{d}_{i}^{\top}=\boldsymbol{d}_{i}^{* \top} \neq \mathbf{0}^{\top} .
$$

Aus (B.13) und (B.14) folgt gemäß der Definition B.1 der Differenzenordnungen, dass für Ausgänge mit $\delta_{i}=0$ im erweiterten System $\tilde{\delta}_{i}=\rho$ gilt.

In ähnlicher Art und Weise werden Ausgänge mit $\delta_{i} \geq 1$ betrachtet. Für sie ist $\boldsymbol{d}_{i}^{\top}=\mathbf{0}^{\top}$, woraus unmittelbar $\tilde{\boldsymbol{c}}_{i}^{\top} \tilde{\boldsymbol{A}}^{k} \tilde{\boldsymbol{B}}=\mathbf{0}^{\top}$ für $k=0, \ldots, \rho-1$ folgt. Für höhere Potenzen in $\tilde{\boldsymbol{A}}$ ist nach (B.12)

$$
\tilde{\boldsymbol{c}}_{i}^{\top} \tilde{\boldsymbol{A}}^{\rho+k} \tilde{\boldsymbol{B}}=\left[\begin{array}{lllll}
\boldsymbol{c}_{i}^{\top} & \boldsymbol{d}_{i}^{\top} & \mathbf{0}^{\top} & \cdots & \mathbf{0}^{\top}
\end{array}\right]\left[\begin{array}{c}
\boldsymbol{A}^{k} \boldsymbol{B} \\
\mathbf{0} \\
\vdots \\
\mathbf{0}
\end{array}\right]=\boldsymbol{c}_{i}^{\top} \boldsymbol{A}^{k} \boldsymbol{B} .
$$

Also ist $\tilde{\boldsymbol{c}}_{i}^{\top} \tilde{\boldsymbol{A}}^{k} \tilde{\boldsymbol{B}}=\mathbf{0}^{\top}$ für $k=0, \ldots, \rho+\delta_{i}-2$ und

$$
\tilde{\boldsymbol{c}}_{i}^{\top} \tilde{\boldsymbol{A}}^{\rho+\delta_{i}-1} \tilde{\boldsymbol{B}}=\boldsymbol{c}_{i}^{\top} \boldsymbol{A}^{\delta_{i}-1} \boldsymbol{B}=\boldsymbol{d}_{i}^{* \top} \neq \mathbf{0}^{\top} .
$$

Dementsprechend wird auch für Ausgänge mit $\delta_{i} \geq 1$ die Differenzenordnung um $\rho$ erhöht und es ist $\tilde{\delta}_{i}=\delta_{i}+\rho$. Aus (B.14) und (B.15) geht darüber hinaus hervor, dass die Zeilen der Entkoppelbarkeitsmatrix des erweiterten Systems denen der Entkoppelbarkeitsmatrix des ursprünglichen Systems entsprechen, was $\tilde{D}^{*}=\boldsymbol{D}^{*}$ bedingt. 
Durch Lemma B.2 ist gezeigt, dass sich durch die vorgeschlagene Systemerweiterung ein entkoppelbares System mit erhöhten Differenzenordnungen ergibt. Das folgende Lemma macht darüber hinaus eine Aussage über die invarianten Nullstellen des erweiterten Systems.

Lemma B.3. Gegeben sei ein System der Form (B.1), welches die Annahmen B.1-B.3 erfüllt. Wird das System um $\rho$ dem Eingang vorgeschaltete Integratorblöcke dynamisch erweitert, so entsprechen die invarianten Nullstellen des erweiterten Systems den invarianten Nullstellen des ursprünglichen Systems.

Beweis. Für die Rosenbrock'sche Systemmatrix des erweiterten Systems gilt

$$
\begin{aligned}
\tilde{\boldsymbol{\Pi}}(s)= & {\left[\begin{array}{cccc}
s \boldsymbol{I}_{n+\rho n_{u}}-\tilde{\boldsymbol{A}} & -\tilde{\boldsymbol{B}} \\
\tilde{\boldsymbol{C}} & \mathbf{0}
\end{array}\right] } \\
= & {\left[\begin{array}{ccccc}
s \boldsymbol{I}_{n}-\boldsymbol{A} & -\boldsymbol{B} & \mathbf{0} & \ldots & \mathbf{0} \\
\mathbf{0} & s \boldsymbol{I}_{n_{u}} & -\boldsymbol{I}_{n_{u}} & \ddots & \vdots \\
\vdots & \ddots & \ddots & \ddots & \mathbf{0} \\
\mathbf{0} & \cdots & \mathbf{0} & s \boldsymbol{I}_{n_{u}} & -\boldsymbol{I}_{n_{u}} \\
\boldsymbol{C} & \boldsymbol{D} & \mathbf{0} & \cdots & \mathbf{0}
\end{array}\right] . }
\end{aligned}
$$

Die schrittweise Entwicklung der Determinante von $\tilde{\boldsymbol{\Pi}}(s)$ nach Spalten, die jeweils nur ein Nichtnullelement enthalten, führt auf

$$
\operatorname{det}(\tilde{\boldsymbol{\Pi}}(s))=\left|\begin{array}{cc}
s \boldsymbol{I}_{n}-\boldsymbol{A} & -\boldsymbol{B} \\
\boldsymbol{C} & \boldsymbol{D}
\end{array}\right|=\operatorname{det}(\boldsymbol{\Pi}(s)) .
$$

Da die Determinanten von $\tilde{\boldsymbol{\Pi}}(s)$ und $\boldsymbol{\Pi}(s)$ übereinstimmen, entsprechen die invarianten Nullstellen des erweiterten Systems denen des ursprünglichen Systems.

Gemeinsam mit Lemma B.2 zeigt Lemma B.3, dass für das dynamisch erweiterte System (B.11) eine statische entkoppelnde Zustandsrückführung entworfen werden kann, welche auf einen stabilen Regelkreis führt, wenn das ursprüngliche System die Annahmen B.1 bis B.3 erfüllt. Mithilfe des folgenden Satzes lässt sich auf dieser Grundlage ein statischer Entkopplungsregler für das erweiterte System entwerfen. Darüber hinaus liefert er eine direkte Parametrierung eines dynamischen Entkopplungsreglers für das ursprüngliche System. 
Satz B.2 (Entwurf dynamischer Entkopplungsregler mit erweiterter Dynamik der Diagonalelemente im Zeitbereich). Gegeben sei ein System der Form (B.1), welches die Annahmen B.1-B.3 erfüllt. Für das um $\rho$ Integratorblöcke erweiterte virtuelle Gesamtsystem (B.11) ist durch

$$
\begin{aligned}
\boldsymbol{\zeta}_{\rho} & =-\boldsymbol{D}^{*-1} \tilde{\boldsymbol{M}} \tilde{\boldsymbol{x}}+\boldsymbol{D}^{*-1} \tilde{\boldsymbol{N}} \boldsymbol{w} \\
& =-\tilde{\boldsymbol{K}} \tilde{\boldsymbol{x}}+\tilde{\boldsymbol{F}} \boldsymbol{w}
\end{aligned}
$$

mit

$$
\begin{aligned}
\tilde{\boldsymbol{M}} & =\left[\begin{array}{c}
\tilde{\boldsymbol{c}}_{1}^{\top}\left(\tilde{\boldsymbol{A}}^{\rho+\delta_{1}}+\sum_{k=0}^{\rho+\delta_{1}-1} q_{1, k} \tilde{\boldsymbol{A}}^{k}\right) \\
\vdots \\
\tilde{\boldsymbol{c}}_{n_{y}}^{\top}\left(\tilde{\boldsymbol{A}}^{\rho+\delta_{n_{y}}}+\sum_{k=0}^{\rho+\delta_{n_{y}-1}} q_{n_{y}, k} \tilde{\boldsymbol{A}}^{k}\right)
\end{array}\right], \\
\tilde{\boldsymbol{N}} & =\operatorname{diag}\left(z_{1,0}, \ldots, z_{n_{y}, 0}\right)
\end{aligned}
$$

ein statisch stabiler Entkopplungsregler gegeben. Mit der Partitionierung

$$
\tilde{\boldsymbol{K}}=\left[\begin{array}{lllll}
\tilde{\boldsymbol{K}}_{x} & \tilde{\boldsymbol{K}}_{u} & \tilde{\boldsymbol{K}}_{\zeta_{1}} & \cdots & \tilde{\boldsymbol{K}}_{\zeta_{\rho-1}}
\end{array}\right]
$$

erhält man durch

$$
\begin{aligned}
& \boldsymbol{A}_{u}=\left[\begin{array}{ccccc}
\mathbf{0} & \boldsymbol{I}_{n_{u}} & \mathbf{0} & \cdots & \mathbf{0} \\
\vdots & \ddots & \ddots & \ddots & \vdots \\
\vdots & & \ddots & \ddots & \mathbf{0} \\
\mathbf{0} & \cdots & \cdots & \mathbf{0} & \boldsymbol{I}_{n_{u}} \\
-\tilde{\boldsymbol{K}}_{u} & -\tilde{\boldsymbol{K}}_{\zeta_{1}} & -\tilde{\boldsymbol{K}}_{\zeta_{2}} & \cdots & -\tilde{\boldsymbol{K}}_{\zeta_{\rho-1}}
\end{array}\right] \\
& \boldsymbol{B}_{u, x}=\left[\begin{array}{c}
\mathbf{0} \\
\vdots \\
\mathbf{0} \\
-\tilde{\boldsymbol{K}}_{x}
\end{array}\right], \boldsymbol{B}_{u, w}=\left[\begin{array}{c}
\mathbf{0} \\
\vdots \\
\mathbf{0} \\
\tilde{\boldsymbol{F}}
\end{array}\right] \text {, } \\
& \boldsymbol{C}_{u}=\left[\begin{array}{llll}
\boldsymbol{I}_{n_{u}} & \mathbf{0} & \cdots & \mathbf{0}
\end{array}\right], \boldsymbol{D}_{u, x}=\mathbf{0}, \boldsymbol{D}_{u, w}=\mathbf{0}
\end{aligned}
$$

die Parametrierung eines dynamischen Entkopplungsreglers für das ursprüngliche System, welcher in $\boldsymbol{G}_{\boldsymbol{y} \boldsymbol{w}}(\mathrm{s})$ auf Diagonalelemente der Form (B.4) führt. 
Beweis. Es werden die zeitlichen Ableitungen der einzelnen Ausgangsgrößen $y_{i}$ betrachtet, wobei aufgrund der in Lemma B.2 ermittelten Differenzenordnungen für das erweiterte System

$$
\begin{aligned}
y_{i}= & \tilde{\boldsymbol{c}}_{i}^{\top} \tilde{\boldsymbol{x}}=\boldsymbol{c}_{i}^{\top} \boldsymbol{x}, \\
y_{i}= & \tilde{\boldsymbol{c}}_{i}^{\top} \tilde{\boldsymbol{A}} \tilde{\boldsymbol{x}}, \\
& \vdots \\
\frac{\left(\rho+\delta_{i}-1\right)}{y_{i}}= & \tilde{\boldsymbol{c}}_{i}^{\top} \tilde{\boldsymbol{A}}^{\rho+\delta_{i}-1} \tilde{\boldsymbol{x}}, \\
{ }^{\left(\rho+\delta_{i}\right)} y_{i} & =\tilde{\boldsymbol{c}}_{i}^{\top} \tilde{\boldsymbol{A}}^{\rho+\delta_{i}} \tilde{\boldsymbol{x}}+\tilde{\boldsymbol{c}}_{i}^{\top} \tilde{\boldsymbol{A}}^{\rho+\delta_{i}-1} \tilde{\boldsymbol{B}} \boldsymbol{\zeta}_{\rho} \\
& =\tilde{\boldsymbol{c}}_{i}^{\top} \tilde{\boldsymbol{A}}^{\rho+\delta_{i}} \tilde{\boldsymbol{x}}+\boldsymbol{d}_{i}^{* \top} \boldsymbol{\zeta}_{\rho}
\end{aligned}
$$

gilt. Für den $i$-ten Übertragungskanal muss entsprechend (B.10) im Zeitbereich

$$
\stackrel{\left(\rho+\delta_{i}\right)}{y_{i}}+q_{i, \rho+\delta_{i}-1} \stackrel{\left(\rho+\delta_{i}-1\right)}{y_{i}}+\ldots+q_{i, 1} \dot{y}_{i}+q_{i, 0} y_{i}=z_{i, 0} w_{i}
$$

gelten. Setzt man in diese Beziehung die zeitlichen Ableitungen (B.16) ein, so ergibt sich für jeden Übertragungskanal eine vom Regler zu erfüllende Gleichung. Fasst man diese Gleichungen in Matrixschreibweise zusammen, so ergibt sich die Bestimmungsgleichung

$$
\tilde{\boldsymbol{M}} \tilde{\boldsymbol{x}}+\boldsymbol{D}^{*} \boldsymbol{\zeta}_{\rho}=\tilde{\boldsymbol{N}} \boldsymbol{w}
$$

wobei $\tilde{\boldsymbol{M}}$ und $\tilde{\boldsymbol{N}}$ in Satz B.2 gegeben sind. Da $\boldsymbol{D}^{*}$ nach Voraussetzung invertierbar ist, gilt $\tilde{\boldsymbol{K}}=\boldsymbol{D}^{*-1} \tilde{\boldsymbol{M}}$ und $\tilde{\boldsymbol{F}}=\boldsymbol{D}^{*-1} \tilde{\boldsymbol{N}}$.

Es verbleibt zu zeigen, dass die angegebene Parametrierung des dynamischen Reglers das gewünschte Übertragungsverhalten gewährleistet. Dazu wird zunächst die Dynamik des geschlossenen Regelkreises bestehend aus der erweiterten Strecke und dem durch $\tilde{\boldsymbol{K}}$ und $\tilde{\boldsymbol{F}}$ parametrierten Regler betrachtet. Sie ergibt sich zu

$$
\begin{aligned}
\dot{\tilde{\boldsymbol{x}}} & =(\tilde{\boldsymbol{A}}-\tilde{\boldsymbol{B}} \tilde{\boldsymbol{K}}) \tilde{\boldsymbol{x}}+\tilde{\boldsymbol{B}} \tilde{\boldsymbol{F}} \boldsymbol{w}, \\
\boldsymbol{y} & =\tilde{\boldsymbol{C}} \tilde{\boldsymbol{x}} .
\end{aligned}
$$


Dabei gilt für die Systemmatrizen

$$
\begin{aligned}
& \tilde{\boldsymbol{A}}-\tilde{\boldsymbol{B}} \tilde{\boldsymbol{K}}=\left[\begin{array}{ccccc}
\boldsymbol{A} & \boldsymbol{B} & \mathbf{0} & \cdots & \mathbf{0} \\
\mathbf{0} & \mathbf{0} & \boldsymbol{I}_{n_{u}} & \ddots & \vdots \\
\vdots & & \ddots & \ddots & \mathbf{0} \\
\mathbf{0} & \cdots & \cdots & \mathbf{0} & \boldsymbol{I}_{n_{u}} \\
\mathbf{0} & \cdots & \cdots & \cdots & \mathbf{0}
\end{array}\right]+\ldots \\
& -\left[\begin{array}{c}
\mathbf{0} \\
\vdots \\
\vdots \\
\mathbf{0} \\
\boldsymbol{I}_{n_{u}}
\end{array}\right]\left[\begin{array}{lllll}
\tilde{\boldsymbol{K}}_{x} & \tilde{\boldsymbol{K}}_{u} & \tilde{\boldsymbol{K}}_{\zeta_{1}} & \cdots & \tilde{\boldsymbol{K}}_{\zeta_{\rho-1}}
\end{array}\right] \\
& =\left[\begin{array}{ccccc}
\boldsymbol{A} & \boldsymbol{B} & \mathbf{0} & \cdots & \mathbf{0} \\
\mathbf{0} & \mathbf{0} & \boldsymbol{I}_{n_{u}} & \ddots & \vdots \\
\vdots & & \ddots & \ddots & \mathbf{0} \\
\mathbf{0} & \cdots & \cdots & \mathbf{0} & \boldsymbol{I}_{n_{u}} \\
-\tilde{\boldsymbol{K}}_{x} & -\tilde{\boldsymbol{K}}_{u} & -\tilde{\boldsymbol{K}}_{\zeta_{1}} & \cdots & -\tilde{\boldsymbol{K}}_{\zeta_{\rho-1}}
\end{array}\right] \\
& \tilde{\boldsymbol{B}} \tilde{\boldsymbol{F}}=\left[\begin{array}{c}
\mathbf{0} \\
\vdots \\
\mathbf{0} \\
\boldsymbol{I}_{n_{u}}
\end{array}\right] \tilde{\boldsymbol{F}}=\left[\begin{array}{c}
\mathbf{0} \\
\vdots \\
\mathbf{0} \\
\tilde{\boldsymbol{F}}
\end{array}\right], \quad \tilde{\boldsymbol{C}}=\left[\begin{array}{lllll}
\boldsymbol{C} & \boldsymbol{D} & \mathbf{0} & \cdots & \mathbf{0}
\end{array}\right] \text {. }
\end{aligned}
$$

Demgegenüber wird das Übertragungsverhalten zwischen den Führungsund den Ausgangsgrößen für das mittels einer dynamischen $\mathrm{Zu}$ standsrückführung geregelte ursprüngliche System betrachtet. Durch Einsetzen von (B.3) in (B.1) ergibt sich

$$
\begin{aligned}
{\left[\begin{array}{c}
\dot{\boldsymbol{x}} \\
\dot{\boldsymbol{\psi}}
\end{array}\right] } & =\left[\begin{array}{cc}
\boldsymbol{A}+\boldsymbol{B} \boldsymbol{D}_{u, x} & \boldsymbol{B} \boldsymbol{C}_{u} \\
\boldsymbol{B}_{u, x} & \boldsymbol{A}_{u}
\end{array}\right]\left[\begin{array}{l}
\boldsymbol{x} \\
\boldsymbol{\psi}
\end{array}\right]+\left[\begin{array}{c}
\boldsymbol{B} \boldsymbol{D}_{u, w} \\
\boldsymbol{B}_{u, w}
\end{array}\right] \boldsymbol{w}, \\
\boldsymbol{y} & =\left[\begin{array}{ll}
\boldsymbol{C}+\boldsymbol{D} \boldsymbol{D}_{u, x} & \boldsymbol{D} \boldsymbol{C}_{u}
\end{array}\right]\left[\begin{array}{l}
\boldsymbol{x} \\
\boldsymbol{\psi}
\end{array}\right]+\boldsymbol{D} \boldsymbol{D}_{u, x} \boldsymbol{w} .
\end{aligned}
$$

Setzt man in dieses System die Parametrierung des dynamischen Reglers 
aus Satz B.2 ein, so folgt aus dem Vergleich mit (B.17)

$$
\begin{aligned}
& {\left[\begin{array}{cc}
\boldsymbol{A}+\boldsymbol{B} \boldsymbol{D}_{u, x} & \boldsymbol{B} \boldsymbol{C}_{u} \\
\boldsymbol{B}_{u, x} & \boldsymbol{A}_{u}
\end{array}\right]=\tilde{\boldsymbol{A}}-\tilde{\boldsymbol{B}} \tilde{\boldsymbol{K}}} \\
& {\left[\begin{array}{c}
\boldsymbol{B} \boldsymbol{D}_{u, w} \\
\boldsymbol{B}_{u, w}
\end{array}\right]=\tilde{\boldsymbol{B}} \tilde{\boldsymbol{F}}} \\
& {\left[\boldsymbol{C}+\boldsymbol{D} \boldsymbol{D}_{u, x} \quad \boldsymbol{D} \boldsymbol{C}_{u}\right]=\tilde{\boldsymbol{C}},} \\
& D D_{u, x}=\mathbf{0} \text {. }
\end{aligned}
$$

Folglich realisieren die dynamisch erweiterte Strecke mit statischem Regler und die ursprüngliche Strecke mit dynamischem Regler das gleiche Übertragungsverhalten. 


\section{Parameter der Beispielsysteme}

C.1 Beispiele zum Entwurf von FIOs für nominale Systeme

C.1.1 Quadratisches System, CE 150 Modellhubschrauber

FIO-Parametrierung

$$
\boldsymbol{L}=\left[\begin{array}{rr}
9,1421 & -1,6875 \\
12,0792 & -12,1150 \\
0,0015 & -0,0000 \\
-0,0367 & 7,6792 \\
-0,0021 & 19,6500 \\
-0,0030 & -0,1396
\end{array}\right], \boldsymbol{V}=\left[\begin{array}{rr}
0,5751 & -0,0000 \\
0,0194 & 0,8927
\end{array}\right]
$$

\section{C.1.2 Erweiterte Diagonalelemente, CE 150} Modellhubschrauber

DFIO-Parametrierung

$$
\begin{array}{rlrl}
\boldsymbol{L}_{p} & =\left[\begin{array}{rr}
-0,0000 & -0,0000 \\
-0,0000 & -0,0000 \\
-0,0000 & -0,0000 \\
-0,0000 & -0,0000 \\
-0,0000 & -0,0000 \\
-0,0000 & -0,0000
\end{array}\right], & \boldsymbol{L}_{i}=\left[\begin{array}{rr}
76,0925 & -23,0837 \\
26,0608 & -111,3328 \\
0,0025 & 0,0000 \\
-0,1469 & 78,5650 \\
-0,5522 & 107,7370 \\
0,0196 & 0,9003
\end{array}\right], \\
\mathbf{\Phi} & =\left[\begin{array}{rr}
-17,1421 & 1,6875 \\
0,0476 & -15,1792
\end{array}\right], \quad \boldsymbol{\Gamma}=\left[\begin{array}{rr}
1,0000 & 0,0000 \\
0,0000 & 1,0000
\end{array}\right] \\
\boldsymbol{V}_{\boldsymbol{\zeta}} & =\left[\begin{array}{ll}
4,0256 & 0,0000 \\
0,1533 & 7,0385
\end{array}\right], & \boldsymbol{V}_{\boldsymbol{y}} & =\left[\begin{array}{ll}
0,0000 & 0,0000 \\
0,0000 & 0,0000
\end{array}\right]
\end{array}
$$


C.1.3 Statisch nicht isolierbares System, DHC-2 Beaver

DFIO 1. Ordnung

$$
\begin{aligned}
\boldsymbol{L}_{p} & =\left[\begin{array}{rr}
0,0000 & 1,0119 \\
0,0000 & -2,0732 \\
0,0000 & -7,0168 \\
0,0000 & 3,6789
\end{array}\right], & \boldsymbol{L}_{i} & =\left[\begin{array}{r}
15,3286 \\
-15,8367 \\
-13,0332 \\
-6,7948
\end{array}\right], \\
\boldsymbol{\Phi} & =\left[\begin{array}{rl}
-64,3722
\end{array}\right], & \boldsymbol{\Gamma} & =\left[\begin{array}{ll}
22,2767 & -1,3300
\end{array}\right], \\
\boldsymbol{V}_{\boldsymbol{\zeta}} & =\left[\begin{array}{r}
0,0365 \\
11,2045
\end{array}\right], & \boldsymbol{V}_{\boldsymbol{y}} & =\left[\begin{array}{ll}
0,0000 & -1,9115 \\
0,0000 & -0,2355
\end{array}\right]
\end{aligned}
$$

\section{C.1.4 Nicht minimalphasiges System, Verladebrücke}

DFIO 2. Ordnung

$$
\begin{aligned}
\boldsymbol{L}_{p} & =\left[\begin{array}{rr}
-36,6101 & 0,0000 \\
2987,9054 & -0,0000 \\
-2123,5550 & -0,0000 \\
2413,4270 & -0,0000
\end{array}\right], & \boldsymbol{L}_{i} & =\left[\begin{array}{rr}
0,0000 & -0,0000 \\
-0,0000 & 0,0000 \\
-0,0000 & -0,0000 \\
-0,0000 & 0,0000
\end{array}\right], \\
\boldsymbol{\Phi} & =\left[\begin{array}{rr}
-6,5000 & 3,2833 \\
-3,2163 & -0,0000
\end{array}\right], & \boldsymbol{\Gamma} & =\left[\begin{array}{rr}
-33,1910 & -0,1037 \\
-2,7401 & 0,0011
\end{array}\right], \\
\boldsymbol{V}_{\boldsymbol{\zeta}} & =\left[\begin{array}{rr}
61,4027 & -0,0000 \\
0,0000 & 0,0000
\end{array}\right], & \boldsymbol{V}_{\boldsymbol{y}} & =\left[\begin{array}{rr}
-0,9796 & 0,9796 \\
79,1035 & 0,0000
\end{array}\right]
\end{aligned}
$$

\section{C.1.5 Doppelt erweitertes System, DHC-2 Beaver}

FIO für doppelt erweitertes Gesamtsystem

$$
\tilde{\boldsymbol{L}}=\left[\begin{array}{rr}
0,9472 & 0,0512 \\
-0,4452 & -0,0102 \\
-1,0264 & 0,0206 \\
-0,5933 & 0,0617 \\
9,2045 & -0,0281 \\
52,7098 & 215,7669
\end{array}\right], \tilde{\boldsymbol{V}}=\left[\begin{array}{ll}
0,0025 & -0,0654 \\
0,5747 & -0,0060
\end{array}\right]
$$

\section{C.1.6 Nichtquadratisches System, Hydraulik}

Parameter des Beispielsystems: $A_{k}=50 \mathrm{~cm}^{2}, \quad m_{1}=2 \mathrm{~kg}, \quad m_{2}=4 \mathrm{~kg}$, $k_{1}=20 \mathrm{~N} / \mathrm{cm}, \quad k_{2}=100 \mathrm{~N} / \mathrm{cm}, \quad R=2 \mathrm{Ns} / \mathrm{cm}, \quad d=0,5 \mathrm{Ns} / \mathrm{cm}$, 
$K_{1}=3 \mathrm{~N} / \mathrm{cm}^{2} \mathrm{~V}, K_{2}=7500 \mathrm{~cm}^{5} / \mathrm{Ns}, T=1,25 \mathrm{~s}$.

Zustandsraummodell

$$
\begin{aligned}
& \boldsymbol{A}=\left[\begin{array}{ccccc}
-0,8 & 0 & 0 & 0 & 0 \\
0 & 0 & 1 & 0 & 0 \\
25 & -60 & -1,4167 & 50 & 0,25 \\
0 & 0 & 0 & 0 & 1 \\
0 & 25 & 0,125 & -25 & -0,625
\end{array}\right], \boldsymbol{B}=\left[\begin{array}{c}
-2,4 \\
0 \\
0 \\
0 \\
0
\end{array}\right] \\
& \boldsymbol{C}=\left[\begin{array}{lllll}
0 & 1 & 0 & 0 & 0 \\
0 & 0 & 0 & 1 & 0 \\
0 & 0 & 0 & 0 & 1
\end{array}\right], \boldsymbol{E}_{a}=\left[\begin{array}{cc}
-2,4 & 0 \\
0 & 0 \\
0 & 0,5 \\
0 & 0 \\
0 & -0,25
\end{array}\right]
\end{aligned}
$$

FIO-Parametrierung

$$
\begin{aligned}
\boldsymbol{L} & =\left[\begin{array}{rrr}
0,0571 & 0,0264 & 0,0000 \\
4,0333 & 1,6480 & -2,0000 \\
-57,3739 & 157,9868 & -2,9167 \\
0,0000 & 10,0000 & 1,0000 \\
25,1292 & -75,6315 & 2,1250
\end{array}\right], \\
\boldsymbol{V} & =\left[\begin{array}{rrr}
-0,1250 & 0,0000 & -0,0000 \\
1,5000 & -0,0000 & -12,0000
\end{array}\right]
\end{aligned}
$$

\section{C.1.7 Teilweise Fehlerisolation, Verladebrücke}

PFIO-Parametrierung

$$
\boldsymbol{L}_{\mathrm{pfi}}=\left[\begin{array}{rr}
-0,0000 & -51,3101 \\
-2,1160 & 3697,6130 \\
9,0000 & -58,8941 \\
5,9579 & 3684,3389
\end{array}\right], \quad \boldsymbol{V}_{\mathrm{pfi}}=\left[\begin{array}{rr}
-192,0000 & 158,0161 \\
-0,0000 & 15,2928
\end{array}\right]
$$




\section{C.2 Beispiele zum Entwurf robuster FIOs}

\section{C.2.1 Quadratisches System, CE 150 Modellhubschrauber}

FIO-Parametrierung mit optimierten Beobachtereigenwerten

$$
\boldsymbol{L}_{\mathrm{opt}, \mathrm{ew}}=\left[\begin{array}{rr}
1,6429 & -1,6875 \\
0,0544 & 3,0697 \\
-0,1122 & 0,0000 \\
0,3953 & -1,3191 \\
-2,6397 & 28,0482 \\
1,5184 & -12,2360
\end{array}\right], \boldsymbol{V}_{\mathrm{opt}, \mathrm{ew}}=\left[\begin{array}{rr}
0,0751 & 0,0000 \\
-0,1076 & 0,8669
\end{array}\right]
$$

DFIO-Parametrierung

$$
\begin{array}{rlrl}
\boldsymbol{L}_{p} & =\left[\begin{array}{rr}
-0,0000 & -0,0000 \\
-0,0000 & -0,0000 \\
-0,0000 & -0,0000 \\
-0,0000 & -0,0000 \\
-0,0000 & -0,0000 \\
-0,0000 & -0,0000
\end{array}\right], & \boldsymbol{L}_{i}=\left[\begin{array}{rr}
115,6176 & -29,0608 \\
108,5355 & -170,9342 \\
-0,0062 & 0,0000 \\
-0,2165 & 115,6554 \\
-0,5123 & 220,0266 \\
0,0116 & 0,5342
\end{array}\right], \\
\boldsymbol{\Phi} & =\left[\begin{array}{rr}
-19,5301 & 1,6875 \\
0,0443 & -17,7212
\end{array}\right], & \boldsymbol{\Gamma} & =\left[\begin{array}{rr}
1,0000 & 0,0000 \\
0,0000 & 1,0000
\end{array}\right], \\
\boldsymbol{V}_{\boldsymbol{\zeta}} & =\left[\begin{array}{rr}
7,1393 & 0,0000 \\
0,2719 & 12,4825
\end{array}\right], & \boldsymbol{V}_{\boldsymbol{y}} & =\left[\begin{array}{ll}
0,0000 & 0,0000 \\
0,0000 & 0,0000
\end{array}\right]
\end{array}
$$

\section{C.2.2 Quadratisches System, Verladebrücke}

FIO-Parametrierung zur exakten Fehlerisolation

$$
\boldsymbol{L}_{\text {exakt }}=\left[\begin{array}{rr}
0,0000 & -41,3101 \\
-2,1160 & 3114,5124 \\
7,0000 & -48,3101 \\
-2,0421 & 3102,5124
\end{array}\right], \quad \boldsymbol{V}_{\text {exakt }}=\left[\begin{array}{rr}
-115,2000 & 115,2000 \\
-0,0000 & 35,6831
\end{array}\right]
$$

FIO-Parametrierung mit relaxierten strukturellen Anforderungen

$$
\boldsymbol{L}_{\text {relax }}=\left[\begin{array}{rr}
-42,7368 & 1,5321 \\
2831,6814 & 271,7471 \\
-35,8422 & -5,2616 \\
2831,3338 & 260,7919
\end{array}\right], \quad \boldsymbol{V}_{\text {relax }}=\left[\begin{array}{rr}
-101,1161 & 90,6482 \\
0,3182 & 34,6557
\end{array}\right]
$$




\section{C.2.3 Nichtquadratisches System, Hydraulik}

FIO-Parametrierung

$$
\begin{aligned}
\boldsymbol{L}_{\mathrm{opt}} & =\left[\begin{array}{rrr}
0,0571 & 0,2861 & 0,0000 \\
4,0333 & -40,5352 & -2,0000 \\
-57,3739 & -146,5418 & -2,9167 \\
0,0000 & 31,3918 & 1,0000 \\
25,1292 & 84,4399 & 2,1250
\end{array}\right], \\
\boldsymbol{V}_{\mathrm{opt}} & =\left[\begin{array}{rrr}
-0,1250 & -0,1923 & -0,0000 \\
1,5000 & 0,0010 & -12,0000
\end{array}\right]
\end{aligned}
$$

FIO-Parametrierung (optimierte Eigenwerte)

$$
\begin{aligned}
\boldsymbol{L}_{\text {opt,ew }} & =\left[\begin{array}{rrr}
0,5242 & -1,1193 & -0,0000 \\
3,6394 & -12,9645 & -2,0000 \\
-49,6616 & -157,3011 & 0,0833 \\
-0,0000 & 44,6316 & 1,0000 \\
25,2674 & 90,0773 & 0,6250
\end{array}\right], \\
\boldsymbol{V}_{\text {opt }, \text { ew }} & =\left[\begin{array}{rrr}
-0,4200 & -2,7725 & -0,0000 \\
0,7500 & 0,0157 & -6,0000
\end{array}\right]
\end{aligned}
$$

\section{C.2.4 Nichtquadratisches System, Hydraulik (beschränkter Frequenzbereich)}

FIO-Parametrierung (Path-Following)

$$
\begin{aligned}
\boldsymbol{L}_{\text {opt,ff,pf }} & =\left[\begin{array}{rrr}
0,0571 & 0,2186 & 0,0000 \\
4,0333 & -46,2987 & -2,0000 \\
-57,3739 & -187,4794 & -2,9167 \\
-0,0000 & 36,7920 & 1,0000 \\
25,1292 & 105,5976 & 2,1250
\end{array}\right], \\
\boldsymbol{V}_{\text {opt,ff,pf }} & =\left[\begin{array}{ccr}
-0,1250 & -0,4913 & -0,0000 \\
1,5000 & -318,7807 & -12,0000
\end{array}\right]
\end{aligned}
$$


FIO-Parametrierung (rangbeschränkte LMI)

$$
\begin{aligned}
\boldsymbol{L}_{\text {opt,ff,rc }} & =\left[\begin{array}{rrr}
0,0571 & 125,4438 & 0,0000 \\
4,0333 & -808,0975 & -2,0000 \\
-57,3739 & -901,5167 & -2,9167 \\
-0,0000 & 43,8465 & 1,0000 \\
25,1292 & 87,4832 & 2,1250
\end{array}\right], \\
\boldsymbol{V}_{\text {opt,ff,rc }} & =\left[\begin{array}{ccr}
-0,1250 & 33,6022 & 0,0000 \\
1,5000 & -503,8981 & -12,0000
\end{array}\right]
\end{aligned}
$$

\section{C.2.5 Nichtquadratisches System, akademisches Beispiel (unsichere Parameter)}

Initiale FIO-Parametrierung

$$
\begin{aligned}
\boldsymbol{L}_{\text {init }} & =\left[\begin{array}{rrr}
6,0000 & 2,0000 & 10,0000 \\
1,1905 & 2,0000 & 4,1905 \\
0,2432 & 0,0000 & 3,2432
\end{array}\right], \\
\boldsymbol{V}_{\text {init }} & =\left[\begin{array}{rrr}
-0,0000 & 4,0000 & -0,0000 \\
-0,7500 & -0,0000 & 0,7500
\end{array}\right]
\end{aligned}
$$

Optimierte FIO-Parametrierung

$$
\begin{aligned}
\boldsymbol{L}_{\mathrm{opt}} & =\left[\begin{array}{rrr}
9,1810 & 2,0000 & 13,1810 \\
-1,3112 & 2,0000 & 1,6888 \\
-1,9332 & 0,0000 & 1,0668
\end{array}\right], \\
\boldsymbol{V}_{\mathrm{opt}} & =\left[\begin{array}{rrr}
9,2848 & 4,0000 & 9,2848 \\
-1,5582 & 0,0000 & -0,0582
\end{array}\right]
\end{aligned}
$$

\section{C.2.6 Partielle Fehlerisolation}

\section{Akademisches Beispiel}

Vergleich der FIO-Parametrierungen

$$
\begin{array}{rlr}
\boldsymbol{L}_{\mathrm{ffi}} & =\left[\begin{array}{rr}
1,2987 & -1,9351 \\
0,5195 & 2,5260
\end{array}\right], \quad \boldsymbol{V}_{\mathrm{ffi}}=\left[\begin{array}{rr}
-1,6623 & -4,8831 \\
-0,3506 & -0,4675
\end{array}\right], \\
\boldsymbol{L}_{\mathrm{pfi}, \mathrm{VMS}} & =\left[\begin{array}{rr}
2,5131 & -0,3158 \\
-0,6950 & 0,9067
\end{array}\right], \quad \boldsymbol{V}_{\mathrm{pfi}, \mathrm{VMS}}=\left[\begin{array}{rr}
1,7662 & -0,3118 \\
-0,3506 & -0,4675
\end{array}\right], \\
\boldsymbol{L}_{\mathrm{pfi}, \mathrm{pf}} & =\left[\begin{array}{rr}
2,1294 & -0,8275 \\
-0,3112 & 1,4184
\end{array}\right], \quad \boldsymbol{V}_{\mathrm{pfi}, \mathrm{pf}}=\left[\begin{array}{rr}
1,6923 & -0,4103 \\
-0,3506 & -0,4675
\end{array}\right]
\end{array}
$$




\section{Verladebrücke}

Vergleich der FIO-Parametrierungen

$$
\begin{aligned}
& \boldsymbol{L}_{\mathrm{ffi}}= {\left[\begin{array}{rr}
0,0000 & -51,3101 \\
-2,1160 & 3697,6130 \\
9,0000 & -60,3101 \\
5,9579 & 3677,6130
\end{array}\right], \quad \boldsymbol{V}_{\mathrm{ffi}}=\left[\begin{array}{rr}
-192,0000 & 192,0000 \\
-0,0000 & 15,2928
\end{array}\right], } \\
& \boldsymbol{L}_{\mathrm{pfi}, \mathrm{VMS}}=\left[\begin{array}{rr}
-0,0000 & -51,3101 \\
-2,1160 & 3697,6130 \\
9,0000 & -60,0311 \\
5,9579 & 3680,5394
\end{array}\right], \quad \boldsymbol{V}_{\mathrm{pfi}, \mathrm{VMS}}=\left[\begin{array}{rr}
-192,0000 & 139,2834 \\
0,0000 & 15,2928
\end{array}\right], \\
& \boldsymbol{L}_{\mathrm{pfi}, \mathrm{pf}}=\left[\begin{array}{rr}
0,0000 & -51,3101 \\
-2,1160 & 3697,6130 \\
9,0000 & -55,6398 \\
5,9579 & 3680,5261
\end{array}\right], \quad \boldsymbol{V}_{\text {pfi,pf }}=\left[\begin{array}{rr}
-192,0000 & 103,1742 \\
-0,0000 & 15,2928
\end{array}\right]
\end{aligned}
$$

\section{C.3 Beispiele zum Entwurf von FIFs}

\section{C.3.1 FIFs für nominale Systeme}

\section{Hydrauliksystem}

FIF voller Ordnung $\left(n_{\text {filt }}=9\right)$

$$
\begin{aligned}
& \boldsymbol{A}_{f}=\left[\begin{array}{rrrrrrrrr}
-3,0110 & 0,0068 & -0,0003 & 0,0003 & 0,0000 & -0,0000 & -0,0000 & 0,0001 & -0,0000 \\
2,9767 & -4,8606 & 0,0945 & 0,0019 & 0,0021 & 0,0000 & -0,0003 & -0,0001 & 0,0000 \\
-0,9110 & 0,7378 & -2,6168 & -0,0133 & -0,0168 & 0,0005 & 0,0002 & 0,0010 & -0,0002 \\
-0,2220 & 4,5945 & 10,9597 & -2,1195 & -0,1661 & 0,0094 & -0,0040 & 0,0065 & -0,0009 \\
-4,2176 & 1,6459 & -3,2347 & 0,5304 & -1,2968 & 0,0057 & -0,0002 & 0,0069 & -0,0012 \\
-0,0214 & -0,2497 & -0,4612 & 0,3073 & -0,7087 & -9,5510 & 5,1927 & 2,7593 & -0,6795 \\
-7,7562 & -0,6636 & -0,2712 & 0,1556 & -0,3554 & -3,6714 & -8,5746 & 0,9172 & -0,9812 \\
14,1962 & 1,0387 & -0,0479 & 0,0582 & -0,1447 & -1,4916 & 0,9257 & -9,7160 & -0,3669 \\
0,2008 & 0,1207 & 0,3186 & -0,1975 & 0,4626 & 4,6661 & 6,7871 & 3,5673 & -8,0215
\end{array}\right], \\
& \boldsymbol{B}_{f, u}=\left[\begin{array}{r}
0,0012 \\
-0,0160 \\
-0,3492 \\
-3,9881 \\
-2,2600 \\
0,0649 \\
0,0055 \\
0,0023 \\
-0,0032
\end{array}\right], B_{f, y}=\left[\begin{array}{rrr}
0,7005 & -0,7937 & 0,0418 \\
1,1039 & 0,9539 & 0,5125 \\
-0,2955 & -0,1102 & -0,1899 \\
-0,0290 & -0,9614 & 0,1664 \\
-0,0715 & -0,9183 & -0,1200 \\
0,0547 & 0,0241 & -0,0047 \\
-0,0033 & 0,0251 & 0,2415 \\
0,0675 & -0,0637 & -0,4533 \\
-0,0350 & 0,0081 & 0,0001
\end{array}\right] \text {, }
\end{aligned}
$$

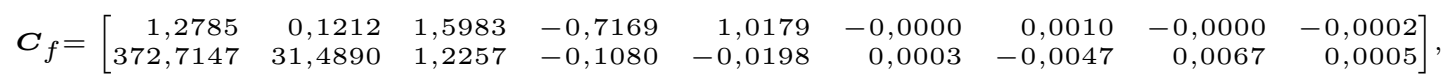

$$
\begin{aligned}
& \boldsymbol{D}_{f, u}=\left[\begin{array}{l}
-0,0000 \\
-0,0000
\end{array}\right], \quad \boldsymbol{D}_{f, y}=\left[\begin{array}{rrr}
-0,1250 & 0,1796 & -0,0000 \\
1,5000 & -2,9773 & -11,9998
\end{array}\right]
\end{aligned}
$$


FIF reduzierter Ordnung bei Erweiterung der Dynamik des zweiten Übertragungskanals um einen zusätzlichen Pol $\left(n_{\text {filt }}=n+1=6\right)$

$$
\begin{aligned}
& \boldsymbol{A}_{f}=\left[\begin{array}{rrrrrr}
-1,0360 & 2,4465 & -0,0006 & -0,0005 & -0,0001 & -0,0000 \\
-2,3795 & -5,9640 & 0,0014 & 0,0008 & -0,0006 & 0,0000 \\
0,0007 & 0,0123 & -0,3072 & -0,9960 & -0,5191 & 0,0012 \\
-0,0021 & 0,0429 & 0,9468 & -1,6734 & -2,6069 & 0,0054 \\
-0,0107 & 0,0072 & -0,2458 & 0,9439 & -4,0250 & 0,0180 \\
0,0001 & 0,0003 & 0,0002 & -0,0013 & -0,0051 & -9,8022
\end{array}\right] \text {, } \\
& \boldsymbol{B}_{f, u}=\left[\begin{array}{r}
0,0000 \\
0,0000 \\
-0,6329 \\
0,7120 \\
-0,2748 \\
0,0002
\end{array}\right], \boldsymbol{B}_{f, y}=\left[\begin{array}{rrr}
9,3551 & -9,2910 & -0,7457 \\
8,7966 & -9,1808 & 2,8450 \\
-0,0064 & 0,0029 & -0,0327 \\
0,1399 & 0,1379 & -0,2019 \\
0,3284 & 0,2530 & -0,2350 \\
0,0004 & 0,0010 & 0,0006
\end{array}\right], \\
& \boldsymbol{C}_{f}=\left[\begin{array}{rrrrrr}
0,0049 & 0,0256 & 0,6338 & 0,7657 & 0,5501 & -0,0012 \\
13,2059 & -13,0292 & 0,0033 & 0,0029 & 0,0005 & 0,0000
\end{array}\right] \text {, } \\
& \boldsymbol{D}_{f, u}=\left[\begin{array}{l}
0,0012 \\
0,0000
\end{array}\right], \quad \boldsymbol{D}_{f, y}=\left[\begin{array}{rrr}
-0,1291 & 0,0000 & -0,0026 \\
0,0154 & -0,0000 & -0,1230
\end{array}\right]
\end{aligned}
$$




\section{DHC-2 Beaver}

Einfach erweitert (invariante Nullstelle kompensiert, $n_{\text {filt }}=n+1$ )

$$
\begin{aligned}
& \boldsymbol{A}_{f}=\left[\begin{array}{rrrrr}
-2,4492 & 0,2851 & -0,3174 & 1,4161 & -1,0310 \\
-3,5134 & -6,1836 & 5,8482 & 2,3393 & 3,7181 \\
3,0461 & 19,4930 & -22,4315 & 6,5726 & 19,1594 \\
0,5906 & -7,0275 & 6,9848 & -7,7712 & -5,7853 \\
-8,7201 & -37,6504 & 40,0675 & -6,9181 & -35,3443
\end{array}\right], \\
& \boldsymbol{B}_{f, u}=\left[\begin{array}{rr}
-0,2855 & -0,0604 \\
-0,2028 & 0,6034 \\
-0,2568 & 0,6789 \\
-0,6268 & -0,0008 \\
0,1474 & 0,1830
\end{array}\right], \boldsymbol{B}_{f, y}=\left[\begin{array}{rr}
-0,4655 & 0,7316 \\
4,8408 & 0,3950 \\
-16,6497 & 1,2755 \\
5,9483 & -0,9273 \\
31,0698 & -1,5655
\end{array}\right] \text {, } \\
& \boldsymbol{C}_{f}=\left[\begin{array}{rrrrr}
6,8995 & 0,0721 & 0,3143 & -3,0816 & 0,8679 \\
0,8320 & -0,2053 & -1,5936 & 1,9559 & 6,8721
\end{array}\right], \\
& \boldsymbol{D}_{f, u}=\left[\begin{array}{rr}
0,0000 & -0,0000 \\
-0,0000 & 0,0000
\end{array}\right], \quad \boldsymbol{D}_{f, y}=\left[\begin{array}{rr}
0,0043 & -1,9088 \\
-0,0103 & -0,2351
\end{array}\right]
\end{aligned}
$$

Doppelt erweitert (invariante Nullstelle nicht kompensiert, $n_{\text {filt }}=n+2$ )

$$
\begin{aligned}
& \boldsymbol{A}_{f}=\left[\begin{array}{rrrrrr}
-1,3194 & 0,1587 & -3,4518 & 2,6202 & 1,2587 & -0,2607 \\
0,0687 & -1,4498 & -0,7070 & 1,5709 & -1,7974 & 0,1582 \\
0,3387 & 0,2073 & -5,8240 & 0,6551 & -0,4680 & -0,7877 \\
-0,0888 & -0,1519 & -2,2268 & -5,2720 & -1,5109 & 1,3581 \\
-0,4028 & 0,3007 & 4,2128 & -0,3380 & -2,8933 & 1,4750 \\
-0,1776 & -0,0114 & -1,6100 & 0,5981 & -0,4955 & -5,9587
\end{array}\right], \\
& \boldsymbol{B}_{f, u}=\left[\begin{array}{rr}
-0,2543 & -0,0042 \\
0,0098 & -0,2483 \\
0,0688 & 0,1334 \\
-0,0738 & 0,0468 \\
-0,1573 & 0,2846 \\
-0,4498 & -0,0415
\end{array}\right], \boldsymbol{B}_{f, y}=\left[\begin{array}{rr}
-0,1985 & -0,1300 \\
0,1717 & -0,0832 \\
-1,7387 & -0,4616 \\
-3,1277 & 0,4256 \\
1,1249 & 0,3232 \\
0,1214 & -0,2022
\end{array}\right], \\
& \boldsymbol{C}_{f}=\left[\begin{array}{rrrrrr}
4,5342 & -0,3946 & 6,6673 & -4,9262 & -2,5787 & 0,1288 \\
0,0228 & 4,7796 & 1,8587 & -3,4893 & 3,8362 & -0,3730
\end{array}\right] \\
& \boldsymbol{D}_{f, u}=\left[\begin{array}{rr}
0,0000 & 0,0001 \\
0,0000 & -0,0000
\end{array}\right], \boldsymbol{D}_{f, y}=\left[\begin{array}{ll}
0,0055 & -0,1357 \\
0,0055 & -0,0082
\end{array}\right]
\end{aligned}
$$




\section{C.3.2 Robustheit von FIFs bezüglich exogener Störungen}

\section{Hydrauliksystem}

FIF voller Ordnung $\left(n_{\text {filt }}=9\right)$

$$
\begin{aligned}
& \boldsymbol{A}_{f}=\left[\begin{array}{ll}
\boldsymbol{A}_{f, 1} & \boldsymbol{A}_{f, 2}
\end{array}\right], \quad \boldsymbol{A}_{f, 1}=\left[\begin{array}{rrrrr}
-3,0948 & -0,1272 & 0,0026 & 0,0111 & -0,0049 \\
-13,0008 & -19,5382 & -1,1962 & 0,0476 & -0,0190 \\
-3,9250 & -4,7568 & -3,4365 & -0,5692 & 0,2538 \\
-7,8152 & 7,7734 & -22,7836 & -20,1523 & 7,6550 \\
4,0431 & -0,7464 & 5,2612 & 10,0115 & -6,2509 \\
41,6326 & -55,9834 & 111,3573 & 148,3390 & -72,4655 \\
-34,1334 & 22,7867 & -45,0573 & -59,5774 & 29,2279 \\
124,7468 & -2,0876 & 2,2655 & 3,1889 & -1,5624 \\
44,4151 & -58,4695 & 117,7362 & 155,5448 & -76,3358
\end{array}\right], \\
& \boldsymbol{A}_{f, 2}=\left[\begin{array}{rrrr}
-0,0013 & -0,0052 & 0,0000 & 0,0001 \\
-0,0062 & -0,0037 & -0,0011 & 0,0001 \\
0,0659 & 0,1099 & 0,0146 & -0,0005 \\
2,1205 & 3,2052 & 0,4440 & -0,0100 \\
-1,1638 & -1,7106 & -0,2400 & 0,0097 \\
-18,8632 & -25,9919 & -3,6117 & 0,0919 \\
6,8681 & -36,3817 & 1,1788 & -1,7925 \\
-0,3677 & 2,5393 & -44,3628 & 0,3391 \\
-17,8967 & 88,6544 & 12,3782 & -27,2275
\end{array}\right] \\
& B_{f, u}=\left[\begin{array}{r}
-0,0012 \\
0,1034 \\
-1,3823 \\
4,6123 \\
9,5129 \\
-9,2297 \\
0,1285 \\
0,0013 \\
-0,0369
\end{array}\right], \quad B_{f, y}=\left[\begin{array}{rrr}
4,0478 & -4,2565 & 0,3839 \\
-3,1226 & -16,3481 & -1,2579 \\
-0,9664 & -4,0523 & -0,6131 \\
4,3582 & -0,9244 & -0,6190 \\
-2,3148 & 2,1079 & 0,0320 \\
-37,6741 & -0,0333 & 0,0078 \\
14,7784 & 0,0589 & 2,8655 \\
1,7621 & -0,0009 & -20,4917 \\
-39,5273 & 0,5477 & 0,0063
\end{array}\right]
\end{aligned}
$$

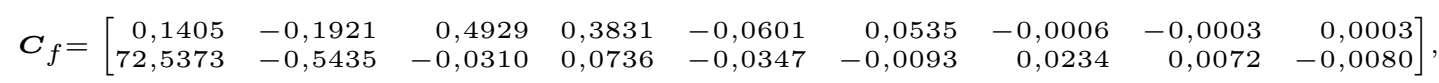

$$
\begin{aligned}
& \boldsymbol{D}_{f, u}=\left[\begin{array}{l}
-0,0000 \\
-0,0000
\end{array}\right], \quad \boldsymbol{D}_{f, y}=\left[\begin{array}{rrr}
-0,1246 & -0,0041 & -0,0001 \\
1,4878 & -0,0023 & -11,9991
\end{array}\right]
\end{aligned}
$$

FIF reduzierter Ordnung $\left(n_{\text {filt }}=n=5\right)$ mit höherer Gewichtung der 
Störungen

$$
\begin{aligned}
& \boldsymbol{A}_{f}=\left[\begin{array}{rrrrr}
-2,9984 & -0,0001 & 0,0093 & -0,0115 & 0,0097 \\
-0,0492 & -0,3785 & -2,3316 & 0,8363 & -0,3469 \\
-0,4748 & 0,7231 & -6,9544 & 5,8060 & -2,3032 \\
0,0960 & 0,6234 & 1,3443 & -4,2260 & 3,0463 \\
-0,0006 & 0,0534 & -1,1495 & 1,0341 & -4,3286
\end{array}\right], \\
& \boldsymbol{B}_{f, u}=\left[\begin{array}{r}
-0,0005 \\
0,5944 \\
-0,3810 \\
-0,4571 \\
-0,1013
\end{array}\right], \boldsymbol{B}_{f, y}=\left[\begin{array}{rrr}
-12,6720 & 12,8697 & -1,2210 \\
-0,2049 & 0,0025 & -0,0481 \\
-1,6008 & 0,4316 & -0,0831 \\
0,5839 & 0,1564 & -0,1127 \\
-0,1916 & -0,1797 & 0,0682
\end{array}\right] \text {, } \\
& \boldsymbol{C}_{f}=\left[\begin{array}{rrrrr}
-0,0536 & -0,6306 & -1,7030 & 0,7653 & -0,2881 \\
-18,1024 & 0,0002 & 0,0278 & -0,0367 & 0,0300
\end{array}\right] \text {, } \\
& \boldsymbol{D}_{f, u}=\left[\begin{array}{r}
-0,0002 \\
0,0001
\end{array}\right], \quad \boldsymbol{D}_{f, y}=\left[\begin{array}{rrr}
-0,2663 & -0,0100 & -0,0508 \\
1,1734 & 0,0153 & -9,3174
\end{array}\right]
\end{aligned}
$$

\section{C.3.3 Robustheit von FIFs bezüglich unsicherer Parameter}

\section{Akademisches Beispiel}

FIF voller Ordnung $\left(n_{\text {filt }}=5\right)$

$$
\begin{aligned}
& \boldsymbol{A}_{f}=\left[\begin{array}{rrrrr}
-2,2267 & 6,8429 & -2,2755 & 1,6256 & -0,0003 \\
-3,0393 & -16,1957 & 3,5274 & -2,6863 & 0,0022 \\
-1,4648 & -1,7787 & -10,2312 & 11,7749 & 0,0003 \\
1,1460 & -3,0646 & 5,0945 & -16,9580 & 0,0004 \\
0,0002 & 0,0014 & 0,0031 & -0,0030 & -18,6386
\end{array}\right], \\
& \boldsymbol{B}_{f, u}=\left[\begin{array}{r}
1,3640 \\
-0,3565 \\
1,3755 \\
0,1978 \\
-0,0001
\end{array}\right], \boldsymbol{B}_{f, y}=\left[\begin{array}{rrr}
-1,2109 & 3,0132 & 2,3868 \\
5,0352 & -1,3549 & 5,0076 \\
1,0707 & 2,1343 & -0,0884 \\
0,3769 & -1,7031 & 0,2137 \\
-0,0004 & -0,0003 & -0,0001
\end{array}\right] \text {, } \\
& \boldsymbol{C}_{f}=\left[\begin{array}{rrrrr}
-4,2211 & 5,9474 & -2,0930 & 1,4651 & -0,0003 \\
0,5344 & 4,1256 & 1,7945 & -0,9904 & -0,0004
\end{array}\right] \text {, } \\
& \boldsymbol{D}_{f, u}=\left[\begin{array}{r}
0,0138 \\
-0,0023
\end{array}\right], \quad \boldsymbol{D}_{f, y}=\left[\begin{array}{rrr}
0,2255 & 4,6723 & 0,1579 \\
-1,2700 & 0,0723 & 0,2543
\end{array}\right]
\end{aligned}
$$




\section{Veröffentlichungen und studentische Arbeiten}

Im Rahmen der Tätigkeit als wissenschaftlicher Mitarbeiter am Fachgebiet Regelungstheorie und Robotik sind die Publikationen [83, 96, 131, 132, 224-242, 250] entstanden. Im unmittelbaren Zusammenhang mit der Arbeit stehen [83, 224-232, 234-241, 250], ebenso wie die in Tabelle D.1 aufgeführten studentischen Arbeiten.

Tabelle D.1: Übersicht studentischer Arbeiten

\begin{tabular}{c|l}
\multicolumn{1}{c|}{ Art } & Titel \\
\hline \hline Studienarbeit & LMI-basierter Entwurf robuster Zustandsbeobachter \\
\hline Studienarbeit & $\begin{array}{l}\text { Robuste Zustandsschätzung mittels dynamisch erweiter- } \\
\text { ter Beobachter }\end{array}$ \\
\hline Studienarbeit & $\begin{array}{l}\text { LMI-basierter Entwurf robuster Fehlerisolationsbeobach- } \\
\text { ter für unsichere lineare Systeme }\end{array}$ \\
\hline Bachelorarbeit & $\begin{array}{l}\text { Beobachterbasierte Fehlerisolation in nicht-quadra- } \\
\text { tischen Systemen }\end{array}$ \\
\hline Studienarbeit & Entkopplungsregelungen für überaktuierte Systeme \\
\hline Projektseminar & $\begin{array}{l}\text { Beobachterbasierte Fehlerisolation am Beispiel einer elek- } \\
\text { tronischen Drosselklappe }\end{array}$ \\
\hline Studienarbeit & Robuste Fehlerisolation mithilfe von Beobachterbänken \\
\hline Bachelorarbeit & $\begin{array}{l}\text { Beobachterbasierte Fehlerisolation nicht-minimalpha- } \\
\text { siger Systeme }\end{array}$ \\
\hline Studienarbeit & Fehlerisolation mittels allgemeiner dynamischer Filter \\
\hline Projektseminar & $\begin{array}{l}\text { Anwendung evolutionärer Algorithmen für die Fehleriso- } \\
\text { lation }\end{array}$ \\
\hline Masterarbeit & $\begin{array}{l}\text { Entwurf robuster Beobachter für die teilweise Fehleriso- } \\
\text { lation }\end{array}$ \\
\hline Projektseminar & $\begin{array}{l}\text { Sensor- und Aktorfehlerlokalisierung am Beispiel einer } \\
\text { Verladebrücke }\end{array}$
\end{tabular}




\section{Literaturverzeichnis}

[1] Adamy, J. : Nichtlineare Regelungen. Springer, 2009

[2] Adamy, J. ; Kempf, R. : Regularity and chaos in recurrent fuzzy systems. In: Fuzzy Sets and Systems 140 (2003), Nr. 2, S. 259-284

[3] AdAmy, J. ; Schwung, A. : Qualitative modeling of dynamical systems employing continuous-time recurrent fuzzy systems. In: Fuzzy Sets and Systems 161 (2010), Nr. 23, S. 3026-3043

[4] Alwi, H. ; Edwards, C. ; Tan, C. P.: Fault Detection and FaultTolerant Control Using Sliding Modes. Springer, 2011

[5] Alwi, H. ; Edwards, C. ; Marcos, A. : Fault reconstruction using a LPV sliding mode observer for a class of LPV systems. In: Journal of the Franklin Institute 349 (2012), Nr. 2, S. 510-530. - Advances in Guidance and Control of Aerospace Vehicles using Sliding Mode Control and Observation Techniques

[6] Alwi, H. ; Edwards, C. ; Tan, C. P.: Sliding mode estimation schemes for incipient sensor faults. In: Automatica 45 (2009), Nr. 7 , S. $1679-1685$

[7] Amato, F. : Robust Control of Linear Systems Subject to Uncertain Time-Varying Parameters. Springer, 2006

[8] Apkarian, P. : Tuning Controllers Againt Multiple Design Requirements. In: Proceedings of the American Control Conference. Washington, 2013, S. 3894-3899

[9] Apkarian, P. ; Noll, D. : Nonsmooth $\mathcal{H}_{\infty}$ synthesis. In: IEEE Transactions on Automatic Control 51 (2006), Nr. 1, S. 71-86

[10] Bahrampour, S. ; Moshiri, B. ; Salahshoor, K. : Weighted and constrained possibilistic C-means clustering for online fault detection and isolation. In: Applied Intelligence 35 (2011), Nr. 2, S. 269-284 
[11] Basseville, M. ; Nikiforov, I. V.: Detection of Abrupt Changes: Theory and Application. Prentice-Hall, 1993

[12] Beale, S. ; Shafai, B. : Robust control system design with a proportional integral observer. In: International Journal of Control 50 (1989), Nr. 1, S. 97-111

[13] BeARD, R. V.: Failure Accomodation in Linear Systems Through Self-Reorganization, Massachusetts Institute of Technology, Diss., 1971

[14] Bernstein, D. S.: Matrix Mathematics. Princeton University Press, 2005

[15] Bland, R. G. ; Goldfarb, D. ; Todd, M. J.: The Ellipsoid Method: A Survey. In: Operations Research 29 (1981), Nr. 6, S. 1039-1091

[16] Blanke, M. ; Kinnaert, M. ; Lunze, J. ; Staroswiecki, M. : Diagnosis and Fault-Tolerant Control. Springer, 2006

[17] Boem, F. ; Ferrari, R. M. G. ; Parisini, T. ; Polycarpou, M. M.: Distributed fault diagnosis for continuous-time nonlinear systems: The input-output case. In: Annual Reviews in Control 37 (2013), Nr. 1, S. 163-169

[18] Bokor, J. ; BAlAs, G. : Detection filter design for LPV systems a geometric approach. In: Automatica 40 (2004), Nr. 3, S. 511-518

[19] Boyd, S. ; Vandenberghe, L. : Convex Optimization. Cambridge University Press, 2004

[20] Boyd, S. ; Ghaoui, L. E. ; Feron, E. ; Balakrishnan, V. : Linear Matrix Inequalities in System and Control Theory. SIAM, 1994

[21] Bronstein, I. N. ; Semendjajew, K. A. ; Musiol, G. ; Mühlig, H. : Taschenbuch der Mathematik. 6. Auflage. Harri Deutsch, 2005

[22] Bureau D'Enquêtes et D'AnAlyses pour la sÉcurité de L'AVION CIVILE: Flugunfalluntersuchung des Airbus A330-203, Flug AF 447, Zusammenfassung. Online verfügbar, Zugriff am 10.06.2013, http://www . bea.aero/fr/enquetes/vol.af .447/ note05juillet2012.de.pdf, 2012 
[23] Casavola, A. ; Gagliardi, G. : Fault Detection and Isolation of Electrical Induction Motors via LPV Fault Observers. In: Proceedings of the IFAC SAFEPROCESS Symposium. Mexico City, 2012, S. $800-805$

[24] Casavola, A. ; Garone, E. : Fault-tolerant adaptive control allocation schemes for overactuated systems. In: International Journal of Robust and Nonlinear Control 20 (2010), Nr. 17, S. 1958-1980

[25] Casavola, A. ; Famularo, D. ; Franzè, G. : Robust fault detection of uncertain linear systems via quasi-LMIs. In: Automatica 44 (2008), Nr. 1, S. 289-295

[26] Castaneda, E. ; Ruiz-Leon, J. : On the decoupling problem of linear multivariable systems by static state feedback. In: Proceedings of the IEEE Conference on Decision and Control. Maui, 2012, S. $3227-3232$

[27] Chang, J.-L. : Applying Discrete-Time Proportional Integral Observers for State and Disturbance Estimations. In: IEEE Transactions on Automatic Control 51 (2006), Nr. 5, S. 814-818

[28] Chen, B. ; Nagarajaiah, S. : Linear-Matrix-Inequality-Based Robust Fault Detection and Isolation Using the Eigenstructure Assignment Method. In: Journal of Guidance, Control, and Dynamics 30 (2007), Nr. 6, S. 1831-1835

[29] Chen, J. : Robust Residual Generation for Model-based Fault Diagnosis of Dynamic Systems, University of York, Diss., 1995

[30] Chen, J. ; Patton, R. J. ; Zhang, H. : Design of unknown input observers and robust fault detection filters. In: International Journal of Control 63 (1996), Nr. 1, S. 85-105

[31] Chen, J. ; Patton, R. : Optimal filtering and robust fault diagnosis of stochastic systems with unknown disturbances. In: IEE Proceedings - Control Theory and Applications 143 (1996), Nr. 1, S. $31-36$

[32] Chen, J. ; Patton, R. J.: Robust Model-Based Fault Diagnosis for Dynamic Systems. Kluwer Academic Publishers, 1999 
[33] Chen, J. ; Zhang, H. : Robust detection of faulty actuators via unknown input observers. In: International Journal of Systems Science 22 (1991), Nr. 10, S. 1829-1839

[34] Chen, J. ; CaO, Y.-Y. : A stable fault detection observer design in finite frequency domain. In: International Journal of Control 86 (2013), Nr. 2, S. 290-298

[35] Chen, J. : Formulating and Solving Robust Fault Diagnosis Problems Based on a $\mathcal{H}_{\infty}$ Setting. In: Proceedings of the IFAC World Congress. Seoul, 2008, S. 7259-7264

[36] Chen, W. ; Saif, M. : Fault Detection and Isolation Based on Novel Unknown Input Observer Design. In: Proceedings of the American Control Conference. Minneapolis, 2006, S. 5129-5134

[37] Chen, W. ; SAIf, M. : A sliding mode observer-based strategy for fault detection, isolation, and estimation in a class of Lipschitz nonlinear systems. In: International Journal of Systems Science 38 (2007), Nr. 12, S. 943-955

[38] Chilali, M. ; Gahinet, P. ; Apkarian, P. : Robust Pole Placement in LMI Regions. In: IEEE Transactions on Automatic Control 44 (1999), Nr. 12, S. 2257-2270

[39] Chow, E. ; Willsky, A. : Analytical redundancy and the design of robust failure detection systems. In: IEEE Transactions on Automatic Control 29 (1984), Nr. 7, S. 603-614

[40] Chowdhury, F. N. ; Chen, W. : A Modified Approach to Observerbased Fault Detection. In: Proceedings of the IEEE Multi-Conference on Systems and Control. Singapur, 2007, S. 539-543

[41] Chowdhury, F. N. ; Chen, W. : Fault Detection in Dynamic Systems: A New Approach via Total Measurable Fault Information. In: Proceedings of the IEEE Multi-Conference on Systems and Control. Singapur, 2007, S. 946-951

[42] Chung, W. H. ; Speyer, J. L.: A game theoretic fault detection filter. In: IEEE Transactions on Automatic Control 43 (1998), Nr. 2, S. $143-161$ 
[43] Clark, R. N.: A Simplified Instrument Failure Detection Scheme. In: IEEE Transactions on Aerospace and Electronic Systems 14 (1978), Nr. 4, S. 558-563

[44] Combastel, C. ; RakA, S.-A. : A Set-Membership Fault Detection Test with Guaranteed Robustness to Parametric Uncertainties in Continuous Time Linear Dynamical Systems. In: Proceedings of the IFAC SAFEPROCESS Symposium. Barcelona, 2009, S. 1192-1197

[45] Commault, C. ; Dion, J. M.: Transfer Matrix Approach to the Triangular Block Decoupling Problem. In: Automatica 19 (1983), Nr. 5, S. 533-542

[46] Cremer, M. : Festlegen der Pole und Nullstellen bei der Synthese linearer entkoppelter Mehrgrößenregelkreise, Teil 1. In: Regelungstechnik und Prozeß-Datenverarbeitung 21 (1973), Nr. 5, S. 144-150

[47] Cremer, M. : Festlegen der Pole und Nullstellen bei der Synthese linearer entkoppelter Mehrgrößenregelkreise, Teil 2. In: Regelungstechnik und Prozeß-Datenverarbeitung 21 (1973), Nr. 6, S. 195-199

[48] Cui, Y. ; Huang, X.-H. ; Wang, M. : Multi-objective Robust Fault Detection Filter Design in a Finite Frequency Range. In: Proceedings of the International Symposium on Neural Networks: Advances in Neural Networks. Wuhan, 2009, S. 733-743

[49] Dai, X. ; Gao, Z. ; Breikin, T. ; Wang, H. : Zero Assignment for Robust $\mathcal{H}_{2} / \mathcal{H}_{\infty}$ Fault Detection Filter Design. In: IEEE Transactions on Signal Processing 57 (2009), Nr. 4, S. 1363-1372

[50] Darouach, M. ; Zasadzinski, M. ; Xu, S. J.: Full-Order Observers for Linear Systems with Unknown Inputs. In: IEEE Transactions on Automatic Control 39 (1994), Nr. 3, S. 606-609

[51] Davoodi, M. R. ; Talebi, H. A. ; Momeni, H. : A Novel Simultaneous Fault Detection and Control Approach Based on Dynamic Observer. In: Proceedings of the IFAC World Congress. Mailand, 2011, S. 12036-12041

[52] De Schutter, B. : Minimal state-space realization in linear system theory: An overview. In: Journal of Computational and Applied Mathematics 121 (2000), Nr. 1-2, S. 331-354 
[53] Deng, H. ; Li, H.-X. : Functional Observers for Linear Systems with Unknown Inputs. In: Asian Journal of Control 6 (2004), Nr. 4, S. 462-468

[54] Descusse, J. ; Lafay, J. F. ; Malabre, M. : Solution to Morgan's Problem. In: IEEE Transactions on Automatic Control 33 (1988), Nr. 8, S. $732-739$

[55] Ding, S. X.: Data-Driven Design of Model-based Fault Diagnosis Systems. In: Proceedings of the IFAC Somposium on Advanced Control of Chemical Processes. Singapur, 2012, S. 840-847

[56] Ding, S. X. ; Ding, E. L. ; Jeinsch, T. : An approach to analysis and design of observer and parity relation based FDI systems. In: Proceedings of the IFAC World Congress. Peking, 1994, S. 37-42

[57] Ding, S. X. ; Zhang, P. ; Frank, P. M. ; Ding, E. L.: Threshold calculation using LMI-technique and its integration in the design of fault detection systems. In: Proceedings of the IEEE Conference on Decision and Control. Maui, 2003, S. 469-474

[58] Ding, S. X.: Model-based Fault Diagnosis Techniques. Springer, 2008

[59] Douglas, R. K. ; Speyer, J. L.: Robust Fault Detection Filter Design. In: Proceedings of the American Control Conference. Seattle, 1995, S. 91-96

[60] Duan, Z. ; Huan, L. ; YaO, Y. ; Jiang, Z.-P. : On the effects of redundant control inputs. In: Automatica 48 (2012), Nr. 9, S. $2168-2174$

[61] Dunia, R. ; Qin, S. J. ; Edgar, T. F. ; McAvoy, T. J.: Identification of faulty sensors using principal component analysis. In: American Institute of Chemical Engineers Journal 42 (1996), Nr. 10, S. $2797-2812$

[62] Ebihara, Y. ; Hagiwara, T. : On $\mathcal{H}_{\infty}$ model reduction using LMIs. In: IEEE Transactions on Automatic Control 49 (2004), Nr. 7, S. $1187-1191$

[63] Edelmayer, A. ; Bokor, J. ; Szigeti, F. ; Keviczky, L. : Robust detection filter design in the presence of time-varying system perturbations. In: Automatica 33 (1997), Nr. 3, S. 471-475 
[64] Edwards, C. ; Alwi, H. ; Tan, C. P.: Sliding Mode Methods for Fault Detection and Fault Tolerant Control with Application to Aerospace Systems. In: International Journal of Applied Mathematics and Computer Science 22 (2012), Nr. 1, S. 109-124

[65] Edwards, C. ; Spurgeon, S. K. ; Patton, R. J.: Sliding mode observers for fault detection and isolation. In: Automatica 36 (2000), Nr. 4, S. 541-553

[66] Emami-Naeini, A. ; Akhter, M. M. ; Rock, S. M.: Effect of Model Uncertainty on Failure Detection: The Threshold Selector. In: IEEE Transactions on Automatic Control 33 (1988), Nr. 12, S. 1106-1115

[67] Falb, P. L. ; Wolovich, W. A.: Decoupling in the Design and Synthesis of Multivariable Control Systems. In: IEEE Transactions on Automatic Control 12 (1967), Nr. 6, S. 651-659

[68] Fazel, M. : Matrix Rank Minimization with Applications, Stanford University, Diss., 2002

[69] Fernando, T. ; MacDougall, S. ; Sreeram, V. ; Trinh, H. : Existence conditions for unknown input functional observers. In: International Journal of Control 86 (2013), Nr. 1, S. 22-28

[70] Finsler, P. : Über das Vorkommen definiter und semi-definiter Formen in Scharen quadratischer Formen. In: Comentarii Mathematici Helvetici 9 (1936), Nr. 1, S. 188-192

[71] Flemming, A. : Zeitkontinuierliche rekurrente Fuzzy-Systeme, Technische Universität Darmstadt, Diss., 2009

[72] Floquet, T. ;Barbot, J. P. ; Perruquetti, W. ; DJemai, M. : On the robust fault detection via a sliding mode disturbance observer. In: International Journal of Control 77 (2004), Nr. 7, S. 622-629

[73] Forsgren, A. ; Gill, P. E. ; Wright, M. H.: Interior Methods for Nonlinear Optimization. In: SIAM Review 44 (2002), Nr. 4, S. $525-597$

[74] Frank, P. M.: Advanced fault detection and isolation schemes using nonlinear and robust observers. In: Proceedings of the IFAC World Congress. München, 1987, S. 63-68 
[75] Frank, P. M. ; Ding, X. : Frequency domain approach to optimally robust residual generation and evaluation for model-based fault diagnosis. In: Automatica 30 (1994), Nr. 5, S. 789-804

[76] Frank, P. M. ; Seliger, R. : Fault detection and isolation in automatic processes. In: Leondes, C. (Hrsg.): Control and Dynamic Systems. Academic Press, 1991, S. 241-287

[77] Frank, P. M. ; Wünnenberg, J. : Robust fault diagnosis using unknown input schemes. In: Patton, R. J. (Hrsg.) ; Frank, P. M. (Hrsg.) ; Clark, R. N. (Hrsg.): Fault Diagnosis in Dynamic Systems: Theory and Application. Prentice-Hall, 1989, S. 47-98

[78] Frank, P. M.: Fault Diagnosis in Dynamic Systems Using Analytical and Knowledge-based Redundancy - A Survey and Some New Results. In: Automatica 26 (1990), Nr. 3, S. 459-474

[79] FRisk, E. ; Nielsen, L. : Robust residual generation for diagnosis including a reference model for residual behavior. In: Automatica 42 (2006), Nr. 3, S. 437-445

[80] Furuta, K. ; Kamiyama, S. : State feedback and inverse system. In: International Journal of Control 25 (1977), Nr. 2, S. 229-241

[81] Gao, Z. ; Ding, S. X.: State and Disturbance Estimator for TimeDelay Systems With Application to Fault Estimation and Signal Compensation. In: IEEE Transactions on Signal Processing 55 (2007), Nr. 12, S. 5541-5551

[82] George, J. : Robust fault detection and isolation in stochastic systems. In: International Journal of Control 85 (2012), Nr. 7, S. 779-799

[83] Gering, S. ; Grieser, J. ; Wahrburg, A. : Using LMIs to Optimize Robustness of Observer-Based State-Feedback for a Synchrotron. In: International Journal of Robust and Nonlinear Control (2013). Online verfügbar, DOI: 10.1002/rnc.3066

[84] Gertler, J. J.: Survey of model-based failure detection and isolation in complex plants. In: IEEE Control Systems Magazine 8 (1988), Nr. 6, S. 3-11

[85] Gertler, J. J.: Modelling errors as unknown inputs. In: Proceedings of the IFAC SAFEPROCESS Symposium. Espoo, 1994, S. 266-271 
[86] Gertler, J. J.: Fault detection and isolation using parity relations. In: Control Engineering Practice 5 (1997), Nr. 5, S. 653-661

[87] Gertler, J. J.: Fault Detection and Diagnosis in Engineering Systems. Marcel Dekker, 1998

[88] Gertler, J. J. ; Kunwer, M. M.: Optimal residual decoupling for robust fault diagnosis. In: International Journal of Control 61 (1995), Nr. 2, S. 395-421

[89] Grant, M. ; Boyd, S. : Graph implementations for nonsmooth convex programs. In: Blondel, V. (Hrsg.) ; BoYd, S. (Hrsg.) ; KimurA, H. (Hrsg.): Recent Advances in Learning and Control. Springer, 2008 (Lecture Notes in Control and Information Sciences), S. 95-110

[90] Grant, M. ; Boyd, S. : CVX: Matlab Software for Disciplined Convex Programming, version 2.0 beta. Online verfügbar, Zugriff am 25.06.2013, http://cvxr.com/cvx, 2012

[91] Gumussoy, S. ; Henrion, D. ; Millstone, M. ; Overton, M. L.: Multiobjective Robust Control with HIFOO 2.0. In: Proceedings of the IFAC Symposium on Robust Control Design. Haifa, 2009, S. 144-149

[92] Hallouzi, R. ; Verdult, V. ; Babuska, R. ; Verhaegen, M. : Fault Detection and Identification of Actuator Faults using Linear Parameter Varying Models. In: Proceedings of the IFAC World Congress. Prag, 2005, S. 119-124

[93] Hansmann, M. : Entwurf, Inbetriebnahme und Untersuchung zur Steuerung und Regelung des Praktikumsversuches Verladebrücke. Studienarbeit, Technische Universität Darmstadt, 1996

[94] Harihara, P. P. ; Kim, K. ; Parlos, A. G.: Signal-based versus model-based fault diagnosis - a trade-off in complexity and performance. In: Proceedings of the IEEE International Symposium on Diagnostics for Electric Machines, Power Electronics and Drives. Stone Mountain, 2003, S. 277-282

[95] Hassibi, A. ; How, J. ; Boyd, S. : A Path-Following Method for Solving BMI Problems in Control. In: Proceedings of the American Control Conference. San Diego, 1999, S. 1385-1389 
[96] Haumann, D. ; Willert, V. ; Wahrburg, A. : Kalman Filtering in Mobile Consensus Networks. In: Proceedings of the IEEE MultiConference on Systems and Control. Dubrovnik, 2012, S. 944-950

[97] Herrera H., A. N. ; Lafay, J. F.: New results about Morgan's problem. In: IEEE Transactions on Automatic Control 38 (1993), Nr. 12, S. $1834-1838$

[98] HoagG, J. B. ; Bernstein, D. S.: Nonminimum-Phase Zeros. In: Control Systems Magazine 27 (2007), Nr. 3, S. 45-57

[99] Horayek, P. : User's Manual for CE 150 Helicopter Model. Humusoft Ltd., Bedienungsanleitung, 2003

[100] Hou, M. ; Müller, P. C.: Design of robust observers for fault isolation. In: Proceedings of the IFAC SAFEPROCESS Symposium. Baden-Baden, 1991, S. 295-300

[101] Hou, M. ; Müller, P. C.: Design of observers for linear systems with unknown inputs. In: IEEE Transactions on Automatic Control 37 (1992), Nr. 6, S. 871-875

[102] Hou, M. ; MülleR, P. C.: Fault detection and isolation observers. In: International Journal of Control 60 (1994), Nr. 5, S. 827-846

[103] Hsiao, T. ; Tomizuka, M. : Threshold selection for timely fault detection in feedback control systems. In: Proceedings of the American Control Conference. Portland, 2005

[104] Hui, S. ; ZaK, S. H.: Low-Order Unknown Input Observers. In: Proceedings of the American Control Conference. Portland, 2005, S. 4192-4197

[105] Ilchmann, A. ; Wirth, F. : On minimum phase. In: at - Automatisierungstechnik (2013). - akzeptiert

[106] Ingimundarson, A. ; Bravo, J. M. ; Puig, V. ; Alamo, T. ; GuerrA, P. : Robust fault detection using zonotope-based setmembership consistency test. In: International Journal of Adaptive Control and Signal Processing 23 (2009), Nr. 4, S. 311-330

[107] Isermann, R. : Fault diagnosis of machines via parameter estimation and knowledge processing. In: Automatica 42 (1993), Nr. 4, S. $815-835$ 
[108] Isermann, R. : Fault-Diagnosis Applications. Springer, 2011

[109] Isermann, R. ; Ballé: Trends in the Application of Model-Based Fault Detection and Diagnosis of Technical Processes. In: Control Engineering Practice 5 (1997), Nr. 5, S. 709-719

[110] Isermann, R. : Process fault detection based on modeling and estimation methods - A survey. In: Automatica 20 (1984), Nr. 4, S. $387-404$

[111] Isermann, R. : Fault-Diagnosis Systems: An Introduction from Fault Detection to Fault Tolerance. Springer, 2006

[112] Iwasaki, T. ; Hara, S. : Generalization of Kalman-YakubovichPopov Lemma for Restricted Frequency Inequalities. In: Proceedings of the American Control Conference. Denver, 2003, S. 3828-3833

[113] IwASAKI, T. ; HARA, S. : Generalized KYP lemma: unified frequency domain inequalities with design applications. In: IEEE Transactions on Automatic Control 50 (2005), Nr. 1, S. 41-59

[114] Jaimoukha, I. ; Li, Z. ; Mazars, E. : Fault isolation filter with linear matrix inequality solution to optimal decoupling. In: Proceedings of the American Control Conference. Minneapolis, 2006, S. 2339-2344

[115] Johansen, T. A. ; Fossen, T. I.: Control Allocation - A Survey. In: Automatica 49 (2013), Nr. 5, S. 1087-1103

[116] Johansson, A. ; Bask, M. ; Norlander, T. : Dynamic threshold generators for robust fault detection in linear systems with parameter uncertainty. In: Automatica 42 (2006), Nr. 7, S. 1095-1106

[117] Jones, H. L.: Failure Detection in Linear Systems, Massachusetts Institute of Technology, Diss., 1973

[118] Kaptipamula, S. ; Brembley, M. R.: Methods for Fault Detection, Diagnostics, and Prognostics for Building Systems - A Review, Part I. In: International Journal of Heating, Ventilating, AirConditioning, and Refrigerating Research 11 (2005), Nr. 1, S. 3-25

[119] Kefferpütz, K. : Regelungen für Systeme unter Stellgrößen- und Stellratenbeschränkungen, Technische Universität Darmstadt, Diss., 2012 
[120] Kempf, R. : Rekurrente Fuzzy-Systeme, Technische Universität Darmstadt, Diss., 2004

[121] KoČvara, M. ; Stingel, M. : PENBMI User's Guide (Version 2.0). Online verfügbar, Zugriff am 24.06.2013, http://www.penopt.com, 2005

[122] Konigorski, U. : Entwurf robuster strukturbeschränkter Zustandsregelungen durch Polgebietsvorgabe mittels Straffunktionen. In: at - Automatisierungstechnik 35 (1987), Nr. 6, S. 250-254

[123] Koussiouris, T. G.: A frequency domain approach to the block decoupling problem - II. Pole assignment while block decoupling a minimal system by state feedback and a constant non-singular input transformation and the observability of the block decoupled system. In: International Journal of Control 32 (1980), Nr. 3, S. 443-464

[124] Kudva, P. ; Viswanadham, N. ; Ramakrishna, A. : Observers for Linear Systems with Unknown Inputs. In: IEEE Transactions on Automatic Control 25 (1980), Nr. 1, S. 113-115

[125] Kugi, A. ; Schlacher, K. : Analyse und Synthese nnichtlineare dissipativer Systeme: Ein Überblick (Teil 2). In: at - Automatisierungstechnik 50 (2002), Nr. 3, S. 103-111

[126] Kwakernaak, H. : Robust Control and $\mathcal{H}_{\infty}$-Optimization - Tutorial Paper. In: Automatica 29 (1993), Nr. 2, S. 255-273

[127] Lens, H. ; AdAmy, J. : Weiche strukturvariable Regelungen mit Beobachtern für einen hydraulischen Aktor. In: at - Automatisierungstechnik 55 (2007), Nr. 8, S. 411-417

[128] LÖFBERG, J. : YALMIP : A toolbox for modeling and optimization in MATLAB. In: Proceedings of the IEEE International Symposium on Computer-Aided Control System Design. Taipei, 2004, S. 284-289

[129] Li, Z. ; Mazars, E. ; Zhang, Z. ; Jaimoukha, I. M.: State-space solution to the $\mathcal{H}_{-} / \mathcal{H}_{\infty}$ fault-detection problem. In: International Journal of Robust and Nonlinear Control 22 (2012), Nr. 3, S. 282299

[130] Li, Z. ; JAimoukhA, I. M.: Observer-based fault detection and isolation filter design for linear time-invariant systems. In: International Journal of Control 82 (2009), Nr. 1, S. 171-182 
[131] Listmann, K. D. ; Wahrburg, A. ; Strubel, J. : Synchronisierung heterogener linearer Systeme. In: 45. Regelungstechnisches Kolloquium. Boppard, 2011

[132] Listmann, K. D. ; Wahrburg, A. ; Strubel, J. ; Adamy, J. ; Konigorski, U. : Partial-State Synchronization of Linear Heterogeneous Multi-Agent Systems. In: Proceedings of the IEEE Conference on Decision and Control and European Control Conference. Orlando, 2011, S. 3440-3445

[133] LiU, B. ; Si, J. : Fault isolation filter design for linear time-invariant systems. In: IEEE Transactions on Automatic Control 42 (1997), Nr. 5, S. $704-707$

[134] Liu, G. P. ; Patton, R. J.: Parametric state feedback controller design of multivariable systems. In: International Journal of Control 61 (1995), Nr. 6, S. 1457-1464

[135] LiU, J. ; WANG, J. L. ; YANG, G.-H. : An LMI approach to minimum sensitivity analysis with application to fault detection. In: Automatica 41 (2005), Nr. 11, S. 1995-2004

[136] Lohmann, B. : Partial decoupling of non-minimum phase systems by constant feedback. In: Kybernetika 27 (1991), Nr. 5, S. 436-445

[137] Lohmann, B. : Vollständige Entkopplung durch dynamische Zustandsrückführung. In: at - Automatisierungstechnik 39 (1991), Nr. 9, S. 459-464

[138] Lohmann, B. : Vollständige und teilweise Führungsentkopplung dynamischer Systeme durch konstante Zustandsrückführung, Teil 1. In: at - Automatisierungstechnik 39 (1991), Nr. 9, S. 329-334

[139] Lohmann, B. : Vollständige und teilweise Führungsentkopplung dynamischer Systeme durch konstante Zustandsrückführung, Teil 2. In: at - Automatisierungstechnik 39 (1991), Nr. 9, S. 376-378

[140] Lohmann, B. : Vollständige und teilweise Führungsentkopplung im Zustandsraum, Universität Karlsruhe, Diss., 1991

[141] LOnG, Y. ; YANG, G.-H. : Fault detection and isolation for networked control systems with finite frequency specifications. In: International Journal of Robust and Nonlinear Control (2012). http://dx.doi.org/10.1002/rnc.2899. - DOI 10.1002/rnc.2899 
[142] Lou, X.-C. ; Willsky, A. S. ; Verghese, G. C.: Optimally Robust Redundancy Relations for Failure Detection in Uncertain Systems. In: Automatica 22 (1986), Nr. 3, S. 333-244

[143] Lunze, J. : Automatisierungstechnik. 3. Auflage. Oldenbourg, 2012

[144] Lunze, J. : Regelungstechnik 2. 3. Auflage. Springer, 2005

[145] MacFarlane, A. G. J. ; Karcanias, N. : Poles and zeros of linear multivariable systems: a survey of the algebraic, geometric and complex-variable theory. In: International Journal of Control 24 (1976), Nr. 1, S. 33-74

[146] Massoumnia, M.-A. ; Verghese, G. C. ; Willsky, A. S.: Failure detection and identification. In: IEEE Transactions on Automatic Control 34 (1989), Nr. 3, S. 316-321

[147] Massoumnia, M.-A. : A Geometric Approach to the Synthesis of Failure Detection Filters. In: IEEE Transactions on Automatic Control 31 (1986), Nr. 9, S. 839-846

[148] Mazars, E. ; Jaimoukha, I. M. ; Li, Z. : Computation of a Reference Model for Robust Fault Detection and Isolation Residual Residual Generation. In: Journal of Control Science and Engineering (2008). - DOI:10.1155/2008/790893

[149] Mazars, E. ; Jaimoukha, I. M. ; Li, Z. ; Zolotas, A. C.: Fault detection and isolation filter design for systems subject to polytopic uncertainties. In: Proceedings of the IEEE Mediterranean Conference on Control and Automation. Athen, 2007, S. 1-5

[150] Meyer, C. D.: Matrix Analysis and Applied Linear Algebra. SIAM, 2001

[151] Moness, M. ; Amin, M. : Minimal-order precompensators for decoupling linear multivariable systems $(\mathrm{A}, \mathrm{B}, \mathrm{C}, \mathrm{E})$. In: International Journal of Control 47 (1988), Nr. 6, S. 1925-1936

[152] Montes de Oca, S. ;Puig, V. ;Witczak, M. ;Dziekan, L. : FaultTolerant Control Strategy for Actuator Faults using LPV techniques: Application to a two degree of freedom helicopter. In: International Journal of Applied Mathematics and Computer Science 22 (2012), Nr. 1, S. 161-171 
[153] Montes De OcA, S. ; Puig, V. ; Blesa, J. : Robust fault detection based on adaptive threshold generation using interval LPV observers. In: International Journal of Adaptive Control and Signal Processing 26 (2012), Nr. 3, S. 258-283

[154] Moore, B. : On the flexibility offered by state feedback in multivariable systems beyond closed loop eigenvalue assignment. In: IEEE Transactions on Automatic Control 21 (1976), Nr. 5, S. 689-692

[155] Morgan, B. S.: The Synthesis of Linear Multivariable Systems by State-Variable Feedback. In: IEEE Transactions on Automatic Control 9 (1964), Nr. 10, S. 468-472

[156] Morse, A. ; Wonham, W. : Triangular decoupling of linear multivariable systems. In: IEEE Transactions on Automatic Control 15 (1970), Nr. 4, S. 447-449

[157] NG, K. Y. ; Tan, C. P. ; Oetomo, D. : Disturbance decoupled fault reconstruction using cascaded sliding mode observers. In: Automatica 48 (2012), Nr. 5, S. 794-799

[158] Nguyen, H. T. ; Su, S. W.: Conditions for triangular decoupling control. In: International Journal of Control 82 (2009), Nr. 9, S. $1575-1581$

[159] Niemann, H. : A Setup for Active Fault Diagnosis. In: IEEE Transactions on Automatic Control 51 (2006), Nr. 9, S. 1572-1578

[160] Noura, H. ; Theilliol, D. ; Ponsart, J.-C. ; Chamseddine, A. : Fault-tolerant Control Systems. Springer, 2009

[161] Oppenheim, A. V. ; Schafer, R. W.: Discrete-Time Signal Processing. 3. Auflage. Prentice-Hall, 2009

[162] ORSI, R. : LMIRank: software for rank constrained LMI problems. Online verfügbar, Zugriff am 24.06.2013, http://users. cecs. anu.edu.au/ robert/lmirank/, 2005

[163] Orsi, R. ; Helmke, U. ; Moore, J. B.: A Newton-like method for solving rank constrained linear matrix inequalities. In: Automatica 42 (2006), Nr. 11, S. 1875-1882 
[164] Ortseifen, A. : Entwurf von modellbasierten Anti-WindupMethoden für Systeme mit Stellbegrenzungen, Technische Universität Darmstadt, Diss., 2013

[165] Ostertag, E. : An Improved Path-Following Method for Mixed $\mathrm{H}_{2} / \mathrm{H}_{\infty}$ Controller Design. In: IEEE Transactions on Automatic Control 53 (2008), Nr. 8, S. 1967-1971

[166] Palade, V. ; Patton, R. J. ; Uppal, F. J. ; Quevedo, J. ; Daley, S. : Fault diagnosis of an industrial gas turbine using neuro-fuzzy methods. In: Proceedings of the IFAC World Congress. Barcelona, 2002, S. $2477-2482$

[167] Park, J. ; Rizzoni, G. : A Closed-Form Expression for the Fault Detection Filter. In: Proceedings of the IEEE Conference on Decision and Control. San Antonio, 1993, S. 259-264

[168] Park, J. ; Rizzoni, G. : An Eigenstructure Assignment Algorithm for the Design of Fault Detection Filters. In: IEEE Transactions on Automatic Control 39 (1994), Nr. 7, S. 1521-1524

[169] Park, J. ; Rizzoni, G. ; Ribbens, W. B.: On the Representation of Sensor Faults in Fault Detection Filters. In: Automatica 30 (1994), Nr. 11, S. 1793-1795

[170] Park, J.-K. ; Shin, D.-R. ; Chung, T. M.: Dynamic observers for linear time-invariant systems. In: Automatica 38 (2002), Nr. 6, S. $1083-1087$

[171] Pasqualetti, F. ; Dorfler, F. ; Bullo, F. : Attack Detection and Identification in Cyber-Physical Systems. In: IEEE Transactions on Automatic Control (2013). - akzeptiert

[172] Patton, R. J. ; Chen, J. : Robust fault detection of jet engine sensor systems using eigenstructure assignment. In: Journal of Guidance, Control, and Dynamics 15 (1992), Nr. 6, S. 1491-1497

[173] Patton, R. J. ; Chen, J. : Optimal unknown input distribution matrix selection in robust fault diagnosis. In: Automatica 29 (1993), Nr. 4, S. $837-841$

[174] Patton, R. J. ; Chen, J. : Observer-based fault detection and isolation: Robustness and applications. In: Control Engineering Practice 5 (1997), Nr. 5, S. 671-682 
[175] Patton, R. J. ; Chen, J. : On eigenstructure assignment for robust fault diagnosis. In: International Journal of Robust and Nonlinear Control 10 (2000), Nr. 14, S. 1193-1208

[176] Pipeleers, G. ; Vandenberghe, L. : Generalized KYP Lemma With Real Data. In: IEEE Transactions on Automatic Control 56 (2011), Nr. 12, S. 2942-2946

[177] Raimondo, D. M. ; Braatz, R. D. ; Scott, J. K.: Active Fault Diagnosis Using Moving Horizon Input Design. In: Proceedings of the European Control Conference. Zürich, 2013. - akzeptiert

[178] Rank, M. L. ; Niemann, H. : Norm based design of fault detectors. In: International Journal of Control 72 (1999), Nr. 9, S. 773-783

[179] Rantzer, A. : On the Kalman-Yakubovich-Popov lemma. In: Systems $\&$ Control Letters 28 (1996), Nr. 1, S. 7-10

[180] Rauw, M. O.: FDC 1.2 - A simulink Toolbox for Flight Dynamics and Control Analysis. 1997. - Online verfügbar unter www.dutchroll.com, Zugriff am 13.03.2013

[181] Roppenecker, G. : Minimum norm output feedback design under specified eigenvalue areas. In: Systems $\&$ Control Letters 3 (1983), Nr. 2, S. 101-103

[182] Roppenecker, G. : Vollständige Modale Synthese linearer Systeme und ihre Anwendung zum Entwurf strukturbeschränkter Zustandsrückführungen, Universität Karlsruhe, Diss., 1983

[183] Roppenecker, G. : Modal Approach to Parametric State Observer Design. In: Electronics Letters 22 (1986), Nr. 3, S. 118-120

[184] Roppenecker, G. : On Parametric State Feedback Design. In: International Journal of Control 43 (1986), Nr. 3, S. 793-804

[185] Roppenecker, G. ; Lohmann, B. : Vollständige Modale Synthese von Entkopplungsregelungen. In: at - Automatisierungstechnik 36 (1988), Nr. 11, S. 434-441

[186] Roppenecker, G. ; Preuss, H.-P. : Nullstellen und Pole linearer Mehrgrößensysteme - Teil 1: Grundlagen und Definitionen. In: Regelungstechnik und Prozeß-Datenverarbeitung 30 (1982), Nr. 7, S. 219-225 
[187] Roppenecker, G. ; Preuss, H.-P. : Nullstellen und Pole linearer Mehrgrößensysteme - Teil 2: Zusammenhänge, Eigenschaften und numerische Berechnung. In: Regelungstechnik und ProzeßDatenverarbeitung 30 (1982), Nr. 8, S. 255-263

[188] Rosa, P. ; Casau, P. ; Silvestre, C. ; Tabatabaeipour, S. M. ; Stoustrup, J. : A Set-Valued Approach to FDI and FTC: Theory and Implementation Issues. In: Proceedings of the IFAC SAFEPROCESS Symposium. Mexico City, 2012, S. 1281-1286

[189] Rosa, P. ; Silvestre, C. ; Shamma, J. ; Athans, M. : Fault Detection and Isolation of LTV systems using Set-Valued Observers. In: Proceedings of the IEEE Conference on Decision and Control. Atlanta, 2010, S. 768-773

[190] Rosenbrock, H. H.: The zeros of a system. In: International Journal of Control 18 (1973), Nr. 2, S. 297-299

[191] Rosenbrock, H. H.: Correction to "The zeros of a system". In: International Journal of Control 20 (1974), Nr. 3, S. 525-527

[192] Scherer, C. ; Weiland, S. : Linear Matrix Inequalities in Control. Vorlesungsskriptum, 2005. - Delft University of Technology

[193] Scherer, C. W.: Lineare Matrixungleichungen in der Theorie der robusten Regelungen. In: at - Automatisierungstechnik 45 (1997), Nr. 7, S. $306-318$

[194] Scherer, C. ; Gahinet, P. ; Chilali, M. : Multiobjective OutputFeedback Control via LMI Optimization. In: IEEE Transactions on Automatic Control 42 (1997), Nr. 7, S. 896-911

[195] Schmid, W. ; ZeItz, M. : Grenzübergang Beobachter - Differenzierer. In: Regelungstechnik und Prozeß-Datenverarbeitung 29 (1981), Nr. 8, S. 270-274

[196] Schrader, C. B. ; Sain, M. K.: Research on system zeros: a survey. In: International Journal of Control 50 (1989), Nr. 4, S. 1407-1433

[197] Schweppe, F. : Uncertain Dynamic Systems. Prentice-Hall, 1973

[198] Schwung, A. : Modellbildung und Fehlerdiagnose mit rekurrenten Fuzzy-Systemen, Technische Universität Darmstadt, Diss., 2011 
[199] Schwung, A. ; Ortseifen, A. : Anwendung zeitdiskreter rekurrenter Fuzzy-Systeme zur Fehlerdiagnose. In: at - Automatisierungstechnik 58 (2010), Nr. 6, S. 322-331

[200] SeBe, N. : A New Dilated LMI Characterization and Iterative Control System Synthesis. In: Proceedings of the IFAC Symposium on Large Scale Systems: Theory and Applications. Gdansk, 2007

[201] Shafai, B. ; Carroll, R. L.: Design of Proportional-Integral Observer for Linear Time-Varying Multivariable Systems. In: Proceedings of the IEEE Conference on Decision and Control. Fort Lauderdale, 1985, S. 597-599

[202] Shafai, B. ; Pi, C. T. ; Nork, S. : Simultaneous Disturbance Attenuation and Fault Detection using Proportional Integral Observers. In: Proceedings of the American Control Conference. Anchorage, 2002, S. 1647-1649

[203] Shamma, J. S. ; Tu, K.-Y. : Set-Valued Observers and Optimal Disturbance Rejection. In: IEEE Transactions on Automatic Control 44 (1999), Nr. 2, S. 253-264

[204] Simani, S. ; Fantuzzi, C. ; Patton, R. J.: Model-based Fault Diagnosis in Dynamic Systems Using Identification Techniques. Springer, 2003

[205] Skelton, R. ; Iwasaki, T. ; Goriadis, K. : A Unified Algebraic Approach to Linear Control Design. Taylor and Francis, 1998

[206] Skogestad, S. ; Postlethwaite, I. : Multivariable Feedback Control: Analysis \& Design. 2. Auflage. Wiley, 2005

[207] Smagina, Y. M.: System zeros. In: ArXiv Mathematics e-prints (2006). - arXiv:math/0605092

[208] Stefanovski, J. : Sufficient conditions for linear control system decoupling by static state feedback. In: IEEE Transactions on Automatic Control 46 (2001), Nr. 6, S. 984-990

[209] Stone, R. B. ; Turner, I. Y. ; Wie, M. van: The Function-Failure Design Method. In: Journal of Mechanical Design 127 (2004), Nr. 3, S. 397-407 
[210] Sturm, J. F.: Using SeDuMi 1.02, a MATLAB toolbox for optimization over symmetric cones. In: Optimization Methods and Software 11 (1999), Nr. 1-4, S. 625-653

[211] SvariceK, F. : Zur numerischen Berechnung der Nullstellen von Zustandsraummodellen mit Matlab. In: GMA-Fachausschuss 1.40 "Theoretische Verfahren der Regelungstechnik", 2012

[212] SvariceK, F. : Zuverlässige numerische Analyse linearer Regelungssysteme. Vieweg \& Teubner, 1995

[213] Tan, C. P. ; Edwards, C. : Robust Fault Reconstruction in Uncertain Linear Systems Using Multiple Sliding Mode Observers in Cascade. In: IEEE Transactions on Automatic Control 55 (2010), Nr. 4, S. $855-867$

[214] TAn, C. P. ; Edwards, C. : Sliding mode observers for detection and reconstruction of sensor faults. In: Automatica 38 (2002), Nr. 10, S. $1815-1821$

[215] Tan, C. P. ; Edwards, C. : Sliding mode observers for robust detection and reconstruction of actuator and sensor faults. In: International Journal of Robust and Nonlinear Control 13 (2003), Nr. 5, S. 443-463

[216] Toh, K. C. ; Todd, M. J. ; TÜтüncü, R. H.: SDPT3 - A Matlab software package for semidefinite programming. In: Optimization Methods and Software 11 (1999), S. 545-581

[217] VanAntwerp, J. G. ; BraAtz, R. D.: A tutorial on linear and bilinear matrix inequalities. In: Journal of Process Control 10 (2000), Nr. 4, S. $363-385$

[218] Varga, A. : The nullspace method - a unifying paradigm to fault detection. In: Proceedings of the IEEE Conference on Decision and Control. Shanghai, 2009, S. 6964-6969

[219] Venkatasubramanian, V. ; Rengaswamy, R. ; Yin, K. ; Kavuri, S. N.: A review of process fault detection and diagnosis - Part I: Quantitative model-based methods. In: Computers and Chemical Engineering 27 (2003), Nr. 3, S. 293-311 
[220] Venkatasubramanian, V. ; Rengaswamy, R. ; Yin, K. ; KavuRI, S. N.: A review of process fault detection and diagnosis - Part II: Qualitative models and search strategies. In: Computers and Chemical Engineering 27 (2003), Nr. 3, S. 313-326

[221] Venkatasubramanian, V. ; Rengaswamy, R. ; Yin, K. ; KavuRI, S. N.: A review of process fault detection and diagnosis - Part III: Process history based methods. In: Computers and Chemical Engineering 27 (2003), Nr. 3, S. 327-346

[222] Viswanadham, N. ; Taylor, J. H. ; Luce, E. C.: A frequencydomain approach to failure detection and isolation with application to GE-21 turbine engine control systems. In: Control-Theory and Advanced Technology 3 (1987), Nr. 1, S. 45-72

[223] Voigt, C. ; Adamy, J. : Formelsammlung der Matrizenrechnung. Oldenbourg, 2007

[224] WahrBurg, A. : Design of robust fault isolation observers for linear systems with bounded uncertain parameters. In: International Journal of Adaptive Control and Signal Processing (2013). - eingereicht

[225] Wahrburg, A. ; AdAmy, J. : LMI-based design of robust fault isolation filters for linear systems. In: Proceedings of the European Control Conference. Zürich, 2013, S. 943-949

[226] Wahrburg, A. ; Adamy, J. : LMI-basierter Entwurf allgemeiner dynamischer Filter zur robusten Fehlerisolation. In: 4\%. Regelungstechnisches Kolloquium. Boppard, 2013

[227] Wahrburg, A. ; AdAmy, J. : On robust fault-isolation observers with relaxed structural constraints. In: Proceedings of the IEEE Mediterranean Conference on Control and Automation. PlataniasChania, 2013, S. 407-412

[228] Wahrburg, A. ; Adamy, J. : Parametric design of robust fault isolation observers for linear non-square systems. In: Systems $\&$ Control Letters 62 (2013), Nr. 5, S. 420-429

[229] Wahrburg, A. ; AdAmy, J. : Robust partial fault isolation for linear systems using observers. In: Proceedings of the American Control Conference. Washington, 2013, S. 2796-2802 
[230] Wahrburg, A. ; Adamy, J. : Entwurf von Fehlerisolationsbeobachtern für lineare Systeme mit unsicheren Parametern. In: at Automatisierungstechnik 62 (2014), Nr. 1, S. 21-33

[231] Wahrburg, A. ; Haumann, D. ; Willert, V. : Minimum-Variance Fault Isolation Observers for Discrete-Time Linear Systems. In: Proceedings of the IEEE Conference on Decision and Control. Florenz, 2013, S. 5643-5649

[232] Wahrburg, A. ; Adamy, J. : Anwendung dynamisch erweiterter Beobachter zur robusten Fehlerisolation. In: GMA-Fachausschuss 1.40"Theoretische Verfahren der Regelungstechnik". Salzburg, 2011

[233] Wahrburg, A. ; Adamy, J. : Observer-Based Synchronization of Heterogeneous Multi-Agent Systems by Homogenization. In: Proceedings of the IEEE Australian Control Conference. Melbourne, 2011, S. 386-391

[234] Wahrburg, A. ; Adamy, J. : Anwendung dynamisch erweiterter Beobachter zur robusten Fehlerisolation. In: at - Automatisierungstechnik 60 (2012), Nr. 8, S. 499-508

[235] Wahrburg, A. ; AdAmy, J. : Beobachterbasierte Fehlerisolation für nicht-quadratische Systeme mit unsicheren Parametern. In: GMAFachausschuss 1.40 "Theoretische Verfahren der Regelungstechnik". Salzburg, 2012

[236] Wahrburg, A. ; AdAmy, J. : Fault isolation for linear non-minimum phase systems using dynamically extended observers. In: Proceedings of the IEEE Conference on Decision and Control. Maui, 2012, S. $1226-1232$

[237] Wahrburg, A. ; Adamy, J. : Observer-based fault isolation for statically non-isolable linear systems. In: Proceedings of the IEEE MultiConference on Systems and Control. Dubrovnik, 2012, S. 1207-1213

[238] Wahrburg, A. ; Adamy, J. : Robust fault isolation observers for non-square systems - a parametric approach. In: Proceedings of the IFAC SAFEPROCESS Symposium. Mexico City, 2012, S. 1275-1280

[239] Wahrburg, A. ; Adamy, J. : Robust Fault Isolation using dynamically extended Observers. In: Proceedings of the IEEE MultiConference on Systems and Control. Dubrovnik, 2012, S. 1201-1206 
[240] Wahrburg, A. ; AdAmy, J. : Entkopplungsregelungen für lineare überaktuierte Systeme. In: at - Automatisierungstechnik 61 (2013), Nr. 1 , S. $28-38$

[241] Wahrburg, A. ; Khodaverdian, S. ; AdAmy, J. : Robust observerbased fault detection and isolation in the standard control problem framework. In: Proceedings of the IFAC Symposium on Robust Control Design. Aalborg, 2012, S. 467-472

[242] Wahrburg, A. ; Listmann, K. D. ; Adamy, J. : Master-Slave Synchronization for Nonlinear Systems based on Reduced Observers. In: Proceedings of the IFAC World Congress. Mailand, 2011, S. 1099410999

[243] Wang, H. ; YAnG, G.-H. : Fault Detection Observer Design in Low Frequency Domain. In: Proceedings of the IEEE Multi-Conference on Systems and Control. Singapur, 2007, S. 976-981

[244] Wang, H. ; YAng, G.-H. : Fault Estimations for Uncertain Linear Discrete-time Systems in Low Frequency Domain. In: Proceedings of the American Control Conference. New York City, 2007, S. 11241129

[245] Wang, H. ; YAnG, G.-H. : A finite frequency approach to filter design for uncertain discrete-time systems. In: International Journal of Adaptive Control and Signal Processing 22 (2008), Nr. 6, S. 533550

[246] Wang, H. ; Yang, G.-H. : A Finite Frequency Domain Approach to Fault Detection Observer Design for Linear Continuous-Time Systems. In: Asian Journal of Control 10 (2008), Nr. 5, S. 1-10

[247] Wang, J. L. ; YAnG, G.-H. ; LiU, J. : An LMI approach to $\mathcal{H}_{-}$index and mixed $\mathcal{H}_{-} / \mathcal{H}_{\infty}$ fault detection observer design. In: Automatica 43 (2007), Nr. 9, S. 1656-1665

[248] Wang, Y. ; MA, G. ; Ding, S. X. ; Li, C. : Subspace aided datadriven design of robust fault detection and isolation systems. In: Automatica 47 (2011), Nr. 11, S. 2474-2480

[249] Wang, Z. ; Schittenhelm, R. S. ; Borsdorf, M. ; RinderKNECHT, S. : Application of Augmented Observer for Fault Diagnosis in Rotor Systems. In: Engineering Letters 21 (2013), Nr. 1, S. 10-17 
[250] Wang, Z. ; Wahrburg, A. ; Rinderknecht, S. : Consideration of gyroscopic effect in fault detection and isolation for unbalance excited rotor systems. In: International Journal of Rotating Machinery (2012). - DOI 10.1155/2012/640794

[251] Watanabe, K. ; Himmelblau, D. M.: Instrument fault detection in systems with uncertainties. In: International Journal of Systems Science 13 (1982), Nr. 2, S. 137-158

[252] WeI, M. ; SHEN, D. : Pole assignment in the regular row-by-row decoupling problem. In: Automatica 49 (2013), Nr. 1, S. 349-359

[253] Wei, M. ; Wang, Q. ; Cheng, X. : Some new results for system decoupling and pole assignment problems. In: Automatica 46 (2010), Nr. 5, S. 937-944

[254] Wei, X. ; Verhaegen, M. : Robust fault detection observer design for linear uncertain systems. In: International Journal of Control 84 (2011), Nr. 1, S. 197-215

[255] White, J. E. ; Speyer, J. L.: Detection Filter Design: Spectral Theory and Algorithms. In: IEEE Transactions on Automatic Control 32 (1987), Nr. 7, S. 593-603

[256] Widodo, A. ; YAng, B.-S. : Support vector machine in machine condition monitoring and fault diagnosis. In: Mechanical Systems and Signal Processing 21 (2007), Nr. 6, S. 2560-2574

[257] Willsky, A. S.: A survey of design methods for failure detection in dynamic systems. In: Automatica 12 (1976), Nr. 6, S. 601-611

[258] Witsenhausen, H. : Sets of possible states of linear systems given perturbed observations. In: IEEE Transactions on Automatic Control 13 (1968), Nr. 5, S. 556-558

[259] Wünnenberg, J. : Observer-based Fault Detection in Dynamic Systems, Universität Duisburg, Diss., 1990

[260] Wünnenberg, J. ; Frank, P. M.: Sensor Fault Detection via Robust Observers. In: Tzafestas, S. (Hrsg.) ; Singh, M. (Hrsg.) ; Schmidt, G. (Hrsg.): System Fault Diagnostics, Reliability and Related Knowledge-Based Approaches. Springer, 1987, S. 147-160 
[261] Wünnenberg, J. ; Frank, P. M.: Model-based residual generation for dynamic systems with unknown inputs. In: Proceedings of the IMACS World Congress on Scientific Computation. Paris, 1988, S. $435-437$

[262] Wojciechowski, B. : Analysis and synthesis of proportionalintegral observers for single-input single-output time-invariant continuous systems, Politechnika Ślaska, Diss., 1978

[263] Wolkowicz, H. (Hrsg.) ; Saigal, R. (Hrsg.) ; Vandenberghe, L. (Hrsg.): Handbook of Semidefinite Programming - Theory, Algorithms, and Applications. Springer, 2000

[264] Wolovich, W. : On determining the zeros of state-space systems. In: IEEE Transactions on Automatic Control 18 (1973), Nr. 5, S. $542-544$

[265] Wolovich, W. A.: Linear Multivariable Systems. Springer, 1974

[266] Wonham, W. ; Morse, A. : Decoupling and Pole Assignment in Linear Multivariable Systems: A Geometric Approach. In: SIAM Journal on Control 8 (1970), Nr. 1, S. 1-18

[267] YAN, X.-G. ; EDWARDS, C. : Robust decentralized actuator fault detection and estimation for large-scale systems using a sliding mode observer. In: International Journal of Control 81 (2008), Nr. 4, S. 591-606

[268] Yang, F. ; Wilde, R. W.: Observers for Linear Systems with Unknown Inputs. In: IEEE Transactions on Automatic Control 33 (1988), Nr. 7, S. 677-681

[269] YAng, H. ; XiA, Y. ; LiU, B. : Fault Detection for T-S Fuzzy Discrete Systems in Finite-Frequency Domain. In: IEEE Transactions on Systems, Man, and Cybernetics 41 (2011), Nr. 4, S. 911-920

[270] Zaccarian, L. : Dynamic allocation for input redundant control systems. In: Automatica 45 (2009), Nr. 6, S. 1431-1438

[271] Zhong, M. ; Ding, S. X. ; LAm, J. ; Wang, H. : An LMI approach to design robust fault detection filter for uncertain LTI systems. In: Automatica 39 (2003), Nr. 3, S. 543-550 
[272] Zhou, K. ; Khargonekar, P. P.: An algebraic Riccati equation approach to $\mathcal{H}_{\infty}$ optimization. In: Systems \& Control Letters 11 (1988), Nr. 2, S. 85-91 



\section{Lebenslauf}

\begin{tabular}{|c|c|}
\hline \multicolumn{2}{|l|}{ Persönliche Daten } \\
\hline Name & Arne Wahrburg \\
\hline Anschrift & Caroline-Herschel-Straße 18 \\
\hline & 64293 Darmstadt \\
\hline Geburtsdatum/-ort & 02.03.1985 in Lüdenscheid \\
\hline \multicolumn{2}{|l|}{ Werdegang } \\
\hline seit Oktober 2013 & $\begin{array}{l}\text { Forschungsingenieur am deutschen Forschungs- } \\
\text { zentrum der ABB AG }\end{array}$ \\
\hline $2010-2013$ & $\begin{array}{l}\text { Wissenschaftlicher Mitarbeiter am Institut für } \\
\text { Automatisierungstechnik und Mechatronik, FG } \\
\text { Regelungstheorie und Robotik an der TU Darm- } \\
\text { stadt }\end{array}$ \\
\hline $2011-2013$ & $\begin{array}{l}\text { Promotionsstipendium der Deutsche Telekom } \\
\text { Stiftung }\end{array}$ \\
\hline $2004-2010$ & $\begin{array}{l}\text { Studium der Elektrotechnik (Vertiefung Mecha- } \\
\text { tronik) an der TU Darmstadt, Abschluss: Dipl.- } \\
\text { Ing. }\end{array}$ \\
\hline $2003-2004$ & $\begin{array}{l}\text { Freiwilliges Jahrespraktikum im Seniorenzen- } \\
\text { trum Bethanien, Halver }\end{array}$ \\
\hline 2003 & $\begin{array}{l}\text { Abitur, Evangelisches Gymnasium Meinerzha- } \\
\text { gen }\end{array}$ \\
\hline
\end{tabular}

